
政

a

$-$$$
\text { . }
$$ 
Digitized by the Internet Archive in 2007 with funding from Microsoft Corporation 


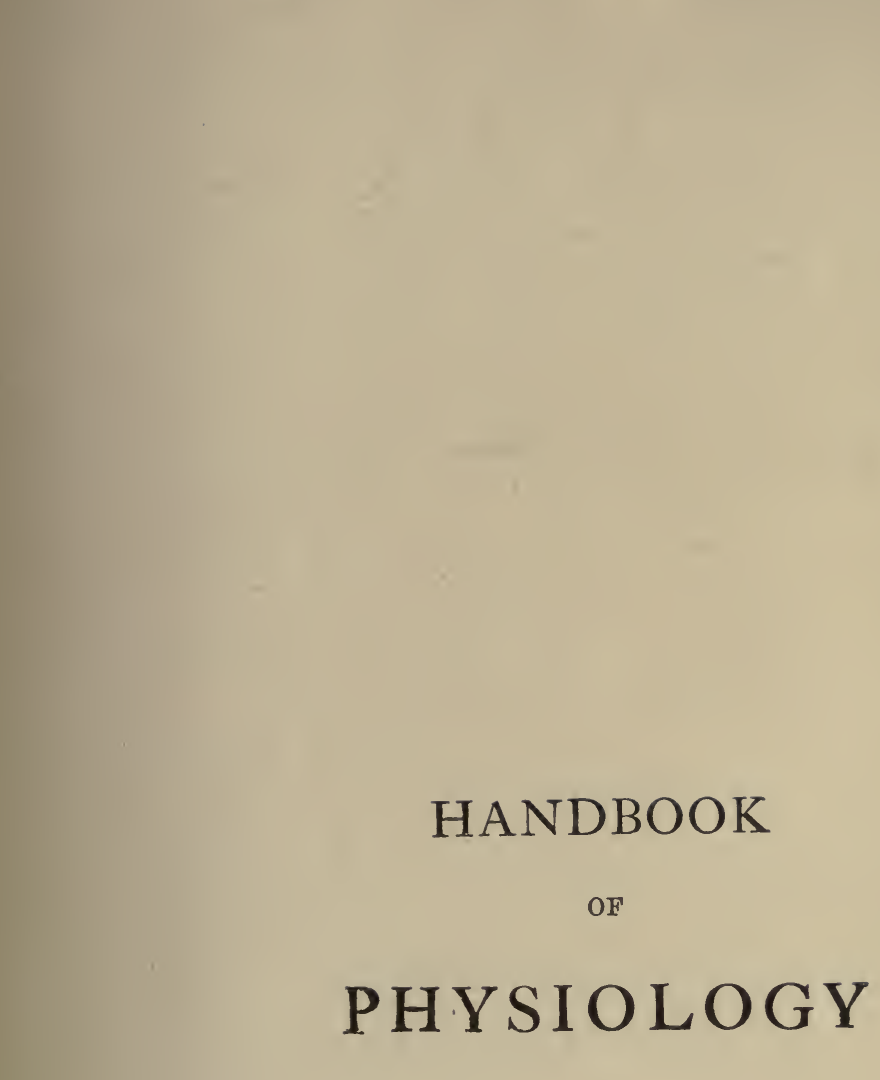

HANDBOOK
PHYSIOLOGY

HANDBOOK
PHYSIOLOGY

PHYSIOLOGY

PHYsiology (n) .

PHYSIOLOGY 


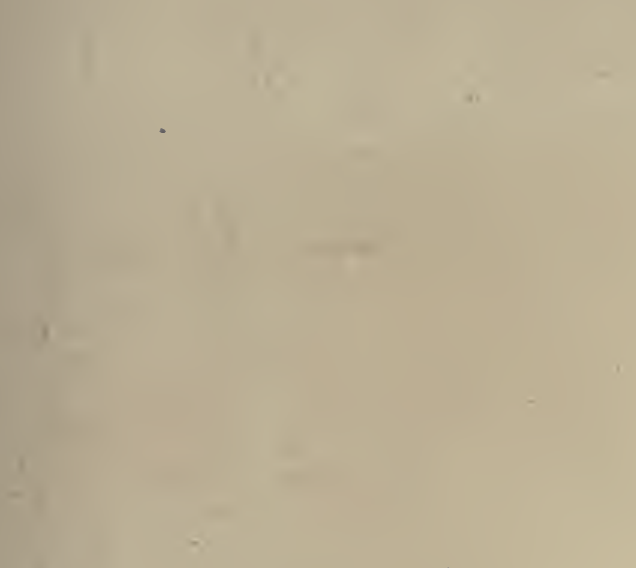
BLDOD-SPECTRA CIMPARED WITH SPECTRUM DF ARGAND - LAMP.

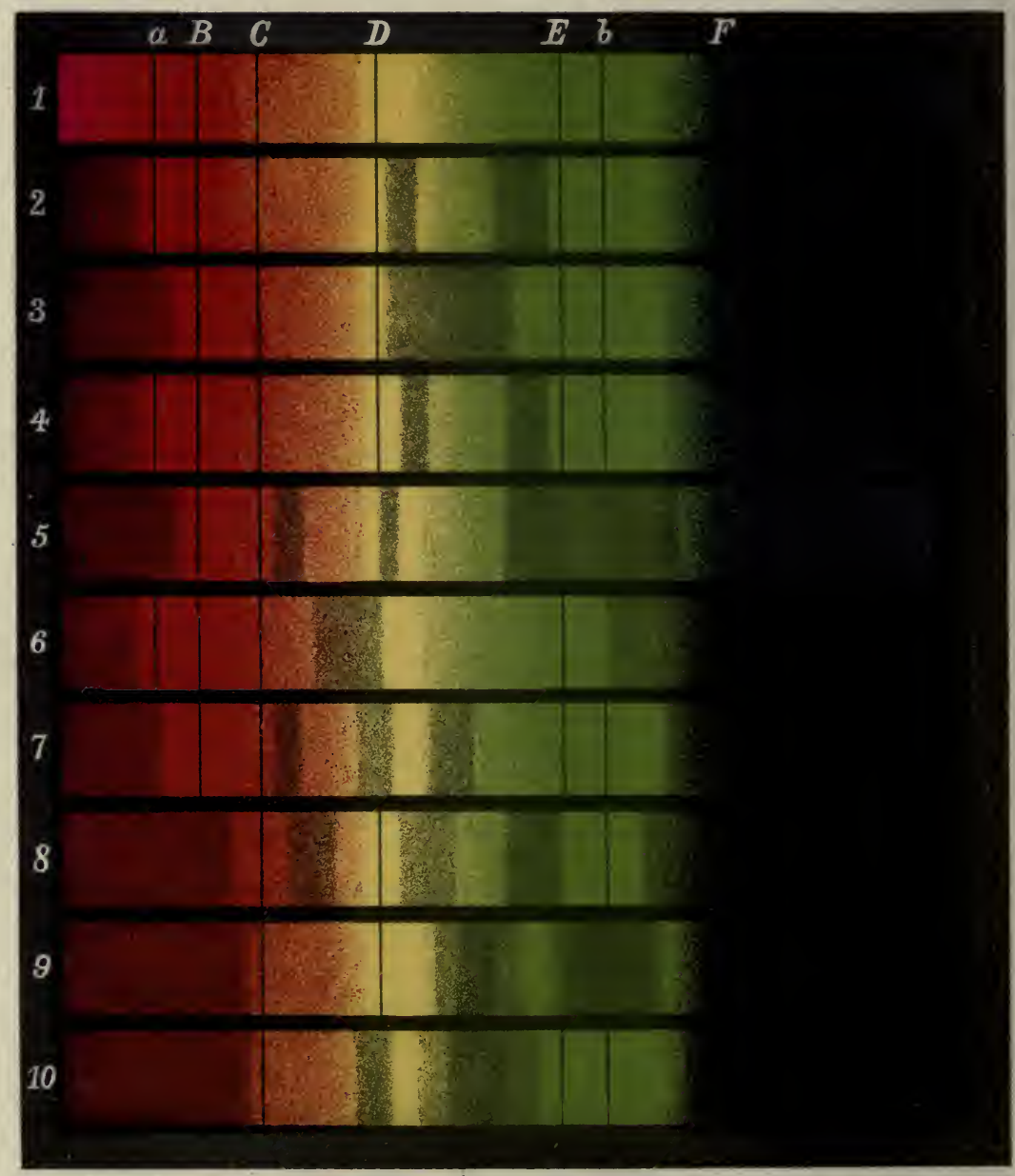

I Spectrum of Aroand-Iamp with Fraunhofers lines in position.

2 spectrum af Dxyhamadabin in diluted blaod.

35 pectrum of Reduced $\mathrm{H}$ 巴maglabin.

4 Spectrum of Carbanic axide Hamodabio.

5 spectrum of Acid Hzmatin in etherial solution.

6 5pectrum of Alkaline $\mathrm{Hzm}$ atin.

7 spectrum of Cbloraform extract of acidulated Dx-Bile.

8 Spectrum of Methmmoglabin.

9 spectrum of Hamachramaḑen.

10 spectrum of Hametoparphyrin.

.Yost of the above Spectra have been drown from observations by MP W.Lepraik F.C.S: 


\title{
KIRKES' HANDBOOK
}

\section{$\mathrm{OF}$ \\ PHYSIOLOGY}

Revised and Rewritten by

CHARLES WILSON GREENE, A. M., Ph. D.

PROFESSOR OF PHYSIOLOGY AND PHARMACOLOGY, UNIVERSITY OF MISSOURI

Tentb Fmerican Revision

WITH FIVE HUNDRED AND TWENTY-FOUR ILLUSTRATIONS, INCLUDING MANY IN COLORS

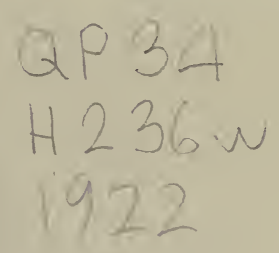

\author{
NEW YORK \\ WILLIAM WOOD AND COMPANY \\ MDCCCCXXII
}





\section{PREFACE}

In this the tenth American edition of the Handbook of Physiology revisions and amplifications have been made throughout the entire text. New subject matter, new illustrations, and the more recent refinements of methods have been freely incorporated. This has unavoidably increased the size of the volume. The chapters on Circulation, Respiration, Internal Secretion, Metabolism and the Autonomic division of the Nervous System in particular have been entirely rewritten and reillustrated.

The chapter on the Circulation has been made to include the newer researches on the development of cardiac physiology as regards rhythm production, the control and finer adjustments of rate, and the conduction phenomena that determine sequence. These factors, represented in the highly differentiated bundle branch system, are given new emphasis and new illustration. Physiological interest in Respiration has been intensified by the practical problems of air navigation and by the newer investigations in the field of oxygen supply and oxygen control in relation to the daily physiological round in both health and disease. The result has been a new impetus to respiratory physiology contributed to by numerous writers of the last decade. The works of Barcroft, Henderson, Schneider, Greene and Gilbert and numerous others have been drawn on from this field.

The science of nutrition has made rapid advances. That subject has been revised to call attention to the very fundamental work of Osborne and Mendel on food factors necessary for growth; of Funk, Voegtlin and others on the vitamines and nutritional diseases; of Lusk and DuBois in the field of basal metabolism; of Van Slyke, Stadie, Harrop, and others on blood gases, and of Banting, Best and Macleod on glycemia and the hormone of the pancreas controlling sugar metabolism.

The giant strides of the science of physiology make it difficult for a textbook to keep pace with the literature, but it is hoped that the newest facts and principles have been incorporated in so far as the limitations of the available space permit. Many of the illustrative laboratory experiments have been again rewritten, and improvements simplifying the experimental technique have been incorporated. It is felt that the student in Physiology gains the greatest strength in laboratory experience when the tests he executes are chosen from the standpoint of the efficiency of the entire work. In this field, under the present day conditions, the deter- 
mining pedagogical horizon includes not only the subject matter of Physiology, but of Physics and Chemistry on which Physiology rests, as well as Clinical Medicine and Surgery for which Physiology furnishes the foundation.

For valuable aids and criticisms in this and recent editions I again acknowledge grateful indebtedness to Professor Robert Banks Gibson, University of Iowa, Dr. Carl Hartley Greene, Mayo Foundation, to my colleague, Professor Addison Gulick, and to my students and assistants of former years, Professor Theodore K. Kruse of the University of Pittsburgh, and Professor Erwin Ellis Nelson of the University of Michigan.

Columbia, Mo.,

Chas. W. Greene.

Sept. I, I922. 


\section{CONTENTS}

ChaPter I-The Phenomena of Life; Properties of Protoplasm, Structure of Protoplasm. . . . . . . . . . . . I

CHAPTER II-Cell Differentiation and the Structure of the Elementary Tissues; The Structure of the Cell, The Structure of the Elementary Tissues. The Epithelial Tissues. The Connective Tissues. Muscular Tissue. Nervous Tissue.

Chapter III-The Chemical Composition of the Body; The Nitrogenous Substances, The Proteins, Classification of the Proteins, Characteristics of the Proteins, The Fats, The Carbohydrates; Inorganic Substances of the Body, Laboratory Experiments.

CHAPTER IV-THE BLOoD; Quantity of the Blood, Coagulation of the Blood, Morphology of the Blood, Chemical Composition of the Blood, Globulocidal and Other Properties of Serum, The Character and Composition of Lymph, Laboratory Experiments

Chapter V-The Circulation of the Blood; Anatomical Considerations, The Action of the Heart, The Regulative Influence of the Central Nervous System, The Circulation through the Bloodvessels, The Pulse, The Peripheral Regulation of the Flow of Blood, Vaso-constrictor and Vaso-dilator Nerves for Individual Organs, Laboratory Experiments . . . . . . . . . . . .

CHAPTER VI-Respiration; The Respiratory Apparatus, The Movements of the Respiratory Mechanism, Respiratory Changes in the Air Breathed, The Respiratory Changes in the Blood, The Nervous Regulation of the Respiratory Apparatus, The Effect of Respiration on the Circulation, Laboratory Experiments in Respiration. . . . . . . . . . . . .

CHAPTER VII-Secretion in General; Organs and Tissues of Secretion, Secreting Glands, The Process of Secretion, Influence of the Nervous System on Secretion. 
CHAPTER VIII-Food AND Digestion; Food and Food Principles, The Process of Digestion, Digestion in the Mouth, Deglutition, Nervous Mechanism of Deglutition, Digestion in the Stomach, Movements of the Stomach, Digestion in the Intestines, Movements of the Intestines, Laboratory Experiments in Digestion, Saliva and Salivary Digestion, Gastric Juice and Gastric Digestion, Pancreatic Juice and Pancreatic Digestion.

CHAPTER IX-ABsorption; Absorption in the Stomach, Absorption in the Intestines, Absorption from the Skin, the Lungs, etc.

CHAPTER X-Excretion; Structure and Function of the Kidneys, General Structure, The Urine, The Method of Excretion of Urine, The Discharge of the Urine, The Structure and Excretory Functions of the Skin, Laboratory Experiments in Excretion

CHAPTER XI-Metabolism, Nutrition, and Diet; Metabolism of Proteids, The Metabolism of Fats, the Metabolism of Carbohydrates, Influence of Minerals, Fasting, Requisites of a Normal Diet, The Vitamines, The Influence of the Ductless Glands on Metabolism

CHAPTER XII-Animal Heat; Heat-producing Organs, Variation in the Loss of Heat, Variation in the Production of Heat, Influence of the Nervous System on Heat Production

CHAPTER XIII-Muscle-nerve Physiology; Chemical Composition of Muscle, The Properties of Living Muscle, Single Muscle Contractions, Conditions which Affect the Irritability of the Muscle and the Character of the Contraction, Tetanic and Voluntary Muscular Contractions, The Type of Contraction in Involuntary Muscle and in Cilia, The Function of Nerve Fiber, Some Special Coordinated Motor Activities, Locomotion, The Production of the Voice, Laboratory Experiments on Muscle and Nerves. 5 Io

CHAPTER XIV-The Nervous System; Function of the Nerve Cell; Specific Energy of the Nerve Impulse, Structure and the Function of the Spinal Cord, Tracts of the Cord, The Functions of the Cord. The Brain, The Medulla Oblongata and Pons, Structure, Functions of the Medulla, The Cerebellum, The Midbrain, The Peduncles of the Cerebrum, Corpora Quadrigemina, Corpora Geniculata, Corpora Striata, The Cerebrum, Structure of the Cortex, General Function of the Cerebrum, Localization of the 
Motor Function, Localization of Sensory Function, Association Centers, The Cranial Nerves, The Sympathetic or Autonomic System, The Physiology of Sleep, Laboratory Experiments on the Nervous System.

CHAPTER XV-The Senses; I. The Senses of Touch, Pain, Temperature, and the Muscle Sense. II. Taste and Smell, The Sense of Taste, The Sense of Smell. III. Hearing and Equilibration, The Anatomy of the Ear, The Physiology of Hearing, The Sense of Equilibrium. IV. The Sense of Sight, The Eye, The Optical Apparatus, Accommodation, Defects in the Optical Apparatus, Visual Sensations from Excitation of the Retina, Color Sensations, Binocular Vision, Visual Judgments, Laboratory Directions for Experiments on the Sense Organs.

CHAPTER XVI-The Reproductive Organs; The Reproductive Organs of the Male, The Reproductive Organs of the Female, Ovulation and Menstruation, Menstrual Life.

CHAPTER XVII-Development; Changes which Occur in the Ovum Prior to Impregnation, Changes Following Impregnation, Circulation of Blood in the Fetus, Parturition, Lactation . . . 78I 



\section{HANDBOOK OF PHYSIOLOGY}

\section{CHAPTER I \\ THE PHENOMENA OF LIFE}

Physiology is the science which treats of the various processes or changes which take place in the organs and tissues of the body during life. These processes, however, must not be considered as by any means peculiar to the human organism, since, putting aside the properties which serve to distinguish man from other animals, the changes which go on in the tissues of man go on in much the same way in the tissues of all other animals as long as they live. Furthermore, it is found that similar changes proceed in all living vegetable tissues; they indeed constitute what are called vital phenomena, and are those properties which mark out living from non-living material.

The lowest types of life, whether animal or vegetable, are found to consist of minute masses of a substance generally known under the name of protoplasm. Each such living mass is called a cell, so that these minute elementary organisms are designated unicellular.

The phenomena of life are exhibited by protoplasm, whether that exists in the simple form typified by a microscopic one-celled animal, or in a more complex mass represented by the organs and tissues of animals and plants. In the lowest type of life the morphological unit of structural organization is represented by the single cell. In the more complex organisms of both animals and plants the total mass represents a great aggregation of more or less distinct cells. A degree of differentiation takes place whereby the tissues and organs of the body of plants and animals present great aggregates of differentiating cells. It must be at once evident that the great mass of knowledge dealing with the nature and activities of protoplasm constitutes the science of physiology. The cell, therefore, is the working unit in physiology no less than in morphology.

The prime importance of the cell as an element of structure was first established by the researches of the botanist Schleiden, and his conclusions, drawn from the study of vegetable histology, were at once extended by Theodor Schwann to the animal kingdom. The earlier observers defined a cell as a more or less spherical body limited by a membrane, and containing a smaller body termed a nucleus, which in its turn incloses one or more still 
smaller bodies or mucleoli. Such a definition applied admirably to most vegetable cells, but the more extended investigation of animal tissues soon showed that in many cases no limiting membranc or cell wall could be demonstrated.

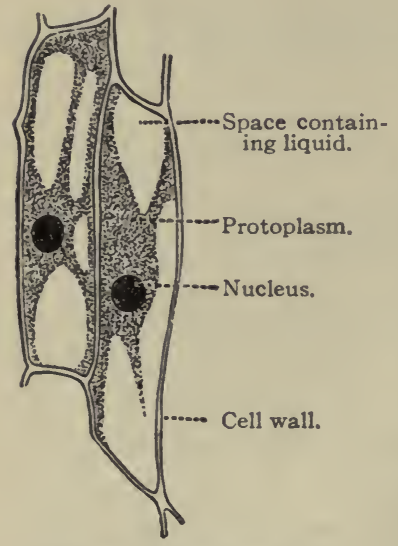

Fig. I.-Vegetable Cells.

The presence or absence of a cell wall, therefore, was then regarded as quite a secondary matter, while at the same time the cell substance came gradually to be recognized as of primary importance. Many of the lower forms of animal life, the Rhizopoda, were found to consist almost entirely of matter very similar in appearance and chemical composition to the cell substance of higher forms; and this from its chemical resemblance to flesh was termed Sarcode by Dujardin. When recognized in vege-. table cells it was called Protoplasm by Mulder, while Remak applied the same name to the substance of animal cells. As the presumed formative matter in animal tissues it was termed Blastema, and in the belief that, wherever found, it alone of all substances has to do with generation and nutrition, Beale has named it Germinal matter or Bioplasm. Of these terms the one most in use at the present day as we have already said, is protoplasm, and inasmuch as all life, both in the animal and vegetable kingdoms, is associated with protoplasm, we are justified in describing it, with Huxley, as the "physical basis of life," or simply "living matter."

General Physical and Chemical Properties of Protoplasm.-Protoplasm is a semifluid substance, which absorbs, but does not mix with water. It is transparent and generally colorless, with refractive index higher than that of

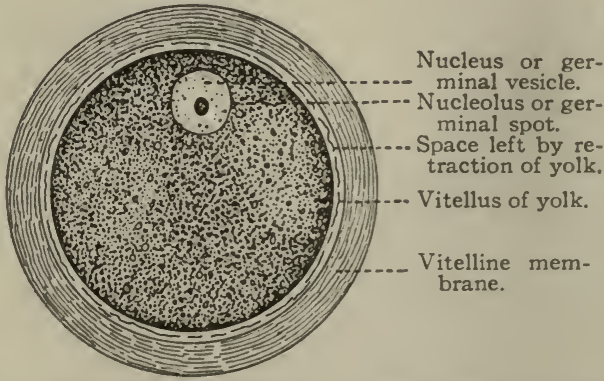

FIG. 2.-Semidiagrammatic Representation of a Human Ovum, showing the parts of an animal cell. (Cadia.) water, but lower than that of oil.

It is neutral or weakly alkaline in reaction, but may under special circumstances be acid, as, for example, after activity. It undergoes heat coagulation at a temperature of about $54.5^{\circ} \mathrm{C}$. ( $130^{\circ} \mathrm{F}$.), and hence no organism can live when its own temperature is raised above that point. It is also coagulated and therefore killed by alcohol, by solutions of 
many of the metallic salts, by strong acids and alkalies, and by many other chemical substances.

Under the microscope it is seen almost universally to be granular, the granules consisting of different substances, albuminous, fatty, or carbohydrate matter. The granules are not equally distributed throughout the whole cell mass, as they are sometimes absent from the outer part or layer and very numerous in the interior. In addition to granules, protoplasm generally exhibits spaces or vacuoles, usually globular in shape, excepting during movement, when they may be irregular, and filled with a watery fluid. These vacuoles are more numerous and pronounced in vegetable than in animal cells. Gas bubbles also sometimes exist in cells.

It is impossible to make any definite statement as to the exact chemical composition of living protoplasm, since the methods of chemical analysis
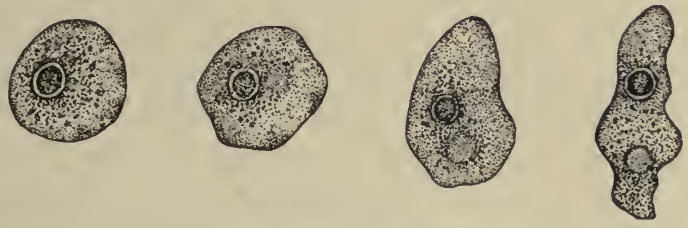

Fig. 3.-Phases of Ameboid Movement.

necessarily imply the death of the cell; it is stated, however, that protoplasm contains 75 to 85 per cent. of water, and of the 15 to 25 per cent. of solids the most important part belongs to the class of substances called proteins or albumins. Proteins contain the chemical elements carbon, hydrogen, nitrogen, oxygen, sulphur, and phosphorus, the last two in very small quantities only. A protein-like substance, nuclein, found in the nuclei of cells, contains phosphorus in greater abundance. In the cell nucleus a compound of nuclein with protein, called nucleoprotein, forms the most abundant protein substance. Other bodies are frequently found associated with the proteins, such as glycogen, starch, cellulose, which contain the elements carbon, hydrogen, and oxygen, the last two in the proportion to form water, and hence are termed carbohydrates; fatty bodies, containing carbon, hydrogen, and oxygen, but not in proportion to form water; lecithin, a complicated fatty body containing phosphorus; cholesterin, a monatomic alcohol; chlorophyll, the coloring matter of plants; hemoglobin, the complex animal pigment; inorganic salts, particularly the chlorides and phosphates of calcium, sodium, and potassium; ferments, and many special substances.

The General Physiological Characteristics of Protoplasm.-The properties of protoplasm may be well studied in the microscopic animal called the ameba, a unicellular organism found chiefly in fresh water. These properties may be conveniently studied under the following heads:

The Power of Spontaneous Movement.-When an ameba is observed with a high power of the microscope, it is found to consist of an irregular mass 
of protoplasm containing one or more nuclei, the protoplasm itself being more or less granular and vacuolated. If watched for a minute or two, an irregular projection is seen to be gradually thrust out from the main body; other masses are then protruded until gradually the whole protoplasmic sub-

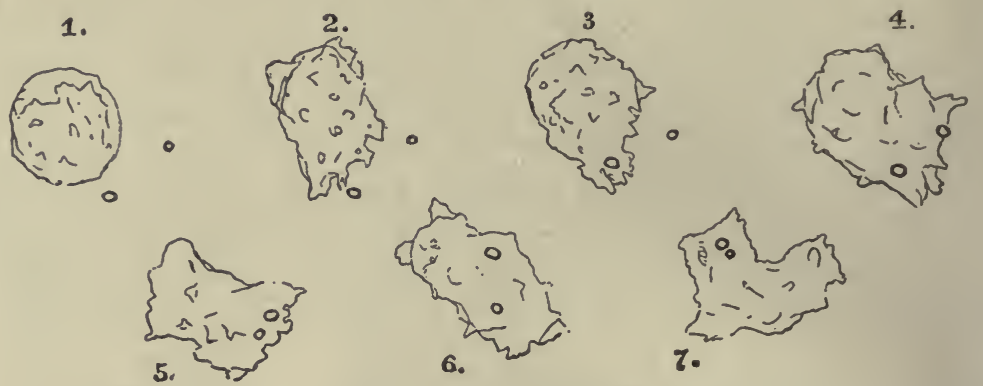

Fig. 4. - Changes of Form of a White Corpuscle, Sketched at Brief Intervals. The figures show also the ingestion of two small granules. (Schäfer.)

stance is, as it were, drawn over to a new position, and when this is repeated several times we have locomotion in a definite direction, together with a continual change of form. These movements, figures 3 and 4 , are observed in such cells as the colorless blood corpuscles of higher animals, in the branched corneal cells of the frog and elsewhere, and are termed ameboid.
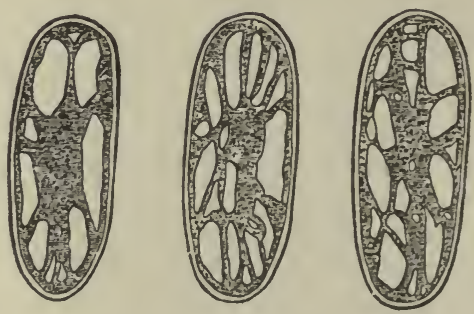

Fig. 5.-Cell of Tradescantia Drawn at Successive Intervals of Two Minutes.-The cell contents consist of a central mass connected by many irregular processes to a peripheral film, the whole forming a vacuolated mass of protoplasm, which is continually changing its shape. (Schofield.)

The remarkable movement of pigment granules observed in the branched pigment cells of the frog's skin by Lister are also probably due to ameboid movement. These granules are seen at one time distributed uniformly through the body and branched processes of the cell, while at another time they collect in the central mass leaving the branches quite colorless.

This movement within the pigment cells might also be considered an example of the so-called streaming movement not infrequently seen in certain of the protozoa, in which the mass of protoplasm extends long and fine proc- 
esses, themselves very little movable, but upon the surface of which freely moving or streaming granules are seen. A gliding movement has also been noticed in certain animal cells; the motile part of the cell being composed of protoplasm bounding a central and more compact mass. By means of the free movement of this layer, the cell may be observed to move along.

In vegetable cells the protoplasmic movement can be well seen in the hairs of the stinging-nettle and Tradescantia and in the cells of Vallisneria. It is marked by the movement of the granules nearly always embedded in it. For example, if part of a hair of Tradescantia, figures 5 and 6 , be viewed under a high magnifying power, streams of protoplasm containing crowds of granules hurrying along, like the foot passengers in a busy street, are seen flowing steadily in definite directions, some coursing round the film which lines the interior of the cell wall, and others flowing toward or away from the irregular mass in the center of the cell cavity. Many of these streams of protoplasm run together into larger ones and are lost in the central mass, and thus ceaseless variations of form are produced. The movement of the protoplasmic granules to or from the periphery is sometimes called vegetable circulation, whereas the movement of the protoplasm round the interior of the cell is called rotation.

The first account of the movement of protoplasm was given by Rösel in I755, as occurring in a small Proteus, probably a large fresh-water ameba. His description was followed twenty years later by Corti's demonstration of the rotation of the cell sap in characeæ, and in the earlier part of the last century by Meyer in Vallisneria, 1827; Robert Brown, I83r, in "Staminal Hairs of Tradescantia." Then came Dujardin's description of the granular streaming in the pseudopodia of Rhizopods and movements in other cells of animal protoplasm (Planarian eggs, von Siebold, I841; colorless blood corpuscles, Wharton Jones, I846).

The Power of Response to Stimuli, or Irritability.-Although the movements of the ameba have been described above as spontaneous, yet they may be increased under the action of external agencies which excite them and are therefore called stimuli. If the movement has ceased for the time, as is the case if the temperature is lowered beyond a certain point, movement may be set up again by raising the temperature. Contact with foreign bodies, gentle pressure, certain salts, and electricity produce or increase the movement in the ameba. The protoplasm is, therefore, sensitive or irritable to stimuli, and shows its irritability by movement or contraction of its mass.

The effects of some of these stimuli may be thus further detailed:

a. Changes of Temperature.-Moderate heat acts as a stimulant; the movement stops below $0^{\circ} \mathrm{C}$. $\left(32^{\circ} \mathrm{F}\right.$.), and above $40^{\circ} \mathrm{C}$. (I04 ${ }^{\circ} \mathrm{F}$.); between these two points the movements increase in activity; the optimum tempera- 
ture is about $37^{\circ}$ to $38^{\circ} \mathrm{C}$. Exposure to a temperature even below $\circ^{\circ} \mathrm{C}$. stops the movement of protoplasm, but does not prevent its reappearance if the temperature is raised; on the other hand, prolonged exposure to a temperature of a little over $40^{\circ} \mathrm{C}$. kills the protoplasm and causes it to enter into a condition of coagulation or heat rigor.

b. Mechanical Stimuli.-When gently squeezed between a cover and object-glass under proper conditions, a colorless blood corpuscle contracts and ceases its ameboid movement.

c. Nerve Influence.-By stimulation of the nerves of the frog's cornea, contraction of certain of its branched cells has been produced.

d. Chemical Stimuli.-Water generally stops ameboid movement, and by imbibition causes great swelling and finally bursting of the cells. In some cases, however (myxomycetes), protoplasm can be almost entirely dried up, but remains capable of renewing its movements when again moistened. Dilute salt solution and many dilute acids and alkalies stimulate the movements temporarily. Strong acids or alkalies permanently stop the movements; ether, chloroform, veratrum, and quinine also stop it for a time.

Movement is suspended in an atmosphere of hydrogen or carbonic acid and resumed on the admission of air or oxygen, but complete withdrawal of oxygen will after a time kill the protoplasm.

e. Electrical.-Weak currents stimulate protoplasmic movement, while
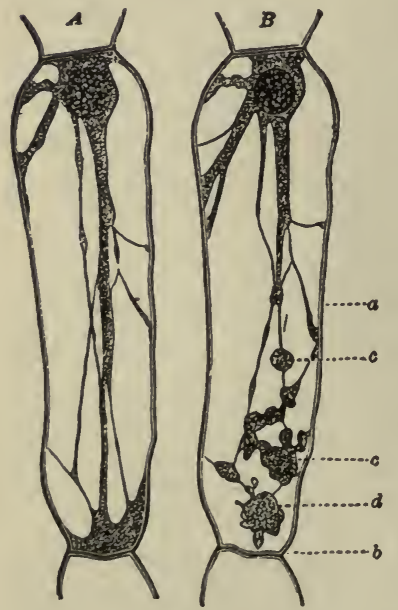

Fig. 6.-Cells from the Staminal Hairs of Tradescantia. $A$, Fresh in water; $B$, the same cell after slight electrical stimulation; $a, b$, region stimulation; $c, d$, clumps and knobs of contracted protoplasm. (Kühne.) strong currents cause the cells to assume a spherical form and to become motionless.

The Power of Digestion, Respiration, and Nutrition.-This consists in the power which is possessed by the ameba and similar animal cells of taking in food, modifying it, building up tissue by assimilating it, and rejecting what is not assimilated. These various processes are effected in some one-celled animals by the protoplasm simply flowing around and enclosing within itself minute organisms such as diatoms and the like. From these it extracts what it requires, and then rejects or excretes the remainder, which has never formed part of the body. This latter proceeding is done by the cell withdrawing itself from the material to be excreted. The assimilation constantly taking place in the body of the ameba is for the purpose of replacing waste of its tissue consequent upon manifestation of energy. The respiratory process of absorbing oxygen goes on at the same time. 
The processes which take place in cells, both animal and vegetable, are summed up under the term metabolism (from $\mu \epsilon \tau a \beta \circ \lambda \eta$, change). The changes which go on are of two kinds, viz., assimilation, or building up, and disassimilation, or breaking down; they may be also called, using the nomenclature of Gaskell, anabolism or constructive metabolism, and catabolism or destructive metabolism. In the direction of anabolism two processes occur, viz., the building up of special though non-living substances from materials which it takes in, and secondly, the building up of its own living substance from those or other materials. As we shall see in a subsequent paragraph, the process of anabolism differs to some extent in vegetable and animal cells. The catabolism of the cell consists in the disintegrative chemical changes which occur in the cell substance itself or in substances in contact with it.

The destructive metabolism of a cell is increased by its activity, but goes on also during quiescence. It is probably of the nature of oxidation, and results in the evolution of carbon dioxide and water on the one hand, and in the formation of various more complex chemical substances on the other, some of which may be stored up in the cell for future use, and are called secretions, and others, like carbon dioxide, for example, and bodies containing nitrogen, are eliminated as excretions.

The Power of Growth.-In protoplasm it is seen that the two processes of waste and repair go on side by side, and so long as they are equal the size of the animal remains stationary. If, however, the building up exceed the
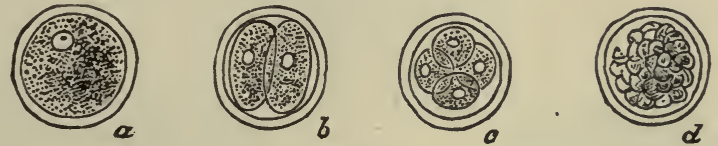

Fig. 7.-Diagram of an Ovum (a) Undergoing Segmentation. In $(b)$ it has divided into two, in $(c)$ into four; and in $(d)$ the process has ended in the production of the so-called "mulberry mass." (Frey.)

waste, then the animal grows; if the waste exceeds the repair, the animal wastes; and if decay goes on beyond a certain point, life becomes impossible and the animal dies.

The power of increasing in size, although essential to our idea of life, is not, it must be recollected, confined to living beings. A crystal of common salt, for example, if placed under appropriate conditions for obtaining fresh material, will increase in size in a fashion as definitely characteristic and as easily to be foretold as that of a living creature; but the growth of a crystal takes place merely by additions to its outside; the new matter is laid on particle by particle, and layer by layer, and, when once laid on, it remains unchanged. In a living structure, where growth occurs, it is by addition of new matter, not to the surface only, but throughout every part of the mass, and this matter becomes an intimate part of the living substance. 
The Power of Reproduction.-The ameba, to return to our former illustration, when the growth of its protoplasm has reached a certain point, manifests the power of reproduction, by splitting up into (or in some other way producing) two or more parts, each of which is capable of independent existence. The new amebæ manifest the same properties as the parent, perform the same functions, grow and reproduce in their turn. This cycle of life is being continually passed through.

In more complicated structures than the ameba, the life of individual protoplasmic cells is probably very short in comparison with that of the organism they compose; and their constant decay and death necessitate constant reproduction. The manner in which this takes place has long been the subject of investigation.

The exact manner of reproduction and growth of protoplasm is a matter of great complexity. Those who have already learned the embryological story have the foundations laid for the physiological uses made of this mate-

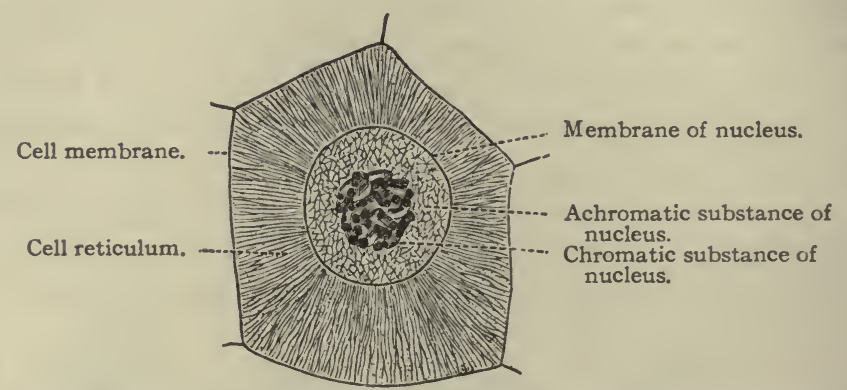

FIG. 8.-Cell with its Reticulum Disposed Radially; from the intestinal epithelium of a worm. (Carnoy.)

rial. Certain of the essential facts are set forth in Chapter II a little later, in so far as the reproduction of the cellular unit is concerned. The reproduction of the animal organism as a whole is a still more complicated story and is presented in the last chapter of this volume.

\section{THE DIFFERENTIATION OF PROTOPLASM}

The Morphological Unit.-Protoplasm was formerly thought to be homogeneous. It is found, however, that every mass of living protoplasm has one or more special structures imbedded in the mass, the nuclei. In most tissues of the mammalian body each mass of protoplasm is more or less distinctly subdivided into elemental divisions, corresponding to the number of nuclei, the cells. The cells present in the mass, therefore, represent the morphological units. The arrangements of these units as to size and space relations constitute the form and mass characteristics of the tissues on the one hand, and of the organs on the other. Where there is no distinct marking off of 
the mass of protoplasm into individual cellular units, there is formed what is known as a syncytium. All syncytial structures are multi-nuclear, otherwise their protoplasm is not unlike that of the cell as a unit.

The fuller detail of cellular types and cell structure is given a little later in Chapter II. However, for the purpose of reference to this point, one may here call attention to the fact that the principal differentiations of the protoplasm of the cell are the nuclei and the cytoplasm. The cytoplasm is differentiated further into two substances, spongioplasm and hyaloplasm. The spongioplasm or reticulum forms a fine network, increases in relative amount as the cell grows older, and has an affinity for staining reagents. The hyaloplasm is less refractile, elastic, or extensile, and has little or no affinity for stains; it predominates in young cells, is thought to be fluid, and fills the interspaces of the reticulum. The nodal points of the reticulum, with the granular microsomes, found in the protoplasm, cause the granular appearance. The arrangement of the reticulum varies considerably in different cells, and even in different parts of the same cell.

In some cells, particularly in plants, but also in some animal cells, there is a tendency toward the formation of a firmer external envelope, constituting in vegetable cells a membrane distinct from the more central and more fluid part of the protoplasm. In such cases the reticulum at the periphery of the cell is made up of very fine meshes. The membrane when formed is usually pierced with pores by which fluid may pass in, or through which protrusion of the protoplasmic filaments forming the cell's connection with other cells surrounding it may take place.

A

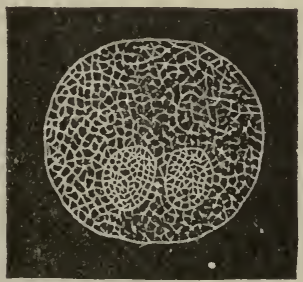

B

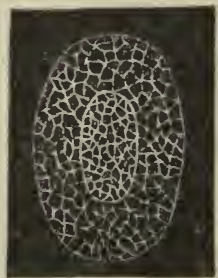

Fig. 9.-A: The Colorless Blood Corpuscle, Showing the Intracellular Network, and two nuclei with intranuclear network. B: Colored blood corpuscle of newt showing the intracellular network of fibrils. Also oval nucleus composed of limiting menibrane and fine intranuclear network of fibrils. $\times 800$. (Klein and Noble Smith.)

All protoplasm at some period of its existence possesses one or more neclei. The origin of a nucleus in a cell is the first sign of the differentiation of protoplasm. The existence of nuclei was first pointed out in the year 1833 by Robert Brown, who observed them in vegetable cells. They are either small transparent vesicular bodies containing one or more smaller particles called nucleoli, and always when in the resting condition bounded by a well-defined 
envelope. In their relation to the life of the cell they are certainly hardly second in importance to the cytoplasm itself, and thus Beale is fully justified in comprising both under the term "germinal matter." The nuclei control the nutrition of the cell, and probably initiate the process of subdivision. If a cell be mechanically divided so that a portion of it possesses the nucleus while other portions have no nucleus, that portion containing the nucleus will live and develop while the parts without nucleus soon die. Concerning this interesting question of the relation of the nuclei and cytoplasm in the cells, Schäfer summarizes as follows: "There are cells and unicellular organisms both animal and vegetable, in which no reticular structure can be made out, and these may be formed of hyaloplasm alone. In that case, this must be looked upon as the essential part of protoplasm. So far as ameboid phenomena are concerned it is certainly so; but whether the chemical changes which occur in many cells are effected by this or by spongioplasm is another matter."

Protoplasmic nuclei are highly differentiated chemically as well as functionally. They contain special structures which react in a characteristic chemical way to staining solutions, and to other chemical treatment. This differential structure is emphasized in Chapter II. There the morphological changes through which the nuclei pass in cell multiplication are given in greater detail. It is the study of these changes that supplies the basis of fact for many of our present conceptions of the physiological importance of nuclei.

Differentiation and Growth of Organized Protoplasm.-The detail of cellular division of protoplasm is more fully given in Chapter II. The morphological fact to which attention is called here is that as we proceed upward in the scale of life from the unicellular organisms, another phenomenon is exhibited in the life history of the higher forms, namely, that of development. The one-celled ameba comes into being derived from a previous ameba; it manifests the properties and performs the functions of its life which have been already enumerated. In the higher organisms it is different. Each, indeed, begins as a single cell, but the cells which result from division and subdivision do not form so many independent organisms but adhere in one differentiated community which ultimately forms the complex but co-ordinated whole, in man the human body.

Thus from the ovum or germ cell which forms the starting-point of an individual animal during development, there is rapidly formed a number of tissues each characterized by its own type of structure, the whole laid down in an orderly manner to form the complicated individual. In the unfolding of this individual growth process, the developing ovum soon forms a complete membrane of cells called the blastoderm, and this speedily differentiates into two and then into three layers, chiefly from the rapid proliferation of the cells of the first single layer. These layers, figure ro, are called the Epiblast, the Mesoblast, and the Hypoblast. In the further development of the animal 
it is found that from each of these layers is produced a very definite part of the completed body. For example, from the cells of the epiblast are derived, among other structures, the skin and the central nervous system; from the mesoblast the muscles and connective tissue of the body, and from the hypoblast the epithelium of the alimentary canal, some of the chief glands, and so on.

The result of this' developmental process therefore is the formation of the adult tissues highly differentiated and specialized in form.

From the physiologist's point of view this anatomical differentiation accomplishes a highly specialized structure which, machine-like, is capable of doing some part of the total work of the body in an especially effective manner. In a word, the differentiated tissues do not altogether lose the general properties which characterize protoplasm, but each tissue develops a structure capable of doing some special part of this activity better than the undifferentiated protoplasm can do it. As an illustration, the muscles, derived chiefly from

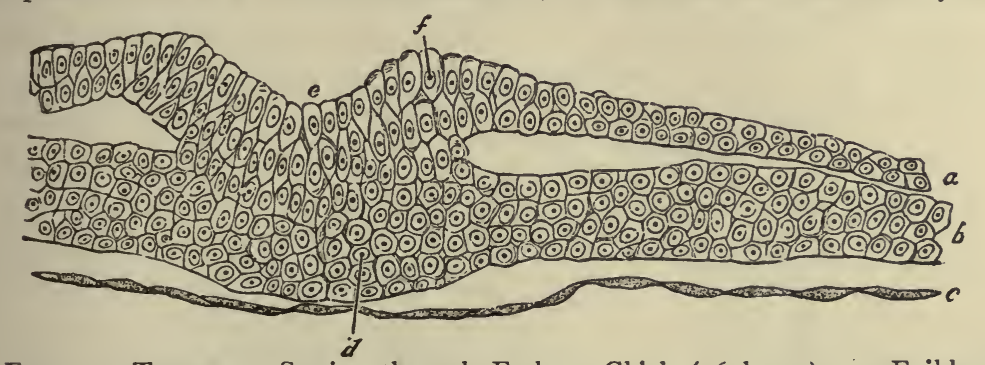

FIG. Io.-Transverse Section through Embryo Chick (26 hours). $a$, Epiblast; $b$, mesoblast; $c$, hypoblast; $d$, central portion of mesoblast, which is here fused with epiblast; $e$, primitive groove; $f$, dorsal ridge. (Klein.)

either epithelial cells or mesoblast, are highly contractile, and especially responsive to stimuli. They have not developed in the same degree the power to produce chemical substances which characterizes the salivary glands. The cells of the liver, on the other hand, in the adult stage have practically lost the property of contractility, but have developed in a high degree the functional properties of nutrition and secretion.

Hand in hand with the anatomical differentiation has gone physiological division of labor. In the adult animal body each type of activity is no longer accomplished by the whole organism as in the case of the ameba, but now some specializing part of the body assumes each chief activity. In other words the muscles contract, the nervous tissue conducts changes from one part of the body to the other, glands secrete, special sense organs respond to the stimuli of the environment, and the reproductive gonads have assumed the chief responsibility in the reproductive process. It is through the high degree of physiological division of labor that the great versatility of the body activity is accomplished by the human organism. It is a matter of great economy and effectiveness in this biological machine, the body. 
Differences between Animals and Plants.-Having considered the vital properties of protoplasm, as shown in cells of animal as well as of vegetable organisms, we are now in a position to discuss the question of the differences between plants and animals. It might at the outset of our inquiry have seemed an unnecessary thing to recount the distinctions which exist between an animal and a vegetable organism as they are in many cases so obvious, but, however great the differences may be between the higher animals and plants, in the lowest of them the distinctions are much less plain.

In the first place, it is important to lay stress upon the differences between vegetable and animal cells, first as regards their structures and next as regards their functions.

It has been already mentioned that in animal cells an envelope or cell wall is by no means always present. In adult vegetable cells, on the other hand, a well-defined wall is highly characteristic; this is composed of cellulose, is non-nitrogenous, and thus differs chemically as well as structurally from the contained protoplasmic mass. Moreover, in vegetable cells, figure Ir, $\mathrm{B}$, the protoplasmic contents of the cell fall into two subdivisions: I, a continuous film which lines the interior of the cellulose wall; and, 2, a reticulate
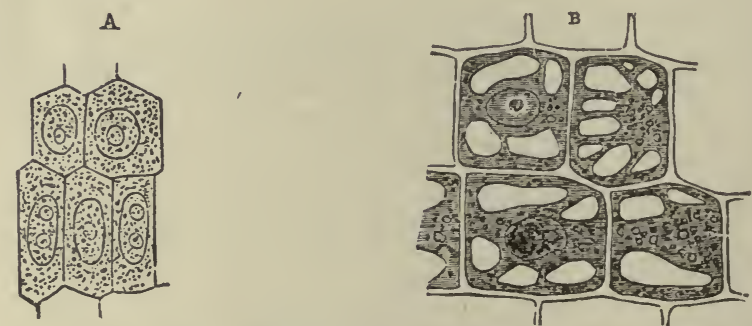

FIG. II.-A. Young Vegetable Cells, Showing Cell Cavity Entirely Filled with Granular Protoplasm Enclosing a Large Oval Nucleus, with one or more Nucleoli. B. Older cells from same plant, showing distinct cellulose wall and vacuolation of protoplasm.

mass containing the nucleus and occupying the cell cavity. The interstices are filled with fluid. In young vegetable cells such a distinction does not exist; a finely granular protoplasm occupies the whole cell cavity, figure I , A. As regards the respective functions of animal and vegetable cells, one of the most important differences consists in the power which vegetable cells possess of being able to build up new complicated nitrogenous and non-nitrogenous bodies out of very simple chemical substances obtained from the air and from the soil. They obtain from the air oxygen, carbon dioxide, and water, as well as traces of ammonia gas; and from the soil they obtain water, ammonium salts, nitrates, sulphates, and phosphates in combination with such bases as potassium, calcium, magnesium, sodium, iron, and others. The majority of plants are able to work up these elementary compounds into other and more complicated bodies. This they are able 
to do in consequence of their containing a certain coloring matter called chlorophyll, the presence of which is the cause of the green hue of plants. In all plants which contain chlorophyll two processes are constantly going on when they are exposed to light: one, which is called true respiration and is a process common to animal and vegetable cells alike, consists in the taking of the oxygen from the atmosphere and the giving out of carbon dioxide; the other, which is peculiar apparently to bodies containing chlorophyll, consists in the taking in of carbon dioxide and the giving out of oxygen. It seems that the chlorophyll is capable of decomposing the carbon dioxide gas and of fixing the carbon in the structures in the form of new compounds, one of the most rapidly formed of which is starch.

Vegetable protoplasm by the aid of its chlorophyll is able to build up a large number of bodies besides starch, the most interesting and important being protein or albumin. It appears to be a fact that the power which plants possess of elaborate chemical synthesis is to a large extent dependent upon the chlorophyll they contain. Thus the power is present to a marked extent only in the plants in which chlorophyll is found, and is absent in those saphrophytic plants which do not possess chlorophyll.

It must be recollected, however, that chlorophyll without the aid of the light of the sun can do nothing in the way of building up substances, and a plant containing chlorophyll when placed in the dark, while it continues to live, though not as a rule long, acts as though it did not contain any of that substance. It is an interesting fact that certain of the bacteria have the chlorophyll replaced by a similar pigment which is able to decompose carbon dioxide gas.

Animal cells do not possess the power of building up or synthesizing from simple materials, though higher organic synthesis can no longer be questioned. Their activity is chiefly exercised in the opposite direction, viz., the oxidations of the complicated compounds produced by the vegetable kingdom which they have brought to them as foods. With thesefoods animals are able to perform their complex functions, setting free energy in the direction of heat, motion, and electricity, and at the same time eliminating such bodies as carbon dioxide and water, and producing other bodies, many of which contain nitrogen but are derived from decomposition.

With reference to the substance chlorophyll it has been noted that ${ }^{\circ}$ the synthetical operations of vegetable cells are peculiarly associated with the possession of chlorophyll and that these operations are dependent upon the light of the sun. It has been further shown that a solution of chlorophyll when examined with the spectroscope reveals a definite absorption spectrum, and that it is particularly those parts of the solar spectrum corresponding to these absorption bands which are chiefly active in the decomposition of carbon dioxide. In the synthetical processes of the plant, then, by aid of its chlorophyll, the radiant energy of the sun's rays becomes stored up or ren- 
dered potential in the chemical products formed. The potential energy is set free, or is again made kinetic, when these products by simple combustion produce heat, or when they are taken into the animal organism and used as food and there later produce heat and motion.

The influence of light is not absolutely essential to animal life; indeed, it is said not to increase the metabolism of animal tissue to any great extent, and the animal cell does not receive its energy directly from the sun's light nor yet to any extent from the sun's heat, but from the potential energy of the food stuffs. But it must be always kept in mind that anabolism is not peculiar to vegetable, or katabolism to animal cells; both processes go on in each. Some of the lowest forms of vegetable life, e.g., the bacteria, will live only in a highly albuminous medium, and in fact seem to require for their growth elements of food stuffs which are essential to animal life. In their metabolism, too, they very closely approximate animal cells, not only requiring an atmosphere of oxygen, but giving out carbon dioxide freely, and secreting and excreting many very complicated nitrogenous bodies, as well as forming protein, carbohydrates, and fat, requiring heat but not light for the due performance of their functions. However, certain bacteria grow only in the absence of oxygen.

There is, commonly, a difference in general chemical composition between vegetables and animals, even in their lowest forms; for associated with the protoplasm of the former is a considerable amount of cellulose, a substance closely allied to starch and containing carbon, hydrogen, and oxygen only. The presence of starch in vegetable cells is very characteristic, though, as we have seen above, it is not distinctive, and a substance, glycogen, similar in composition to starch, is very common in the organs and tissues of animals.

Inherent power of movement is a quality which we so commonly consider an essential indication of animal nature that it is difficult at first to conceive of its existence in any other. The capability of simple motion is now known, however, to exist in so many vegetable forms that it can no longer be held as an essential distinction between them and animals, and ceases to be a mark by which one can be distinguished from the other. Thus the zoöspores of many of the Cryptogams exhibit ciliary or ameboid movements of a like kind to those seen in amebæ; and even among the higher orders of plants, many, e.g., Dionca muscipula (Venus's fly-trap), and Mimosa sensitiva (Sensitive plant) exhibit such motion, either at regular times or on the application of external irritation. Were this fact taken by itself, it might lead one to regard them as sensitive organisms. Inherent power of movement, then, although especially characteristic of animal nature, is, when taken by itself, no proof of it.

Sources and Utilization of Physiological Material.-In studying the functions of the human body it is necessary first of all to know of what it is 
composed, of what tissues and organs it is made up; this can of course be ascertained only by the dissection of the dead body, and thus it comes that Anatomy, the science which treats of the structure of organized bodies, is closely associated with physiology, which treats of the functions of these structures. So close, indeed, is the association that Histology, which is especially concerned with the minute or microscopic structure of the tissues and organs of the body and which is, strictly speaking, a department of anatomy, is often included in works on physiology. There is much to be said in favor of such an arrangement, since it is impossible to consider the changes which take place in any tissue during life, apart from the knowledge of the structure of the tissues themselves. There is indeed an almost inseparable relation between the structure and the function of the differentiated animal body in which the one is made the means to a knowledge of the other as an end, and vice versa, according to the aims and purposes of the student.

An equally important essential to the right comprehension of the changes which take place in the living organism is a knowledge of the chemical composition of the body. Here, however, we can deal directly only with the composition of the dead body, and it is well at once to admit that there may be many chemical differences between living and non-living tissues; but as it is impossible to ascertain the exact chemical composition of the living tissues, the next best thing which can be done is to find out as much as possible about the composition of the same tissues after they are dead. This is the assistance which the science of Chemistry can afford to the physiologist.

Having considered the structure and composition of the body, we' are brought face to face with physiology proper, and have to investigate the vital changes which go on in the tissues, the various actions taking place as long as the organism is at work. The subject includes not only the observation of the manifest processes which are continually taking place in the healthy body, but the conditions under which these are brought about, the laws which govern them and their effects.

It may be well to mention as a preliminary that the physiological information which we have at our disposal has been derived from many sources, the chief of which are as follows: I, From actual observation of the various phenomena occurring in the human body from day to day, and from hour to hour, as, for example, the estimation of the amount and composition of the ingesta and egesta, the respiration, the beat of the heart, and the like; 2, from observations upon other animals, the bodies of which we are taught by comparative anatomy approximate the human body in structure, and may be supposed to be similar in function; 3, from observations of the changes produced by experiment upon the various processes in such animals, or in the organs and tissues of animals; 4 , from observations of the changes in the working of the human body produced by diseases; and 5 , from observations upon the gradual changes which take place in the functions of organs when 
watched in the embryo from their earliest beginnings to their completed development.

The physiologist, in order to utilize the sources of material, must be familiar with the gross structure of the animals or parts of animals which he proposes to use in experimental procedure. So simple a matter as the determination of arterial blood pressure involves familiarity with extensive anatomical structure. Experimental procedure must also draw on the field of microscopic structure or histology, and many of the most instructive bodies of physiological knowledge have come directly from the utilization of the facts of comparative anatomy and of biology. The problems in animal nutrition which are under such extensive investigation at the present time require for their solution not only the use of the most complex methods of chemistry, both analytic and synthetic, but also the principles and methods of physics. Indeed, since the work of Helmholz, the interpretation of physiological phenomena by means of physical methods and laws has contributed more than any other means toward the prominent scientific position of physiology at the present time. In a word, physiology must utilize the facts of anatomy, histology, biology, physics, and chemistry to interpret the phenomena of life. 


\section{CHAP'TER II}

\section{CELL DIFFERENTIATION AND THE TYPICAL STRUCTURE OF THE ELEMENTARY TISSUES}

In the preceding discussion a general view of the type of cell activity and the structural basis therefor has been briefly presented. Emphasis has been laid on the fact that the complicated phenomena of life are manifested through the agency of the.tissues and cells. The histological cells, alone or in combination, are capable of all the activities manifested by the living body. Throughout the different phases of the physiological discussions which follow it will be assumed that the reader has some knowledge of this structural basis. However, for the purpose of reference there is presented in this chapter a brief but elementary review of the characteristic cytological structure of the tissues and cells of the animal body.

\section{THE ESSENTIAL STRUCTURE OF THE TYPICAL CELL}

The typical cell is a spherical or ovoid mass of protoplasm. It is of microscopic size and varies from 6 or 7 micra in diameter for the lymphocytes and erythrocytes to 150 to 200 micra for the diameters of the larger cell bodies of the neurones. Its structure is quite complex, but the most general differentiation is into the cell-mass or cytoplasm, and its contained nucleus. The cytoplasm is sometimes bounded by a definite cell membrane, but in differentiated animal tissues this membrane is usually not present.

The Cell Body. - The cell body or ctyoplasm is a complex semi-fluid mass, the determination of the detailed relations of which has presented many difficulties. The cell cytoplasm is usually described as having a framework of spongioplasm supporting a homogeneous hyaloplasm. In some cells there are formed materials resulting from the cellular activity called metaplasm, figure i2.

Cell protoplasm includes several kinds of stainable granules and fibrils, some are essential constituents while others are formed by the reactions of the protoplasm and are in a sense extraneous material. These structural features are made more evident by their selective affinity for certain staining reagents.

The exact form of the spongioplasm or reticulum varies greatly in different types of cells, and even in different parts of the same cell. Its affinity for stains discloses a fine network, the reticulum, which increases in amount and also in constancy in the type of arrangement in the older cells. 
The hyaloplasm is more fluid, less refractile, and stalns with greai diffculty. It fills the interspaces of the spongioplasm. In this material may be embedded such substances as the metaplasts mentioned above.

The hyaloplasm contains in solution the various nutritive constituents brought to the cell as well as the soluble end products of its chemical activity. Here, too, are found the various hormones, oxidases and enzymes which play so important a part in the cellular reactions in the different types of cells.

Structure of the Nucleus. - The nucleus when in a condition of rest is bounded by a distinct membrane, the nuclear membrane, possibly derived from the spongioplasm of the cell, which encloses the nuclear contents, mucleoplasm or karyoplasm. The membrane consists of an inner, or chromatic, and of an outer, or achromatic layer, so called from their reaction to stains. The nucleoplasm is made up of a reticular network, or chromoplasm, whose interspaces are filled by the karyolymph, or muclear matrix, a homogeneous substance which is rich in proteins, has but slight affinity for stains, and is supposed to be fluid in consistency.

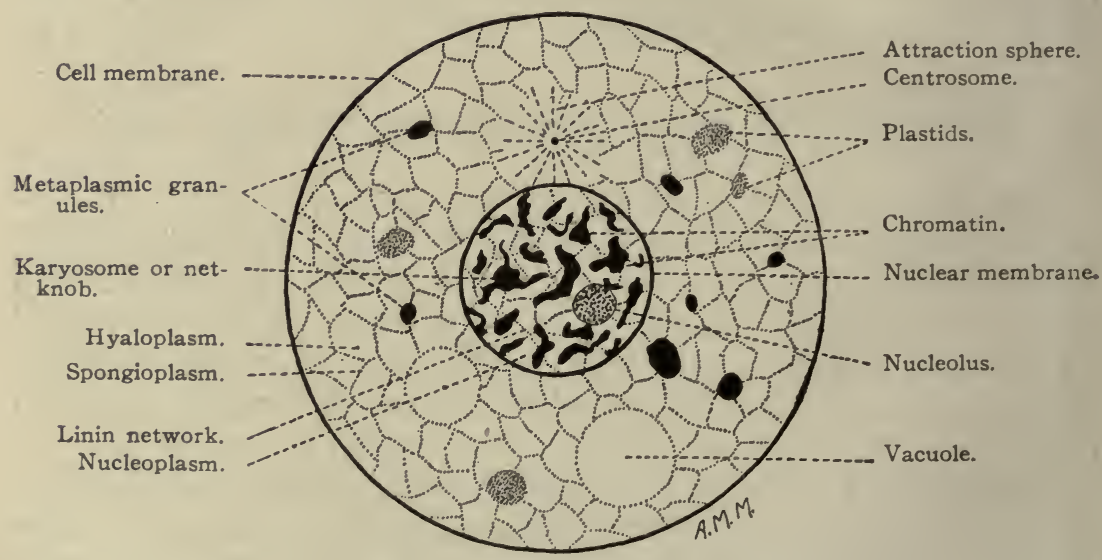

Fig. 12.-Diagram of a Typical Cell. (Bailey.)

The network is composed of linin or achromatin, a transparent unstainable framework, and of chromatin, which stains deeply. It is supported by the linin, and occurs sometimes in the form of granules, but usually as irregular anastomosing threads, both thicker primary fibers and thinner connecting branches. The threads often form thickened nodes, karyosomes or false nucleoli, at their points of intersection. It is now quite generally believed that the chromatin occurs as short, rod-like, and highly refractive masses, which are embedded in the linin in a regular series.

The nucleoli, or plasmosomes, are spherical bodies which stain deeply, and may either lie free in the nuclear matrix or be attached to the threads of the network. 
The Centrosome and Attraction Sphere.-In addition to the nucleus, a minute spherical body called the centrosome is believed to be constantly present in animal cells, though sometimes too small to be demonstrated. The centrosome is smaller than the nucleus, close to which it lies, and exerts a peculiar attraction for the protoplasmic filaments and granules in its vicinity, so that it is surrounded by a zone of fine radiating fibrils, forming the attraction sphere or archoplasm. Some authorities assert that the centrosome lies within the nucleus in the resting state, and passes into the cell proper only in the earlier stages of cell division. The attraction sphere is most distinctly seen in cells about to divide. It plays an important rôle in nuclear division, but it is doubted if it gives the initial impulse to the process.

Cell Multiplication.-Cells increase in number by a process known as cell division, of which the first act is nuclear division. In fact the nucleus is the center of control of the cell mass in the process of division. Cell multiplication takes place by two recognized methods, direct or amitosis, in which there is little disturbance of the nuclear network, and indirect or mitosis, in which there is a complex series of nuclear network changes.

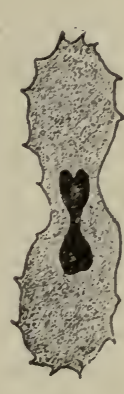

a
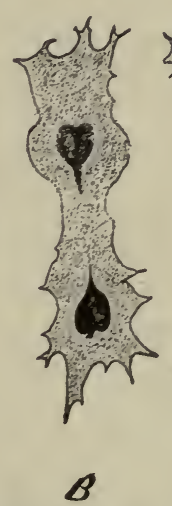

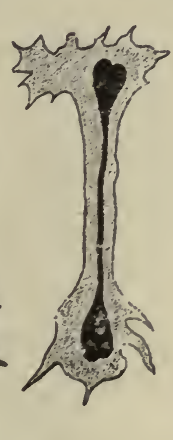

c

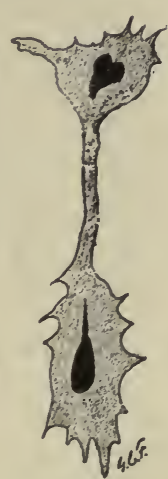

$\not \dot{D}$

FIg. 13.-Akinesis, Amitosis, or Direct Cell Division. A, Constriction of nucleus; $B$, division of nucleus and constriction of cell body; $C$, daughter nuclei still connected by $a$ thread, division being delayed; $D$, division of cell body nearly complete. (After Arnold.)

Direct Cell Division or Amitosis.-The division of a cell is preceded by division of its nucleus. Direct or simple division, amitosis or akinesis, see figure 13 , occurs without any change in the arrangement of the intranuclear network. A constriction develops at the center of the nucleus, possibly preceded by division of the nucleoli, and gradually divides it into two equal daughter nuclei. A similar constriction of the protoplasm of the cell occurs between the daughter nuclei and divides it into two parts.

Indirect Cell Division or Mitosis. - Indirect division, mitosis, or karyokinesis is the usual method of cell division, and consists of a series of changes in the arrangement of the intranuclear network, resulting in the exact division 
of the chromatic fibers into two parts, which form the chromoplasm of the daughter nuclei. The changes follow a closely similar course in both plant and animal cells.

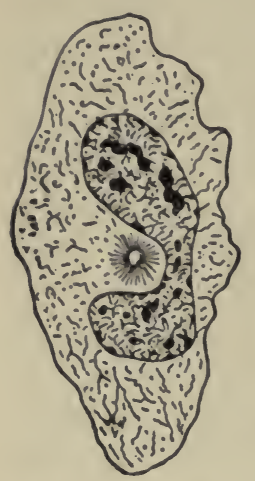

Fig. 14.-Leucocyte of Salamander Larva, Showing Attraction Sphere. (After Flemming.)

The process may be divided into the following stages:

Prophase.-The resting nucleus becomes somewhat enlarged, and the centrosome (according to those who regard it as lying normally within the nucleus) migrates into the cell protoplasm. The centrosome then divides into two daughter centrosomes which lie near the nucleus but are separated by a considerable interval. Each is surrounded by the radiating fibrils of the attraction sphere, and some of these fibrils pass continuously from one centrosome to the other, forming the achromatic spindle. At the same time the intranuclear network becomes converted into a fine convoluted coil, the spirem or skein, which may be either continuous or else broken up into several threads. The thread or threads then shorten and become thicker, while the convolutions, which have become less numerous, arrange themselves in a series of connecting loops, forming the wreath. The nuclear membrane and the nucleolus disappear, the latter passing at times into the cell protoplasm and disintegrating. The wreath then breaks up into V-shaped segments, the chromosomes, of which each species of animal has a constant and characteristic number. This varies in the different animals, but is sixteen in man.

The two centrosomes migrate to the poles of the nucleus, while the achromatic spindle which connects them occupies the long axis of the nucleus
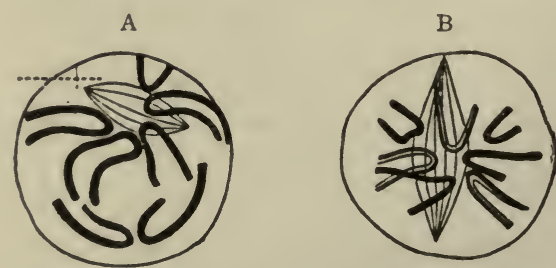

FIG. 15.-Early Stages of Karyokinesis. A. The thicker primary fibers remain and the achromatic spindle appears. B. The thick fibers split into two and the achromatic spindle becomes longitudinal. (Waldeyer.)

The chromosomes, becoming much shorter and thicker, gather around the spindle in its equatorial plane, with their angles directed toward the center, forming the aster or monaster.

Metaphase. - The actual division of the nucleus is begun at this time by the splitting of each chromosome longitudinally into halves which lie at first close together so that each seems doubled. Soon afterward the fibrils of the 
achromatic spindle begin to contract, and thus separate the halves of the chromosomes in such a way that one-half of each is turned toward one pole, and the other half toward the other. As this continues, the two groups, which are equal in size, draw away from each other and from the equator, each group being formed of daughter chromosomes.

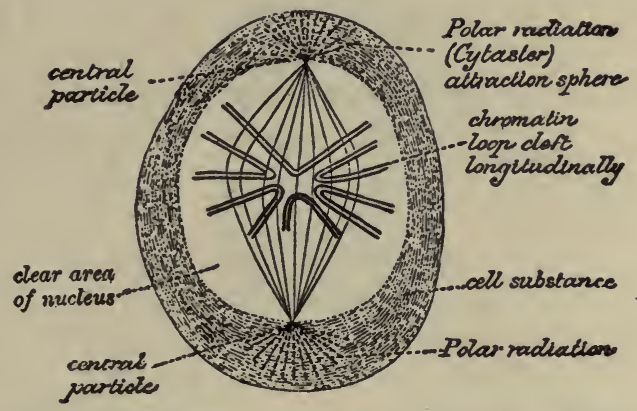

FIG. 16.-Monaster Stage of Karyokinesis. (Rabl.)

Anaphase.-The two groups (daughter chromosomes) now gradually approach their respective poles, or centrosomes, and the equator becomes free. On reaching the pole, each group gathers in a form which is similar in arrangement to the monaster and is known as the diaster. During this time the cell body becomes slightly constricted by a circular groove at its equatorial plane.

Telophase.-Soon afterward the fibrils of the chromatic spindle which connect the two groups begin to grow dim and finally disappear. The daugh-
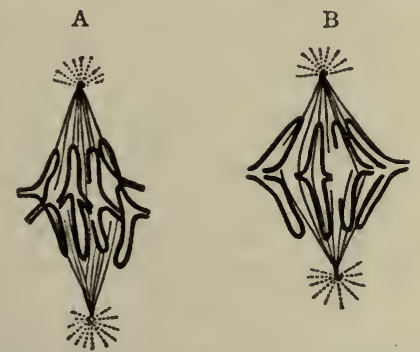

C

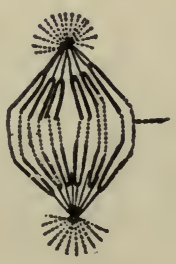

FIG. I7.- Stages of Karyokinesis. A. Commencing separation of the split chromosomes. B. The separation further advanced. C. The separated chromosomes passing along the fibers of the achromatic spindle. (Rabl.)

ter chromosomes assume the form of threads twisted in a coil and develop each a nuclear membrane and a nucleolus, forming a daughter nucleus. The nuclei enlarge and the nuclear threads assume the appearance of the resting state of the nucleus. Meanwhile, the constriction about the body of the cell cytoplasm has become deeper and deeper until the protoplasm is divided into two equal parts, or daughter cells, each with its daughter nucleus, and the process of karyokinesis is completed. 
The Cell Types.-All of the elementary tissues consist of cells and of their altered equivalents. It will be as well therefore to indicate some of the differences between the cells of the body. They are named in various ways, according to their shape, origin, and functions.

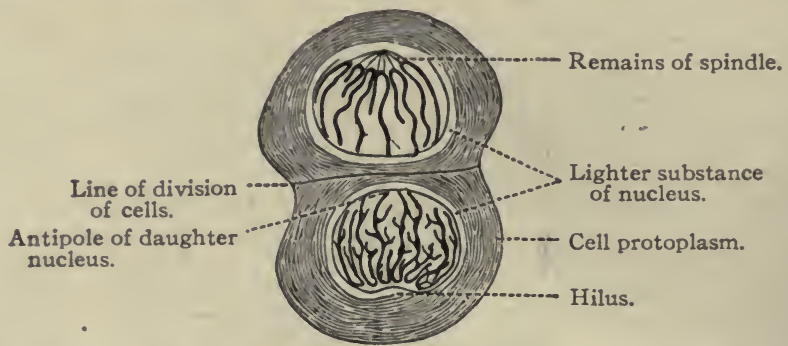

FIG. 18.-Final Stages of Karyokinesis. In the lower figure the changes are still more advanced than in the upper. (Waldeyer.)

From their shape, cells are described as spherical or spheroidal, which is the typical shape of the free cell; this may be altered to polyhedral when the pressure on a mass of cells in all directions is nearly the same; of this the primitive segmentation cells afford an example. The discoid form is seen
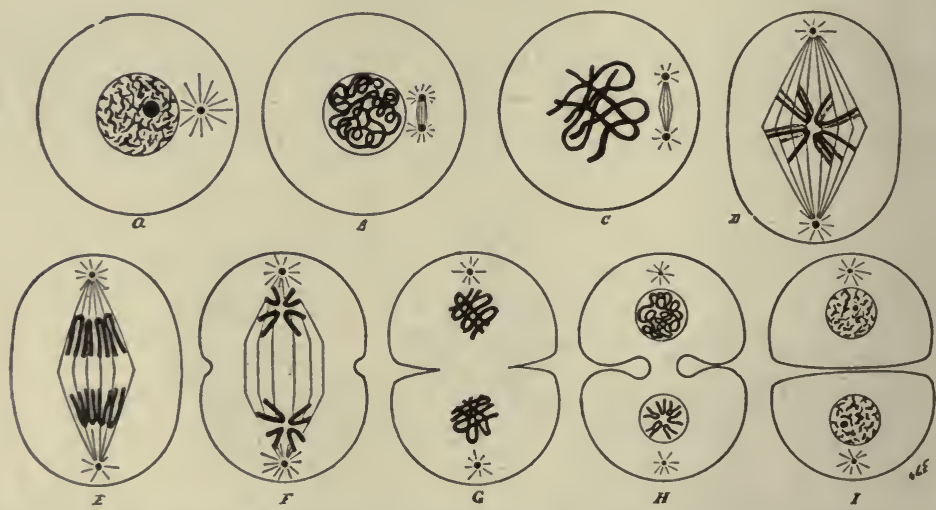

Fig. 19.-Karyokinesis, Mitosis, or Indirect Cell Division (diagrammatic). $A$, Cell with resting nucleus; $B$, wreath, daughter centrosomes and early stage of achromatic spindle; $C$, chromosomes; $D$, monaster stage, achromatic spindle in long axis of nucleus, chromosomes dividing; $E$, chromosomes moving toward centrosomes; $F$, diaster stage, chromosomes at poles of nucleus, commencing constriction of cell body; $G$, daughter nuclei beginning return to resting state; $H$, daughter nuclei showing monaster and wreath; $I$, complete division of cell body into daughter cells whose nuclei have returned to the resting state. (After Böhm and von Davidoff.)

in blood corpuscles, and the scale-like form in superficial epithelial cells. Some cells have a jagged outline and are then called prickle cells. Cells of cylindrical, conical, or prismatic form occur in various places in the body. Such cells may taper at one or both ends into fine processes, in the former case 
being caudate, in the latter fusiform, or they may be greatly elongated so as to become fibers. Cells with hair-like processes, or cilia, projecting from their free surfaces, are a special variety. The cilia vary greatly in size, and may even exceed in length the cell itself. Finally, cells may be branched or stellate with long outstanding processes.

From their function cells are called secreting, protective, sensitive, contractile, and the like.

From their origin cells are called epiblastic and mesoblastic and hypoblastic, ectodermic, mesodermic, and endodermic.

Modes of Cell Connection.-Cells are connected together to form tissues in various ways. They are connected by means of a cementing intercellular substance. This is probably always present as a transparent, colorless, viscid, albuminous substance, even between the closely apposed cells of epithelium; while in the case of cartilage it forms the main bulk of the tissue, and the cells only appear as embedded in, not as cemented together by, the intercellular substance. This intercellular substance may be either homogeneous or fibrillated. In many cases, e.g., the cornea, it can be shown to contain a number of irregular branched cavities, which communicate with each other, and in which branched cells lie. Nutritive fluids can find their way through these branching spaces into the very remotest parts of a non-vascular tissue. The basement membrane, membrana propria must be mentioned as a special variety of intercellular substance which is found at the base of the epithelial cells in most mucous membranes, and especially as an investing tunic of gland follicles which determines their shape.

Cells are connected by anastomoses of their processes. This is the usual way in which stellate cells, e.g., of the cornea, are united. The individuality of each cell is thus to a great extent lost by its connection with its neighbors to form a reticulum. As an example of a network so produced we may cite the anastomosing cells of the reticular tissue of lymphatic glands.

The intercellular substance sometimes forms so great a part of the tissue as to overshadow the cells proper. Examples of this type of structure are found in the matrix of cartilage, the fibers of connective tissue, bone, etc.

Decay and Death of Cells.-There are two chief ways in which the comparatively brief existence of cells is brought to an end, i.e., by mechanical abrasion and by chemical transformation.

The various epithelia furnish abundant examples of mechanical abrasion. As it approaches the free surface, the epidermal cell becomes more and more flattened and scaly in form and more horny in consistency, till at length it is simply rubbed off. Hence we find free epithelial cells in the mucus of the mouth, in the intestine, and in the genito-urinary tract, as well as on the surface of the outer skin.

In the case of chemical transformation the cell contents undergo a degeneration which, though it may sometimes be pathological, is very often 
a normal process. Thus we have cells by fatty metamorphosis producing oil globules in the secretion of milk, fatty degeneration of the muscular fibers of the uterus after the birth of the fetus. Calcareous degeneration is common in the cells of many cartilages.

As the cells approach decay and death their normal physiological processes diminish in intensity and finally cease. This occurs early in the transformation and function is lost before the cell form is destroyed beyond recognition.

\section{THE STRUCTURE OF THE ELEMENTARY TISSUES.}

In the differentiation of the protoplasm of the body, great masses of cells are formed of the same elemental structure and typical functional properties. These are the elementary tissues. The tissues alone or in combination in varying proportions constitute the organs of the body. These elementary tissues are: The Epithelial, The Connective, The Muscular, and The Nervous Tissues. To these four some would add a fifth, looking upon the Blood and Lymph, containing, as they do, formed elements in a fluid menstruum, as a distinct tissue.

\section{THE EPITHELIAL TISSUES.}

Epithelium is a tissue composed almost wholly of cells, with a very small amount of intercellular substance which glues the cells together. In general it includes all those cellular membranes which cover either an external or an internal free surface, together with the cellular portions of the glands which are connected with, or developed from, these free surfaces.

Epithelium clothes (I) the whole exterior surface of the body, forming the epidermis with its appendages; becoming continuous at the chief orifices of the body -nose, mouth, anus, and urethra-with (2) the epithelium which lines the whole length of the respiratory, alimentary, and genito-urinary tracts, together with the ducts and secretory cells of their various glands. Epithelium also lines the cavities of (3) the brain and the central canal of the spinal cord, (4) the serous and synovial membranes, and (5) the interior of blood vessels and lymphatics.

Epithelial cells vary in size and shape, pressure being the main factor in this variation. The protoplasm may be granular, reticular, or fibrillar in appearance. The nucleus is spherical or oval, usually there is only one, but there may be two or more present.

Epithelial tissues are non-vascular, that is to say, do not contain blood vessels, but in some varieties minute channels exist between the cells of certain layers. Nerve fibers are supplied to the cells of many epithelia.

\section{CLASSIFICATION OF EPITHELIA.}

As to form and arrangement of cells.

I. Epithelia in the form of membranes (covering surfaces).

I. Simple epithelium. Cells only one layer in thickness. 
(I) Squamous or pavement. Cells flattened.

(a) Non-ciliated. Alveoli of lungs, also includes endothelium, lining the blood vessels, and mesothelium, lining the large serous spaces.

(b) Ciliated. The peritoneum of some forms at breeding season.

(2) Cubical epithelia. Cells with the three dimensions approximately equal, mainly glandular.

(a) Non-ciliated. The usual type. It is found lining both ducts and secretory portions of most glands, the pigmented layer of the retina, etc.

(b) Ciliated. Not common. Lining of some of the smaller bronchial tubes.

(3) Columnar. Cells may be cylindrical, conical, or goblet-shaped.

(a) Non-ciliated. Intestinal.

(b) Ciliated. Fallopian tube and uterus.

(c) Pseudo-stratified. Smaller bronchi, nasal duct, etc.

2. Stratified epithelia. Cells more than one layer in thickness.

(I) Squamous. Surface cells flattened.

(a) Non-ciliated. Skin, mouth, vagina, etc.

(b) Ciliated. Pharynx of embryo.

(2) Columnar. Surface cells columnar.

(a) Non-ciliated. Portions of male urethra.

(b) Ciliated. Trachea, bronchi, etc.

II. Epithelia not in the form of membranes, but in solid masses or cords, usually glandular.

(I) Cells spheroidal. Ova.

(2) Cells polyhedral. Liver, suprarenal, etc.

Epithelia, classified mainly as to function.

I. Protective. Skin, mouth, alimentary canal.

I. Cornified. Skin, nails, hair.

2. Cuticular border. Columnar cells of intestine.

II. Glandular.

I. Secretory. Cells of salivary glands, pancreas, etc.

2. Excretory. Cells of kidney.

3. Absorptive. Cells of alimentary canal.

III. Sensory Epithelium. Cells of olfactory membrane, organ of Corti, taste buds, etc.

IV. Reproductive. Sex cells.

V. Pigmented. Pigmented layer of retina.

VI. Ciliated. Trachea, uterus, Fallopian tube, etc.

Only a few of the more important of the above-mentioned types of epithelium will be described here.

Simple Epithelium.-Simple Squamous.-This form of epithelium 
is found arranged in a single layer of flattened cells, for example, the lining of the alveoli of the lungs and of the descending arm of Henle's loop of the kidney tubule. Aside from endothelium as mesothelium it has very limited distribution in man. Endothelium and mesothelium are typical simple squamous epithelia. They consist of much flattened cells with clear or slightly granular protoplasm and oval bulging nuclei, the edges of the cells are peculiarly wavy or serrated.

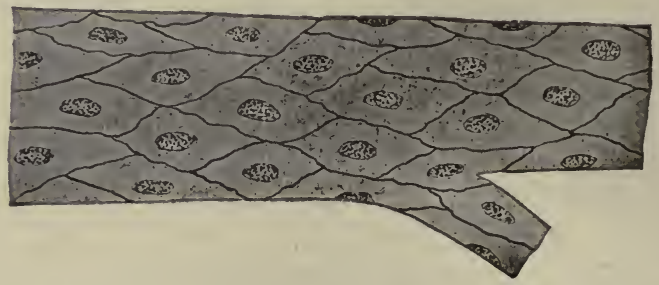

F1G. 20. - The Endothelium of a Small Blood Vessel. Silver-nitrate stain. $\times 350$.

The presence of endothelium in any locality may be demonstrated by staining with silver nitrate, which brings into view the intercellular cement substance. When a small portion of a perfectly fresh serous membrane, for example, figure 20 , is immersed for a few minutes in a solution of silver nitrate, and exposed to the action of light, the silver is precipitated in the intercellular cement substance, and the endothelial cells are thus mapped out by fine, dark, and generally sinuous lines of extreme delicacy.

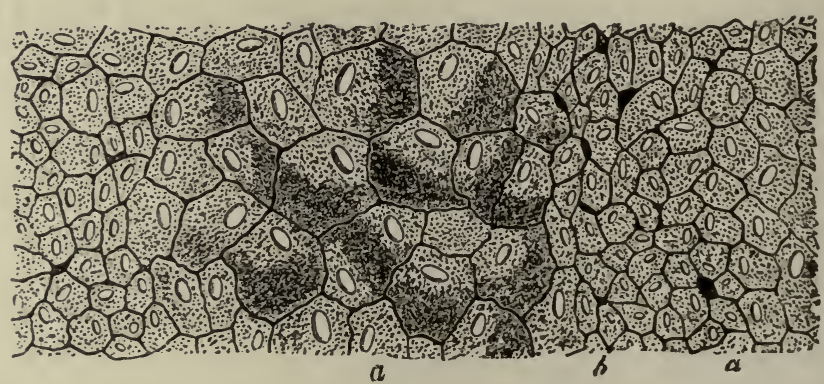

Fig. 2I.-Abdominal Surface of Central Tendon of the Diaphragm of Rabbit, showing the general polygonal shape of the endothelial cells; each cell is nucleated. (Klein.) $\times 300$.

Endothelial cells in certain situations may be ciliated, e.g., those of the mesentery of the frog, especially during the breeding season.

On those portions of the peritoneum and other serous membranes in which lymphatics abound, apertures, figure 22, are found surrounded by small, more or less cubical, cells. These apertures are called stomata. They are particularly well seen in the anterior wall of the great lymph sac of the frog, figure 22, and in the omentum of the rabbit. These are really the open mouths of lymphatic vessels or spaces, and through them lymph corpuscles and the serous fluid from the serous cavity pass into the lymphatic system. 
Simple Non-ciliated Columnar Epithelium, figure 23, lines, $a$, the mucous membrane of the stomach and intestines as a single layer, from the cardiac orifice of the stomach to the anus, and $b$, wholly or in part all the ducts of the glands opening on its free surface, and $c$, many gland ducts in other regions of the body, e.g., mammary, salivary, etc. The intracellular and intra-

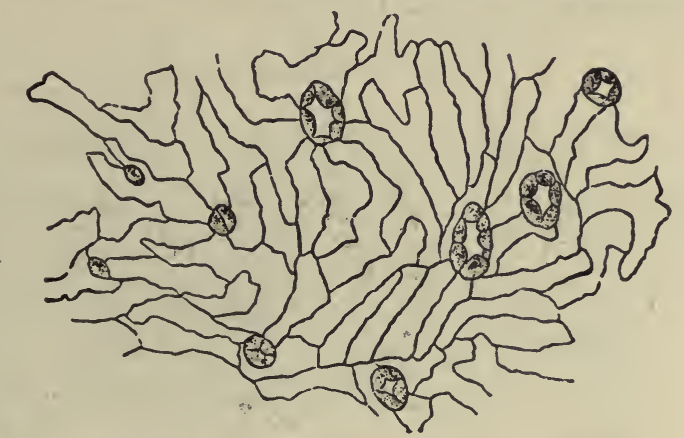

FIG. 22.-Peritoneal Surface of a Portion of the Septum of the great Lymph Sac of Frog. The stomata, some of which are open, some collapsed, are surrounded by endothelial cells Klein.) $\times 160$.

nuclear networks are well developed, and in some cases the spongioplasm is arranged in rods or longitudinal striæ at one part of the cell, as in the cells of the ducts of salivary glands. The protoplasm of columnar cells may be vacuolated and may also contain fat or other substances seen in the form of granules. Certain columnar cells transform a large part of their protoplasm

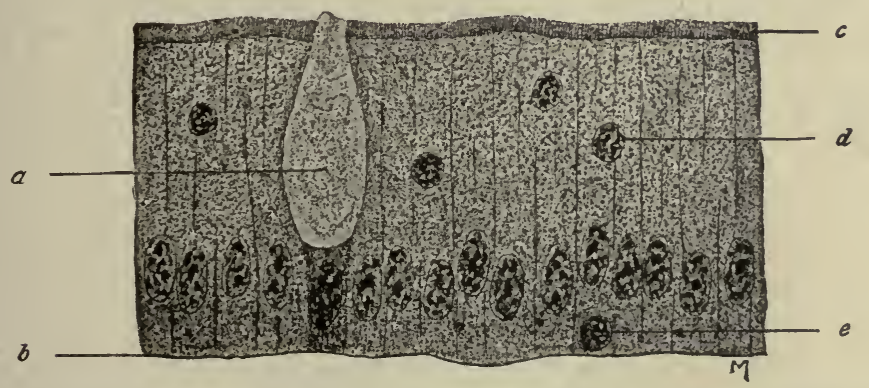

Frg. 23.- Simple Columnar Epithelial Cells from the Human Intestinal Mucous Membrane. $a$, Mucous (goblet) cell; $b$, basement membrane; $c$, cuticle; $d$, leucocyte nucleus; $e$, germinating cell. (Bailey.)

into mucin, goblet cells, figure 24 , which is discharged by the open mouth of the goblet, leaving only a nucleus surrounded by the remains of the protoplasm in its narrow stem. This transformation is a normal process which is continually going on during life, the cells themselves being supposed to regenerate into their original shape.

Stratified Epithelium.-The term stratified epithelium is employed 
to describe the type found in the skin or its derivatives in which the cells forming the epithelium are arranged in a considerable number of superimposed layers. The shape and size of the cells of the different layers, as well as the number of layers, vary in different situations. Thus the superficial cells may be either squamous or columnar in shape and the deeper cells range from polygonal to columnar in form.
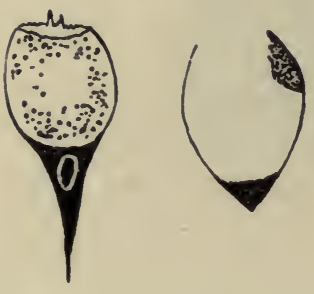

FIG. 24 .

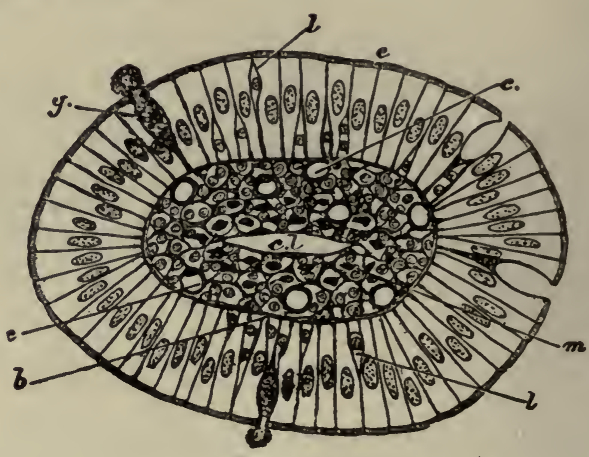

Fig. 25.

Fig. 24.-Goblet Cells. (Klein.)

FIG. 25.-Cross-section of a Villus of the Intestine. $e$, Columnar epithelium with striated border; $g$, goblet cell, with its mucus partly extruded; $l$, lymph corpuscles between the epithelial cells; $b$, basement membrane; $c$, sections of blood capillaries; $m$, section of plain muscle fibers; $c l$, central lacteal. (Schäfer.)

Stratified Squamous. - The intermediate cells are polygonal in shape and approach more to the flat variety the nearer they are to the surface, and to the columnar as they approach the lowest layer. In many of the deeper layers of epithelium in the mouth and skin, the outline of the cells is very irregular, in consequence of processes passing from cell to cell across these intervals. Such cells, figure 28 , are termed "prickle" cells. These "prickles" are the

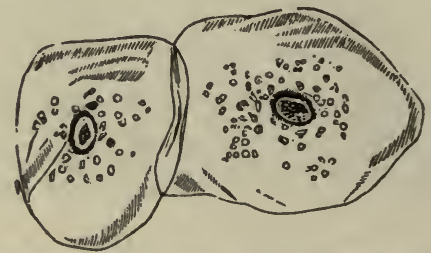

FIg. 26. -Squamous Epithelium Scales from the Inside of the Mouth. $\times 260 . \quad$ (Henle.)

intercellular bridges which run across from cell to cell, the interstices being filled by the transparent intercellular lymph. When this increases in quantity in inflammation the cells are pushed further apart, and the connecting fibrils or "prickles" are elongated and more clearly visible.

The columnar cells of the deepest layer are distinctly nucleated; they multiply rapidly by division; and as new cells are formed beneath, they press the older cells forward, to be in turn pressed forward themselves toward the 
surface, gradually altering in shape and chemical composition until they die and are cast off from the surface.

Stratified squamous epithelium is found in the following situations: I. Forming the epidermis, covering the whole of the external surface of the body; 2. Covering the mucous membrane of the nose, tongue, mouth, pharynx, and esophagus; 3. As the conjunctival epithelium, covering the cornea; 4 . Lining the vagina and the vaginal part of the cervix uteri.

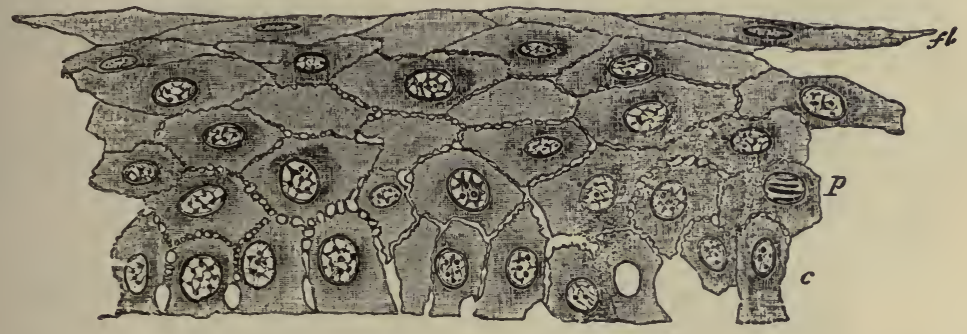

FIG. 27.-Vertical Section of the Stratified Epithelium Covering the Front of the Cornea. Highly magnified. (Schäfer.) $c$, Lowermost columnar cells; $p$, polygonal cells above these; $f$, flattened cells near the surface. The intercellular channels, bridged by minute cell processes, are well seen.

Stratified Columnar Epithelium.-In this type of epithelium, the surface cells alone are columnar, the deeper cells being irregular in shape. From the surface cells long processes extend down among the underlying cells. This type of epithelium is usually ciliated, as in the trachea, bronchi, etc., but may be non-ciliated, as in portions of the human male urethra.

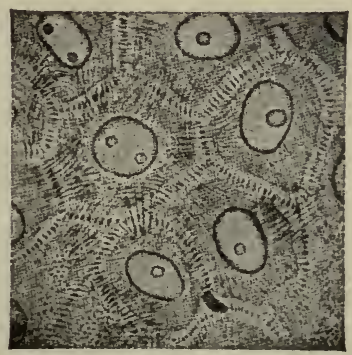

FIG. 28.-Epithelial Cells from the Stratum Spinosum of the Human Epidermis, Showing "Intercellular Bridges." × 700 . (Szymonowicz.)

Transitional Epithelium.-This is a stratified epithelium consisting of only three or four layers of cells. The superficial cells are large and flat, often containing two nuclei. The under surfaces of these cells are hollowed out, and into these depressions fit the large ends of the pyriform cells which form the next layer. Beneath the layer of pyriform cells are from one to 
four layers of polyhedral cells. This type of epithelium occurs in the bladder, ureter, and pelvis of the kidney.

Specialized Epithelium.-Glandular epithelium forms the active secreting agent in the glands; the cells are usually spheroidal, but may be polyhedral from mutual pressure, or even columnar; their protoplasm is generally occupied by the materials which the gland secretes. Examples of glandular

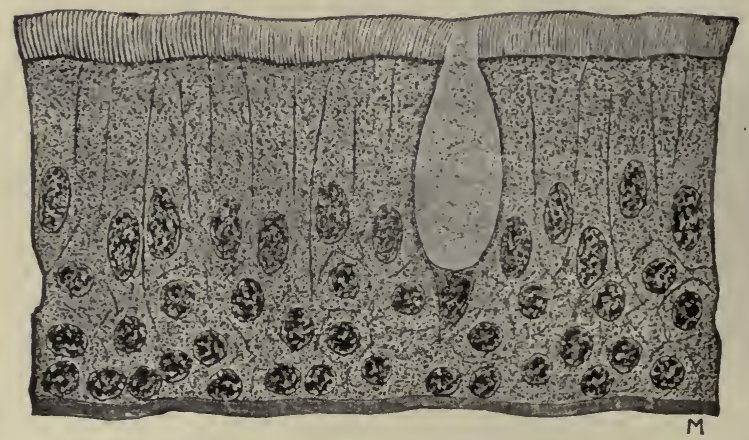

FIG. 29.- Stratified Columnar Ciliated Epithelium from the Human Trachea. A mucous (goblet) cell also is present.

epithelium are to be found in the liver, figure $3 \mathrm{I}$, in the secreting tubes of the kidney, and in the salivary, figure 32 , and gastric glands.

Ciliated epithelium consists of cells which are generally cylindrical in form, figures 29,30 , but may be spheroidal or even squamous.

This form of epithelium lines: $a$. The mucous membrane of the respiratory tract beginning just beyond the nasal aperture, and completely covers the nasal passages, except the upper part to which the olfactory nerve is

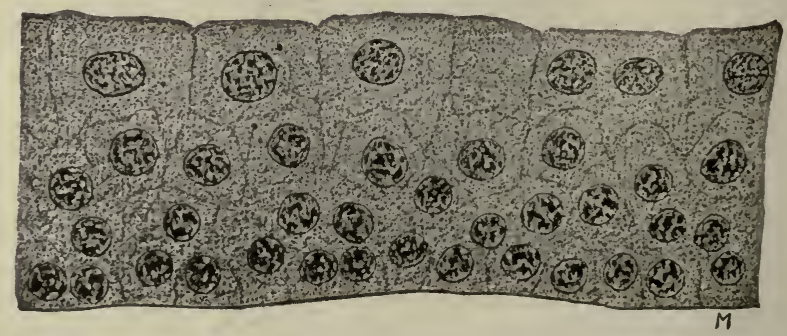

Frg. 30.- Transitional Epithelium from the Human Bladder. (Bailey.)

distributed, and also the sinuses and ducts in connection with it and the lachrymal sac, the upper surface of the soft palate and the naso-pharynx, the Eustachian tube and tympanum, the larynx, except over the vocal cords, to the finest subdivisions of the bronchi. In part of this tract, however, the epithelium is in several layers, of which only the most superficial is ciliated, 
so that it should more accurately be termed transitional, page 24 , or stratified. $b$. Some portions of the generative apparatus in the male, viz., lining the vasa efferentia of the testicle, and their prolongations as far as the lower

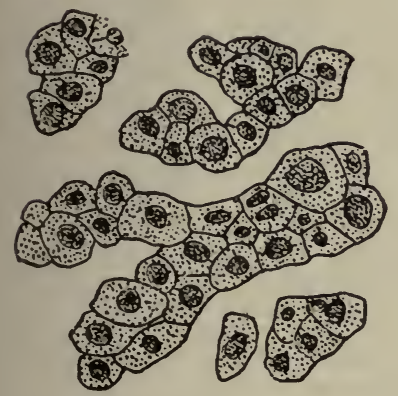

FIG. 3 I.

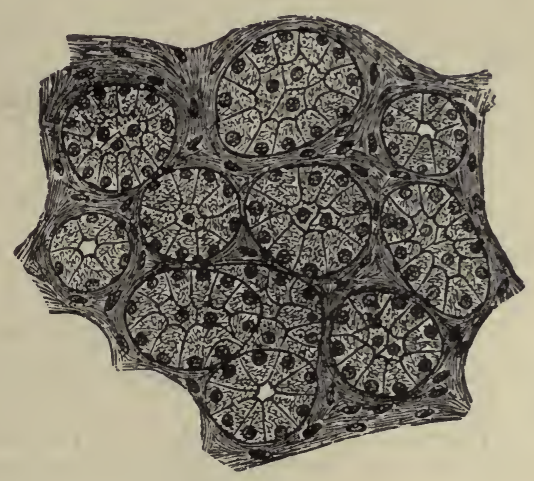

FIG. 32.

FIG. 3 x.-A Small Piece of the Liver of the Horse. (Cadiat.)

FIG. 32.-Glandular Epithelium. Small lobule of a mucous gland of the tongue, showing nucleated glandular cells. $\times 200 . \quad$ (V. D. Harris.)

end of the epididymis, and much of the vas deferens; in the female, $c$, commencing about the middle of the neck of the uterus, and extending throughout the uterus and Fallopian tubes to their fimbriated extremities, and even for

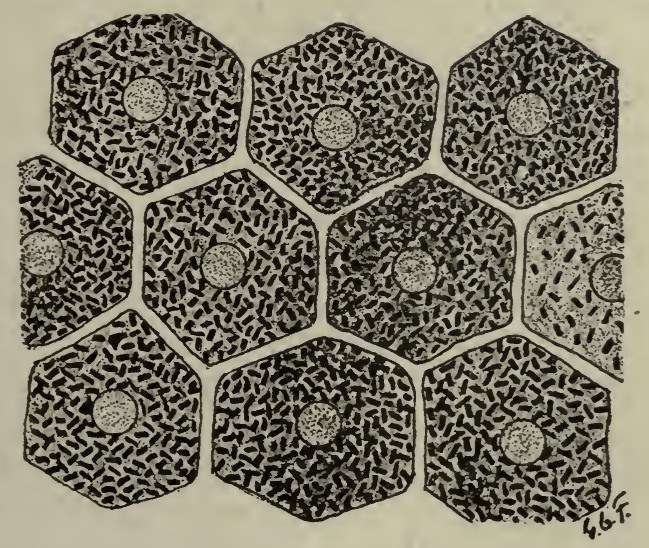

FrG. 33.-Specialized Pigmented Epithelial Cells of Retina.

a short distance on the peritoneal surface of the latter. $d$. The ventricles of the brain and the central canal of the spinal cord are clothed with ciliated epithelium in the child, but in the adult this epithelium is limited to the central canal of the cord.

The cilia themselves are fine rounded or flattened homogeneous processes. 
According to some observers, these processes are connected with longitudinal fibers which pass to the other end of the cell, but which are not connected with the nucleus.

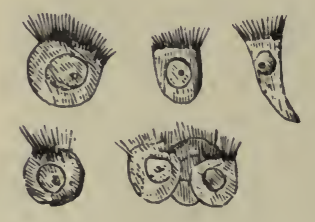

Fig. 34 .

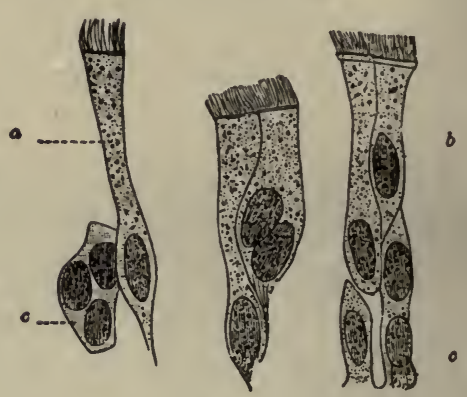

Fig. 35 .

Fig. 34. - Spheroidal Ciliated Cells from the Mouth of the Frog. $\times 300$ diameters. (Sharpey.)

FIG. 35.-Ciliated Epithelium from the Human Trachea. a, Large, fully formed cell; $b$, shorter cell; $c$, developing cells with more than one nucleus. '(Cadiat.)

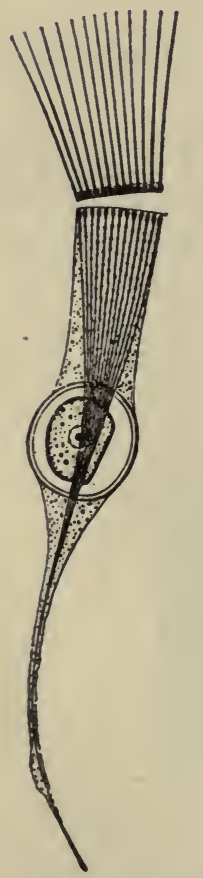

FIG. 36.-Ciliated Cell of the Intestine of a Mollusk. (Engelmann.)
Functions of Epithelium.-According to function, epithelial cells may be classified as: I, protective, e.g., in the skin, mouth, blood vessels, etc.; 2, protective and motive, ciliated epithelium; 3, secreting, glandular epithelium; 4, germinal, as epithelium of testicle producing spermatozoa; 5, absorbing and secreting, e.g., epithelium of intestine; 6, sensory, e.g., olfactory cells, organ of Corti.

Epithelium forms a continuous smooth investment over the whole body, being thickened into a hard, horny tissue at the points most exposed to pressure, and developing various appendages, such as hairs and nails. Epithelium lines also the sensorial surfaces of the eye, ear, nose, and mouth, and thus serves as the medium through which all impressions from the external world-touch, smell, taste, sight, hearing-reach the delicate nerve endings, whence they are conveyed to the brain.

The ciliated epithelium which lines the air passages serves to promote currents of the air in the bronchial tubes and to propel fluids and minute particles of solid matter out of the body. In the case of the Fallopian tube, the cilia assist the progress of the ovum toward the cavity of the uterus.

The epithelium of the various glands, and of the whole intestinal tract, has the power of secretion, i.e., of producing certain materials by processes of metabolism in its protoplasm. 
Epithelium is likewise concerned in the processes of transudation, diffusion, and absorption.

\section{THE CONNECTIVE TISSUES.}

This group of tissues forms the skeleton with its various connectionsbones, cartilages, and ligaments-and also affords a supporting framework and investment to the various organs composed of nervous, muscular, and glandular tissue. Its chief function is the mechanical one of support, and for this purpose it is so intimately interwoven with nearly all the textures of the body that if all other tissues could be removed, and the connective tissues left, we should have a wonderfully exact model of almost every organ and tissue in the body.

General Structure of Connective Tissue.-The connective tissue is made up of two chief elements, namely, cells and intercellular or formed substance.

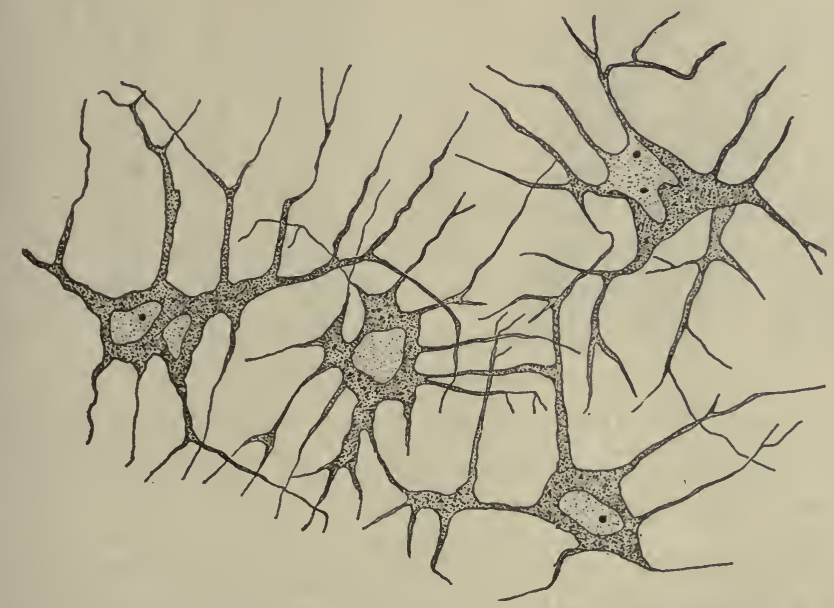

FIG. 37.- - Horizontal Preparation of the Cornea of Frog, Stained in Gold Chloride; showing the network of branched corneal corpuscles. The ground substance is completely colorless. $\times 400 . \quad$ (Klein.)

Cells.-The cells are usually of an oval shape, often with branched processes, which are united to form a network. They are most readily observed in the cornea, in which they are arranged, layer above layer, parallel to the free surface. They lie in spaces in the intercellular or ground substance, which form by anastomosis a system of branching canals freely communicating, figure 37 .

The flattened tendon corpuscles which are arranged in long lines or rows parallel to the fibers belong to this class of cells, figure 39 .

These branched cells often contain pigment granules, giving them a dark appearance; they form one variety of pigment cell. Pigment cells of this kind are found in the outer layers of the choroid. In many of the lower ani- 
mals, such as the frog, they are found widely distributed not only in the skin, but also in internal parts, the mesentery, sheaths of blood vessels, etc. Under the action of light, electricity, and other stimuli, the pigment granules become massed in the body of the cell, leaving the processes quite hyaline; if the stimulus be removed, they will gradually be distributed again throughout the processes. Thus the skin in the frog is sometimes uniformly dusky and sometimes quite light colored, with isolated dark spots.

Intercellular Substance.-This is fibrillar, as in the fibrous tissues and in certain varieties of cartilage; or homogeneous, as in typical mucoid tissue.

The fibers composing the former are of two kinds, white fibrous and yellow elastic tissue.

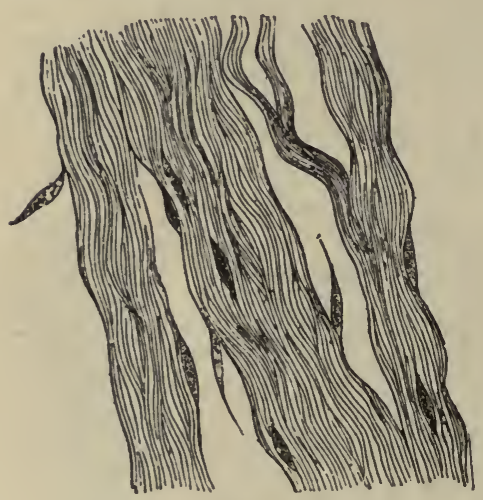

FIG. 38.

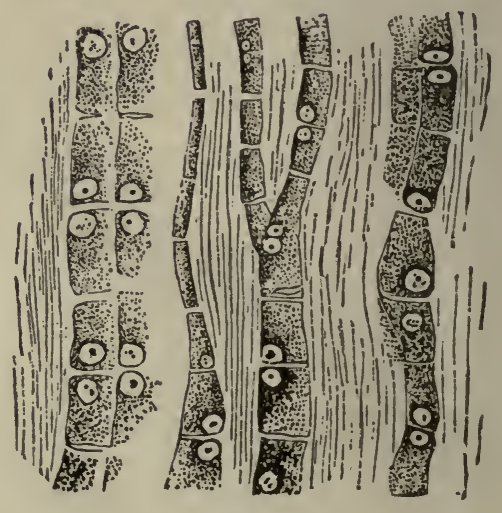

FIG. 39.

FIG. 38.-Mature White Fibrous Tissue of Tendon, Consisting Mainly of Fibers with a Few Scattered Fusiform Cells. (Stricker.)

FIG. 39.-Caudal Tendon of Young Rat, Showing the Arrangement, Form, and Structure of the Tendon Cells. $\times 300$. (Klein.)

The chief varieties of connective tissues may be thus classified:

White fibrous.

Elastic.

Areolar.

Gelatinous.

Adenoid or retiform.

Adipose.

Neuroglia.

Cartilage.

r. Hyaline.

2. White fibrous.

3. Elastic.

Bone and dentine.

The White Fibrous Tissue.-It is found typically in tendon; also in ligaments, in the periosteum and perichondrium, the dura mater, the peri- 
cardium, the sclerotic coat of the eye, the fibrous sheath of the testicle, in the fascix and aponeuroses of muscles, and in the sheaths of lymphatic glands.

Structure.-To the naked eye, tendons and many of the fibrous membranes, when in a fresh state, present an appearance as of watered silk. This is due to the arrangement of the fibers in wavy parallel bundles. Under the microscope the tissue appears to consist of long, often parallel, bundles of fibers of different sizes. The cells in tendons, figure 39, are arranged in long chains in the ground substance separating the bundles of fibers, and are more or less regularly quadrilateral with large round nuclei containing nucleoli, generally placed so as to be contiguous in two cells. Each of these cells consists of a thick body from which processes pass in various directions into, and partially fill up the spaces between, the bundles of fibers. The rows of cells are separated from one another by lines of cement substance.

Yellow Elastic Tissue.-Yellow elastic tissue is found chiefly in the ligamentum nuchæ of the ox, horse, and other animals; the ligamenta subflava of man; the arteries, constituting the fenestrated coat of Henle; the veins in the lungs and trachea; the stylo-hyoid, thyro-hyoid, and cricothyroid ligaments; in the true vocal cords; and in areolar tissue.

Structure.-Elastic tissue occurs in various forms, from a structureless, elastic membrane to a tissue whose chief constituents are bundles of fibers crossing each other at different angles; when seen in bundles elastic fibers are yellowish in color, but individual fibers are not so distinctly colored. The varieties of the tissue may be classified as follows:

a. Fine elastic fibrils, which branch and anastomose to form a network. This variety of elastic tissue occurs chiefly in the skin and mucous membranes, in subcutaneous and submucous tissue, in the lungs and true vocal cords.

$b$. Thick fibers, sometimes cylindrical, sometimes flattened, which branch, anastomose and form a network: these are seen most typically in theligamenta subflava and also in the ligamentum nuchæ of such animals as the ox and horse, in which that ligament is largely developed, figure 40 .

A certain number of connective-tissue cells are found in the ground substance between the elastic fibers which make up this variety of connective tissue, page 33 .

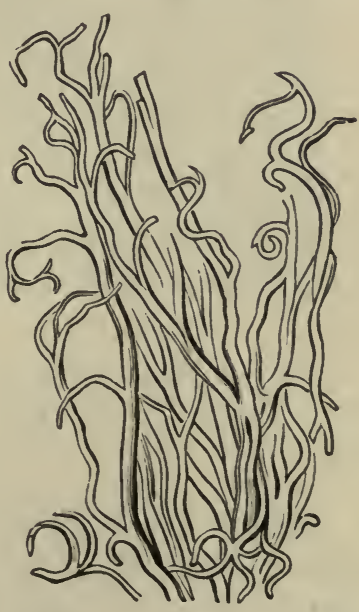

Fig. 40.-Elastic Fibers from the Ligamenta Subflava. $\times$ 200. (Sharpey.)

Areolar Tissue.- This variety of fibrous tissue has a very wide distribution and constitutes the subcutaneous, subserous, and submucous tissue. It is found in the mucous membranes, in the true skin, and in the outer 
sheaths of the blood vessels. It forms sheaths for muscles, nerves, glands, and the internal organs, and, penetrating into their interior, supports and connects the finest parts.

Structure.-To the naked eye it appears, when stretched out, as a fleecy, white, and soft meshwork of fine fibrils, with here and there wider films joining in it, the whole tissue being evidently elastic. The openness of the meshwork varies with the locality from which the specimen is taken. Under the microscope it is found to be made up of fine white fibers, which interlace in a most irregular manner, together with a variable number of elastic fibers. On the addition of acetic acid, the white fibers swell up, and become gelatinous in appearance; but as the elastic fibers resist the action of the acid, they may still be seen arranged in various directions, sometimes appearing to pass in a more or less circular or spiral manner round a small gelatinous mass of changed white fiber. The cells of areolar tissues are connective-tissue corpuscles.

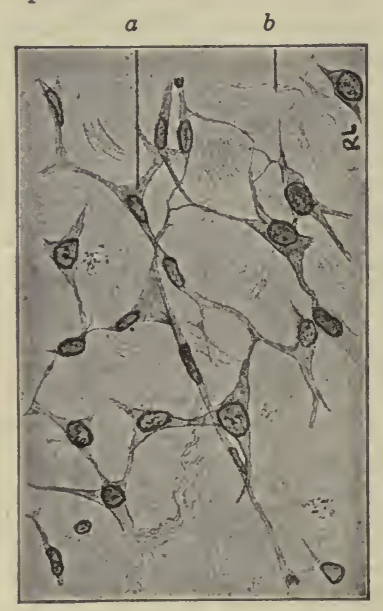

Fig. 4r.

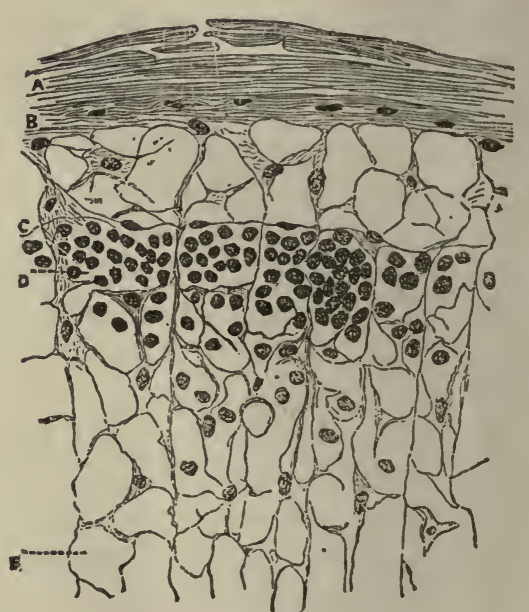

FIG. 42.

Fig. 4I. - Mucous Connective Tissue from the Umbilical Cord. $a$, Cells; $b$, fibrils.

FIG. 42.-Part of a Section of a Lymphatic Gland, from which the corpuscles have been for the most part removed, showing the Adenoid Reticulum. (Klein and Noble Smith.)

Gelatinous Tissue.-Gelatinous connective tissue forms the chief part of the bodies of such marine animals as the jelly-fish. It is found in many parts of the human embryo. It may be best seen in the "Whartonian jelly" of the umbilical cord and in the enamel organs of developing teeth.

Structure.-It consists of cells, which in the jelly of the enamel organ are stellate, embedded in a soft jelly-like intercellular substance which forms the bulk of the tissue.

Adenoid or Lymphoid Tissue.-Distribution.-This variety of tissue makes up the stroma of the spleen and lymphatic glands, and is found also 
in the thymus, in the tonsils, and in the follicular glands of the tongue; in Peyer's patches, in the solitary giands of the intestines, and in the mucous membranes generally.

Structure.-Adenoid or retiform tissue consists of a very delicate network of minute fibrils, figure 42 . The network of fibrils is concealed by being covered with flattened connective-tissue corpuscles, which may be readily dissolved in caustic potash, leaving the network bare. The network consists of white fibers, the interstices of which are filled with lymph corpuscles. The cement substance of adenoid tissue is very fluid.

Neuroglia.-This form of connective tissue found in the nervous system is described on page 78 .

Development of Fibrous Tissues.-In the embryo the place of the fibrous tissues is at first occupied by a mass of roundish cells, derived chiefly from

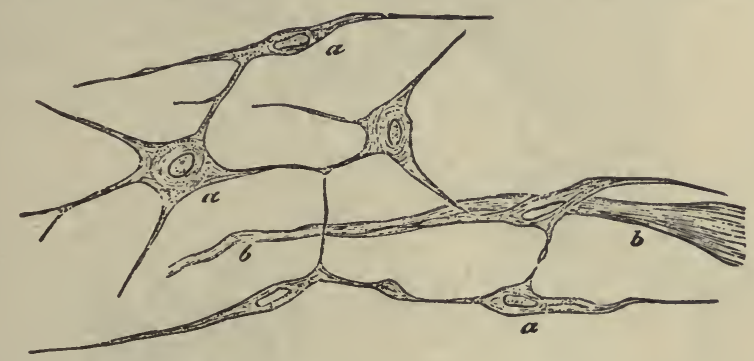

FIG. 43.--Portion of Submucous Tissue of Gravid Uterus of Sow. $a$, Branched cells, more or less spindle-shaped; $b$, bundles of connective tissue. (Klein.)

the mesoderm, but also from ectoderm and from entoderm. These develop either into a network of branched cells or into groups of fusiform cells, figure 43 .

The cells are embedded in a semifluid albuminous substance derived probably from the cells themselves. Later this formed material is converted into fibrils under the influence of the cells. The process gives rise to fibers arranged in the one case in interlacing networks, areolar tissue, in the other in parallel bundles, white fibrous tissue. In the mature forms of purely fibrous tissue not only the remnants of the cell substance, but even the nuclei, may disappear. The embryonic tissue, from which elastic fibers are developed, is composed of fusiform cells and a structureless intercellular substance. The fusiform cells dwindle in size and eventually disappear so completely that in mature elastic tissue hardly a trace of them is to be found; meanwhile the elastic fibers steadily increase in size.

Adipose Tissue.-In almost all regions of the human body a larger or smaller quantity of adipose or fatty tissue is present. Adipose tissue is almost always found seated in areolar tissue, and forms in its meshes little masses of unequal size and irregular shape, to which the term lobules is commonly applied. 
Structure.-Adipose tissue consists essentially of cells which present dark, sharply defined edges when viewed with transmitted light; each consisting of a structureless and colorless membrane or bag formed of the remains of the original protoplasm of the cell, filled with fat. A nucleus is always present in some part or other of the cell protoplasm, but in the ordinary condition of the loaded cell it is not easily or always visible. This membrane and the nucleus can generally be brought into view by extracting the fat with ether and by staining the tissue.

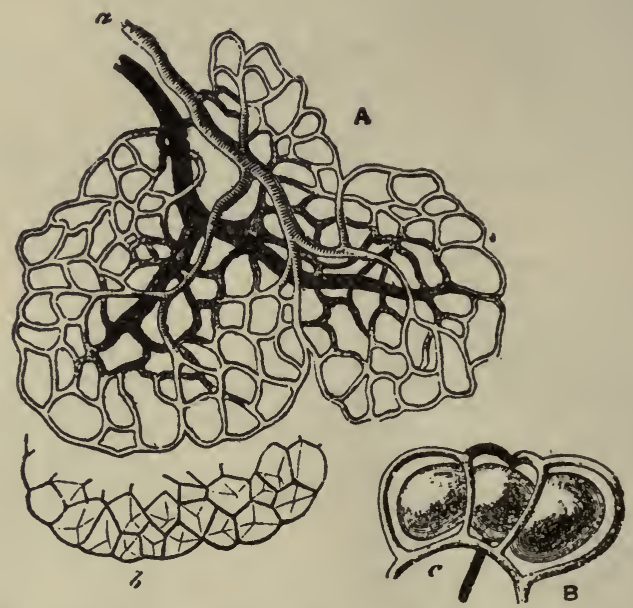

Fig. 44. - Blood Vessels of Adipose Tissue. A, Minute flattened fat lobule, in which the vessels only are represented. $a$, The terminal artery; $v$, the primitive vein; $b$, the fat vesicles of one border of the lobule separately represented. $\times$ Iо0. B, Plan of the arrangement of the capillaries. $c$, On the exterior of the vesicles; more high y magnified. (Todd and Bowman.)

The ultimate cells are held together by capillary blood vessels, figure 44; while the little clusters thus formed are grouped into small masses, and held so, in most cases, by areolar tissue. The oily matter contained in the cells is composed chiefly of the compounds of fatty acids with gylcerin, olein, stearin, and palmitin.

Development of Adipose Tissue.-Fat cells are developed from connectivetissue corpuscles. In the infra-orbital connective tissue there are cells exhibiting every intermediate gradation between an ordinary branched connective-tissue corpuscle and mature fat cells. Their developmental appearance is as follows: a few small drops of oil make their appearance in the protoplasm, and by their confluence a larger drop is produced, figure 45. This gradually increases in size at the expense of the original protoplasm of the cell, which becomes correspondingly diminished in quantity till in the mature cell it forms only a thin crescentic film with a nucleus closely pressed against the cell wall. Under certain circumstances this process may be reversed.

A large number of blood vessels are developed in adipose tissue, which 
subdivide until each lobule of fat contains a fine meshwork of capillaries ensheathing each individual fat globule, figure 44 .

Adipose tissue serves as a storehouse of combustible matter which may be reabsorbed into the blood when occasion requires, and, being used up in the metabolism of the tissues, may help to preserve the heat of the body.

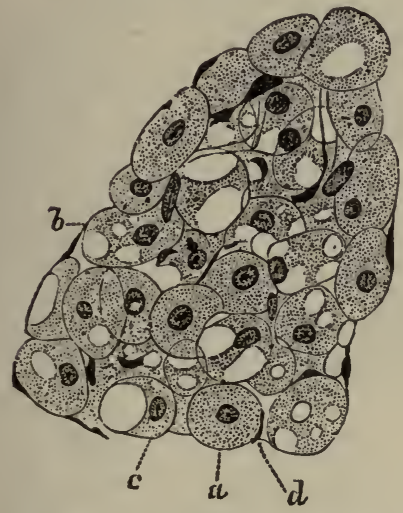

Fig. 45.-A Lobule of Developing Adipose Tissue from an Eight-months Fetus. $a$, Spherical or, from pressure, polyhedral cells with large central nucleus, surrounded by a finely reticulated substance staining uniformly with hematoxylin. $b$, Similar cells with spaces from which the fat has been removed by oil of cloves. $c$, Similar cells showing how the nucleus with enclosing protoplasm is being pressed toward periphery. $d$, Nucleus of endothelium of investing capillaries. (McCarthy.)

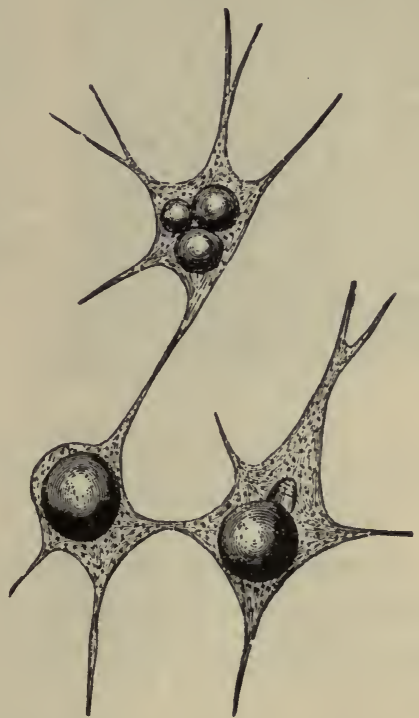

Fig. 46.-Branched Connectivetissue Corpuscles, Developing into Fat Cells. (Klein.)

That part of the fat which is situated beneath the skin must, by its want of conducting power, assist in preventing undue waste of the heat of the body by escape from the surface.

\section{CARTILAGE.}

All kinds of cartilage are composed of cells embedded in a substance called the matrix. The apparent differences of structure met with in the various kinds of cartilage are more due to differences in the character of the matrix than of the cells. With the exception of the articular variety, cartilage is invested by a thin but tough firm fibrous membrane called the perichondrium.

Cartilage exists in three different forms in the human body, viz., hyaline cartilage, yellow elastic cartilage, and white fibro-cartilage.

Hyaline Cartilage.-This variety of cartilage is met with largely in the human body where it invests the articular ends of bones, and forms the 
costal cartilages, the nasal cartilages, and those of the larynx with the exception of the epiglottis and cornicula laryngis, the cartilages of the trachea and bronchi.

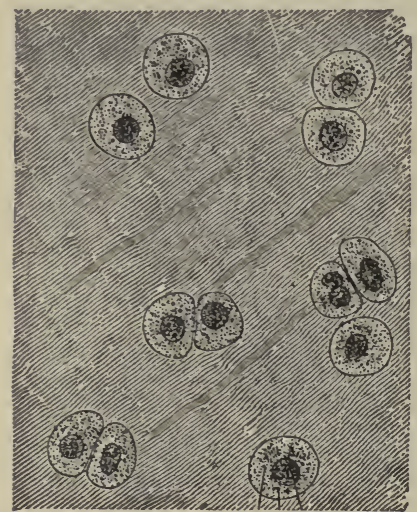

FIG. 47.

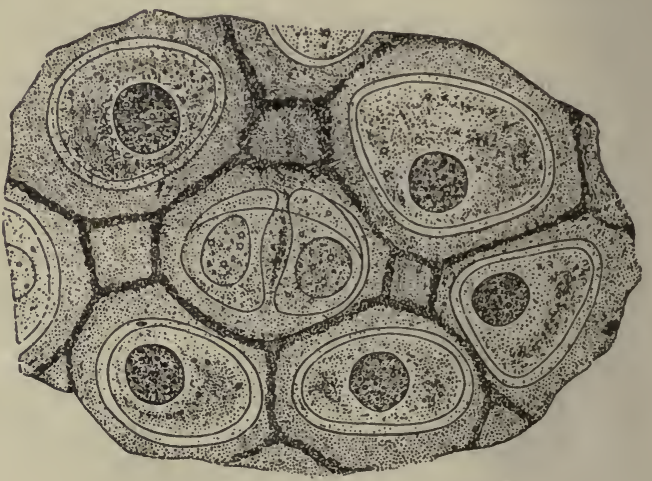

FIG. 48.

FIG. 47.-Hyaline Articular Cartilage (Human). The cell bodies entirely fill the spaces in the matrix. $\times 340$ diams. (Schäfer.)

FIG. 48.-Fresh Cartilage from the Triton. (A. Rollett.)

Structure.-Like other cartilages, it is composed of cells embedded in a matrix. The cells are irregular in shape, generally grouped together in patches, figure 47. The patches are of various shapes and sizes and placed

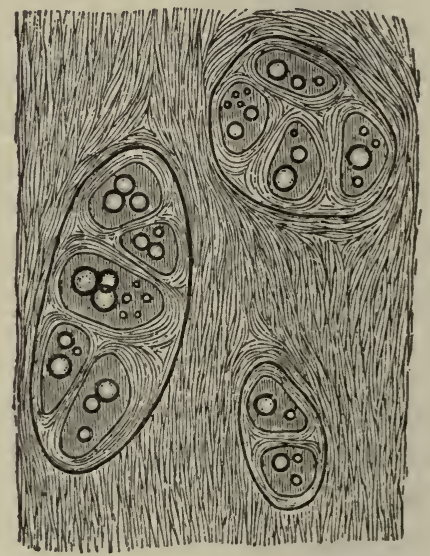

FIG. 49. - Costal Cartilage from an Adult Dog, showing the Fat Globules in the Cartilage Cells. (Cadiat.)

at unequal distances apart. They generally appear flattened near the free surface of the mass of cartilage, and more or less perpendicular to the surface in the more deeply seated portions. 
The intercellular substance of hyaline cartilage, when viewed fresh or after ordinary fixation, appears homogeneous. However, when subjected to special methods, the seemingly homogeneous intercellular substance can be shown to be made up of fibers, comparable with those found in white fibrous tissue, embedded in the homogeneous matrix.

In the hyaline cartilage of the ribs the cells are mostly larger than in the articular variety, and there is a tendency to the development of fibers

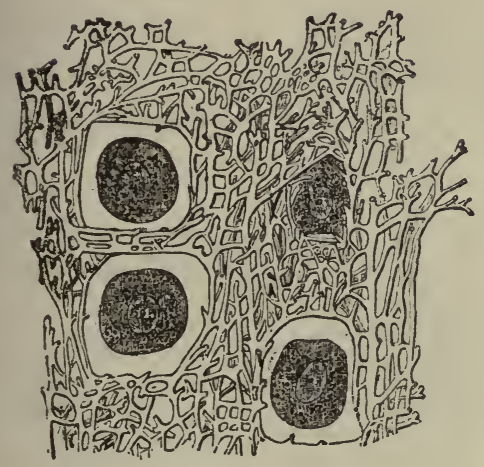

FIG. 50.- Yellow Elastic Cartilage of the

Ear. Highly magnified. (Hertwig.)

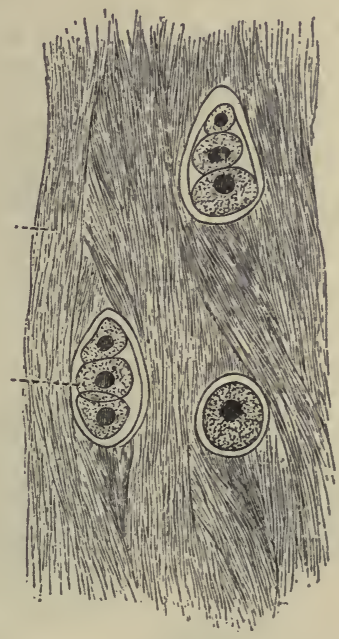

FIG. 51.-White Fibro-cartilage. (Cadiat.)

in the matrix, figure 49. The costal cartilages also frequently become calcified in old age, as also do some of those of the larynx.

In the fetus cartilage is the material of which the bones are first constructed; the "model" of each bone being laid down, so to speak, in this substance. In such cases the cartilage is termed temporary. It closely resembles the ordinary hyaline cartilage, but the cells are more uniformly distributed throughout the matrix.

Elastic and White Fibro-cartilage.-The first variety is found in the cartilage of the external ear; the latter in portions of the joints, the intervertebral cartilages, etc.

Structure.-Elastic and white fibro-cartilage are composed of cells and a matrix; the latter being made up almost entirely of fibers closely resembling those of fibrous connective tissue.

Development of Cartilage.-Cartilage is developed out of mesoblast cells with a very small quantity of intercellular substance. The cells multiply by fission within the cell capsules. 


\section{BONE.}

The characteristic of bone is that the matrix is solidified by a deposit of earthy salts, chiefly calcium phosphate, but some magnesium phosphate and calcium carbonate.

To the naked eye there appear two plans of structure in different bones, and in different parts of the same bone, namely, the dense or compact, and the spongy or cancellous tissue. In a longitudinal section of a long bone, as the humerus, the articular extremities are found capped on their surface by a thin shell of compact bone, while their interior is made up of the spongy or cancellous tissue. The shaft is formed almost entirely of a thick layer of the compact bone which surrounds a central canal, the medullary cavity, so called from its containing the medulla, or marrow. In the flat bones, the parietal bone or the scapula, a layer of cancellous structure lies between two layers of the compact tissue. In the short and irregular bones, as those of the carpus and tarsus, the cancellous tissue alone fills the interior, while a thin shell of compact bone forms the outside.

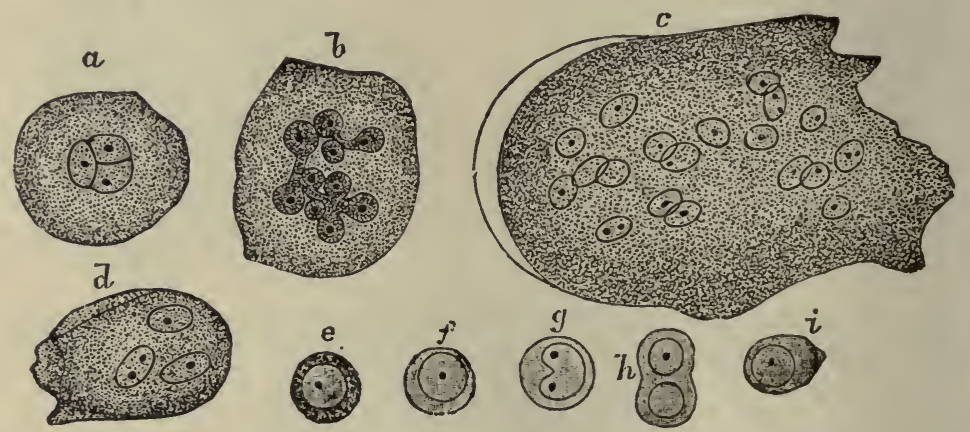

FIG. 52.-Cells of the Red Marrow of the Guinea-pig, highly magnified. a, A large cell, the nucleus of which appears to be partly divided into three by constrictions; $b$, a cell, the nucleus of which shows an appearance of being constricted into a number of smaller nuclei; $c$, a so-called giant cell, or myeloplaxe, with many nuclei; $d$, a smaller myeloplaxe, with three nuclei; $e-i$, proper cells of the marrow. (Schäfer.)

The Marrow.-There are two distinct varieties of marrow-the $r c d$ and the yellow.

Red marrow is that variety which occupies the spaces in the cancellous tissue; it is highly vascular, and thus maintains the nutrition of the spongy bone, the interstices of which it fills. It contains a few fat cells and a large number of marrow cells, many of which are undistinguishable from lymphoid corpuscles, and has for a basis a small amount of fibrous tissue. Among the cells are some nucleated cells containing hemoglobin like the blood corpuscles. There are also a few large cells with many nuclei, termed giant cells or myeloplaxes, which are probably derived from the ordinary marrow cells, figure 52 . 
Yellow marrow fills the medullary cavity of long bones, and consists chiefly of fat cells with numerous blood vessels. Many of its cells are in every respect similar to lymphoid corpuscles.

From these marrow cells, especially those of the red marrow, the red blood corpuscles are derived.

The Periosteum and Nutrient Blood Vessels.-The surfaces of bones, except the part covered with articular cartilage, are clothed by a

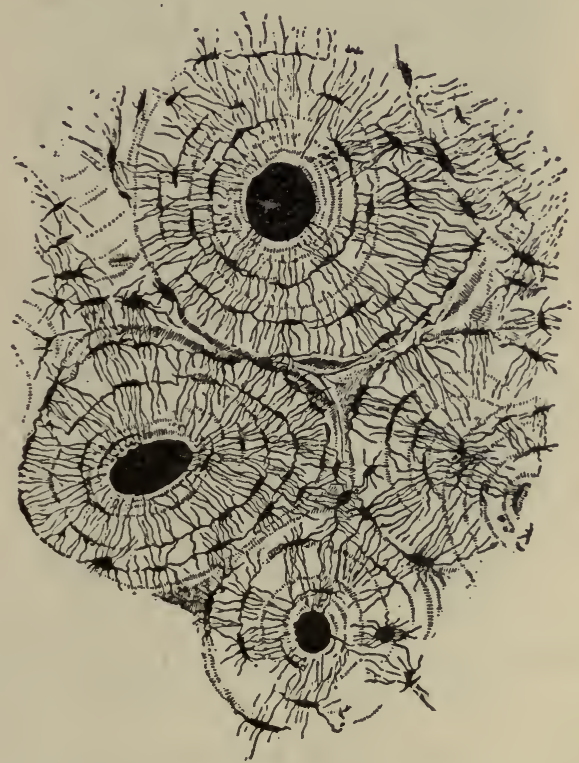

Fig. 53. - Transverse Section of Compact Bone (of humerus). Three of the Haversian canals are seen, with their concentric rings; also the lacunæ, with the canaliculi extending from them across the direction of the lamello.. The Haversian apertures were filled with débris in grinding down the section, and therefore appear black in the figure, which represents the object as viewed with transmitted light. The Haversian systems are so closely packed in this section, that scarcely any interstitial lamelle are visible. $\times{ }_{50}$. (Sharpey.)

tough, fibrous membrane, the periosteum, which is closely attached to the surface of the bone. Blood vessels are distributed in this membrane, and minute branches from these periosteal vessels enter the Haversian canals to supply blood to the solid part of the bone. The long bones are supplied also by a proper nutrient artery which, entering at some part of the shaft so as to reach the medullary canal, breaks up into branches for the supply of the marrow, from which again small vessels are distributed to the interior of the bone. Other small nutrient vessels pierce the articular extremities for the supply of the cancellous tissue. 
Microscopic Structure of Bone.-Notwithstanding the differences of arrangement just mentioned, the structure of all compact bone substance is found under the microscope to be essentially the same.

Examined with a rather high power its substance is found to contain a multitude of small irregular spaces, approximately fusiform in shape, called lacunce, with very minute canals or canaliculi, as they are termed, leading

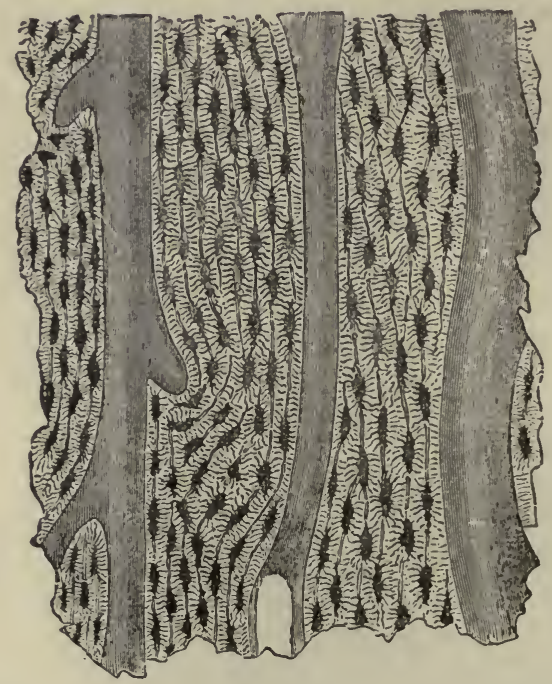

FIG. 54.-Longitudinal Section from the Human Ulna, Showing Haversian Canals, Lacunæ, and Canaliculi. (Rollett.)

from them, and anastomosing with similar prolongations from other lacunæ, figure 53. In very thin layers of bone, no other canals than these may be visible; but on making a transverse section of the compact tissue of a long bone, as the humerus or ulna, the arrangement shown in figure 53 can be seen. The bone seems mapped out into small circular districts, at or about the center of each of which is a hole, around which are concentric layers, the lamella, the lacunce and canaliculi following the same concentric distribution around the center, with which indeed they communicate.

On making a longitudinal section, the central holes are shown to be simply the cut extremities of small canals which run lengthwise through the bone, anastomosing with each other by lateral branches, figure 54, and are called Haversian Canals, after the name of the physician, Clopton Havers, who first accurately described them.

The Haversian Canals. - The average diameter of the Haversian canals is $50 \mu$. They contain blood vessels, and by means of them blood is conveyed to even the densest parts of the bone; the minute canaliculi and lacunæ 
absorbing nutrient matter from the Haversian blood vessels and conveying it still more intimately to the very substance of the bone which they traverse. The blood vessels enter the Haversian canals both from without from the periosteum, and from within from the medullary cavity or from the cancellous tissue. The arteries and veins usually occupy separate canals.

The lacunce are occupied by branched cells, the bone cells or bone corpuscles, figure 55 , which very closely resemble the ordinary branched connectivetissue corpuscles. The processes of the bone cells extend into the canaliculi. Each cell controls the nutrition of the bone immediately surrounding it. Each lacunar corpuscle communicates with the others in its surrounding

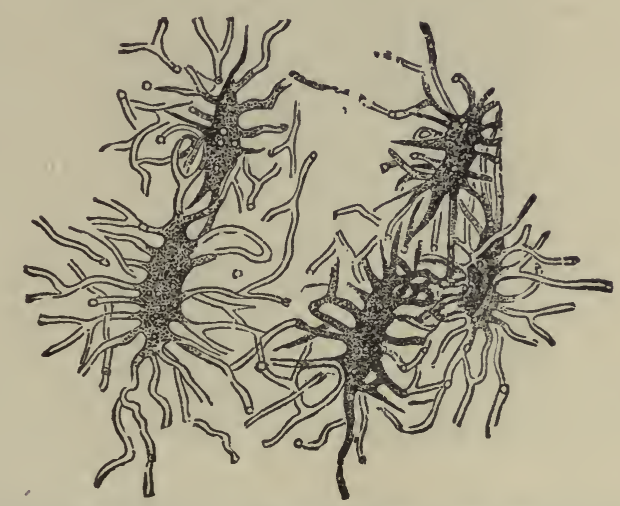

Fig. 55.-Bone Corpuscles with their Processes as seen in a Thin Section of Human Bone. (Rollett.)

district, and with the blood vessels of the Haversian canals by means of the ramifications just described.

It will be seen from the above description that bone bears a very close structural resemblance to what may be termed typical connective tissue. The bone corpuscles with their processes occupying the lacunæ and canaliculi correspond exactly to the cornea corpuscles lying in the branched spaces.

The Lamelle of Compact Bone.-In the shaft of a long bone three distinct sets of lamellæ can be clearly recognized: General or fundamental lamellæ, which are just beneath the periosteum and parallel with it, and around the medullary cavity; Special or Haversian lamellæ, which are concentrically arranged around the Haversian canals to the number of six to eighteen around each; Interstitial lamellæ, which connect the systems of Haversian lamellæ, filling the spaces between them, and consequently attaining their greatest development where the Haversian systems are few.

The ultimate structure of the lamellæ appears to be fibrous. A thin film peeled off the surface of a bone, from which the earthy matter has been removed by acid, is composed of a finely reticular structure, formed ap- 
parently of very slender fibers decussating obliquely, but coalescing at the points of intersection, as if here the fibers were fused rather than woven together. The reticular lamellæ are perforated by the perforating fibers of Sharpey, which bolt the neighboring lamellæ together, and may be drawn out when the latter are torn asunder, figure 56 . These perforating fibers originate from ingrowing processes of the periosteum, and in the adult still retain their connection with it.

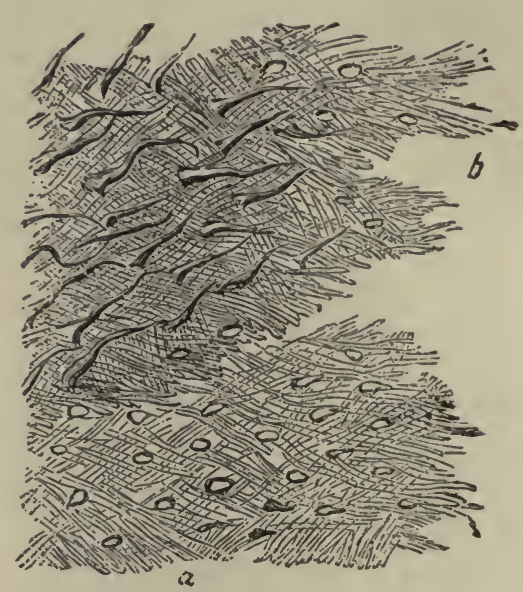

Fig. 56.-Lamellæ Torn off from a Decalcified Human Parietal Bone at some Depth from the Surface. $a, a$, Lamellæ, showing reticular fibers; $b, b$, darker part, where several lamellæ are superposed; $c$, perforating fibers. Apertures through which perforating fibers had passed, are seen especially in the lower part, $a, a$, of the figure. (Allen Thomson.)

Development of Bone.-From the point of view of their development all bones may be subdivided into two classes:

Those which are ossified directly in membrane or fibrous tissue, e.g., the bones forming the vault of the skull, parietal, frontal, and a certain portion of the occipital bones.

Those whose form, previous to ossification, is laid down in hyaline cartilage, e.g., humerus, femur, etc.

The process of development, pure and simple, may be best studied in bones which are not preceded by cartilage, i.e., membrane-formed. Without a knowledge of ossification in membrane it is difficult to understand the much more complex series of changes through which such a structure as the cartilaginous femur of the fetus passes in its transformation into the bony femur of the adult (ossification in cartilage).

Ossification in Membrane.-The membrane, afterward forming the periosteum, from which such a bone as the parietal is developed, consists of two layers, an external fibrous and an internal cellular or osteogenetic. 
The external layer consists of ordinary connective tissue, with branched corpuscles here and there between the bundles of fibers. The internal layer consists of a network of fine fibrils with nucleated cells and ground or cement substance between the fibrous bundles. It is more richly supplied with capillaries than the outer layer. The relatively large number of its cellular elements, together with the abundance of blood vessels, clearly mark it as the portion of the periosteum which is immediately concerned in the formation of bone.

In such a bone as the parietal there is first an increase in vascularity, followed by the deposition of bony matter in radiating spicula, starting from a center of ossification. These primary bony spicula are osteogenetic fibers, composed of osteogen, in which calcareous granules are deposited. Calcareous granules are deposited also in the interfibrillar matrix. By the junction of the osteogenetic fibers and their resulting bony spicula a meshwork of bone is formed. The osteoblasts, being in part retained within the bone trabeculæ thus produced, form bone corpuscles. Lime salts are deposited in the circumferential part of each osteoblast, and thus a ring of osteoblasts gives rise to a ring of bone with the remaining uncalcified portions of the osteoblasts embedded in it as bone corpuscles. At the same time the plate increases at the periphery by the extension of the bony spicula and by deposits taking place from the osteogenetic layer of the periosteum. The bulk of the primitive spongy bone is gradually converted into compact bony tissue of the Haversian systems.

Ossification in Cartilage.-Under this heading, taking the femur as a typical example, we may consider the process by which the solid cartilaginous rod which represents the bone in the fetus is converted into the hollow cylinder of compact bone with expanded ends formed of cancellous tissue in the adult long bone.

The fetal cartilage is sheathed in a membrane termed the perichondrium, which resembles the periosteum described above. Thus, the differences between the fetal perichondrium and the periosteum of the adult are such as usually exists between the embryonic and mature forms of connective tissue.

There are several steps in the transformation of the fetal cartilage to the adult bone, due to the fact that there is first an impregnation of the cartilage with lime salts, followed later by the resorption of this entire material with formation of the embryonic spongy bone, which is still later replaced by the permanent bone. The complicated phenomenon takes place in steps or sagest as follows:

Stage of Proliferation and Calcification.-The cartilage cells in and near the center of ossification become enlarged, proliferate, and arrange themselves in rows in the long axis of the fetal cartilage, figure 57 . Lime salts are next deposited in fine granules in the hyaline matrix of the cartilage, and this 
gradually becomes transformed into calcified trabeculx, figure 57 . The enlarging cartilage cells become more transparent, and finally disintegrate, the spaces occupied by them forming the primordial marrow cavities. During this stage the perichondrium has become the periosteum, and is beginning to deposit bone on the outside of the cartilage.

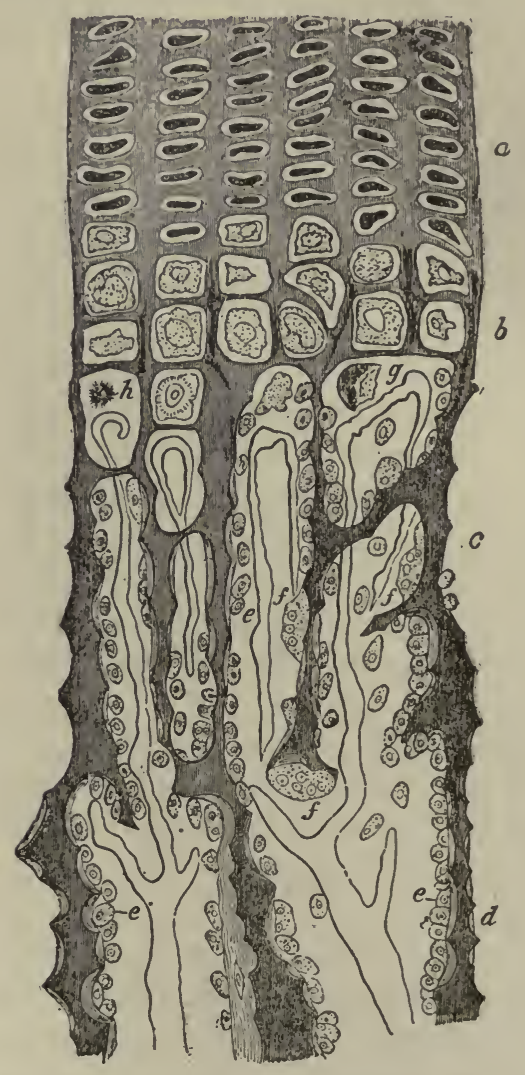

Fig. 57.-Developing Bone of Femur of the Rabbit. $\times 350$. a, Cartilage cells; $b$, cartilage cells enlarged in the region of calcifying matrix; $c, d$, trabeculæ of calcifying cartilage covered with $e$, osteoblasts; $f$, osteoclasts eroding the trabeculx; $g, h$, disappearing cartilage cells. The osteoblasts are seen to be depositing layers of bony substance. Loops of blood vessels extend to the limit of the region in which the bone is forming. (Schäfer, from Klein.)

Stage of Vascularization of the Cartilage.-Processes from the osteogenetic layer of the periosteum containing blood vessels break through the bone into the primordial marrow cavities and form the primary marrow, beginning at the centers of ossification, and spreading chiefly up and down the shaft.

Stage of Substitution of Embryonic Spongy Bone for Calcified Cartilage.The cells of the primary marrow arrange themselves as a continuous epi- 
thelium-like layer on the calcified trabeculæ and deposit a layer of bone, and ensheath them. The encased trabeculæ are gradually absorbed by the osteoclasts of Kölliker.

These stages are precisely similar to what goes on in the growing shaft of a bone which is increasing in length by the advance of the process of ossifi-

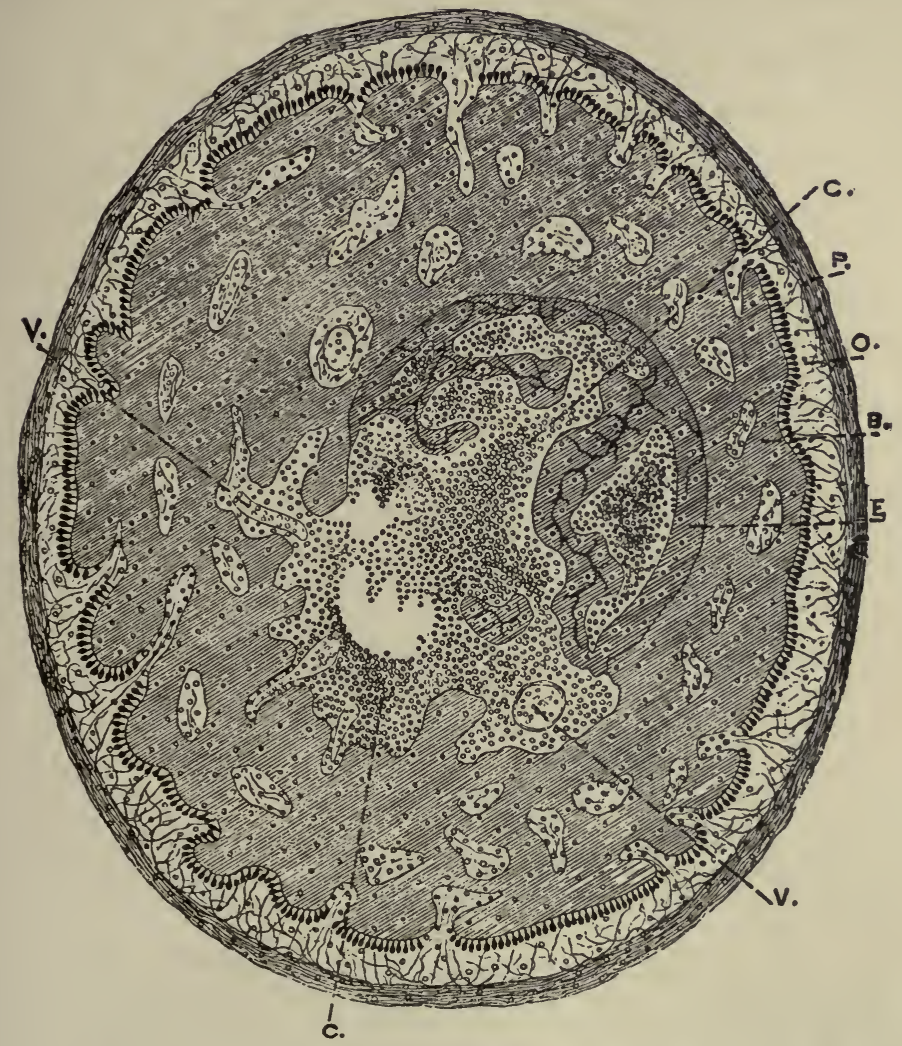

Frg. 58. - Transverse Section through the Tibia of a Fetal Kitten, semidiagrammatic. $\times$ 6o. P, Periosteum. O, Osteogenetic layer of the periosteum, showing the osteoblasts arranged side by side, represented as pear-shaped black dots on the surface of the newly formed bone. B, The periosteal bone deposited in successive layers beneath the periosteum and ensheathing E, the spongy endochondral bone; represented as more deeply shaded. Within the trabeculæe of endochondral spongy bone are seen the remains of the calcified cartilage trabeculæ represented as dark wavy lines. C, The medulla, with V, V, veins. In the lower half of the figure the endochondral spongy bone has been completely absorbed. (Klein and Noble Smith.)

cation into the intermediary cartilage between the diaphysis and epiphysis. In this case the cartilage cells become flattened and, multiplying by division, are grouped into regular columns at right angles to the plane of calcification while the process of calcification extends into the hyaline matrix between them. 
The embryonic spongy bone, formed as above described, is simply a temporary tissue occupying the place of the fetal rod of cartilage; the preceding stages show the successive changes at the center of the shaft. Periosteal bone is at the same time deposited in successive layers beneath the periosteum at the circumference of the shaft, exactly as described in the section on ossification in membrane, and thus a casing of periosteal bone is formed around the embryonic endochondral spongy bone. The embryonic spongy bone is absorbed, through the agency oi the osteoclasts, until the trabeculæ are replaced by one great cavity, the medullary cavity of the shaft.

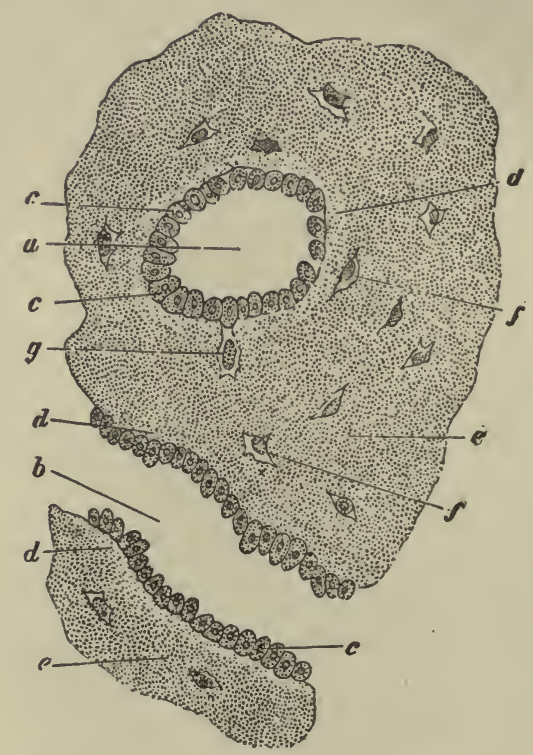

Fig. 59. - Transverse Section of Femur of a Human Embryo about Eleven Weeks Old. $a$, Rudimentary Haversian canal in cross-section; $b$, in longitudinal section; $c$, osteoblasts; $d$, newly formed osseous substance of a lighter color; $e$, that of greater age; $f$, lacunæ with their cells; $g$, a cell still united to an osteoblast. (Frey.)

Stage of Formation of Compact Bone.-The transformation of spongy periosteal bone into compact bone is effected in a manner exactly similar to that which has been described in connection with ossification in membrane, page 46 . The irregularities in the walls of the spongy periosteal bone are absorbed by the osteoclasts, while the osteoblasts which line them are developed in concentric layers, each layer in turn becoming ossified till the comparatively large space in the center is reduced to a well-formed Haversian canal, figure 59. When once formed, bony tissue grows to some extent interstitially, as is evidenced by the fact that the lacunæ are rather further apart in full-formed than in young bone. 
It will be seen that the common terms ossification in cartilage and ossification in membrane are apt to mislead, since they seem to imply two processes radically distinct. The process of ossification, however, is in all cases one and the same, all true bony tissue being formed from membrane, perichondrium or periosteum; but in the development of such a bone as the femur, lime salts are first of all deposited in the cartilage; this calcified cartilage, however, is gradually and entirely reabsorbed, replaced by bone formed from the periosteum. Thus calcification of the cartilaginous matrix precedes the real formation of bone. We must, therefore, clearly distinguish between calcification and ossification. The former is simply the infiltration of an animal tissue with lime salts, while ossification is the formation of true bone.

Growth of Bone.-Bones increase in length by the advance of the process of ossification into the cartilage intermediate between the diaphysis and epiphysis. The increase in length indeed is due entirely to growth at the two ends of the shaft. Increase in thickness in the shaft of a long bone occurs by the deposition of successive layers beneath the periosteum. If a thin metal plate be inserted beneath the periosteum of a growing bone it will soon be covered by osseous deposit, but if it be put between the fibrous and osteogenetic layers it will never become enveloped in bone, for all the bone is formed beneath the latter.

\section{THE TEETH.}

During the course of his life, man, in common with most other mammals, is provided with two sets of teeth; the first set, called the temporary or milkteeth of infancy, are shed and replaced by the second or permanent set.

\section{Temporary Teeth.}

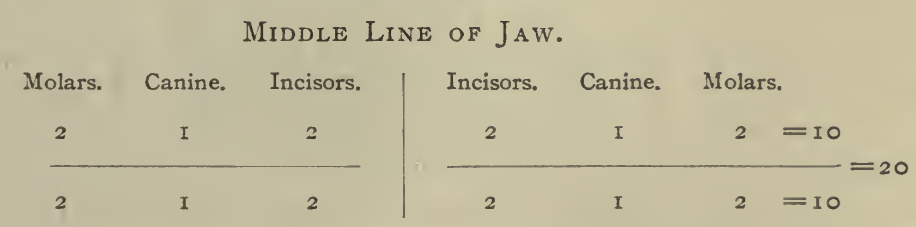

The figures indicate in months the age at which each tooth appears:

\begin{tabular}{c|c|c|c|c}
\hline $\begin{array}{c}\text { Lower central } \\
\text { incisors }\end{array}$ & Upper incisors & $\begin{array}{c}\text { First molar and } \\
\text { lower lateral } \\
\text { incisors }\end{array}$ & Canines & Second molar \\
\hline 6 to 9 & 8 to I 2 & I 2 to I 5 & I 8 to 24 & 24 to 30 \\
\hline
\end{tabular}




\section{Permanent Teeth.}

\section{Middle Line of Jaw.}

\begin{tabular}{cccc|cccr}
$\begin{array}{l}\text { True } \\
\text { Molars. }\end{array}$ & $\begin{array}{c}\text { Bicuspids or } \\
\text { Premolars. }\end{array}$ & Canine. & Incisors. & Incisors. & Canine. & $\begin{array}{c}\text { Bicuspids or } \\
\text { Premolars. }\end{array}$ & $\begin{array}{c}\text { True } \\
\text { Molars }\end{array}$ \\
3 & 2 & I & 2 & 2 & 1 & 2 & 3 \\
\hline 3 & 2 & I & 2 & 2 & I & 2 & 3
\end{tabular}

The age at which each permanent tooth is cut is indicated in this table in years:

\begin{tabular}{|c|c|c|c|c|c|c|c|}
\hline \multirow{2}{*}{ First molars } & \multicolumn{2}{|c|}{ Incisors } & \multicolumn{2}{|c|}{$\begin{array}{l}\text { Bicuspids or } \\
\text { premolars }\end{array}$} & \multirow{2}{*}{ Canines } & \multirow{2}{*}{$\begin{array}{l}\text { Second } \\
\text { molars }\end{array}$} & \multirow{2}{*}{$\begin{array}{l}\text { Third } \\
\text { molars or } \\
\text { wisdoms }\end{array}$} \\
\hline & Centrals & Laterals & First & Second & & & \\
\hline 6 & 7 & 8 & 9 & Io & I 2 to 14 & I 2 to I 5 & I 7 to 25 \\
\hline
\end{tabular}

Structure.-A tooth is generally described as possessing a crown, neck, and root or roots. The crown is the portion which projects beyond the level of the gum. The neck is that constricted portion just below the crown which is embraced by the free edges of the gum, and the root includes all below this.

On making longitudinal and transverse sections through its center, figure $6 \mathrm{r}, \mathrm{A}, \mathrm{B}, \mathrm{a}$ tooth is found to be principally composed of a hard superficial

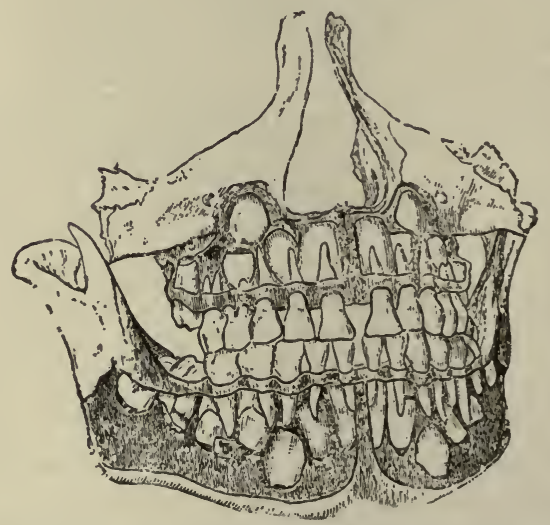

FIg. 60.-Normal Well-formed Jaws, from which the Alveolar Plate has been in great part removed, so as to expose the Developing Permanent Teeth in their Crypts in the Jaws. (Tomes.)

material, dentine or ivory, which is hollowed out into a central cavity which resembles in general shape the outline of the tooth, and is called the pulp cavity.

The tooth pulp is composed of fibrous connective tissue, blood vessels, nerves, and large numbers of cells of varying shapes. On the surface in 
close connection with the dentine there is a specialized layer of cells called odontoblasts, which are elongated columnar cells with a large nucleus at the tapering ends farthest from the dentine. The cells are all embedded in a mucoid gelatinous matrix.

The blood vessels and nerves enter the pulp through a small opening at the apical extremity of each root.

A layer of very hard calcareous matter, the enamel, caps the dentine of the crown; beneath the level of the gum is a layer of true bone, called the cement or crusta petrosa. The enamel and cement are very thin at the neck of the tooth where they come in contact, the cement overlapping the enamel. The enamel becomes thicker toward the crown, and the cement toward the lower end or apex of the root.
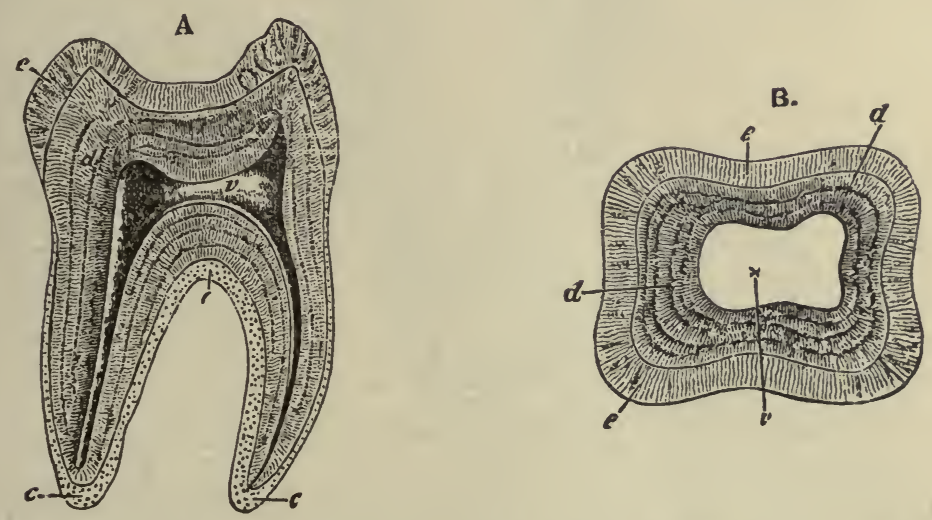

FIG. 61.-A. A Longitudinal Section of a Human Molar Tooth. $c$, Cement; $d$, dentine; $e$, enamel; $v$, pulp cavity. B.-Transverse section. The letters indicate the same as in $A$ (Owen).

Dentine or Ivory.-Dentine closely resembles bone in chemical composition. It contains, however, rather less animal matter.

Structure.-Dentine is finely channelled by a multitude of delicate tubes, which by their inner ends communicate with the pulp cavity, and by their outer extremities come into contact with the under part of the enamel and cement, and sometimes even penetrate them for a greater or less distance, figures $6_{3}, 64$. The matrix in which these tubes lie is composed of "a reticulum of fine fibers of connective tissue modified by calcification, and, where that process is complete, entirely hidden by the densely deposited lime salts" (Mummery).

The tubules of the dentine contain fine prolongations from the tooth pulp, which gives the dentine a certain faint sensitiveness under ordinary circumstances and, without doubt, have to do also with its nutrition. They are probably processes of the dentine cells or odontoblasts lining the pulp 
cavity. The relation of these processes to the tubules in which they lie is precisely similar to that of the processes of the bone corpuscles to the canalic-

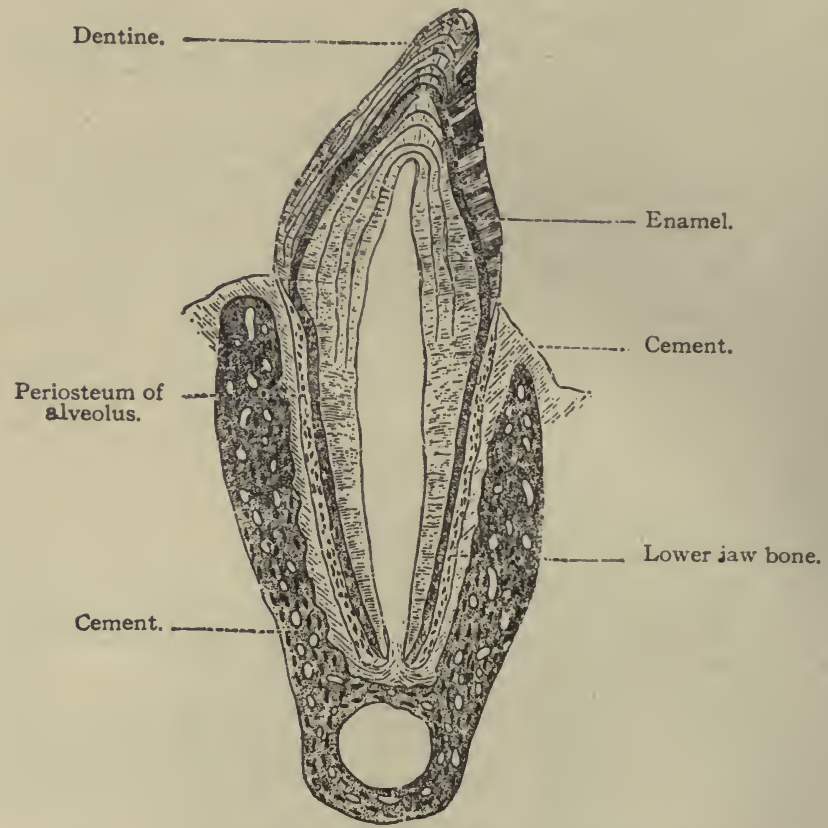

FIG. 62.-Premolar Tooth and Surrounding Bone of Cat.

ali of bone. The outer portion of the dentine, underlying the cement and the enamel, figure $6_{3}, b, c$, contains cells like bone corpuscles.

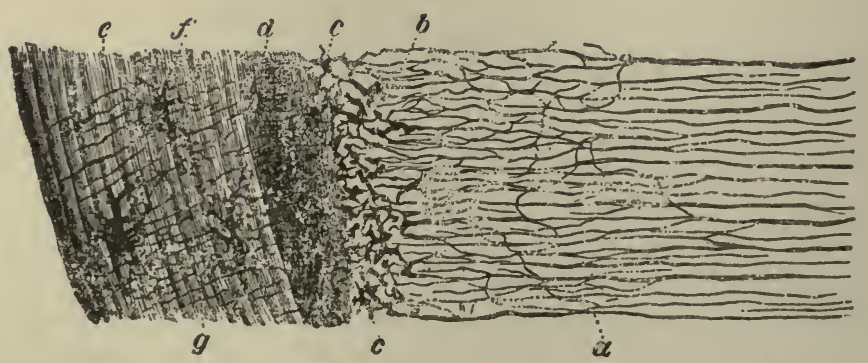

Fig. 63.-Section of a Portion of the Dentine and Cement from the Middle of the Root of an Incisor Tooth. $a$, Dental tubuli ramifying and terminating, some of them in the interglobular spaces $b$ and $c$, which somewhat resemble bone lacunæ; $d$, inner layer of the cement with numerous closely set canaliculi; $e$, outer layer of cement; $f$, lacunæ; $g$, canaliculi. $\times 350$. (Kölliker.)

Enamel.-The enamel, which is by far the hardest portion of a tooth, is composed chemically of the same elements that enter into the composition 
of dentine and bone, but the animal matter amounts only to about 2 or 3 per cent. It contains a larger proportion of inorganic matter and is harder than any other tissue in the body.

Structure.-Enamel is composed of fine hexagonal fibers, figures 64,65 .

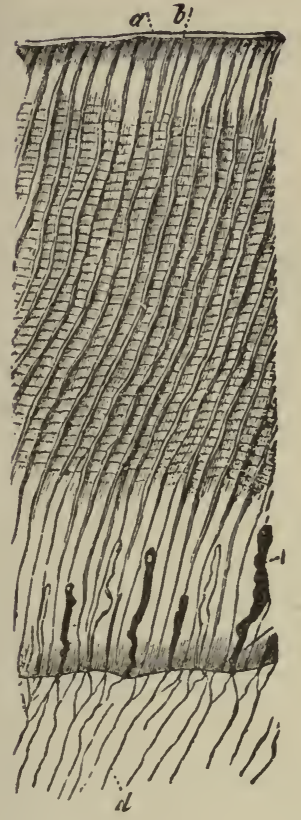

FIG. 64

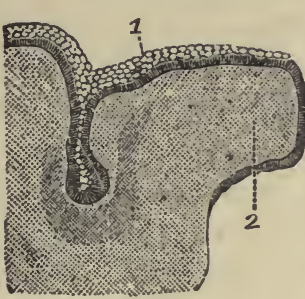

A

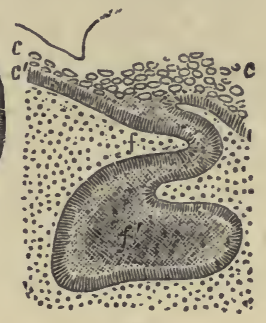

B

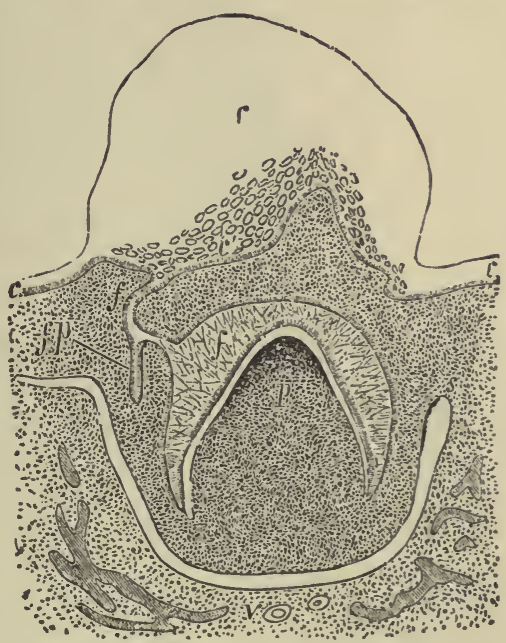

C

FIG. 65 .

FIG. 64.- Thin Section of the Enamel and a Part of the Dentine. $a$, Cuticular pellicle of the enamel (Nasmyth's membrane); $b$, enamel fibers, or columns with fissures between them and cross striæ; $c$, larger cavities in the enamel, communicating with the extremities of some of the dentinal tubuli $(d) . \quad \times 350 . \quad$ (Kölliker.)

FIG. 65.-Section of the Upper Jaw of a Fetal Sheep. A.- I, Common enamel germ dipping down into the mucous membrane; 2, palatine process of jaw; 3, rete Malpighi. (Waldeyer.) B.-Section similar to A, but passing through one of the special enamel germs here becoming flask-shaped; $c, c$, epithelium of mouth; $f$, neck; $f^{\prime}$, body of special enamel germ. (Rose.) C.-A later stage; $c$, outline of epithelium of gum; $f$, neck of enamel germ; $f^{\prime}$, enamel organ; $p$, papilla; $s$, dental sac forming; $f p$, the enamel, germ of permanent tooth; $m$, bone of jaw; $v$, vessels cut across. (Kölliker.) Copied from Quain's "Anatomy."

These are set on end vertical to the surface of the dentine, and fit into corresponding depressions in the same.

Like the dentine tubules, they are disposed in wavy and parallel curves. 
The fibers are thus marked by transverse lines. They are mostly solid, but some of them may contain a very minute canal.

The enamel prisms are connected together by a trace of hyaline cement substance.

Development.-The first step in the development of the teeth consists in a downward growth, figure $65, \mathrm{~A}, \mathrm{r}$, from the deeper layer of stratified epithelium of the mouth, which first becomes thickened in the neighborhood of the maxillæ or jaws, now also in the course of formation. This epidermal papilla grows downward into a recess of the imperfectly developed tissue of the embryonic jaw. It forms the primary enamel organ or enamel germ, and its position is indicated by a slight groove in the mucous membrane of the jaw. The next step consists in the elongation and the inclination outward

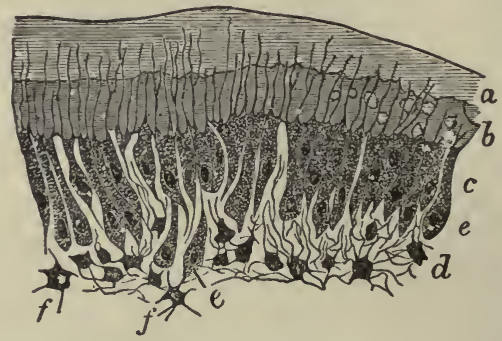

Fig. 66.-Part of Section of Developing Tooth of a Young Rat, showing the Mode of Deposition of the Dentine. Highly magnified. $a$, Outer layer of fully formed dentine; $b$, uncalcified matrix with one or two nodules of calcareous matter near the calcified parts; $c$, odontoblasts sending processes into the dentine; $d$, pulp; $e$, fusiform or wedge-shape cells found between odontoblasts; $f$, stellate cells of pulp in fibrous connective tissue. The section is stained in carmine, which colors the uncalcified matrix but not the calcified part. (E. A. Schäfer.)

of the deeper part, figure $65, \mathrm{~B}, f^{\prime}$, of the enamel germ, followed by an increased development at certain points corresponding to the situations of the future milk-teeth. The enamel germ becomes divided at its deeper portion, or extended by further growth, into a number of special enamel germs corresponding to each of the milk-teeth, and connected to the common germ by a narrow neck. Each tooth is thus placed in its own special recess in the embryonic jaw, figure $65, \mathrm{c}, f^{\prime}$.

As these changes proceed, tnere grows up from the underlying tissue into each enamel germ, figure $65, \mathrm{c}, p$, a distinct vascular papilla, dental papilla, and upon it the enamel germ becomes molded, and presents the appearance of a cap of two layers of epithelium separated by an interval, figure $65, \mathrm{c}, f^{\prime}$. While part of the subepithelial tissue is elevated to form the dental papillæ, the part which bounds the embryonic teeth forms the dental sacs, figure $65, \mathrm{c}$, s; and the rudiment of the jaw sends up processes forming partitions between the teeth. The papilla, which is really part of the dental sac, is composed of nucleated cells arranged in a meshwork, in 
the outer layer of which are the columnar cells called odontoblasts. The odontoblasts form the dentine, while the remainder of the papilla forms the pulp. The method of the formation of the dentine from the odontoblasts is said to be as follows: The cells form elongated processes at their outer surfaces which are directly converted into the tubules of dentine, figure $66, c$, and into the contained fibrils.

Each papilla early takes the shape of the crown of the tooth to which it corresponds, but as the dentine increases in thickness and papilla diminishes until when the tooth is cut only a small amount remains as the pulp. It is supplied by vessels and nerves which enter at the end of the root. The roots are not completely formed at the time of the eruption of the teeth.

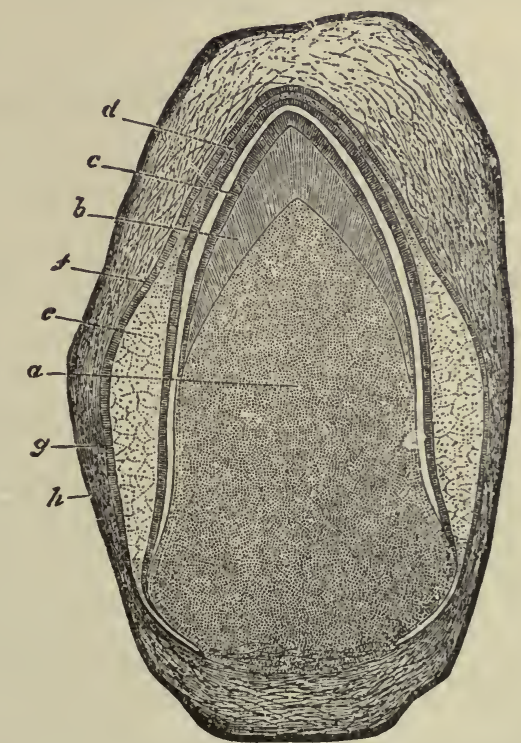

FIg. 67.-Vertical Transverse Section of the Dental Sac, Pulp, etc., of a Kitten. a, Dental papilla or pulp; $b$, the cap of dentine formed upon the summit; $c$, its covering of enamel; $d$, inner layer of epithelium of the enamel organ; $e$, gelatinous tissue; $f$, outer epithelial layer of the enamel organ; $g$, inner layer, and $h$, outer layer of dental sac. $\times \mathbf{r} 4$. (Thiersch.)

The enamel cap is formed by the enamel cells, by the deposit of a keratinlike substance, which subsequently undergoes calcification. Other layers are formed in the same manner meanwhile.

The temporary or milk-teeth are speedily replaced by the growth of the permanent teeth.

The development of the temporary teeth commences about the sixth week of intra-uterine life, after the laying down of the bony structure of the jaws. Their permanent successors begin to form about the sixteenth week of intra-uterine life. 


\section{MUSCULAR TISSUE.}

There are two chief kinds of muscular tissue, differing both in minute structure as well as in mode of action, viz., (I) the smooth or non-striated, and (2) the striated.

\section{SMOOTH OR NON-STRIATED MUSCLE.}

Non-striated muscle forms the proper muscular coats of the digestive canal from the middle of the esophagus to the internal sphincter ani; of the uterus and urinary bladder; of the trachea and bronchi; of the ducts

Fig. 68. - Isolated Smooth Muscle Cells from Human Small Intestine. $\quad \times_{400 .}$ Rodshaped nucleus surrounded by area of finely granular protoplasm; longitudinal striations of cytoplasm.

of glands; of the gall-bladder; of the vesiculæ seminales; of the uterus and Fallopian tubes; of the blood vessels and lymphatics; and of the iris and some other parts of the eye. This form of tissue also enters largely into the composition of the tunica dartos of the scrotum. Unstriped muscular tissue

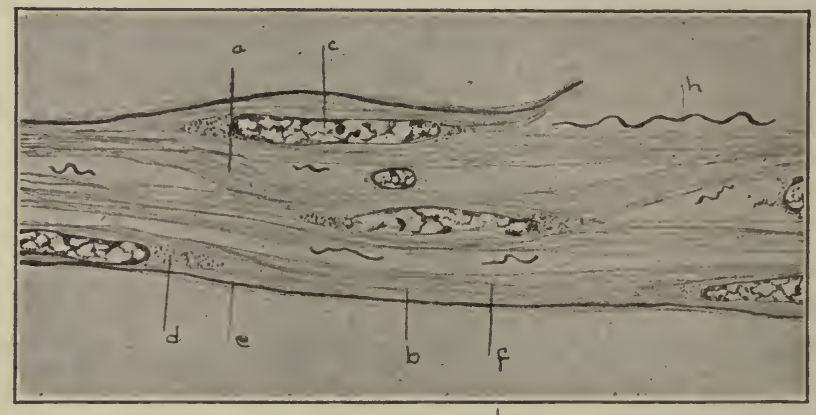

Fig. 69.-Smooth Muscle from Intestine of Pig, Showing Syncytial Structure. $a$, Protoplasmic process connecting two muscle fibers; $b$, end-to-end union of two muscle fibers, showing the continuity of protoplasm and myofibrils; $c$, nucleus of muscle fiber; $d$, granular protoplasm at the end of muscle nucleus; $e$, coarse myofibril; $f$, fine myofibril; $g$, connective-tissue cell with connective-tissue fibrils surrounding it; $h$, elastic fiber. (New figure by Caroline McGill.)

occurs largely also in the true skin generally, being especially abundant in the interspaces between the bases of the papillæ, and, when it contracts, the papillæ are made unusually prominent, giving rise to the peculiar roughness of the skin termed cutis anserina, or goose flesh. It also occurs in all parts 
where hairs occur, in the form of flattened roundish bundles which lie alongside the hair follicles and sebaceous glands.

Structure.-Unstriated muscle fibers are elongated, spindle-shaped

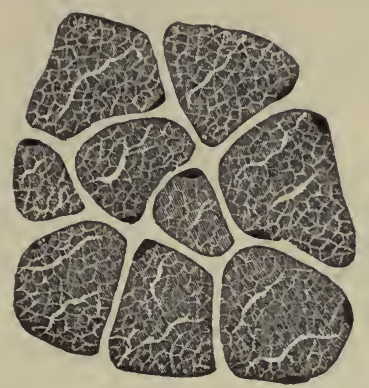

FIg. 70.-Transverse Section through Muscular Fibers of Human Tongue. The deeply stained nuclei are situated at the inside of the sarcolemma. Each muscle fiber shows "Cohnheim's fields," that is, the sarcous elements in transverse section separated by clear (apparently linear) interstitial substance. $\times 45^{\circ}$. (Klein and Noble Smith.)

mononucleated cells, 7 to $8 \mu$ in diameter by 40 to $200 \mu$ in length, figures 68 and 69. The protoplasm of each cell, the contractile substance, is marked by longitudinal striations representing fibrils which have been described as contractile. The nucleus is an oblong mass placed near the

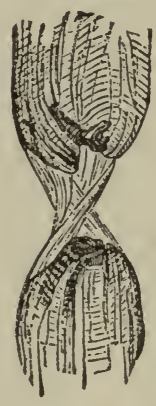

FIG. 71 .

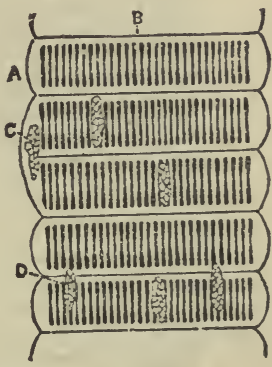

FIG. 72.

FIg. 7 r.-Muscle Fiber Torn Across; the sarcolemma still connects the two parts of the fiber. (Todd and Bowman.)

FIg. 72. - Part of a Striped Muscle Fiber of a Water Beetle prepared with Absolute Alcohol. A, Sarcolemma; $B$, Krause's membrane. The sarcolemma shows regular bulgings. Above and below Krause's membrane are seen the transparent "lateral discs." The chief mass of a muscular compartment is occupied by the contractile disc composed of sarcous elements. The substance of the individual sarcous elements has collected more at the extremity than in the center hence this latter is more transparent. The optical effect is that the contractile disc appears to possess a "median disc." (Disc of Hensen). Several nuclei, $C$ and $D$, are shown, and in them a minute network. $\times 300$. (Klein and Noble Smith.)

center of the cell. It is covered by a nuclear membrane which encloses a network of anastomosing fibrils.

Development.- In the pig the smooth muscle of the alimentary canal originates in the syncytium of the mesodermal cells which surround the 
entoderm. The cells soon begin to grow into the adult spindle-shaped form and the fibrils make their appearance. Even in the adult muscle the syncytial connections are retained, according to Dr. McGill.

\section{STRIATED MUSCLE.}

Striated or striped muscle constitutes the whole of the muscular apparatus of the skeleton, of the walls of the abdomen, the limbs, etc.-the whole of those muscles which are under the control of the will and hence termed voluntary; also the muscle of the heart.

For the sake of description, striated muscular tissue may be divided into two classes, (a) skeletal, which comprises the whole of the striated muscles of the body except (b) the heart.

A
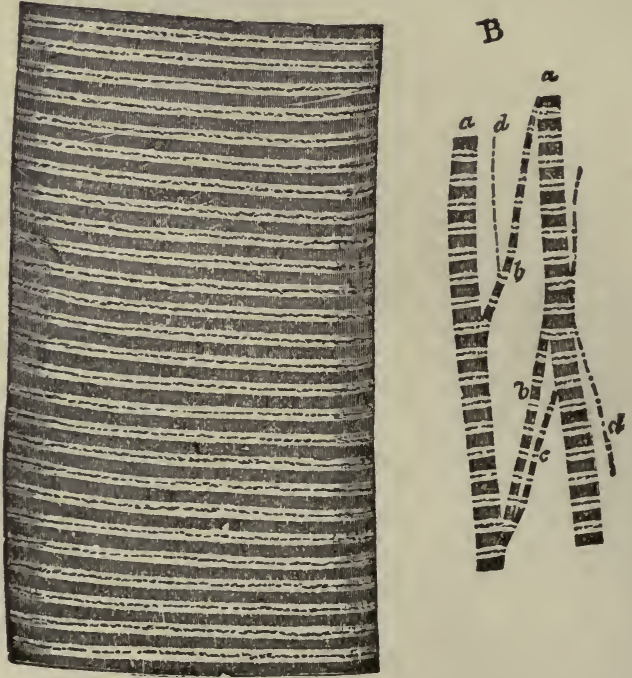

FIG. 73.- $A$, Portion of a Medium-sized Human Muscle Fiber. $B$, Separated bundles of fibrillæ equally magnified; $a, a$, larger, and $b, b$, smaller collections; $c$, still smaller; $d, d$, the smallest which could be detached, possibly representing a single series of sarcous elements. $\times 800$. (Sharpey.)

Skeletal Muscle.-The muscle fibers of the skeletal muscles are usually grouped in small parallel bundles, fasciculi. The fasciculi extend through the muscle, converging to their tendinous insertions. Connective-tissue sheaths, endomysium, surround the fasciculi and support the blood vessels, while a stronger sheath, the perimysium, encases the entire muscle.

The unit of muscular structure is the fiber. Each muscle fiber is a long cylinder with fusiform ends. The fibers vary in diameter from 1o to $100 \mu$, while the length may reach as much as $40 \mathrm{~mm}$. Each fiber is enclosed in 
a distinct sheath, the sarcolemma. The sarcolemma is a transparent structureless sheath of great resistance which surrounds each fiber, figure $7 \mathrm{I}$.

The substance of the fiber enclosed by the sarcolemma, the contractile substance, contains a number of oval nuclei distributed along the length of the fiber and lying just under the sarcolemma or through the sarcoplasm. Each nucleus is accompanied by a small mass of granular protoplasm at its poles. The main mass of the fiber is characterized by transverse light and dark bands, figure 73, from which the name striated muscle arises.

Longitudinal striation is also apparent under certain modes of treatment, figure $8 \mathrm{r}$. The muscle fibers can be split longitudinally into fibrils, called sarcostyles, figures 73 and 74 , each of which exhibits the characteristic striation of the whole fiber. Under certain treatment the sarco-

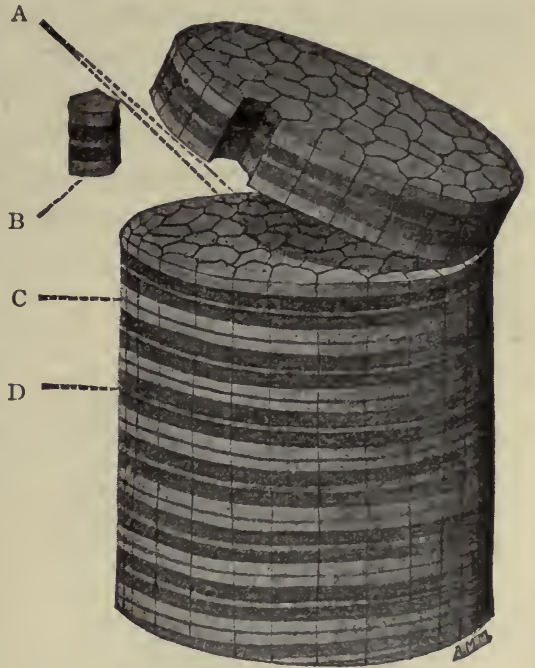

Fig. 74.-Diagram of Segment of Muscle Fiber, showing Sarcostyle $A$, Sarcous element $B$, Krause's line $C$, Hensen's line $D$. styles break transversely into smaller discs by cleavage at the line of Krause's membrane.

The sarcostyle is, therefore, composed of a number of smaller elements

FIG. 75 .

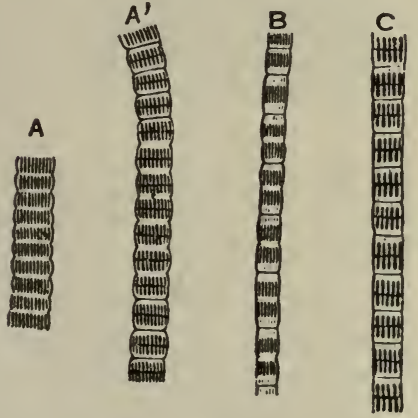

FIG. 75.-Sarcostyles from the Wing Muscles of a Wasp. $A, A^{\prime}$, Sarcostyles showing degrees of retraction; $B$, a sarcostyle extended with the sarcous elements separated into two parts; $C$, sarcostyles moderately extended (semidiagrammatic). (E. A. Schäfer.)

FIG. 76.- Diagram of a Sarcomere in a Moderately Extended Condition, B. K, K, Krause's membranes; $H$, plane of Henson; $S, E$, poriferous sarcous element. (E. A. Schäfer.)

joined end to end. These are the sarcous elements of Bowman. The sarcous element has a highly refractive denser middle piece surrounded by a 
less refractive more fluid material. The polarizing microscope reveals the fact that the middle piece which corresponds in position to the dark transverse band is doubly refractive, anisotropic, while the surrounding material, the light band, is singly refractive, isotropic.

In transverse section, figure 70 , the area of the muscle substance is mapped out into small polygonal areas by a network of clear lines called Cohnheim's areas. The lines represent the substance between the sarcostyles. This substance probably represents the less differentiated contractile sub-

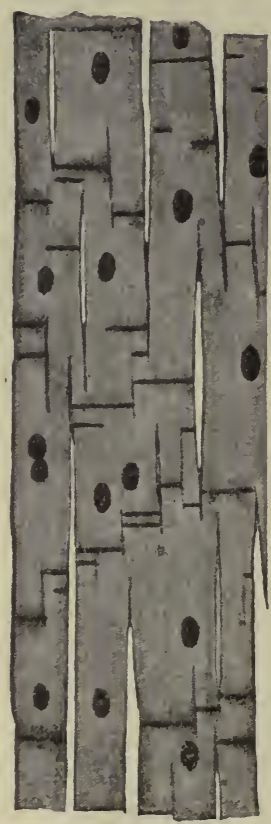

FIG. 77 . stance, called sarcoplasm. In figure $8 \mathrm{I}$ the interfibrillar sarcoplasm is indicated by the longitudinal and transverse lines.

Heart Muscle.-The muscle substance of the heart is composed of

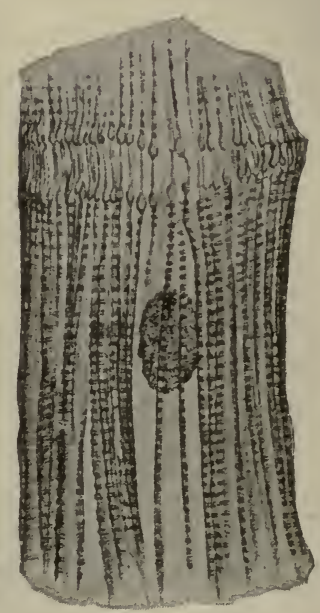

FIG. 78.

FIG. 77.-A Section of Cardiac Muscle, Diagrammatic. (From E. A. Schäfer, after Heidenhain.)

FIg. 78. - Intercellular Continuity of Muscle Fibrils in Cardiac Muscle. (From E. A. Schäfer after Przewosky.)

mononucleated masses of protoplasm, cardiac muscle cells, in which the substance of the cell presents the transversely striated appearance characteristic of the voluntary muscle just described. But the heart muscle is physiologically much more like an involuntary muscle. The cells are rather small, two to four times as long as thick, and the nucleus is usually situated near the middle of the cell, figure 79. There is no sarcolemma; on the other hand, the cells present branched and irregular outlines, but adjacent cells interlock in close-fitting contact.

Certain observers have described fibrils as extending across the so-called cell boundary and noted that not all such boundaries enclose nuclei. These 
observations suggest that cardiac muscle belongs to the group of tissues possessing a syncytium. However, the section of cardiac tissue may very possibly cut many cells without enclosing a nucleus. The continuity of fibrils is an important observation from the physiological point of view; see Circulation chapter.

In certain parts of the heart, the cardiac tissue is not completely differentiated and retains in the adult somewhat embryonic characters; for example, the bundle of His running in the septum from the auricles to the ventricles and the cells containing Purkinje's fibers lying immediately under the endocardium.

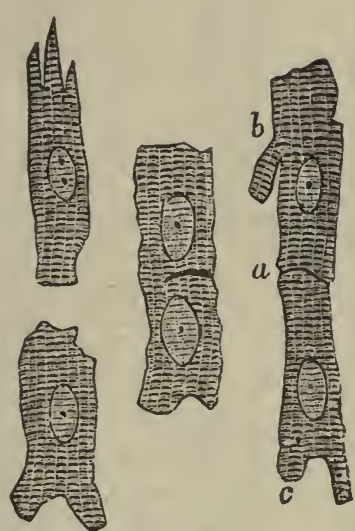

FIG. 79.

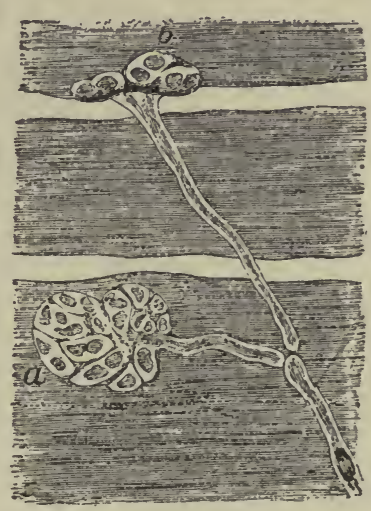

FIG. 80.

Fig. 79.-Muscular Fiber Cells from the Heart. (E. A. Schäfer.)

Fig. 80.- From a Preparation of the Nerve Termination in the Muscular Fibers of a Snake. $a$, End-plate seen only broad-surfaced; $b$, end-plate seen as narrow surface. (Lingard and Klein.)

Blood and Nerve Supply.-The muscles are freely supplied with blood vessels; the capillaries form a network with oblong meshes around the fibers. Nerves also are supplied freely to muscles; the striated voluntary muscles receiving them from the cerebro-spinal nerves, and the cardiac muscle from both the cerebro-spinal and the sympathetic nerves.

In striped muscle the nerves end in motor end-plates. The nerve fibers are medullated; and when a branch passes to a muscle fiber, its primitive sheath becomes continuous with the sarcolemma, and the axis-cylinder forms a network of its fibrils on the surface of the muscle fiber. This network lies embedded in a flattened granular mass containing nuclei of several kinds; this is the motor end-plate, figures 80 and $8 \mathrm{r}$. There is considerable variation in the exact form of the nerve end-plate in the muscle. In batrachia the nerve fiber ends in a brush of branching nerve fibrils which are accompanied here and there by attached oval nuclei. 
Development.-The striated muscle of the voluntary variety is usually developed from the mesoderm. The embryonic cells increase enormously in size, the nuclei multiply by fission and distribute themselves beneath the

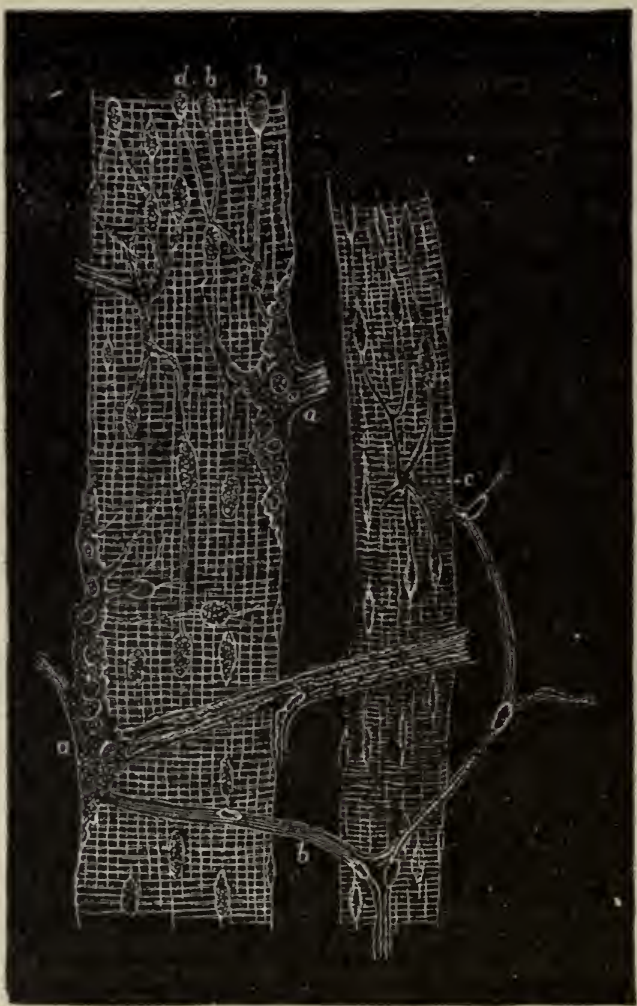

FIG. $8 \mathrm{I}$.
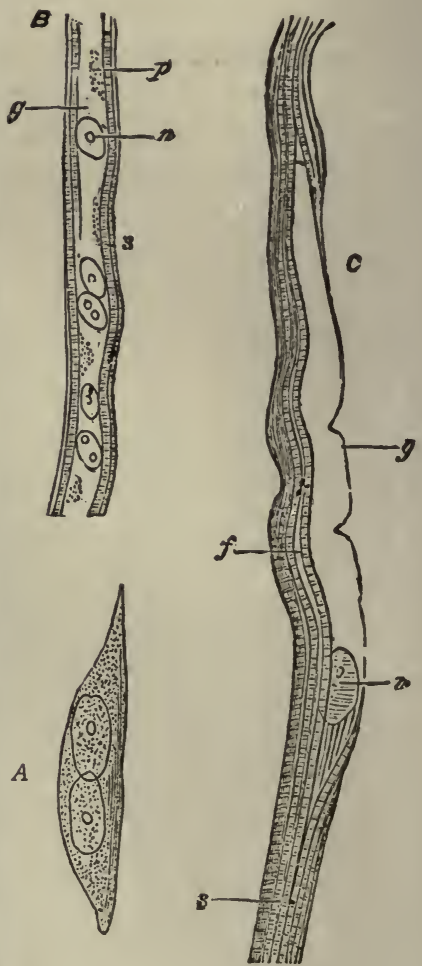

FIG. 82.

FIG. 8I.-Two Striped Muscle Fibers of the Hyoglossus of Frog. $\quad a$, Nerve end-plate; $b$, nerve fibers leaving the end-plate; $c$, nerve-fibers terminating after dividing into branches; $d$, a nucleus in which two nerve fibers anastomose. $\times 600$. (Arndt.)

FIG. 82.-Developing Striated Muscular Fibers, Showing Different Stages of Development and Different Positions of the Unstriated Protoplasm. A.-Elongated cell with two nuclei; the longitudinal striation is beginning to show on the right side. From a fetal sheep. (Wilson Fox.) B.-Developing muscular fiber, showing both longitudinal and transverse striations at the periphery, and a central unstriated cylinder of protoplasm containing several nuclei. From a human fetus near the third month. (Ranvier.) $n$, Nucleus (there is usually a mass of glycogen near each nucleus); $p$, central unstriated protoplasm; $s$, peripheral striated substance. C.-Developing muscular fiber, showing a lateral position of the unstriated protoplasm. From a three months' human fetus. (Ranvier.) $n$, Nucleus; $g$, unstriated protoplasm at one side of the fiber; $s$, striated sarcous substance with longitudinal and transverse striations.

sarcolemma. There is a differentiation of the cell protoplasm which takes place by the formation of sarcostyles. This begins nearest the surface of the cells and proceeds toward the center of the mass. 
The sarcolemma is apparently produced from embryonic connective tissue.

The cardiac muscle cells are at first spindle-shaped embryonic cells which elongate more and more. In further differentiation their protoplasm exhibits faint striations which pervade the cell as it grows in the great increase in size. The rhythmic contractions begin long before the striations appear.

\section{NERVOUS TISSUE.}

Nervous tissue has usually been described as being composed of two distinct substances, nerve fibers and nerve cells. The modern view of the nature of nerve tissue is, however, that the nerve cell and the nerve fibers are to be considered together as one unit, called the neurone. The neurone

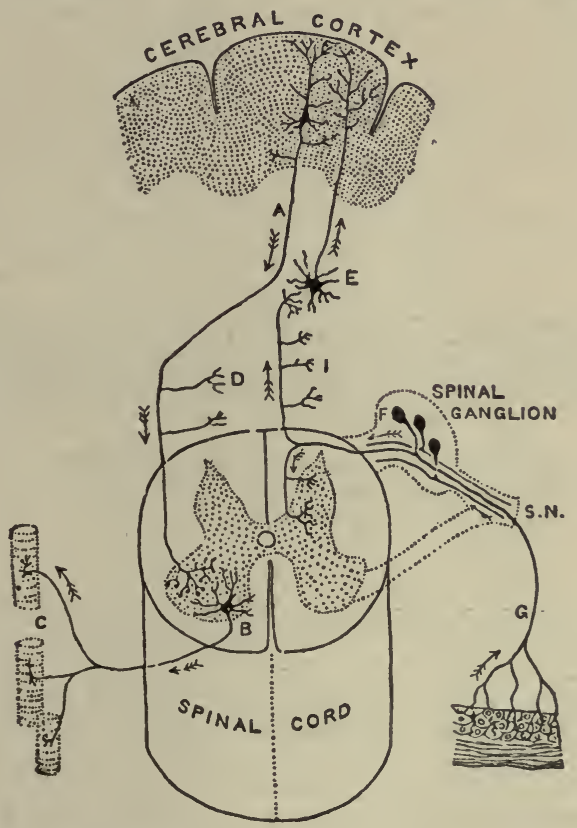

Fig. 83.-Diagram Showing the Arrangement of the Neurones or Nerve Units in the Architecture of the Nervous System. (Raymón y Cajàl.) $A$, Pyramidal neurone of cerebral cortex; $B$, anterior horn motor cell of spinal cord; $D$, collateral branches of $A ; E$, medullary neurone with ascending axone; $F$, spinal-ganglion neurones; $G$, sensory axones of $F ; I$, collaterals of $F$ in the cord.

is embedded in, and supported by, a substance called neuroglia. This neurone consists of a cell body, a number of branching processes termed dendrites, and a long process running out from it, the neuraxone, or axone, which becomes eventually a nerve fiber. The nerve cell and the nerve fiber are parts of the same anatomical unit, and the nervous centers are made up of those units, arranged in different ways throughout the nervous system, figure 83 . 


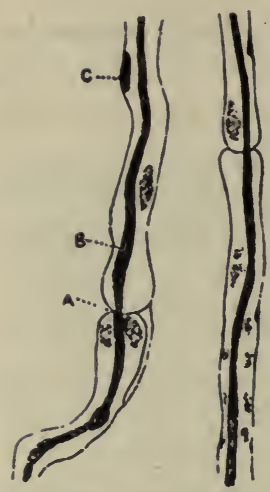

Fig. 84.-Two Nerve Fibers of the Sciatic Nerve. $A$, Node of Ranvier; $B$, axis-cylinder; $C$, sheath of Schwann with nuclei. $\times 300$. (Klein and Noble Smith.)

\section{NERVE FIBERS.}

While the nerve fiber is really to be considered as a process of the nerve cell, it is convenient to describe it separately. Nerve fibers are of two kinds, medullated or white fibers, and non-medullated or gray fibers.

Medullated Fibers.-Each medullated nerve fiber is made up of the following parts: An external sheath, called the primitive sheath, neurilemma, or nucleated sheath of Schwann; an intermediate, known as the medullary or myelin sheath, or white substance of Schwann; and a central thread, the axis-cylinder, or axial fiber.

The Primitive Sheath.-This is a pellucid membrane forming the outer investment of the nerve fiber. The sheath is constricted at intervals of a millimeter or less, the nodes of Ranvier. Each internodal segment bears a single nucleus surrounded by a variable amount of protoplasm. This membrane is described as having its origin in the

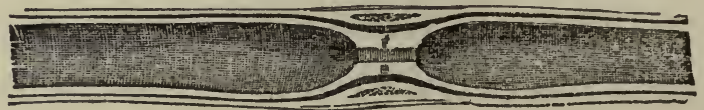

Fig. 85.-A Node of Ranvier in a Medullated Nerve Fiber, viewed from above. The medullary sheath is interrupted and the primitive sheath thickened. Copied from Axel Key and Retzius. $\times 750$. (Klein and Noble Smith.)

$\Delta$

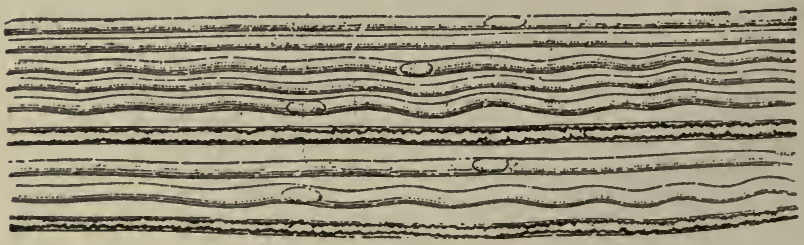

B

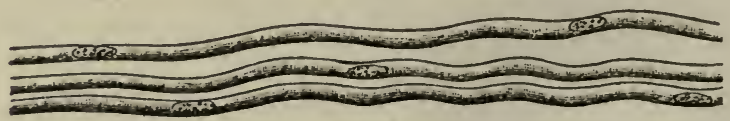

Fig. 86.-Gray, Pale, or Gelatinous Nerve Fibers. $A$, From a branch of the olfactory nerve of the sheep; two dark-bordered or white fibers from the fifth pair are associated with the pale olfactory fibers, $B$, from the sympathetic nerve. $\times 45^{\circ}$. (Max Schultze.)

mesoblastic cells, and the nuclei are the indications of the cellular nature of each nodal segment.

The Medullary or Myelin Sheath.-This is the part to which the peculiar 
opaque white aspect of medullated nerves is due. The thickness of this layer of nerve fiber varies considerably. It is a semifluid, fatty substance of high refractive power. It possesses a fine reticulum (Stilling, Klein), in the meshes of which is embedded the fatty material. It stains well with osmic acid.

The Axis-cylinder.-The central thread of a medullated nerve fiber is the axis-cylinder. It is the prolongation of a nerve cell and extends uninterrupted for the full length of the fiber. It consists of a large number of primitive fibrilla, as shown in the cornea, where the axis-cylinders of nerves break up into minute fibrils which form terminal networks. From various considerations, such as its invariable presence and unbroken continuity in all nerves, there can be no doubt that the axis-cylinder is the essential con-

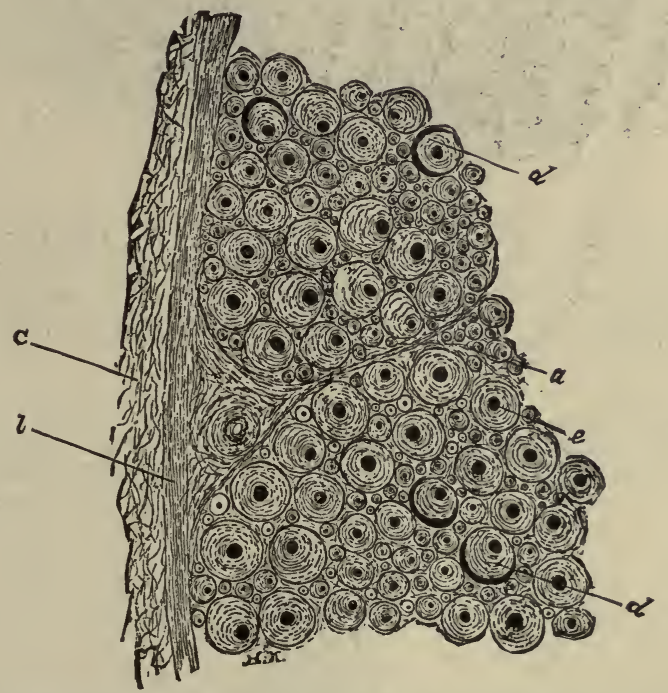

FIG. 87. - Transverse Section of a Portion of the Sciatic Nerve of the Rabbit, Hardened in Chromic Acid and Stained with Picro-carmine, to show medullated fibers in end view. $\times 275$. $a$, Perifascicular connective tissue; $b$, lamellar sheath; $e$, axis-cylinder.

ducting part of the fiber, the other parts having the subsidiary function of support and possibly of insulation.

The size of the nerve fibers varies, figure 87 . The largest fibers are found within the trunks and branches of the spinal nerves, in which the majority measure from $\mathrm{I} 4 \mu$ to $\mathrm{I} 9 \mu$ in diameter. In the so-called visceral or autonomic nerves of the brain and spinal cord medullated nerves are found, the diameter of which varies from $\mathrm{x} .8 \mu$ to $3.6 \mu$. In the hypoglossal nerve they are intermediate in size, and generally measure $7.2 \mu$ to $10.8 \mu$.

Non-medullated Fibers. - The fibers of the second kind, figure 86, which are also called fibers of Remak, constitute the principal part of the trunk and branches of the sympathetic nerves, the whole of the olfactory 
nerve, and are mingled in various proportions in the cerebro-spinal nerves. They differ from the preceding chiefly in not possessing the outer layer of medullary substance; their contents being composed exclusively of the axiscylinder.

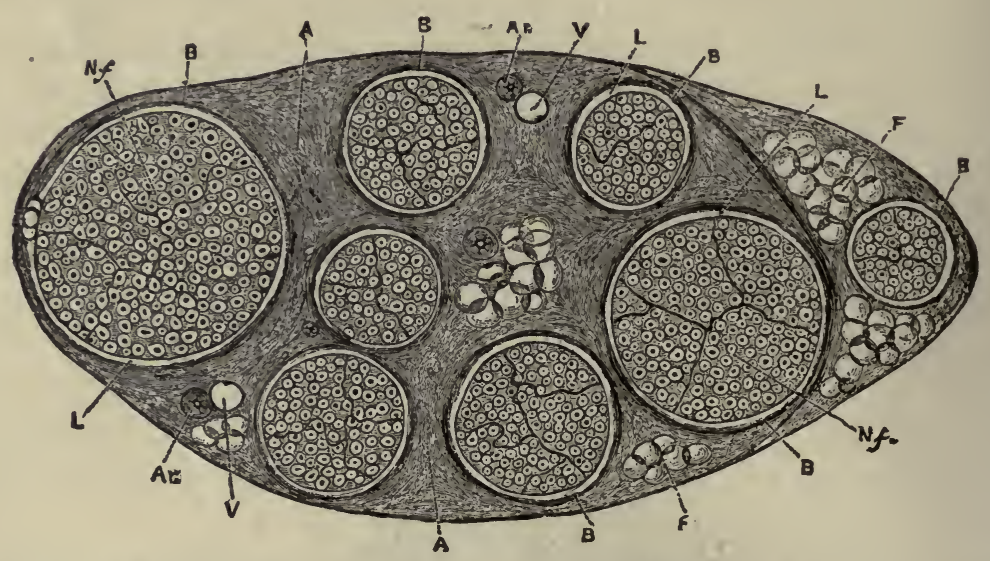

Fig. 88. - Transverse Seciion of the Sciatic Nerve of a Cat, about $X$ roo. It consists of bundles (funiculi) of nerve fibers ensheathed in a fibrous supporting capsule, epineurium, $A$; each bundle has a special sheath (not sufficiently marked out from the epineurium in the figure) or perineurium, $B$, the nerve fibers, $N f$; $L$, lymph spaces; $A r$, artery; $V$, vein; $F$, fat. Somewhat diagrammatic. (V. D. Harris.)

The non-medullated nerves are only about one-third to one-half as large as the medullated nerves, they do not exhibit the double contour, and

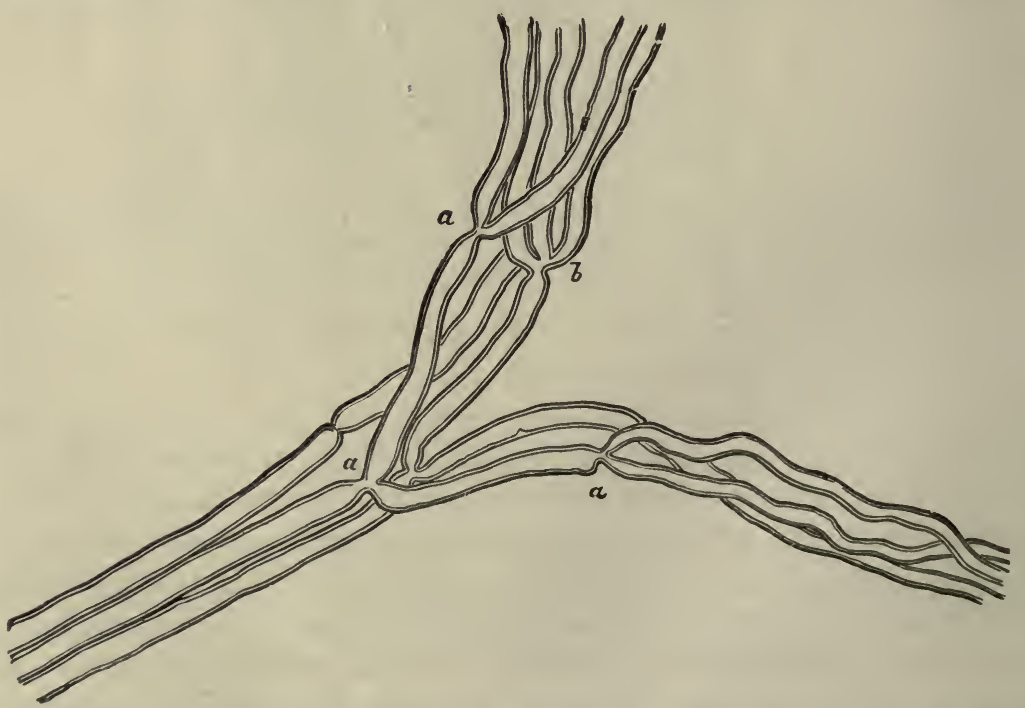

FIG. 89. - Small Branch of a Motor Nerve of the Frog, near its Termination, Showing Divisions of the Fibers: $a$, into two; $b$, into three. $\times 350$. (Kölliker.) 
they are grayer than the medullated nerves. The non-medullated fibers frequently branch.

It is worthy of note that in the fetus, at an early period of development, all nerve fibers are non-medullated.

Nerve Trunks.-Each nerve trunk is composed of a variable number of different-sized bundles, funiculi, of nerve fibers which have a special sheath, perineurium. The funiculi are enclosed in a firm fibrous sheath, epineurium; this sheath also sends in processes of connective tissue which connect the bundles together. In the funiculi between the fibers is a delicate supporting tissue, the endoneurium. There are numerous lymph spaces both beneath the connective tissue investing individual nerve fibers and also beneath that which surrounds the funiculi.

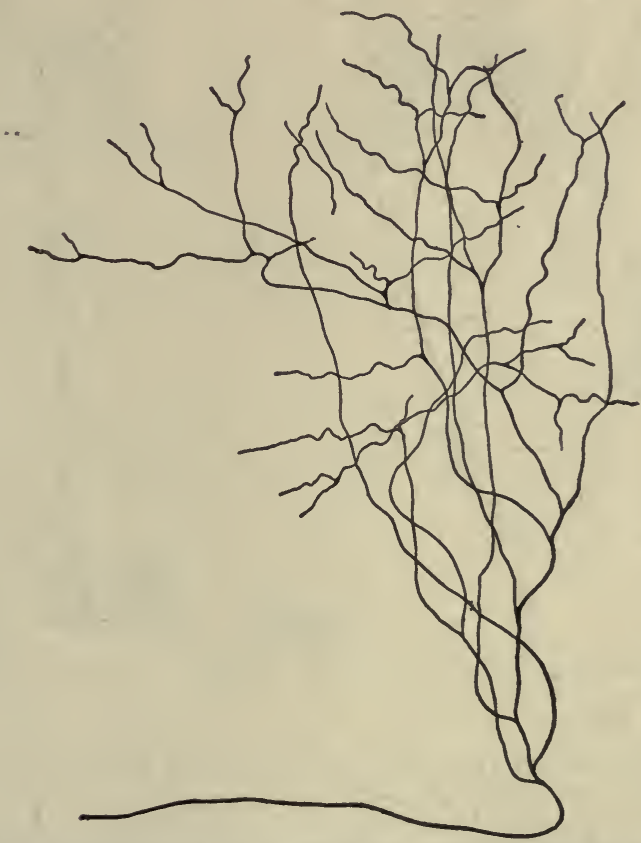

Fig. 90.-Terminal Ramifications of a Collateral Branch Belonging to a Fiber of the Posterior Column in the Lumbar Cord of an Embryo Calf.

Bundles of fibers run together in the nerve trunk, but they merely lie in approximation to each other, they do not unite. Even when nerves anastomose, there is no union of fibers, but only an interchange of fibers between the anastomosing bundles. Although each nerve fiber is thus single through most of its course, yet, as it approaches the region in which it terminates, it may break up into several subdivisions before its final ending.

Nerve Collaterals.-It has been discovered through the researches of Golgi, and confirmed by the further studies of Cajal and other anatomists, 
that each individual nerve fiber in the central nervous system gives off in its course branches which pass out from it at right angles for a short distance, and then may run in various directions. These branches are called collaterals. They end in fine, brush-like terminations known as end-brushes, or in little

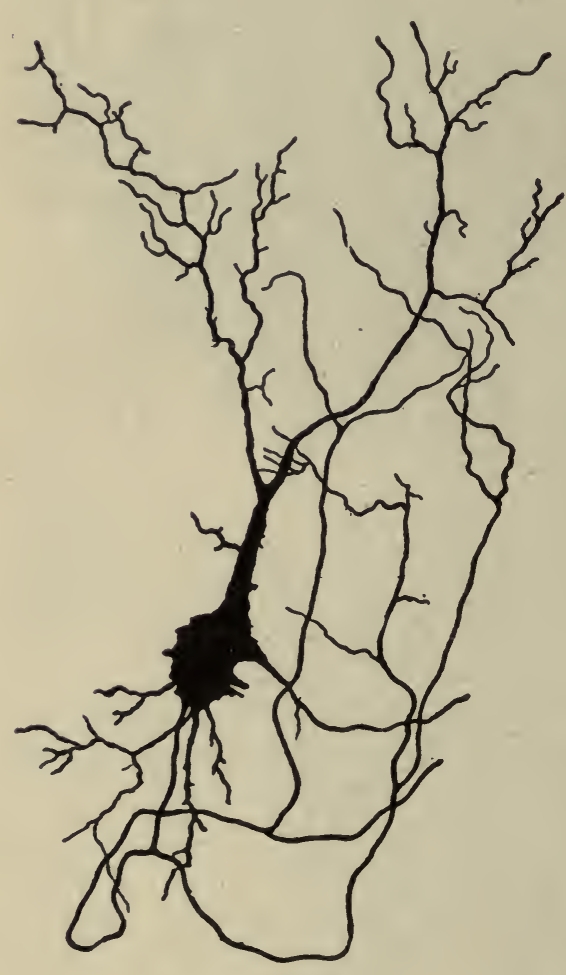

FIG. 9 r.

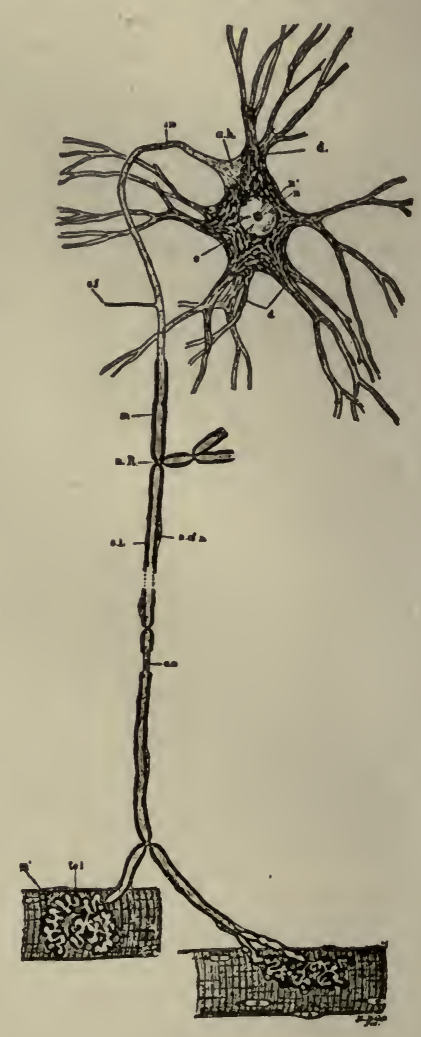

FrG. 92.

FIG. 9r.-Nerve Cell with Short Axis-cylinder from the Posterior Horn of the Lumbar Cord of $0.55 \mathrm{~cm}$. Embryo Calf. (After Van Gehuchten.)

FIG. 92.- Scheme of Lower Motor Neurone. The cell body, protoplasmic processes, axone, collaterals, and terminal arborizations in muscle are all seen to be parts of a single cell and together constitute the neurone. $c$, Cytoplasm of cell body containing chromophilic bodies, neurofibrils, and perifibrillar substance; $n^{\prime}$, nucleus; $n$, nucleolus; $d$, dendrites; $a h$, axone hill free from chromophilic bodies; $a x$, axone; $s f$, side fibril (collateral); $m$, medullary sheath; $n R$, node of Ranvier where side branch is given off; $s l$, neurilemmaand incisures of Schmidt; $m$, striated muscle fiber; $t e l$, motor end-plate. (Barker.)

bulbous swellings which come in close contact with other nerve cells, figures $8_{3}$ and 90 .

In the nerve centers, that is, in the brain and spinal cord, the different nerve fibers end just as the collaterals do, by splitting up into fine branches which form the end-brushes. Collaterals of the nerve fibers and end-brushes 
are chiefly found in the nervous centers. The nerve fibers of the peripheral nerves end in the muscles, glands, or special sensory organs, such as the eye and ear, each by its own special type of ending. Here, however, some

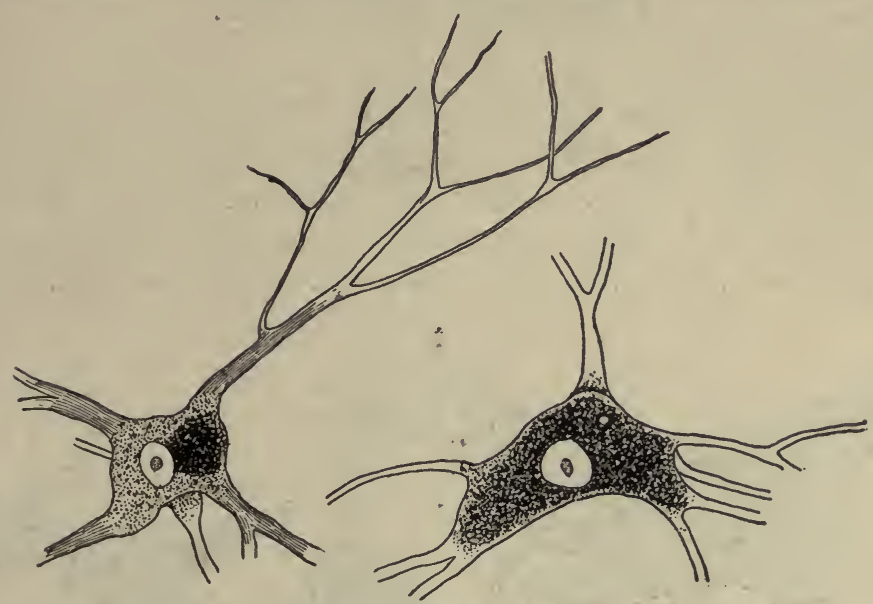

Fig. 93.-Large Nerve Cells with Processes, from the Ventral Cornua of the Cord of Man. $\times 350$. On the cell at the right two short processes of the cell body are present, one or the other of which may have been an axis-cylinder process (Deiters). A similar process appears also on the cell at the left.

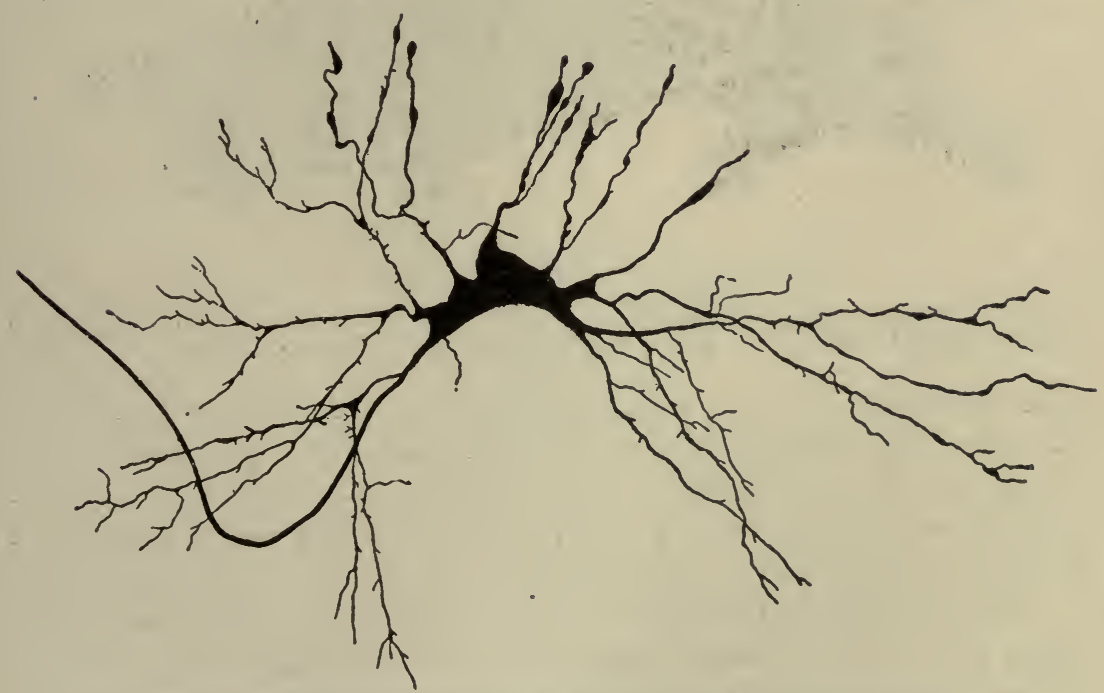

Fig. 94.-Multipolar Nerve Cell of the Cord of an Embryo Calf.

analogy to the end-brush can also be discovered. As the peripheral nerve fibers approach their terminations, they lose their medullary sheath, and consist then merely of an axis-cylinder and primitive sheath. They may even lose the latter, and only the axis-cylinder be left. Finally, the axis- 
cylinder breaks up into its elementary fibrillæ, to end in various ways to be described later.

\section{THE NERVE-CELL BODY.}

The nerve-cell body is the nodal and important part of the neurone, and from it are given off the dendrites and axis-cylinder process or axone. It consists of a mass of protoplasm, of varying shape and size, containing within it a nucleus and nucleolus. All nerve cells give off one or more processes which branch out in various directions, dividing and subdividing like the branches of a tree, but never anastomosing with each other or with other cells.

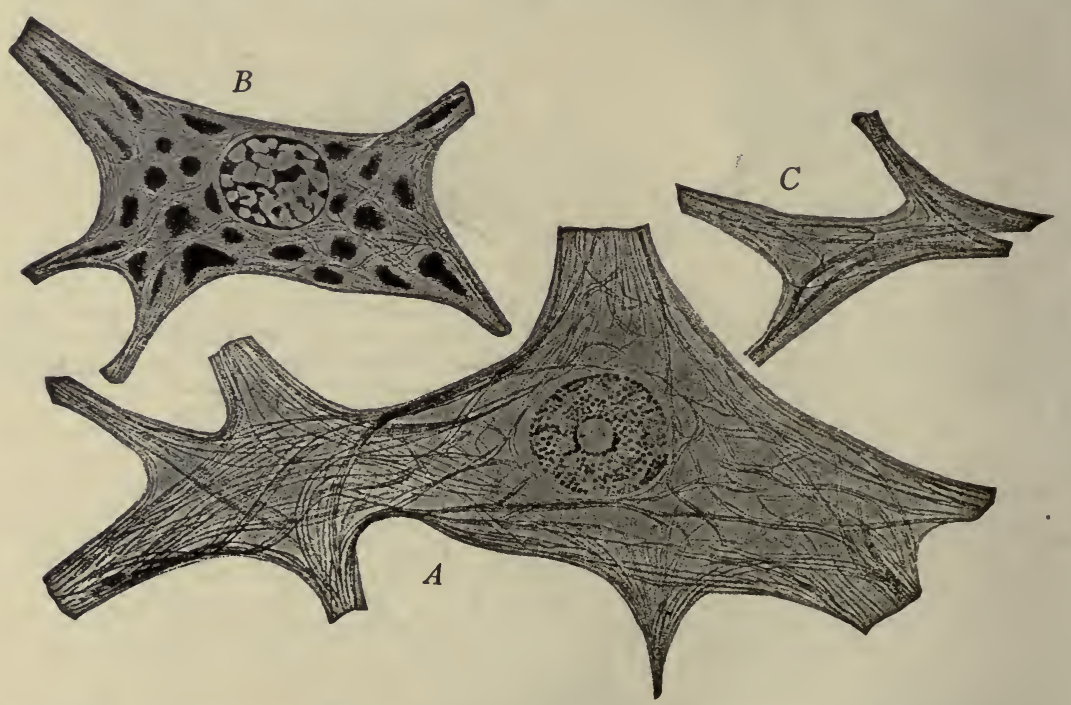

FIG. 95.-Ganglion Cells, Showing Neurofibrils. $A$, Anterior-horn cells of human; $B$, cell from the facial nucleus of rabbit; $C$, dendrite of anterior horn cell of human. (Bethe.)

These branches are what have already been referred to as the dendrites of the cell. They were formerly called the protoplasmic processes, figures $9 \mathrm{I}$, 93. It is thus seen that the neurone or nerve unit consists of a number of subdivisions, namely, the cell body, with its nucleus and nucleolus, the dendrites or protoplasm processes, and the axone or axis-cylinder process.

The protoplasm of the cells is shown by various dyes to consist of neurofibrils, perifibrillar substance, and in most cells chromophilic bodies. Apáthy and others have demonstrated that a network of interlacing and anastomosing fibrils traverses both the cell body and its branches, figure 95 .

The perifibrillar substance is a fluid or semifluid substance in which the fibrils are embedded. By treating nerve cells with special stains granular bodies varying in size are found embedded in the cytoplasm. These bodies are the chromophilic bodies, figure 96 . 
Ganglion cells are generally enclosed in a transparent membranous capsule similar in appearance to the external nucleated sheath of nerve fibers; within this capsule is a layer of small flattened cells.

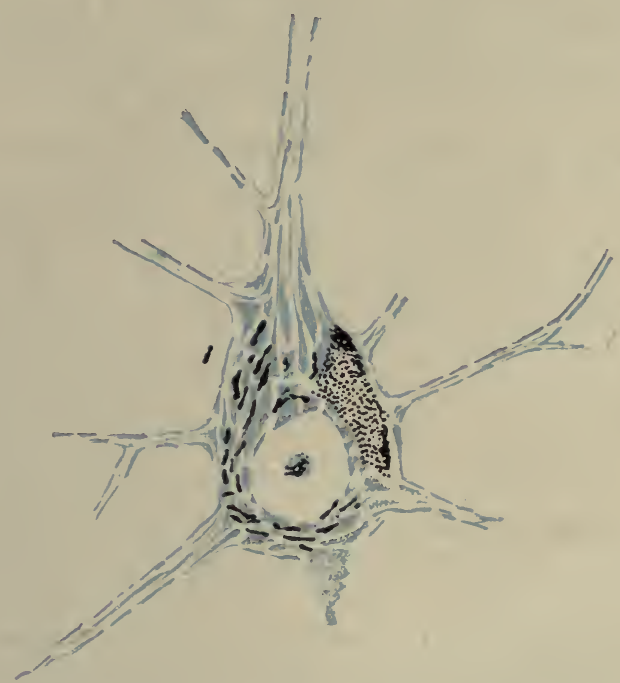

Fig. 96.-Cell of the Anterior Horn of the Human Spinal Cord, Stained by Nissl's Method, showing chromophiles in blue, and pigment in black. (After Edinger.)

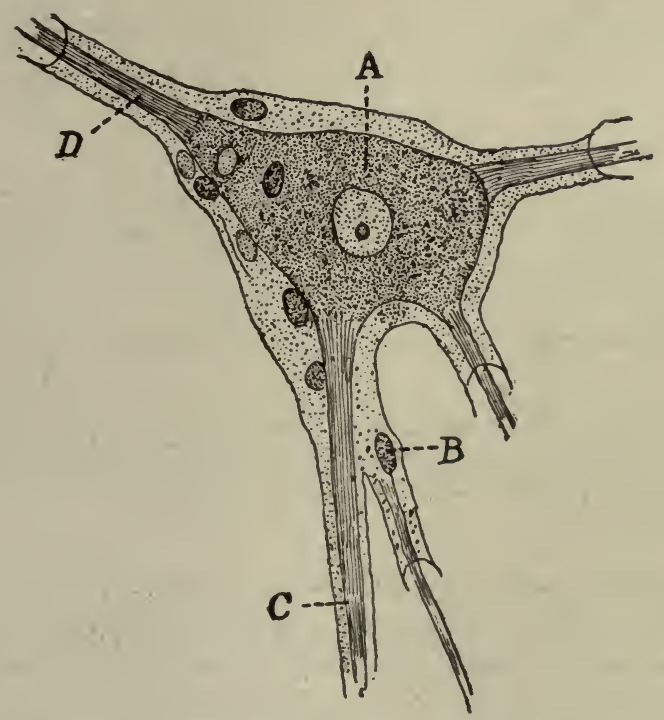

FIG. 97.-An Isolated Sympathetic Ganglion Cell of Man, Showing Sheath with Nucleated Cell Lining, $B$. A, Ganglion cell, with nucleus and nucleolus; $C$, branched process or dendrite; $D$, unbranched process or axone. (Key and Retzius.) $\times 750$. 


\section{Nerve Terminations.}

Nerve fibers terminate peripherally in four different ways: $\mathrm{I}$, by the terminal subdivisions which pass in between epithelial cells, and are known as interepithelial arborizations; 2, by motor-plates which lie in the muscles; 3 , by special end-organs, connected with the sense of sight, hearing, smell, and taste; and 4 , by various forms of tactile corpuscles.

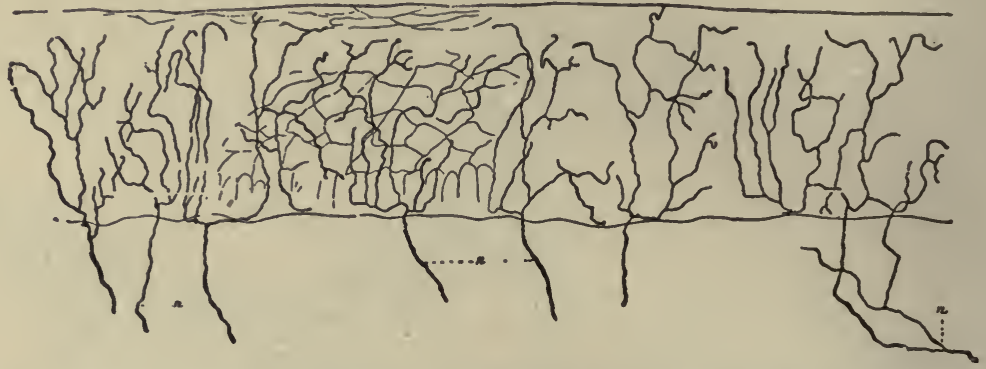

FIG. 98.-Sensory Nerve Terminations in Stratified Pavement Epithelium. Golgi's rapid method. (After G. Retzius.)

The Interepithelial Arborizations. - This forms a most common mode of termination of the sensory nerves of the body. The nerve fibers to the surface of the skin or mucous membrane lose their neurilemmæ and myelin sheaths, the bare axis-cylinder divides and subdivides into minute ramifications among the epithelial cells of the skin and mucous membrane. In the various glands of the body this form of termination also prevails. The hair bulbs, the teeth, and the tendons of the body are supplied by this same process of terminal arborization, figures $98,99$.

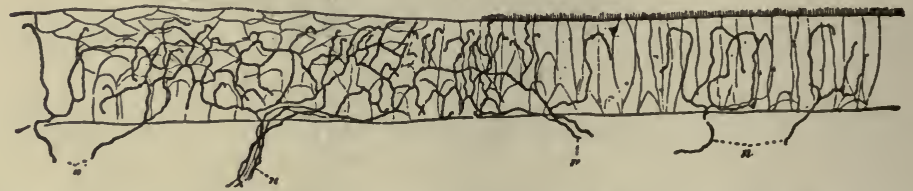

FIG. 99.-Sensory Nerve Termination in the Epithelium of the Mucosa of the Inferior Vocal Cord and in the Ciliated Epithelium of the Subglottic Region of the Larynx of a Cat Four Weeks Old. Golgi's rapid method. n, Nerve fibers rising from the connectivetissue layer into the epithelial layer, where they terminate in ramified and free arborizations. (After G. Retzius.)

The motor nerves to the muscles end in what are known as muscle plates, the details of whose structure have been already described.

The special sensory end-organs will be described later in the chapter on the Special Senses.

A fourth form of termination consists of corpuscles that are more or less encapsulated, and these are known as the corpuscles of Pacini, the tactile 
corpuscles of Meissner, the tactile corpuscles of Krause, the tactile menisques, and the corpuscles of Golgi.

The Pacinian Corpuscles.-These nerve endings, named after their discoverer Pacini, are elongated oval bodies situated on some of the cerebrospinal and sympathetic nerves. They occur on the cutaneous nerves of the hands and feet, the branches of the large sympathetic plexus about the abdominal aorta, the nerves of the mesentery, and have been observed also in the pancreas, lymphatic glands, and thyroid glands, figure roo. Each corpuscle is attached by a narrow pedicle to the nerve on which it is

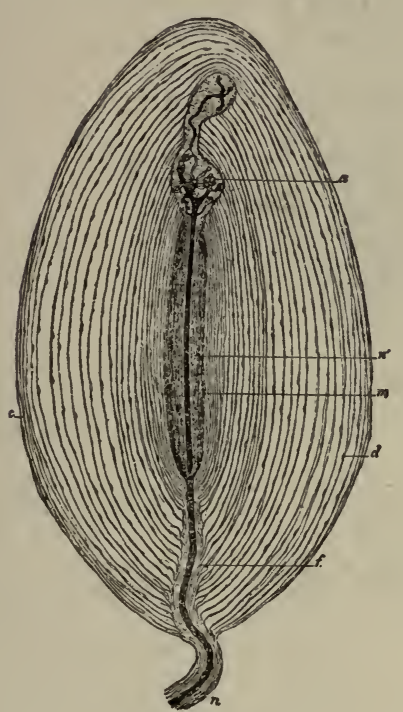

FIG. I00.

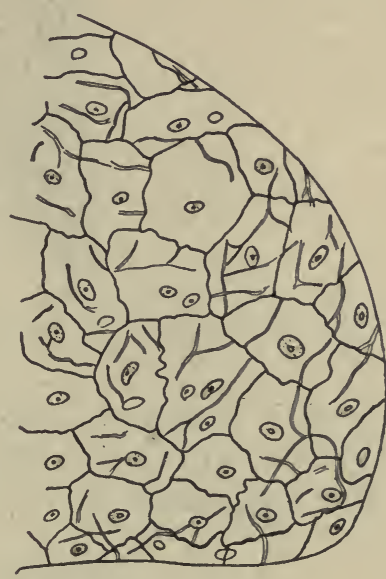

FIG. Ior.

FIG. I00.-Pacinian Corpuscle of the Cat's Mesentery. The stalk consists of a nerve fiber, $n$, with its thick outer sheath. The peripheral capsules of the Pacinian corpuscle are continuous with the outer sheath of the stalk. The intermediary part becomes much narrower near the entrance of the axis-cylinder into the clear central mass. A hookshaped termination with the end-bulb, $a$, is seen in the upper part. (Ranvier.)

FIG. IOI.-Summit of a Pacinian Corpuscle of the Human Finger, showing the Endothelial Membranes Lining the Capsules. $\quad \times 220 . \quad$ (Klein and Noble Smith.)

situated, and is formed of several concentric layers of fine membrane, each layer being lined by endothelium, figure ror. A single nerve fiber passes through its pedicle, traverses the several concentric layers, enters a central cavity, and terminates in a knob-like enlargement or in a bifurcation.

The physiological import of these bodies is still obscure.

The Tactile Corpuscles of Meissner.-They are found in the papillæ of the skin of the fingers and toes or among its epithelium. When simple they are small, slightly flattened transparent bodies composed of nucleated cells enclosed in a capsule. When compound, the capsule contains several 
small cells. The nerve fiber penetrates the corpuscles, loses its myelin sheath, and divides and subdivides to form a series of arborizations. The terminal arborizations occupy the central part of the corpuscle, and are

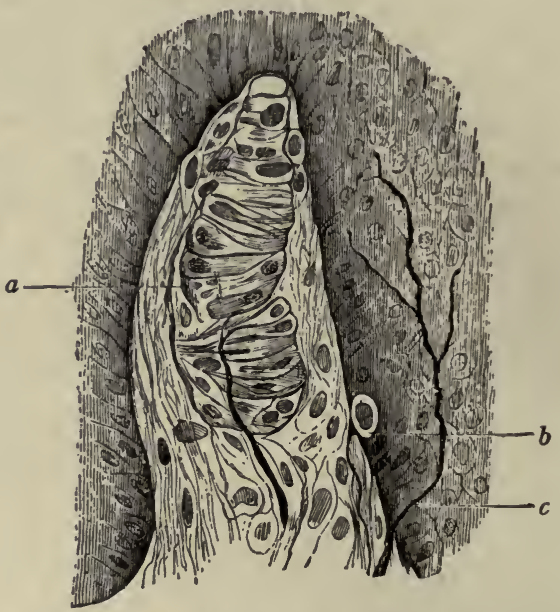

FIG. 102.- Tactile Corpuscle of Meissner, Tactile Cell, and Free Nerve Ending. $a$, Corpuscle proper, outside of which is seen the connective-tissue capsule; $b$, fiber ending on tactile cell; $c$, fiber ending freely among the epithelial cells. (Merkel-Henle.)

surrounded by a great number of marginal cells. The tactile corpuscles of Meissner serve for the special purpose of touch.

The Corpuscles of Krause or End-bulbs.-These exist in great

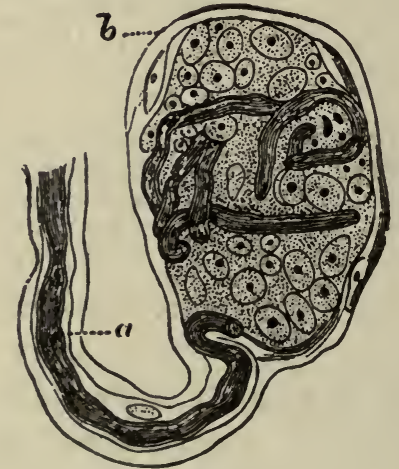

FIG. I03.

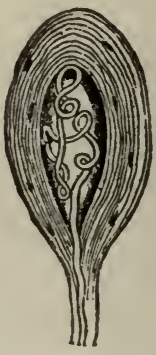

FIG. 104 .

FIG. I03.-End-bulb of Krause. $a$, Medullated nerve fiber; $b$, capsule of corpuscle.

FIg. I04.-A Termination of a Medullated Nerve Fiber in Tendon, lower half with Convoluted Medullated Nerve Fiber. (Golgi.)

numbers in the conjunctiva, the glans penis, clitoris, lips, skin, and in tendon of man. They resemble the corpuscles of Pacini, but have much fewer concentric layers to the corpuscle, and contain a relatively voluminous central 
mass composed of polyhedral cells. In man these corpuscles are spherical in shape, and receive many nerve fibers which wind through the corpuscles and end in the free extremities, figure ro3.

Tactile Menisques.-In different regions of the skin of man, one meets, in the superficial layers and in the Malpighian layers, nerves which, after having lost their myelin sheath, divide and subdivide to form extremely beautiful arborizations. The branches of these arborizations are the tactile menisques. These menisques, which simulate the form of a leaf, represent a mode of terminal nervous arborization (Ranvier).

The Corpuscles of Golgi.-These are small terminal plaques placed at the union of tendons and muscles, but belonging more properly to the

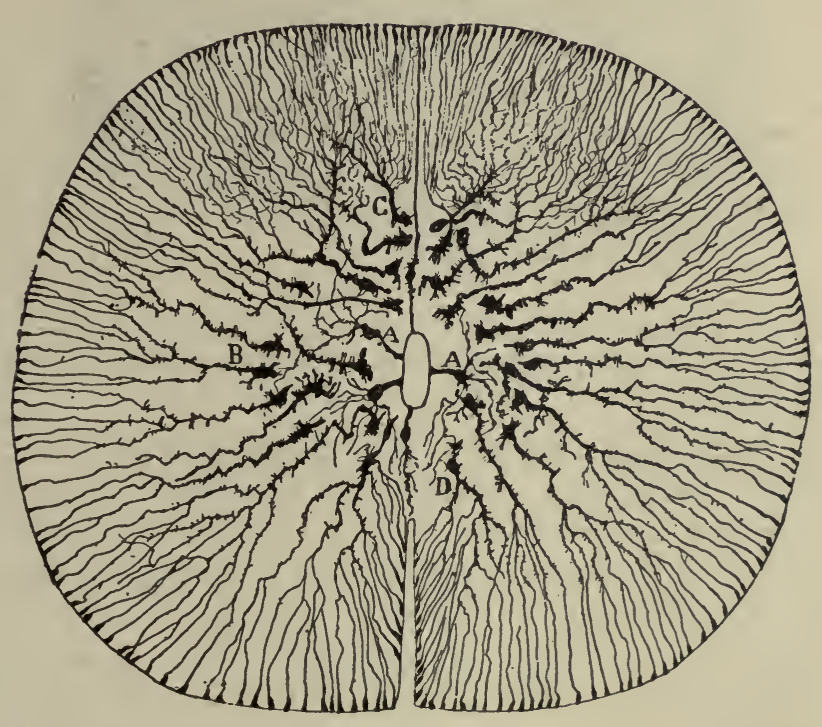

FIg. I05.-Neuroglia Cells in the Cord of an Adult Frog. $A$, Ependyma cells with their peripheral extremities atrophied and ramified; $B, C, D$, neuroglia cells in different degrees of emigration and separation from the ependymal canal; their central extremity is atrophied and much contracted; their peripheral extremity, on the other hand, is greatly extended; the ramifications of the latter, terminating in conical buttons, $I$, end under the pia mater. (After Cl. Sala.)

tendon. They are fusiform in shape and are flattened upon the surface of the tendon close to its insertion into the muscular fibers. They are composed of a granular substance, enveloped in several concentric hyaline membranes which contain some nuclei. The nerve fiber passes into this little corpuscle, splitting itself up into fine terminals. The corpuscles of Golgi are believed to be related to the muscular sense, figure 104 . 
The Muscle Spindles.-Voluntary muscles are supplied with nerve terminations of a sensory nature ending in Pacinian corpuscles, in end bulbs, and in special structures known as neuromuscular bundles or muscle spindles. A muscle spindle consists of one or more muscle fibers somewhat smaller than the typical fibers of that particular muscle, and containing a relatively great amount of sarcoplasm, and many nuclei. These fibers are intimately bound with nerve terminations as shown in the figure ro6. Certain of the voluntary muscles, particularly those of the arms and legs, contain large num-

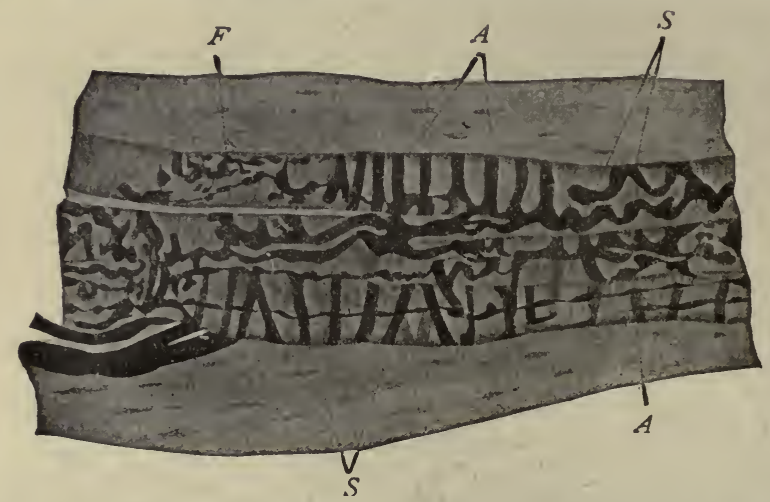

FIG. 106.-A section of a muscle spindle from a voluntary muscle of the cat. $A$, Annular terminations; $S$, spiral terminations; $F$, arborescent terminations. (From Barker, after Ruffini.)

bers of muscle spindles. In the tendons of these muscles also are numerous muscle-tendon organs of the Golgi type. Sherrington conclusively demonstrated the sensory nature of these fibers and terminations by showing that they did not undergo Wallerian degeneration when the corresponding anterior spinal nerve roots were cut and allowed to degenerate.

\section{THE NEUROGLIA.}

The neuroglia, while not a nervous tissue, is closely mingled with it and forms an important constituent of the nervous system. It consists of cells giving off a fine network of richly branching fibers. Neuroglia is a form of connective tissue, and it is in its functions strictly comparable to the connective tissue which supports the special structures of other organs, like the lungs and kidneys. In the adult animal the neuroglia tissue is composed of cells from which are given off immense numbers of fine processes. These extend out in every direction, and intertwine among the nerve fibers and nerve cells, figure 105. The neuroglia cell differs in size and shape very much in different parts of the nervous system in accordance with the arrangement of the nervous structures about it. The cell is composed of granular protoplasm, and lying in it is a large nucleus, within which is a nucleolus. The body of the cell is small in proportion to the nucleus. 


\section{CHAPTER III.}

\section{THE CHEMICAL COMPOSITION OF THE BODY.}

OF the eighty chemical elements which have been isolated, no less than seventeen combine in varying quantities to form the chemical basis of the animal body. The substances which contribute the largest share are the non-metallic elements, Nitrogen, Oxygen, Carbon, and Hydrogen-oxygen and carbon making up altogether about 85 per cent. of the whole. The most abundant of the metallic elements are Calcium, Sodium, and Potassium.*

These elements do not exist in the animal body in the free state, but are combined into complex chemical compounds.

The first step in the act of separating the composition products of protoplasm produces changes which destroy the chemical and physical relations of these products which maintain the state of life. Dead protoplasm, however, yields a number of substances which must be very directly derived from the living protoplasm. On the other hand, certain products can be isolated from the animal body which are evidently not a part of the protoplasm itself, but products of protoplasmic activity. Some of these, like fat, glycogen, etc., are constructive products, others are disintegration products of protoplasmic activity.

\section{THE NITROGENOUS SUBSTANCES.}

The nitrogenous substances in the body consist chiefly of the proteins or of substances which are derived from the proteins. Nitrogenous substances occur in the solid tissues of the body and are found also to a considerable extent in the circulating fluids (the blood and lymph) and in the secretions and excretions.

* The following table represents the relative proportion of the various elements in the body. (Marshall.)

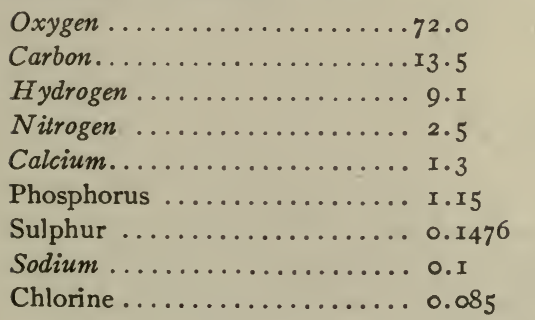

Fluorine $\ldots \ldots \ldots \ldots \ldots \ldots \ldots, 0.08$

Potassium .................. 0.026

Iron................... or

Magnesium .............. 0.012

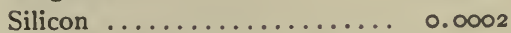

(Traces of copper, lead, and aluminum)..................

I00 


\section{THE PROTEINS.}

These nitrogenous substances constitute the most important and complex compounds in the body. They are essentially the organic basis of all living substance. At the same time they are the most important of our organic food stuffs. The proteins are necessary as food material for the continuance of life and cannot be replaced in the diet by any other organic or inorganic substances. Without them all life, whether animal or vegetable, is impossible.

The proteins are substances containing carbon, hydrogen, and oxygen (which are present in fats and carbohydrates). The proteins also contain nitrogen and sulphur. Phosphorus and certain metallic elements are present as constituents of some proteins. The elementary composition of most protein substances falls within the following percentages:

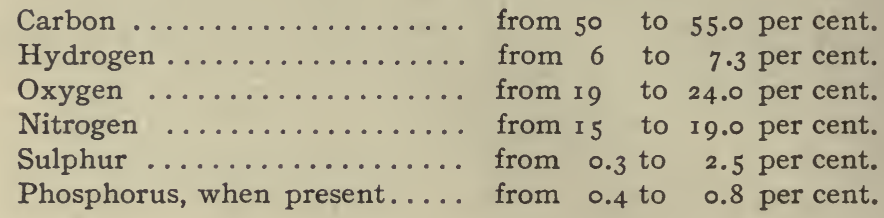

The individual protein substances are chemical entities. As individuals of a group they differ in elementary composition and in the derivatives which they yield on cleavage of the protein molecule.

Chemical Structure of Proteins.-Proteins are combinations of $\alpha$-amino acids, the simplest example of which is glycocoll or $\alpha$-amino acetic acid. Acetic acid has the formula $\mathrm{CH}_{3} \mathrm{COOH}$; if the $\mathrm{NH}_{2}$ group is substituted for one of the H's in the $\mathrm{CH}_{3}$ radical it is an amino acid. The introduction of the amino group in this way yields bodies which combine both with acids and with bases. It is also possible for the amino acids to combine with one another, with the elimination of water. The reaction, however, can only be brought about under certain conditions. For instance, glycocoll can be combined with itself as a dipeptid or combined with any other amino acid. The combination may be indicated by the following:

$$
\begin{aligned}
& \begin{array}{c:c:c}
\mathrm{CH}_{2}-\mathrm{NH}_{2}-\mathrm{CO} & \mathrm{OH}+\mathrm{H} & \mathrm{HN}-\mathrm{CH}_{2}-\mathrm{COOH}= \\
\text { glycocoll } & \text { plus } & \text { glycocoll }
\end{array} \\
& \underset{\text { glycyl-glycine }}{\mathrm{CH}_{2}}-\underset{\text { plus }}{\mathrm{NH}_{2}}-\mathrm{CO}-\mathrm{NH}-\underset{\text { water }}{\mathrm{CH}_{2}}-\mathrm{COOH}+\mathrm{H}_{2} \mathrm{O}
\end{aligned}
$$

A glance at the chemical formulæ of the resulting dipeptid indicates that in this new substance there is still an amino group $\left(\mathrm{NH}_{2}\right)$ and a carboxyl group $(\mathrm{COOH})$ which are not combined. Another amino acid may be joined on to the carboxyl root, and yet a fourth on to the remaining amino 
group, so that the still more complex peptids can be formed. When this is done there is yet a carboxyl and an amino group to which other amino acids can similarly be joined. The structure of the protein molecule accordingly may be represented as follows:<smiles>[R]C([R])NOC([R])NC</smiles>

In which $\mathrm{R}$ indicates here the rest of the formula for any of the $\alpha$-amino acids entering into the constitution of protein.

At least eighteen amino acids have been found to enter into the composition of the proteins. The list includes glycocoll, alanine, serine, phenylalanine, tyrosine, tryptophane, cystine, leucine, isoleucine, amino-butyric acid, aspartic acid, glutaminic acid, proline, oxyproline, histidine, argenine, lysine, and diaminotrihydroxydodecanoic acid. Associated with the amino acids there is usually a detectable amount of amino-carbohydrate. The chemical formulæ for the more important of these are given below:<smiles>NCC(=O)O</smiles><smiles>CCC(N)C(=O)O</smiles><smiles>CC1CCCCCC1</smiles>

Glycocoll. Alanine.

Phenylalanine.

(amino acetic acid.) ( $\alpha$-amino proprionic acid.) (Phenyl $\alpha$-amino proprionic acid.)<smiles>CC(O)C(N)C(C)C(O)CCCC(C)C(N)C(=O)O</smiles>

Serine. $(\alpha$-amino $\beta$-hydroxy proprionic acid.)
Tyrosine. (p-oxyphenyl $\alpha$-amino proprionic acid.)<smiles>NC(CSSCC(=O)O)C(N)C(=O)O</smiles>

Cystine. 
<smiles>NC(C(=O)O)C1CNC2CCCC21</smiles>

Tryptophane.

(Indol amino proprionic acid.)<smiles>CC(C)C(N)C(=O)O</smiles>

Valine. ( $\alpha$-amino isovalerianic acid.)<smiles>CC(C)C(N)C(=O)O</smiles>

Leucine. ( $\alpha$-amino isobutylacetic acid.)<smiles>NC(Cc1c[nH]cn1)C(=O)O</smiles>

Histidine. ( $\alpha$-amino $\beta$-imidazol proprionic acid.)<smiles>NC(C(=O)O)C(=O)O</smiles>

Aspartic acid. (amino succinic acid.) $\quad(\alpha$-amino normal glutaric
acid).<smiles>NC(CC(=O)O)C(=O)O</smiles>

Glutaminic acid.<smiles>NCCCCCC(N)C(=O)O</smiles>

The amino acids belong either to what is known in organic chemistry as the aliphatic, carbocyclic, or heterocyclic series; that is, they are derivations either of the hydrocarbons, of benzene or of closed-ring compounds not composed wholly of carbon atoms, but in which one or more of the links in the closed chain are supplied by other polyvalent elements (in the proteins by nitrogen). Thus tyrosine and phenylalanine are carbocyclic compounds; histidine, proline, and tryptophane are heterocyclic compounds, and the remaining members of the list are aliphatic derivatives.

Of the elements of the protein molecule, nitrogen is by far the most 
characteristic and important. The animal organism is unable to construct the amino acid molecules and hence cannot build up protein material from nitrogen of the atmosphere or from combinations, such as ammonia, nitrates, and nitrites. Plants, however, have the property of synthesizing proteins from inorganic nitrogen. The nitrogen of amino acids and protein is directly utilizable by the body, so that the animals are ultimately dependent upon plants for their protein-supply.

Nitrogen in the protein molecule occurs in four different forms:

r. The monamino acid nitrogen, or the nitrogen that is in the $\mathrm{NH}_{2}$ (amino) group of the $\alpha$ position.

2. The diamino acid nitrogen, or the basic nitrogen, as shown in the amino group in lysine.

3. Amide nitrogen; the $\mathrm{OH}$ of the second $\mathrm{COOH}$ group in the dibasic glutaminic and aspartic acids in protein may be replaced by the amino group. On cleavage, the $\mathrm{NH}_{2}$ is split off from the acid amide as ammonia.

4. The guanidine residue as in arginine.

The distribution of nitrogen in the protein accordingly depends on the amino acids entering into its composition.

Sulphur of the protein is present in the amino acids, cystine and cystein.

It has been stated that the amino acids are combined together in a protein molecule, carboxyl with amino radical. On boiling the proteins with mineral acids, the reaction is reversed and the protein substances are split, with the combining of water, into the individual amino acid components. This change is termed a "hydrolytic cleavage." The qualitative and quantitative determination of the products thus obtained have shown us that the proteins differ chemically both as to the individual amino acids which enter into the complex protein molecule and the amount of each acid present. Proteins, then, which may give exactly the same percentage composition and elementary analysis and which show practically the same physical properties are found to be actually different individuals of the protein group when the products of their hydrolytic cleavage are investigated. For example, the following tables give the elementary composition and the amino acids obtained from three proteins which are present in wheat flour.

Elementary Composition of Wheat Proteins.

\begin{tabular}{|c|c|c|c|}
\hline & Gliadin & Glutenin & Leucosin \\
\hline Carbon ............. & $5^{2} .7^{2}$ & $5^{2} \cdot 34$ & 53.02 \\
\hline Hydrogen ......... & 6.86 & 6.83 & 6.84 \\
\hline Nitrogen ... & 17.66 & 17.49 & I 6.80 \\
\hline Sulphur ... . & I.०3 & I.०8 & 1.28 \\
\hline \multirow[t]{2}{*}{ Oxygen $\ldots \ldots \ldots \ldots$} & 21.73 & 22.26 & 22.06 \\
\hline & 100.00 & I 00.00 & 100.00 \\
\hline
\end{tabular}


Amino Acids Obtained on Hydrolytic Cleavage of Wheat Protein.

\begin{tabular}{|c|c|c|c|}
\hline Percent of & Gliadin & Glutenin & Leucosin \\
\hline Glycocoll ............ & 0.00 & 0.89 & 0.94 \\
\hline Alanin $\ldots \ldots \ldots \ldots \ldots$ & 2.00 & 4.65 & 4.45 \\
\hline Amino valerianic acid..... & $0.2 \mathrm{I}$ & 0.24 & 0.18 \\
\hline 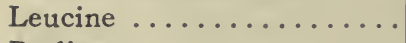 & $5.6 \mathrm{I}$ & 5.95 & II.34 \\
\hline Proline ............... & 7.06 & 4.23 & 3.18 \\
\hline Phenylalanine .......... & 2.35 & I.97 & 3.83 \\
\hline Aspartic acid.......... & $0.5^{8}$ & $0.9 \mathrm{I}$ & 3.35 \\
\hline Glutaminic acid $\ldots \ldots \ldots$ & $37 \cdot 33$ & 23.42 & 6.73 \\
\hline 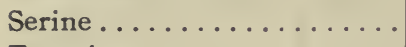 & 0.13 & 0.74 & 0.00 \\
\hline Tyrosine.............. & 1.20 & 4.25 & $3 \cdot 34$ \\
\hline 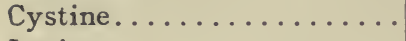 & 0.45 & 0.02 & 0.00 \\
\hline Lysine ................ & 0.00 & 1.92 & 2.75 \\
\hline Histidine $\ldots \ldots \ldots \ldots \ldots$ & $0.6 \mathrm{I}$ & 1.76 & 2.83 \\
\hline Arginine.............. & 3.16 & $4 \cdot 72$ & $5 \cdot 94$ \\
\hline 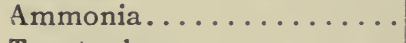 & $5 . \mathrm{II}$ & 4.01 & $\mathrm{I} .4 \mathrm{I}$ \\
\hline Tryptophane........... & present & present & present \\
\hline
\end{tabular}

It has been indicated that the synthesis in very simple proteins can be attained by combining amino acids. A synthesis, however, can be brought about by the reversible action of the digestive enzymes. Recently Taylor has been able to synthesize a simple protein, a protamine, by the reversible action of a trypsin on amino acids which were previously obtained by the digestion of the protamine. Cleavage by enzymes is a hydrolysis of the same type as that mentioned above by the use of the mineral acids. In the concentration of the products of a digestion the enzymes act in the reverse manner and resynthesize these substances on which they have previously acted. Robertson has demonstrated a similar reversible action of pepsin on paranuclein derived from a digestion of casein. The reversible action may be indicated by the equation:

\section{PROTEIN + WATER $\rightleftarrows$ AMINO ACIDS.}

These experiments lend a new stimulus to the efforts to build up proteins in the chemical laboratory along the lines of catalytic action of enzymes. The proteins of the various tissues of the body are quite different in character and chemical constitution and are different from the protein of the diet. In digestion there is a breaking down of the protein in the food into simple combinations of amino acids or the acids themselves, and subsequently there is a selection of certain amino acids and resynthesis of characteristic proteins by the cells of the tissues. 
It is customary to assign to a compound having an unknown molecular mass, i.e., relative weight in units of the weight of an atom of hydrogen, a formula representing the least mass which the substance could have and preserve its characteristic properties. The simplest formula for oxyhemoglobin, the compound protein of the red blood cells, is $\mathrm{C}_{658} \mathrm{H}_{1181} \mathrm{~N}_{207} \mathrm{~S}_{2} \mathrm{FeO}_{210}$. This formula is based on the relative proportion that the $\mathrm{C}, \mathrm{H}, \mathrm{N}, \mathrm{S}$, and $\mathrm{O}$ bear to the $\mathrm{Fe}$ as determined by analysis. This protein contains iron, and the least $\mathrm{Fe}$ that one molecule can contain is one atom. By addition of the atomic masses of the total number of atoms of each element, the least possible molecular mass for oxyhemoglobin is about 15,000. It might just as well be 30,000 with two atoms of iron in the compound. The following formulæ have been proposed for ovalbumin and seralbumin:

$$
\begin{aligned}
& \text { Ovalbumin, } \mathrm{C}_{239} \mathrm{H}_{386} \mathrm{~N}_{58} \mathrm{~S}_{2} \mathrm{O}_{78} \\
& \text { Seralbumin, } \mathrm{C}_{450} \mathrm{H}_{720} \mathrm{~N}_{116} \mathrm{~S}_{6} \mathrm{O}_{140}
\end{aligned}
$$

the molecular masses being in the neighborhood of 5000 and 6000 , respectively.

Besides the amino acids other radicals are present in some proteins. A carbohydrate moiety is evidently present in certain proteins and phosphoric acid in others (as in the milk protein casein). Such proteins are to be distinguished from those which exist as combinations with definite chemical entities, as hematin, nucleic acid, amino sugars, lecithins, etc.

Properties of Protein.-Many proteins have been prepared in crystalline form, especially the reserve proteins from various seeds. Very few animal proteins have ever as yet been crystallized-seralbumin, lactalbumin, ovalbumin and hemoglobin are examples.

The majority of the proteins are soluble in water or in dilute solutions of neutral salts of strong bases with alkalies. The proteins do not form solutions as do, for instance, the inorganic salts, but are to be regarded as a suspension of the molecules or molecular aggregates. Such a solution is known as a colloidal solution, and the proteins are frequently spoken of as colloids. Colloidal solutions of the heavy metals can be formed by the interrupted contact of metal electrodes under water. The metallic colloidal solutions and the protein solutions have many properties in common.

When a true solution of a chemical substance of relatively small molecular weight is placed within a parchment or animal membrane, and the whole immersed in water, the substance in solution will diffuse through the pores of the membrane into the water external to it; similarly, water will pass through the membrane to the interior. After some time the system will come into equilibrium. The force which drives the dissolved substance from the more concentrated to the less concentrated solution is known as osmotic pressure. The large protein molecules and molecular aggregates cannot pass through the pores of the membrane, or, in other words, they are not 
diffusible. Their osmotic pressure is very slight. Some of the simpler derived proteins, however, are diffusible.

Proteins in aqueous solution rotate polarized light to the left. The specific rotation of the individual proteins varies. The compound proteins, hemoglobin and nucleoproteins are dextrorotatory.

Proteins chemically are rather unstable bodies and are easily hydrolyzed, through heating and standing in alcohol, into modifications which differ only slightly from the original substances. This change is ordinarily termed coagulation.

Proteins may be precipitated from their solutions by the addition of the neutral inorganic salts in high concentration or to saturation. This precipitation is essentially a physical one, the protein remaining unchanged. Salting out is, then, a most valuable method for the separation and purification of the protein substances. The proteins form insoluble combinations with the so-called alkaloidal reagents; phosphotungstic acid, phosphomolybdic acid, picric acid, trichloracetic acid, potassium mercuric iodide, and tannic acid. The protein also forms insoluble albuminates with the salts of the heavy metals.

The color reactions for the proteins, which are given in the laboratory experiments at the end of this chapter are due to a reaction between some one or more of the constituent radicals of the complex protein molecule and the chemical reagent or reagents used. Thus certain color tests are due to the presence of individual amino acids in the protein molecule, and the intensity of the reaction obtained will vary with the amount of the amino acids present. The negative results for a certain test will indicate the absence of the particular amino acid in the molecular complex. The color tests, then, are important because they throw light on the chemical constitution of the protein under observation.

\section{CLASSIFICATION OF THE PROTEINS.}

The following classification is that adopted by the American Physiological Society and the American Society of Biological Chemists:

I. Simple Proteins.-Protein substances which yield only $\alpha$-amino acids or other derivatives on hydrolysis.

a. Albumins.-Soluble in pure water; e.g., ovalbumin, seralbumin, and the vegetable albumins.

b. Globulins. - Insoluble in pure water, but soluble in neutral solutions of strong bases with strong acids; e.g., ovoglobulin, edestin, and other vegetable globulins.

c. Glutelins.-Simple proteins insoluble in neutral solvents, but readily soluble in very dilute acids and alkalies. These substances occur abundantly in the seeds of cereals. 
All of the above are coagulable by heat.

d. Prolamins or Alcohol-soluble Proteins.-Soluble in 70 to 80 per cent. alcohol; insoluble in water, absolute alcolol, and other neutral solvents; e.g., zein from corn, gliadin from wheat, and hordein from barley.

e. Albuminoids.-Simple proteins characterized by a pronounced insolubility in all neutral solvents. These form the principal organic constituents of the connective tissues of animals including their external covering and its appendages. Examples: elastin, collagen, and keratin.

The above sub-classes are characterized by physical rather than by chemical differences. When the protein, for instance, is termed a globulin it means that it is a typical simple protein with certain characteristic solubilities. Proteins intermediate in character between albumins and globulins are met with, and the use of these terms as a hard-and-fast classification has led to considerable confusion.

f. Histones. On hydrolysis these yield a large number of amino acids, among which the basic ones predominate. The histones stand chemically between the typical simple proteins and the following group of protamins. Examples are: globin, thymus histone, scombrone.

g. Protamines.-Simpler polypeptids than the proteins included in the preceding groups. They yield comparatively few amino acids, among which the basic ones predominate. They are the simplest natural proteins. Examples are: salmin, sturine, clupeine, and scombrine.

II. Conjugated Proteins.- Substances which contain the protein molecule united to some other molecule or molecules otherwise than as a salt.

a. Nucleoproteins.-Compounds of one or more protein molecules with nucleic acid; e.g., nucleohistone.

b. Glycoproteins.-Compounds of the protein molecule with a substance or substances containing a carbohydrate group other than a nucleic acid; e.g., mucins and mucoids.

c. Phosphoproteins.-Compounds of the protein molecule with phosphorous containing substances other than a nucleic acid or lecithin; e.g., casein, ovovitellin.

d. Hemoglobins.-Compounds of a protein molecule with hematin or some similar substance. These include the respiratory pigments; e.g., hemoglobin and hemocyanin.

e. Lecithoproteins. - Compounds of the protein molecule with the lipoid lecithin; e.g., lecithans, phosphatides.

III. Derived Proteins. Class I. Primary Protein Derivatives.Derivatives of the protein molecule apparently formed by hydrolytic changes which involve only slight alteration of the protein molecule.

f. Proteans.-Insoluble products which apparently result from the incipient action of water, from dilute acids or enzymes; e.g., myosan, edestan.

g. Metaproteins.-Products of the further action of acids and alkalies 
whereby the molecule is so far altered as to form products soluble in very weak acids and alkalies, or insoluble in neutral solutions; e.g., acid metaprotein, acid albuminate, alkali metaprotein or alkali albuminate.

h. Coagulation Proteins.-Insoluble products resulting from $\mathrm{r}$, the action of heat on protein in solution or, 2, the action of alcohol on the protein.

Class 2. Secondary Protein Derivatives.-Products of more extensive hydrolytic cleavage of the protein molecule than that in the preceding class.

i. Proteoses. - Soluble in water, non-coagulable by heat, and precipitated by saturating their solutions with ammonium or zinc sulphate.

j. Peptones.-Soluble in water, non-coagulable by heat, and not precipitated by saturating their solutions with ammonium sulphate.

$k$. Peptides.-Definitely characterized combinations of two or more amino acids, the carboxyl group of one being united with the amino group of the other with the elimination of a molecule of water.

\section{CHARACTERISTICS OF THE VARIOUS CLASSES OF PROTEINS.}

Albumins.-Albumins constitute the first class of simple proteins. They may be defined as simple proteins which are coagulable by heat and are soluble in pure (salt-free) water. As a rule, they are not precipitated on saturating their solutions with sodium chloride or magnesium sulphate, unless the solution be then acidified with dilute acid. They do not coagulate out on heating their solution unless a trace of a salt is present. Some albumins have been prepared in crystalline form; e.g. ovalbumin, serumalbumin, and lactalbumin. Crystallization is obtained on adding ammonium sulphate to the protein solution until slight turbidity results, then clearing the solution by adding a little water and acidifying slightly with acetic acid. The albumins, as a rule, are precipitated on saturating with neutral ammonium sulphate. Zinc sulphate may be employed when it is desired not to introduce ammonium salts into the precipitation. The proteins are precipitated and subsequently coagulated by the addition of an excess of alcohol. They remain in solution on removal of inorganic salts by dialysis.

On heating a solution of an albumin (or a globulin) the turbidity and flocking out of the coagulum occur at a temperature which is more or less characteristic for the individual protein. However, the coagulation temperature can be varied according to the concentration of the protein solution, the presence of inorganic salts, and by the reaction of the solution. The coagulation temperatures, then, cannot be given as definite characters for individual proteins unless the conditions under which the figures were obtained are comparable.

The albumins differ among themselves in the cleavage products they yield on hydrolysis, in their elementary composition, and in the specific rotation and coagulation temperatures. The serum albumin and lact- 
albumin are quite closely related chemically, though differing in their specific rotation of polarized light. The albumins contain, as a rule, more sulphur than do the other classes of proteins.

Globulins.-The globulins are simple proteins which are insoluble in pure (salt-free) water, but which are soluble in neutral solutions of salts of strong bases with strong acids. Most globulins are precipitated from their solutions on slight acidification and on saturation with sodium chloride and magnesium sulphate. They are precipitated also from other solutions on adding equal volume of saturated ammonium sulphate solution; this precipitation is commonly termed "precipitation at half saturation ammonium sulphate." Since they are insoluble in pure water, dilution of the weak salt solution containing protein causes precipitation. The globulins are especially predominant in the vegetable kingdom. They occur in relatively large amounts as the reserve protein in seeds of various sorts. There are, however, no essential differences as a class between the globulins of animal and of vegetable origin.

The globulins are precipitated from weak salt solutions on dialysis in pure water. The inorganic salts diffuse through parchment, and with the reduction in salt content the globulins are precipitated. Many of the vegetable globulins can be obtained in crystalline form by precipitating them in this way.

As a class the globulins are relatively less stable than the albumins. They are converted over into proteans on successive reprecipitation or simply by standing under water.

Albuminoids. - The albuminoids yield similar amino acids on hydrolysis to those obtained from the simple proteins. They possess essentially the same general chemical structure. They differ from all other proteins in that they are insoluble in neutral solvents. The classification, then, is based purely on this property, though they are characterized by their occurrence as the principal organic constituents in the structure of the supporting tissues of the body and of the skin and its appendages. The individual albuminoids differ from each other fundamentally in certain chemical characteristics. The albuminoids also are differentiated along with the morphological variations of the connected tissues in which they occur. For example, the keratins occur in the skin and its appendages. Collagen is the principal albuminoid of white fibrous tissue, though found also in cartilage and bone. Elastin characterizes the yellow elastin tissue, as, for instance, the nuchal tendon, the elastic tendon so well developed in the neck of the ox.

Keratins.-The epidermis of the skin, the nails, hair and horn, feathers, tortoise shell, silk, and the supporting neuroglia of nervous tissue may be considered to be keratins in relatively pure form. The keratins take the form of the tissue from which they are prepared. On heating they are decomposed with the odor of burnt horn. They are insoluble in water, 
alcohol, and ether, and in the ordinary protein solvents. They are not acted upon by gastric or pancreatic juices. On heating to 150 to $200^{\circ} \mathrm{C}$. in water the protein is hydrolyzed and dissolves. The keratins are also soluble in the caustic alkalies, especially on heating. Keratins from any source may be prepared in pure form by treating with artificial gastric juice, artificial pancreatic juice, boiling alcohol, and boiling ether, from twentyfour to forty-eight hours being devoted to each process. Several keratins, so far as their chemical structure is concerned, exist.

Collagen.-Collagen can be most satisfactorily prepared from the tendo achillis of the ox. It forms the principal organic constituent of this and other white fibrous tissues, as shown by the analysis given in the following table:

\section{Chemical Composition of the White Fibrous Tissue: Tendo Achillis (BERg AND Gies).}

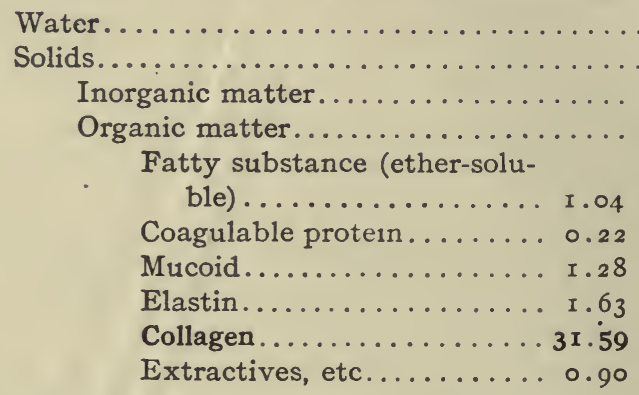

The collagen from various sources in common with the keratin is not identical in composition. It differs from the keratin in containing less sulphur. It does not give the reaction for tryptophane and contains but little tyrosin. It is dissolved by pepsin and hydrochloric acid, but not by pancreatic juice.

The general characteristic of collagen is that it is hydrolyzed into gelatin by boiling with water or dilute acid. Gies has shown that ammonia is liberated by this procedure. Gelatin is soluble in hot water, but its solutions form a jell when cooled. Inasmuch as tyrosin and tryptophane are not present in the gelatin molecule, this albuminoid is not a satisfactory substitute for the protein constituents in the normal diet.

Elastin.-Elastin is the principal solid constituent of yellow elastic tissue; e.g., the ligamentum nuche. It gives the ordinary protein color reactions. It contains, however, a relatively small amount of sulphur. Elastin is dissolved by pepsin hydrochloric acid and by pancreatic juice, and unlike collagen it is not converted into gelatin on prolonged boiling with water or dilute acids. 
Composition of Yellow Elastic Tissue: Ligamentum nuche (Berg and Gres).

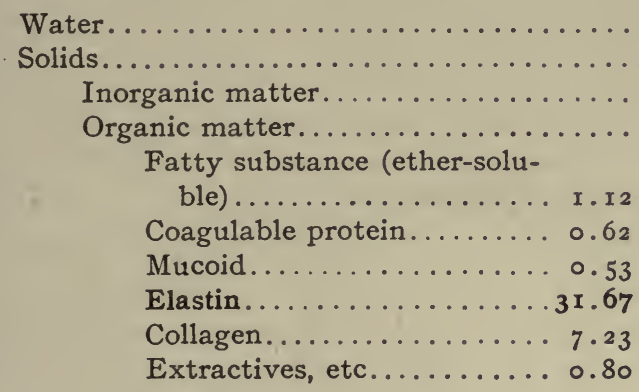

Histones. - The histones are proteins which stand in their chemical structure between the true proteins and the protamines. On hydrolysis they yield a large number of amino acids, among which the basic ones predominate. The basicity, however, is only slight. They are precipitated by dilute ammonia and this precipitate is soluble in an excess of ammonia in the absence of ammonium salts. They are precipitated by nitric acid, the precipitate dissolving on heating and again appearing on cooling. They give precipitates with solutions of other proteins. On heating, the histones yield a coagulum which is easily soluble in very dilute acids

The histones are found in the nuclei of the red blood cells of birds, the unripe testis in salmon and mackerel and in the ripe spermatozoön of the sea-urchin. The thymus contains histone. The liver, kidneys, ox pancreas, and testis of mammals contain no histone-like substances. The globin of the compound protein hemoglobin is to be regarded as a histone.

Protamines.-The protamines are basic proteins which are combined with nucleic acid and form the chief constituent of the spermatozoa of the salmon and other fish. They are relatively simple proteins yielding comparatively few amino acids on hydrolysis, among which the basic ones predominate. From elementary analyses the following formula has been suggested for the platinum salt of salmine. $\mathrm{C}_{30} \mathrm{H}_{57} \mathrm{~N}_{17} \mathrm{O}_{6} \cdot 4 \mathrm{HCl} .2 \mathrm{PtCl}_{4}$. As seen from the formula, the protamines are extremely rich in nitrogen which comprises from 25 to 32 per cent. of their weight. The protamines dissolve in water and give a faintly alkaline reaction. They are precipitated in acid solution by platinic chloride. The protamines, after the addition of ammonia, precipitate true proteins, proteoses, and nucleic acid. On hydrolysis all protamines yield relatively large amounts of argenine and varying amounts of histone, lysin, proline, alanine, valine, leucin, tyrosin, and apparently also tryptophane. Protamines also do not contain sulphur or a carbohydrate moiety. They are not changed by digestion with pepsin hydrochloric acid. 
Conjugated proteins consist of a protein molecule united with some other molecule or molecules otherwise than as a salt. There are nucleoproteins, glycoproteins, phosphoproteins, hemoglobins or chromoproteins and lecithoproteins-five classes of conjugated proteins.

Nucleoproteins.-Nucleoproteins contain phosphorus and in most instances iron. They are combinations of simple proteins with a substance known as nucleic acid. On boiling with strong acids they undergo hydrolytic cleavage, yielding the ordinary protein cleavage products from the protein in the combination, and purine and pyrimidine bases, carbohydrates and phosphoric acid from the nucleic acid moiety. Nucleoproteins are differentiated from the phosphoproteins in that the latter do not yield purine and pyrimidine bases on hydrolytic cleavage. Nucleoproteins are the essential organic constituents of cell nuclei. They go into solution when the tissues are extracted with cold water or dilute salt solution. They are precipitated from these extracts by careful acidification and dissolved if an excess of acid is added. The solutions of nucleoproteins coagulate on heating. They give the color reactions of proteins.

By boiling nucleoproteins with water or very dilute acetic acid, some of the protein is split off. There result substances which are precipitated by very dilute acids. These bodies are known as $\beta$-nucleoproteins and have a smaller carbon and higher phosphorus content than the original nucleoprotein. On digestion of the original nucleoprotein or of the $\beta$-nucleoprotein with pepsin hydrochloric acid, a precipitate of nuclein is obtained. On further digestion with pepsin hydrochloric acid, or with trypsin, or on cleavage with acids and alkalies, there is a complete splitting away of the protein, and substances are formed known as nucleic acids. The structure and cleavage of the nucleoprotein is indicated in the following diagram.

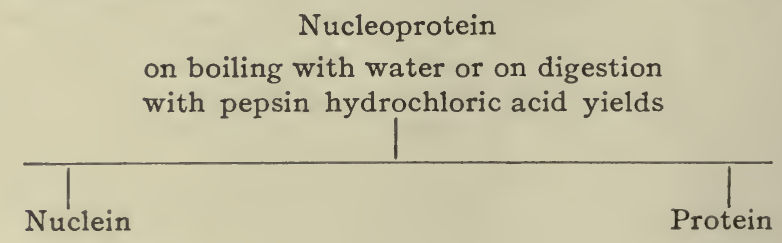

On long boiling with water, $\mathbf{r}$

per cent. hydrochloric acid, or

dilute alkalies gives

$\prod_{\text {Nucleic acid }}^{\mid}$

Nucleic acids are not merely known as cleavage products of nucleoprotein, but occur preformed in the cell nuclei. A special group of nucleic acids are bound with protamines to form the principal constituent of the spermatozoa. 
Nucleic acids are white, amorphous substances containing 9 to 10 per cent. of phosphorus. According to Levine the composition corresponds to $\mathrm{C}_{43} \mathrm{H}_{67} \mathrm{~N}_{15} \mathrm{P}_{4} \mathrm{O}_{30}$. Nucleic acids give none of the protein reactions. In the sperm they are united with the strongly basic protamines and so are acid in character. They may be precipitated, however, by tannic acid, picric acid, or phosphotungstic acid as are other organic bases.

Nucleic acid has been isolated from many different tissues and apparently is a uniform constituent of the nuclei of all cells. The nucleic acids from different animal tissues are apparently identical and similarly the nucleic acids of vegetable origin are identical, though there are marked chemical differences between these two types.

The structure of plant nucleic acid is the better known of the two. Thanks to the extensive investigations of Levine, and Jones, and their co-workers, plant nucleic acid has been shown to be composed of four different nucleotides. These nucleotides are composed of a purine or pyrimidine base linked to phosphoric acid by carbohydrate groups. In the case of yeast or plant nucleic acid, the carbohydrate is a pentose (d. Ribose). The first of the nucleotides studied was guanylic acid which contains the purine base quanine or 2 amino- 6 oxypurine.

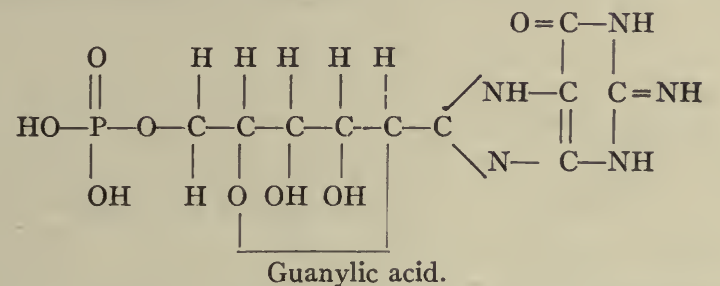

The other three nucleotides contain adenine and the pyramidine bases uracil and cytosine respectively. The tentative structure of yeast nucleic acid is probably that of a tetra nucleotide, though the mode of linkage of the different groups is still in doubt.

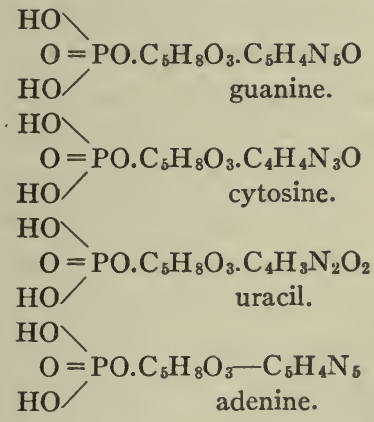

The structure of animal nucleic acid is less well known. It contains a hexose instead of a pentose group and thymine in place of uracil.

Purines.- To the purines belong a number of extremely important anima! and plant substances, including adenine, guanine, hypoxanthine, xanthine, and uric acid, and the methyl purines caffeine, theobromine, and theophylline.

The mother substance of the purines is known as purine, which has the following structure:<smiles>C1CNC2NC(C[NH2+]2)N1</smiles>

Purine ring.<smiles></smiles>

Purine. 
Adenine and guanine may be converted into hypoxanthine and xanthine, respectively, when added to extracts of tissues, such as the liver and the thymus, spleen and pancreatic glands. The "deamidization" is brought about by specific enzymes or non-living ferments which have been termed adenase and guanase. On slight oxidation, hypoxanthine is converted into xanthine, and the latter into uric acid. This change can also be brought about in the body by oxidizing enzymes. The relation of the purine bases to uric acid is indicated in the following scheme:

Adenine.

(6-amino purine.)<smiles>NC1=NC2N=CN=C2N1</smiles><smiles>Nc1nc2nc(N)[nH]c2c(=O)[nH]1</smiles>

Guanine.

(2-amino 6-oxypurine.)
Hypoxanthine.

(6-oxypurine.)

$\mathrm{HN}-\mathrm{C}=\mathrm{O}$<smiles>O=C1NC=NC2=NCNC12</smiles>

on oxi- $\mid$ dation

Glycoproteins.-The glycoproteins are to be considered as compounds of protein and considerable quantities of a carbohydrate complex. The carbohydrate group can be split from the protein by boiling with mineral acids or by the action of alkalies. The group of glycoproteins includes a number of proteins, of which the mucines and mucoids are the most important.

Mucines are very widely distributed. They give the mucilaginous character to many secretions and are formed and discharged through the respiratory, digestive, and other tracts, partly by mucous cells and in part by the mucous glands, especially by the submaxillary and sublingual salivary glands, and in the bile passages. On hydrolysis the mucines are split, the carbohydrate moiety yielding glucose amine or galactose amine.

Mucoids occur in the connecting tissues along with the albuminoids. They are found especially in the tendon, bone, and cartilage. They are combinations of protein and a carbohydrate containing ethereal sulphuric acid known as chondroitin sulphuric acid. On cleavage, besides the products formed from the protein, they yield sulphates and a reducing substance.

Phosphoproteins. - The phosphoproteins, sometimes called nucleoalbumins, are compounds of the protein molecule with some as yet undefined phosphorus-containing substance other than a nucleic acid or lecithin. While the phosphorus content of these substances is quite similar to that 
of the nucleoproteins, they do not yield any purine or pyrimidine bases on hydrolytic cleavage. Two of the best known phosphoproteins are the casein of milk, and vitellin of the egg yolk. The phosphorus is apparently present as a phosphoric acid ester.

Hemoglobins.-These are compounds of the simple protein histone, with an iron-, or in some lower animals, copper-, or manganese-containing pigment substance. The hemoglobins are more fully discussed in the chapter on the Blood.

Lecithoproteins. - These are combinations of proteins and a fat-like substance, lecithin. Lecithin is a compound of fatty acids, glycerin, phosphoric acid, and an ammonium-like organic base, choline. The combination of lecithin and protein is apparently a loose one: the lecithin ordinarily can be split off by boiling alcohol. The lecithoproteins include substances commonly termed lecithans and phosphatids.

The derived proteins are formed as intermediate products in the hydrolytic cleavage of the original protein molecule. The primary protein derivatives are "apparently formed through hydrolytic changes which involve only slight alteration of the protein molecule."

Metaproteins.- These are formed from the simple proteins by the action of weak acids and alkalies. This class comprises what have commonly been termed acid and alkali albuminates. The metaproteins are soluble in acid or alkaline solution, but are insoluble in neutral solutions. In the formation of alkali metaproteins, the sulphur in organic combination is split off. Thus the alkali metaprotein differs from the acid metaprotein in that the former contains little or no sulphur. It is impossible, then, to transform an alkali metaprotein into an acid metaprotein, though the acid metaprotein can be changed into the other modification. Acid metaproteins are the first products formed in the pepsin hydrochloric acid digestion of proteins.

Coagulated Proteins.-Unaltered typical simple proteins in solution are altered when heated or by long standing under alcohol. They are transformed into a coagulated modification no longer soluble in water or dilute salt solutions. A similar change occurs when solutions of the proteins are continuously shaken or by the action of enzymes, as in the formation of fibrin from fibrinogen in the clotting of the blood. On treating coagulated proteins with acids or alkalies they are converted into the respective metaproteins.

Secondary Protein Derivatives.-Secondary protein derivatives are intermediary cleavage products which result from a more profound change than occurs in the formation of the primary derived protein.

Proteoses or albumoses are intermediate products in the digestion of proteins by proteolytic enzymes or in the cleavage with acids. Peptones are yet more simple products than the proteoses and are to be regarded as relatively simple polypeptides which still retain some of the protein 
characteristics. A number of proteoses and peptones have been described. However, there is no sharp dividing line between the more simple proteoses and more complex peptones, or between the simple peptones and the peptides. (The term peptide as at present understood designates only those combinations of amino acids possessing a known definite structure.) The peptones differ from the proteoses in being more diffusible, being non-precipitable on saturation with ammonium sulphate, and by their failure to give certain protein reactions. As a class, proteoses and peptones are relatively very soluble and are non-coagulable by heat.

Melanins are the pigmentary substances found in the hair, feathers, skin, the choroid coat of the eye, and in some tumors. Products similar to the naturally occurring melanins are obtained on hydrolizing nearly all proteins with acids. The melanins are sulphur-containing acid-like substances, and seem to be combinations of amino-sugars (glucosamine) with certain aminoacids, especially tyrosine, tryptophane, and lysine. Iron is found in some of the melanins.

\section{THE FATS.}

Fats occur very widely distributed in the plant and animal kingdom, and constitute one of the four classes of food stuffs. Fats are esters or ethereal salts consisting of an organic radical (glycerol) united with the residue of an organic acid. Ethyl alcohol may be combined as an ester with acetic acid.

$$
\underset{\text { Acetic acid }}{\mathrm{CH}_{3} \mathrm{COOH}}+\underset{\text { Alcohol }}{\mathrm{C}_{2} \mathrm{H}_{5} \mathrm{OH}}=\underset{\text { Etyhl acetate }}{\mathrm{CH}_{3} \mathrm{COOC}_{2} \mathrm{H}_{5}}+\mathrm{H}_{2} \mathrm{O}
$$

Similarly the triatomic alcohol glycerol may be combined with the higher fatty acids to form the true fats.

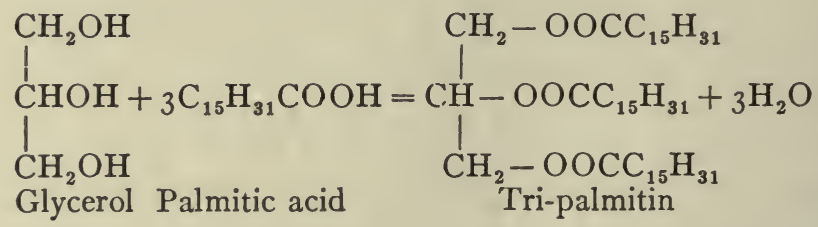

The animal fats are for the most part mixtures of tri-palmitin, tri-stearin, and tri-olein, the last two being esters of glycerol with stearic acid, $\mathrm{C}_{17} \mathrm{H}_{35}$ $\mathrm{COOH}$, and, with the unsaturated oelic acid, $\mathrm{C}_{17} \mathrm{H}_{33} \mathrm{COOH}$. Human fat consists of a mixture of which tri-palmitin and tri-stearin comprise threefourths of the whole. The fat in milk and butter is in part tri-butyrin, the ester of glycerol with butyric acid, $\mathrm{C}_{2} \mathrm{H}_{5} \mathrm{COOH}$. The percentage of any individual fat in animal tissue depends on, and is characteristic of, the particular species of animal from which the fat was obtained. Ordinary mutton fat contains more tri-stearin and less tri-olein than pork fat, and the mutton fat is stiffer because the melting-point of the tri-stearin is the highest of the fats.

The pure fats are odorless, tasteless, and generally colorless. They 
are insoluble in water and cold alcohol, but are dissolved by acetone, hot alcohol, benzol, chloroform, and ether. When shaken with water, protein solutions, soap, or gum arabic, the fats assume a finely divided condition known as an emulsion. The suspension in water is only temporary, while the emulsions are permanent.

The fats are hydrolyzed or saponified by superheated steam into glycerol and the fatty acids, the reaction being the reverse of that indicated in the equation above. On boiling with caustic alkalies, they are similarly saponified; the fatty acids are then combined with the bases to form salts or soaps.

Lecithins are tri-glycerides in which the $\mathrm{H}$ atom of two instead of three groups of the glycerol is replaced by a fatty acid radical; for the $\mathrm{H}$ of the third hydroxyl $(\mathrm{OH})$ group there is substituted an ester-like combination of phosphoric acid with a nitrogen-containing organic base, choline.

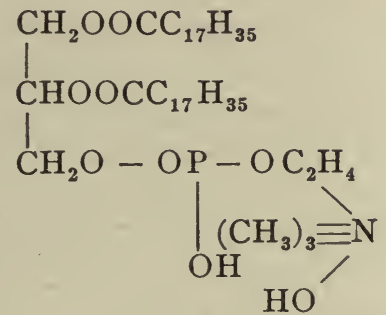

Lecithin

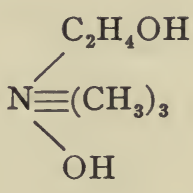

Choline

On saponification the di-stearyl lecithin molecule above combines with three molecules of water and is split into two molecules of stearic acid, one of glycero-phosphoric acid and one of choline.

The lecithins are soluble in alcohol, benzene, chloroform, and ether. They are precipitated from chloroform or alcohol-ether solution by acetone.

The lecithins are found in nearly all animal and vegetable tissues, especially in nervous tissues. They are essential constituents of the cell. Kephalin is of more than passing interest in that its presence hastens blood clotting. It accomplishes this result by removing the restraints of antithrombin on fibrin formation.

Cholesterol is a complex alcohol with the elementary formula $\mathrm{C}_{27} \mathrm{H}_{45} \mathrm{OH}$, and related to the vegetable terpenes, being grouped with the fats solely because of its physical properties. Accordingly, it cannot be saponified. It crystallizes in the form of thin, colorless, transparent plates usually notched in one corner. It exists in the tissues in part in the form of esters with the complex fatty acids. Cholesterol is an essential cell constituent; it is present in relatively large amounts in nervous tissue. It occurs also in wool fat, eggs, milk, and blood plasma.

Cholesterol and the lecithins are often termed lipoids or fat-like substances. 


\section{CARBOHYDRATES.}

The typical carbohydrates contain carbon combined with hydrogen and oxygen in the proportion to form water. Other substances, such as acetic acid, $\mathrm{CH}_{3} \mathrm{COOH}$, lactic acid, $\mathrm{CH}_{3} \mathrm{CHOHCOOH}$, and inosit, $(\mathrm{CHOH})_{6}$, which contain hydrogen and oxygen in the proportion to form water, are not carbohydrates. Certain true carbohydrates also do not fulfill this condition. Chemically, the carbohydrates are aldehyde or ketone derivatives of complex alcohols; i.e., they have the structure

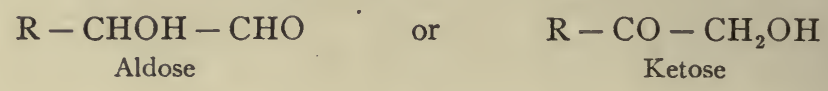

Accordingly, the carbohydrates are termed aldoses or ketoses. They are commonly classified by the number of carbon atoms in the molecule; e.g., pentoses are those containing five carbon atoms, and hexoses have six carbons. Each member of the carbohydrate class, with the exception of the pentoses, may be regarded as containing the saccharide group, $\mathrm{C}_{6} \mathrm{H}_{10} \mathrm{O}_{5}$. The monosaccharides are then $\mathrm{C}_{6} \mathrm{H}_{10} \mathrm{O}_{5}+\mathrm{H}_{2} \mathrm{O}$; the di-saccharides contain two saccharide groups with water, $\left(\mathrm{C}_{6} \mathrm{H}_{10} \mathrm{O}_{5}\right)_{2}+\mathrm{H}_{2} \mathrm{O}$, while the polysaccharides contain this group taken a large number of times, $\left(\mathrm{C}_{6} \mathrm{H}_{10} \mathrm{O}_{5}\right)_{n}$.

In general, the solubility of the saccharides varies inversely with the number of saccharide groups present: the mono-saccharides, as a class, being the most soluble and the poly-saccharides being the least so. On boiling in the autoclave or with mineral acids, and by the action of amylolytic and inverting enzymes, the poly-saccharides are, as a rule, hydrolyzed into the simple carbohydrates. The reaction may be indicated

$$
\underset{\text { Poly-saccharide }}{\left(\mathrm{C}_{6} \mathrm{H}_{10} \mathrm{O}_{5}\right)_{n}}+\underset{\text { Water }}{\mathrm{nH}_{2} \mathrm{O}}=\underset{\text { Mono-saccharide }}{\mathrm{nC}_{6} \mathrm{H}_{12} \mathrm{O}_{6}}
$$

Simple poly-saccharides, and di-saccharides are formed as intermediate products in the cleavage and in turn these are further hydrolyzed.

$$
\begin{aligned}
& \mathrm{C}_{12} \mathrm{H}_{22} \mathrm{O}_{11}+\mathrm{H}_{2} \mathrm{O}={ }_{2} \mathrm{C}_{6} \mathrm{H}_{12} \mathrm{O}_{6} \\
& \text { Di-saccharide Water Mono-saccharide }
\end{aligned}
$$

The color reactions with iodine, fermentation with yeast and bacteria, the formation of characteristic crystalline osazones with phenylhydrazine and the reducing of alkaline solutions of the oxides of metals like copper, bismuth, mercury, and ammoniacal silver solutions, are the most widely used reactions for testing and differentiating the carbohydrates. The reduction of alkaline solutions of the metallic oxides is due to the easily oxidized aldehyde and ketone structure of the sugar. 
The more common carbohydrates may be listed as follows:

I. Mono-saccharides.

Hexoses, $\mathrm{C}_{6} \mathrm{H}_{12} \mathrm{O}_{6}$ - dextrose, levulose, galactose.

Pentoses, $\mathrm{C}_{5} \mathrm{H}_{10} \mathrm{O}_{5}$-arabinose, xylose, rhamnose (methylpentose, $\mathrm{C}_{6} \mathrm{H}_{12} \mathrm{O}_{5}$ ).

2. Di-saccharides, $\mathrm{C}_{12} \mathrm{H}_{22} \mathrm{O}_{11}$,

Maltose, saccharose (cane-sugar), lactose.

3. Poly-saccharides, $\left(\mathrm{C}_{6} \mathrm{H}_{10} \mathrm{O}_{5}\right)_{n}$.

Dextrin group-dextrins.

Starch group-starch, inulin, lichenin, glycogen.

Cellulose group-cellulose.

Dextrose (glucose, grape-sugar) is an aldose found in honey and in many fruit juices where it is usually associated with levulose. It is present in the blood in small amounts, O.I to O.I5 per cent., in normal urine in minute traces, and in diabetic urine. It is not as sweet as canesugar. Glucose is produced on boiling starch with dilute acids. It is very soluble in water and is slightly soluble in alcohol. It crystallizes from water in leaves or plates and from alcohol in anhydrous needles. Dextrose rotates the plane of polarized light to the right-its specific rotation is given by the expression $[\alpha]_{\mathrm{d}}=+52.5^{\circ}$. It forms a characteristic glucosazone when boiled with phenylhydrazine in the presence of acetic acid; the osazone crystallizes from the hot solution. Glucose reduces metallic oxides in alkaline solution. It undergoes alcoholic fermentation with yeast and acid fermentation with certain bacteria.

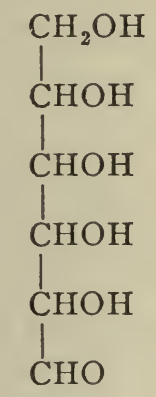

Dextrose.

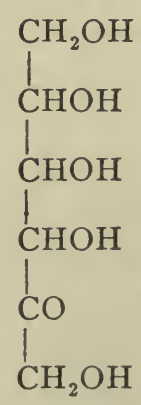

Levulose.

Levulose (fructose) is a ketose and is found associated with dextrose in many fruits, the mixture probably being produced by the hydrolysis of, or preceding the synthesis of, cane-sugar. It may be prepared by the hydrolysis of inulin and, along with dextrose, by the inversion of cane-sugar on boiling with dilute mineral acids or through the action of specific enzymes. It is 
levorotatory, $[\alpha]_{\mathrm{d}}=-92^{\circ}$. Levulose may be crystallized with difficulty in needles. It has a sweet taste. It reduces alkaline solutions of the metallic oxides, but not so much as dextrose, and yields an osazone identical with glucosazone. It undergoes fermentation, but less readily than dextrose.

Galactose is obtained with dextrose from milk-sugar or lactose on boiling with dilute mineral acids. It is less soluble in water than levulose or glucose. It reduces metallic oxide in alkaline solution, forms a characteristic osazone which melts at $193-4^{\circ} \mathrm{C}$. and it undergoes slow fermentation by yeast. It is dextrorotatory.

It is also obtained on hydrolysis of cerebrin, a glucoside occurring in nervous tissue.

Maltose (malt-sugar) is produced by the action of amylolytic enzymes on starch and glycogen. It crystallizes in small needles, is strongly dextrorotatory, $[\alpha]_{\mathrm{d}}=+\mathrm{I} 40.6^{\circ}$, reduces alkaline copper solutions much feebler than dextrose, and does not reduce bismuth oxide. It forms a characteristically crystalline osazone, melting at $206^{\circ} \mathrm{C}$. It is readily soluble in water and only slightly soluble in alcohol. Maltose is not so sweet as cane-sugar. It does not undergo alcoholic fermentation with yeast unless first split into dextrose. On boiling with dilute mineral acids or through the action of inverting enzymes, it is hydrolyzed into dextrose.

Saccharose (cane-sugar) is obtained from many plants, such as the sugar beet and sugar cane, and from the sap of certain trees, as the sugar maple. It crystallizes in prisms, is soluble in water, and only very slightly soluble in alcohol. Cane-sugar is not directly fermentable by yeast. It is dextrorotary. Saccharose has no reducing action on alkaline copper solution -the dextrose and levulose yielded on inversion probably being united together by the aldehyde and ketone radicals, respectively. Similarly, it does not form an osazone. In strong solutions, saccharose acts as a food preservative against bacterial or other decomposition through organic agencies. On boiling with dilute mineral acids or through the action of inverting enzymes, one molecule of saccharose yields one molecule of dextrose and one molecule of levulose.

Lactose (milk-sugar) is present as the chief carbohydrate of milk. It may be separated from the whey-the product remaining after skimming milk and precipitating the proteins therein. It can be prepared in large hard crystals. It is much less soluble in water than cane-sugar, and has but a slightly sweet taste. Lactose is strongly dextrorotatory, $[\alpha]_{d}=+5^{2} \cdot 5^{\circ}$. It reduces alkaline copper solution and forms a characteristic osazone. It is not fermented by ordinary yeast; certain bacteria easily convert it into lactic and other simple organic acids. The inverted milk-sugar undergoes alcoholic fermentation readily, and kumiss and kephir, made from mare's and cow's milk, respectively, are prepared in this way. On hydrolyzing by boiling with mineral acids or through the action of inverting enzymes, the lactose is split into dextrose and galactose. 
Dextrins are a series of intermediate poly-saccharides between the disaccharides and the starches. They are non-crystalline, are soluble in water, and are precipitated on the addition of alcohol in excess. They are dextrorotatory and are not fermented by yeast. Their power to reduce alkaline copper solution has been questioned, but they yield osazones which are relatively soluble. They have a slightly sweet taste. The more complex dextrins give a red reaction with iodine.

Starch is found in various parts of plants, especially in the tubers and seeds. It is a form of storage carbohydrate, and serves as a source of material for the development of the young plant. Starch is obtained commercially from potatoes, rice, corn, wheat, sago, and the like. It constitutes the greater proportion of our food.

Starch as obtained is a soft white powder which on microscopic examination is found to consist of small granules. These are often characteristic in shape and size according to the origin of the material. The granules appear to be built up of concentric layers of two varieties of starch. The more soluble form, known as amylose, is ensheathed by the less soluble variety of starch, the amylopectin. This sheath is ruptured by boiling the aqueous suspension of starch granules and an opalescent solution or starch paste is obtained. . On standing in dilute solution, the opalescent material settles to the bottom, but the clear fluid above still gives the blue reaction with iodine. This color is characteristic for starch; it disappears on heating, but returns when the liquid cools. Starch will not diffuse through a semi-permeable membrane. On boiling with dilute mineral acids starch is hydrolyzed to dextrose. The dextrins and maltose are formed as intermediate products. With amylolytic enzymes, the change practically only goes as far as the maltose stage.

Glycogen (animal starch) is the reserve form of carbohydrates in animals. It is synthesized from dextrose and can again be hydrolyzed to dextrose for transportation or for oxidation to yield energy to the tissues. The following table shows the per cent. and distribution of glycogen in the various tissues of the dog (Schöndorff):

\begin{tabular}{|c|c|c|}
\hline Organ & Per cent. glycogen & $\begin{array}{c}\text { Per cent. of total } \\
\text { glycogen. }\end{array}$ \\
\hline Blood ............... & $0.004 \mathrm{I}$ & 0.015 \\
\hline 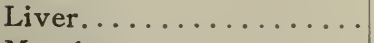 & $7 \cdot 297$ & $37 \cdot 97$ \\
\hline Muscles............. & 0.7599 & 44.23 \\
\hline 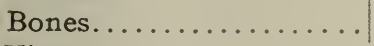 & 0.2736 & 9.25 \\
\hline Viscera ............. & 0.2024 & $3.8 \mathrm{I}$ \\
\hline Skin............ & 0.0859 & $4 \cdot 49$ \\
\hline Heart.......... & $0.23 \mathrm{I}$ & 0.17 \\
\hline Brain....... & 0.1984 & 0.09 \\
\hline
\end{tabular}


It occurs in relatively large amounts in some invertebrates, especially in moluscs and in intestinal worms. The muscles and reproductive organs of oysters, clams, and scallops are very rich in this substance.

Glycogen resembles starch in forming opalescent solutions. It may be prepared from the liver or muscle of a freshly killed animal by boiling the tissue to coagulate the proteins, grinding with sand, boiling with water slightly acidified with acetic acid and precipitating the filtrate with an excess of alcohol; dilute or concentrated solutions of caustic alkalies may be used to extract all the glycogen. Glycogen is a white, tasteless, and amorphous powder. It gives a maroon color with iodine, and does not reduce alkaline copper sulphate solution. It is completely precipitated by saturating its solution with solid ammonium sulphate, by tannic acid, or by ammoniacal basic lead acetate. On hydrolysis with mineral acids or on digestion with amylolytic enzymes it yields the same series of products as ordinary starch.

Inulin is the reserve carbohydrate of the Compositc, occurring in the tubers of the artichoke and dahlia and in the roots of the dandelion and burdock. On hydrolysis with acids or the enzyme inulase, it yields levulose. Inulin is slightly soluble in cold and easily soluble in hot water. It is precipitated from its solution by an excess of alcohol. The digestive enzymes of the body do not act on inulin.

Lichinin is obtained from the Cetraria Islandica (Iceland moss). It forms a difficultly soluble jelly in cold water and an opalescent solution in hot water. On hydrolysis with dilute acids, it yields dextrines and dextrose. The ordinary digestive enzymes have no action on lichinin.

Cellulose forms a large portion of the cell wall or the woody structure of plants. It is extremely insoluble. Chemically, it is more complex than the common starch molecule. The hydrochloric and hydrofluoric acid extracted (ash-free) filter-papers and absorbent cotton are examples of practically pure cellulose.

\section{INORGANIC SUBSTANCES OF THE BODY.}

Salt.-The inorganic principles of the human body are numerous. They are derived, for the most part, directly from food and drink and pass through the system unaltered. Some radicals are newly formed by oxidation within the body, as, for example, a part of the sulphates and carbonates from the sulphur of the proteins and the carbon of protein, fat, and carbohydrate.

Much of the inorganic saline matter found in the body is a necessary constituent of its structure, as necessary in its way as protein or any other organic principle. Another part is important in regulating or modifying various physical processes, as absorption, solution, and the like. A part must be reckoned only as matter which is, so to speak, accidentally present, whether derived from the food or the tissues, and which will, at the first 
opportunity, be excreted from the body. The principal salts present in the body are:

Sodium and Potassium Chlorides. - These salts are present in nearly all parts of the body. The former seems to be especially necessary, judging from the instinctive craving for it on the part of animals in whose food it is deficient, and from the condition which is consequent on its withdrawal. The quantity of sodium chloride in the blood is greater than that of all its other saline ingredients taken together, but it is present chiefly in the fluids of the body. In the tissues, the muscles for example, the quantity of sodium chloride is less than that of the chloride of potassium, which forms a constant ingredient of protoplasm.

Calcium Fluoride.-It is present in minute amount in the bones and teeth, and traces have been found in the blood and some other fluids.

Calcium, Potassium, Sodium, and Magnesium Phosphates.-These phosphates are found in nearly every tissue and fluid. In some tissues-the bones and teeth-tricalcium phosphate exists in very large amount. The phosphate of calcium is intimately incorporated with the organic basis or matrix, but it can be removed by acids without destroying the general shape of the bone. After the removal of its inorganic salts, a bone is left soit, tough, and flexible.

Potassium and sodium phosphates, with the carbonates, maintain the alkalinity of the blood.

Calcium Carbonate.-It occurs in bones and teeth, but in much smaller quantity than the phosphate. It is found also in some other parts. The small concretions of the internal ear of some fishes (otoliths) are composed of crystalline calcium carbonate, and form the only example of inorganic crystalline matter existing as such in the body.

Potassium and Sodium Carbonates and Sulphates.-These are found in the blood and most of the secretions and tissues.

Silicon.-A very minute quantity of silica exists in the urine and in the blood. Traces of it have been found also in bones, hair, and some other parts.

Iron.-The especial place of iron is in hemoglobin, the coloring-matter of the blood, of which a full account will be given with the chemistry of the blood. Iron is found, in very small quantities, in the ashes of bones, muscles, and many tissues, and in lymph and chyle, albumin of serum, fibrin, bile, milk, and other fluids.

Iodine occurs as an iodized protein in the thyroid gland. Biologically, it is found as a tri-iodotyrosin in sponges and the Gorgonian corals.

Water.-Water forms a large proportion, more than two-thirds, of the weight of the whole body. Its relative amount in some of the principal solids and fluids of the body is shown in the following table (from Robin and Verdeil): 


\section{Quantity of Water in Per Cent.}

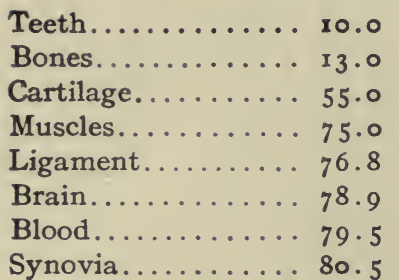

Bile.......... 88.0

Milk......... 88.7

Pancreatic juice... 90.0

Urine........... 93.6

Lymph........ 96.0

Gastric juice..... 97.5

Perspiration...... 98.6

Saliva......... $99 \cdot 5$

In all the fluids and tissues of the body-blood, lymph, muscle, gland, etc. - water acts the part of a general solvent, and by its means alone circulation of nutrient matter is possible. It is the medium also in which all fluid and solid aliments are dissolved before absorption, as well as the means by which all, except gaseous, excretory products are removed. All the various processes of secretion, transudation, and nutrition depend of necessity on its presence for their performance.

The greater part, by far, of the water present in the body is taken into it as such from without, in the food and drink. A small amount, however, is the result of the chemical union of hydrogen with oxygen in the oxidations of the body.

The loss of water from the body is intimately connected with excretion from the lungs, skin, and kidneys, and, to a less extent, from the alimentary canal. The loss from these various organs may be thus apportioned (quoted by $\mathrm{Dalton}$ from various observers):

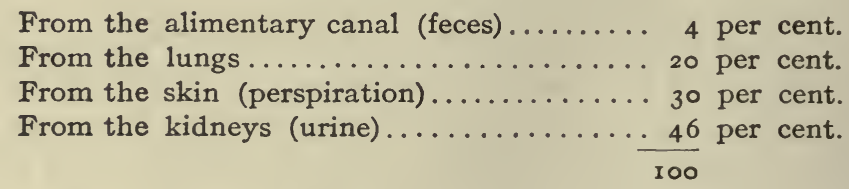

Under some conditions the loss of water from the alimentary canal may be enormously increased, as in acute diarrheas. In young children and babies in particular this fact is often not realized and not enough water is given by the mouth to supply the loss. The result is a considerable concentration of the blood and tissues, a relative dessication that may prove very injurious. 


\section{LABORATORY EXPERIMENTS ON THE CHEMISTRY OF THE BODY.}

This list will serve as basal for the guidance of students and teachers. The experiments listed are to be supplemented by technical laboratory guides and references to fuller discussion in the literature.

\section{The Proteins.}

I. Preparation of Proteins.-The most convenient source of proteins for laboratory work are blood serum, egg white, or commercial preparations of the milk protein casein. Protein may also be prepared from various plant seeds, especially cereals. Hempseed contains a globulin, edestin, which is very easily isolated in the laboratory.

a. Preparation of Edestin.-Grind up some hemp-seed in an ordinary meat chopper and extract the resulting meal with 5 per cent. salt solution, warming to $60^{\circ}$. The solution should not be heated above $65^{\circ}$ because the protein will be coagulated. Filter while hot. On cooling slowly the edestin will crystallize out. Examine some of the precipitate with a microscope and sketch the crystals. The edestin is soluble in ro per cent. salt solution without warming, and solutions for laboratory use can be prepared in this way.

b. Preparation of Egg Albumin.-The yolk should be separated from the white of fresh eggs and the reticulum in the egg white broken up with a wire egg-beater. Egg white can then be diluted as desired and the precipitate globulin filtered off. Crystals of ovalbumin can be prepared as follows:

The egg white, beaten as directed, is strained through gauze and an equal volume of saturated ammonium sulphate solution is added. After twenty-four hours the globulin precipitate is filtered off and concentrated ammonium sulphate solution added until the mixture becomes turbid. Then distilled water is added very carefully until turbidity has disappeared. The solution is then acidified with acetic acid, which has been saturated with ammonium sulphate, until a precipitate is obtained. 'The precipitate is at first amorphous, and on standing becomes crystalline. Examine the crystals under the microscope and sketch them.

c. Other Protein Crystals. - Crystals of hemoglobin may be demonstrated by adding a drop of ether to diluted dog blood on the microscope slide and allowing the mixture to dry around the edge. Hemoglobin crystals may be observed under the microscope to have formed where the solution has concentrated and dried.

Crystals of seralbumin and of lactalbumin can be obtained in essentially the same manner already described for ovalbumin. 
2. Elementary Composition of the Proteins.-a. Nitrogen.-Make an intimate mixture of some dry protein, preferably a casein preparation, with sodalime and place it in a dry test-tube. Warm gently over a Bunsen flame. Hold a piece of moistened red litmus-paper over the mouth of the test-tube. The ammonia split off from the protein will color the litmus blue.

Warm together carefully in a dry test-tube a few particles of dry protein and a small cube of metallic sodium. (Do not place the tube in the flame.) When the fusion is complete and the tube is cooled somewhat, plunge the end into a small amount of water in a casserole. The glass of the test-tube will probably break. When the fused mass has dissolved, filter, and to the filtrate add a few drops of ferric chloride and ferrous sulphate solution. On acidifying with hydrochloric acid a precipitate of Prussian blue is obtained. Sodium ferricyanide is formed in the reaction and in consequence gives a Prussian blue with the excess of iron present.

b. Carbon.-Place some desiccated casein in the end of a piece of glass tubing, tap it down gently so that the lumen of the tube will not be obstructed. Heat the casein gently over a small Bunsen flame, inclining the tube so that there will be a slight current of air passing upward through it. The casein will char, indicating the presence of carbon.

c. Hydrogen.-Note the clear fluid that has condensed in the upper portion of the tubing from the preceding experiment. If a little anhydrous copper sulphate is introduced into the tube, the fluid of condensation coming in contact with it will become blue, indicating that water has been formed in the charring of the casein. Hydrogen in protein has been oxidized to form water.

d. Sulphur.-Boil some casein or some egg-white solution with sodium hydroxide after adding a few drops of lead acetate solution. The presence of sulphur is shown by the formation of a black-lead sulphide. On adding hydrochloric acid the lead sulphide formed will be decomposed and the odor of hydrogen sulphide will be noted.

e. Phosphorus.- - Heat some casein, preferably in a nickel crucible, with a fusion mixture composed of three or four parts of caustic soda and one part of potassium nitrate, warming cautiously until the mass becomes coloriess. Dissolve the residue when cool in a small volume of water, neutralize and acidify with nitric acid slightly, and add about 5 c.c. of ammonium molybdate solution. Warm for some minutes at $80^{\circ}$. A yellow precipitate of ammonium phosphomolybdate is obtained.

3. Color Reactions of the Proteins.-a. Millon's Reaction.-To about 5 c.c. of a dilute solution of egg albumin in a test-tube add a few drops of Millon's reagent. A white precipitate forms which turns red when heated. This test can be used with advantage on solid proteins. In this case the 
reagent is added to suspensions of the solid substance. Such proteins as are not precipitated by the mineral acids yield a red solution instead of a red precipitate. Millon's reagent consists of one part mercury dissolved in two parts by weight of concentrated nitric acid; the resulting solution is diluted with two volumes of water.

This reaction is due to the presence of the hydroxyphenyl group or $\mathrm{C}_{6} \mathrm{H}_{5} \mathrm{OH}$ in the protein molecule. Accordingly, certain non-protein substances give this reaction; i.e., tyrosine, phenol (carbolic acid), thymol, etc. The reaction given by the protein is due to the presence of the amino acid tyrosine, and it is evident that the test is really an indication of the presence of the tyrosine complex in the protein molecule.

b. Xanthoproteic Reaction.-To 2-3 c.c. of egg-albumin solution or of some dry casein in the test-tube, add concentrated nitric acid and heat until the protein dissolves, forming a yellow solution. Cool the solution and carefully add ammonium hydroxide in excess. The yellow color changes to an orange. This reaction is due to the presence in the protein molecule of the phenyl group in phenyalanine, tyrosine, or tryptophane; with the phenyl group nitric acid forms certain nitro-derivatives of benzene.

c. Adamkiewicz or Hopkins-Cole Reaction.-Mix a couple of c.c. of concentrated sulphuric acid with 4 or 5 c.c. of glacial acetic acid in a test-tube. Add a few drops of egg-albumin solution and warm gently. A reddishviolet color is produced. This reaction is due to the presence of tryptophane in the protein and the test depends on the presence of glyoxylic acid $\left(\mathrm{CHOH}_{2} \mathrm{COOH}\right)$ in the acetic acid. The Hopkins-Cole reagent, a glyoxylic acid solution, may be used instead of the glacial acetic acid. The reagent is prepared by adding sodium amalgam to a saturated solution of oxalic acid and allowing the mixture to stand until the evolution of gas ceases. In making the test the protein solution and Hopkins-Cole reagent are mixed thoroughly in a test-tube, and concentrated sulphuric acid poured gently into the tube which has been inclined somewhat so that it forms a layer in the bottom of the test-tube. The acid and protein solutions will be stratified and a reddish-violet ring is developed where the two fluids come in contact.

This reaction is due to the presence of tryptophane in the protein molecule. Gelatin does not respond to this test, for it does not yield this substance as a cleavage product.

d. Liebermann's Reaction.-Add a few drops of egg-white solution or a little dry casein to about 5 c.c. of concentrated hydrochloric acid in a testtube and boil the mixture until a pinkish-violet color results. It was formerly thought that this reaction indicated the presence of a carbohydrate group in the protein molecule, but this is now considered uncertain.

e. Biuret Reaction.-To 2 or 3 c.c. of egg-white solution in a test-tube an 
equal volume of concentrated potassium hydroxide solution is added and mixed thoroughly. Very dilute ( 2 per cent.) copper sulphate solution is added until a purplish-violet or pinkish-violet color is produced. This reaction is given by substances containing two amino groups in the molecule, these groups being joined directly together or through a single atom of nitrogen or carbon. Non-protein substances that contain the necessary groups will of course respond to this test, which derives its name from the fact that it is given by biuret, a substance formed on heating urea to $180^{\circ}$.

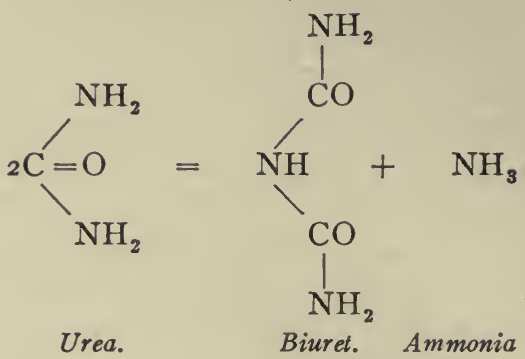

Proteins give this reaction since there are more than one $\mathrm{CONH}_{2}$ group in the protein molecule. Proteoses and peptones give a pink biuret reaction, gelatin a rather blue reaction, and the ordinary proteins a purple.

f. Molisch Reaction.-This reaction is really a carbohydrate test, but is given by some proteins and interpreted as indicating that such proteins contain a carbohydrate moiety. The test is made as follows:

Place about 5 c.c. of the solution to be tested in a test-tube, and add a couple of drops of a I5 per cent. alcoholic solution of $\alpha$-naphthol. Incline the tube and pour very carefully down the side about 5 c.c. of concentrated sulphuric acid so that the two solutions are stratified. A blue or violet-red ring is obtained in the area of contact of the solutions.

4. Precipitation Reactions of the Proteins.-a. Precipitation with Concentrated Mineral Acids.-Prepare four test-tubes which contain about 5 c.c. of egg-white solution. To these respectively add drop by drop concentrated sulphuric acid, hydrochloric acid, nitric acid, and acetic acid. Note that the mineral acids precipitate the protein. The precipitation with nitric acid is a frequently used protein reaction, and when carried out as follows is known as Heller's ring test. The solution to be tested is placed in a test-tube, the tube is inclined and about 5 c.c. of concentrated nitric acid is poured carefully down the side of the tube so that the solution and acid stratify. A white zone of precipitated protein is obtained between the strata. An instrument known as the albumiscope has been devised to facilitate the making of the ring tests. Heller's ring test is most commonly used to determine the presence of protein in urine. 
b. Precipitation with Heavy Metals.-Proteins form insoluble compounds with the metals when mercuric chloride, lead acetate, copper sulphate, silver nitrate, etc., are added to protein solutions.

c. Acetic Acid and Potassium Ferrocyanide Test.-To about 5 c.c. of eggwhite solution in a test-tube add five to ten drops of acetic acid and then potassium ferrocyanide drop by drop until a precipitate forms. This test is very delicate.

d. Precipitation with the Alkaloidal Reagents. - Prepare six tubes containing about 3 c.c. egg-white solution. To the first add picric acid drop by drop until excess of the reagent has been added, noting the changes with care. Repeat the experiment with trichloracetic acid and tannic acid. Acidify the remaining tubes with hydrochloric acid, and repeat the experiment with phosphotungstic acid, phosphomolybdic acid, and potassium mercuric iodide.

e. Heat Coagulation.-Take about Io c.c. of egg-white solution in a testtube and heat to boiling. Then add a few drops of dilute acetic acid. The protein will be coagulated. The acetic acid should be added after heating, since otherwise acid metaprotein might be formed. The presence of some neutral inorganic salts tends to give a sharper test. The addition of the acid also will dissolve the earthy phosphates which are often precipitated from the urine on heating. Proteoses, peptones, the casein of milk, and a few other proteins are not coagulated by heat.

f. Precipitation by Alcohol.-Add some 95 per cent. alcohol to a test-tube containing about 3 c.c. of egg-white solution. The protein is precipitated, and on standing it is coagulated so that it can no longer be dissolved in neutral solvent.

g. Salting-out Experiments.-Add to some diluted blood-serum in a small beaker, crystals of magnesium sulphate until no more of the salt will go into solution. After standing for a few minutes, filter and test the filtrate and residue for protein by some of the precipitation or color reactions given above. It is found that the filtrate still contains some protein. Further, that this protein can be precipitated on adding a few drops of dilute acetic acid. When the blood serum is similarly saturated with ammonium sulphate, there will be no protein found in the filtrate. If to the blood serum an equal volume of saturated ammonium sulphate solution is added, the result will be the same as that already obtained with magnesium sulphate. Some proteins then are precipitated on saturating their solutions with magnesium sulphate or by adding an equal volume of saturated ammonium sulphate solution; albumins, globulins, and proteoses, however, are all precipitated by saturation with the more soluble ammonium sulphate. 


\section{Albumins and Globulins.}

5. Properties of Albumins and Globulins.-Try out the solubility and the precipitation tests indicated in the following table for a solution of ovalbumin, and on edestin furnished by the instructor.

\begin{tabular}{|c|c|c|c|c|c|c|c|c|c|c|c|}
\hline \multirow[b]{2}{*}{ Protein } & \multicolumn{4}{|c|}{ Soluble in } & \multicolumn{5}{|c|}{ Precipitated } & \multicolumn{2}{|c|}{$\begin{array}{c}\text { Coagulated } \\
\text { by }\end{array}$} \\
\hline & 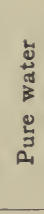 & 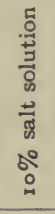 & 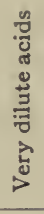 & 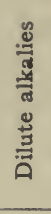 & 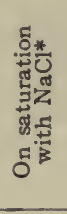 & 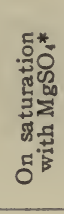 & 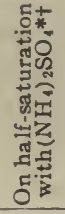 & 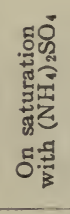 & 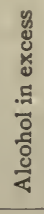 & 苞 & $\begin{array}{l}\frac{+}{0} \\
\frac{0}{0} \\
\frac{0}{4}\end{array}$ \\
\hline $\begin{array}{l}\text { Albumin } \\
\text { (ovalbumin }\end{array}$ & & & & & & & & & & & \\
\hline $\begin{array}{l}\text { Globulin } \\
\text { (edestin) }\end{array}$ & & & & & & & & & & & \\
\hline
\end{tabular}

Albuminoids.

6. Keratin.-Horn shavings are most conveniently used for the experiments with keratin.

$a$. Try the solubility of keratin in water, ro per cent. salt solution, dilute hydrochloric acid, and dilute potassium or sodium hydroxide.

b. Make a test for loosely combined sulphur as in experiment $2, d$, page ro6.

c. Try Millon's reaction and the biuret reaction, putting the undissolved shavings directly into the reagent.

$d$. Try the solubility of keratin in the artificial gastric and pancreatic juice furnished by the instructor.

7. Collagen.-The tendo achillis of the ox may be used for the preparation of collagen.

$a$. Clean the tendon and cut it into small pieces. Wash the pieces in dilute salt solution in order to remove the soluble protein, and then wash with distilled water. Transfer the pieces of washed tendon to a flask and add too c.c. of saturated lime-water and same amount of distilled water. The flask should be shaken at intervals for twenty-four hours. The limewater dissolves the mucoid in the tendon. Filter off the pieces of tendon and save the filtrate for a later experiment. The residue of the tendon consists of the albuminoid collagen and a little elastin. We may consider the tests to follow as being made upon collagen.

$b$. Cut the collagen into very fine pieces and try the solubility as in $6 a$ above.

* If not precipitated, acidify and note result.

† See page 89.

$\ddagger$ On standing under alcohol. 
c. Test for loosely combined sulphur in collagen. Heat with the alkali until the large piece of collagen used is partly decomposed, then add one or two drops of lead acetate and again heat to boiling.

d. Try Millon's reaction, the biuret test, the xanthoproteic reaction, and the Hopkins-Cole reaction.

e. Formation of gelatin from collagen. Transfer the remainder of the collagen to a casserole, fill about two-thirds with water, and boil for several hours, replacing enough of the water that is lost by evaporation to keep the pieces well covered. Filter and cool. Collagen is transformed into gelatin and the characteristic gel is obtained. Try this experiment also with some cartilage from the trachea and with some 0.2 per cent. $\mathrm{HCl}$ extracted bone.

f. The properties of gelatin. Try the solubility as in $a$ above on some gelatin furnished by the instructor. Try the solubility also in hot water.

g. Try the tests for loosely combined sulphur. Try Millon's HopkinsCole, and the biuret reactions. Try the solubility in artificial gastric and pancreatic juices.

8. Elastin.-a. Preparation of Elastin.-Cut the ligamentum nucha of the ox into strips and run it through a meat chopper. Wash the finely divided material with salt solution and in running water for some hours. Transfer it to a flask and add about 200 c.c. of half-saturated lime-water and extract for forty-eight hours. Filter off the lime-water which has dissolved the mucoid present, saving the filtrate, and wash the residue and tendon with water. Boil the material in dilute acetic acid for some hours to convert the collagen present into gelatin. Wash the residue thoroughly with water. It may be dried by washing it with boiling alcohol, pressing it out between filters, and manipulating it in the air.

b. Try the solubility of elastin as in $a$. Test for loosely combined sulphur. Try Millon's, biuret, and the Hopkins-Cole reactions. Try the solubility in artificial gastric and pancreatic juices.

c. Make a table comparing the properties of keratin, collagen, and elastin.

\section{GLycoproteins.}

9. Mucoid.-a. Unite the lime-water of filtrates obtained in the extraction of the tendons in the preceding experiment and acidify them with dilute acetic acid until precipitation occurs. The mucoid is precipitated. Avoid adding excessive acid or the mucoid will again go into solution. Allow the mucoid precipitate to settle and decant the supernatant fluid and filter off the precipitate.

$b$. Try the biuret test on a portion of the mucoid.

c. Place the remainder of the mucoid in a beaker, add about 25 c.c. of water and 2 c.c. of dilute hydrochloric acid; boil until the solution becomes dark. To a portion of the mixture add a few drops of barium chloride. A 
white precipitate shows the presence of sulphate. Neutralize to litmus-paper with solid potassium or sodium hydroxide and test for sugar with Fehling's solution (see Experiment 12, d). The mucoid has been split by boiling with acid into protein, protein cleavage products, and carbohydrate or a reducing substance. The presence of oxidized sulphur in the molecule has also been shown.

\section{Derived Proteins.}

Io. Metaproteins.-a. Acid Metaproteins or Acid Albuminate.-Acid metaprotein or acid albumin may be prepared as follows:

Dilute solution of white of egg with several volumes of .4 per cent. hydrochloric acid. Allow it to stand for some time at $40^{\circ}$ Centigrade. Filter and neutralize the filtrate. A precipitate of acid metaprotein is obtained. The acid albuminate then is insoluble in neutral dilute salt solutions, but it dissolves in acidified solutions. Filter off some of the precipitated acid albuminate and test it for loosely combined sulphur.

b. Alkali metaprotein is formed on treating proteins with alkali. To some undiluted egg white in an evaporating dish add sodium hydroxide solution slowly with constant stirring. The mixture forms a stiff gel known as Lieberkühn's jelly. Wash the gel, which has been broken into small pieces, with running water until the excess of alkali is removed. Warm it in a small amount of water and dissolve by heating gently. Neutralize the solution carefully with acid, noting the odor of the hydrogen sulphide that is given off. The precipitate which appears when the neutral point is reached is alkali metaprotein or alkali albuminate. Filter off the precipitate, wash it in water, and try the test for loosely combined sulphur. A weak reaction or negative result shows that the loosely combined sulphur has been split off by treating the protein with the alkali, a change which has not occurred in the formation of the acid albuminate above.

\section{Secondary Protein Derivatives.}

II. Proteose and Peptone.-Commercial proteose-peptone preparations, such as Witte peptone or Armour's peptone, may be employed for the separation of proteoses and peptones.

a. Take about 5 grams of the proteose-peptone mixture and dissolve it in Io० c.c. of water.

Try the biuret reaction, Millon's reaction, and Heller's ring test. Do the proteoses and peptones coagulate on heating?

$b$. Place the remainder of the solution in the beaker and add dry ammonium sulphate in excess. Note that before the solution is completely saturated with the salt, the precipitation of the primary proteoses (proto proteose and hetero proteose). Completely saturate the solution with the ammonium sulphate, warming it gently to facilitate the separation. At full saturation the second- 
ary proteoses (deutero-proteoses) are precipitated. The peptones remain in solution. Try the biuret test on the precipitate and on the filtrate containing the peptone, in the latter instance making the solution strongly alkaline with solid sodium or potassium hydroxide.

$d$. From what you have learned of the properties of the derived proteins in the text and in the laboratory, prepare a chart (Exp. 5) in which the properties of these substances are indicated. Compare the results in this chart and the results in this table with the similar one that you made for the albumins and globulins

\section{CARBOHYDRATES.}

I2. Starch.- $a$. Examine under the microscope and sketch the starch granules obtained by grinding some potato scrapings in a mortar with a little water; examine and sketch also the granules of corn, wheat (flour), and arrowroot starch.

b. Starch Paste.-Make a suspension of I gram of arrowroot starch in a little distilled water by grinding in a mortar, and pour slowly into some boiling water. Heat for a few minutes longer, cool and make up to roo c.c.

c. Iodine Test.-Shake up three or four drops of dilute iodine solution with 2 c.c. starch. A deep blue color appears. The color is discharged in dilute alkali and reappears on acidifying again. Heat also discharges the color.

d. Fehling's Test.-Fill a test-tube about a fourth full of Fehling's solution and heat to boiling for a minute or two. Add a few drops of starch paste. The red precipitate of copper (cuprous) oxide should not be obtained, for pure starch does not reduce Fehling's solution.

e. Hydrolysis of Starch.-Boil starch solution with 5 per cent. sulphuric acid for fifteen minutes. Test with Fehling's solution, first neutralising the excess of acid. A copious precipitate of cuprous oxide shows that the starch has been converted to reducing sugar.

I3. Dextrins.-Make a 5 per cent. solution of dextrin in distilled water and try:

a. Iodine Test.-This gives a red which is characteristic.

b. Fehling's Test.

I4. Dextrose.-Test a 5 per cent. solution of dextrose:

a. Iodine Test.-No reaction.

b. Fehling's Test.

I5. Glycogen.-Use I per cent. solution of glycogen. Note the characteristic opalescence of the solution.

a. Iodine Test.-A wine-red, somewhat like that given by dextrin.

b. Fehling's Test.-Glycogen does not reduce the copper solution.

c. Hydrolysis.-Test as in $e$. The glycogen is hydrolyzed to dextrose. 


\section{FATS.}

r6. Neutral Fat.-a. Melting-point.-Compare neutral olive oil, some fresh rendered lard, and some tallow. The former is fluid at ordinary room temperature. Determine the melting-points of the lard and of the tallow by the method of Wiley. Fill a test-tube one-half full of water and add a two-inch top layer of alcohol. Prepare a thin flake of fat and suspend it in the test-tube at the dividing line of the water and alcohol. Insert the bulb of a thermometer at the same level. Mount the test-tube with the thermometer in a beaker on a ring stand, fill the beaker with water above the level of the content of the test-tube, and gradually heat with stirring of the water in the beaker. At the melting temperature the flake of fat will run into a round drop.

b. Solubility.-Fat is insoluble in water, but soluble in acetone, ether, chloroform, benzol, and in alcohol.

c. Saponification.-Heat some fat in an evaporating-dish, add sodium hydrate, and boil. Saponification takes place. The soap is soluble in water. Add 25 per cent. sulphuric acid to some of the soap, the fatty acid is liberated and collects on the surface of the solution.

I7. Fatty Acids.-Collect some of the fatty acids, wash to remove excess of sulphuric acid, and dissolve in ether.

a. Acid Reaction.-Add an ether solution of the fatty acid to neutral litmus, or to faintly alkaline phenolphthalein. The former turns red, and the red of the alkaline solution of the latter is discharged.

b. Acrolein Test.-Evaporate the ether from 2 c.c. of the solution, add potassium bisulphate crystals to the acid in a test-tube, and raise to a high heat over a Bunsen. No acrolein is given off. Repeat on neutral fat and on glycerin. Both liberate the irritating fumes of acrolein.

I8. Emulsification.- $a$. Shake up neutral olive oil and water, no emulsion is formed and the oil quickly separates.

b. Add a couple of drops of fatty acid, a very good but temporary emulsion is now formed.

c. Use rancid fat, a temporary emulsion is formed.

$d$. Add a little soap to each of the above. A good permanent emulsion is now formed, but best in $c$.

19. Lipoids.- $a$. Grind some pig's or calf's brain with ether in a mortar, place in a flask with sufficient ether to make a thin suspension of the material and set aside until the undissolved material has sedimented completely to the bottom. Decant the clear ether and add acetone until the lecithins have been precipitated. Cholesterol will crystallize from the filtrate on spontaneous evaporation of the latter. Sketch the cholesterol crystals.

b. Show the presence of nitrogen in lecithin (see Exp. $2 a$ ). 
:. Try the acrolein test as in Exp. I 7 .

d. Fuse some lecithin in a metal crucible with a fusion mixture of 3 parts of caustic potash and I part of potassium nitrate. Dissolve in a small volume of water, acidify slightly with nitric acid, add a few drops of ammonium molybdate solution, and warm to $75^{\circ} \mathrm{C}$. for a few minutes. A yellow precipitate of ammonium phosphomolybdate indicates the presence of phosphorus in the lecithin.

e. Try to saponify some cholesterol as in Exp. 16 c. As cholesterol is not a fat, saponification does not take place.

20. The Salts.-Coagulate the protein in Io grams of chopped meat or 25 c.c. of blood by boiling for a few minutes with 25 c.c. of water to which a few drops of acetic acid have been added. Filter off the coagulated protein, wash the precipitate on the paper with a very little hot water, and add the washings to the original filtrate. The coagulum should filter off quickly and the filtrate should be perfectly clear; otherwise repeat the experiment. Make the following tests:

a. Chlorides.-Acidify a small portion of the filtrate with nitric acid and add a few drops of silver nitrate solution. A white precipitate, which dissolves on adding ammonia and reappears on acidification with nitric acid, shows the presence of chlorides.

b. Sulphates.-Acidify a portion of the filtrate with hydrochloric acid and add a few drops of barium chloride solution. A white precipitate of barium sulphate indicates that sulphates are present. A much stronger reaction can be obtained when the ash or alkali fusion of a tissue is tested, the sulphur in the protein then having been oxidized to a sulphate.

c. Phosphates.-Acidify with nitric acid and then add a few drops of ammonium molybdate solution. Warm in the water-bath at $75^{\circ} \mathrm{C}$. for a few minutes. A yellow precipitate of ammonium phosphomolybdate indicates the presence of phosphate.

d. Calcium.-Add a few drops of ammonium oxalate solution to a portion of the filtrate. A white precipitate of calcium oxalate forms. On microscopic examination this is found to consist of characteristic minute octahedrals.

e. Iron.-Acidify with hydrochloric acid and add a few drops of potassium ferrocyanide. A Prussian blue color indicated the presence of iron. Try the reaction on some blood which has been ashed in a crucible and dissolved out in a little dilute hydrochloric acid. The iron is present in the compound protein, hemoglobin, of the red corpuscles.

f. Magnesium.-Tease out some fibers of frog muscle in a few drops of water on a microscope slide and invert a beaker containing a piece of filterpaper moistened with ammonia over it. After a few minutes, examine under the microscope. Characteristic star-shaped or fern-like crystals of ammonium magnesium phosphate will be seen. 


\section{PLATE II}

\section{VARIETIES OF LEUCOCYTES}

Plate II is reproduced by the kind permission of Dr. Cabot. It illustrates certain typical varieties of leucocytes. All are stained with the Ehrlich triacid stain, and drawn with camera lucida. Oil immersion objective $\frac{1}{12}$ and ocular No. iii of Leitz. (Cabot.)

I. Small Lymphocytes.-In the cell at the left note the transparent protoplasm; in the cell next to it note the very pale pink of protoplasm around the nucleus which is deeply stained, especially at the periphery. The next cell has an indented nucleus; its protoplasm relatively distinct. The cell on the extreme right shows no protoplasm and is probably necrotic. In all note absence of gramules with this stain. With basic stains a blue network appears in the protoplasm.

2. Large Lymphocytes.-Note the pale stain of nuclei and protoplasm, regularity of outline; indented nucleus in one. Every intermediate stage between these and the "small" lymphocytes occurs, and the distinction between them is arbitrary.

3. Polymorphonuclear Neutrophiles. - Note the varieties in size and shape of granules, the regular staining of the nuclei, the light space around them, their relatively central position in the cell.

- 4. Myelocytes.-Note the identity of granules with those just described; the even, pale stain of nuclei, their position near the surface (edge) of the cell. The two cells figured indicate the usual variations in size of the whole cell.

5. Eosinophile.-Note regular shape, loose connection of granules, their copper color, their uniform and relatively large size, and spherical shape.

6. Eosinophilic Myelocyte.-Note similarity to the ordinary myelocytes $b$, except as regards granules. Colors of granules may be, as in $e$, ordinary eosinophile. 


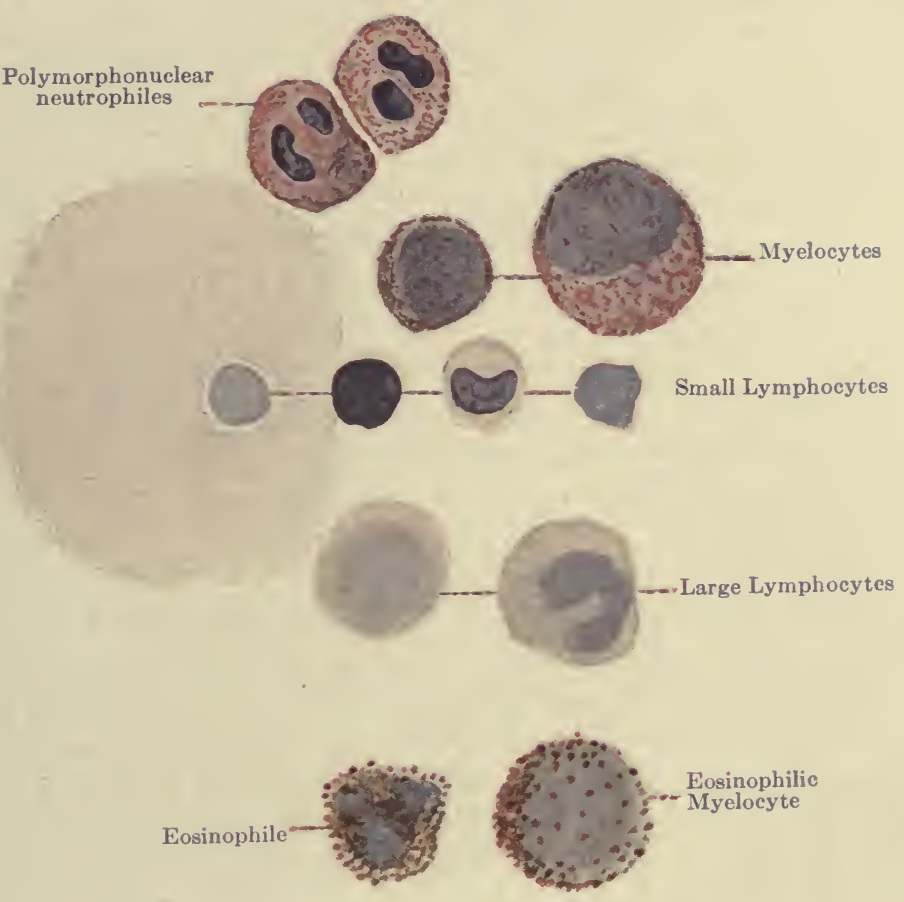

Varieties of Leucocytes

R. C. Cabot fec. 



\section{CHAP'TER IV}

\section{THE BLOOD}

THE blood is the fluid medium from which all the tissues of the body are nourished. By means of the blood, materials absorbed from the alimentary canal as well as oxygen taken from the air in the lungs are carried to the tissues, while substances which result from the metabolism of the tissues are carried to the excretory organs to be removed from the body. The blood acts as a medium of exchange between the various tissues themselves. A good example is the activity of the blood in regulating the reaction of the body in balanced neutrality. The blood is also an important factor in the regulation of the body temperature.

The blood is a somewhat viscid fluid, and in man and in all other vertebrate animals, with the exception of the two lowest, is red in color. The exact color of blood is variable; that taken from the systemic arteries, from the left side of the heart and from the pulmonary veins is of a bright scarlet hue; that obtained from the systemic veins, from the right side of the heart, and from the pulmonary artery is of a much darker color, which varies from bluish-red to reddish-black. At first sight the red color appears to belong to the whole mass of blood, but on further examination this is found not to be the case. In reality blood consists of an almost colorless fluid, called plasma or liquor sanguinis, in which are suspended numerous minute masses of protoplasm, called blood corpuscles. The corpuscles are of the two varieties, the white ameboid corpuscles, or leucocytes, and the red corpuscles, erythrocytes. The latter compose by far the larger mass of blood-cells. They contain the red pigment, hemoglobin, to which the color of the blood is due.

The plasma or fluid part of the blood is a remarkably complex chemical mixture. It is kept in constant rapid circulation through the blood vessels of the body and is, therefore, thoroughly mixed and homogeneous in character.

Quantity of the Blood.-The quantity of blood in any animal under normal conditions bears a fairly constant relation to the body weight. The amount of blood in man averages $\frac{1}{11}$ to $\frac{1}{13}$ of the body weight. In other mammals the proportion of blood is also fairly constant, varying from $\frac{1}{12}$ to $\frac{1}{20}$ of the body weight. In many of the lower vertebrates, the fishes for example, the relative quantity of blood is very much less.

It is difficult to make an exact estimate of the quantity of blood in animals or in man though the blood volume in man is of great importance from the standpoint of disease. Measurements are constantly given 
for the number and distribution of the corpuscles and of the blood volume index. This data, to be of value, must be compared with the total proportionate amount of blood. A comparatively recent determination of this question was made by methods that are modern by Keith, Rowntree, and Geraghty. They injected a known quantity of a dye that is absorbed with difficulty, vital red. As soon as the dye was distributed, three minutes, they withdrew blood into powdered oxylate from the corresponding vein of the other arm, centrifuged quickly and compared the stained plasma obtained from the arm with a standard dilution of the dye, using the colorimetric method. Computation yielded the following facts. The total blood averaged 8.8 per cent., $\frac{1}{11.4}$ of the body weight. This is about 85 cubic centimeters per kilo. The plasma averaged about $5^{\circ}$ cubic centimeters per kilo.

This quantity of blood is distributed in the different parts of the body, chiefly in the muscles, the liver, the heart, and larger blood vessels, as shown by the following figures determined on the rabbit by Ranke (from Vierordt):

\begin{tabular}{|c|c|}
\hline & Per cent. \\
\hline$\ldots \ldots \ldots \ldots \ldots$ & 0.23 \\
\hline Brain and cord. & I. 24 \\
\hline Kidney........... & x. 63 \\
\hline 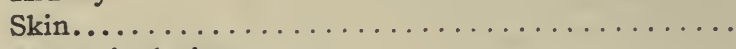 & 2. 10 \\
\hline Abdominal viscera $\ldots \ldots \ldots \ldots \ldots \ldots \ldots \ldots$ & 6.30 \\
\hline 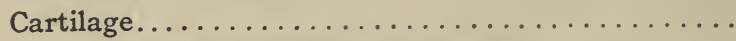 & 8.24 \\
\hline Heart, lungs, and large blood vessels........... & $22 \cdot 76$ \\
\hline 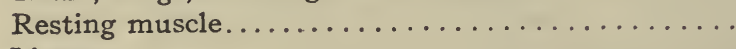 & 29.20 \\
\hline 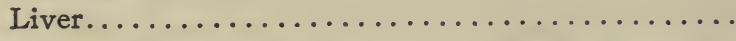 & $29 \cdot 30$ \\
\hline
\end{tabular}

The normal blood volume varies somewhat with relation to the intake of food and drink. Relative body dessication would appear in extreme thirst or under conditions of unusual loss of water without increasing the intake, as in extreme perspiration, diarrheas, etc.

\section{COAGULATION OF THE BLOOD.}

The most characteristic property which the blood possesses is that of clotting or coagulating. This phenomenon may be observed under the most favorable conditions in blood which has been drawn into an open vessel. In about two or three minutes, at the ordinary temperature of the air, the surface of the fluid is seen to become semisolid or jelly-like, and this change takes place, in a minute or two afterward, at the sides of the vessel in which it is contained and then quickly extends throughout the entire mass. The time which is occupied in these changes is about eight or nine minutes. The solid mass is of exactly the same volume as the previously liquid blood, and adheres so closely to the sides of the containing vessel that if the latter be inverted none of its contents escape. The solid mass is the crassamentum or clot. If the clot be watched for a few minutes, drops of a light, strawcolored fluid, the serum, may be seen to make its appearance on the surface, 
and, as it becomes greater and greater in amount, to form a complete superficial stratum above the solid clot. At the same time the serum begins to transude at the sides and at the under surface of the clot, which in the course of an hour or two floats in the liquid. The appearance of the serum is due to the fact that the clot contracts, thus squeezing the fluid out of its mass. The first drops of serum appear on the surface about eleven or twelve minutes after the blood has been drawn; and the fluid continues to transude for from thirty-six to forty-eight hours.

The clotting of blood is due to the development in the plasma of an insoluble substance called fibrin. This fibrin forms threads or strands through the mass in every direction. The strands adhere to each other wherever they come in contact, thus forming a very dense tangle and meshwork which incloses within itself the blood-corpuscles. The clot when first formed, therefore, includes the whole of the blood in an apparently solid mass, but soon the fibrinous meshwork begins to contract and the serum is squeezed out. When a large part of the serum has been squeezed out the clot is found to be smaller, but firmer and harder, as it is now made up more largely of fibrin and blood corpuscles. Thus in coagulation there is a rearrangement of the constituents of the blood; liquid blood consisting of plasma and blood corpuscles, and clotted blood of serum and clot. These relations are roughly shown in the following diagram:

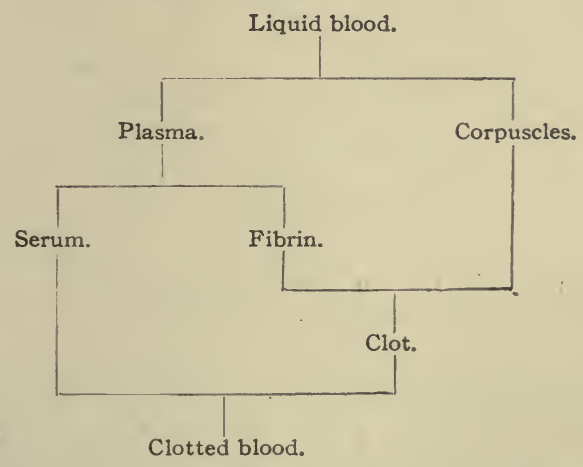

The rapidity with which coagulation takes place varies greatly in different animals and at different times in the same animal. Where coagulation is very slow the red corpuscles, which are somewhat heavier than plasma, often have time to settle considerably before the fibrin is formed. If the blood is rapidly cooled to a temperature approaching $0^{\circ} \mathrm{C}$. then the clot is very greatly delayed. Horse's blood is particularly favorable for demonstrating this point. In it clotting occurs so slowly that very often the red corpuscles will completely settle out, and when the blood is again warmed and the clotting takes place there is a superficial stratum differing in appearance from the rest of the clot, having a grayish-yellow color. This is known 
as the buffy coat or crusta phlogistica. The buffy coat, produced in the manner just described, commonly contracts more than the rest of the clot, on account of the absence of colored corpuscles from its meshes. When the clot is allowed to stand, the white corpuscles which have escaped the clot by ameboid movement settle on its surface often in such numbers that they form a distinct superficial layer, grayish-white in appearance.

That the clotting of blood is due to the gradual appearance in it of fibrin may be easily demonstrated, For example, if recently drawn blood be whipped with a bundle of twigs or wires, the fibrin may be withdrawn from the blood before it can entangle the blood corpuscles within its meshes. It adheres to the twigs in stringy threads relatively free from corpuscles. The blood from which the fibrin has been withdrawn no longer exhibits the power of spontaneous coagulability and it is now called defibrinated blood. Although these facts have long been known, the closely associated problem as to the exact manner in which fibrin is formed is by no means so simple.

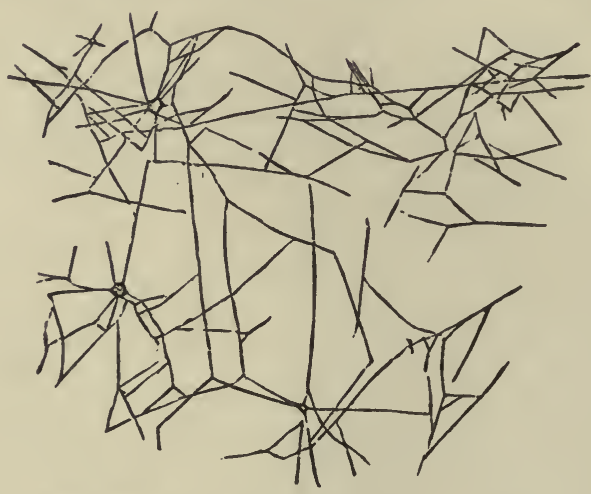

FIG. 107.- Reticulum of Fibrin, from a Drop of Human Blood, after Treatment with Rosanilin. (Ranvier.)

Fibrin is derived from the plasma. Pure plasma may be procured by delaying coagulation in blood by keeping it at a temperature slightly above freezing-point, until the colored corpuscles have subsided to the bottom of the containing vessel. The blood of the horse is specially suited for the purposes of this experiment. A portion of the colorless supernatant plasma, if decanted into another vessel and exposed to the ordinary temperature of the air, will coagulate, producing a clot similar in all respects to blood clot, except that it is colorless from the absence of red corpuscles. If some of the plasma be diluted with twice or three times its bulk of normal saline solution ( 0.9 per cent.), coagulation is delayed, and the stages of the gradual formation of fibrin in it may be conveniently watched. The viscidity which precedes the complete coagulation may be actually seen to be due to the formation of fibrils of fibrin-first of all at the edge of the fluid-containing 
vessel, and then gradually extending throughout the mass. If a portion of plasma, diluted or not, be whipped with a bundle of twigs or wire during the process of clotting, the fibrin will be obtained as a stringy, insoluble mass, just in the same way as from the entire blood. The resulting fluid no longer retains its power of spontaneous coagulability and is in fact now a typical serum.

Theories of Coagulation.-It is evident that the blood plasma contains some substance or substances which take part in the formation of fibrin. By numerous investigations it has been found that the direct antecedent of the fibrin is the protein substance, fibrinogen. This fibrinogen exists in the blood plasma at all times, but is somewhat increased under certain conditions. The fibrinogen is reacted on by another substance known as thrombin. We shall not present the numerous theories which have been held concerning blood coagulation, many of which have been more or less disproven, but shall try to present the condensed statement of the present explanations of this

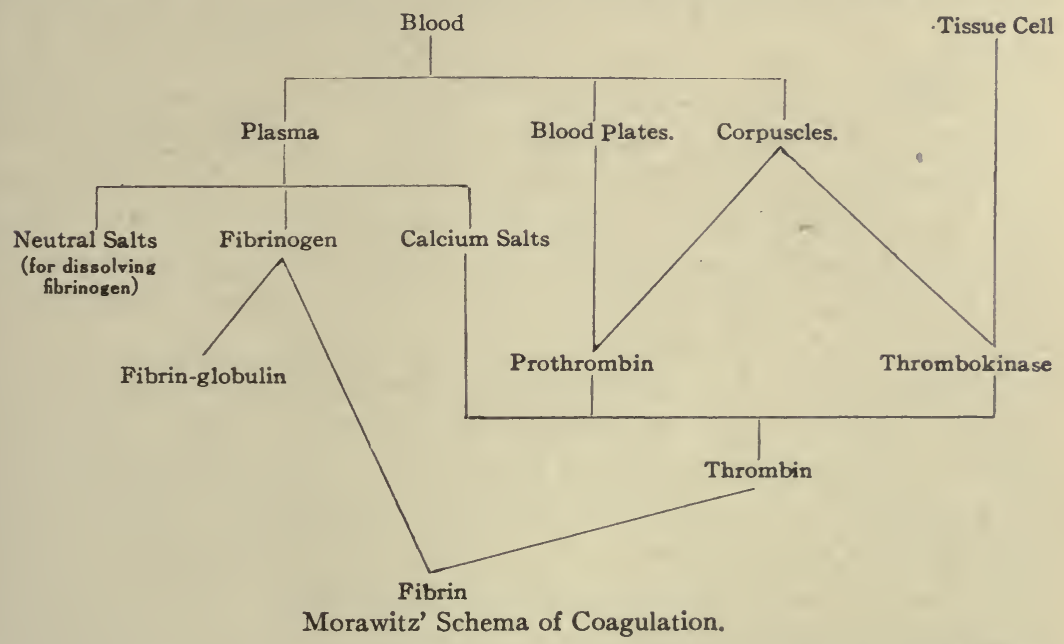

intricate phenomenon. One may start from the statement that the fibrinogen of the plasma when acted upon by the thrombin, also of the plasma, produces an insoluble substance, fibrin. The chief interest centers around the origin and character of the firbinogen, the origin and nature of the thrombin, and the conditions which influence its activity.

The fibrinogen is present in blood plasma of the circulating blood of the body at all times. It can be separated from plasma by various chemical means, and when purified can be made to form fibrin under proper conditions. All observers are agreed that this protein is the immediate precursor of the insoluble fibrin. Its origin in the blood has been traced by Matthews to the disintegration of the white blood corpuscles.

The thrombin is the substance which reacts on the fibrinogen in the 
processes of fibrin formation. It does not exist in the living blood vessels, or at least is present only in minute quantities, but makes its appearance immediately the blood is drawn.

The sources of these substances and the part taken by each in the process of coagulation are given by Morawitz. If blood be drawn, centrifugalized, and the leucocytes and blood plates separated from the plasma and suspended in water, their solution will cause the formation of fibrin from fibrinogen in the presence of calcium. The leucocytes and platelets are, therefore, the source of thrombin. The thrombin can be isolated in a stable condition, and when its solutions are added to fibrinogen solutions fibrin is formed. By Morawitz' view the appearance of thrombin in the blood is due to the interaction of at least three antecedent substances. These are, I, prothrombin (thrombogen), 2, calcium, and 3, thrombokinase (cytozym). If the blood is drawn from vessels with due precautions, i.e., not to allow it to come in contact with the cut vessel or other tissue, clotting is very much delayed. The plasma if separated by the centrifuge will remain unclotted for a long time as shown by Howell for the terrapin's plasma. This plasma will quickly clot at any time if a few drops of tissue extract in salt solution be added. When blood is drawn over the lacerated tissue of a wound it clots more quickly. These observations have led to the assumption of an activating substance or kinase to which the name thrombokinase has been given by Morawitz. It is assumed to have its origin in tissue cells and in the cells of the blood, especiallv the leucocytes.

If precautions are taken to draw the blood in such a manner as to remove the calcium from the plasma, no clot is formed. The calcium which exists in solution in the plasma to the extent of 0.026 per cent. (measured as calcium chloride) can be removed by precipitation with oxalate solution or by the action of fluorides or citrates which bind the calcium so that it is no longer available to the prothrombin. Such plasma contains fibrinogen, prothrombin, and thrombokinase, and whenever calcium chloride is added to excess, coagulation takes place.

L. J. Rettger has recently made a re-examination of the conditions for the clotting of blood. He questions the interpretation of the facts on the basis of which the assumption of a kinase is made. He says:

"Terrapin's blood taken carefully through an oiled cannula and put into a perfectly clean beaker will remain fluid for days. The blood may be centrifuged, and the clear supernatant plasma is then equally or more resistant to spontaneous clotting. If, however, tissue extracts or pieces of tissue be added, the coagulation is pronounced and immediate. The most apparent explanation is that a thrombin or coagulin or kinase is present in the extract. But this blood or plasma can be made to clot equally well and equally rapidly in ways which preclude the presence of such a definite agent. If, for instance, 
dust particles, loose sweepings, or linty shreds be added, the coagulation is equally prompt and in a number of experiments was more rapid than with tissue extract." "The bird's blood or plasma clots with practically the same rapidity and firmness if dust particles are generously added. Bits of down or feathers introduced bring about a speedy clotting. Surely there can be no question of a 'kinase' in these instances." "The existence of 'kinase' or 'coagulins' in the various tissues is improbable. Using carefully prepared fibrinogen solutions, extracts of tissues, irrigated to remove every trace of thrombin-containing blood, cause no clotting. When the addition of such extracts produces coagulation in bloods of bird or reptile, similar results can be secured by the addition of substances, such as dust, lint, shreds, etc., which preclude the presence of definite coagulating agents. These results render very probable the assumption that in such plasmas all the factors of coagulation are in reality present, and the addition of tissue extract or other foreign substance brings into the mixture nothing that can be regarded as a coagulin or as a kinase."

Howell has been studying the phenomena of coagulation for a number of years. On the basis of his work he makes a somewhat different interpretation of the facts on which the theory of Morawitz is founded. By Howell's view, "Circulating blood contains normally all the necessary fibrin factors, namely, fibrinogen, prothrombin, and calcium. These substances are prevented from reacting, and the normal fluidity of the blood is maintained, by the fact that antithrombin is also present, and this substance prevents the calcium from activating the prothrombin to thrombin. In shed blood the restraining effect of the antithrombin is neutralized by the action of a substance (thromboplastin), furnished by the tissue elements. In the mammalia the thromboplastin is derived, in the first place, from the elements of the blood itself (blood platelets). In the lower vertebrates the supply of this material, in normal clotting, comes from the external tissues." Howell's thromboplastin and Morawitz' thrombokinase are probably one and the same substance, it being an enzyme by Morawitz' view, a property denied by Howell and by Rettger.

Quite recently Howell has thrown light on the nature of his thromboplastic substance. He finds that the lipoid, kephalin, present in many tissues of the body possesses the power of neutralizing antithrombin and is comparable, in relation to blood clotting, to the action of thromboplastin. Lecithin does not possess this property.

One may restate Morawitz' view in a word as follows: The coagulation of the blood takes place because of the formation of fibrin from fibrinogen by the action of thrombin. The fibrinogen is constantly present in the plasma. The thrombin is formed by the interaction of three substances, prothrombin, calcium, and thrombokinase. The prothrombin arises chiefly from the disintegration of the blood platelets and leucocytes when the blood leaves the 
blood vessels. The calcium is present in the blood plasma at all times. The thrombokinase originates in tissue cells of the blood and of the organs of the body in general.

Rettger's view is best given in his own words: "On the basis of the work here presented it is not necessary to assume the existence of a kinase in explaining the clotting of shed blood. The prothrombin formed from the platelets and leucocytes by secretion or by processes of disintegration is activated to thrombin by the calcium salts present, and the thrombin so formed combines in quantitative fashion with the fibrinogen to form fibrin."

Howell's demonstration of antithrombin offers a new factor in the problem of blood clotting. For example, the hastening influence of kephalin on blood clotting is probably due to its action on antithrombin, rather than on either of the other clotting complexes.

The Coagulation Time of Blood.-The rapidity with which blood coagulates varies greatly in different animals. In the majority of mammals the coagulation time varies from 2.5 to 5 minutes. In man this time is about 3 to $3: 5$ minutes in normal blood. In recent experiments by Cannon and Mendenhall, on the coagulation time of the blood from the dog and cat, determined by a new and mechanically accurate method, the normal coagulation time is given as from 3.5 to 4.5 minutes. This coagulation time however varies under different conditions of the animal, especially under conditions which affect the activities of the glands, in particular the epinephros. Stimulation of this gland either directly by stimulation of the splanchnic nerves, or indirectly through conditions that arouse fear, etc., leads to a sharp diminution in the coagulation time of the blood. This decrease in some instances is well within I minute, less than $\frac{1}{2}$ minute in their experiment 3 , which was an experiment after emotional excitement. The coagulation time is retarded by the elimination of the circulation of the intestine and of the liver, also by nephrectomy.

Conditions Affecting Coagulation.-From the preceding discussion it is evident that the rapidity of the coagulation of the blood will be influenced by anything that will influence the formation of the fibrin factors or their interaction. The most important influences are the following:

Condition of the Blood.- The blood varies greatly through a wide range in its ability to form fibrin. This depends upon the interaction of those tissues that produce the fibrin factors. An efficient circulation through the abdominal viscera is necessary to maintain the clotting properties of the blood. The rapidity of clotting is increased following the process of digestion. It is also increased (Cannon) by the injection of epinephrin or by the stimulation of the splanchnic nerves which increase the output of epinephrin by the suprarenal bodies. (See Influence of the Ductless Glands, page 482). If those glands be removed, the time of blood clotting 
increases. The exclusion of the abdominal circulation tends to increase the time of blood clotting, and if the liver circulation be eliminated, the influence of epinephrin is lost.

Hemorrhage increases the rapidity of coagula tion, apparently by stimulating the production of the fibrin factors.

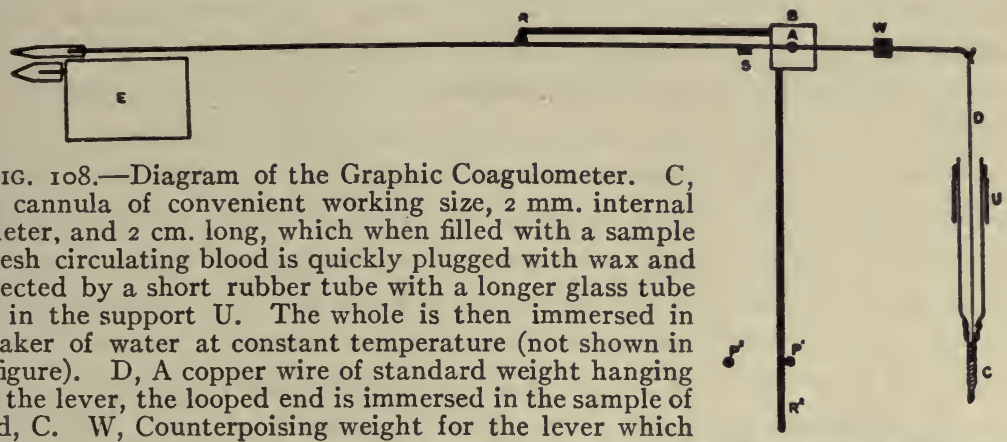

Fig. I08.-Diagram of the Graphic Coagulometer. C In a cannula of convenient working size, $2 \mathrm{~mm}$. internal diameter, and $2 \mathrm{~cm}$. long, which when filled with a sample of fresh circulating blood is quickly plugged with wax and connected by a short rubber tube with a longer glass tube held in the support $U$. The whole is then immersed in a beaker of water at constant temperature (not shown in the figure). D, A copper wire of standard weight hanging over the lever, the looped end is immersed in the sample of blood, C. W, Counterpoising weight for the lever which rotates on the axis A. S, Lever support. $R^{1}-R^{2}$, Short $L$-shaped arm which when moved from $\mathrm{P}^{1}$ to $\mathrm{P}^{2}$ releases the lever at $\mathrm{R}^{1}$. To use the instrument, draw the sample of blood at a signal, insert it in the apparatus, and at regular intervals release the lever by moving the arm $\mathrm{R}^{2}$. If the blood is fluid, the counterpoising lever will make a vertical stroke through its free range. If threads of fibrin have formed, these counteract the movement of the lever. E, Time signal. From Cannon.

Condition of the Blood-vessel Walls.-Intravascular clotting often takes place upon injury of the endothelial lining of the blood vessels, either from the liberation of thrombokinase in quantity too great for elimination by the healthy portion of the wall (Morawitz), or by the disturbance of the equilibrium of the forces which prevent the interaction of the fibrin factors present in the blood (Rettger). The healthy endothelium no doubt is an important factor in controlling the relative amounts of the fibrin factors that must be constantly forming. The open wounds and lacerations of tissue that accompany the loss of blood by accident are the very conditions most favorable to clotting, since large amounts of tissue extract are set free under these conditions.

Temperature.-Cold retards coagulation. Gentle warmth, $40^{\circ} \mathrm{C}$, hastens, but a temperature above $56^{\circ} \mathrm{C}$. destroys clotting, since that temperature heat-coagulates the fibrinogen.

Contact with Foreign Bodies.-Such contact hastens clotting. This is due to the influence of such bodies in hastening the formation of fibrin factors, especially the substances that arise from the disintegration of leucocytes.

Neutral Salts. - The additon of neutral salts in the proportion of 2 or 3 per cent. and upward delays coagulation. When added in large proportions, most of these saline substances prevent coagulation altogether. Coagulation, however, ensues on dilution with water. The time during which salted blood can be thus preserved in a liquid state, and coagulated 
by the addition of water, is quite indefinite, if measures be taken to prevent putrefaction.

Oxalates and Fluorides.-Oxalates to the extent of o.I per cent. concentration prevent clotting by the removal of calcium, one of the factors in the formation of thrombin. Once thrombin is formed, clotting will take place in the absence of soluble calcium. This is proven by the fact that solutions of pure fibrinogen and thrombin form fibrin clots.

Flourides, on the other hand, not only precipitate soluble calcium but fix the blood platelets from which the prothrombin is formed.

Peptone.-The injection of commercial peptone (a mixture of proteoses and peptones) in the blood vessels of an animal to the extent of 0.5 gram of peptone per kilo weight of the body of the animal will deprive the blood of the power of coagulation. If a smaller quantity be injected the coagulation of the blood will be delayed. If peptone blood is drawn and centrifuged, the plasma obtained is called peptone plasma. Howell has shown that peptone contains antithrombin in a relatively large quantity. The increase of antithrombin acts to restrain the reaction of prothrombin in the formation of thrombin. Peptone plasma in the blood vessels of the animal gradually regains the power to coagulate. When blood is drawn into a physiological salt solution of proteose-peptone clotting occurs.

\section{MORPHOLOGY OF THE BLOOD.}

The corpuscles floating in the fluid plasma of the blood, when separated by a centrifugal machine are found to make up 45 to 50 per cent. of the total mass of the blood. These corpuscles, or formed elements, are of three varieties, the red corpuscles or erythrocytes, the white corpuscles leucocytes, and the blood platelets which have been called thrombocytes.

Red Corpuscles or Erythrocytes.-Human red blood corpuscles are circular, biconcave discs with rounded edges, from $7 \mu$ to $8 \mu$ in diameter, and about $2 \mu$ in thickness. When viewed singly they appear of a pale yellowish tinge; the deep red color which they give to the blood being observable in them only when they are seen en masse. They are composed of a colorless, structureless, and transparent filmy framework or stroma, infiltrated in all parts by the red coloring matter, the hemoglobin. The stroma is tough and elastic, so that as the corpuscles circulate they admit of elongation and other changes of form in adaption to the vessels, yet recover their natural shape as soon as they escape from compression.

Number and Character of the Red Corpuscles.-The normal number of red blood-cells in a cubic millimeter of human blood was estimated by Welcker, in $\mathrm{r} 854$, to be $5,000,000$ in men and 4,500,000 in women. Numerous recent observations, however, have shown that these estimates are a little low, especially in men, and the average number has been placed by different authorities at various points between 5,000,000 and 5,500,000. Still the original numbers as given by Welcker are accepted at the present 
day as being sufficiently accurate for ordinary purposes. It has been also shown that there are many distinct physiological variations in the number, depending on the time of day, digestion, sex, etc. The number of red cells usually diminishes in the course of each day, while the leucocytes increase in number. It has been suggested that this is due to the influence of digestion and of exercise.

It has generally been found that within half an hour or an hour after a full meal the number of red cells begins to diminish, and that this keeps up for from two to four hours, when it is followed by a gradual rise to the normal. The usual fall is 250,000 to 750,000 per cubic millimeter. These results are most marked after a largely fluid meal, and are probably due to dilution of the blood as a result of the absorption of fluids. In animals the number of red cells is increased by fasting, but in man the results are variable, some

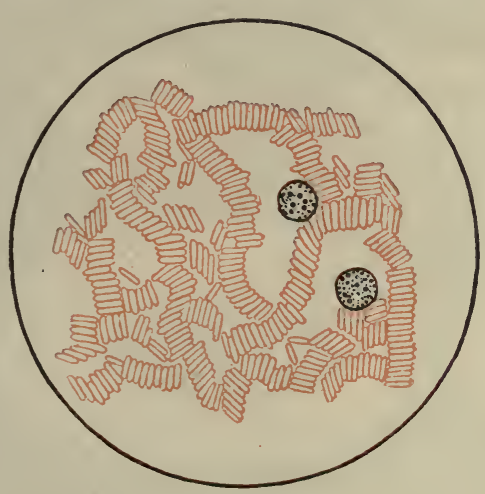

FIG. I09.

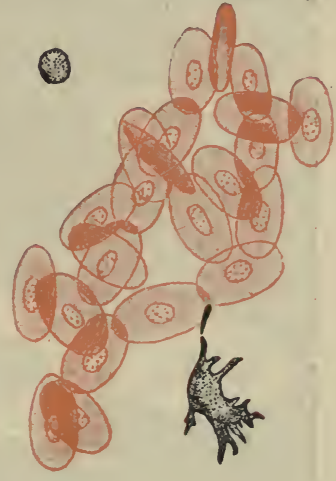

Fig. IIo.

Fig. rog.- Red Corpuscles in Rouleaux. The rounded or uncolored corpuscles are leucocytes.

FIG. Iro.-Corpuscles of the Frog. The central mass consists of nucleated colored corpuscles. The other corpuscles are two varieties of the colorless form.

authorities claiming an increase and others a decrease. In childhood there is no difference between the sexes in the number of red cells per cubic millimeter, but after menstruation is established a relative anemia develops in women. Welcker's original estimate placed the difference at 500,000 per cubic millimeter, and these figures have been generally accepted, though Leichtenstein asserts that the difference is $1,000,000$.

Menstruation in healthy subjects has practically no effect, as not more than 100-200 cubic centimeters of blood are lost normally in the course of several days. Under such circumstances the normal diminution of red cells per cubic millimeter is probably less than 150,000 , though Sfameni has placed the loss at about 225,000 . The leucocytes are slightly increased during menstruation. It is now the general opinion that pregnancy has little or no effect on the number of red cells, and that any anemia must be due to abnormal conditions. Post-partum anemia should not last longer than two weeks. 
The red corpuscles are not all alike. In almost every specimen of blood a certain number of corpuscles smaller than the rest may be observed. They are termed microcytes, or hematoblasts, and are probably immature corpuscles.

A peculiar property of the red corpuscles, which is exaggerated in inflammatory blood, may be here again noticed, $i . e$. , their great tendency to

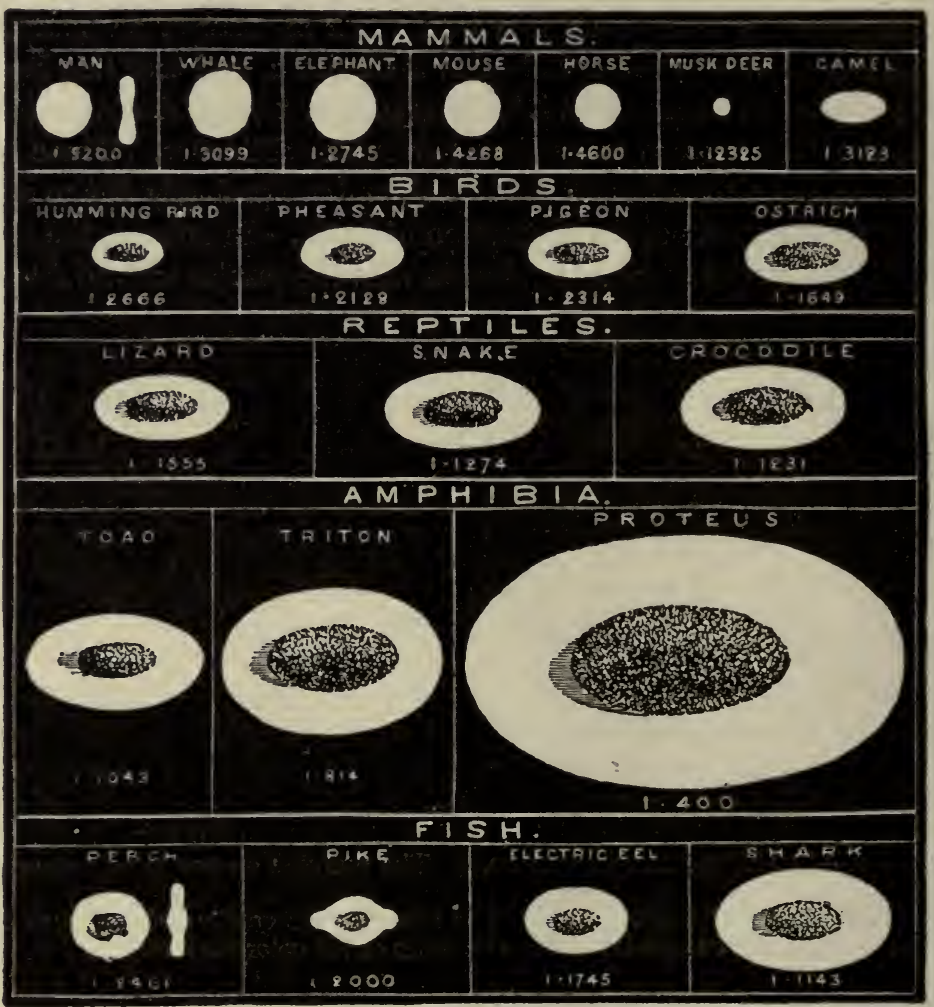

FIG. III.-Illustration exhibiting the typical characters of the red blood-cells in the main Divisions of the Vertebrata. The fractions are those of an inch, and represent the average diameter. In the case of the oval cells, only the long diameter is here given. It is remarkable, that although the size of the red blood-cells varies so much in the different classes of the vertebrate kingdom, that of the white corpuscles remains comparatively uniform, and thus they are, in some animals, much greater, in others much less, than the red corpuscles existing side by side with them. Modified from Gulliver.

adhere together in rolls or columns (rouleaux), like piles of coins. These rolls quickly fasten together by their ends, and cluster; so that, when the blood is spread out thinly on a glass, they form a kind of irregular network, with crowds of corpuscles at the several points corresponding with the knots of the net, figure rog. Hence the clot formed in such a thin layer of blood looks mottled with blotches of pink upon a white ground. 
The red corpuscles are constantly undergoing disintegration in different parts of the circulatory system, particularly in the spleen. The liberated hemoglobin contributes to the formation of the bile pigments in the liver.

Development of the Red Blood Corpuscles. - The first formed blood corpuscles of the human embryo differ much in their general characters from those which belong to the later periods of intra-uterine, and to all periods of extra-uterine life. Their manner of origin is at first very simple.

Surrounding the early embryo is a circular area, called the vascular area in which the first rudiments of the blood vessels and blood corpuscles are developed. Here the nucleated embryonal cells of the mesoblast, from which the blood vessels and corpuscles are to be formed, send out processes in various directions, and these, joining together, form an irregular mesh-work. The nuclei increase in number, and collect chiefly in the larger masses of protoplasm, but partly also in the processes. It appears that hemoglobin then makes its appearance in certain of these nucleated embryonal cells, which thus become the earliest red blood corpuscles.

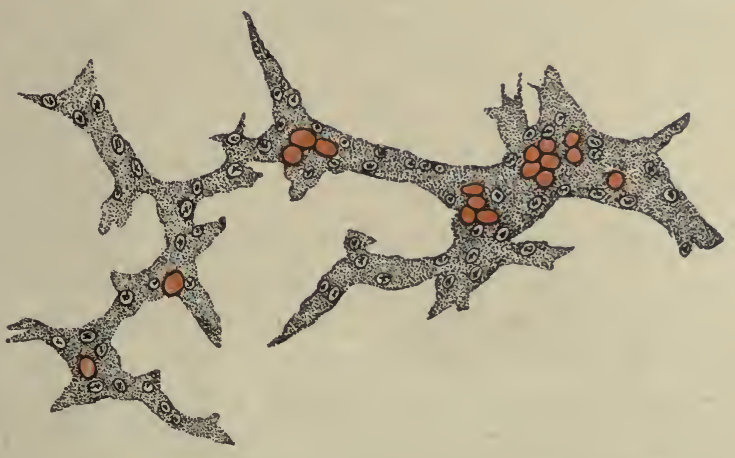

FIG. II 2.-Part of the Network of Developing Blood Vessels in the Vascular Area of a Guinea-pig. Showing blood corpuscles becoming free in an enlarged and hollowed-out part of the network and processes of protoplasm. (E. A. Schäfer.)

The protoplasm of the cells and their branched network in which these, corpuscles lie then become hollowed out into a system of canals enclosing fluid in which the red nucleated corpuscles float. The corpuscles at first are from about Iо $\mu$ to $16 \mu$ in diameter, mostly spherical, and with granular contents, and a well-marked nucleus. Their nuclei, which are about $5 \mu$ in diameter, are central, circular, very little prominent on the surfaces of the corpuscles, and apparently slightly granular.

The corpuscles then strongly resemble the colorless corpuscles of the fully developed blood but for their color. They are capable of ameboid movement and multiply by division.

When, in the progress of embryonic development, the liver is formed, the multiplication of blood-cells in the whole mass of blood ceases, and new blood-cells are produced by this organ, and also by the spleen. These are at first colorless and nucleated, but afterward acquire the ordinary blood tinge, and resemble very much those of the first set. They also multiply by division. The bone marrow also begins to form red corpuscles, though at 
first in small amounts only. This function develops rapidly, however, so that at birth the marrow represents the chief seat of production of the red cells. Nevertheless, nucleated red cells are usually found at birth, sometimes in considerable quantities in the liver and in the spleen. Non-nucleated red cells begin to appear soon after the first month of fetal life, and gradually increase so that at the fourth month they form one-fourth of the whole amount of colored corpuscles. At the end of fetal life they almost completely replace the nucleated cells. In late fetal life the red cells are formed in almost the same way as in extra-uterine life.

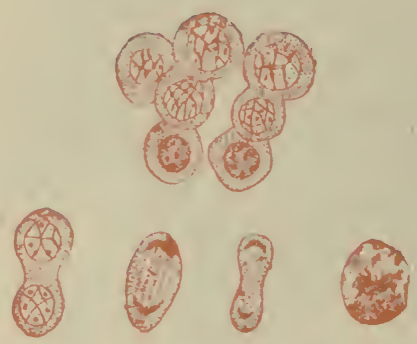

FIG. II3.
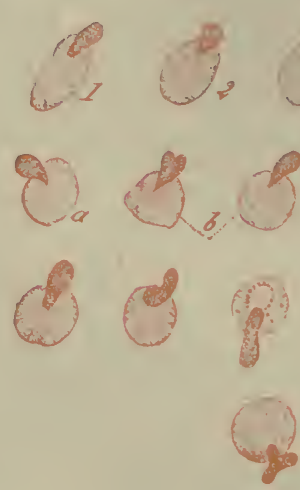

Fig. 114 .

Fig. II3.-Multiplication of the Nucleated Red Corpuscles. Marrow of young kitten after bleeding, showing above karyokinetic division of erythroblast, and below the formation of mature from immature erythrocytes. (Howell.)

FIg. Ir4. - Shows the Way in which the Nucleus Escapes from the Nucleated Red Corpuscles. I, 2, 3, 4, represent different stages of the extrusion noticed upon the living corpuscles. $a$, Specimen from the circulating blood of an adult cat, bled four times; $b$, specimen from the circulating blood of a kitten forty days old, bled twice; $c$, specimens from the blood of a fetal cat, $9 \mathrm{~cm}$. long. Others from the marrow of an adult cat, two of the figures showing the granules present in the corpuscles, which have been interpreted erroneously as a sign of the disintegration of the nucleus. (Howell.)

Various theories have prevailed as to the mode of origin of the nonnucleated colored corpuscles. For a time it was thought that they were of endoglobular origin, and merely fragments of some original cell, being produced by subdivision of the cell body itself. This theory easily accounted for the absence of the nuclei, but it has not been supported by recent investigations. At present it is the general belief that the non-nucleated cells, or erythrocytes, are derived from nucleated cells by a process of mitotic division, and further that their nuclei gradually shrink or fade and are then extruded. The use of some of the more recent stains seems to prove that there are traces of nuclear material in the non-nucleated corpuscles.

After infancy and early childhood the origin of erythrocytes is practically limited to the red marrow of the bones. The mother cells, or erythroblasts, are constantly forming and setting free erythrocytes, the rate varying greatly at different periods. 
The Colorless Corpuscles or Leucocytes.-In human blood the white corpuscles, leucocytes, are nearly spherical masses of granular protoplasm without cell wall. In all cases one or more nuclei exist in each corpuscle. The corpuscles vary considerably in size, but average $10 \mu$ in diameter.

The number of leucocytes in a cubic millimeter of blood is estimated at 7,500 to 8,000 . The proportion of white corpuscles to red, therefore, is about one of the former to 700 of the latter. This proportion is not very constant in health and great variations occur under the influence of disease

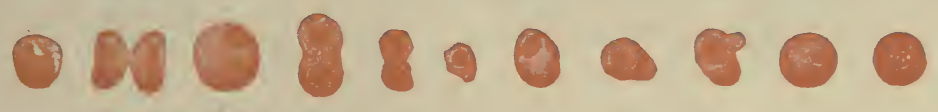

FIG. II5.-Colored Nucleated Corpuscles, from the Red Marrow of the Guinea-pig. (E. A. Schäfer.)

especially in certain infectious diseases in which the number of white corpuscles is markedly increased.

After a full meal the white cells in a healthy adult are increased in number about one-third, the increase beginning within an hour, attaining a maximum in three or four hours, and then gradually falling to normal. This process is frequently modified by the character of the food, the greatest increase occurring with an exclusively meat diet, while a purely vegetarian diet has usually no effect. The increase is also more marked in children, and especially in infants. The essential factor is probably the absorption of albuminous matter in considerable quantities. This causes proliferation of leucocytes in the lymphoid tissue of the gastro-intestinal tract.

In pregnancy there is often a moderate increase in the number of white cells during the later months. This does not begin until after the third month, and is most marked and constant in primiparæ. After parturition the leucocytes gradually diminish under normal conditions, and usually reach the normal within a fortnight. The essential factor is probably the general stimulation in the maternal organism. It is well established that the white cells are very numerous in the new-born, though different observers have made very conflicting estimates. Still all agree that there is a very rapid decrease in their numbers during the first few days, and that this is followed by a less marked increase, which continues for many months. According to Rieder there are at birth from 14,200 to 27,400 per cubic millimeter, and after the fourth day from I 2,400 to 14,800 .

The colorless corpuscles present a great diversity of form. There are certain constant types found in fairly definite proportions in normal blood, but in pathological bloods a long series of variants have been described and figured by such authors as Wood, Webster, and Simon. In histological and clinical examination the white corpuscles are classified 
according to their size, structure, and staining reaction. Some of these cells are mononuclear, others polynuclear and many charged with special types of granules that take now basic, now acid dyes, presumably according to their clinical composition. The following varieties may be listed for normal adult blood.

I. Small mononuclear leucocytes, 22-25.

2. Large mononuclear leucocytes, $\mathbf{I}-2$.

3. Polynuclear neutrophilic leucocytes, $65^{-75}$.

4. Polynuclear eosinophilic leucocytes, $\mathrm{I}-4$.

5. Polynuclear basophilic leucocytes, mast cells, 0.2 to 0.5 .

The small mononuclear leucocytes, or lymphocytes, are about the size of or smaller than the red corpuscles, a single nucleus with very little nongranular protoplasm, staining deeply with methyline blue with a lighter staining nucleus, 22 to 25 per cent.

The large mononuclear leucocytes are double the size of the small leucocytes or even larger. They have a single nucleus about the size of the preceding type but a much larger relative development of protoplasm. Their cytoplasm is not granular and they are weakly basophilic. These cells like the small leucocytes arise in the lymphoid tissue.

The polynuclear neutrophilic leucocytes are about the size of a red blood corpuscle and are granular in appearance. The nuclei take basic dyes. The cytoplasm is slightly acidic but the granules imbedded in it are basophilic. These leucocytes constitute from 65 to 75 per cent. of the total number of white corpuscles. This class is most actively phagocytic and is increased in number in response to most infections.

The polynuclear eosinophilic leucocytes. - The cells of this type are the largest cells of the white corpuscle group. Their cytoplasm is crowded with granules which stain deeply with eosin and other acid dyes. From this characteristic they get their name. The eosinophiles constitute I to 4 per cent. of the total. They are extremely motile and phagocytic and are very greatly increased in number in certain diseases.

The mast cells are much fewer in number except in certain particular diseases. They have a polymorphic nucleus characteristic in appearance. They take basic dyes with difficulty. These cells are also granular. The granules do not take eosin but are basic in type. They vary in size, being rather larger than eosinophilic granules. In normal blood one mast cell occurs in from 200 to 250 leucocytes.

The relative number of leucocytes varies in children as compared with normal adults. The small mononuclear lymphocytes are practically double the adult number and the polymorphonuclears about half the number in the adult. The eosinophiles are also more frequent though still relatively rare. 


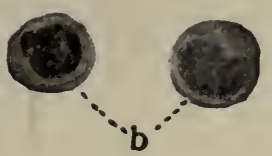

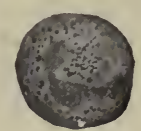

d

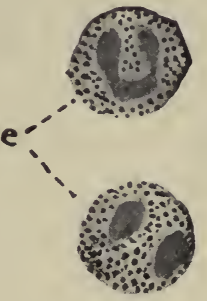

FIG. I I6.-(a) Red blood corpuscle for comparison; (b) Small lymphocyte; (c) Large lymphocyte (myelocyte); $(d)$ Fine and $(e)$ coarse eosinophiles; $(f)$ Basophile. (F. C. Busch.)

A meboid Movement and Phagocytic Action of Leucocytes.-The remarkable property of the colorless corpuscles of spontaneously changing their shape was first demonstrated by Wharton Jones in the blood of the skate. If a drop of blood be examined with a high power of the microscope, under conditions by which loss of moisture is prevented, and at the same time the temperature is maintained at about that of the body, $37^{\circ} \mathrm{C}$., the colorless corpuscles will be observed slowly to alter their shapes, and to send out processes at various parts of their circumference. The ameboid movement which can be demonstrated in human colorless blood corpuscles can be most conveniently studied in the newt's blood. Processes are sent out from the corpuscle. These

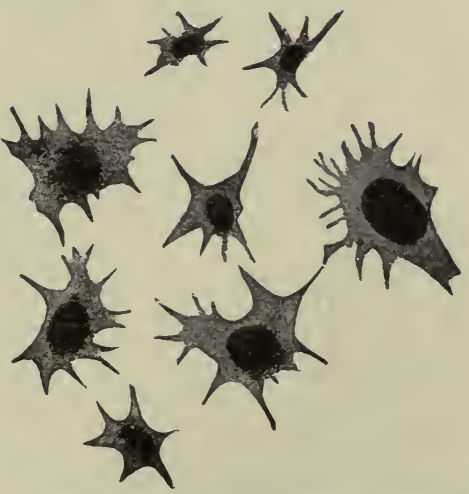

FIG. II8.-Blood Plates, showing chromatic centers regarded by some as nuclei, and exhibiting ameboid movement. (Schäfer, from Kopsch.) may be withdrawn, but more often the protoplasm of the whole corpuscle flows gradually forward to the position occupied by the process, thus the corpuscle changes its position. The change of position of the corpuscle can also take place by a flowing movement of the whole mass, and in this case the locomotion is comparatively rapid. The activity both in the processes of change of shape and also of change in position is much more marked in some corpuscles than in others. Klein states that in the 


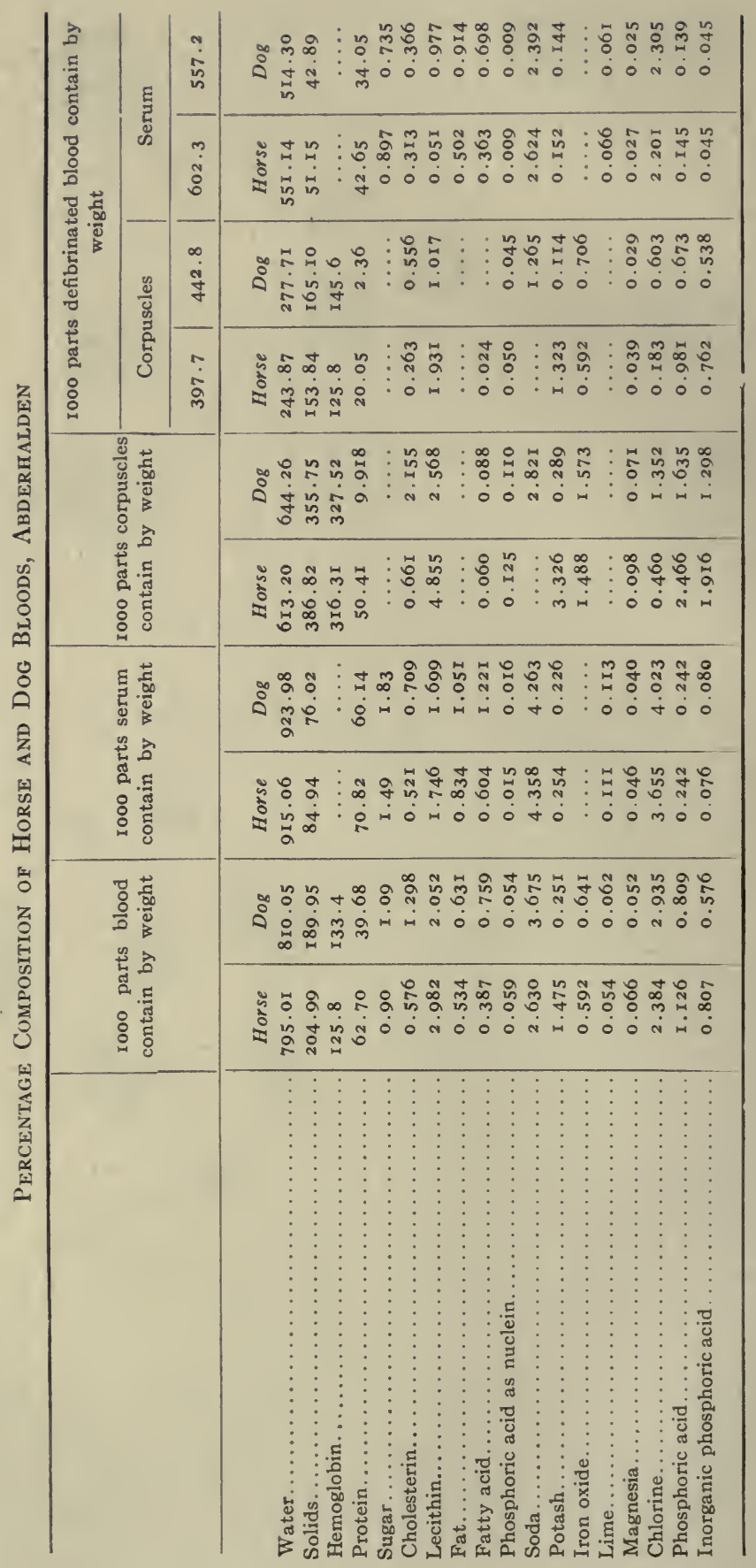


newt's blood the changes are especially noticeable in a variety of colorless corpuscle, which consists of a mass of finely granular protoplasm with jagged outline and contains three or four nuclei, or in large irregular masses of protoplasm containing from five to twenty nuclei.

The blood leucocytes are the phagocytes of the body. By means of their ameboid movements they surround or engulf foreign bodies including bacteria. These they digest, therefore destroy. It is through the activity of the leucocytes that the body gains its relative immunity, since these cells are able to overcome to a certain extent bacterial invasion. The blood largely depends on the polymorphonuclear leucocytes for phagocytosis, hence these cells are found to be increased in number under the stimulus of infectious processes. The relative numbers of leucocytes in clinical blood counts have their value in part in this fact. However, in clinical bloods many pathological types appear which are of special significance.

The Blood Platelets or Thrombocytes. - A third variety of corpuscle found in the blood is known as the blood platelets. They are circular or elliptical in shape, of nearly homogeneous structure, and vary in size from 0.5 to $5 \mu \mu$. Hence they are smaller than the red corpuscles. They vary in number from 5,000 to 45,000 per cubic millimeter and are preserved by drawing fresh blood directly into Hayem's or other preserving fluid. When fresh blood is drawn the blood platelets, coming in contact with foreign bodies, rapidly disintegrate and give rise to one of the antecedents of blood plasma concerned in clotting, prothrombin, p. I2I.

\section{CHEMICAL COMPOSITION OF THE BLOOD.}

The chemical composition of the blood as a whole may be presented by consideration of the constituents of the plasma and of the corpuscles. The available blood analyses have dealt with whole blood, with either plasma or serum and with whole corpuscles, although separate analyses of red and white corpuscles have been made.

The classical analyses in the literature that have become the reliance for teaching purposes are those of Schmidt for the blood of man and of Abderhalden for dog's and horse's blood. These analyses are quoted in tabular form below.

\section{ANALYSES OF BLOOD OF MAN-KARL SCHMIDT}

One Thousand Grammes of Blood.

Blood-Corpuscles........... 5 5 I $3.02^{2}$

Water................. 349.69

Substances not vaporizing at $\mathrm{I}_{20^{\circ}} \quad 163.33$

Hematin...

7.70 (including 0.512 iron)

Blood casein, etc........... I51.89

Inorganic constituents.

$3 \cdot 74$ (excluding iron) 
Chlorine

Sulphuric acid.............

Phosphoric acid.

Potassium.

Sodium .

Phosphate of lime.

Phosphate of magnesium

Oxygen

Blood Plasma. 486.89

Water. 439.02

Substances not vaporizing at $120^{\circ}$

Fibrin $47 \cdot 96$

Protein, etc.

3.93

Inorganic constituents.

Chlorine.

Sulphuric.

Phosphoric acid

Potassium.

Sodium

Phosphate of lime.

Phosphate of magnesium

Oxygen .

Specific Gravity = I.0599. 39.89

4. 14
0.898 Chloride of potassium.

I. 887

0.031

0.695

I. 586

$0.24 \mathrm{I}$

0.048

0.031

0.206

I. 722

0.063

0.071

o. 153

I. $66 \mathrm{I}$

o. 145

०. 106

0.221

Sulphate of potassium.

0.068

Phosphate of potassium......... I. 202

Phosphate of sodium.

0.325

Soda.

0.175

Phosphate of lime.

0.048

Phosphate of magnesium.

0.031

Total............... $3 \cdot 736$

Sulphate of potassium.

O. 137

Chloride of potassium

o. 175

Chloride of sodium.

2. 701

Phosphate of sodium.

0.132

Soda.................... 0.746

Phosphate of lime.

0.145

Phosphate of magnesium.

०. 106

The Composition of Plasma.-The plasma is the liquid part of the blood in which the corpuscles float. It differs from the serum only in that the fibrin factors have been removed during the process of clotting. To all intents and purposes the chemistry of the plasma and serum are identical. Plasma may be freed from the corpuscles by the centrifugal machine in the interval before clotting forms. However, it is customary to delay clotting by means enumerated below.

Cooled Plasma.-If blood is drawn directly into a chilled vessel and kept at a temperature of about $0^{\circ} \mathrm{C}$., or only two or three degrees higher, the corpuscles will settle out, leaving a clear supernatant plasma. This plasma will clot promptly on raising the temperature. This method yields a pure plasma for isolation of fibrinogen and for quantitative analysis.

Salted Plasma.-Blood will not clot if it is mixed with sodium sulphate or magnesium sulphate in adequate proportion, one part to twelve parts of blood of the former, and one part to six parts of blood of the latter. Salted plasma obtained by this method will coagulate on dilution.

Oxalated Plasma.- In experimental work it is customary to prevent blood clotting by receiving it into an oxalate solution or over a powder of an oxalate salt. It is necessary that the blood contain at least one tenth per cent. of oxalate. The oxalate removes the soluble calcium salts before the prothrombin is converted into thrombin. Oxalated plasma will not 
clot until a calcium salt is added. The oxalate does not interfere with gas absorption and gas determination tests.

Hirudin Plasma.-Leach extract contains an anticoagulant which prevents clotting of blood. Such blood clots readily on adding serum or other solution containing thrombokinase which neutralizes the hirudin effect as it does the antithrombin when blood is drawn.

Peptone Plasma.-Peptone solution injected directly into the blood stream renders the blood non-coaguable when it is afterward drawn. Peptone does not prevent blood from clotting when mixed in vitro. However, when blood serum or a pure solution of fibrin ferment is added, both of which neutralize the antithrombin, then peptone plasma will form a clot.

Water of Blood Plasma.-The water of the plasma varies in amount through a wide range. During absorption of food and drink the water increases temporarily in the blood, though at this time it is flowing into the tissues and being more rapidly excreted by the kidneys. In a few minutes, say 15 or 20 , after a glass of water the blood constituents will be diluted and the red corpuscles demonstrably swollen. This condition is quickly equalized. On the other hand the rapid loss of water in evaporation of sweat following vigorous exercise quickly leads to loss of water from and concentration of the plasma. These variations occur around an average content of 90 per cent. of plasma water.

Proteins.-The chief proteins of plasma are serum albumin, serum globulin, and fibrinogen, a total of from 6 to 8 per cent. of the plasma. Fibrinogen is the part of plasma that is converted into fibrin when blood clots. It is a globulin. Fibrinogen is precipitated from plasma by half jaturation with sodium chloride and along with globulin by full saturation with sodiurn chloride or magnesium sulphate. It is soluble in dilute salt solutions but not soluble in water.

Serum globulin or paraglobulin is completely precipitated by saturation by magnesium sulphate, incompletely by sodium chloride, and coagulates at a temperature of $75^{\circ} \mathrm{C}$. It is likewise soluble in dilute salt solutions, but insoluble in water. It is present in plasma in from 3.5 to 4 per cent., but varies greatly.

Serum albumin is the protein which predominates in human plasma. It is readily obtained in crystalline form; is soluble in saturated magnesium sulphate and sodium chloride solutions, but insoluble in saturated ammonium sulphate solutions. It coagulates in neutral or acid solutions at from $73^{\circ}$ to $75^{\circ} \mathrm{C}$.

Extractives.-The extractives are the nitrogen-containing substances, such as urea, uric acid, creatin, creatinin, etc., and the non-nitrogenous glycogen, dextrose, cholesterin, etc., a total of 0.5 to 0.6 per cent. The dextrose content amounts to from O.I to o.I 5 per cent.

Among the extractives must be classed the various hydrolytic ferments such as the diastatic ferments that react with the carbohydrates of the 
blood, liver, etc.; the lipolytic or fat-splitting ferments, and the proteolytic ferments. From their property of producing not only hydrolytic cleavage but the reverse reactions of synthesis these ferments hold one of the most significant positions among the plasma constituents.

Inorganic Substances. - The total inorganic salts of human plasma amount to from 0.80 to 0.89 per cent. By the recent titration method of Cramer, the sodium ran 323 to 344 milligrams per roo cubic centimeters of serum. The chief salt is sodium chloride which constitutes over half the total and contributes largely to the osmotic pressure of the blood. Curiously enough only a minimal quantity of potassium salt is present in the plasma, from 18 to $2 \mathrm{I}$ milligrams in a hundred grams. The potassium exists as sulphate and basic phosphate. The calcium is very constant in normal serum, from 9.3 to 9.9 milligrams in a hundred cubic centimeters of serum. It is probably in the blood as a phosphate. The calcium content varies greatly in disease, is depressed in parathyroid tetany.

The Serum.-The serum is the liquid part of the blood or of the plasma which remains after the fibrin has been formed and removed. It is a transparent yellowish fluid with a specific gravity of 1025 to 1032. Serum is ordinarily obtained free from blood corpuscles by whipping the blood, that is removing the fibrin as fast as it forms and then sedimenting the corpuscles in the centrifugal machine. It may be obtained by allowing blood to clot in a test tube or beaker and then stand in the cold. The clot contracts, squeezing out the clear yellowish straw-colored serum. In quantitative chemical analysis the serum is essentially of the same composition as plasma, in fact serum is usually taken for such quantitative work. It differs from plasma only in the loss of the fibrin or the fibrin factors which go to form fibrin. It is usually rich in thrombokinase or fibrin ferments. The percentages given above for the salts of plasma were actually determined on serum.

The Composition of the White Corpuscles.-The white corpuscles are comparatively undifferentiated cellular elements, hence possess the chemical composition of protoplasm. Lillienfeld has made an analysis of the leucocytes of thymus gland from the calf, which contain Ir.49 per cent. of solids, as follows:

In roo Parts of Dry Substance of White Corpuscles of Calf.

Per cent.

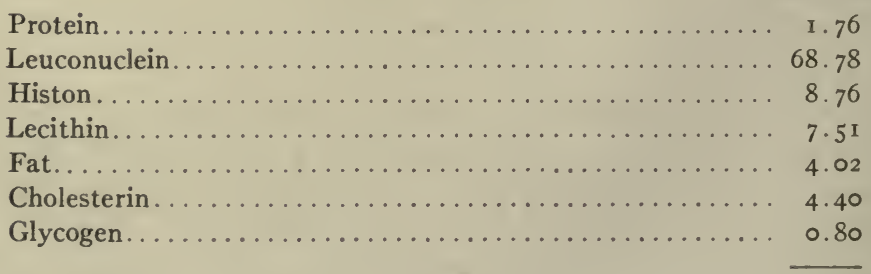


Most noteworthy substances in this table are the nuclein and histon, first isolated by Kossel and Lillienfeld as nucleohiston. Besides the substances in the table, the white corpuscles contain salts of potassium, sodium, calcium, and magnesium, with potassium phosphate present in greatest amount.

The Composition of the Red Corpuscles.-Analysis of moist blood corpuscles shows the following results:

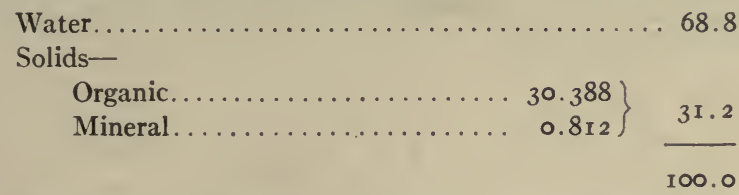

Of the solids the most important is the respiratory pigment, hemoglobin, the substance to which the blood owes its color. It constitutes, as will be seen from the appended table, more than 90 per cent. of the organic matter of the corpuscles. Besides hemoglobin the corpuscles contain protein and fatty matters, the former chiefly consisting of globulins, and the latter of cholesterol and lecithin.

In roo parts of organic matter are found:

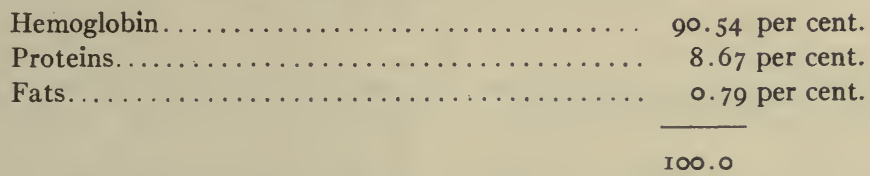

The inorganic salts of the red corpuscles differ from the salts of serum in that the ash of corpuscles contains a high content of those salts that tend to form fixed organic compounds. For example, iron is present as a part of the hemoglobin molecule. There is an excess of potassium in corpuscles, present in fixed organic compounds. Only a small amount of sodium is present, and of calcium only a trace.

Hemoglobin.-Of the substances in the erythrocytes, by far the most important from every point of view is the pigment, hemoglobin. It composes about 90 per cent. of the total solids of the corpuscles; therefore, between 14 and 15 per cent. of the blood itself. Hemoglobin is the most complex compound in the body, having a molecule of the enormous molecular weight of 16,669 . Hemoglobin is intimately distributed throughout the stroma of the corpuscle, and when dissolved out it can be crystallized.

Its percentage composition is $\mathrm{C}, 53.85 ; \mathrm{H}, 7.32 ; \mathrm{N}, \mathrm{I} 6.17 ; \mathrm{O}, 21.84 ; \mathrm{S}, 0.63$ $\mathrm{Fe}$, 0.42. Jacquet gives the empirical formula for the hemoglobin of the dog, $\mathrm{C}_{758} \mathrm{H}_{1205} \mathrm{~N}_{195} \mathrm{~S}_{3} \mathrm{FeO}_{218}$. The most interesting of the properties of hemoglobin are its powers of crystallizing and its attraction for oxygen and other gases under certain pressure relations.

Hemoglobin Crystals. - The hemoglobin (oxyhemoglobin) of the blood of various animals possesses the power of crystallizing to very different ex- 
tents. In some the formation of crystals is almost spontaneous, whereas in others it takes place either with great difficulty or not at all. Among the animals whose blood coloring-matter crystallizes most readily are the guinea-pig, rat, squirrel, and dog; and in these cases to obtain crystals it is generally sufficient to dilute a drop of recently drawn blood with water and to expose it for a few minutes to the air. In many instances other means must be adopted; e.g., the addition of alcohol, ether, or chloroform, rapid freezing and then thawing, the application of an electric current, a tempera-

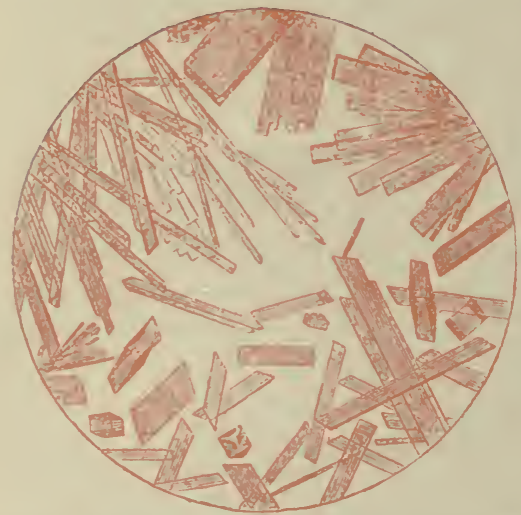

Fig. Irg.-Crystals of Oxyhemoglobin -Prismatic, from Human Blood.

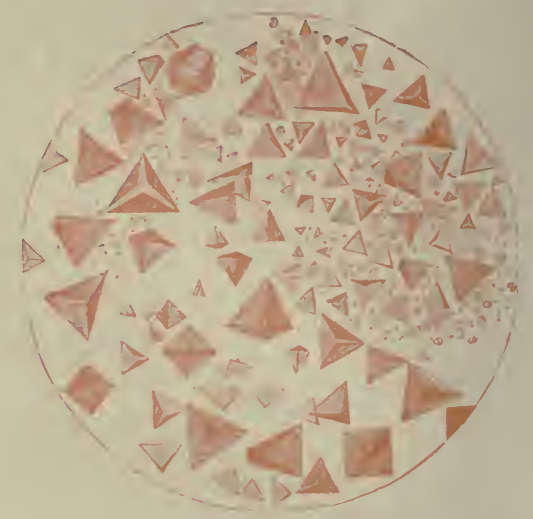

FIG. I20.-Oxyhemoglobin CrystalsTetrahedral, from Blood of the Guinea-pig.

ture of $60^{\circ} \mathrm{C}$., the addition of sodium sulphate, or the addition of decomposing serum of another animal.

The hemoglobin of human blood crystallizes with difficulty, as does also that of the ox, the pig, the sheep, and the rabbit.

The forms of hemoglobin crystals, as will be seen from figures II 9 and 120, differ greatly. Hemoglobin crystals are soluble in water. Both the crystals themselves and also their solutions have the characteristic color of arterial blood.

A dilute solution of oxyhemoglobin gives a characteristic appearance with the spectroscope. Two absorption bands are seen between the solar lines $D$, which is the sodium band in the yellow, and $E$, see the frontispiece, one in the yellow, with its middle line some little way to the right of $D$. 'This band is very intense, but narrower than the other, which lies in the green near to the left of $E$. Each band is darkest in the middle and fades away at the sides. As the strength of the solution increases, the bands become broader and deeper. Both the red and the blue ends of the spectrum become encroached upon until the bands coalesce to form one very broad band when only a slight amount of the green and part of the red remain unabsorbed. Any further increase of strength leads to complete absorption of the spectrum.

If crystals of hemoglobin are exposed to an atmosphere of oxygen they take up oxgyen and form oxyhemoglobin, each gram of the pigment fixing 
a definite amount oxygen, see chapter on Respiration. When subjected to a mercurial air-pump the oxygen is given off, and the crystals become of a purple color. A solution of the oxyhemoglobin in the blood corpuscles may be made to give up oxygen, and to change color in a similar manner. One gram of oxyhemoglobin liberates I . 59 c.c. oxygen, or, according to Hüfner's later determinations, I. 34 C.C., see page 292.

This change may be also effected by passing through the solution of blood or of oxyhemoglobin, hydrogen or nitrogen gas, or by the action of

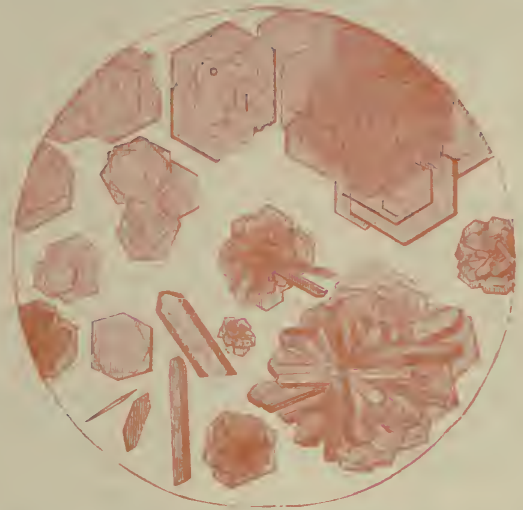

FIG. I2I.- Hexagonal Oxyhemoglobin Crystals, from Blood of Squirrel. On these hexagonal plates prismatic crystals, grouped in a stellate manner, not unfrequently occur (after Funke).

reducing agents, of Stokes's fluid* or ammonium sulphide are the most convenient.

With the spectroscope, a solution of deoxidized or reduced hemoglobin is found to give an entirely different appearance from that of oxidized hemoglobin. Instead of the two bands at $\mathrm{D}$ and $\mathrm{E}$, we find a single broader but fainter band occupying a position midway between the two, and at the same time less of the blue end of the spectrum is absorbed. Even in strong solutions this latter appearance is found, thereby differing from the strong solution of oxidized hemoglobin which lets through only the red and orange rays; accordingly, to the naked eye the one (reduced-hemoglobin solution) appears purple, the other (oxyhemoglobin solution) red. The deoxidized crystals or their solutions quickly absorb oxygen on exposure to the air, becoming scarlet. If solutions of blood be taken instead of solutions of hemoglobin, results similar to the whole of the foregoing can be obtained.

Venous blood never, except in the last stages of asphyxia, fails to show the oxyhemoglobin bands, inasmuch as the greater part of the hemoglobin even in venous blood exists in the more highly oxidized condition.

*Stokes's fuid consists of a solution of ferrous sulphate, to which ammonia has been added and sufficient tartaric acid to prevent precipitation. Another reducing agent is a solution of stannous chloride, treated in a way similar to the ferrous sulphate, and a third reagent of like nature is an aqueous solution of yellow ammonium sulphide, $\left(\mathrm{NH}_{4}\right)_{2} \mathrm{~S}+\mathrm{S}$. 
Action of Gases on Hemoglobin.-Carbon monoxide gas passed through a solution of hemoglobin causes it to assume a cherry-red color and to present a slightly altered spectrum; two bands are still visible but are slightly nearer the blue end than those of oxyhemoglobin, see Plate I. The amount of carbon monoxide taken up is equal to the amount of the oxygen displaced. Carbon monoxide gas readily displaces oxygen under the ordinary respiratory conditions. It is less readily displaced by excess of oxygen and by carbon dioxide, hence the poisonous effects of coal gas which contains much carbon monoxide. Carbon monoxide hemoglobin is not an oxygen carrier, and death may result from suffocation due to the want of oxygen, notwithstanding the free entry of pure air into the lungs. Crystals of carbon monoxide hemoglobin closely resemble in form those of oxyhemoglobin.

Nitric oxide produces a similar compound to the carbon monoxide hemoglobin, which is even less easily reduced.

Sulphuretted hydrogen, if passed through a solution of oxyhemoglobin, reduces it and an additional band appears in the red. If the solution be then shaken with air, the two bands of oxyhemoglobin replace that of reduced hemoglobin, but the band in the red persists.

Methemoglobin.-If an aqueous solution of oxyhemoglobin is exposed to the air for some time, its spectrum undergoes a change; the two $d$ and $e$ bands become faint and a new line in the red at $C$ is developed. The solution, too, becomes brown and acid in reaction, and is precipitable by basic lead acetate. This change is due to the decomposition of oxyhemoglobin, and to the production of methemoglobin. On adding ammonium sulphide, reduced hemoglobin is produced, and on shaking this up with air, oxyhemoglobin is again produced. Methemoglobin is probably a stage in the deoxidation of oxyhemoglobin. It appears to contain less oxygen than oxyhemoglobin, but more than reduced hemoglobin. Its oxygen is in more stable combination, however, than is the case with the former compound.

Estimation of Hemoglobin.-The most exact method is by the estimation of the amount of iron (dry hemoglobin containing 0.42 per cent. of iron) in a given specimen of blood, but as this is a somewhat complicated process, various methods have been proposed which, though not so exact, have the advantage of simplicity. Of the several varieties of hemoglobinometer, one of the oldest adapted to its purpose is that invented by professor Fleischl, of Vienna. In this instrument the amount of hemoglobin in a solution of blood is estimated by comparing a stratum of diluted blood with a standard solid substance of uniform tint similar spectroscopically to diluted blood. The instrument has been modified and made more accurate by Miescher. The Fleischl-Miescher apparatus consists of a stand with a metal plate having a circular opening and a 
plaster mirror below, $S$, figure 122 , which casts light through the opening. Beneath the plate is a metal framework containing a colored glass wedge, and along the side of the same is a scale graduated so as to indicate the percentage of hemoglobin corresponding to the shades of the different parts of the wedge. This framework can be moved by the wheel $T$ which fits into a rack on its lower surface. The scale can be read through a small opening $M$ in the plate. Into the large circular opening of the plate fits a cylindrical metal cell $G$ with a glass bottom and divided by a metal partition into two equal parts. One of these halves lies over the wedge and is filled with distilled water. The other contains the solution of blood in which the hemoglobin is to be estimated. The appar-

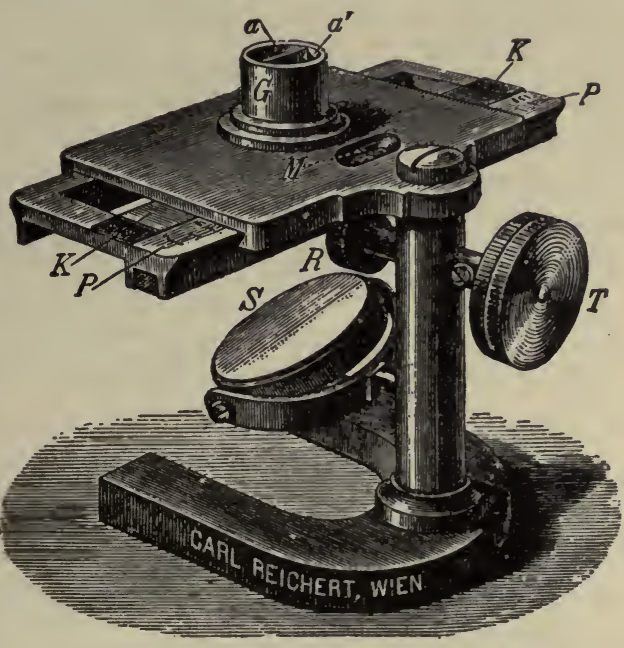

Fig. 122.-Fleischl's Hemoglobinometer. atus is usually supplied with three cells. Of these, the first two are used in estimating the hemoglobin according to Miescher. This is the method now generally used. These cells are furnished with a glass cover having a groove whicn fits upon the partition of the cell. Over this cover is placed a diaphragm with a longitudinal slit, which only permits of the central part of each side of the cell being seen.

The patient's ear or finger is pricked, and the blood from the wound sucked up into the graduated pipet until it reaches the mark $\frac{1}{2}, \frac{2}{3}$, or $\frac{1}{1}$, a I per cent. solution of sodium carbonate is then sucked in until the upper mark is reached. The pipet is then well shaken in order to mix the blood thoroughly. One-half of each of the two cells, which are, respectively, I2 and I5 millimeters high, is then filled with the mixture, the other half being filled with water. An important point is that the liquids should completely fill the cells. The cover-glasses and diaphragms are then applied and the cells are ready for examination. This must be done by artificial light. Moreover, in order to have accurate results, light of the same intensity should be always used. One of the cells is placed on the plate and the wheel $T$ turned until the colors of the two halves exactly correspond. When this point is reached, the result is read off on the scale through the opening $M$. This should be repeated several times with each of the cells, and the average of the readings taken. The result obtained with the 12 -millimeter 
cell should be multiplied by $\frac{5}{4}$ to bring it up to that of the larger. For example, suppose the result of several readings to be:

With the large cell (I $5 \mathrm{~mm}$.).............. 54.00

With the small cell (I $2 \mathrm{~mm}.) \ldots \ldots \ldots \ldots \ldots \ldots 4^{2} .00$

If the readings obtained with the large cell are exactly correct, then the readings with the smaller one should be 43.2 , since $54 \times \frac{4}{5}=43.2$. Or, if the readings with the smaller cells are exact, the readings with the larger should be 52.5 , since $42 \times \frac{5}{4}=52.5$. Hence the mean of 54 and 52.5 , namely 5.25 , should be taken as the correct figure. On looking at the corrected table of hemoglobin values supplied with each instrument, we would find that this number on the scale corresponds to a solution containing 400 milligrams of hemoglobin per rooo cubic centimeters of solution. But our original dilution was either I: $200, I: 300$, or I: 400 , according as our pipet had been filled with blood up to the mark $\frac{1}{1}, \frac{2}{3}$, or $\frac{1}{2}$; so that in order to obtain the actual percentage of hemoglobin in the blood under examination we should be obliged to multiply our results by 200,300 , or 400 . In the example we have taken, the amount of hemoglobin would be, if our dilution was I: $200,400 \times$ $200=80,0.00$ milligrams $=80$ grams in $\mathrm{r}, 000$ cubic centimeters $=8$ grams in roo cubic centimeters, or 8 per cent.

The Dare's hemoglobinometer avoids the error of diluting blood by comparing undiluted blood under artificial light with a colored scale which is graduated after standardization against a hemoglobin content of normal blood, i.e., $\mathrm{I} 3.77$ grams of hemoglobin per roo cubic centimeters. The instrument, see figure I $22 a$, consists of a blood pipette, a case inclosing the color comparison disc, and is provided with a small telescope for reading the color contrasts against an artificial candle light. A drop of blood is drawn with a lancet from a finger tip or the lobe of the ear, is allowed to run directly between the plates of the pipette where it spreads by capillarity. The gradations on the comparison scale are read under candle light and the computations for the percentage of hemoglobin in the sample made against the normal. Its ease of manipulation and comparative accuracy gives to this method the status of a clinical favorite. Another clinical method somewhat less readily manipulated is that of Sahli.

The Talquist method enables one to make a quick approximation of the hemoglobin content. It is valuable as a preliminary test to the Dare and is sufficiently accurate for many clinical determinations.

This consists of a series of shades of color corresponding to undiluted blood of various hemoglobin values, ranging from to to roo per cent. of an arbitrary scale. This scale is included in a book, the remaining pages of which consist of filter-paper, which is used for absorbing the specimen of blood whose hemoglobin percentage is to be estimated. The bloodstained filter-paper is compared with the hemoglobin scale by direct day- 
light until a shade is found with which it corresponds. For approximate results this method has proved very satisfactory.

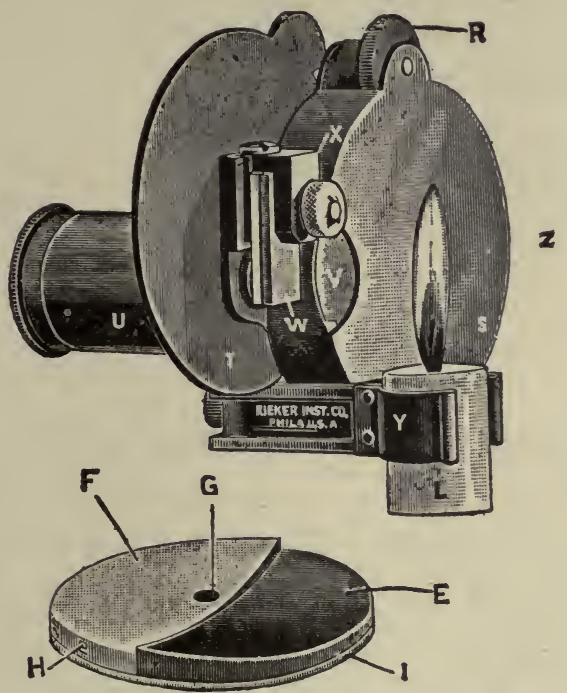

Fig. I 22a.-Dare Hemoglobinometer. Instrument ready for use, illustration onehalf actual size. $R$, Milled wheel by which the color prism is rotated by friction exerted upon its edge. $S$, Case enclosing color prism, showing stage upon which the blood pipet slides. $T$, Movable wing pivoted to case. When drawn outward screens the eyes of observer from the light. When not in use lies superimposed upon the circular prism case, occupying no extra space. $U$, Telescoping camera tube in position for examination. $\quad V$, Opening in prism case, admitting light for illumination of color prism. The white glass disc of prism is seen inside. $W$, White glass of blood pipet. $X$, Pipet clamp held in position on the stage by grooves and guides. $Y$, Detachable candleholder. $Z$, Rectangular opening in edge of case for reading hemoglobin percentage indicated by beveled blade. $L$, Light (candle or electric.) Color prism. $E$, Prism of colored glass. $F$, Semi-circle of white glass, the edge carrying the index of hemoglobin percentage in black; this edge also serves as a friction surface for the rubber-covered roller by which the prism is rotated. $G$, Hole in which hub is fixed. $H$, Index of hemoglobin percentage etched in black. $I$, Disc of white glass which serves to break the glare of direct light and furnishes a white background to view the shades of color.

Derivatives of Hemoglobin.-Hematin.-By the action of heat or of acids or alkalies in the presence of oxygen, hemoglobin can be split up into a substance called hematin, which contains all the iron of the hemoglobin from which it was derived, and a protein residue, a histone, globin. If there be no oxygen present, instead of hematin a body called hemochromogen is produced, which, however, will speedily undergo oxidation into hematin.

Hematin is a dark brownish or black non-crystallizable substance of metallic luster. Its percentage composition is $\mathrm{C}, 64.30 ; \mathrm{H}, 5.50 ; \mathrm{N}, 9.06$; $\mathrm{Fe}, 8.82 ; \mathrm{O}, \mathrm{I} 2.32$; which gives the formula $\mathrm{C}_{68} \mathrm{H}_{70} \mathrm{~N}_{8} \mathrm{Fe}_{2} \mathrm{O}_{10}$ (HoppeSeyler). It is insoluble in water, alcohol, and ether; soluble in the caustic alkalies; soluble with difficulty in hot alcohol to which is added sulphuric acid. The iron may be removed from hematin by heating it with fuming 
hydrochloric acid to $160^{\circ} \mathrm{C}$., and a new body, hematoporphyrin, the so-called iron-free hematin, is produced. Hematoporphyrin $\left(\mathrm{C}_{68} \mathrm{H}_{74} \mathrm{~N}_{8} \mathrm{O}_{12}\right.$, HoppeSeyler) may also be obtained by adding blood to strong sulphuric acid, and if necessary filtering the fluid through asbestos. It forms a fine crimson solution, which has a distinct spectrum, viz., a dark band just beyond $\mathrm{D}$, and a second all but midway between $\mathrm{D}$ and $\mathrm{E}$. It may be precipitated from

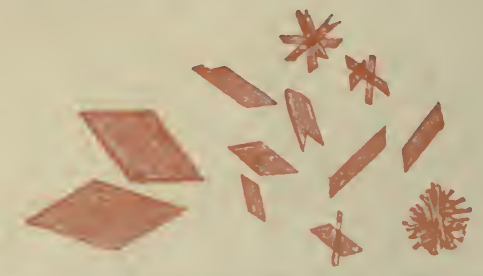

Fig. 123.-Hematoidin Crystals. (Frey.)

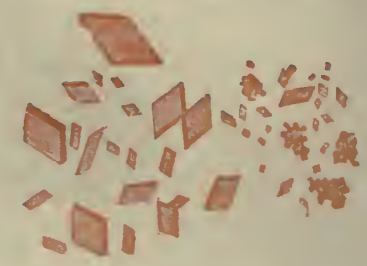

Frg. I23a.-Hemin Crystals. (Frey.)

its acid solution by adding water or by neutralization, and when redissolved in alkalies presents four bands, a pale band between $\mathrm{C}$ and $\mathrm{D}$, a second between $\mathrm{D}$ and $\mathrm{E}$, nearer $\mathrm{D}$, another nearer $\mathrm{E}$, and a fourth occupying the chief part of the space between $b$ and $\mathrm{F}$.

Hematin in Acid Solution. - If an excess of acetic acid is added to blood, and the solution boiled, the color alters to brown from decomposition of hemoglobin and the setting free of hematin; by shaking this solution with ether, a solution of hematin in acid solution is obtained. The spectrum of the ethereal solution shows no less than four absorption bands, viz., one in the red between $\mathrm{C}$ and $\mathrm{D}$, one faint and narrow close to $\mathrm{D}$, and then two broader bands, one between $\mathrm{D}$ and $\mathrm{E}$, and another nearly midway between $b$ and $\mathrm{F}$. The first band is by far the most distinct, and the acid aqueous solution of hematin shows it plainly.

Hematin in Alkaline Solution.-If a caustic alkali is added to blood and the solution is boiled, alkaline hematin is produced, and the solution becomes olive-green in color. The absorption band of the new compound is in the red, near to $\mathrm{D}$, and the blue end of the spectrum is absorbed to a considerable extent. If a reducing agent be added, two bands resembling those of oxyhemoglobin, but nearer to the blue, appear; this is the spectrum of reduced hematin, or hemochromogen. On violently shaking the reduced hematin with air or oxygen the two bands are replaced by the single band of alkaline hematin.

Hematoidin.-This substance is found in the form of yellowish crystals, figure I $_{2}$, in old blood extravasations and is derived from the hemoglobin. Their crystalline form and the reaction they give with fuming nitric acid seem to show them to be closely allied to bilirubin, the chief coloring matter of the bile, and in composition they are probably either identical or isomeric with it.

Hemin.-One of the most important derivatives of hematin is hemin. It is usually called hydrochloride of hematin, but its exact chemical com- 
position is uncertain. Its formula is said to be $\mathrm{C}_{32} \mathrm{H}_{30} \mathrm{~N}_{4} \mathrm{FeO}_{3} \mathrm{HCl}$, and it contains 5.18 per cent. of chlorine, but by some it is looked upon as simply crystallized hematin. Although difficult to obtain in bulk, a specimen may be easily made for the microscope in the following way: A small drop of dried blood is finely powdered with a few crystals of common salt on a glass

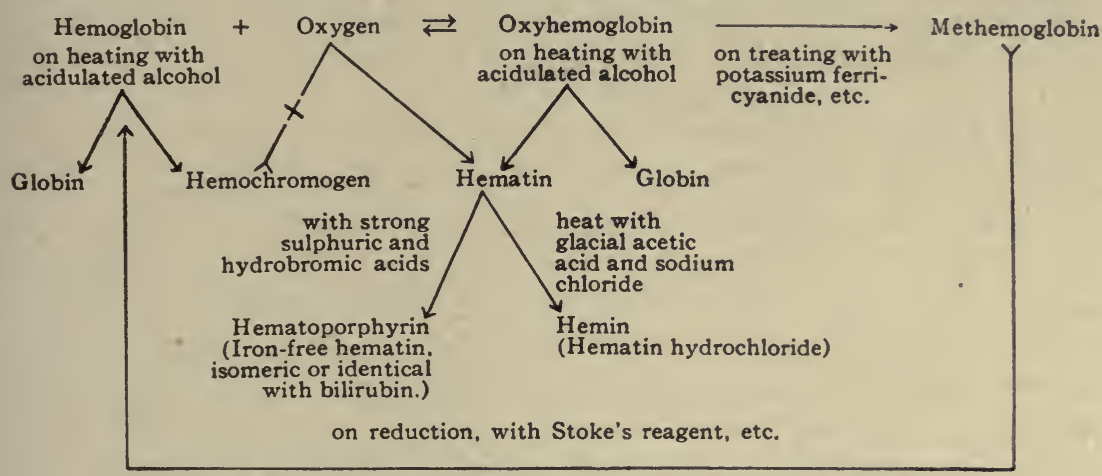

Scheme to Show the Relations of Hemoglobin and its Derivatives.

slide and spread out; a cover-glass is then placed upon it, and glacial acetic acid added by means of a capillary pipet. The blood at once turns a brownish color. The slide is then heated, and the acid mixture evaporated to dryness at a high temperature. The excess of salt is washed away with water from the dried residue, and the specimen may then be dried and mounted. A large number of small, dark, reddish-black crystals of a rhombic shape, sometimes arranged in bundles, will be seen if the slide be subjected to microscopic examination, figure $123 \mathrm{a}$.

The formation of these hemin crystals is of great interest and importance from a medico-legal point of view, as it constitutes the most certain and delicate test we have for the presence of blood (not of necessity the blood of man) in a stain on clothes, etc. It exceeds in delicacy even the spectroscopic test. Compounds similar in composition to hemin, but containing hydrobromic or hydriodic acid, instead of hydrochloric, may be also readily obtained.

Variations in the Composition of Healthy Blood.-The conditions which appear most to influence the composition of the blood in health are these: Diet, Exercise, Sex, Pregnancy, and Age.

Sex.-The blood of men differs from that of women, chiefly in being of somewhat higher specific gravity, from its containing a relatively larger quantity of red corpuscles.

Pregnancy.-The blood of pregnant women has rather lower than the average specific gravity. The quantity of the colorless corpuscles is increased in the later months, especially in primiparæ; it is also claimed that the fibrin is increased in amount. 
Age. - The blood of the fetus is very rich in solid matter, and especially in colored corpuscles; and this condition, gradually diminishing, continues for some weeks after birth. The quantity of solid matter then falls during childhood below the average, rises during adult life, and in old age falls again.

Diet.-Such differences in the composition of the blood as are due to the temporary presence of various matters absorbed with the food and drink, as well as the more lasting changes which must result from generous or poor diet, respectively, need be here only referred to.

Effects of Bleeding.-The result of bleeding is to diminish the specific gravity of the blood, and so quickly that in a single venesection the portion of blood last drawn has often a less specific gravity than that of the blood that flowed first. This is, of course, due to absorption of fluid from the tissues of the body. The physiological import of this fact, namely, the instant absorption of liquid from the tissues, is the same as that of the intense thirst which is so common after either loss of blood or the abstraction from it of watery fluid, as in cholera, diabetes, and the like.

For some little time after bleeding, the want of colored corpuscles is well marked, but with this exception no considerable alteration seems to be produced in the composition of the blood for more than a very short time; the loss of the other constituents, including the colorless corpuscles, being very quickly repaired.

Variations in Different Parts of the Body. - The composition of the blood, as might be expected, is found to vary in different parts of the body. Thus arterial blood differs from venous; and although its composition and general characters are uniform throughout the whole course of the systemic arteries, they are not so throughout the venous system, the blood contained in some veins differing markedly from that in others.

Differences between Arterial and Venous Blood.-The differences between arterial and venous blood are these:

Arterial blood is bright red, from the fact that almost all its hemoglobin is combined with oxygen, oxyhemoglobin, while the dark red tint of venous blood is due to the deoxidation of a certain quantity of its oxyhemoglobin, and its consequent reduction to the hemoglobin.

Arterial blood coagulates somewhat more quickly.

Arterial blood contains more oxygen than venous and less carbon dioxide gas.

Some of the veins contain blood which differs from the ordinary standard considerably. These are the portal, the hepatic, and the splenic veins.

Pertal Blood.-The blood which the portal vein conveys to the liver is supplied from two chief sources; namely, from the gastric and mesenteric veins, which contain the soluble elements of food absorbed from the stomach and intestines during digestion, and from the splenic vein. It must, therefore, combine the qualities of the blood from each of these sources. 
The blood in the gastric and mesenteric veins will vary much according to the stage of digestion and the nature of the food taken, and can therefore be seldom exactly the same. Speaking generally and without considering the sugar and other soluble matters which may have been absorbed from the alimentary canal, this blood appears to be deficient in solid matters, especially in colored corpuscles, owing to dilution by the quantity of water absorbed, to contain an excess of protein matter, and to yield a less tenacious kind of fibrin than that of blood generally.

The blood of the portal vein, combining the peculiarities of its two factors the splenic and mesenteric venous blood, is usually of lower specific gravity than blood generally, is more watery, contains fewer colored corpuscles, more proteins, and yields a less firm clot than that yielded by other blood, owing to the deficient tenacity of its fibrin.

Guarding (by ligature of the portal vein) against the possibility of an error in the analysis from regurgitation of hepatic blood into the portal vein, recent observers have determined that hepatic venous blood contains less water, proteins, and salts than the blood of the portal veins; but that it yields a much larger amount of extractive matter, in which is one constant element, namely, glucose, which is found whether carbohydrates have been present in the food or not.

\section{GLOBULOCIDAL AND OTHER PROPERTIES OF SERUM.}

Cytolysis.- It has been known for some time that the sera of certain animals when injected into the circulation of animals of another species will cause destructive changes in the blood corpuscles, accompanied by symptoms of poisoning, which may even end fatally. These results served to bring into disrepute the use of foreign blood in transfusion, which has consequently been practically abandoned. The discharge of the hemoglobin of the red blood corpuscles and their solution in the plasma (laking) is now included in the general term Cytolysis, and more specifically known as Hemolysis. Agents which produce such an effect are known as hemolytic or hemotoxic agents.

Transfusion of the blood of one animal into the vessels of another is often quickly fatal because of the hemolytic reactions of the two bloods. Transfusions between different species or distantly related animals are as a rule not possible. But the blood of different individuals of the same species are usually not lytic. This subject possesses great importance to man because of the growing practice of blood transfusions in man. However not all human bloods can be blended without great danger though as a rule members of the same family are miscible. Human bloods must first be tested or typed, as it is called, to determine whether or not lysis 
would occur on transfusion. Four human types have been described by serologists. No transfusion is now ever performed without this preliminary test which is absolutely necessary lest immediate solution of the red corpuscles be produced and the death of the recipient follow.

The serum of one animal may be made to acquire lytic properties for the blood of another. This adaptation is brought about in the following way: For instance, the blood of the guinea-pig, which is not normally lytic for the red cells of the rabbit, may be adapted to the latter by previously, at several successive intervals (three to seven days) injecting into the abdominal cavity or subcutaneous tissues of the guinea-pig small quantities of rabbit's blood. If now a small quantity of serum be obtained from the guinea-pig by the usual methods and mixed in a test-tube with some of the rabbit's blood diluted with physiological salt solution, hemolysis occurs; that is, the coloring matter of the rabbit's red blood-cells goes into solution and the cells appear under the microscope as shadow corpuscles or ghosts, devoid of hemoglobin. Such an artificially produced hemolytic serum is only lytic for the blood of the animal species for which it has been adapted. It is true that it may also show slightly lytic properties for closely allied species. It has therefore been suggested as a possible valuable aid in determining relationships of various animal species.

Concerning the nature of the lytic substance, it has been found that it probably consists of two bodies acting conjointly, for if the serum be heated to $56^{\circ} \mathrm{C}$. for a short time, its lytic powers are lost, but may be restored by adding a little serum of another animal of the same species which has not been adapted, and whose serum is consequently not in itself lytic. Of these two bodies, therefore, one is stable and is formed only in the adapted serum, while the other is more unstable or labile, destroyed at $56^{\circ} \mathrm{C}$., and exists normally in the blood plasma. The former is known as the immune body, the amboceptor, and the latter as the alexin, or complement. Lysis occurs only when both are present at the same time, and not through the agency of one or the other singly.

This cytolytic adaptation has been extended to include other cells besides the red blood corpuscles. Thus in a similar manner leucolytic, hepatolytic, nephrolytic, and a number of other lytic sera have been developed.

It is further possible, under certain circumstances, that substances may be developed in the tissues which are lytic for other tissue cells of the same animal, autolytic substances. This may be a physiologically important process in the elimination of worn-out tissue cells, cellular elements in injury, inflammation, etc.

Agglutinative Substances.-A further property of adapted sera is that of agglutination. The adaptation is secured in the same way as in 
the production of cytolysins. In fact, both cytolysis and agglutination may occur at the same time. The normal blood serum of some animals may be agglutinative for the blood-cells of some other species. In normal serum, agglutinative and cytolytic properties may be present together or one only may be normally present.

The activity of agglutinative substances is not destroyed at a temperature of $56^{\circ} \mathrm{C}$. They do become inert, however, at $70^{\circ} \mathrm{C}$., and, furthermore, they cannot be restored by adding normal serum, as is the case with cytolysins.

Hemagglutinative substances are found in certain plant seeds; e.g., in castor oil beans (Ricinus communis), in cotton seed, and in the legumes.

Precipitins.-Other forms of adaptive substances which may be found in animal serum are those which, when mixed with the substances by means of which adaptation has been secured, form a precipitate. By this means blood of different species of animals may be detected even when in a dried state. It has been suggested as a possible valuable aid in medico-legal cases, since human blood in a dilution of $\mathrm{I}$ to 50,000 has been recognized by this means.

Opsonins.-Wright and Douglass have shown that there are certain substances in the serum that affect bacteria in such a way that they are more easily taken up and destroyed by leucocytes. The phagocytic power of the leucocytes in destroying toxic bacteria is not made to increase by stimulative substances, as Metchnikoff believed, but rather by those materials in the serum diminishing the resisting power of the bacteria. These substances are called by their discoverers opsonins. They found opsonin present in normal serum, but also found that its quantity varies under certain conditions. They suggested that the opsonins could be measured by determining the phagocytic power. The ratio of the average number of bacteria taken up by leucocytes in normal serum to the number taken up in the immune serum, they called the opsonic index.

Antitoxins.-Certain kinds of bacteria, notably the diphtheria and tetanus organisms, elaborate poisonous substances known as toxins. The pathological conditions resulting from such infections are produced by the poisons so formed. Behring first showed that immunity to diphtheria was due to the presence in the blood plasma and blood serum of substances which apparently combine with and so prevent the toxic action of the bacterial products. This antitoxic power of the blood can be artificially developed by injecting small doses of the toxins into an animal, usually a horse, at intervals of some days. The protective power of the blood against the toxins can thus be developed to a relatively enormous degree. The serum of an artificially immunized animal can be injected into other individuals of the same or other species and an immunity will be conferred on the person or animal so treated. Similarly, the antitoxic sera have a curative effect in infected individuals if the disease is not too far advanced. Antitoxic sera are specific 
for the particular toxin used for the immunization. Antitoxins can be similarly prepared for the naturally occurring vegetable toxins, ricin and abrin, for snake venoms, etc.

Anaphylaxis.-It has been found that if an animal, especially the guinea pig, be injected with even the minutest quantity of protein material, that after ten to fourteen days, the animal becomes susceptible to a second injection of the same material if made intravascularly. Thus a guinea pig may be sensitized with $0.00000 \mathrm{r}$ of a c.c. of blood serum; after two weeks have elapsed, the introduction of a half c.c. of the same serum into the circulation will in a few minutes lead to respiratory failure and death. This phenomenon is relatively specific for foreign protein substances. Guinea-pigs which have recovered from the second injection acquire a temporary immunity against a third injection of the same protein. It has been found that this peculiar susceptibility is transferred from the mother guinea-pig to successive litters.

Nature of the Antisubstances in Blood.-The lecithins and fatty acids, especially oleic acid, will in a measure replace a hemolytic complement. The antitoxins and agglutinins in the blood seem either to be associated with, or actually are a portion of, the para- or pseudoglobulin. During immunization, the pseudoglobulin of the blood may be twice the normal content; coincidently the per cent. of the serumalbumin will diminish. The protein changes in the blood of horses and the antitoxic variations, however, are not parallel, and no quantitative relationship has been established.

\section{PHYSICAL FACTORS OF BLOOD PLASMA OR SERUM.}

Diffusion, Osmosis, Dialysis.-The term diffusion has long been applied to the regular mixing of the molecules of two gases when brought into contact in a confined space, this interpenetration being due to the to-and-fro movements of their molecules. More recently it has been applied to the mixing of the molecules of two solutions when brought into contact, as it has been found that they act in the same way and obey the same laws as gases. If, however, the two solutions are separated by a membrane, permeable to the solutions, diffusion will still occur. To this form of diffusion the term osmosis has been applied in the case of water, and dialysis in the case of diffusible substances. All bodies can be divided into two groups, crystalloids and colloids. To the former group belong bodies having a crystalline form, which readily go into solution in water. All such bodies are diffusible (dialyzable), their power of dialysis, however, varying considerably. To the second group belong such bodies as have no crystalline form (amorphous). These are generally bodies with a large molecule, which form colloidal suspensions in water and are only slightly or not at all diffusible. An exception to the first group is hemoglobin, which has a very large molecule, and is crystalline but is 
not diffusible. The following may serve as simple illustrations:

Take a jar and divide it in two equal parts by an animal membrane, $M$, figure 124, and place an equal amount of distilled water in the two sides, $A$ and $B$. Now, since the molecules of water act like those of a gas and are continually moving to and fro, bombarding all the surfaces of their retainer, the molecules of water in $A$ and $B$ will be continually striking all the surfaces of $A$ and $B$; but since the membrane is permeable to the water molecules, there will be a continual interchange of molecules between $A$ and $B$. If now, in one side $A$ we place a solution of sodium chloride, still keeping water in $B$, the membrane being permeable to the sodium chloride, the first thing we should notice would be an increase in the amount of water in $A$. For-

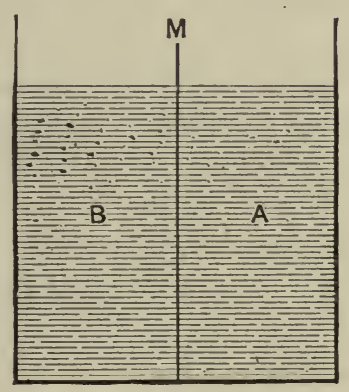

FIG. 124

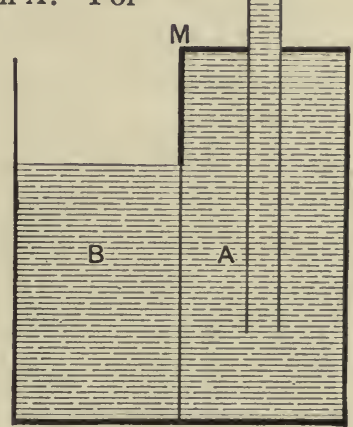

Fig. 125.

merly it would have been said that "the salt had attracted the water." Now we should say that the salt had a certain osmotic pressure. The salt, however, being able to pass (dialyse) through the membrane, will do so, and this will continue until the strength of the two salt solutions, and therefore the osmotic pressure on both sides, is equal.

Osmotic Pressure.-If now in $A$ we place a solution of some soluble colloidal substance to which the membrane is impermeable, or else replace the membrane, $M$, we used in our former experiment by one which is not permeable to the sodium chloride, and arrange our jar as in figure 125 , so as to be able to read off any increase of water which may pass into $A$, we will notice that the amount of liquid in $A$ will continue to increase up to a certain point. Once that point is reached, there will be no further change, since the substance in solution, in $A$, cannot pass through the membrane as in the previous example. This pressure can be measured and expressed in millimeters of mercury. It is constant for all solutions of this substance that are of the same concentration when measured under like conditions of temperature and pressure, and is called the osmotic pressure of this solution.

Of the numerous explanations regarding the nature of osmotic pressure which has been more or less satisfactory, a simple one, and one that can 
be easily understood, is as follows: In figure 125 one surface of the membrane is being bombarded by the molecules of a non-diffusible substance mixed with those of a diffusible one (water); while the other surface is being bombarded entirely by water molecules. The former condition permits only a fraction of the molecules to diffuse out, since fewer water molecules get to the surface of the membrane; while the latter permits all of the molecules which reach it to pass through.

Osmotic pressure can be estimated in several different ways in addition to the above, viz., the determination of the freezing-point of the solution, determination of the boiling-point, determination of the electrical conductivity. The results obtained with the various methods agree very closely. The following solutions have the same osmotic pressure: Sodium chloride, I.64 per cent.; potassium nitrate, I.09 per cent.; sugar 5.5 per cent.

Isotonic Solutions.-Solutions that have the same osmotic pressure are called isotonic. The term isotonic is a relative one, implying a comparison with some other solution taken as a standard. In physiology it has been customary to take blood plasma as a standard. A solution of 0.64 per cent. sodium chloride is isotonic for the blood plasma of the frog, and a 0.9 per cent. solution for that of man. Further, any solution which is of a lower osmotic pressure than the standard solution is said to be hypoisotonic (hypotonic) in relation to that solution. A solution of a higher osmotic pressure is said to be hyperisotonic (hypertonic).

Water passes in the Direction of the Arrows.

Hypertonic saline solution............ (2 per cent.)

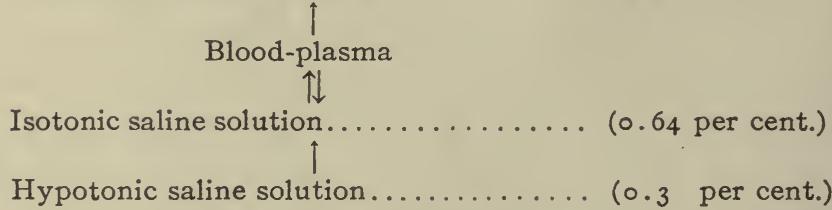

If a hypotonic solution be mixed with blood, water from the hypotonic solution passes through the cell membrane of the red corpuscles into the stroma and causes it to swell. The hemoglobin at the same time passes out and goes into solution in the diluted plasma. On the other hand, the addition of a hypertonic solution to the plasma causes the red corpuscles to lose their water and become crenated. The principles of osmosis have been derived from the action of substances separated by dead animal or plant membranes. It must be, however, remembered that in the application of these principles to processes occurring in the living organism, the cells, forming the various membanes, are an important modifying factor. It is probable that physico-chemical processes, occurring in the protoplasm of the cell, may change its permeability to the same substance at different times.

\section{THE CHARACTER AND COMPOSITION OF LYMPH.}

The lymph is the fluid which immediately surrounds the tissue cells of 
the living body. It fills up the spaces between the cells themselves and between the cells and the blood vessels which ramify among the cell masses. The lymph, therefore, is an intermediate fluid between blood plasma on the one hand and the tissue cells on the other, receiving its ingredients by the passage of fluid from the plasma through the walls of the finer blood vessels in the one direction and by the discharge of the substances from the cells themselves in the other.

The Chemical Composition of the Lymph.-Since the chief source of the lymph is the blood plasma, one would naturally expect that its chemical composition would be very similar to that of plasma, which is in fact the case. The variations that are noted in lymph taken from definite sources no doubt have their origin in the fact that the lymph passes through these organs slowly, and that ingredients peculiar to the necessities of the function and growth of the differentiated tissue of the organ are taken from the lymph in special organs. Lymph obtained from a human lymphatic fistula has been analyzed; the figures from Hammarsten are as follows, though considerable variations appear in the analyses from other authorities:

ANALYSIS OF LYMPH.

\begin{tabular}{|c|c|c|}
\hline \multirow{2}{*}{ 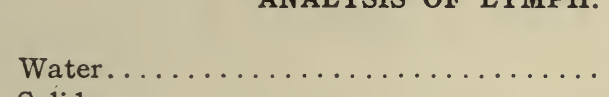 } & \multicolumn{2}{|c|}{ Per cent. } \\
\hline & $94 \cdot 5$ & to 96.5 \\
\hline Solids.................. & $3 \cdot 5$ & to $5 \cdot 5$ \\
\hline Pro & $3 \cdot 4$ & to $4 \cdot I$ \\
\hline Ethereal extract. & 0.06 & to 0.13 \\
\hline$\ldots \ldots \ldots \ldots \ldots$ & 0.1 & \\
\hline Salts.......... & 0.8 & to 0.9 \\
\hline Sodium chloride........... & 0.55 & to $0.5^{8}$ \\
\hline Sodium carbonate........... & 0.24 & \\
\hline Disodic phosphate. . & 0.02 & \\
\hline
\end{tabular}

The most notable fact to be derived from this composition table is the low percentage of proteins present in the lymph.

The Formation of Lymph.-The manner in which the substances in the lymph pass through the walls of the capillaries from the plasma is a question which has been surrounded with considerable difficulty. It was thought by Ludwig and many of his followers that the process involved is merely one of filtration. Certainly the blood-pressure in the capillaries is in the main greater than that of the pressure of the lymph in the surrounding tissues, and this positive pressure will contribute so much to the direct ingredients of the blood plasma through the capillary walls. It is true, as a matter of experiment, that anything which contributes to an increase in the capillary pressure is very apt to produce an edema of the corresponding tissues. Since the colloidal materials represented by the protein are nondiffusible, one would by this theory expect to find a diminished percentage in the lymph, which is true, though not to the extent which the theory demands. 
Heidenhain was the first to question the adequacy of the blood-pressure and filtration hypothesis. He showed that many of the conditions under which lymph formation takes place are not sufficient to produce filtrations of the material found. He advanced the hypothesis that the living endothelial lining of the blood vessels exerted a secretory activity in lymph production. He discovered that various substances known as lymphagogues when introduced into the circulatory system produce a remarkable increase in the flow of lymph from the thoracic duct. Further, he noticed that the concentration of the lymph was changed; i.e., increased. Heidenhain thought that the lymphagogues acted directly on the capillary and endothelial lining stimulating these cells to produce a greater quantity of lymph. He divided such substances into two classes. The best known representatives of the "first class" are such as proteoses and peptones, leech extract, extract of crustacean tissue, etc. The lymphagogues of the second class are the neutral inorganic salts, sugars, and other crystalline substances. These all cause a marked flow of lymph. The lymphagogues as a class cause fall of blood-pressure; for example, proteose-peptone injections. This fact argues against their purely physical action. Many drugs act to increase the flow of lymph in a way which cannot be presumed to be other than normal; i.e., they stimulate the physiological processes going on in the endothelial cells. Such observations contribute strongly to the view advanced by Heidenhain. Many investigations have been brought to the support of the hypothesis that lymph formation is largely a process of secretion, yet it seems at the present time that we cannot wholly deny that filtration and osmosis play a part in the processes.

In following the action of peptones and proteoses, Pick and Spiro came to the conclusion that it was not peptone, but some contaminating substance which produced the characteristic action. This hypothetical substance they called peptozyme. Underhill re-examined the influence of peptones, using preparations made from plant proteins by hydration with enzymes, heat, and acid, carrying the hydrolysis to a greater extent than did Pick and Spiro. Underhill still obtained the great increase in the flow of lymph together with the usual fall of blood-pressure. Mendel published the result of a demonstration of post-mortem lymph flow in which he showed that "the lymph continued to flow for four hours without any extraordinary mechanical assistance" after the death of the animal. This observation would seem to give complete refutation of the filtration hypothesis. A similar post-mortem salivary secretion has been observed, and in each case the processes involved must be assumed to be physiological rather than purely physical phenomena. Certainly the permeability or activity of the endothelial lining of the blood vessels varies greatly at different times in the life of an individual, and this variation in function is associated with the marked change in the character and quantity of lymph produced. 


\section{LABORATORY EXPERIMENTS FOR THE EXAMINATION OF THE BLOOD.}

I. Microscopical Examination of the Blood.-Mount a drop of frog's blood in 0.7 per cent. sodium chloride and examine with the high power of a compound microscope. The red corpuscles will appear as oval nucleated discs with a faint yellowish color, figure I ro. Here and there white granular cells of irregular outline will be noted, the white corpuscles. Examine the drop of blood with a high magnifying power (oil-immersion lens) and sketch the outline of the blood-cells. Select the white corpuscle which is most irregular in outline and make a series of outline drawings once every minute to show its ameboid movements, figure II 7 .

Draw a drop of your own blood by puncturing the tip of the finger, under sterile conditions, and mount in a drop of 0.9 per cent. physiological saline. Examine with a high power, note the small biconcave red corpuscles which appear faintly yellow in color and even adhere in rouleaux, figure rog. The white corpuscles will appear as somewhat larger granular discs differing in form and size. By mounting a drop of blood on a warm stage the ameboid movements of the white corpuscles can be observed with comparative ease.

2. Action of Fluids on the Red Corpuscles.-Water.-When water is added gradually to frog's blood, the oval disc-shaped corpuscles become spherical and gradually discharge their hemoglobin, a pale, transparent stroma being left behind. Human red blood-cells change from a discoidal

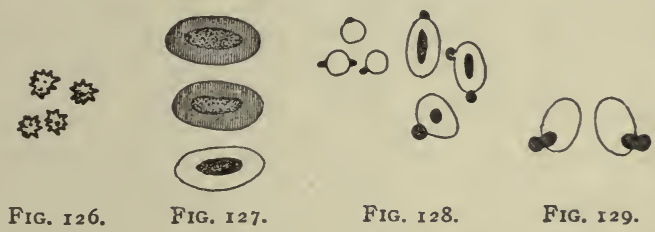

Fig. I 26.-Effect of Hypertonic Salt Solution on the Red Blood Corpuscles of Man. Fig. 127.-Effect of Acetic Acid. FIG. I 28.-Effect of Tannin. FIG. I29.-Effect of Boric Acid.

to a spheroidal form and discharge their cell contents, becoming quite transparent and all but invisible (ghost corpuscles).

Hypertonic Salt Solutions. - Mount a drop of human blood in 2 per cent. sodium-chloride solution. The red blood-cells lose their disc shape and become spherical with spinous projections or crenations, figure $\mathrm{x} 26$.

The original form of the red blood-cells can be restored by transferring them to isotonic salt solution.

Dilute Acetic Acid.-This reagent causes the nucleus of the red bloodcells in the frog to become more clearly defined; if the action is prolonged, the nucleus becomes strongly granulated, and all the coloring matter seems to be concentrated in it, the surrounding cell substance and outline of the cell becoming almost invisible; after a time the cells lose their color altogether. 
The cells in figure 127 represent the successive stages of the change. $\Lambda$ similar loss of color occurs in the red cells of human blood, which, from the absence of nuclei, seem to disappear entirely.

Alkalies. - Alkalies cause the red blood corpuscles to absorb water and finally to disintegrate.

Chloroform and Ether.-These reagents when added to the red bloodcells of the frog cause them to part with their hemoglobin; the stroma of the cells becomes gradually broken up. A similar effect is produced on the human red blood-cell.

Tannin and Boric Acid.-When a 2 per cent. fresh solution of tannic acid is applied to frog's blood it causes the appearance of a sharply defined little knob, projecting from the free surface (Roberts' macula). The coloring matter becomes at the same time concentrated in the nucleus, which grows more distinct, figure I28. A somewhat similar effect is produced on the human red blood corpuscle.

A 2 per cent. solution of boric acid applied to nucleated red blood-cells of the frog will cause the concentration of all the coloring matter in the nucleus; the colored body thus formed gradually quits its central position, and comes to be partly, sometimes entirely, protruded from the surface of the now colorless cell, figure I29. The result of this experiment led Brücke to distinguish the colored contents of the cell (zoöid) from its colorless stroma (ecoid). When applied to the non-nucleated mammalian corpuscle its effect merely resembles that of other dilute acids.

3. Phagocytosis in White Corpuscles.-Mix some very fine pigment granules, bacterial emulsion, or charcoal with a few drops of frog's blood, let stand for ro or 20 minutes, then mount a drop on the glass slide or make a smear and stain and examine under a high-magnifying microscope. Or inject a few drops of one of these pigments suspended in physiological saline and after a few minutes examine drops of the blood as above. In a favorable field here and there will be found some white corpuscles which have taken up the pigment. Colored corpuscles have been observed with fragments of pigment embedded in their substance. White corpuscles have also been seen in diseased states of the body to contain micro-organisms, for example bacilli, and have the power of destroying these organisms, which gives them the name phagocytes.

4. Enumeration of the Blood Corpuscles.-Several methods are employed for counting the blood corpuscles, most of them depending upon the same principle; i.e., the dilution of a minute volume of blood with a given volume of a colorless solution similar in specific gravity to blood plasma, so that the size and shape of the corpuscles are altered as little as possible. A minute quantity of the well-mixed solution is then taken, examined under the microscope, either in a flattened capillary tube (Malassez) or in a cell (Hayem and Nache, Gowers) of known capacity, and the number of 
corpuscles in a measured length of the tube or in a given area of the cell is counted. The length of the tube and the area of the cell are ascertained by means of a micrometer scale in the microscope ocular; or, in the case of

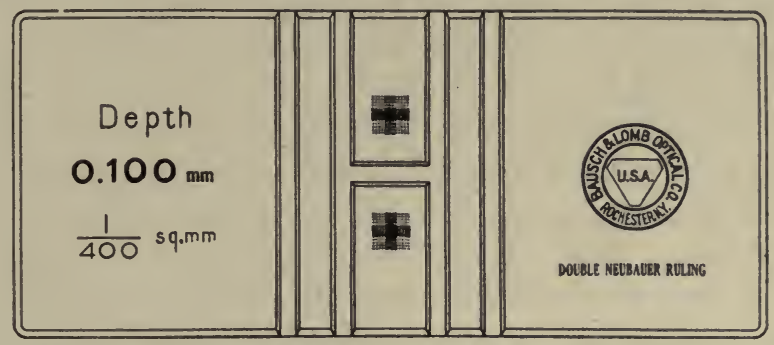

Fig. I30.- Section of the new Bausch and Lomb form of the Thoma-Zeiss Hemacytometer. This form has the advantage of having the two standard graduated scales reproduced in the next figure ground in the glass slide itself.

Gowers's modification, by the division of the cell area into squares of known size. Having ascertained the number of corpuscles in the diluted blood, it is easy to find out the number in a given volume of normal blood.

The hemacytometer, which is most used at the present time, is known as the Thoma-Zeiss hemacytometer. It consists of a carefully graduated pipet, in which the dilution of the blood is done; this is so formed that the capillary stem has a capacity equaling one-hundredth of the bulb above it. If the blood is drawn up in the capillary tube to the line marked I, figure I3I, the saline solution may afterward be drawn up the stem to the line ror; in this way we have Ior parts of which the blood forms I. As the content of the stem can be displaced unmixed we shall have in the mixture the proper dilution. The blood and the saline solution are well mixed by shak-

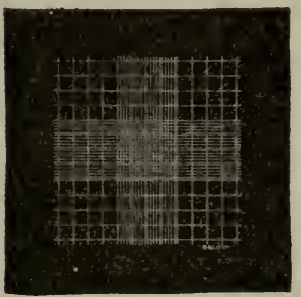

Neubauer Ruling FIG. I30a.-Standard hemacytometer rulings, I/400 square millimeter.

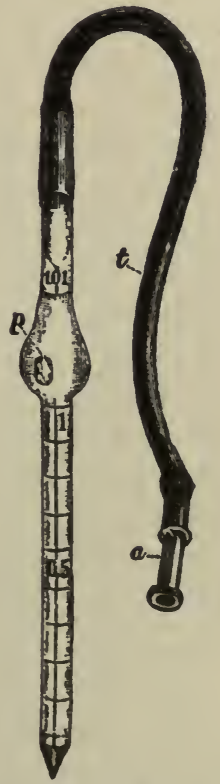

FIG. I3I.-ThomaZeiss Hemacytometer, pipet.

ing the pipet, in the bulb of which is contained a small glass bead for the purpose of aiding the mixing. The counting instrument consists of a glass slide, figure $\mathrm{I} 30$, provided with a depressed area the surface of which is 
accurately ruled so as to present one square millimeter divided into 400 squares of one-twentieth of a millimeter each. In the older instruments the rulings are on the cover disc. The micrometer surface is ground below the general surface exactly one-tenth millimeter. If a drop of the diluted blood be placed upon the ruled surface, and covered with a perfectly flat cover-glass, the volume of the diluted blood above each of the squares of the micrometer, i.e., above each $1 / 400$ square millimeter area will be $1 / 4000$ of a cubic millimeter. An average of ten or more squares is then counted, and this number multiplied by $4000 \times$ roo gives the number of corpuscles in a cubic millimeter of undiluted blood. A separate pipet is used for making dilutions for counts of leucocytes. In this, the dilution is made of one part of blood and ten parts of diluting fluid. Acetic acid, 0.2 of one per cent., is usually employed for this purpose.

\section{The Percentage of Corpuscles and Plasma in Human Blood.-} Fill the two graduated capillary tubes of a hematocrite with blood drawn from the tip of your own finger, insert into the instrument, and centrifuge as rapidly as possible. The experiment must be performed within the time limit of clotting in order to be successful. The corpuscles will be thrown down and the percentage of plasma and corpuscles can be read off directly. Should one fail to fill the tube exactly full, then the percentage of plasma and corpuscles can be calculated from the proportion which each bears to the quantity in the tube.

6. Estimation of the Percentage of Hemoglobin.-The percentage of hemoglobin in a sample of blood can best be obtained by either the Dare or the Sahli hemoglobinometer. The principle of the Dare is given in the text. It rests on a comparison of the color of a drop of undiluted blood with a standard color scale when illuminated by candle light. Sterilize a lobe of the ear or finger tip, draw a drop of blood, bring the edge of the Ware blood pipette carefully against the blood drop, allowing it to flow between the plates. Place this in the instrument and examine either in a dark room or with the instrument turned toward a dark wall or paper.

A sample of blood may be taken by a Miescher pipette diluted in the Miescher hemoglobinometer, and its percentage of hemoglobin computed. See text, page I40, for diagram of instrument and description of the method.

Perhaps a more convenient and certainly a quicker method for determining the percentage of hemoglobin is Talquist's hemoglobinometer. By this method a drop of blood is drawn directly on to absorbent-paper furnished with the instrument, and the resulting stain is compared directly with a paper color scale which is graduated in percentage. In this method the comparison is made in ordinary daylight, and because of its rapidity it is very convenient for clinical examinations, though it is less accurate. 
7. Reaction of Blood Plasma.-Wet a piece of red litmus-paper in saturated magnesium sulphate solution, then touch one end of the strip with a drop of blood drawn from your finger under sterile conditions. After a few moments wash off the excess of corpuscles in neutral distilled water. The blue at the point of contact with the blood indicates alkalinity.

8. The Specific Gravity of Blood.-From standard mixtures of chloroform and benzol with specific gravity of I.050, I.060, and I.070 make up a set of specific-gravity solutions of I.050, I.052, I.054, etc., to I.070. These standards may be kept in stoppered 4 -dram vials, or in test-tubes. The specific gravity of blood is determined by inserting with a pipet a drop of freshly drawn blood into the middle of one of the solutions, say r.056. Since the blood does not mix with the chloroform and benzol, the drop will rise or sink according to its relative specific gravity. By a few trials one may quickly find a specific gravity in which the drop of blood floats without rising or sinking. This represents the specific gravity of the drop of blood.

This method permits rapid clinical application and has proven of considerable interest in the hands of clinicians.

9. The Isotonicity of Blood.-The absorption or loss of water by the corpuscles of blood in solutions of other concentrations than that of blood plasma can be used as a means of determining the isotonicity of blood. Make up a series of solutions of sodium chloride, varying by tenths, from 0.5 to 1.2 per cent. Prepare a series of slides with vaselin rings and mount drops of human blood in drops of saline of $0.4,0.6,0.8, \mathrm{I}$, I.2 and I.4 per cent., examine immediately then every ten minutes under a high-power microscope. The corpuscles of some of the slides will swell up and may disintegrate, others will show crenation as in figure $\mathrm{I} 26$. In the isotonic solutions the corpuscles will appear of their normal size and condition.

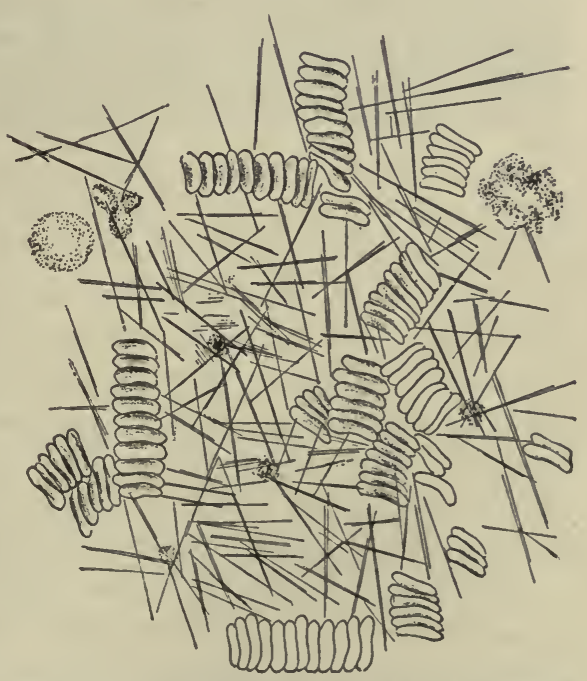

FIG. 132.-Microscopic View of Clot Showing Fibrin Network.

ro. Coagulation of Blood.- $a$. Normal Clot.-Anesthetize a dog, insert a cannula into the carotid or femoral artery, and draw samples of blood into two or three clean, dry test tubes. Draw one sample into a testtube that has had its sides oiled. Note the exact time at which the blood 
was drawn into the test-tubes and set the test-tubes in a test-tube rack. Examine at intervals of 30 seconds by gently inclining the test tubes. Presently it will be noted that the blood becomes more viscous and does not flow freely up the sides of the test-tubes. Later the whole mass will become jelly-like and will retain the form of the test-tube. Note the time of the first slight change, and also when the clot becomes more perfect. The sample in the oiled test-tube will be found to clot more slowly.

If the test-tubes of clotted blood are left standing for a day, the coagulum will become smaller in size and a transparent yellowish blood will make its appearance on the surface or between the sides of the clot and the testtube wall. This fluid is the serum and it is squeezed out by the shrinking of the fibrin which holds the corpuscles in its meshes.

b. The Time of Blood Clotting.-The speed of clotting is measured more accurately by Cannon's coagulometer, see figure ro8. A sample of blood is carefully drawn from an artery under conditions which insure fresh circulating blood (Cannon and Mendenhall, American Journal of Physiology, Vol. 34, p. 225, for fuller details). This sample is inserted in the coagulometer and successive tests for coagulation made every 30 seconds. Even a thread or two of fibrin is indicated by the apparatus if the lever is accurately counterpoised.

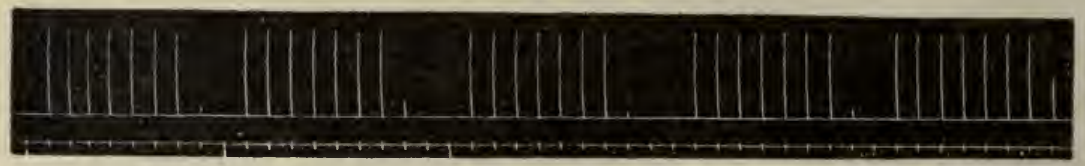

FIG. I $_{32}$ a.-Successive tests of the coagulation of blood drawn from the femoral artery of an animal in uniform condition. The mark below the time record signifies when the sample was drawn. Time in 30 second intervals. (From Cannon and Mendenhall.)

c. Microscopic Examination of the Process of Clotting.-Take a drop of fresh blood from the tip of your finger under sterile conditions and mount on a microscopic slide in a few drops of salt solution, and examine immediately under the high power. Small threads of fibrin will presently be seen to form across the field, usually being most clearly obvious where fragments of white corpuscles are noted, see figures I07 and I32. The threads of fibrin become more apparent when stained with rosanilin.

d. Whipped Blood.-Draw a sample of blood into a glass tumbler, enough to fill it one-half or two-thirds full. Immediately begin vigorously stirring the blood with a bunch of stiff wires or a pencil, and keep it up until the time of clotting has passed, 5 or ro minutes. In this instance the wires will break up and collect the fibrin as fast as it forms, and no firm mass will be produced. The remaining fluid is called whipped blood. The fibrin can be removed from the wires and washed in tap water until all the adherent red corpuscles are removed. This mass of fibrin is white, elastic, and composed of a network of thread-like fibers. It is these fibers extending 
through and through the mass of blood which makes it retain the form of the vessel when undisturbed clotting occurs.

$e$. The Influence of Salt Solution on Blood Clotting.-Add 2 cc.of saturated magnesium sulphate, I per cent. sodium oxalate, and 0.9 per cent. sodium chloride to each of three test-tubes. Draw into each test-tube 5 to $6 \mathrm{cc}$. of blood and immediately mix thoroughly and let stand. The magnesium and oxalate test-tubes will not coagulate even though they stand for days, but the sodium-chloride blood will clot in a few minutes.

The magnesium-sulphate blood will coagulate if diluted with a sufficient amount of distilled water or physiological saline solution. Make a series of dilutions and note when coagulation takes place. The sodium-oxalate blood will coagulate when a sufficient excess (of I per cent. solution) of calcium chloride is carefully added to neutralize the excess of sodium oxalate. Demonstrate these on a series of samples.

If a quantity of magnesium or oxalate blood is secured and separated by a centrifuge or by letting stand for a sufficient time, a sample of uncoagulated plasma will be obtained. This sample will coagulate when it is treated as just described above for blood, showing that the antecedents of fibrin are found in the plasma.

f. Action of Tissue Extracts on Coagulation.-Wash out the blood of a small animal by circulating 0.9 per cent. saline through the arteries until the outflowing fluid from the veins is clear. Take an organ, the liver for example, grind it up in a sausage mill by running it through the mill two or three times, then extract with 0.9 per cent. physiological saline. The macerating mass should be shaken up at intervals, and may be kept from spoiling by adding an excess of chloroform or by keeping on ice. A few cubic centimeters of this fluid extract added to a sample of freshly drawn blood will very greatly hasten the rapidity of coagulation. This tissue extract contains the thromboplastin of Howell (Thrombokinase of Morawitz), and hastens the formation of thrombin from thrombogen.

II. The Chemistry of Blood Plasma (or Serum).-The blood plasma contains all the chemical substances which are utilized by the tissues in their nutrition or which are thrown off by the tissues as a result of their activity. It is therefore a very complex mixture. The serum contains the same substances in the same proportion, with the exception of the antecedents of fibrin. It may, therefore, be used as a substitute for plasma in most cases.

a. Proteins of Plasma.-There are three principal proteins in blood plasma: serum-albumin, serum-globulin, and fibrinogen. These may be isolated as follows: To a sample of blood plasma add an equal quantity of sodium-chloride solution that has been saturated at $40^{\circ} \mathrm{C}$. A white flocculent precipitate of fibrinogen comes down. Filter off, and add to the filtrate 
an equal volume of saturated ammonium sulphate. A second heavier precipitate of serum-globulin separates out. When this is separated, and crystals of ammonium sulphate are added to the filtrate to complete saturation at $40^{\circ} \mathrm{C}$., a third precipitate of serum-albumin separates.

Each of these precipitates may be redissolved and purified by reprecipitation and can be tested by the characteristic protein reactions, see page 107, which they all give.

b. Sugars of Blood Plasma or Serum.-If a quantity of blood serum is diluted with about 5 to ro times its volume of water, and the proteins are removed by slight acidulation with acetic acid and boiling and filtering, the filtrate will contain reducing sugar and the various salts of blood plasma. To a concentrated sample of the filtrate add Fehling's solution and boil. A reddish precipitate indicates the presence of reducing sugar. If this experiment is done quantitatively, about from o.I to 0.2 per cent. of sugar will be found. The sugar may be separated from the serum by dialysis through collodian membranes.

c. The Salts of Blood Plasma.-The salts of blood plasma are tested best by evaporating some of the blood serum to dryness, and burning the residue to oxidize the organic matter and dissolving the ash in water. Test as follows: To a sample add I per cent. of silver nitrate; a white precipitate soluble in an excess of ammonia, but not soluble in nitric acid, indicates chlorides.

To a second sample add I per cent. barium chloride. If sulphates are present there will be a white precipitate which settles out quickly.

Acidify a third sample with nitric acid and add ammonium molybdate and heat. A yellow precipitate indicates the presence of phosphates.

To the fourth sample add an excess of strong ammonia and I per cent. ammonium oxalate, heat. A white precipitate indicates the presence of calcium.

I2. Blood Corpuscles.- The characteristic substance in the composition of the blood corpuscles is the pigment known as hemoglobin, and this is the only chemical factor that will be considered in these experiments.

a. Hemoglobin Crystals. - Take a sample of dog's blood, or if a centrifuge is available separate and wash a sample of blood corpuscles, and mix with about three volumes of saturated ether water, or if blood is used dilute with two or three volumes of water and add about Io per cent. by volume of pure ether and shake thoroughly. Crystals of oxyhemoglobin will be formed, and these can be mounted and examined with a microscope.

b. Spectrum of Hemoglobin and its Compounds.

I. Oxyhemoglobin.-Dilute a sample of defibrinated blood with about ten volumes of distilled water. From this stock solution make five solutions all differing by $33^{1} \frac{1}{3}$ per cent. Examine these with a direct-vision spectroscope. Make a drawing showing the absorption spectrum of each sample 
as compared with the solar spectrum. Compared with the spectrum shown in the frontispiece.

2. Hemoglobin.-The oxygen can be driven out from the oxyhemoglobin by adding to the above samples a few drops of ammonium sulphide and gently warming. Re-examine with the direct-vision spectroscope and map as before.

3. Carbon-monoxide Hemoglobin.-Pass a stream of ordinary illuminating gas through the dilutions of hemoglobin. The carbon monoxide of the gas will form a compound with the hemoglobin, which now turns a bright scarlet color. When examined with the spectroscope, the absorption bands are found to be very similar to those of oxyhemoglobin. However, map the spectrum to the scale as usual. Add the reducing agent, warm, and shake vigorously and re-examine. It is very difficult to break up the combination of hemoglobin with carbon monoxide, hence the poisonous action of this gas. 


\section{CHAPTER V.}

\section{THE CIRCULATION OF THE BLOOD}

THE blood is contained in a system of closed vessels through which it is kept in circulation during the life of the individual. The energy to keep up this motion is supplied by the heart, which is a large muscular organ consisting of four great divisions, the right and left auricles and right and left ventricles. The right ventricle discharges its blood into the pulmonary

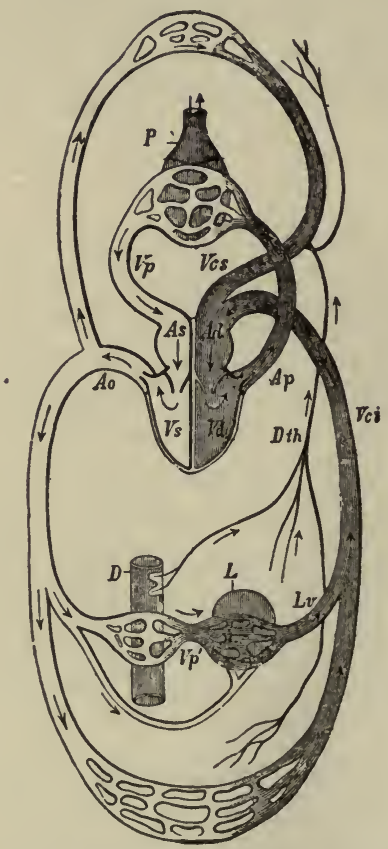

Fig. I33.-Diagram of the Circulation in an Animal with a Completely Separated Right and Left Ventricle and a Double Circulation. $A d$, Right auricle receiving the superior and inferior venæ cavæ, $V c s$ and $V c i$; $D t h$, thoracic duct, the main trunk of the lymphatic system; $A d$, right auricle; $V d$, right ventricle; $A p$, pulmonary artery; $P$, lung; $V p$, pulmonary vein; $A s$, left auricle; $V s$, left ventricle; $A o$, aorta; $D$, intestine; $L$, liver; $V p$, portal vein; $L v$, hepatic vein. (After Huxley.)

artery, through which it passes to the lungs, returning through the pulmonary veins to the left auricle, and into the ventricle. From the left ventricle the blood is pumped into the great aorta, and through its branches distributed to the entire body. The terminal arteries are continuous with the 
general capillaries of the body, and these in turn with the veins, which conduct the blood back to the right side of the heart again. It will be seen, therefore, that the circulatory apparatus consists of two great divisions, the pulmonary and the systemic circulation. This arrangement is illustrated by the accompanying figure. A study of this figure will show that in certain regions of the systemic circulation there are two capillary beds between the main arteries and the main veins. This subordinate stream through the liver is called the portal circulation, and the similar arrangement existing in the kidney is called the renal circulation. This, in general, is the outline of the course of the blood in its circulation.

To make a study of the various phenomena manifested in the physiology of the circulatory apparatus, it is obvious that we have to do with certain fundamental activities; first, the physiology of the pumping organ, the heart; second, the movement of the blood in the arteries, capillaries, and veins; third, the co-ordination of these various divisions of the apparatus through the nervous system. To understand this it will be necessary to have in mind in detail the anatomical structure of the apparatus itself.

\section{ANATOMICAL CONSIDERATIONS.}

The Heart.-The heart is contained in the chest or thorax, and lies between the right and left lungs, figure $\mathrm{I}_{34}$, enclosed in a membranous sac, the pericardium. The pericardium is made up of two distinct parts, an external fibrous membrane and an internal serous layer which not only lines the fibrous sac, but also is reflected on to the heart, which it completely invests. These form a closed sac, the cavity of which contains just enough pericardial fluid to lubricate the two surfaces, and thus to enable them to glide smoothly over each other during the movements of the heart. The vessels passing in and out of the heart receive investments from this sac to a greater or less degree.

The heart is situated in the chest behind the sternum and costal cartilages, being placed obliquely from right to left. It is of pyramidal shape, with the apex pointing downward, outward, and toward the left, and the base backward, inward, and toward the right. The heart is suspended in the chest by the large vessels which proceed from its base, but, excepting at the base, the organ itself hangs free within the sac of the pericardium. The part which rests upon the diaphragm is flattened, and is known as the diaphragmatic surface, while the free upper part is called the sternocostal surface.

On examination of the external surface the division of the heart into parts which correspond to the chambers inside of it may be traced. A deep transverse groove, called the coronary sulcus, divides the auricles from the ventricles; and the anterior longitudinal sulcus runs between the ventricles, both in 
front and in the back, separating the one from the other. The anterior groove is nearer the left margin, and the posterior nearer the right, as the front surface of the heart is made up chiefly of the right ventricle and the posterior surface of the left ventricle. The coronary vessels which supply the tissue of the heart with blood run in the furrows or sulci; also the nerves and lymphatics, which are embedded in more or less fatty material, are found in this groove.

The Chambers of the Heart.-The interior of the heart is divided by a longitudinal partition in such a manner as to form two chief chambers or cavities, the right and the left. Each of these chambers is again subdivided transversely into an upper and a lower portion, called, respectively, the auricle

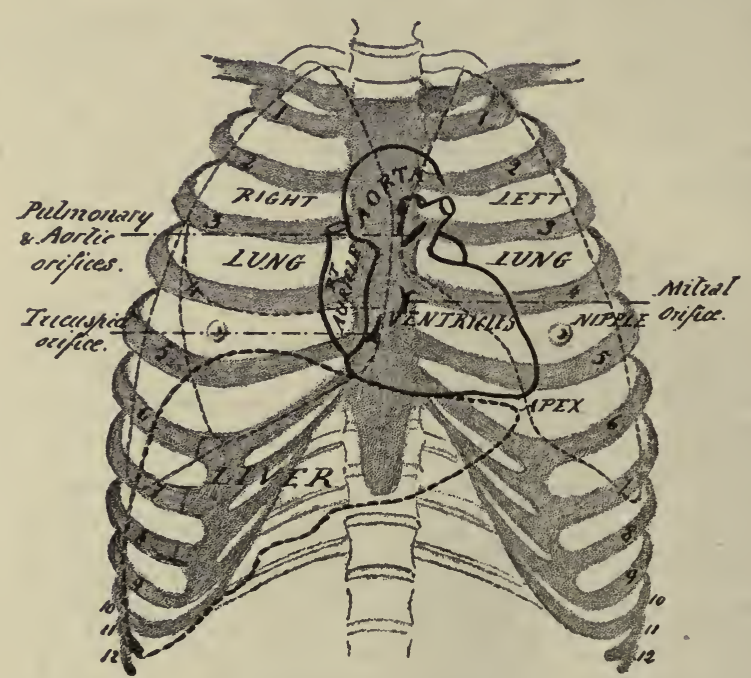

FIG. 134.- Outline of Heart, Lungs, and Liver to Show their Relations to each other and to the Chest Wall. (Heusman and Fisher's "Anatomical Outlines.")

and the ventricle, which freely communicate. The aperture of communication, however, is guarded by valves so disposed as to allow blood to pass freely from the auricle into the ventricle, but not in the opposite direction. There are thus four cavities in the heart, the auricle and ventricle of one side being quite separate from those on the other, figure $\mathrm{I} 35$.

The right auricle, the right part of the base of the heart as viewed from the front, is a thin-walled cavity of more or less quadrilateral shape, prolonged at one corner into a tongue-shaped portion, the right auricular appendix, which slightly overlaps the exit of the aorta from the left ventricle.

The interior of the auricle is smooth, being lined with the general lining membrane of the heart, the endocardium. The superior and inferior vence cavce open into the auricle. The opening of the inferior cava is protected 
and partly covered by a membrane called the Eustachian valve. In the posterior wall of the auricle is a slight depression called the fossa ovalis, which corresponds to an opening between the right and left auricles, existing in fetal life the foramen ovale. The foramen fails to close in many individuals. Statistics from observations of hearts from the dissecting rooms show as many as forty out of a hundred hearts with more or less. open interauricular foramina. In the appendix are closely set elevations of the muscular tissue covered with endocardium, and on the anterior

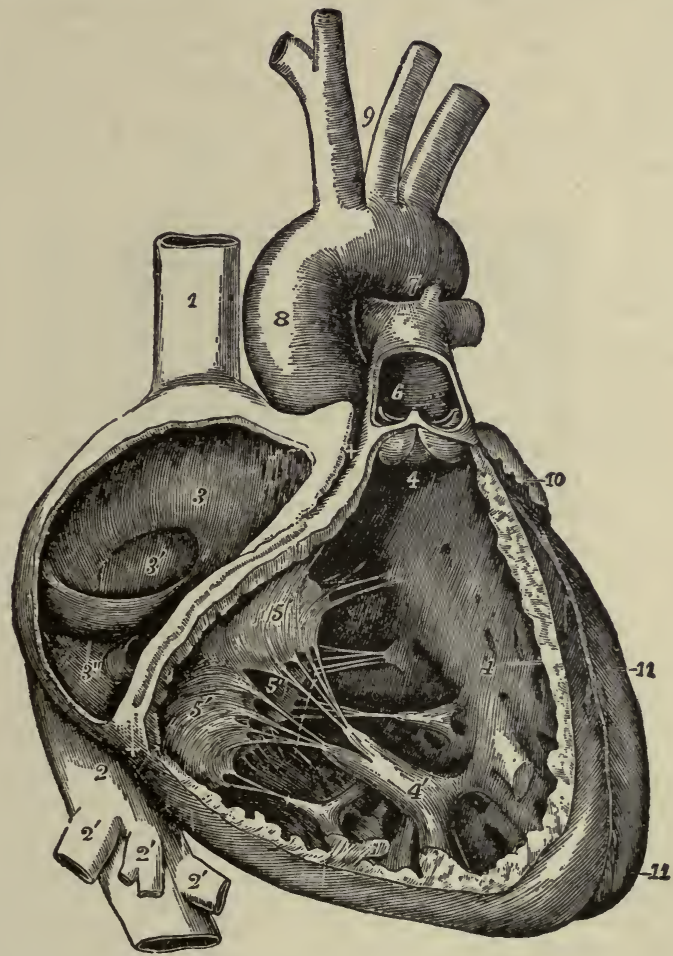

FIg. 135.-The Right Auricle and Ventricle Opened and a Part of their Right and Anterior Walls Removed so as to Show their Interior. I, Superior vena cava; 2, inferior vena cava; $2^{\prime}$, hepatic veins cut short; 3 , right auricle; $3^{\prime}$, placed in the fossa ovalis, below which is the Eustachian valve; $3^{\prime \prime}$, is placed close to the aperture of the coronary vein; $t, t$, placed in the auriculo-ventricular groove, where a narrow portion of the adjacent walls of the auricle and ventricle has been preserved; 4, 4, cavity of the right ventricle, the upper figure is immediately below the semilunar valves; $4^{\prime}$, large columna carnea or musculus papillaris; $5,5^{\prime}, 5^{\prime \prime}$, tricuspid valve; 6 , placed in the interior of the pulmonary artery, a part of the anterior wall of that vessel having been removed and a narrow portion of it preserved at its commencement where the semilunar valves are attached; 7 , concavity of the aortic arch, close to the cord of the ductus arteriosus; 8, ascending part or sinus of the arch covered at its commencement by the auricular appendix and pulmonary artery; 9 , placed between the innominate and left carotid arteries; ro, appendix of the left auricle; II, II, outside of the left ventricle the lower figure near the apex. (Allen Thomson.)

wall of the auricle are similar elevations arranged parallel to one another, called musculi pectinati. 
The right ventricle forms the right margin of the heart. It takes no part in the formation of the apex. On section its cavity is semilunar or crescentic, figure I35. Into it are two openings, the venous orifice at the base, and the arterial orifice of the pulmonary artery, also at the base but more to the left. The part of the ventricle leading to the pulmonary artery is called the conus arteriosus. Both orifices are guarded by valves, the former called the tricuspid and the latter the semilunar. In this ventricle are also the projections of the muscular tissue called the trabecula carnea.

The left auricle is situated at the left and posterior part of the base of the heart. The left auricle is only slightly thicker than the right and its form and structure are the same as in the right. The left venous orifices are oval and a little smaller than those on the right side of the heart. There is a slight vestige of the foramen on the septum between the auricles.

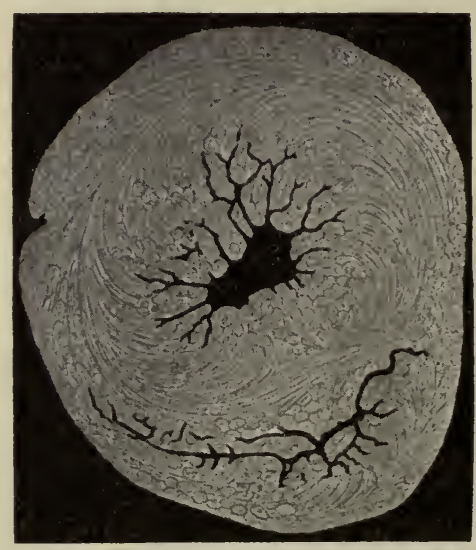

Fig. 136.-Cross-section of a Completely Contracted Human Heart, at the Level of the Lower and Middle Thirds. (According to Krehl.)

The left ventricle occupies the posterior and apical portion of the heart, and is connected directly with the great aorta. It is separated from the auricle by the bicuspid or mitral valve, and the opening into the great aorta is guarded by the semilunar valves. The walls of the left ventricle are two or three times as heavy as those of the right, and may be as much as half an inch in total thickness.

The left ventricle is capable of containing 90 to r 20 c.c. of blood. The capacity of the auricles is considerably less after death owing to their contracted condition. The whole heart is about $\mathrm{I} 2 \mathrm{~cm}$. long by $8 \mathrm{~cm}$. at its greatest width, and $6 \mathrm{~cm}$. in thickness. The average weight in the adult is about 300 grams.

The walls of the heart are constructed almost entirely of layers of muscular fibers; but a ring of connective tissue, to which some of the muscular 
fibers are attached, is inserted between each auricle and ventricle and forms the boundary of the venous opening. Fibrous tissue also exists at the origins of the pulmonary artery and aorta. The muscular fibers of each auricle are in part continuous with those of the others and in part separate; and the same holds true for the ventricles. The fibers of the auricles are,
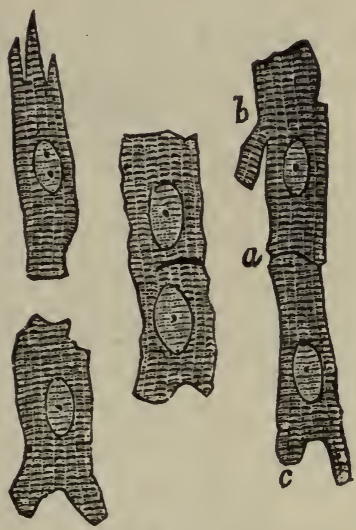

Fig. 137.

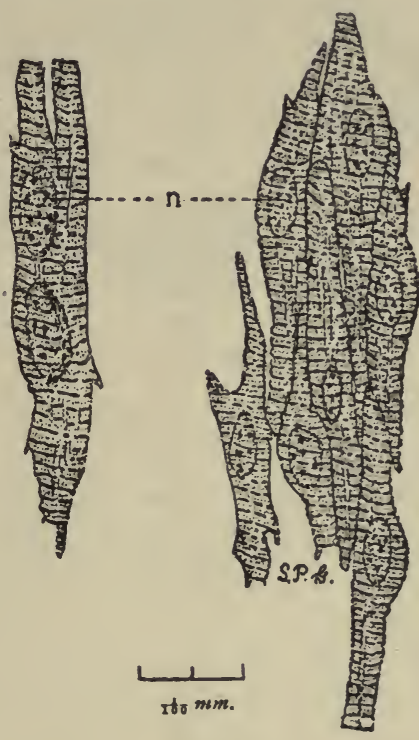

FIG. 138 .

FIG. I37.-Cardiac Muscle Cells, Showing their Form, Branches, Nuclei, and Strix. From the heart of a young rabbit. Magnified 425 diameters. $a$, Line of junction between the cells (intercellular cement); $b, c$, branches of the cells. (Schäfer.)

FIG. I38.-Cardiac Muscle Cells of the Left Ventricle of a Child at Birth (full term), to show the form of the cells, their structural details, their relations to one another, and their general agreement with those of cold-blooded vertebrates. $A$, Large cell with two nuclei; this cell has nearly the proportions of those of the adult; $B$, group of cells in their natural relation. At the right of the middle cell are two spaces or fissures. $n$, Nucleus. The transverse striations cross the nuclei in all the cells, and each nucleus possesses several nucleoli. (Gage.)

however, quite separate from those of the ventricles. The bond of connection between the auricles and the ventricles is made by the Purkinje fibers, an embryonic muscular type of tissue composing the auriculoventricular strand in the septum called the bundle of His.

The development of the heart shows that it is derived from an embryonic tube, which in its growth becomes twisted upon itself and divided into the two main divisions that we know in the adult. Anatomical dissections have shown that the muscles of the ventricles form spiral sheaths extending from the base of the two ventricles in spiral bands toward the apex. These bands of muscle are wound about the surface of the ventricles in the right- 
to-left direction. At the apex they extend up into the deeper tissue. If the superficial muscles are dissected off, there is left a great central core of muscle, which is described by MacCallum as running more transversely

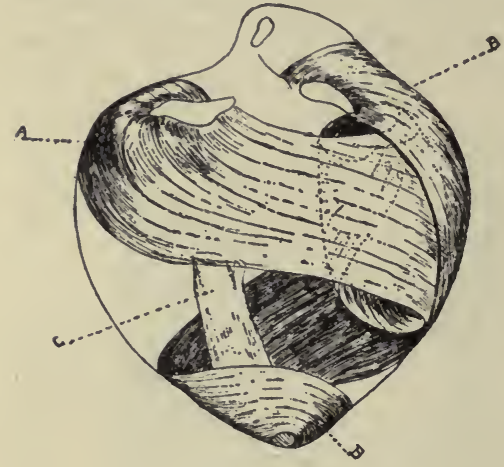

FIG. 139.

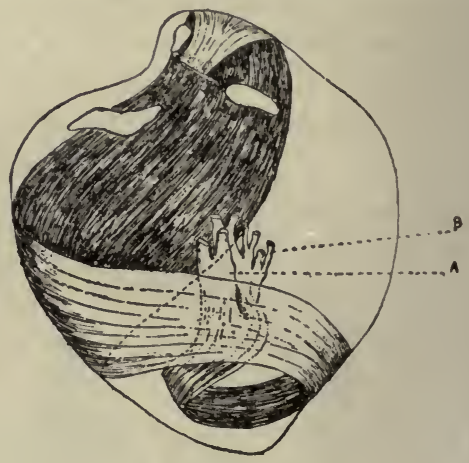

FIG. 140.

FIG. I39.-Diagram of the Course of the Superficial Muscle Layers Originating in the Right and Left Coronary Sulci and in the Posterior Half of the Tendon of the Conus. $C$, Anterior papillary muscle. (After MacCallum.)

FIG. I40.-Diagram of the Course of the Superficial Muscle Layers Originating in the Anterior Half of the Tendon of the Conus. A, Posterior papillary muscle; $B$, papillary muscle of the septum. (After MacCallum.)

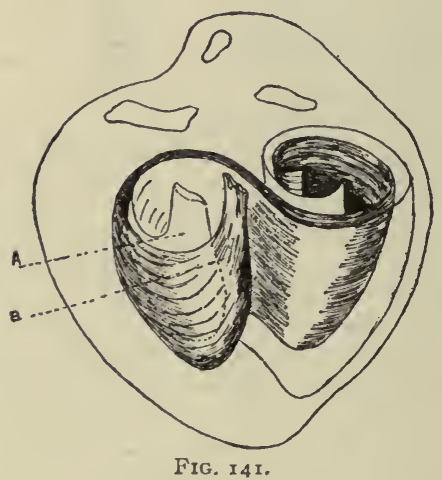

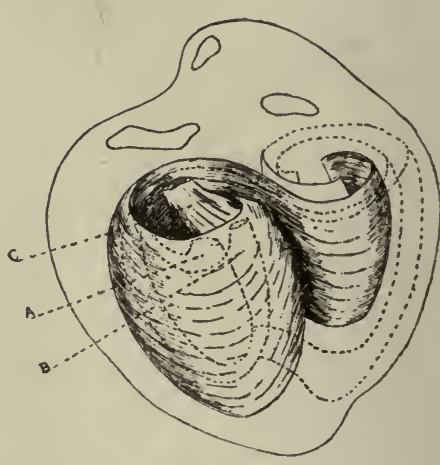

FIG. 142.

FIG. I4I.-Diagram of the Course of the Layer Superficial to the Deepest Layer of the Muscle of the Left Ventricle, which is shown in outline. The deepest layer is also shown. $A$, Posterior papillary muscle; $B$, papillary muscle of the septum. (After MacCallum.)

FIG. I42.-Diagram of a Layer still more Superficial to that Shown in Fig. I4I, and Ending in the Anterior Papillary Muscle. The deeper layers are represented in dotted lines. $A$, Posterior papillary muscle; $B$, papillary muscle of septum; $C$, anterior papillary muscle. (After MacCallum.)

around the wall of one ventricle, then through the septum and around the other in a reverse scroll, figure I4I.

The Valves of the Heart.-The valves of the heart are arranged so that the blood can pass only in one direction. These are the tricuspid 
valve, between the right auricle and right ventricle, figure $\mathrm{I}_{35}$, and the semilunar valve of the pulmonary artery, the mitral valve between the left auricle and ventricle, and semilunar valve of the aorta. The bases of the tricuspid, figure $\mathrm{I}_{52}$, and mitral valves are attached to the walls of the venous orifices respectively. Their ventricular surfaces and borders are fastened by slender tendinous fibers, the chorde tendinea, to the internal surface of the walls of the ventricles at points which project into the ventricular cavity in the form of bundles or columns, the musculi papillares.

The semilunar valves guard the orifices of the pulmonary artery and of the aorta. They are nearly alike on both sides of the heart, but the aortic valve is altogether thicker. Each valve consists of three parts which are of similunar shape, the convex margin of each being attached to a fibrous ring at the place of junction of the artery to the ventricle, and the concave or nearly straight border being free, so as to form a little pouch like a pocket, figure $15 \mathrm{I}$. In the center of each free edge of the valves which contains a fine cord of fibrous tissue is a small fibrous nodule, the corpus Arantii of the valves.

The Arteries.-The arterial system begins at the left ventricle in a single large trunk, the aorta, which, almost immediately after its origin, gives off in the thorax three large branches for the supply of the head, neck, and upper extremities; it then traverses the thorax and abdomen, giving off branches, some large and some small, for the supply of the various organs and tissues it passes on its way. In the abdomen it divides into two chief branches. The arterial branches, wherever given off, divide and subdivide until the caliber of each subdivision becomes very minute. These smallest arteries are called arterioles. These arterioles are continuous into the capillaries. Arteries frequently communicate or anastomose with other arteries. The arterial branches are usually given off at an acute angle, and the areas of the branches of an artery generally exceed that of the parent trunk, and, as the distance from the origin is increased, the area of the combined branches is increased also. As regards the arterial system of the lungs, the pulmonary artery and its subdivisions, they are branched in much the same manner as the arteries belonging to the general systemic circulation.

The walls of the arteries are composed of three principal coats, the external, or tunica adventitia, the middle, or tunica media, and the internal, or tunica intima. The external coat, figures $\mathrm{I} 43$ and $\mathrm{r} 44, a$, the strongest and toughest part of the wall of the artery, is formed of areolar tissue, with which is mingled throughout a network of elastic fibers. The middle coat, figure I $44, m$, is composed of both muscular and elastic fibers with a certain proportion or areolar tissue. In the larger arteries, its thickness is comparatively as well as absolutely much greater than in the small arteries, constituting, as it does, the greater part of the arterial wall. The muscular fibers are unstriped, figure 145 , and are arranged, for the most part, trans- 
versely to the long axis of the artery, figure I $43, m$, while the elastic element, taking also a transverse direction, is disposed in the form of closely interwoven and branching fibers intersecting in all parts the layers of muscular fiber. In arteries of various size there is a difference in the proportion of the muscular and elastic element, elastic tissue preponderating in the largest arteries and unstriped muscle in those of medium and small size. The arteries are quite elastic in both large and small vessels. The internal coat is formed by a layer of elastic tissue, called the fenestrated membrane of Henle. It is peculiar in its tendency to curl up when peeled off from the artery, and

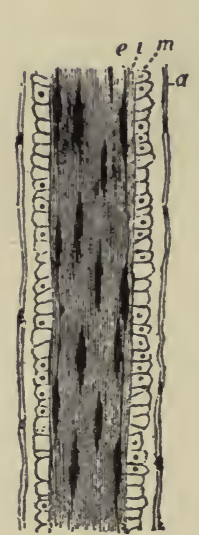

FIG. 143.

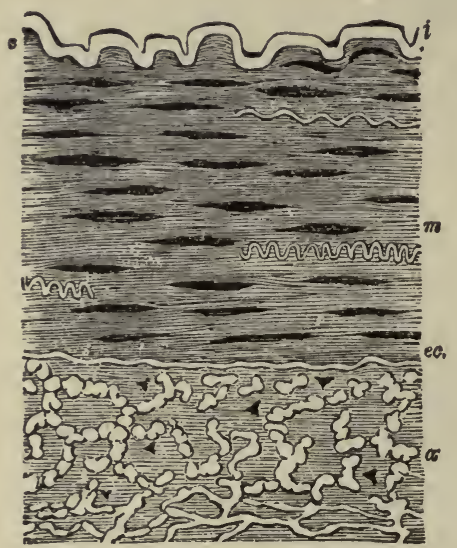

FIG. 144 .
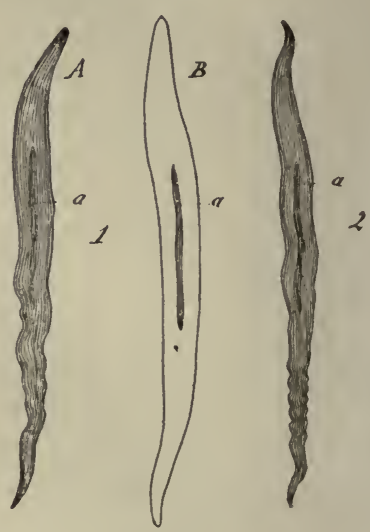

FIG. 145.

FIG. 143.-Minute Artery Viewed in Longitudinal Section. $e$, Nucleated endothelial membrane, with faint nuclei in lumen, looked at from above; $i$, thin elastic tunica intima; $m$, muscular coat or tunica media; $a$, tunica adventitia. (Klein and Noble Smith.)

FIG. I44.- Transverse Section through a Large Branch of the Inferior Mesenteric Artery of a Pig. $e$, Endothelial membrane; $i$, tunica elastica interna, no subendothelial layer is seen; $m$, muscular tunica media, containing only a few wavy elastic fibers; $e, c$, tunica elastica externa, dividing the media from the connective-tissue adventitia, $a$. Magnification, $35^{\circ}$ diameters. (Klein and Noble Smith.)

Fig. I45.-Muscular Fiber Cells from Human Arteries. Magnified $35^{\circ}$ diameters. $a$, Nucleus; $B$, a fiber cell treated with acetic acid. (Kölliker.)

in the perforated and streaked appearance which it presents under the microscope. The inner surface of the artery is lined with a delicate layer of elongated endothelial cells which make it smooth and polished and furnish a nearly impermeable surface along which the blood may flow with the smallest possible amount of resistance from friction.

Many of the arteries are accompanied by a plexus of vaso-motor nerves. In the smaller arteries these nerves consist of few fibers that form a delicate network over the walls of the vessels. Many fibers appear to end in the muscle cells of the arterioles in the proximity of the nuclei.

The Capillaries.- In all vascular textures, except some parts of the corpora cavernosa of the penis, of the uterine placenta, and of the spleen, 
the transmission of the blood from the minute branches of the arteries to the minute veins is effected through a network of capillaries. They may be seen in all minutely injected preparations.

The point at which the arteries terminate and the capillaries commence cannot be exactly defined, for the transition is gradual. The capillaries maintain essentially the same diameter throughout. The meshes of the

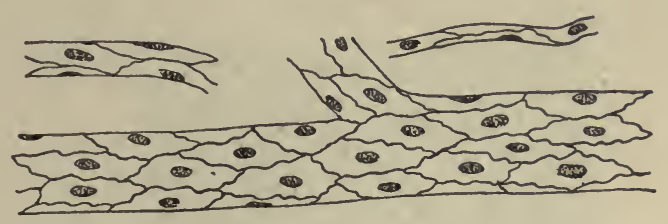

FIG. I46.-Vein and Capillaries. Silver-nitrate and hematoxylin stain, to show outlines of endothelial cells and their nuclei. (Bailey.)

network that they compose are more uniform in shape and size than those formed by the anastomoses of the minute arteries and veins.

The walls of the capillaries are composed of a single layer of elongated or radiate, flattened and nucleated endothelial cells, so joined and dovetailed together as to form a continuous transparent membrane, figure ${ }_{4} 6$.

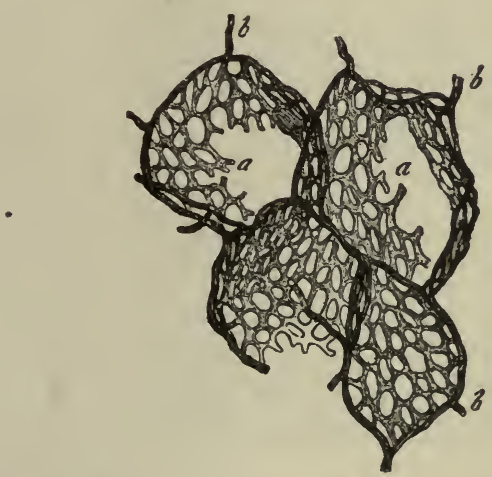

FIG. 147.-Network of Capillary Vessels of the Air Celis of the Horse's Lung Magnified. $a, a$, Capillaries proceeding from $b, b$, terminal branches of the pulmonary artery. (Frey.)

Outside these cells in the larger capillaries there is a structureless supporting membrane on the inner surface of which they form a lining.

The diameter of the capillary vessels varies somewhat in the different textures of the body, the most common size being about 12 micromillimeters, $\frac{1}{2000}$ of an inch. Among the smallest may be mentioned those of the 
brain and of the follicles of the mucous membrane of the intestines; among the largest, those of the skin and especially those of the medulla of the bones.

The form of the capillary network differs in the different organs of the body, but is usually adjusted to the structural arrangement of the cells of any given organ.

The capillary network is closest in the lungs and in the choroid coat of the eye. In the human liver the interspaces are of the same size, or even

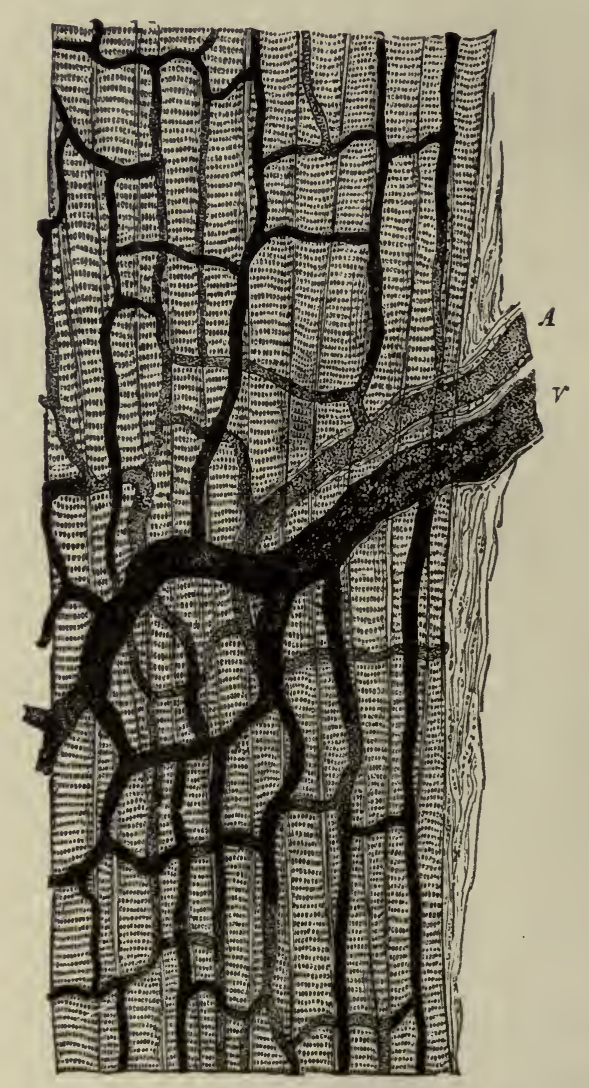

FIg. I48.-Capillaries of Striated Muscular Tissue. From a cat. Magnified 300 diameters. A, Artery; $V$, vein. (Heitzmann.)

smaller than the capillary vessels themselves. In the human lung the spaces are smaller than the vessels; in the human kidney and in the kidney of the dog the diameter of the injected capillaries, compared with that of the interspaces, is in the proportion of one to four, or one to three. The brain receives a very large quantity of blood; but its capillaries are very minute and are less numerous than in some other parts. In the mucous mem- 
branes, for example in the conjunctiva and in the cutis vera, the capillary vessels are much larger than in the brain and the interspaces narrower, namely, not more than three or four times wider than the vessels. In the periosteum and in the external coat of arteries the meshes are much larger, their width being about ten times that of the vessels. It may be held as a general rule that the more active the functions of an organ are, the more vascular it is.

The Veins.-The venous system begins in small vessels which are slightly larger than the capillaries from which they spring. These vessels

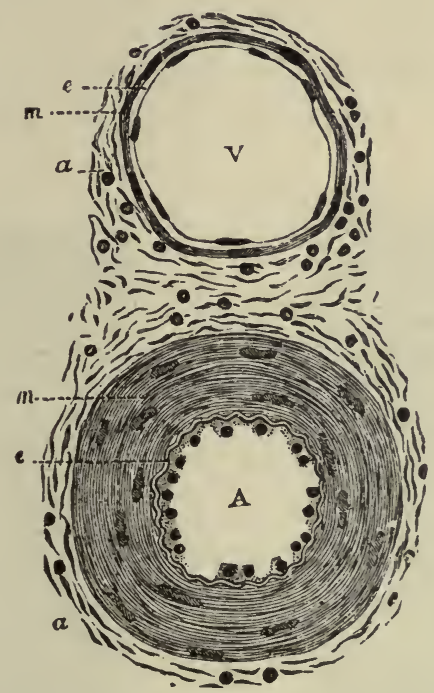

Fig. I $\$ 9$. - Transverse Section through a Small Artery and Vein of the Mucous Membrane of a Child's Epiglottis; the artery is thick-walled and the vein thin-walled. $A$, Artery; the letter is placed in the lumen of the vessel. $e$, Endothelial cells with nuclei clearly visible; these cells appear very thick from the contracted state of the vessel. Outside it a double wavy line marks the elastic tunica intima. $m$, Tunica media consisting of unstriped muscular fibers circularly arranged; their nuclei are well seen. $a$, Part of the tunica adventitia, showing bundles of connective-tissue fiber in section, with the circular nuclei of the connective-tissue corpuscles. This coat gradually merges into the surrounding connective tissue. $\quad V$, The lumen of the vein. The other letters indicate the same as in the artery. The muscular coat of the vein, $m$, is seen to be much thinner than that of the artery. $\times 350$. (Klein and Noble Smith.)

are gathered up into larger and larger trunks until they terminate in the two venæ cavæ and the coronary vein which enter the right auricle, and in four pulmonary veins which enter the left auricle. The total capacity of the veins diminishes as they approach the heart; but their capacity exceeds by two or three times that of their corresponding arteries. The pulmonary veins, however, are an exception to this rule. The veins are found after 
death more or less collapsed and often contain blood. They are usually distributed in a superficial and a deep set which anastomose frequently in their course.

The coats of veins bear a general resemblance to those of arteries, figure I49. Thus, they possess outer, middle, and inner coats. The outer coat is of areolar tissue like that of the arteries, but is relatively thicker. In some veins it contains a few musclar longitudinal cells. The middle coat is considerably thinner than that of the arteries; it contains circular unstriped muscular fibers mingled with a large proportion of yellow elastic and white fibrous connective tissue. In the large veins near the heart the middle coat is replaced for some distance from the heart by circularly arranged striped muscular fibers continuous with those of the auricles. The internal coat of veins consists of a fenestrated membrane lined by endothelium. The fenestrated membrane may be absent in the smaller veins.

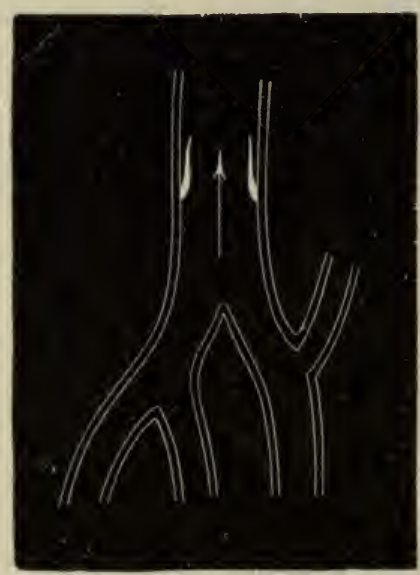

A

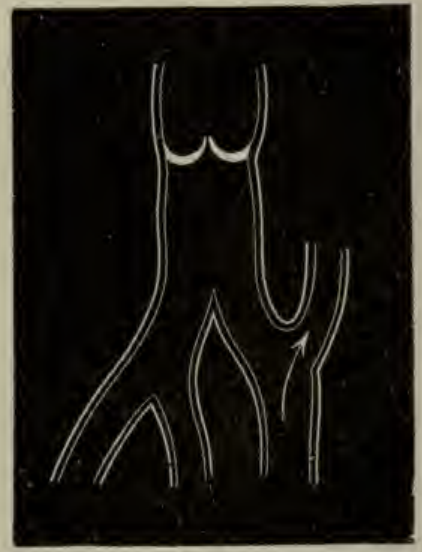

B

FIG. 150.- $A$, Vein with valves open. $B$, vein with valves closed; stream of blood passing off by lateral channel. (Dalton.)

The veins are supplied with valves in certain regions of the body, especially in the arms and legs. The general construction of these valves is similar to that of the semilunar valves of the aorta and pulmonary artery already described. Their free margins are turned in the direction toward the heart, so as to prevent any movement of blood backward. They are commonly placed in pairs, at various distances in different veins. In the smaller veins single valves are often met with, and three or four are sometimes placed together or near one another in the larger veins, such as in the subclavians at their junction with the jugular veins. During the period of their inaction, when the venous blood is flowing in its proper direction, 
they lie by the sides of the walls of the veins; but when in action they come together like valves of the arteries, figure I $_{5}$. Their situation in the superficial veins of the forearm is readily discovered by pressing along its surface, in a direction opposite to the venous current, i.e., from the elbow toward the wrist, when little swellings, figure $\mathrm{I} 5 \mathrm{\circ}, B$, will appear in the position of each pair of valves.

Lymphatic spaces are present in the coats of both arteries and veins; but in the tunica adventitia or external coat of the large vessels they form a distinct plexus of more or less tubular vessels. In smaller vessels they appear as sinuses lined by endothelium. Sometimes, as in the arteries of the omentum, mesentery, and membranes of the brain, the pulmonary, hepatic, and splenic arteries, the spaces are continuous with vessels which distinctly ensheath them, perivascular lymphatic sheaths. Lymph channels are said to be present also in the tunica media.

\section{THE ACTION OF THE HEART.}

The heart's action in propelling the blood consists in the successive alternate contraction, systole, and relaxation, diastole, of the muscular walls of the auricles and the ventricles. This activity furnishes the power which keeps the blood moving through the arteries, capillaries, and veins. The heart in its activity is like a great force pump in that it injects a certain quantity of blood at each contraction into the great arteries. Owing to the interaction between this heart-beat and the peripheral resistance to the flow of blood, together with the elasticity of the vessels themselves, a high pressure in the arteries is maintained all the time. The heart's contractions pumping against this high arterial tension, are sufficient to maintain a constant flow of blood through the capillaries, and therefore through the active tissues.

The heart beats at an average rate of about 72 times per minute during life. Each successive contraction really begins in the great veins, the superior vena cava and extends over the auricles and ventricles in regular sequence. The contraction of each successive part is called its systole and the relaxation its diastole. The diastole covers the period of active relaxation of the muscle and the pause before beginning its next contraction. Each muscular chamber of the heart may, therefore, be said to have its own systole and diastole. The whole series of events from the beginning of one contraction until the corresponding event in the next contraction is described as the cardiac cycle.

Action of the Auricles.-The description of the action of the heart may be commenced at that period in each cycle in which the whole heart is at rest. The heart is then in a passive state. The auricles are gradually 
filled with the blood flowing into them from the veins, and a portion of this blood passes at once through the auricles into the ventricles, the opening between the cavity of each auricle and that of its corresponding ventricle being free during the pause of the entire heart. The auricles, however, receive more blood than at once passes through them to the ventricles. Near the end of the pause they become passively distended. At this moment a contraction wave begins at the bases of the venæ cavæ and, running down over the walls about the mouths of the veins, passes to the muscular walls. The contraction of the auricles, the right and left contracting at the same time, forces the blood into the ventricles.

The contraction of the muscular walls at the mouths of the great veins and of the sinus region maintains a condition of constriction during the time of the auricular contraction. This hinders the reflux of blood from the auricles into the veins during the auricular systole. Any slight regurgitation from the right auricle is limited by the valves at the junction of the subclavian and internal jugular veins beyond which the blood cannot move backward, and by the coronary vein which is supplied with valve-like fold at its mouth. The force of the blood propelled by the auricle into the ventricle at each auricular systole is transmitted in all directions, but, being insufficient to open the semilunar valves, it is expended in distending the walls of the ventricle.

Action of the Ventricles.-The dilatation of the ventricles which occurs during the latter part of the diastole of the auricles, is completed by the forcible injection of the contents of the latter. The ventricles, now distended with blood, immediately begin to contract. The tricuspid and mitral valves are closed by the initial reflux of blood, or possibly by the currents of blood formed by the sudden injection of the ventricles by the auricular contractions. The ventricular systole follows the auricular systole so closely that it seems continuous with it. As a result of the ventricular systole, sufficient pressure is produced on its contents to overcome the pressure against the semilunar valves of the aorta and the pulmonary artery, and the ventricles are then emptied completely. After the whole of the blood has been expelled from the ventricles, the walls remain contracted for a brief period.

The form and position of the fleshy columns on the internal walls of the ventricles no doubt help to produce the obliteration of the ventricular cavities during contraction. The completeness of the closure may often be observed on making a transverse section of a heart shortly after death in any case in which rigor mortis is very marked, figure 136 . In such a case only a central fissure may be discernible to the eye in the place of the cavity of each ventricle. The arrangement of the muscles of the heart, as described on page I $\mathrm{I}$, is such as to expend the whole force of the contraction in diminishing the cavity of the ventricle, or, in other words, in expelling the contents of blood. 
On the conclusion of the systole the ventricular diastole begins. The muscular walls relax and, by virtue of their elasticity, a slight negative pressure may be set up. This negative or suctional pressure on the left side of the heart may be of importance in helping the pulmonary circulation. It is somewhat inconstant in appearance, but has been found to be equal to as much as $20 \mathrm{~mm}$. of mercury, and is said to be quite independent of the aspiratory power of the thorax itself, which will be described in a later chapter. The ventricles now remain in a state of relaxation or rest until the next systole begins.

The duration of the ventricular systole and the diastole has been variously estimated. A computation of the time of these two phases, for man, in figure ${ }_{153}$, reproduced from Hürthle, gives for the systole 0.38 of a second and for the diastole 0.4 of a second, with a total of 0.78 of a second. This is equivalent to a rate of 77 per minute. Variation in the time of the systole and the diastole of the ventricle falls chiefly on the pause of the diastole.

The ventricles undergo little or no change of shape in the unopened chest. At the moment in the systole when the ventricles begin to discharge their

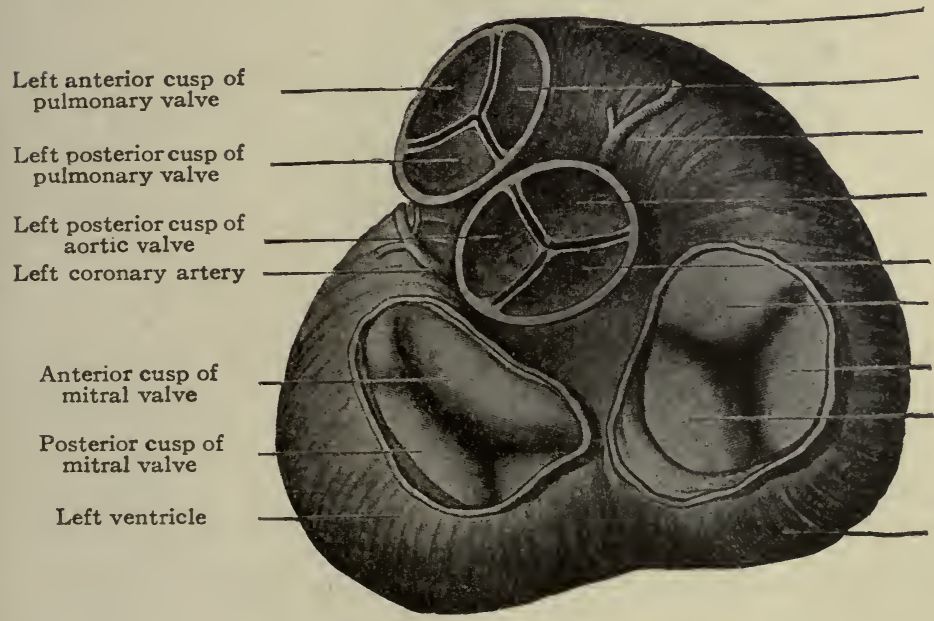

Conus arteriosus

Right anterior cusp of pulmonary valve

Right coronary artery

Anterior cusp of aortic valve

Right posterior cusp of aortic valve Anterior (infundibular) cusp of tricuspid valve

Right (marginal)

cusp of tricuspid valve

Posterior (septal)

cusp of tricuspid valve

Right ventricle

FIG. I5I.-The Bases of the Ventricles of the Heart, showing the auriculo-ventricular, aortic, and pulmonary orifices and their valves. (Cunningham.)

contents into the aorta and pulmonary arteries, respectively, there is a sharp decrease in size of the ventricles. This decrease takes place in all dimensions.

Action of the Valves.-The Tricuspid Valve.-During the diastole of both auricles and ventricles blood flows directly through the auricles into the ventricles, the auricles during this period acting as continuations of the large veins which empty into them. At the end of the period the ventricle 
on each side has already been filled and distended by the pressure of blood from the veins. The systole of the auricle completes this filling and slightly overdistends the ventricle. When the force of the auricular contraction is spent, the ventricular walls rebound slightly toward their former position and in so doing exert some pressure upon the ventricular side of the tricuspid valve which floats the cusps upward toward the auricle. In this connection another force comes into play, viz., vortex or back currents resulting from the flow of the blood into the ventricle under the pressure of the auricular systole. These currents aid in floating the valve cusps into apposition. Thus the venous orifices of the ventricles are closed at the end of the auricular systole; i.e., the end of the ventricular diastole. The ventricular systole which follows simply serves to place the valves under greater tension thus closing them still more firmly. It should be recollected that the diminution in the breadth of the base of the heart in its transverse diameters during

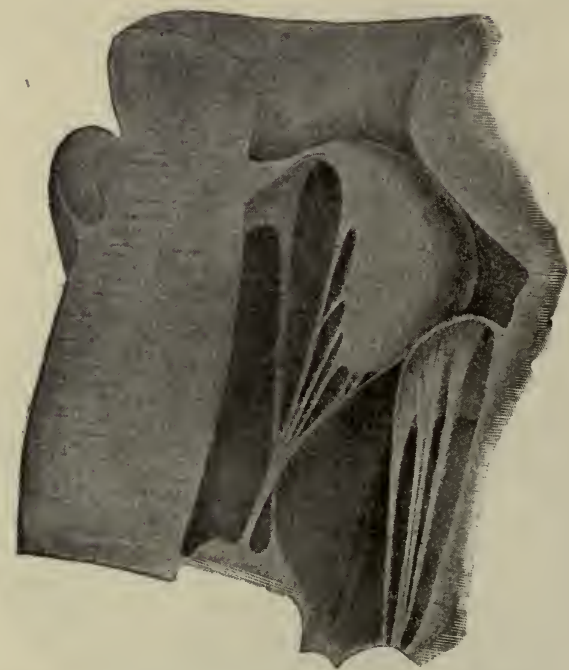

FIG. I52. - The Tricuspid Valves of the Ox, Closed. Vertical section. (Krehl.)

the ventricular systole is especially marked in the neighborhood of the venous orifices, and this aids in rendering the tricuspid valve competent to close the openings by greatly diminishing the diameter. The cusps of the valve meet not by their edges only, but by the opposed surfaces of their thin outer borders. The margins of the valve are still more secured in apposition with one another by the simultaneous contraction of the papillary muscles, whose tendinous chords have a special mode of attachment for this very object. They compensate for the shortening of the ventricular walls and thus prevent the valve cusps from being everted into the auricles, an event that does occur in certain valvular lesions. 
The actions of the tricuspid and mitral valves on the right and left sides of the heart are essentially the same.

The Semilunar Valves. - The commencement of the ventricular systole precedes the opening of the semilunar valve by a fraction of a second. The intraventricular pressure increases with the progress of the systole until there is a distinct increase over the arterial pressure, then the opening of the valves takes place at once. The valves remain open as long as this difference continues. When the diastole of the ventricles begins and the arterial blood pressure exceeds the intraventricular pressure, there is an initial reflux of blood toward the heart which closes the semilunar valve.

The dilatation of the arteries is peculiarly adapted to bring this about. The lower borders of the semilunar valves are attached to the inner surface of the tendinous ring which bounds the orifice of the artery. The tissue of this ring is tough and inelastic and the valves are equally inextensible, being formed mainly of tough fibrous tissue with strong interwoven cords. the effect, therefore, of each propulsion of blood from the ventricle into the artery is to dilate the wall of the first portion of the artery and the three pouches behind the valve cusps while the free margins of the cusps are drawn inward toward the center. This position of the valves and arterial walls is maintained while the ventricle continues in contraction; but as it relaxes, and the dilated arterial walls recoil by their elasticity, the blood is forced backward toward the ventricles and onward in the course of the circulation. Part of the blood thus forced back lies in the pouches (sinuses of Valsalva) between the valve cusps and the arterial walls; and the cusps are pressed together till their thin lunated margins meet in three lines radiating from the center to the circumference of the artery, figure I5I. The corpora Arantii at the middle of the free margins insure a more effective closure.

The Sounds of the Heart.-When the ear is placed on the chest over the heart, two sounds may be heard at every beat. They follow in quick succession and are succeeded by a pause or period of silence. The first sound is dull and prolonged; its commencement coincides with the impulse of the heart against the chest wall, and just precedes the pulse at the wrist. The second sound is shorter and sharper, with a somewhat flapping character. The periods of time occupied, respectively, by the two sounds taken together and by the pause between the second and the first are unequal. According to Foster, the interval of time between the beginning of the first sound and the second sound is 0.3 of a second, while between the second and the succeeding first it is nearly 0.5 of a second, see figures I 53, I $_{54}$, and I 67 . The relative length of time occupied by each sound, as compared with the other, may be best appreciated by considering the different forces concerned in the production of the two sounds. In one case there is a strong, comparatively slow contraction of a large mass of 
muscular fibers, urging forward a certain quantity of fluid against considerable resistance; while in the other it is a strong but shorter and sharper recoil of the elastic coat of the large arteries-shorter because there is no resistance to the flapping back of the semilunar cusps as there was to their opening. The sounds may be expressed by the words $l \bar{u} b b-d \breve{u} b$. The beginning of the first sound corresponds in time with the three coincident events, namely, the beginning of the contraction of the ventricles, the closure of the tricuspid and mitral valves, and the first part of the dilatation of the auricles. The sound continues through a somewhat longer interval than the second sound. The second sound, in point of time, immediately

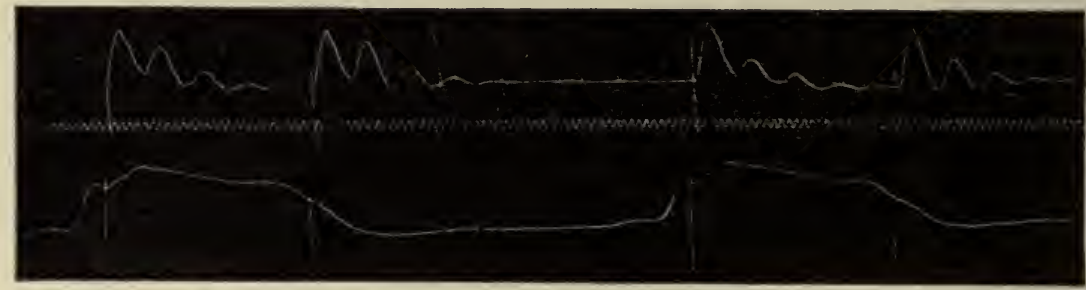

FIG. 153.-Simultaneous Tracings of the Cardiac Impact, or Cardiogram (lower), and the Heart Tones (upper), of Man. The cross strokes at the beginning of the cardiac sound tracing and on the cardiogram mark the synchronous events. (Hürthle.)

follows the cessation of the ventricular contraction, and corresponds with the commencing dilatation of the ventricles and the opening of the semilunar and mitral valves, figure ${ }_{54}$.

The exact cause of the first sound of the heart is not absolutely known. Two factors probably enter into it. First, the vibration of the semilunar and mitral valves and of the chordæ tendineæ. Second, the vibration of the

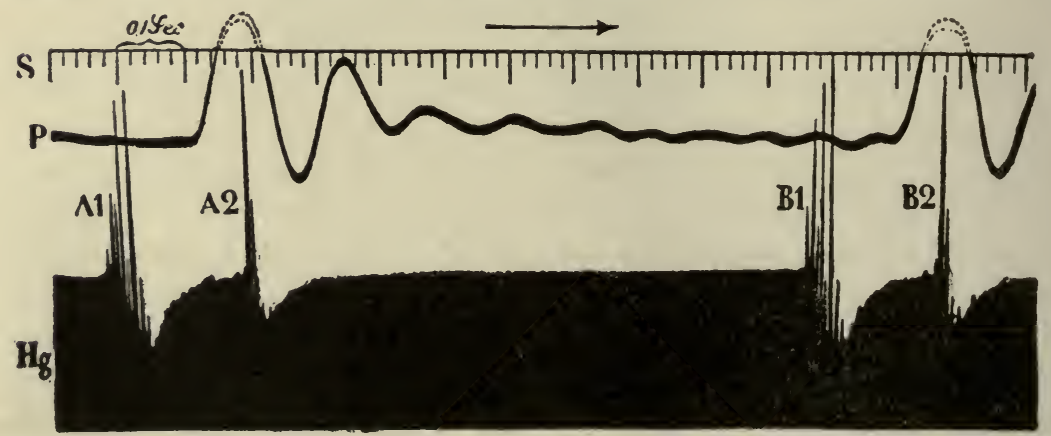

FIG. I54.-Simultaneous Tracings of the Heart Tone and Pulse of the Carotid in the Dog. $A \mathrm{r}$ and $A_{2}$, First and second sounds; $P$, pulse; $S$, time in tenths and fiftieths of a second. (Einthoven and Geluk.)

muscular mass of the ventricles themselves. The same mechanical conditions produce equal tension on the ventricular muscle itself and, according 
to the second view this is sufficient to account for the first sound. Looking upon the contraction of the heart as a simple contraction and not as a series of contractions, or tetanus, it is at first sight difficult to see why there should be any muscular sound when the heart contracts.

The cause of the second sound is more simple and definite than that of the first. It is entirely due to the vibration consequent on the sudden closure of the semilunar valves when they are pressed down across the orifices of the aorta and pulmonary artery. The influence of these valves in producing the sound was first demonstrated by Hope who experimented with the hearts of calves. In these experiments two delicate curved needles were inserted, one into the aorta and another into the pulmonary artery below the line of attachment of the semilunar valves. After being carried upward about half an inch the needles were brought out again through the coats of the respective vessels, so that in each vessel one valve was held back against the arterial walls. Upon applying the stethoscope to the vessels it was found that after such an operation the second sound had ceased to be audible.

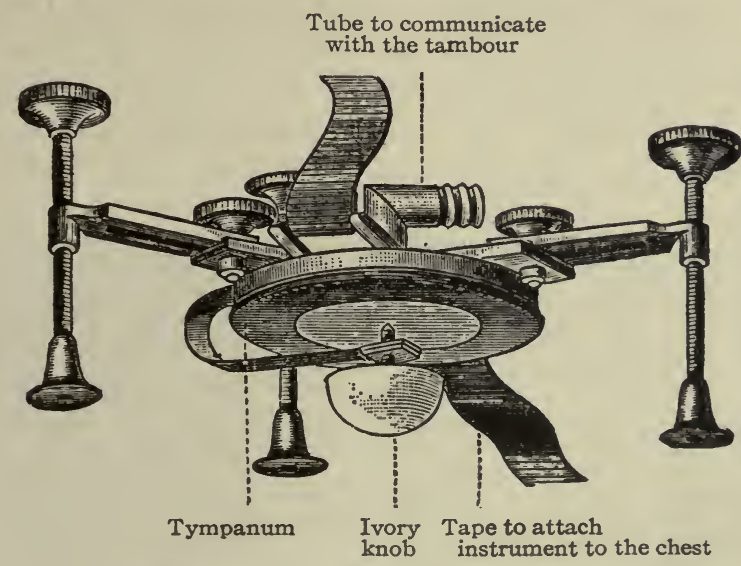

FIG. I55-Cardiograph. (Sanderson's.)

Disease of these valves, when sufficient to interfere with their efficient action, also demonstrates the same fact by modifying the second sound or destroying its distinctness.

The Cardiac Impulse.-The heart may be felt to beat with a slight shock or impulse against the walls of the chest at the level of the fifth intercostal space on the left side. Its extent and character vary in different individuals, a factor of considerable clinical significance, and therefore especially discussed in works on clinical diagnosis. The cause of the cardiac impulse has been variously described. It will be remembered that during the period which precedes the ventricular systole the relaxed heart rests quietly in the pericardial cavity and with its apex exerting no pressure 
against the wall of the chest. When the ventricles contract, their walls suddenly become firm and tense. Being firmly attached to the base the effect of the movement is to press the hardened ventricle against the

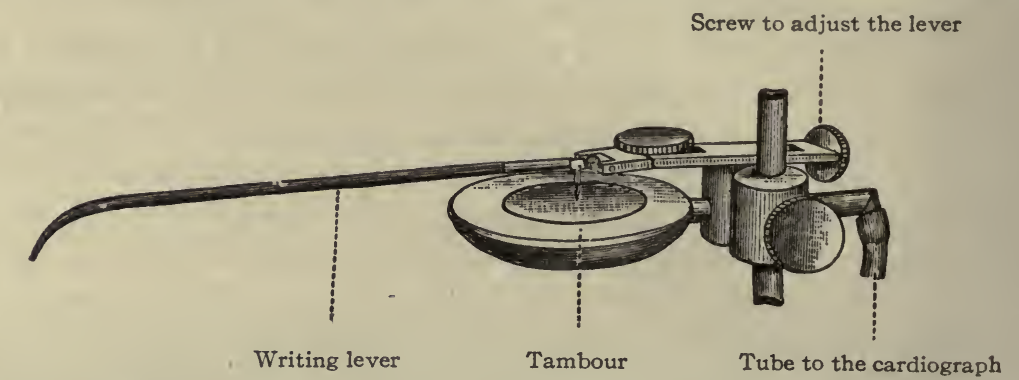

Fig. I56.-Marey's Tambour, to which the Movement of the Column of Air in the Cardiograph is Conducted by a Tube, and from which it is Communicated by the Lever to a Revolving Cylinder so that the tracing of the movement of the cardiac impulse is obtained.

chest wall. The discharge of the contents of the ventricle into the curved aorta intensifies this pressure by its mechanical effect in tending to straighten the curve of that vessel and thus holds the ventricle in firm contact with the chest. It is this sudden pressure of the contracting heart against the chest wall that is felt on the outside. The impact or

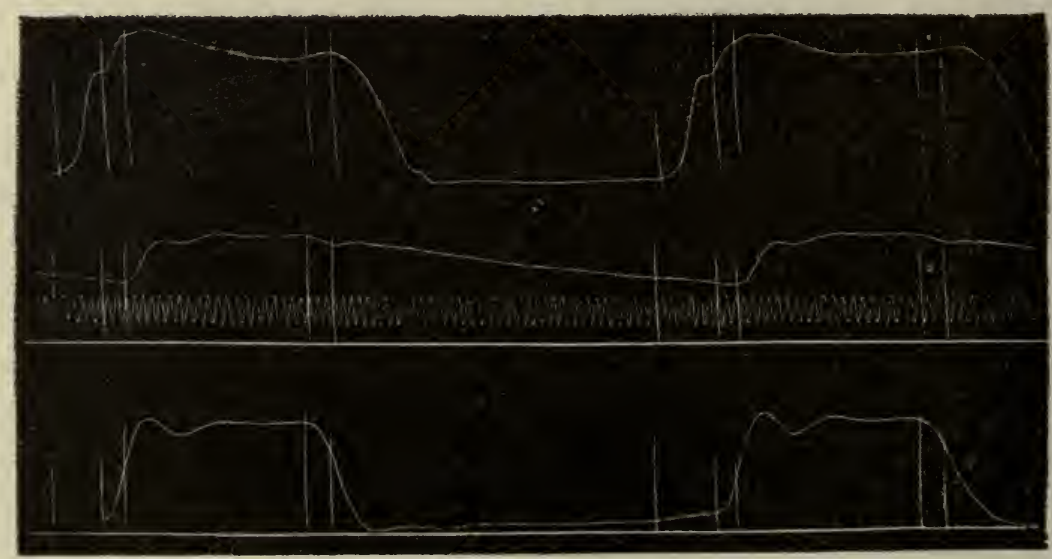

FIG. I57- - Typical Cardiogram (upper trace) from the Dog. Taken simultaneously with the aortic pressure (middle) and intraventricular pressure (lower) tracings. 'I'ime in 0.01 of a second. (Hürthle.)

shock is possibly more distinct because of the partial rotation of the whole heart toward the right and front along its long axis. The movement of the chest wall produced by the ventricular contraction against it may be registered by means of an instrument called the cardiograph; 
and the record or tracing, called a cardiogram, corresponds almost exactly with a tracing obtained by an instrument applied over the contracting ventricle itself.

The cardiograph, figure $\mathrm{r}_{55}$, consists of a cup-shaped metal box over the open front of which is stretched an elastic india-rubber membrane upon which is fixed a small knob of hard wood or ivory. This knob, however, may be attached, as in the figure, to the side of the box by means of a spring, and may be made to act upon a metal disc attached to the elastic membrane.

The knob is for application to the chest wall over the place of the greatest impulse of the heart. The box or tambour communicates by means of an air-tight tube with the interior of a second or recording tambour supplied with. a long and light writing lever figure ${ }_{5} 6$. The shock of the heart's impulse being communicated to the ivory knob, and through it to the first tambour, the effect is, of course, at once transmitted by the column of air in the elastic tube to the interior of the second recording tambour, also closed, and through the elastic and movable disc of the latter to the writing lever which is adjusted to a registering apparatus. This latter generally consists of a cylinder or drum covered with smoked paper and revolves by clock-work with a definite velocity. The point of the lever writing upon the paper produces a tracing of the heart's impulse, a cardiogram.

Endocardiac Pressure.-The effect of the muscular contractions and relaxations of the walls of the heart during its systole and diastole is to produce changes of pressure on its content of blood. When this pressure is measured by the proper instrument it is found that the pressure in the

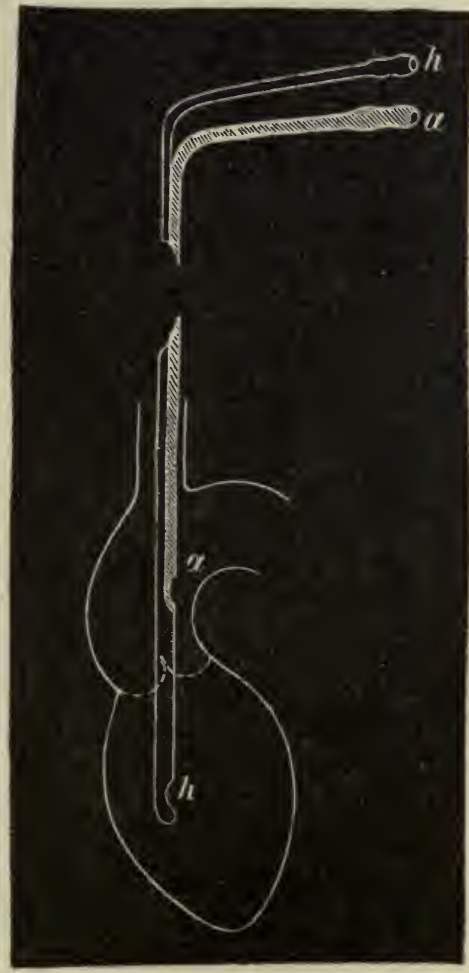

Fig. I58.-Double Cardiac Sound for Simultaneous Registration of the Blood Pressure in the Right Auricle and Ventricle, or in the Aorta and Left Ventricle. (Hürthle.) left ventricle varies between wide ranges. With the beginning of the muscular contraction, the pressure rises till it slightly exceeds that of the pressure of the aorta, remains high for a brief interval of time, then slowly 
and quietly decreases to less than that of atmospheric pressure. It remains low until the beginning of the next systole. For the right ventricle the events and variations are relatively the same.

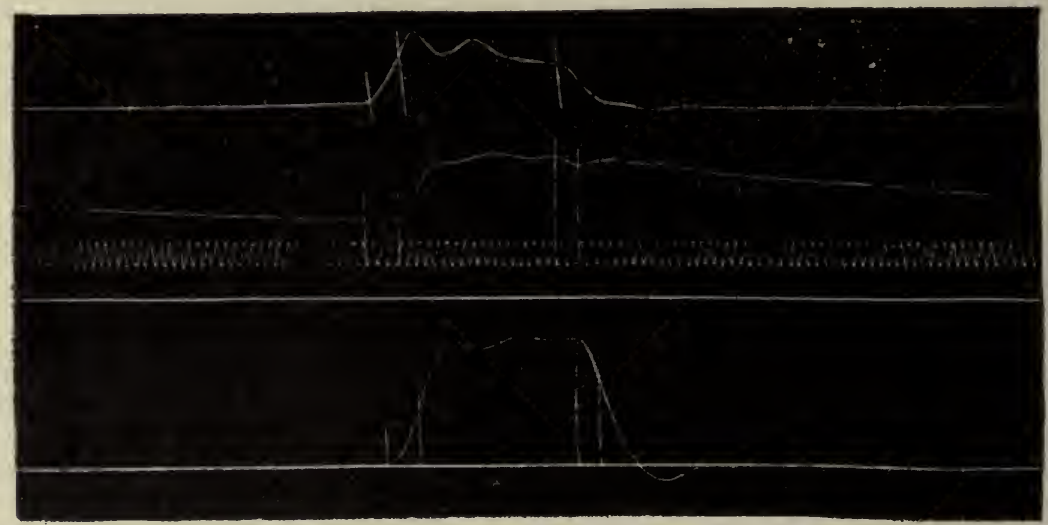

FIG. I59.-Simultaneous Registration of Curves of the Left Intraventricular Pressure (lower), the Aortic Pressure (middle), and the Cardiac Impact (upper). Time, o.or of a second. (Hürthle.)

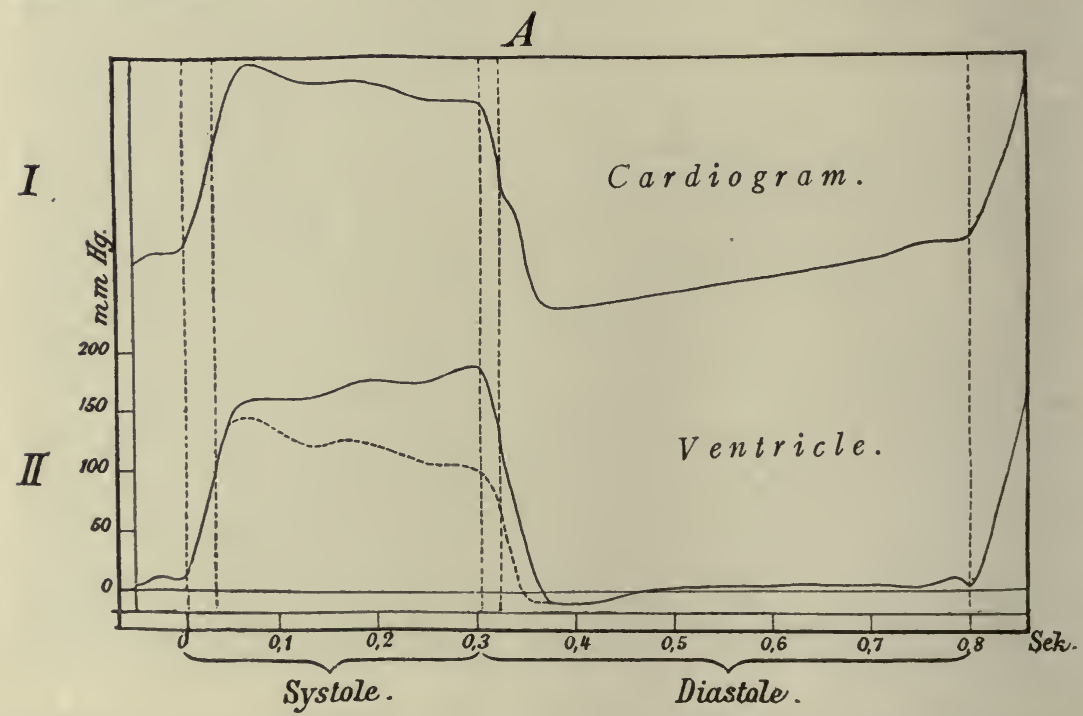

FIG. r6o.-Schematic Cardiogram I, and Intraventricular Pressure Curves II, from the Dog. The ventricular pressure curve of the descending type is represented by the dotted line. Pressure in millimeters of mercury, time in tenths of a second. (Hürthle.)

In order to determine the endocardiac pressure communication must be established with the cavities of the heart. This is accomplished by a tube known as a sound, which is introduced into the left ventricle by passing it down the common carotid artery, or into the right auricle and 
ventricle through the jugular vein. When a cardiac sound is introduced and connected with some form of pressure-recording apparatus, accurate tracings of the variations in pressure during the heart-beat are obtained.

Chauveau and Marey recorded and measured with accuracy the variations of the endocardiac pressure and the comparative duration of

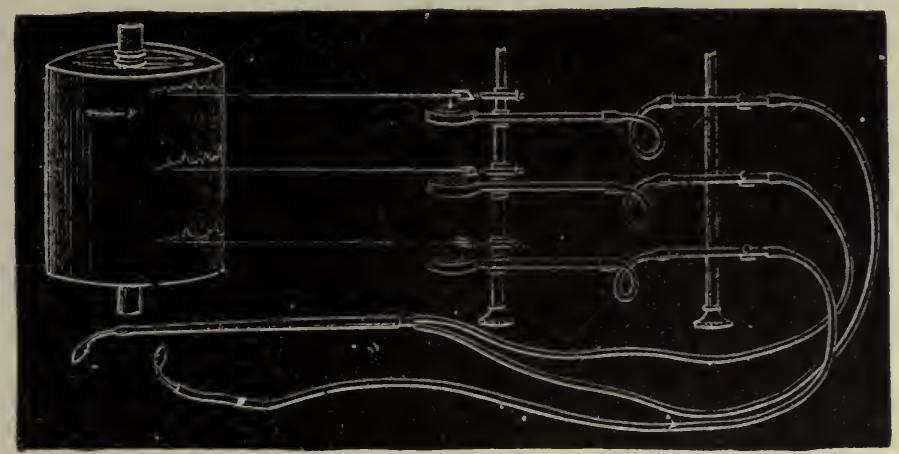

FIG. I6r.-Apparatus of MM. Chauveau and Marey for Estimating the Variations of Endocardiac Pressure, and Production of the Impulse of the Heart.

the contractions of the auricles and ventricles. They placed three small india-rubber air-bags or sounds in the interior, respectively, of the right auricle, the right ventricle, and in an intercostal space in front of the heart of living animals-the horse. These bags were connected by means of

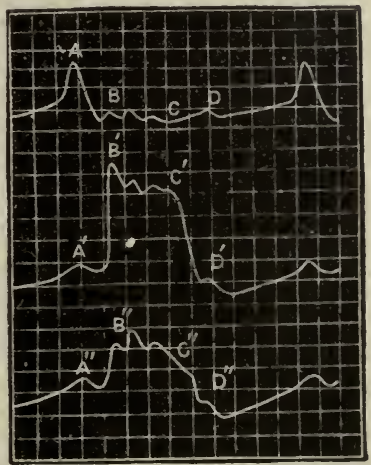

FIG. I62.-Tracings of I, Intra-auricular; 2, Intraventricular Pressures; and 3, of the Cardiac Impulse of the Heart. To be read from left to right. Obtained by Chauveau and Marey.

long narrow tubes with three levers arranged one over the other in connection with a registering apparatus, figure I6r. The synchronism of the impulse with the contraction of the ventricles is also well shown by means of the same apparatus, and the causes of the several vibrations of which it is really composed have been demonstrated. 
In the tracing, figure 162 , the intervals between the vertical lines represent periods of a tenth of a second. The parts on which any given vertical line falls represent simultaneous events. It will be seen that the contraction of the auricle, indicated by the marked curve at $A$ in the first tracing, causes a slight increase of pressure in the ventricle which is shown at $A^{\prime}$ in the second tracing, and produces also a slight impulse, which is indicated by $A^{\prime \prime}$ in the third tracing. The closure of the semilunar valves causes a momentarily increased pressure in the ventricle at $D^{\prime}$, affects the pressure in the auricle $D$, and is also shown in the tracing of the cardiac impulse $D^{\prime \prime}$.

The large curve of the ventricular and the cardiac impulse tracings, between $A^{\prime}$ and $D^{\prime}$, and $A^{\prime \prime}$ and $D^{\prime \prime}$, are caused by the ventricular contraction, while the smaller undulations, between $B$ and $C, B^{\prime}$ and $C^{\prime}, B^{\prime \prime}$ and $C^{\prime \prime}$, are caused by the vibrations consequent on the tightening and closure of the tricuspid and mitral valves.

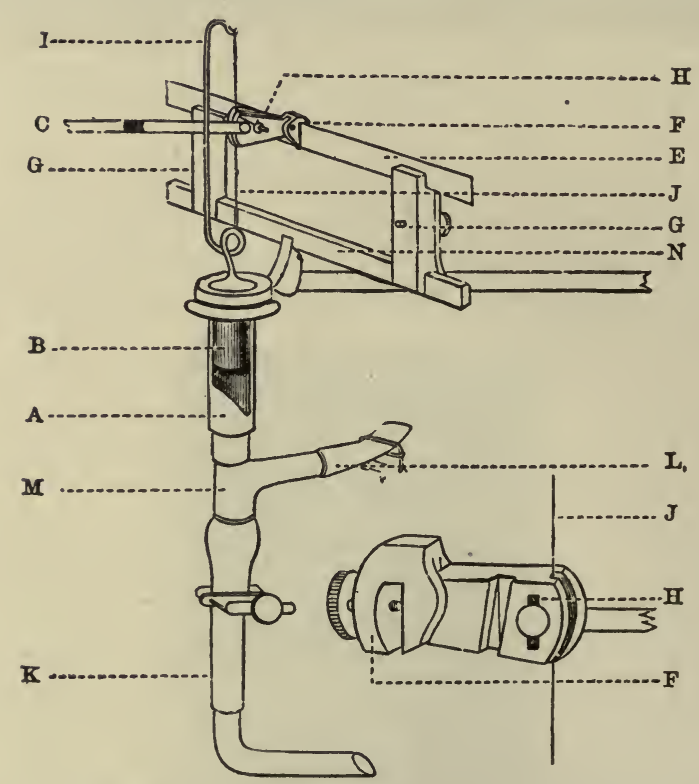

FIG. 163.-Apparatus for Recording the Endocardiac Pressure. (Rolleston.)

It seems by no means certain that Marey's curves properly represent the variations in intraventricular pressure. Objection has been taken to his method of investigation: First, because his tambour arrangement does not admit of both positive and negative pressure being simultaneously recorded; second, because the method is applicable only to large animals, such as a horse; third, because the intraventricular changes of pressure 
are communicated to the recording tambour by a long elastic column of air; and fourth, because the tambour arrangement has a tendency to record inertia vibrations. H. D. Rolleston, who has pointed out the above in perfections of Marey's method, has reinvestigated the subject with a more suitable apparatus.

The method adopted by Rolleston is as follows:

A window is made in the chest of an anesthetized and curarized animal, and an appropriately curved glass cannula introduced through an opening in the auricular appendix. The cannula is then passed through the auriculo-ventric-

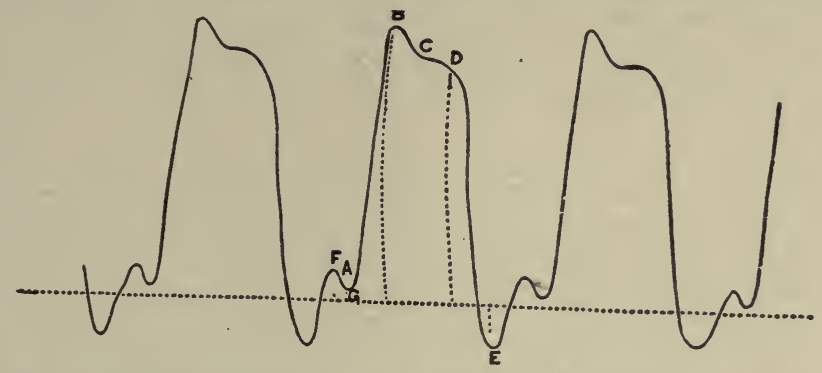

FIG. 164.-Endocardiac Pressure Curve from the Left Ventricle of the Dog. The thorax was opened and a cannula introduced through the apex of the ventricle; the abscissa is the line of atmospheric pressure. $G$ to $D$ represents the ventricular contraction; from $D$ to the next rise at $G$ represents the ventricular diastole. The notch, at the top of which is $F$, is a post-ventricular rise in pressure from below that of the atmosphere and not a presystolic or auricular rise in pressure.

ular orifice without causing any appreciable regurgitation into the auricle. or it may be introduced into the cavity of the right or left ventricle by an opening made in the apex of the heart. In some experiments the trocar is pushed through the chest wall into the ventricular cavity. His apparatus is filled with a solution of leech extract in 0.75 per cent. saline solution, or with a solution of sodium bicarbonate of specific gravity 1.083 .

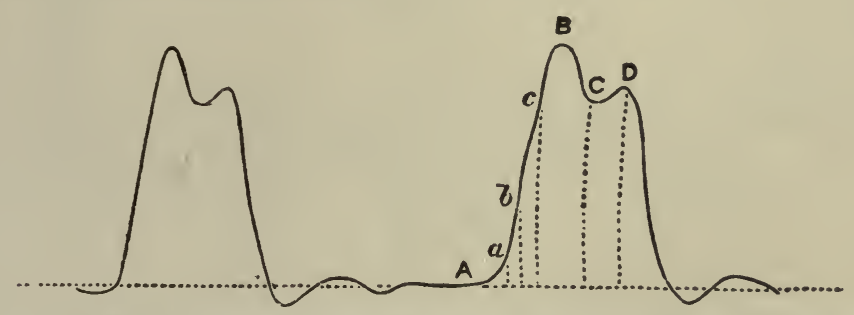

FIG. 165.-Curve with a Dicrotic Summit from the Left Ventricle; the Abscissa Shows the Atmospheric Pressure.

The animals employed were chiefly dogs. The movement of the column of blood is communicated to the writing lever by means of a vulcanite piston which moves with little friction in a brass tube connected with a glass cannula by means of a short connecting tube.

When the lower part of the tube, $A$, is placed in communication with one 
of the cavities of the heart, the movements of the piston are recorded by means of the lever, $C$. Attached to the lever is a section of a pulley, $H$, the axis of which coincides with that of the steel ribbon, $E$; while, firmly fixed to the piston, is the curved steel piston rod, $l$, from the top of which a strong silk thread, $J$, passes downward into the groove on the pulley.

This thread, $J$, after being twisted several times around a small pin at the side of the lever, enters the groove in the pulley from above downward, and then passes to be fixed to the lower part of the curve on the piston rod as shown in the smaller figure.

The movement of the lever, $C$, is controlled by the resistance to torsion of the steel ribbon, $E$, to the middle of which one end of the lever is securely fixed by a light screw clamp, $F$. At some distance from this clamp, the distance varying with the degree of resistance which it is desired to give to the movements of the lever, are two holders, $G, G^{\prime}$, which securely clamp the steel ribbon.

As the torsion of a steel wire or strip follows Hooke's law, the torsion being proportional to the twisting force, the movements of the lever point are proportional to the force employed to twist the steel strip of ribbon-in other words, to the pressures which act on the piston, $B$. To make it possible to record satisfactorily the very varying ventricular and auricular pressures, the resistance to torsion of a steel ribbon adapts itself very conveniently.

This resistance can be varied in two ways, Ist, by using one or more pieces of steel ribbon or by using strips of different thicknesses; or $2 \mathrm{~d}$, by varying the distance between the holders, $G, G^{\prime}$, and the central part of the steel ribbon to which the lever is attached.

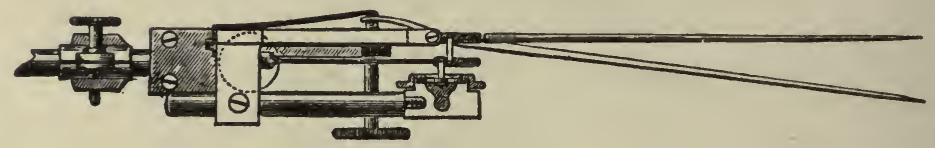

A

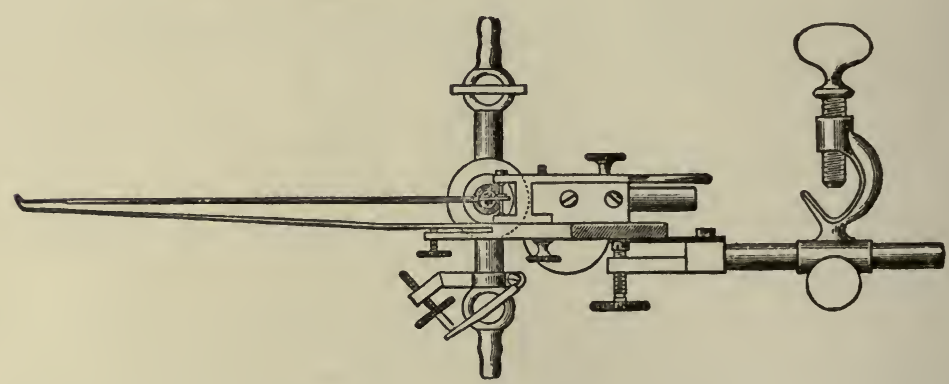

B

FIG. I66.-Hürthle's Spring Manometer. $A$, Viewed from the side; $B$, viewed from the top.

Rolleston's conclusions are: That there is no distinct and separate auricular contraction marked in the pressure curves obtained from the right or the left ventricle, the auricular and ventricular rises of pressure being merged into one continuous rise. He concludes that the tricuspid and mitral valves are closed before there is any great rise of pressure within the ventricle above that which results from the auricular systole, $a$, figure $6_{5}$. The 
closure of the valve occurs probably in the lower third of the rise $A B$, figure 165 , and does not produce any notch or wave. It is shown that the semilunar valve opens at the point in the ventricular systole, situated at $c$, about or a little above the junction of the middle and upper thirds of the ascending line $A B$, and the closure about or a little before the shoulder, $D$. The figures show, finally, that the minimum pressure in the ventricle may fall below that of the atmosphere, but that the amount varies considerably.

On the whole, the most satisfactory recording instrument for the measurement of endocardiac pressures is the membrane manometer devised by Hürthle. This instrument avoids mechanical errors in a most satisfactory manner. By simultaneous tracings of the pressure in the ventricle and in the aorta by Hürthle's differential manometer, the exact moment of the

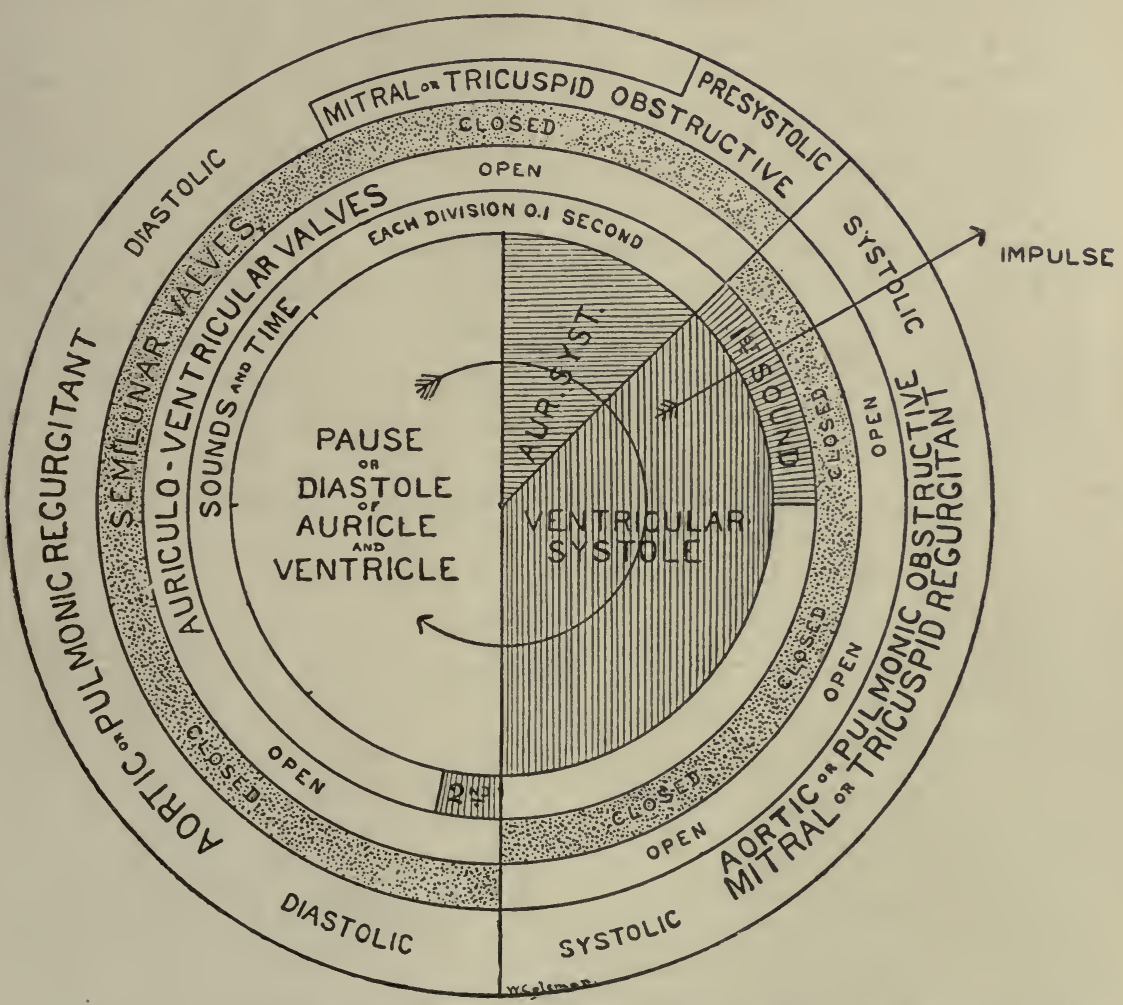

FIG. I67.-Diagrammatic Representation of the Events of the Cardiac Cycle. For events which occur in sequence, read in the direction of the curved arrow; for synchronous events, read from the center to the periphery in any direction. (Coleman.)

opening and closing of the semilunar valve has been determined. By similar methods we have been able to fix synchronism between other events occurring during the beat. These we will summarize in the following section. 
Cardiac Cycle.-The entire series of occurrences in a single heart-beat is called the cardiac cycle. If the condition of the heart is considered at that moment when its muscular walls are at rest it will be found that the tricuspid and mitral valves are open, that the blood is flowing from the great veins into the auricle and the ventricle, which form a continuous cavity, and that the pressure is about that of the atmosphere, but slowly rising. Now a wave of contraction begins at the sinus node and extends over the auricles, which immediately contract and discharge their blood into the ventricles, somewhat distending their walls. At this moment the ventricular systole begins, the tricuspid and mitral valves are closed, the flow of blood into the ventricles is checked, and the first heart sound is heard. The contraction of the ventricles produces a rapidly rising pressure on the enclosed contents until the pressure exceeds that in the pulmonary artery (and aorta), the semilunar valves open, and the blood is discharged into the arteries. The ventricles ordinarily remain contracted for a brief moment after their contents are emptied.

The ventricular diastole begins next. With the initial relaxation and the first slight fall of the intraventricular pressure below that of the aorta, the semilunar valves close and the second sound is heard. The relaxation rapidly proceeds and the intraventricular pressure drops to below atmospheric pressure, the auriculo-ventricular valves fall open, the blood that has been accumulating in the auricles flows into the ventricles and the whole heart is in the state of pause described as the point of beginning of the cycle.

The duration of the cardiac cycle varies with the heart rate. With a rate of 75 per minute, the cardiac cycle will take 0.8 of a second. In round numbers the systole of the auricle takes 0 . I of a second with a diastole of 0.7 of a second, 0.6 of which is in the pause or rest period. The ventricle requires about 0.3 of a second for the systole, 0.5 of a second for the diastole, with 0.2 to 0.3 of this for the pause. It is evident that the whole heart is at rest at the same instant for from 0. I to 0.2 of a second.

The relations of the cardiac sounds to the systole and the diastole have been graphically recorded by Hürthle, figure 153 , and by Einthoven and Geluk, figure $x_{54}$. The former found that in a heart-beat lasting 0.76 of a second the interval of time between the beginning of the first and second sounds was 0.25 of a second, and that the sounds occur just at the beginning of the ventricular systole and diastole, respectively.

During the cardiac cycle the ventricles are completely closed from the moment of the beginning of the ventricular systole until the pressure amounts to a little greater than the pressure in the corresponding arteries, which takes about 0.2 of a second. From the opening of the semilunar valves until the closure of those valves, about 0.15 of a second, the ventricular cavity is in open communication with the arteries. There is, during the diastole, 
a second moment of complete closure of the ventricles, from the time of the closing of the semilunar valves until the ventricular pressure falls below the auricular pressure which permits the tricuspid and mitral valves to open.

The Force of the Cardiac Action.-In estimating the amount of work done by a machine it is usual to express it in terms of work units. A convenient work unit for this purpose is the amount of energy required to lift a unit of weight, i.e., I gram or I kilogram, through a unit of height; i.e., I centimeter or I meter, the work required being I gramcentimeter for small units, and I kilogrammeter for large units, respectively. The average work done by the heart at each contraction can be readily computed by multiplying the weight of blood expelled by the ventricles by the height through which it would have to be lifted to overcome the resistance to its discharge from the cavities into the arteries.

The quantity of blood expelled and the pressure of the arteries can only be estimated for man. But the computations from indirect observations on other mammals indicate that the quantity of blood discharged from each ventricle at a single contraction is from 80 to roo c.c. The pressure of the aorta, see page $22 \mathrm{I}$, is an average of, say, $\mathrm{I} 20 \mathrm{~mm}$. of mercury, or $\mathrm{I} 26 \mathrm{~cm}$. of blood. The pressure in the pulmonary artery is much less, say, $30 \mathrm{~mm}$. (20 to 40 ), of mercury or $40 \mathrm{~cm}$. of blood. Collecting these facts, we have the following computation:

\begin{tabular}{|c|c|c|c|}
\hline & $\begin{array}{c}\text { Blood } \\
\text { discharged. }\end{array}$ & $\begin{array}{l}\text { Against } \\
\text { pressure } \\
\text { column of } \\
\text { blood. }\end{array}$ & $\begin{array}{l}\text { Work in } \\
\text { gramcenti- } \\
\text { meters. }\end{array}$ \\
\hline $\begin{array}{l}\text { The left ventricle } \ldots \ldots \ldots \ldots \ldots \ldots \\
\text { The right ventricle } \ldots \ldots \ldots \ldots \ldots\end{array}$ & $\begin{array}{l}90 \text { c.c. } \\
90 \text { c.c. }\end{array}$ & $\begin{array}{l}156 \mathrm{~cm} \\
40 \mathrm{~cm}\end{array}$ & $\begin{array}{r}14,040 \\
3,600\end{array}$ \\
\hline Total. & 90 c.c. & $240 \mathrm{~cm}$ & r 7,640 \\
\hline
\end{tabular}

This computation shows that each heart-beat expends $I_{7}, 640$ gramcentimeters ( 7.64 grammeters) of work. The amount of energy developed in the contractions of the auricles may be ignored in this calculation, which is at best only of relative value. Calculations based on the determinations of Vierordt, also other earlier determinations, give much higher figures than are presented here.

The Properties of the Heart Muscle.-It is evident that if we are to arrive at any adequate explanation of the action of the heart, one of the first questions that must be considered is, what are the fundamental properties of heart muscle as such? 
It has already been shown, page 62 , that the muscular fibers of the heart differ in structure from skeletal muscle fibers on the one hand, and from unstriped muscle on the other, occupying an intermediate position

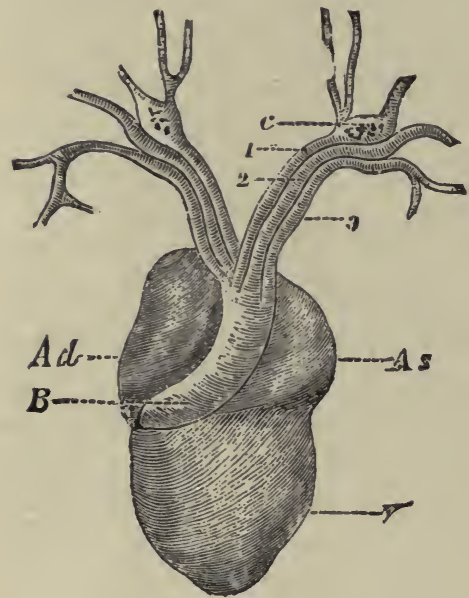

Fig. 168.

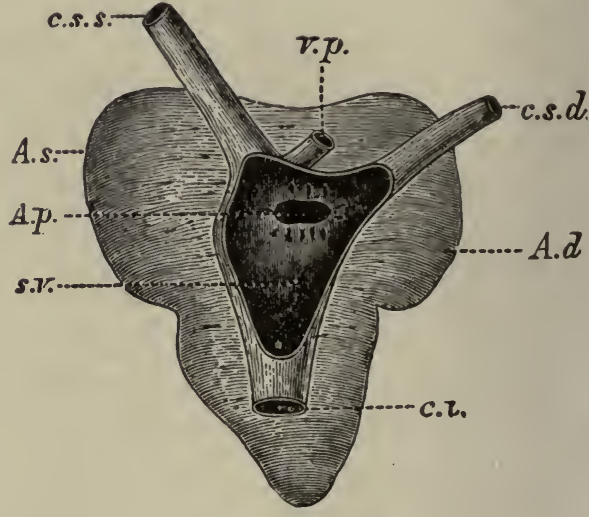

Fig. I69.

FIG. I68. - The Heart of a Frog (Rana esculenta), from the Front. $V$, Ventricle; Ad right auricle; $A s$, left auricle; $B$, bulbus arteriosus, dividing into right and left aortæ (Ecker.)

FIG. I69. - The Heart of a Frog (Rana esculenta), from the Back. s. v., Sinus venosus opened; $c . s$. s., left vena cava superior; $c . s$. $d$., right vena cava superior; $c$. $i$., vena cava inferior; $v_{\text {. }} p_{\text {., }}$ vena pulmonales; $A . d_{\text {. }}$, right auricle; $A$. s. left auricle; $A_{\text {. }} p_{.}$, opening of communication between the right auricle and the sinus venosus. $\times 2 \frac{1}{2}-3$. (Ecker.)

between the two varieties. The heart muscle, however, possesses a property which is not possessed by skeletal muscle, or by unstriped muscle to such a degree, namely, the property of contracting rhythmically.

Rhythmicity.-The property of rhythmic contraction is shown by the action of the heart within the body; its systole is followed by its diastole in

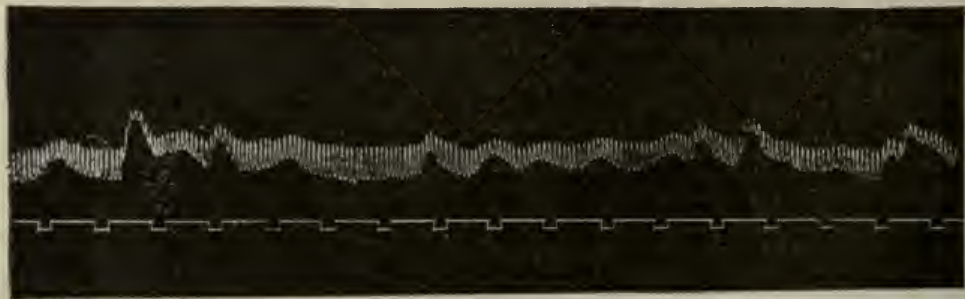

FIG. I70.-Automatic Contractions of Sinus Muscle from the Terrapin's Heart in 0.7 per cent. Sodium Chloride. Time in minutes. (New figure by L. Frazier.)

regular sequence throughout the life of the individual. The force and frequency of the systole may vary from time to time as occasion requires, but there is no interruption to the action of the normal heart or any interfer- 
ence with its rhythmical contractions. Further, in an animal rapidly bled to death, the heart continues to beat for a time, varying in duration with the kind of animal experimentally dealt with and depending on whether or not there is entire absence of blood within the heart chambers. Furthermore, if the heart of an animal be removed from the body, it still continue its alternate systolic and diastolic movements for a varying time. Thus we see the power of rhythmic contraction depends neither upon connection with the central nervous system nor yet upon the stimulation produced by the presence of blood within its chambers. Whether or not rhy thmicity is a property of heart muscle, as such, was conclusively settled by Gaskell and by numerous later investigators by a very simple experimental procedure. Gaskell cut thin strips of the apex of the ventricle of the terrapin, which is free from the nerve cells, at least nerve ganglia, and found that they contracted rhythmically for hours. This experiment has become a classic one for the study of the cardiac muscular tissue. Strips of cardiac muscle cut from the auricle and from the contractile walls of the venæ cavæ, or sinus venosus, of the terrapin also contract rhythmically. If the strips of muscle are kept moist with the same blood or serum, then the rhythm of the sinus is greater than that of the auricle, and that of the auricle greater than that of the ventricle, a difference that is based on a physiological differentiation of the tissue. The sinus muscle is also more delicately responsive to stimuli than is the ventricular muscle; i.e., it is more irritable.

Porter first performed the more difficult experiment of isolating a small disc of muscle from the ventricle of the dog, leaving only the delicate nutrient artery through which the muscle was fed with defibrinated blood. This isolated small piece of ventricle contracted vigorously for many minutes. Moorhouse has recently shown that various portions of the auricle, the sinus, and the veins contract in good rhythm. They also respond to various drugs in a characteristic
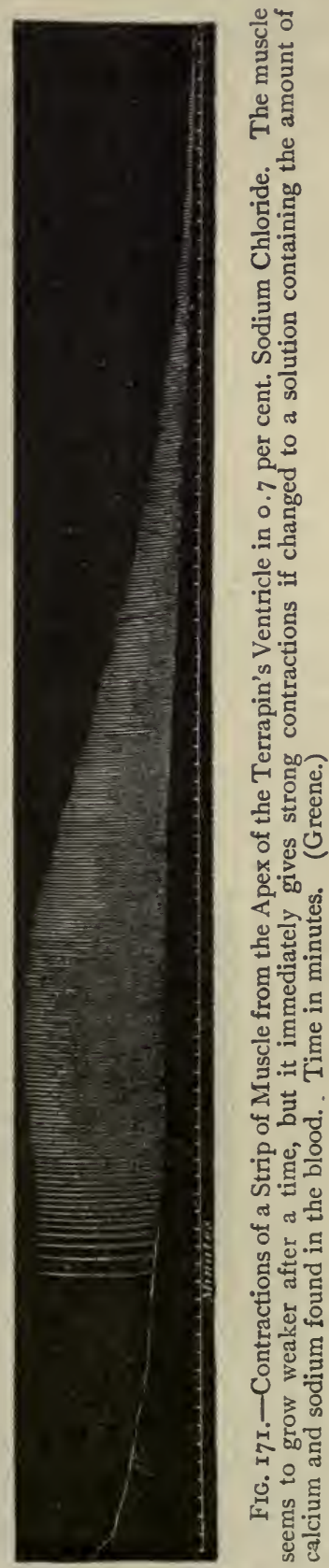
fashion. We may safely conclude, therefore, that the mammalian heart muscle is also automatically rhythmic.

Tonicity.-Cardiac muscle is characterized by its maintaining a constant degree of partial contraction described as muscle tone, or tonicity.

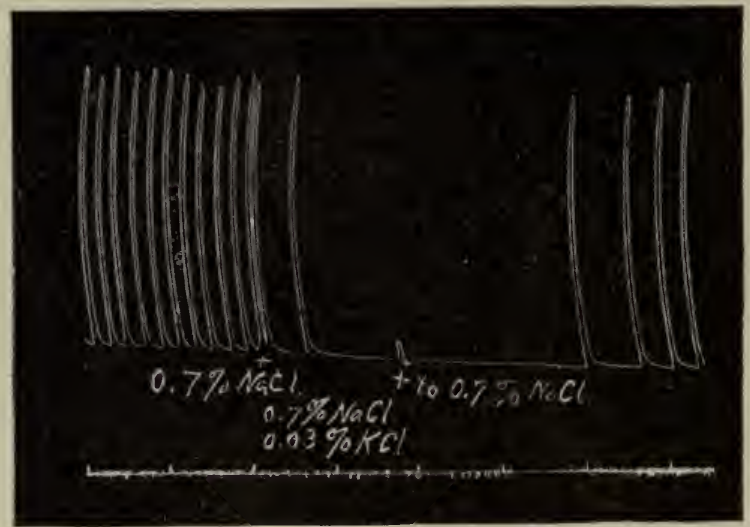

Fig. I 72.-Automatic Contractions of a Strip of Ventricular Muscle from the Apex of the Terrapin's Heart contracting in 0.7 per cent. Sodium Chloride; from + to +0.03 per cent. Potassium Chloride is added to the Sodium Chloride. The thythm is recovered very slowly when the muscle is returned to 0.7 per cent. sodium chloride. Time in minutes (upper) and seconds (lower stroke). (Watkins and Elliott.)

This property is possessed by all parts of the heart. In the auricle, however, and especially in the muscular walls of the sinus and veins, there is considerable variation in tonicity. Botazzi showed that in the auricle of

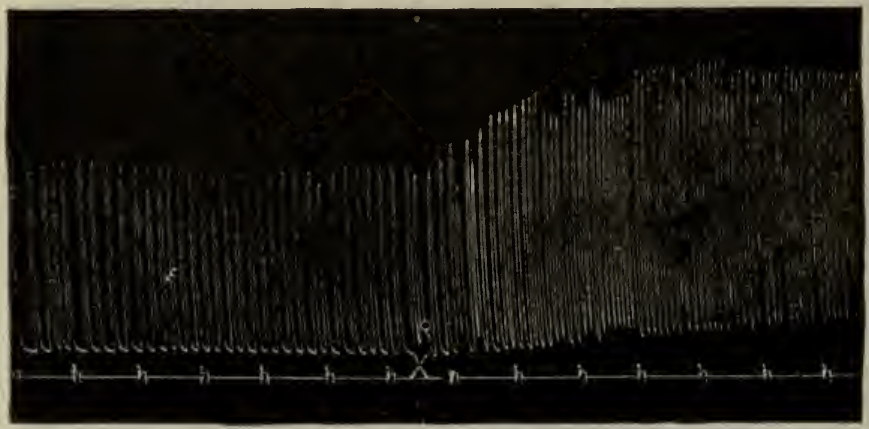

Frg. I 73.-Automatic Contractions of a Strip of Ventricular Muscle from the Apex of the Terrapin's Heart. $a$, Contracting in 0.7 per cent. sodium chloride; $b$, when 0.03 per cent. calcium chloride solution is added. Time in minutes. (Frazier.)

the toad the variations of tone were wave-like and periodic, even though the auricle were contracting with its ordinary fundamental rhythm. Howell has published numerous experiments showing tone waves in auricular and sinus muscle of the terrapin, in which muscle there may or may 
not be occurring at the same time the ordinary fundamental rhythmic contractions, figure $\mathrm{I} 70$.

Irritability of Heart Muscle.-Mention was made above of the difference in irritability of heart muscle chosen from different parts of the heart. The irritability of the muscle of each part also varies during the different stages of the contraction. Experiment shows that the muscle is not irritable to a stimulus applied at any time from the beginning of the contraction until the summit of the contraction is reached. This is called the refractory period. From the summit, through the relaxation and succeeding pause, the irritability rapidly increases until the beginning of the next contraction. Considering the automatically contracting muscle, the point in which the automatic contraction is released, i.e., begins, is the point of maximal irritability. It is the moment when the irritability is so great that the muscular equilibrium is no longer stable, and the physiological contraction results.

The irritability of heart muscle is very sharply influenced by its condition of nutrition, especially by the inorganic salts present in the blood and lymph, see page 207. The salt content of the blood comprises about 0.7 per cent. sodium chloride, 0.03 per cent. potassium chloride, and 0.025 to 0.03 per cent. calcium (phosphate probably), as well as traces of other metal bases.

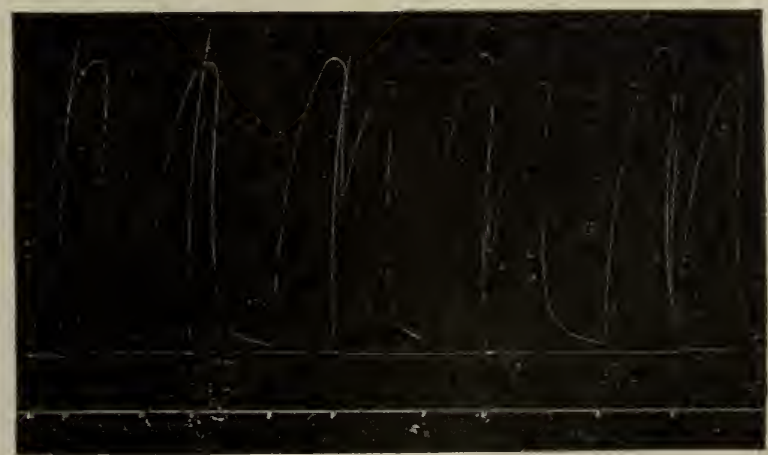

FIG. I74.-Refractory Period in the Ventricular Strip of the Terrapin.

The heart muscle has been shown by numerous investigators to be delicately responsive to the proportions of these salts in the blood, or in any artificial solution which may be substituted for blood. If the rhythm is to be taken as an index of the irritability, then an increase of sodium and calcium salts increases the irritability (rhythm), while the influence of an increase in potassium is to depress the irritability.

Cardiac Contractions Always Maximal. - The heart muscle exhibits another property which distinguishes it from ordinary skeletal muscle, viz., the way in which it reacts to stimuli. The latter, Chapter XIII, reacts slightly to a stimulus little above the minimal, and with an increase of the 
strength of the stimulus will give contractions of increasing amplitude until the maximum contraction is reached. In the case of the heart-beats this is not so, since the minimal stimulus which has any effect is followed by the maximum contraction; in other words, the weakest effectual stimulus brings out as great a contraction as the strongest. If a contraction is induced earlier than it would automatically occur, then the succeeding pause is longer; i.e., there is a compensatory pause. Also the contraction induced is smaller and the one following the compensatory pause is proportionately larger. This observation can easily be demonstrated on the heart strip, see figure I 74, or on the whole ventricle of the frog, which was originally used by Bowditch.

Nerve influence, nutrition, temperature, etc., will of course affect the extent of the contractions, but under a given set of conditions it is held that the contractions which occur are maximal for the particular set of nutritive and other conditions. This is more readily understood when taken in connection with the fact that when a contraction originates in a cardiac cell it is conducted throughout the continuity of all the cells of the mass.

Theories of the Heart-beat.-The cause of the rhythmic power of the heart as a whole has been the subject of much discussion and experimental observation. Two leading hypotheses have given inspiration to investigators, and now one, now the other theory has attracted followers as new facts have been discovered. These are known as the neurogenic theory and the myogenic theory, respectively, though neither is proven correct.

The heart has long been known to have the power of rhythmic contractions after removal from the body and after all connection with the central nervous system has been destroyed.

The heart can be taken entirely away from the body of an animal and kept beating rhythmically with ease. This is true for many invertebrates and for all vertebrates examined including fishes, frogs, turtles, snakes, birds, and numerous mammals including man himself. It is only necessary to supply the heart through its local circulatory vessels with the proper nutritive fluid well aerated with oxygen and at the normal temperature of the animal from which the heart is taken. The question long debated is this. What initiates these wonderfully persistent and regularly repeated contractions?

If the frog's or terrapin's heart is removed from the body entire, it will continue to contract for many hours and even days, and the contractions have no apparent difference from the contractions of the heart before removal. The contractions will take place, as we have mentioned, without the presence of blood or other fluid within its chambers. Not only is this the case, but the auricles and ventricle may be cut off from the sinus, and all parts continue to pulsate. Further, the auricles may be divided 
from the ventricle with the same result. If the heart be divided lengthwise, its parts will continue to pulsate rhythmically. The ventricle remains comparatively quiet, contractions occurring at longer intervals, if at all. However, the isolated ventricle remains irritable so long as bathed in blood or in a balanced Ringer solution, and will contract upon receiving a slight stimulus. In fact, a single stimulus will often call forth a series of contractions of the ventricle. The frog's ventricle, when its muscular
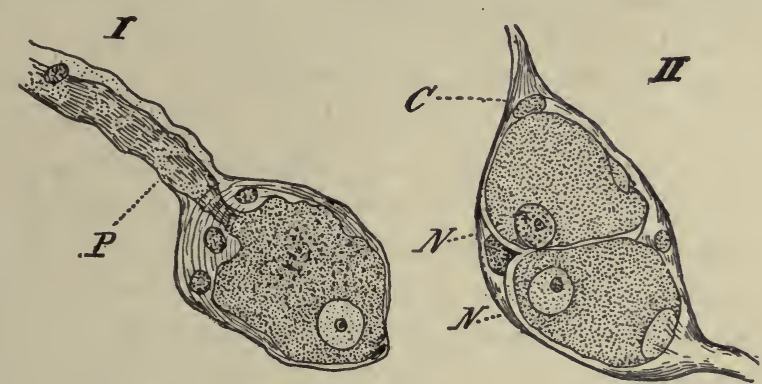

FIG. 175.-Isolated Nerve Cells from the Frog's Heart. I, Usual form; II, twin cell; $C$, capsule; $N$, nucleus; $P$, process. (From Ecker.)

and nervous connections with the auricle are physiologically severed, as by crushing, will remain quiet when fed by its own blood, though it will contract rhythmically when fed with physiological salt solution.

It is thus seen that the rhythmical movements of these parts of the heart appear to be more marked in the parts at the venous end of the organ, i.e., the sinus and auricle, and less marked in the ventricular end. Ventricular pieces contract when still connected with the auricles but do not readily contract in the ordinary condition even when irrigated with blood. These are regarded as facts peculiarly in favor of the view that the rhythm is inherent in the special nervous elements of the heart.

This view which has long been known as the neurogenic theory, attributes the remarkable power of the heart to continue contractions after removal from the body, and presumably while in the body, to the presence of the collections of nerve cells within the walls of the heart itself. The local nervous mechanism in the frog consists of three chief groups of cells or ganglia. The first group is situated in the wall of the sinus venosus at the junction of the sinus with the right auricle, Remak's ganglion. The second group is placed near the junction between the auricles and ventricles, Bidder's ganglion. The third is in the septum between the auricles, von Bezold's ganglion. Small ganglia have been described for the base of the ventricle, but no ganglia are present in the apical part of the ventricles, though isolated neurones have been found. The nerve cells of which these ganglia are composed are generally unipolar, seldom bipolar. Some- 
times two cells are said to exist in the same envelope, constituting the twin cells of Dogiel. The cells are large, and have very large round nuclei and nucleoli, figure I75. The neurogenic theory assumes that the periodic discharge of motor nerve impulses takes place from these neurones thus stimulating the musculature of the heart to rhythmic contractions. The stimuli start at the region of the sinus and are conducted over the heart in orderly sequence, but their origin in nerves is questioned.

In the myogenic theory the heart's rhythmical contractions are explained as due to the inherent property of the cardiac muscle itself. Most convincing facts in support of this theory have been arrived at by a study of cardiac muscle, as such, and by studies on the whole heart, particularly by Gaskell's method of blocking. The term blocking is explained as follows: It will be remembered that under normal conditions the wave of the contractions in the heart starts at the sinus and travels down over the auricles to the ventricles. The irritability of the muscle and its power of rhythmic contractions is greatest in the sinus, less in the auricles, and least in the ventricles. By an arrangement of ligatures or by a system of clamping, one part of the heart may be more or less isolated from any other portion. With such a clamp the contraction waves can be more or less completely interrupted in their passage from the sinus end of the heart past the clamp toward the ventricular end. If the clamp is complete, so as to interrupt the physiological continuity between the parts, then any contractions in the apical portion will be entirely independent of those in the sino-auricular portion. If the blocking is partial only, then the ventricular end of the heart ordinarily contracts in unison with the sino-auricular, although its rate may be as I to 2, I to 3 , etc. In other words, only every second or every third sino-auricular contraction will be able to pass the block to the ventricle.

The effects of blocking are due to the interruption of muscle continuity rather than to nerve continuity. This is beautifully demonstrated by an experiment of zigzag cutting of the ventricle in the terrapin, since it cannot be supposed that any nerves would pass through the ventricular mass by such a zigzag course. In this experiment the contraction wave passes down over the muscle and around the end of the cuts until it reaches the apex. The apex muscle contracts in sequence with the auricle and successive pieces of the ventricle. If the zigzag cuts are made almost complete so as to reduce to a minimum the muscular tissue which bridges from one cut to the next, then it happens that occasional contractions will be unable to pass and the apex contracts after its preceding piece in the ratio of $\mathrm{I}$ to 2 , or $\mathrm{I}$ to 3 , etc., as described above Thus, division of the muscle has the same effect as partial clamping in the same position. These facts all point to a greater power of rhythmicity in the cardiac tissue nearer the venous end of the heart. This difference of rhythmicity 
is not due to the nerves of the heart, say the myogenists, but to the inherent property of the muscle itself.

It was thought for a long time that in the mammal there was no muscular continuity between the auricles and ventricles to conduct the contraction wave and that this was an insurmountable difficulty in the way of accepting the myogenic theory of the heart beat. In I 893 Kent described a bundle of muscle fibers arising in the wall of the right auricle and near the septum and running down into and forming a muscular connection with the ventricles. This bundle was also independently described by His, Jr., and generally bears his name. This band is called the auriculo-ventricular bundle.

It is now generally recognized that the early embryonic cardiac tissue undergoes differentiation in two directions. Out of one of these types of tissue there is produced the wellknown cardiac muscular tissue which makes up the mass of the auricles and the ventricles. Out of the other differentiation is produced the type of tissue which constitutes the auriculo-ventricular conducting or bundle system. This conducting type of tissue is striated like the ordinary cardiac tissue but in general appearance is more embryonic in type. Its cells constitute what is known as the Purkinje fibers. The main bundle described by His, Jr., runs in the interventricular septum somewhat lightly buried in the tissue beginning at the base of the auricle on the right side and running down through the interauricular tissue to the septum of the ventricles where it divides into a right and left branch. Strands of this tissue extend somewhat up into the auricles but are elaborately developed in to a net work lying just underneath the endothelium of the right and left ventricles. The branching net work of these cells shades into and is continuous with cells of the ordinary cardiac type. Miss De Witt (I909) made an excellent model of this system which has become classic in the literature and is reproduced in figure I06. The bundle system contains two regions known as nodes. the sino-auricular node imbedded in the wall of the right auricle just in the angle where it is joined by the superior vena cava; and the auriculoventricular node, described first by Tawara, which lies in the upper end of the His bundle. The physiological differentiation of this tissue is in the direction of rhythm production and facilitated conduction.

The demonstration of the auriculoventricular bundle has proven to be of the strongest support to the myogenic theory. Erlanger has shown, by an ingenious device for partially clamping this muscular band, that even the mammalian ventricle exhibits the phenomenon of heart block. The sequence of auricle and ventricle can be perfectly controlled by the degree of compression exerted by the clamp. In his experiments the ventricle contracts in unison with every auricular contraction, or only every second or every third, according to the degree of blocking. 
It was shown along ago (by Merunowicz in 1875 ) that the isolated apex of the ventricle of the frog remains quiet when filled with blood, but readily gives good rhythmic contractions in physiological saline and other artificial solutions. The inactivity in blood is not necessarily, therefore, due to nervous isolation from the ganglionated parts of the heart nor to the bundle system alone. Contractions occur in the small bits of ventricular muscle as isolated by Gaskell, and these may continue for hours. It is well known also that the embryonic heart contracts rhythmically before nerve cells have reached the organ or even before any blood is formed, as shown in the embryos of certain fishes.

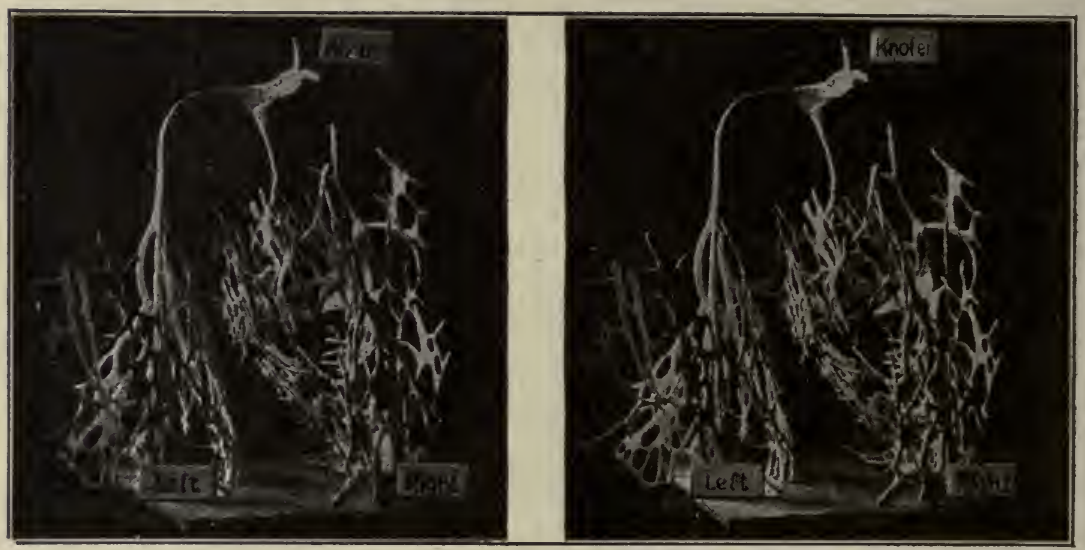

FIG. I76.- Stereoscopic photograph of a model of the atrioventricular nodal system in the calf's heart. Viewed from behind. The auricular network is not shown. Should be examined through a stereoscope. (Lydia M. DeWitt.)

The phenomena of heart block, the contractions of the ventricular apex when physiologically isolated from the parts of the heart which contain the ganglia, the behavior of isolated strips of the heart, especially of the ventricle and the rhythm of the embryonic heart are all held to be in favor of the myogenic theory. However, in light of recent developments we must find our explanation of the block phenomena as well as of other facts used in argument for the myogenic theory in the physiology of this differentiation from the embryonic muscle, namely, the bundle system.

\section{PRESENT VIEWS OF THE AUTOMATICITY OF THE HEART.}

Whatever view one adopts of the heart's beat he has to explain not only the periodic origin of the rhythm but also to explain the orderly sequence of auricles and ventricles. Keith and Flack (I906) have ascribed the initial rhythm to a center or node, the sino-auricular node as given above. In the mammalian heart the normal beat under normal conditions is 
generated at this point and conduction proceeds in an orderly manner in all directions not only toward the ventricle but out over the atria and on to the veins themselves. These last points have been most carefully studied and

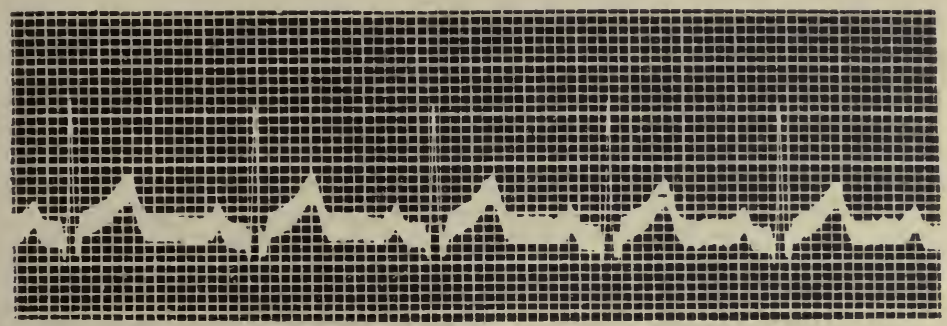

FIG. I 76a.-Normal electrocardiogram of man, lead II.

established by Lewis and a number of his associates. Tawara, Eyster and Meek, Wilson, Greene and Gilbert, and others have explained that under certain conditions rhythm may arise lower down in the conducting tissue, namely, at the auriculo-ventricular node or center or even as low as the bundle branch (Greene and Gilbert). In this case the conduction is from the point of rhythm production not only toward the ventricles which contract together in response to the stimulus reaching them from the auriculo-ventricular node, but conduction is reversed toward the auricle leading to delay in the auricular contraction in comparsion with the normal.

It is obvious that the tissue of the bundle system is differentiated highly in the direction of rhythm production and of conductivity. When once the rhythm arises in the sino-auricular node the stimulus is conducted five to ten times more rapidly over the bundle system to the proper auricular tissue and to the larger ventricular mass than would be possible through the slower conducting cardiac muscle. This insures the contraction of the entire

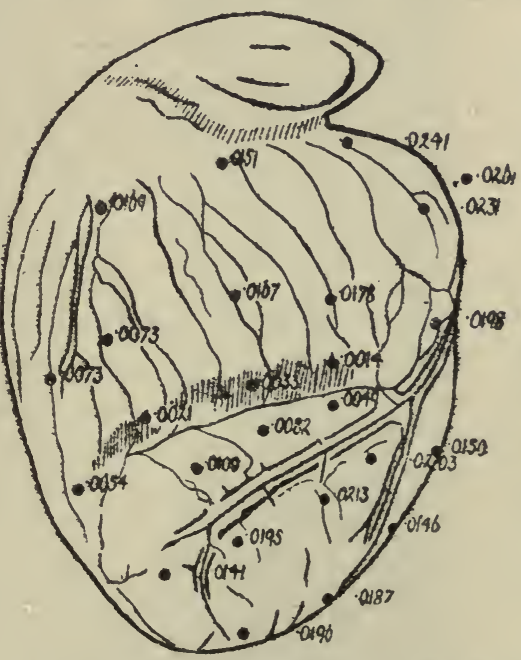

FIG. I $76 b$.-The figure gives the times of beginning contraction of the respective points measured in fractions of a second after the beginning of the $\mathrm{R}$ in lead II. The contraction of the right ventricular wall begins in a horizontal band about the central area and occurs latest near the base of the aorta. (Lewis.) ventricular walls at more nearly the same instant than would otherwise be the case. In fact, the arrangement of muscle bands of the ventricle 
does not materially influence the spread of the conduction wave over the ventricular walls (Lewis) as was once supposed. The Purkinje system furnishes the shorter pathway. These facts have been demonstrated

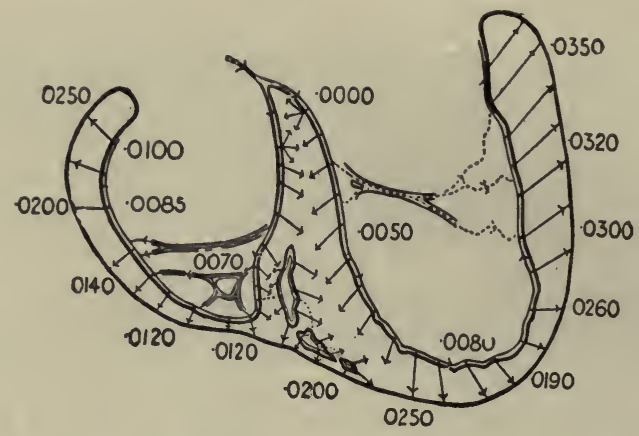

FIG. I 76c.-Transection of the dog's heart to show the spread of the conduction stimulus from the bundle to the different parts of the ventricular walls, and the delay in conduction through the walls by muscle paths. (Lewis.)

by recording the time of arrival of the stimulus at different points on the ventricular wall, figure $176 b$. The companion figure, $176 c$, gives a schematic transection of the dog's heart to indicate the direction

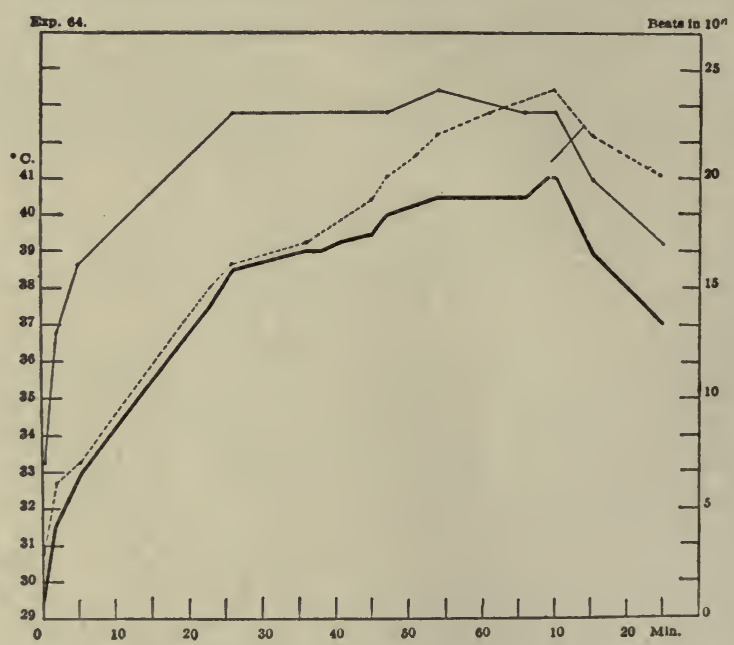

Fig. I77.- The rate of different isolated strips of mammalian ventricle under the influence of changes of temperature. coronary strip, ture in degrees centigrade, rate in beats per ten seconds. (Moorehouse.)

of spread of the stimulus from the inner surface into and through the muscle walls of the two ventricles. The delay in sequence between the auricles and ventricles is represented by this rate of conduction through 
the bundle system. It is approximately o.I6 seconds. The time at which the contraction stimulus arrives at different points on the ventricles, measured from the moment of the beginning of the $R$ wave is illustrated in Figure $176 c$.

This newer conception of the heart's differentiations gives to the true cardiac muscle a position in energy production under the direct control of the conducting system for its coordination. To the conducting system is relegated the function of stimulus production and a rapid distribution of the stimulus that still preserves a mechanically efficient sequence.

The function of the nerve elements by this view is neither to initiate rhythm production nor control the orderly sequence of the beats but to regulate the whole organ as regards its four main functions, namely, rhythm, conduction, energy production, and irritability. The details of this regulation are discussed later.

Relation of Rhythm to Nutrition.-The whole heart, like the muscular parts of which it is composed, responds delicately to its condition of nutrition. In the frog and the turtle hearts the muscular fibers are brought in intimate contact with the blood contained within the cavities. In the mammalian heart, on the other hand, a distinct system of vessels, the coronary vessels and the vessels of Thebesius, supply blood to the walls. If the heart is supplied with nutrient fluid similar to its normal blood, and with proper aeration to insure plenty of oxygen, it contracts with a strong rhythm for many hours. This rhythm, however, responds quickly to changes in the composition of the nutrient fluid. An abundant supply of oxygen is absolutely necessary to the maintenance of rhythm in the mammalian heart, though the heart, especially a cold-blooded heart, will contract for a time in an atmosphere of hydrogen. No doubt the organic constituents of blood are very essential to the prolonged maintenance of rhythm in the heart, but the heart is not dependent on these ingredients for its stimulus

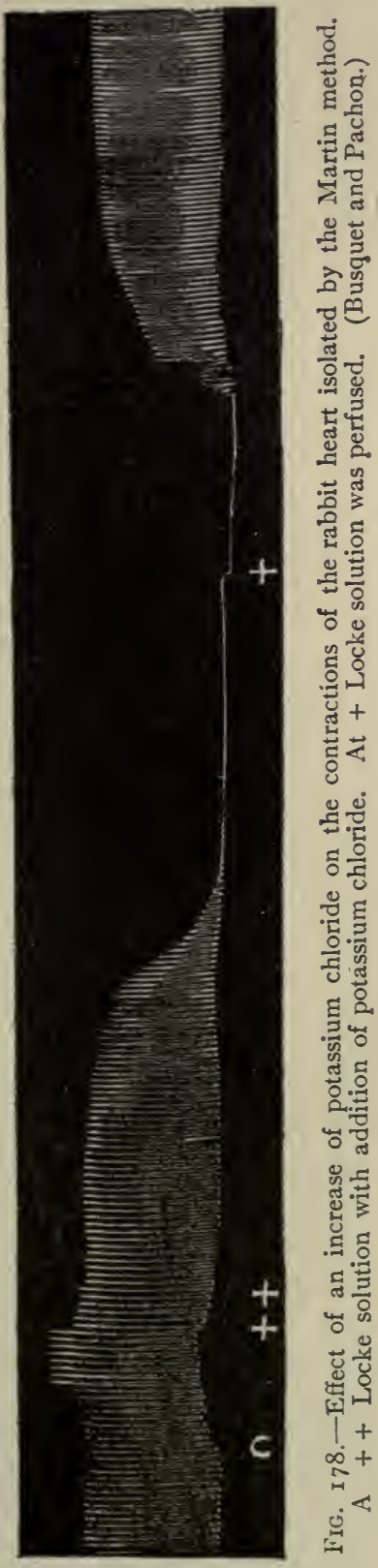


production. The inorganic salts seem to be peculiarly closely related to the development and character of the cardiac rhythm, figures I 7 I I I 72 , and I73. Both the cold-blooded heart and the mammalian heart respond very quickly to the influence of these salts. The details of this influence have been discussed on page I99. It is somewhat surprising, however, that the highly organized mammalian heart will contract rhythmically for hours on purely inorganic nutrient fluid, provided only that the oxygen be supplied in sufficient quantity and under high enough tension. The isolated mammalian heart also responds sharply to a change in the salt content of the perfusing solution. For example, addition of potassium chloride to a Locke solution slows or even suppresses the rate, as is shown in figure 178 .

Irregularities of Cardiac Rhythm.-There are a number of cardiac irregularities in rhythm that are due to variations in irritability, conductivity, or other of the normal properties of the differentiated tissue of the heart itself. The phenomena of this type of most common occurrence are heart block, extra ventricular systoles, and auricular fibrillation.

Heart Block.-It occasionally happens that the heart rate becomes very slow, 30 or 40 a minute, and the rate does not vary much from this

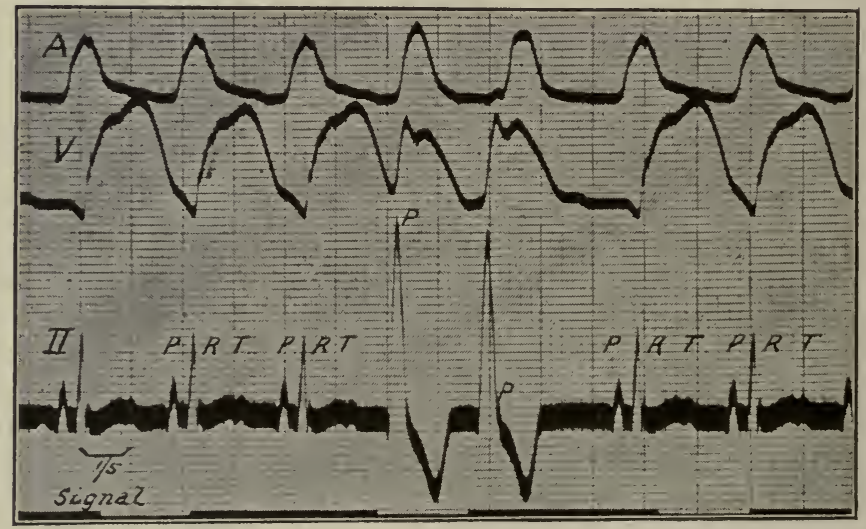

FIG. I 78a.-An electrocardiogram, lead II, and auricular and ventricular muscle tracings, A and V taken simultaneously from the dog's heart. Two extra ventricular contractions are artificially produced by stimulation of the right ventricle. They show the characteristic right ventricular dominance. The first is taller because the $\mathrm{P}$ of the natural stimulus and the $R$ of the artificial coincide. Time, fifths and twentyfifths of a second. (Lewis.)

low level. On careful examination it is found that the rate is ventricular only and that the auricle is contracting much faster. The latter may contract in multiples of the ventricular rate, $2-1,3^{-1}$, or $4^{-1}$ rhythm in which case there is said to be partial block. Only every second, third, etc., contraction reaches the ventricle. Or the two rates may be wholly independent as in total block. 
The conducting bundle or path between the sino-auricular node where the normal beat arises and the auriculo-ventricular node is usually at fault in partial block. In complete block the independent ventricular beats start from a rhythmic center in the auriculo-ventricular node as a rule. But the block may be in the His bundle itself in which case the rhythm production is low in the conducting system or even in the ventricular muscle.

Extra Ventricular Systoles.-When for any reason some portion of the ventricular complex becomes excessively irritable its rhythmicity may be so much increased that it starts an independent contraction before the normal ventricular stimulus reaches the muscle. This leads to a contraction with the shortened period between beats and is called an extraventricular systole. As a rule there is a longer or compensatory pause following an extra systole after which the regular rhythm again becomes dominant. Most people of middle or old age experience occasional extrasystoles. In disease they may become frequent and troublesome but they are of no particular importance.

Auricular Flutter and Fibrillation.-A type of irregularity that is more common is that of an auricular rate far above the normal, i.e., $\mathrm{r} 50$ or even more a minute. These are due to hyperirritability of the auricle often

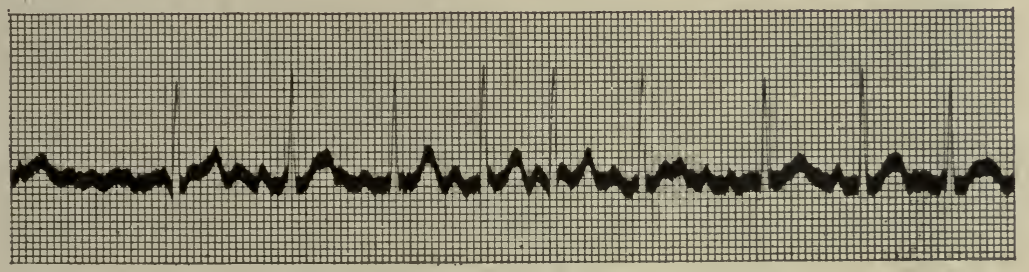

FIG. I78b.-Electrocardiogram of auricular fibrillation.

with an ectopic center of rhythm production. When such hearts are examined with the electrocardiograph or with the polygraph it becomes evident that the auricle is contracting at a very much higher rate than the ventricle. The ventricle does not respond to every auricular contraction, i.e., in this kind of block the stimulus falls within the refractory period of the ventricles. Each auricular contraction is complete and the series is regular but the rate is above that which can be conducted to the ventricle. Auricular flutter is the term that describes this phenomenon.

The auricular contractions are not always coordinated and complete. They sometimes begin in apparently many foci at once so that no rhythmic center controls the entire auricular muscle. Such contractions are called fibrillation. In auricular fibrillation individual muscle 
cells may contract and relax at as high as 400 a minute. These contractions spread to the conducting bundle system at irregular intervals and the ventricle contracts at unevenly spaced intervals. The arterial pulse beats are also irregularly spaced, figure $x 78 b$. When ventricular fibrillation occurs it quickly produces death, but auricular fibrillation only reduces the efficiency of filling the ventricle. The physician controls auricular fibrillation by reducing the irritability of the auricle and the conductivity of the bundle system.

\section{THE REGULATIVE INFLUENCE OF THE CENTRAL NERVOUS SYSTEM ON THE HEART.}

The heart is capable of automatic rhythmic movement, yet while in the body its beats are under the constant control of the central nervous system. The influence which is exerted by the central nervous system is of two kinds: first, in the direction of slowing or inhibiting the beats, and second,

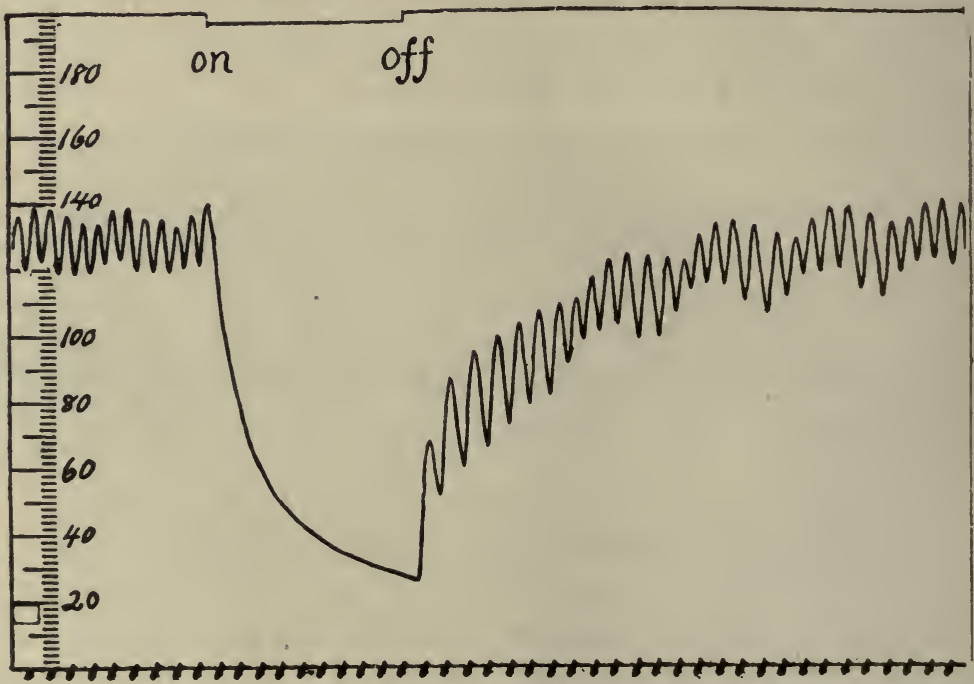

Fig. I79.-Effect on the Heart Rate and on the Arterial Blood Pressure of Stimulating the Right Vagus of the Dog. Stimulus applied at the mark "on" and removed at "off." Pressure in millimeters of mercury shown by the scale to the left. Time in seconds. (Hill and Chilton.)

in the direction of accelerating or augmenting the beats. The infuence of the first kind is brought to bear upon the heart through the fibers of the pneumogastric or vagus nerves, and that of the second kind through the sympathetic nerves.

The Inhibitory Nerves. - It has long been known, indeed since the experiments of the Weber brothers in 1845 , that stimulation of one or 
both vagi produces slowing of the rhythm of the heart. It has since been shown, in all of the higher vertebrate animals experimented with, that this is the normal reaction to vagus stimulation. Moreover, a section of one vagus, or at any rate of both vagi, produces acceleration of the

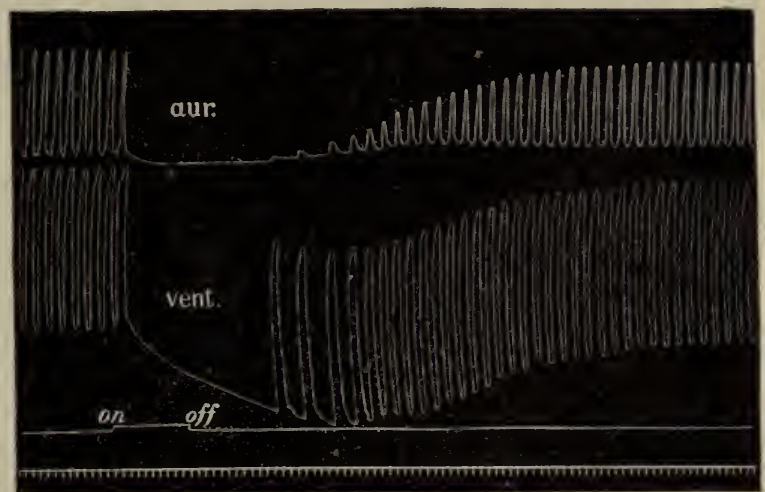

Fig. 180.- Tracing Showing Action of the Vagus on the Heart of the Terrapin. Aur, Auricular; vent, ventricular tracing. The part between words "on " and "off" indicates a period of vagus stimulation. The part of tracing to the left shows the regular contractions before stimulation. During stimulation, and for some time after, the beats of the auricle and ventricle are arrested. After they commence again the auricle contracts weakly at first, but soon acquires a much greater amplitude. The ventricular contractions that follow the first weak auricular contractions are maximal in the terrapin, but not so in the frog. See next figure. Time in seconds. (Carr.)

pulse by breaking the pathway from the vagus center to the heart; stimulation of the distal or peripheral end of the divided nerve normally produces slowing or stopping of the heart beats, showing that the fibers are efferent and thus carry the nerve impulses toward the heart.

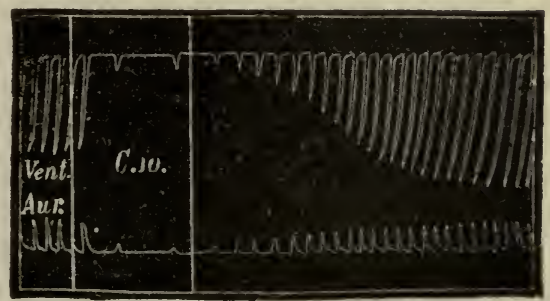

FIG. I8I-Tracing Showing Diminished Amplitude and Slowing of the Pulsations of the Auricle and Ventricle of the frog without Complete Stoppage during Stimulation of the Vagus. (Gaskell.)

It appears that any kind of stimulus, either chemical, mechanical, electrical, or thermal, produces the same effect, but that of these the most potent is a rapidly interrupted induction current. A certain amount of confusion has arisen as to the effects of vagus stimulation in consequence of the fact that fibers of the sympathetic nerve run within the trunk of the 
vagus nerves of some animals, for example, the frog. Speaking generally, however, excitation of any part of the trunk of the vagus produces inhibition, the stimulus being particularly potent if applied to the points where the nerves enter the substance of the heart at the situation of the sinus ganglia. The stimulus may be applied to either vagus with like effect. There are quantitative differences, however, between the right and left vagi. The right vagus usually has the greater effect on rhythm.

The effect of the stimulation of the vagus is threefold - to slow the rate, or even to bring the heart to a complete standstill, to produce a decrease in the amplitude, and to delay conduction through the bundle system. The slowing does not take place until after the lapse of a short latent period during which one or more contractions may occur. The stoppage may be due either to prolongation of the diastole or to diminution of the systole. Vagus stimulation inhibits the spontaneous beats of the heart only, it does not entirely suppress the irritability of the heart muscle, since mechanical stimulation may bring out a beat during the pause caused by vagus stimulation. The inhibition of the beats varies in duration according to the strength of the stimulus and the animal stimulated. The heart of the terrapin can be completely inhibited for hours with a strong stimulus. This phenomenon is shown in figure 180 , which illustrates the action of the vagus on the terrapin's heart.

The heart of a dog escapes from complete inhibition in a few seconds. When the beats reappear, the first few are usually feeble, after a time the contractions become more and more strong, and may soon exceed both in amplitude and frequency those which occurred before the application of the stimulus. If the stimulation is prolonged, the inhibition escapes to a slow rate, much under the normal rate. It is held there with some variations until the stimulus ceases. This is due to the fact that, in the dog at least, the stimulation reacts more strongly on rhythm production at the sino-auricular nodal center, holding it in check with a strength that does not inhibit the auriculo-ventricular nodal rhythm. The fundamental rhythm of the latter center is at a slower rate. The escape is to the auriculo-ventricular nodal rhythm.

The inhibitory fibers have their origin in nerve cells in the nucleus of the vagus, and of the glosso-pharyngeal, located in the floor of the fourth ventricle. These cells have not been exactly identified, but the center is called the cardio-inhibitory center. The center is a bilateral one and the fibers from it pass into the great vagus trunk to be distributed to the heart through superior and inferior cardiac branches which help to form the cardiac plexus. Within the heart the inhibitory fibers form synapses with cells whose axones reach the cardiac muscle cells. The cardiac-inhibitory center is in more or less constant tonic activity, and the tonic influence is eliminated when both nerves are cut, figure 182 . 
Inhibitory Reflexes.-The inhibitory center is influenced by afferent nerve impulses which may reach it from the heart itself by the depressor nerve, or from other parts of the body. These reflex stimulations of the vagus center are constantly occurring during our daily life and are the most potent factors in co-ordinations going on between the heart and the rest of the body.

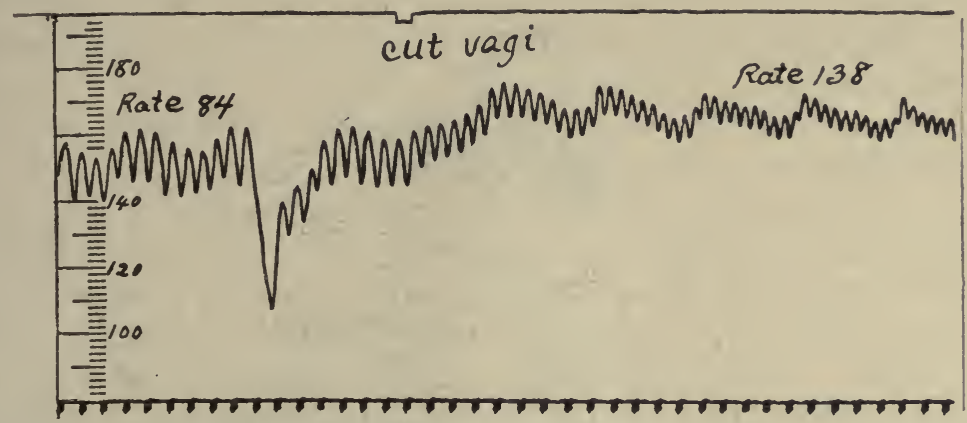

FIG. 182.- Arterial Blood Pressure of the Dog, Showing the Effect on the Heart Rate of Cutting both Vagus Nerves as marked. The scale to the left shows the pressure in millimeters of mercury. Time in seconds. The momentary inhibition just before the nerves were cut is probably due to mechanical stimulation of the nerves. (Hill and Chilton.)

The vagus trunk itself contains afferent fibers, the depressor nerves, that arise from sensory endothelia in the heart itself and in the aortic arch. These endings are stimulated by excessive mechanical pressure. Their nerve impulses react on the vagal motor cells to produce reflex inhibition, hence the cardiac slowing that relieves the pressure that produced the reflex. This reflex apparatus is one of the most interesting self protecting mechanisms in the mammalian body.

Rhythmical alterations of the heart rate occur in association with the effects of the mechanical variations of pressure of the thorax on the heart and blood vessels. Apparently the cardio-inhibitory center is stimulated during the rise of blood pressure. The activity of the center produces a slower rate of the heart during expiration, shown in figure 243. This variation in heart rate disappears when the vagi are cut off from the center. The variations from this cause are called sinus arrhythmia in clinical literature. Such variations are purely physiological and normal in character.

The Accelerator Nerves.-The influence of the accelerator nerves distributed to the heart through the thoracic sympathetic, is the reverse of that of the vagus. Stimulation of the sympathetic, even of one side, produces acceleration of the rate of the heart-beats, augmentation of the amplitude, or force, and better or at least faster conduction through the nodal system according to certain observers. Section of the nerve produces 
slowing. The action of the nerve is more properly termed augmentor. The sympathetic or augmentor differs from the vagus in several particulars. First, the stimulus required to produce any effect must be more powerful than is the case with vagus stimulation. Second, a longer time elapses before the effect is manifest. Third, the augmentation is followed by exhaustion, the beats becoming after a time feeble and less frequent.

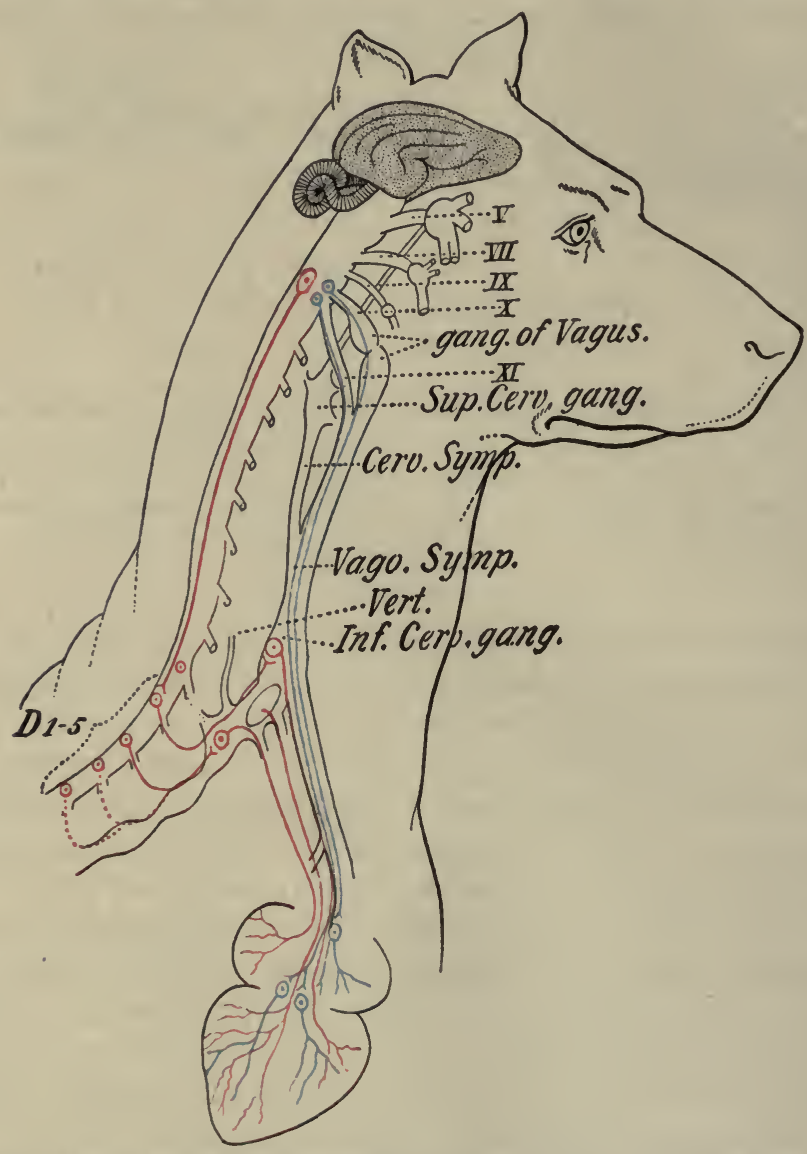

FIG. 183.-Diagrammatic Representation of the Origin and Course of the Cardiac Nerves in the Dog, showing the Constituent Neurones. D r-5, First to fifth dorsal spinal nerves. Inhibitory fibers in blue, accelerators in red. (Modified from Moret.)

The fibers of the sympathetic system, which influence the heart-beat in the frog, leave the spinal cord by the anterior root of the third spinal nerve. They pass by the ramus communicans to the third sympathetic ganglion, thence to the second ganglion, the annulus or ansa (around the subclavian artery), through the first ganglion, and along the main trunk to the exit of the vagus from the cranium. There the two nerves join 
and run down to the heart within a common sheath, forming the vagosympathetic trunk. Stimulation of the accelerators of the frog must be applied to the pathway before the fibers join the common trunk if uncomplicated augmentation is to be secured. On stimulation of the mixed vago-sympathetic trunk inhibition ordinarily occurs at once. Augmentor effects come on only after the inhibition has disappeared, usually fifteen or twenty seconds later. If the vagal influence is first removed by a specific poison, atropine, then on stimulation pure augmentation results at once. This method applied to the frog is one of the most satisfactory methods of illustrating the different elements in cardiac augmentation.

In the dog the augmentor fibers leave the cord by the anterior roots of the second and third dorsal nerves, and possibly also by the first, fourth, and fifth dorsal nerves. They pass by the rami communicantes to the ganglion stellatum, or first thoracic ganglion, and around the ansa to the inferior cervical ganglion of the sympathetic. Fibers from the ansa or from the inferior cervical ganglion proceed to the heart, figure 183 . The course of the augmentor fibers in the spinal cord is not so well known except that they originate in an augmentor center in the medulla. The circulation of venous blood appears to stimulate the augmentor center, and of highly oxygenated blood the inhibitory center.

The accelerator center, like the inhibitory, is in constant tonic activity; and the cardiac acceleration on cutting the vagi, shown in figure 182 , is in part to be ascribed to this tone. When both nerves are stimulated together, the resulting rate is the algebraic sum of the opposed influences, according to Hunt. The accelerator center is influenced by afferent impulses arising throughout the body, and these reflexes contribute to the general co-ordination of the chest with the activities of the body.

In addition to direct and reflex stimulation, impulses passing down from the cerebrum may have a similar effect, psychic stimulation.

Other Influences which Affect the Heart.-A great variety of special conditions influence the heart's action in the normal body, conditions that are not discussed directly under any of the categories treated above. Of these may be mentioned the coronary circulation, temperature, mechanical tension, age.

The Coronary Circulation.-The contractions of the heart cannot long be maintained without a due supply of blood or other nutrient fluid. The nutrient fluid for the heart of man and the mammals is supplied from the coronary arteries and the vessels of Thebesius. The coronary arteries arise from the base of the aorta, where they receive the benefit of the highest arterial pressure. The coronary arteries are terminal arteries; that is, they do not permit the establishment of a collateral circulation when one of their branches is blocked. If the block be complete, that portion of the heart wall supplied by the branch dies. The immediate effect of the 
closure of a large coronary branch, in the dog, may be occasional and transient irregularity or arrest of the ventricular contractions preceded by irregularities in the force of the contractions and a diminution in the amount of work performed. The force, rather than the rate, of the ventricular contractions is closely dependent upon the blood supply to the coronary arteries. Porter and others have shown that the pressure in the coronary vessels follows closely the pressure in the aorta and that there is not, as formerly claimed, a closure of these vessels by the pressure of the systole of the ventricle.

The vessels of Thebesius, which have been demonstrated to open both into the auricular and ventricular cavities, must now be looked upon, according to the investigations of Pratt, as an important source of cardiac nutrition. Blood may pass through them by way of connecting branches to the coronary arteries and veins. Pratt succeeded in maintaining cardiac contractions for several hours when the only source of nutrition was from these vessels. This source of nutrition may account for the survival of hearts for years where pronounced arterio-sclerosis of the coronary arteries exists.

Alteration of Temperature.-The effect of cold is to slow the rate of the heart-beat. If the heart of a frog be cooled down to $0^{\circ} \mathrm{C}$. it will stop beating, but when the temperature of the surrounding lymph or blood is again raised, it will renew its spontaneous beats. The effect of heat is to quicken and shorten the heart-beats, but at a moderate temperature, $20^{\circ}$ C., the contractions are increased in force, figure I77.

The isolated mammalian heart is influenced by temperature variations in much the same way as that of the frog. It will contract slowly in a low temperature and rapidly in a temperature higher than that normal to the body. The very rapid heart in some high fevers is in part due to the increase in temperatures which affects the heart directly.

Mechanical Tension.-The mechanical factors produced by the heartbeat are so prominent that it would be surprising indeed if there were no reaction of these mechanical conditions on the heart itself. The isolated cardiac muscle responds very quickly to variations in tension. Beginning with a low tension the activity of heart muscle is increased up to a certain optimum tension, after which further increase is unfavorable to the development of automatic rhythm. A quite strong stretching will paralyze the muscle.

Tension on the whole heart influences its activity, not only through the effects on the muscle, but indirectly through the nervous mechanism. High tension, such as contracting against a high aortic pressure, stimulates sensory nerves of the heart which, acting through the depressor nerve on the inhibitory center, produce reflex slowing of the heart. It also produces reflex vaso-dilatation. Both reflexes relieve the high tension on the heart. This 
nerve reaction takes place with a tension which still mechanically stimulates the cardiac-muscle substance, and the inhibitory effects must therefore overcome the direct stimulating effect of the tension on the muscle fibers.

Age, Sex, etc.-The average heart rate for the normal adult man is 72 times a minute, but this rate will vary much in different individuals according to the age, sex, size, and personal equation. The frequency of the heart's action gradually diminishes from the commencement to near the end of life, but is said to increase again somewhat in extreme old age, thus:

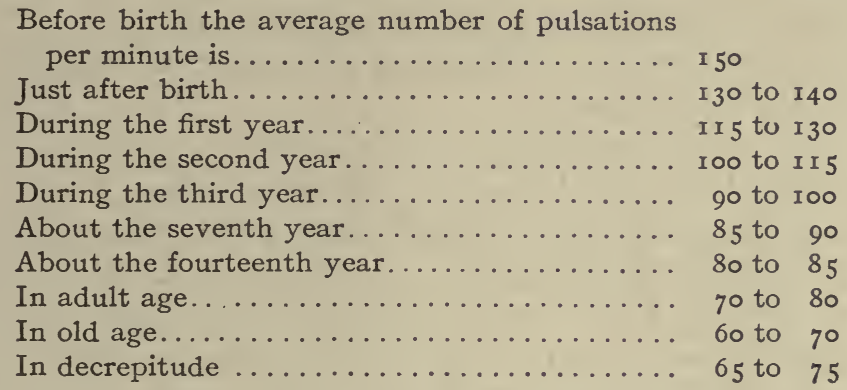

The heart rate is greater in woman than in man. It is also greater in small than in large individuals. The rate varies from the type in certain individuals where no cause can be assigned other than personal equation.

Poisons and Other Chemical Substances.-A large number of chemical substances have a distinct effect upon the cardiac contractions. Of these the most important are atropine, muscarine, digitalis, barium, nicotine, caffeine, etc.

Atropine produces considerable augmentation of the heart-rate, and when acting upon the heart prevents inhibition by vagus stimulation. Its effects are produced by poisoning the nerve endings of the vagus within the heart. When these endings are poisoned stimuli arising in the inhibitory center of the medulla (tonic activity), or artificially applied to the vagus, cannot reach the heart muscle, and inhibition is impossible.

Muscarine, which is obtained from various species of poisonous fungi, produces marked slowing of the heart-beats, and, in larger doses, stoppage of the heart. It produces an effect similar to that of prolonged vagus stimulation. The effect can be removed by the action of atropine, hence is supposed to stimulate the nerve endings of the vagus.

Digitalis slows the heart by stimulating the vagi at their origin in the inhibitory center in the medulla. The heart muscle itself is also rendered more excitable.

Veratrine and aconitine have a somewhat similar effect.

Nicotine and caffeine are both very powerful cardiac stimulants. The great injurious effects of nicotine on the heart are due to two causes, first, 
to paralysis of the nervous mechanism and relative loss of control, second, to the great direct stimulation of the cardiac muscle. The constant overuse of tobacco, therefore, very sharply weakens the efficiency of the heart. Caffeine does not lead to so great disturbance of the heart's nutrition as does nicotine.

\section{THE CIRCULATION THROUGH THE BLOOD VESSELS.}

Blood-Pressure.-The subject of blood-pressure has been already incidentally mentioned more than once in the preceding pages; the time has now arrived for it to receive more detailed consideration.

That the blood exercises pressure upon the walls of the vessels containing it is due to the following facts:

The heart at each contraction forcibly injects a considerable amount of blood, 80 to roo c.c., suddenly and quickly into the arteries.

The arteries are highly distensible and stretch to accommodate the extra amount of blood forced into them. The arteries are already full of blood at the commencement of the ventricular systole, since there is not sufficient time between the heart-beats for the blood to pass into the veins.

There is a distinct resistance interposed to the passage of the blood from the arteries into the veins by the enormous number of minute vessels, small arteries (arterioles) and capillaries, into which the main artery has been ultimately broken up. The sectional area of the capillaries is several hundred times that of the aorta, and the friction generated by the passage of the blood through these minute channels opposes a considerable hindrance or resistance in its course. The resistance thus set up is called peripheral resistance. The friction is greater in the arterioles, where the current is comparatively rapid, than in the capillaries, where it is slow.

The interaction of these factors-heart-beat, elastic vessels, and peripheral resistance-is sufficient to maintain a flow of blood through the entire circulatory system. It is the interrelation of these factors which maintains an even and steady flow through the capillaries and past the tissues, where it is desirable that the conditions of blood flow should be most constant if the purposes of nutrition are to be best accomplished. In fact, we shall even find that it is the interaction of these same factors, together with the possibility of variations through regulation by their nerve-motor mechanisms, that we have the great variations and adjustments of bloodpressure, speed of flow, volume of flow, and the regulation of volume in particular parts of the body or local control.

Arterial Blood-Pressure.- That the blood exerts considerable pressure upon the arterial walls in keeping them in a stretched or distended condition may be readily shown by puncturing any artery. The blood is instantly projected with great force through the opening, and the jet rises to a considerable height, the exact level of which varies with the size of the 
artery experimented upon. If a large artery be punctured the blood may be projected upward for several feet, whereas if it is a small artery the jet does not rise so high. Another characteristic of the jet of blood from a cut artery, particularly well marked if the vessel be a large one and near the heart, is the intermittent character of the outflow. If the artery be cut across, the jet issues with force, chiefly from the central end. If there is considerable anastomosis of vessels in the neighborhood the jet from the peripheral end may be almost as forcible and as intermittent as that from the central end. The intermittent flow in the arteries due to the action of the heart, and which represents the systolic and diastolic alterations of blood-pressure, may be felt if the finger be placed upon a sufficiently superficial artery. The finger is apparently raised and lowered by the intermittent distention of the vessel occurring at each heart-beat. This intermittent distention of the artery is what is known as the pulse, to the further consideration of which we shall presently return, but we may say here that in the normal condition the pulse is a characteristic of the arterial, and is absent from the venous, flow.

At the same time it must be recollected that in the veins also the blood exercises a pressure on the containing vessels, though it is small when compared with the arterial pressure. As might be expected, therefore, the blood is not expelled with so much force if a vein be punctured or cut. The flow from the cut vein is continuous and not intermittent, and the greater amount of blood comes from the peripheral and not from the central end, as is the case when an artery is severed.

Methods of Measuring Arterial Blood-Pressure.-The pressure in an artery may be measured by cutting the vessel and introducing into it a carnula and connecting the cannula with a tall vertical glass tube. When the blood in the vessel is released to the cannula, a column of blood will rise in the tube at once to the height that can be supported by the pressure in that particular vessel. If the vessel be an artery, the blood will rise several feet, according to the distance of the vessel from the heart, and when the pressure has reached its highest point it will be seen to oscillate with the heart-beats. This experiment shows that the pressure which the blood exerts upon the walls of the containing artery equals the pressure of a column of blood of a certain height. In the case of the rabbit's carotid it is equal to 90 to $120 \mathrm{~cm}$. of blood, or rather more than the same height of water. In the case of the vein, if a similar experiment be performed, blood will rise in the tube only for 8 or $10 \mathrm{~cm}$. or less.

The usual method of estimating the amount of blood-pressure differs somewhat from the foregoing simple experiment. Instead of a simple straight tube or glass manometer for measuring the pressure, a U-shaped tube containing mercury, the mercury manometer, is employed. The artery is connected with the manometer by means of the cannula inserted into the vessel as before, an arrangement being made whereby the cannula, tubes, etc., 
are first filled with a half-saturated solution of magnesium sulphate or other saline to prevent the clotting of blood when it is allowed to pass from the artery into the apparatus. The loss of blood is prevented during the preparation of the details of the experiment by a clamp or bull-dog forceps. The free end of the U-tube of mercury contains a very fine glass or metal rod with a bulb which floats upon the surface of the mercury and oscillates with the oscillations of the mercury. As soon as there is free communication between the artery and the tube of mercury, the blood rushes out and pushes before it the column of mercury. The mercury will therefore rise in the free limb of the tube, and will continue to do so until a point is reached which corresponds to the mean pressure of the blood-vessel used. The blood-pressure is thus communicated to the near limb of the column of mercury; and the depth to which the latter sinks, added to the height to which it rises in the other limb, the weight of the saline solution being subtracted, will give the height of the column of mercury which the blood-pressure balances. For the estimation of the amount of blood-pressure one can make direct readings at any given moment and no further apparatus

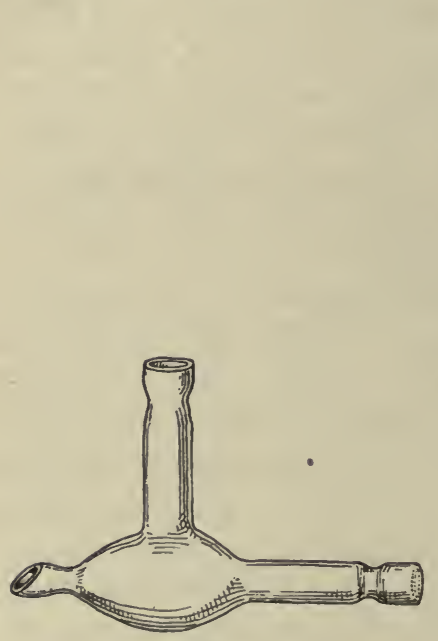

FIG. I 84 .

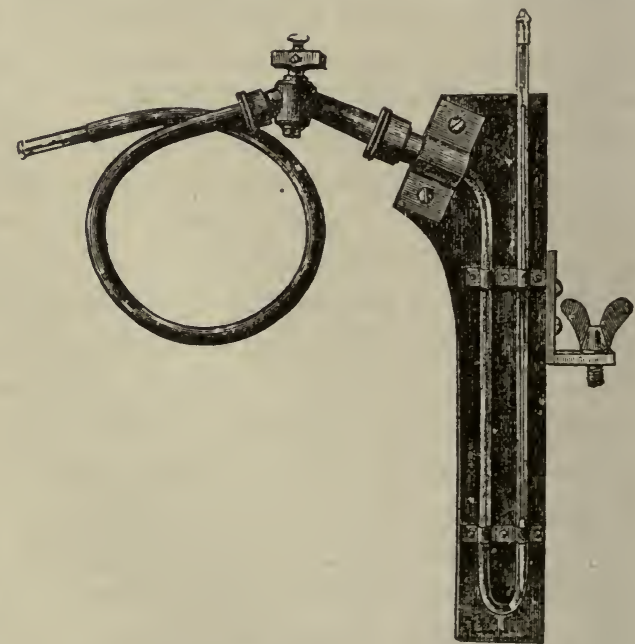

Fig. 185 .

FIG. I84.-Arterial Cannula. T-form for convenience in washing out clots.

FIG. I85.-Ludwig's Mercury Manometer. The mercury which partially fills the tube supports a float in the form of a piston, nearly filling the tube; a wire is fixed to the float, and the writing style or pen is guided by passing through the brass cap of the manometer tube; the pressure is communicated to the mercury by means of a flexible metal tube filled with fluid.

than this is necessary. But in the more accurate study of the variations of pressure in the arterial system, as well as its absolute amount, the instrument is usually combined with a recording apparatus, called a kymograph. Numerous forms of recording kymographs are to be had in the market. These instruments, while all constructed on the same principle, vary chiefly in the accuracy of their construction and convenience of their adjustments. 
The essential part of a recording kymograph consists of a uniformly revolving cylinder accurately centered and carrying a paper on which a record is made of the physiological change which is being studied. This cylinder or drum may be driven by a weight, clock spring, electric motor, or other mechanical device that insures uniformity of speed and which is capable of speed regulation. The cylinder is covered with glazed paper, blackened in the flame of a lamp, and the mercury manometer is so supported that its float, provided with a style, writes on the cylinder as it revolves. In some of the instruments, especially Ludwig's continuous paper kymograph, a long paper band is made to pass over the recording surface and the record itself is written by various devices carrying ink.

There are also many ways in which the mercury manometer may be varied; in figure 185 is seen a form which is known as Ludwig's. In order to obviate the necessity of a large quantity of blood entering the tube of the apparatus and being lost to the animal, it is usual to have some arrangement by means of which the mercury may, previous to the experiment, be forced

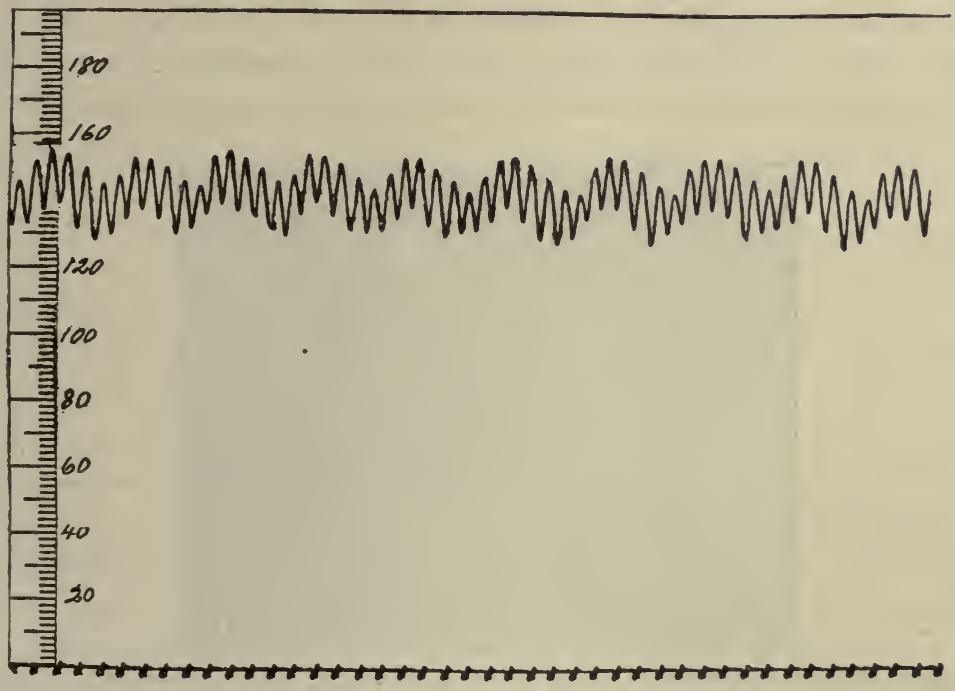

FIG. I86.-Tracing of Normal Arterial Pressure in the Dog, Obtained with the Mercurial Manometer. The smaller undulations correspond with the heart-beats; the larger curves with the respiratory movements. Pressure is in millimeters of mercury as shown by the scale to the left. Time in seconds.

up in the tube of the manometer to the pressure level corresponding to approximately the mean pressure of the artery experimented with, so that the writing style simply records the variations of the blood-pressure above and below the mean pressure. This is done by causing the anti-coagulant solution, generally a saturated solution of sodium carbonate or of Io per 
cent. magnesium sulphate, to fill the apparatus from a bottle suspended at a height about that of the pressure to be measured, and capable of being raised or lowered as required for the purpose.

The cannula inserted and tied into the artery may be of several different kinds. A glass T-tube with the end drawn out and cut so that it is oblique, and provided with a slightly constricted neck to prevent its coming out of the artery easily, is a very convenient form, figure 184 . Of the two free ends of the T-cannula one is connected with the manometer, the other with the pressure bottle. The peripheral end of the cut artery is tied to obviate the escape of blood. By this means, the presssure communicated to the column of mercury is the forward, and not the lateral, pressure of blood, but there is very little if any difference.

As soon as the experiment is begun, the writing float is seen to oscillate in a regular manner, and a curve of blood pressure is traced upon the smoked paper by the style (or, if a continuous roll of unsmoked paper be used, the trace is made by an inked pen) when a figure similar to figure 186 will be obtained. This indicates two main variations of the blood pressure. The smaller excursions of the lever correspond with the systole and diastole of the heart, and the larger curves correspond with the respirations, being called the respiratory undulations of blood-pressure, to which attention will be directed

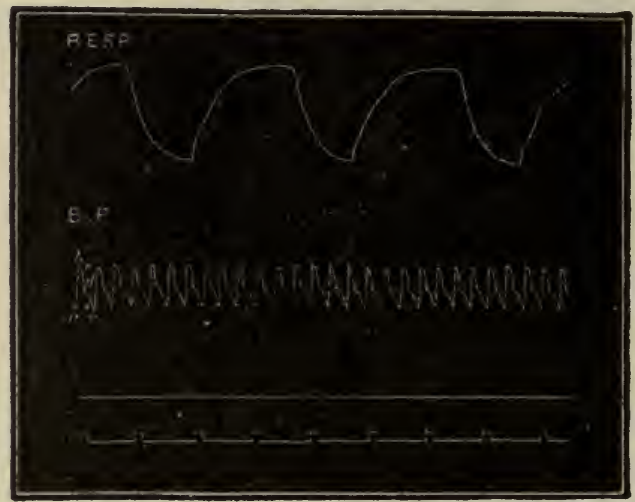

FIG. 187.- Tracing of Normal Arterial Pressure Taken from the Rabbit with a Hürthle Manometer. The horizontal lines show zero pressure. Time in seconds. (Dreyer.)

in the next chapter. Of course, the undulations spoken of are seen only in records of arterial blood-pressure. They are more clearly marked in the arteries nearer the heart than in those more remote. The amount of the pressure in the smaller arteries as well as the indication of the systolic rise of pressure is, comparatively speaking, small.

In order to record the details of the undulations of arterial pressure, it is better for some purposes to use the Hürthle membrane manometer than the mercurial manometer. Two views of this instrument are shown in figure 166. 
The instrument consists of a hollow tube and cup covered with rubber sheet against which a disc supported by a metal spring is adjusted. The apparatus is filled with fluid, the interior of which is connected with the artery by means of a metal tube and cannula. The pressure transmitted to the apparatus tends to stretch the rubber and bend the spring, and the movement thus produced is communicated by means of a lever to a writing style and so to a recording apparatus. This instrument obviates the errors which might be caused by the inertia of the mercury in the mercurial manometer; it alsoshows in more detail the variations of the blood pressure in the vessel during, and after each individual beat of the heart.

As regards the actual amount of blood-pressure, from observations which have been made by means of the mercurial manometer, it has been found that the pressure of blood in the carotid of a rabbit is capable of supporting a column of 90 to $120 \mathrm{~mm}$. of mercury; in the dog 100 to $175 \mathrm{~mm}$.; in the horse I 52 to $200 \mathrm{~mm}$.; and in man the pressure is estimated to be about the same as in the dog. To measure the absolute amount of this pressure in any artery multiply the area of its transverse section by the height of the column of mercury which is already known to be supported by the blood pressure in any part of the arterial system. The weight of a column of mercury thus found will represent the absolute pressure of the blood. Calculated in this way, the blood pressure in the human aorta is equal to I. 93 kilogrammeters; that in the aorta of the horse being 5.2 kilogrammeters; and that in the radial artery at the human wrist only 0.08 kilogrammeter. Supposing the muscular power of the right ventricle to be one-fourth that of the left, absolute pressure in the pulmonary artery will be only 0.5 kilogrammeter. The amounts above stated represent the arterial tension at the time of the ventricular contraction.

The arterial pressure is greatest at the beginning of the aorta, and decreases toward the capillaries. It is greatest in the arteries at the period of the ventricular systole and least during the diastole. The blood-pressure gradually lessens as we proceed from the arteries near the heart to those more remote, and again from these to the capillaries, as it does also from the capillaries along the veins to the right auricle.

Arterial Blood-Pressure Measurements in Man.-A number of instruments have been devised for estimating blood-pressure in man for clinical purposes. Some of these, though excellent in principle, are too complicated for general use. The first simple and approximately accurate form of apparatus was that devised by Riva-Rocci in 1896 . This has been modified and improved in minor points since, but the principles of the original instrument remain practically the same.

In brief, the apparatus, figure $\mathrm{I} 88$, consists of an elastic tube ending in a rubber bag which can be adjusted about the arm, and a mercury manometer connected with this tube and also with some form of air pump. 
used for inflating the tube about the arm and thus exerting pressure upon its blood-vessels. The elastic tube is covered by some inelastic tissue, usually a leather cuff, in order that the inflation of the bag may cause the full increase of pressure to be exerted upon the encased arm. By inflating the bag until the pulse at the wrist just disappears, and reading the height of the column of mercury in the manometer, the maximum or systolic pressure is obtained in millimeters of mercury. If now the pressure on the arm is reduced until the widest oscillations of the mercury column are obtained, the lowest position of the mercury meniscus represents the diastolic pressure.

The apparatus depends on the principle that an external pressure just equal to the maximal pressure within an artery will hold the vessel in the collapsed condition, a fact that has been proven for vessels that are exposed. An external pressure that will just equal the minimal or diastolic pressure will cause a complete collapse of a vessel during diastole and will allow a complete expansion of an artery to its maximal limits during the systolic period of pressure. In other words, the mercury of the manometer will oscillate to its maximal. If the pressure is reduced to a still lower point, it

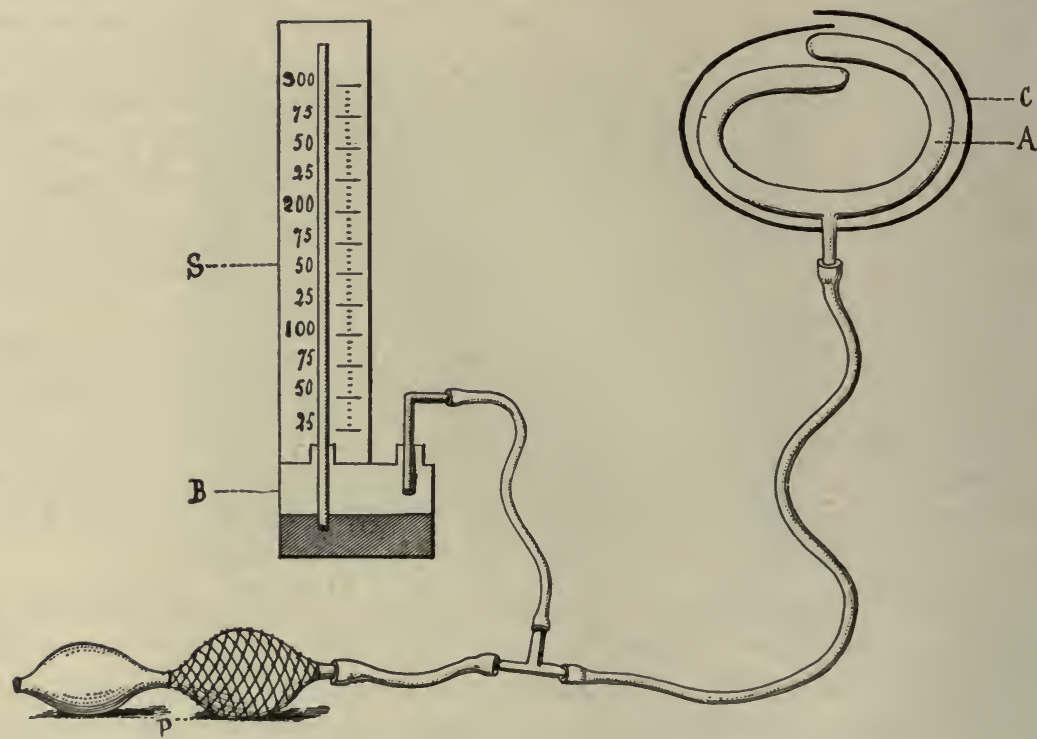

FIG. 188.-Riva-Rocci Apparatus (schematic) for Determining Blood Pressure in Man.

will not be sufficient to compress the artery completely, and the mercury oscillations will again become smaller. In applying the instrument to the brachial artery, one must, of course, deal with a vessel deeply buried in muscular and other tissues. These latter tissues probably consume a certain 
small percentage of the pressure, an error which may be ignored for all comparative purposes.

Erlanger has perfected a form of sphygmomanometer which contains a very ingenious and compactly arranged recording device, figure 189 . This instrument has a mercury manometer from which the pressures are read off directly. On a side limb of the manometer there is a rubber bag enclosed in a glass bell. The cavity of the bell outside of the rubber bag is connected

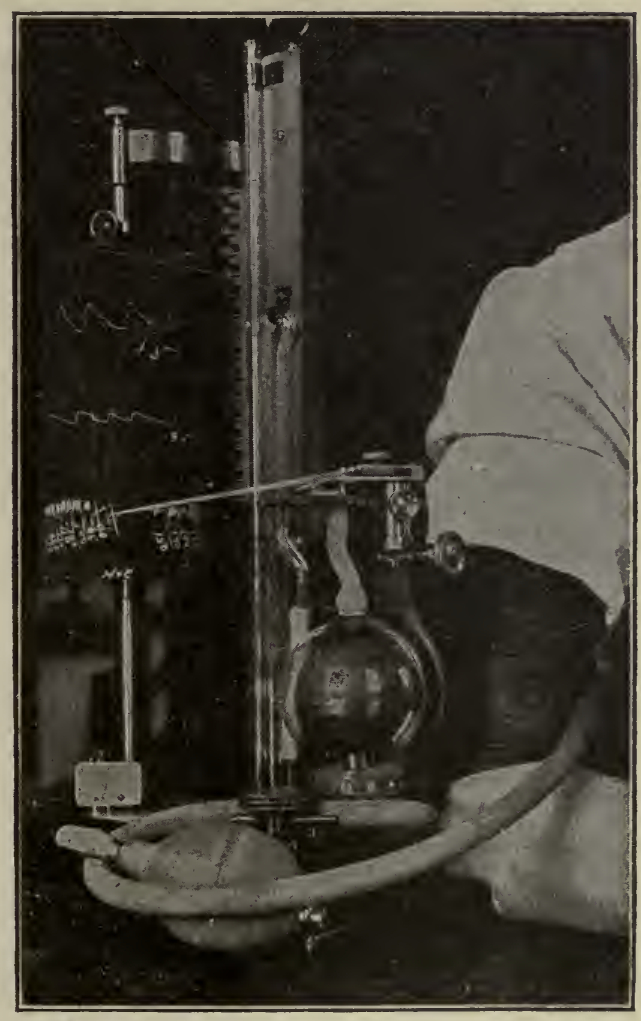

FIG. I89.-Erlanger's Sphygmomanometer, Shown with the Rubber Bag Attached to the Arm. The picture is taken at the end of an experiment after the pressure in the instrument is run up again to above the systolic pressure. The upper part of the cylinder shows a sphygmogram taken with the instrument. (Experiment and photo by Hill and Watkins.)

with a recording tambour, the entire apparatus being fully supplied with the necessary valves and adjusting devices which make it mechanically very perfect. The instrument is mounted on a stand with a small clock and recording cylinder adapting it to convenient clinical use.

The brachial arterial pressure of man when taken by this form of apparatus has been found to vary greatly, but Erlanger gives I Io mm. of mercury as the average of observations on young adults in the determi- 
nation of the systolic pressure; i.e., the maximal arterial pressure. He gives for the diastolic pressure 40 to $45 \mathrm{~mm}$. of mercury below the systolic pressure. Other observers using the same method find a somewhat higher average pressure, see figure 190 , which represents a fair type of observation.

The form of sphygmomanometer in almost universal clinical and laboratory use for determining the arterial blood-pressure of man is the aneroid type of Dr. Rogers. This instrument or its various modifications measures the pressure by means of the expansion of an aneroid coupled with a mechanical lever and gage device. The most widely distributed forms of instruments of this type are known as the Tyco and Faught. These instruments use an arm belt and bag of the Riva-Rocci type. The rubber bag is inclosed in a cloth belt which is conveniently wrapped around the arm above the elbow. The bag contains two connections

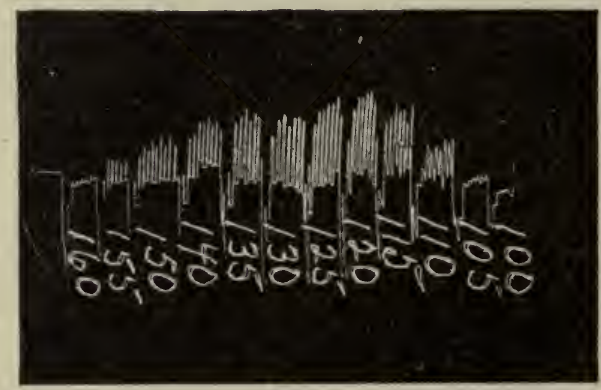

FIG. I90.- Tracing taken with Erlanger's Sphygmomanometer. The figures indicate pressure in millimeters of mercury. Systolic pressure 160; diastolic pressure, I20. (New figure by Hill.)

one of which is attached to the pressure gage, the other connected with a convenient pump made of either metal of rubber. The pressure measurement can be made directly from the oscillations of the dial as described for the Erlanger or Riva-Rocci apparatus. Readings may also be obtained by the palpation of the artery at the wrist as the pulse breaks through during gradual reduction of the pressure in the arm bag. However, the most accurate determinations are made by the auscultatory method (Goodman and Howell). A Bowles sphygmometroscope, which is a stethoscope modified by a button attached to the center of the disc, is attached to the arm just below the arm band at the inner angle of the elbow with the button of the diaphragm directly over the brachial artery near its division into the ulnar and radial.

In operation the arm band is pumped to a pressure above that of the underlying artery and then the pressure very gradually released. When the external pressure just equals to or is slightly less than the maximum pressure in the artery, some fluid will escape into the occluded 
limb of the artery below the band. The flow of this fluid produces a very definite first sound which is used to determine the moment of systolic pressure. The sounds in the brachial artery, known as Karatkoff sounds, have been described as going through five phases before the circulation is fully established in the cut off artery. The first phase is the initial development of a clear cut and sharp sound. It is the index of the systolic pressure. The first sound is followed by a series of murmurs called the second sound, and that by a more definite tone, the third phase. The third phase will vary in character with certain abnormalities in the vessel wall-thickening, sclerosis, etc.

The fourth phase is the appearance of a duller tone of diminishing intensity which rapidly fades into no sound, the so-called fifth phase. The fourth phase is taken as the index of minimal or diastolic arterial pressure.

If the ascultatory reading and the palpation reading are made at the same time the latter usually gives a slightly lower systolic pressure than the former. In other words, the stethoscopic reading by the sound is the more accurate. The diastolic reading is far more accurately determined by the ascultatory method. Woley gives the average systolic pressure as I $27 \mathrm{~mm}$. for all ages. But it is well known that the pressure increases with age from 75 at one year, to 105 in youth and $140 \mathrm{~mm}$. or more at the age of fifty.

Average Blood Pressure Measurements of Man

\begin{tabular}{|c|c|c|c|}
\hline & Standing & Sitting & $\begin{array}{c}\text { Immediately } \\
\text { after } \\
\text { Exercise }\end{array}$ \\
\hline \multicolumn{4}{|l|}{ Systolic Blood Pressure: } \\
\hline Men............... & $\mathrm{x} 20$ to $\mathrm{x} 35$ & 110 to 133 & 129 to 160 \\
\hline Women............. & 105 to 127 & 105 to 125 & 118 to 150 \\
\hline \multicolumn{4}{|l|}{ Diastolic Blood Pressure: } \\
\hline Men............... & 68 to 90 & 65 to 85 & 70 to 120 \\
\hline Women. . & 65 to 75 & 60 to 80 & 70 to 98 \\
\hline
\end{tabular}

The Venous Blood-Pressure and Capillary Pressure.-The bloodpressure in the veins is nowhere very great, but is greatest in the small veins, while in the large veins near the heart the pressure may become negative. In other words, when a vein is put in connection with a mercurial manometer the mercury may fall in the arm farthest away from the vein and will rise in the arm nearest the vein, the action being that of suction rather than pressure. In the large veins of the neck the tendency to suck in air is especially marked, and is the cause of death in some accidents or operations in that region. The amount of pressure in the brachial vein is said to 
support $9 \mathrm{~mm}$. of mercury, whereas the pressure in the veins of the neck may fall to a negative pressure of from -3 to $-8 \mathrm{~mm}$.

The variations of venous pressure during systole and diastole of the heart are very slight, and a distinct pulse is never seen in veins except under extraordinary circumstances. In certain forms of cardiac valvular insufficiency there may be considerable regurgitation of the blood with a strong venous pulse.

Careful observations upon the web of the frog's foot, the tongue and mesentery of the frog, the tails of newts and small fishes, and upon the skin of the finger behind the nail (Hooker); as well as estimations of the amount of pressure required to empty the vessels of blood under various conditions, all indicate that the capillary blood-pressure is subject to very great variations. Apparently the variations follow the variations of pressure in the arteries, though the measurements of the capillary pressure of the skin in man indicate that it is occasionally markedly influenced by the venous pressure variations (Hough). In the skin in man it is from 30 to $50 \mathrm{~mm}$. mercury.

The pulse in the arterioles, capillaries, and venules becomes more and more evident as the extravascular pressure is increased. The pressure in the web of the frog's foot has been found to be equal to about I4 to $20 \mathrm{~mm}$. of mercury; in other capillary regions the pressure is found to be equal to from one-fifth to one-half of the ordinary arterial pressure.

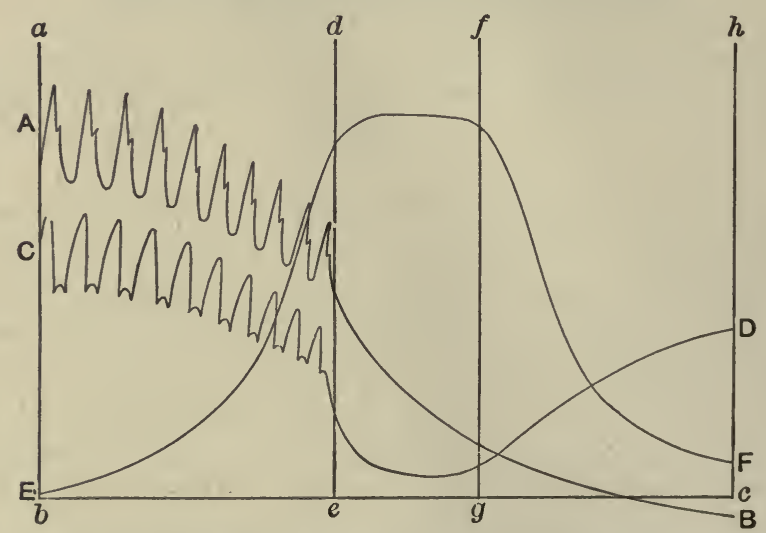

Fig. I9r.-Schema Showing the Relation between Blood Pressure, Velocity of Flow, and Vascular Area, in the Arteries, Capillaries, and Veins. Ordinates represent height of pressure and speed of flow. The abscissa, $b-c$, represents zero pressure and speed. Space between lines $a-b$ and $d-e$ represents arterial system; between $d-e$ and $f-g$, capillary system, and between $f-g$ and $h-i$, the venous system. Line $A-B$ equals pressure; line $C-D$, speed of flow; and line $E-F$, vascular area. (Modified from Gad.)

General Variations in Blood-Pressure.-The arterial blood-pressure may be made to vary by alterations in either of the chief factors upon which 
the pressure in the vessels depends, but primarily by the cardiac contractions and the peripheral resistance. Thus, increase of blood-pressure may be brought about by either, I, a more frequent or more forcible action of the heart, or 2 , by an increase of the peripheral resistance. On the other hand, diminution of the blood-pressure may be produced, either by, $a$, a diminished force or frequency of the contractions of the heart, or by $b$, a diminished peripheral resistance. These different factors, however, although varying constantly, are so combined that the general arterial pressure remains fairly constant. For example, the heart may, by increased force or frequency of its contractions, distinctly increase the blood pressure, but this increased action is almost certainly followed by diminished peripheral resistance, and thus the two altered conditions may balance, with the result of bringing back the blood-pressure to what it was before the heart began to beat more rapidly or more forcibly.

It will be clearly seen that the circulation of the blood within the bloodvessels must depend upon the diminution of the pressure from the heart to the capillaries, and from the capillaries to the veins, the blood flowing in the direction of least resistance. We shall presently see further that the local flow also depends upon the relations between the heart's action and the peripheral resistance both general and local.

The Arterial Flow. - The character of the flow of blood through the arterial system depends to a very considerable extent upon the structure of the arterial walls, and particularly upon the elastic tissue which is so highly developed in them.

The elastic tissue of the arteries, first of all, guards them from the suddenly exerted pressure to which they are subjected at each contraction of the ventricles. In every such contraction, as is above seen, the contents of the ventricles are forced into the arteries more quickly than they are discharged through the capillaries. The blood, therefore, being for an instant resisted in its onward course, a part of the force with which it is impelled is directed against the sides of the arteries; under this force their elastic walls dilate, stretching enough to receive the blood, and becoming more tense and more resisting as they stretch. Thus by yielding they break the shock of the force impelling the blood. On the subsidence of the pressure, should the ventricles cease contracting, the arteries are able by the same elasticity to resume their former caliber.

The elastic tissue in the same way equalizes the current of blood by maintaining pressure on it in the arteries during the period at which the ventricles are at rest or are dilating. If the arteries were rigid tubes, the blood, instead of flowing as it does in a constant stream, would be propelled through the arterial system in a series of spurts corresponding in time to the ventricular contractions and with intervals of almost complete rest during the inaction of the ventricles. But in the actual condition of the vessels, the force of the successive contractions of the ventricles is expended partly in the 
direct propulsion of the blood and partly in the dilatation of the elastic arteries; and in the intervals between the contractions of the ventricles, the force of the recoil is employed in continuing the flow onward. Of course the pressure exercised is equally diffused in every direction, and the blood tends to move backward as well as onward. All movement backward, however, is prevented by the closure of the semilunar valves, which takes place at the very commencement of the recoil of the arterial walls.

The Arterial Flow is Rhythmic.-By the exercise of the elasticity of the arteries, all the force of the ventricles is expended upon the circulation. That part of the force which is used up or rendered potential in dilating the arteries is restored or made active or kinetic when they recoil. There is no loss of force, neither is there any gain; for the elastic walls of the artery cannot originate any force for the propulsion of the blood; they only restore that which they receive from the ventricles.

Since the ventricular discharge is intermittent, there will be intermittent accessions of pressure, and therefore the flow of blood in the arteries will be periodically accelerated. The volume of blood discharged from a cut artery increases and decreases with the systole and diastole of the ventricles, or with the systolic and diastolic pressures of the arteries themselves, the maximal speed being at the moment of maximal systolic pressure, see page 228 .

The equalizing influence of the resistance of the successive arterial branches reacts so that at length the intermittent accelerations produced in the arterial flow by the discharge of the heart cease to be observable, and the jetting stream is converted into the continuous and even movement of the blood which characterizes the flow in the capillaries and veins. The resistance which is offered to the flow of the blood stream in these vessels is a necessary agent in the production of a continuous stream of blood in the smaller arteries and capillaries. Were there no greater obstacle to the escape of blood from the larger arteries than exists to its entrance into them from the heart, the stream would be intermittent, notwithstanding the elasticity of the walls of the arteries.

The muscular element of the middle coat co-operates with the elastic element in adapting the caliber of the vessels to the quantity of blood which they contain; for the amount of fluid in the blood-vessels varies quite considerably even from hour to hour, and can never be quite constant. Were the elastic tissue only present, the pressure exercised by the walls of the containing vessels on the contained blood would be sometimes very small and sometimes inordinately great. The presence of a muscular element, however, provides for a certain uniformity in the amount of pressure exercised: the muscles are in constant action or tone, and it is by this adaptive, uniform, gentle muscular contraction that the normal tone of the blood-vessels is maintained. Deficiency of this tone is the cause of the soft and yielding arterial pulse, and the sluggish blood flow through the arterioles. 
Incidentally it may be mentioned that the elastic and muscular contraction of an artery may also be regarded as fulfilling a natural purpose when, the artery being cut, the sudden contraction at first limits, and then, in conjunction with the coagulating blood, completely arrests, the flow of blood. It is only in consequence of such contraction and coagulation that we are free from danger through even very slight wounds; for it is only when the artery is closed that the processes for the more permanent and secure prevention of bleeding are established.

The Velocity of the Arterial Blood Flow.-The velocity of the blood current at any given point in the various divisions of the circulatory system is inversely proportional to the united sectional area at that point. If the united sectional area of all the branches of a vessel were always the same as the area of the vessel from which they arise, and if the aggregate sectional area of the capillary vessels were equal to that of the aorta, the mean rapidity of the blood's motion in the small arteries and in the capillaries would be the same as in the aorta. If a similar correspondence of capacity existed in the veins there would be an equal correspondence in the rapidity of the circulation in them. But the volume of the arterial and venous systems may be represented by two truncated cones with their apices directed toward the heart; the area of their united bases, the sectional area of the capillaries, being about eight hundred times as great as that of the truncated apex representing the aorta. Thus the velocity of blood in the smallest arterioles and the capillaries is about one-eight-hundredth of that in the aorta.

The velocity of the stream of blood is greatest in the neighborhood of the heart. The rate of movement is greatest during the ventricular systole and diminishes during the diastole. The rate of flow also decreases along the arterial system, becoming least in the parts of the system most distant from the heart. Chauveau has estimated the rapidity of the blood stream in the carotid of the horse at over 20 inches per second during the heart's systole, and nearly 6 inches during the diastole $(520-150 \mathrm{~mm}$.) see figure I I I.

The Capillary Flow.-It is in the capillaries that the chief resistance is offered to the progress of the blood; for in them the friction of the blood is greatly increased by the enormous multiplication of the surface with which it is brought in contact.

When the capillary circulation is examined in any transparent part of a full-grown living animal by means of the microscope, figures I93, I94, the blood is seen to flow with a constant equable motion; the red blood corpuscles moving along, mostly in single file, and bending in various ways to accommodate themselves to the tortuous course of the capillary, but instantly recovering their normal outline on reaching a wider vessel.

At the circumference of the stream and adhering to the walls of the larger capillaries, but especially well marked in the small arteries and veins, there is a layer of plasma which appears to be motionless. The existence 
of this still layer, as it is termed, is inferred both from the general fact that such a one exists in all fine tubes traversed by fluid, and from what can be seen in watching the movements of the blood corpuscles. The red corpuscles occupy the middle of the stream and move with comparative rapidity; the colorless corpuscles run much more slowly by the walls of the vessels; while next to the wall there is a transparent space in which the fluid appears to be at rest; for if any of the corpuscles happen to be forced within it, they move more slowly than before, rolling lazily along the side of the vessel and often adhering to its wall, figure r94. Part of this slow movement of the colorless corpuscles and their occasional stoppage may be

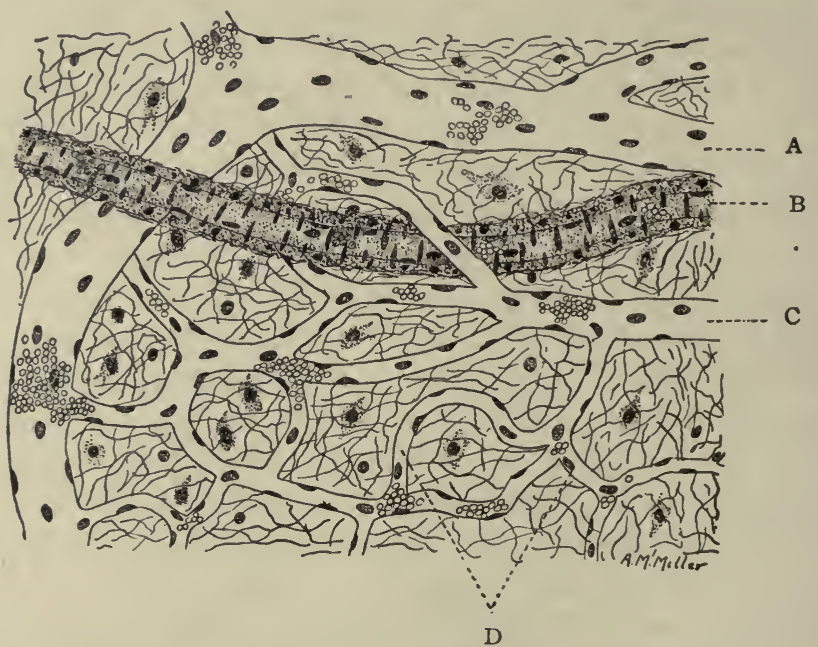

Fig. I93.-Capillary Network from Human Pia Mater, Showing also an Arteriole in "Optical Section"; and a Small Vein. $\times 35$. $A$, Vein; $B$, arteriole; $C$, large capillary; $D$, small capillaries. (Bailey.)

due to their having a tendency to adhere to the walls of the vessels. Sometimes, indeed, when the motion of the blood is not strong, many of the white corpuscles collect in a capillary vessel, and for a time entirely prevent the passage of the red corpuscles.

When the peripheral resistance is greatly diminished by the dilatation of the small arteries and capillaries, so much blood passes on from the arteries into the capillaries at each stroke of the heart that there is not sufficient remaining in the arteries to distend them. Thus, the intermittent current of the ventricular systole is not always converted into a continuous stream by the elasticity of the arteries before the capillaries are reached. The intermittency of the flow occurs both in capillaries and veins and a venous pulse is produced. The same phenomenon may occur when the arteries become rigid from disease, and when the beat of the 
heart is so slow or so feeble that the blood at each cardiac systole has time to pass on to the capillaries before the next stroke occurs. The amount of blood sent at each stroke is not sufficient properly to distend the elastic arteries.

It was formerly supposed that the occurrence of any transudation from the interior of the capillaries into the midst of the surrounding tissues was confined, in the absence of injury, strictly to the fluid part of the blood; in other words, that the corpuscles could not escape from the circulating stream, unless the wall of the containing blood vessel was ruptured. It is true that the English physiologist Augustus Waller affirmed in 1846 that he had seen blood corpuscles, both red and white, pass bodily through the wall of the capillary vessel in which they were contained (thus confirming what had been stated a short time previously by Addison). He said that no opening could be seen before their escape and that none could be observed afterward, so rapidly was the part healed. But these observations did not attract much notice until the phenomenon of escape of the blood corpuscles from the capillaries and minute veins, apart from mechanical injury, was rediscovered by Cohnheim in 1867.

Cohnheim's experiment demonstrating the passage of the corpuscles through the wall of the blood vessel is performed in the following manner: A frog is curarized; that is to say, paralysis is pro-

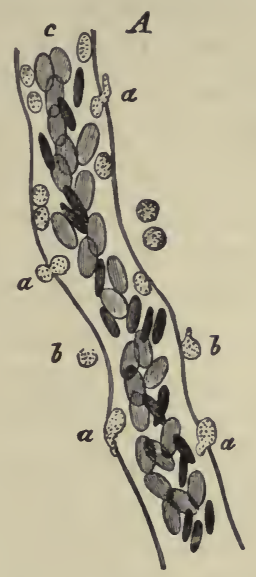

Fig. 194.-A Large Capillaryfrom the Frog's Mesentery Eight Hours after Irritation had been set up, Showing Emigration of Leucocytes. $a$, Cells in the act of traversing the capillary wall; $b$, some already escaped. (Frey.) duced by injecting under the skin a minute quantity of the poison called curare. The abdomen is then opened, a portion of the small intestine is drawn out, and its transparent mesentery spread out under a microscope. After a variable time, occupied by dilatation following contraction of the minute vessels and the accompanying quickening of the blood stream, there ensues a retardation of the current and the red and white blood corpuscles begin to make their way through the capillaries and small veins.

The white corpuscles pass through the capillary wall chiefly by the ameboid movement with which they are endowed. This migration occurs to a limited extent in health, but in inflammatory conditions is much increased.

The process of diapedesis of the red corpuscles, which occurs under circumstances of impeded venous circulation, and consequently increased blood pressure, resembles closely the migration of the leucocytes, with the exception that they are squeezed through the wall of the vessel, and do not, like the leucocytes, work their way through by ameboid movement. 
Various explanations of these remarkable phenomena have been suggested. It is no longer believed that pseudo-stomata between contiguous endothelial cells provide the means of escape for the blood corpuscles. The chief share in the process is probably due to mobility and contraction of the parts concerned, both of the corpuscles and of the capillary wall itself.

The Speed of the Blood in the Capillaries.-The velocity of the blood through the capillaries must, of necessity, be largely influenced by that which occurs in the vessels on both sides of them, in the arteries and the veins. Their intermediate position causes them to respond at once to any alteration in the size or rate of the arterial or venous blood stream. Thus, the apparent contraction of the capillaries, on the application of certain irritating substances or during certain mental states, and their dilatation in blushing may be referred primarily to the corresponding action of the small arteries.

The Measurement of Velocity in the Capillaries.-The observation of Hales, E. H. Weber, and Valentin agree very closely as to the rate of the blood current in the capilaries of the frog. The mean of their estimates gives the velocity of the systemic capillary circulation at about $0.5 \mathrm{~mm}$. per second. The velocity in the capillaries of warm-blooded animals is greater, in the dog 0.5 to $0.75 \mathrm{~mm}$. per second. This may seem inconsistent with the facts, which show that the whole circulation is accomplished in about half a minute. But the whole length of capillary vessels, through which any given portion of blood has to pass, probably does not exceed $0.5 \mathrm{~mm}$. Therefore the time required for each quantity of blood to traverse its own appointed portion of the general capillary system will scarcely amount to more than a second. This comparatively slow velocity is evidently favorable to the nutritive interchanges that go on through these thin-walled vessels between the blood within the capillaries and the outside active tissues.

The Venous Flow.-The blood current in the veins is maintained, $a$, primarily by the contractions of the left ventricle; but very effectual assistance to the flow is afforded, $b$, by the action of the muscles capable of pressing on the veins with valves, and $c$, by the aspiration of the thorax and possibly, $d$, by the aspiration of the heart itself.

The effect of muscular pressure upon the circulation may be thus explained: When pressure is applied to any part of a vein, and the current of blood in it is obstructed, the portion behind the seat of pressure becomes swollen and distended as far back as the next pair of valves, which are in consequence closed. Thus, whatever force is exercised by the external pressure of the muscles on the veins, is distributed partly in pressing the blood onward in the proper course of the circulation, and partly in pressing it backward and closing the valves behind. 
The circulation might lose as much as it gains by such an action if it were not for the numerous communications, or venous anastomoses. Owing to these anastomoses the closing up of the venous channel by the backward pressure is prevented from being any serious hindrance to the circulation, since the blood which is arrested in its onward course by the closed valves can at once pass through some anastomosing channel and proceed on its way by another vein. Thus the effect of muscular pressure upon veins which have valves is turned almost entirely to the advantage of the circulation. The pressure of the blood onward is all advantageous, and the pressure of the blood backward is prevented from being a hindrance by the closure of the valves and by the anastomoses of the veins.

The venous flow is also assisted by the aspiration of the thorax and to some extent by that of the heart, since at some time during every cardiac cycle the intra-auricular and intra-ventricular pressure falls below that of the atmosphere. This activity will be considered more fully in the chapter on Respiration. In this connection it may be said, however, that the pressure in the great veins falls during inspiration and rises during expiration.

The Velocity in the Veins. - The velocity of the blood is greater in the veins than in the capillaries, but less than in the arteries; this fact depending upon the relative capacities of the arterial and venous systems. If an accurate estimate of the proportionate areas of arteries and the veins corresponding to them could be made, we might, from the velocity of the arterial current, calculate that of the venous. The usual estimation is that the capacity of the veins is about two or three times as great as that of the arteries, and that the velocity of the blood's motion is, therefore, about one-half or one-third as great in the veins as in the arteries, i.e., $200 \mathrm{~mm}$. a second. The rate at which the blood moves in the smallest venules is only slightly greater than that in the capillaries, but the speed of flow gradually increases the nearer the vessel approaches to the heart. The total sectional area of the venous trunks, compared with that of the branches opening into them, becomes gradually smaller as the trunks advance toward the heart, figure I9I.

The Velocity of the Circulation as a Whole.-It would appear that a portion of blood can traverse the entire course of the circulation, in the horse, in half a minute. Of course it would require longer to traverse the vessels of the most distant part of the extremities than to go through those of the neck, but taking an average length of the vessels to be traversed it may be concluded that half a minute represents the average rate. Stewart estimated that the circulation time in man is probably not less than twelve nor more than fifteen seconds.

Satisfactory data for these estimates are afforded by the results of.experiments to ascertain the rapidity with which chemicals introduced in to the blood are transmitted from one part of the vascular system to another. The 
time required for the passage of solutions of potassium ferrocyanide, mixed with the blood, from one jugular vein, through the right side of the heart, the pulmonary vessels, the left cavities of the heart, and the general circulation, to the jugular vein of the opposite side, varies from twenty to thirty seconds in the dog. The same substance is transmitted from the jugular vein to the great saphenous vein in twenty seconds; from the jugular vein to the mesenteric artery in between fifteen and thirty seconds; to the facial artery, in one experiment, in between ten and fifteen seconds; in another experiment, in between twenty and twenty-five seconds; in its transit from the jugular vein to the metatarsal artery, it occupies between twenty and thirty seconds. The result is said to be nearly the same whatever the rate of the heart's action. In more recent methods some innocuous dye like methylene blue is used, since it permits the determination without the loss of blood, the change in color being visible through the walls of the blood vessels.

Stewart has made most accurate measurements of the circulation time by the electrical-resistance method. Strong salt solutions injected into the jugular vein on one side when they reach the other jugular (or any other vessel) are instantly detected by a decrease in the electrical resistance through the vessel when it is laid between the poles of the proper conductivity apparatus.

In all these experiments it is assumed that the substance injected moves with the blood and at the same rate, and does not move from one part of the organs of circulation to another by diffusing itself through the blood or tissues more quickly than the blood moves. The assumption may be accepted that the times above mentioned as occupied in the passage of the injected substances are the times in which the portion of blood itself is carried from one part to another of the vascular system.

Another mode of estimating the general velocity of the circulating blood is by calculating it from the quantity of blood supposed to be contained in the body and from the quantity which can pass through the heart in each of its contractions. But the conclusions arrived at by this method are less satisfactory. For the total quantity of blood and the capacity of the cavities of the heart have as yet been only approximately ascertained. Still the most careful of the estimates thus made accord very nearly with those already mentioned; and it may be assumed that the blood may all pass through the heart in man in about thirty seconds or even less.

\section{THE PULSE.}

The most characteristic feature of the arterial pressure and blood flow is its intermittency, and this intermittent flow is seen or felt as waves of change in diameter of the arteries, known as the Pulse. 
The pulse is generally described as a wave-like expansion of the artery produced by the injection of blood at each ventricular systole into the already full aorta. The force of the left ventricle is expended in pressing the blood forward and in dilating the aorta. With the injection of each new quantity of blood into the aoria there is a wave of dilatation which passes on, expanding the arteries as it goes, running as, it were, over the more slowly traveling blood contained in them, and producing the pulse as it proceeds. A sharp distinction must be made between the passage of the pulse wave along an artery and the rate of flow of the blood in the vessel. The pulse produced by any given beat of the heart is not felt at the same moment in all parts of the body. Thus, it can be felt in the carotid a short time before it is perceptible in the radial artery, and in this vessel before it occurs in the dorsal artery of the foot. Careful measurements of the intervals between the time of the pulse at the carotid and at the wrist shows that the delay in the beat is in proportion to the distance of the artery from the heart. The difference in time between the pulse of any two arteries probably never exceeds one-sixth to one-eighth of a second. The rate at which the pulse travels in the arteries is from five to ten meters per second.

The distention of each artery increases both its length and its diameter. In their elongation the arteries change their form, the straight ones becoming slightly curved, and those already curved becoming more so; but they recover their previous form as well as their diameter when the ventricular contraction ceases, and their elastic walls recoil. The increase of their curves which accompanies the distention of arteries, and the succeeding recoil, may be well seen in the prominent temporal artery of an old person. In feeling the pulse, the finger cannot distinguish the sensation produced by the dilatation from that produced by the elongation and curving. That which it perceives most plainly, however, is the dilatation and return more or less to the cylindrical form, of the artery which has been partially flattened by the finger.

The Sphygmograph.-Much light has been thrown on what may be called the form of the pulse wave by an instrument called the sphygmograph, figures 195 and 196 . The principle on which it acts will be seen on reference to the figures.

A small button replaces the finger in the act of taking the pulse. This button is made to rest lightly on the artery the pulsations of which it is desired to investigate. The up-and-down movement of the button is communicated to the lever, to the hinder end of which is attached a light spring. The spring is adjusted to the proper tension to follow the movements of the artery wall during the pulse wave. The sphygmograph is bound on the wrist while taking a record.

It is evident that the beating of the pulse will cause an up-and-down movement of the lever, the pen of which will write the effect on a smoked 
card moved by the clock-work of the instrument.

Thus a tracing of the pulse is obtained, and in this way much more delicate changes can be seen than can be felt by the mere application of the finger.

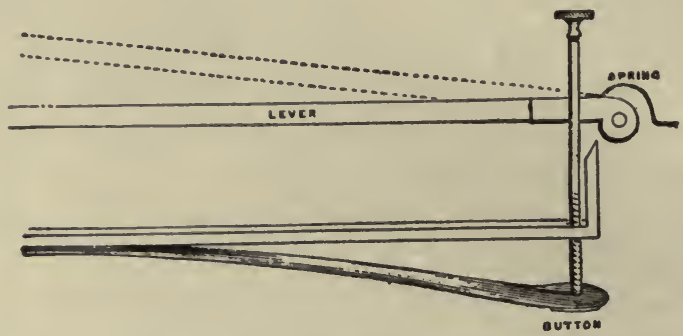

FIG. I95.-Diagram of the Lever of the Sphygmograph.

The principle of the sphygmometer of Roy and Adami is shown in the diagram, figure 197.

The apparatus consists of a box, $a$, which is moulded to fit over the end of the radius so as to bridge over the radial artery. Within this is a flexible bag, $q$, filled with water,

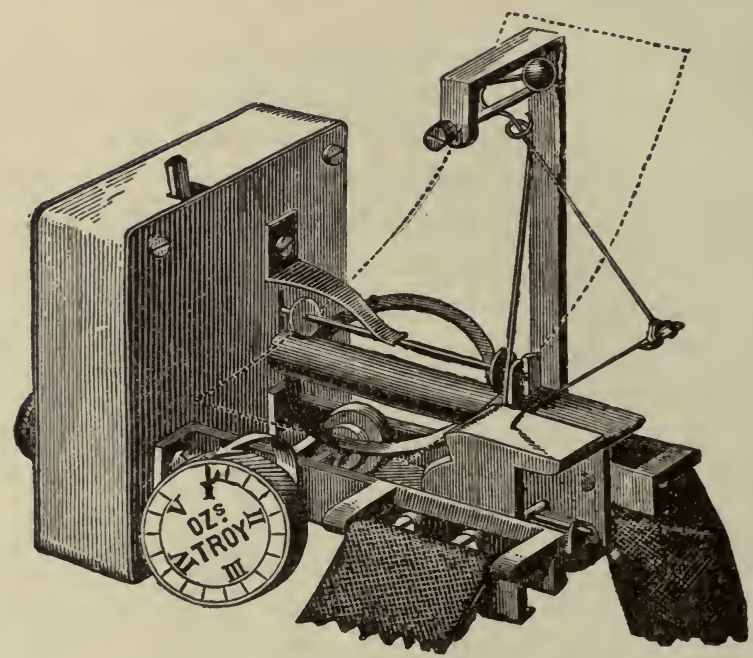

FIG. I96.-Dudgeon's Sphygmograph.

and connected by a T-tube with a rubber bag, $h$, and mercurial manometer. The fluid in the box may be raised to any desired pressure, and may then be shut off by tap, $c$. At the upper part of the box is a circular opening, and resting upon $b$ is a flat button, $d$, which by means of a short light rod, $e$, communicates the movement of $b$ to the lever, $f$. At the axis of rotation of this lever is a spiral watch-spring, $g$, which can be tightened at will, so that the lever can be made to take a vertical position at any desired hydrostatic pressure within the box. The movements of the lever are recorded upon a piece of blackened glazed paper made to move in a vertical direction past it. When in use, the box is fixed upon the wrist by an appropriate holder, and the pressure is raised to 
any desired height to which the lever is adapted by tightening or slackening the spring; the tap, $c$, is then closed. The pressure within the box acts in all directions, and is correctly indicated by the manometer.

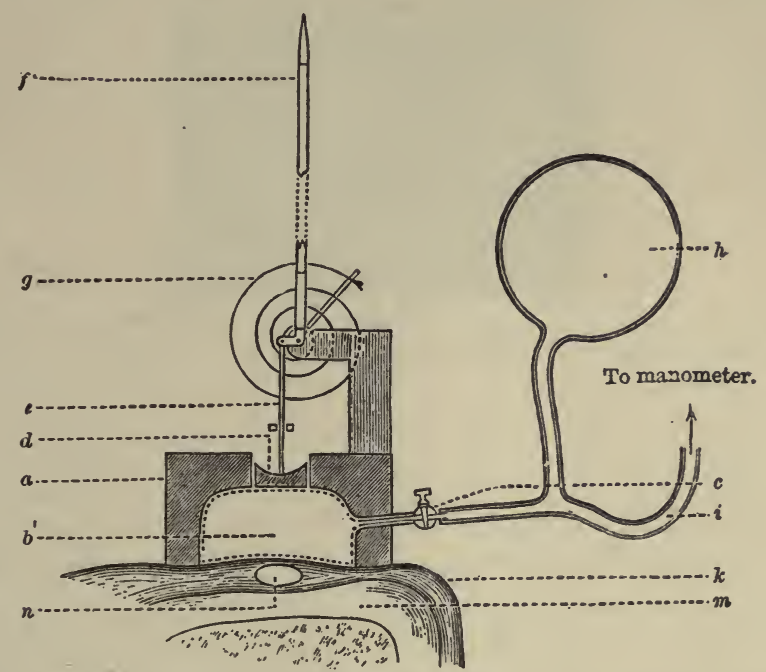

Fig. 197.-Diagrammatic Sectional Representation of the Sphygmometer. a, Box by which the portion of the artery is covered; $b$, thin-walled india-rubber bag filled with water, and communicating through tap, $c$, with the manometer and thick-walled rubber bag, $h ; d$, piston connected by rod, $e$, with recording lever, $f ; g$, spiral spring, attached to axis of lever, and by which the pressure in $b$, against the piston, $d$, is counterbalanced; $k$, skin and subcutaneous tissue; $m$, end of radius seen in section; $n$, radial artery seen in section. (Roy and Adami.)

Sphygmogram.-The tracing of the pulse obtained by the use of the sphygmograph, called a sphymogram, differs somewhat according to the artery from which it is taken, but its general characters are much the same in all cases. It consists of a sudden upstroke, or anacrotic limb, figure I98, $A$, which is somewhat higher and more abrupt in the pulse of the carotid and of other arteries near the heart than in the radial and other arteries more remote; and a gradual decline or catacrotic limb, $P$, less abrupt, and taking a longer time than $A$. It is seldom, however, that the decline is an uninterrupted fall; it is usually marked about half-way by a distinct notch, $C-D$ the dicrotic notch, followed immediately by a second more or less marked ascent of the lever called the dicrotic wave, $D$. Not infrequently there is also at the beginning of the descent a slight wave previous to the dicrotic notch; this is called the pre-dicrotic wave, and in addition there may be one or more slight waves after the dicrotic, called post-dicrotic, $E$. The interruptions in the downstroke are called the catacrotic waves to distinguish them from an interruption in the upstroke, called the anacrotic wave, which is sometimes met with. 
The explanation of these tracings present some difficulties, not, however, as regards the two primary factors, viz., the upstroke and downstroke,

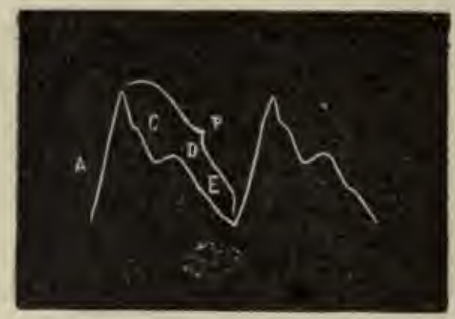

FIG. 198.-Diagram of Pulse Tracing. $A$, upstroke or anacrotic limb; $P$, downstroke or katacrotic limb; $C$, pre-dicrotic wave; $D$, dicrotic; $E$, post-dicrotic wave.

because they are universally taken to mean the sudden injection of blood into the already distended arteries, and the gradual recovery of the arteries by their recoil. These points may be demonstrated on a system of elastic tubes, with a pump to inject water at regular intervals, just as well as on the radial artery, or on the arterial schema, a more complicated system of tubes in which the heart, the arteries, the capillaries, and veins are represented. If we place two or more sphygmographs upon such a system of tubes at increasing distances from the pump, we may demonstrate, first, that the rise

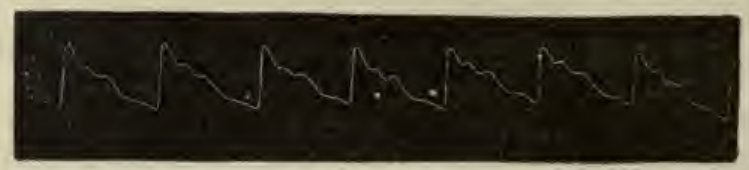

FIG. 199.-Sphygmogram from the Radial Artery Taken with Marey's Sphygmograph. (Langendorff.)

of the lever commences earliest in that nearest the pump, and, second, that it is higher and more sudden. So in the arteries of the body the wave gradually gets less and less as we approach the periphery of the arterial system, and it is lost in the capillaries.

The origin of the secondary waves is to some extent a matter of uncertainty. The anacrotic wave occurs when the peripheral resistance is high; that is, when, for some time during the systole, the flow from the aorta toward the periphery is slower than the flow from the ventricle into the aorta. Thus it is seen in some cases of nephritis where the arteries are rigid and the peripheral resistance is high.

The dicrotic wave is the most important of the secondary waves, and has been the subject of much discussion. It is constantly present in pulse tracings, but varies in height. In point of time the dicrotic wave occurs immediately after the closure of the aortic semilunar valves. In certain 
conditions, generally of disease, it becomes so marked as to be quite plain to the unaided finger. Such a pulse is called dicrotic. The generally accepted explanation of the cause of the dicrotic wave is that it represents a
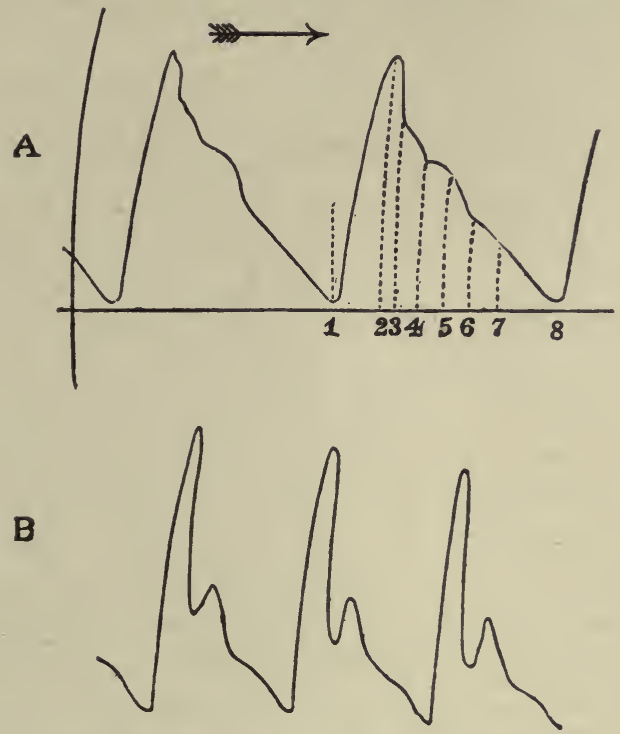

FIG. 200.- $A$, Normal Pulse Tracing from Radial of Healthy Adult Obtained by the Sphygmometer; $B$, from same artery, with the same extra-arterial pressure, taken during acute nasal catarrh. I-2 Anacrotic limb; 2-8 Catacrotic limb; 3 Predicrotic notch; 5 Dicrotic crest; 6 Postdicrotic notch; 7 Postcrotic crest; 4 Dicrotic notch.

rebound of the overdistended artery at the time of the closure of the aortic valves. During systole, as the blood is forcibly injected into the aorta, there is an overdistention of the artery. The systole suddenly ends, the aorta by reason of its elasticity tends to recover itself, the blood is driven back against the semilunar valves, closing them and at the same time giving rise to a wave, the dicrotic wave, which begins at the heart and travels onward toward the periphery like the primary wave. According to Foster, the conditions favoring the development of dicrotism are: I, a highly extensible and elastic arterial wall; 2, a comparatively low mean blood-pressure, leaving the extensible reaction free scope to act; 3 , a vigorous and rapid stroke of the ventricle discharging into the aorta a considerable quantity of blood. The other secondary waves are probably due to the oscillations in the elastic recoil of the arteries, though some of them at least may be due to the inertia of the instruments used.

In the use of the sphygmograph care must be taken in the regulation of the pressure of the spring. If the pressure be too great, the characters of the pulse may be almost entirely obscured or the artery may be completely obstructed and no tracing is obtained. On the other hand, if the pres- 
sure is too slight, a very small part of the characters may be represented on the tracing.

\section{THE PERIPHERAL REGULATION OF THE FLOW OF BLOOD.}

The flow of blood through the circulatory system depends on the interaction of several factors which have already been mentioned in another connection: The rate and volume of the heart-beat, the elasticity of the blood vessels, the resistance of the microscopic peripheral vessels, and the volume of blood in the body. We have already learned, page 205; that both the rate and volume of the contractions of the heart are under very minute and intimate regulation and control through the cardiac nervous mechanism. Also we have found that there is intimate co-ordination between the activity of the heart and the activity of all other parts of the body, a co-ordination secured through the nervous system. All regulation which affects the heart must of necessity affect the general blood-pressure and, therefore, not directly any particular part.

The general elasticity of the blood vessels, and of the arteries in particular, which makes the general arterial pressure possible, is dependent primarily on the presence of a large amount of elastic connective tissue in the walls of the vessels. The elasticity of this tissue is a purely passive property which can be utilized only by some positive source of energy, in this instance the heart.

The Variations in Peripheral Resistance.-Certain arteries and veins, especially the smallest ones, the arterioles and venules, are supplied with muscular tissue in their walls. The activity of these muscles in the vascular complex makes the peripheral regulation of the flow of blood possible. This muscular tissue not only exhibits a passive elasticity comparable to that of the yellow elastic connective tissue, but upon the proper stimulation it actively contracts or relaxes, thus securing to the peripheral resistance of the vessels an active adjustment to the ever-varying dynamic conditions of the vascular apparatus.

The muscular tissue in the vessels increases relatively in amount as the vessels become smaller. In the arterioles it is developed out of all proportion to the other elements. In fact, in passing from the arterioles to the capillary vessels, made up as we have seen of endothelial cells with a supporting ground substance only, the last change on the side of the arteries, which occurs as the vessels become smaller, is the disappearance of muscular fibers.

The office of the muscular coat is to adjust the size of the arterioles and, therefore, the flow of the blood. It is to regulate the quantity of blood to be received by each part or organ, and to adjust this quantity to the requirements of each, according to various circumstances, but chiefly according to the de- 
gree of activity which each organ at different times exhibits. The amount of work done by each organ of the body constantly varies, and the variations often quickly succeed each other, so that, as in the muscles for example, within the same hour a part may be now very active and now quite inactive. In all its active exercise of function, such an organ requires a larger supply of blood than is sufficient for it during the times when it is comparatively inactive.

It is evident that the heart cannot regulate the blood-supply to each part of the body at different periods independently of the other parts. Neither could this be regulated by any general and uniform contraction of the arteries. But it may be regulated by the power which the arteries of each part have, through their muscular tissue, of contracting or relaxing so as to diminish or increase their size. Since the general blood pressure is fairly constant the size of the local vessels controls the supply of blood to the particular part of the body to which the vessels are distributed. Thus, while the ventricles of the heart determine the total quantity of blood to be sent onward to each contraction and the force of its propulsion, and while the large and merely elastic arteries distribute the blood and equalize its stream, the smaller arteries regulate and determine the.proportion of the whole quantity of blood which shall be distributed to each particular organ.

The variation of the size of arterioles and, therefore, of the resistance to the flow of the blood in them is secured by the contractions of the muscular tissue, but the muscles are regulated in their contractions by the nervous system. The muscular tissue in the blood vessels of the organs of the different parts of the body is also co-ordinated by the same regulative and controlling influence of the nervous system.

The Discovery of the Vaso-motor Nerves.-More than half a century ago (I85I) it was shown by Claude Bernard that if the cervical sympathetic nerve is divided, the blood vessels of the corresponding side of the head and neck become dilated. This effect is best observed in the ear, which if held up to the light is seen to become redder and the arteries to become larger. The whole ear is distinctly warmer than the opposite one. This effect is produced by removing the arteries from the tonic influence of the central nervous system, which influence normally passes along the course of the divided nerve.

If the peripheral end of the divided nerve be stimulated in its course toward the organ, i.e., that farthest from the brain, the arteries which were before dilated return to their natural size, and the parts regain their former condition. And, besides, if the stimulus is very strong or very long-continued, the amount of normal constriction is passed and the vessels become much more contracted than before. The natural condition, which is midway between extreme contraction and extreme dilatation, is called the natural 
tone of an artery. If this is not maintained, the vessel is said to have lost tone, or, if it is exaggerated, the tone is said to be too great. The effects described as having been produced by section of the cervical sympathetic and by subsequent stimulation are not peculiar to that nerve and the vessels to which it is distributed.

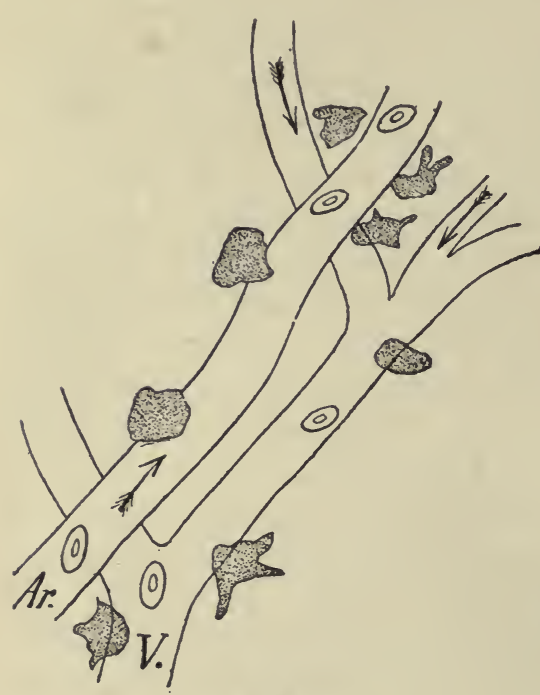

A

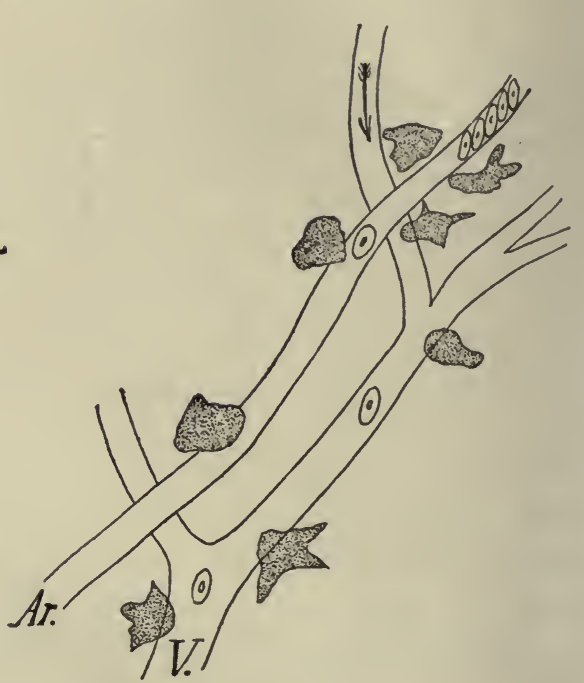

B

FIG. 20x.-Small Artery and Vein of the Frog's Web. $A$, Under normal conditions; $B$, upon stimulation of the sciatic nerve; $A r$, artery; $V$, vein. In this experiment the vein also showed well-marked vaso-constriction. (Greene.)

It has been found that for every part of the body there exists a nerve the division of which produces the same effects, viz., dilatation of the vessels. Such may be cited as the case with the sciatic, the splanchnic nerves, and the nerves of the brachial plexus; when these are divided, dilatation of the blood vessels in the parts supplied by them takes place. It appears, therefore, that nerves exist which have a distinct control over the vascular supply of every part of the body. These are called vaso-motor or vasoconstrictor nerves. But the arterioles are also under the influence of a second set of nerves, also discovered by Claude Bernard, which produce exactly the opposite influence, i.e., dilatation. These nerves are called vasodilator nerves.

Mall has also shown that veins, at least the portal vein, possess a vasomotor nerve supply as well as arteries.

Vaso-constrictor Nerves.-The presence of vaso-constrictor nerves can be shown in several different ways, of which the most convincing is that of direct inspection. If a vascular membrane, like the web of the frog's 
foot or the bat's wing, be adjusted on the stage of a microscope for direct inspection, and the smaller arterioles are under observation, then upon the stimulation of the general nerve supplying the part these arterioles will sharply decrease in size. In fact, the vaso-constriction is often so great as completely to occlude the vessel. Very soon after the stimulation the vessel again dilates to its normal size.

The presence and course of the vaso-constrictor nerve supply to the organs of the body have been demonstrated not by direct inspection, but by the use of various forms of the plethysmograph. A plethysmograph is an

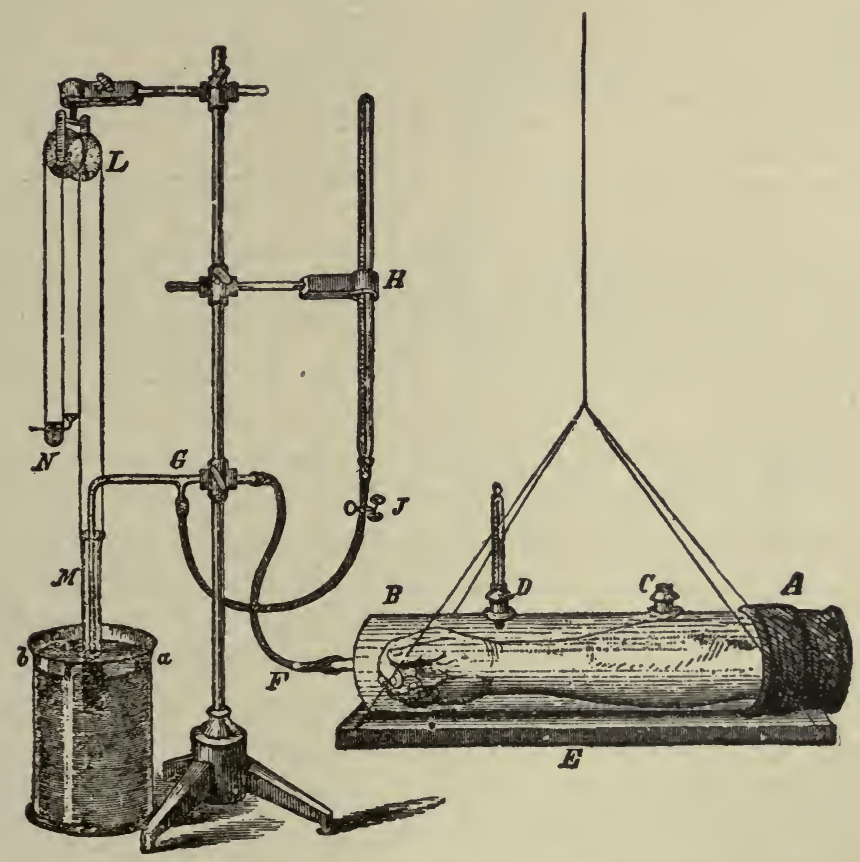

Fig. 202.-Arm Plethysmograph. Apparatus for measuring the change in volume in the arm due to variation in the blood supply. The arm is enclosed in a glass cylinder which is completely filled with fluid, the opening through which the arm is inserted being closed by a rubber sleeve, $A$. The cavity of the glass cylinder communicates through the tube, $F, G$, with the test-tube, $M$, which is supported in the jar, $P$. Any variation in volume in the arm will cause water to flow out or into the test-tube, $M$, which is lowered as the tube fills, and raised as it empties. The rise and fall of the test tube, $M$, is communicated over the pulley, $L$, to the writing pen, $N$, which records the movements on the smoked cylinder. Kymograph not shown. (Mosso.)

instrument designed to measure the variations in the volume of an organ. If the finger, the whole hand, the spleen, or the kidney be placed in such an instrument and the proper steps be taken to record the volume changes, it will be found that the volume of the enclosed organ is constantly changing with every variation of the blood-pressure. If the nerves to the organ are 
stimulated by the usual rapidly interrupted induction current, for example the splanchnics to the kidney, then there is a decrease in the volume of the organ. This decrease takes place even when there is a simultaneous increase of the arterial blood-pressure, a result that can be explained only on the assumption of vascular decrease in the organ. The decrease in the flow of blood to the specific organ can be induced only by a great decrease in the size of the arterioles produced by contractions of the circular muscles of their walls.

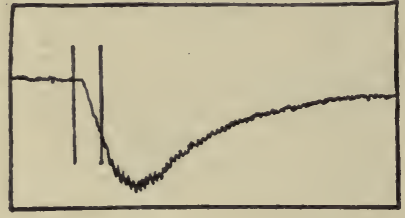

$a$

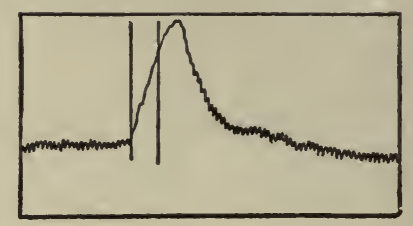

$b$

FIG. 203.- Plethysmograms of the Hind Leg of the Cat, showing $a$, vaso-contriction on stimulating the sciatic at the rate of 15 stimuli per second for twenty seconds. In $b$, the dilation of the blood vessels of the opposite leg of the same animal is shown on stimulating the sciatic which had been cut four days previously. The vaso-constrictor nerves were degenerated and the vaso-dilators still active. (Bowditch and Warren.)

Vaso-motor Tone.-Vaso-constrictor changes are constantly occurring in the blood vessels of the organs of the body, a fact that has been abundantly demonstrated by the plethysmographic experiments just mentioned. Direct inspection of the ear of an albino rabbit will show that the arteries, and veins as well, are now full and large and red, and the interspaces filled with blood, and now pale and constricted, and the interspaces relatively bloodless. If the cervical sympathetic is cut as in Bernard's experiment, then the ear vessels remain dilated, that is they lose their tone. This shows that the condition is dependent primarily on the constant discharges of nerve impulses from the nervous system. It is said that the vessels regain their tone after a time. when the nerves are cut. The regained power may be ascribed to the muscle fibers themselves.

Vaso-constrictor Center.-When the tonic influence exerted by the nerve fibers on the arterioles is traced back into the central nervous system, it is found to be associated with the activity of certain groups of nerve cells, or centers, which are called the vaso-constrictor centers. This determination is made in part by the method of sectioning. A lesion of the cerebro-spinal axis below the corpora quadrigemina is followed by partial or complete general dilatation of the blood vessels and a great fall of bloodpressure. This is due to the isolation of the vaso-constrictor center, which lies in the floor of the fuurth ventricle, a few millimeters caudal to the corpora quadrigemina, and extends longitudinally over an area of about three millimeters. Owsjannikow has shown that the center is composed of 
two halves, each half lying in the lateral column to the side of the median line. This center is in constant action during life, and its discharges are responsible for the vascular tone described in the previous paragraph. The vaso-constrictor center varies in its activity, sometimes producing wave-like contractions with relaxations of the arterial walls, producing variations in the blood-pressure known as TraubeHering waves. These waves are more often observed in mammalian blood-pressure experiments after prolonged operations, when the center may be supposed to be itself in a weakened condition.

Secondary vaso-motor centers are present in the spinal cord as proven by Goltz. Under normal conditions they do not act independently of the medullary center; but when the function of the latter has been interrupted by section of the cord, then after a few days the spinal cells below the section take on central functions and bring about a re-establishment of the lost vascular tone. If these centers be destroyed by the destruction of the cord, then the tone of the vessels immediately disappears, but is regained after the lapse of a much longer time. This can be ascribed to the presence of possible sympathetic constrictor centers or more probably to a fundamental property of the muscles themselves. This experiment was carried out by Goltz and Oswald, who found that after destruction of the lower part of the spinal cord, the tone of the vessels of the hind limbs, lost as a result of the operation, was later re-established.

Vaso-constrictor Reflexes.-Under normal conditions the medullary center responds to afferent stimuli by vaso-motor reflexes. The secondary vasomotor centers in the spinal cord, when removed from the influence of the bulbar center, can and do respond to afferent impulses by similar vaso-motor action.

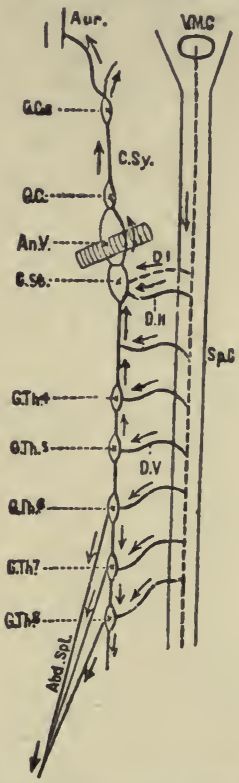

Fig. 204.-Diagram Showing the Paths of the Vaso-constrictor Fibers along the Cervical Sympathetic and the Abdominal Splanchnic. Aur. Artery of ear; $G$. $C s$, superior cervical ganglion; $A n$. $V$, annulus of Vieussens; $G$. St, stellate ganglion; $D$. $I, D . I I, D . V$, thoracic spinal nerves; $A b d$. Spl, abdominal splanchnic. The arrows indicate the direction of vaso-constrictor impulse.

The afferent impulses which excite reflex vaso-motor action may proceed from the terminations of sensory nerves in general, or possibly from the blood vessels themselves, and the constriction which follows generally occurs in the area whence the impulses arise. Yet the reflex may appear elsewhere. Impulses proceeding to the vaso-motor center from the cerebrum may cause vaso-dilatation, as in blushing, or vaso-constriction, as in the pallor of fear or of anger. 
Afferent influence upon the vaso-motor centers is well shown by the action of the depressor nerve, the existence of which was demonstrated by Cyon and Ludwig. The depressor is a small afferent nerve which passes up to the medulla from the heart, in which it takes its origin. It runs upward in the sheath of the vagus or in the superior laryngeal branch of the vagus or as an independent branch, as in the rabbit, communicating by filaments with the inferior cervical ganglion as it proceeds from the heart. If, in a rabbit, this nerve be divided and the central end stimulated during a blood-pressure observation, a remarkable fall of blood-pressure takes place, figure 205 .

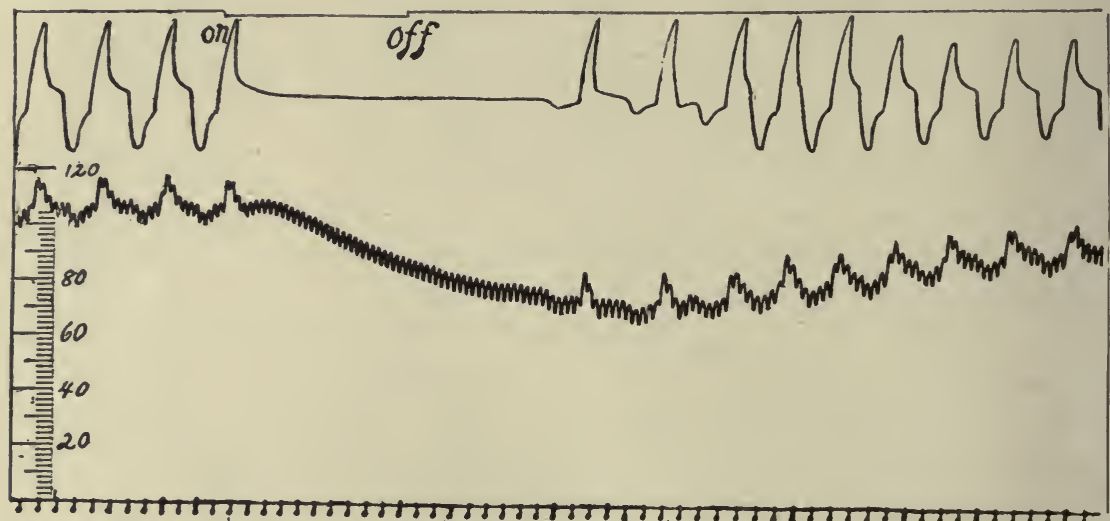

FIG. 205.-Blood-pressure Record (lower) and Respiratory Record (upper) Obtained from a Dog upon Stimulating the Central End of the Divided Vagus, Both Vagi being Cut. The marked fall in blood-pressure is due to the effect of stimulating the depressor fibers contained in the vagus trunk of the dog. (New figure by Dooley and Dandy.)

The cause of the fall of blood pressure is found to proceed primarily from the dilatation of the vascular district within the abdomen supplied by the splanchnic nerves, in consequence of which the vessels hold a much larger quantity of blood than usual. The engorgement of the splanchnic area very greatly diminishes the amount of blood in the vessels elsewhere, and so materially diminishes the blood-pressure. The function of the depressor nerve is that of conveying to the vaso-motor center afferent nerve impulses from the heart, which produce an inhibition of the tonic activity of the vaso-motor center and, therefore, a diminution of the tension in the blood vessels. This diminishes the overstrain on the heart in propelling blood into the already too full or too tense arteries. It has been shown by Porter and Beyer that the fall in blood-pressure, following stimulation of the depressor nerve, will still occur, even when the abdominal vaso-constriction is kept constant by a simultaneous stimulation of the splanchnics. It is therefore evident that the inhibitory effect of depressor-nerve stimulation is a general one and not confined to the splanchnic area alone. 
The action of the depressor nerve in causing an inhibition of the vasomotor center illustrates the more unusual effect of afferent impulses; that is, inhibition of the vaso-constrictor tone. As a rule, the stimulation of the central end of an afferent nerve, such as the sciatic or the internal saphenous, produces the reverse, i.e., a pressor effect, and increases the tonic influence of the center which by causing constriction of the arterioles raises the blood pressure. Thus the reflex effects of stimulating an afferent nerve may be either to constrict or to dilate the arteries. These reflexes may be general enough to influence the general blood-pressure or they may be limited to definite local areas, but the local effects are the all-important ones, since by these the local regulation of the blood flow is accomplished.

Traube-Hering Curves. - The vaso-motor center sends out rhythmical impulses by which undulations of blood-pressure of a large and sweeping character are produced, quite independent of the so-called respiratory undulations. The action of this center in producing such undulations is demonstrated in the following observations. In an animal under the influence of curare and with both vagi cut, and a record of whose blood-pressure is being taken, if artificial respiration be stopped, the blood-pressure rises sharply at first. After a time the rhythmical undulations shown in figure 206 occur. These variations are called Traube's or Traube-Hering curves. There may be upward of ten of the respiratory undulations in one TraubeHering curve. They continue until the vaso-motor center is asphyxiated and the heart exhausted, when the pressure falls. The undulations cannot be caused by anything but the vaso-motor center, as the mechanical effects of respiration have been eliminated by the curare and by the cessation of artificial respiration, and the effect of the cardio-inhibitory center has been removed by the division of the vagi. The rhythmic rise of blood-pressure is most likely due to a rhythmic constriction of the arterioles followed by a corresponding relaxation and fall of pressure, both being due to the action of the vaso-motor center. The vaso-motor center, therefore, is capable of producing rhythmical discharges of vaso-constrictor nerve impulses that result in the undulations of blood-pressure.

Vaso-dilator Nerves.-Claude Bernard discovered (1856) that the blood flow was increased through the salivary glands by stimulation of the nerves (the chorda tympani for the submaxillary, and the tympanic branch of the glossopharyngeal for the parotid), thus proving that the arteries have not only vaso-constrictors, but also vaso-dilator nerves. Vaso-dilator nerves have been described for most parts of the body. In general they are distributed in the same nerve trunks which bear the vaso-constrictors.

It is not supposed that the vaso-dilators produce widening of the arterioles by stimulation to active muscular contraction; in fact, the circular arrangement of the muscle fibers would seem to exclude such a deduction. It is probable that there is local inhibition of the tonic contraction of the 
muscles, thus allowing the mechanical factor of the general blood-pressure to dilate the vessels. The vaso-dilator nerves are characterized by their response to slowly developed stimuli, shown by Bowditch and Warren, and by the retention of irritability after degeneration of the constrictors has taken place, see figure 203 .

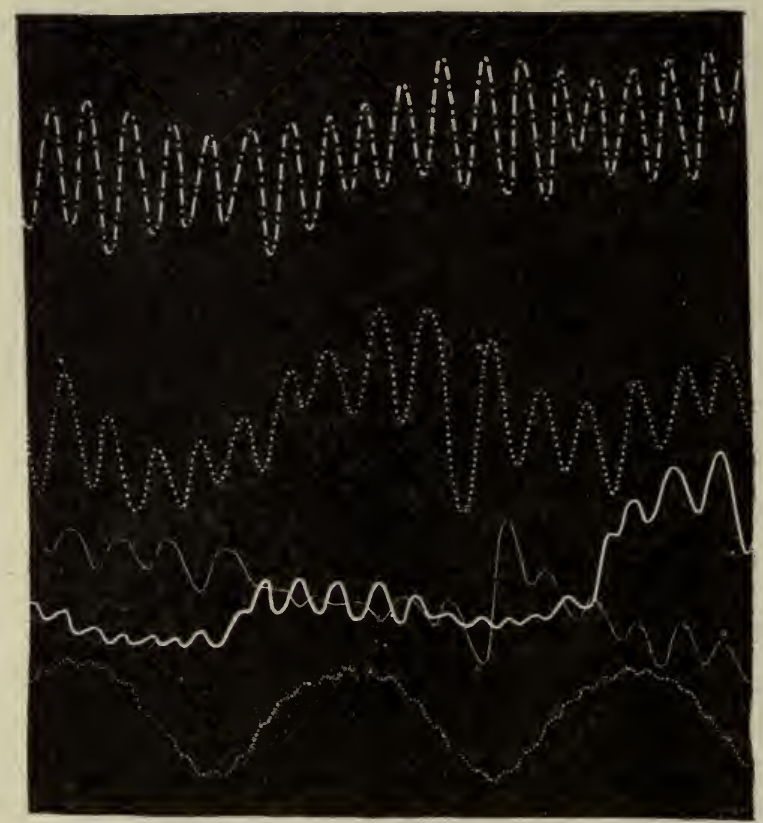

4 .

FIG. 206.- Traube-Hering's Curves. (To be read from left to right.) The curves $\mathrm{x}, 2,3,4$, and 5 are portions selected from one continuous tracing forming the record of a prolonged observation, so that the several curves represent successive stages of the same experiment. Each curve is placed in its proper position relative to the base line, which is omitted; the blood-pressure rises in stages from I to 2, 3, and 4, but falls again in stage 5 . Curve $I$ is taken from a period when artificial respiration was being kept up, but, the vagi having been divided, the pulsations on the ascent and descent of the undulations do not differ; when artificial respiration ceased, these undulations for a while disappeared, and the blood-pressure rose steadily while the heart-beats became slower. Soon, as at 2, new undulations appeared; a little later, the blood-pressure was still rising, the heart-beats still slower, but the undulations still more obvious 3 ; still later 4 , the pressure was still higher, but the heart-beats were quicker, and the undulations flatter; the pressure then began to fall rapidly 5 , and continued to fall until some time after artificial respiration was resumed. (M. Foster.)

Vaso-dilator Centers. - No distinct medullary center has yet been shown to regulate the vaso-dilator nerve activity. Such centers, if they exist, should be influenced by isolating them from their efferent paths, on the one hand, or by stimulation by afferent channels, on the other. The former method of study has revealed nothing that can be compared to the tonic ac- 
tivity of the constrictor center. Efferent dilator-nerve impulses can be reflexly produced by sensory stimulation. The isolated lumbar cord of a dog is capable of reflex vaso-dilator activity, since stimulation of the skin of the penis leads to reflex vaso-dilatation, indicating the presence of local vaso-dilator reflex mechanisms or paths through the lumbo-sacral portion of the spinal cord.

Table Showing the Influence' of Rate and Strength of Stimulation of the Sciatic Nerve on the Blood Vessels of the Leg (Bowditch and Warren)

\begin{tabular}{|c|c|c|c|c|c|c|c|c|c|}
\hline \multirow{2}{*}{$\begin{array}{l}\text { Stimu- } \\
\text { lation, } \\
\text { rate per } \\
\text { second }\end{array}$} & \multicolumn{3}{|c|}{ Weak Ind. = ro-r9 } & \multicolumn{3}{|c|}{ Medium Ind. $=100-250$} & \multicolumn{3}{|c|}{ Strong Ind. $=300-800$} \\
\hline & $\begin{array}{l}\text { No. of } \\
\text { obs. }\end{array}$ & $\begin{array}{c}\text { Contr. } \\
\mathrm{mm} \text {. }\end{array}$ & $\begin{array}{l}\text { Dilat. } \\
\mathrm{mm} .\end{array}$ & $\begin{array}{l}\text { No. of } \\
\text { obs. }\end{array}$ & $\begin{array}{c}\text { Contr. } \\
\mathrm{mm} \text {. }\end{array}$ & $\begin{array}{l}\text { Dilat. } \\
\mathrm{mm} .\end{array}$ & $\begin{array}{l}\text { No. of } \\
\text { obs. }\end{array}$ & $\begin{array}{c}\text { Contr. } \\
\mathrm{mm} \text {. }\end{array}$ & $\begin{array}{l}\text { Dilat. } \\
\text { mm. }\end{array}$ \\
\hline$I / I 0-I$ & 45 & I. 2 & 6.2 & 146 & I. 9 & 4.5 & 85 & 2.6 & 3.2 \\
\hline $2-4$ & 63 & 3.6 & 5.6 & 72 & $4 \cdot 4$ & $5 \cdot 4$ & 57 & 7.6 & 6.4 \\
\hline $5-8$ & 20 & $4 \cdot 5$ & $4 \cdot 5$ & 49 & 6.3 & 3.2 & 75 & $8 \cdot 7$ & $4 \cdot 0$ \\
\hline $10-16$ & 49 & 10.3 & 2.4 & I04 & 7.8 & 2.8 & 34 & I I. 4 & 2.7 \\
\hline $30-64$ & 62 & $14 \cdot 3$ & $2 \cdot 1$ & 40 & 17.6 & $3 \cdot I$ & 8 & 14.6 & I. 4 \\
\hline
\end{tabular}

Vaso-dilator Reflexes.-Perhaps the only unquestioned case of reflex vaso-dilatation is that of the lumbar cord just mentioned. It is true that many apparent reflexes can be noted, for example the increased flow of blood in the salivary glands under gustatory reflexes, the blushing of the skin on exposure to sudden warmth, or even the blushing of emotional origin. These on first thought would be regarded as vaso-dilator reflexes. In all these cases there is a widening of the peripheral arterioles with a great increase in the volume of blood flowing through the local vascular bed. But each instance can be just as readily explained as inhibition of the vaso-constrictor tonic activity. This double explanation can, as a matter of fact, be applied to the action of the depressor nerve described above, page 248. However, the confusion is in part due to the difficulty of analyzing the two classes of nerves in the same nerve trunk. All the thoracic spinal nerves and the upper lumbar trunks contain both vaso-constrictor and vaso-dilator nerves. The usual methods of physiological stimulation with rapidly interrupted currents we now know are normal stimuli for the constrictors only. Stimulations at the rate of one or two per second call forth responses in the dilator fibers. Also, the vasodilator fibers degenerate more slowly and retain their irritability longer than the constrictor fibers when both are isolated from their cell bodies by sectioning. The method of slow stimulation and the method of differential degeneration were given us by Bowditch and Warren. 
The Relation of Vaso-constrictor and Vaso-dilator Activity.-The distribution of two sets of regulative fibers for the muscular walls of the blood vessels, when considered in connection with the other factors of the vascular apparatus, gives a wonderfully complete mechanism for the coordination of the vascular supply with the activity of the different organs. General and broadly distributed activity of the constrictors produces increase of general blood pressure and of the dilators decrease of pressure, but local activity of either set will produce a great reduction or increase of blood in the local organ with little or no effect on the general pressure. When a vaso-dilatation is produced locally in one organ and there is an accompanying vaso-constriction in other regions, as usually happens, it is evident that the result may be a flooding of the local region. This is exactly the thing that is accomplished in the muscles in violent exercise, in the glands during secretion, in the stomach during digestion. It is this mechanism that is utilized to throw a large volume of blood to the skin when the temperature of the body is above the average, or to blanch the skin when the temperature is low.

Normally certain regions of the body are associated in that when vasodilatation occurs $n$ one region, vaso-constriction occurs in the other. This is particularly true with the skin or surface of the body and the viscera or deeper organs. The same relation is said to exist between some of the visceral organs.

\section{General Course of the Vaso-constrictor and Vaso-dilator Nerves.-} The cell bodies forming the medullary vaso-motor center give off axones, axis-cylinder processes, some of which go to the nuclei of origin of certain cranial nerves, while others pass down the cord to end at different levels in contact with certain cells, probably small cells in the anterior horn and lateral part of the gray matter. These cord cells constitute the spinal centers. The neuraxones of the spinal cells leave the cord in certain spinal nerves in the anterior roots, pass by the white rami to the sympathetic ganglionic chain, where they end in physiological connection with the ganglionic cell. Axones from these latter cells pass by an uninterrupted course to their terminations on the blood vessel walls. The vaso-constrictor fibers leave the central nervous axis by the ventral roots of all the dorsal nerves and the first two lumbar roots, a comparatively restricted region. The vaso-dilators have the same origin with two exceptions, viz., the vaso-dilators of the salivary glands found in the seventh and ninth cranial nerves, and the nervi erigentes, which arise in the roots of the second and third sacrals.

The nerves to the viscera pass direct to their blood vessels, but the vascular nerves for the skin, muscles, limbs, etc., rejoin the main divisions of the spinal nerves through the gray rami, see figures $4 \mathrm{I} 7$ and $4 \mathrm{I} 8$, and pass to the blood vessels along with the general nerves of the organ or organs. 


\section{VASO-CONSTRICTOR AND VASO-DILATOR NERVES FOR INDIVIDUAL ORGANS.}

The particular paths for the vaso-motor nerves has been pretty definitely established by numerous researches, especially by those of Langley and his students.

The course of the vaso-constrictor and the vaso-dilator nerve fibers has been followed satisfactorily in many of the important parts of the body, though the supply for some regions is yet obscure. This is particularly true for the brain, where such supply is apparently absent. The two groups of fibers run the same course, except in the cephalic and sacral regions already mentioned They may, therefore, be described together.

The Vascular Nerve Supply for the Head.-The vascular nerves for the head, face, and mouth have their origin in the cord from the first to the fifth dorsal spinal nerves. They pass through the white rami to sympathetic ganglia, through the stellate ganglion, and up the cervical sympathetic nerve to the superior cervical ganglion. From this ganglion they run to their distribution, either along with the arteries, as with the salivary supply, or with the sensory nerves, as in the nerves to the mucous membrane of the mouth, etc. The vascular nerves supplied to the base of the ear follow the above course, but the nerves for the tip leave the stellate ganglion in the ramus vertebralis, run to the third cervical nerve, and pass with its auricular branch to the ear, a circuitous route determined by Fletcher.

The great exception to the above origin is with the vaso-dilator group. Dilator fibers leave the base of the brain in the direct path of the seventh cranial nerve to supply the submaxillary and sublingual glands, in the ninth cranial nerve to the parotid gland, and in both these to the tongue.

The Vascular Regulation in the Brain.-The brain requires a large and uniform supply of blood for the due performance of its functions. This object is effected through the number and size of its arteries, the two internal carotids and the two vertebrals. It is also desirable that the force with which this blood is sent to the brain should be subject to less variation from external circumstances than it is in other parts, an effect that is accomplished by the free anastomoses of the large arteries in the circle of Willis. This arrangement insures that the supply of blood will be uniform in both hemispheres even though it may be limited through operation or accident to one or more of the four principal arteries. Uniformity of supply is further insured by the arrangement of the vessels in the pia mater. Previous to their distribution to the substance of the brain the large arteries break up and divide into innumerable minute branches. These arterioles after frequent communication with one another enter the brain in a very uniform and equable distribution. The arrangement of the veins within the cranium is also peculiar. The large venous trunks or sinuses are formed so as to be scarcely capable of change of size; and composed, as they are, of the tough tissue of the dura mater, and in someinstances bounded by the bony cranium, 
they are not compressible by any force which the fullness of the arteries might exercise through the substance of the brain. Nor do they admit of distention when the flow of venous blood from the brain is obstructed.

The mechanical conditions in the brain and skull formerly appeared enough to justify the opinion that the quantity of blood in the brain must

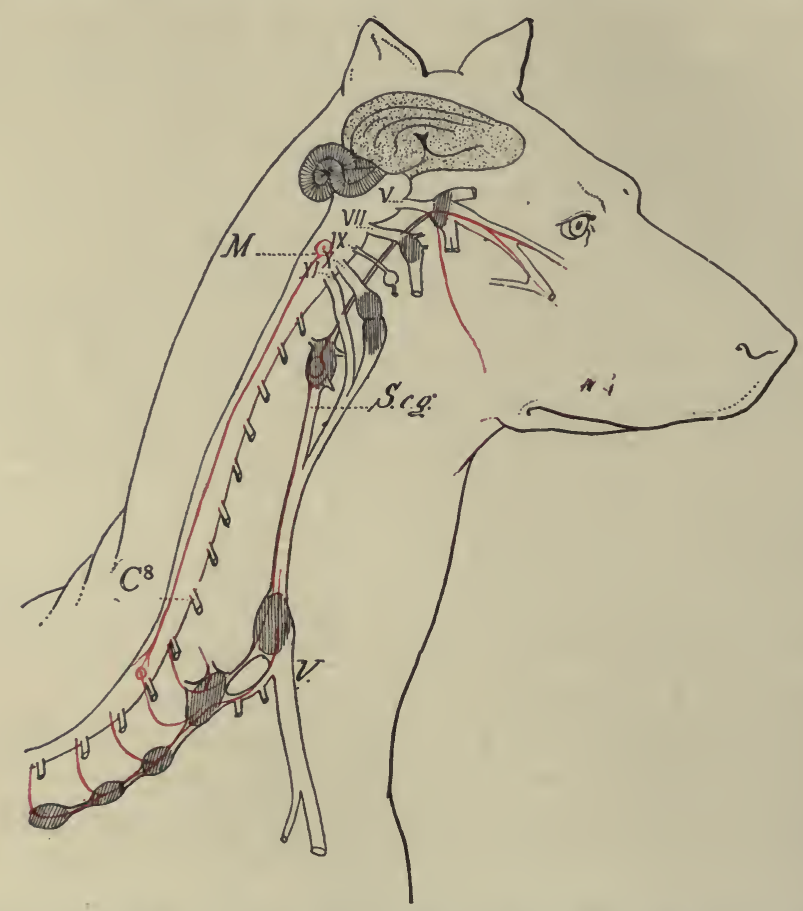

FI G. 208.- Showing the Origin and Course of the Vaso-constrictor Nerves for the Head. $M$, medulla; $C^{8}$, eighth cervical spinal nerve; $V$, vagus, $S . c . g$. , superior cervical ganglion. Modified from Moret.

be at all times the same. But it was found that in animals bled to death without any aperture being made in the cranium, the brain became pale and anemic like other parts. And in death from strangling or drowning, there was congestion of the cerebral vessels; while in death by prussic acid, the quantity of blood in the cavity of the cranium was determined by the position in which the animal was placed after death, thê cerebral vessels being congested when the animal was suspended with its head downward, and comparatively empty when the animal was kept suspended by the ears. Thus, although the total volume of the contents of the cranium is probably nearly always the same, yet the quantity of blood in it is liable to variation, its increase or diminution being accompained by a simultaneous diminution or increase in the quantity of the cerebro-spinal fluid. The cerebro-spinal fluid being readily removed from one part of the brain and spinal cord to 
another, and capable of being rapidly absorbed and as readily effused, would serve as a kind of supplemental fluid to the other contents of the cranium to keep it uniformly filled. Although the arrangement of the blood vessels insures to the brain an amount of blood which is tolerably uniform, yet with every beat of the heart, and every act of respiration, and under many other circumstances, the quantity of blood in the cavity of the cranium is constantly varying. Roy and Sherrington are responsible for the view now generally current that the brain, therefore, is largely if not entirely dependent. upon the general blood-pressure for variations in the quantity of blood which it receives. During a high blood-pressure the amount of blood that flows in a given unit of time is greater, and during low blood-pressure less. Howell has shown that in the decapitated dog's brain the flow of blood is directly proportional to the difference in pressure.

Numerous attempts have been made to show vaso-motor mechanisms. for the cerebral arteries, but with generally unconvincing success. Huber and others have shown nerve endings in such arteries by histological methods. Bayless, Hill, and Gulland make the statement that "no. evidence has been found of the existence of cerebral vaso-motor nerves, either by means of stimulation of the vaso-motor center or central end of the spinal cord, after division of the cord in the upper dorsal region, or

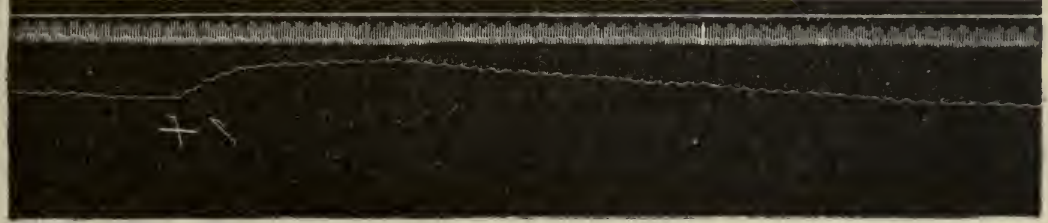

Fig. 209.-Vaso-dilatation in the Brain from Stimulation of the Cerebral Cortex in the Presence of Complete Destruction of the Medulla in the Cat. The upper trace is of the carotid pressure; the lower trace is of the brain oncometer. (Weber.)

by stimulation of the stellate ganglion, and that is to say the whole sympathetic supply to the carotid and vertebral arteries." However, Ernest Weber (I908) reinvestigated the control of the blood flow in the brain. He admits that the blood flow in the brain is sharply dependent on the general blood-pressure, but he presents plausible evidence that both vaso-constrictors and vaso-dilators exist for the brain vessels. The most striking facts are obtained upon stimulating general sensory nerves, the central end of the sectioned cord, the cerebral cortex, and the cervical sympathetic. The stimulation of the cerebral cortex calls forth vasodilatation in the brain even when the medulla is completely destroyed. Weber, therefore, concludes that these stimuli act reflexly through a cerebral vascular center located at some as yet undetermined point in the brain stem above the general medullary center. An active cerebral vaso-dilatation may be accomplished through this center even in the 
presence of an accompanying fall in general blood-pressure. More often the type of vascular reflex is that of dilatation followed by constriction of the brain vessels. If there is an accompanying sharp rise in general blood-pressure, then the reflex cerebral vascular dilatation is followed by
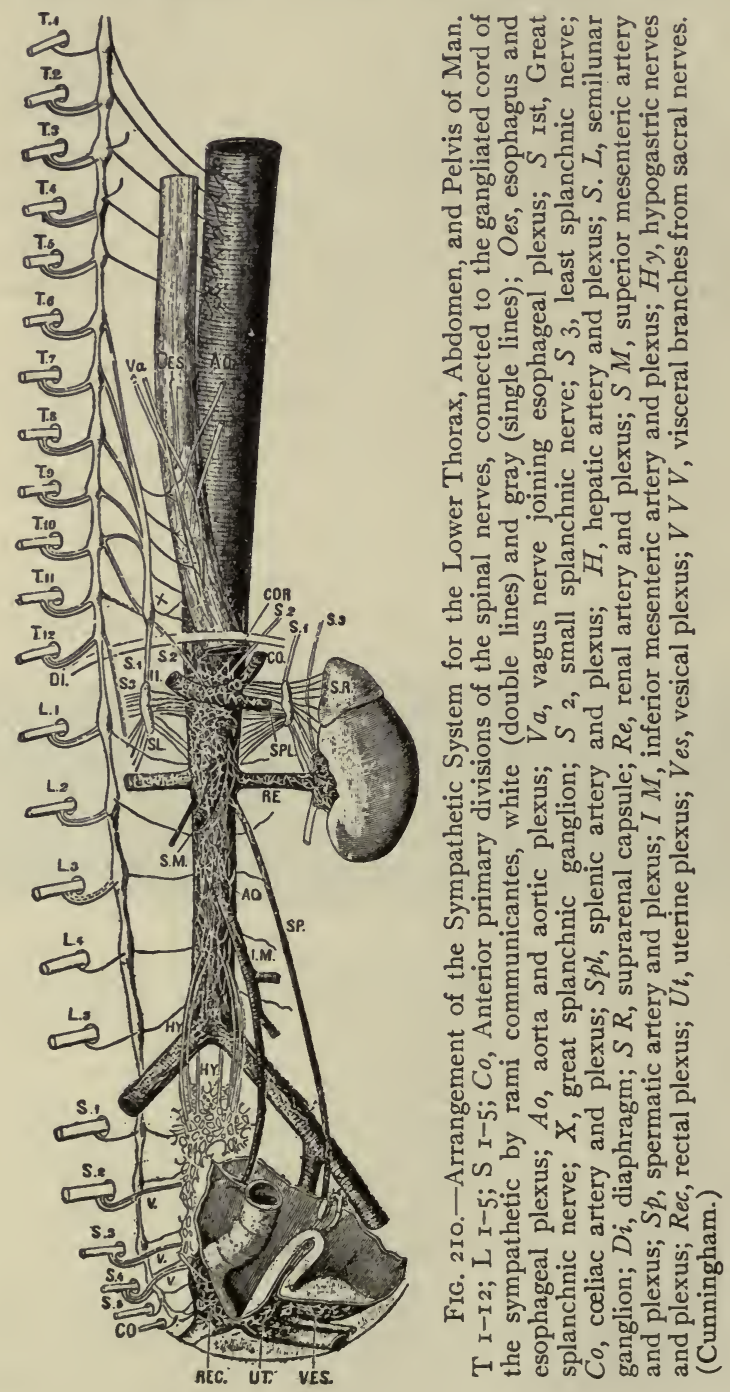

vaso-constriction which takes place while the general blood-pressure is yet high. Weber suggests that this local vaso-constrictor mechanism provides an apparatus for the regulation and control of the cerebral congestion that results from the rise of general blood-pressure due to the operation of the general medullary vaso-motor center. 
The question may be summarized by the statement that the regulation of the flow of blood through the brain is accomplished by the interaction of two factors: First, the indirect regulation of blood-pressure through the variations in the heart's activity, and through the general action of the medullary vaso-motor apparatus producing vaso-constrictions or dilatations in areas other than the brain. Second, the local and direct regulation of the brain ressels through reflex action on a special local vascular center.

The Vascular Nerves for the Thoracic Viscera. - Numerous efforts have been made to determine the vaso-motor nerve supply for the thoracic organs, the heart and lungs. In the heart the observation is rendered complex by the fact of the rhythmic contractions which produce mechanical pressure on the coronary arteries. Martin, by direct observation through a lens, and Porter, by measuring the outflow of the coronaries upon vagus stimulation, came to exactly opposite views: the former that the vagus contained vaso-dilators, the latter that it contained vaso-constrictors. Still other experiments have been made to prove either constrictor or dilator nerves for the coronary arteries, but the fact is still undetermined.

The lesser circulation through the lungs has also proven a difficult situation to interpret as regards any nervous regulation of the pulmonary arterioles. The evidence, while not conclusive, is that the vaso-constrictor supply to the lungs is from the third to the fifth thoracic nerves, but that the vasoconstriction produced is slight in comparison with regions of the systemic circulation.

The Vascular Nerves for the Abdominal Viscera.-The vaso-constrictors and the vaso-dilators for the organs of the abdominal cavity have a broad origin in the cord, from the first dorsal to the fourth lumbar in the dog and cat. The nerves pass to the organs by the splanchnic nerves, and by the solar, celiac, and mesenteric ganglia. The vascular nerves for the different organs may be given in tabulated form:

Vascular Nerves for the Abdominal Viscera.

Organ.

$\left.\begin{array}{l}\begin{array}{l}\text { Stomach and in- } \\ \text { testine. }\end{array} \\ \text { Spleen.......... }\end{array}\right\}$

Liver..........

Kidney........ $\}$

Pelvic viscera......
Spinal origin of the vascular nerves.

$5,6,7,8,9$, ro, $\mathbf{I}$ I, I2, I $3_{3}$ I $\mathrm{L}$

$3,4,5,6,7,8,9$, хо, I I, I 2,$\}$ I3 $\mathrm{D}, \mathrm{I} \mathrm{L}$

$3,4,5,6,7,8,9$, то, I I D .

$4,5,6,7,8,9$, IO 11 I I 2,13

D. I, 2, 3, 4 L.

I, 2, 3, $4 \mathrm{~L}$
Course to the organ.

Splanchnic nerves and solar and celiac ganglia. Splanchnic nerves and solar and celiac ganglia. Splanchnic nerves and solar and celiac ganglia. Splanchnic and celiac ganglia.

Inferior splanchnic and inferior mesenteric ganglia. 
The Vascular Nerves for the External Genital Organs.-The vasodilators for these organs arise from the second and third sacral nerves and pass to the organs by the nervi erigentes and the pelvic plexus. They form the second great exception to the region of general outflow of vascular nerves. The constrictors, on the other hand, arise in the spinal nerves from the last dorsal and first four lumbar. They run the same course as given in the table for the pelvic viscera.

The greatest variations in the quantity of blood contained at different times in the external genital organs are found in certain structures which contain what is known as erectile tissue. These organs, under ordinary circumstances, are soft and flaccid, but at certain times they receive an unusually large quantity of blood, become distended and swollen by it, and pass into the state termed erection. Such structures are the corpora cavernosa and corpus spongiosum of the penis of the male, and the clitoris in the female. The nipple of the mammary gland in both sexes, and, according to some authors, certain nasal membranes contain erectile tissue.

The corpus cavernosum of the penis, which is the best example of an erectile structure, has an external fibrous membrane or sheath. From the inner surface of the sheath numerous fine lamellæ project into the cavity, dividing it into small compartments, like cells when they are inflated. Within these cells there is a plexus of veins upon which the erectile property of the organ mainly depends. The plexus consists of short veins with very close interlacings and anastomoses with very elastic walls admitting of great variations in size. They collapse in the passive state of the organ, but are capable of an amount of dilatation which exceeds beyond comparison that of the arteries and veins which convey the blood to and from them. The strong fibrous tissue lying in the intervals of the venous plexuses, and the external fibrous membrane or sheath with which it is connected, limit the distention of the vessels and give to the organ its condition of tension and firmness. The same general condition of vessels exists in the corpus spongiosum urethræ, but the fibrous tissue around the urethra is much weaker than around the body of the penis, while around the glans there is none. The venous blood is returned from the plexuses by comparatively small veins; all of which are liable to the pressure of muscles where they leave the penis. The muscles chiefly concerned in this action are the erector penis and accelerator urinæ. Erection results from the distention of the venous plexuses by a sudden influx of blood resulting from the action of the nervous vascular reflexes. It is facilitated by the special muscular mechanism which prevents the outflow of blood.

The Vascular Nerves for the Trunk and Limbs.-The skin and muscles of the trunk receive their cutaneous and motor nerves by a segmental arrangement in which the innervation is by bands corresponding with the segments of the cord and the spinal nerves. It is much the same 
with the vascular nerves; they are distributed to the skin and walls of the trunk in the same segment in which they arise. Langley says that the successive bands overlap somewhat.

In the fore legs or arms the vascular nerves arise from the first to the sixth dorsal spinal nerves, run to the stellate ganglia, then by the gray rami back through the ramus vertebralis to join those cervical nerves that enter into the brachial plexus, figure $2 \mathrm{II}$.

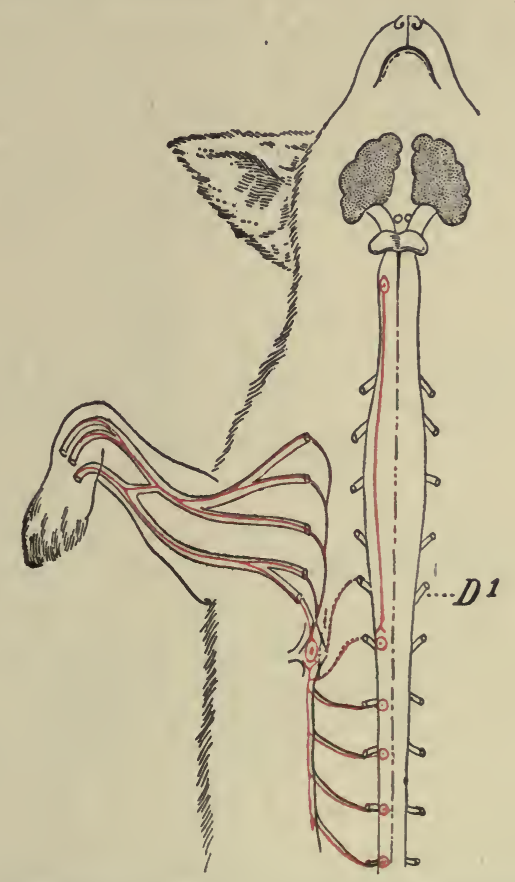

Fig. 21 I.-Plan of Distribution of Vaso-constrictor Nerves for the Fore Limbs.

(Modified from Moret.)

The nerves for the blood vessels of the lower limbs arise from the tenth dorsal to the second lumbar nerves. These pass to the ganglionic chain, and gray rami are given off which join the lumbo-sacral plexus and run with the divisions of that nerve complex to their distribution in the skin and muscles. Vaso-constrictors and vaso-dilators have a common course to the lower limbs.

The Vaso-constrictor Nerves for the Veins.-Mall has proven that vaso-constrictors are present for the portal vein. These fibers are present in the splanchnic nerves. Other evidences have been observed which render the view probable that vaso-motors for the veins in general exist. Hough, for example, in an extended study of the capillary pressure found many variations which were readily explained only on the assumption of veno-motor activity, see figure 201. 


\section{LABORATORY EXPERIMENTS ON THE CIRCULATION}

I. The Rate of the Human Heart-beat.-Determine the rate of the heart-beat per minute by counting the radial pulse, using a watch for the time. Make the determination after sitting quietly for five minutes. Take the average of at least ten determinations for your own case. Determine the heart-rate under the same conditions for as many different persons as you can. Tabulate these rates to show age, sex, weight, and height of the different classes of individuals, and compute general averages for your sets. Count the rate in children and in aged.

Note the effect on the averages obtained above after lying down for five minutes, after standing quietly for the same time, and after five minutes' brisk walk. Tabulate as directed.

Count the heart-rate by successive I 5 second periods immediately upon standing from the reclining position and until the quarter minute rates are constant; repeat immediately after two minutes of fast running. Tabulate these results and compare the graphs obtained from several different individuals. This method measures the character of the vascular control in certain clinical states.

Count your own heart-rate at one-hour intervals during one entire day, giving special attention to the rate just before and just after meals, but in every case make the count on the fifth minute while sitting quietly. A marked diurnal variation will usually appear. Determine these rates on several individuals, and tabulate as before.

2. Human Cardiogram.-Apply a Burdon-Sanderson cardiograph to the thorax over the point between the fifth and sixth ribs of the left side, where the cardiac impulse is felt most distinctly. Connect the cardiograph with a recording tambour, Marey's form, adjust the tension of the cardiograph and the pressure of the air within the system, and take a tracing of the movements of the lever of the recording tambour. The recording cylinder should travel at the rate of about two centimeters per second. Take the time of the movements of the kymograph by an electric seconds magnet. The proper description should be written on the smoked paper, the paper removed carefully and the whole record fixed in shellac.

Count the rate of the heart-beat from the record. Compute the time of the cardiac systole and diastole, and of the pause at the end of the diastole in seconds to three decimals. Records secured under different conditions of exercise, etc., brought together in a table will usually show that the higher heart-rates decrease the time of the cardiac cycle at the expense of the diastole. In other words, the time of the systole remains fairly constant, while the time of the diastole increases or decreases inversely with the rate, a fact to which Hürthle has drawn attention, figure ${ }_{57} 7$. 
3. The Rate and Sequence of the Contractions of the Frog's Heart. Destroy the brain of the frog and open the thorax, but do not destroy the pericardium. Count the rate of the heart per minute, then remove the pericardium and make a second determination after the heart is exposed to the air. The different parts of the heart are easily identified and the contractions in definite sequence can be determined without difficulty. Make this determination for the ventricle, auricle, and sinus venosus by direct observation.

Prepare a cardiac lever as shown in figure $2 \mathrm{I} 2$ or $2 \mathrm{I} 6$, taking special care to arrange the foot so that it will not bind when in motion. Adjust the foot of the lever on the exposed ventricle and bring its point to write

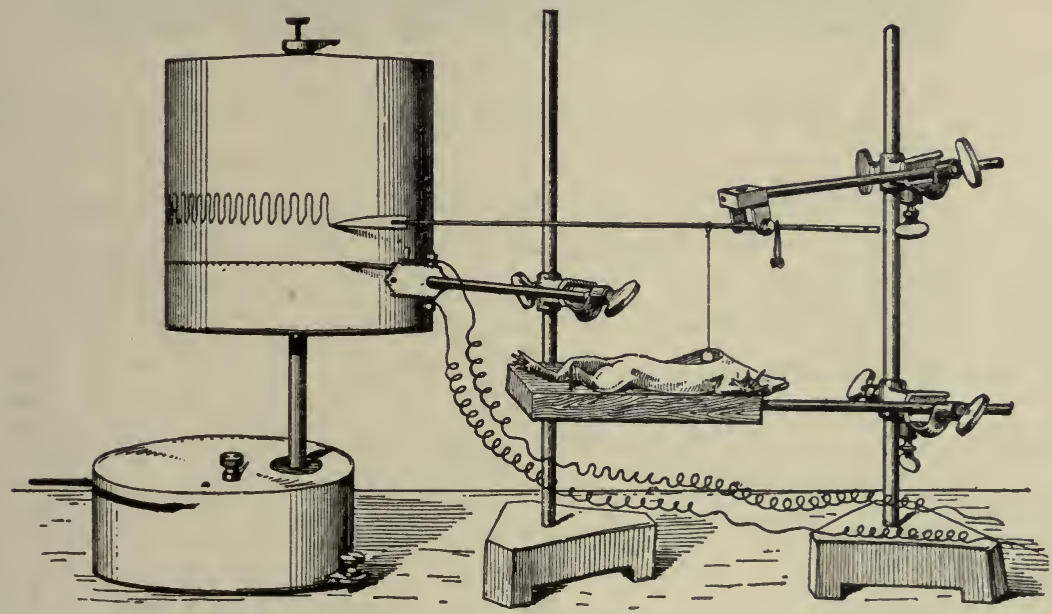

FIG. 21 2.- Heart Lever for Frog or Turtle Hearts. This lever rests directly on the surface of the heart, the foot consisting of a tiny piece of dry cork bark. The lever can also be used as in figure 216 , in this case by attaching the tip of the apex of the ventricle by a tiny hook and thread to the short axis of the lever. Either device can be used for taking records of cardiac sequence.

lightly on the smoked paper of a recording cylinder. This cylinder should travel at the rate of about $2 \mathrm{~cm}$. per second and its speed be marked by the writing point of an electric magnet. Take care to adjust the time magnet in a vertical line with the writing point of the heart lever, placing the heart lever about I $\mathrm{cm}$. above. The tracing of the ventricle, the cardiogram, will show rhythmic contraction, relaxation, and pause of the ventricle. It will also enable one to measure the exact proportion of the total time of the cardiac cycle consumed by the systole and diastole, and also that portion of the diastole in which the ventricle is wholly at rest. A drum rate of $2 \mathrm{~mm}$. per second gives a more satisfactory record of variations when amplitude and rate alone are studied. 
After one has obtained ventricular tracings and has learned the difficulties of adjusting the apparatus, a second heart lever should be adjusted to the auricle, and the auricular movements recorded at the same time as those of the ventricle. If some care is taken to adjust these two writing points in a vertical line a splendid tracing showing synchronism between

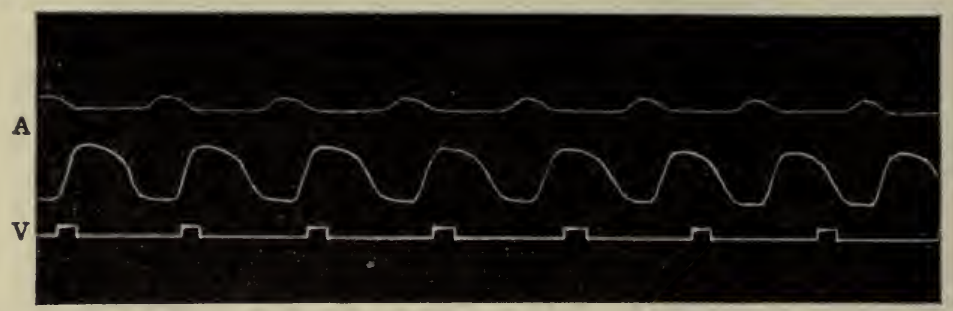

FIG. 213.-Cardiogram Showing Contractions of the Auricle, $a$, and Ventricle, $v$ of a Frog. Time in seconds. The record shows the sequence of the auricle and ventricle, (New figure by Dooley.)

auricle and ventricle is obtained. Measure the rate and the time of the different phases of the contractions of the auricle and ventricle and tabulate them in the following form, always expressing fractions in the decimal system:

\begin{tabular}{l|c|c|c|c}
\hline & $\begin{array}{c}\text { Rate per } \\
\text { minute }\end{array}$ & $\begin{array}{c}\text { Time of systole } \\
\text { in seconds }\end{array}$ & $\begin{array}{c}\text { Time of diastole } \\
\text { in seconds }\end{array}$ & $\begin{array}{c}\text { Time of pause } \\
\text { in seconds }\end{array}$ \\
\hline Auricle............................. & & & & \\
\hline Ventricle...... & & & \\
\hline
\end{tabular}

4. Sequence, Conduction and Heart Block, Turtle Heart.-Prepare a turtle, expose the heart and determine the rate and sequence of the parts. Observe that the veins are contractile.

$a$. Make simultaneous records of the right auricle and the ventricle, use speeds of $2 \mathrm{~mm}$. and $2 \mathrm{~cm}$. per second.

$b$. Attach a Gaskell's clamp close against the ventricle in the $a-v$ groove. While taking records slowly compress the clamps by steps until the ventricular rhythm begins to slow down. Produce partial block through 2 auricles to I ventricle, 3 to I, 4 to I, etc., rhythms, until complete block is obtained. Note recovery on removing the clamp.

$c$. While recording the contractions of the right and left auricles by separate levers split the heart into right and left halves. The right half will continue the usual rhythm, the left will be slower but show more tone. These halves will respond to nerve control and to block tests. 
5. The Contractions of the Excised Heart of the Frog-Pith a frog and expose the heart, as described in the preceding experiment. Remove it completely from the body by first cutting the arteries at their branching in front of the bulbus arteriosus, then carefully lifting up the parts of the heart and cutting away the great veins where they enter the sinus. This will remove the entire heart, including all its contractile parts. The frog's heart, when thus removed and still wet with its own blood, will continue contracting rhythmically and in its natural sequence for some hours. Place such an isolated heart in a watch-glass and take a record of its contractions by the apparatus described in the preceding experiment. (The same phenomena may be studied on a heart isolated and mounted in a Williams' apparatus.)

Set this watch-glass on the metal warming-box supplied, and arrange for the circulation of water of different temperatures through the box. Vary the temperature of the box, and therefore of the heart placed upon it, by allowing water of $0^{\circ} \mathrm{C}$., $10^{\circ} \mathrm{C}$., $20^{\circ} \mathrm{C}$, $30^{\circ} \mathrm{C}$., $40^{\circ} \mathrm{C}$, to flow through it. Or place the heart in a watch-glass over a drinking glass of water of the proper temperature. Record the contractions of the heart at each of these temperatures. The exposed heart will not take the same absolute temperature as the box, but the relative temperature will be decreased or increased. Tabulate the rates at these different temperatures by the plan previously described.

6. The Perfused Heart, Influence of Different Nutrient Fluids. - Expose a frog's heart, as previously described, and insert a 4-way cannula into the ascending vena cava where it enters the sinus. Connect the limbs of the venous cannula with Mariotte's bottles. Fill one with Ringer's solution, the other with comparison fluids. Adjust the constant level tube for a pressure of $4 \mathrm{~cm}$. of fluid and allow it to flow through the heart. The heart will continue its contractions in good sequence and with a uniform rate. Record the contractions by the Engelmann lever method on smoked paper, together with a time tracing in seconds. Set the drum at the rate of about $2 \mathrm{~mm}$. per second. After each comparison is made Ringer's fluid should

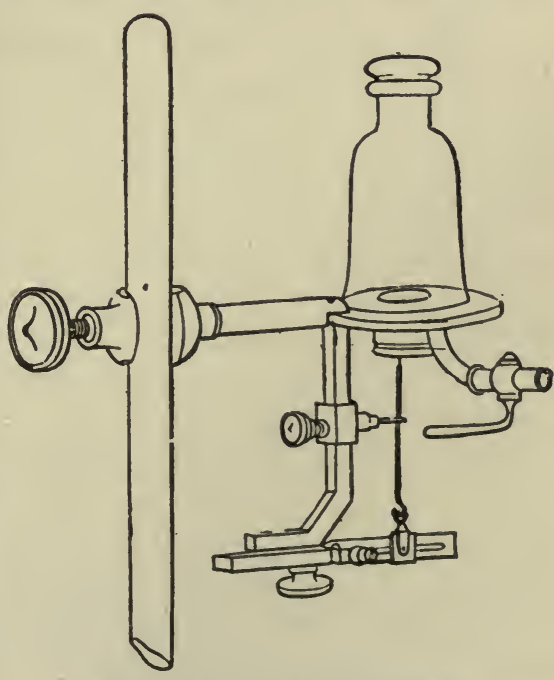

FIG. 2r 4.-Roy's Tonometer. be perfused to secure a return of the normal contractions. 
Use the tracing obtained under the influence of Ringer's solution as a normal and compare it with the rate and amplitude of the contractions. when the heart is perfused with:

a. Physiological saline solution, then return to Ringer's solution;

$b$. With saline and potassium chloride in the proportions found in Ringer's solution (.7 per cent sodium chloride +.03 per cent potassium chloride);

c. With saline and calcium chloride in the proportions found in Ringer's. solution (.7 per cent sodium chloride +.026 per cent calcium chloride);

d. With Locke's solution;

$e$. With milk diluted 4 volumes with physiological saline;

$f$. With normal serum, or blood;

g. With blood, or serum, diluted four times with saline.

Tabulate the rates and amplitudes of the heart under these different influences by the method previously followed.

7. The Heart Volume.-Isolate a frog's heart by the method described for perfusing it with fluid in the preceding experiment. Connect it in a Roy's tonometer, see figure 214, adjust the lever of the tonometer for a tracing on smoked paper. This instrument records the change in volume with each heart contraction. The influence of pressure, varied between 2 and $10 \mathrm{~cm}$., and of nutrient fluids on the heart volume may be determined.

An instructive demonstration can be had by placing the heart of the cat or dog in a Henderson plethysmograph and recording the volume changes by the tambour method.

8. The Isolated Heart of the Terrapin.-The heart of the terrapin, being somewhat larger and somewhat more responsive than the heart of the frog, may be substituted in the two immediately preceding experiments. The facts obtained from it will be essentially the same as those obtained from the frog's heart.

9. The Isolated Mammalian Heart.-The mammalian heart may be isolated from the body and kept alive and contracting for many hours, as has been demonstrated by numerous observers. It is only necessary to keep the temperature approximately that of the normal body and to perfuse the heart through the coronary circulation with aerated blood, or diluted blood, containing sufficient, aerated hemoglobin to supply the heart with the requisite amount of oxygen. Or the heart may be kept alive on the inorganic salt solutions, provided these are supplied with oxygen under considerable tension (Porter, Howell). Even the human heart has been isolated and kept contracting for some hours in the above manner (Kuliabko). The method used is to insert a cannula into the aorta and perfuse the heart through the coronary circulation under adequate pressure, as described by Martin. Many interesting experiments 
and demonstrations can be made on the mammalian heart, but, as this experiment is usually a demonstration experiment, the detail of procedure is left to be supplied by the demonstrator.

Io. Automatic Contractions of Cardiac Muscle.-Isolated portions of the dog's ventricle have been kept in rhythmic contraction by Porter. But the best laboratory material is supplied by the heart of the terrapin. Cut a strip from the ventricle of the terrapin extending around its curved apex, as shown by the dotted line in the accompanying figure, 21 5. Split this strip longitudinally into two parts, each of which will then be about 3 to $4 \mathrm{~mm}$. in diameter by $3 \mathrm{~cm}$. long. Use care to cut smooth strips. Tie a silk thread around the extreme tips of each end of the strip, tying a loop of about $\mathrm{I} \mathrm{cm}$. long at one end, and about Io $\mathrm{cm}$. long at the other. Suspend the strip over a glass hook, figure 216, by the short loop, and connect it with a heart lever by the long loop, as shown in the same figure. Use a tension of I gram. Contractions of this strip as arranged will be recorded with a magnification of about five and with the upstroke of the lever, which is convenient for reading and interpretation. Keep the strip moist with physiological saline in a specimen tube I by 3 inches in size. The arrangement of apparatus figured makes it possible easily and quickly to change this solution for any other that may be desired.

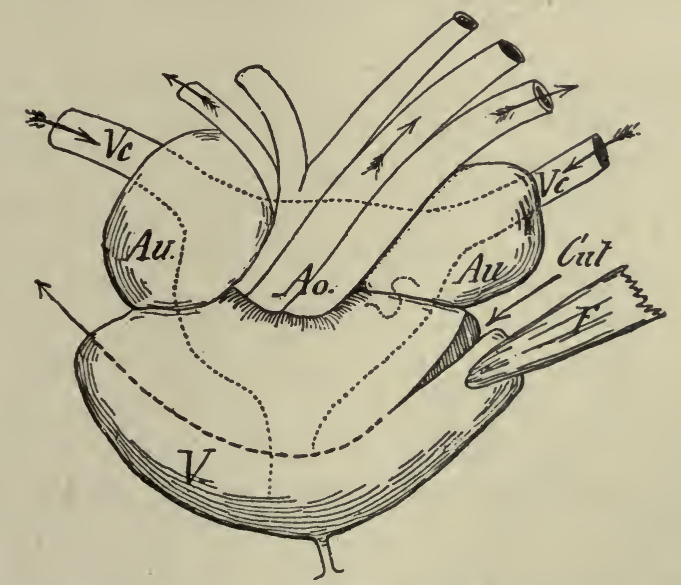

FIG. 215.-Heart of the Terrapin to Show the Method of Cutting the Apex Strip. V, Ventricle; $A u$, auricles; $V c$, venæ cavæ; $A o$, aorta.

Contractions of the ventricular strip in saline begin in from to to 40 minutes after the preparation is made, and go through a regular sequence of slight increase in rate and amplitude for from to to 20 minutes, followed by a very constant rate, but gradually decreasing amplitude for a period of from 2 to 3 hours, figure I7I. Saline is used for the ventricular muscle because it increases the rhythmicity to a degree convenient for study. 
In pure serum or blood the rhythmicity is entirely absent though it is significant that the few sporadic contractions that do occur are large.

This preparation makes possible many instructive experiments tending to show fundamental properties of cardiac muscle. The preparation contains no nervous mechanism except terminal fibers, and its behavior may be safely attributed to the muscle substance itself.

Try the following experiments: I. Submit the strip to saline solutions of different temperatures, varying through steps of 10 degrees from $0^{\circ} \mathrm{C}$. to $30^{\circ} \mathrm{C}$. 2. Try the effect of the different ingredients in Ringer's solution using physiological saline as the standard normal for the ventricle, see Exp. $6, b, c$, etc.

a. Combine potassium chloride with saline, figure 172 ;

b. Calcium chloride with saline, figure I73;

c. Potassium and calcium chloride and saline;

$d$. Locke's solution;

$e$. Solution of blood diluted with saline;

$f$. Solution of milk with saline in the proportion of one part milk to four of saline.

Cut and mount strips from the auricle and from the sinus, letting the latter extend out on to the vena cava. In these last preparations care must be taken to balance the lever, as a slight overtension paralyzes the muscle.

Immerse these strips in pure serum, or Ringer but not physiological saline, and compare their behavior with that of the ven tricle in pure serum. The sinus and usually the auricle will be found rhythmic in serum, while the ventricle, if it contracts at all, will contract at irregular periods. Often there is a distinct progressive decrease in the rhythm, the sinus having the same rhythm as the whole heart, the auricle a considerably slower rhythm, and the ventricle slow and aperiodic. The sinus preparation will show beside the fundamental rhythm a characteristic slow contraction and relaxation, which has been described as tone, figure I 70 . Repeat Exp. 6, $a, b, c$, etc., on the auricle and sinus.

II. Influence of the Cardiac Nerves on the Frog's Heart.-Carefully pith a frog with the minimal loss of the blood of the animal. Expose the heart as previously described, make a cut through the manubrium, continue it through the skin and muscles at the angle of the jaw, thus exposing the vagus nerve. The vagus runs along the edge of a delicate muscle diagonally downward and backward toward the heart. The glosso-pharyngeal is just in front of the vagus and the hypoglossal just behind it. The latter runs parallel with the vagus near its origin, but lower down turns across the vagus and runs to its distribution in the tongue muscles. These two nerves serve to aid the student in the identification of the vagus, see figure 217 . It is usually better to cut the 
hypoglossal away, and also to cut the brachial and the laryngeal nerves to prevent the stimulation of undesired structures.

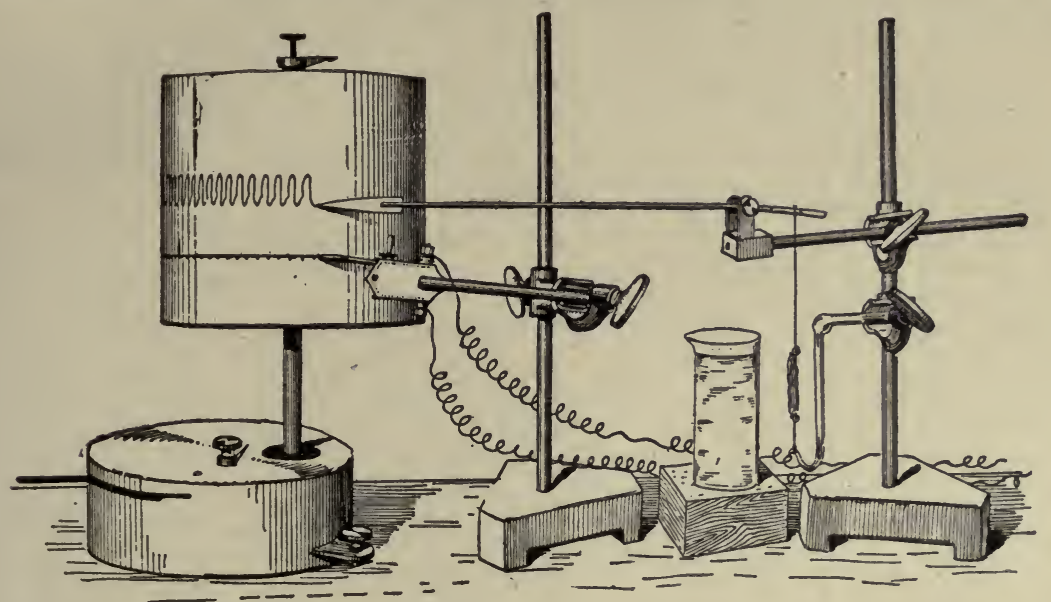

FIG. 216.-Arrangement of Apparatus for Studying the Contractions of the Strip of the Apex of the Ventricle.

Prepare an induction coil, see laboratory experiments on muscle. Use platinum electrodes of the Harvard pattern, set the coil for a mild stimulus tested by the lips or the tongue, lift up the vagus gently and lay it on the platinum tips of the electrodes. Take care that the electrodes do not come in contact with the adjacent tissue. Arrange a signal magnet as shown in the diagram, so that the magnet and the stimulating key of the induction coil may be closed and opened at the same instant. When all is ready (a) secure a normal record, then (b) stimulate the vagus for five to ten seconds, recording the time with the signal magnet and allowing the record to continue until the heart has returned to its normal rate and amplitude, i.e., usually one circuit of the drum. Most students fail in this experiment by 'not allowing sufficient

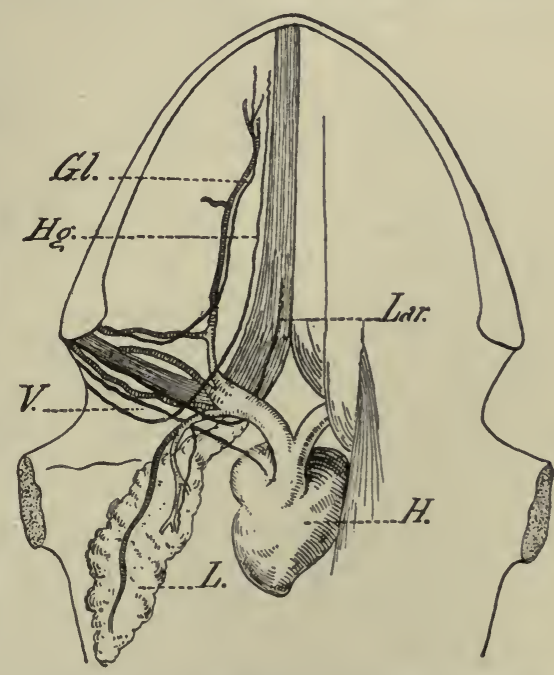

FIG. 217.-Diagram Showing the Relations of the Vago-sympathetic Nerve to the Heart, in the Frog. $H y$, Hypoglossal; $G l$, glosso-pharyngeal; Lar, laryngeal; $V$, vagosympathetic; $H$, heart; $L$, lung. time in the record for a normal before stimulation, and by not allowing 
sufficient time after stimulation for a return to the normal. It will be better to take one good tracing, showing all the facts of the experiment, than several partial tracings, none of which are complete.

With these suggestions in mind, $(c)$ repeat the above experiment, using stimulating currents of increasing intensity until complete cardiac inhibition is produced. (d) Perform experiments showing the influence of the duration of the stimulation on the inhibition; i.e., stimuli of I second, 2 seconds, ro seconds, and 30 seconds.

In the frog the vagus, or inhibitory, and sympathetic, or accelerator, fibers, are found in one trunk, the vago-sympathetic, bitt stimuli will usually produce inhibitions and not acceleration. Occasionally with very weak preparations direct acceleration may be produced. To get the pure inhibitory or pure accelerator effects one must dissect back to and $(e)$ stimulate the trunk of the vagus before it is joined by the sympathetic fibers; or to the sympathetic trunk and $(f)$, stimulate between the third spinal nerve and the point where it joins the vagus trunk. Pure accelerator effects may be demonstrated by $(g)$, stimulating the vago-sympathetic after applying I c.c. of o.I per cent. atropine to the heart to poison the vagus endings. ( $h$ ) perfusion of I c.c. of o.or per cent. epinephrin will chemically stimulate the accelerator endings in the presence of normal vagus endings.

In the study of the above experiments one should note the rates per minute and the amplitude of the normal period just before stimulation, the rate and amplitude during the period of stimulation, and the same at different times after the stimulation until constant results are obtained. A tabulation of these results will usually enable one to judge the influence of each of the various factors recommended in the experiment.

I2. Influence of the Cardiac Nerves on the Terrapin's Heart.Instead of the frog one may use the terrapin in the above experiment. In this animal the vagus and sympathetic in the neck-can very readily be isolated. It is usually quite impossible to demonstrate any cardiac acceleration. But the vagus produces inhibitions which differ from the effects in the frog in that during the recovery from complete inhibitions the ventricular contractions are apparently at once maximal, see figures 180 and $18 \mathrm{r}$. In the frog the ventricular contractions when they reappear are at first slight, but gradually increase in amplitude until they have their former value. The student should explain the significance of these observations.

I3. Arterial Blood-pressure in Man.-The arterial blood-pressure in man can be measured indirectly by measuring the pressure which it takes around the arm completely to close the artery. Some form of the Riva-Rocci type of apparatus, preferably the Tyco or Faught, should be used. Two points fundamental to physiology and to clinical diagnosis can be determined, the systolic or maximum pressure in the artery, and the diastolic or minimum pressure. 
$a$. Bare the left arm, and wrap the rubber sleeve band snugly just above the elbow and turn the free end under at the top. Connect the Tyco or Faught manometer with one entrance tube and the bulb pump with the other.

Lightly bind the bell of a stethoscope over the brachial artery on the inner surface of the forearm just under the border of the arm band.

$b$. Quickly fill the band to a pressure of $150 \mathrm{~mm}$., i.e., completely compress the brachial artery. Slowly allow the air to escape, listening carefully with the stethoscope for the first appearance of a pulse murmur in the artery. At the same time watch for the appearance of oscillations in the dial of the manometer. At a certain pressure the hand of the dial will suddenly begin to oscillate and a distinct intermittent sound clear and sharp in tone will be heard. This is the moment when the first escape of blood through the compressed artery takes place. It measures the systolic pressure. $c$. Continue to reduce the pressure. The oscillations of the dial will increase up to a certain point. An intermittent pulse will be heard passing into a loud and sharp snappy sound. At a certain point the sound suddenly becomes dull and low and disappears. The point of maximum oscillation and of disappearance of the intermittent sound marks the diastolic pressure.

Measure the pressures in the standing position, in sitting position, and after a short run. Tabulate the results and draw averages. Repeat the measurements on children and elderly people, using care not to prolong the total compression of the artery.

I4. The Arterial Blood-Pressure in a Mammal.-The arterial bloodpressure may be measured on the anesthetized cat, dog, or rabbit. Simple blood-pressure was originally measured by Hale's method of connecting the artery with a vertical tube and allowing the blood to flow freely into the tube until a column was raised to the height which balanced the pressure in the vessel. This simple method is decidedly the best for the beginner, since it does not necessitate the use of very complicated apparatus. At the same time it gives practice in anesthesia and in operations under anesthetics, and therefore serves as a good preparation for the more complicated experiments which follow.

The necessary apparatus should first be prepared as follows: A vertical tube supported on a stand with a scale graduated in the metric system, assorted cannulæ of approximately the size of the carotid artery of the animal to be operated on, linen-thread ligatures, dissecting set in good condition, an animal-holder with strings or straps firmly to fasten the anesthetized animal, a chloroform-ether mixture for dogs (or other anesthetic according to the animal to be used). Four men should be assigned to perform this experiment. While two are chloretonizing, anesthetizing and preparing the animal, two should arrange the apparatus as nearly ready for con- 
necting with the artery as possible. When all the apparatus is arranged and the animal anesthetized, it should be tied firmly to the animal-holder. Let one experimenter attend strictly and at all times to anesthetizing the animal; recovery to light anesthesia must not occur. Let the operator quickly expose about $3 \mathrm{~cm}$. of the carotid artery (or the femoral artery if circulation time is also to be tested on this animal), by making an incision through the skin of the neck $5 \mathrm{~cm}$. long, and dissecting down between the muscles. Separate the carotid from the adherent vagus nerve by tearing the connective tissue with the scalpel handle, freeing the vessel for about 2 to $3 \mathrm{~cm}$. of its length. Lay two loose ligatures of linen thread around the vessel, place a small bulldog forceps on the exposed artery nearest the heart, and ligate the end nearest the head with one of the ligatures. Lift up the intervening artery with strong forceps and make a $V$-shaped cut near the ligature pointing the cut toward the heart, let it extend about half-way across the artery. Introduce a cannula toward the heart, and tie it firmly with the second ligature. Connect the cannula with the rubber tubing to the vertical glass tube.

When all is ready remove the bulldog forceps from the artery. The blood will flow freely from the artery into the tube, rising by rapid spurts until the pressure from the column of liquid is just equal to that inside the artery itself. If an anticoagulant-like powdered potassium oxalate is first introduced into the vertical tube, probably clotting at the cannula will be delayed for some minutes. The mounting of the blood into the empty tube makes, indeed, a most striking demonstration.

An accurate measure of the height of the top of the column above the level of the cannula at the artery represents the arterial blood-pressure in terms of blood. The specific gravity of blood is 1.056; of mercury, r3.6. Record the pressure you obtain in terms of blood and of mercury. Note also the variations in pressure and account for the rhythm of each. There will be a general variation of pressure, depending upon the degree of anesthesia. If anesthesia is light and muscular movements happen, there will be an increase in the blood-pressure. If the anesthesia is heavy, then the blood-pressure falls. These points of variation should be marked and recorded at once in note-books. Make full notes of all accessory facts which would aid in explaining the variations in bloodpressure, such as size of the animal, rate of respiration, rate of heart-beat, the variations in anesthesia, the presence of the reflexes, etc., etc.

Chloroform the animal to kill it, and note the change in blood-pressure during the process, but first do experiment 15 .

I5. The Circulation Time.-The circulation time is most satisfactorily determined in the laboratory by introducing a saline solution of methylene blue into the jugular vein on one side. Note the exact time with a stopwatch until the color appears in the carotid artery, and in the jugular vein of the opposite side. 
Anesthetize a cat or dog with a chloroform-ether mixture, tie it on the animal-holder and, when the eye reflexes are lost, expose the jugular vein on the right side, the carotid artery and the jugular vein on the left. Fill a $2-\mathrm{cm}$. hypodermic syringe with I per cent. methylene blue in physiological saline, insert the needle into the right jugular vein, pointing it toward the heart. Lift the left carotid artery and place under it a strip of moist white paper $2 \mathrm{~cm}$. wide; prepare the left jugular vein in the same way. Place the animal so that these vessels are lighted to the best advantage. At a given moment inject the contents of the hypodermic syringe, noting the time with a stop-watch. Observe the color of the left carotid and the left jugular, respectively, very carefully, and take the time of the first appearance of the methylene blue. The color will appear first in the artery, second in the vein. The difference in time between the moment of injection and the moment of color in the artery represents, with a slight correction, the circulation time of the pulmonary or lesser circulation. The time from the injection until the color in the other jugular vein represents the total time of circulation.

Stewart has made these determinations even more correctly by the electrical-resistance method. He injected ro per cent. salt solution and determined the variation in resistance by a galvanometer. If the galvanometer is available, then check the above determinations by the electrical method, arranging the apparatus under the direction of an instructor.

I6. The Blood-pressure Model.-An artificial model of the circulatory apparatus, which illustrates all mechanical parts involved, has been arranged by Porter, or can be easily constructed. The model should have the following possibilities: A pump, which permits of rhythmic action at a varying rate and varying force; a resistance to the outflow liquid which can be increased or decreased; and an elastic set of vessels into which the pump discharges.

If Porter's schema is used, determine the following points. (a) The pressure in terms of mercury in the arterial and venous limbs of the apparatus when the pump makes a rate of 72 per minute; $(b)$ the influence on these two pressures when the rate is increased, when it is decreased, (c) the effect on these pressures when the peripheral resistance is great, when it is low. With a sphygmograph, $(d)$ take a tracing of the pulse in the elastic tube representing the arterial side of the schema.

If an ordinary bulb syringe and simple apparatus is used, then determine the following: (e) The character and rate of the outflow when water is pumped into the rigid glass tube with no resistance to the outflow; $(f)$ when a glass tube of smaller caliber is connected with the end of the larger glass tube so as to produce a high resistance to the outflow. (g) Pump the water into a rubber tube of smaller size and compare with the proceeding when there is no resistance to the outflow; also $(h)$ when a glass tube of 
small caliber is introduced into the end in order to produce high resistance to the outflow. Determine the amount of resistance necessary to produce a constant outflow when the pump has a rate of 72 beats per minute.

In this experiment what effect is produced on the outflow if the rate of the pump is varied? if the force of the stroke is varied? if the elasticity of the rubber tube representing the artery is varied? If the resistance represented by the size of the glass tube at the outflow is varied?

I7. The Arterial Pulse.-The form of the arterial pulse may be taken by one of the various sphygmographs applied to the radial artery at the wrist or the common carotid in the neck. If the tambour method is used, apply a sphygmographic tambour on the wrist adjusting the central pressure over the radial artery. Fasten it in place by the proper bands, adjusting the tension by flexing the wrist. Connect the receiving tambour with a delicately balanced, small-sized recording tambour, which should write its movements on a cylinder revolving at the rate of $\mathrm{I}$ to $2 \mathrm{~cm}$. per second.

A convenient clinical instrument is the Dudgeon or the Jacquet sphygmograph. These are to be applied at the wrist and give tracings showing delicate variations in the form of the pulse wave with great magnification and a considerable degree of accuracy. Make a comparison of the form of the pulse wave from tracings taken from at least six different individuals.

The sphygmogram from the carotid artery may best be taken by applying an air sphygmograph to the neck over the carotid and fastening it in position by a spring. Record by the tambour method.

I8. The Rate of Propagation of the Pulse Wave.-Apply tambour sphygmographs to the carotid in the neck and to the radial at the wrist, and make simultaneous records on a drum, adjusting the writing levers of the two recording tambours in an exact vertical line. Let the recording drum travel at the speed of $2 \mathrm{~cm}$. or more per second, and record the speed by a 50 double-vibration tuning-fork. The carotid pulse will be found to precede the radial pulse by the fraction of a second. This short interval, which can be determined in thousandths of a second by comparison with the time tracing below, represents the time required for the pulse wave to traverse the distance from the carotid to the radial. Measure the distance used in the experiment and calculate the rate of propagation in centimeters per second.

If the writing points of the recording levers in this experiment are made of very delicate strips of note paper or of thin photographic film celluloid, so as to offer little resistance to the surface of the drum, the detail of the pulse wave at the two points will be accurately transcribed and may be compared.

I9. The Capillary Circulation.-The capillary circulation is best 
demonstrated in the laboratory by direct observation on the web of the frog's foot by the use of the compound microscope. Give a $40-\mathrm{gm}$. frog a hypodermic injection of 0.3 c.c. of ether under the skin of the back. Wet a piece of cheesecloth the size of a handkerchief with tap water and wrap the etherized frog so as to cover the entire body with the exception of the foot. When the anesthesia has progressed so as to destroy voluntary movements, bind the foot on an ordinary frog board and spread the web over the window in the board. Choose an area of the skin which shows small arteries, capillaries, and veins, and in which the blood is flowing freely and rapidly. Examine with the low-power of a compound microscope. In a favorable field small arteries, capillaries, and veins with blood flowing rapidly through them will be easily found. Choose one such field, cover with a piece of thin cover-glass, moisten with a drop of water if necessary, and examine with a high power. Note in the small artery the pulsating current; the border of clear fluid along the side of the main stream of blood; the white corpuscles along the clear borders of the current. In the small veins there are usually no pulsations and the speed of the current is somewhat less. Careful examination of the capillaries will reveal a delicate wall, the individual corpuscles, and the fact that the red corpuscles are actually larger than the diameter of the capillary at some points and must be bent to pass through. Note that the capillaries form an intricate and anastomosing network; that the current may occasionally be reversed in some of the anastomoses.

The anesthetizing effect of the ether recommended will usually continue about ro to 20 minutes. If the observation is more prolonged, a second dose of ether should be given. The capillaries in the tails of small fish are often very readily observed, and these may be substituted for the frog's web.

20. Capillary Blood-Pressure of Man.-Measure the capillary bloodpressure in your own finger by von Krie's method. This apparatus consists of a small piece of glass an inch square, or less, which is placed across the knuckle of the finger just back of the nail. A small weight pan is suspended by a loop of thread over this glass plate so that weights put in the pan will bring varying pressure on the plate above. Add weights to the pan until an area of the skin, about $5 \mathrm{~mm}$. in diameter, is blanched by the pressure. Mark the outline of this bloodless area on the glass, take off the apparatus and measure the exact area of the glass so marked, weigh the entire apparatus and compute the pressure in grams per square centimeter for the area. This pressure in terms of mercury represents the capillary blood-pressure in the vessels of the skin of the finger at that level. Measure the pressure when the finger is held at the level of the top of the head; with the finger held as low as possible; held at the level of the heart. Tabulate the measurements. The capillary blood-pressure at the level of the heart is usually from 40 to $50 \mathrm{~mm}$. of mercury. 
Lombard determines the end point in this experiment more accurately by the aid of a low power binocular microscope after rubbing vaseline into the skin to increase its transparency. Hooker's method of compressing the capillaries by an air system controlled and measured by a manometer allows measurement both during compression and in decompression. This method has been adapted to the mammalian mesentery by Ellis.

21. The Arterial Blood-Pressure in a Mammal and Its Nervous Regulation.-After the student has measured the arterial blood-pressure by Hale's method, described above, he is in a position to study the variations and co-ordinations in the blood circulatory apparatus. The recording apparatus consists of writing pens, seconds time marker, signal marker, blood-pressure manometer preferably Ludwig's mercury manometer, and a continuous paper kymograph preferably Ludwig's weightdriven form or the Harvard belt kymograph for a continuous record of the arterial blood-pressure. Connect the cannula with the mercury manometer which is provided with a pressure bottle. Use a cannula of the form shown in figure 185 , connecting the side limb of the cannula with the mercury manometer, and the end limb with the pressure bottle. When the apparatus is ready chloretonize and anesthetize a mammal (dog, cat, or rabbit), and bind it to the animal-holder. Let one operator attend strictly and at all times to the anesthetic, for the animal must not under any condition recover consciousness during the experiment.

Expose the carotid artery in the neck, as described in Experiment 13 above, arrange it with ligatures for inserting the cannula, expose the vagus nerve with the same care, and throw ligatures around it for convenience in lifting it out of its bed. Make a V-shaped cut in the carotid directed toward the heart, insert and ligate the cannula as previously described. Before beginning the experiment one should see that all the tubes are filled with the anticoagulating liquid and that the manometer is under pressure from $\mathrm{I} 30$ to $\mathrm{I} 40 \mathrm{~mm}$. mercury. When all is ready start the kymograph, see that the recording pens are properly adjusted, remove the bulldog forceps from the artery, and the pressure record will begin.

$a$. Take a tracing of the normal arterial pressure and heart rhythm with the recording paper moving at the rate of $0.5 \mathrm{~cm}$. per second.

$b$. Stimulate the right vagus nerve with a mild induction current for ro seconds. If this stimulus is strong enough to produce change in bloodpressure or inhibitions of the heart-rate, then allow sufficient time following the stimulus for the blood-pressure to return to the previous normal. Observing these rules, vary the intensity of the stimulus from that which produces no apparent effect to that which produces complete inhibition of the heart. Vary the time of the stimulus through I, 5, ro, and 20 seconds, using different strengths. Do not allow the nerve to cool, become dry, or to be unduly stretched. 
$c$. Test the sensitiveness of the left vagus.

$d$. Allow the vagus to fall back in its warm bed and stimulate the skin of the animal at some sensory surface, say the lips, the ear, or the foot. By varying the intensity of the stimulus, a strength will be found which will produce no reflexes of the voluntary muscles, but will produce marked effects on the heart rate and on the blood-pressure.

Expose the sciatic nerve, or any other nerve trunk containing afferent or sensory fibers, cut it, and stimulate the central end for five seconds. With a proper strength of stimulus a greater effect is produced on the heart and on the blood-pressure than by stimulating a small spot of skin. This stimulus will also reflexly accelerate respiration.

$e$. Cut the right vagus nerve and mark the exact time on the tracing by the signal marker. Do not disturb the animal of the record until stable equilibrium is again reached.

$f$. Now lift up the distal end of the divided right vagus, and stimulate it with the strength which previously just produced inhibition. Repeat the experiment on the proximal end of the divided vagus. The reflex effects are still threefold, cardiac, vasomotor and respiratory. The stimulation of the proximal end of the vagus produces effects on the heart rate when one vagus is still intact. See Experiment $l$ below.

$g$. After ro to 5 seconds cut the left vagus, marking the time of cutting on the tracing with the same care as before. As soon as the vagus nerves are cut, the heart-rate will be observed to increase sharply and the bloodpressure to rise. The respirations also change in rate and depth, a fact which can be noted directly and by its influence on the blood-pressure tracing.

$h$. Lift up the distal end of the left vagus, and stimulate it with an electric current of the strength which previously just produced inhibition. Stimulate the proximal end of the divided vagus. The stimulation produces no direct inhibitory effect on the heart rate when both vagi are cut, but does produce profound changes in the blood-pressure owing to vaso-motor reflexes.

Occasionally an animal will be found in which one or both vagi are comparatively inactive.

$k$. If the rabbit is used, stimulate the depressor nerve, which produces a marked fall in blood-pressure from reflex effects, explain.

$l$. Repeat the stimulation of the central end of the sciatic as described in $f$, now that the vagus nerves are cut. The stimulation of this nerve no longer produces decrease in the heart-rate, but occasionally an acceleration. The blood-pressure is influenced as before, showing that the vasomotor centers are reflexly stimulated.

$m$. When you have finished the outline of experiments, give an excess of ether to kill the animal and continue the record until it is dead. The 
blood-pressure will fall rapidly, the heart-rate will become slower but does not cease for a long time after respirations stop.

Should a clot form in the cannula, put a bulldog forceps on the artery, disconnect the manometer tube, and wash the clot out by a stream of liquid from the pressure bottle. Use care not to allow this fluid to enter the exposed wound.

Represent the results of each individual experiment in the above series in tabulated form which shall show: $\mathrm{I}$, the blood-pressure and heart-rate just before each experiment; 2, during the experiment; and 3 , at different times after the experiment until the normal is reached. After the data are taken from the tracings and arranged in tabular form, make a study of these facts and draw all the conclusions you can concerning the nervous regulations of the heart and of the blood-pressure. Make a written report.

22. The Vaso-motor Changes in the Finger, the Plethysmogram.-Insert the finger in the Porter finger plethysmograph, fill the tube with warmed water, and connect it with a small-sized air tambour. The variations in volume of the finger are slight, so that one must use a most delicate recorder. Take a tracing at a slow speed, I mm. per second. The finger and its plethysmograph should be suspended so that no mechanical movements will destroy the accuracy of the record. Observations through several minutes will usually show variations in volume of the finger, which will be recorded by the tambour. The reagent must be warm and relaxed.

Try a short mental problem. Cold air or cold water in the face will usually be marked by a decrease in volume indicating vaso-constriction. Warmth will lead to increase in volume indicating vaso-dilatation. In sleep there is the greatest relaxation and a large volume pulse will be present.

23. The Vaso-motors of the Frog's Web.-Prepare a frog for observation of the circulation of the web under the microscope, as described above, giving it 3 drops of ether, or just enough I per cent. curare to destroy voluntary movements. Quickly dissect the sciatic nerve in the thigh, using extreme care not to interfere with the circulation. Mount the preparation, pick out an active field of capillaries, small arteries, and veins under the low power of the microscope, then adjust the high power to a field which shows one or more small arteries. Make a drawing to record the diameter of these arteries, using pigment cells for land-marks, or measure with an ocular micrometer. Now quickly stimulate the exposed sciatic nerve while keeping the selected artery under constant observation. After stimulation for Io seconds the diameter of the vessels will be seen to decrease considerably, sometimes to the point of complete occlusion. When the stimulation ceases, the vessel will remain contracted for a few seconds, then will slowly regain its usual caliber, figure 201 . 
This is an exceptionally good method for direct observation of the vasomotor changes.

24. The Plethysmogram of the Kidney.-Anesthetize a dog or cat, see Experiments I 2 and I9 above, and take continuous blood-pressure tracings. Now open the abdominal wall by an incision along the median line, expose the left kidney and carefully dissect off its capsule, taking care not to injure its artery and vein. Enclose the kidney in the renal onkometer and carefully seal with vaseline and cover with omentum. Connect it with a delicate volume recording apparatus. Brodie's bellows recorder or a large air tambour is the best for this purpose. Adjust the recording apparatus in the vertical line with the manometer and signal pens.

Stimulation of the nerves which affect general blood-pressure through the medium of the heart will produce changes in the volume of the kidney in the same direction as the blood-pressure. Stimuli which give variations of the blood-pressure without direct change in the heart itself affect the volume of the kidney independent of the blood-pressure:

$a$. Dissect out and stimulate the splanchnic nerves just where they pass through the pillars of the diaphragm. They cause vaso-constriction in the kidney without sharply affecting the blood-pressure.

$b$. Stimulate the depressor nerve, or the central end of the divided vagus. The volume of the kidney will increase though the general bloodpressure decreases, showing that the fall of blood-pressure is due to peripheral vascular dilatation.

$c$. Stimulate the peripheral end of the divided vagus so as to slow or even completely to stop the heart. The sharp fall in blood-pressure is now accompanied by decrease in the volume of the kidney, showing that the kidney volume is merely passively following the blood-pressure. 


\section{CHAPTER VI}

\section{RESPIRATION}

THE maintenance of animal life necessitates the continual absorption of oxygen and the excretion of carbon dioxide by the living tissues. The blood is the medium in all animals which possess a well-developed blood-vascular system by which these gases are carried. Oxygen is absorbed by the blood from without and conveyed to all parts of the organism; and carbon dioxide which comes from the cells within is carried by the blood to the surfaces from which it may escape from the body. The two processes-absorption of oxygen and excretion of carbon dioxide-are complementary, and their sum is termed the process of Respiration.

In all Vertebrata and in a large number of Invertebrata certain parts, either lungs or gills, are especially constructed for bringing the blood into proximity with the aërating medium (atmospheric air, or water containing air in solution). In some of the lower Vertebrata (frogs and other naked Amphibia) the skin is important as a respiratory organ, and is capable of supplementing to some extent the functions' of the proper breathing apparatus.

A lung or a gill is constructed essentially of a fine transparent membrane, one surface of which is exposed to the air or water, as the case may be, while on the other surface is a network of blood vessels. The only separation between the blood and aërating medium is the thin wall of the blood vessels and the thin membrane on which the vessels are distributed. The difference between the simplest and the most complicated respiratory membrane is one of degree only.

In the mammals and the higher vertebrates the respiratory membrane is included within a respiratory cavity, the chest or thorax, which carries on regular movements, the respiratory movements, to bring changes of air into close contact with the respiratory surface.

The complexity of the respiratory membrane, the kind of aërating medium, and the respiratory movements are not, however, the only conditions which cause a difference in the respiratory capacity of different animals. The quantity and composition of the blood, especially as regards the number and size of the red corpuscles, and the vigor and efficiency of the circulatory apparatus in driving the blood to and fro between the lungs and the active tissues, these are conditions of equal, if not greater, importance.

It may be as well to state here that the lungs are only the medium for the exchange, on the part of the blood, of carbon dioxide for oxygen. The 
living tissues are the seat of those combustion processes which consume oxygen and produce carbon dioxide. These processes occur in all parts of the body in the substance of the living active tissues, and are the true respiratory processes, sometimes called internal or tissue respiration.

\section{THE RESPIRATORY APPARATUS.}

The object of the respiratory movements being the interchange of gases in the lungs, it is necessary that the atmospheric air shall pass into them and that the changed air shall be expelled from them. The lungs are contained in the chest or thorax, which is a closed cavity having no communication with the outside except by means of the respiratory passages. The air enters these passages through the nostrils or through the mouth, thence it passes through the larynx into the trachea or windpipe, which about the middle of the chest divides into two tubes, the bronchi, one to each lung.

The Larynx.-The upper part of the passage which leads exclusively to the lung is formed by the thyroid, cricoid, and arytenoid cartilages, figure $2 \times 8$, and contains the vocal cords, by the vibration of which the voice is chiefly produced. These vocal cords are ligamentous bands covered with mucous membrane and attached to certain cartilages which are capable of movement by muscles. By their approximation the cords can entirely close the entrance into the larynx; but under ordinary conditions the entrance of the larynx is formed by a more or less triangular opening between them, called the rima glottidis. Projecting at an acute angle between the base of the tongue and the larynx to which it is attached, is a leaf-shaped cartilage with its larger extremity free. This

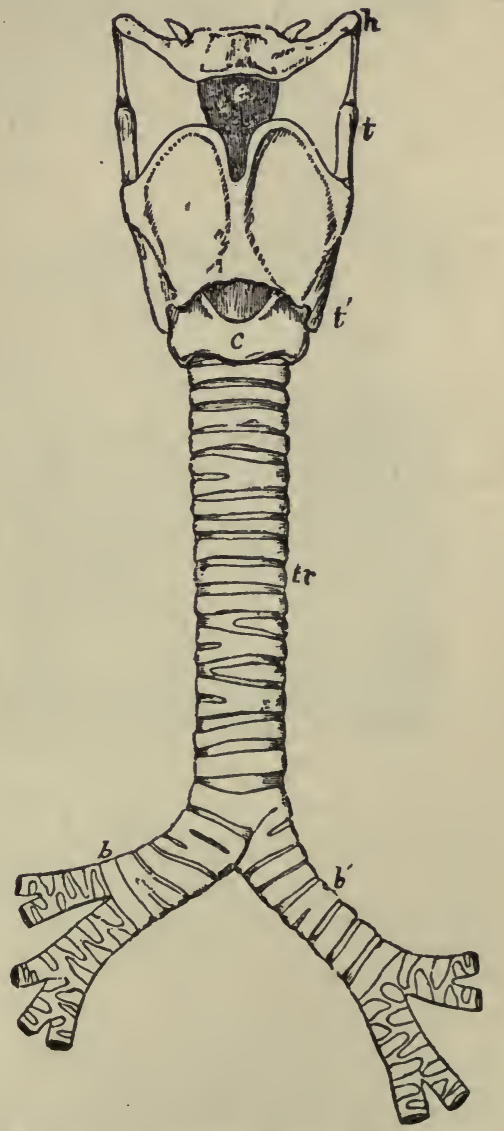

FIG. 218.-Outline Showing the General Form of the Larynx, Trachea, and Bronchi, as seen from Before. $h$, The great cornu of the hyoid bone; $e$, epiglottis; $t$, superior, and $t^{\prime}$, inferior cornu of the thyroid cartilage; $c$, middle of the cricoid cartilage; $t r$, the trachea, showing sixteen cartilaginrus rings; $b$, the right, and $b^{\prime}$, the left bronchus. $\times \frac{1}{2}$. (Allen Thomson.) 
is called the epiglottis. The whole of the larynx is lined by mucous membrane, which, however, is extremely thin over the vocal cords. At its lower extremity the larynx joins the trachea.

Taste buds have been found in the epithelium of the posterior surface of

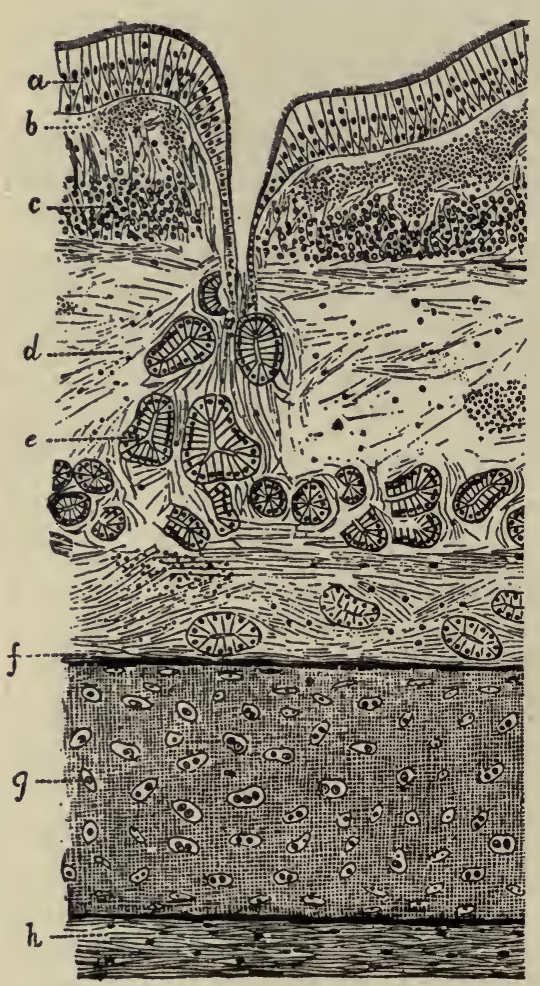

FIG. 219.-Section of the Trachea. a, Columnar ciliated epithelium; $b$, and $c$, proper structure of the mucous membrane, containing elastic fibers cut across transversely; $d$, submucous tissue containing mucous glands, $e$, separated from the hyaline cartilage, $g$, by a fine fibrous tissue, $f, h$, external investment of fine fibrous tissue. (S. K. Alcock.) the epiglottis, and in several other situations in the laryngeal mucous membrane.

The Trachea and Bronchi.The trachea extends from the cricoid cartilage, which is on a level with the fifth cervical vertebra, to a point opposite the third dorsal vertebra, where it divides into the two bronchi, one for each lung, figure 218 . The trachea measures, on an average, four or four and a half inches, 12 to $14 \mathrm{~cm}$., in length, and from three-quarters of an inch to an inch, 2 to $2.5 \mathrm{~cm}$., in diameter, and is essentially a tube of fibroelastic membrane within the layers of which are enclosed a series of cartilaginous rings, from sixteen to twenty in number. These rings extend only around the front and sides of the trachea, about twothirds of its circumference, and are deficient behind; the interval between their posterior extremities being bridged over by a continuation of the fibrous membrane in which they are enclosed, figure 219, $h$.

Immediately within this tube and at the back is a layer of unstriped muscular fibers. This muscular layer extends transversely between the ends of the cartilaginous rings to which it is attached, and also opposite the intervals between them; its evident function being to diminish the caliber of the trachea by approximating the ends of the cartilages. Outside there are a few longitudinal bundles of muscular tissue, which, like the preceding, are attached both to the fibrous and to the cartilaginous framework.

The mucous membrane, figures 219 and 220 , consists largely of adenoid tissue, separated from the stratified columnar epithelium, which lines it, by a 
homogeneous basement membrane. This is penetrated here and there by channels which connect the adenoid tissue of the mucosa with the intercellular substance of the epithelium. The stratified columnar epithelium is formed of several layers, of which the most superficial layer is ciliated and

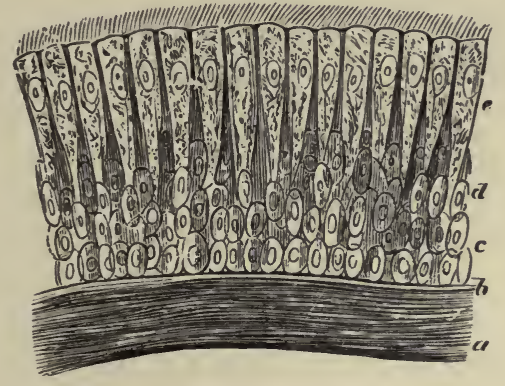

Fig. 220.-Ciliary Epithelium of the Human Trachea. $a$, Layer of longitudinally arranged elastic fibers; $b$, basement membrane; $c$, deepest cells, circular in form; $d$, intermediate elongated cells; $e$, outermost layer of cells fully developed and bearing cilia. $X$ 350. (Kölliker.)

the cells often branched downward. Many of the superficial cells are of the goblet variety. In the deeper part of the mucosa are many elastic fibers between which lie connective-tissue corpuscles and capillary blood vessels.

Numerous mucous glands are situated on the exterior and in the substance of the fibrous framework of the trachea, their ducts perforating the various

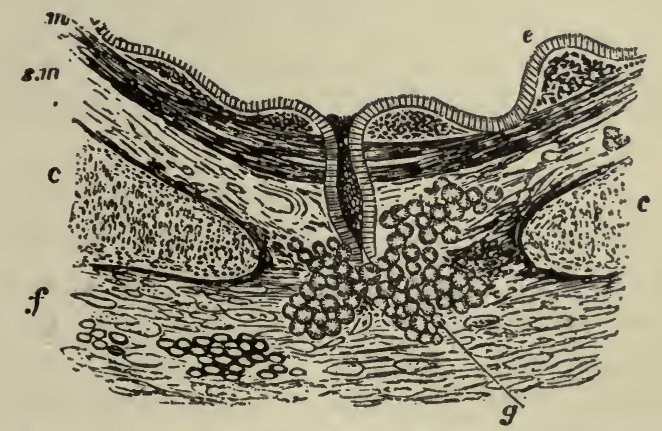

Fig. 221.-Transverse Section of a Bronchus, about $\frac{1}{2}$ inch in Diameter. e, Epithelium (ciliated); immediately beneath it is the mucous membrane or internal fibrous layer, of varying thickness; $m$, muscular layer; $s, m$, submucous tissue; $f$, fibrous tissue; $c$, cartilage enclosed within the layers of fibrous tissue; $g$, mucous gland. (F. E. Schulze.)

structures which form the wall of the trachea, and opening through the mucous membrane into the cavity of the trachea.

The two bronchi into which the trachea divides resemble the trachea in structure, with the difference that in them there is a distinct layer of unstriped muscle arranged circularly beneath the mucous membrane, forming 
the muscularis mucosa. On entering the substance of the lungs the cartilaginous rings, although they still form only larger or smaller segments of a circle, are no longer confined to the front and sides of the tubes, but are distributed impartially to all parts of their circumference.

The bronchi divide and subdivide in the substance of the lungs into smaller and smaller branches, which penetrate into every part of the organ until at length they end in the smaller subdivisions of the lungs called lobules.

All the larger branches have walls formed of tough membrane, containing portions of cartilaginous rings, by which they are held open, and unstriped muscular fibers, as well as longitudinal bundles of elastic tissue. They are lined by mucous membrane, the surface of which, like that of the larynx and trachea, is covered with ciliated epithelium; but the several layers become less and less distinct until the lining consists of a single layer of more or less cubical cells covered with cilia, figure $22 \mathrm{I}$. The mucous membrane is abundantly provided with mucous glands.

As the bronchi become smaller and smaller and their walls thinner, the cartilaginous rings become fewer and more irregular, until in the smaller bronchial tubes they are represented only by minute and scattered cartilaginous flakes. And when the bronchi by successive branches are reduced to about $\frac{1}{40}$ of an inch, $0.6 \mathrm{~mm}$., in diameter, they lose their cartilaginous element altogether and their walls are formed only of a tough, fibrous, elastic membrane with circular muscular fibers. They are still lined, however, by a thin mucous membrane with ciliated epithelium, the length of the cells bearing the cilia having become so far diminished that the cells are almost cubical. In the smaller bronchi the circular muscular fibers are relatively more abundant than in the larger bronchi and form a distinct circular coat.

The Lungs and Pleuræ.-The lungs occupy the greater portion of the thorax. They are of a spongy elastic texture, and on section appear to the naked eye as if they were in great part solid organs, except where branches of the open bronchi or air-tubes may have been cut across and show on the surface of the section. In fact, however, the lungs are hollow organs composed of a mass of air cavities all of which communicate finally with the common air-tube, the trachea.

Each lung is enveloped by a serous membrane, the pleura, which adheres closely to its surface and provides it with its smooth and slippery covering. This same membrane lines the inner surface of the chest wall. The continuity of this membrane, which forms a closed sac as in the case of other serous membranes, will be best understood by reference to figure 222 . The appearance of a space, however, between the pleura which covers the lung, visceral layer, and that which lines the inner surface of the chest, parietal layer, is inserted in the drawing only for the sake of distinctness. These 
layers are, in health, everywhere in contact, one with the other; and between them is only just as much fluid as will insure frictionless movement in their expansion and contraction.

When considering the subject of normal respiration, one may discard altogether the notion of the existence of any space or cavity between the lungs and the wall of the chest. If, however, an opening be made so as to permit air or fluid to enter the pleural sac, the lung in virtue of its elasticity recoils, and a considerable space is left between it and the chest wall. In other words, the natural elasticity of the lungs would cause them at all times to contract away from the ribs were it not that the contraction is resisted by atmospheric pressure which bears only on the inner surface of the air-tubes and air-cells.

The pulmonary pleura consists of an outer or denser layer and an inner looser tissue in which there is a lymph-canalicular system. Numerous

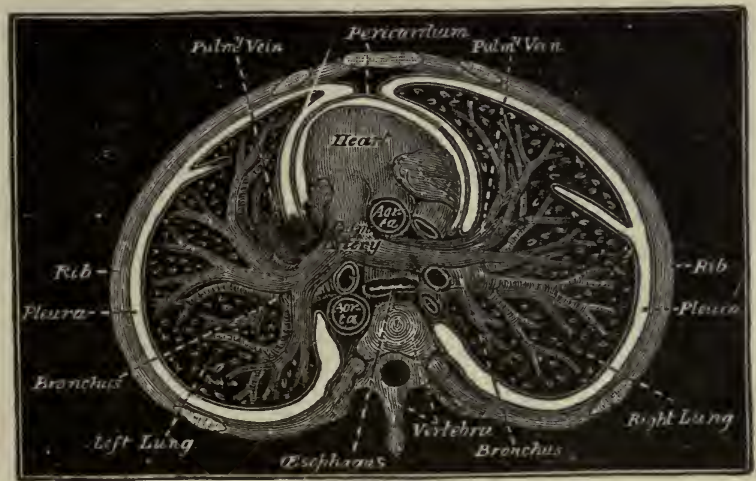

FIG. 222.- Transverse Section of the Chest.

lymphatics are to be met with, which form a dense plexus of vessels, many of which contain valves. They are simple endothelial tubes, and take origin in the lymph-canalicular system of the pleura proper. Scattered bundles of unstriped muscular fiber occur in the pulmonary pleura. They are especially strongly developed on the anterior and internal surfaces of the lungs, the parts which move most freely in respiration. Their function is doubtless to aid in expiration.

The Finer Structure of the Lung.-Each lung is partially subdivided into separate portions called lobes: the right lung into three lobes and the left into two. Each of these lobes, again, is composed of a large number of minute parts, called lobules. Each pulmonary lobule may be considered to be a lung in miniature, consisting, as it does, of a branch of the bronchial tube, of air-cells, blood vessels, nerves, and lymphatics, with a small amount of areolar tissue. 
On entering a lobule, the small bronchial tube, the structure of which has just been described, $a$, figure 223 , divides and subdivides; its walls at the same time becoming thinner and thinner, until at length they are formed only of a thin membrane of areolar and elastic tissue, lined by a layer of squamous epithelium, no longer provided with cilia. At the same time they are altered in shape; each of the minute terminal branches widening out funnel-wise, and its walls being pouched out irregularly into small saccular dilatations, called air-cells, figure 223, $b$. Such a funnel-shaped terminal branch of the bronchial tube, with its group of pouches or air-cells, has been called an infundibulum, figures 223 and 224, and the irregular oblong space in its center, with which the air-cells communicate, an intercellular passage.

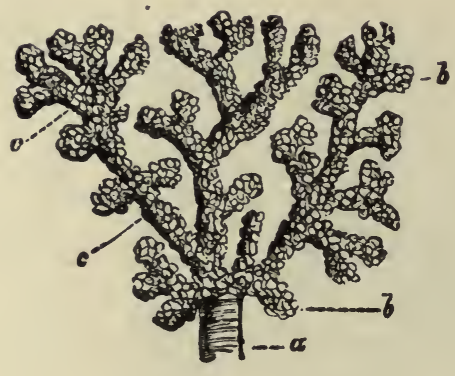

FIG. 223.

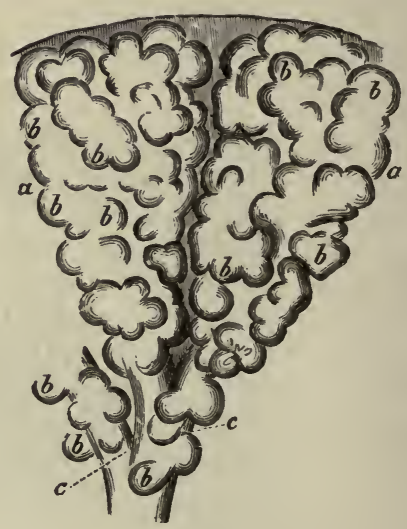

FIG. 224.

Fig. 223. - Terminal Branch of a Bronchial Tube, with its Infundibula and Air-cells, from the Margin of the Lung Injected with Quicksilver; Monkey. $a$, Terminal bronchial twig; $b, b$, infundibula and air-cells. $\times$ ro. (F. E. Schulze.)

FIG. 224.- Two Small Infundibula, $a, a$, with air-cells, $b, b$, and the ultimate bronchial tubes, $c, c$, with which the air-cells communicate. From a new-born child. (Kölliker.)

An inflated and dried turtle's lung illustrates the homologue of a lobule. Such a preparation can be cut across to illustrate the intercellular passage, the infundibulum, and the air-cells.

The air-cells, or air-vessels, are sometimes placed singly, like recesses from the intercellular passage, but more often they are arranged in groups or even rows, like minute sacculated tubes, so that a short series of vesicles all communicating with one another open by a common orifice into the tube. The vesicles are of various forms according to the mutual pressure to which they are subject. Their walls are nearly in contact, and they vary from 0.3 to $0.5 \mathrm{~mm}$. in diameter. Their walls are formed of fine membrane similar to that of the intercellular passages and continuous with it. The membrane is folded on itself so as to form a sharp-edged border at each circular orifice 
of communication between contiguous air-vesicles, or between the vesicles and the bronchial passages. Numerous fibers of elastic tissue are spread out in the walls between contiguous air-cells, and many of these are attached to the outer surface of the wall of which each cell is composed, imparting to it additional strength and the power of recoil after distention.

The air-cells are lined by a layer of epithelium, figure 225, the cells of which are very thin and plate-like. The thin epithelial membrane is free on one side, where it comes in contact with the air of the lungs, but on the other

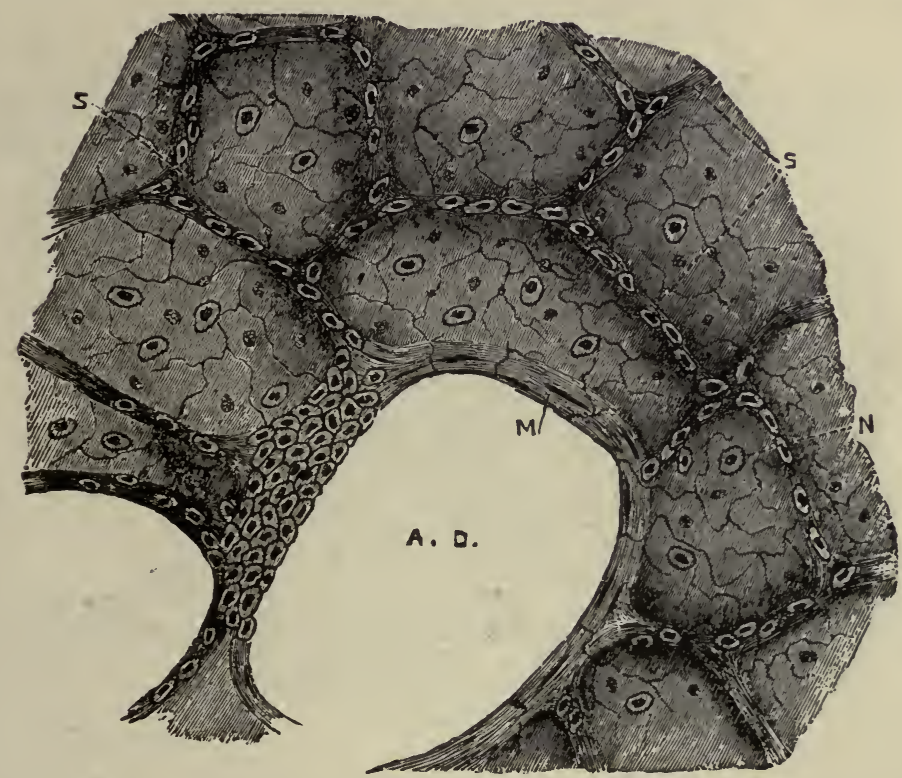

Fig. 225.-From a Section of the Lung of a Cat, Stained with Silver Nitrate. A. D, Alveolar duct or intercellular passage; $S$, alveolar septa, $N$, alveoli or air-cells, lined with large, flat, nucleated cells, with some smaller polyhedral nucleated cells; $M$, unstriped muscular fibers. Circular muscular fibers are seen surrounding the interior of the alveolar duct, and at one part is seen a group of small polyhedral cells continued from the bronchus. (Klein and Noble Smith.)

side a network of pulmonary capillaries is spread out so densely, figure 226 that the interspaces or meshes are even narrower than the vessels. These are on an average $\frac{1}{3000}$ of an inch, or 8 micromillimeters, in diameter. Between the atmospheric air-cells and the blood in these vessels, nothing intervenes but the thin walls of the cells and capillaries. The exposure of the blood to the air is the more complete because the wall between contiguous air-cells, and often the spaces between the walls of the same, contain only a single layer of capillaries both sides of which are at once exposed to the air.

The air-vesicles situated nearest to the center of the lung are smaller and their networks of capillaries are closer than those nearer to the circum- 
ference. The vesicles of adjacent lobules do not communicate. Those of the same lobule or proceeding from the same intercellular passage communicate, as a general rule, only near angles of bifurcation, so that when any bronchial tube is closed or obstructed the supply of air is lost for all the blood vessels of that lobule and its branches.

Blood Supply. - The lungs receive blood from two sources: $a$, the pulmonary artery; $b$, the bronchial arteries. The former conveys venous blood to the lungs for its oxidation, and this blood takes no share in the

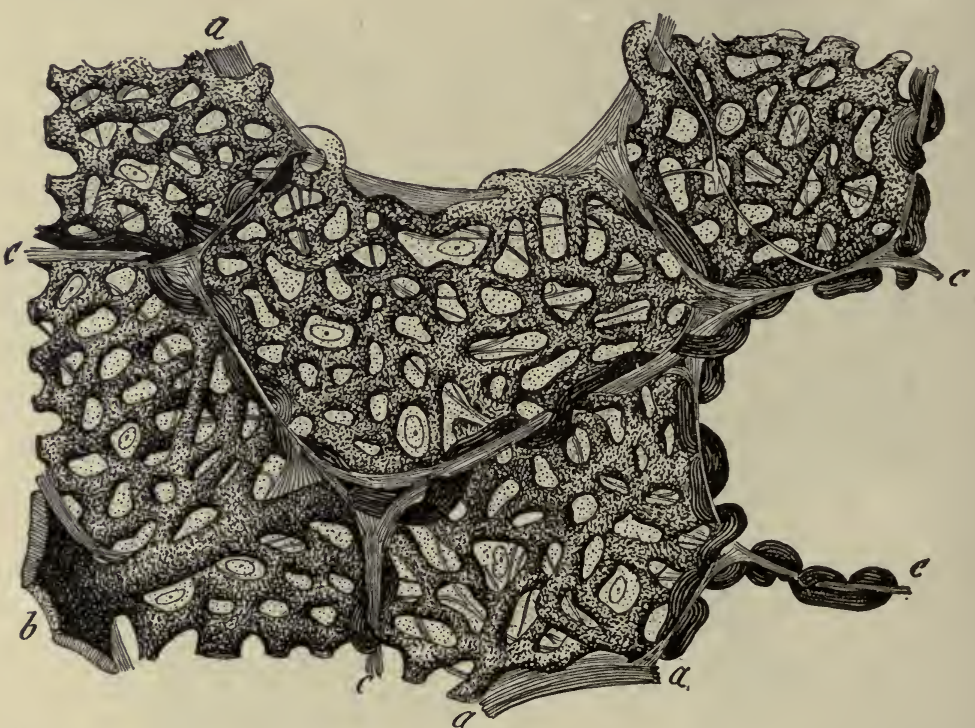

Fig. 226.- Section of Injected Lung, Including Several Contiguous Alveoli. (F. E. Schulze.) Highly magnified. $a, a$, Free edges of alveoli; $c, c$, partitions between neighboring alveoli, seen in section; $b$, small arterial branch giving off capillaries to the alveoli. The looping of the vessels to either side of the partitions is well exhibited. Between the capillaries is seen the homogeneous alveolar wall with nuclei of connective-tissue corpuscles and elastic fibers.

nutrition of the deeper pulmonary tissues through which it passes. The branches of the bronchial arteries are nutrient arteries which ramify in the walls of the bronchi, in the walls of the larger pulmonary vessels, and in the interlobular connective tissue, etc. The blood of the bronchial vessels is returned chiefly through the bronchial, but partly through the pulmonary, veins.

Lymphatics.- The lymphatics are arranged in three sets: I. Irregular lacunæ in the walls of the alveoli or air-cells. The lymphatic vessels which lead from these accompany the pulmonary vessels toward the root of the lung. 2, Irregular anastomosing spaces in the walls of the bronchi. 3, Lymph spaces in the pulmonary pleura. The lymphatic vessels from all 
these irregular sinuses pass in toward the root of the lung to reach the bronchial glands.

Nerves.-The nerves of the lung are to be traced from the anterior and posterior pulmonary plexuses, which are formed by branches of the vagus and sympathetic. The nerves follow the course of the blood vessels and bronchi, and many small ganglia are situated in the walls of the latter.

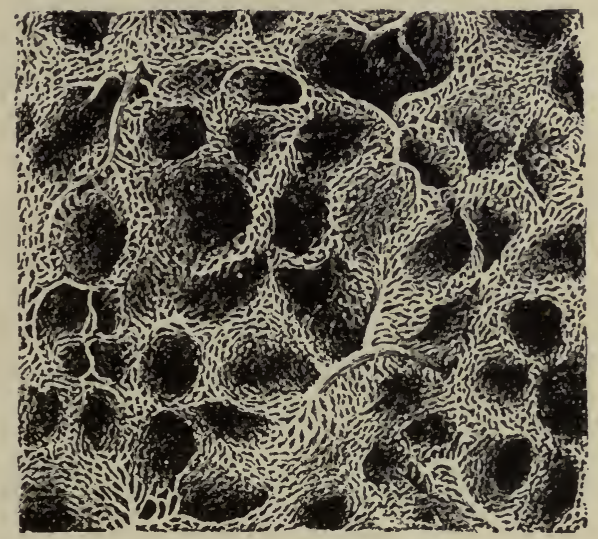

Fig. 227. - Capillary Network of the Pulmonary Blood Vessels in the Human Lung. $\quad \times 60$. (Kölliker.)

\section{THE MOVEMENTS OF THE RESPIRATORY MECHANISM.}

Respiratory movement consists of the alternate expansion and contraction of the thorax, by means of which air is drawn into, or expelled from, the lungs.

A movement of the side walls or floor of the chest to increase its diameter or length will enlarge the capacity of the interior. By such an increase of capacity there will be of course a diminution of the pressure of the air in the lungs, and a fresh quantity of air will enter through the larynx and trachea to equalize the pressure on the inside and outside of the chest. This movement is called inspiration.

The movement which diminishes the capacity of the chest and increases the pressure in the interior expels air until the pressure within and that without the chest are again equal. This movement is called expiration. In both cases the air passes through the trachea and larynx, whether in entering or leaving the lungs, there being no other communication with the exterior of the body. And the lung, for the same reason, remains closely in contact with the walls and floor of the chest under all the circumstances described. To speak of expansion of the chest is to speak also of expansion of the lung, and vice versa. 
Inspiration.-The enlargement of the chest during inspiration is due to muscular action, which brings about an increase in the size of the chest cavity through the contraction of the inspiratory muscles, the rôle played by the lungs being a passive one. The chest cavity is increased in its three axes, the vertical, lateral, and antero-posterior diameters. The muscles engaged in ordinary inspiration are: the diaphragma, the intercostales externi, and the scaleni and levatores costarum. During forced inspiration every muscle is brought into play which by its contraction tends to elevate the ribs

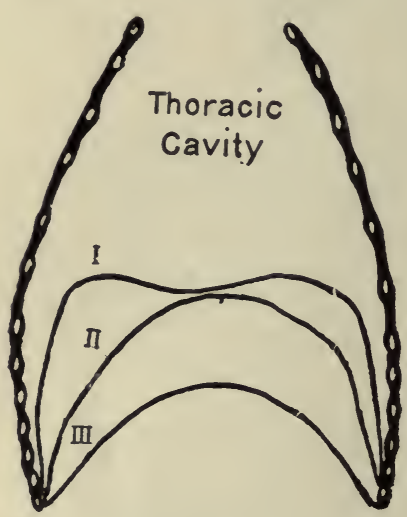

FIG. 228.-Schematic Representation of Diaphragm. In expiration $(I)$, quiet inspiration $(I I)$, and deep inspiration $(I I I)$. (After Schäffer.) and sternum or which will fix points against which these muscles can act. This includes almost every muscle of the trunk and neck.

Changes in the vertical diameter are due, first, to the contraction of the diaphragm. This muscle has the shape of a flattened dome, its highest point being the central tendon. While passive its lower portions are in apposition with the chest walls, figure $228, I$. On contraction, the dome is pulled downward and the lower portion is pulled away from the chest walls, the downward displacement varying from 6 to $12 \mathrm{~mm}$. in normal respiration, and in forced respiration may amount to as much as $45 \mathrm{~mm}$. The tendency of the diaphragm to pull the lower ribs and lower part of the sternum inward is counteracted by the outward pressure of the abdominal viscera, and by the action of the quadrati lumbori, which by their attachment to the last ribs fix these and, in case of deep inspiration, may even pull them downward. The serrati postici inferiores also aid, being attached to the four lower ribs.

Changes in the lateral and antero-posterior diameters are effected by the raising of the ribs, which are attached very obliquely to the spine and sternum. The elevation of the ribs takes place both in front and at the sides-the hinder ends being prevented from performing any upward movement by their pivot attachment to the spine. The movement of the front extremities of the ribs is of necessity limited by an upward and forward movement of the sternum to which they are attached, the movement being greater at the lower end than at the upper end of the sternum.

The axes of rotation in these movements are two: one corresponding with a line drawn through the two articulations which the rib forms with the spine, $a, b$, figure 230 , and the other with a line drawn from one of these (head of rib) to the sternum, $A B$, figure 230; the motion of the rib around the latter axis being somewhat after the fashion of raising the handle of a 
bucket. The elevation of the ribs is accompanied by a slight opening out of the angle which the bony part forms with its cartilage, and thus an additional means is provided for increasing the antero-posterior diameter of the chest. The movements of all the ribs except the twelfth consist of a rotation up-

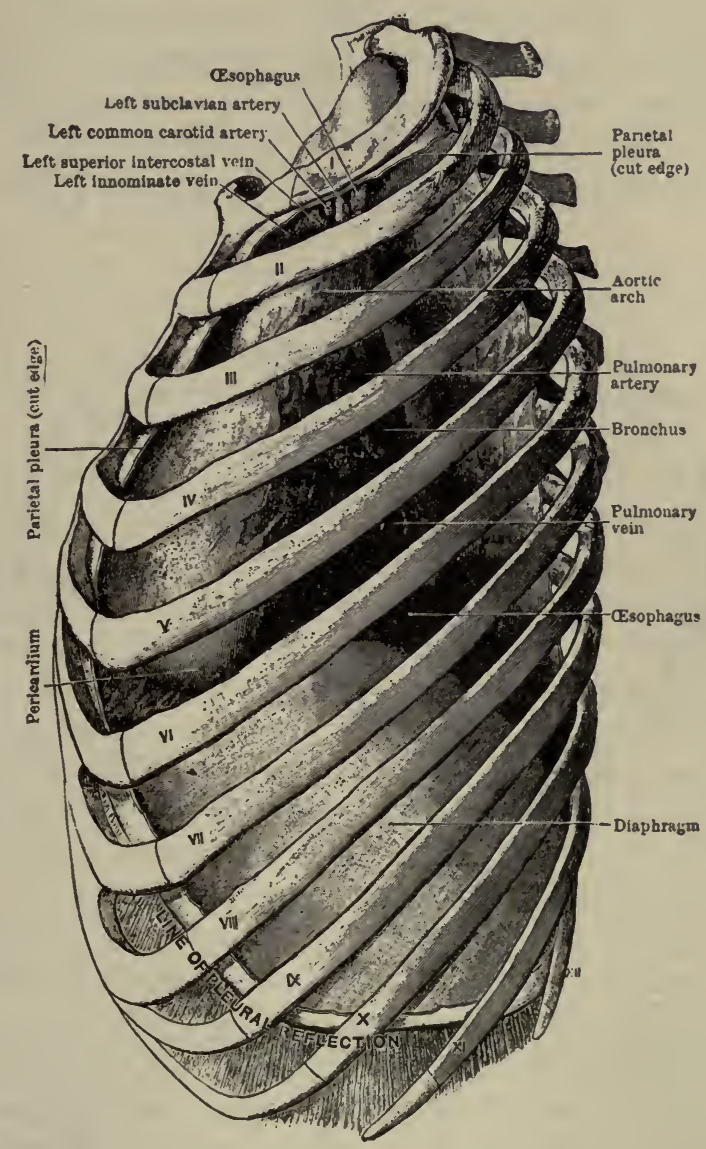

FIG. 229.-Thorax from the Left, Showing Left Pleural Sac, and the Diaphragm. The lung is removed. (Cunningham.)

ward, forward, and outward. The twelfth presents only rotation downward and backward.

The muscles involved in these movements of the ribs are the external intercostals and the part of the internal intercostals situated between the costal cartilages. Their action is to widen the intercostal spaces. The scaleni fix the first and second ribs, thereby making a fixed point of action 
for the other muscles involved. The serrati postici superiores assist the above and also raise the third, fourth, and fifth ribs. The levatores costarum longi and brevi elevate and evert all the ribs from the first to the tenth.

In extraordinary or forced inspiration, which may be due either to violent exercise or to interference with the due entrance of air into the lungs, all the above muscles act more strongly. The diaphragm descends lower, the scaleni raise the first and second ribs instead of merely fixing them, as in ordinary respiration, as do also the sterno-cleido-mastoids. These, together with the sacro-spinales which straighten the spine, increase the vertical diameter. The trapezii and the rhomboidii assist in increasing the antero-

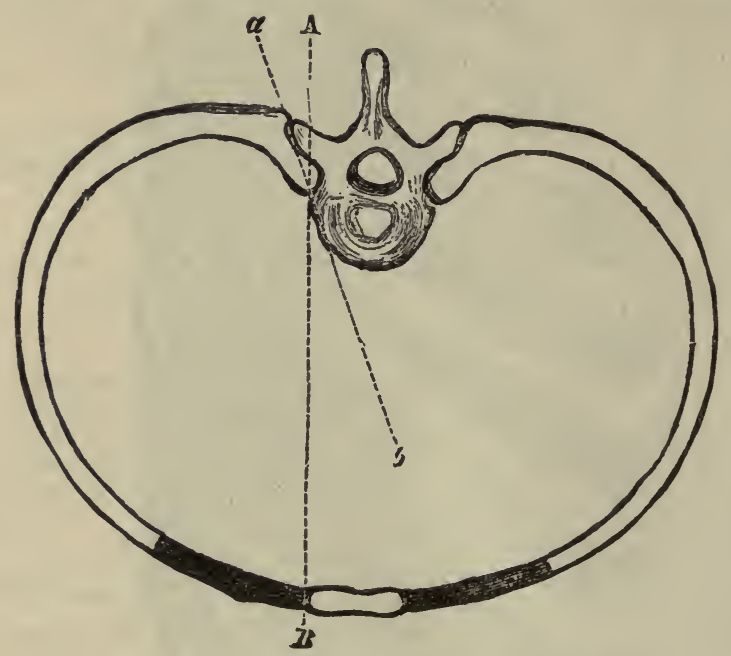

Fig. 230.-Diagram of Axes of Movement of Ribs.

posterior and lateral diameters by fixing the shoulders and thus giving a fixed point for the action of the pectorales and latissimi dorsi.

The enlargement of the chest during inspiration presents peculiarities in different persons. In children of both sexes the principal muscle involved seems to be the diaphragm, and this type of breathing is known as abdominal breathing. In men, the chest and sternum, together with the front wall of the abdomen, are subject to a wide movement; this type of breathing is called the inferior costal. In women, the movement appears less extensive in the lower and more extensive in the upper part of the chest, which is called the superior costal type. This has been shown to be due rather to mode of dress than to a real difference in the sexes (Mosher).

Expiration.-Quiet expiration is a passive act due to the return of the thorax and its contained lungs to their normal position when the muscles involved in inspiration relax. This elastic recoil is sufficient in ordinary quiet breathing to expel air from the lungs. In forced expiration, however, 
which may occur to a slight degree in speaking, singing, etc., as well as in the case of many involuntary and reflex acts, such as coughing, sneezing, etc., other muscles are involved. Of these the principal are the abdominal muscles, obliquus externus and intermus, rectus abdominis, transversus abdominis and pyramidalis. These act, first, by pressing the abdominal viscera against the diaphragm and thereby forcing it up, their descent into the pelvic cavity being prevented; second, by their attachments to the lower ribs and cartilages, the muscles draw these downward and inward, thereby lessening the size of the thoracic cavity; lastly, by their contraction, they form a fixed point for the action of that part of the internal intercostals, not involved in inspiration, to approximate the ribs.

When by the efforts of the expiratory muscles the chest has been squeezed to less than its average diameters, it again, on relaxation of the muscles, returns to the normal dimensions by virtue of its elasticity. The construction of the chest walls, therefore, admirably adapts them for recoiling against and resisting as well undue contraction as undue dilatation.

Respiratory Movements of the Nostrils and of the Glottis.-During the action of the inspiratory muscles which directly draw air into the chest, those which guard the opening through which the air enters are also active. In hurried breathing the dilatation of the nostrils is well seen, although under ordinary conditions it may not be noticeable. The opening at the upper part of the larynx, however, the rima glottidis, is dilated at each inspiration for the more ready passage of air, and becomes smaller at each expiration; its condition, therefore, corresponds during respiration with that of the walls of the chest. There is a further likeness between the two acts in that, under ordinary circumstances, the dilatation of the rima glottidis is a muscular act and its contraction chiefly an elastic recoil; although, under various special conditions to be hereafter mentioned, there may be considerable muscular contraction exercised.

Methods of Recording Respiratory Movements.-The movements of respiration may be recorded graphically in several ways. The ordinary method is to introduce a tube into the trachea of an animal, and to connect this tube by some gutta-percha tubing with a $\mathrm{T}$-piece, the side branch of which is connected with a Marey's tambour, which may be made to write on a recording surface, figure ${ }_{15} 6$. If the tube attached to the free limb of the T-piece be partially closed with a screw compress, the movements of inspiration and expiration are larger than if it were open. The alteration of the pressure within the lungs on inspiration and expiration is shown by the movement of the column of air in the trachea and in its extension to the T-piece. By these means a record of the respiratory movements may be obtained in experimental animals.

Various instruments have been devised for recording the movements of the chest by application of apparatus to the exterior. Such is the stethometer of Burdon-Sanderson, figure 233. This consists of a frame formed of two parallel steel bars joined by a third at one end. At the free end of the bars is attached a leather strap, by means of which the apparatus may be suspended 
from the neck. Attached to the inner end of one bar is a tambour and ivory button, to the end of the other an ivory button. The apparatus is suspended with the transverse bar.posteriorly, the button of the tambour is placed on the part of the chest the movement of which it is desired to record, and the other button is made to press upon the corresponding side of the chest, so that the chest

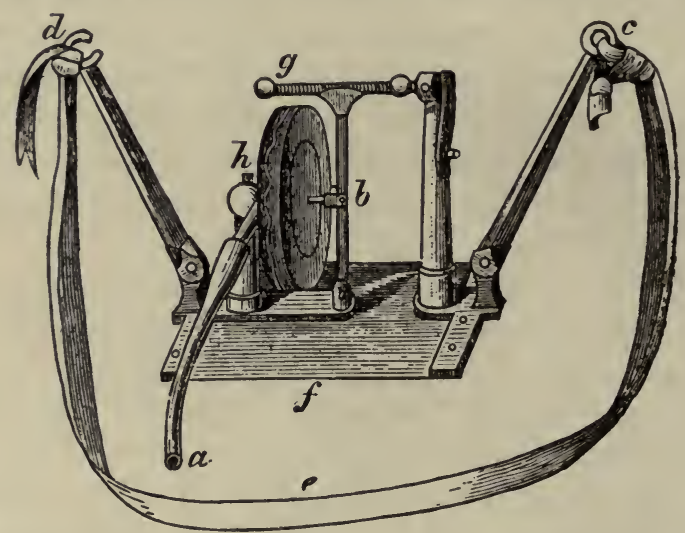

FIG. 231.- Stethograph or Pneumograph. $h$, Tambour fixed at right angles to plate of steel, $f ; c$ and $d$, arms by which instrument is attached to chest by belt, $e$. When the chest expands, the arms are pulled asunder, which bends the steel plate, and the tambour is affected by the pressure of $b$, which is attached to it on the one hand, and to the upright in connection with horizontal screw, $g$. (Modified from Marey's instrument.)

is held as between a pair of calipers. The receiving tambour is connected through a T-piece with a recording tambour of Marey's and with a bulb by means of which air can be squeezed into the cavity of the typanum. When adjusted the tube connected with the air ball is shut off by means of a screw clamp. The movement of the chest is thus communicated to the recording tambour.

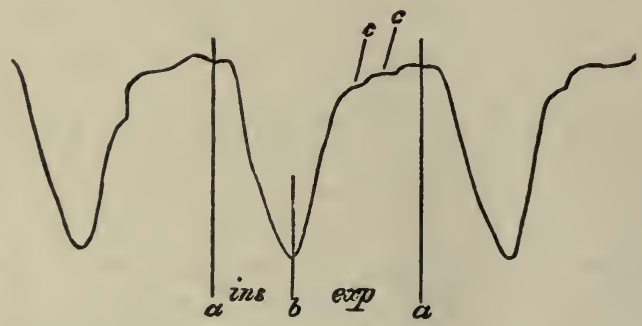

FIG. 232.-Tracing of Thoracic Respiratory Movements obtained by means of Marey's Pneumograph. A whole respiratory phase is comprised between $a$ and $a$; inspiration during which the lever descends, extending from $a$ to $b$, and expiration from $b$ to $a$. The undulations at $c$ are caused by the heart's beat. (Foster.)

A simpler form of this apparatus, called a pneumograph or stethograph, consists of a thick india-rubber bag of elliptical shape about three inches long, to one end of which a rigid gutta-percha tube is attached. This bag may be fixed at any required place on the chest by means of a strap and buckle. By means of the gutta-percha tube the variations of the pressure of air in the bag, 
produced by the movements of the chest, are communicated to a recording tambour. This principle is applied in a modified form in Marey's pneumograph, figure $23 \mathrm{I}$.

The variations of intrapleural pressure may be recorded by introducing a cannula into the pleural or pericardial cavity. The cannula should be previously connected with a mercury or other form of manometer by tubing filled with physiological saline.

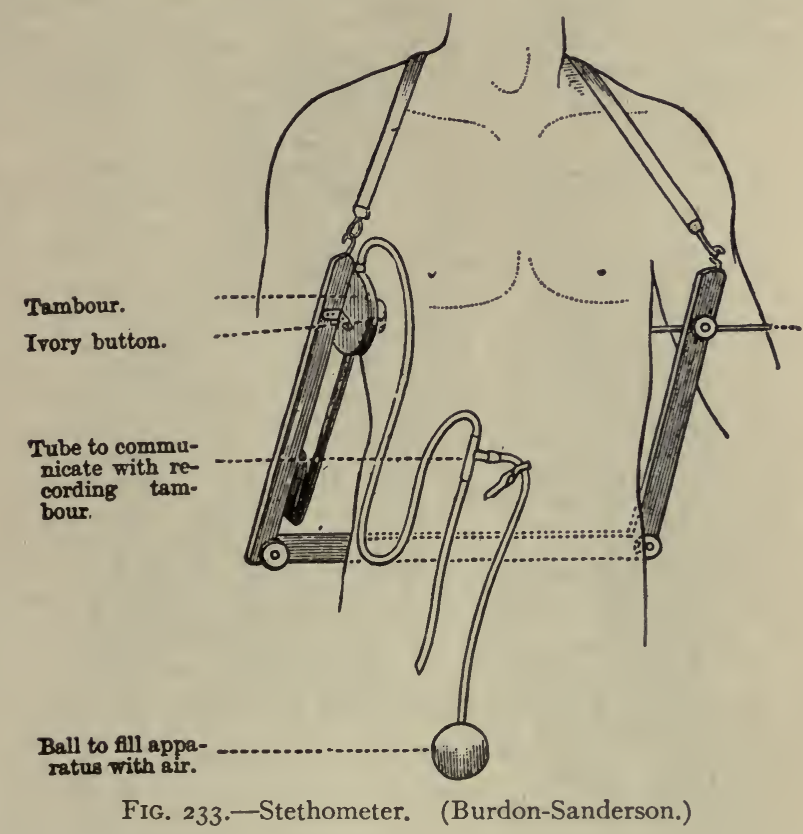

Finally, it has been found possible in various ways to record the diaphragmatic movements. This can be done by inserting a receiving tambour into the abdomen below the diaphragm, by the insertion of needles into different parts of the diaphragm and recording the movement of the free ends of needles about the fulcrum formed where the chest wall is pierced, or by recording the contraction of isolated strips of the diaphragm directly. These records all give an accurate picture of the movements of the diaphragm.

The Relative Time of Inspiration and Expiration and the Respiratory Movement.-The acts of inspiration and expiration take up, under ordinary circumstances, a nearly equal time. The time of inspiration, however, especially in women and children, is a little shorter than that of expiration, and there is commonly a very slight pause between the end of expiration and the beginning of the next inspiration, see figure 232. The ratio of the respiratory rhythm may be thus expressed:

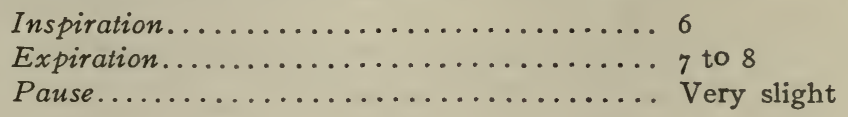


If the ear be placed in contact with the wall of the chest or be separated from it only by a good conductor of sound or a stethoscope, a faint respiratory murmur is heard during inspiration. This sound varies somewhat in different parts, being loudest or coarsest in the neighborhood of the trachea and large bronchi (tracheal and bronchial breathing), and fading off into a faint sighing as the ear is placed at a distance from these (vesicular breathing). It is heard best in children. In them a faint murmur is heard in expiration also. The cause of the vesicular murmur has received various explanations. Most observers hold that the sound is produced in the glottis and larger

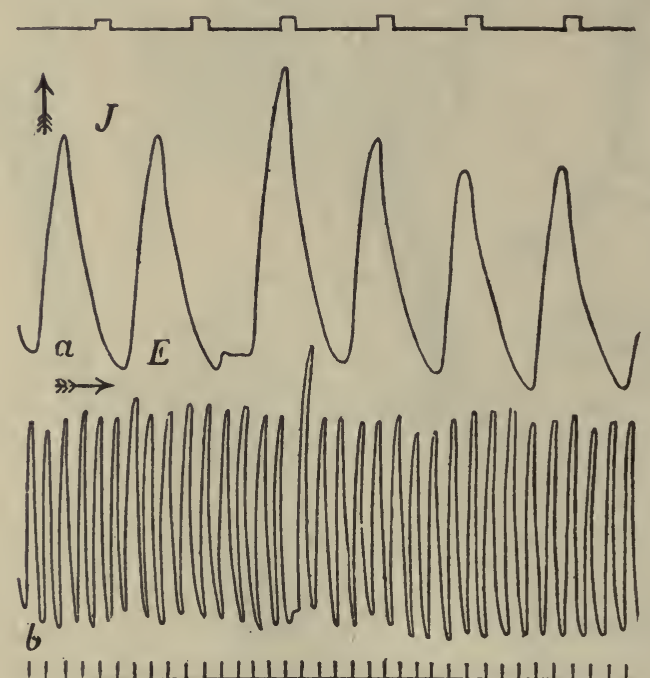

FIG. 234.-Tracing of the Normal Diaphragm Respirations of the Rabbit. $a$, With quick movement of drum; $b$, with slow movement; $J$, inspiration; $E$, expiration. To be read from left to right. (Marckwald.)

bronchial tubes, but that it is modified in its passage to the pulmonary alveoli. In disease of the lungs the vesicular murmur undergoes various modifications, for a description of which one must consult text-books on physical diagnosis.

- The Quantity of Air Breathed.-Tidal air is the quantity of air which is habitually and almost uniformly changed in each act of breathing. In a healthy adult man it is about 30 cubic inches, or about $500 \mathrm{cc}$. or half a liter. In college students the tidal air is somewhat less, varying from 300 to $400 \mathrm{cc}$. while at rest.

The complemental air is the quantity of air which can be drawn into the lungs by the deepest inspiration over and above that which is in the lungs at the end of an ordinary inspiration. Its amount varies, but may be reckoned as roo cubic inches, or about 1,600 c.c. 
The reserve air is that which may be expelled by a forcible and deeper expiration, after an ordinary expiration, such as that which expels the tidal air. The reserve air amounts to from $\mathrm{r}, 200$ to $\mathrm{I}, 500 \mathrm{cc}$. This is also termed the supplemental air.

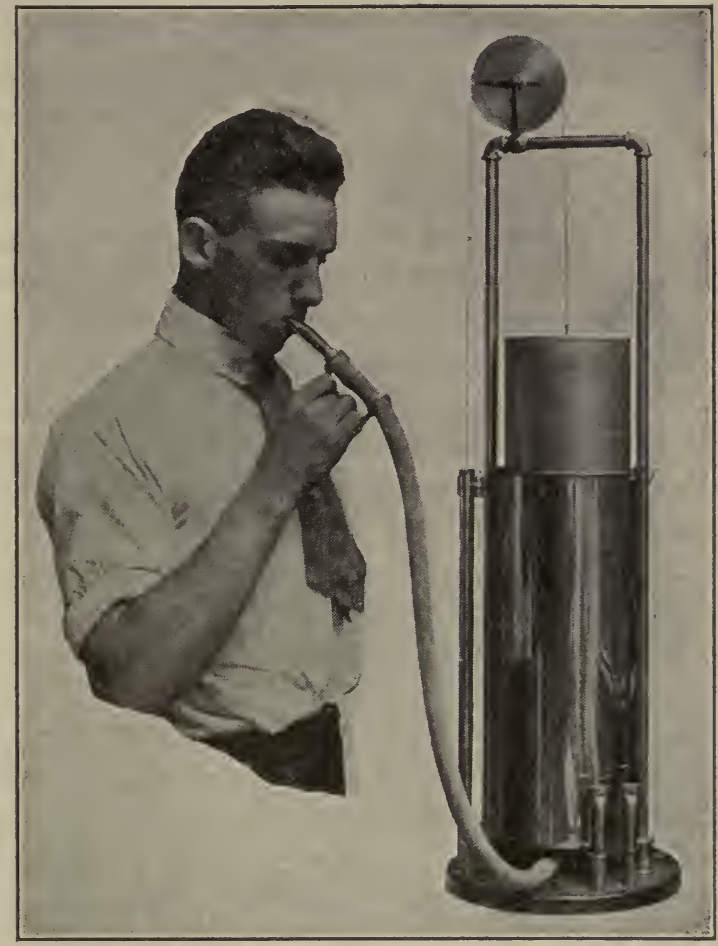

Fig. 235.-Photograph of the Sanborn Company form of spirometer. The graduated disc records the volume of air exhaled. In using the spirometer the reservoir is first filled with water to form a water seal for the air chamber. To perform a respiratory volume test the instrument is set automatically at zero, though it is well to begin with the air chamber empty. Place the previously sterilized mouth piece between the lips and if necessary, close the nostrils with pinch cock or with the hand. The receiving chamber is delicately balanced and as the breath is forced through the tube into this bell it rises, recording the movement on the circular index, from the scale of which the volume is read off directly. For very accurate comparative determinations the expired air should be allowed to stand long enough to come to constant temperature, and corrections for variations from the standard temperature and pressure should be made. This is not necessary in the routine laboratory and gymnasium measurements.

The residual air is the quantity which still remains in the lungs after the most violent expiratory effort. Its amount depends in great measure on the absolute size of the chest, but may be estimated at about I,000 cc. to $\mathrm{I}, 200 \mathrm{cc}$.

The quantity of air breathed per minute, called the minute volume, varies in the adult at rest according to size. But the average may be set down as between 5 and 8 liters. In 24 hours this would amount to from 
7,200 to II,500 liters. However, many factors lead to great variations in this volume. Vigorous exercise will increase the minute volume to I 2 to I5 or more liters per minute. Breathing a rarefied air will produce the same result, as in mountain climbing or aviation. The lack of oxygen or anoxemia augments the minute volume both by accelerating the respiratory rate and increasing the depth. Excess of carbon dioxide, or after moderate carbon monoxide poisoning, similar response is given, though the rate is more vigorously affected on breathing air rich in carbon dioxide.

The Respiratory Capacity.-The greatest respiratory capacity or vital capacity of the chest is indicated by the quantity of air which a person can expel from his lungs by a forcible expiration after the deepest possible inspiration. The vital capacity is the sum of the reserve, tidal, and complemental airs. It expresses the power which a person has of breathing in the emergencies of active exercise, violence, and disease. The average capacity of an adult, at $15.4^{\circ} \mathrm{C}$. $\left(60^{\circ} \mathrm{F}\right.$.), is about 225 to $250 \mathrm{cc}$, , or 3,500 to $4,000 \mathrm{cc}$. In healthy men, the respiratory capacity varies chiefly with the stature, weight, and age.

Circumstances Affecting the Amount of Respiratory Capacity.-John Hutchinson states that for every centimeter of height above the standard the respiratory capacity is increased, on an average, by $50 \mathrm{cc}$.

The influence of weight on the capacity of respiration is less manifest, and considerably less than that of height. It is difficult to arrive at any definite conclusions on this point, because the natural average weight of a healthy man in relation to stature has not yet been determined.

The capacity appears to be increased by age from about the fifteenth to the thirty-fifth year, at the rate of $80 \mathrm{cc}$. per year; from thirtyfive to sixty-five it diminishes at the rate of about $25 \mathrm{cc}$. per year; so that the capacity of respiration of a man sixty years old would be about $480 \mathrm{cc}$. less than that of a man forty years old, of the same height and weight.

The number of respirations in a healthy adult person usually ranges from 14 to 18 per minute. It is greater in infancy and childhood. It varies also much according to different circumstances, such as exercise or rest, health or disease, etc. Variations in the number of respirations correspond ordinarily with similar variations in the pulsations of the heart. In health the proportion is about I to 4 , or I to 5 ; and when the rapidity of the heart's action is increased, that of the chest movement is commonly increased also, but not in every case in equal proportion. It happens occasionally in disease, especially of the lungs or air-passages, that the number of respiratory acts increases in quicker proportion than the beats of the pulse; and, in other affections, much more commonly, 
that the number of the pulses is greater in proportion than that of the respirations.

The Force of Inspiratory and Expiratory Muscles.-The force which the inspiratory muscles are capable of exerting on the chest is greatest in muscular individuals of the mean height of about five feet seven or eight inches and is equal to a column of two and a half to three inches of mercury. The force manifested in the strongest expiratory acts is, on the average, one-third greater than that exercised in inspiration. But this difference is in a great measure due to the power exerted by the elastic reaction of the walls of the chest; and it is also much influenced by the disproportionate strength which the expiratory muscles attain from their being called into use for other purposes than that of simple expiration.

Within the limits of ordinary tranquil respiration the elastic resilience of the walls of the chest favors inspiration. It is only in deep inspiration that the ribs and rib cartilages offer an opposing force to dilatation. In other words, the elastic resilience of the lungs, at the end of an act of ordinary exhalation has drawn the chest walls within the limits of their normal degree of expansion. Under all circumstances, of course, the elastic tissue of the lungs opposes inspiration and favors expiration.

It is possible that the contractile power which the bronchial tubes and air-vesicles possess, by means of their muscular fibers may assist in expiration. But it is more likely that its chief purpose is to regulate and adapt, in some measure, the quantity of air admitted to the lungs, and to each part of them, according to the supply of blood. The muscular tissue contracts upon and gradually expels collections of mucus, which may have accumulated within the tubes, and which cannot be ejected by forced expiratory efforts, owing to collapse or other morbid condition of the portion of lung connected with the obstructed tubes (Gardner). Apart from any of the before-mentioned functions, the presence of muscular fiber in the walls of a hollow viscus, such as a lung, is only what might be expected from analogy with other organs. Subject as the lungs are to such great variation in size, it might be anticipated that the elastic tissue, which enters so largely into their composition, would be supplemented by the presence of much muscular fiber.

\section{RESPIRATORY CHANGES IN THE AIR BREATHED.}

Composition of the Atmosphere.-The atmosphere we breathe has, in every situation in which it has been examined in its natural state, a nearly uniform composition. It is a mixture of oxygen, nitrogen, carbon dioxide, and watery vapor, with, commonly, traces of other gases, as argon, ammonia, sulphureted hydrogen, etc. Of every too volumes of pure atmospheric air, 79 volumes, on an average, consist of nitrogen and argon, the remaining 
$2 \mathrm{I}$ of oxygen. The proportion of carbon dioxide is extremely small; 10,000 volumes of atmospheric air contain only about 4 of that gas.

The quantity of watery vapor varies greatly according to the temperature and other circumstances, but the atmosphere is never without some. In this country the average quantity of watery vapor in the atmosphere varies greatly according to the region. In some of our Western arid plains in the dry season the air is almost free of moisture.

Character and Composition of Air which has been Breathed.The changes effected by respiration in the atmospheric air are: $I$, an increase of temperature; 2, a diminution in the quantity of oxygen; 3 , an increase in the quantity of carbon dioxide; 4 , a diminution of volume; 5 , an increase in the amount of watery vapor; 6 , the addition of a minute amount of organic matter and of free ammonia.

Temperature of the Expired Air.-Expired air, after its contact with the Interior of the lungs, is hotter (at least in most climates) than the inspired air. its temperature varies between $36^{\circ}$ and $37.5^{\circ} \mathrm{C}$. $\left(97^{\circ}\right.$ and $99.5^{\circ} \mathrm{F}$.), the lower temperature being observed when the air has remained but a short time in the lungs. Whatever may be the temperature of the air when inhaled, it acquires nearly that of the blood before it is expelled from the chest.

The Oxygen of Expired Air.-Pettenkofer and Voit have found that the mean consumption of oxygen during 24 hours by a man weighing 70 kilos is about 700 grams or 490 liters. The quantity of oxygen absorbed increases with muscular exercise, and falls during rest. In general terms the quantity absorbed varies with the activity of the metabolic processes, following very closely the variation of carbon dioxide under the conditions outlined below.

The Carbon Dioxide of Expired Air.-The percentage of carbon dioxide is increased in expired air, but the total quantity of carbon dioxide exhaled in a given time is subject to change from various circumstances. From every volume of air inspired 4 to 5 per cent. of oxygen is abstracted; while a rather smaller quantity, 4.38 per cent., of carbon dioxide is added in its place. The expired air will contain, therefore, 438 volumes of carbon dioxide in ro, 000 . The total quantity of carbon dioxide exhaled into the air breathed by a healthy adult, calculating that $\mathrm{I} 5.4 \mathrm{cc}$. of the $35^{\circ} \mathrm{cc}$. of the average air exhaled at each expiration consists of carbon dioxide, and that the rate of respiration per minute is on an average 16 , would be about 400 liters in twenty-four hours. From actual experiment this amount seems to be a trifle too great, since from the average of many investigations the total amount of carbon dioxide excreted per day by the entire body has been found to be about 400 liters, weighing 800 grams, and consisting of 218 grams of carbon, and $5^{82}$ grams of oxygen. From the 218 grams of carbon must be deducted about Io grams excreted in other ways than by the lungs, which leaves about 215 grams as the amount of carbon excreted by the average healthy man by respiration each day and night. These quantities 
must be considered approximate only, inasmuch as various circumstances, even in health, influence the amount of carbon dioxide excreted, and, correlatively, the amount of oxygen absorbed.

The total amount of carbon dioxide excreted is influenced sharply by a number of factors: First, the depth and volume of respiratory movements. The greater the volume of air breathed, the greater the total output of carbon dioxide, though the percentage per unit of expired air is decreased. This influence depends upon the more efficient oxidative processes in the presence of more thorough ventilation of the lungs and blood. Second, the carbon dioxide output varies with age. It is greater with children and youth than with the old. In extreme old age the total output may not exceed that of the ten-yearold child. Third, there is a diurnal variation in carbon dioxide output. The respiratory quotient, i.e., the ratio between carbon dioxide eliminated and oxygen absorbed, is greater during the day than during the night. In the day, therefore, the carbon dioxide exhaled in relation to the oxygen absorbed is increased, and it is diminished during the night. This is probably due to the increased production of carbon dioxide as a result of increased tissue activity during the day, and, consequently, the breaking down or katabolism of more substances. Fourth, the character and quantity of the food greatly influence the proportion of carbon dioxide as indicated by the respiratory quotient. It is greater with carbohydrate foods. During fasting there is for the first two or three days an increased carbon dioxide output, but later this is decreased. Fifth, the bodily exercise, in moderation, increases the quantity of carbon dioxide ex-

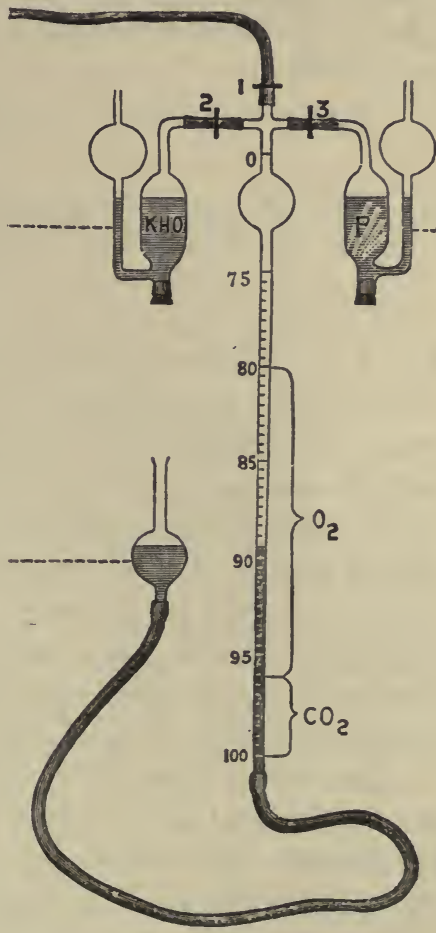

Fig. 236.-Apparatus for Est1mating $\mathrm{O}_{2}$ and $\mathrm{CO}_{2}$ in Expired Air. (Waller.) pired by at least one-third more than it is during rest. For about an hour after exercise the volume of the air expired in the minute is increased nearly $2,000 \mathrm{cc}$., or $\times 18$ cubic inches; and the quantity of carbon dioxide about I $25 \mathrm{cc}$, or 7.8 cubic inches per minute. Violent exercise, such as full labor or athletic competition, still further increases the amount of the carbon dioxide exhaled. Sixth, the observations made by Vierordt at various temperatures between $3.4^{\circ}-23.8^{\circ} \mathrm{C} .\left(3^{\circ} \mathrm{F}\right.$. and $75^{\circ} \mathrm{F}$.) show, for warm-blooded animals, that within this range every rise equal to $5 \cdot 5^{\circ} \mathrm{C}$. $\left(10^{\circ} \mathrm{F}\right.$.) causes a diminution of about $33 \mathrm{cc}$. (2 cubic inches) in the quantity of carbon dioxide exhaled per minute.

The Volume of the Respired Air is Diminished.-When allowance has been made for the expansion in heating, the volume of expired air is decreased, 
the loss being due to the fact that a portion of the oxygen absorbed is not returned in the form of carbon dioxide. Since the oxygen of a given volume of carbon dioxide would have the same volume as the carbon dioxide itself at a given temperature and pressure, a portion of the oxygen absorbed must be used for other purposes than the formation of carbon dioxide. In fact, some of it is used in the formation of urea, some in the formation of water, etc. The volume of the carbon dioxide exhaled, divided by the volume of the oxygen absorbed, gives what is known as the respiratory quotient; thus

$$
\frac{\mathrm{CO}_{2} \text { exhaled }}{\mathrm{O}_{2} \text { absorbed }}
$$

Normally in man on a mixed diet the respiratory quotient averages 0.82

$$
\frac{4.0 \text { to } 4.5}{5}=0.8 \text { to } 0.9 \text {. }
$$

But it is subject to variation through several causes; for example, through variation in the composition of the diet. On a pure carbohydrate diet the respiratory quotient will rise above 0.9 , i.e., to $\mathrm{I} .0$, since carbohydrates contain enough oxygen to oxidize the hydrogen in the molecule. On a diet containing much fat the quotient is lowest, since relatively more oxygen is needed completely to oxidize fat. The theoretical respiratory quotient for fats is 0.7 . The same is true, but to a less degree, in the case of proteins which also require much oxygen for their complete oxidation. Muscular exertion raises the respiratory quotient, because in its performance carbohydrates are used up in relatively greater quantity.

The Watery Vapor in Respired Air.-The quantity of water vapor emitted is, as a general rule, sufficient to saturate the expired air, or very nearly so. Its absolute amount is, therefore, influenced by the following circumstances: I. By the quantity of air respired; for the greater the volume of air, the greater also will be the quantity of moisture exhaled; 2 . by the quantity of water vapor contained in the air previous to its being inspired; because the greater the moisture inhaled, the less will be the amount to complete the saturation of the air; 3 . by the temperature of the expired air; for the higher the temperature the greater will be the quantity of water vapor required to saturate the air; 4 . by the length of time which each volume of inspired air is allowed to remain in the lungs; for although, during ordinary respiration, the expired air is always saturated with water vapor, yet, when respiration is performed very rapidly, the air has scarcely time to be raised to the highest temperature or be fully charged with moisture ere it is expelled.

The quantity of water exhaled from the lungs in 24 hours ranges (according to the various modifying circumstances already mentioned) from about 200 to $800 \mathrm{cc}$., the ordinary quantity being about 400 to $500 \mathrm{cc}$. Some of this is probably formed by the chemical combination of oxygen with hydro- 
gen in the system; but the far larger proportion of it is water which has been absorbed, as such, into the blood from the alimentary canal, and which is exhaled from the surface of the air-passages and cells, as it is from the free surfaces of all moist animal membranes, particularly at the high temperature of warm-blooded animals.

A small quantity of ammonia is added to the ordinary constituents of expired air. It seems probable, however, both from the fact that this substance cannot be always detected and from its minute amount when present, that the whole of it may be derived from decomposing particles of food left in the mouth or the teeth, and that it is, therefore, only an accidental constituent of expired air.

The Organic Matter in Expired Air.- It was formerly supposed that this organic matter was injurious and gave rise to the unpleasant symptoms which are experienced in badly ventilated rooms. But this has been strongly questioned so that the matter cannot be considered settled at the present time.

\section{THE RESPIRATORY CHANGES IN THE BLOOD.}

Pressure and Diffusion of the Air.-It must be remembered that the tidal air in the lungs amounts only to from 300 to $500 \mathrm{cc}$. at each inspiration. This amount at once mixes with the reserve and the residual air already in the lungs. The mixture is facilitated by the air currents set up in the deeper parts of the lungs by the sudden entrance of the tidal air; but, after all is considered, it will be found that diffusion is the greatest factor in producing a uniform mixture of the gases in the alveoli and in the air-cells of the lungs. Just as a fresh supply of oxygen introduced within the door of a closed room will quickly diffuse throughout the space of the entire room so will the fresh tidal air diffuse into the space of the lungs. When the tidal air is expired its average composition has been changed so it has only about 16 per cent. of oxygen instead of the usual 20.96 per cent. of oxygen in air. The oxygen content of the air still left in the lungs is probably somewhat less than the percentage in this expired air for the reason that the air of the respiratory tree, the trachea, bronchi, and bronchioles, is never fully mixed with the alveolar air.

The partial pressure of the oxygen of the air measured under standard conditions is $159 \mathrm{~mm}$. of mercury; that is, 20.96 per cent. of $760 \mathrm{~mm}$. of mercury, the standard pressure of one atmosphere. The partial oxygen pressure in expired air with I6 per cent. of oxygen is only I $22 \mathrm{~mm}$. of mercury. These figures show a diffusion pressure of at least $37 \mathrm{~mm}$. of mercury to carry oxygen into the deeper recesses of the lungs. The constant loss of oxygen to the blood probably keeps the mean difference greater.

The Gases of the Blood.-Turning now to the consideration of the gases of the blood in the lungs, a somewhat different picture presents itself. 
The blood consists of a fluid plasma with a mass of corpuscles floating in it. The gas analysis of the blood shows that it contains oxygen, carbon dioxide, nitrogen, and traces of other inert gases. The blood gases are measured by the method of extracting them, measuring the volume and computing the volume to standard temperature and pressure.

Numerous analyses of the blood from the arteries and veins of normal men have recently been obtained by Stadie, Harrop, and others, made possible by the development of the micro-analytical methods and apparatus introduced by Van Slyke, Fig. 237. Arterial blood obtained by puncture from the radial artery with a slender hypodermic needle and syringe have yielded on analysis the following average volumes per cent. of oxygen.

Arterial and Venous Oxygen, Total Oxygen Capacity, and Arterial and Venous Oxygen Unsaturation in Five normal Individuals (Stadie)

\begin{tabular}{|c|c|c|c|c|c|c|c|}
\hline \multirow{3}{*}{ Individual number } & \multicolumn{2}{|c|}{ Oxygen content } & \multirow{3}{*}{$\begin{array}{c}\text { Oxygen } \\
\text { capacity } \\
\text { per Ioo cc. } \\
\text { of blood }\end{array}$} & \multicolumn{4}{|c|}{ Unsaturation } \\
\hline & \multirow{2}{*}{$\begin{array}{l}\text { Arterial, } \\
\text { per Ioo cc. } \\
\text { of blood }\end{array}$} & \multirow{2}{*}{$\begin{array}{l}\text { Venous, } \\
\text { per roo cc. } \\
\text { of blood }\end{array}$} & & \multicolumn{2}{|c|}{ Arterial } & \multicolumn{2}{|c|}{ Venous } \\
\hline & & & & $\begin{array}{l}\text { Per roo cc. } \\
\text { of blood }\end{array}$ & $\begin{array}{l}\text { Per } \\
\text { cent. }\end{array}$ & $\begin{array}{l}\text { Per roo cc. } \\
\text { of blood }\end{array}$ & $\begin{array}{l}\text { Per } \\
\text { cent. }\end{array}$ \\
\hline I & 17.9 & 12.8 & I9. I & I. 2 & 6.3 & 6.3 & 33.0 \\
\hline 2 & 21.0 & 16.7 & 21.6 & 0.6 & 2.8 & 4.9 & 22.7 \\
\hline 3 & $22 . \mathrm{I}$ & 17.2 & 23.3 & I. 2 & 5.2 & $6 . \mathrm{I}$ & 26.2 \\
\hline 4 & 20.2 & 15.6 & 21.6 & I. 4 & 6.5 & 6.0 & 27.8 \\
\hline 5 & 19.5 & 15.4 & $20 \cdot 3$ & 0.8 & 3.9 & 4.9 & $24 \cdot I$ \\
\hline Mean.. & 20.2 & 15.6 & 21.2 & I.O & 5.0 & 5.6 & 26.8 \\
\hline
\end{tabular}

The amount of oxygen per unit quantity of blood varies with the concentration of hemoglobin. In blood from the radial artery the sample is under normal respiratory conditions about 93 to 97 per cent saturated, See Harrop, Table I.

The amount of carbon dioxide in the total blood averages about 40 volumes per cent. in arterial blood and 46 to 58 volumes per cent. in venous blood. Venous blood may contain as much as 65 volumes per cent. of carbon dioxide. The carbon dioxide-carrying bases are largely in the plasma and increase or decrease with variations of acid or alkali production, thereby maintaining equilibrium. The amount of nitrogen in solution in the blood follows closely its ratio of physical absorption by fluids. Saturated arterial blood contains I.52 volumes per cent. of nitrogen. Venous blood contains somewhat less, about I.36 volumes per cent. (Van Slyke and Stadie). 
For extracting the gases from the blood the older methods using the mercurial air pumps of Ludwig, Geissler, or Sprengel have given place to much simpler and more convenient microapparatus of Van Slyke, Fig. 237. The Van Slyke apparatus can be used for the analysis of oxygen, or of carbon dioxide, and of the inert residue of nitrogen by difference.

The Van Slyke Blood Gas Apparatus.-The Van Slyke apparatus consists of a $50 \mathrm{cc}$. pipette with three-way stop cocks, $e$ and $f$, at the top and bottom. The top of the pipette is graduated in I cc. and .02 cc. divisions. A . reservoir of $80 \mathrm{cc}$. capacity is connected with the bottom of the apparatus by a heavy black rubber tubing of small bore and the whole apparatus filled with mercury. The sample of blood to be analyzed is introduced through the cup $b$ into the pipette and the gases evacuated and measured according to the following technique.

The solutions required are ammonia solution to which is added the soluble saponine from 5 grams of commercial soap bark and $4 \mathrm{cc}$. concentrated ammonia per liter; redistilled caprillic alcohol to prevent foaming; and ro per cent. potassium ferricyanide

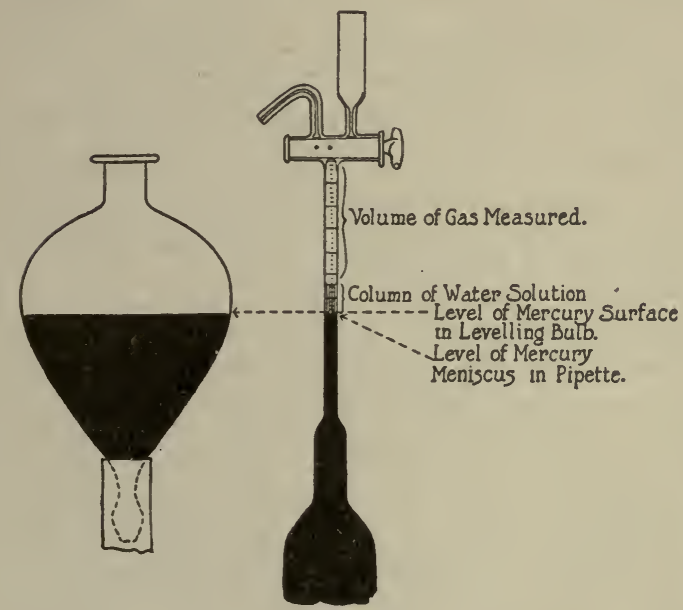

FIG. $237 a$.

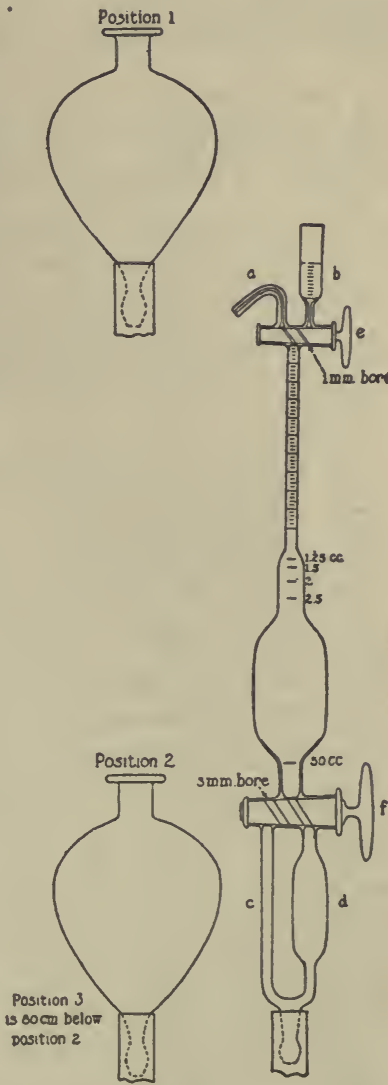

FIG. 237

in normal potassium hydrate. A determination is made by the following steps. Introduce 3 drops of caprillic alcohol and $6 \mathrm{cc}$. ammonia into the pipette, evacuate and wash out the dissolved air, run $2 \mathrm{cc}$. of air-free ammonia back up in the cup as a seal. A $2 \mathrm{cc}$. sample of fresh arterial blood drawn under oil is run from a $2 \mathrm{cc}$. graduated pipetteinto the cup under the ammonia solution and drawn down into the pipette. Mix the solutions until the blood is completely laked, which occurs in a few seconds. Next introduce $0.4 \mathrm{cc}$. of Io per cent. ferricyanide to set the oxygen free from the hemoglobin. This dissociation is facilitated by lowering the mercury to about the $50 \mathrm{cc}$. mark on the pipette thus producing a Toricellian vacuum in which the blood and reagent mixture is 
shaken vigorously for about one minute. Make a preliminary reading of the liberated gas and repeat the evacuation until the readings check. For final reading draw the fluids into the chamberd below the lower stopcock, using care not to trap any gas, and run the mercury around the side tube $c$, level the mercury bulb against the mercury meniscus in the graduated limb as in Fig. $237 b$ and read. The gas of the 2 c.c. sample of blood consists of the oxygen bound by the hemoglobin and of the air in solution at the temperature and barometer of the analysis. The corrections for dissolved air including nitrogen are readily made from tables of solubility. (See Van Slyke in Journal of Biological Chemistry, Vol. 33, p. I 26; also Vol. 49, p. r.)

The large quantity of oxygen found in arterial and in venous blood is the more striking when the facts of absorption of gases by liquids are reviewed. A liquid such as water will, when exposed to a gas, take up the gas by absorption according to definite physical laws. Under constant temperature the amount of gas absorbed, oxygen for example, varies directly as the pressure of the gas, or partial pressure if the gas is in a mixture. The oxygen absorbed by water from pure air is in direct proportion to the partial pressure of oxygen in the air, which is $159 \mathrm{~mm}$. mercury.

The amount of gas absorbed by I c.c. of water under standard pressure (one atmosphere at $\circ^{c}$ C.) is termed the absorption coefficient. The absorption of oxygen by water for one atmosphere of oxygen is .048 c.c. For blood plasma the coefficient is a little less than for water. The amount of oxygen in simple solution in 100 c.c. of blood at the partial pressure of oxygen in alveolar air is therefore only about 0.32 c.c. The actual amount of oxygen in solution in any particular specimen of plasma is rather less and is determined by the oxygen tension.

The saturation of oxygen in arterial whole blood is measured by the method of subjecting the blood to an atmosphere in which the oxygen tension is accurately known. The instrument is called a tonometer. The procedure depends upon the fact that a thin film of blood exposed to mixtures of gases in air gives up gases to or absorbs them from the air until an equilibrium is established. When a sample of whole blood is exposed to atmospheric air in a tonometer the blood becomes fully saturated with oxygen and the volume it contains is spoken of as the capacity. When such blood has its gases extracted by the Van Slyke apparatus and the results computed to standard, the volumes per cent. contained are such as indicated in the table, page 302. When alveolar airs are used the degree of saturation is of course proportionately less than the saturation against pure air because of the diminished per cent. of alveolar oxygen. The volumes per cent. of oxygen absorbed is found to vary also according to the per cent. of oxygen in the sample of air and the content of hemoglobin in the blood.

By means of the tonometer observers have measured the tension of 
blood gases. The oxygen tension has been found to be from 4 (Strassburg) to ro (Herter) per cent. of an atmosphere. Many determinations have been given of both lower and higher-percentages, but, accepting the above limits for a working average, the oxygen tension in arterial blood would be from 30.4 to $76 \mathrm{~mm}$. of mercury or more.

Blood plasma exposed to an air with a partial pressure of 30 to $76 \mathrm{~mm}$. of mercury would absorb only from 0.10 to 0.32 (0.26 c.c. Pflüger) c.c. of oxygen for 100 c.c. of blood. As a matter of fact Ioo c.c. of whole

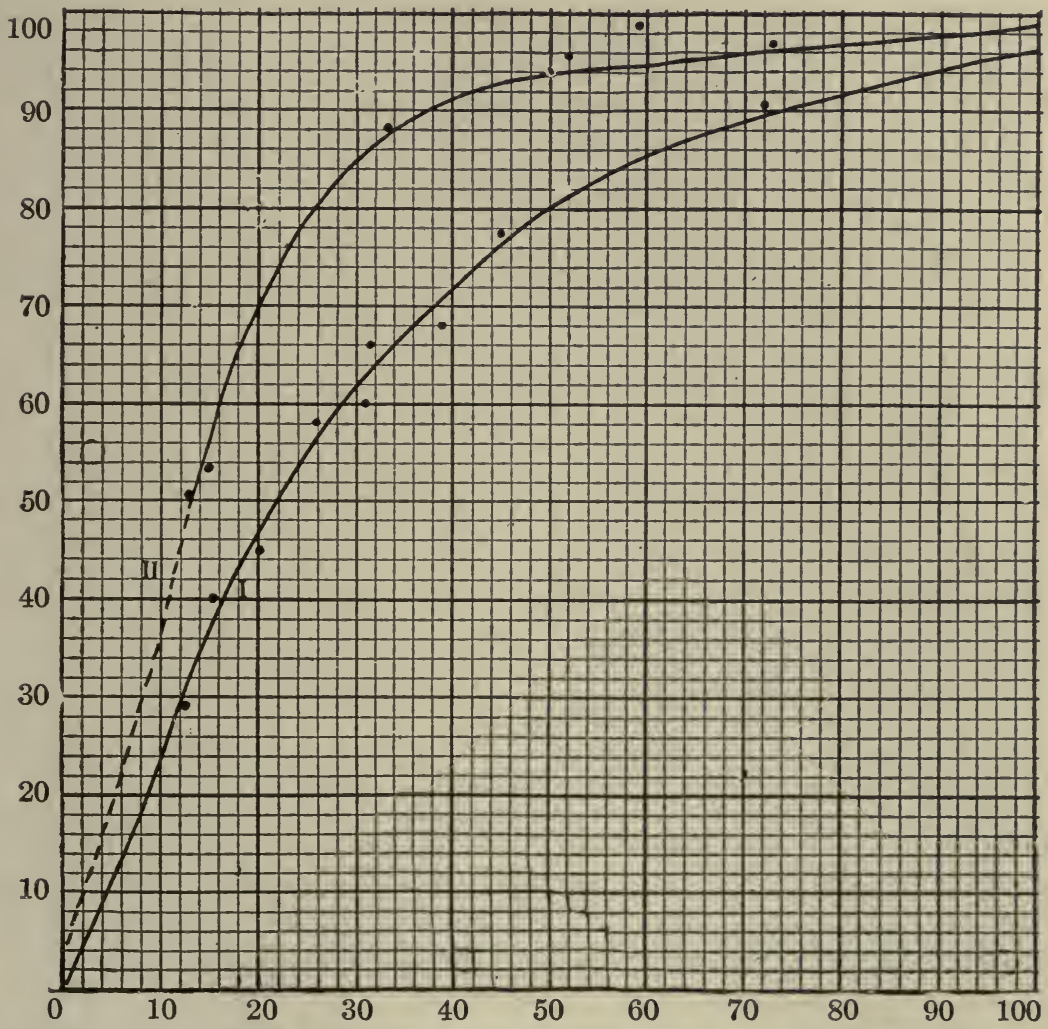

FIG. 238.-Dissociation curves of oxyhemoglobin. The figures along the ordinates represent percentages of saturation of hemoglobin by oxygen. The figures along the abscissæ represent $\mathrm{mm}$. of oxygen pressure in mercury.

I. Bohr's dissociation curve of oxyhemoglobin dissolved in water.

II. Dissociation curve of oxyhemoglobin dissolved in Ringer's Solution. (After Barcroft and Camis.)

blood has a capacity of an average of from 18.5 c.c. (the Haldane standard) to 22.6 c.c. or more of oxygen. It is evident that blood carries far more oxygen than can be held in simple solution. The red blood corpuscles carry their enormous excess of oxygen by virtue of the special respiratory pigment, hemoglobin. 
Combining Power of Hemoglobin with Oxygen.-One hundred cubic centimeters of blood contain about $\mathrm{I}_{4}$ grams of hemoglobin, page I37. Each gram of hemoglobin, when fully saturated with oxygen, according to Hüfner's earlier determination, combines with $\mathrm{r} .56 \mathrm{cc}$. of oxygen. By later work he gets the determination of $\mathrm{I} .34 \mathrm{cc}$. for hemoglobin of ox blood. This last figure indicates that the combining power of the hemoglobin is dependent upon the iron in the molecule, in which one atom of iron combines with one atom of oxygen. A number of investigators have

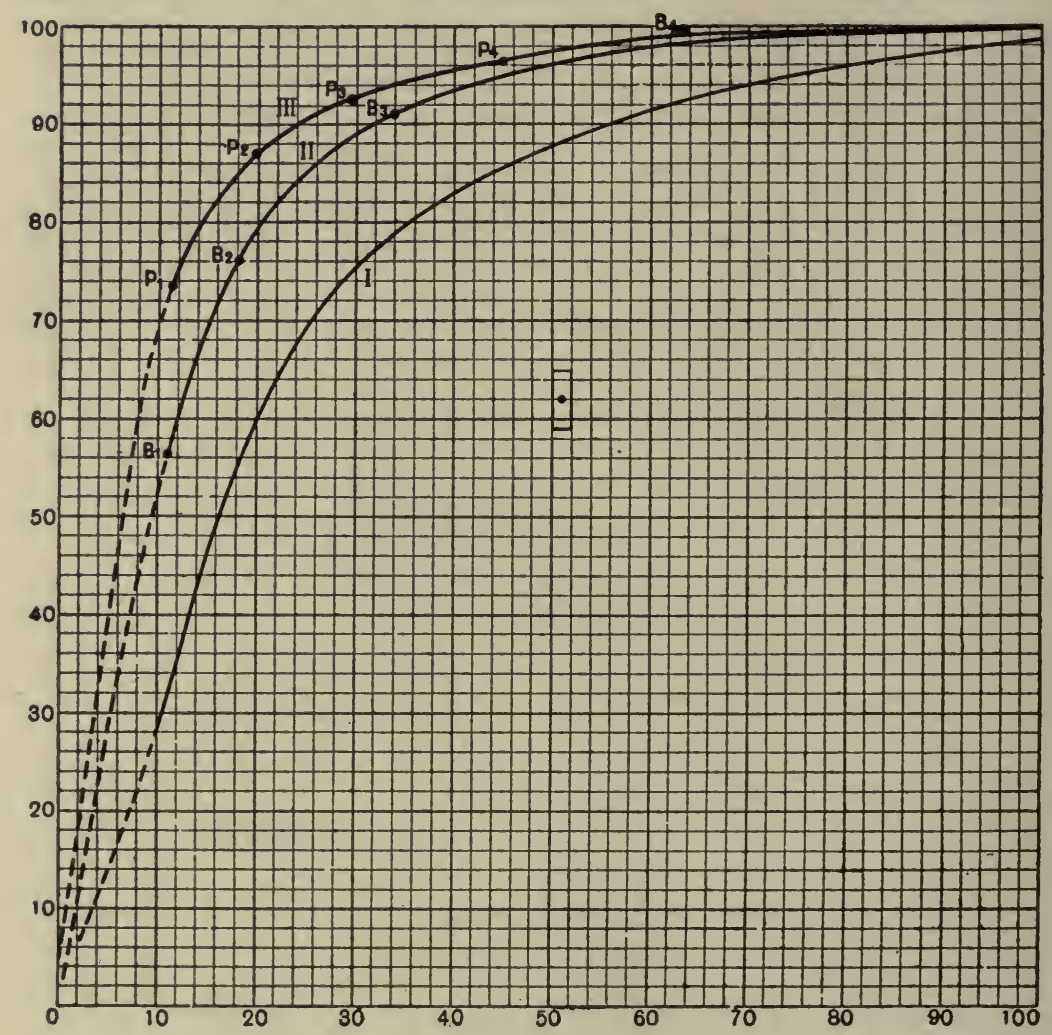

FIG. 239.-Dissociation curves of oxyhemoglobin in: I, 0.7 per cent. sodium chloride; II, in sodium bicarbonate, and III, in disodium phosphate. The figures along the ordinates represent percentages of saturation of hemoglobin by oxygen. The figures along the abscissæ represent $\mathrm{mm}$. of oxygen pressure in mercury. (Barcroft and Camis.)

examined the conditions under which hemoglobin combines with oxygenHüfner, Bohr, Löwy, and Barcroft and Camis. Hüfner, working with purified hemoglobin in watery solution, found that when the oxygen tension in the air in contact with the hemoglobin was increased above zero by graded stages, the amount of oxygen that was combined was very great per unit of increased pressure at the low pressures, but relatively less at the 
higher pressures. Or, which amounts to the same, if hemoglobin saturated with oxygen be subjected to decreasing oxygen pressure, it sets free the combined oxygen, at first slowly, then more rapidly. By consulting the typical curve showing this relation, it will be evident that the critical partial oxygen pressures influencing this combination fall at about 30 to $35 \mathrm{~mm}$. mercury of oxygen tension and below. See figure 238 .

A number of factors influence the dissociation of oxygen from hemoglobin at a given oxygen tension. Of prime importance is the influence of the presence of carbon dioxide gas as shown by Bohr and confirmed by Barcroft and Camis. With an increase in the tension of carbon dioxide there is a decrease in the fixation of oxygen.

Table Showing the Dissociation of Oxhemoglobin in the Presence of Varying Tensions of Carbon Dioxide. (Barcroft and Camis.)

\begin{tabular}{|c|c|c|c|c|c|c|c|c|c|c|c|c|c|}
\hline \multirow{2}{*}{$\begin{array}{c}\text { Tension of car- } \\
\text { bon dioxide }\end{array}$} & \multicolumn{13}{|c|}{$\begin{array}{c}\text { Tensions of oxygen in mm. mercury and the per cent. of saturation } \\
\text { of the hemaglobin at each pressure }\end{array}$} \\
\hline & ro & I 5 & 20 & 25 & 30 & 35 & 40 & 45 & 50 & 60 & 70 & 80 & 100 \\
\hline $5 \mathrm{~mm} . \mathrm{CO}_{2}$ & 28 & 35 & 47 & $5^{8} .5$ & 70 & $8 x$ & 89 & 93 & 95 & 96 & 98 & 99 & \\
\hline IO $\mathrm{mm} . \mathrm{CO}_{2}$ & II & 26 & 38.5 & $5 I$ & $6_{3}$ & $74 \cdot 5$ & 83 & 88 & 91.5 & 94.5 & 96.5 & 97.5 & \\
\hline $20 \mathrm{~mm} ; \mathrm{CO}_{2}$ & $\circ$ & 10 & 25 & 49 & 53.8 & $65 \cdot 5$ & 74.5 & 80.5 & 84.5 & 90 & 93 & 95 & 97.5 \\
\hline $40 \mathrm{~mm} . \mathrm{CO}_{2}$ & $\circ$ & $\circ$ & II & 26 & $42 \cdot 5$ & 56 & 65 & 72 & 77 & $83 \cdot 5$ & 88.5 & 93 & 95.5 \\
\hline $80 \mathrm{~mm} . \mathrm{CO}_{2}$ & $\circ$ & $\circ$ & I & 12.5 & $3 I$ & $45 \cdot 5$ & 56.5 & 64 & 69.5 & 77 & 83 & 87.5 & 92.5 \\
\hline
\end{tabular}

The salts of the blood also influence the oxygen fixation by hemoglobin under a given tension as indicated in the following table:

Table Showing the Influence of the Presence of Different Salts on the Percentage of Saturation of Hemoglobin Under a Constant Oxygen Tension of 30 mm. Mercury.

(BARCROFT AND CAMIS.)

r. Hemoglobin in water dissociation. ..........6 62 per cent.

2. Hemoglobin in 0.7 per cent. $\mathrm{NaCl}$ dissociation... 75 per cent.

3. Hemoglobin in Ringer's solution dissociation..... 85 per cent.

4. Hemoglobin in $\mathrm{NaHCO}_{3}$ solution dissociation.... 89 per cent.

5. Hemoglobin in 0.9 per cent. $\mathrm{KCl}$ dissociation..... 9 9 per cent.

6. Hemoglobin in $\mathrm{Na}_{2} \mathrm{HPO}_{4}$ solution dissociation... 93 per cent.

Barcroft and Camis find that the dissociation curve also varies in the blood of different animals. Strassburg gives the oxygen tension of arterial blood as $29.64 \mathrm{~mm}$. of mercury, and for venous blood $22.04 \mathrm{~mm}$. of mercury. That is to say, during the brief interval in which the blood is in the pulmonary capillaries the oxygen tension has increased by $7.6 \mathrm{~mm}$. of mercury, an increase of tension which would produce very little increase in simple absorption of oxygen. Yet it is sufficient to cause fixation of from four to five volumes per cent. of oxygen by the hemoglobin.

It is evident that there will be diffusion of oxygen from the high tension 
toward the lower and in the direction indicated by the arrows in the table below. As fast as the oxygen diffuses into the venous blood, thus tending to raise the pressure of the gas in solution, it is taken up and fixed by the hemoglobin. This process proceeds far enough during the interval the blood is in the pulmonary capillaries to raise the oxygen tension from $22.04 \mathrm{~mm}$. of mercury to $29.64 \mathrm{~mm}$. of mercury, and also far enough to permit of the fixation of from four to five volumes per cent. of oxygen. The oxygen diffusion pressures are indicated as follows:

Oxygen pressure in the atmosphere 2 I per cent. or $159 \mathrm{~mm}$. of mercury

Oxygen pressure in the alveolar air 16 per cent. or $122 \mathrm{~mm}$. of mercury

Oxygen pressure in the venous blood 3 per cent. or $22.04 \mathrm{~mm}$. of mercury

Liberation of Oxygen in the Tissue Capillaries. - When the arterial blood reaches the capillaries of the tissues, then the situation which we have just found holding good in the lungs is reversed. As rapidly as the oxygen reaches the living protoplasm of the tissues it enters into fixed combination, thus rendering it inert. The oxygen tension in the tissue cells will, therefore, be zero. Under these conditions the difference in pressure level between the oxygen tension in the blood and that in the tissues is sufficient to cause a rapid diffusion of oxygen through the capillary walls with corresponding liberation of the oxygen from the hemoglobin according to the laws of combination given in the curves above. The total effect of this process is to maintain a relatively high and constant diffusion pressure of the oxygen in the blood. During the time the blood remains in the capillaries the total oxygen tension will have been lowered from 29.64 to $22.04 \mathrm{~mm}$. of mercury, yet this slight lowering of tension is sufficient to liberate from four to five volumes per cent: of oxygen. This figure, of course, is comparative. In many of the very active tissues, such as in muscle, a much larger per cent. of oxygen will have been dissociated and the oxygen tension correspondingly lowered so that the venous blood returning through such an active organ may not have more than half the average amount of oxygen found in venous blood.

Considering the pressure relations of oxygen from the time of its introduction into the body with the fresh air to its fixation in the tissues we have the following schema:

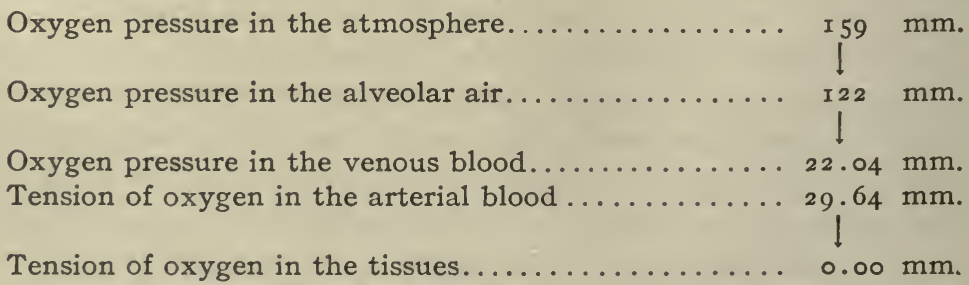


Elimination of Carbon Dioxide by the Blood and the Respiratory Apparatus.-The principles of absorption of gas by liquids discussed in the preceding pages apply equally well for carbon dioxide with the exception that carbon dioxide is about three times as soluble in blood as is oxygen. The carbon dioxide results from the oxidative processes going on in the tissues, and this gas is present in large quantities in the tissues and their immediately surrounding lymph. An analysis of the carbon-dioxide content of venous blood reveals the presence of about $45 \mathrm{cc}$. of the gas in Ioo cc. of blood. This gas, like oxygen, is held in such large quantity by virtue of the fact that it forms loose chemical combinations in the blood. Of the total quantity not more than 5 per cent. is held in simple solution. From to to I5 per cent. of the total volume is found in firm combination in such forms as carbonates, bicarbonates, etc. The remaining 85 and more volumes per cent. is held in loose chemical combination, a combination which is broken up under the same conditions of variation in carbon-dioxide tension as were found to exist for oxygen in combination with hemoglobin. In the case of carbon dioxide an analysis of plasma reveals the fact that the gas is in combination with some compound of the plasma, probably a protein. In fact, there is some evidence to show that carbon dioxide combines with the globulin group. Carbon dioxide also forms loose chemical compounds with the constituents of the red corpuscles, probably with the protein portion of the hemoglobin molecule. The pressure relations of this gas as regards its diffusion in the process of elimination are shown in the following table:

Carbon-dioxide tension in the tissues..... $5^{8} \mathrm{~mm}$. of mercury

Carbon-dioxide tension in the venous blood $45 \mathrm{~mm}$. of mercury

Carbon-dioxide tension in the alveolar air. . 23 to $38 \mathrm{~mm}$. of mercury

Carbon-dioxide tension in the expired air... $5.8 \mathrm{~mm}$. of mercury

Theories of Interchange of Gases in the Lungs and in the Tissues.The above discussion is on the basis of the mechanical interpretation of the transfer of gases in the lungs and in the tissues. By this theory it is assumed that the oxygen passes from the air in the lungs through the moist pulmonary membrane of the alveoli through the capillary walls and into the blood plasma, obeying the physical laws of gas diffusion. Likewise in the tissues this theory presupposes that the difference in the mechanical tension in the capillary blood plasma, the lymph, and the living tissue will lead to diffusion of the oxygen in the direction of lowest pressure, i.e., toward the tissues.

Some facts have indicated that we cannot account for the transference of oxygen by the purely mechanical theory. The idea has been advanced that the living epithelial wall of the lung, as well as that of the capillaries, exerts a distinct influence on the passage of oxygen of such nature that it 
might be regarded as a secretion of this gas. This theory finds some support in that a distinct secretion of oxygen in the air bladders of certain fishes has been proven by Bohr. The theory apparently does not apply to mammals.

\section{THE NERVOUS REGULATION OF THE RESPIRATORY APPARATUS.}

Respiratory movement is essentially an involuntary act. Unless this were the case, life would be in constant danger, and would cease on the loss of conciousness for a few moments, as in sleep. It is, however, of advantage to the body that co-ordination of respiratory movements should be to some extent under the control of the will. For, were it not so, it would be impossible to perform those voluntary respiratory acts, such as speaking, singing, and the like.

The Respiratory Nerve Center. - It has been known for centuries that there exists a region of the central nervous system on the destruction of which both respiration and life cease. Flourens, I842, after many series of experiments as to the exact position of what he called the "knot of life" (naud vital), placed it in the floor of the fourth ventricle, at the point of the $\mathrm{V}$ in the gray matter at the lower end of the calamus scriptorius; a district of considerable size, some $5 \mathrm{~mm}$. in extent on each side of the middle line. Observers subsequent to Flourens have attempted to show that the chief respiratory center, on the one hand, is situated higher up in the nervous system, in the floor of the third ventricle (Christiani), or in the corpora quadrigemina (Martin and Booker, Christiani, and Stanier), or lower down in the spinal cord. The balance of experimental evidence, however, is to prove that the sole centers for respiration are in a limited district in the medulla oblongata in close connection with the vagus nucleus on each side. They are approximately identical in location. The destruction of this region stops respiration. If the center be left in connection with the muscles of respiration by their nerves, although the remainder of the central nervous system be separated from it, respiration continues. It may be considered almost certain that the medullary center is the only true respiratory center. Langendorff states that in newly born animals in which the medulla has been immediately cut across at a level a few millimeters below the point of the calamus scriptorius, respiration continues for some time, but this is questionable. Normal respiration does not occur after separation of the bulb from the cord, and the so-called respiratory movements noticed by Langendorff are merely tetanic contractions of the respiratory muscles in which often enough other muscles take part.

The action of the medullary center is to send out impulses during inspiration, which cause contractions of the inspiratory muscles- $a$, of the nostrils and jaws, through the facial and inferior division of the fifth nerves; $b$, of the glottis, chiefly through the inferior laryngeal branches of the vagi; $c$ 
of the intercostal and other muscles which produce raising of the ribs, chiefly through the intercostal nerves, and $d$, of the diaphragm, through the phrenic nerves. If any one of these sets of nerves be divided, respiratory movements of the corresponding muscles cease. Similarly it may be supposed that the center sends out impulses to certain other muscles during expiration.

It has been suggested, however, that the center is double, that it is made up of inspiratory cells which are constantly in action, and of an expiratory group of cells which act less generally, inasmuch as ordinary tranquil expiration is seldom more than an elastic recoil, and not a muscular act to any marked degree.

The respiratory center is also bilateral, as has been proven by the method of antero-posterior section of the medulla. The tracts from each half of the center are separate and distinct. If the cervical cord be split into a right and left half, and one side sectioned at the level of the second cervical vertebra, then the respiratory movements of that side of the diaphragm cease while on the opposide side they continue their rhythm.

Assuming this view of the quadruple nature of the respiratory centers to be correct, there is some difference of opinion as to the exact working of the mechanism in its reactions. It is thought that the center may act automatically, but normally its automatic discharges of nerve impulses are

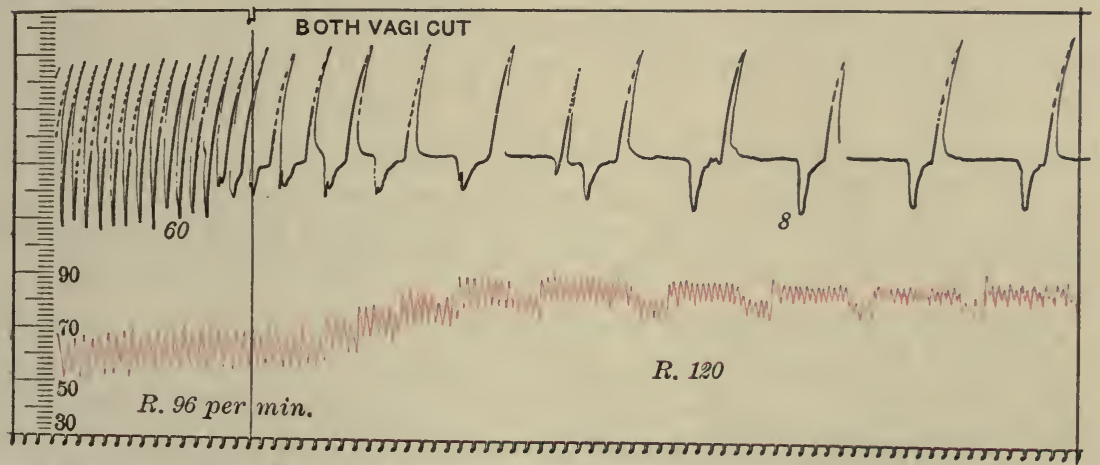

FIG. 240.-The Effect on the Respiratory Rate of Cutting Both Vagi in the Dog. The rate of 60 respirations per minute before the section of the nerves drops to 8 per minute afterward. The arterial blood-pressure is also shown, the pressure in mm. mercury is shown in the scale to the left. Compare with figure 182 .

influenced by afferent impulses from the periphery, as well as by impulses passing down from the cerebrum. The center is, in other words, both automatic and reflex. It will be simplest to discuss its reflex function first.

Action of Afferent Stimuli on the Respiratory Rhythm.-Action of the vagi. If both vagi be divided in the neck, the respirations become much slower and deeper, figure 240. This may be the case, but to a less marked degree, if one of the nerves is divided instead of both. If the central end of the divided nerve be stimulated with a weak but properly adjusted 
strength of interrupted current, the effect is to quicken the respirations. And if the stimuli are properly regulated the normal rhythm of respiration may be approached. If the stimuli be repeated with stronger currents, the breathing is brought to a standstill, sometimes at the height of inspiration, by tetanus of the diaphragm. Usually, however, stimulation of the central end of the divided vagus produces still greater slowing than that which follows the division, so that the respirations cease with the diaphragm in a condition of complete relaxation, figures 205 and $24 \mathrm{r}$.

The sensory action of the vagus may therefore be to call forth either inspiration or expiration - the impulses passing up the vagi being factors for the production and regulation of the normal variations in respiratory rhythm. The fibers of the vagus are stimulated under the following circumstances: one set of fibers, those which tend to inhibit expiration and to stimulate inspiration, are stimulated at their origin in the lung when the lung tissue is under least tension, i.e., in a condition of expiration. The fibers which tend to inhibit inspiration and to promote expiration are stimulated when the lung is fully expanded. The afferent impulses by this view are the results of mechanical stimulation, and do not depend upon the chemical nature of the gases within the pulmonary alveoli.

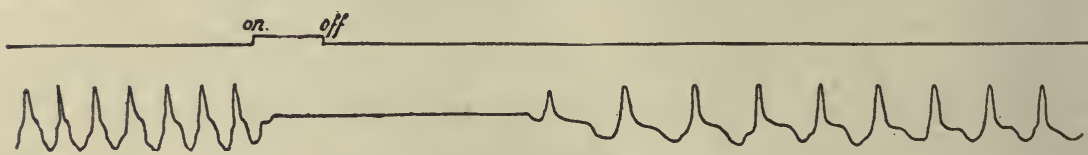

Fig. 24r. - The Effect of Stimulating the Vagus on Respiratory Rate. The stimulus was applied between the points "on" and "off." The inhibition lasts some seconds after the stimulus is removed. Time in seconds. The intra-tracheal pressure is recorded

The Respiratory Action of the Superior Laryngeal Nerves.-If the superior laryngeal branch of the vagus be divided, which usually produces no apparent effect, and the central end be stimulated, the reaction is very constant, respirations are slowed, and there is a distinct tendency toward expiration, as shown by the contractions of the abdominal muscles. Thus the superior laryngeal fibers inhibit inspiration and stimulate expiration, while the deep branch of the vagus contains fibers which stimulate inspiration and inhibit expiration.

The superior laryngeal nerves are true expiratory nerves, and are normally set in action when the mucous membrane of the larynx is irritated. They are not in constant action like the vagi.

Action of the Glosso-pharyngeal Nerves.-It has been ascertained, by the researches of Marckwald, that while division of the glosso-pharyngeal nerves produces no effect upon respiration, stimulation causes inhibition of inspiration for a short period. This action accounts for the very necessary 
cessation of breathing during swallowing. The effect of the stimulation is only temporary, and is followed by normal breathing movements.

Action of Other Sensory Nerves.-The respiratory center is influenced strongly by afferent nerve impulses having their origin in general sensory nerves, particularly the nerves of the skin. Cold water suddenly dashed on the skin is followed by a deep inspiration. Stimulation of the splanchnics or of the abdominal branches of the vagi produces expiration. Stimulation of the isolated sciatic nerve of the dog or of the rabbit causes a marked acceleration both of the rate and of the amplitude of the respiratory movements, see figure $246 \mathrm{~b}$. This acceleration is due to afferent impulses which reach the respiratory center in the medulla over sensory paths, paths which are not necessarily special respiratory afferent paths, but rather are general afferent paths which affect the respiratory center through their numerous collaterals in the brain stem.

It must be remembered that, although many sensory nerves may on stimulation be made to produce an effect upon the respiratory center, yet there is no evidence to show that any one of them, except the vagus, is constantly in action. The vagi indeed are, as far as we know, the normal regulators of respiratory movements, yet it is possible reflexly to influence the respiratory rate and depth through impulses that may have their origin in any sensory part of the body.

The respiratory center is also influenced by nerve activity of the cerebral cortex, psychic activity. This is illustrated by the limited voluntary control of the respiratory movements.

Automatic Action of the Respiratory Centers.-Although it has been very definitely proved that the respiratory centers may be affected by afferent stimuli, and particularly by those reaching them through the vagi, there is reason for believing that the center is capable of sending out efferent nerve impulses to the respiratory muscles without the action of any afferent stimuli. Thus, if the brain be removed above the bulb, respiration continues. If the spinal cord be divided immediately below the bulb, the facial and laryngeal respiratory movements continue, although no afferent impulses can reach the center except through the cranial sensory nerves, and these indeed may be divided without producing any effect, when the bulb and cord are intact. As has been shown, too, respiration continues when the vagi are divided. Isolation of the respiratory center from its sensory relations does not destroy respiratory movements so long as the motor paths through the phrenic nerves are intact. All of these experiments render it highly probable that afferent impulses are not required in order that the respiratory centers should send out efferent impulses to the respiratory muscles. The center, then, is automatic.

Method of Automatic Stimulation of the Respiratory Center.-The respiratory center is capable of working automatically apart from afferent 
impulses, and this fact has been explained by the supposition that it is stimulated to action by the condition of the blood circulating through it. When the blood becomes more and more venous the action of the center becomes more and more energetic, and if the air is prevented from entering the chest, the respiration in a short time becomes very labored. If the aëration of the blood is much interfered with, not only are the ordinary respiratory muscles employed, but also those muscles of extraordinary inspiration and expiration which have been previously enumerated. Thus, as the blood becomes more and more venous, and by venous we mean that the blood contains a relatively large amount of carbon dioxide and a diminished amount of oxygen, the action of the medullary center becomes more and more profound. The question has been much debated as to what quality of the venous blood it is which causes this increased activity; whether it is its deficiency of oxygen or its excess of carbon dioxide. It has been answered to some extent by experiments which offer no obstruction to the exit of carbon dioxide, as when an animal is placed in an atmosphere of nitrogen. Under these conditions dyspnea occurs, showing that blood which contains a diminished amount of oxygen stimulates the cells of the respiratory center. On the other hand, if the normal amount of oxygen is supplied while the carbon dioxide of the blood is prevented from escaping and thus allowed to accumulate in the blood, there is also a great increase in the respiratory activity of the center; an excess of carbon dioxide in the blood, flowing through the respiratory center, stimulates the cells to greater activity. It is highly probable, therefore, that the respiratory centers may be stimulated to action both by the absence of sufficient oxygen in the blood circulating in it, and by the presence of an excess of carbon dioxide.

These facts are particularly well supported by the experiments of Zuntz who varied the oxygen and the carbon-dioxide content of the air breathed, and measured the volume breathed per minute. When the oxygen of the air breathed was reduced by 10 to 50 per cent., the air breathed was increased only slightly, 5 to ro per cent. When the oxygen of the air was reduced by 60 per cent., the volume of air breathed was increased 30 to 40 per cent., and even more. Other observations show us that the oxygen in the blood in these experiments will fall in much less per cent. than the reduction in the oxygen of the air would lead us to suspect.

When Zuntz kept the oxygen content of the air about constant, but increased the carbon-dioxide content, then the amcunt of air breathed was greatly increased. Air containing I 8.4 per cent. of oxygen and I I .5 per cent. of carbon dioxide caused an increase in the amount breathed per minute from 7.5 liters to 32.5 liters. These experiments indicate that within the limits of its normal variations in blood the carbon dioxide has a much greater influence than oxygen on the irritability of the cells of the respiratory center.

But this is not all, since it has been observed by Marckwald that the 
medullary center is capable of acting for some time in the absence of any circulation and after excessive bleeding. The view taken by this author with regard to the action of the center is as follows: The respiratory center is set to act by the condition of its metabolism, much in the same way as the heart is set to beat rhythmically. When anabolism is completed, katabolism or discharge occurs, and this alternate but crude and spasmodic action will occur without a definite blood supply so long as the centers are properly nourished and stimulated by their own intercellular fluid.

It is also apparent that the respiratory center is dependent on the character of the blood supply, both as regards quantity and quality of the blood. It has been shown that the presence in the blood of the products of vigorous muscular metabolism will greatly increase the irritability of the respiratory center, even if the blood itself be not particularly venous in character as regards its gaseous content.

The Establishment of Respiratory Movements at Birth.-From the preceding paragraph it appears that the regulation of the respiratory movements is normally due to the automaticity of the respiratory center as influenced, first, by the blood flowing through it and, second, by the afferent nerve impulses which reach the center. The fetus in the womb is supplied by arterial blood from the blood vessels of the mother. The fetus does not ordinarily give respiratory movements before birth, but it may be made to do so by experimental procedure. At birth the placental circulation is suddenly interrupted, and the blood rapidly increases in venosity until the skin, lips, and mucous membranes are very cyanotic in appearance. It is at this time that the respiratory center begins its rhythmic discharges, being aroused by the direct stimulating effects of the great excess of carbon dioxide in the strongly venous blood. The irritability of the respiratory center is also increased by the stimulation of the skin by the air, the contact with clothing, and by the hands of the nurse. We have already seen that cutaneous stimulation leads to an increase in both respiratory rate and amplitude even in the adult, a reaction that is more pronounced in the child. The primary stimulus for the establishment of the respiratory rhythm at first, then, is the venosity of the blood, but this cause is supported by the afferent cutaneous impulses producing reflexes through the respiratory center.

Certain Special Types of Respiration.-Whatever the exact quality of the venous blood which excites the respiratory center to produce normal respirations, there can be no doubt that, as the blood becomes more and more venous from obstruction to the entrance of air into the lungs or from the blood not taking up from the air its usual supply of oxygen, the respiratory center becomes either less or more active and excitable. Conditions ensue which have received the names Apnea, diminished breathing; Hyperpnea, excessive breathing; Dyspnea, difficult breathing; and Asphyxia, suffocation. 
A pnea.-This is a condition of diminished respiratory movement. When we take several deep inspirations in rapid succession by voluntary effort, we find that we can do without breathing for a much longer time than usual; in other words, several rapid respirations seem to inhibit for a time normal respiratory movements. The reason for this partial cessation of respiration, or apnea, is not that we overcharge our blood with oxygen, as was once thought, for Hering has shown that animals in a condition of apnea may have less oxygen in their blood than in a normal state, although the carbon dioxide is less. It is probable that the cause of apnea is that by rapid inflations of the lungs impulses pass up by the vagi by means of which inspiration is after a while inhibited; or that by the repeated stimulation of the center by vagus impulses which result in rapid respiratory movements, anabolism is at last arrested. Apnea is with difficulty produced, if at all, when the vagi are divided.

Asphyxia.-The condition of stress in the respiratory apparatus brought about by insufficient respiratory activity leads to a condition of asphyxia. Progressive asphyxiation may be brought on by anything which interferes with the normal interchange of the respiratory gases of the blood.

Asphyxia may be produced by the prevention of the due entry of oxygen into the blood, either by direct obstruction of the trachea or other part of the respiratory passages, or by introducing instead of ordinary air a gas devoid of oxygen, or by interference with the due interchange of gases between the air and the blood.

The respiratory symptoms of progressive asphyxiation may be divided into three groups, which correspond with the stages of the condition most readily recognized; these are: $x$, the stage of exaggerated breathing, hyperpnea; 2 , the stage of convulsions, dyspnea; 3 , the stage of exhaustion, asphyxiation.

In the first stage the breathing becomes more rapid and at the same time deeper than usual, the inspirations at first being especially exaggerated and prolonged. This is soon followed by a similar increase in the expiratory efforts being aided by the muscles of extraordinary expiration. This stage is usually called hyperpnea. Hyperpnea soon passes into a condition of labored breathing in which there is marked increase of the force of the expiratory as well as of the inspiratory act, a condition described as dyspnea. All the muscles capable of aiding either directly or indirectly in respiration are ultimately brought into action. These respiratory convulsions are followed by rather sudden onset of paralysis of the respiratory center and ultimate death.

The conditions of the vascular system in asphyxia are: I, more or less interference with the passage of the blood through the systemic and the pulmonary blood vessels; 2, accumulation of blood in the right side of the heart and in the systemic veins; 3 , circulation of impure (non-aërated) blood in all parts of the body, especially through the respiratory center; 4 , great 
slowing of the heart by stimulation of the vagus center from lack of oxygen.

It must be kept clearly in mind that the respiratory changes just described as characteristic of asphyxiation are the secondary results of the primary general tissue asphyxiation. If an animal is deprived of its income of oxygen, and its ability to eliminate the product of oxidation, carbon dioxide, the nutritive balance around the peripheral tissues is immediately disturbed. The result is that tissue metabolism such as occurs is deranged. If oxidations are incomplete, intermediary products accumulate and on the whole the physiological life of the tissue is rapidly blocked. The average organ of the human body can endure only a certain degree of asphyxiation before changes occur which destroy, in whole or in part, the protoplasmic organization. Of all the tissues the nervous tissues are most susceptible to asphyxiation. The generalized tissues, the epidermis, connective tissue, etc., are most resistant. Life is jeopardized by injury to the weakest point, hence the body as a whole will not recover from complete asphyxiation which endures for a time greater than that which the nervous tissues will withstand. Stewart, Guthrie, Burns and Pike have set the limits very low for this tissue, from 7 to 16.5 minutes. However, incomplete asphyxiation is a condition difficult to determine, and the less complete the asphyxiation, the greater the probability of recovering the normal tissue activity. In all those conditions of life in which accidental asphyxiation occurs, it must be assumed that we are dealing with one of partial asphyxiation, especially in all efforts at resuscitation.

Cheyne-Stokes' breathing is a rhythmical irregularity in respirations which has been observed in various diseases. Respirations occur in groups. At the beginning of each group the inspirations are very shallow, but each successive breath is deeper than the preceding, until a climax is reached. The inspirations then become less and less deep, until they cease altogether for a time, after which the cycle is repeated. This phenomenon appears to be due to the want of action of some of the usual cerebral influences which pass to, and regulate the discharges of, the respiratory center.

Effects of Vitiated Air.-Ventilation.-As the air expired from the lungs contains a large proportion of carbon dioxide and a minute amount of organic matter, it is obvious that if the same air be breathed again and again, the proportion of carbon dioxide and organic matter in it will constantly increase till it becomes unfit to breathe; long before this point is reached, however, sensations of uneasiness occur, such as headache, languor, and a sense of oppression. It is a remarkable fact, however, that the organism after a time adapts itself to a very vitiated atmosphere, and that a person soon comes to breathe, without sensible inconvenience, an atmosphere which, when he first enters it, feels intolerable. Such an adaptation, however, can take place only at the expense of a depression of all the vital functions, which 
must be injurious if long-continued or often repeated. This power of adaptation is well illustrated by an experiment of Claude Bernard. If a sparrow is placed under a bell-glass of such size that it will live for three hours, be taken out at the end of the second hour (when it could have survived another hour), and a fresh healthy sparrow introduced, the latter will die at once.

It must be evident that provision for a constant and plentiful supply of fresh air, and the removal of that which is vitiated, are of greater importance than the actual cubic space per person of occupants. Not less than 2,000 cubic feet per individual should be allowed in sleeping apartments (barracks, hospitals, etc.), and with this allowance the air can be maintained at the proper standard of purity only by such a system of ventilation as provides for the supply of $I, 500$ to 2,000 cubic feet of fresh air per person per hour.

Effects of Breathing Gases Other than the Atmosphere.-Asphyxiation is produced by the direct poisonous action of such gases as carbon monoxide, which is contained to a considerable amount in common coal gas. The fatal effects often produced by this gas (as accidents from burning charcoal stoves in small, close rooms) are due to its entering into combinations with the hemoglobin of the blood corpuscles and thus preventing the formation of oxyhemoglobin because of the more stable carbon-monoxide hemoglobin. The partial pressure of oxygen in the atmosphere may be considerably increased without much effect in displacing the carbon monoxide, hence this is rapidly fatal when breathed. Hydrogen may take the place of nitrogen with no marked ill effect, if the oxygen is in the usual proportions. Su phureted hydrogen destroys the hemoglobin of blood and thus produces oxygen starvation. Nitrous oxide acts directly on the nervous system as a narcotic, and may also form a compound with hemoglobin. Certain gases, such as carbon dioxide in more than a certain proportion, sulphurous acid gases, ammonia, and chlorine, produce spasmodic closure of the glottis and prevent respiration.

Alteration in the Atmospheric Pressure.-Lower barometric pressures than the normal occur in high altitudes, for example in mountain climbing or in aërial navigation. The susceptibility to decrease in barometric pressure varies in different individuals. At an altitude of about I0,000 feet many persons begin to experience mountain sickness, though most individuals are not so affected until they ascend to $\mathrm{I}_{5}, 000$ feet or more. The symptoms that develop are nausea, dizziness, palpitation of the heart, headache, and muscular weakness. The oxygen partial pressure of the atmosphere is reduced to half at about 15,000 feet elevation. At this pressure the body begins to show some stress from inability to get an adequate quantity of oxygen. The tension of the oxygen in the alveolar air is not great enough, see figure 238 showing the relation of the partial pressure of oxygen and the percentage of hemoglobin saturation, 
to allow the blood of the pulmonary capillaries to combine with its usual quantity of oxygen. There is enough oxygen absorbed, however, to satisfy the amount used by the tissues under ordinary circumstances. It is only when an extra amount of activity is called for that stress is observed at this level. At still greater altitudes the oxygen of the arterial blood is further reduced until a level is reached at which the total amount of oxygen absorbed by the pulmonary blood is less than that normally lost in the tissues. This produces a real tissue oxygen want. The concondition receives the technical name anoxemia.

Progressive anoxemia sets into activity a number of physiological mechanisms which aid the body to absorb its maximum of oxygen from the alveolar air. These reactions are called compensatory. They have been described by a number of workers in the Medical Division of the United States Army who developed a technique for testing the ability of the

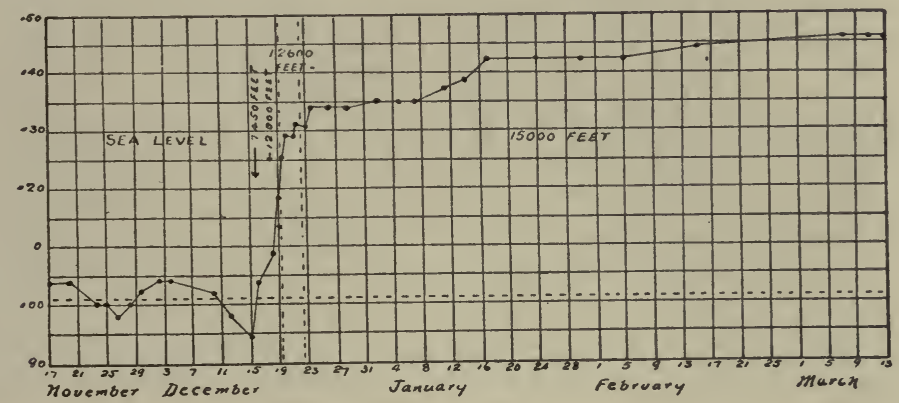

FIG. 24I $a$.-The progressive increase of the amount of hemoglobin in the blood during a journey from England to the high Andes (Richards).

human body to withstand low atmospheric pressures in aviation. The compensatory factors lead to great increase in respiratory rate, an increase in the tidal air, therefore, a great increase in the respiratory minute volume of air breathed. The heart rate is also greatly increased thus maintaining a higher systolic blood pressure notwithstanding the fact that there is vascular dilation, therefore, increased volume of blood flow. Also, the percentage of hemoglobin in the blood is increased if the low oxygen pressure acts through sufficient time, as in mountain residence, figure 24I $a$. When the limit of compensation is reached, then the body quickly succumbs through the following symptoms. Respiration decreases in rate and in amplitude and stops. The blood pressure at first becomes high, then falls slowly at first, but more rapidly later to a final low level. The heart rate, which is greatly accelerated in the early stage of anoxemia, is enormously slowed in the late stages and especially at the time of and following the stopping of respiration. It would seem that the lack of oxygen at first strongly stimulates the medullary centers of respiration, of cardiac acceleration, and of vaso constriction. But in extreme anoxemia 
the respiratory center is no longer supported in activity, and the cardiac inhibitory center is stimulated to inhibitory spasm. This is shown in figure $24 \mathrm{I} b$. This figure represents only the terminal effect of anoxemia in the dog under chloretone anesthesia. The stopping of respiration is shown in the top trace. The blood pressure tracing shows an enormous slowing of the heart rate by anoxemial stimulation of the vagus medullary center. The proof is found in the rapid heart rate after the vagus nerves are both cut.

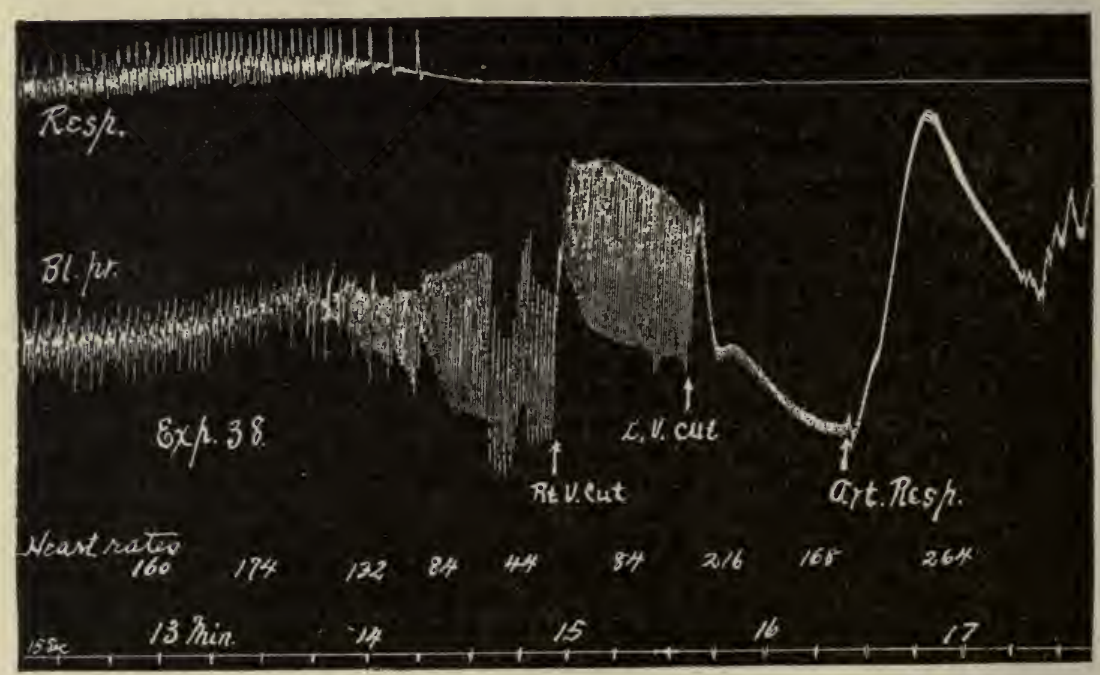

FIG. $24 \mathrm{I} b$. - The effects of extreme anoxemia in the dog.

The compensatory increase in respiratory rate and volume during oxygen want disturbs the factor of carbon dioxide balance in the blood. The carbon dioxide is lost more rapidly than usual, hence its concentration in the blood is diminished, often from twenty to fifty per cent. That carbon dioxide is the chemical stimulator of the respiratory center has long been known, but Henderson has more recently given evidence of the stimulating effect of carbon dioxide on certain other functions of the nervous and muscular mechanisms. He has called the condition of reduced carbon dioxide acapnia, and offers the suggestion that lack of sufficient carbon dioxide may contribute to the complex of symptoms associated with coincident lack of oxygen.

Men are often subjected to higher than normal barometric pressures in caisson work, diving, etc. Paul Bert has found in experimenting with animals that the oxygen pressures may be gradually increased to a considerable extent without marked effect, even to the extent of 8 or ro atmospheres, but when the oxygen pressure is increased up to 20 atmospheres the oxygen becomes poisonous and the animals experimented upon died with 
severe tetanic convulsions. However, caisson workers often experience very severe symptoms, such as bleeding from the nose, dyspnea, vascular inco-ordination, etc. These symptoms are due not so much to the great increase in pressure as to the release from the pressure. When the pressure is released too rapidly, the excess of gases in the tissues and in the blood are set free more rapidly than they can be thrown off by excretion processes. Gases, as such, gather in the blood vessels and form embolisms which occlude the finer vessels. This, of course, produces serious disturbances in the nutrition of the parts involved. If these parts happen to be vital, death may result.

Resuscitation from Electric Shock and Drowning.-Of the numerous conditions which lead to accidental asphyxiation, electric shock on the one hand, and drowning on the other are of great scientific and practical importance in the present day. These special conditions of asphyxiation necessarily involve problems of general tissue asphyxiation and resuscitation. Under the influence of electric shock of sufficient intensity, an immediate result is paralysis of the nervous respiratory control, with whatever else may be directly or indirectly involved. This condition quickly brings on asphyxiation with all of its train of perverted functional activity. So also in drowning, suspension under water blocks respiratory activity and induces asphyxiation. Within the last few years careful investigation of this condition has been made by Stewart, Guthrie and Pike, by Crile and Dolley, and by numerous others. The work has tended to set the time limits of tissue asphyxiation after which recovery is impossible or at most incomplete. The nerve tissues are most susceptible to injury here, see page 303. Within the nervous tissues, the different functional centers manifest different degrees of susceptibility. Those immediately involved in the injuries are the respiratory, vaso-motor, and cardiac regulative centers. If all the activities of these nervous mechanisms can be re-established, control of general visceral reactions will be insured. The delicate functional activity of the higher or cortical regions of the brain are even more susceptible to asphyxiation, and recover more slowly if at all.

In the condition of drowning there are no highly important special injuries. In electric shock on the other hand, there may be a series of local injuries from electric burns, etc. These may injure only the point of contact between the surface of the body and the electric conductor, but it is perfectly possible that the injury may be intense on some deep-seated vital structure. In such cases, recovery of the respiration or of the circulation will not necessarily insure ultimate success in the efforts to revive the individual.

Considering the fact that the nervous tissue cannot be safely recovered beyond the limit of $\mathrm{I}_{5}$ minutes (this is a fair maximum average from our most reliable authorities) it follows that immediate and careful steps must 
be taken to eliminate the conditions producing tissue asphyxiation, i.e., to re-establish both respiration and circulation. Artificial respiration in one form or another is the first aid to be given in drowning, and in other types of asphyxiation, since the technique is equally effective after the lungs are emptied of fluid. The method at present most relied upon is that of Schäfer which includes both the artificial respiration and indirect heart massage. The procedure to be followed in Schäfer's method in condensed statement is given as follows by Dolley:

"The patient is rolled upon his belly, the face turned to one side, and the arms are extended as straight forward as possible. The extension of the arms is a very important improvement, introduced by the Commission, on the original Schäfer method. The operator kneels straddling the patient's thighs and facing his head; he places his palms on the muscles of the small of the back with the fingers spread over the lowest ribs. Then holding his arms straight, he swings forward so that his weight is gradually brought to bear upon the subject. This should take from two to three seconds, and must not be violent. It compresses both the chest and the abdomen. The result is that not only is the chest compressed from front to back, but the pressure on the abdominal viscera tends to force the diaphragm upward. The air is forced out of the lungs, expiration. The operator then immediately swings back to his starting position. Through their elasticity the chest walls expand and air is inspired. A two-second interval should follow the forced expiration so that the rate is from twelve to fifteen a minute. The method not only accomplishes safely and easily ventilation of the lungs, but it must affect a fair amount of compression and relaxation of the heart, especially in young or thin individuals. This so-called indirect heart massage, which will be more emphasized later, is a valuable stimulant to a failing heart." Artificial respiration should be kept up for from two to four hours. A slight but temporary circulation of the blood may produce a partial oxidation which only very slowly recovers sufficient vital activity to bring the nerve centers up to the automatic and reflex level of activity required.

Some cases of so-called drowning are in reality death from cardiac failure. Any hope of resuscitation in this type depends upon vigorous indirect cardiac massage. This is accomplished more effectively in the above method of artificial respiration by allowing the palms of the hands to slide around to the sides of the body, presssing near the ends of the free ribs. In this position it is easy to give pressure with the finger tips under the ribs and against the heart. In extreme, and perhaps in surgical cases, direct massage may be given. ${ }^{1}$

1 Fuller discussion of the conditions involving drowning and the procedure looking toward recovery are available in the following references: C. C. Guthrie, Bloodvessel Surgery, chapter on Resuscitation, page 300 . Report of the Commission on Resuscitation from Electric Shock, W. B. Cannon, Chairman. Medical Handbook for the Use of Lighthouse Vessels, etc., published by the U. S. Public Health and Marine-Hospital Service. D. H. Dolley, On Resuscitation, Bulletin of the University of Missouri, Medical Series, No. 4 . 
Apparent recuperation with the re-establishment of both normal and respiratory activity may occur, yet later stoppage will come about. Prolonged asphyxiation leaves the body and tissues so clogged with carbon dioxide and other waste products, that the renewal of the vital activity of the nervous centers is under a weakened condition. In such cases it is highly necessary to prolong artificial respiration. Even after asphyxiation of short duration it may be some hours or even days before the body is brought back to its normal level of functional efficiency.

\section{THE EFFECT OF RESPIRATION ON THE CIRCULATION.}

As the heart, the aorta, and pulmonary vessels are situated in the airtight thorax, they are exposed to a certain alteration of pressure when the capacity of the latter is varied during respiration. The disturbance of pressure which occurs during inspiration causes, first, a decrease in the intra-

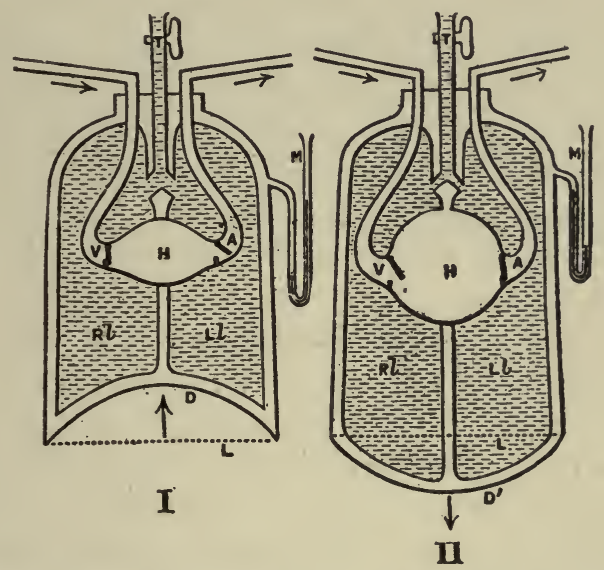

FIG. 242.-Diagram of an Apparatus Illustrating the Effect of Inspiration upon the Heart and Great Vessels within the Thorax. I, The thorax at rest; II, during inspiration; $D$ represents the diaphragm when relaxed; $D^{\prime}$, when contracted (it must be remembered that this position is a mere diagram), i.e., when the capacity of the thorax is enlarged; $H$, the heart; $V$, the veins entering it, and $A$, the aorta; $R l, L l$, the right and left lung; $T$, the trachea; $M$, mercurial manometer in connection with pleura. The increase in the capacity of the box representing the thorax is seen to dilate the heart as well as the lungs, and so to pump in blood through $V$, whereas the valve prevents reflux through $A$. The position of the mercury in $M$ shows also the suction which is taking place. (Landois.)

thoracic pressure, a decrease which affects all the organs of the thorax-the lungs, the great blood-vessels, the heart. The expansion of the elastic lungs counterbalances this change in pressure in part, but it never does so entirely, since part of the pressure within the lungs is expended in overcoming their elasticity. The amount thus used up increases as the lungs become more and more stretched, so that the intrathoracic pressure during inspiration, as 
far as the heart and great vessels are concerned, never quite equals the intrapulmonary pressure, and at the conclusion of inspiration is considerably less than the atmospheric pressure. It has been ascertained that the amount of the pressure used up in the way above described varies from 5 to $7 \mathrm{~mm}$. of mercury in ordinary inspiration, to $30 \mathrm{~mm}$. of mercury at the end of a deep inspiration. So it will be understood that the pressure to which the heart and great vessels are subjected diminishes as inspiration progresses, and at its summit is less by from 7 to $30 \mathrm{~mm}$. than the normal atmospheric pressure, $760 \mathrm{~mm}$. of mercury. It will be understood from the accompanying diagram how an increase in the volume of the thorax will have the effect of pumping blood into the heart from the veins. During inspiration the pressure outside the heart and great vessels is diminished, and they, by virtue of their elasticity, have therefore a tendency to expand and to diminish the intravascular pressure. The diminution of pressure within the veins passing to the right auricle and within the right auricle itself, will draw the blood into the thorax, and so assist the circulation. This suction action of the thorax is the cause of the slight negative pressure of the ventricles previously described. The effect of more blood in the right auricle will, cateris paribus, increase the amount passing through the right ventricle, and through the lungs into the left auricle and ventricle, and thus into the aorta. This all tends to increase the blood-pressure. The effect of the diminished pressure upon the pulmonary vessels will also help toward the same end, an increased flow through the lungs, so that, as far as the mechanical effects on the heart and its veins are concerned, inspiration increases the blood-pressure in the arteries. The effect of inspiration upon the aorta and its branches within the thorax would be, however, contrary; for as the external pressure is diminished, the vessels would tend to expand, and thus to diminish the tension of the blood within them, but, inasmuch as the relative variation in pressure on the large arteries is slight, the diminution of arterial tension caused by this means will be insufficient to counteract the increase of blood-pressure produced by the effect of inspiration upon the volume of discharge of the veins of the chest, and the balance of the whole action would be in favor of an increase of blood-pressure during the inspiratory period. When a bloodpressure tracing is taken at the same time that the respiratory movements are being recorded, it will be found that, although, speaking generally, the arterial tension is increased during inspiration, the maximum of arterial tension does not correspond. with the acme of inspiration, figure 243. In fact, at the beginning of inspiration the pressure continues to fall for a brief moment, then gradually rises until the end of inspiration, and continues to do so for a moment after expiration has commenced. For explanation of the influence of heart rate in this variation of blood-pressure, associated with the respiratory movement, see page 2 I 2 .

In ordinary expiration all this would be reversed, but if the abdominal 
muscles are violently contracted, as in extraordinary expiration, the same relative effect would be produced as by inspiration. The immediate effect during inspiration of the diminished intra-thoracic pressure upon the pulmonary vessels is to produce an initial dilatation of both artery and veins, and this delays for a moment the passage of blood toward the left side of the heart, resulting in an initial fall in the arterial pressure, but the fall of blood-pressure is immediately followed by a steady rise, since the flow is increased by the initial dilatation of the vessels. The converse is the case with expiration. As, however, the pulmonary veins are more easily dilatable than the pulmonary artery, their greater distensibility increases the

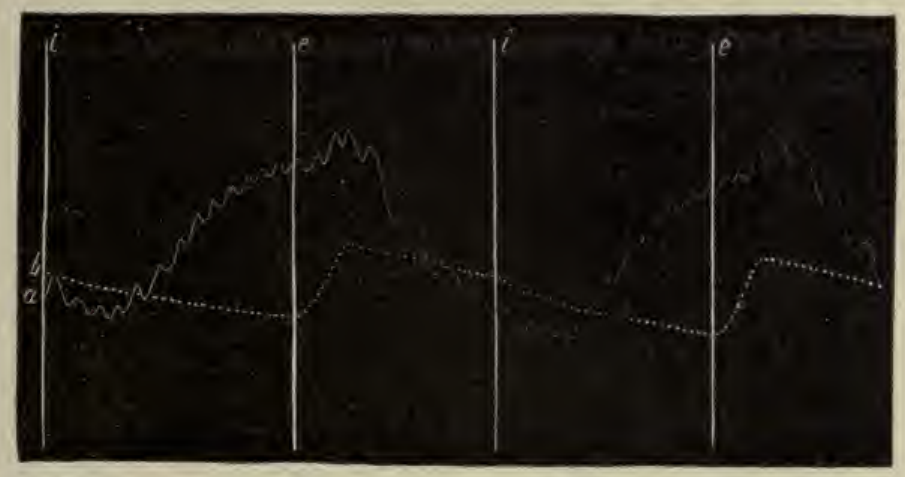

FIG. 243.-Comparison of Blood-pressure Curve with Curve of Intra-thoracic Pressure. (To be read from left to right.) $a$ is the curve of blood-pressure with its respiratory undulations, the slower beats on the descent being very marked; $b$ is the curve of intrathoracic pressure obtained by connecting one limb of a manometer with the pleural cavity. Inspiration begins at $i$ and expiration at $e$. The intra-thoracic pressure rises very rapidly after the cessation of the inspiratory effort, and then slowly falls as the air issues from the chest; at the beginning of the inspiratory effort the fall becomes more rapid. (M. Foster.)

flow of blood as inspiration proceeds, while during expiration, except at its beginning, this property of theirs acts in the opposite direction, and diminishes the flow. Thus, at the beginning of inspiration the diminution of blood pressure, which commenced during expiration, is continued, but after a time the diminution is succeeded by a steady rise. The reverse is the case with expiration, i.e., there is at first a rise and then a fall of blood pressure.

As regards the effect of expiration, the capacity of the chest is diminished and the intra-thoracic pressure returns to the normal, which is still slightly below the atmospheric pressure. The effect of this on the veins is to increase their extravascular and so their intravascular pressure, and to diminish the flow of blood into the left side of the heart. This will, of course, react to decrease the general blood-pressure. Ordinary expiration does not produce a distinct obstruction to the circulation, as even when the expiration is at an end the intra-thoracic pressure is less than the atmospheric pressure. The effect of violent expiratory efforts, however, does have a distinct action 
in obstructing the current of blood through the lungs, as seen in the congestion in the exaggerated condition of straining, this condition being produced by pressure on the entire group of pulmonary vessels.

There are other mechanical factors, such, for example, as the effect of the abdominal movements, both in inspiration and in expiration, upon the arteries and veins within the abdomen and of the lower extremities. Also the influence of the varying intrathoracic pressure upon the pulmonary vessels, which ought to be taken into consideration. The effect of the abdominal movements during inspiration is twofold. On the one hand, blood is sent upward into the chest by compression of the vena cava inferior; on the other hand, the passage of blood downward from the chest through the abdominal aorta, and upward in the veins of the lower extremity, is to a certain extent obstructed. 


\section{LABORATORY EXPERIMENTS IN RESPIRATION.}

I. Respiratory Rate in Man.-Count your respirations for from 2 to 4 minutes while sitting quietly, and determine the average number per minute. Repeat the counting after standing for 5 minutes, and after brisk exercise. These determinations involve the element of consciousness, under which condition it is difficult for a person to breathe with his normal rate and depth.

Make a series of determinations of respiratory rates of persons who are sitting quietly but unconscious of your determinations. Count the rates in a number of persons of different ages; where possible, take into consideration height, weight, etc. Tabulate the results for a comparison and for future reference.

\section{The Character of Respiratory Movements} in Man.-A number of instruments have been devised for measuring human respiratory movement, many of which measure the change in diameter of the chest in respiratory movement. Adjust one of these, for example Burdon-Sanderson's stethograph, to the thorax, and record the movement of the receiving tambour on a smokedpaper kymograph which travels at the rate of I $\mathrm{cm}$. per second. This record, called a stethogram, will exhibit the respiratory rate, the relative time of the inspiratory and expiratory phases, and the character of each.

3. The Actual Change of Diameter in the Chest in Respiration.-Use a caliper provided for the purpose and measure the dorso-ventral diameter of the chest at a series of points along the sternum, taking the reading at the height of the inspiratory phase and of the expiratory phase in ordinary respiration.

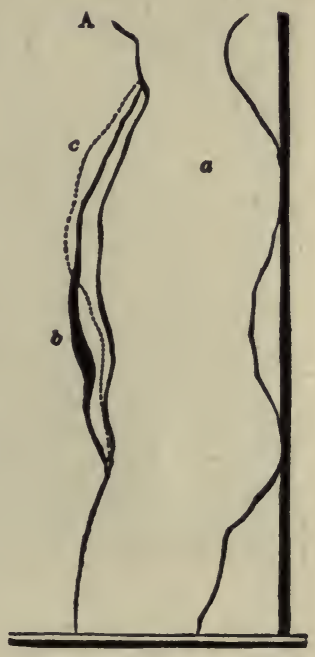

FIG. 244.- Change in Diameter of the Body in Respiration, Costal Type. $a$, Outline of the body in forced expiration. In the heavy continuous line, $b$, the outer margin indicates the contour of the body in ordinary inspiration and the inner margin that of ordinary expiration. $c$, Contour of forced inspiration. (After Hutchinson.) ment in forced respiration. Map the results on millimeter paper, as indicated in figure 244 .

Repeat these measurements in the transverse diameter at the first, fifth, and tenth ribs.

Using the thoracograph, figure 245 , record the outline of the cross section of the chest at the level of the mammæ, tenth rib, and the umbilicus, showing the volume changes in the following four positions: (I) Ordinary expiration, (2) ordinary inspiration, (3) forced expiration, (4) forced inspiration, see figure 246 . 
4. The Volume of Air Breathed by Man.-Determine the average volume of air breathed per respiration, using Hutchinson's spirometer, figure 235, set the instrument at the zero point, exhale into the instrument through the tube, using all possible care

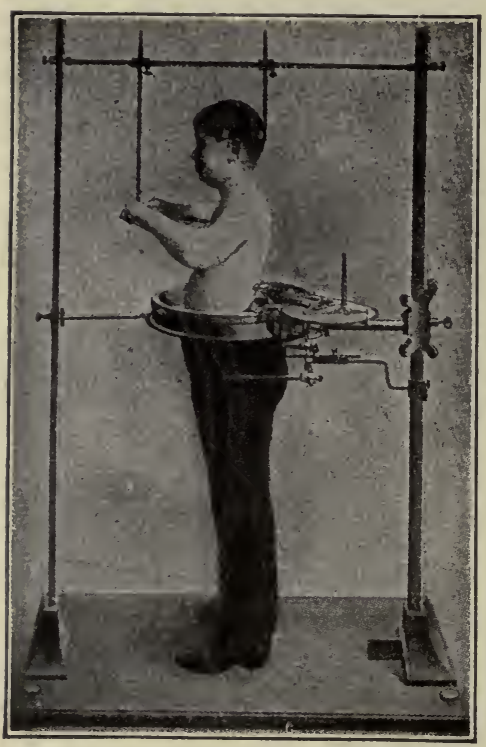

FIG. 245.-Thoracograph of Deufestel. to breathe with your normal rate and depth. Better results will be obtained by taking the average from sets of ten consecutive expirations into the instrument. From the average of the volume per respiration, and the average number of respirations per minute, determined in Experiment I, calculate the amount of air breathed per hour and per day.

5. Vital Capacities.- Using the spirometer as in the preceding experiment, set the instrument at zero and exhale into it:

a. Begin with the fullest possible inspiration and exhale the greatest possible amount of air from the lungs. This is known as the vital capacity.

$b$. Beginning at the end of an ordinary expiration exhale into the instrument the greatest possible amount.

This is called the reserve air.

c. Following ordinary inspiration exhale into the instrument until you reach the ordinary state of expiration. This involves the conscious fixing of two points in the respiratory act, namely, the summit of inspiration and of expiration, which are ordinarily automatically adjusted by the body. The error of the determination is therefore great. It is better to make this measurement in sets of ten, as in the preceding experiment, and take the average. This reduces the error. This quantity of air is known as the tidal air. One can measure the tidal air and the reserve air together, check them against the sum of the two, as in $a$ and $b$.

$d$. The sum of the tidal and reserve air taken from the vital air will leave the amount which one may inspire over and above that in the chest at the end of ordinary inspiration. This is called complemental. The complemental can be measured by inspiring the air from the spirometer, but this is not good hygienic practice where large numbers are using the same instrument, unless the instrument be thoroughly cleaned before the inspiration is taken.

6. The Respiratory Pressure in Man.-Measure there spiratory pressure, the variation in pressure of the air in the air-passages, by means 
of the mercury manometer or by a graduated Marey's tambour. Connect a piece of gas tubing with the proximal limb of the mercury manometer and provide it with a glass mouthpiece. Insert this mouthpiece well back into the cavity of the mouth, closing the lips firmly about it, keeping the pharynx relaxed. Note the variations in pressure at the height of ordinary inspiration and expiration through the nasal passages. Repeat with forced inspiration and expiration, close the nasal passages, and make the

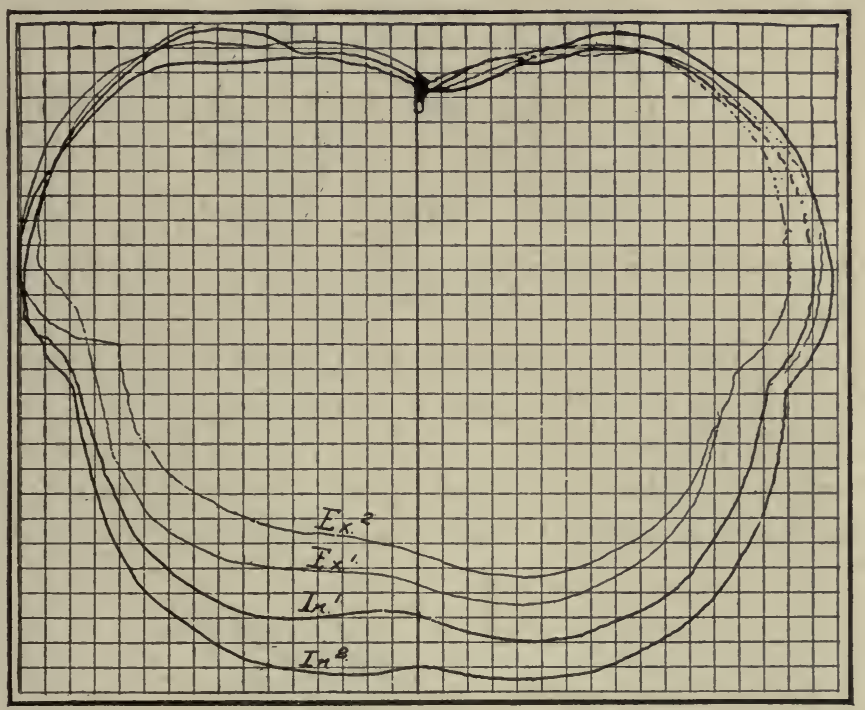

FIG. 246.-Chart showing transverse section of the chest at the level of the mamma of a distance runner, age twenty-one, height, 5 feet, I I inches, net weight, I 40 pounds. $E x^{1}$, ordinary expiration; $E x^{2}$, forced expiration; $I n^{1}$, ordinary inspiration; $I n^{2}$, forced inspiration. Scale $\frac{1}{1}$.

maximal expiratory and inspiratory effort. The manometer may be adjusted to write on the smoked paper or read directly.

7. Demonstration of Carbon Dioxide in Expired Air.-Arrange two flasks, as in figure 246 , filling each one-third full of baryta-water, or lime-water. Close the lips around the mouthpiece of the apparatus and inhale and exhale the air through it. The inspired air will come through $a$, the expired air out through $b$. The baryta water in $b$ will quickly become clouded with a white precipitate of barium carbonate while that in $a$ will remain clear or only very slightly clouded, showing the excess of carbon dioxide in expired air.

\section{Quantitative Determination of Carbon Dioxide and Oxygen in} Inspired Air and in Expired Air.-Inspired Air.-Fill a gas-analysis apparatus, the Guthrie or any modern modification of the Haldane or the Orsat analyzer, with air from outside the laboratory. Read the volume 
at room temperature and pressure. Wash the sample back and forth through the potash bulb as directed ten times and read for absorption of the carbon dioxide. Next wash in ten per cent. pyrogallic acid in potassium hydroxide until constant readings show that all oxygen is absorbed.

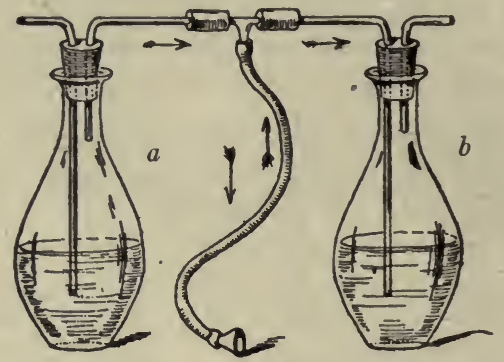

FIG. 246a.-Apparatus for Demonstrating Excess of $\mathrm{CO}_{2}$ in Expired Air. Flasks filled with lime-water.

The nitrogen residue is calculated by difference. Compute the percentages of carbon dioxide, oxygen and nitrogen.

Expired Air.-Exhale ten expirations controlled by one-way valves into an ordinary respirometer or a Tissot apparatus. Now fill the Guthrie apparatus with a sample of this expired air and analyze as before, first for
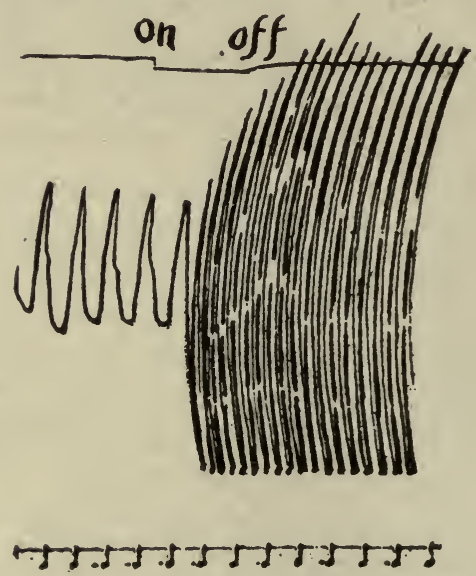

FIG. 246b.-Change in Respiration on Stimulating the Central End of the Sciatic Nerve. The rate is sharply increased and the amplitude more than doubled. The stimulation is between the points marked on and off, time in seconds. The inspiratory movement following the stimulation was greater than the limit of the recording tambour.

carbon dioxide, then for oxygen; compute the percentage of each gas, including nitrogen. The expired air will usually be found to have lost from 4 to 5 per cent. of oxygen and have gained a little more than that quantity of carbon dioxide. 
From the percentages obtained in these experiments, and the volume of air breathed per unit of time, computed in Experiment 4 above, determine the amount of carbon dioxide exhaled per hour per kilogram of weight for your own body. Compute also the amount of oxygen consumed per kilo per hour; per square meter of surface per hour.

9. The Rate and Character of the Respiratory Movements in the Mammal.- $a$, The rate of respiration can be best determined by direct count per minute, an effort being made to maintain as nearly normal

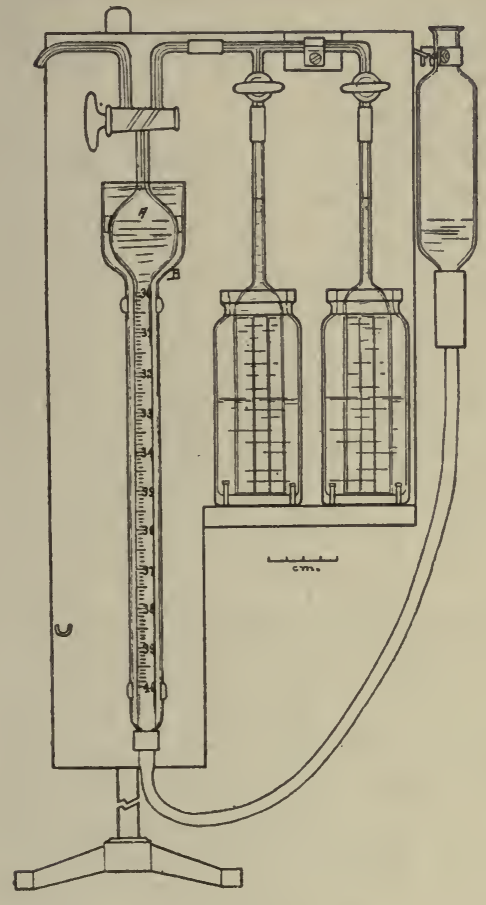

$c$

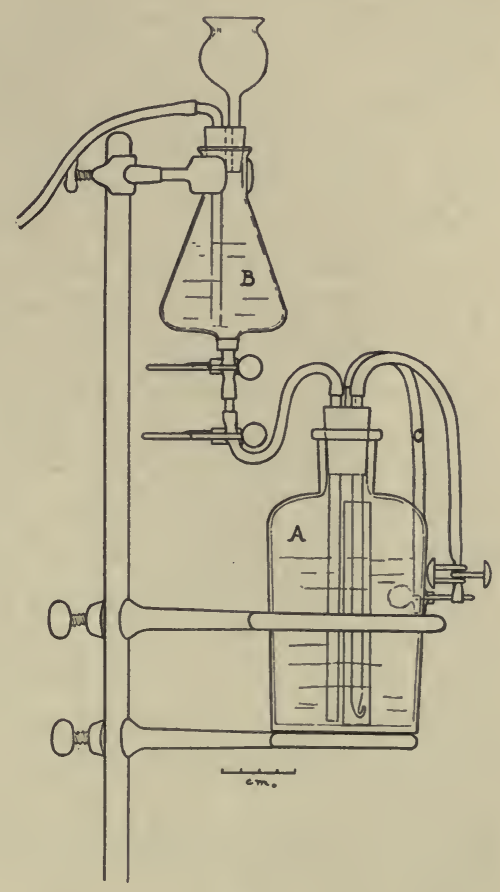

$d$

FIG. 246c.-Oxygen and carbon dioxide analyzer, Guthrie form.

FIG. 246d.-Receiver for air sample, Guthrie form.

conditions as possible. Make the determinations on a cat, a dog, and a guinea-pig. $b$, The character of the respiratory movements can be recorded by one of the various forms of stethograph adapted to the size of the animal, or by the arrangement shown in figure 233. It is necessary to make the determination with an animal under the influence of an anesthetic.

Io. The Nervous Mechanism of Respiratory Movements.- $a$. The Effect of Stimulating Cutaneous Nerves.-Use a small dog or a cat for this experiment; anesthetize and introduce a tracheal tube with a side 
branch adapted for measuring the variations of pressure during respiration. Connect the free limb of the tracheal tube with an ether apparatus and adjust to secure constant anesthesia. Connect the side branch of the tracheal tube with a Marey's recording tambour of medium size and supplied with a comparatively delicate membrane. The amplitude of the movements of the tambour may be regulated by a screw compress on a connecting tube. Arrange an induction coil with platinum electrodes in the usual manner for stimulating by means of the interrupted current. Record the results of the experiment along with the variations of bloodpressure on a continuous-paper kymograph; the instrument should be supplied with a time signal, a stimulating signal, etc.

Now stimulate the skin of the abdominal region, the groin, with a comparatively strong medium induction current, figure $246 b$. Dissect out the sciatic nerve, cut it, stimulate the central end with a mild to medium strength of current. The stimulus should be graduated carefully, for there is often such a great increase in respiratory rate and volume that the animal may become overanesthetized.

b. The Effect of Stimulating the Vagus Nerve.-Isolate and stimulate the vagus nerve with a medium strength of stimulus. The effect is usually complete inhibition of respiratory movements. By means of graduated stimuli one may demonstrate the accelerator effects from the stimulation of the vagus. Stimulate also the superior laryngeal, and compare with the effects of stimulating the whole vagus.

c. The Effect of Cutting the Vagus Nerves.-Isolate both vagus nerves and section them as nearly at the same moment as possible. Be sure to mark on the tracing the exact moment at which the nerves are cut. This experiment should be performed with every accessory condition as constant as possible, and the animal should not be disturbed for one or two minutes so that the effects of the section will be recorded, figure 240 . The result is always a marked deepening and slowing of the respiratory movements.

d. The Effect of Stimulating the Central End of the Vagus.-Upon stimulating the central end of the vagus after section, the respiration rate will be inhibited as in $b$, showing that the vagus nerves carry afferent respiratory fibers, figure 24I.

e. The Effect of Stimulation of the Phrenic Nerves.-Isolate the right phrenic nerve at its origin from the brachial plexus and stimulate it with a medium strength of stimulus. Upon stimulating the nerve the diaphragm will remain in contraction and the record will show that the thorax is in the inspiratory phase. The phrenic is therefore a simple motor nerve.

Section this nerve and note the change in the character of respiratory movements; make direct observations on the diaphragm, examining from the abdominal side. 
II. Demonstration of Apnea, Dyspnea, and Asphyxia.-Produce deep anesthesia, then disconnect the ether bottle and connect the tracheal tube with a hand bellows. Produce deep and forced artificial respiration for ten to twenty seconds. Stop artificial respiration; the animal will remain quiet and without any effort at breathing. This is the condition of apnea. Allow the animal to recover its normal respiratory rate and again produce deep anesthesia. Now clamp off the tracheal tube so that the animal can no longer receive air and leave it so until death. As the blood becomes more and more venous there will first be a marked increase in the respiration rate and depth. This is known as hyperpnea. This stage is followed by one of increasing respiratory amplitude in which the accessory respiratory muscles not previously active are brought into forcible contractions, both inspiratory and expiratory phases are now forced, dyspnea. The movements continue to increase, and the muscles of the neck, larynx, mouth, and nostrils now take part. There is a rather sudden decrease in the respiratory movements, an extension of the limbs, and gasping movements, after which the animal remains quiet, death being produced by asphyxia.

I2. Respiratory Exchange and Calorimetry.-Indirect calorimetry is made the basis for determining the metabolic rate in man and mammals. Measurement of the oxygen intake and of the carbondioxide output under standard conditions suffices for the computation from the data of the metabolic rate of heat production per kilo or per square meter of surface per hour. This principle is used in the rapid methods of human clinical calorimetry.

Generally a man is made to exhale into a large collecting chamber (Tissot apparatus), or breathe through a closed chamber (rebreather) provided with absorbers and either one-way valves or a pump to circulate the air of the chamber.

In man the test is made with the body at maximum rest. Test (I) after a I 2 hour fast; (2) measure the height in centimeters and weight in kilos; (3) provide 20 to 30 minutes complete relaxation lying at rest; (4) measure systolic and diastolic blood pressures and mouth temperature after the rest period; (5) Adjust the face mask and run the test of oxygen consumption. Use a modified Henderson rebreather or a Benedict filled with excess of pure oxygen, or a Tissot apparatus. Continue the test for ten minutes or more. In the dog use a rebreather type of apparatus of adapted size. Attach a face mask to a ten kilo dog trained to the experiment. Record all rebreather movements.

From the data for man, compute the metabolic rate per square meter per hour for man by DuBois' formula.

In man the surface area in sq. $\mathrm{cm} .=$ The Weight in kilos ${ }^{0.425} \times$ the Height in $\mathrm{cm} .{ }^{0.725} \times$ a constant 71.84 . 
Compute the rate per kilo per hour for the dog. Compare with the rate per kilo on man and explain the difference observed.

13. Gases of Arterial and Venous Blood.-Tissue respiration is intimately dependent on the capacity of the blood to carry oxygen and carbon dioxide by chemical fixation, chiefly by the respiratory pigment hemoglobin. Measure the gaseous content of arterial and venous blood simultaneously drawn from a dog under local analgesia. Draw the samples under petrolatum and analyze promptly for the three gases, carbon doxide, oxygen and nitrogen, in a Van Slyke apparatus, page 303. Also consult the Journal of Biological Chemistry, vol. 30 , page 347 , and vol. 33 , page I 27 . 


\section{CHAPTER VII}

\section{SECRETION IN GENERAL}

All tissues of the body produce certain chemical changes as a result of their protoplasmic activity. But in certain cells chemical elaborations have come to be the chief function, the cells have been differentiated in that direction, and the name secreting tissue or gland tissue is applied. The end result of metabolism in gland tissue is the extrusion on the free borders of the cells of the products of their metabolism. The products are known as secretions and the process itself is the act of secretion. Certain secretions which are in the nature of waste products to the body as a whole, such as urine in the kidney, are often called excretions, but the use of the term should not be allowed to confuse the general similarity of this to other secretions as regards the physiological changes involved in its production.

Most secretions accomplish some definite office in the economy of the body. Those that are discharged on some free mucous surface, as the saliva, gastric juice, tears, etc., are called external, or true secretions, or merely secretions. Substances that are discharged back into the blood stream later to influence the metabolism of tissues other than the ones which produced them are called internal secretions.

Gland cells, like other tissues, draw their nourishment from the blood and lymph. The product or secretion of gland cells may, in fact usually does, contain some of the substances found in the blood, but there are also present new materials elaborated by the cells, and even where the same substance exists both in the secretion and in the blood and lymph it can make its appearance in the secretion only under the control of the protoplasm of the gland cells. The saliva secreted by the salivary cells, for example, consists of about 98 to 99 per cent. water containing in solution small quantities of certain salts, also found in the lymph, and a small percentage of the enzyme, ptyalin. This enzyme is peculiar to the salivary secretion and is manufactured by the salivary-cell protoplasm. As is well known, it acts vigorously in extreme dilution, hence the high per cent. of water in the secretion. The passage of water from a solution as concentrated as blood plasma to a solution as dilute as saliva requires a high amount of osmotic energy, an amount that can be supplied only from the chemical energy liberated by the cell in its protoplasmic activity. After the removal of the special organ by which each secretion is manufactured, the secretion is no longer formed. Cases sometimes occur in which the secretion continues to be formed by the natural 
organ, but, not being able to escape toward the exterior, on account of some obstruction, is reabsorbed and accumulates in the blood. It may be discharged from the body in other ways; but these are not instances of true vicarious secretions, and must not be so regarded.

Organs and Tissues of Secretion.-The principal secreting organs are the following: $r$. The serous and synovial membranes; 2 . the mucous membranes with their special glands, e.g., the buccal, gastric, and intestinal glands; 3 . the salivary glands and pancreas; 4 . the liver; 5 . the mammary glands; 6 . the lachrymal glands; 7 . the kidney and skin; 8 . the testes and ovaries, and 9. thyroid, supra-renal, etc.

The special structure and functions of the secreting organs will be given in greater detail in the chapters which immediately follow. The general types of structure and general conditions that influence the functions are introduced at this point.

Structural Types of Secreting Organs.-Serous and Synovial Type.The serous membranes form closed sacs lining visceral cavities like the abdominal, pericardial, or pleural cavities. The organs are, as it were, pushed into this sac and carry before them an investment of membrane. The serous membranes consist of a single layer of flattened polygonal cells resting on a supporting membrane of connective tissue, supporting a ramification of blood vessels, lymphatics, and nerves.

In some instances, i.e., synovial membranes, the secreting layer is increased by finger-like elevations. This type of secreting organ produces ordinarily only enough secretion to keep the surface moist.

The Mucous Type.-The mucous tracts, and different portions of each of them, present certain structural peculiarities adapted to the functions which each part has to discharge; yet in some essential characters the mucous membrane is the same, from whatever part it is obtained. In all the principal and larger parts of the several tracts it presents an external layer of epithelium, situated upon a basement membrane, and beneath this a stratum of vascular tissue of variable thickness, containing lymphatic vessels and nerves. The vascular stratum, together with the basement membrane and epithelium, in certain cases is elevated into minute papillæ and villi, in others depressed into involutions in the form of glands. But in the invaginations of the secreting membrane of gland cells, the supporting basement membrane and the network of capillaries are still retained in their relative position. With increasing complexity of involution the simple mucous membrane becomes packed away in an apparently solid mass. The equivalent of a large amount of secreting surface is thus condensed into a small space. In the process of invagination some differentiation occurs in that certain of the gland cells become conducting and have their secretory activity somewhat reduced.

Secreting Glands.-The secreting glands present, amid manifold diversities of form and composition, a general plan of structure; but all are 
constructed with particular regard to the arrangement of the cells which has just been described.

Secreting glands are classified according to certain structural types, as: I. The simple tubular gland, A, figure 247 , examples of which are furnished by the follicles of Lieberkühn, and the tubular peptic glands of the stomach. They are simple tubes of mucous membrane, the walls of which are lined with
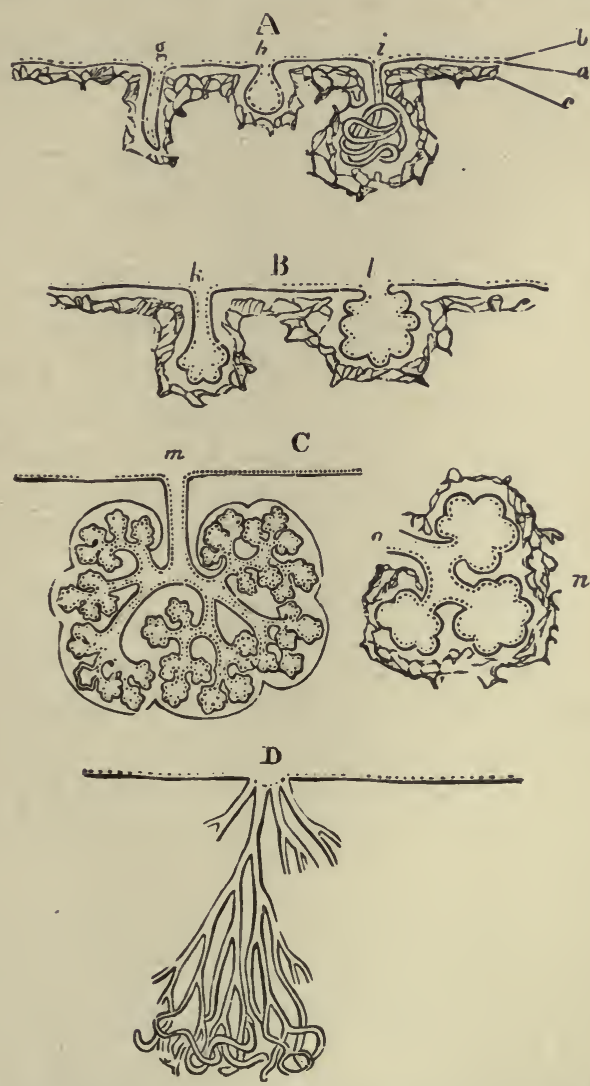

FIG. 247.-Plans of Extension of Secreting Membrane by Inversion or Recession in the Forms of Cavities. A, Simple glands, viz., $g$, straight tube; $h$, sac; $i$, coiled tube. B, Multilocular crypts; $k$, of tubular form; $l$, saccular. C, Racemose or saccular compound gland; $m$, entire gland, showing branched duct and lobular structure; $n$, a lobule, detached with $o$, branch of duct proceeding from it. D, Compound tubular gland. (Sharpey.)

secreting cells arranged as an epithelium. To the same class may be referred the elongated and tortuous sudoriferous glands.

2. The compound tubular glands, D, figure 247 , form another division. These consist of main gland tubes, which divide and subdivide. Each gland may be made up of the subdivisions of one or more main tubes. The ultimate subdivisions of the tubes are sometimes highly convoluted. They are 
formed of epithelium of various forms, supported by a basement membrane. The larger tubes may have an outside coating of fibrous areolar or muscular tissue. The salivary glands, pancreas, Brunner's glands, kidney, testes, with the lachrymal and mammary glands, are examples of this type, but present more or less marked variations among themselves.

3. The racemose glands, in which a number of vesicles or acini are arranged in groups of lobules, $C$, figure 247. The Meibomian follicles are examples of this kind of gland. There seem to be glands of mixed character, combining some of the characters of the tubular with others of the racemose type; these are called tubulo-racemose or tubulo-acinous glands. The acini are formed by a kind of fusion of the walls of several vesicles, which thus combine to form one large cavity with recesses lined or filled with secreting cells. The smallest branches of the gland-ducts sometimes open into the centers of these cavities; sometimes the acini are clustered round the extremities or by the sides of the ducts; but, whatever secondary arrangement there may be, all have the same essential character of rounded groups of vesicles containing gland cells, and opening by a common central cavity into minute ducts, which in the large glands converge and unite to form larger and larger branches, and at length one common trunk which opens on a free surface.

The Process of Secretion.-The process of secretion is dependent upon the activity of the secreting cells. In the case of the water and salts the physical processes of filtration and diffusion may play a part.

The chemical processes constitute the process of secretion properly so called, as distinguished from mere transudation spoken of above. In the process of secretion various materials which do not exist as such in the blood are manufactured by the agency of the gland cells, using as a nutrient fluid the blood or, to speak more accurately, the lymph which fills the interstices of the gland textures.

Evidences in favor of this view are: I. That gland cells are constituents of all glands, however diverse their outer forms and other characters, and they are placed in all glands on the surfaces or in the cavity whence the secretion is poured. 2. That certain materials of secretions are visible with the microscope in the gland cells before they are discharged. Thus, granules probably representing the precursors of the ferments of the pancreas may be discerned in the cells of that gland. Granules of uric acid are found in the cells of the kidneys of birds and fish, and fatty particles, like those of milk, in the cells of the mammary gland.

Certain secreting cells, like the cells of the sebaceous glands, appear to develop, grow, and attain their individual perfection by appropriating nutriment from the fluid exuded by adjacent blood vessels and building it up so that it shall form part of their own substance. In this perfected state the cells subsist for some brief time and then appear to dissolve, wholly or in part, and yield their contents to the peculiar material of the secretion. The changes 
which have been noticed from actual experiment in the cells of the salivary glands, pancreas, and peptic glands will be described more fully in the chapter on Digestion.

Discharge of secretions from the glands may either take place as soon as formed, or the secretion may be long retained within the gland or its ducts. The former is the case with the sweat glands. But the secretions of those glands whose activity of function is periodical are usually retained in the cells in an undeveloped form during the period of the gland's inaction.

When discharged into the ducts, the further course of secretions is affected: (I) partly by the pressure from behind; the fresh quantities of secretion propelling those that were formed before. In the larger ducts, its propulsion is (2) assisted by the contraction of the walls. All the larger ducts, such as the ureter and common bile duct, possess in their coats plain muscular fibers; they contract when irritated, and sometimes manifest peristaltic movements. Rhythmic contractions in the pancreatic and bile ducts have been observed, and also in the ureters and vasa deferentia. It is probable that the contractile power extends along the ducts to a considerable distance within the substance of the glands whose secretions can be rapidly expelled. Saliva and milk, for instance, are sometimes ejected with much force.

Circumstances Influencing Secretion.-The principal conditions which influence secretion are variations in the quantity of blood and variations in nerve impulses passing to the gland cells over secretory nerve fibers.

An increase in the quantity of blood traversing a gland, as in nearly all the instances before quoted, coincides generally with an augmentation of its secretion. Thus the mucous membrane of the stomach becomes florid when, on the introduction of food, its glands begin to secrete. The mammary gland becomes much more vascular during lactation. All circumstances which give rise to an increase in the quantity of material secreted by an organ produce, coincidently, an increased supply of blood. But we shall see that a discharge of saliva may occur under extraordinary circumstances without increase of blood supply, and so it may be inferred that this condition of increased blood supply is not absolutely essential to the immediate formation of secretion, but that it favors the prolonged activity of glands.

Influence of the Nervous System on Secretion.-The process of secretion is largely regulated through the nervous system. The exact mode in which the influence is exhibited must still be regarded as somewhat obscure. In part it exerts its influence by increasing or diminishing the quantity of blood supplied to the secreting gland, in virtue of the power which it exercises over the contractility of the smaller blood vessels. It also has a more direct influence, as is described at length in the case of the submaxillary gland, upon the secreting cells themselves. This may be called trophic influence. Its influence over secretion, as well as over other functions of the body, may be excited by causes acting directly upon the nervous centers, upon the nerves 
going to the secreting organ, or upon the nerves of other parts. In the latter case a reflex action is produced. Thus the impression produced upon the sensory nerves by the contact of food in the mouth leads to afferent nerve impulses to the secretory center in the central nervous system, impulses which are reflected by the nerves supplying the salivary glands, and produce, through these, a more abundant secretion of the saliva.

Through the nerves, various conditions of the brain also influence the secretions. Thus, the thought of food may be sufficient to excite an abundant flow of saliva. And, probably, it is the mental state which excites the abundant secretion of urine in hysterical paroxysms, as well as the perspirations, and occasionally diarrheas, which ensue under the influence of terror, and the tears excited by sorrow or excess of joy. The quality of a secretion may also be affected by mental conditions, as in the cases in which, through grief or passion, the secretion of milk is altered, and is sometimes so changed as to produce irritation in the alimentary canal of the child. 


\section{CHAPTER VIII}

\section{FOOD AND DIGESTION}

THE term digestion includes those changes taking place in the body which bring the materials of the food into such condition that they may be taken up by the blood and lymphatic vessels and thus rendered available for the metabolism of the tissues. In the process the foods are rendered more soluble and more diffusible. Certain bodies which are already soluble and diffusible are converted into forms which are more available for the tissues; as an example, cane-sugar, although both soluble and diffusible, cannot be readily used by the body until it is converted from a disaccharide to a monosaccharide. In fact, few of the food materials are fit for immediate use when taken into the body and are therefore practically useless until digested.

\section{FOOD AND FOOD PRINCIPLES.}

We have been accustomed to classify foods into certain main groups, chiefly according to their chemical character, as follows:

Proteins.-Such as albumin, myosin, gluten, casein, etc.; gluco-protein, nucleoprotein, etc.; gelatin, elastin, etc. These furnish nitrogen in available form.

Carbohydrates.- Such as starch, dextrose, cane-sugar, etc.

Fats.-Such as tristearin, tripalmitin, triolein.

Minerals.-The various salines found in animal and vegetable food. Water.

The classes of foods just enumerated usually exist in mixtures rather than in simple forms, as, for example, a beef roast contains a representative of each of the five classes enumerated, though it is composed chiefly of water, proteins, and fats. The human body is capable of using materials of a great variety of forms, but most of these have the foods mixed in such a way as to give representatives of each of the classes above in certain general proportions.

Nitrogenous Foods.-The Flesh of Animals, e.g., beef, veal, mutton, pork, bacon, ham, chicken, eggs, milk, etc., are typical nitrogenous foods.

Of these, beef and eggs are richest in nitrogenous matters, containing about 20 per cent. Mutton contains about 18 per cent., veal 16.5 , and pork ro. Beef is firmer, more satisfying, and is supposed to be more strengthening than mutton, whereas the latter is more digestible. The flesh of young animals, such as lamb and veal, is less digestible and less nutritious. Pork contains a large amount of fat and is, therefore, comparatively indigestible. 
Percentage Composition and Fuel Value per Pound of Some Common Food Stuffs. (Atwater and Bryant.)

\begin{tabular}{|c|c|c|c|c|c|c|}
\hline & $\begin{array}{l}\text { Water. } \\
\text { Per } \\
\text { cent. }\end{array}$ & $\begin{array}{l}\text { Pro- } \\
\text { tein. } \\
\text { Per } \\
\text { cent. }\end{array}$ & $\begin{array}{l}\text { Fat. } \\
\text { Per } \\
\text { cent. }\end{array}$ & $\begin{array}{c}\text { Carbo- } \\
\text { hy- } \\
\text { drate. } \\
\text { Per } \\
\text { cent. }\end{array}$ & $\begin{array}{c}\text { Ash. } \\
\text { Per } \\
\text { cent. }\end{array}$ & $\begin{array}{c}\text { Fuel } \\
\text { Value. } \\
\text { Calories } \\
\text { per } \\
\text { Pound }\end{array}$ \\
\hline Meat (beef, round) & 73.6 & 22.6 & 2.8 & $\ldots \ldots$ & I. 3 & 540 \\
\hline Meat (pork loin). & $5^{2} \cdot 0$ & I 6.6 & 30.1 & & 1.0 & $1,5^{80}$ \\
\hline Fish (king salmon) .......... & 63.6 & $\mathrm{I} 7.8$ & I 7.8 & $\ldots$ & I . I & $\mathrm{I}, 080$ \\
\hline 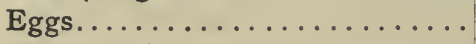 & $73 \cdot 7$ & $13 \cdot 4$ & 10.5 & $\ldots$ & I. 0 & 720 \\
\hline Milk (cow's) & $87 \cdot 0$ & $3 \cdot 3$ & $4 \cdot 0$ & $5 \cdot 0$ & 0.7 & 325 \\
\hline Milk (human) .............. & $89 \cdot 7$ & 2.0 & $3 \cdot I$ & 6.0 & 0.2 & $\ldots$ \\
\hline Cheese (American)........... & 31.6 & 28.8 & $35 \cdot 9$ & 0.3 & $3 \cdot 4$ & 2,055 \\
\hline 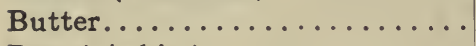 & II.O & I.० & 85.0 & ... & $3 \cdot 0$ & $3,6 \circ 5$ \\
\hline Bread (white) ............. & $33 \cdot 2$ & 10.9 & $\mathrm{I} \cdot 3$ & $53 \cdot 6$ & I. .0 & 1,255 \\
\hline 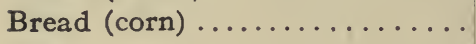 & 38.9 & $7 \cdot 9$ & $4 \cdot 7$ & $46 \cdot 3$ & 2.2 & $x, 205$ \\
\hline 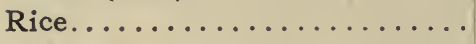 & $\mathrm{I} 2 \cdot 3$ & 8.0 & 0.3 & 79.0 & 0.4 & $x, 630$ \\
\hline Oatmeal.. & $7 \cdot 3$ & I6. I & $7 \cdot 2$ & $67 \cdot 5$ & I.9 & 1,860 \\
\hline Beans (dry) ........... & 12.6 & $22 \cdot 5$ & I. 8 & $59 \cdot 6$ & $3 \cdot 5$ & 1,605 \\
\hline Potatoes (white) & $78 \cdot 3$ & 2.2 & $0 . \mathrm{I}$ & 18.4 & I. 0 & 385 \\
\hline Potatoes (sweet) & 69.0 & r. 8 & 0.7 & $27 \cdot 4$ & $\mathrm{I} .2$ & 570 \\
\hline Fruit (strawberries) ......... & 90.4 & I.० & 0.6 & $7 \cdot 4$ & 0.6 & I 80 \\
\hline Watermelon (edible portion) .... & $92 \cdot 4$ & 0.4 & 0.2 & 6.7 & 0.3 & 140 \\
\hline
\end{tabular}

Meat contains: (I) Muscle proteins, chiefly myosin, blood proteins, collagen (from the interstitial fibrous connective tissue), elastin (from the elastic tissue), as well as traces of hemoglobin. (2) Fats, including the lipoids lecithin and cholesterol. (3) Extractives, some of which are agreeable to the palate and others weakly stimulating. These are divided into the non-nitrogenous: glycogen, dextrose, lactic acid, inosit, etc., and into the nitrogenous: consisting chiefly of creatin, and the purine bases. (4) Salts, chiefly chlorides and phosphates of potassium, calcium, and magnesium. (5) Water, the amount of which varies from I 5 per cent. in dried bacon to 39 in pork, $5 \mathrm{I}$ to 53 in fat beef and mutton, and 72 per cenț. in lean beef and mutton.

Table of Percentage Composition of Beef, Mutton, Pork, and Veal. (Letheby.)

\begin{tabular}{|c|c|c|c|c|}
\hline & Water. & Protein. & Fats. & Salts. \\
\hline Beef-lean. & 72 & 19.3 & 3.6 & $5 \cdot x$ \\
\hline Beef-fat... & $5 \mathrm{I}$ & $\mathrm{r} 4.8$ & 29.8 & $4 \cdot 4$ \\
\hline 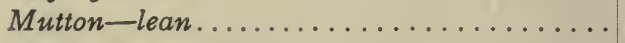 & 72 & $\mathrm{I} 8 \cdot 3$ & $4 \cdot 9$ & 4.8 \\
\hline Mutton-fat. ... & 53 & 12.4 & $3 \mathrm{r} \cdot \mathrm{x}$ & $3 \cdot 5$ \\
\hline Veal........... & 63 & I 6.5 & I 5.8 & $4 \cdot 7$ \\
\hline Pork-fat .... & 39 & 9.8 & 48.9 & $2 \cdot 3$ \\
\hline
\end{tabular}


Table of Percentage Composition of Poultry and Fish. (Letheby.)

\begin{tabular}{|c|c|c|c|c|}
\hline & Water. & Protein. & Fats. & Salts. \\
\hline Poultry. & 74 & $2 \mathrm{I} .0$ & 3.8 & 1.2 \\
\hline White fish & 78 & I $8 . \mathrm{I}$ & 2.9 & I.० \\
\hline 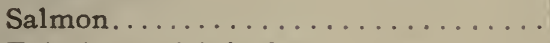 & 77 & I6. I & $5 \cdot 5$ & $\mathrm{I} .4$ \\
\hline Eels (very rich in fat).. & 75 & $9 \cdot 9$ & $\mathrm{r} 3.8$ & $\mathrm{r} \cdot 3$ \\
\hline Oysters............. & $75 \cdot 74$ & II. 72 & $2 \cdot 42$ & 2.73 \\
\hline
\end{tabular}

The flesh of nearly all animals has been occasionally eaten, and we may presume that except for difference of flavor, etc., the average composition, aside from the fat, is nearly the same in most cases.

Milk.-Milk is the entire food of young animals, and contains all the elements of a typical diet. Albuminous substances are represented in the form of caseinogen, and serum or lactalbumin; fats in thecream; carbohydrates in the form of lactose or milk-sugar; salts, chiefly as calcium phosphate; and water. From milk we obtain a number of food preparations, such as cheese rich in protein and fat, butter and cream, buttermilk rich in proteins and peculiarly well adapted for invalid diet, and whey which contains all the sugar, salts and the albumin.

Table of Composition of Milk, Buttermilk, Cream, and Cheese.

(Letheby and Payen.)

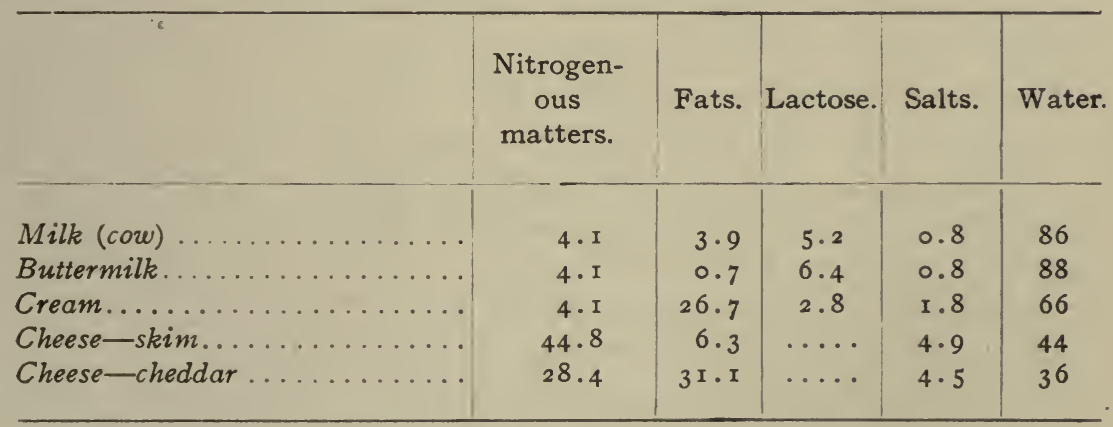

Eggs.-The yolk and albumin of eggs of oviparous animals bear the same relation as food for the embryos that milk bears to the young of mammalia, and affords another example of the natural admixture of the various alimentary principles. The proteins of eggs are ovalbumin and ovoglobulin and phosphoprotein, the vitellin of the yolk. In addition to the three common fats there is a yellow fatty pigment, lutein (lipochrome), lecithin, and cholesterol, a small quantity of dextrose, and inorganic salts, chiefly calcium, potassium, sodium, chlorides, and phosphates. 
Table of the Percentage Composition of Fowls' Eggs.

\begin{tabular}{|c|c|c|c|c|}
\hline 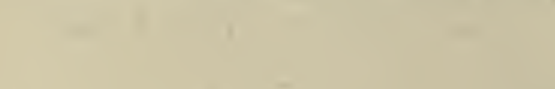 & $\begin{array}{l}\text { Nitrogenous } \\
\text { substances. }\end{array}$ & Fats. & Salts. & Water \\
\hline 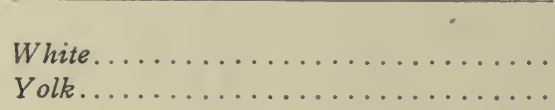 & $\begin{array}{l}20.4 \\
\text { I } 6 .\end{array}$ & … & $\begin{array}{l}\text { I. } 6 \\
\text { I. } 3\end{array}$ & $\begin{array}{l}78 \\
52\end{array}$ \\
\hline
\end{tabular}

Legumes are used by vegetarians as the principal source of the nitrogen of the food. Those chiefly used are peas, beans, lentils, etc.; they contain a nitrogenous substance called legumin, allied to albumin. Legumes contain about 25.30 per cent. of this nitrogenous body and twice as much nitrogen as wheat. Nuts also form a very nutritious article of diet.

Carbohydrate Foods. - Bread, made from ground grain obtained from the various so-called cereals, viz., wheat, rye, maize, barley, rice, oats, etc., is the direct form in which the carbohydrate is supplied in an ordinary diet. It contains starch, dextrin, and a little sugar. It also contains gluten, composed of vegetable proteins, and a small amount of fat.

Table of Percentage Composition of Bread and Flour.

\begin{tabular}{|c|c|c|c|c|c|}
\hline & $\begin{array}{l}\text { Nitrogenous } \\
\text { matters. }\end{array}$ & $\begin{array}{r}\text { Carbo- } \\
\text { hydrates. }\end{array}$ & Fats. & Salts. & Water \\
\hline 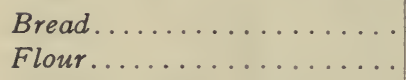 & $\begin{array}{r}8 . \mathrm{I} \\
\text { IO } 8\end{array}$ & $\begin{array}{l}51 \\
70.85\end{array}$ & $\begin{array}{l}\text { I. } 6 \\
2.0\end{array}$ & $\begin{array}{l}2 \cdot 3 \\
1 \cdot 7\end{array}$ & $\begin{array}{l}37 \\
\text { I } 5\end{array}$ \\
\hline
\end{tabular}

Various articles besides bread are made from flour, e.g., spaghetti, macaroni, etc. Dextrin and a small amount of dextrose are present in bread, particularly in the crust.

Vegetables, especially potatoes. They contain starch and sugar. In cabbage, turnips, etc., the salts of potassium are abundant.

Fruits contain sugar and organic acids, tartaric, malic, citric, and others.

Sugar, chiefly saccharose, used pure or in various sweetmeats.

Oils and Fats.-The substances supplying the oils and fats of the food are chiefly butter, bacon and lard, suet (beef and mutton fat), and vegetable oils. These contain the fats olein, stearin, and palmitin. Butter contains some tributyrin, while vegetable oils, as a rule, contain no stearin.

Mineral or Inorganic Foods. - The salts of the food. Nearly all the substances in the preceding classes contain a greater or less amount of the salts required in food. Green vegetables and fruit contain certain salts, chiefly potassium. Sodium chloride is an essential food; it is contained in 
nearly all solid foods, but so much is required that it has also to be taken as a condiment. Potassium salts are found in muscle, nerve, and in meats generally, and in potatoes and other vegetables. Calcium salts are contained in eggs, blood of meat, wheat, and vegetables. Iron is contained in hemoglobin, in milk, eggs, and vegetables.

Liquid Foods. - Water is essential to life, and from two to two and a half pints a day must be consumed in addition to that taken mixed with solid food. Of the non-alcoholic substances which may be added to it for flavoring purposes, such as tea, coffee, cocoa, etc., the last can alone be considered to have a certain food value, as it contains fats, albuminous material, and starch, the other constituents of such substances being a volatile oil, an alkaloid caffeine, and tannic acid. The food value of alcoholic beverages, which has long been a subject of controversy, as now generally agreed is but slight. Beer, wines, and spirits contain ethyl alcohol, the amount varying from I.5 to 4.5 per cent. in beer to 40 to 80 per cent. in spirits.

The Effect of Cooking on Foods.-In general terms cooking may be said to render food more easily digestible, both directly and indirectly, through increased palatability. Subjecting food to high degrees of heat also serves to kill parasites, such as trichinæ and the various tapeworms, which may be present.and alive in raw meats. In the case of meats various methods of cooking are employed. In roasting, the meat in bulk is subjected to a high temperature in an oven for a short time, $250^{\circ} \mathrm{C}$. for $\mathrm{I} 5$ minutes, followed by a somewhat lower temperature, $\mathrm{I} 75^{\circ} \mathrm{C}$., until the cooking is completed. This causes a coagulation of the outer layers of albumin so that the juices of the meat are retained until the center of the mass is cooked to the stage desired, i.e. raised to a temperature of $63^{\circ}$ to $65^{\circ} \mathrm{C}$. when medium done. In boiling, the meat is first immersed in boiling water for a time and then the cooking continues at a lower temperature. In a broth, the extractives may be obtained by heating the meat in water for a long period. Such a broth contains the flavoring and the stimulating extracts of the meat, but is of only slight nutritive value. A temperature below the coagulation point, at $60^{\circ} \mathrm{C}$., will extract more nutritive protein substance. For small pieces of meat, broiling practically serves the same purpose as does roasting for larger pieces. Frying, as usually employed, is the least serviceable method of preparation, since the fat or other oily material used so permeates the food as to render it difficult of penetration by the digestive juices.

Cooking produces upon vegetables the necessary effect of rendering them softer, so that they can be more readily broken up in the mouth. It also causes the starch grains to swell and burst, and so aids the digestive fluids in penetrating into their substance. The albuminous matters are coagulated, and the gummy, saccharine, and saline matters are removed. The conversion of flour into dough is effected by mixing it with water, and adding a little salt and a certain amount of yeast. Yeast consists of the cells of an 
organized ferment (Torula cerevisia); this plant in its growth changes by ferment action the sugar produced from the starch of the flour, and a quantity of carbon dioxide and some alcohol is formed. The gas together with the action of heat during baking causes the dough to rise, and the gluten being coagulated, the bread sets as a permanently vesiculated mass.

\section{THE PROCESS OF DIGESTION.}

The Enzymes.-The digestive process involves both mechanical and chemical changes. The former are secured by the crushing and grinding in the mouth, together with the mixing and kneading that come from the peristalses of the stomach and intestine. The chemical changes are the most important factors of the digestive process. The various secretions that are poured into the mouth, stomach, and intestines all contain substances which react on the foods to render the latter more soluble. The special agency in each secretion is the presence of representatives of the chemical groups known as enzymes. These enzymes, or unorganized ferments, are the essential factors in the secretions which produce the chemical changes in the foods. Their predominant action is one of hydrolytic cleavage; that is, the substance acted upon takes up water and then splits into two different substances, usually of the same class. The chemical nature of the enzymes is as yet undetermined because of the difficulty of getting absolutely pure specimens. Their mode of action is at present regarded in the nature of catalysis. That is to say, the enzymes by their presence facilitate reactions that would otherwise take place but very slowly. Practically all are formed in the glands as zymogens, which bear the same relation to enzymes as fibrinogen does to fibrin; they are transformed to enzymes by the proper stimulus, but never exist as such in the glands.

Each enzyme has a special point of temperature at which it acts best, and any change in the temperature retards its action; the action is suspended at a definite point of low temperature, but the enzyme is not destroyed by cold. The action is suspended at a somewhat higher temperature, and at a still higher point the enzyme is destroyed. Some enzymes act only in an alkaline medium, being destroyed in an acid medium, and vice versa. Others act in either alkaline, or neutral, or acid media. Enzymes are hindered in their action by the accumulation of the products of their activity. Most of them cease acting altogether when these products reach a certain concentration, but will begin acting again on the removal of these products or if the mixture be simply diluted.

The quantity of the enzyme determines the rapidity of the action, but not the amount; a small quantity will digest as much as a large quantity, but will take longer. The enzymes are not used up in the course of their activity, as far as can be seen, and do not seem to undergo any change in their composition. 
Enzymes are more or less specific in their action. That is, each enzyme is supposed to produce its change in only one particular substance, as in starch, maltose, protein, fat, etc. An enzyme that can cause cleavage of the starch molecule will not act on fat or protein or even on other members of the starch group. This specific action is doubtless expressive of a definite relation between the structure of the enzyme and the substance acted on.

An interesting fact as to enzyme action is its reversibility - a phenomenon now well known and well established for carbohydrates and fats. Kastle and Löwenhart have shown that lipase, which acts to split neutral fats into fatty acid and glycerin, will also produce a synthesis, at least of butyric acid and alcohol into ethylbutyrate. Taylor and Robertson in independent papers have recently made the far-reaching discovery that the protein molecule can be synthesized by the agency (apparent reversible action) of enzymes.

Enzymes are classified either according to the chemical nature of their action or according to the class of substances on which they act; the former classification is more logical, but the latter is more convenient and more generally used.

\section{Table of Digestive Enzymes.}

\section{Amylolytic.}

Ptyalin of saliva, and amylopsin of pancreatic juice, change starch to maltose. Maltase in the saliva, and pancreatic juice in the small intestine, change maitose to dextrose. Lactase splits lactose to galactose and dextrose, and invertase splits cane-sugar to levulose and dextrose in the small intestine.

\section{Lipolytic.}

Steapsin or lipase, found in the pancreatic juice, splits neutral fats into glycerin and fatty acid.

\section{Proteolytic.}

Pepsin of the gastric secretion, and trypsin of the pancreatic secretion, change proteins to proteoses and peptones, trypsin breaking the protein down to simpler nitrogenous products. Erepsin of the intestine splits peptones to simpler products.

\section{Coagulating.}

Rennin of the gastric juice coagulates milk.

\section{Activating.}

Enterokinase of the intestinal juice converts trypsinogen to trypsin. (Thrombokinase of the blood is of this class.)

\section{DIGESTION IN THE MOUTH.}

The food is received into the mouth and is subjected to the action of the teeth and tongue, being at the same time mixed with the first of the digestive juices, the saliva. It is then swallowed, and, passing through the pharynx and esophagus into the stomach, is subjected to the action of the gastric juice, the second digestive juice. Thence it passes into the small intestines, where it meets with the bile, the pancreatic juice, and the intestinal juices, all 
of which exercise a digestive influence upon the portion of the food not already digested and absorbed. In the large intestine some further digestion and absorption take place, and the residue of undigested matter leaves the body in the form of feces.

Mastication.-The act of mastication is performed by the biting and grinding movement of the lower range of teeth against the upper. The simultaneous movements of the tongue and cheeks assist by crushing the softer portions of the food against the hard palate and gums, thus supplementing the action of the teeth, and by returning the morsels of food to the action of the teeth as they are squeezed out from between them until they have been sufficiently chewed.

The simple up-and-down or biting movements of the lower jaw are performed by the temporal, masseter, and internal pterygoid muscles, the action of which in closing the jaws alternates with that of the digastric and other muscles passing from the os hyoides to the lower jaw, which open the jaws. The grinding or side movements of the lower jaw are performed mainly by the external pterygoid muscles, the muscle of one side acting alternately with the other. When both external pterygoids act together, the lower jaw is pulled directly forward, so that the lower incisor teeth are brought in front of the level of the upper.

The act of mastication is voluntary. It will suffice here to state that the afferent nerves chiefly concerned are the sensory branches of the fifth, ninth, and tenth, and the efferent are the motor branches of the fifth and the twelfth cerebral nerves.

The act of mastication is much assisted by the saliva, which is secreted by the salivary glands in largely increased amount during the process. The intimate incorporation of the saliva with the food is termed insalivation.

The Salivary Glands. - The glands which secrete the saliva in the human subject are the salivary glands proper, the parotid, the submaxillary, and the sublingual, and numerous smaller bodies of similar structure and with separate ducts, which are scattered thickly beneath the mucous membrane of the lips, cheeks, soft palate, and root of the tongue.

Histological Structure.-The salivary glands are compound tubular or tubulo-racemose glands. They are made up of lobules. Each lobule consists of the branchings of a division of the main duct of the gland, which are generally more or less convoluted toward the extremities, that form the alveoli, or proper secreting parts of the gland. The salivary secreting cells are of cubical or columnar form and are arranged around a central canal. The granular appearance frequently seen in the salivary cells is due to the numerous zymogen granules which they contain.

During the rest period the cells are larger, highly granular, with obscured nuclei and smaller lumen. During activity the cells become smaller and their contents more opaque. 
When the mucous type of gland is secreting, or on stimulation of the nerve, mucinogen is converted into mucin, the cells swell up, appear more transparent and stain deeply in logwood, figure 249. After stimulation, the cells become smaller, more granular, and more easily stained from having discharged their contents, and the nuclei appear more distinct.

Nerves of large size are found in the salivary glands. They are principally contained in the connective tissue of the alveoli, and certain glands, especially in the dog, are provided with ganglia. Some nerves have special

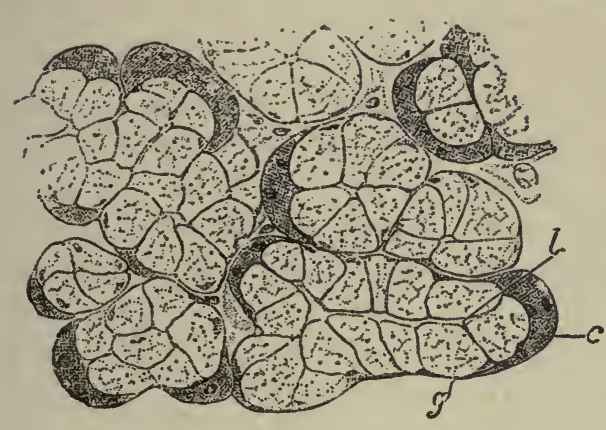

FIG. 248.

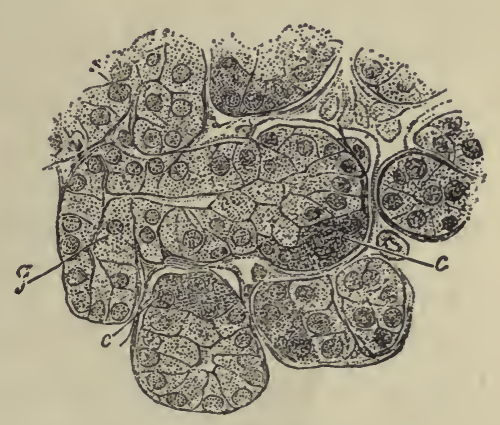

FIG. 249.

Fig. 248.-Section of the Submaxillary Gland of a Dog, Resting Stage. Most of the alveolar cells are large and clear, being filled with the material for secretion (in this case, mucigen), which obscures their protoplasm; some of the cells, however, are small and protoplasmic, forming the crescents seen in most of the alveoli. (Ranvier.)

FIG. 249. - Section of a Similar Gland after a Period of Activity. The mucigen has been discharged from the mucin-secreting cells, which consequently appear shrunken and less clear. Both the cells and the alveoli are much smaller, and the protoplasm of the cells is more apparent. The crescents of Gianuzzi are enlarged. $c$, Crescent cells; $g$, mucussecreting cells; $l$, lumen of alveolus. (Ranvier.)

endings in Pacinian corpuscles, some supply the blood vessels, and others penetrate the basement membrane of the alveoli and end upon, but not in, the salivary cells.

The blood vessels form a dense capillary network around the ducts of the alveoli, being carried in by the fibrous trabeculæ between the alveoli, in which also the lymphatics begin by lacunar spaces.

The Nervous Mechanism of the Secretion of Saliva.-The secretion of saliva is under the control of the nervous system. Under ordinary conditions it is excited by the stimulation of the peripheral branches of two nerves, the gustatory or lingual branch of the inferior maxillary division of the fifth nerve, and of the glosso-pharyngeal, which are distributed to the mucous membrane of the tongue and pharynx conjointly. The stimulation occurs on the introduction of sapid substances into the mouth, and the secretion is brought about in the following way: From the terminations of the above-mentioned sensory nerves distributed in the mucous membrane 
an impression is conveyed upward (afferent) to the special nerve center situated in the medulla oblongata which controls the process, and by it is reflected to certain nerves supplied to the salivary glands, which will be presently indicated. In other words, the center, when stimulated to action by the sensory impressions carried to it, sends out impulses along efferent or secretory nerves supplied to the salivary glands. These cause the saliva to be secreted by, and discharged from, the gland cells. Other stimuli, however, besides that of the food, and other sensory nerves than those mentioned may reflexly produce the same effects. For example, saliva may be caused to flow by irritation of the mucous membrane of the mouth with mechanical, chemical, electrical, or thermal stimuli, also by the irritation of the mucous membrane of the stomach in some way, as in nausea which precedes vomiting when some of the peripheral fibers of the vagi are irritated. Stimulation of the olfactory nerves by smell of food, of the optic nerves by the sight of it, and of the auditory nerves by the sounds which are known by experience to accompany the preparation of a meal may also stimulate the nerve center to action. In addition to these, as a secretion of saliva follows the movement of the muscles of mastication, it may be assumed that this movement stimulates the secreting nerve fibers of the gland, direct or reflexly. From the fact that the flow of saliva may be increased or diminished by mental states, it is evident that impressions from the cerebrum also are capable of stimulating the center to action or of inhibiting its action.

Influence of Nerves on the Submaxillary Gland.-The submaxillary gland has been the gland chiefly employed for the purpose of experimentally demonstrating the influence of the nervous system upon the secretion of saliva, because of the comparative facility with which the gland, with its blood vessels and nerves, can be exposed to view in the dog, rabbit, and other animals.

The chief nerves supplied to the gland are: (I) the chorda tympani, a branch given off from the facial in the canal through which it passes in the temporal bone; and (2) branches of the sympathetic nerve from the plexus around the facial artery and its branches to the gland. The chorda, figure 250, passes downward and forward, under cover of the external pterygoid muscle, and joins the lingual or gustatory nerve, proceeds with it for a short distance, and then passes along the submaxillary-gland duct, giving branches to the submaxillary ganglion, and sending others to terminate in the superficial muscles of the tongue. It consists of fine medullated fibers which lose their medullæ in the gland. If this nerve be exposed and divided anywhere in its course from its exit from the skull to the gland no immediate result will follow, nor will stimulation either of the lingual or of the glosso-pharyngeal produce a flow of saliva. But if the peripheral end of the divided nerve be stimulated, an abundant secretion of saliva ensues, and the blood supply is enormously increased by dilatation of the arteries. 
The veins may even pulsate, and the blood contained within them is more arterial than venous in character.

When, on the other hand, the stimulus is applied to the sympathetic filaments (mere division producing no apparent effect), the arteries contract, and the blood stream is in consequence much diminished; and only a sluggish stream of dark blood escapes from the veins. The saliva, instead of being abundant and watery, becomes scanty and tenacious. If both chorda tympani and sympathetic branches be divided, the gland, released from nervous control, may secrete continuously and abundantly (paralytic secretion).

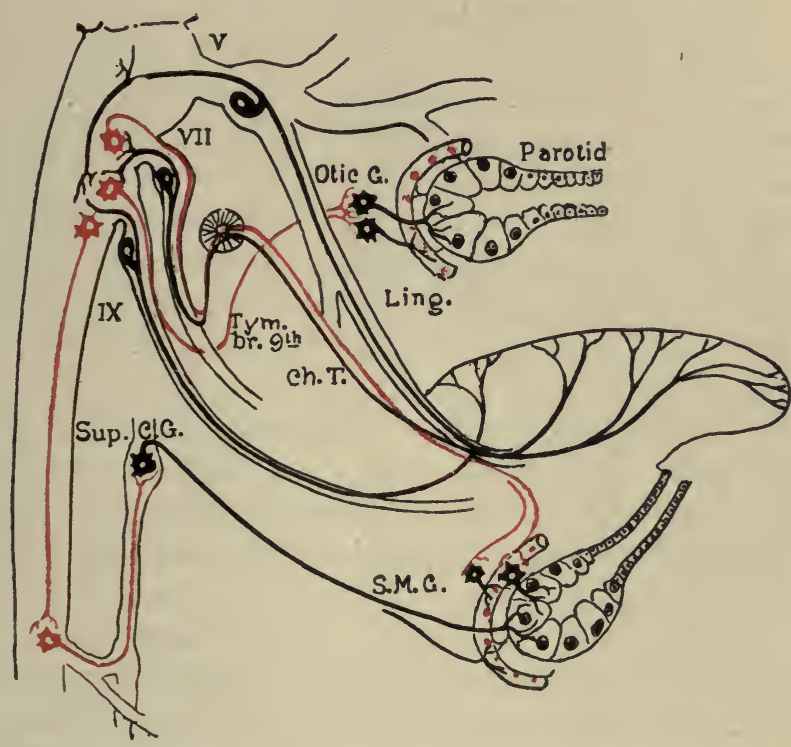

FxG. 250.-Diagram showing the distribution of the cranial and sympathetic secretory and vaso-motor nerves for the parotid and submaxillary glands. The post-ganglionic neurones are in black; the pre-ganglionic neurones including the central neurone of the sympathetic path are in red. (Diagram based on figures by Sheldon, Brubaker, and Starling.)

The abundant secretion of saliva which follows stimulation of the chorda tympani is not merely the result of a filtration of fluid from the blood vessels, in consequence of the largely increased circulation through them. This is proved by the fact that, when the main duct is obstructed, the pressure within may considerably exceed the blood-pressure in the arteries; and also that, when some atropine has been previously injected into the veins of the animal experimented upon stimulation of the peripheral end of the divided chorda produces all the vascular effects as before, without any secretion of saliva accompanying them. Again, if an animal's head be cut off, and the chorda be rapidly exposed and stimulated with an interrupted current, a secretion of 
saliva ensues for a short time, although the blood supply is necessarily absent. These experiments serve to prove that the chorda contains two sets of nerve fibers: one set, vaso-dilator, which, when stimulated, act upon a local vasomotor center for regulating the blood supply, inhibiting its action, and causing the vessels to dilate, and so producing an increased supply of blood to the gland; while another set, which are paralyzed by injection of atropine, directly stimulate the cells themselves to activity, whereby the cells secrete and discharge the constituents of the saliva which they produce, the secretory nerves. These latter fibers very possibly terminate on the salivary cells themselves. If, on the other hand, the sympathetic fibers be divided, stimulation of the tongue by sapid substances, or electrical stimulation of the trunk of the lingual or of the glosso-pharyngeal, continues to produce a flow of saliva. From these experiments it is evident that the chorda-tympani nerve is the principal nerve through which efferent impulses proceed from the center to excite the secretion of this gland.

The sympathetic nerve also contains two sets of fibers, vaso-constrictor and secretory. But the flow of saliva upon stimulating the sympathetic is scanty, and the saliva itself viscid. At the same time the vessels of the gland are constricted. The secretory fibers may be paralyzed by the administration of atropine.

Nerves of the Parotid Gland.-The nerves which influence secretion in the parotid gland are branches of the facial (lesser superficial petrosal) and of the sympathetic. The former nerve, after passing through the otic ganglion, joins the auriculo-temporal branch of the fifth cerebral nerve, and, with it, is distributed to the gland. The nerves by which the stimulus ordinarily exciting secretion is conveyed to the medulla oblongata are, as in the case of the submaxillary gland, the fifth and the glosso-pharyngeal. The pneumogastric nerves convey a further stimulus to the secretion of saliva when food has entered the stomach; the nerve center is the same as in the case of the submaxillary gland.

Changes in the Gland Cells. - The method by which the salivary cells produce the secretion of saliva appears to be divided into two stages, which differ somewhat according to the class to which the gland belongs, viz., whether to (I) the true salivary or to (2) the mucous type. In the former case it has been noticed, as already described, that during the rest which follows an active secretion the lumen of the alveolus becomes smaller, the gland cells larger and very granular. During secretion the alveoli and their cells become smaller, and the granular appearance in the latter to a considerable extent disappears, and at the end of secretion the granules are confined to the inner part of the cell nearest to the lumen, which is now quite distinct, figure $25 \mathrm{r}$.

It is supposed from these appearances that the first stage in the act of secretion consists in the protoplasm of the salivary cell taking up from the 
lymph certain materials from which it manufactures the elements of its own secretion, and which are stored up in the form of granules in the cell during rest; the second stage consists of the actual discharge of these granules, with or without previous change. The granules are zymogen granules, and represent the chief substance of the salivary secretion, ptyalin. In the case of the submaxillary gland of the dog, at any rate, the sympathetic nerve fibers appear to have to do with the first stage of the process, and when stimulated the protoplasm is extremely active in manufacturing the granules, whereas the chorda tympani is concerned in the production of the second act, the actual discharge from the cells of the materials of secretion, together with a

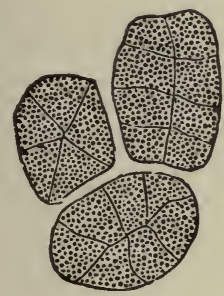

A

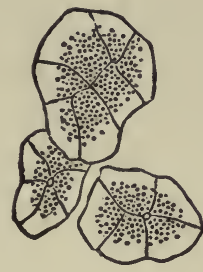

B

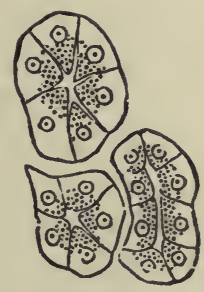

C

FIG. 251.-Alveoli of True Salivary Gland. A, At rest; B, in the first stage of secretion; $\mathrm{C}$, after prolonged secretion. (Langley.)

considerable amount of fluid. The latter is an actual secretion by the protoplasm, as it ceases to occur when atropine has been subcutaneously injected.

In the mucus-secreting gland, the changes in the cells during secretion have been already spoken of. They consist in the gradual production by the protoplasm of the cell of a substance called mucigen, which is converted into mucin, and discharged on secretion into the canal of the alveoli. The mucigen is, for the most part, collected into the inner part of the cells during rest, pressing the nucleus and the small portion of the protoplasm which remains against the limiting membrane of the alveoli.

The process of secretion in the salivary glands is identical with that of glands in general. The cells which line the ultimate branches of the ducts are the agents by which the special constituents of the saliva are formed. The material which they have incorporated within themselves, which is doubtless a product of the metabolism of the protoplasm of the cells, is given up again almost at once in the form of a fluid, secretion, which escapes from the ducts of the gland. The cells themselves undergo diminution in the mass of their protoplasm, which is again renewed in the intervals of the active exercise of the functions. The source whence the cells obtain the materials for the construction of secretion is the blood plasma, which is filtered off from the circulating blood into the interstices of the glands, as in all living tissues. 
Saliva.-Saliva, as it commonly flows from the mouth, is the mixed secretion of the salivary glands proper and of the glands of the buccal mucous membrane and tongue. When obtained from parotid ducts, and free from mucus, saliva is a transparent watery fluid, the specific gravity of which varies from $\mathrm{I} .004$ to $\mathrm{I} .008$ and in which, when examined with the microscope, are found floating a number of minute particles, derived from the secreting ducts and vesicles of the glands. In the impure or mixed saliva are found, besides these particles, numerous epithelial scales separated from the surface of the mucous membrane of the mouth and tongue, and the socalled salivary corpuscles, discharged probably from the mucous glands of the mouth and the tonsils. These subside when the saliva is collected in a deep vessel and left at rest. They form a white opaque sediment leaving the supernatant fluid transparent and colorless, or with a pale bluish-gray tint. Saliva also contains various kinds of micro-organisms (bacteria). The saliva, when first secreted, appears to be always alkaline in reaction; the alkalinity is about equal to 0.08 per cent. of sodium carbonate, and is due to the presence of disodium phosphate, $\mathrm{Na}_{2} \mathrm{HPO}_{4}$.

The presence of potassium sulphocyanide, $\mathrm{KCNS}$, in saliva may be shown by the blood-red coloration which the fluid gives with a solution of ferric chloride, $\mathrm{Fe}_{2} \mathrm{Cl}_{6}$, and which is bleached on the addition of a solution of mercuric chloride, $\mathrm{HgCl}_{2}$, but not by hydrochloric acid.

\section{Chemical Compositron of Human Saliva. (Hammerbacher.)}

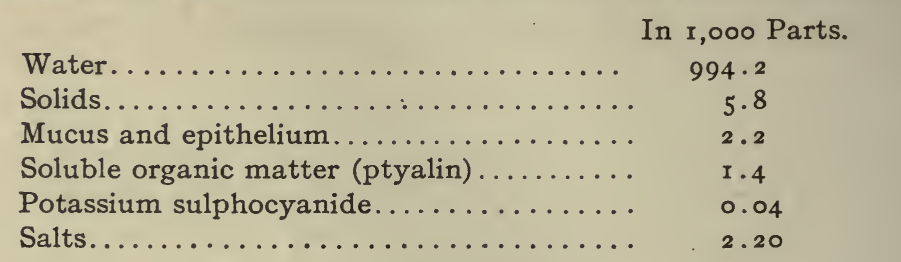

Saliva from the parotid is less viscid; less alkaline, the first few drops discharged in secretion being even acid in reaction; clearer, although it may become cloudy on standing from the precipitation of calcium carbonate by the escape of carbon dioxide; and more watery than that from the submaxillary. It has, moreover, a less powerful action on starch. Sublingual saliva is the most viscid, and contains more solids than either of the other two, but has little diastasic action.

Rate of Secretion and Quantity of Saliva.-The rate at which saliva is secreted is subject to considerable variation. When the tongue and muscles concerned in mastication are at rest, and the nerves of the mouth are subject to no unusual stimulus, the quantity secreted is not more than sufficient with the mucus to keep the mouth moist. During actual secretion the flow is much accelerated. 

I liter.

The quantity secreted in twenty-four hours varies greatly, but is at least

Function of Saliva.-The purposes served by saliva are mechanical and chemical.

Mechanical.-(I) It keeps the mouth in a due condition of moisture, facilitating the movements of the tongue in speaking and in the mastication of food. (2) It serves also in dissolving sapid substances, and renders them capable of exciting the nerves of taste. (3) But the principal mechanical purpose of the saliva is that, by mixing with the food during mastication, it

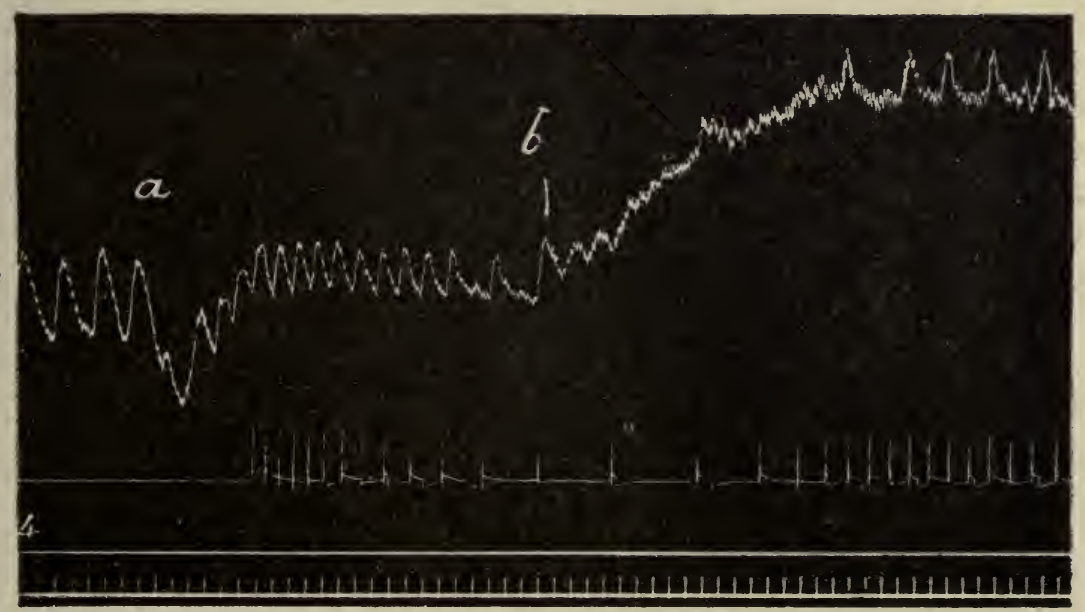

Fig. 252.- Showing the variation of the rate of secretion of saliva, second line from the top, and variation of blood pressure, top line. At $a$, an injection of 0.2 mgr. pilocarpine. At $b, 50$ c.c. oxygenated blood was injected into the jugular vein. (Jonescu.)

makes a soft pulpy or creamy mass such as may be easily swallowed. To this purpose the saliva is adapted both by quantity and quality. For, speaking generally, the quantity secreted during feeding is in direct proportion to the dryness and hardness of the food.

Chemical. - The chemical action which the saliva exerts upon the food in the mouth is to convert the starchy materials which it contains into soluble starch and then into sugar. This power the saliva owes to the enzyme ptyalin. Certain investigators have of late asserted that saliva contains another enzyme, known as maltase, which has the power of splitting the disaccharides into monosaccharides, or maltose into dextrose. The action of this ferment is certainly very limited. The conversion of the starch under the influence of the ferment into sugar takes place in several stages, and in order to understand it a knowledge of the structure and composition of starch granules is necessary. A starch granule consists of two parts: an en- 
velope of cellulose, which does not give a blue color with iodine except on addition of sulphuric acid, and of gramulose, which is contained within, and which gives a blue color with iodine alone. Brücke states that a third body is contained in the granule, which gives a red color with iodine, viz., erythrogramulose. The granulose swells up on boiling, bursts the envelope, and the whole granule is more or less completely converted into a paste or gruel which is called gelatinous starch.

When ptyalin acts upon boiled starch, it first changes the latter, by hydrolysis, into soluble starch, or amidulin; this is more limpid and more like a true solution, though it still gives the blue coloration on the addition of iodine. This stage is very brief, only thirty seconds being sometimes required in laboratory experiments to render a stiff starch paste completely fluid when a few drops of saliva are added at body temperature. This rapidity of action is of great importance, as under proper conditions of mastication practically all the boiled starch of the food ought to enter the stomach as soluble starch. When the starch has not been previously boiled, the envelope of cellulose retards the action of the ptyalin to a very marked degree.

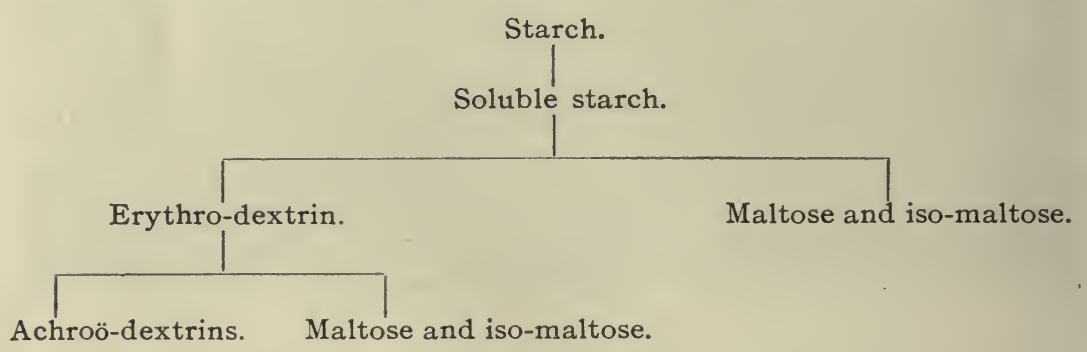

The further stages of hydrolytic cleavage result in the formation of a variable mixture of maltose and iso-maltose with a series of dextrins, but apparently never result (in laboratory experiments) in the complete conversion of the dextrins into sugars. Gradually, as the starch is converted, the blue coloration with iodine is replaced by a purplish-red and finally by a red color: the latter color is produced by erythro-dextrin (so called from the color). In the later stages no coloration is obtained with iodine, and for this reason the dextrins formed are known as achroö-dextrins; there are probably several of these, but they have not yet been sufficiently isolated. As sugar appears very early in the process, even at the stage of erythro-dextrin, and gradually increases in amount, it is generally concluded that maltose is formed early in the decomposition of the starch molecule. The process is usually represented schematically as above.

The sugars formed are maltose $\left(\mathrm{C}_{12} \mathrm{H}_{22} \mathrm{O}_{11}\right)$ and a closely allied sugar known as iso-maltose. A small percentage of dextrose has been found by some observers, and this is due to the action of maltase. Maltose is allied 
to saccharose or cane-sugar more nearly than to glucose; it is crystalline; its solution has the property of polarizing light to the right to a greater degree than solutions of glucose ( 3 to $\mathrm{I}$ ); it is not so sweet, and reduces copper sulphate less easily. It can be converted into glucose by boiling with dilute acids and by the action of the enzyme maltase present in saliva.

According to Brown and Heron, the reactions may be represented thus:

One molecule of gelatinous starch is converted by the action of an amylolytic ferment into $n$ molecules of soluble starch.

One molecule of soluble starch $=\left(\mathrm{C}_{12} \mathrm{H}_{20} \mathrm{O}_{10}\right)_{10}+8 \mathrm{H}_{2} \mathrm{O}$, which is further converted by the ferment into

r. Erythro-dextrin, $\left(\mathrm{C}_{12} \mathrm{H}_{20} \mathrm{O}_{10}\right)_{9}$ (giving red with iodine) + Maltose $\left(\mathrm{C}_{12} \mathrm{H}_{22} \mathrm{O}_{11}\right)$.

then into 2. Erythro-dextrin $\left(\mathrm{C}_{12} \mathrm{H}_{20} \mathrm{O}_{10}\right)_{8}$ (giving yellow with iodine) + Maltose $2\left(\mathrm{C}_{12} \mathrm{H}_{22} \mathrm{O}_{11}\right)$.

next into 3. Achroö-dextrin $\left(\mathrm{C}_{12} \mathrm{H}_{20} \mathrm{O}_{10}\right)_{7}+$ Maltose $3\left(\mathrm{C}_{12} \mathrm{H}_{22} \mathrm{O}_{11}\right)$.

And so on; the resultant being:

Soluble starch $\left(\mathrm{C}_{12} \mathrm{H}_{20} \mathrm{O}_{10}\right)_{10}+$ Water $8 \mathrm{H}_{2} \mathrm{O}=$ Maltose $8\left(\mathrm{C}_{12} \mathrm{H}_{22} \mathrm{O}_{11}\right)+$ Achrö̈-dextrin $\left(\mathrm{C}_{12} \mathrm{H}_{20} \mathrm{O}_{10}\right)_{2}$.

Many observers, however, believe that the maltose simultaneously present with erythro-dextrin is not actually split off from the starch molecule in the formation of erythro-dextrin, but that it is the product of more advanced hydrolysis in other starch molecules. They point out that in such a chemical reaction of considerable time duration, it is improbable that all the starch molecules are attacked at the same rate or are, at any given moment, equally advanced in cleavage. Their theory is that there is a series of more and more simple dextrins formed giving rise finally to the disaccharides.

The presence of sugar in such an experiment is at once discovered by the application of Trommer's test, which consists in the addition of a drop or two of a solution of copper sulphate, followed by a larger quantity of caustic potash. When the liquid is boiled, an orange-red precipitate of copper suboxide indicates the presence of sugar.

Influences which Affect the Action of Saliva on Starch.-Moderate heat, about $37.8^{\circ}$ to $40^{\circ} \mathrm{C}$., is most favorable to the rapid cleavage of starch by the ptyalin. Cold retards and $\circ^{\circ} \mathrm{C}$. suspends the action but does not destroy the ferment. A temperature of $60^{\circ} \mathrm{C}$. destroys the ptyalin.

Removal of the products of salivary digestion as they are formed facilitates the action of the enzyme, as an excess of these products is detrimental to further action.

The reaction between starch and saliva takes place best in a neutral or very faintly alkaline medium and is inhibited by strong alkalies and especially by acids even as weak as the acidity of the gastric juice. This last is of particular importance since it raises the question as to how long the ptyalin may act. 
The action of saliva on starch is not limited to the brief interval during which food remains in the mouth, as is now well known, but may continue for a time in the stomach.

Ptyalin is strictly an amylolytic ferment.

Starch appears to be the only principle of food with the exception of the dextrins and glycogen, upon which the saliva acts chemically. The secretion has no apparent influence on gum, cellulose, or on fat, and is equally destitute of power over albuminous and gelatinous substances.

The salivary glands of children do not produce functionally active saliva till the age of 4 to 6 months, and hence the bad effects of feeding them before this age on starchy food, corn-flour, etc., which they are unable to render soluble and capable of absorption.

Salivary Digestion in the Stomach.-Laboratory experiments have demonstrated that while the addition of even 0.05 per cent. of hydrochloric acid will inhibit the action of ptyalin on a solution of starch, if any proteins be present in the solution much more acid must be added before the action of the ptyalin is stopped. The explanation of the latter fact is that the acid unites with the proteins in some chemical combination forming "combined acid," which has little effect, comparatively, on ptyalin. This "combined acid" gives a red color with litmus, but is distinguished from free acid by giving a brownish instead of a bluish color with Congo red. When food enters an empty stomach, as happens at the beginning of a meal the acid first combines with the protein food stuffs and so does not at once affect the ptyalin.

A still more important fact in its bearing on this subject was recently discovered by Cannon, who showed experimentally that starchy foods mixed with weak alkali remain alkaline in the stomach for as much as an hour and a half. Such foods when swallowed into the stomach are packed away in that organ in a mass. The secretion of the acid gastric juice comes in contact only with the outer surface of the mass, which is not materially disturbed by the stomach peristalses. The center of the mass may, therefore, remain alkaline until the outer layers are completely eroded away, and the ptyalin may continue to act on starch during the whole time.

\section{DEGLUTITION.}

When properly masticated, the food is transmitted in successive portions to the stomach by the act of deglutition or swallowing. The following account of deglutition is based upon the researches of Kronecker and Meltzer, whose experiments seem to modify in some details the earlier theory of Magendie:

The mouth is closed, and the food after thorough mixing with the saliva is rolled into a bolus on the dorsum of the tongue. The tip of the tongue is pressed upward and forward against the hard palate, thus shutting off the anterior part of the mouth cavity. The mylo-hyoid muscles then suddenly 
contract, the bolus of food is put under great pressure and shot backward and downward through the pharynx and into the esophagus and, if the food be fluid enough, even to the cardiac orifice of the stomach. Coincidently with the contraction of the mylo-hyoid muscles, the hyoglossi are thrown into action, drawing the tongue backward and downward, not only increasing the pressure upon the food, but forcing the epiglottis over the glottis, closing the larynx.

It has been shown by the Roentgen-ray method that the character of the food determines somewhat its passage through the esophagus. The dry and semisolid foods are seized by the musculation of the esophagus and passed down that organ by a peristaltic wave. The longitudinal muscles contract, tending to enlarge the diameter of the esophagus in advance of the food, while contractions of the circular muscles produce pressure on the bolus just behind, thus forcing it along to the cardia. This wave reaching the cardiac orifice about six seconds after the commencement of the act of deglutition, forces the food into the stomach, the sphincter having previously relaxed. The interval of time between the commencement of the act of deglutition and the arrival of the more fluid food at the cardiac orifice of the stomach may not be more than one-tenth second, though it remains at the cardiac orifice without entering the stomach until the first parts of the act of swallowing is reinforced by the subsequent contraction of the constrictors of the pharynx and the passage of a peristaltic wave down the esophagus. In some cases, however, the liquid food is not stopped at the cardiac orifice, but is sent through the relaxed sphincter by the original force of the mylohyoid contraction.

In man the esophagus was said to contract in three separate segments, the first segment lying in the neck and being about six centimeters long, the second being the next ten centimeters of the tube, and the third the remaining portion to the stomach. But the later Roentgen-ray observations show no break in the continuous passage of the food, though the movement of the food is slower in the lower segment of the esophagus.

The act of swallowing consists, then, of the contraction in sequence of the mylo-hyoids, the constrictors of the pharynx, and of the esophagus. The computed time of contraction is as follows:

Seconds.

Contraction of mylo-hyoids and constrictors of the pharynx... 0.3

Contraction of the first part of the esophagus......... 0.9

Contraction of the second part of the esophagus......... I.8

Contraction of the third part of the esophagus.......... 3.0

6.0

If a second attempt at swallowing be made before the first has been completed (that is, before six seconds have elapsed), the remaining portion of the 
first act is inhibited, and the contraction wave reaches the stomach six seconds after the commencement of the second act.

During the act of deglutition the posterior nares are closed through the action of the levator palati and tensor palati muscles, which raise the velum; the palato-pharyngei, drawing the posterior pillars of the fauces together; and the azygos uvulæ, which raises the uvula-thus forming a complete curtain. Otherwise the food would pass into the nose, as happens in the case of cleft palate. At the same time the larynx is closed by the adductor muscles of the vocal cords and the descent of the epiglottis, the larynx being drawn upward as a whole through the action of the mylo-hyoid, genio-hyoid, thyro-hyoid, and digastric muscles. The presence of the epiglottis is not necessary for the completion of the act of deglutition.

Nervous Mechanism of Deglutition.-The sensory nerves engaged in the reflex act of deglutition are branches of the fifth cerebral, supplying the soft palate; the glosso-pharyngeal, supplying the tongue and pharynx; the superior laryngeal branch of the vagus, supplying the epiglottis and the glottis. The motor fibers concerned are branches of the fifth, supplying part of the digastric and mylo-hyoid muscles and the muscles of mastication; the facial, supplying the levator palati; the glosso-pharyngeal, supplying the muscles of the pharynx; the vagus, supplying the muscles of the larynx through the inferior laryngeal branch; and the hypoglossal, the muscles of the tongue. The nerve center by which the muscles are harmonized in their action is situated in the medulla oblongata. It cannot be definitely circumscribed, but is in the general level of the vagus origin. The movements of the esophagus are co-ordinated by the complex of sensory and motor fibers of the fifth and the ninth to twelfth cranial nerves, which all take some part in this complicated reflex.

Cannon has demonstrated that the smooth muscle of the lower end of the esophagus and around the cardiac orifice is maintained in contraction by a local reflex mechanism. This prevents regurgitation of the foods. The local apparatus is brought into action by the stimulation of sensory cells in the mucous membrane of this region of the stomach by the acid of the gastric secretion. The reflex is assumed to be a local one taking place through the intrinsic nervous mechanism. This acid closure of the cardiac sphincter is to be compared with the similar mechanism for the pylorus, see page $35^{6}$.

\section{DIGESTION IN THE STOMACH.}

The stomach of man and the carnivora is the dilated portion of the alimentary canal following the esophagus. The esophagus enters the stomach at the cardiac end and the pyloric end of the stomach is continuous with the duodenal part of the intestine. It varies in shape and size according to its state of distention. It is supplied with nerves from the vagus and from the sympathetic and receives a special artery, the gastric artery. 
Structure of the Stomach.-The stomach is composed of four coats, called, respectively, the external or peritoneal, the muscular, the submucous,

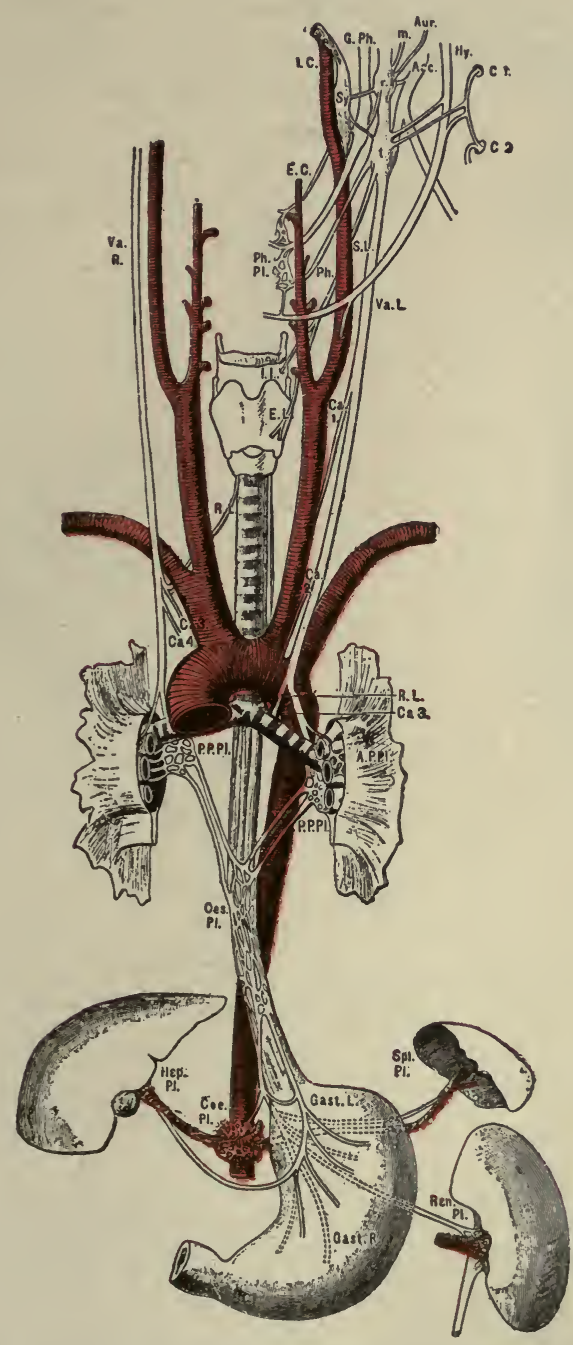

FIG. 253. - The Human Stomach and the Vagus Distribution. R. L., Recurrent laryngeal; $\mathrm{Ca}_{2}$, inferior cervical cardiac branch; $\mathrm{Ca}_{3} ; \mathrm{Ca}_{4}$, cardiac branches of vagus; A.P.Pl., P.P.Pl., anterior and posterior pulmonary plexuses; Oes. $P l$., esophageal plexus; Gast. $R$. and $L$., gastric branches of vagus, right and left; $\mathrm{Coe}, \mathrm{Pl}$., coeliac plexus; $\mathrm{Hep}$. pl., hepatic plexus.

and the mucous coat. Blood vessels, lymphatics, and nerves are distributed in and between them. 
The muscular coat consists of three separate layers of fibers which, according to their several directions, are named the longitudinal, circular, and oblique. The longitudinal set are the most superficial and are continuous with the longitudinal fibers of the esophagus and spread out in a diverging

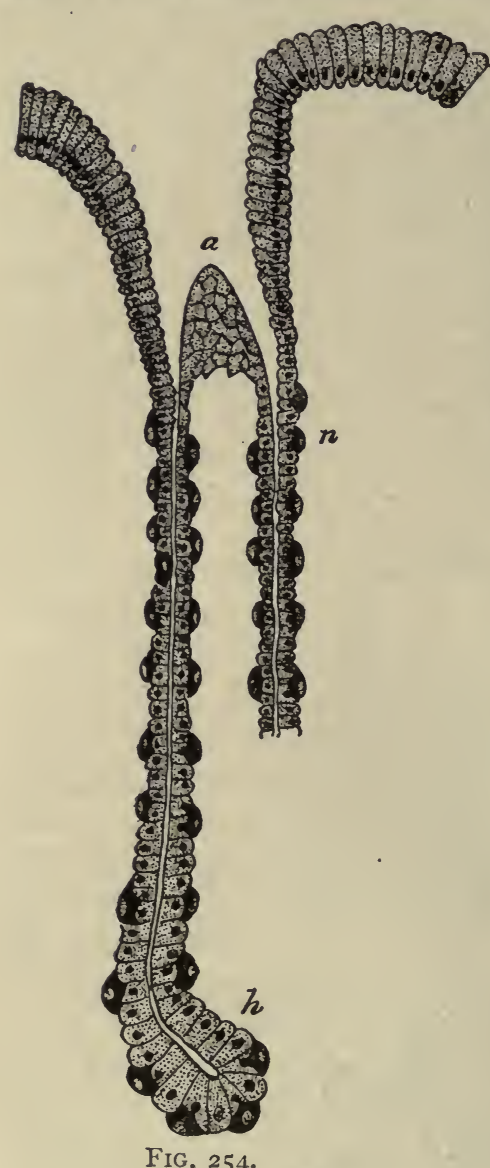

manner over the cardiac end and sides of the stomach to the pylorus. The circular or transverse coat more or less completely encircles all parts of the stomach; this coat is thickest at the middle and in the pyloric portion of the organ, and forms the chief part of the thick ring of the pylorus. The next and consequently deepest coat, the oblique, is continuous with the circular muscular fibers of the

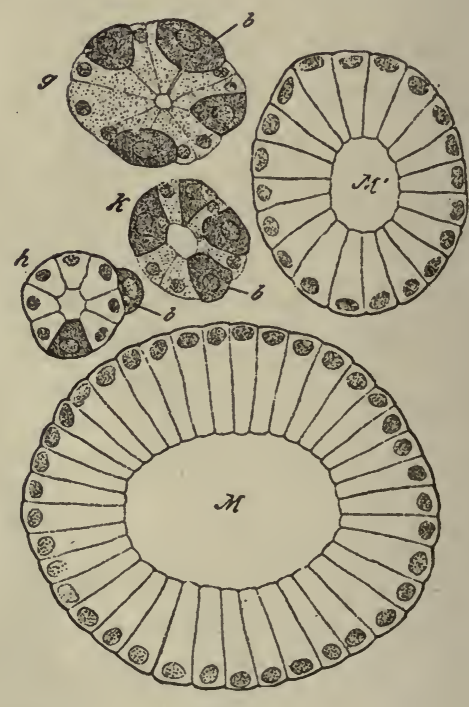

FIG. 255 .

FIg. 254.-From a Vertical Section through the Mucous Membrane of the Cardiac End of Stomach. Two peptic glands are shown with a duct common to both, one gland only in part. $a$, Duct with columnar epithelium becoming shorter as the cells are traced downward; $n$, neck of gland tubes, with central and parietal or so-called peptic cells; $h$, fundus with curved cecal extremity - the parietal cells are not so numerous here. $\times 400$. (Klein and Noble Smith.)

FIG. 255.-Cross-sections at Various Levels of Peptic Glands of Stomach. $\times 400$. $M$, Section through gastric pit near surface; $M^{\prime}$, section through gastric pit near bottom; $h$, mouth of gland; $k$, neck; $g$, body near fundus; the chief cells are shaded lightly; $b$, parietal cells. (Kölliker.)

esophagus at the cardiac orifice of the stomach. This coat is quite interrupted and more or less incompleie. The muscular fibers of the stomach and intestinal canal are unstriated. 
The mucous membrane of the stomach, which rests upon a layer of loose cellular membrane, or submucous tissue, is smooth, soft and velvety. It is of a pale pink color during life, and in the contracted state is thrown into numerous longitudinal folds or rugæ, which disappear when the organ is distended. It is composed of a mass of short tubular secreting glands.

The Gastric Glands. - The glands of the mucous membrane of the stomach are of two varieties, Cardiac and Pyloric.

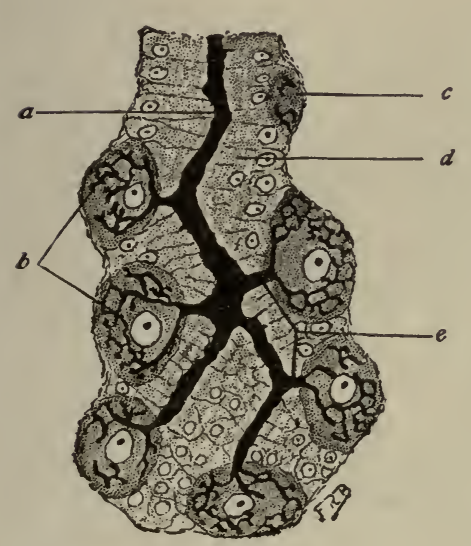

FIG. 256.

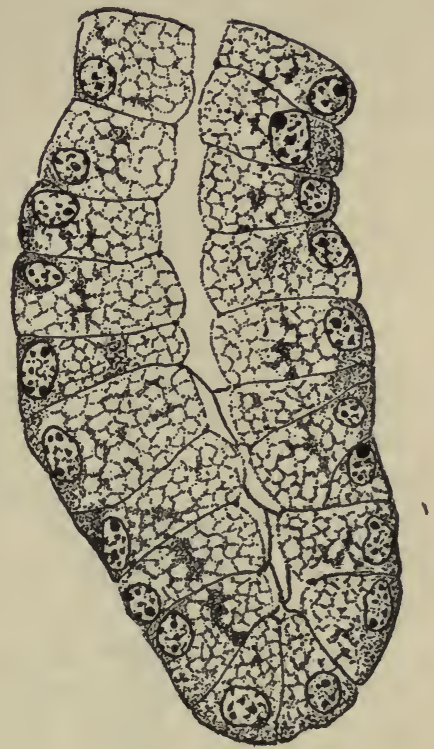

FIG. 257 .

FIG. 256.-Longitudinal Section of Fundus of Gland from Dog's Stomach. $a$, Lumen of gland; $b$, intracellular canals in parietal cells; $c$, cut-off portion of parietal cell; $d$, chief cells; $e$, intercellular canals leading from lumen of gland to canals in parietal cells. (Bailey.)

FIG. 257.-Tubule of Pyloric Gland of Man. Note the thin basal layer of cytoplasm; the reticular cell body containing secretion; the subdivision of the latter in some cells into proximal and distal masses. Highly magnified. (Bailey.)

Cardiac glands are found throughout the whole of the cardiac end of the stomach. They are arranged in groups of four or five, which are separated by a fine connective tissue. Two or three tubes often open into one duct, figure 254 , which forms about a third of the whole length of the tube and opens on the surface. The ducts and the free surface are lined with columnar epithelium. The body of the gland is composed of granular secreting cells, called chief cells or peptic cells. Between these cells and the membrana propria of the tubes are large oval or spherical cells, granular in appearance with clear oval nuclei; these cells are called parietal cells. They do not form a continuous layer, figure 254. Intercellular tubules extending from 
the duct of the gland between the chief cells and connecting with intracellular secretory tubules in the parietal cells have been shown by the Golgi silver method, by napthol blue, etc., figure 256 .

As the pylorus is approached the gland ducts become longer and the tube proper becomes shorter, and occasionally branched at the fundus.

The Pyloric Glands. - These glands have much longer ducts and larger mouths than the peptic glands.

The parietal cells are absent in the pyloric glands. The pyloric glands become larger as they approach the duodenum, also more convoluted and more deeply situated. They are directly continuous with Brunner's glands in the duodenum (Watney).

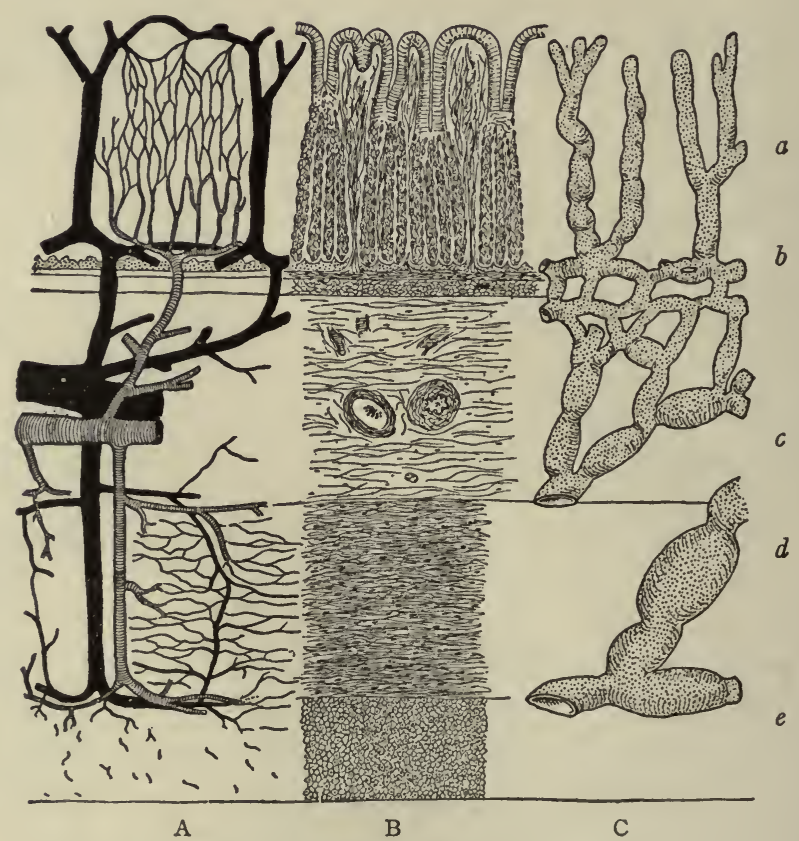

FIG. 258.-Scheme of Blood Vessels and Lymphatics of Stomach. $\times$ 70. a, Mucous membrane; $b$, muscularis mucosæ; $c$, submucosa; $d$, inner circular muscle layer; $e$, outer longitudinal muscle layer; $A$, blood vessels; $B$, structure of coats; $C$, lymphatics. (Szymonowicz, after Mall.)

Blood vessels and Lymphatics. - The blood vessels of the stomach first break up in the submucous tissue and send branches upward between the closely packed glandular tubes, which anastomose around them by a fine capillary network with oblong meshes. Contiguous with this deeper plexus, or prolonged upward from it, so to speak, is a more superficial network of larger capillaries, which branch densely around the orifices of the tubes and form the framework on which are molded the small elevated ridges of mucous 
membrane. From this superficial network the veins chiefly take their origin, pass down between the tubes, with no very free connection with the deeper intertubular capillary plexus, and open finally into the venous network in the submucous tissue.

The lymphatic vessels surround the gland tubes with a network. Toward the fundus of the peptic glands are masses of lymphoid tissue which may appear as distinct follicles, somewhat like the solitary glands of the small intestine.

\section{Microscopic Changes in the Gastric Glands During Secretion.-} Langley has made a study of the histological changes in the glandular tissues in the fresh state. He finds that during fasting or when the glands are at rest the chief cells are granular throughout, being crowded with large highly refractive granules. During activity these granules gradually disappear progressively from the base toward the border of the cell on the lumen of the tube. They no doubt represent the zymogen substances from which the first discharge of enzyme is derived during the activity of secretion. The parietal cells are finely granular throughout, though they decrease in size during activity, as in fact do the chief cells. Macallum by the use of microchemical tests has shown the presence of excess of chlorides in the ducts and intracellular canals, and in the parietal cells. The pyloric cells do not undergo such marked changes, and the mucous cells of the more superficial layers of the mucosa cannot be said to show any special changes at the time of digestional activity of the other layers. During periods of rest the gastric cells increase in size and again become charged with granules as before.

The Act of Secretion of Gastric Juice.-The gastric glands undergo periods of rest and activity. The active secretion of normal gastric juice takes place when food is introduced into the mouth, or in fact the mere sight of appetizing food is followed by an abundant secretion of gastric juice, as shown by Bidder and Schmidt on the dog with a gastric fistula. Such observations strongly indicate that the act is a nervous phenomenon, at least under nervous control.

Quite recently Pavlov has proved that secretory fibers are carried to the gastric glands in the vagus trunk. His experiment consisted in establishing a gastric fistula, and some days later in dividing the esophagus in the neck in such a manner that any food swallowed would be diverted to the exterior through the cut end. A "fictitious meal" could then be given to the animal, and the effect upon the stomach noted. As long as the vagi were intact, certain foods (meats) caused a flow of gastric juice, though none of the food reached the stomach. The secretion of gastric juice continued for hours with the production of a large quantity of secretion. When the vagi had been cut, no secretion occurred. Moreover, he found that direct stimulation of the vagus produced a flow of gastric juice.

Khigine placed foods in an isolated gastric pouch prepared with care to 
maintain the nervous relations intact, and it led to secretion of gastric juice in the main part of the stomach. This is undoubtedly a nervous reflex effect.

Recently observations on a case of stricture of the human esophagus which prevented food from reaching the stomach have shown that an abundant flow of gastric juice takes place when food is taken into the mouth.

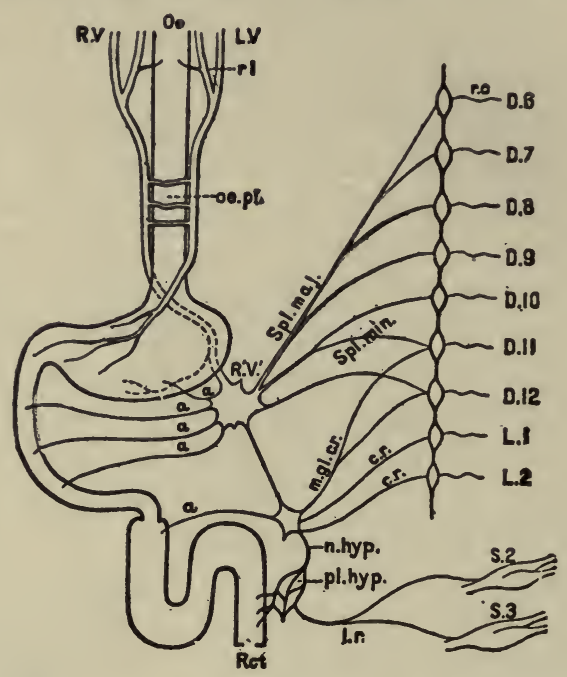

FIG. 259.-Very Diagrammatic Representation of the Nerves of the Alimentary Canal. $O e$ to $R c t$, the various parts of the alimentary canal from esophagus to rectum; $L$. $V$, left vagus, ending on front of stomach; $r l$, recurrent laryngeal nerve, supplying upper part of esophagus; $R$. $V$, right vagus, joining left vagus in esophageal plexus; $\propto$. pl., supplying the posterior part of stomach, and continues as $R^{\prime} V^{\prime}$ to join the solar plexus, here represented by a single ganglion, and connected with the inferior mesenteric ganglion, $m$. $g l . ; a$, branches from the solar plexus to stomach and small intestine, and from the mesenteric ganglia to the large intestine; $S p l$. maj., large splanchnic nerve, arising from the thoracic ganglia and rami communicantes; $r$. $c$., belonging to dorsal nerves from the 6 th to the gth (or Ioth); Spl. min., small splanchnic nerve similarly from the roth and IIth dorsal nerves. These both join the solar plexus, and thence make their way to the alimentary canal; $c . r$., nerves from the ganglia, etc., belonging to $\mathrm{IIth}$ and $\mathrm{r} 2 \mathrm{th}$ dorsal and Ist and $2 \mathrm{~d}$ lumbar nerves, proceeding to the inferior mesenteric ganglia (or plexus), $m . g l$., and thence by the hypogastric nerve, $n$. hyp., and the hypogastric nerve, $n$. hyp., and the hypogastric plexus, pl. hyp., to the circular muscles of the rectum; $l$. $r$., nerves from the $2 \mathrm{~d}$ and $3 \mathrm{~d}$ sacral nerves, S. 2, S. 3 (nervi erigentes) proceeding by the hypogastric plexus to the longitudinal muscles of the rectum. (M. Foster.)

It seems conclusively established at the present time that the secretion of gastric juice is a reflex act controlled by a definite nervous mechanism. This reflex can be aroused by the sensory stimuli of taste, smell, and even sight. It can also be initiated by stimuli arising in the stomach itself by the effects of ingredients of the food or by the products of digestion. Indeed, it has been shown that peptone is a very efficient stimulus for this stomach reflex.

Edkins, however, has recently shown that the contact of certain food products with the pyloric end of the stomach, where they are slightly absorbed, gives rise to some chemical substance-a gastric hormone or secre- 
tagogue-which acts as a powerful stimulus to gastric secretion when it is introduced into the circulation. Such food substances are dextrins, maltose and dextrose, proteoses, and above all meat extract.

The influence of the higher nerve centers on gastric digestion, as in the case of emotions, is too well known to need more than a reference.

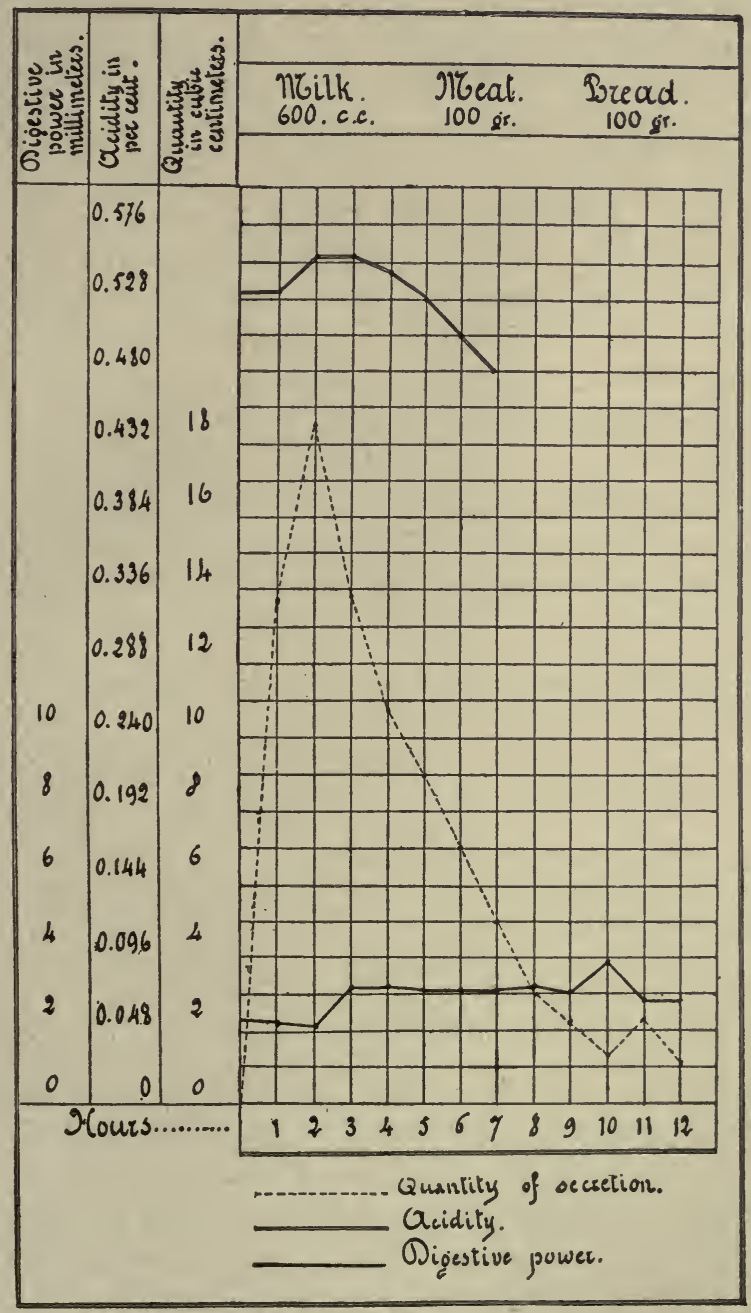

FIg. 260.-Table to show the Secretion of Gastric Juice by the Dog. (Lliffine.)

Immediately on the introduction of food or other stimulating substance, the mucous membrane, which was previously quite pale, becomes slightly turgid and reddened with the influx of a larger quantity of blood, and the gastric glands commence actively to secrete. An acid fluid is poured out in minute drops and the secretion may continue for hours. 
The Gastric Juice.-The first analysis of gastric juice was made by Prout on a small and impure specimen. Beaumont made an elaborate and classic series of observations on the gastric secretion of Alexis St. Martin, in whom there existed, as the result of a gunshot wound, an opening leading directly into the stomach near the upper extremity of the great curvature and three inches from the cardiac orifice. The introduction of any mechanical irritant, such as the bulb of a thermometer, into the stomach through this artificial opening excited the secretion of gastric fluid. This was drawn off, and was often obtained to the extent of nearly an ounce.

The chemical composition of human gastric juice has been also investigated by Schmidt. The fluid in this case also was obtained by means of an accidental gastric fistula. The mucous membrane was excited to action by the introduction of some hard matter, such as dry peas, and the secretion was removed by means of an elastic tube. The fluid obtained was found to be acid, limpid, odorless, with a specific gravity of $\mathrm{I} .002$ to $\mathrm{I}$. oro. It contained a few cells and some fine granular matter. The analysis of the fluid obtained in this way is given below. Essentially it is a weakly acid fluid containing hydrochloric acid and enzymes, of which pepsin and rennin are the chief, though lipase and maltase are both present. The gastric juice obtained from gastric fistulas of dogs and other animals shows some difference in composition.

Chemical Composition of Gastric Juice (Schmidt).

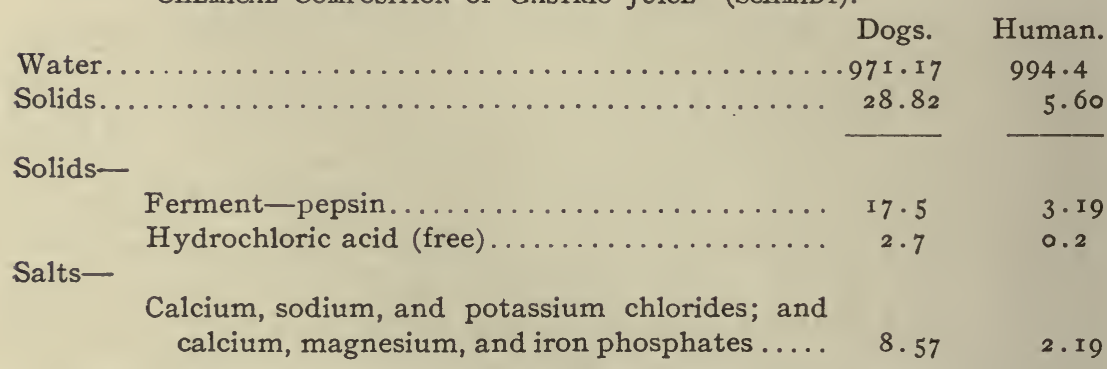

The quantity of gastric juice secreted daily has been variously estimated; but the average for a healthy adult may be assumed to range from 2,000 to 3,000 cubic centimeters in the twenty-four hours.

The Nature and Origin of the Acid of Gastric Juice.-The acidity. of the fluid is due to free hydrochloric acid, although other acids, e.g., lactic, acetic, butyric, are not infrequently to be found therein as products of gastric digestion or abnormal fermentation. In healthy gastric juice the amount of free hydrochloric acid is usually about 0.2 per cent., but may be as much as 0.3 per cent. In pathological conditions it may be entirely absent, or may amount to 0.5 per cent., or even more.

Hydrochloric acid is the proper acid of healthy gastric juice, and various tests have been used to prove this. The tests depend upon changes produced 
in aniline colors by the action of hydrochloric acid even in minute traces, whereas lactic and other organic acids have no such action. An aqueous solution of oo-tropeolin, a bright yellow dye, is turned red on the addition of a minute trace of hydrochloric acid, and aqueous solutions of methyl violet and gentian violet are turned blue under the same circumstances.

The protein matter in the food combines to some extent with the hydrochloric acid, which then is known as combined acid and does not redden litmuspaper. As this combination is immediate, it follows that no free acid is found in the gastric contents until the amount secreted is more than enough to saturate the various albuminous affinities. It is partly for this reason that, as already mentioned, salivary digestion may continue in the stomach for some time after the commencement of gastric digestion. According to Ehrlich, the amount necessary to saturate the affinities of 100 grams of various articles of diet is as follows:

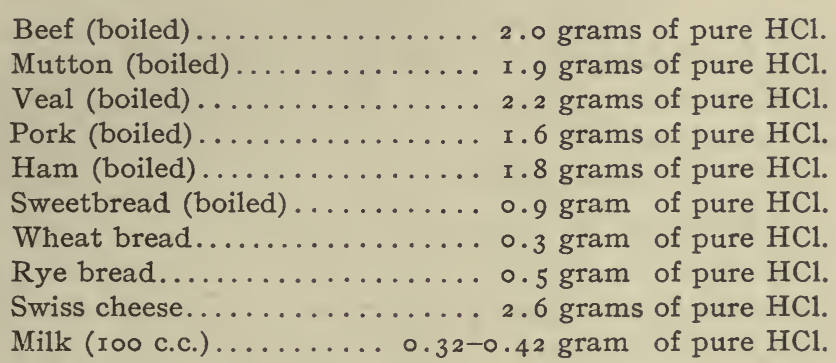

The acid of the gastric juice is not found until after the secretion is poured out on the surface of the mucous membrane of the stomach. Thus Claude Bernard after microscopic examination said that there was no acid in the gastric glands, that "the acid of the gastric juice is formed only after the secretion of the juice, the glands secreting a liquid which breaks up into an acid fluid and another product as yet not definitely determined." Harvey and Bensley, from whom the translation just given is quoted, confirm Bernard's views completely. They find by an exhaustive study and by ingenious staining methods for identifying alkalinity and acidity, that the acid of gastric juice does not make its appearance until the secretion reaches the open mouths of the glands and the surface of the mucosa. They observe that in the gland ducts the secretion is viscid, adherent, stainable, and "breaks up into round droplets which maintain their individuality for several minutes," noting "the red reaction also at the same time slowly changing to the blue acid reaction, if the secretion has been stained with cyanamin." "From these observations we are obliged to conclude that the secretion formed in the gland possesses a relatively high content of solids, and that the bulk of the water found in the gastric secretion is added at the level of the glandular foveolæ." The parietal cells are alkaline in reaction and not acid, as are in fact all the tissues of the gland. However, the observation is well established that the 
paretal cells are peculiarly rich in chlorides, and these chlorides enter into the composition of the secretion and apparently are the final source of the hydrochloric acid formed in the secretion.

Malay holds that the acid probably results from a combination of common salt with monosodic phosphate, $\mathrm{NaH}_{2} \mathrm{PO}_{4}+\mathrm{NaCl}=\mathrm{Na}_{2} \mathrm{HPO}_{4}+\mathrm{HCl}$; the disodic phosphate is then reconverted by the action of carbonic acid and water, $\mathrm{Na}_{2} \mathrm{HPO}_{4}+\mathrm{CO}_{2}+\mathrm{H}_{2} \mathrm{O}=\mathrm{NaH}_{2} \mathrm{PO}_{4}+\mathrm{NaHCO}_{3}$. All these salts are found in the gastric secretion. However, Harvey and Bensley believe that the hydrochloric acid is derived from an organic combination of the chlorides in the secretion, the nature of which is not determined.

The Pepsin.-The pepsin of the gastric juice is derived from the activity of the chief cells of the fundic glands. The zymogen, pepsinogen, which is its immediate precursor, is in all probability represented by the granules of the resting cells. The ferment pepsin does not exist as such in the cells, for an extract of peptic glands in 0.2 per cent. soda solution kept at $40^{\circ} \mathrm{C}$. retains for hours its power to digest protein when added to 0.2 per cent. hydrochloric acid. If the extract be first treated with acid till it is active, then neutralized and kept, it quickly loses its power to digest. The enzyme is destroyed by the treatment, but the pro-enzyme is not so injured.

Digestive Action of Pepsin and Hydrochloric Acid.-The chief function of gastric juice is to alter the protein food stuffs so that they may be readily absorbed. Less important functions are the antiseptic action of the hydrochloric acid and the coagulation of milk. The chief digestive power of the gastric juice depends on the pepsin and acid contained in it, both of which are necessary for the process in the stomach.

This action on proteins may be shown by adding a little gastric juice (natural or artificial) to some flakes of fibrin or to diluted egg albumin, and keeping the mixture at a temperature of about $37.8^{\circ} \mathrm{C}$. (I00 $\mathrm{F}$.). It is soon found that the fibrin goes into solution and that the albumin cannot be precipitated on boiling. If the solution be neutralized with an alkali, a precipitate of acid metaprotein is thrown down. After a while the acid metaprotein disappears, so that no precipitate results on neutralization, and proper analysis will show that all the fibrin or albumin has been converted into other protein ubstances, viz., proteoses and peptones. The process, as in the case of salivary digestion, is never complete and the final result is always a mixture of peptones with proteoses which cannot be further peptonized. The relative proportions, of course, depend on the duration of the process. A side product is found (as an insoluble residue) in artificial gastric digestion which gives practically all the protein reactions and is soluble in dilute alkali, though insoluble in water, sodium chloride, or dilute acid. This is known as anti-albumid and may be changed into peptone by prolonged digestion; it does not occur in physiological gastric digestion. The commonest proteose is the one formed from albumin and is known as albumose, or by the more 
general name proteose; this name is used in the subsequent descriptions of the digestive processes.

All classes of proteins are digested by gastric juice, leading to the production of proteoses and peptones. The change is indicated best by the characters of the new protein formed. Peptones have certain characteristics which distinguish them from other proteins. They are diffusible; i.e., they possess the property of passing through animal membranes. In their diffusibility peptones differ remarkably from egg albumin, and on this diffusibility depends one of their chief uses. Egg albumin as such, even in a state of solution, would be of little service as food, inasmuch as its diffusibility renders difficult its absorption or in the case of insoluble proteins effectually prevents absorption into the blood vessels of the digestive canal. When completely changed by the action of the gastric juice into peptones, albuminous matters diffuse readily, and can be then absorbed. Peptones, however, are not found in the blood, even of the vessels immediately concerned in absorption from the stomach and intestines. As will be shown, the proteins are broken down into their simpler cleavage products in the intestine.

Products at Different Stages of Gastric Digestion.-The protein is first changed into syntonin, or acid metaprotein, by the combined action of the pepsin and acid. Though the acid alone is capable of accomplishing this step, the fact that it does not do so physiologically is proven by the great length of time required in laboratory experiments for the change. The acid is absolutely essential to the action of pepsin.

The next change is the conversion of the syntonin into proteoses which, according to Neumeister, occurs in two successive stages. The first of these stages is the conversion of syntonin into the primary proteoses; $i . e$., protoproteose and hetero-proteose. The second is the conversion of both protoproteose and hetero-proteose into the secondary proteoses; i.e., deuteroproteose. The last change is the conversion of the deutero-proteose into the end product peptone. This last change does not occur to any great extent and the proteoses always predominate in the digesting mass. The action of pepsin is one of hydrolysis and the products are hydrated forms of protein. Schematically the changes in the proteins may be represented as follows:

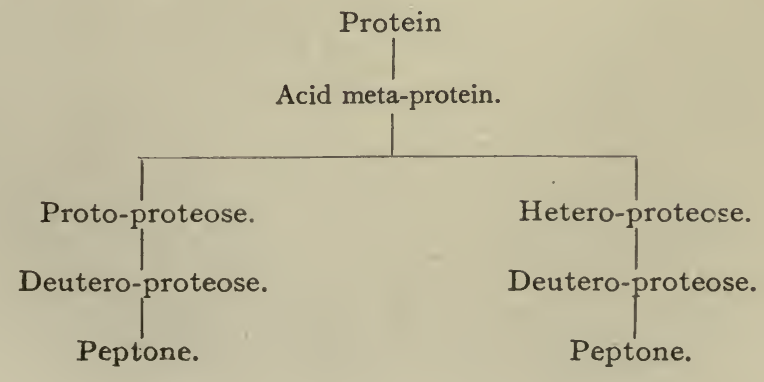


Circumstances Influencing Gastric Digestion.-A temperature of about $40^{\circ} \mathrm{C}$. is most favorable to gastric digestion. The pepsin is destroyed by a temperature of $55^{\circ}$ (neutral) to $65^{\circ} \mathrm{C}$. (acid solution) and its action is retarded and suspended by low temperatures. It is inactive in neutral or alkaline solution, for an acid medium is necessary. Hydrochloric is the best acid for the purpose, but other mineral acids or the organic acids may be substituted for the hydrochloric. Excess of peptone delays the action, and the removal of the products of digestion facilitates the process.

Action of Rennin.-Milk is curdled by the action of gastric juice, the casein being first precipitated, and then dissolved. The curdling is due to a special ferment of the gastric juice, rennin, and is not due to the action of the free acid alone. The effect of rennin, which is obtained commercially from the fourth stomach of the calf, has long been known, as it is used extensively to cause precipitation of casein in cheese manufacture. The ferment rennin is active in a neutral solution as well as in acid.

The Action of Gastric Lipase.-For many years it has been known that fats were digested in the stomach, but it has been a more difficult matter to definitely prove the source of the lipase, most physiologists holding that the lipase is regurgitated from the intestine. In I880 Cash proved that extracts of the gastric mucosa contained an active lipase which experimentally caused the dissociation of neutral fats as tested by the increased amount of fatty acid. He removed the pancreas and showed that fats were still digested. Ogata made the tests in the living stomach of the dog, closing off the opening into the intestine. The stomach thus isolated and washed out with physiological saline repeatedly caused the appearance of fatty acid when olein was introduced and brought into contact with the living gastric mucosa. It is evident that the secretion of the gastric glands contains an active lipase.

The well known observation of Pawlow showing that the secretion of pepsin is inhibited by an excess of fat in the stomach, when taken in connection with other facts showing the specific nature of the digestive secretions, see figure 268 , suggests that lipase secretion may be thus stimulated.

Pancreatic Digestion in the Stomach.-Boldyroff has recently shown that after the ingestion of fats or fatty foods in sufficient amounts, that the secretion of gastric juice is inhibited and that the presence of the pancreatic and intestinal secretions can be demonstrated in the stomach contents.

The accuracy of this observation as suggested from the preceding paragraph may be held in question to some extent, since the fat enzymes are present in the gastric juice itself. But that there may be regurgitation of the intestinal juices into the stomach is further supported by numerous clinical observations. This regurgitation may, often does, take place in great amount in the later stages of gastric digestion, at the time when the 
pyloric valve is least vigorously active. The presence of bile under these conditions is usually taken as indicative of this regurgitation. Under these conditions, the pancreatic juice is present in amounts sufficient to have a considerable proteolytic and fat-splitting action.

Time Occupied in Gastric Digestion.-Under ordinary conditions, from three to four hours may be taken as the average time occupied by the digestion of a meal in the stomach. But many circumstances will modify the rate of gastric digestion. The chief are: The nature of the food taken and its quantity (the stomach should be fairly filled, not distended); the time that has elapsed since the last meal, which should be at least enough for the stomach to be quite clear of food; the amount of exercise previous and subsequent to a meal (gentle exercise being favorable, overexertion injurious, to digestion); the state of mind; and the bodily health.

Summary of Changes in the Food in Gastric Digestion.-Briefly summarizing the action of gastric juice, the facts appear as follows: I Gastric juice has a specific digestive action on protein foods of all kinds, converting them into the more soluble proteoses and peptones. The action is due to an enzyme, pepsin, acting in and with an acid, hydrochloric acid. 2. The lipase in gastric juice produces a small amount of fat cleavage, tending to convert the fats into fatty acids and glycerin in which condition they are absorbed. The presence of fat tends to inhibit the gastric digestion of proteins. 3. Milk is first coagulated by a special enzyme, rennin, and then digested as any other protein. 4. Gastric juice dissolves soluble substances like salts, saccharides, etc.

5. The enzyme, ptyalin, continues the digestion of the carbohydrates in the stomach so long as the food remains neutral or alkaline, but they are not digested under the influence of any gastric enzymes. However, maltase is present in the gastric juice and aids in the last step in carbohydrate hydrolysis. It is significant that outside the body digestion takes place best at the temperature of the body, is destroyed by high heat and suspended by cold, $0^{\circ} \mathrm{C}$. Putrefaction is prevented by the acid of natural gastric juice.

\section{MOVEMENTS OF THE STOMACH.}

Attention has been called to the fact that the stomach is a muscular sac capable of holding quite a large mass of food. During a full meal as much as one to two liters or more of semi-solid food is packed away in the organ in a comparatively short space of time. The gastric juice is secreted by the mucous membrane which surrounds the surface of the food mass. The result is that the secretion begins to soften and digest the food over its surface, thus tending to liquefy and erode away layer after layer of the food mass. The picture is made clearer if one remembers that the food mass is retained almost wholly in the fundus of the stomach. The pyloric portion of the stom- 
ach is quite strongly muscular and quite definitely marked off by the strong transverse band at its union with the fundus.

Acid Closure of the Cardiac and Pyloric Orifices.-The gastric juice is assisted in accomplishing digestion by the movements of the stomach itself. When digestion is not going on, the stomach is uniformly contracted, its orifices not more firmly than the rest of its walls; but, if examined shortly after the introduction of food, it is found closely encircling its contents, and its orifices are firmly closed like sphincters. The cardiac orifice, every time food is swallowed, opens to admit its passage to the stomach, and immediately closes again. This closure of the cardiac orifice is accomplished by a local reflex. The stimulus is the acid secretion covering the mucous membrane in the immediate neighborhood.

At the taking of food or immediately thereafter the content of the stomach begins to pass through the pyloric orifice into the intestine. But the pylorus is quickly closed so completely that little of the contents escape at this time. The pylorus is automatically regulated as demonstrated by Cannon. The acid gastric content in the duodenum sets up a local reflex that closes the pylorus until the bile and pancreatic juice have neutralized the acid. When an alkaline reaction is produced the pylorus relaxes and at the next peristaltic wave of contraction is opened again. Indeed it is claimed that in the human the pylorus takes more or less part in each peristaltic wave passing from the stomach on over the duodenum.

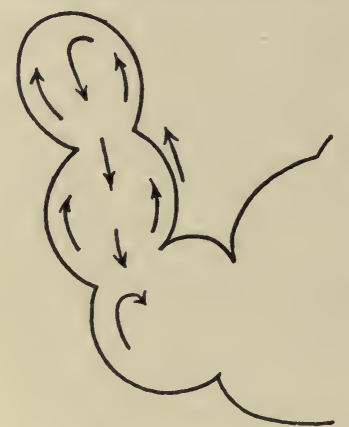

Fig. 26r.-Diagram to show the movement of food in the pylorus at times when the pyloric sphincter is closed.

The Peristalsis of the Stomach. - The character of stomach movements has been admirably determined by recent observers using the $\mathrm{X}$-ray method. Thus Cannon, working with cats, has shown that in from five to ten minutes after a meal slight rings or constrictions occur in the pyloric antrum and travel slowly toward the pyloric sphincter in the form of a peristaltic wave. Successive waves begin a little further back toward the fundus each time and follow over the pyloric antrum with clock-like regularity, in the cat one wave in ten seconds, which requires in each case about twenty seconds for its completion. In man they are doubtless slower. These peristalses continue during the whole period of digestion for as much as seven or even more hours.

These peristaltic contractions aid the gastric juice in carrying away the softened layers of food by propelling it into the pylorus. There it is thoroughly mixed with the gastric juice, forming the chyme. Figure 26I gives an idea of the movement of the food in the antrum. The peristaltic contractions carry it forward, but if the valve does not open to permit passage to the duodenum, then the pressure will force the chyme back through the center toward the 
fundus. After several minutes, i.e., when the secretion of alkaline bile and pancreatic juice into the duodenum is well established, the pyloric sphincter will relax more often to allow fluid food to pass to the duodenum, but when the more solid particles come up against the sphincter it promptly contracts and remains so for some time. Toward the completion of digestion even solid undigested particles are carried on into the intestine.

Hunger Contraction.-Our present knowledge of the character and nervous regulation of the peristaltic contractions of the stomach has been recently immeasurably advanced by the work of Carlson. His studies have been on man, with and without gastric fistula, and on dogs. As a result there can no longer be doubt that the sensation of hunger is attended with characteristic contractions of the empty stomach. Carlson's studies of the contractions of the empty stomach has established a number of points in the nervous control. For example, the vagus nerves have a tonic influence over the organ, and their section "leaves the empty stomach on the whole permanently hypotonic." This control does not readily yield to the usual reflexes. The splanchnic nerves are, on the other hand, inhibitory for the stomach. It is through this channel that the psychic and other reflexes act to control the organ. Carlson studied the phenomena in dogs which had

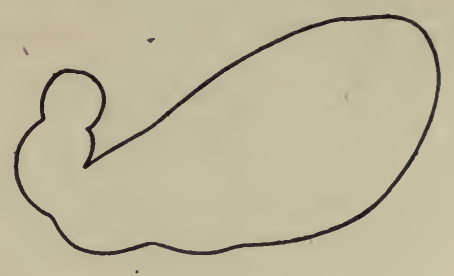

11A.M.

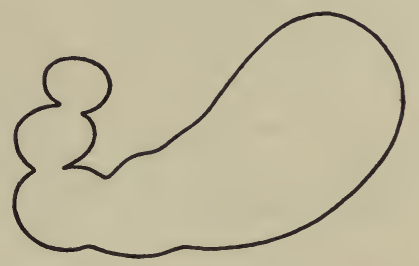

$12 M$.

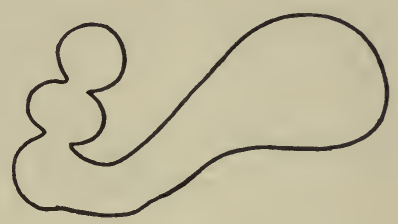

2P.M.

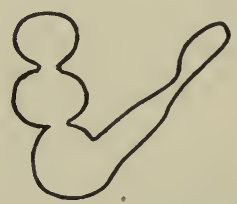

5P.M.

FIG. 262.- Outlines of the Roentgen-ray Shadows of the Stomach Content as Digestion Progresses. (Cannon.) both the ragi and the splanchnics sectioned. The stomach thus isolated manitests the usual hunger peristalses and often "the hunger contractions are identical in rate and character with those of the intact stomach in normal (strong) tonus." This indicates that "the primary stimulus to these contractions is not to be sought in the extrinsic nerves." It follows therefore that the extrinsic nerves are merely regulative and modifying for the contractions of the otherwise automatic organ. Of the two mechanisms, the vagus is the more vital and least readily disturbed in its control. 
Vomiting. - The expulsion of the contents of the stomach in vomiting is preceded by a deep inspiration with closure of the glottis, followed immediately afterward by strong contractions of the muscles of the abdomen, diaphragm, and stomach. The diaphragm forms an unyielding surface against which the stomach can be pressed. In this way as well as by

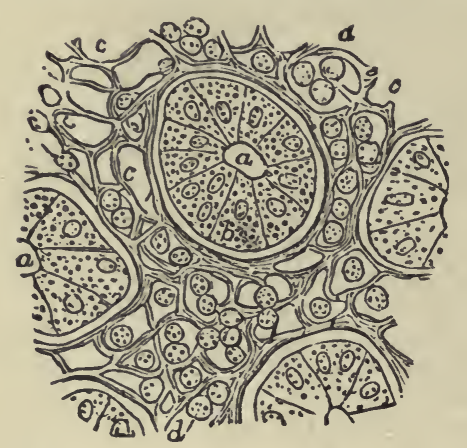

FIG. 263.-Horizontal Section of a Small Fragment of the Mucous Membrane, including one entire crypt of Lieberkühn and parts of several others. its own contraction the diaphragm is fixed, to use a technical phrase. At the same time the cardiac sphincter muscle is relaxed, and the orifice which it naturally guards is actively dilated. The pylorus is closed and, the stomach itself also contracting, the action of the abdominal muscles produces strong compression which expels the contents of the organ through the esophagus, pharynx, and mouth. Reversed peristaltic action of the esophagus probably increases the effect.

It has been frequently stated that the stomach itself is quite passive during vomiting, and that the expulsion of its contents is effected solely by the pressure exerted upon it when the capacity of the abdomen is diminished by the contraction of the diaphragm. It is true that facts are wanting to demonstrate with certainty the contraction of the stomach in vomiting; but cases of fistulous opening into the organ appear to support the belief that it does take place; and the analogy of the case of the stomach with that of the other hollow viscera, as the rectum and bladder, may also be cited in confirmation.

Vomiting is a reflex act. It can be excited by irritation of the lining of the stomach, which is perhaps the normal stimulus. It is excited by stimulation or irritation of other parts of the alimentary tube; i.e., the pharynx, the uvula, the intestine, etc. Vomiting may occur from stimulation of sensory nerves from many organs, e.g., kidney, testicle, etc., or by impulses arising in the organs of special sense, the eye, olfactory membrane, etc. The sensory impulses are co-ordinated by a nerve center located in the medulla. The center may also be stimulated by impressions from the cerebrum and cerebellum or by changes arising in the center itself, the so-called central vomiting occurring in disease of those parts. The efferent impulses are carried by the phrenics and other spinal nerves and by the vagus.

\section{DIGESTION IN THE INTESTINES.}

The food that enters the small intestine has already been subjected to two digestive enzymes. The ptyalin of the saliva and the pepsin of the gastric juice together with the mechanical processes involved have reduced the food 
to a pulpy mass, the chyme. This peptonized food contains most of the total quantity of food eaten, little having been absorbed, as we shall see later, but

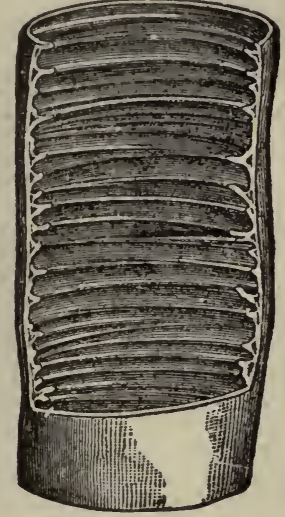

FIG. 264 .

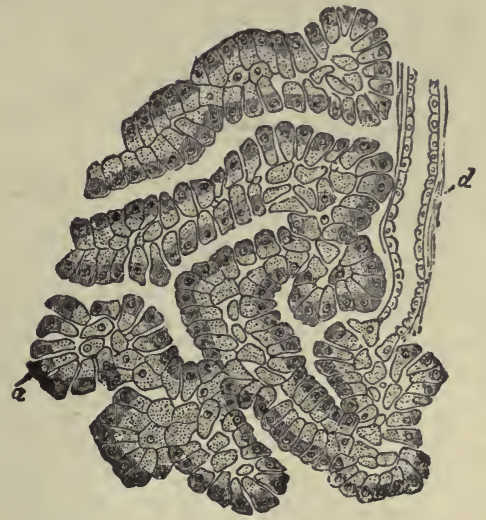

FIG. 265 .

FIG. 264.-Piece of Small Intestine (previously distended and hardened by alcohol), Laid open to Show the Normal Position of the Valvulæ Conniventes.

FIG. 265. - Section of the Pancreas of a Dog During Digestion. a, Alveoli lined with cells, the outer zone of which is well stained with hematoxylin; $d$, intermediary duct lined with squamous epithelium. $\times 350$. (Klein and Noble Smith.)

much of the starch has been changed to soluble maltose and dextrose and the protein to albumoses and peptones. The discharge from the stomach through the pyloric valve to the duodenum has been going on through three or four hours on an average for each full meal. This stream of food passing down the small intestine, slowly because of the valvulæ conniventes, meets a number of secretions which contain enzymes which act on each of the three great food principles, proteins, fats, and carbohydrates. These secretions are the pancreatic fluid, the succus entericus, and the bile.

The Pancreas. - The pancreas is situated within the curve formed by the duodenum, and its main duct opens into that part of the small intestine through a duct common to it and to the liver and about two and a half inches from the pylorus.

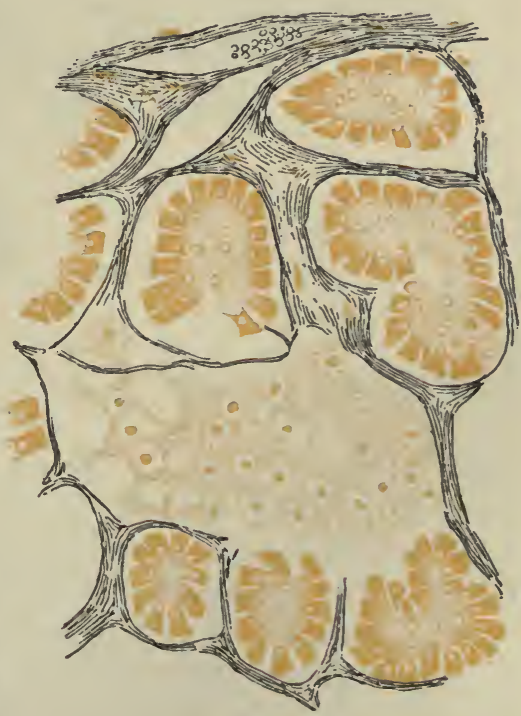

FIG. 266.-Section of the Pancreas of Armadillo, Showing the Two Kinds of Gland-structure. (V. D. Harris.)

The pancreas bears some resem- 
blance in structure to the salivary glands. Its capsule and septa, as well as the blood vessels and lymphatics, are similarly distributed. It is, however, looser, the lobes and lobules being less compactly arranged.

Heidenhain has observed that the alveolar cells in the pancreas of a fasting dog consist of two zones, an inner or central zone which is finely granular, and which stains feebly, and a smaller parietal zone of finely striated proto-

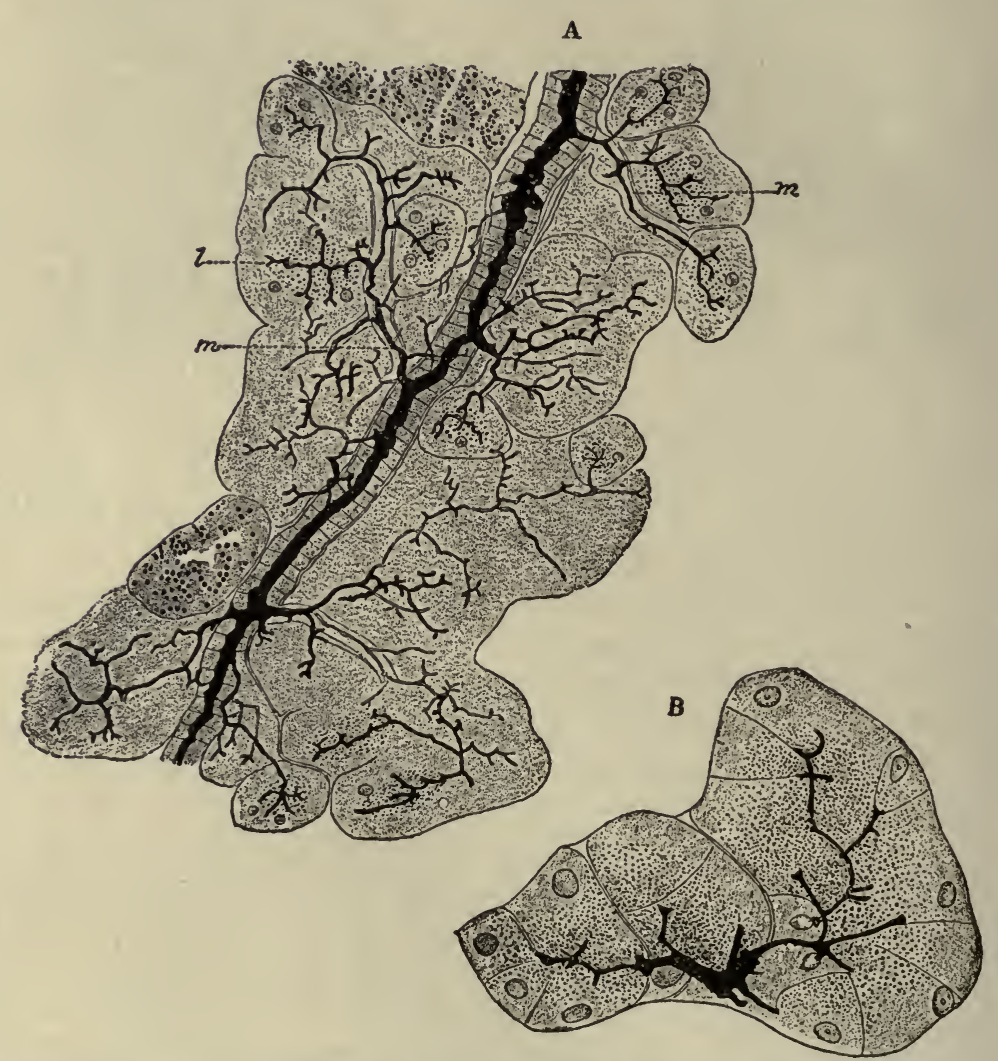

FIG. 267.-Duct with Laterals to the Alveoli. Silver method of Golgi (E. Muller). A Duct with branches; $m$, between the cells. $B$, Laterals more strongly magnified.

plasm which stains easily. The nucleus is partly in one, partly in the other zone. During secretion it is found that the outer zone increases in size, and the central granular zone diminishes, as in the case of the salivary glands. The pancreatic cell itself becomes smaller from the discharge of the secretion. During a period of rest the granular zone again increases in size and the outlines of the cells become full and indistinct. The granules, as in the salivary cells, are the material from which, under certain conditions, the ferments of the gland are developed, and which are therefore a zymogen. In addition to the ordinary alveoli of the pancreas there are distributed irregu- 
larly in the gland other collections of cells of a different character, the islands of Langerhans. These cells are considerably smaller, their protoplasm is more granular and less easily stained with hematoxylin, and their nuclei are small and stain deeply. The collections of cells vary in size and shape. The islands of Langerhans' cells are not concerned with the production of the pancreatic juice. The special form of nerve terminations, called Pacinian corpuscles, are often found in the pancreas.

The secretion of the pancreas has been obtained for purposes of experiment from the lower animals and from man in at least one case. A pancreatic fistula is established in the dog by opening the abdomen and exposing the duct of the gland which is then made to communicate with the exterior. In Pawlow's method a circular bit of the intestinal mucous membrane around the mouth of the duct in the intestine is brought to the surface and stitched into the wound. The secretion is then easily collected into a vessel suspended under the opening.

The Pancreatic Juice.-Pancreatic juice is colorless, transparent, slightly viscid, and alkaline in reaction. It varies in specific gravity from r.oro to $\mathrm{r} .03$, according as it is obtained from a permanent fistula, when it is more watery, or from a newly opened duct. The solids vary in a temporary fistula from 80 to roo parts per thousand, and in a permanent one from 16 to 50 per thousand. It is characterized by having three distinct and important enzymes known as trypsin, amylopsin, and steapsin, whose actions are, respectively, proteolytic, amylolytic, and lipolytic (fat-splitting). Maltase, which inverts the disaccharides, is also present, and a rennin is found in the pancreatic juice.

Chemical Composition of Pancreatic Juice. (C. Schmidt.)

\begin{tabular}{|c|c|c|}
\hline From a dog. & $\begin{array}{l}\text { Recent } \\
\text { fistula. }\end{array}$ & $\begin{array}{c}\text { Perma- } \\
\text { nent } \\
\text { fistula. }\end{array}$ \\
\hline Water. & $900 \cdot 76$ & 980.45 \\
\hline 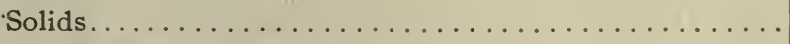 & 99.24 & $19 \cdot 55$ \\
\hline Organic substances... & 90.44 & 12.71 \\
\hline Ash............. & 8.80 & 6.84 \\
\hline 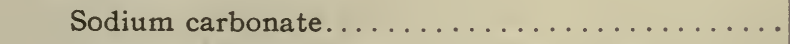 & $0.5^{8}$ & $3 \cdot 3^{I}$ \\
\hline 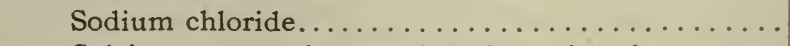 & $7 \cdot 35$ & $2 \cdot 50$ \\
\hline Calcium, magnesium, and sodium phosphates .... & 0.53 & 0.08 \\
\hline
\end{tabular}

An extract of pancreas made from the gland which has been removed from an animal killed during digestion possesses the active properties of pancreatic secretion. It is made by first dehydrating in absolute alcohol the gland which has been cut up into small pieces. After the entire removal 
of the alcohol the gland is pulverized and extracted in strong glycerin. The amount of the ferment greatly increases if the gland be exposed to the air for three or four hours before placing in alcohol; indeed, a glycerin extract made from the gland immediately upon the removal from the body often appears to contain none of the ferments. The conversion of zymogen in the gland into the ferment takes place only after the gland stands a while.

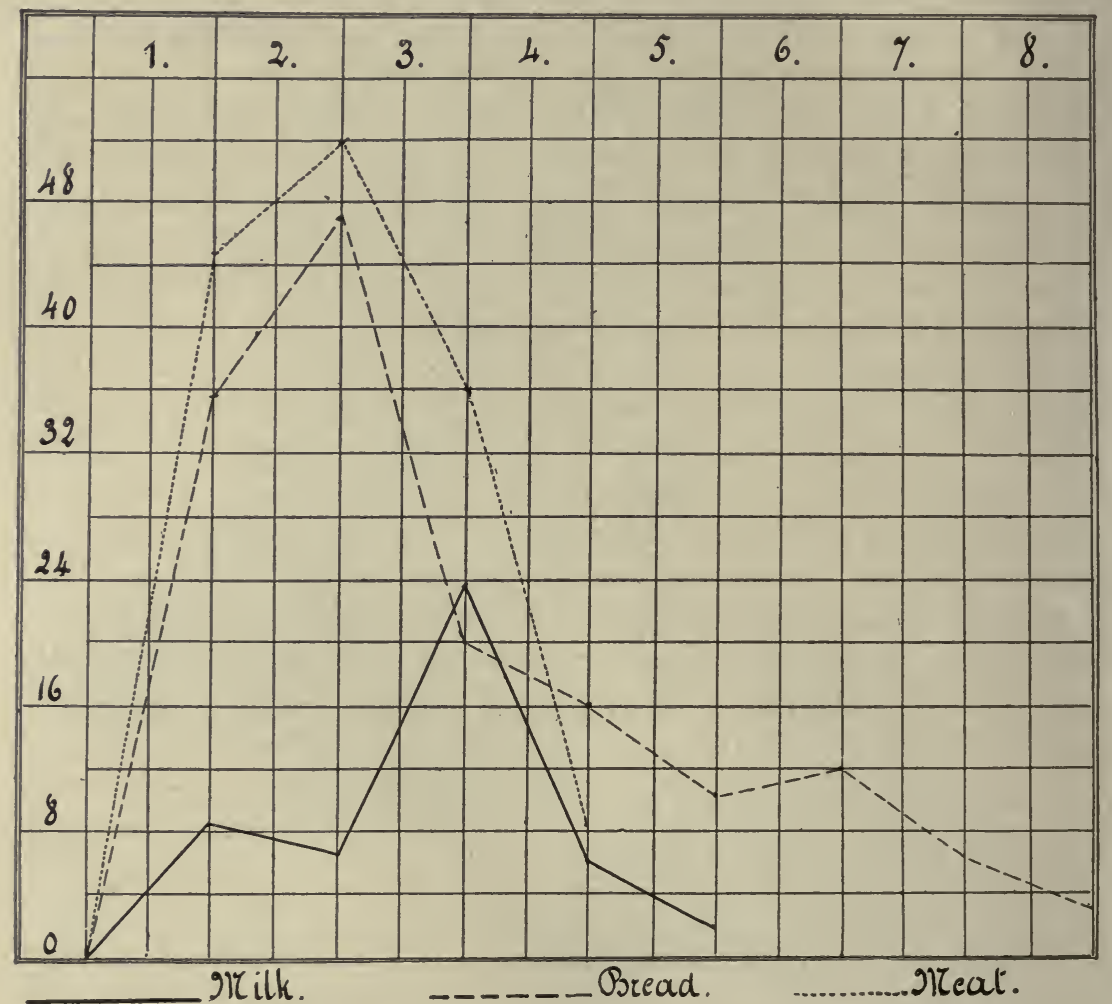

FIg. 268.-Three Curves Showing the Secretion of Pancreatic Juice upon a Diet (I) of $600 \mathrm{cc}$. of milk; (2) of $250 \mathrm{gm}$. of bread; (3) of $100 \mathrm{gm}$. of meat. The divisions along the abscissæ represent hours after the beginning of the meal; the figures along the ordinates represent the quantity of the secretion in cubic centimeters. (Walter.)

Dilute acid assists or accelerates the conversion, and if a recent pancreas be rubbed up with dilute acid before dehydration, a glycerin extract made afterward, even though the gland may have been only recently removed from the body, is very active.

Nervous Regulation of the Secretion of the Pancreas.-Fibers from the vagus and from the splanchnics are distributed to the pancreas. In Pawlow's laboratory it has been found that stimulation of these nerves leads to the increased secretion of the pancreas. Popielski, in studying the effects 
of dilute hydrochloric acid solution in the duodenum, which resulted in a marked increase of pancreatic secretion, explained the phenomenon as a local nerve reflex.

Doubt has been cast on the whole question of nervous control by the recent discovery of the fact that acid ( 0.4 per cent. hydrochloric acid) in the duodenum results in the production of a chemical substance, by the duodenal mucous membrane. This substance secretin, is absorbed into the circulation and acts specifically on the pancreas to produce increased activity of the pancreatic cells. Acid extracts of the duodenal mucous membrane produce the same effects on the pancreas, in fact this is the current method of experimentally stimulating the flow of pancreatic juice at the present time, the secretion being collected from a tube introduced into the duct.

Under the normal stimulus of food, the flow of pancreatic juice is greatly increased. The increase continues to a maximum in from two to three hours, after which it gradually decreases through the period of digestion. Pawlow has found a certain amount of adaptation, not only of the quantity but of the enzyme composition of the pancreatic secretion, to the kind and character of the food (in dogs).

Action of the Enzymes of Pancreatic Juice.-The secretion of the pancreas accomplishes its digestive action by means of the enzymes given above, viz., trypsin, amylopsin, steapsin, and maltase.

Trypsin.-Trypsin is a proteolytic enzyme. Strange to say, it does not exist in the fresh pancreatic juice as such, but makes its appearance only when there is an admixture with the succus entericus, the secretion of the mucous membrane of the intestine. The succus entericus contains an activating enzyme, enterokinase, which converts the inactive and stable trypsinogen of the pancreatic juice into the active but less stable trypsin. This fact is another of the wonderful series of contributions to the exact knowledge of the subject of digestion made from Pawlow's laboratories.

Trypsin, like pepsin-hydrochloric acid, converts proteins into proteoses and peptones. The change, however, does not stop here; the hydrolysis with trypsin goes much further. While simple amino-acids, with the exception of traces of tyrosine, are not found in gastric digestions, these are rapidly split off in the tryptic cleavage. Thus in tryptic digestion are formed: tyrosine, leucine, cystine, amino-valerianic acid, asparaginic acid, glutaminic acid, histidine, lysine, and arginine. A portion of the protein, however, is not completely broken down, the residue consisting of polypeptids containing proline and phenyl-alanine combined with small amounts of alanine, leucine, aspartic acid, and glutaminic acid. Glycocoll, when present in the protein digested, is also combined in the resistant polypeptids. Crystals of leucine and of tyrosine, especially, can be found in tryptic digestion mixtures. The products formed from protein in tryptic digestion may be given in the following graphic scheme: 


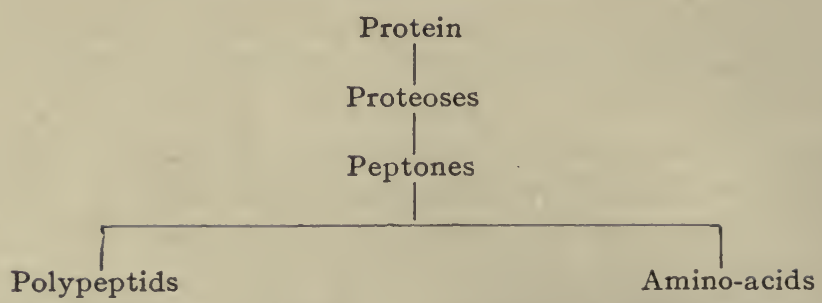

Combinations of proline, phenylalanine and glycocoll, with relatively small amounts of alanine, leucine, asparaginic acid, and glutaminic acid.

Tyrosine, tryptophane, cystine, alanine, amino-valerianic acid, leucine, aspartic acid, glutaminic acid, histidine, lysine, arginine.

The ferment trypsin acts best in an akaline medium, but will act also in a neutral medium, or in the presence of a very small amount of combined acid; it will not work in the presence of free acid. It therefore differs from pepsin in being able to act without the aid of any other substance than water. In the process of tryptic digestion, protein matter does not swell up at first, but seems to be corroded at once.

Amylopsin.-Starch is converted by amylopsin into maltose by hydrolytic action similar to that of ptyalin, erythro-dextrin and one or more achroö-dextrins being the intermediate products. The amylolytic enzyme of the pancreatic juice, which cannot be distinguished from ptyalin, is called amylopsin. The maltose thus formed is converted to dextrose by the maltase, in which form it is ultimately absorbed.

Pancreatic juice, according to certain observers, possesses the property of curdling milk. It contains a special ferment, rennin, for that purpose. The ferment is distinct from trypsin, and will act in the presence of an acid (W. Roberts). The milk-curdling ferment of the pancreas is, in some pancreatic extracts, said to be quite powerful, insomuch that I cc. of a brine extract will coagulate $50 \mathrm{cc}$. of milk in a minute or two.

Steapsin or Lipase. - Oils and fats are emulsified and saponified by the pancreatic secretion. The terms emulsification and saponification may need a little explanation. The term emulsification is used to signify an important mechanical change in oils or fats, whereby they are made into an emulsion or, in other words, are minutely subdivided into small microscopic particles. If a drop of an emulsion be looked at under the microscope, an immense number of minute rounded particles of oil or fat of varying sizes will be seen. The more complete the emulsion the smaller are these particles. In milk, which is a splendid example of an emulsion, the fat droplets vary in diameter between I and $5 \mu$. An emulsion is formed at once if oil, which when old is slightly acid from the presence of free fatty acid, is mixed with an alkaline solution.

Saponification signifies a distinct chemical change in the composition of oils and fats. An oil or fat being made up chemically of glycerin, a 
triatomic alcohol, and three fatty-acid radicles which may or may not be identical, when an alkali (potassium hydrate) is added to it two changes take place; first, the oil or fat is split up into glycerin and its fatty acid; second, the fatty acids combine with the alkali to form soaps which are chemically known as stearate, oleate, or palmitate of potassium according to the particular fatty acid or acids involved. Saponification thus means a chemical splitting up of oils or fats into new compounds, and emulsification means merely a mechanical splitting up into minute particles. The pancreatic juice has been for many years credited with the possession of a special ferment, which was called by Claude Bernard steapsin, and which is a lipase or fat-splitting ferment. This ferment has not been isolated, butits presence may be demonstrated by adding portions of the fresh pancreas to butter or other fat and maintaining the proper temperature. Its action is made manifest by the liberation of butyric acid, which imparts the typical odor to rancid butter.

The older theory was that only a small portion of the fat eaten was thus changed into soap, and that the function of the saponified fat was to assist in the emulsification of the remaining major part, a process favorably influenced by the bile. Although the proper emulsification of fat is indeed a preliminary step favoring more effective contact of the fat splitting enzyme, lipase, we now know that all the fat is dissociated into fatty acid and glycerin before abosrption can occur. When in disease the entrance of the pancreatic juice and the bile into the intestine is interfered with, the feces contain an excess of fat.

All recent experiments, however, tend to support the view of Pfluiger that the entire fat of the food is changed into fatty acids and glycerin; that the fatty acids are entirely, or in part, changed to soaps; and that these soaps, or mixture of soaps and free fatty acids, are absorbed in solution. The chief facts favoring this view are that: (I) The reaction of lipase is sufficiently rapid to allow the saponification of a full fatty meal within the ordinary period of digestion; (2) histological examination has never shown that fat particles can pass into a columnar cell, and droplets have not been found in the broad striated border of the cell; (3) the fat globules found in columnar cells after a fatty meal grow steadily larger as the period of absorption progresses, indicating that they are deposited from solution; (4) the fatty acids are easily soluble in bile solutions, and the solubility of the soaps is greatly increased by the presence of bile. The fat constituents, according to this theory, are recombined in the columnar cells to form neutral fats where their presence can be easily demonstrated by methods of staining.

Conditions which Influence the Action of the Pancreatic Enzymes. - The various pancreatic enzymes are influenced by heat, by the presence of an excess of digestion products, etc., in the same way as ptyalin and pepsin. Pancreatic enzymes act in a neutral, but best in an alkaline solution. The 
trypsin, strange to say, is quickly destroyed by the alkaline solution (Bayliss and Starling). The pancreatic juice offers the special case of a secretion of

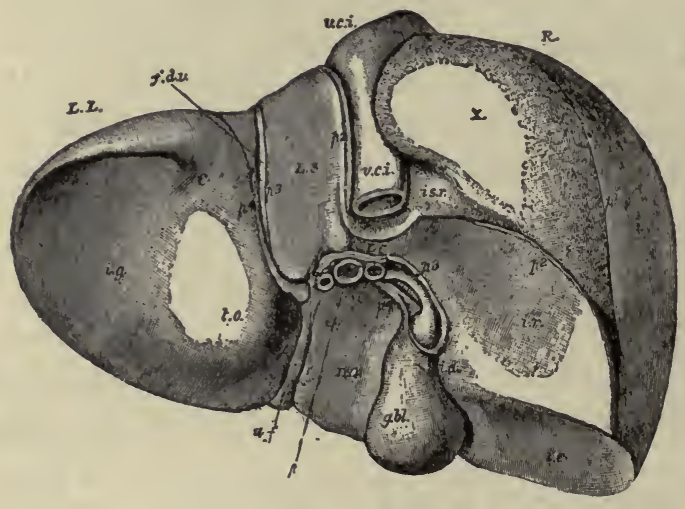

Fic. 269 .

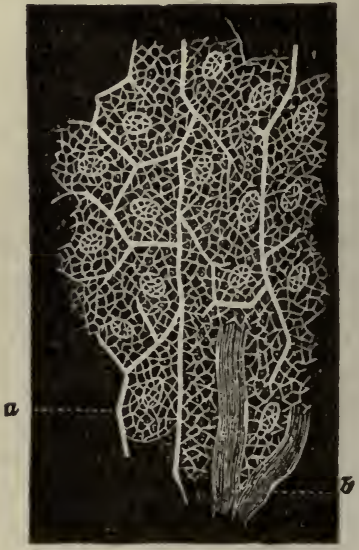

FIG. 270.

Fig. 269. - The Liver from Below and Behind. L. S., Spigelian lobe; L. C., caudate lobe; $L . Q$., quadrate lobe; R.L., right lobe; L.L., left lobe; g. bl., gall-bladder; v.c.i., inferior vena cava; $u$. $f$., umbilical fissure; $f . d . v$., fissure of the ductus venosus; $p$, portal fissure with portal vein, hepatic artery, and bile-duct. (Wesley, from a His model.)

FIG. 270.-Portion of a Lobule of Liver. a, Bile capillaries between liver cells, the network in which is well seen; $b$, blood capillaries. $\times 35^{\circ}$. (Klein and Noble Smith.)

proenzyme which is stable in alkaline solution until acted on by enterokinase. The amount of kinase present will, therefore, markedly influence the amount of digestion of protein per unit of time.
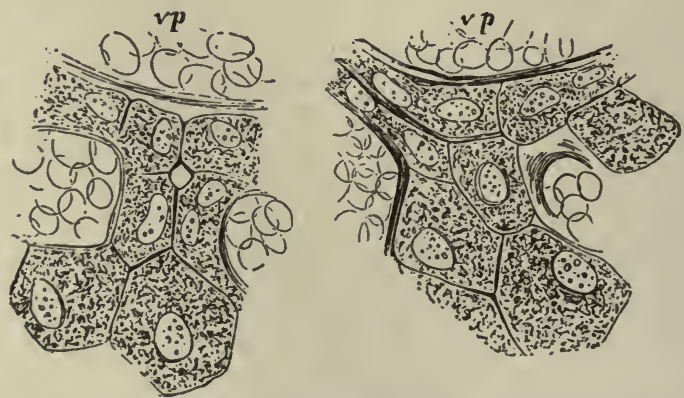

FIG. 271.- - Hepatic Cells and Bile Capillaries, from the Liver of a Child Three Months Old. Both figures represent fragments of a section carried through the periphery of a lobule. The red corpuscles of the blood are recognized by their circular contour; $v p$ corresponds to an interlobular vein in immediate proximity with which are the epithelial cells of the biliary ducts. (E. Hering.)

The Secretions of the Liver.-The liver, the largest gland in the body, situated in the abdomen on the right side chiefly, is an extremely vascular organ, and receives its supply of blood from two distant sources, viz., from the portal vein and from the hepatic artery, while the blood is returned from it into the vena cava inferior by the hepatic veins. Its secretion, the bile, 
is conveyed from it by the hepatic duct, either directly into the intestine or when digestion is not going on, into the cystic duct, and thence into the gallbladder, where it accumulates until required. The portal vein, hepatic artery, and hepatic duct branch together throughout the liver, while the hepatic veins and their tributaries run by themselves. The interstices of the vessels are filled by the liver cells.

Structure of the Liver.-The liver is made up of small roundish or oval portions called lobules, each of which is about $\frac{1}{20}$ of an inch (about

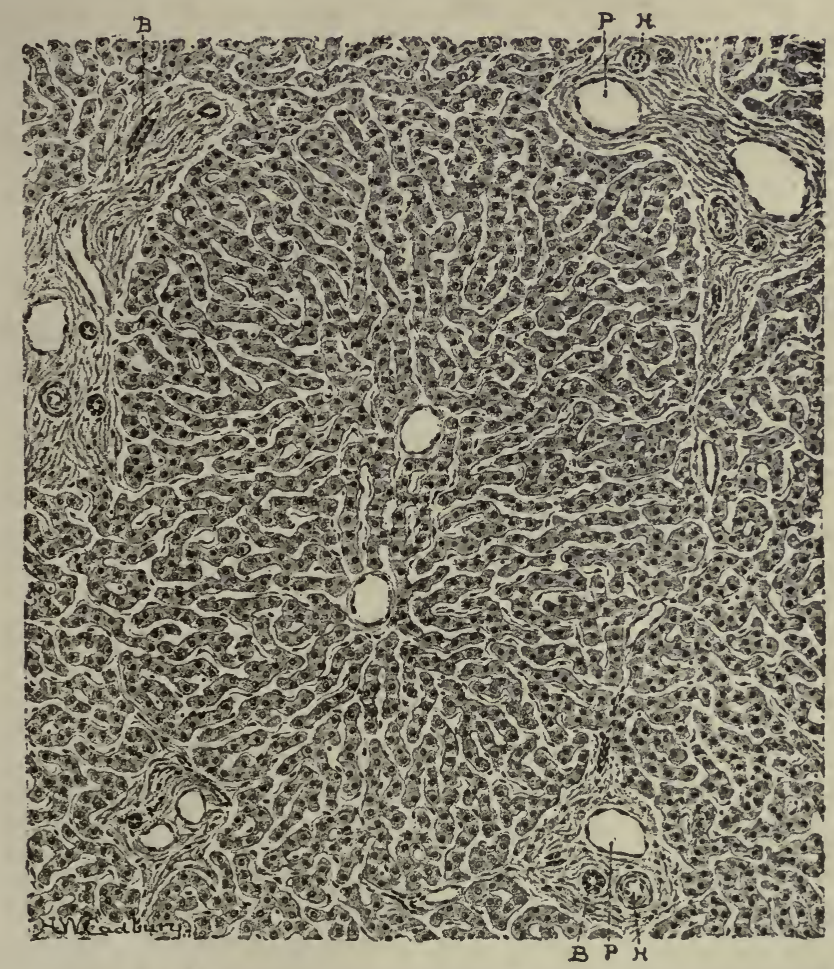

FIG. 272.- Section of Liver. $\times 80 . \quad P$, Portal vein; $H$, hepatic artery; $B$, bile-duct. (Hendrickson.)

I $\mathrm{mm}$.) in diameter, and includes the minute hepatic artery and hepatic duct. The hepatic cells, which form the glandular or secreting part of the liver, are of spheroidal form, somewhat polygonal from mutual pressure, about 25 to $30 \mu$ in diameter, and possess one, sometimes two nuclei. The cell substance contains a variable amount of glycogen and often some fatty globules and possibly some granules of bile pigment.

The bile capillaries commence between the hepatic cells, and are bounded by a delicate membranous wall of their own. They appear to be always bounded by hepatic cells on all sides, and are thus separated from the nearest blood capillary by at least the breadth of one cell, figures $27 \mathrm{I}$ and 272 . 
The gall-bladder, $g . b l$, figure 269 , is a pyriform sac attached to the under surface of the liver, and supported also by the peritoneum. The larger end, or fundus, projects beyond the front margin of the liver, while the smaller end contracts into the cystic duct. It is a muscular walled reservoir covered with a serous epithelium and lined by mucous membrane. The function of the gall-bladder is to retain the bile during the interval of digestion.

The Bile.-The bile is a somewhat viscid fluid, of a yellow, reddishyellow, or green color, a strongly bitter taste, and, when fresh, with a scarcely perceptible odor; it has a neutral or slightly alkaline reaction, and its specific gravity is about 1.020 . Its color and consistency vary much, quite independent of disease; but, as a rule, bile becomes gradually more deeply colored and thicker as it advances along its ducts, or when it remains long in the gallbladder where it becomes more viscid and ropy, darker, and more bitter. This is on account of its greater degree of concentration, from resorption of its water; and also from being mixed with mucus, lipoids, and phosphatid proteins secreted by the lining membrane of the gall-bladder.

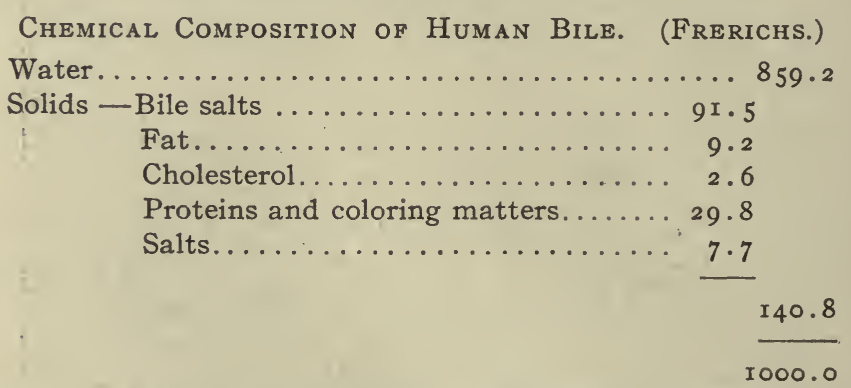

Bile salts can be obtained as colorless, exceedingly deliquescent crystals, soluble in water, alcohol, and alkaline solutions, giving to the watery solution the taste and general characters of bile. They consist of sodium salts of glycocholic and taurocholic acids; the formula of the former being $\mathrm{C}_{26} \mathrm{H}_{42} \mathrm{NaNO}_{6}$, and of the latter $\mathrm{C}_{28} \mathrm{H}_{44} \mathrm{NaNSO}_{7}$.

The bile acids are easily decomposed by the action of dilute acids or alkalies thus:

$$
\begin{aligned}
& \mathrm{C}_{26} \mathrm{H}_{43} \mathrm{NO}_{6}+\mathrm{H}_{2} \mathrm{O}=\mathrm{C}_{24} \mathrm{H}_{40} \mathrm{O}_{5}+\mathrm{CH}_{2} \cdot \mathrm{NH}_{2} \cdot \mathrm{COOH} \text {. } \\
& \text { Glycocholic Acid Cholic Acid Glycocoll } \\
& \mathrm{C}_{26} \mathrm{H}_{45} \mathrm{NSO}_{7}+\mathrm{H}_{2} \mathrm{O}=\mathrm{C}_{24} \mathrm{H}_{40} \mathrm{O}_{5}+\mathrm{CH}_{2} \cdot \mathrm{NH}_{2} \cdot \mathrm{CH}_{2} \mathrm{SO}_{3} \mathrm{H} \text {. } \\
& \text { Taurocholic Acid Cholic Acid Taurine }
\end{aligned}
$$

Glycocoll is amido-acetic acid, i.e., acetic acid, $\mathrm{CH}_{3} \mathrm{COOH}$ with one of the atoms of $\mathrm{H}$ replaced by the radical amidogen $\mathrm{CH}_{2} \cdot \mathrm{NH}_{2} \cdot \mathrm{COOH}$. Taurine likewise is amino-ethylsulphonic acid. Accordingly, it has the formula $\mathrm{CH}_{2} \mathrm{NH}_{2} \mathrm{CH}_{2} \mathrm{SO}_{3} \mathrm{H}$. The proportion of these two salts in the bile of different animals varies, e.g., in the ox bile the glycocholate is in great excess, whereas the bile of the dog, cat, bear, and other carnivora contains taurocholate alone. In human bile the glycocholate is in excess ( 4.8 to r.5).

The yellow coloring matter of the bile of man and the carnivora is termed 
bilirubin, $\mathrm{C}_{16} \mathrm{H}_{18} \mathrm{~N}_{2} \mathrm{O}_{3}$, is crystallizable and insoluble in water, and soluble in chloroform or carbon disulphide. A green coloring matter, biliverdin, $\mathrm{C}_{18} \mathrm{H}_{18} \mathrm{~N}_{2} \mathrm{O}_{4}$, which always exists in large amount in the bile of herbivora, is formed from bilirubin on exposure to the air or by subjecting the bile to any other oxidizing agency, as by adding nitric acid. Biliverdin is soluble in alcohol, glacial acetic acid, and strong sulphuric acid, but insoluble in water, in chloroform, and ether. It is usually amorphous, but may sometimes crystallize in green rhombic plates.

There is a close relationship between the coloring matters of the blood and of the bile and, it may be added, between these and that of the urine, urobilin, and of the feces, stercobilin. It is probable they are, all of them, varieties of the same pigment, or derived from the same source. Cholesterol $\mathrm{C}_{27} \mathrm{H}_{45} \mathrm{OH}$, and lecithin, $\mathrm{C}_{43} \mathrm{H}_{84} \mathrm{NPO}_{8}$ are constant constituents of bile. Iron is found among the salts of the ash.

\section{The Role of Bile in Intestinal Digestion.} - Though it is not a true digestive fluid, in that it has no ferment and digests nothing itself, yet it must be regarded as an important aid to digestion for the following reasons: (a) Bile assists in emulsifying the fats of the food, and dissolves the fatty acids thus rendering them more capable of absorption. For it has

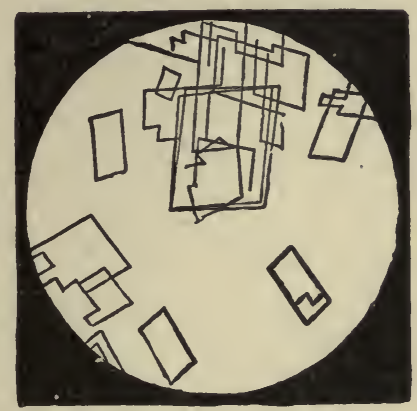

FIG. 273.-Crystalline Scales of Cholesterol: appeared in some experiments in which the common bile-duct was tied that, although the process of digestion in the stomach was unaffected, chyle was no longer well formed. The contents of the lacteals consisted of clear, colorless fluid, instead of being opaque and white, as they ordinarily are after feeding. It is, however, the combined action of the bile with the pancreatic juice to which the emulsification is due rather than to that of the bile alone. The bile itself has a very feeble emulsifying power. If the theory be accepted that fats are absorbed as fatty acids and soaps, in solution, the action of the bile becomes very important because solutions of bile salts have the power of dissolving the fatty acids. The moistening of the mucous membrane of the intestines with bile, for this very reason, facilitates absorption of fatty matters through it.

(b) The bile, like the gastric fluid, has a certain but not very considerable antiseptic power, and may serve to prevent the decomposition of food during the time of its sojourn in the intestines. Experiments show that the contents of the intestines are much more fetid after the common bile-duct has been tied than at other times. Moreover, it is found that the mixture of bile with a fermenting fluid stops the process of fermentation. Contact with bile also destroys the digestive action of pepsin on protein. 
Bile is also an excretive fluid carrying waste products thrown off by the liver. The liver during fetal life is proportionately larger than it is after birth, and the secretion of bile is active, although there is no food in the intestinal canal upon which it can exercise any digestive property. At birth, the intestinal canal contains concentrated bile, mixed with intestinal secretion and this constitutes the meconium, or feces of the fetus. In the fetus, therefore, the main purpose of the secretion of bile must be directly excretive. Probably all the residue of the bile secreted in fetal life is incorporated in the meconium, and with it discharged.

Mode of Secretion and Discharge of Bile.-In considering the flow of bile into the intestine, two factors are involved. These are the emptying of the gall-bladder and an increased secretion by the hepatic cells.

The secretion of bile can be studied by tying the common bile-duct of a dog and then making a fistulous opening between the skin and the gallbladder; all the bile secreted is then discharged at the surface. In such animals it has been found that the secretion of bile is continuous. With

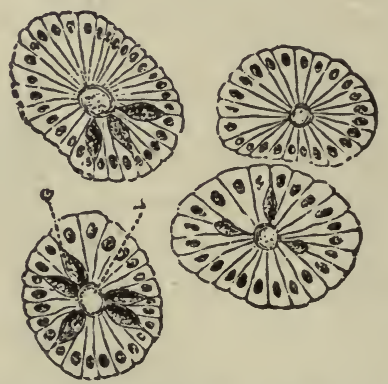

FIG. 274. - Transverse Section through Four Crypts of Lieberkühn, from the Large Intestine of the Pig. They are lined by columnar epithelial cells, the nuclei being placed in the outer part of the cells. The divisions between the cells are seen as lines radiating from L, the lumen of the crypt; $\mathrm{G}$, epithelial cells, which have become transformed into goblet cells. $\times 35 \circ$. (Klein and Noble Smith.)

the great discharge of bile into the intestine that occurs during the third hour after a meal, there is an increased secretion of this fluid. This increased secretion of bile can also be evoked by the introduction of 0.4 per cent. hydrochloric acid into the duodenum, and occurs even after the division of all connections between the liver and the central nervous system. There is evidence that the increased secretion of bile is brought about through a mechanism identical with that for the secretion of pancreatic juice, and that in each case one and the same substance-secretin-is formed by the action of the cells of the mucous membrane and absorbed into the blood stream and excites both the liver and pancreas to increased activity.

The emptying of the gall-bladder has been investigated on dogs with a Pavlov fistula. In this operation, the orifice of the duct, with the mucous membrane around it, is cut out of the wall of the intestine and the latter 
again closed. The excised portion with the opening of the bile duct is stitched into the abdominal wound. The natural orifice of the duct is thus made to open externally. The discharge of bile is found to begin almost immediately after taking food; it attains its maximum during the third hour, coincident with the pancreatic flow, and then rapidly diminishes. Dale has shown that the muscular fibers of the wall of the gall-bladder are supplied by nerves from both the vagus and the sympathetic. The former are motor, while the latter convey inhibitory impulses. The contractions of the gallbladder are provoked reflexly on the passage of the acid chyme into the intestine. The gall-bladder acts as a reservoir for the bile during the intervals when digestion is not in progress. The mechanism by which the bile passes into the gall-bladder is simple. The orifice through which the common bile-duct communicates with the duodenum is narrower than the duct, and appears to be closed, except when there is sufficient pressure behind to force the bile through it. The pressure exercised upon the bile secreted during the intervals between periods of digestion appears insufficient to overcome the force of the sphincter by which the orifice of the duct is closed; and the bile in the common duct traverses the cystic duct and so passes into the gall-bladder. It is probably aided in this retrograde course by the peristaltic action of the ducts.

The bile is discharged from the gallbladder and enters the duodenum on the introduction of food into the small intestine. It is pressed on by the contraction of the coats of the gall-bladder and of the common bile-duct.

When the discharge of the bile into the intestine is prevented by an obstruction of some kind, as by a gall-stone blocking the

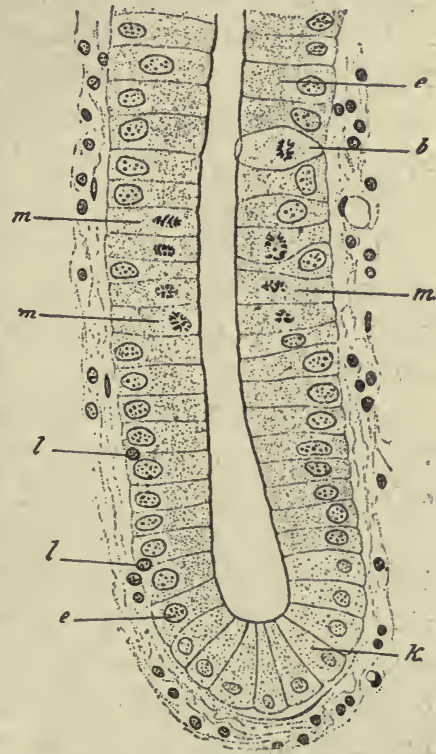

FIG. 275.-Longitudinal Section of Fundus of Crypt of Lieberkühn. $b$, Goblet cell showing mitosis; $e$, epithelial cell; $k$, cell of Paneth; $l$, leukocyte in epithelium; $m$, mitosis in epithelial cell. Surrounding the crypt is seen the stroma of the mucous membrane. $\times 53$. (Kölliker.) hepatic duct, it is reabsorbed in great excess into the blood, and, circulating with it, gives rise to the well-known phenomena of jaundice. This is explained by the fact that the pressure of secretion in the ducts, although normally very low, not exceeding 15 millimeters of mercury in the $\operatorname{dog}$, is still higher than that of the portal veins. If the pressure exceeds $15 \mathrm{~mm}$. the secretion continues to be formed, but passes into the blood vessels through the lymphatics. 
The Intestinal Secretion, or Succus Entericus.-It is impossible to isolate the secretion of the glands of Brunner or of the glands of Lieberk ühn, but the total secretion of the intestinal mucosa can be secured by isolating a loop of intestine by the operation known as the Thiry fistula. A few drops of secretion, the succus entericus, can be obtained by this means. Intestinal juice is a yellowish alkaline fluid with a specific gravity of I.OII and contains about 2.5 per cent. of solid matters.

Intestinal juice has only slight digestive action. It contains a weak proteolytic enzyme and a weak amylolytic enzyme. Maltase is also present. But the chief and most profound importance is given to the intestinal juice by the discovery of the activating enzyme, enterokinase. This specific activating enzyme for the trypsinogen of the pancreatic juice places the intestinal secretion in the rank of necessary secretion for efficient digestion. Enterokinase can be prepared by extracting the superficial scrapings of the intestinal mucous coat. The duodenal region is richest in enterokinase, but the secretion of the lower intestinal lengths also contains the enzyme.

Extracts of the mucosa of the intestine have been found to contain another substance which has the specific action of splitting peptones into simpler polypeptids and amino-acids. This substance has been called erepsin.

There are, therefore, three important new substances in the succus entericus (or in the extract of the glands), secretin (page 360), erepsin, and enterokinase, in addition to the proteolytic and diastatic enzymes.

Summary of the Digestive Changes in the Small Intestine.-The thin chyme, which, during the whole period of gastric digestion, is being constantly squeezed or strained through the pyloric orifice into the duodenum, consists of albuminous matter that is breaking down, dissolving and half dissolved; of fatty matter that is mechanically separated and melted, but not dissolved at all; of starch in various stages of the process of conversion into sugar, and as it becomes sugar dissolving in the fluids with which it is mixed; and with these are mingled gastric juice and fluid that has been swallowed, together with such portions of the food as are not digestible.

The chyme in the duodenum is subjected to the influence of the bile and pancreatic juice and also to that of the succus entericus. All these secretions have a more or less alkaline reaction, and at once neutralize the acid of the gastric chyme.

The special digestive changes in the small intestine are: (I) The fats are changed by the bile and pancreatic juice in two ways: (a) They are chemically decomposed by the alkaline secretions, and a soap and glycerin are the result. (b) They are emulsified; i.e., their particles are minutely subdivided and diffused, so that the mixture assumes the condition of a milky fluid or emulsion. (2) The albuminous substances which have been 
partly dissolved in the stomach are subjected chiefly to the action of the pancreatic juice. The pepsin is rendered inert by the bile. The pancreatic trypsin proceeds with the further conversion of the proteoses into peptones, and, with the erepsin, of the peptones into leucin, tyrosin, and the other amino-acids. (3) The starchy portions of the food are now acted on briskly by the pancreatic juice and the succus entericus, and are changed to maltose and dextrose. (4) Salines are usually in a state of solution before they reach the intestine.

Digestive Changes in the Large Intestine.-The changes which take place in the chyme in the large intestine are probably only the continuation of the same changes that occur in the course of the food's passage through the upper part of the intestinal canal. No special enzymes have been clearly shown for the mucous membrane of the large intestine. The enzymes of the small intestine may continue their action here, being hindered only by the acid developed from fermentation processes.

Action of Micro-organisms in the Intestines.-Certain changes take place in the intestinal contents independent of, or at any rate supplemental to, the action of the digestive ferments. These changes are brought about
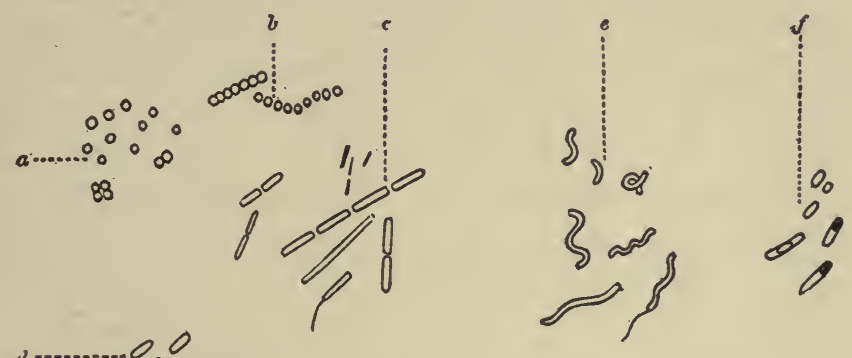

Fig. 276.-Types of Micro-organisms. a, Micrococci arranged singly; in twos, diplococci-if all the micrococci at $a$ were grouped together, they would be called staphylococci-and in fours, sarcinæ; $b$, micrococci in chains, streptococci; $c$, and $d$, bacilli of various kinds, one is represented with flagellum; $e$, various forms of spirilla; $f$, spores, either free or in bacilli.

by the action of micro-organisms or bacteria. We have indicated elsewhere that the digestive ferments are examples of unorganized ferments, so bacteria are examples of organized ferments. Organized ferments, of which the yeast plant may be taken as a typical example, consist of unicellular vegetable organisms, which when introduced into a suitable medium grow with remarkable rapidity. By their growth they produce new substances from those supplied to them as food. Thus, for example, when the yeast cell is introduced into a solution of grape-sugar, it grows, and alcohol and carbon dioxide are produced. The alcohol and carbon dioxide arise from the formation by the cell of some chemical substances which are allied to the unorganized ferments and which greatly increase in amount with the multiplication of the original cell. In 
all such fermentative processes organisms analogous to the yeast cell are present, and it is not strange that if the ferment cell is introduced into a suitable medium it may by its rapid growth convert an unlimited amount of one substance into another. Speaking generally, a special variety of cell is concerned with each ferment action, thus one variety has to do with alcoholic, another with lactic, and another with acetous fermentation.

A considerable number of species of bacteria exist in the body during life chiefly in connection with the mucous membranes, particularly of the digestive tract. Many forms of bacteria have been isolated from the mouth, a few varieties from the stomach, and a very large number from the intestines. It is only in the last-named locality that their multiplication has much effect from a physiological point of view. The normal (hydrochloric acid) acidity of the stomach usually destroys all the micro-organisms taken in with the food, but when the amount of this acid is deficient (and sometimes even when it is normal) some of the spores may escape. On reaching the small intestine these spores begin to develop in its alkaline medium, and may increase to such an extent as to stop all intestinal digestion; the point where this occurs varies from day to day. The large intestine always swarms with micro-organisms, though they do not readily pass the ileocecal valve into the small intestine. Some species of bacteria found in the intestine are anaërobic; i.e., they do not develop in the presence of free oxygen.

The changes induced in the intestine by the activity of micro-organisms are of two kinds, fermentation and putrefaction; the former of these results in the breaking-down of carbohydrate matter, and the latter in the disintegration of protein matter. The process of fermentation is the less complex and probably occurs normally in the small intestine. The lactic acid fermentation is the most important, though the butyric acid fermentation is next; under the influence of these bacteria the carbohydrates are butyrates broken down into lactic and butyric acids, and perhaps into acetic acid also. Carbonic acid gas may be formed at the same time and cause flatulence. Cellulose and other insoluble carbohydrates are decomposed, with the formation of marsh gas and hydrogen, which escape by the rectum.

In putrefaction the process is somewhat similar to that in tryptic digestion, the proteins being broken down into peptones, leucin, tyrosin, and a long row of similar substances, including ammonia in relatively large amount from the large intestine. Folin and Denis discovered over four times as much ammonia nitrogen in the mesenteric vein as in the carotid, 0.38 to 0.44 milligrams in Ioo centimeters of the blood of the vein and from 0.03 to 0.08 milligrams in the same amount of arterial blood from the carotid. It also results in the production of various gases, such as carbon dioxide, sulphureted hydrogen, ammonia, hydrogen, and methane (marsh gas), and of a high percentage of the volatile fatty acids, valerianic and butyric. Of the aromatic substances the most important are some phenol derivatives and indol and skatol, though their toxicity has been greatly 
overestimated. Indol and skatol undergo oxidation in the liver after absorption, forming indoxyl and skatoxyl. They are in part carried off in the. feces, but when the bowel is obstructed they are absorbed and eventually appear in the urine, indoxyl and skatoxyl forming, respectively, indoxyland skatoxyl-sulphuric acids and their salts. Tyrosin is broken down into para-oxy-phenol-propionic acid, paracresol, and phenol; para-oxy-phenolacetic acid is also formed. The phenols, after absorption, are in part conjugated with glycuronic acid which is formed by the incomplete oxidation of dextrose and are eliminated into the urine. Experiments have been performed to determine whether or not the intestinal bacteria are necessary to normal digestion. The weight of evidence is in favor of the view that they are not.

The Feces.-The contents of the large intestine, as they proceed toward the rectum, become more and more solid, lose more liquid and nutrient parts, and gradually acquire the odor and consistency characteristic of feces. After a sojourn of uncertain duration in the sigmoid flexure of the colon, or in the rectum, they are finally expelled by the act of opening the bowels, or defecation. The average quantity of matter evacuated by the human adult in twenty-four hours is about 200 to 250 grams, but the amount and character vary exceedingly according to the food eaten. Vegetable foods contain much indigestible matter, while meats and meat diets leave very little unabsorbed material to be expelled in the feces.

\section{Table of Composition of Feces.}

The amount of water varies considerably, from 68 to 82 per cent. and upward. The following table gives about an average composition:

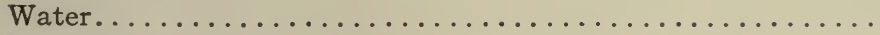

Solids, comprising:

a. Insoluble residues of the food, uncooked starch, cellulose, woody fibers, cartilage, horny matter, mucin, seldom muscular fibers and other proteins, fat, and cholesterin.

b. Certain substances resulting from decomposition of foods, such as indol, skatol, fatty and other acids; calcium and

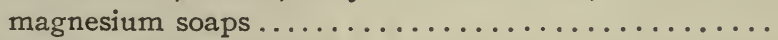

c. Special excretion,-excretin, excretoleic acid (Marcet),

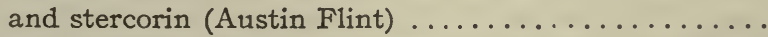

d. Salts-chiefly phosphate of magnesium and phosphate of calcium, with small quantities of iron, soda, lime, and

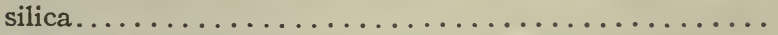

e. Insoluble substances accidentally introduced with the food.

f. Mucus, epithelium, altered coloring matter of bile, fatty

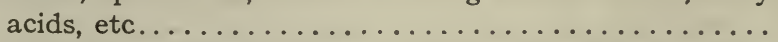

g. Varying quantities of other constituents of bile and the

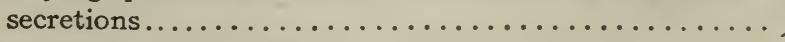


Intestinal Gases.-Under ordinary circumstances, the alimentary canal contains a considerable quantity of gases. The presence of gas in the intestines is so constant and the amount in health so uniform that there can be no doubt that its existence is a normal condition.

The gas contained in the stomach and bowels is from air swallowed with either food or saliva, gases developed by the decomposition of foods, or of the secretions and excretions thrown into the intestines. The decomposition of foods is the chief source. The following table, compiled by Brinton, is a collection of analyses that have been made and is chiefly valuable as showing the kinds of gases present, although the amounts of the gases vary with the diet.

Gases Found in the Alimentary Canal.

\begin{tabular}{|c|c|c|c|c|c|c|}
\hline \multirow[b]{2}{*}{ Whence obtained } & \multicolumn{6}{|c|}{ Composition by volume } \\
\hline & Oxygen & Nitrog. & $\begin{array}{l}\text { Carbon } \\
\text { dioxide }\end{array}$ & Hydrogen & $\begin{array}{l}\text { Carburet. } \\
\text { hydrogen }\end{array}$ & $\begin{array}{l}\text { Sulphuret. } \\
\text { hydrogen }\end{array}$ \\
\hline Stomach. & I I & $7 \mathrm{I}$ & I4 & 4 & $\ldots \ldots$ & \\
\hline Small intestines....... & $\ldots$ & 32 & 30 & $3^{8}$ & $\ldots \ldots$ & \\
\hline 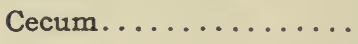 & $\ldots$ & 67 & I2 & 8 & $\mathrm{r} 3$ & Trace \\
\hline Colon............... & $\ldots$ & 35 & $5^{\mathrm{I}}$ & 6 & 8 & Trace. \\
\hline 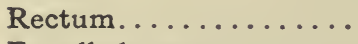 & $\ldots$ & 46 & 43 & $\ldots \ldots$ & II & \\
\hline Expelled per anum..... & $\ldots$ & 22 & 40 & I9 & & 0.5 \\
\hline
\end{tabular}

An analysis of the intestinal gases by Ruge in man is as follows:

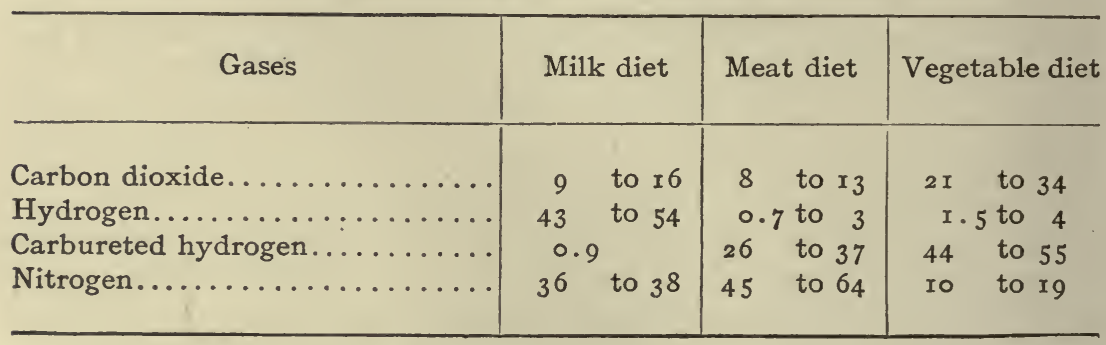

\section{MOVEMENTS .OF THE INTESTINES.}

The muscular activity of the intestines accomplishes two important functions; i.e., it thoroughly mixes the digesting food and secretions and it carries the content along the tract. Intestinal peristalses have been described for a long time. These peristalses begin as contractions of the circular muscles, producing ring-like constrictions that are propagated as waves over the intestine from above downward. Such constrictions carry the intestinal contents forward. The longitudinal muscles by their contraction produce pendular motion of the intestine.

A most instructive contribution to the knowledge of intestinal movements 
has been made by Cannon. He fed cats food mixed with ro to 33 per cent. of subnitrate of bismuth, and observed the shadows of the food when subjected to the X-rays. A length of food in the intestine was seen to be constricted into a series of oval masses, figure 277. Each of these oval masses is quickly constricted in the middle, and neighboring halves of adjacent masses flow together. After this process is repeated a number of times a peristaltic wave of the type previously described sweeps the whole content of the loop down the intestinal tract. The segmentations of the intestine are facilitated by pressure from within, perhaps stimulated by the direct pressure of the food. In general, hollow organs receive their most effective motor stimulus in this manner, i.e., by distention. This rule applies in the lengths of the small intestine.

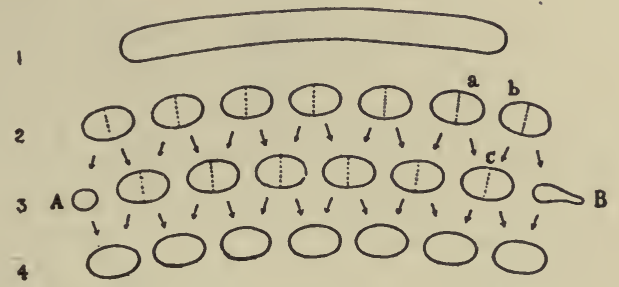

FIG. 277.-Diagram Illustrating the Segmentation of the Food in the Small Intestine. (Cannon.)

Peristaltic contractions of the same general type as in the small intestine also occur in the large intestine. Cannon has noted a variation here also. The ascending and the transverse loops of the colon sometimes exhibit rhythmic antiperistalses which keep the content moving against the iliocecal sphincter for several minutes at a time. In the meantime digesting material is being received into the ascending colon through the ilio-cecal sphincter, being slowly forced on by the peristalses of the lower loops of the small intestine. From time to time strong general peristalses in the colon slowly force the food onward. Under ordinary circumstances remnants of unabsorbed material in the colon do not pass beyond the pelvic colon, being held at this point until the greater portion of the entire colon is moderately filled. When sufficient material has accumulated here, it is evacuated by strong peristalses combined with compression by the contracting abdominal muscles.

Reverse peristalsis, antiperistalsis, does not commonly occur in the small intestine, but large nutrient enemata introduced into the rectum and colon may be forced by antiperistaltic waves in the large intestine against the ilio-cecal sphincter at a time when it is atonic and relaxed thus allowing materials to pass through the ilio-cecal valve into the small intestine. In normal healthy individuals this sphincter reacts, according to Hertz, like the sphincter of the pylorus effectively closing the tube. 
Influence of the Nervous System on Intestinal Peristalsis.-As in the case of the esophagus and stomach, the peristaltic movements of the intestines may be directly set up in the muscular fibers by the presence of food acting as the stimulus. Few or no movements occur when the intestines are empty, vigorous contractions when filled. The intestines are connected with the central nervous system both by the vagi and by the splanchnic nerves, as well as by other branches of the sympathetic which come to them from the celiac and other abdominal plexuses. The relations of these nerves respectively to the movements of the intestine and the secretions are probably the same as in the case of the stomach already considered.

The vagus carries the motor fibers for the intestine, while the sympathetics are inhibitory. Various states of the central nervous system, such as fear, anger, etc., inhibit the intestinal movements. The intestine, and stomach, too, carries out peristalses when isolated from the central nervous system and indeed from the body, so that the central connections do not originate the rhythmic stimulus but are only regulative. The intestinal movements are essentially automatic. It has long been known that isolated portions of the intestine contract rhythmically and automatically. This has been proven for both the longitudinal and circular muscles, and in the absence of the mucous membrane. According to Magnus, rhythmic contractions do not occur in the muscle if the plexus of Auerbach be removed. He concludes that the automatic rhythm is inherent in the local nerve ganglia, that it is not a reflex since it occurs in the absence of the mucosa, and that the contractions of the smooth muscles are directly dependent upon the local nerve distribution. Others contend that the contractions are independent; depending on the rhythmic property of the muscle itself, but co-ordinated by the complex local nervous mechanism. By either conception the nerve connections with the central nervous system are regulative and co-ordinative.

The innervation of the large intestine is also double in character and the relations are doubtless the same as in the small intestine.

Defecation.-The emptying of the bowels is essentially an involuntary act which has acquired a certain amount of voluntary regulation. The act is accomplished wholly reflexly in dogs with isolated lumbar cord, in fact has been observed when the lumbar spinal cord was removed. In the latter case defecation occurs by automatic peristalsis of the rectum, and colon, while in the former reflexes through the lumbar cord carry out the act. When the material that has accumulated in the colon descends into the rectum, which is normally empty, it initiates the reflex stimulus which culminates in opening the bowel, or defecation. Hertz and others have taken X-ray photographs of the human immediately before and after opening the bowels, with a view to a better understanding of what structures take part in the process. It is found that the entire colon as far back as the ilio-cecal sphincter may be emptied during the act, see figure $276 a$. 
Normally in man the rectal stimulus gives rise to the consciousness of the desire to defecate and to the initiation of efferent nerve impulses that may increase the contraction of the external sphincter and inhibit the act temporarily. During opening of the bowels however, the voluntary effort leads to relaxation of the external sphincter, and the normal peristalsis of the rectum is suppported by contractions of the abdominal musculature so as greatly to increase the abominal pressure, thus aiding the involuntary reflex which controls the relaxation of the internal sphincter and the contractions of the colon, and rectum.

The Time of Passage of Food Through the Alimentary Canal.-It is important to understand the rapidity with which food passes along the different divisions of the alimentary tube.

The effectiveness of digestion, the dangers from fermentation and putrefaction, and the ills that follow the absorption of toxic by-products are largely dependent upon the time element.

At the beginning of a meal the activity of the EXAMPLE 1
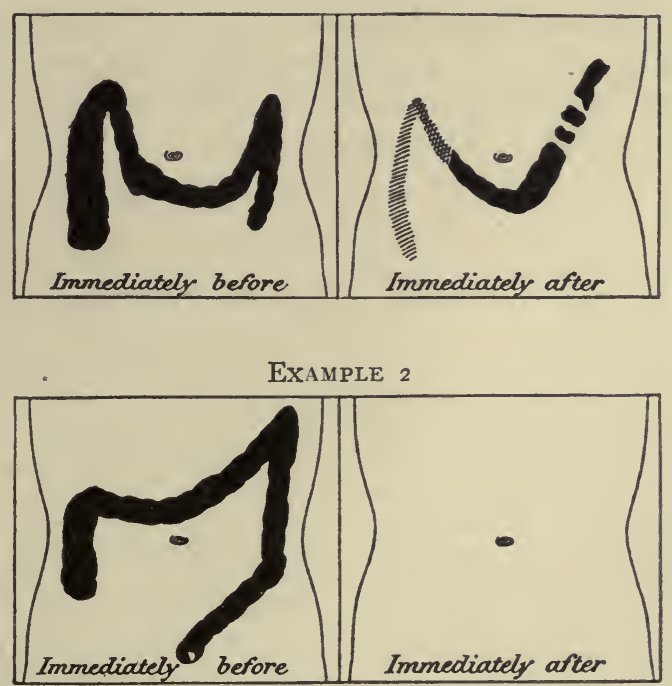

FIG. $276 a$.- The upper pair of figures show the bismuth shadows for normal defecation, the lower after the use of magnesium sulphate. (From Hertz, Cook, and Schlesinger.) entire alimentary canal is low, and one may assume that the various muscular mechanisms are relatively relaxed. When food is swallowed, immediately small portions begin to pass through the stomach and pyloric sphincter. However, the main portion of the food passes out of the stomach as it undergoes digestion and solution during the succeeding 4 or 5 hours. Hertz, who has given us the most accurate picture of the elements in this process allows $4 \frac{1}{2}$ hours for the passage of food along the 22 feet of the small intestine to the iliac sphincter. This he has determined by X-ray photographs after a bismuth meal. This is a comparatively rapid passage averaging about $2 \frac{1}{2} \mathrm{~cm}$. per minute. The rate is much slower in the large intestine. He allows an average speed of 2 hours each for the ascending, transverse, and descending colon. In the short lengths of the iliac and pelvic colons an equal or even greater time is consumed. Thus he would allow an average of eighteen 
hours for the first appearance of the food remnants at the end of the pelvic colon where it is retained until the bowels are opened. Of course, assuming an empty canal ahead of the moving material, the undigested and waste products of the food require on an average from eighteen to twenty-two hours for the passage of the entire alimentary tract. The earliest possible moment at which food remnants can appear in the feces will be when defecation occurs just after those remnants which passed immediately through the stomach have reached the ascending colon, say five hours. On the other hand, assuming that a meal is taken on an entirely empty canal, the food remnants will not produce the reflex for opening the bowels until they reach the pelvic colon. Hence if the bowels are open only once in twenty-four hours, it is obvious that the remnants of a meal may not be passed before thirty-six to thirty-eight hours. 


\section{LABORATORY EXPERIMENTS IN DIGESTION.}

\section{SALIVA AND SALIVARY DIGESTION.}

I. Reflex Salivary Secretion.-Saliva, which is the mixed secretion of the salivary and buccal glands, is produced more or less intermittently. Examine, taste, or smell appetizing food, for example, an apple, the salivary glands begin to discharge secretion which is poured into the mouth more rapidly than under ordinary conditions. This increased activity is a reflex secretion. It is brought about by the stimulation of sensory structures which lead to afferent nerve impulses reacting on nerve centers in the medulla to cause secretory nerve impulses to the glands. The stimulating effect of food in the mouth causes the most rapid reflex secretion, which may last through several minutes or even hours. Especially stimulating substances are, beside food, such substances as tartaric acid, lemon juice, ether, alcohol, etc., in fact, anything that produces strong local irritation will lead to reflex secretion.

2. The Secretory Nerves of the Salivary Glands of the Dog.-The nervous mechanism for the salivary glands is well known, and the anatomical relations are such as to make these glands favorable for studying the nervous mechanism of glands in general.

Anesthetize a dog and bind it to a suitable holder. Expose the nerves to the submaxillary gland as follows: cut through the skin of the lower jaw along the inner border for about 3 inches. Isolate and double ligate the jugular vein and any other veins in the field except the ones coming from the submaxillary gland. Isolate and cut the digastric muscle, also the mylohyoid, using pains not to injure the duct of the gland or its arteries. When the muscles are laid back; the artery and accompanying sympathetic nerve branches, the hypoglossal and the lingual nerves, the submaxillary duct and the submaxillary gland, will all be exposed. Isolate and introduce a very fine glass cannula into the submaxillary duct tying it firmly in place. A small nerve filament branches from the lingual nerve and runs to the hilus of the gland, the chorda tympani. Carefully expose the chorda, place a silk ligature under it for convenience in handling. Also expose the sympathetic filaments with the artery.

Stimulate the chorda tympani with a mild induction current for a few - minutes at a time at intervals, and note that the secretion which is absent or forming slowly before stimulation now gathers quickly and leaves the end of the cannula in a series of drops. Collect the saliva in a small beaker. One can measure the rate of flow by collecting the saliva in a small graduated cylinder or, by changing the beaker every ten minutes, making a record of the quantity of secretion formed. Stimulate the sympathetic fibers, cutting the hypoglossal nerve if necessary, and note that the secretion is very slightly 
increased, but the increase lasts for only a few minutes. If the sympathetic fibers are stimulated before the chorda, then the sympathetic secretion is relatively less than if the order of stimulation is reversed.

Connect the duct with a mercury manometer and record the secretion pressure, compare with arterial blood pressure. It is often greater. Explain.

During stimulation of the nerves, note the relative flow of blood through the organ. During chorda stimulation the flow is increased; during sympathetic stimulation it is decreased, as these nerves contain vaso-dilator and vaso-constrictor fibers, respectively.

Inject 5 milligrams of pilocarpine. This drug stimulates the secretory nerve endings and thus causes a copious flow of saliva.

3. Microscopic Changes in the Gland Cells.-Make a histological preparation (by any standard method of fixing and staining) of the submaxillary gland of the cat, $a$, taken after a period of several hours' fasting when the gland cells may be assumed to be at rest; and $b$, immediately after a period of activity (from eating, or activity secured by the stimulation of the chorda tympani) and note: $a$, The cells from the resting gland are relatively larger, the nuclei are pushed back against the basement membrane, they have sparsely sustaining protoplasm, and the cells are crowded with large granules, which in a fortunate preparation fill the entire cell. The outlines of the cells are relatively indistinct and the lumen of the gland is small. $b$, The cells of the active gland are relatively small, the nuclei are centrally placed, the protoplasm stains more definitely, the granules are usually present but limited to the side of the cell next to the lumen, the outlines of the cells are distinct, and the lumen is often quite large.

4. The Chemical Composition of Saliva.-Collect several cubic centimeters of saliva as follows: Wash the mouth thoroughly with water, then induce secretion of saliva by chewing a bit of paraffin or a piece of thoroughly washed rubber. The inhalation of ether vapor will often facilitate the reflex secretion. One should avoid strong acids to induce secretion unless their presence is to be taken into consideration afterward. Make the following tests:

Reaction.-A slip of neutral litmus-paper when introduced into freshly collected saliva, or for convenience simply held in the mouth during salivary secretion, shows an alkaline reaction.

Mucin.-To 3 or 4 cc. of saliva add 2 per cent. acetic acid drop by drop . until distinct acidity is obtained. On stirring the saliva with a glass rod a sticky mucin makes its appearance.

Potassium Sulphocyanide.-To 2 cc. of saliva in a test-tube add 2 or 3 drops of ferric chloride solution, slightly acidulated with hydrochloric acid, a reddish-brown coloration indicates the presence of potassium sulphocyanide. One should run a blank test on distilled water for comparison. 
Chlorides. - Add silver nitrate to 2 cc. of saliva after first removing the proteins. A white, cloudy precipitate, which disappears on adding ammonia and reappears on adding nitric acid, indicates the presence of chlorides.

Proteins.- Remove the mucin from a sample of saliva, as above, and test by the characteristic protein reactions. A faint trace of protein can usually be demonstrated.

5. Digestive Action of Saliva on Starch.-Review the tests for starch, dextrin, and dextrose, as preparation for an identification of these products of salivary digestion. To Io cc. of I per cent. starch paste in the water-bath at $40^{\circ} \mathrm{C}$., add $2 \mathrm{cc}$. of saliva, and mix thoroughly with a glass rod. Immediately begin two series of tests: $a$, for the presence of starch; $b$, for the presence of reducing sugar. The tests for starch can be made by adding to 3 drops of solution, on a porcelain plate, an equal quantity of dilute iodine in potassium iodide solution. Use a glass rod. Make the tests every 2 minutes for 20 minutes. The tests for reducing sugar are best made by placing $2 \mathrm{cc}$. of Fehling's solution in each of a series of test-tubes and adding to successive tubes, at intervals of 5 minutes, $\mathrm{I}$ cc. portions of the digest from a dropping pipet and boiling. If the tests are set away as fast as they are prepared, a reddish-yellow cuprous oxide will settle out, and the series will give a rough comparison as to the quantity of reducing sugar present.

In the first series the deep blue of the starch reaction quickly changes to a reddish-blue, a red, a reddish-brown, until finally no change in color other than that produced by the mixture of the iodine occurs, showing that the starch has passed the second stage of erythro-dextrin in its disappearance. The indication of reducing sugar in the second series shows that this erythrodextrin has been transformed into reducing sugar, and also that the amount of sugar is greatly increased during the progress of the test.

6. The Influence of Temperature on Salivary Digestion.-Prepare three test-tubes, $a, b, c$, containing $i$ cc. each of saliva. Boil $a$, place $b$ in a water-bath at $40^{\circ} \mathrm{C}$., and place $c$ in ice water. After $c$ has been cooled down to the temperature of the ice-bath, add to each $2 \mathrm{cc}$. of I per cent. starch solution and mix. At intervals of 5 minutes test these three samples for the disappearance of starch and appearance of reducing sugar, as in Experiment 5. No change will take place in $a ; b$ will be quickly digested, and the digestion in $c$ will be slight or suspended. Upon placing $c$ in a warm bath digestion will quickly occur.

7. Influence of Acids and Alkalies on Salivary Digestion.-To each of 5 test-tubes, $a, b, c, d, e$, add 2 cc. saliva and water, or the solutions given in the table, so that the saliva will be uniformly diluted. Then add quickly I cc. of I per cent. starch paste to each. Run parallel tests for the appearance of reducing sugar and disappearance of starch. 
The results obtained in Experiments 5, 6, and 7 show that starch is converted into reducing sugar, and furthermore, that the conditions for its conversion indicate that the change is accomplished by an amylolytic enzyme which in this case is called ptyalin.

\begin{tabular}{|c|c|c|c|c|c|}
\hline Steps & A & B & $\mathrm{C}$ & $\mathrm{D}$ & $\mathrm{E}$ \\
\hline $\begin{array}{l}\text { I. Prepare and } \\
\text { set in water- } \\
\text { bath at } 40^{\circ} \mathrm{C} \text {. }\end{array}$ & $\begin{array}{lll}2 & c c . & \text { saliva } \\
2 & \text { cc. } & \text { water }\end{array}$ & $\begin{array}{l}2 \text { cc. saliva } \\
2 \text { cc. } 0.5 \text { per } \\
\text { cent. sodium } \\
\text { bicarbonate }\end{array}$ & $\begin{array}{c}2 \text { cc. saliva } \\
2 \mathrm{cc} . \\
\text { strong } \\
\text { sodium } \\
\text { hydrate }\end{array}$ & $\begin{array}{l}2 \text { cc. saliva } \\
2 \text { cc. } 0.4 \text { per } \\
\text { cent. hydro- } \\
\text { chloric acid }\end{array}$ & $\begin{array}{l}2 \text { cc. saliva } \\
2 \text { cc. strong hy- } \\
\text { drochloric acid }\end{array}$ \\
\hline 2. Then add & I cc. starch & I cc. starch & I cc. starch & I cc. starch & I cc. starch \\
\hline $\begin{array}{l}\text { 3. Test for } \\
\text { starch and } \\
\text { sugar } \\
\text { immediately }\end{array}$ & & & & & \\
\hline $\begin{array}{l}\text { 4. After ro } \\
\text { minutes }\end{array}$ & & & & & \\
\hline $\begin{array}{l}\text { 5. After } 20 \\
\text { minutes }\end{array}$ & & $=$ & & & \\
\hline $\begin{array}{l}\text { 6. After } 40 \\
\text { minutes }\end{array}$ & & & $v$ & & \\
\hline
\end{tabular}

8. The Action of Ptyalin is Favored by the Removal of the End Products.-Place $50 \mathrm{cc}$. of 2 per cent. starch paste in a dialyzing tube or paper, suspend in a beaker of running water. Take $50 \mathrm{cc}$. of the same solution in a beaker, to each add 2 cc. of saliva and mix thoroughly. Test for the disappearance of starch at intervals of 20 minutes. The starch in the dialyzing tube will disappear first because the reducing sugar passes out through the dialyzer, while in the beaker it is retained and hinders the further action of ptyalin.

\section{GASTRIC JUICE AND GASTRIC DIGESTION.}

9. The Secretion of Gastric Juice.-The conditions which influence gastric secretion can be readily observed on the dog with a gastric fistula. Take a dog which has had a gastric fistula prepared some weeks before and which is in a condition of hunger, place him in a holder with a cup suspended to collect the gastric juice, and exhibit before the dog some fresh meat or other food which he enjoys, but do not allow him to eat it. After teasing the 
animal for 5 to ro minutes, an abundant flow of gastric juice will begin. Pawlow calls this the psychic secretion.

If an esophageal fistula has also been performed the dog may be allowed to eat the meat, of course swallowing it out of the esophageal fistula back into the plate. In this experiment an abundant flow of gastric secretion takes place and may continue for an hour or more.

If a gastric pouch has been performed by Pavlov's method, the animal may be allowed to eat the food, swallowing it into the stomach. In this case the reflex secretion just described takes place as usual, but is followed after an hour or an hour and a half by a second increase in the quantity of secretion. This second increase has been ascribed to the reflexes origi-

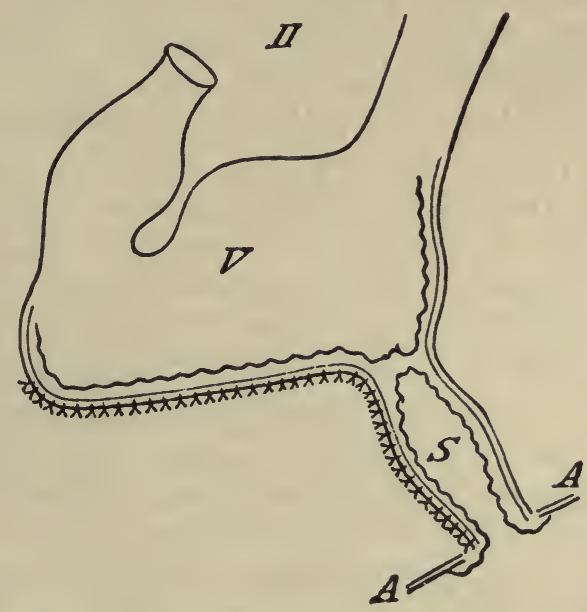

FIG. 278.-Operation on the Stomach to Form an Isolated Pouch with Nerves Intact. $S$, Isolated sac; $V$, cavity of stomach; $A, A$, opening at the abdominal wall. nating in the stomach, possibly from the reflex stimulating action of the digestive products themselves.

I0. Composition of Gastric Juice.-Test a sample of gastric juice obtained from a gastric fistula, as follows:

Keaction.-Gastric juice is strongly acid. Test for free hydrochloric acid as iollows: Gastric juice turns congo-red to a blue color. Gastric juice plus 0.5 per cent. alcoholic solution of dimethyl-amido-azobenzol develops a cherry-red color, a reaction that is given by free hydrochloric acid. Combined hydrochloric acids and organic acids do not give the color. Günzburg's reagent, consisting of 2 per cent. phloroglucin and I per cent. vanillin in 80 per cent. alcohol, produces a rose-colored mirror on porcelain in the presence of free hydrochloric acid. The test is very delicate.

The lactic acid sometimes present in the contents of the stomach is derived partly from the sarcolactic acid of muscle and partly from lactic-acid fermentation of carbohydrates. Lactic acid $\left(\mathrm{C}_{3} \mathrm{H}_{3} \mathrm{O}_{3}\right)$, if present, gives the following test: A solution of to cubic centimeters of a 4 per cent. aqueous solution of carbolic acid, 20 cubic centimeters of water, and one drop of ferric chloride is made; forming a blue-colored mixture. A mere trace of free lactic acid added to such a solution causes it to become yellow. Inasmuch as mineral acids also discharge the color, the lactic acid should first be removed from the gastric contents by shaking with ether and the test tried out with a solution of the residue after evaporation of the ether. 
Proteins.-The usual protein tests (page ro7) can be applied to gastric juice and show that it contains small quantities.

I1. Artificial Gastric Juice.-The active principle, pepsin, of gastric juice can be obtained by extracting the gastric mucous membrane of the dog, pig, etc. Scrape off the mucous membrane, grind it to a fine pulp by repeatedly running it through a sausage machine or by pounding in a mortar with clean sand. The mucous membrane should be allowed to stand for three or four hours before extraction, otherwise the zymogen, and not the enzyme, will be obtained. Extract a portion of this gastric pulp in water and filter. Or extract with glycerin for several weeks and filter. Either of these extracts contains the enzyme. A solution of the glycerin extract in 0.2 per cent. hydrochloric acid contains all the properties of gastric juice. This solution is known as artificial gastric juice.

Commercial pepsin already prepared can be obtained on the market. Artificial gastric juice is made from commercial pepsin by adding 0.5 grams scale pepsin per hundred cc. of 0.2 per cent. hydrochloric acid, which gives a very active preparation.

I2. Digestive Action of Gastric Juice, or Artificial Gastric Juice on Proteins.-The chief digestive action of gastric juice is on proteins. Shreds of fibrin which permit the gastric juice to come in intimate contact with all parts of the material form the best protein for testing the action of this enzyme. Prepare a series of test-tubes, $a, b, c, d$, each containing $5 \mathrm{cc}$. of artificial gastric juice. Add to $a$ some shreds of fibrin; to $b$ some boiled white of egg; to $c$ some fibers of boiled meat; to $d$ some fibers of raw meat; place in a warm bath at $40^{\circ} \mathrm{C}$. and examine at intervals of 20 minutes. Tabulate the results by the plan indicated in Experiment $\mathrm{I}_{3}$, noting particularly the rapidity with which the different proteins go into solution.

13. Condition Affecting the Enzyme Action of Gastric Juice.-Prepare a series of test-tubes containing $5 \mathrm{cc}$. each of gastric juice, according to the table on the next page. Add fibrin threads to each and note the changes at intervals of ro minutes.

I4. The Effect of Bile on Peptic Digestion.-The influence of bile on the activity of pepsin-hydrochloric acid is demonstrated in the following steps:-r. Place in a series of test tubes, $A, B, C, D, 2$ cc. each of 0.5 per cent. pepsin hydrochloric acid solution. 2. Add to $A$ 2 cc. of water; $B_{2}$ cc. water, o.I cc. bile solution (5 per cent. dried bile); $C$ r. 5 cc. water, 0.5 cc. bile solution; $D_{2} \mathrm{cc}$. bile solution. 3. Add to each test tube $2 \mathrm{cc}$. of 0.4 per cent. hydrochloric acid and shake thoroughly. Drop in each test tube 4 fibrin threads, and set in water bath at $40^{\circ} \mathrm{C}$. Tabulate the results at intervals of ro minutes.

15. Cleavage Products of Gastric Digestion.-Add 5 to ro grams of fibrin to $500 \mathrm{cc}$. of artificial gastric juice in a flask and place in a waterbath at $40^{\circ} \mathrm{C}$. After one hour filter off roo cc. Exactly neutralize this 
filtrate with I per cent. potassium hydrate. A precipitate makes its appearance, and can be collected on the filter-paper, washed with distilled water, and dissolved in I per cent. hydrochloric acid, acid metaprotein. Test for the protein reactions.

\begin{tabular}{|c|c|c|c|c|c|c|}
\hline & A & B & $\mathrm{C}$ & $\mathrm{D}$ & $\mathrm{E}$ & $\mathrm{F}$ \\
\hline Prepare & $\begin{array}{c}2 \text { cc. pepsin } \\
\text { solution } \cdot 5 \\
\text { per cent. in } \\
\text { water } \\
\text { (neutral) }\end{array}$ & $\begin{array}{l}2 \mathrm{cc} \text {. hydro- } \\
\text { chloric acid } \\
0.4 \text { per cent. }\end{array}$ & $\begin{array}{l}\text { 2cc. pepsin } \\
\text { hydro- } \\
\text { chloric } \\
\text { acid solu- } \\
\text { tion. (arti- } \\
\text { ficial gas- } \\
\text { tric juice) }\end{array}$ & $\begin{array}{l}\text { 2cc. pepsin } \\
\text { hydro- } \\
\text { chloric } \\
\text { acid solu- } \\
\text { tion in ice } \\
\text { bath }\end{array}$ & $\begin{array}{l}\text { 2cc. pepsin } \\
\text { hydro- } \\
\text { chloric } \\
\text { acid solu- } \\
\text { tion, } \\
\text { boiled }\end{array}$ & $\begin{array}{l}\text { 2cc. pepsin- } \\
\text { alkali solu- } \\
\text { tion. }(0.5 \text { per } \\
\text { cent. } \\
\left.\mathrm{NaHCO}_{3}\right)\end{array}$ \\
\hline Then add & $\begin{array}{l}4 \text { fibrin } \\
\text { threads }\end{array}$ & $\begin{array}{l}4 \text { fibrin } \\
\text { threads }\end{array}$ & $\begin{array}{l}4 \text { fibrin } \\
\text { threads }\end{array}$ & $\begin{array}{l}4 \text { fibrin } \\
\text { threads }\end{array}$ & $\begin{array}{l}4 \text { fibrin } \\
\text { threads }\end{array}$ & $\begin{array}{l}4 \text { fibrin } \\
\text { threads }\end{array}$ \\
\hline $\begin{array}{l}\text { Changes } \\
\text { after ro } \\
\text { minutes }\end{array}$ & & & & & & \\
\hline $\begin{array}{l}\text { after } 20 \\
\text { minutes }\end{array}$ & & & & & & \\
\hline $\begin{array}{l}\text { after } 40 \\
\text { minutes }\end{array}$ & & & & & & \\
\hline $\begin{array}{l}\text { after } 60 \\
\text { minutes }\end{array}$ & & & & & & \\
\hline
\end{tabular}

After twelve hours or more filter the remaining $400 \mathrm{cc}$, exactly neutralize to remove any traces of acid albumin, and filter. The filtrate contains proteoses and peptones. Concentrate the filtrate over a water-bath to onefourth its volume, add an equal quantity of saturated ammonium sulphate solution, a sticky precipitate of primary proteoses separates out. Collect on a filter-paper or in a centrifuge, wash with half-saturated ammonium sulphate, redissolved in very dilute salt solution, and test for protein reactions. The primary proteoses are precipitated by nitric acid.

To the filtrate from the half-saturated ammonium sulphate add crystals of ammonium sulphate until complete saturation with the salt. Deuteroalbumoses separate out. Collect on a filter-paper, wash, dissolve, and test for proteins. The secondary proteoses are not precipitated by nitric acid.

Finally the filtrate contains peptone. It can be isolated and tested by concentrating over the water-bath, adding barium hydrate to slight excess 
to remove the sulphate, filtering, and precipitating the excess of barium by exact neutralization with I per cent. sulphuric acid. Test for protein reactions. Peptone gives a rose color in the biuret reaction. The xanthoproteic reaction gives the color change, but not the usual precipitate. Peptone is redissolved from its alcoholic precipitate without change. It is dialyzable.

I6. Action of Rennin.-Add a solution of commercial rennin (junket powder), or of the extract of gastric mucous membrane of the fourth stomach of the calf, to $5 \mathrm{cc}$. of milk and let stand for a few minutes. Repeat the test with artificial gastric juice. Also with neutral gastric juice. In each case the milk will form a jelly-like clot, which is firmer in the test-tube containing commercial rennin. In the test-tube containing artificial gastric juice the milk is first coagulated, then slowly dissolved or digested. This clotting is due to the special coagulating enzyme, rennin.

\section{PANCREATIC JUICE AND PANCREATIC DIGESTION.}

I7. The Secretion of Pancreatic Juice.-If a dog containing a pancreatic fistula made by Pavlov's method is available, then try the experiment of feeding the animal and noting the rate of secretion of pancreatic juice through a period of two hours. When the gastric digestion has proceeded to the point where the acid chyme may be supposed to have entered the duodenum, then a sharp increase in the flow of pancreatic juice takes place. This increased activity will last through a period of two or three hours or more. It is produced either by nerve reflexes (Pavlov) or by the influence of the secretin produced by the intestinal mucous membrane when stimulated by acid.

I8. Influence of Secretin on the Rate of Secretion.-Make an extract of the intestinal mucous membrane from the duodenum, by scraping off the membrane, grinding it to a pulp, and extracting it over a waterbath in 0.2 per cent. hydrochloric acid, and filtering.

Anesthetize a large dog, open the abdomen, isolate the pancreatic duct, introduce a cannula, and arrange for the collection of pancreatic juice. Introduce a cannula into the saphenous vein and connect it with a buret containing the extract of secretin already prepared. Inject 5-cc. quantities of the secretin solution into the vein at intervals of ten minutes. Measure the rate of secretion of pancreatic juice by counting the drops per minute or, if the secretion is rapid enough, by collecting it at intervals of five or ten minutes and measuring it in a graduated pipet.

This method will often yield enough pancreatic juice in the course of a couple of hours to make the pancreatic experiments which follow. Bayless and Starling call it secretin juice.

I9. Chemical Characters of Pancreatic Juice.-Test the reaction, protein, salt, etc., content of the sample of pancreatic juice collected in the last experiment. 
20. Artificial Pancreatic Juice.-Artificial pancreatic juice can be prepared from the pancreas by grinding and macerating and extracting a pancreas with water or glycerin, as described for the gastric glands in Experiment II above. Commerical preparations of pancreatic enzyme can be obtained on the market. A solution of a glycerin extract of pancreatic gland or of commercial pancreatin in 0.2 per cent. sodium carbonate is known as artificial pancreatic juice.

2I. The Enzymes of Pancreatic Juice.-The pancreatic juice contains enzymes which exert a digestive action on starches, fats, and proteins. This fact can be tested as follows: $a$, to $5 \mathrm{cc}$. of artificial pancreatic juice add $2 \mathrm{cc}$. of I per cent. starch paste, mix and set in the water-bath at $40^{\circ} \mathrm{C}$.; $b$, to I cc. of pancreatic juice (artificial juice is not active), collected in Experiment I 7 , add $0.5 \mathrm{cc}$. of neutral olive oil, and place over a water-bath; $c$, to $5 \mathrm{cc}$. of artficial pancreatic juice add a few flocks of fibrin. Test the digestive action on starch by the iodine test for the disappearance of starch, or by the copper-reduction test for the presence of reducing sugar. Test the fat by its reaction, noting that the neutral or slightly alkaline solution has become acid, also by the fact that an emulsion has been formed. Note that the protein has gone into solution.

The digestive action on starch is due to the enzyme amylopsin, or pancreatic diastase, as it is sometimes called. The fat-splitting effect is due to the enzyme lipase, and the solution of the fibrin is accomplished by the proteolytic enzyme, trypsin.

22. Conditions which Influence the Action of the Enzymes of Pancreatic Juice.-Prepare each of 5 test-tubes, $a, b, c, d, e$, as shown in the table on the next page. Place $a, b, c, d$ in the water-bath at $40^{\circ} \mathrm{C}$., and $e$ into an ice-bath. Add to each tube $2 \mathrm{cc}$. of I per cent. starch paste. Follow the digestive changes by the tests previously outlined and tabulate the results.

Repeat this experiment with a second set of test-tubes containing 4 threads of fibrin in each. Lipase is not very active in artificial pancreatic juice and may be omitted, but if pancreatic juice is available make a third set containing fat.

23. Cleavage Products of Pancreatic Digestion.-To $400 \mathrm{cc}$. of artificial pancreatic juice add 25 grams of moist fibrin and place in a water-bath at $40^{\circ} \mathrm{C}$., add $2 \mathrm{cc}$. of chloroform or of thymolin alcoholic solution to prevent putrefactive changes. After three or four hours filter off roo cc. and place the remainder on the water-bath for one or two days. Test the filtrate for alkali ablumin, albumoses, and peptones by the method outlined in Experiment 14 above.

Filter the second portion and concentrate to a syrupy mass on the waterbath. Crystals make their appearance. Pour off the fluid, wash the crystals with cold water, and examine under the microscope for sheaves of tyrosin. The filtrate contains leucin. 
If the digestion had been allowed to proceed without the antiseptic, bacteria would have appeared in the solution, and protein cleavage products, due to their action, would be found, notably indol, phenols, and volatile fatty acids.

\begin{tabular}{|c|c|c|c|c|c|}
\hline & A & B & $\mathrm{C}$ & $\mathrm{D}$ & $\mathrm{E}$ \\
\hline Take & $\begin{array}{l}2 \text { cc. neutral } \\
\text { pancreatin, } \\
2 \text { cc. } 0.5 \text { per } \\
\text { cent. } \mathrm{Na}_{2} \mathrm{CO}_{3}\end{array}$ & $\begin{array}{l}2 \text { cc. neutral } \\
\text { pancreatin, } \\
2 \text { cc. water }\end{array}$ & $\begin{array}{l}2 \text { cc. neutral } \\
\text { pancreatin, } \\
2 \text { cc. } 0.4 \text { per } \\
\text { cent. } \mathrm{HCl}\end{array}$ & $\begin{array}{l}2 \text { cc. neutral } \\
\text { pancreatin, } \\
2 \text { cc. water. } \\
\text { Boil }\end{array}$ & $\begin{array}{l}2 \mathrm{cc} \text {. neutral } \\
\text { pancreatin, } \\
2 \mathrm{cc} . \mathrm{Na}_{2} \mathrm{CO}_{3} \text {. } \\
\text { Keep at } 0^{\circ} \mathrm{C} \text {. }\end{array}$ \\
\hline Then add & 2 cc. of starch & 2 cc. of starch & 2 cc. of starch & $2 \mathrm{cc}$. of starch & $2 \mathrm{cc}$. of starch \\
\hline \multicolumn{6}{|l|}{$\begin{array}{l}\text { Note after } \\
20 \mathrm{~min}- \\
\text { utes }\end{array}$} \\
\hline \multicolumn{6}{|l|}{$\begin{array}{l}\text { After } 40 \\
\text { minutes }\end{array}$} \\
\hline $\begin{array}{c}\text { After } 60 \\
\text { minutes }\end{array}$ & & & & & \\
\hline
\end{tabular}

\section{BILE AND INTESTINAL JUICE.}

24. Bile.-Secure bile from the gall-bladder of a pig or dog, or, if it is possible, a sample of human bile. Test the reaction which, in fresh bile, is neutral. Test for mucin, albumin, and for iron; hydrochloric acid and ferrocyanide of potassium give a blue color when iron is present.

BileSalts.-Evaporate io c.c. of bile to complete dryness, mix with animal charcoal, add 50 c.c. of absolute alcohol, filter; add an excess of ether to the filtrate, which gives a white precipitate of bile salts. Crystals will form on standing in a well-stoppered flask for a day or two.

Bile Acids.-A drop of syrup of cane-sugar in a test-tube of bile forms a deep red-purple color at the line of separation from concentrated sulphuric acid. Furfur aldehyde with cholalic acid gives the color.

Bile Pigments. -With I c.c. of bile in a test-tube strong nitroso-nitric acid produces a play of colors beginning with green, blue, red, and yellowGmelin's test.

Bile does not contain digestive enzymes, but the bile wets the mucous surface of the intestine and facilitates the solution and absorption of fat and fatty acids.

25. Intestinal Juice.-The secretion of the mucous membrane of the small intestine has been proven to have a weak digestive action on proteins and perhaps on starches. It can be obtained from an intestinal fistula. 
Its chief digestive importance consists in the presence of the activating enzyme, enterokinase. Enterokinase can be prepared by extracting the mucous membrane of the small intestine by the method outlined for making a pancreatic extract.

To two test-tubes containing $2 \mathrm{cc}$. of artificial pancreatic juice, or preferably containing secretin pancreatic juice, add 2 threads of fibrin. Keep one for the control, to the other add 2 cc. of enterokinase solution. The testtube containing enterokinase will digest more rapidly and more effectively than the other.

\section{ALIMENTARY MECHANISMS.}

26. Normal Peristalsis and the Vagus Control of the Frog Stomach and Intestine.-Pin a pithed frog supine on a frog board. Expose the stomach and isolate the vagus. Attach a lever to the outer curvature of the stomach by S-shaped hook and thread, so that the contractions register as usual. Anchor the gastric mesentery by pin but avoid blood vessels.

a. Make a continuous record on a slow drum of the normal peristalsis of the stomach for 30 minutes.

b. Stimulate the vagus nerve for 5 to ro seconds with weak induction shocks, as tested by your tongue. Mark the time of stimulation by a signal magnet. Allow a long recovery period after each stimulation. Repeat with stronger stimuli.

c. Compare by direct inspection the general characteristics of gastric peristalsis both before and following vagus stimulation. Does stimulation induce a single contraction or a rhythm?

$d$. Transfer the lever to the duodenum and record its normal contractions. Then stimulate the vagus to test extrinsic motor nerve control.

$e$. Expose the small intestine of the frog just used and make observations by inspection without registration. Lightly pinch the pyloric stomach or duodenum with a forceps. Note the time with watch and determine the rate of peristalsis. Observe the final swinging movements of the cloaca or rectum. Remove the intestine and measure its total length. Calculate the rate of propagation per centimeter per second and the total time for the wave to pass the length of the intestine.

27. Gastro-intestinal Movements of the Cat.-Use a two kilo cat which has been fed ground meat four hours before. Give Io cc. of a ro per cent. chloral hydrate solution per rectum, or 4 grams of urethane, twenty minutes before the experiment. Very little ether is then required for narcosis. Open the abdomen along the midline to expose the stomach and intestine. Protect the visceral organs by covering with very thin wax paper. 
$a$. Observe two kinds of intestinal movements, segmenting movements of the intestine, and peristaltic movements, i.e., rings of contractions moving downward. Compare these movements carefully, noting the rate of propagation, the sequence, etc.

$b$. Note especially the movements of the stomach. Compare the pylorus with the fundus. Where does peristalsis commence in the cat stomach?

c. Insert a stomach tube and fill with warm water until distended, record any reactions.

$d$. Stimulate the vagus in the neck with interrupted induction shocks of moderate intensity.

e. Cut the splanchnics, then stimulate the vagus again. Explain the variations.

$f$. Inject I cc. of a o.I per cent. nicotine solution intravenously, explain.

g. Inject I cc. of o.I per cent. epinephrin.

28. Pancreatic and Bile Secretion.-Anesthetize and tracheotomize a dog and connect with the ether apparatus. Expose the jugular for venous injections. Ligate the vagus on the same side and section. Open the abdomen along the upper half of the linea alba, 6 or 8 inches. Expose the duodenum and insert a cannula into the common bile duct at the point where it joins the intestine. The greater pancreatic duct along the posterior border of the pancreas enters the intestine below the bile duct. Carefully dissect and insert a cannula. Provide all the cannulæ with small rubber tubes long enough to extend to the surface through the wound. Carefully adjust the parts and close the wound by stitches.

a. Record the rate of pancreatic and bile secretions by signal key.

$b$. Stimulate the peripheral end of the vagus in the neck. Give time for recovery from the vascular effects and for the secretion to develop.

c. Inject 20 to $30 \mathrm{cc}$. of 0.4 per cent. hydrochloric acid by hypodermic needle into the duodenum. The reaction is slow. Allow I $_{5}$ minutes or more and repeat.

$a$. Inject $40 \mathrm{cc}$. of "secretin" solution prepared by extracting the macerated mucosa of the duodenum with 0.4 per cent. $\mathrm{HCl}$ and neutralizing carefully and filtering. Secretin is in solution in the filtrate. Repeat in 20 to 30 minutes (Journal of Physiology, Vol. 28, p. 235, I920). Read the topic of chemical control and hormones. 


\section{CHAPTER IX}

\section{ABSORPTION}

THE term absorption in its restricted physiological use means the process by which the digested foods pass through the walls of the alimentary canal and into the circulation. In a more general sense absorption is the process by which substances pass from one part of the body to another by means other than the blood and lymph vessels. Usually absorption takes place from a free surface, such as the mucosa of the alimentary canal, the surface of the skin, and from the lungs.

The alimentary canal is lined throughout with a continuous layer of epithelial tissue. This layer is only a single cell thick in most of its extent, but nevertheless it effectively separates the food inside the canal from the lymph in the tissue interspaces on the outside of the mucous membrane. These spaces are separated from the blood in the adjacent blood vessels by a second continuous layer, the endothelial walls of the capillaries. The food, therefore, during its absorption from the alimentary canal must pass through two layers of tissue to reach the blood stream. But the submucous lymphatic spaces and vessels furnish channels which may carry substances into the blood by way of the thoracic duct. The mucous membrane is, therefore, the one strict barrier through which the food must pass in the act of absorption.

The exact methods by which absorption takes place have long been a subject of controversy and of research. But this problem is of such difficulty that it is yet, in the main, unsolved. Known physical and chemical laws are thought to explain the facts of absorption. Some of the physical factors concerned in absorption and elimination have already been considered in a former chapter, osmosis and diffusion, Chapter IV. A third factor, filtration, consists in the passage of a fluid under pressure through a membrane. These factors undoubtedly play an important rôle in the passage of solutions through the alimentary mucous membrane and the walls of the blood vessels. The part which the physical factors play is probably more pronounced in the absorption of water and crystalloids. The nature of the fluid within the digestive tract, and the movements of the walls of the stomach and intestines by means of which the material to be absorbed is brought into intimate contact with the absorbing membrane, are additional factors which influence absorption.

But the mechanical and physical factors do not fully explain the observed facts of absorption. It becomes more and more evident that there is an unexplained factor bound up in the characteristics of the living protoplasm of the epithelial cells themselves. When isotonic blood serum is introduced 
into the intestine the salts and water are at once absorbed, also the albumins, but more slowly. In this experiment the osmotic conditions are in balance and the pressure is greater on the side of the blood vessels, so that absorption takes place with the actual expenditure of energy. The important fact here is that the absorption through a living membrane is influenced by the membrane in ways that we cannot yet explain. It is this factor which determines the different rate of absorption and the so-called selective absorption in different regions of the alimentary canal.

As a rule, the current of absorption is from the stomach or intestine into the blood; but the reversed action may occur, as, for example, when sulphate of magnesium is taken into the alimentary canal. In this case there is a rapid discharge of water from the blood vessels into the canal. The rapidity with which matters may be absorbed and diffused through the textures of the body has been found by experiment. It appears that lithium chloride may be diffused into all the vascular textures of the body, and into some of the non-vascular, as the cartilage of the hip-joint, as well as into the aqueous humor of the eye, in a quarter of an hour after being given by way of the mouth and on an empty stomach. Lithium carbonate, when taken in fiveor ten-grain doses on an empty stomach, may be detected in the urine in five or ten minutes; or, if the stomach be full at the time of taking the dose, in twenty minutes.

Absorption in the Mouth.-The epithelial lining of the mouth is of the thicker stratified type and the conditions are otherwise unfavorable for absorption. Little, if any, absorption normally takes place in the mouth, and the same is true for the esophagus.

Absorption in the Stomach.-The mucous and submucous coats of the stomach, see figure 258 , are well supplied with blood vessels and lymphatics. The mucous membrane is, however, so crowded with the peptic glands that the relative amount of absorbing surface is small. It is limited to the mucous membrane around the mouths of the glands.

Recent experiments have shown that though absorption does take place in the stomach, it is not as active as was formerly supposed, even in the case of water. Von Mering has found that water begins to pass from the stomach into the intestine almost as soon as it is swallowed, and that very little of it is absorbed from the stomach. Of $500 \mathrm{cc}$. given by the mouth to a large dog with a duodenal fistula, only $5 \mathrm{cc}$. were absorbed in 25 minutes, the rest having passed into the intestine. Peptones, sugars, and salts are absorbed in the stomach, but only to a limited extent. Peptones are not absorbed in appreciable amount unless present to as much as 5 per cent. or more. Examination of the mucous membrane during the stage of active digestion has revealed the presence of albumoses. Sugars, like peptones, are absorbed by the stomach only to a slight extent in the weaker solutions, but are readily absorbed when the more concentrated solutions are intro- 
duced into the stomach, 5 per cent. and over (von Mehring). That fats are absorbed in the stomach was clearly indicated by von Kölliker as far back as I 857 , although the fact seems to have been more or less ignored all these years. He observed an increase in the amount of fat in the gastric mucosa of both young and old animals after feeding. This observation has been confirmed under carefully guarded experimental conditions, not only for the different experimental laboratory animals, but for snakes and a number of fishes. The relative amount of fat absorbed through the gastric mucosa is small, however, compared with that absorbed by the intestinal villi. Even

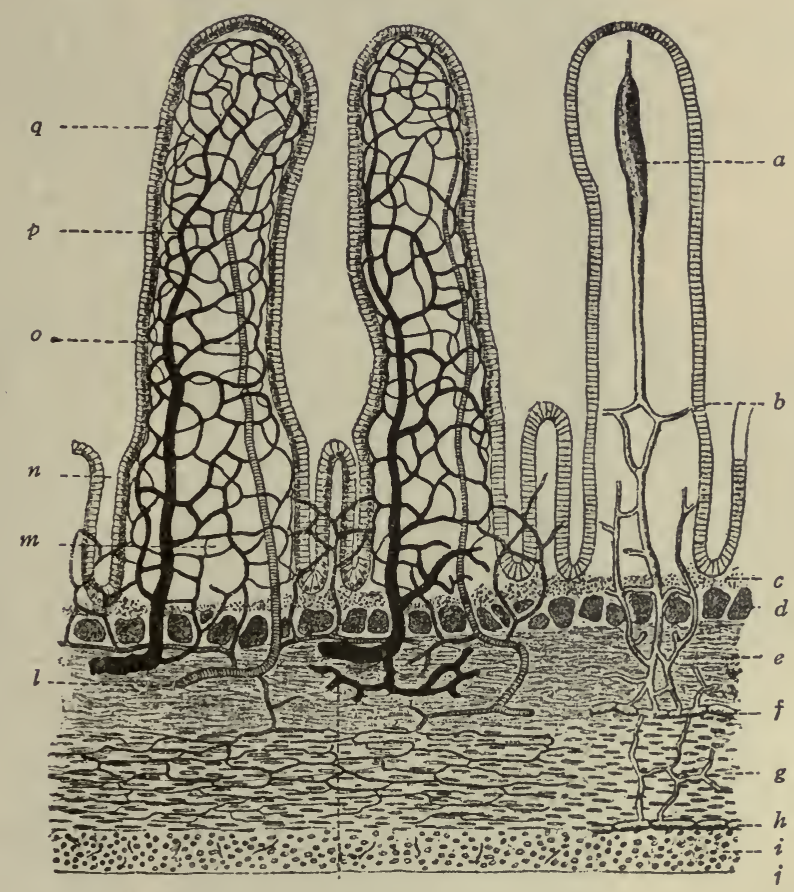

FIG. 279. - Scheme of Blood Vessels and Lymphatics of Human Small Intestine. $a$, Central lacteal of villus; $b$, lacteal; $c$, stroma; $d$, muscularis mucosæ; $e$, submucosa; $f$, plexus of lymph vessels; $g$, circular muscle layer; $h$, plexus of lymph vessels; $i$, longitudinal muscle layer; $j$, serous coat; $k$, vein; $l$, artery; $m$, base of villus; $n$, crypt; $o$, artery of villus; $p$, vein of villus; $q$, epithelium. (Mall.)

salts in the stomach are not readily absorbed until the concentration is from three to four times that of the blood. This fact is in direct opposition to the popular views on the subject.

While some absorption does take place in the stomach it is evidently not of any great importance under normal conditions. The presence of alcohol has been shown to increase the amount of absorption, and pepper, mustard, and such drugs as produce mild local irritation accomplish the same result. 
Absorption in the Intestines. - The products of digestion are all readily absorbed in the small intestine, as is abundantly shown by experiments. Absorption from the small intestine has been studied in the human subject in the case of a patient who had a fistulous opening in the lower part of the ileum. For example, 85 per cent. of the protein of a test meal was absorbed before the food reached the fistula. The food passes slowly down the length of the small intestine, and the digestive changes produce a series of cleavages

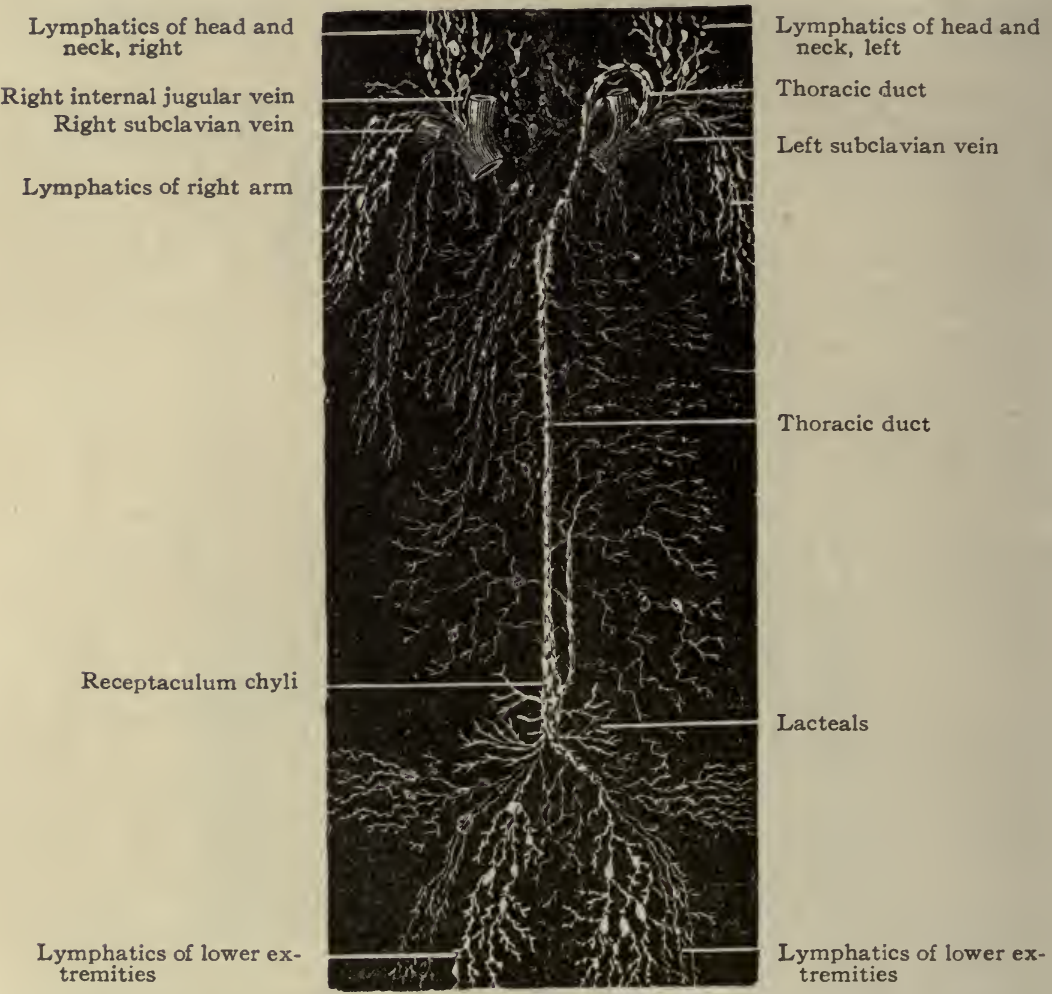

FIG. 280.-Diagram of the Principal Groups of Lymphatic Vessels. (From Quain.)

which have known osmotic and diffusion properties. The question has been to determine which of the cleavage products are most favorable for absorption and the details of the mechanism.

The mucous membrane of the small intestine possesses special structures for absorption, the villi. Each villus projects as a finger-like process into the lumen of the intestine. Its single-layered covering of epithelial cells supported by a connective-tissue framework brings a relatively large extent of surface into contact with the digesting food, which is thus separated from a loop of capillaries and lymphatic radicals. 
The capillaries of the villus are connected with the veins which contribute to the portal vein, hence carry blood to the liver. The lacteals of the villus contribute to the mesenteric lacteal system, hence the chyle and lymph pass through the mesenteric glands and the thoracic duct to the subclavian vein

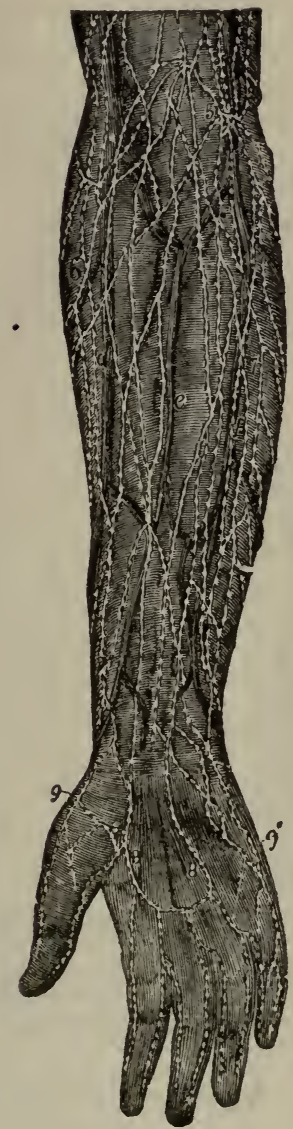

FIG. 281 .

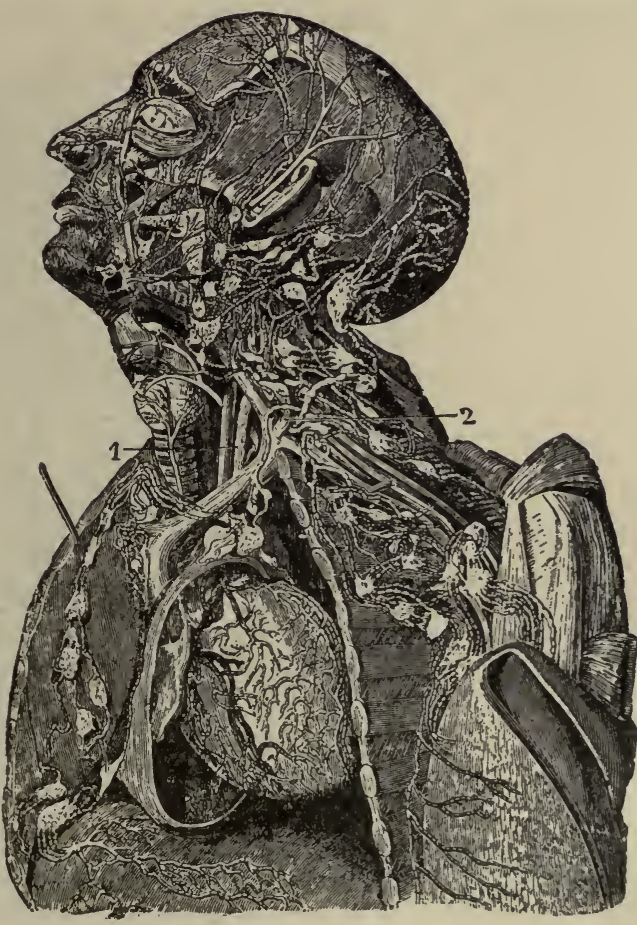

FIG. 282 .

FIG. 28I.-Superficial Lymphatics of the Forearm and Palm of the Hand, $\frac{1}{5} .-5$, Two small glands at the bend of the arm; 6 , radial lymphatic vessels; 7 , ulnar lymphatic vessels; 8,8 , palmar arch of lymphatics; $9,9^{\prime}$, outer and inner sets of vessels; $b$, cephalic vein; $d$, radial vein; $e$, median vein; $f$, ulnar vein. The lymphatics are represented as lying on the deep fascia. (Mascagni.)

FIG. 282.- Lymphatic Vessels of the Head and Neck and the Upper Part of the Trunk. (Mascagni.) $\frac{1}{6}$. - The chest and pericardium have been opened on the left side, and the left mamma detached and thrown outward over the left arm, so as to expose a great part of its deep surface. The principal lymphatic vessels and glands are shown on the side of the head and face, and in the neck, axilla, and mediastinum. Between the left internal jugular vein and the common carotid artery, the upper ascending part of the thoracic duct marked $x$, and above this, and descending to 2 , the arch and last part of the duct. The termination of the upper lymphatics of the diaphragm in the mediastinal glands, as well as the cardiac and the deep mammary lymphatics, is also shown. 
in the neck. There are thus two routes by which absorbed foods may reach the general circulation. These paths can be independently isolated; and a study of the composition of their discharge during active absorption contributes to our knowledge of the course taken by the different absorption products.

Absorption of Proteins from the Intestines.-Protein is absorbed chiefly in the small intestine, though just exactly how cannot at present be affirmed. In the preceding chapter the cleavage products of protein digestion have been discussed. It was shown there that proteoses, peptones,

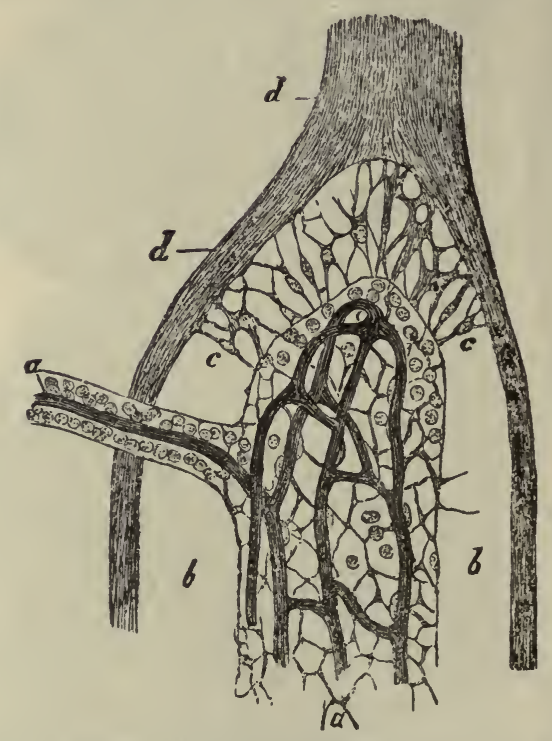

FIg. 283.-A Small Portion of Medullary Substance from a Mesenteric Gland of the Ox. $d, d$, Trabeculæ; $a$, part of a cord of glandular substances from which all but a few of the lymph corpuscles have been washed out to show its supporting meshwork of retiform tissue and its capillary blood vessels (which have been injected and are dark in the figure); $b, b$, lymph sinus, of which the retiform tissue is represented only at $c, c . \times 300$. (Kölliker.)

peptids, and the amino-acids are derived from the proteins as digestion products. It has, in the past, been assumed that peptone represents the form most freely absorbed. No peptone has, however, been isolated from the blood or lymph on the vascular side of the epithelial membrane. The present supposition is that the protein cleavage products are taken up by the epithelium and synthesized into other and more complex forms before being discharged into the blood; or that they are resynthetized into the charac teristic tissue proteins after absorption. The digestion cleavages not so utilized are desamidized by the liver, and the ammonia so formed subsequently converted into and eliminated as urea.

In animal foods, such as eggs, meat, etc., it is estimated that about 98 per cent. of the protein is absorbed; whereas in vegetable foods, where the pro- 
tein is often protected from the action of the digestive enzymes, there may be Io to I 5 per cent. loss. Analysis of the total lymph discharged by the thoracic duct fails to show any increase of proteins during active digestion, from which it is inferred that proteins pass by way of the liver.

The non-nitrogenous residue is oxidized or temporarily stored as glycogen.

From 12 to $x 5$ per cent. of the protein remains in the food as it passes the ileocecal valve. This amount, less the loss in the feces, is absorbed in the large intestine.

Absorption of Carbohydrates by the Intestines.-Carbohydrates are broken down to dextrose, levulose, etc., and are absorbed as such. Even

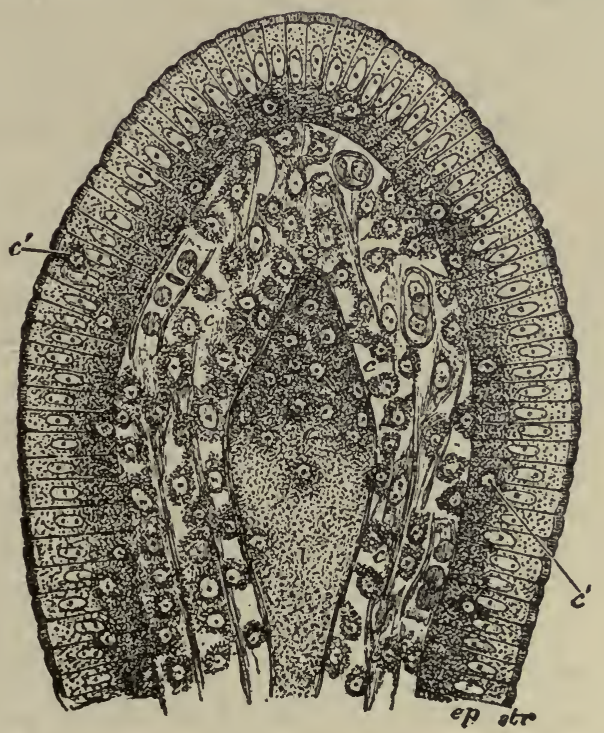

Fig. 284.-Section of the Villus of a Rat Killed during Fat Absorption. ep, Epithelium; str, striated border; $c$, lymph cells; $c^{\prime}$, lymph cells in the epithelium; $l$, central lacteal containing disintegrating lymph corpuscles. (E. A. Schäfer.)

the soluble cane-sugar is split by the invertase of the intestine into the monosaccharides, dextrose and levulose. Starch is the source of most of the 500 grams of dextrose absorbed in an average diet per day. During the absorption of a carbohydrate meal the percentage of dextrose in the blood of the portal vein is increased over the normal, which is O. I to I. 5 per cent. This excess of dextrose passes through the liver and is temporarily stored in the liver cells as glycogen. In the case of a fistula in the receptaculum chyli, the chyle contained less than a half per cent. of the total dextrose absorbed.

Experiments on the rate of absorption of the different sugars seem to indicate that their absorption does not follow known physical laws and that we must assume an unknown chemical factor in the living protoplasm.

Dextroses are absorbed readily by the large intestine. 
Fermentation processes from bacterial growth produce certain acids from the carbohydrates, chiefly in the large intestine. These are readily absorbed.

Absorption of Fats by the Intestines.-Fats reach the absorbing epithelium in two forms, as soluble glycerol and soaps and as finely emulsified fats. The first two are taken up by the epithelium readily enough, but the proof of absorption of emulsified fats is not so clear. It is comparatively easy to demonstrate the presence of microscopic globules of fat in the intestinal mucosa both in the epithelial cells themselves and to a less degree

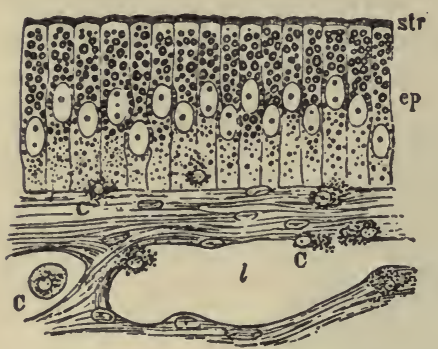

Fig. 285.-Mucous Membrane of Frog's Intestine during Fat Absorption. ep, Epithelium; str, striated border; $C$, lymph corpuscles; $l$, lacteal. (E. A. Schäfer.) in the intercellular substance. But it has been constantly noticed that there is a clear zone along the inner or free borders of the cells. Fat drops exist in the adjacent digesting mass, and in the deeper parts of the cells, but not in this border zone. The demonstration of the reversible action of lipase, has strengthened Pflüger's fat dissociation theory which holds that before absorption the emulsified fats too must be decomposed. They can then pass through the cell border and are resynthesized in the cell protoplasm. This is of course against the strictly, mechanical view, which must be abandoned in the presence of the evidence supporting the newer conception. The decrease in efficiency of fats as foods when the bile, which wets the mucous surface and dissolves the fatty acids, is withheld from the intestine, supports this view. As absorption progresses the size of the fat drops in the epithelial cells increases, a fact that is readily explained by supposing a continued synthesis and accumulation of fat. Pflüger's view of absorption has recently received strong support in the observations of Bloor that isomannid esters of fatty acids when fed to animals were digested but could not be recovered after absorption. Supposing that lipolytic cleavage occurred in this fat during digestion, it would of course not be rebuilt in the cells of the epithelium after absorption. On the theory that the absorption of fats takes place in the emulsified form, this compound should have reappeared in the chyle, but it did not.

The fat is ultimately discharged into the connective-tissue spaces, passes through thelymph channels, the thoracic duct, and into the blood of the subclavian vein. This is the course taken by the larger percentage of the fat. However, during absorption some of the fat enters the capillaries of the villi and passes through the liver. The presence of fat drops in the liver cells at certain times can be ascribed to storage of this absorbed fat, the liver exhibiting not only a glycogenic but a lipogenic function.

It is said that the more readily emulsified fats, those that melt readily at 
the body temperature, are the more completely absorbed. The efficiency of such absorption is as high as 96 to 98 per cent. for the oils, and decreases sharply for such fats as the tallows.

The large intestine is capable of absorbing fats, though not so readily as the small intestine.

Absorption of Minerals and Water in the Intestines.-The salts common in the foods are most of them readily soluble, dissociate quite completely in the dilute solutions, and diffuse and dialyze readily. Of the salts of the foods, the sodium and potassium cations and chlorine anion are the most readily dissociated and are most diffusible, while the calcium and magnesium cations and the sulphate anion are least diffusible. The substances pass through the intestinal epithelial cells and the intercellular substance; at least salts easily recognized by microchemical means have been found in both localities during absorption. It seems probable that the forces concerned are largely osmosis and diffusion.

Yet observers have not been able to show that the rate and character of the absorption of even the salines obey the known physical laws. In fact, there is evidence that some of the salts, iron for example, are taken up as organic compounds (hematogens of Bunge). The activity of the epithelial cells is to be taken into account, even in the absorption of salts.

Water, which we have seen is not absorbed in the stomach, is readily absorbed in the small intestine. Perhaps the bulk of water taken into the system is absorbed in the upper part of the small intestine. In the large intestine, too, it is absorbed with facility and in considerable quantities. The content of the bowel is still quite fluid when it enters the ascending colon, but the feces are quite firm on discharge from the rectum. There are many analogies by which we may suppose a controlling influence of the epithelium over the process of water absorption. Among the fishes there are species, the salmon for example, in which the blood maintains a relatively constant osmotic pressure, and therefore salt content. In the salmon this is about the same as that of human blood. The blood is separated in the gills by an extremely thin epithelium from the water in which the animals live, yet these fishes go with impunity from sea-water, with two and a half times more salt than the blood, to fresh water with practically no salt at all. The epithelium of the gills permits the passage of oxygen, but it does not permit the diffusion or dialysis of the salts or the water in either direction. It is possible that there is a certain amount of resistance to the passage of water through the walls of the stomach, while the intestinal epithelium permits water to pass readily.

The factors active in absorption are under searching investigation at the present time, so that it is reasonable to hope that the near future will give a more exact understanding of this intricate subject. 


\section{ABSORPTION FROM THE SKIN, THE LUNGS, ETC.}

The dry corneous stratified epithelium covering the human body possesses great resistance to the absorption of most substances. The sebaceous secretion keeps the surface slightly oily. Watery solutions do not readily wet the surface and therefore do not penetrate. There is some absorption of water on prolonged contact with the skin, but the amount is insignificant. Medicated baths, especially hot baths, may be accompanied by some slight absorption of the substances dissolved in the waters, though it must be confessed that the primary good effects of such treatment come from other sources.

On the other hand, oily substances come in more intimate contact with the skin and penetrate deeper and more readily. Therefore, lotions containing medicines are occasionally applied to the skin, and slow but gradual absorption occurs. The volatile oils penetrate the skin readily.

The epithelial lining of the lungs seems peculiarly adapted to the quick absorption of gases and volatile substances. This is illustrated by the rapidity of adjustment of the body to variations in pressure in the inert nitrogen of the air in caisson work and in aviation. The volatile anesthetics, ether and chloroform, penetrate the pulmonary epithelium with greatest facility. The same is true for other volatile substances. There is increasing evidence that most substances soluble in water penetrate the pulmonary epithelium with facility, and that this may in the future become a more used channel for administering medicines.

Solutions injected into or otherwise brought into contact with the subdermal connective tissue, the surface of the body of a muscle, or intramuscularly, or within the peritoneal or thoracic cavities, very quickly pass into the general circulation. They are practically injected into the lymphatic interceliular spaces in these instances and, of course, are very readily carried through the lymphatic vessels, figures 280 and 282 , to the thoracic duct and into the blood. Readily diffusible substances, however, pass directly through the blood capillary walls and do not necessarily follow the longer lymph channel route. Comparing the rapidity of absorption in the cases mentioned, that from the muscle is most rapid, a fact of medical importance in the use of the hypodermic needle for the giving of medicines in emergency. 


\section{CHAPTER X.}

\section{EXCRETION.}

EVERY substance taken into the body, in whatever form, must, in the end, be cast off again, no matter how great the change that may be wrought during its sojourn. We have already found that in the lungs the expired air, and in the intestine the feces, carry from the body waste matters of no further use. We have now to find that the kidneys, separating the urine, and the skin, separating the sweat and the sebum, are likewise channels by which the body throws off water, salts, and broken-down organic matters of no further use to the organism. Of these two organs, the skin and the kidney, the latter is by far the more important in so far as the quantity and complexity of its secretion is concerned.

\section{STRUCTURE AND FUNCTION OF THE KIDNEYS.}

General Structure.-The kidneys are two in number, and are situated deeply in the lumbar region of the abdomen on either side of the spinal column behind the peritoneum. They correspond in position to the last two dorsal and two upper lumbar vertebræ, the right slightly below the left in consequence of the position of the liver on the right side of the abdomen. They are about 4 inches long, $2 \frac{1}{2}$ inches broad, and $x^{\frac{1}{2}}$ inches thick. The weight of each kidney is about $4 \frac{1}{2}$ ounces, I 40 grams.

On dividing a kidney into two equal parts by a section carried through its long convex border, figure 286, the main part of its substance is seen to be composed of two chief portions called, respectively, cortical and medullary, the latter being also sometimes called pyramidal, from the fact of its being composed of about a dozen conical bundles of uriniferous tubules, each bundle forming what is called a pyramid. The upper part of the ureter, or duct of the organ, is dilated into the pelvis; and this, again, after separating into two or three principal divisions, is finally subdivided into 8 to $\mathrm{I} 2$ smaller portions, calyces, each of which receives the pointed extremity or papilla of a pyramid. Sometimes, however, more than one papilla is received by a calyx.

The kidney is a compound tubular gland. Both its cortical and its medullary portions are composed essentially of numerous tubes, the tubuli uriniferi, which begin at the opening on the Malpighian pyramid and, after a devious course, end in the capsule of the glomerulus. 
Tubuli Uriniferi.-The tubuli uriniferi, figure 287 , are composed of a nearly homogeneous membrane, and are lined internally by epithelium. They vary considerably in size in different parts of their course, but are, on an average, about $40 \mu$ in diameter, and are found to be made up of several distinct sections. The first section or part to be identified is the $\mathrm{Mal}$ pighian, or Bowman's capsule, figure 287 . It is composed of a hyaline membrana propria, thickened by a varying amount of fibrous tissue, and lined by

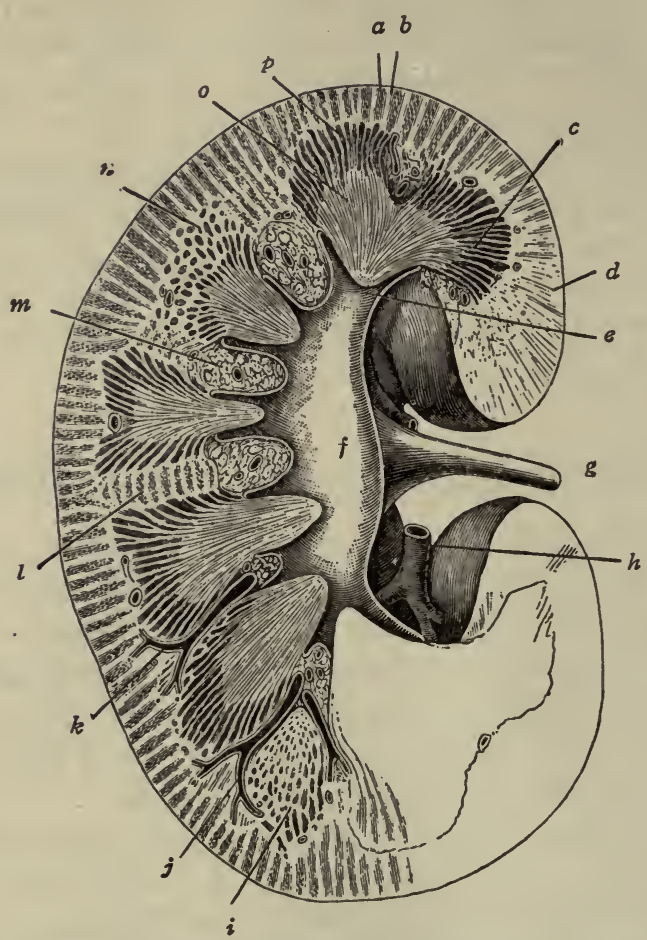

Frg. 286.- - Longitudinal Section of Kidney through Hilum. $a$, Cortical pyramid; $b$, medullary ray; $c$, medulla; $d$, cortex; $e$, renal calyx; $f$, hilum; $g$, ureter; $h$, renal artery; $i$, obliquely cut tubules of medulla; $j$ and $k$, renal arches; $l$, column of Bertini; $m$, connective tissue and fat surrounding renal vessels; $n$, medulla cut obliquely; 0 , papilla; $p$, medullary pyramid. (Merkel-Henle.)

flattened nucleated epithelial plates. This capsule is the dilated extremity of the uriniferous tubule which is invaginated to receive the glomerulus of convoluted capillary blood vessels. The invaginated portion of the tubule is of particular importance since it is the membrane through which a large part of the urine is secreted. The glomerulus is connected with an efferent and an afferent blood-vessel. The Malpighian capsule is connected by a constricted neck, figure $287, N$, with the proximal convoluted tubule. This forms several distinct curves and is lined with short columnar cells. The tube 
next passes almost vertically downward toward the medulla, forming the spiral tubule, still within the cortex of the kidney, which is of much the same diameter. The loop of Henle, $L$, in the medulla, is a very narrow tube lined with flattened nucleated cells. Passing vertically upward from the loop of

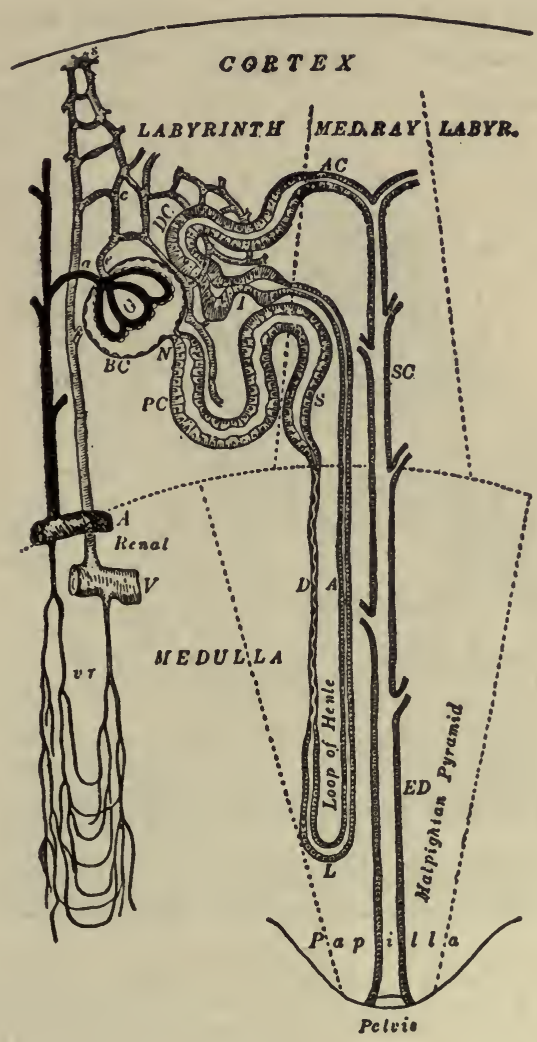

Fig. 287.-Scheme of Uriniferous Tubule and of the Blood Vessels of the Kidney, Showing Their Relation to Each Other and to the Different Parts of the Kidney. $G$, Glomerulus; $B C$, Bowman's capsule; $N$, neck, $P C$, proximal convoluted tubule; $S$, spiral tubule; $D$, descending arm of Henle's loop; $L$, Henle's loop; $A$, ascending arm of Henle's loop; $I D C$, distal convoluted tubule; $A C$, arched tubule; $S C$, straight collecting tubule; $E D$, duct of Bellini; $A$, arcuate artery, and $V$, arcuate vein, giving off interlobular vessels to cortex and vasa recta to medulla; $a$, afferent vessel of glomerulus; $e$, efferent vessel of glomerulus; $c$, capillary network in cortical labyrinth; $s$, stellate veins; $v r$, vasa recta and capillary network of medulla. (Pearsol.)

Henle, the tubule varies somewhat in histological character, but the irregular tubule and the distal convoluted tube, identical in all respects with the proximal convoluted tube, are to be noted. The proximal convoluted tubes pass into the curved and straight collecting tubes, the latter running vertically downward to the papillary layer, and, joining with other collecting 
tubes, form larger ducts which finally open at the apex of the papilla. These collecting tubes are lined with nucleated columnar or cubical cells.

Renal Blood Supply. - The renal artery divides into several branches which pass in at the hilus of the kidney and are covered by a fine sheath of areolar tissue derived from the capsule. They enter the substance of the organ chiefly in the intervals between the papillæ and at the junction between the cortex and the boundary layer. The main branches then pass almost horizontally, forming more or less complete arches and giving off branches

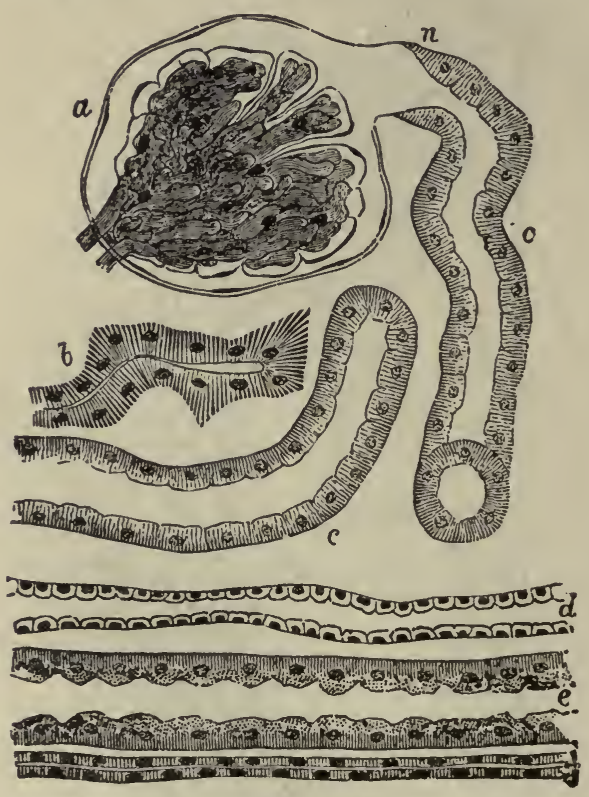

FIG. 288. - From a Vertical Section through the Kidney of a Dog, the Capsule of which is Supposed to be on the Right. $a$, The capillaries of the Malpighian capsule, the glomerulus, are arranged in lobules; $n$, neck of capsule; $c$, convoluted tubes cut in various directions; $b$, irregular tubule; $d, e$, and $f$ are straight tubes running toward capsules forming a so-called medullary ray; $d$, collecting tube; $e$, spiral tube; $f$, narrow section of ascending limb. $\times 380$. (Klein and Noble Smith.)

upward to the cortex and downward to the medulla. The former are for the most part straight; they pass almost vertically to the surface of the kidney, giving off laterally in all directions longer and shorter branches, which ultimately supply the glomerulus. The small afferent artery, figures $287, a$, $290, d$, which enters the Malpighian capsule, breaks up in the interior into a dense convoluted and looped capillary plexus, which is ultimately gathered up again into several efferent vessels, comparable to minute veins, which leave the capsule at one or more places near the point at which the afferent artery enters it. On leaving, they do not immediately join other small veins as might have been expected, but again break up into a second set 
of capillary vessels which form an interlacing network around the uriniferous tubules. This second capillary plexus terminates in small veins which, by union with others, help to form the radicles of the renal vein. These form venous arches corresponding to the arterial arches situated between the medulla and cortex.

Thus, in the kidney, the blood entering by the renal artery traverses two sets of capillaries before emerging by the renal vein, an arrangement which may be compared to the portal system.

The tuft of vessels within the Malpighian capsule in the course of development has been thrust into the dilated extremity of the urinary tubule, which finally completely invests it. Thus within the Malpighian capsule

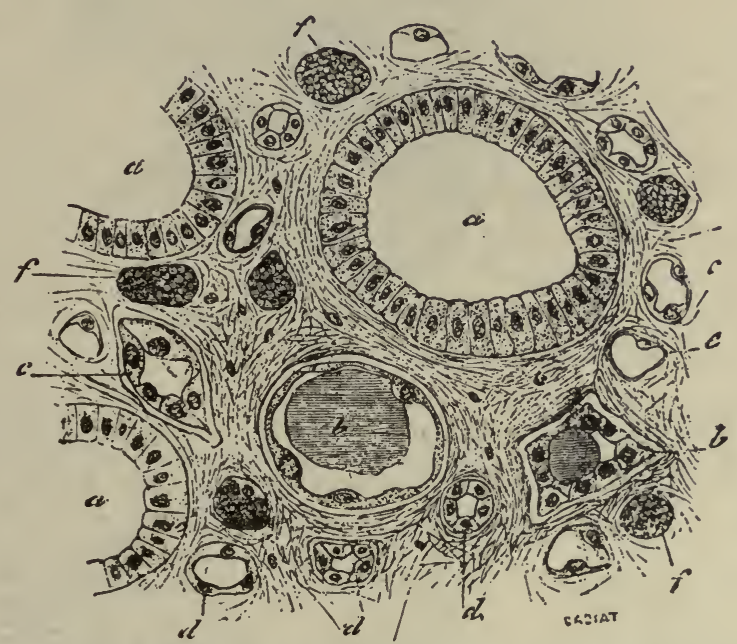

FIG. 289.- Transverse Section of a Renal Papilla. a, Large tubes or papillary ducts; $b, c$, and $d$, smaller tubes of Henle; $e, f$, blood capillaries, distinguished by their flatter epithelium. (Cadiat.)

there are two layers of squamous epithelium, a parietal layer lining the capsule proper, and a visceral or reflected layer immediately covering the vascular tuft, figure 290 , and sometimes dipping down into its interstices. This reflected layer of epithelium is readily seen in young subjects, but cannot always be demonstrated in the adult, figures 290 and $29 \mathrm{r}$.

The vessels which enter the medullary layer break up into smaller arterioles, which form a fine arterial meshwork around the tubes of the papillary layer and end in a similar plexus from which the venous radicles arise. The vessels do not form a double set of capillaries.

Besides the small afferent arteries of the Malpighian bodies there are, of course, others which are distributed in the ordinary manner, for the nutrition of the different parts of the organ; and there are numerous straight 
vessels, the vasa recta, in the pyramids between the tubes. Some of these are branches of the vasa efferentia from Malpighian bodies, and therefore comparable to the venous plexus around the tubules in the cortical portion, while others arise directly as small branches of the renal arteries.

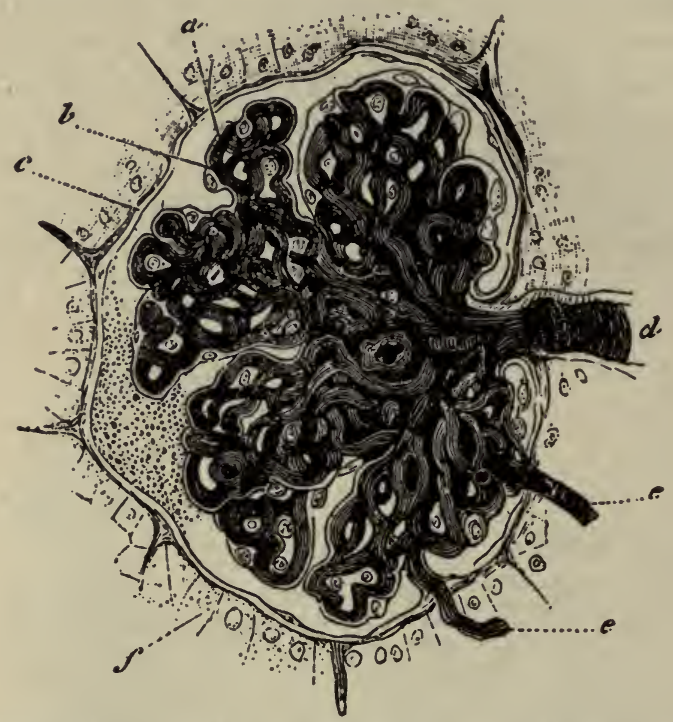

Fig. 290.-Malpighian Capsule and Tuft of Capillaries, Injected through the Renal Artery with Colored Gelatin. $a$, Glomerular vessels; $b$, capsule; $c$, anterior capsule; $d$, glomerular artery; $e$, efferent veins; $f$, epithelium of tubes. (Cadiat.)
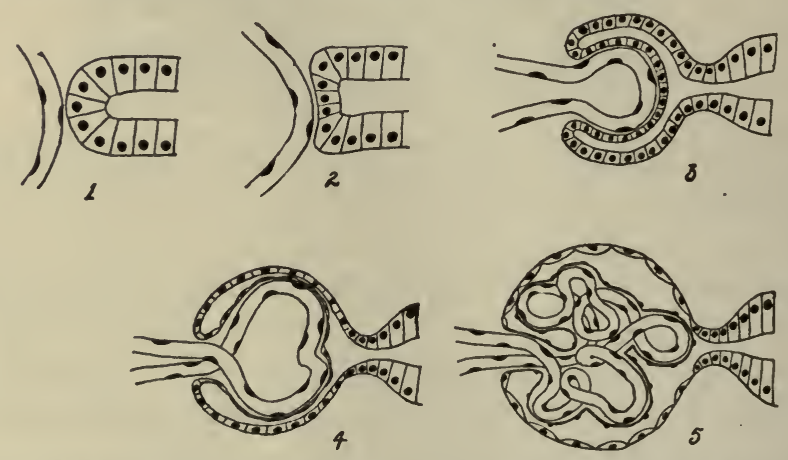

FIG. 291.-Diagrams Illustrating Stages in the Development of the Malpighian Capsule. In $I$ and 2 the developing blood vessel is approaching the blind end of the capsule. In 3 the tubule is beginning to invaginate and enclose the capillary. In 4 and 5 later stages are shown. The cells forming the two layers of the capsule grow very thin. (Bailey.)

Renal Nerves.-Vaso-constrictor and vaso-dilator nerves are supplied to the blood vessels of the kidney, but no clearly defined secretory nerves have yet been demonstrated for the organ. The vascular nerves arise out of the anterior spinal roots (Bradford), chiefly the eleventh to the 
thirteenth dorsal nerves. They reach the kidney by way of the splanchnic nerves and the renal plexus to the renal artery along which they run into the substance of the kidney. Berkely has demonstrated nerve plexuses about the arterioles and around Bowman's capsule. Terminal knob-like endings of nerve fibrils were shown. Some authors have claimed renal vasoconstriction following vagus stimulation, but the fact seems not to be universally admitted.

The Ureters and Urinary Bladder.-The duct of each kidney, the ureter, is a tube about the size of a goose-quill and from twelve to sixteen inches in length. It is continuous above with the pelvis of the kidney, and ends below by obliquely perforating the walls of the bladder and opening on its internal surface. It has three principal coats, an outer fibrous, a middle muscular, of which the fibers are unstriped and arranged in three layers. The fibers of the central layer are circular, and those of the other two layers longitudinal in direction. It has an internal mucous lining continuous with that of the pelvis of the kidney above and the lining of the urinary bladder below. The urinary bladder, which forms a receptacle for the temporary lodgment of the urine in the intervals of its expulsion from the body is more or less pyriform. Its widest part, which is situated above and behind, is termed the fundus; and the narrow constricted portion in front and below, by which it becomes continuous with the urethra, is called its cervix or neck. It is constructed of four principal coats: serous, muscular, areolar or submucous, and mucous. The fibers of the muscular coat deserve special mention. They are unstriped, are arranged in three principal layers, of which the external and internal have a general longitudinal, and the middle layer a circular, direction. The latter are especially developed around the cervix of the organ, and are described as forming a sphincter vesicæ. The mucous membrane is provided with mucous glands, which are more numerous near the neck of the bladder.

The bladder is well provided with blood and lymph vessels and with nerves. The latter are from the sacral plexus (spinal) and hypogastric plexus (sympathetic). Ganglion cells are found, here and there, in the course of the nerve fibers.

\section{THE URINE.}

Quantity and General Properties.-Healthy urine is a perfectly transparent amber-colored liquid, with a peculiar but not disagreeable odor, a bitterish salty taste, and a specific gravity of from I.020 to I.025. The urine consists of water holding in solution certain organic and saline matters as its ordinary constituents, and occasionally various other matters. Some of the latter are indications of diseased states of the system and others are derived from unusual articles of food or drugs taken into the stomach. 
The total quantity of urine passed in twenty-four hours is influenced by numerous circumstances. In adults of average size and medium activity the daily amount of urine may be given as from I, $200 \mathrm{cc}$. to I,500 cc. In Chittenden's recent observations on nine athletic students and on eight soldiers the average daily output of urine through a period of about five months was for the students $I, 215 \mathrm{cc}$. with average specific gravity of $I .020$, and for the soldiers $\mathrm{I}, 042 \mathrm{cc}$. with specific gravity of $\mathrm{I} .023$.

General Chemical Composition of the Urine.

Water..................................... 967 Solids:

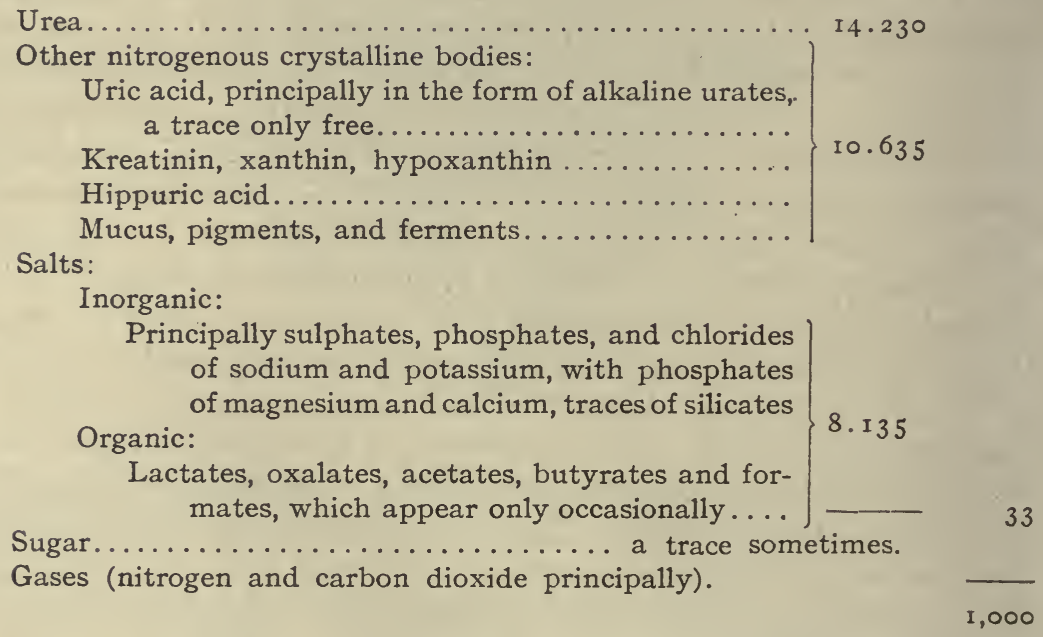

Reaction.-The normal reaction of the urine is slightly acid. This acidity is due to carbon dioxide and to acid phosphate of sodium, and is less marked soon after meals. After a time, varying in length according to the temperature, the reaction becomes strongly alkaline from the change of urea into ammonium carbonate, due to the presence of one or more specific micro-organisms (micrococcus urea). In the process of fermentation the urea takes up two molecules of water, a strong ammoniacal and fetid odor appears, and there are deposits of triple phosphates and alkaline urates. This does not occur unless the urine is freely exposed to the air, or, at least, until air has had access to it.

In most herbivorous animals the urine is alkaline and turbid. The difference depends not on any peculiarity in the mode of secretion, but on the difference in the food on which the two classes of animals subsist. For when carnivorous animals, such as dogs, are restricted to a vegetable diet, their urine becomes pale, turbid, and alkaline like that of herbivorous animals, while the urine voided by the herbivora, e.g., rabbits, fed for some 
time exclusively upon animal substances, presents the acid reaction and other qualities of the urine of carnivora, and its ordinary alkalinity is again restored only on the substitution of a vegetable for the animal diet. Human urine is not usually rendered alkaline by vegetable diet, but it becomes so after the free use of alkaline medicines or of the alkaline salts with carbonic or vegetable acids. These latter are changed into alkaline carbonates previous to elimination by the kidneys.

Specific Gravity of Urine.-The average specific gravity of the human urine is about I.020 to I.025. The relative quantity of water and of solid constituents of which it is composed is materially influenced by the condition and occupation of the body during the time at which it is secreted; by the length of time which has elapsed since the last meal; by the amount of water taken; and by several other less important circumstances. The morning urine is the best adapted for analysis in health, since it represents the simple secretion unmixed with the elements of food or drink. If it is not used the whole of the urine passed during the period of twenty-four hours should be taken. The specific gravity of the urine may thus, consistently with health, range widely on both sides of the usual average. It may vary from I.OI 5 in the winter to $\mathrm{I} .025$ in the summer; but variations of diet and exercise, and many other circumstances, may make even greater differences than these. The variations may be extreme in disease, sometimes decreasing in chronic nephritis to I.004, and frequently increasing in diabetes, when the urine is loaded with sugar, to I.050 or even to I.060.

Average Daily Quantity of the Chief Urinary Constituents (ModiFIED FROM PARKES.)

\begin{tabular}{|c|c|c|c|c|}
\hline & & & $\begin{array}{r}\text { Per ki } \\
\text { Body w }\end{array}$ & $\begin{array}{l}\text { o of } \\
\text { eight. }\end{array}$ \\
\hline ater. & $\mathrm{I}, 500$. & c.c. & 23.0000 & grams \\
\hline olids $\ldots \ldots \ldots \ldots \ldots \ldots \ldots \ldots \ldots$ & 72 . & grams & 0.8800 & grams \\
\hline 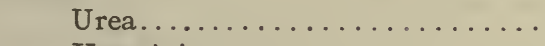 & $33 \cdot 180$ & grams & 0.5000 & grams \\
\hline Kreatinin.............. & .9 ro & grams & 0.0140 & grams \\
\hline Uric acid $\ldots \ldots \ldots \ldots \ldots \ldots$ & .555 & grams & 0.0084 & grams \\
\hline Hippuric acid. ............ & .400 & grams & 0.0060 & grams \\
\hline Pigment and extractives....... & I0.000 & grams & 0.1510 & grams \\
\hline Sulphuric acid........... & 2.012 & grams & 0.0480 & grams \\
\hline Phosphoric acid $\ldots \ldots \ldots \ldots \ldots$ & $3 \cdot 164$ & grams & 0.0305 & grams \\
\hline Chlorine............... & 7.000 & grams & 0.1260 & grams \\
\hline 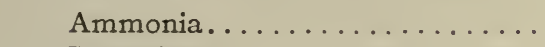 & .770 & grams & & \\
\hline 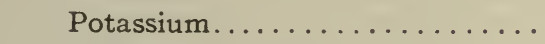 & $2 \cdot 500$ & grams & & \\
\hline 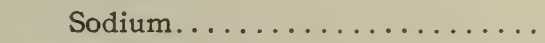 & II . 090 & grams & & \\
\hline Calcium................. & .260 & grams & & \\
\hline 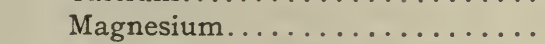 & .207 & grams & & \\
\hline
\end{tabular}

Variations in the Constituents of Urine.-Most of the constituents are, even in health, liable to variations from the proportions given in the above table. The variations of the quantity of water in different seasons 
and according to the quantity of drink and exercise have just been mentioned. The water of the urine is also liable to be influenced by the condition of the nervous system, being sometimes greatly increased, e.g., in hysteria and in some other nervous affections, and at other times diminished. The increase in water may be either attended with an augmented quantity of solid matter in some diseases, as in ordinary diabetes, or may be nearly the sole change, as in the affection termed diabetes insipidus. A febrile condition almost always diminishes the quantity of water; and a like diminution

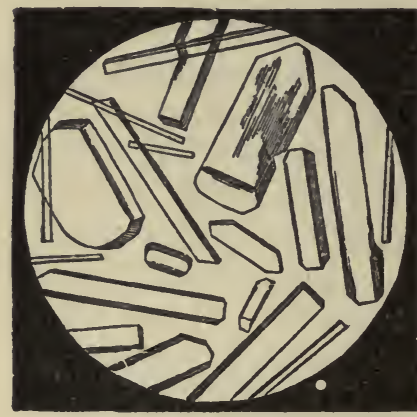

Fig. 292.-Crystals of Urea. is caused by any affection which draws off a large quantity of fluid from the body through any other channel than that of the kidneys, e.g., the bowels or the skin.

In disease or after the ingestion of special foods, various abnormal substances occur in urine, of which the following may be mentioned: Seralbumin, serum globulin, enzymes (apparently some are present in health also), proteoses, blood, sugar, bile acids and pigments, casts, fats, various salts taken as foods or as medicines, micro-organisms of various kinds.

The Nitrogenous Substances in Urine.-The nitrogenous waste products which are formed in the body in the metabolism of the protein foods are ultimately eliminated chiefly through the kidney, to some extent through the bowel, and slightly through the skin. The total nitrogen in the urine and in the feces multiplied by the factor 6.25 is a measure of the nitrogenous foods, i.e., proteins, metabolized by the body. The nitrogen excreted in the urine is in the form of urea 87.5 per cent., ammonia 4.3 per cent., kreatinin 3.6 per cent., uric acid 0.8 per cent., and undetermined forms 3.73 per cent., according to Folin. The total quantity of nitrogen eliminated in all these forms per day is given as about 18 grams. In Chittenden's experiments this quantity is reduced to as low as 6 grams or even less per day.

Urea.-Urea, $\mathrm{CON}_{2} \mathrm{H}_{4}$, is the principal solid constituent of the urine, forming nearly one-half of the total quantity. It is also the most important ingredient, since it is the chief form in which the waste nitrogen which is derived from protein metabolism is excreted from the body.

Properties.-Urea, like other solid constituents of the urine, exists in a state of solution. When in the solid state, it appears in the form of delicate silvery acicular crystals, which, under the microscope, are seen as foursided prisms, figure 292. It readily combines with some acids, like a weak base, and may thus be conveniently procured in the form of crystals of nitrate or oxalate of urea, figures 293 and 294.

Urea is colorless when pure; when impure it may be yellow or brown. 
It is without odor and of a cooling niter-like taste. It has neither an acid nor an alkaline reaction, and deliquesces in a moist and warm atmosphere. At $15^{\circ} \mathrm{C}$. it requires for its solution less than its own weight of water. It is soluble in all proportions of boiling water, and requires five times its weight of cold alcohol for its solution. It is insoluble in ether. At $120^{\circ} \mathrm{C}$. it melts, and at a still higher temperature decomposes.

Urea is decomposed by sodium hypochlorite or hypobromite or by nitrous acid, with evolution of nitrogen. It forms compounds with acids, of which the chief are urea hydrochloride, $\mathrm{CON}_{2} \mathrm{H}_{4}$. $\mathrm{HCl}$; urea nitrate, $\mathrm{CON}_{2} \mathrm{H}_{4}$.$\mathrm{HNO}_{3}$; and urea phosphate, $\mathrm{CON}_{2} \mathrm{H}_{4} \cdot \mathrm{H}_{3} \mathrm{PO}_{4}$. It forms compounds with

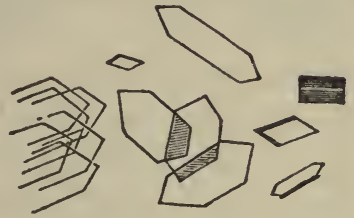

FIG. 293.-Crystals of Urea Nitrate.

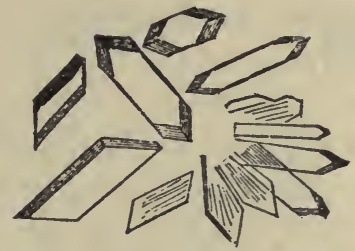

FIG. 294.-Crystals of Urea Oxalate.

metals such as $\mathrm{HgO} . \mathrm{CON}_{2} \mathrm{H}_{4}$, (or with silver, $\mathrm{CON}_{2} \mathrm{H}_{2} \mathrm{Ag}_{2}$ ). Urea is isomeric with ammonium cyanate, $\mathrm{NH}_{4} \mathrm{CNO}$, and was first prepared artificially from that substance.

The Formation of Urea.-Proteins in the body have their nitrogenous moiety split off as ammonia, by what Folin considers essentially a series of hydrolytic cleavages; this is then built up into urea, as described more fully in the chapter on Metabolism. This last step is essentially a synthetic process which, from the fact that ammonium carbonate introduced into the blood is eliminated as urea, may be supposed to occur as follows:

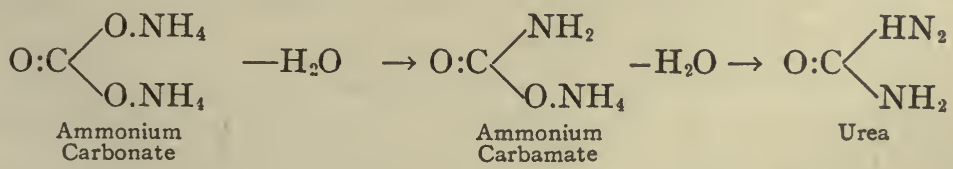

Urea is present in varying amounts in all organs and fluids of the body, as shown by the following determinations of Schoendorff on the dog:

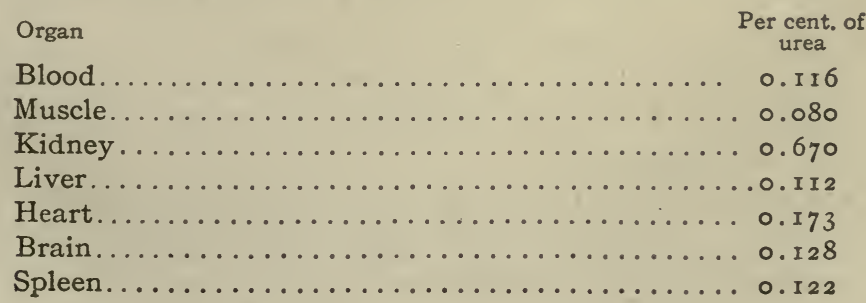


It has peen proven that the kidney does not form urea; in fact the kidneys may be removed from the body, and urea will continue to accumulate in the blood. Urea is formed chiefly in the liver, but may in part be constructed in other organs, as described more fully on page 408. It follows that the kidney is only the channel for the elimination of this nitrogenous compound.

Decomposition of the urea with development of ammonium carbonate takes place from the action of bacteria (micrococcus urex) when urine is kept for some days after being voided, which explains the ammoniacal odor then evolved. The urea is sometimes decomposed before it leaves the bladder, when the mucous membrane is diseased and the mucus secreted by it is abundant; but decomposition does not occur unless atmospheric germs have had access to the urine.

Quantity Excreted.-The quantity of urea excreted is, like that of the urine itself, subject to considerable variation. For a healthy adult about 30 grams per day may be taken as rather a high average. Its percentage in healthy urine is from 2 to 2.5 . Its amount is materially influenced by diet, being greater on a diet of high protein content. The quantity of urea excreted by children, relatively to their body weight, is much greater than by adults; thus the quantity of urea excreted per kilogram of weight was found to be, in a child, 0.8 gram; in an adult only 0.4 gram. Regarded in this way, too, the excretion of carbonic acid gives similar results, the proportions in the child and adult being as 82 to 34 .

Uric Acid.-Uric acid, $\mathrm{C}_{5} \mathrm{H}_{4} \mathrm{~N}_{4} \mathrm{O}_{3}$, see page $4 \mathrm{IO}$, is present in the urine of man and other animals. In birds and reptiles uric acid or its salts is the chief form in which nitrogen is eliminated from the body.

Properties.-Uric acid is a colorless, crystalline compound of the purin group, figure 295. It is odorless and tasteless. It is very slightly soluble in water, quite insoluble in alcohol and ether, and freely soluble in solutions of the alkaline carbonates and other salts.

A study of the elimination of nitrogen in birds, i.e., geese, has shown that uric acid, like urea in mammals, is formed largely in the liver from antecedent protein nitrogen. In man the elimination of uric acid $(0.3$ to 0.6 gram per diem) is more or less constant and characteristic for the individual; it increases or decreases somewhat with the nucleoprotein and purin content of the daily diet. This observation has led to the inference that uric-acid nitrogen is derived from nuclear metabolism, page 94 .

A certain amount of nuclear purin is taken in the food, called exogenous purin to distinguish it from the endogenous purin that is derived from the body nuclear metabolism.

Other representatives of the purin group are adenin, guanin, xanthin, hypoxanthin, etc. Chemically, caffeine from coffee is a trimethyl xanthin.

The most common form in which uric acid is deposited in urine is that 
of a brownish or yellowish powdery substancé, consisting of amorphus ammonium or sodium urate. Urate sediments are commonly deposited on cooling the urine; they are redissolved on warming it slightly. When deposited in crystals, uric acid is most frequently obtained in rhombic or diamond-shaped laminæ, but other forms are not uncommon, figure 295 . When deposited from urine, the crystals are generally more or less deeply colored, from being combined with the coloring principles of the urine.

Hippuric Acid.-This compound, $\mathrm{C}_{6} \mathrm{H}_{5}$. CO.NH. $\mathrm{CH}_{2} \mathrm{COOH}$, has long been known to exist in the urine of herbivorous animals in combination with sodium It also exists naturally in the urine of man, in a quantity equal to,

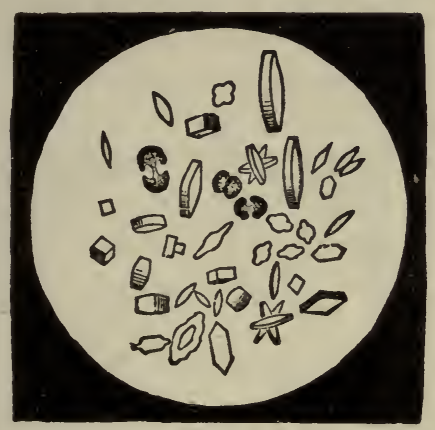

Fig. 295.-Various Forms of Uric Acid Crystals.

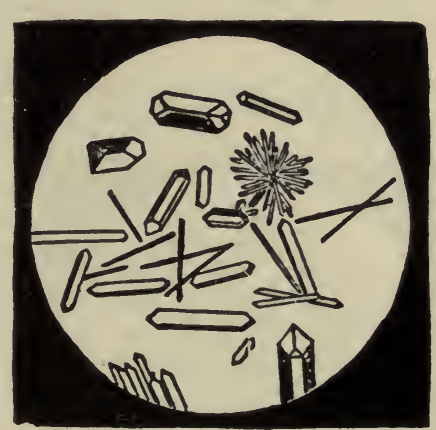

Fig. 296.-Crystals of Hippuric Acid.

or rather exceeding, that of the uric acid. The quantity excreted is increased by a vegetable diet.

Hippuric acid appears to be formed in the body from benzoic acid. The benzoic acid unites with glycocoll, and hippuric acid and water are formed thus:

$$
\mathrm{C}_{6} \mathrm{H}_{5} \cdot \mathrm{COOH}+\mathrm{CH}_{2} \cdot \mathrm{NH}_{\text {Glycin }} \cdot \mathrm{COOH}=\mathrm{C}_{6} \mathrm{H}_{5} \cdot \underset{\text { Hippuric acid }}{\mathrm{CO}} \cdot \mathrm{NH} \cdot \mathrm{CH}_{2} \cdot \mathrm{COOH}+\mathrm{H}_{2} \mathrm{O} \text {. }
$$

Hippuric acid is the first substance which was demonstrated to be synthetized in the body.

Kreatinin.-This substance, which is the anhydride of kreatin, is present in urine in a remarkably constant quantity, as shown recently by Folin's analyses. Its daily excretion quantity is from $I$ to $I .5$ grams according to the amount of active tissue in the individual. It is of especial importance as a measure of the metabolism of muscle protoplasm.

Ammonia.-A considerable daily quantity of ammonia (about $0.7 \mathrm{gram}$ ) in combination, as chloride, phosphate, or sulphate, is found in the normal urine, showing that this is an important method of nitrogen elimination.

Pigments.-The pigments of the urine are the following: I. Urochrome: a yellow coloring matter, giving no absorption band; of which but little is known. Urine owes its yellow color mainly to the presence of this body. 2. Urobilin, an orange pigment, of which traces may be found in nearly ali 
urines, and which is especially abundant in the urines passed by febrile patients. It is characterized by a well-marked spectroscopic absorption band at the junction of green and blue. Those who believe urobilin to be identical with hydrobilirubin suppose that the bilirubin is reduced by the putrefactive processes in the intestines, and is conveyed in its reduced form by the blood stream to the kidneys. 3. Uroerythrin, occasionally found.

Mucus.-Mucus sediment in the urine consists principally of mucin and of the epithelial débris from the mucous surface of the urinary passages. Particles of epithelium, in greater or less abundance, may be detected in most samples of urine, figure 297. As urine cools, the mucus is sometimes seen suspended in it as a delicate opaque cloud, but generally it falls. In inflammatory affections of the urinary passages, especially of the bladder, mucus is secreted in large quantities.

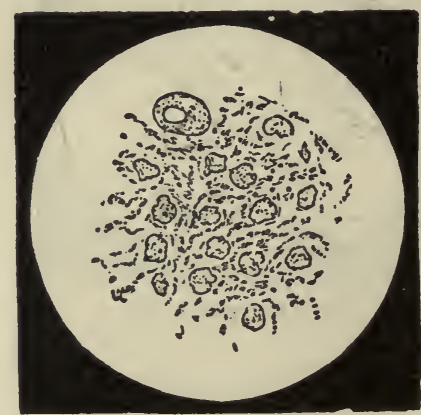

Frg. 287.

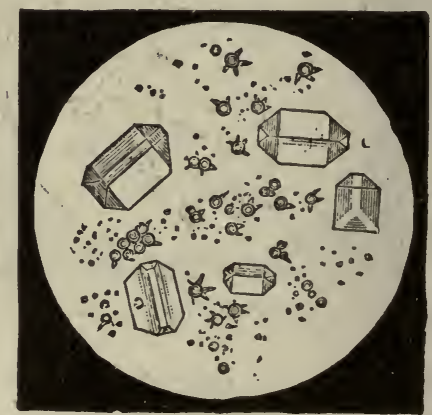

FIG. 298.

Frg. 297.-Urinary Deposit of Mucus, etc.

FIG. 298.-Urinary Sediment of Triple Phosphates (large prismatic crystals) and Urate of Ammonium, from urine which had undergone alkaline fermentation.

Saline Matter.-Sulphuric acid, in the form of salts, is taken in very small quantity with food. Sulphur is also a constituent part of the protein molecule; hence its elimination, like that of nitrogen, gives a certain measure of protein metabolism. It is excreted as inorganic sulphates of sodium and potassium, and as eth eal sulphates, compounds of phenol, cresol, skatol, i.e., cresol sulphuric acid $\left(\mathrm{C}_{7} \mathrm{H}_{7} \mathrm{OSO}_{2} \mathrm{OH}\right)$, etc.

The phosphoric acid in the urine is combined partly with the alkalies, partly with the alkaline earths,-about four or five times as much with the former as with the latter. In blood, saliva, and other alkaline fluids of the body phosphates exist in the form of alkaline, neutral, or acid salts. In the urine they are acid salts, viz., the sodium, ammonium, calcium, and magnesium phosphates, the excess of acid being (Liebig) due to the appropriation of the alkali with which the phosphoric acid in the blood is combined, by the several new acids which are formed or discharged at the kidneys, namely the uric, hippuric, and sulphuric acids. 
The phosphates are taken largely in both vegetable and animal food. Some are excreted at once; others only after being transformed and incorporated with the tissues. Calcium and magnesium phosphates form the principal earthy constituents of bone, and from the decomposition of the osseous tissue the urine derives a quantity of this salt. The decomposition of other tissues also furnishes large supplies of phosphorus to the urine, which phosphorus is supposed, like the sulphur, to be united with oxygen, and then combined with bases. The quantity is, however, liable to considerable variation. The earthy phosphates are more abundant after meals, whether of animal or vegetable food, and are diminished after long fasting. The alkaline phosphates are increased after animal food, diminished after vege-

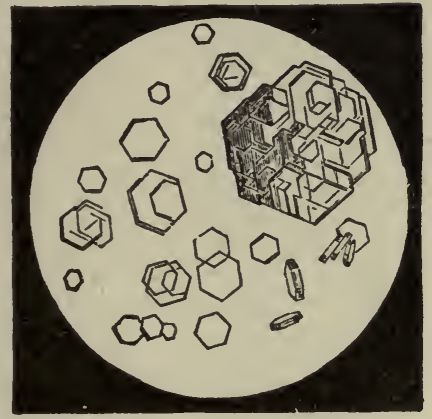

FIG. 299.-Crystals of Cystin.

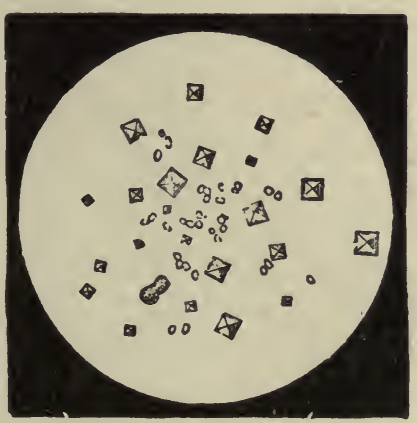

FIG. 300.-Crystals of Calcium Oxalate.

table food. Phosphorus uncombined with oxygen appears, like sulphur, to be excreted in the urine. When the urine undergoes alkaline fermentation, phosphates are deposited in the form of a urinary sediment, consisting chiefly of ammonio-magnesium phosphate (triple phosphate), figure 298.

The chlorine of the urine occurs chiefly in combination with sodium. Next to urea, sodium chloride is the most abundant solid constituent of the urine. As the chlorides exist largely in food, and in most of the animal fluids, their occurrence in the urine is easily understood.

Occasional Constituents of Urine. Cystin, $\mathrm{C}_{3} \mathrm{H}_{7} \mathrm{NSO}_{2}$, figure 299, is an occasional constituent of urine.

Another constituent of the urine is oxalic acid (about 0.02 gram per diem), which is frequently deposited in combination with calcium, figure 300 , as a urinary sediment. Pathologically, oxalic acid is found to be increased in diabetes mellitus, in organic diseases of the liver, and in various other conditions accompanied by derangement of the oxidation mechanism.

Dextrose and albumin are sometimes present in pathological urine, and are of particular interest from the clinical point of view. See the subject Internal Secretions of the Pancreas, page 49I. 


\section{THE METHOD OF EXCRETION OF URINE.}

The secretion of urine is an act the complexity of which can be profitably discussed only after a clear understanding of three main factors which have already been presented, viz., the chemical composition of the urine secreted, the structure of the kidney tubule as a secreting organ, and, finally, the chemical composition of the blood which supplies the materials to the kidney for the formation of the urine. The substances found in the urine are, for the most part, also to be found in the blood plasma. But the relative percentage composition is very different. The amount of urea in the blood is only a fractional part as concentrated as in the urine, while albumins and sugars, which are so plentiful in the blood, are normally present in the urine only in traces. The presence of the glomerulus with its special vascular supply, and the different loops of the tubule, with its gland-like epithelial wall, would, $a$ priori, lead one to suspect special functions for each.

Theories of the Secretion of Urine.-Bowman in 1842 , wholly on structural grounds, advanced a theory of urinary secretion. This theory was given an experimental basis and elaborated by Heidenhain and generally bears his name.

Heidenhain's theory is stated as follows:

I. The secretion in the kidney depends upon the physiological activity of special secreting cells which are of two kinds. 2. The first type of cell is represented by the single layer of epithelium covering the glomerular capillaries. These cells secrete especially water and salts. 3. The second type of cell is represented by the gland-like epithelial cells which form the convoluted tubules and the loop of Henle. These cells secrete the urea, uric acid, and other specific constituents of the urine. 4. The activity of each kind of cell is influenced by the chemical composition of the blood and by the flow of blood through the kidney. 5. The relative secretory activity of the glomerular cells and the tubule cells is sufficient to account for the variation in the chemical composition of the urine.

Ludwig's theory, advanced in 1844 , is a strictly mechanical theory of urinary secretion based on experiments which he presented. I. He considered the glomerulus and Bowman's capsule as a filtering apparatus in which substances present in the blood are driven through the epithelium of the capsule into the renal tubule by the positive pressure of the blood in the glomerular capillaries. 2. This very dilute urine in the capsule is supposed to be concentrated by the resorption of water as it flows down the tubule. Ludwig originally considered this resorption of water an imbibition process in which the greater saturation of salts in the blood caused water to be taken up through the renal tubule walls, an osmotic process. At present most observers who accept the view that filtration takes place at the glomerulus explain the resorption of water down the tubules as an act of cellular resorption or secretion. 
Experimental Observations.-There are numerous nerves to the kidney, but no proven secretory influence has been shown. The variations in the secretion of urine that follow nervous stimulation are quite satisfactorily explained by the changes in the blood flow.

The kidney can be placed in an onkometer and its variation in volume measured directly, figures 301 and 302 . This volume measurement, when taken with the arterial pressure, gives a very good index of the volume of blood flowing through the kidney. Now when the kidney is inserted in an onkometer and the urine collected from the ureter, it is found in general that

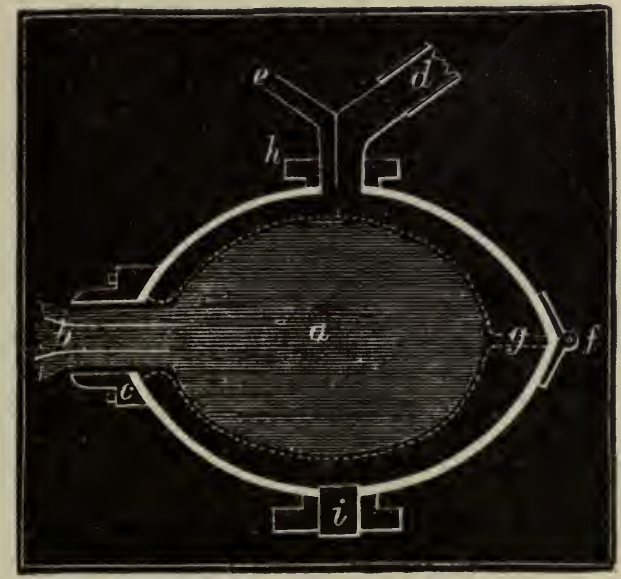

FIG. 301.-Diagram of Roy's Onkometer. $a$, Represents the kidney enclosed in a metal box, which opens by hinge $f ; b$, the renal vessels and ducts. Surrounding the kidney are two chambers formed by membranes, the edges of which are firmly fixed by being clamped between the outside and inside metal capsules (the latter not represented in the figure), the two being firmly screwed together by screws at $h$, and on the opposite side. The membranous chamber below is filled with a varying amount of warm oil, according to the size of the kidney experimented with, through the opening then closed with the plug $i$. After the kidney has been enclosed in the capsule, the membranous chamber above is filled with warm oil through the tube $e$, which is then closed by a tap (not represented in the diagram); the tube $d$ communicates with a recording apparatus, and any alteration in the volume of the kidney is communicated by the oil in the tube to the chamber $d$ of the Onkograph, figure 302 .

the greater the pressure and flow of blood the greater the secretion of urine, as would follow if the glomerulus were a filtering mechanism. However, if the renal vein is partially obstructed, even though the blood pressure be increased, the amount of urine secreted is sharply decreased. If the vein is completely occluded, the secretion of urine not only ceases for the time but does not immediately begin again when the blood pressure and flow are reestablished. The closure of the vein for only one or two minutes is said to stop the flow of urine for as much as forty-five minutes. This short interruption of the circulation is sufficient to bring about other changes in the 
glomerular epithelium, for it now excretes albumin, which it did not previously let pass. Therefore, it is not pressure merely that favors the secretion, there must also be an efficient flow of blood. The secretion is influenced especially by the amount of blood flowing through the kidney in a given time.

In the frog the kidney has a double blood supply. The renal artery supplies the glomeruli, while a branch of the renal-portal vein supplies the tubules. Nussbaum ligated the renal artery in one kidney of the frog, while leaving the circulation of the other kidney undisturbed. He found that the operated kidney secreted little or no urine, but that it could be made to secrete by injections of urea, but not by injections of albumin or sugar as in the nor-

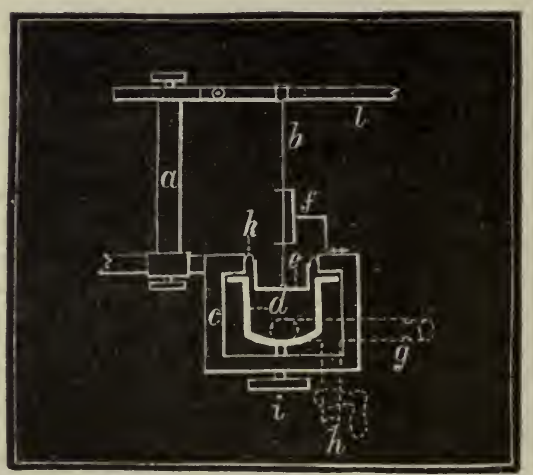

FIG. 302.-Roy's Onkograph, or Apparatus for Recording Alterations in the Volume of the Kidney, etc., as shown by the onkometer. $a$, Upright, supporting recording lever $l$, which is raised or lowered by the needle $b$, which works through $f$, and which is attached to the piston $e$, working in the chamber $d$, with which the tube from the onkometer communicates. The oil is prevented from being squeezed out as the piston descends, by a membrane, which is clamped between the ring-shaped surfaces of the cylinder by the screw $i$ working upward; the tube $h$ is for filling the instrument.

mal kidney. Ligation of the renal-portal vein, which supplies the tubules in the frog, caused a decrease in the quantity of the secretion, whereas, according to Ludwig's view, it ought to have increased the quantity, since obviously resorption could not take place with any degree of efficiency. In the main, the evidence is in favor of the view that even the glomerular epithelium does not filter merely, but that it, as living protoplasm, regulates and controls the quantity and kind of material passing through it.

Microchemical observations have been enlisted to demonstrate more fully, if possible, the activity of the different parts of the epithelial tubule. Heidenhain, by injections of indigo-blue into the blood stream, followed by rapid fixation of the kidney in alcohol at the proper stage of elimination, has demonstrated crystals of the pigment in the renal epithelial cells and in the lumen of the tubule. He concluded that these cells were actively eliminating the pigment by a secretory process. This observation has been questioned. 
But Heidenhain's view is strengthened by Bowman's observation that in birds crystals of uric acid are to be seen in the cells of the convoluted tubules, and in the lumen adjacent.

Only traces of the sugars and proteins of the blood are found in normal urine, but when either cane sugar, peptone, or egg albumin is introduced into the blood it is rapidly eliminated by the kidney. Egg albumin is not essentially different from the serum albumin of the blood, but the serum albumin is not excreted. These are both non-dialyzable compounds. Dextrose and urea, both readily dialyzable, present the same comparison, i.e., urea is excreted, while dextrose is not. If, however, the percentage of this sugar is high, 0.25 per cent. or more, it is then eliminated. The excretion of the highly diffusible sodium chloride bears a similar quantitative relation to excretion. If present in the blood in relatively low amounts it is not secreted, while if the concentration is slightly greater it may be quickly eliminated.

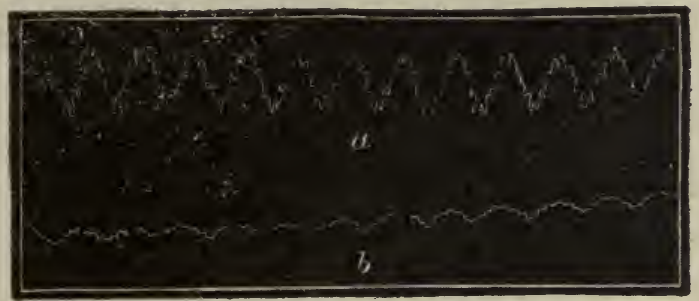

Fig. 303.-Curve Taken by Renal Onkometer Compared with that of an Ordinary Bloodpressure Curve. a, Kidney curve; b, blood-pressure curve. (Roy.)

Other inorganic salts present only in traces, are meanwhile rapidly eliminated. Even the rapid elimination of a slight excess of water in the blood can scarcely be explained on purely physical grounds. To discharge the water across the glomerulus from the blood to the urine requires an expenditure of osmotic pressure much greater than that balanced by the blood pressure. That is, the epithelial cells must do work, and the energy is dependent on metabolism in the cells.

It would seem, therefore, that the separation of urine in the kidney is a secretory process dependent on the protoplasmic activity of the living renal cells, that the apparent selective property of the cells is a manifestation of such activity, and that even water is actively secreted.

Diuretics.-Certain substances increase the flow of urine and are called diuretics. They act directly on the renal epithelium, for example urea, or indirectly on the circulatory system to increase the flow of blood. Digitalis is a well-known diuretic which increases the efficiency of the circulation. It also stimulates the renal epithelium with the production of a marked increase in the flow of urine. Caffeine diuresis can best be explained on an assumed stimulating action of the renal epithelium. Urea introduced into 
the blood produces a copious secretion of urine. Both urea and the saline diuretics induce a flow of urine out of all proportion to the osmotic changes produced, and they may be regarded as direct stimulators of the renal epithelium, a view supported by their stimulative action on other tissues.

\section{THE DISCHARGE OF THE URINE.}

As each portion of urine is secreted, it propels that which is already in the uriniferous tubes onward into the pelvis of the kidney. Thence it passes through the ureter into the bladder, from which at intervals it is discharged to the exterior. The rate and mode of entrance of urine into the bladder has been watched in cases of ectopia vesicæ, i.e., cases in which fissures in the anterior or lower part of the walls of the abdomen and of the front wall of the bladder expose to view the orifices of the ureters. The urine does not enter the bladder at any regular rate, nor is there a synchronism in its movement through the two ureters. Ordinarily two or three drops enter the bladder every minute, each drop as it enters first raising up the little papilla on which the ureter opens, and then passing through the orifice, which at once again closes like a sphincter. Its flow is aided by the peristaltic contractions of the ureters, and is increased in deep inspiration or by straining. The urine collected in the bladder is prevented from regurgitation into the ureters by the mode in which these pass through the walls of the bladder, namely, by their lying a half to three-quarters of an inch between the muscular and mucous coats before they turn rather abruptly forward and open through the latter into the interior of the bladder.

Micturition.-The contraction of the muscular walls of the bladder may by itself expel the urine with little or no help from other muscles. The vesicular pressure is increased in the voluntary act by the contraction of the abdominal and other expiratory muscles which bear on the abdominal viscera, thus aiding in the expulsion of the contents of the bladder. The diaphragm is at the same time fixed in contraction and the sphincter of the bladder relaxes. The pressure within the bladder under the combined contractions of these expulsive muscles sometimes amounts to 8 to $10 \mathrm{~cm}$. of mercury. The act is completed by the accelerator urinæ muscle, which, as its name implies, quickens the stream and expels the last drop of urine from the urethra. The act is under the regulative control of a nervous center in the lumbar spinal cord, through which, as in the case of the similar center for defecation, the various muscles concerned are coordinated in their action. It is well known that the act may be reflexly induced, e.g., in children who suffer from intestinal worms or other such irritation. Generally the afferent impulses which set up the reflexes leading to the desire to micturate are excited by overdistention of the bladder, or sometimes by a few drops of urine passing into the urethra. This impulse passes up to the lumbar center or 
centers, and reflexly produces on the one hand inhibition of the sphincter and on the other contraction of the muscles of the fundus and other necessary muscles for the expulsion of the contents of the bladder. In the voluntary act these motor centers are stimulated to activity by impulses coming from the higher cerebral centers.

\section{THE STRUCTURE AND EXCRETORY FUNCTIONS OF THE SKIN.}

The skin serves, $\mathrm{I}$, as an external integument for the protection of the deeper tissues, and 2, as a sensitive organ in the exercise of touch, a subject to be considered in the chapter on the Special Senses. It is also, 3, an important secretory and excretory organ; and 4 , an absorbing organ. 5 , It plays an important part in the regulation of the temperature of the body by

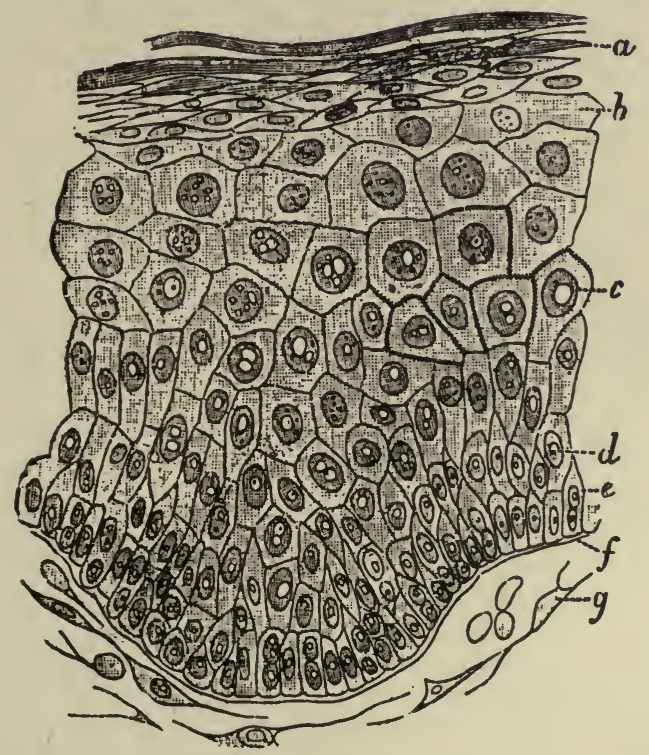

FIG. 304.-Vertical Section of the Epidermis of the Prepuce. a, Stratum corneum, of very few layers, the stratum lucidum and stratum granulosum not being distinctly represented; $b, c, d$, and $e$, the layers of the stratum Malpighii, a certain number of the cells in layers, $d$; and $e$ showing signs of segmentation; layer $c$ consists chiefly of prickle or ridge and furrow cells; $f$, basement membrane; $g$, cells in cutis vera. (Cadiat.)

controlling the loss of heat, i.e., a temperature-regulating function, discussed in the chapter on Animal Heat.

Structure.-The skin consists principally of a vascular tissue named the corium, derma, or cutis vera, and of an external covering of epithelium termed the epidermis or cuticle. Within and beneath the corium are embedded several organs with special functions, namely, sudoriferous glands, cesbaeous glands, and hair follicles; and on its surface are sensitive papille. 
The so-called appendages of the skin-the hair and nails-are modifications of the epidermis.

The epidermis is composed of several strata of cells of various shapes and sizes; it closely resembles in its structure the epithelium of the mucous membrane that lines the mouth or covers the cornea. The following four layers may be distinguished.

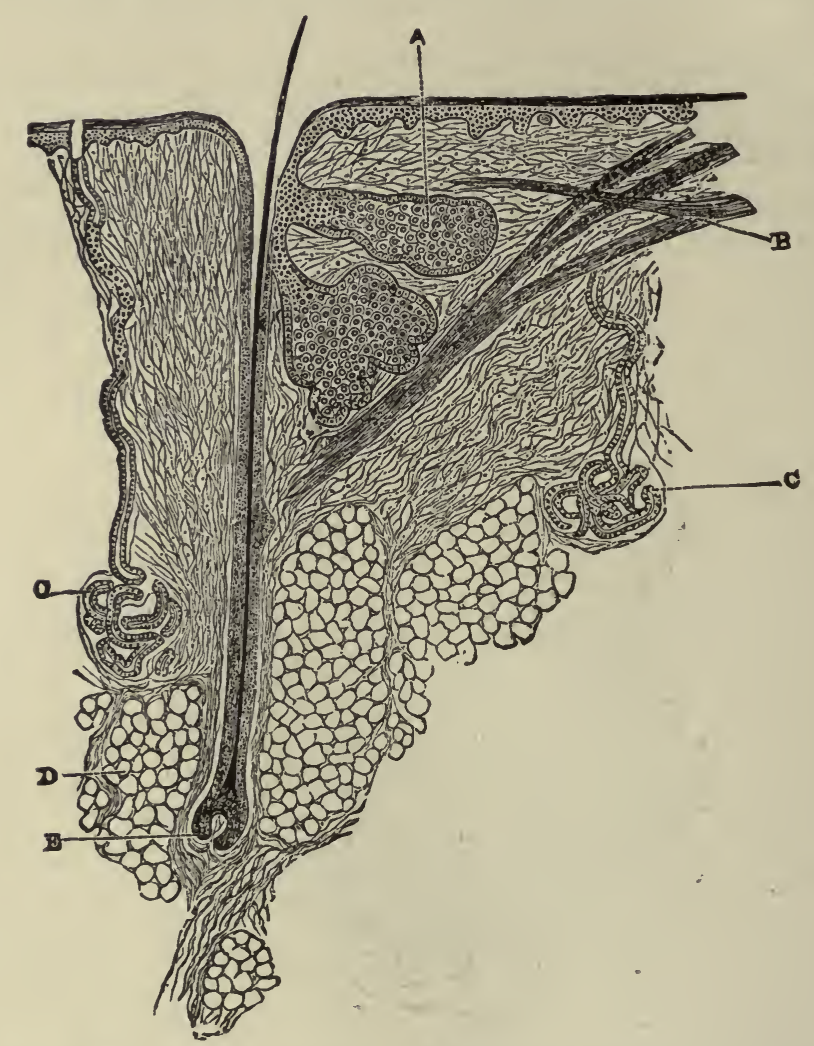

FIG. 305.-Vertical Section of Skin. A, Sebaceous gland opening into hair follicle; $B$, muscular fibers; $C$, sudoriferous or sweat gland; $D$, subcutaneous fat; $E$, fundus of hair follicle, with hair-papillæ. (Klein.)

The stratum lucidum, a homogeneous membrane, consisting of squamous cells closely arranged, in some of which a nucleus can be seen. Stratum granulosum, consisting of one layer of flattened, fusiform, distinctly nucleated cells. Stratum Malpighii or rete mucosum consists of many strata of cells. The deepest cells, placed immediately above the cutis vera, are columnar with oval nuclei, succeeded by a number of layers of more or less polyhedral cells with spherical nuclei; the more superficial layers are considerably flattened. The deeper surface of the rete mucosum is accurately adapted 
to the papillæ of the true skin, being, as it were, molded on them. It is very constant in thickness in all parts of the skin. The cells of the middle layers of the stratum Malpighii are connected by processes, and thus form prickle cells, figure 28 . The pigment of the skin, in the deeper cells of the rete mucosum, causes the various tints observed in different individuals and different races. The epidermis maintains its thickness in spite of the constant wear and tear to which it is subjected. The columnar cells of the deepest layer of the rete mucosum elongate, multiply by division, the new cells produced being pushed toward the free surface of the skin. There is thus a constant production of fresh cells in the deeper layers, and a constant throwing off of old ones from the free surface. When these two processes are accurately balanced, the epidermis maintains its thickness. When by intermittent pressure a more active cell growth is stimulated, the production of cells exceeds their waste and the epidermis increases in thickness, as we see in the horny hands of the laborer.

The dermis, or cutis vera or true skin, is a dense and tough, but yielding and highly elastic structure supporting the epidermis. It is composed of

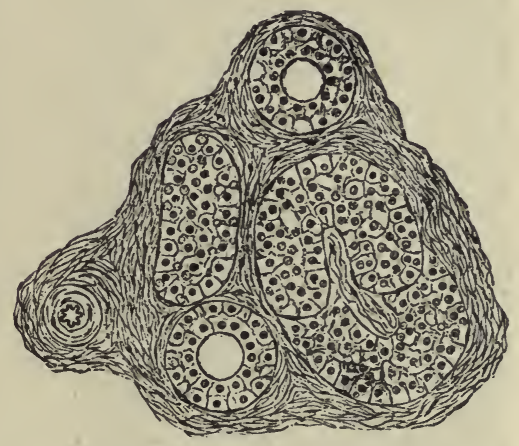

Fig. 306.-Terminal Tubules of Sudoriferous Glands, Cut in Various Directions. From the skin of the pig's ear. (V. D. Harris.) areolar connective tissue interwoven in all directions and forming numerous spaces by its interlacements. These areolæ in the deeper layers of the cutis are usually filled with masses of fat cells, figure 305 . Unstriped muscular fibers are also abundantly present, especially in the skin of animals which erect the hairs with greater ease than is usually the case with man.

There is a rich network of blood vessels to the dermis. In the dermal papillæ and about the sweat glands there are special loops of capillaries. Nerve fibers are also distributed to the papillæ.

The special nerve terminations in the skin have been described on page 75

Glands of the Skin.-The skin possesses glands of two kinds: Sudoriferous or sweat glands, and the sebaceous or oil glands.

A sudoriferous or sweat gland consists of a small lobular mass, formed of a coil of a simple tubular gland, surrounded by blood vesséls, and embedded in the subcutaneous adipose tissue, figure $305, C$. The duct ascends from this coiled mass for a short distance in a spiral manner through the cutis and the epidermis, and then opens on the surface of the skin. In the parts where the epidermis is thin, the ducts themselves are thinner and more nearly straight in their course.

The duct is lined with a layer of columnar epithelium continuous with 
the epidermis. The coiled or secreting portion of the gland is lined with at least two layers of short columnar cells with very distinct nuclei, figure 306 . The lumen is distinctly bounded by a special lining of cuticle.

The sudoriferous glands are abundantly distributed over the whole surface of the body; but are especially numerous, as well as very large, in the skin of the palm of the hand and of the sole of the foot. The glands by which the peculiarly odorous matter of the axillæ and groin is secreted form

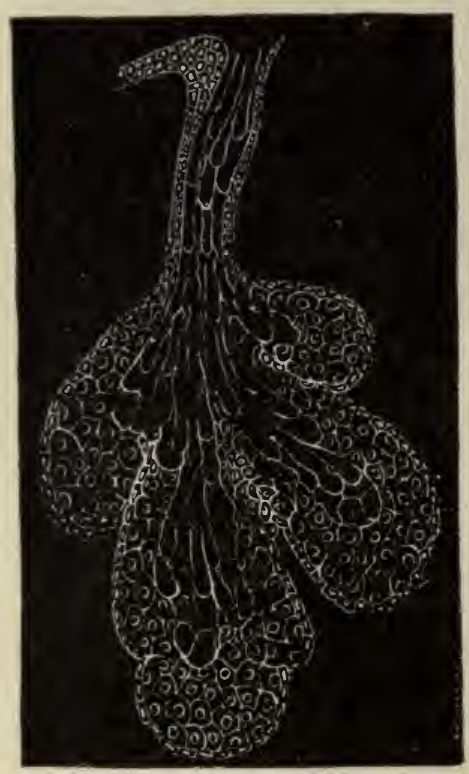

FiG. 307.-Sebaceous Gland from Human Skin. (Klein and Noble Smith.) a nearly complete layer under the cutis, and are like the ordinary sudoriferous glands, except in being larger and having very short ducts.

The peculiar bitter yellow substance secreted by the skin of the external auditory passage is named cerumen, and the glands themselves ceruminous glands; but they do not much differ in structure from the ordinary sudoriferous glands.

The sebaceous glands, figures 305 and 306, like sudoriferous glands, are abundant in most parts of the surface of the body, particularly in parts largely supplied with hair, as the scalp and face. They are thickly distributed about the entrance of the various passages into the body, as the anus, nose, lips, and external ear. They are entirely absent from the palmar surface of the hand and the plantar of the foot. They are racemose glands composed of an aggregate of small tubes or sacculi lined with columnar epithelium and filled with an opaque white substance, like soft ointment, which consists of broken-up epithelial cells which have undergone fatty degeneration. Minute capillary vessels overspread them; and their ducts open on either the surface of the skin, close to the hair, or, which is more usual, directly into the follicle of the hair. In the latter case, there are generally two or more glands to each hair, figure 306 .

The story of the structure and development of such epithelial structures as the hair and nails is best left to the histologist, to whom the student is referred.

The Excretory Function of the Skin.-The function of the skin which is of special interest to this chapter is that of the secretion of the sweat. The fluid secreted by the sweat glands is usually formed so gradually that the watery portion of it escapes by evaporation as fast as it reaches the surface. 
But during strong exercise, exposure to great external warmth, in some diseases, and when evaporation is prevented, the secretion becomes more sensible and collects on the skin in the form of drops of fluid.

The perspiration, as the term is sometimes employed in physiology, includes all that portion of the secretions and exudations from the skin which are thrown on the surface by the sweat glands. As a matter of fact, this is mingled with various substances lying on the surface of the skin. The contents of the sweat are, in part, matters capable of assuming the form of vapor, such as carbonic acid and water, and in part other matters which are deposited on the skin, and mixed with the sebaceous secretions.

The secretion of the sebaceous glands and hair follicles consists of castoff epithelium cells, with nuclei and granules, together with an oily material and extractive matter. In certain parts, also, it is mixed with a peculiar odorous principle, which contains caproic, butyric, and other fatty acids. It is similar in composition to the unctuous coating, or vernix caseosa, which is formed on the body of the fetus while in the uterus, and which contains ordinary fat and cholesterol esters with fatty acids. This sebaceous secretion serves the purpose of keeping the skin moist and supple, and, by its oily nature, of both hindering the evaporation from the surface and guarding the skin from the effects of the long-continued action of moisture. But while it thus serves local purposes, its removal from the body entitles it to be listed among the excretions of the skin.

Chemical Composition of Sweat.

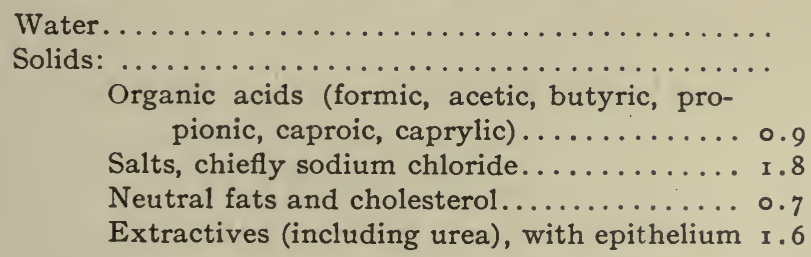

pionic, caproic, caprylic) ........... 0.9

Salts, chiefly sodium chloride............. I.8

Extractives (including urea), with epithelium I.6

1000

The sweat is a colorless, slightly turbid fluid, alkaline, neutral or acid in reaction, of a saltish taste, and peculiar characteristic odor.

Of the several substances it contains, however, only the carbon dioxide and water need particular consideration.

The quantity of water vapor excreted from the skin is, on an average, between $75^{\circ}$ and $\mathrm{r}, 000$ c.c. daily. This subject has been very carefully investigated by Lavoisier and Sequin. The latter chemist enclosed his body in an air-tight bag provided with a mouthpiece. The bag was closed by a strong band above, and the mouthpiece adjusted and gummed to the skin around the mouth. He was weighed, then remained quiet for several 
hours, after which time he was again weighed. The difference in the two weights indicated the amount of loss by pulmonary exhalation. Having taken off the air-tight dress, he was immediately weighed again, and a fourth time after a certain interval. The difference between the two weights last ascertained gave the amount of the cutaneous and pulmonary exhalation together; by subtracting from this the loss by pulmonary exhalation alone, while he was in the air-tight dress, he ascertained the amount of cutaneous transpiration. The average loss by cutaneous and pulmonary exhalation in a minute during a state of rest was eighteen grains-the minimum eleven grains, the maximum thirty-two grains. Of the eighteen grains, eleven passed off by the skin and seven by the lungs.

The quantity of watery vapor lost by transpiration is, of course, influenced by all external circumstances which affect the exhalation from evaporating surfaces, such as the temperature, the hygrometric state, and the stillness of the atmosphere. But, of the variations to which it is subject under the influence of these conditions, no calculation has been exactly made.

The quantity of carbon dioxide exhaled by the skin on an average is said to be about one two-hundredth of that eliminated by the pulmonary respiration.

The cutaneous exhalation is most abundant in the lower classes of animals, more particularly the naked amphibia, as frogs and toads, whose skins are thin and moist, and readily permit an interchange of gases between the circulating blood and the surrounding atmosphere. Bischoff found that, after the lungs of frogs had been tied and cut out, from 3 to 4 c.c. of carbon dioxide gas was exhaled by the skin in eight hours. And this quantity is very large, when it is remembered that a full-sized frog will generate only about to c.c. of carbon dioxide by his lungs and skin together in six hours.

The importance of the respiratory function of the skin, which was once thought to be proved by the speedy death of animals whose skins, after removal of the hair, were covered with an impermeable varnish, has been shown by further observations to have no foundation in fact. The immediate cause of death in such cases is the interference with temperature regulation.

Influence of the Nervous System on Sweat Secretion.-The secretion of sweat is closely connected with the quantity of blood flowing through the cutaneous vessels. The quantity of sweat increases with vaso-dilatation and diminishes with vaso-constriction. The sweat glands are also under the control of efferent impulses passing to them from the special sweat centers in the brain and spinal cord through special sweat nerves. Thus, if the sciatic nerve be divided in a cat and the peripheral end be stimulated, beads of sweat are seen to appear upon the pad of the corresponding foot. The sweat appears even though at the same time the blood vessels are constricted, or the blood flow entirely stopped by compression of the aorta, whereas if atropin is injected previously to the stimulation, no sweat appears, although dila- 
tation of the vessels may be present. Secretion of sweat, too, may be brought about reflexly.

The circulation of venous blood in the spinal bulb causes the sweating of phthisis and of dyspnea generally, by stimulating the sweat center. If the cat whose sciatic nerve is divided be rendered dyspneic, abundant sweat occurs upon the foot of the uninjured, and none on the injured, side. The effect of heat in producing sweating may be both local and general, and, again, the various drugs which produce an increased secretion of sweat do not all act in the same way; thus, there is reason for thinking that pilocarpine acts upon the local apparatus, that strychnine and picrotoxin act upon the sweat centers, and that nicotine acts both upon the central and upon the local apparatus.

The special sweat nerves belong to the thoracid autonomics or sympathetic nerves, and issue from the spinal cord with this outflow, page 636. The fibers join the chain ganglia by way of the white rami and are thence distributed to all parts of the body. In the case of the hind limb of the cat the sweat nerves arise from the cord by the last two or three dorsal and first two to four lumbar nerves, pass to the sympathetic chain, and from thence to the sciatic nerve, following the general course of the autonomic nerves for this region. In the case of the fore limb, the nerves leave the cord by the first to the sixth dorsal roots pass into the thoracic sympathetic and then join the brachial plexus, reaching the arm through the median and ulnar nerves.

The Pilo-motor Nerves to the Skin.-Small groups of smooth muscle fibers are found generally distributed in the skin. They are attached to the hair follicles and by their contractions produce erection of the hairs or in man the roughness called goose-flesh. These muscles are innervated by nerve fibers that belong to the thoracic autonomics. The origin and path of distribution corresponds closely with that of the sweat nerves.

It will be as well to restate here the other functions which the skin subserves. In addition to its excretory office, we have seen that it acts as a channel for absorption. It is also concerned with the special senses, that of touch and temperature, to the consideration of which as well as to its function of regulating the temperature of the body we shall presently return. By its general impermeability it prevents the loss of moisture of the body by direct evaporation from the tissues. It should be recollected, however, that apart from these special functions, by means of its toughness, flexibility, and elasticity, the skin is eminently qualified to serve as the general integument of the body, for defending the internal parts from external violence, while readily yielding and adapting itself to their various movements and changes of position. 


\section{LABORATORY EXPERIMENTS IN EXCRETION.}

\section{PHYSIOLOGICAL REACTIONS.}

\section{The Relation of Blood Flow through the Kidney to the Secre-} tion of Urine.-Properly to check this experiment one should make three determinations: I, the general blood pressure; 2, the volume of the kidney; 3 , the amount of urine secreted. Anesthetize a dog and arrange the apparatus for taking the blood pressure as directed in experiment Ig. Prepare a renal onkometer, see figures 301 and 302 , and an onkograph for recording the variations in the volume of the kidney. The renal onkometer consists of a double metal box to fit the form of a kidney. The inner halves of this box should be covered so loosely with very thin sheet rubber that the rubber can be fitted into the bottom of the cup without undue tension. The rubber must be sealed to the outer edges of this inner cup with rubber cement and allowed to dry. When it is completely dried the inner cup should be adjusted to the outer, and the spaces enclosed by the rubber sheet filled with water. Or the onkometer may be closed with parchment and filled with oil as described in experiment 23 on the Circulation. The half of the onkometer that comes against the wall of the body cavity of the animal should be completely closed with a stopper before the instrument is adjusted to the kidney. Now adjust the onkometer to the kidney, taking care to place the renal arteries, veins, and ureter in the tube in such a way as not to compress them. Fill the outer cup with water and connect this cavity by a two-way cannula with the recording onkograph. In practice it is more satisfactory if one introduces between the onkometer and onkograph an overflow bottle or bulb, adjusted to maintain the constant pressure on the kidney. This direction varies from the usual one in that rubber sheeting instead of parchment is used to cover the inner cup of the onkometer, a method that permits the use of water instead of oil. Recently Jackson has introduced an air onkometer that is simpler to prepare and adjust. The seal is secured by wrapping it in folds of omentum after inserting the kidney. An air recorder must of course be used.

Isolate and insert a small cannula into the úreter. This cannula should be clamped in a stand at a level as little above that of the kidney as possible. The urine secreted may be collected in a roc.c. graduated cylinder and measured at intervals of 5 or Io minutes. Or, if the outflow is scanty, it may be allowed to drop on a tambour recording apparatus, the rate of dropping being indicative of the rapidity of secretion.

Determine the normal rate of secretion of a dog under constant anesthesia. The anesthesia should be medium to light, but should be kept very uniform so as to maintain a strong blood pressure. Note the effect on secretion and the corresponding effect on blood pressure and the kidney volume produced by vagus inhibitions. Section the vagus nerves and produce in- 
hibition by stimulating the peripheral end of the vagus. In this instance there are no reflexes to complicate the experiment, so that the fall in blood pressure is a direct cardiac effect. Stimulate the central end of the vagus which produces a fall of blood pressure through the vaso-motor system. There should be a normal period of at least ten minutes following each experiment to allow the secretion of the kidney to return to the normal.

Expose the splanchnic nerves at the point where they pass beneath the diaphragm into the abdominal cavity. Adjust a pair of shielded electrodes, close the cavity, and, when the animal has returned to the normal uniform rate of secretion and of blood pressure, stimulate the splanchnic nerves. The splanchnics contain vaso-constrictor nerves for the kidney. The onkometer experiment should, therefore, demonstrate a sharp decrease in the volume of the organ, while the blood pressure is only slighty changed. Inject ro cubic centimeters of 5 per cent. potassium nitrate or other inorganic diuretics intravenously. These tests should be repeated with long intervals for readjustment.

In this connection demonstrate the influence of deep chloroform anesthesia on urinary secretion. The chloroform should be pushed to the danger limit and maintained there for a couple of minutes or more. Compare the rapidity of the recovery of blood pressure with the recovery of the rate of secretion.

2. Secretory Nerves for the Sweat Glands.-Langley has mapped out the paths of the secretory nerves for the sweat glands. He has shown that in the cat these fibers are distributed to the hind limb through the sciatic. Anesthetize a half-grown cat, isolate the sciatic nerve, cut it and stimulate the peripheral end with a medium to strong induction current. After a few moments beads of perspiration will appear on the pads of the foot, which should therefore be carefully examined before the experiment.

\section{URINE ANALYSIS.}

3. Daily Quantity.-Determine the total quantity, for 24 hours, of urine secreted through a period of 3 or 4 days, beginning and ending the period at a definite hour in the day, preferably on rising in the morning. The daily secretion varies through wide extremes, depending upon the quantity of liquid taken in the food, the daily exercise, the temperature, etc., etc. In the analysis of urine it is always better to take a mixed 24-hour sample.

4. Specific Gravity.-Determine the specific gravity of 24-hour urine. This is done by the instrument known as the urinometer which carries a graduated scale at the neck. Care should be taken to float the urinometer so that it does not come in contact with the measuring cylinder. The scale should be read at the bottom of the meniscus.

5. Reaction.-Determine the reaction of perfectly fresh urine, using litmus paper. The normal urine is slightly acid under ordinary conditions, due to the presence of acid phosphates or perhaps in some cases to traces of free organic acid. 
After standing some time the reaction is usually alkaline, owing to fermentation processes. The reaction may vary also according to the food, vegetable foods tending to produce alkaline urine, while with animal foods the reaction is acid.

6. The Total Quantity of Solids.-Determine the solids of urine by evaporating $25 \mathrm{cc}$. of a mixed sample of urine to dryness in a weighed platinum or porcelain dish over a water bath. The residue should be dried to constant weight in a drying oven at $105^{\circ} \mathrm{C}$.

A useful rule for approximately estimating the total solids in any given specimen of healthy urine is to multiply the last two figures representing the specific gravity by 2.33 . Thus, in urine of specific gravity $1025,2.33 \times 25=$ 58.25 grains of solids are contained in $I, 000$ grains of the urine. Or the total solids are 5.825 per cent. In using this method it must be remembered that the limits of error are much wider in diseased than in healthy urine.

The solids of urine consist of inorganic salts of sodium, potassium, and calcium, and of a long list of organic compounds, chiefly nitrogenous.

7. Chlorides.-Large quantities of sodium chloride are always present in the normal urine. Add ammonia to 25 or $50 \mathrm{cc}$. of albumin-free urine

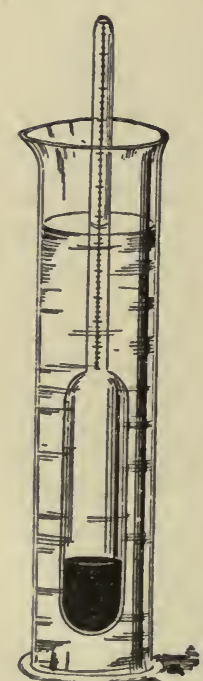

Fig. 308.-The Urinometer. and heat to precipitate earthy phosphates, filter. To a sample of the filtrate add an excess of strong nitric acid and a few drops of $\mathrm{I}$ per cent. silver nitrate. A white flocculent precipitate of silver chloride comes down. This precipitate is soluble in an excess of ammonia. Reprecipitate by'adding nitric acid again. The test may be made without removing the phosphates, though in this case, upon adding ammonia, the disappearance of the silver precipitate is complicated by the appearance of insoluble phosphates.

The chlorides may be estimated quantitatively by Volhard's method, or some one of its modifications, which depends upon the determination of the amount of chloride precipitated by the silver. The student is referred to chemical text-books for this and other quantitative methods.

8. Sulphates.-Sulphates exist in the urine both in inorganic and organic compounds, chiefly the former. Add a few drops of hydrochloric acid to a sample of urine in a test-tube, then a solution of barium chloride, the insoluble barium sulphate settles out. If the test is made on the normal urine without the addition of the acid, the inorganic sulphate will be precipitated, while the ethereal or compound sulphate will remain in solution and can be filtered off. This filtrate, when boiled with strong hydrochloric acid to ro per cent. over a water bath for a short time, will have the sul- 
phates split off from the organic radicle and may be precipitated by the addition of barium chloride in hot solution.

9. Phosphates.-The phosphates of urine consist of the earthy and alkaline salts, the latter predominating. Take a $50 \mathrm{cc}$. sample of urine, add strong ammonia, and heat. The phosphates of calcium and magnesium separate out, as they are insoluble in alkaline solution. Filter.

To the filtrate add a solution of magnesium sulphate. This precipitates the sodium and potassium phosphates as a triple phosphate of magnesium which is insoluble. Test for phosphates in general are:

Add nitric acid to a sample of urine, warm gently, then add a few drops of ro per cent. ammonium molybdate; a yellow precipitate of ammonium phospho-molybdate is formed. Or, add acetic acid, then a few drops of uranium acetate; a bright yellow precipitate of uranium ammonium phos-

$=$ phate is formed. These two reactions are used as the basis

\begin{tabular}{c} 
for a quantitative determination of phosphorus. \\
Io. The Preparation of Urea. - Take Ioo cc. of normal \\
urine, evaporate to one-half its quantity, and precipitate the \\
phosphates and sulphates by adding a mixed solution of barium \\
\hline 0
\end{tabular}

\begin{tabular}{c|c} 
& for a quantitative determination of phosphorus. \\
Io. The Preparation of Urea. - Take I0o cc. of normal \\
urine, evaporate to one-half its quantity, and precipitate the
\end{tabular}

\begin{tabular}{c|c} 
& for a quantitative determination of phosphorus. \\
ro. The Preparation of Urea.-Take roo cc. of normal \\
urine, evaporate to one-half its quantity, and precipitate the
\end{tabular}

\begin{tabular}{c|c} 
& for a quantitative determination of phosphorus. \\
Io. The Preparation of Urea. - Take I0o cc. of normal \\
urine, evaporate to one-half its quantity, and precipitate the
\end{tabular}

\begin{tabular}{c|c} 
& for a quantitative determination of phosphorus. \\
ro. The Preparation of Urea.-Take roo cc. of normal \\
urine, evaporate to one-half its quantity, and precipitate the
\end{tabular} 0.02
0.02
0.02 $=0$ cent. alcohol, and refilter. I: Doremus Ureometer. Crystals of urea separate out when the alcohol is
$0=0.03$
$0+$

$\therefore \quad$ Evaporate a large sample, 200 cc., of urine to a syrupy mass, add nitric acid. Crystals of urea nitrate are formed. Wash the crystals in dilute nitric acid, then dissolve in water. The urea is set free by adding barium carbonate until the carbon dioxide ceases to FIG. 309.-Doremus' Ureometer. come off. Filter, evaporate over a water bath to dryness, and dissolve the urea in 95 per cent. alcohol; decant, and recrystallize by evaporating off the alcohol.

II. Urea Determination by Doremus' Ureometer.-Fill the ureometer with hypobromite of sodium solution. Take a sample of urine in the pipet which accompanies the instrument, drawing it exactly to the mark. Insert the pipet past the bend of the ureometer and slowly and carefully empty the urine so as not to lose any of the liberated nitrogen. The instrument is graduated to read off the percentage of urea directly.

I2. Uric Acid.-Concentrate over a water bath $500 \mathrm{cc}$. of urine to I00 cc. and boil with Io cc. or more of strong hydrochloric acid. Upon cooling, crystals of uric acid are formed. Decant the supernatant liquid and wash the crystals with a few cubic centimeters of ro per cent. hydrochloric acid. Dissolve the crystals and test. 
The Murexide Test.-Add to $2 \mathrm{cc}$. of uric acid solution in a test-tube an equal quantity of nitric acid. Heat gently, a reddish ring forms at the point of contact between the nitric acid and uric acid solution. Cool and add ammonia carefully. The color ring deepens to a purple color. This test succeeds well by evaporating a few drops of uric acid on a porcelain plate. Add to the stain a drop of concentrated nitric acid and evaporate. Concentric rings of reddish color will be formed. This color deepens to reddishpurple when a drop of ammonia is added.

I3. Creatinin.-Test $20 \mathrm{cc}$. of urine in a beaker for creatinin by adding a cubic centimeter of dilute solution of sodium nitroprusside and then weak sodium hydrate. A ruby-red color, which quickly turns yellow, indicates the presence of creatinin (Weyl's reaction). If the yellow solution has an excess of acetic acid added and is then boiled, it turns first green and later blue, forming ultimately a precipitate of Prussian blue. Urine mixed with picric acid gives a red coloration when made alkaline with caustic alkali solution.

I4. Total Nitrogen in Urine.-Determine the total nitrogen in a sample of urine by the Kjeldahl method. This method depends upon the conversion of all the nitrogen to ammonia, the distillation of this ammonia into a known quantity of sulphuric acid, and the final titration of the excess of sulphuric acid when the distillation is complete. The computation is made on the basis that I cc. of a normal sulphuric acid is equivalent to I cc. normal sodium hydrate, and that in turn to $\mathrm{I}$ cc. of ammonium hydrate. The ammonia neutralizes a portion of the sulphuric acid in the distillation. One cc. of normal ammonium hydrate contains 0.014 gram nitrogen, from which the total nitrogen in the sample used can be readily computed.

I5. Pigments of Urine.-The normal color of the urine is due to the presence of a pigment, urobilin. Prepare urobilin by adding lead acetate to a $200 \mathrm{cc}$. sample of urine. A precipitate forms which carries down the coloring mater. Filter. Add acid alcohol to the precipitate to extract the coloring mater, refilter, which gives a deep yellow solution. Shake up with a few cubic centimeters of chloroform which dissolves the pigment. Draw off the chloroform solution and allow to evaporate. The residue is a brownish mass of urobilin.

I6. Test for Indican, Obermayer's.-Take in a test tube I cc. Obermayer's reagent (.2 to .4 per cent. ferric chloride in concentrated hydrochloric acid) and an equal part urine to be tested, then add 2 cc. of chloroform. Thoroughly shake. A blue color will develop if indican be present.

\section{ABNORMAL CONSTITUENTS OF URINE.}

Many abnormal constituents may appear in the urine under pathological conditions, only two of which will be mentioned here. 
I7. Albumin in the Urine.-The detection of the presence of albumin, albuminuria, is of considerable clinical importance. The following are the standard tests which present no special difficulty except when traces only are present.

Heat Coagulation.-Take a half test-tube of urine, boil, and add a drop of dilute acetic acid. A white coagulum indicates the presence of albumin. A faint cloudy appearance indicates traces.

Nitric Acid Test.-To $5 \mathrm{cc}$. of strong nitric acid in a conical test-tube add Io or 5 c.c. of urine, pouring it gently down the inclined side of the glass. Allow the glass to stand for a few minutes, when a white coagulum appears just above the line of contact of the acid with the urine. This test, known as Heller's test, will usually indicate the presence of traces of albumin.

Picric Acid Test.-Add picric acid to a sample of urine. A whitish precipitate of albumin will appear at the line of contact, as in the preceding test.

Citric acid two parts and picric acid one part, when boiled with urine will coagulate minute traces of the protein.

I8. Detection of Sugar in the Urine.-Fehling's Test.-The presence of sugar in the urine can usually be detected by Trommer's test, which depends upon the reduction of copper sulphate in the presence of strong alkali. Boil fresh Fehling's solution and add to it a few drops of urine. When sugar is present a reddish-yellow precipitate of copper oxide comes out. The test should be set away for a few minutes when, if only traces of the reduction are present, a reddish-brown stain will appear on the bottom of the test-tube. Uric acid, if present in excess, may produce a slight precipitation of the copper.

Fermentation Test.-If sugars are present in the urine, they can be detected by adding yeast to a fermentation tube filled with urine, the liberation of carbon dioxide indicating the presence of sugar. Cane sugar does not support the growth of yeast, so it forms an exception by this test.

Phenyl-Hydrazin Test.-Phenyl-hydrazin forms crystals of phenylglucosazone. To ro cc. of urine in a small beaker add o. I of a gram of phenyl-hydrazin hydrochloride and a double quantity of sodium acetate. Heat in the water bath for 20 minutes. Upon cooling a deposit of yellow crystals of phenyl-glucosazone takes place if glucose is present.

I9. Quantitative Determination of Sugar in the Urine.-Fill a to cc. graduated pipet with freshly prepared Fehling's solution. Take ro cc. of urine, measured with a dropping-pipet into a small beaker, and boil. While continuing to boil, add Fehling's solution slowly and cautiously so long as the color is discharged. The amount of Fehling's required to reduce the sugar is a measure of the quantity of reducing sugar present-I cc. of Fehling's being equivalent to 5 milligrams of dextrose.

For the presence of blood pigments and other abnormal constituents of the urine, the student is referred to special handbooks on the subject. 


\section{CHAPTER XI}

\section{METABOLISM, NUTRITION, AND DIET.}

THE term metabolism means, literally, an exchange of material. In its broadest physiological sense it includes the study of the exchange of material between the living tissues of the body and their surrounding media. This includes the study of the income and outgo of material; the storing of energyyielding materials in the body; the transfer of this potential energy into kinetic energy; and the nutritional processes within the various tissues. The building up of absorbed food material into the protoplasm of the cell or of simpler compounds into more complex ones, which may be stored in the cell, is known as anabolism, and the compounds themselves as anabolites. The breaking down of these substances into simpler forms, whereby the potential energy of the anabolites is transformed into kinetic energy, is known as katabolism, and its products as katabolites.

In order to form an estimate of these processes going on in the body, the amount and nature of the ingested material must be known, as well as the amount of refuse or unused material that passes out of the alimentary canal as feces, and the amount of excreted material from the various excretory organs. It is also necessary to know the potential energy of the ingested materials, and the possible potential energy must be checked against the actual energy liberated.

The food is intended to supply the place of the material which has been utilized by the body, and, in a simpler form, eliminated in the excretions. But in the choice of a diet this is not enough; the food should be sufficient to supply such need without waste and without unduly increasing the output of excreta, while at the same time the body should be maintained in health, without increase or loss of weight. The food must also supply the energy liberated without undue waste of the tissues themselves.

These requisites of a diet scale then allow for wide alterations in the amount of different kinds of foods under different circumstances. Numerous and most valuable experiments have been performed in recent years to determine just what each article of the common food materials contributes to the growth of the tissues and to the kinetic energy liberated by the tissues. The potential energy of the food can also be checked against the kinetic energy liberated. A single illustration of this class will serve. In an experiment with mixed food lasting through four days, on a man with body weight of 64 kilograms, and doing a minimum amount of work, Atwater made the following determinations: 
Weight, Composition, and Heat of Combustion of Foods and Excreta PER DAY.

\begin{tabular}{|c|c|c|c|c|c|c|c|c|c|}
\hline & 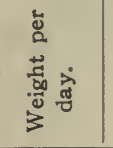 & 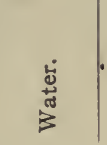 & 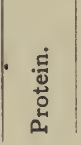 & 离 & 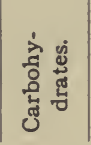 & 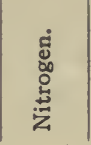 & हึ่ & 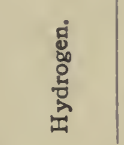 & 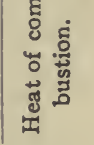 \\
\hline Food & Grms. & Grms. & Grms. & Grms. & Grms. & Grms. & Grms. & Grms. & $\begin{array}{c}\text { Calor- } \\
\text { ies }\end{array}$ \\
\hline Beef........... & 100 & $6 \mathrm{I} \cdot 2$ & $35 \cdot r$ & $3 \cdot I$ & $\ldots \ldots$ & 5.62 & 20.05 & 2.90 & 227 \\
\hline Butter......... & 25 & 2.6 & $\cdot 5$ & $2 I . I$ & $\cdots$ & .08 & $15 \cdot 77$ & $2 \cdot 50$ & r 94 \\
\hline Whole milk..... & $85^{\circ}$ & 726.8 & $32 \cdot 3$ & 45.1 & $39 \cdot 9$ & $5 \cdot 10$ & $67 \cdot 74$ & 9.94 & 768 \\
\hline Bread ........... & 300 & 123.9 & 22.2 & 12.0 & 139.2 & $3 \cdot 90$ & $82 \cdot 53$ & 12.24 & 835 \\
\hline $\begin{array}{l}\text { Shredded wheat } \\
\text { biscuit. }\end{array}$ & 50 & $4 \cdot I$ & 4.8 & $\cdot 7$ & $39 \cdot 7$ & .84 & 20.46 & 2.87 & 204 \\
\hline Ginger snaps.... & 50 & $3 \cdot 3$ & 2.8 & 3.6 & 39.2 & .50 & 2 I.I2 & 3.07 & 12 \\
\hline Sugar............. & 20 & $\cdots \cdot$ & . & . & 20.0 & $\cdots$ & 8.42 & I. 30 & 9 \\
\hline $\begin{array}{l}\text { Total food per } \\
\text { day. }\end{array}$ & $\mathrm{r}, 395$ & $921 \cdot 9$ & $97 \cdot 7$ & 85.6 & 278.0 & 16.04 & 236.09 & 34.82 & 2,519 \\
\hline $\begin{array}{l}\text { Average feces per } \\
\text { day. }\end{array}$ & 98.8 & $77 \cdot 7$ & $7 \cdot 7$ & 4.0 & $6 \cdot 3$ & I.23 & $9 \cdot 98$ & I. 42 & \\
\hline $\begin{array}{l}\text { Average urine per } \\
\text { day. }\end{array}$ & 1420.8 & 1363.0 & . & . & .. & I 5.85 & II.79 & 2.98 & 135 \\
\hline $\begin{array}{l}\text { Excretions-lungs } \\
\text { and skin. }\end{array}$ & $\ldots$. & $88 \mathrm{I} .0$ & . & . & $\cdots$ & $\cdots$ & $22 \mathrm{I} .5$ & $\ldots \ldots$ & 2,397 \\
\hline $\begin{array}{l}\text { Total excreta per } \\
\text { day. }\end{array}$ & $\ldots$ & 2322.6 & . & . & $\cdots$ & 17.08 & 243.27 & $4.40^{\circ}$ & 2,6 \\
\hline Balc & & & .. & . & . & -1.04 & $-7 \cdot 18$ & +30.42 & - \\
\hline
\end{tabular}

Careful analyses of the excreta, many of which we have already had occasion to call attention to, show that they are made up, besides water, chiefly of the chemical elements carbon, hydrogen, oxygen, and nitrogen, but that they also contain, to a less extent, sulphur, phosphorus, chlorine, potassium, sodium, calcium, magnesium, iron, and certain other of the elements. Since this is the case it must be evident that, to balance this waste, foods must be supplied containing all these elements to a certain degree, and some of them in large amount, viz., those which take a principal part in forming the excreta.

The waste products of the body are eliminated through the lungs, the skin, the alimentary canal, and the kidneys. In the lungs the chief waste product is water and carbon dioxide gas. Some carbon dioxide gas and small quantities of urea and salts are eliminated through the skin. From the alimentary canal there are lost, through the feces, the indigestible and un- 
absorbed substances from the food, together with products secreted into the canal by the liver, pancreas, and mucous membrane. The secretion lost daily by the kidney, aside from a large quantity of water, consists of nitrogenous waste products, chiefly urea, and inorganic solids, as were mentioned in the chapter on Excretion.

The relations between the amounts of the chief elements contained in these various excreta in twenty-four hours may be thus summarized:

\begin{tabular}{|c|c|c|c|c|c|}
\hline - & Water. & C. & H. & N. & O. \\
\hline 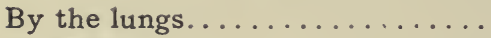 & 330 & 248.8 & $\ldots$ & . & $65 x \cdot 15$ \\
\hline By the skin $\ldots \ldots \ldots \ldots \ldots \ldots$ & 660 & 2.6 & $\ldots$ & $\ldots$ & $7 \cdot 2$ \\
\hline 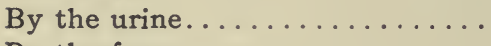 & 1,708 & 9.8 & $3 \cdot 3$ & I 5.8 & I I. I \\
\hline 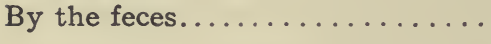 & I 20 & 20.0 & $3 \cdot 0$ & $3 \cdot 0$ & 12.0 \\
\hline Grams.... & 2,8 I 8 & 281.2 & $6 \cdot 3$ & I 8.8 & $68 I \cdot 45$ \\
\hline
\end{tabular}

From the water in this table should be subtracted the 296 grams of water which are produced by the union of hydrogen and oxygen in the body during the process of oxidation, and there should be added to the respective columns the corresponding amounts of the constituent elements, i.e., 33 grams of hydrogen and 262 grams of oxygen. There are 26 grams of salts eliminated through the urine, and 6 by the feces; a total of 32 grams.

The quantity of carbon daily lost from the body amounts to about $28 \mathrm{I} .2$ grams and of nitrogen 18.8 grams, and if a man could be fed by these elements, as such, the problem would be a very simple one; a corresponding weight of charcoal and, allowing for the oxygen in it, of atmospheric air would be all that is necessary. But an animal can live upon these elements only when they are arranged in a particular manner with others, in the form of such food stuffs as we have already enumerated, page 322 et seq.; moreover, the relative proportion of carbon to nitrogen in either of these compounds alone is by no means the proportion required in the diet of man. Thus, in protein, the proportion of carbon to nitrogen is only as 3.5 to $\mathrm{I}$. If, therefore, a man took into his body, as food, sufficient protein to supply him with the needed amount of carbon, he would receive more than four times as much nitrogen as is needed; and if he took only sufficient to supply him with nitrogen, he would be starved for want of carbon. It is plain, therefore, that he should take with the albuminous part of his food, which contains so large an amount of nitrogen in proportion to the carbon he needs, substances in which the nitrogen exists in relatively much smaller quantities than the carbon. 
It is, therefore, evident that the diet must consist of several compounds, not of one alone.

Many valuable observations have been made with a view of ascertaining the effect upon the metabolism of a variation in the amount and nature of food. These are of great assistance in the consideration of dietetics.

\section{METABOLISM OF PROTEINS.}

Nitrogenous Equilibrium.-Experiments have been made, to a considerable extent upon dogs, which demonstrate the necessity for protein food. After a preliminary period without food, during which the output of nitrogen as shown by the urea has diminished to a comparatively constant amount, an animal is fed with a diet of lean meat which would suffice to produce the amount of urea, and so of flesh, which it has been losing during its starvation period. The effect of this, however, is at once to send up the amount of urea excreted to a point above that which had been lost previous to the commencement of the flesh diet. Thus the output of nitrogen still exceeds its income, and the weight of the animal continues slowly to diminish. It is only after a considerable increase of the flesh given in the food that a point is reached where the income and the expenditure of nitrogen are equal, and at which the animal is not using up or storing the nitrogen in its own tissue, and is no longer losing flesh. This condition in which the nitrogen of the egesta equals the nitrogen of the ingesta is known as nitrogenous equilibrium.

Experiment in Nitrogenous Equilibrium.

\begin{tabular}{|c|c|c|c|c|c|}
\hline Days of experiment. & $\begin{array}{l}\mathrm{N} \\
\text { Intake. } \\
\text { Grams. }\end{array}$ & $\begin{array}{l}\text { N } \\
\text { Output. } \\
\text { Grams. }\end{array}$ & $\begin{array}{c}\text { Per cent. } \\
\text { Differ- } \\
\text { ence. }\end{array}$ & $\begin{array}{l}\text { S } \\
\text { Intake. } \\
\text { Grams. }\end{array}$ & $\begin{array}{l}\text { S } \\
\text { Output. } \\
\text { Grams. }\end{array}$ \\
\hline I. $\mathrm{x}-5 \ldots$ & 90.00 & $89.8 \mathrm{I}$ & $-0.2 x$ & $\cdots \cdots \cdots$ & $\ldots \ldots \ldots$ \\
\hline $6-12 \ldots \ldots$ & 131.60 & $132 \cdot 75$ & +0.88 & $\ldots \ldots$ & $\ldots \ldots$ \\
\hline II. $I-2 \ldots \ldots \ldots \ldots$ & 35.80 & 36.16 & $+x .00$ & $\ldots \ldots$ & $\ldots \ldots$ \\
\hline 3-II . . . . . . . & $\mathrm{I} 44 \cdot 5^{\circ}$ & $143 \cdot 13$ & -0.86 & $\ldots \ldots$ & $\ldots \ldots$ \\
\hline III. $I-7 \ldots \ldots \ldots \ldots$ & $\mathrm{I} 54.8 \mathrm{I}$ & I 53.02 & $-0.5^{1}$ & $\ldots \ldots$ & $\ldots \ldots$ \\
\hline $8-17 \ldots \ldots$ & $213 \cdot 72$ & $2 \times 3 \cdot 26$ & -0.21 & 12.77 & I 2.79 \\
\hline
\end{tabular}

In the dog, according to Waller, nitrogenous equilibrium does not occur until the amount of flesh of the food is over three times as great as would be necessary to supply the nitrogen of the urine during a period of starvation. Thus a dog excretes during a starvation period 0.50 gram of urea per kilo 
of body weight. In order to satisfy this waste it would be necessary to administer I.50.grams per kilo of meat protein. This at once increases the urea excreted to about 0.75 gram per kilo of body weight, and nitrogenous equilibrium is not attained until over three times, viz., 3 grams per kilo of body weight, of meat protein is given. Foster gives even a larger figure. The immediate effect, therefore, of increasing the protein food is largely to increase the excretion of urea.

Studies in nitrogenous equilibrium are based on the fact that when an animal is given a diet with a constantly increasing amount of protein food from day to day, after a few days the total nitrogen found in the excreta exactly balances that taken in the food. This condition of nitrogenous equilibrium is established at different levels, varying sometimes according to the individual and with the kind and quantity of other food principles taken at the same time as the nitrogenous foods.

Chittenden's later metabolism experiments have shown that with free choice, but moderate use, of accessory articles of diet, the human body can maintain itself in nitrogenous equilibrium for at least several months on an average of 6 to Io grams of nitrogen per day, the equivalent of 37.5 to 62.5 grams of dry protein or four times as much lean beef.

The Role of Proteins in Metabolism.-The proteins of food are described by Voit as having two relations to the protein metabolism, also to outgoing urea. The first part of the protein of the food goes to maintain the ordinary and quiet metabolism of the tissues, for which purpose it is actually built up into the living protoplasmic molecule. The second part being more directly oxidized causes a more rapid formation of urea, but never becomes a part of the actual protoplasmic molecule. The former proteins are called morphotic or tissue proteins, the latter circulating or floating proteins. Normally more protein is eaten than is needed to supply protein to the protoplasm for growth, as has just been stated. Pflüger takes the view, however, that the tissues must have an excess of protein to destroy in order to perform their metabolic processes normally. This use of the proteins to form heat by their oxidation, and not to produce tissue, was looked upon by the older physiologists as a wasteful use of good material, and was called luxus consumption. This use is now disproved.

Folin has recently announced a theory of protein metabolism in which he calls special attention to the relation of the nitrogenous excretion products to the nitrogenous intake. He has presented evidence to show that the urea contained in the urine varies almost directly with the quantity of protein in the food; that the ammonia also varies with the protein in the food; that the uric acid decreases (and increases) with the protein in the food, but not in direct ratio; while the creatinin excreted is "wholly independent of quantitative changes in the total amount of nitrogen eliminated." 
Table Showing the Output of Nitrogen in a Normal, Healthy Individual on a Food Rich in Nitrogen, July r 3 th, and Poor in Nitrogen JULY 2OTH (FOLIN).

$$
\text { July I } 3^{\text {th. }}
$$

Volume of urine... I, I 70 c.c. .

Total nitrogen..... 6.08 grams

July 20 th.

Urea nitrogen.... I4.70 grams $=87.5$ per cent. Ammonia nitrogen. 0.49 grams $=3.0$ per cent. Uric-acid nitrogen. 0.18 grams $=1$. I per cent. Creatinin nitrogen. 0.58 grams $=3.6$ per cent. Undetermined nitro- 0.85 grams $=4.9$ per cent. gen.

385 c.c.

3.60 grams

Folin states this theory as follows: "It is clear that the metabolic processes resulting in the end products which tend to be constant in quantity appear to be indispensable for the continuation of life; or, to be more definite, those metabolic processes probably constitute an essential part of the activity which distinguishes living cells from dead ones. I would therefore call the protein metabolism which tends to be constant, tissue metabolism, or endogenous metabolism; the other, the variable protein metabolism, I would call the exogenous or intermediate metabolism.

"The endogenous metabolism sets a limit to the lowest level of nitrogen equilibrium attainable. Just where that level is fixed will depend on how much, if any, urea is derived from the same katabolic processes that produce the creatinin. If this can be determined, we shall have a formula expressing more or less definitely the point of lowest attainable protein katabolism, because at such a point the percentage composition of the urine should be practically constant. The total nitrogen eliminated when this constant composition of the urine has been reached will indicate the lowest attainable level of nitrogen equilibrium."

The condition of nitrogenous equilibrium, therefore, is one which may be maintained even if the amount of protein taken as diet far exceeds the necessities of the economy, the urea being excreted in excessive amount. The wasteful use of protein food which is so prevalent may not be attended with harmful consequences, so long as the excreting organs are able to eliminate nitrogen from the body but it overworks those organs.

It is only in cases of growth, by putting on of flesh, as in growing children, that nitrogen is retained in the body in health, except to a very small amount. According to calculations which have been made, it appears that the body puts on 30 grams of flesh for every gram of nitrogen so retained.

Proteins as Fat- and as Glycogen-Formers.-Protein food is undoubtedly a source of energy in the body; and one can say that such protein as is, according to Voit's view, metabolized without becoming part of the tissue may be considered a source of energy. If this be true, one might ex- 
pect that proteins could be metabolized into other forms, such as carbohydrates and fats. Bernard long ago stated that protein was a glycogenformer; that abundant glycogen was stored in the liver when flesh diet was fed, and argued that protein was the source of the glycogen. The careful work of a number of investigators has not obtained sufficient evidence to clear up this question absolutely, but the weight of evidence is in favor of the view that in the body sugar can be formed from proteins. Lusk has recently shown that some of the amino acids must have been converted into dextrose after deamidization in the body. Whether or not protein can be metabolized into fat, and stored as such, seems at present an open question, notwithstanding the immense amount of work expended in trying to solve the problem.

Cramer fed 450 grams of lean meat per day to a cat in a respiraticn chamber for 8 days. The daily excretion of nitrogen was 13 grams, of carbon 34.3 grams; calculating the amount of carbon in the food as 4 I. 6 grams daily, this would leave 7.3 grams retained. This carbon might be stored in the form of glycogen or as fat. Calculated as glycogen, it gives an amount greater than an animal of that size could retain. Therefore, the probabilities are that the carbon is deposited in the form of fat.

In the examination of the fat formed in the larvæ of blow-flies developing in a quantity of coagulated blood, Hoffmann found ten times more fat than existed in the blood. These experiments point in the direction of fat formation from protein.

The Effect of a Gelatin Diet.-The albuminoid eaten in greatest quantity is gelatin. Though gelatin closely resembles the protein molecule chemically, it cannot replace entirely the protein of the food. As was stated in the chapter on the Chemistry of the Body, gelatin is deficient in certain amino acids, notably tryptophane which is not present at all. It is probable that because of the absence of this "building stone," the body tissues cannot reform their characteristic proteins for which this amino acid would be an essential constituent. In other words, nitrogenous equilibrium cannot be maintained on a diet consisting of gelatin, carbohydrates, and fats. Gelatin, then, is a substance whose food value in part is comparable to that of carbohydrates and fats, as the following experiments will prove: On a diet of 500 grams of meat, without any gelatin, the subject lost nitrogen to the equivalent of 22 grams of protein, but when 200 grams of gelatin were added the subject gained 54 grams. In another experiment, when the diet consisted of 2,000 grams of meat without gelatin, the gain was the equivalent of 30 grams of protein, but when 200 grams of gelatin were added the gain became 376 grams. The lack of a mixed protein food value is proven by still a third experiment in which the diet consisted at first of 200 grams each of meat and of gelatin; here the gain was the equivalent of 25 grams of protein, but, when the meat was omitted and the gelatin 
alone given, there was a loss of I 8 grams. In these cases gelatin did not take the place of protein in any sense, but rather saved it from oxidation as a source of energy. The protein was so protected that, instead of being used up, it helped to form tissue and increased the body weight. Gelatin, therefore, saved the protein material for constructive processes.

Murlin has investigated more exactly the substitution of gelatin for the mixed proteins in the food. In a series of experiments on dogs, the nitrogen output was first determined during fasting periods. Varying amounts of gelatin containing from a fourth to two-thirds of this amount of nitrogen were then fed, the remaining three-fourths to one-third of the fasting quantity being supplied in meat or other proteins. The calorific requirement of the animal was made up in each experiment with fats and carbohydrates. Results show an equal sparing of body protein, whether one-fourth, onethird, or one-half of the fasting nitrogen was fed in the form of gelatin, the coincident sparing of protein by fats and carbohydrates being the same. When the coincident sparing of protein by non-nitrogenous food was increased by feeding a larger percentage of carbohydrates and less fat, the fraction of the fasting nitrogen fed in the form of gelatin could be raised to two-thirds, the other one-third being fed in meat. Nitrogenous equilibrium was maintained on this diet for several days. The same result was obtained on man. The evidence at hand indicates that other individual proteins, in which certain amino acid constituents are deficient in quantity or which are absent, like gelatin would not replace entirely the ordinary mixed protein requirements.

The Formation of Urea.-The nitrogenous fraction of the protein molecule is in the end converted largely into urea and is excreted from the body in that form, as described in the chapter on Excretion. The method of formation of urea as well as the place where this occurs has given rise to great controversy, while the intermediate products between proteins and urea have not as yet been fully determined. We can state with certainty that urea is not formed in the kidneys, since it is not only found in the blood of the renal artery, but it accumulates in the blood if the kidneys are diseased or removed and the separation of the urine is interfered with. Circulation of blood through the kidney does not result in the formation of more urea than is present in the blood to begin with.

There are a number of experiments that prove that urea is formed in the liver. The power of the liver cells to form urea is shown by the increase of urea in the blood leaving an isolated living liver through which an artificial circulation is kept up. When ammonium carbamate and other ammonium salts are added to the blood, the urea increases more rapidly and to a greater extent. This change occurs even when the living hepatic tissue is chopped up and simply mixed with the ammonium compounds in a beaker.

If blood from a well-fed animal be circulated through the isolated liver, 
there is a distinct increase in the amount of urea it contains. On the other hand, if the blood be from a fasting animal there is little or no increase of urea. Evidently, then, the blood from a well-fed animal contains something which the liver cells are capable of transporting into urea. And, finally, if the liver be removed and the animal kept alive, as has been done by Pawlow, there is a marked diminution in the quantity of urea in the urine. The power of the liver to form urea is thus demonstrated. The question which now presents itself is, what is this antecedent substance or substances?

It has already been indicated that urea follows closely the amount of protein taken with the food, hence we must look directly to the nitrogenous fraction of protein cleavage as the final source of urea. While the different steps in the process of cleavage, probably hydrolytic (Folin), are yet very obscure, still it is believed that ammonia is split off from the proteincleavage products and is then built up into urea by the liver. It is now believed that ammonium carbamate is at least one true antecedent of urea.

In these experiments the liver is first shut out of the general circulation by an Eck's fistula connecting the portal vein with the vena cava. This operation cuts off the chief blood supply of the liver, viz., the portal blood, but it leaves the small hepatic artery with its oxygen supply to the liver. When animals survive this operation it is found that they can live only when fed very carefully on a mixed diet from which proteins are almost entirely eliminated, and that, if the food contain an excess of proteids, convulsions ensue with fatal termination. Investigation of the composition of the urine and of the blood, with the Eck's fistula, shows that the end product of protein metabolism is represented by ammonium carbonate and carbamate and that there is a considerable decrease in urea. If ammonium salts are injected into the blood of normal animals in a larger quantity than the liver can dispose of, death ensues, following convulsions of the same nature as those produced by an excess of protein food in the animals operated on.

Ammonium carbamate is shown to be, in part at least, the direct antecedent of urea. The reaction by which the liver changes it to the inert form of urea is as follows:

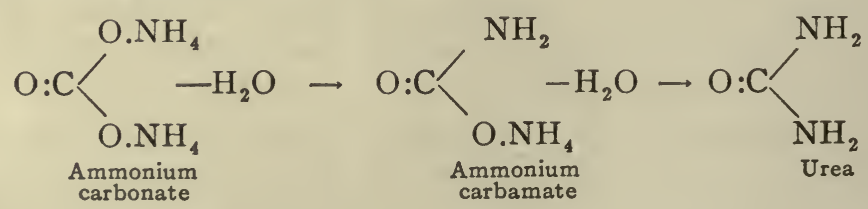

The elimination of urea is increased very slightly by muscular activity. But there is no direct relationship between the amount of work done and the amount of nitrogen excreted as urea.

There is experimental evidence to show that while the liver produces the 
major part of the urea eliminated, other organs or tissues are capable of forming it to a limited degree.

Formation of Uric Acid.-The relation which uric acid and urea bear to each other in different animals, as we have seen, is still obscure. The fact that they exist together in the same urine makes it seem probable that they have different origins. The entire replacement of one by the other, as of urea by uric acid in the urine of birds, serpents, and many insects, shows their close relationship. But although it is true that uric acid on oxidation yields urea, this is not evidence that uric acid is an antecedent of urea in the nitrogenous metabolism of the body. The chemical structure of the uric acid shows it has a nucleus of purin, and therefore is a close relative of adenin, guanin, hypoxanthin, xanthin, theobromin, caffein, etc. The nucleins on cleavage yield members of this group, hence may be looked to as the primary source of uric acid in man. Uric acid, according to Chittenden, has a double origin - endogenous from nuclear metabolism, and exogenous from metabolism of foods rich in nuclear and other purin compounds. In man, at least, the uric acid is to be ascribed to these two sources.

Operative experiments on birds tend to show that the final step in uricacid formation takes place chiefly in the liver, for on the removal of this organ ammonium compounds, i.e., lactates, accumulate in the blood.

Hippuric Acid, Creatinin.-The hippuric acid found in the urine. is derived in part from aromatic constituents of vegetable diet which can be transformed into benzoic acid in the body. It is derived in part also from the phenyl propionic acid formed in the intestinal putrefaction of protein. Hippuric acid is formed from the union of benzoic acid with glycocoll $\left(\mathrm{C}_{2} \mathrm{H}_{5} \mathrm{NO}_{2}+\mathrm{C}_{7} \mathrm{H}_{6} \mathrm{O}_{2}=\mathrm{C}_{9} \mathrm{O}_{9} \mathrm{NO}_{3}+\mathrm{H}_{2} \mathrm{O}\right)$. This union, in dogs, takes place under experimental conditions in the kidneys themselves, but in other animals the synthesis will occur after nephrectomy.

The source of the nitrogenous extractives of the urine is chiefly from the metabolism of the nitrogenous foods and tissues, but we are unable to say whether these nitrogenous bodies have merely resisted further decomposition into urea, or whether they are the representatives of the decomposition of special tissues, or of special forms of metabolism of the tissues. There is, however, one exception, and that is in the case of creatinin. This represents not only the creatinin which enters the body in ordinary flesh food, but is a nitrogenous waste which Folin regards as a measure of muscle metabolism. The creatinin eliminated is almost a constant quantity in a given individual, irrespective of the quantity of protein in the diet. Koch has shown some relation of creatinin excretion to the amount of lecithin in the food.

\section{THE METABOLISM OF FATS.}

Fats, with carbohydrates, are the direct source of most of the energy manifested by the body, a fact demonstrated by numerous observations. 
The Energy Value of Fats in Metabolism.-Fats, in comparison with other food principles, are of especial value as sources of energy. They are completely oxidized in the body to carbon dioxide and water, and yield, therefore, as much energy to the body as they yield upon oxidation outside the body. The energy equivalent of I gram of fat is 9.3 large Calories, more than twice that of starch, which in the body yields only 4.I Calories per gram, or of protein with 4.I Calories available yield of energy.

A study of the elimination of nitrogen and of carbon during fasting shows that the fats contribute to energy formation for many days. This is illustrated by the following computation by Voit:

Metabolism in a Dog during Fasting. (Voit.)

\begin{tabular}{|c|c|c|c|}
\hline Loss per kilogram of live weight. & $\begin{array}{l}\text { Proteins } \\
\text { in grams }\end{array}$ & $\begin{array}{c}\text { Fats } \\
\text { in grams }\end{array}$ & $\begin{array}{c}\text { Total } \\
\text { weight }\end{array}$ \\
\hline Second day.... & $2.2 \mathrm{I}$ & 2.62 & 32.87 \\
\hline 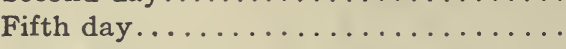 & I. I3 & $3 \cdot 25$ & $3 x \cdot 67$ \\
\hline Eighth day $\ldots \ldots \ldots \ldots \ldots$ & 0.96 & $3 \cdot 25$ & 30.54 \\
\hline
\end{tabular}

The amount of fat metabolized is sharply influenced by the amount and kind of other food. For example, if the amount of fat metabolized per day in fasting is first determined, then a ration of protein given for a few days, followed by a second fasting period, it will be found that the metabolism of body fat is sharply increased in the second period, due to the stimulating influence of the protein. This is demonstrated by the following determination of Rubner:

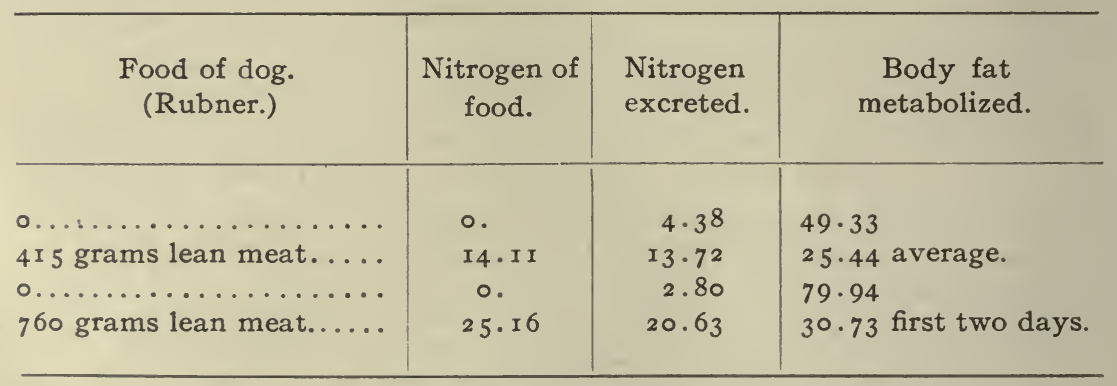

The fat of the ordinary daily diet is absorbed into the blood and no doubt contributes directly to oxidation processes. In some of the lower carnivorous vertebrates fats are undoubtedly the primary and direct source of the energy of daily activity. Just the steps in this oxidation process cannot at present be given. If the fat of the food is insufficient, then the body store is immediately drawn upon; if in excess, then it is stored in the body. This 
observation is strongly supported by the work of Eckles on the variation in the percentage of fat in the milk of dairy cows under the influence of temporary short feed. If a cow is given an insufficient amount of food immediately the percentage of fat in the milk sharply increases, sometimes amounting to almost double the original fat content of the milk.

Source of the Body Fat.-Excess of fat in the food can be stored as fat in the body. This fact is demonstrated by Voit, Hoffmann, Rubner, and others. Rubner states that 82 to 92 per cent. of the fat excess can be stored. The fat stored was long thought to be the same kind given in the food, even though the usual fat of the animal was different. The melting point of dog's fat is about $20^{\circ} \mathrm{C}$., but by feeding an excess of mutton iat the melting point has been raised to $40^{\circ} \mathrm{C}$. The subcutaneous fat of pigs subjected to this experiment is more or less fluid according to the melting point of the fat fed. However, we now know that this conception is only partially correct. The physical and chemical constants show that the fat laid down approaches in characteristics the fat fed, but that it is not the same. Even in the alimentary canal during digestion, changes occur which modify the melting point and other properties of remnants of the fat not absorbed. Fat that is resynthesized in the mucous epithelium after absorption is similar but not identical with the fats of the food. This possibly rests upon a variation in the number and character of the unsaturated bonds during fat mobilization in the body.

The body fat can also be derived from carbohydrate food, a fact which the practices of the stock feeder and dairyman constantly verify. The experiments will present the matter more vividly than pages of description.

Gain in Fat of a Pig Fed on Rice. (Meissl and Strohmer.)

\begin{tabular}{l|c|c|c|c|c|c}
\hline Pig weight. & $\begin{array}{c}\text { Rice fed } \\
\text { daily. }\end{array}$ & $\begin{array}{c}\text { Fat in } \\
\text { food. }\end{array}$ & $\begin{array}{c}\text { Protein } \\
\text { in food. }\end{array}$ & $\begin{array}{c}\text { Protein } \\
\text { gain. }\end{array}$ & $\begin{array}{c}\text { Carbon } \\
\text { gain. }\end{array}$ & $\begin{array}{c}\text { Net gain } \\
\text { in carbon. }\end{array}$ \\
\hline I0 $\mathrm{kgm}$. & $2 \mathrm{kgm}$. & $5.3 \mathrm{gm}$. & $104 \mathrm{gm}$. & $38 \mathrm{gm}$. & $289 \mathrm{gm}$. & $269 \mathrm{gm}$. \\
\hline
\end{tabular}

It is obvious that the 5.3 grams of fat and the 66 grams of protein cannot account for the carbon retained, and one must look to the carbohydrate as the source of the fat.

Jordan placed a Jersey cow on a feed of hay and grain from which the fat was extracted. The cow in 95 days assimilated 5.7 pounds of fat, increased 47 pounds in weight, and produced 62.9 pounds of fat in the milk. The nitrogen excreted was the equivalent of 33.3 pounds of protein. The non-nitrogenous moiety of the protein, if its carbon had all gone into fat, 
could not have produced over $\mathrm{I} 7$ pounds. Summarized, this experiment shows conclusively that fat is synthesized from carbohydrate. It requires about 2.7 grams of dextrose to form I gram of fat, and this condensation takes place with the formation of carbon dioxide and water and the liberation of about 15 per cent. of the available heat of oxidation.

Persistent excess of carbohydrate food produces an accumulation of fat, which may not only be an inconvenience causing obesity, but may interfere with the proper nutrition of muscles, produce a feebleness of the action of the heart, and other troubles.

The formation of fat from protein is discussed on page 459 .

Fat Mobilization in the Body.-Increasing numbers of recent studies are throwing new light on the phenomenon of mobilization of fat in the body. In I900 Kastle and Loevenhart discovered the reversible action of lipase which opened the door to a more fitting conception of how the body is enabled to handle this ordinarily insoluble constituent. Just as fats must be dissociated in order to be absorbed, a step that converts the insoluble fat to a soluble form, so the body transference from one tissue to another, from the storehouse of fats to the lymph and blood, or vice versa, requires that the fats must be in a soluble form, i.e., dissociated. The facility with which fats can be mobilized depends upon the presence and activity of fat lipases. These have been shown to be present in practically all tissues of the body, greater in some, as in the liver, certain glands, etc. Under the influence of the enzyme a fairly constant concentration of fats is normally present in the blood and in the body fluids, and is always available as a source of energy to the oxidizing tissues. The above is the explanation of the increase of fat in the milk previously referred to. When no food is coming, the animal draws immediately on the carbohydrates which are always present in the blood. This store is rapidly exhausted. As tissue hunger approaches, an increase in lipase production undoubtedly takes place, and this dissolves and sets in motion storage fat. Under these conditions the amount of fat in circulation rapidly increases and becomes available to the muscles, glands, etc., as illustrated by the secretion of the mammary gland where the output can be quantitatively tested. In starvation also the general distribution of fat in the form of liposomes increases in many tissues, particularly the glands - a fact readily determined by studies of the microscopic fat in fresh tissues. This phenomenon is not a fatty degeneration as is sometimes held, it is only the redistribution of fat under the law of reversible action of lipase to meet the emergency of tissue starvation.

Lipogenesis.-Noël Patton has shown a distinct accumulation of fat in the liver of the frog in relation to fat feeding. For this phenomenon Loevenhart has suggested the name lipogenesis. The idea is that the liver in particular serves to a degree as a storehouse of fats, comparable to the phenomenon of glycogenesis which characterizes the organ. The liver is 
relatively rich in lipase, hence is to that extent peculiarly fitted for storing and distributing fats.

Whether or not there are other special tissues involved in this immediate problem is not adequately studied. It is quite possible that many of the adipose tissues are involved. Certain tissues of the body are great fat storage tissues - subcutaneous, intermuscular, peritoneal, etc. These tissues readily yield their fat in time of starvation but it is not known that they are concerned in the production of lipase at this time.

Destination of Fats, Obesity, Diet, etc.-It has already been emphasized that fats are a direct source of energy of oxidation. This is their primary function in the body. If fats are taken in excess in the food, or are produced in excess from carbohydrates of the food, then they accumulate in great quantity, especially in middle and old age. This accumulation gives rise to the condition of obesity which is a condition of excessive fat storage. In many of these cases there is persistent storing of fat in the presence of a diet of low energy value and when considerable physical labor is expended. It seems that such persons have a perverted or at least disturbed fat metabolism. The explanation is very often found in deviation of function of some endocrine gland. For example, in decreased secretion of the posterior lobe of the pituitary there is an increase in sugar tolerance associated with a tendency to accumulate large quantities of fat. Defects of this character are met by attempts to supply the deficient internal secretion.

Obesity may result purely from over eating, in which case the person must use self restraint to restrict the quantity of food and the amount of fat and of carbohydrates from which fats are easily produced.

In many persons and under certain conditions, the oxidation of the fats is incomplete. This is indicated by an increase in the intermediary products, acetone, etc. This phenomenon gives rise to the condition of malnutrition expressed by the term acidosis which belongs to the field of pathology to which the reader is referred.

\section{THE METABOLISM OF CARBOHYDRATES.}

Energy Value.-The nutritive function of carbohydrates in the body is to serve as a source of energy. They are oxidized, with the ultimate production of carbon dioxide and water, and must liberate the same amount of energy as when burned outside the body, i.e., 4.I Calories per gram. A given weight of dextrose, therefore, furnishes a little less than half the energy of a corresponding weight of fat.

Carbohydrates are strictly energy-formers and may be regarded as the chief immediate source of the energy of oxidations, although they may be synthesized into fats and possibly even contribute to protein formation. 
Dextrose is a constant constituent of the blood to the extent of about o.I to 0.15 per cent. When this percentage is increased above 0.25 , the dextrose is either stored as glycogen, i.e., in the case of the portal blood during the absorption of a carbohydrate meal, or eliminated by the kidney, i.e., as in pancreatic diabetes.

The Formation of Glycogen-Glycogenesis:-The important fact that the liver normally forms sugar, or a substance readily convertible into it, was discovered by Claude Bernard in the following way: He fed a dog for seven days with food containing a large quantity of sugar and starch; and, as might be expected, found sugar in both the portal and hepatic blood. But when the dog was fed with meat only, to his surprise, sugar was still found in the blood of the hepatic veins. Repeated experiments gave invariably the same result. No excess of sugar was found in the portal vein under a meat diet, if care was taken to prevent reflux of blood from the hepatic venous system. Bernard found sugar also in the substance of the liver. It thus seemed to him certain that the liver formed sugar even when, from the absence of saccharine and amyloid matters in the food, none could be brought directly to it from the stomach or intestines.

Bernard subsequently found that a liver removed from the body, and from which all sugar had been completely washed away by injecting a stream of water through its blood vessels, contained sugar in abundance after the lapse of a few hours. This post-mortem production of sugar was a fact which could be explained only on the supposition that the liver contained a substance readily convertible into sugar. This theory was proved correct by the discovery of a substance in the liver allied to starch, termed glycogen.

Bernard's brilliant researches led him to announce the thecry that the carbohydrate which is periodically absorbed in large amount is stored in the liver only to be reconverted to dextrose and discharged back into the blood stream whenever the percentage in the blood falls below a certain level. He regarded the liver as a storehouse which regulated the blood dextrose to a constant level. This is the glycogenic function of the liver.

Source of Glycogen.-The greatest amount of glycogen is produced by the liver upon a diet of starch or sugar, but a certain quantity is, or at least may be, produced upon a protein diet. The glycogen, when stored in the liver cells, may readily be demonstrated in sections of liver containing it by its reaction (red or port-wine color) with iodine, and, moreover, when the hardened sections are so treated that the glycogen is dissolved out, the protoplasm of the cell is so vacuolated as to appear little more than a framework. There is no doubt that in the liver of a hibernating frog the amount of glycogen stored up in the liver cells is very considerable. 
Averagb amount of Glycogen in the Liver of Dogs under Various Diets. (PAvy.)

Diet. Amount of glycogen in the liver.

Flesh food..................... 7.19 per cent.

Flesh food with sugar................... 4.5 per cent.

Vegetable diet, i.e., potatoes with bread or barley meal. I7.23 per cent.

The dependence of the formation of glycogen on the kind of food taken is also shown by the following results, obtained by the same experimenter:

Average Quantity of Glycogen Found in the Liver of Rabbits after Fasting, and After a Diet of Starch and Sugar Respectively.

After three days' fasting............... Practically absent.

After diet of starch and grape sugar......... r 5.4 per cent.

After diet of cane sugar............ r6.9 per cent.

Glycerol injected into the alimentary canal may increase the glycogen of the liver. Observations indicate that glycogen may be formed in the turtle liver when perfused with very dilute formaldehyde solutions. The diet most favorable to the production of a large amount of glycogen is a mixed diet containing a large amount of carbohydrate, but with some protein. Glycogen is stored in other organs of the body. Of these the muscles are deserving of special mention. The amount of glycogen in the muscles of young animals is often considerable. The placenta is also a storehouse of glycogen.

Glycogenesis Controlled by Hormone.-Glycogenesis and its storage is strictly dependent on chemical control. In disease of the pancreas leading to its degeneration, or in surgical removal of the pancreas the liver is unable to store glycogen. Even when the blood is rich in sugar to the level at which its excretion into the urine occurs, no storage of glycogen occurs. The body seems quite unable to metabolize sugar or to convert it into glycogen as in the normal. From such observations physiologists have long coupled glycogenesis with the function of the pancreas. This association depends upon an internal secretion of the islands of Langerhans. Extracts of pancreas have from time to time been experimentally prepared that seemed to lower the amount of sugar lost during glycosuria. Such preparations have peculiar general toxicity and little progress has resulted in explanation of the mechanism of sugar control.

However, Banting and Best have very recently, 1922, announced brilliant results in the solution of this problem. They demonstrated that intravenous injections of a watery extract of pancreas in which the parenchyma had been degenerated by previous operative procedure, see discussion of internal secreting glands, when injected into the circulation of a depancreatized dog quickly lead to the disappearance of the excess 
of sugar from the blood and to a decrease in its loss in the urine. In later epoch making announcements from the University of Toronto laboratories it was shown that glycogen was actually stored in the diabetic liver under the influence of this pancreatic extract. These observations confirm the hypothesis that glycogenesis in the liver and body tissues is dependent on the presence of a hormone from the pancreatic gland.

The Destination of Glycogen.-The chief theories concerning the use of glycogen in the organism are advanced by Bernard and by Pavy. The former considers glycogen as a reserve supply of carbohydrate. Whenever the glycogen of the blood is reduced below the normal level, i.e., about O. I to o. I 5 per cent., there is a conversion of glycogen into sugar. The sugar enters the blood and passes to the tissues where its oxidation is a source of energy. Pavy considers glycogen to be a stage in the synthesis of carbohydrate into fat and protein. Bernard's theory is more generally accepted. It explains more satisfactorily why the sugar content of the blood is so constant. The conversion of glycogen to sugar takes place by the action of an intracellular ferment in the glycogenic cells. Such an enzyme has been isolated for the liver. It is this enzyme that converts the liver glycogen to dextrose after death, and which is destroyed by boiling in the usual process of isolating glycogen from the liver or other tissues.

Glycemia and Glycosuria.-Sugar may be present to excess not only in the hepatic veins, but in the systemic blood. When such is the case, the sugar is excreted by the kidneys, and appears in variable quantities in the urine. This condition is known as glycosuria.

Glycemia and glycosuria may occur in the normal animal or man during the absorption of the products of digestion following a meal rich in carbohydrates. The concentration of sugars in the blood occurs because of the greater rate of intake than rate of glycogen storage. This leads to a digestion glycemia and glycosuria. However, a more permanent glycosuria may be produced experimentally. The operative removal of the pancreas as related above, leads to an immediate glycemia and glycosuria. This condition is known as pancreatic diabetes. In medicine it forms one of the most difficult and at the same time interesting classes of clinical patients. Puncture of the medulla in the region of the vasomotor center will also produce diabetes. In fact, any sharp disturbance of the central nervous system may be followed by a corresponding disturbance in sugar metabolism. The administration of drugs, such as phloridzin, strychnine, glycosides, morphine, adrenalin, amyl nitrite, or reduction of oxygen or excess of carbon dioxide may be followed by discharge of glycogen from the liver store houses and by glycosuria. Some of these agencies react on the central nervous system influencing either hormone production or liver activity itself. Other agencies like adrenalin stimulate the peripheral hepatic nervous mechanism. In any event, the normal cycle of carbohydrate metabolism is upset. 


\section{THE INFLUENCE OF MINERALS, OF FASTING, ETC.}

Mineral Matters, Water, Etc.-The chief mineral constituents of the foods are sodium, potassium, calcium, magnesium, and iron, together with chlorine, sulphur, and phosphorus. The inorganic substances are not a source of heat. They may supply a certain amount of energy, as osmotic energy, but this is of no significance as compared with their influence on the metabolism of organic substances. An animal fed on a normal food deprived of the mineral constituents survives only a few weeks at most.

The amount of mineral matter in the tissues of the human body, exclusive of the skeletal parts, is about one per cent. It is safe to say that this is chiefly in complex organic combination in the body. The daily quantity excreted is about twenty to thirty grams. This quantity enters the body in the food, chiefly in combination with complex compounds. It is a question as to what per cent. of organic salts, like the calcium, the phosphates, and the iron, is available when taken into the body in inorganic form.

We have discussed in previous chapters the rôle of certain salts in their influence on metabolism; for example, of sodium, potassium, calcium, iron, etc. Foods like milk and eggs are especially rich in calcium and phosphorus and are particularly desirable for young children, the former for its influence on the growth of the skeleton, the latter for the same reason and as a stimulator of growth of protoplasm in general. Lack of mineral constituents, especially calcium compounds, in food shows its influence on metabolism in the disease known as rickets.

Nutrition Experiment in Five-months-old Pigs. (E. B. Forbes.)

\begin{tabular}{|c|c|c|c|c|}
\hline \multirow{2}{*}{ Rations. } & \multirow{2}{*}{$\begin{array}{c}\text { Per cent. gain } \\
\text { in live } \\
\text { weight in } \\
60 \text { days' } \\
\text { feeding. }\end{array}$} & \multicolumn{3}{|c|}{$\begin{array}{l}\text { Per cent. gain in certain } \\
\text { tissues corresponding to } \\
\text { I per cent. gain in live } \\
\text { weight. }\end{array}$} \\
\hline & & $\begin{array}{l}\text { Psoas } \\
\text { muscle. }\end{array}$ & $\begin{array}{l}\text { Ash of } \\
\text { humer- } \\
\text { us. }\end{array}$ & $\begin{array}{l}\text { Thick- } \\
\text { ness of } \\
\text { back fat. }\end{array}$ \\
\hline $\begin{array}{l}\text { Hominy; blood flour; bran extract. } \\
\text { (Phosphorus mostly as phytin.) }\end{array}$ & $69 . I$ & $.8 \mathrm{I}$ & $\cdot 59$ & .64 \\
\hline $\begin{array}{l}\text { Hominy; blood flour; bone flour. } \\
\text { (Phosphorus mostly as tricalcic } \\
\text { phosphate). }\end{array}$ & 61.0 & $.6 \mathrm{I}$ & $\cdot 72$ & .82 \\
\hline $\begin{array}{l}\text { Hominy; blood flour. (Low phos- } \\
\text { phorus ration). }\end{array}$ & 41.6 & $\cdot 72$ & .08 & 1.04 \\
\hline
\end{tabular}


Numerous imvestigations are in progress which may demonstrate more fully the specific influence of phosphorus on animal nutrition and on growth. Tunnicliff has demonstrated that an increase of the phosphorus content of the food of children, if given in complex organic form, increases the efficiency of the metabolism of nitrogen by as much as ro per cent. If given to children as calcium phosphate it has no beneficial influence in this regard. Forbes, in his experiments on the nutrition of pigs, shows that the individuals fed with food to which phosphorus was added, as ground fresh bone, grew larger and stronger skeletons, but that the presence of organic phosphorous (phytin) led to the greatest general growth.

Percentage of Phosphoric Acid $\left(\mathrm{P}_{2} \mathrm{O}_{3}\right)$ in Some Fresh Foods. (Quoted fROM Girard, by Hutchinson, in "FoOd and Dietetics.")

\begin{tabular}{|c|c|c|c|}
\hline Vegetable. & Per cent. & Animal. & Per cent. \\
\hline Carrot........ & $\ldots 0.036$ & Pork. & 0. \\
\hline Turnip...... & .. $0.05^{8}$ & Milk. & \\
\hline Cabbage.... & .0 .089 & Beef. & 0.2 \\
\hline Potato...... & .. 0.140 & Eggs..... & 0.3 \\
\hline Chestnuts.... & .0 .200 & White chees & 0.3 \\
\hline Barley meal. & .0 .230 & Mutton. . & \\
\hline
\end{tabular}

Salts in the body not only take part in the reactions themselves, but they stimulate in other substances reactions that are of incalculable benefit to the body.

The necessity for the taking of water in order to balance the daily excretion, is sufficiently obvious. Man will live only a few days if deprived of water.

Effects of Deprivation of Food.-The animal body deprived of all food dies from starvation in the course of a variable time. The length of time that any given animal will live in such a condition depends upon many circumstances, the chief of which are the nature and activity of the metabolism of its tissues.

The effect of starvation on the lower animals is, first of all, as might be expected, a loss of weight. The loss is greatest at the beginning of the deprivation period, but afterward decreases to a level from which it does not vary much day by day until death ensues. Chossat found that the ultimate proportional loss in different animals experimented on was almost exactly the same, death occurring when the body had lost 40 per cent. of its original weight. Different parts of the body lose weight in very different proportions. The following most noteworthy losses are taken, in round numbers, from the table given by Chossat:

\begin{tabular}{|c|c|c|c|}
\hline \multicolumn{2}{|c|}{ Per cent. } & \multicolumn{2}{|c|}{ Per cent. } \\
\hline 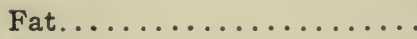 & 93 & Liver.. & $5^{2}$ \\
\hline 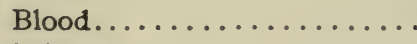 & 75 & 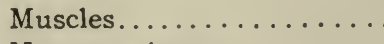 & 43 \\
\hline Spleen................. & 71 & Nervous tissues......... & 2 \\
\hline 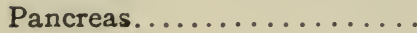 & 64 & & \\
\hline
\end{tabular}


These figures are in practical agreement with those of later experimenters. They show that the chief losses are sustained by the adipose tissue, the muscles and glands. The nervous structures and the heart are maintained at the expense of the other tissues and show but little change.

The effect of starvation on the temperature of the various animals experimented on by Chossat was very distinct. For some time the variation in the daily temperature was more marked than its absolute and continuous diminution, the daily fluctuation amounting to $3^{\circ} \mathrm{C}$. instead of $0.5^{\circ}$ to $\mathrm{I}^{\circ} \mathrm{C}$.,

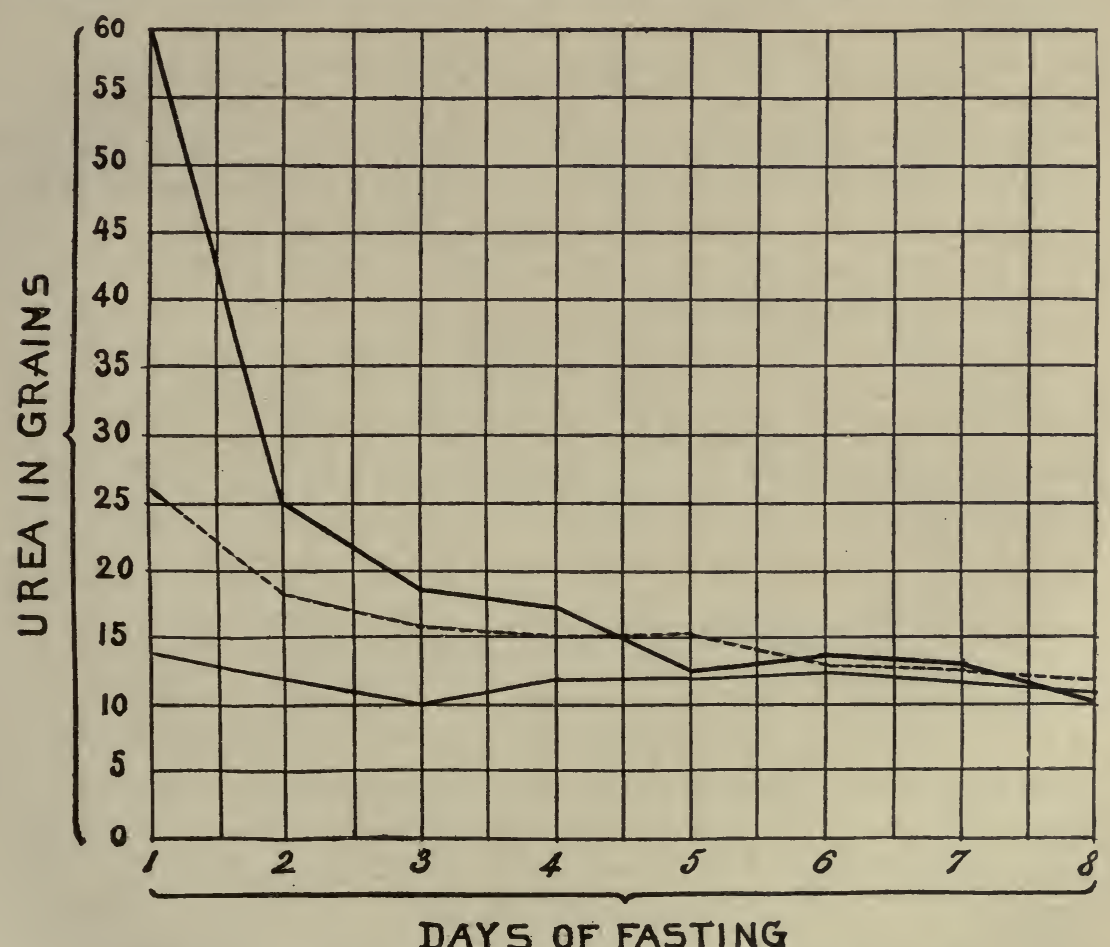

FIG. 3ro.-The Elimination of Urea by Dogs during Fasting. (Voit.)

Following 2,500 grams of meat in the food.

....-........ Following $\mathrm{I}, 500$ grams of meat in the food.

- Following minimal amount of protein in the food.

as in health. The temperature fell very rapidly a short time before death, and death ensued when the loss had amounted to about $16.2^{\circ} \mathrm{C}$. It has been often said, and with truth, that death by starvation is really death from want of heat. The effect of the application of external warmth to animals cold and dying from starvation is more effectual in reviving them than the administration of food.

The symptoms produced by starvation in the human subject are hunger, accompanied, or it may be replaced, by pain, referred to the region of the stomach; insatiable thirst; sleeplessness; general weakness, and emaciation. The exhalations both from the lungs and from the skin are fetid, indicating 
the tendency to decomposition which belongs to badly nourished tissues; and death occurs often with symptoms of nervous disorder, delirium, or convulsions. Death commonly occurs within from six to ten days after total deprivation of food. This period may be considerably prolonged by taking a very small quantity of food, or even by water alone. The cases so frequently related of survival after many days or even some weeks of abstinence have been due either to the last-mentioned circumstances, or to other no less effectual conditions which prevented the loss of heat and moisture.

During the starvation period the excretions diminish. The urea, as representing the nitrogen, falls quickly in amount, reaches a minimum where it remains fairly constant for several days before death. The sulphates and phosphates undergo much the same type of reduction. The carbon dioxide given out and the oxygen taken in diminish. The feces diminish as well as the bile. It is highly probable that the greater part of the nitrogen represents the loss of weight of the muscles.

In starvation, then, we see that the only income consists of water and the inspired oxygen. The whole of the energy of the body given out in the form of heat and mechanical labor is obtained at the expense of its own tissues, there being as a result a constant drain of the nitrogen and carbon, not to mention the other elements of which the tissues are composed. It is obvious that such a condition cannot be endured for any length of time.

\section{REQUISITES OF A NORMAL DIET.}

For many years the dictum has been accepted that it is only necessary that a normal diet should be made of the various classes of food in sufficient quantity to supply to the normal adult animal body the amount of energy that is lost, due to the daily round of activity. In addition, in young and growing animals an excess of the classes of foods used in the construction of new tissue must also be given. No doubt these desiderata may be satisfied by many combinations of foods, and it would be unreasonable to expect the diet of every adult to be the same. The age, sex, strength, and circumstances surrounding each individual must ultimately determine what he takes as food. A dinner of bread and cheese with an onion contains all the requisites for a meal, but such diet would be suitable only for those possessing strong digestive powers. It is a well-known fact that the diet of the continental nations differs from that of our own country, and the diet of dwellers in cold from that of those who live in hot climates, but the same principle underlies all, viz., the replacement of the energy losses of the body in the most convenient and economical way possible. Any one in active work requires more food than one at rest, and growing children require more food in proportion to body weight than adult men and women, but of a different variety. 
The chief diet-scales which have in the past been drawn up with the object of supplying the proximate principles in the required proportions are given in the table below:

Standard Dietaries.

\begin{tabular}{|c|c|c|c|c|}
\hline Author. & Protein. & Fat. & $\begin{array}{c}\text { Carbohy- } \\
\text { drate. }\end{array}$ & Calories \\
\hline Voit............. & I 8 grams & 56 grams & 500 grams & 3,055 \\
\hline Rubner............ & I27 grams & $5^{2}$ grams & $5 \circ 9$ grams & 3,092 \\
\hline Moleschott.......... & I30 grams & 40 grams & $55^{\circ}$ grams & 3,160 \\
\hline Munk........ & ro5 grams & 56 grams & 500 grams & 3,022 \\
\hline Wolff... & I25 grams & 35 grams & 540 grams & 3,030 \\
\hline Playfair.... & II9 grams & $5 \mathrm{r}$ grams & 53 I grams & 3,140 \\
\hline Atwater.... & 125 grams & I 25 grams & $45^{\circ}$ grams & 3,520 \\
\hline Aver & I2I grams & 59 grams & 5ro grams & 3,135 \\
\hline
\end{tabular}

The basis of computation for these diets is to supply the necessary protein nitrogen consumed by the tissues of the body first of all, and then to supply enough potential energy to balance the energy expended per day.

The amount of the excreted carbon and nitrogen is not always the same. It has been proven possible, for example, to subsist on a diet containing 9 or Io grams of protein nitrogen and 200 grams of carbon in the form of carbohydrates and fats per diem, the ordinary diet for needle-women in London, and the average of the cotton operatives in Lancashire during the famine of 1862 . The amount of these elements excreted falls to figures corresponding to such an income. Of course, upon such a diet the metabolism is low, and persistent physical weakness must be the result, probably from insufficient carbon. The 9 or to grams of nitrogen in such a semi-starvation diet would be equivalent to 58.5 to 65 grams of protein, whereas the amount of protein in some diets may be as high as 150 and more grams per day. Chittenden's nutritional experiments, so often referred to in these pages, have proven that adult men can subsist in nitrogenous equilibrium and do vigorous work and maintain good health, on a protein diet below that given in the above example, i.e., on 6 to ro grams of nitrogen. In such diets a plentiful supply of carbohydrates is permitted, but the caloric value of the diet is less than those in the table above.

Not only the proteins but also the fats may vary. The amount may be as low as 35 grams and as high as 125 grams The carbohydrates may vary from 200 grams to 500 grams and upward. Sometimes, with a small proportion of fats, the carbohydrates may be correspondingly increased to make up the necessary quantity of available potential energy. 
From the data on page $34^{2}$, it is possible to form various diet-scales which shall supply the needs of different conditions of growth and decay of the body. In an economical daily ration consisting of meat, bread, butter, cheese, potatoes and vegetables one might supply the requisite amount of protein nitrogen as follows:

\begin{tabular}{|c|c|c|c|c|}
\hline & \multicolumn{2}{|c|}{ N. } & \multicolumn{2}{|c|}{ Proteins. } \\
\hline$s$ lean $u$ & 10.0 & grams & 62.5 & grams \\
\hline is bread........ & 6.0 & grams & $37 \cdot 5$ & grams \\
\hline o grams butter... & 0.5 & grams & 3.1 & grams \\
\hline 28 grams cheese $\ldots \ldots \ldots \ldots \ldots \ldots \ldots \ldots \ldots$ & I. 5 & grams & $9 \cdot 4$ & grams \\
\hline 5 grams potatoes $\} \ldots \ldots \ldots \ldots \ldots \ldots$ & I. 0 & grams & 6.2 & grams \\
\hline & 19.0 & ns & I 8.7 & \\
\hline
\end{tabular}

The 30 grams of salts necessary to replenish the daily loss by excretion in the urine are contained in the meat 16 grams, the bread 12 grams, and vegetables about 4 grams.

The fluid should consist of about 2,500 to 2,800 grams, and might be given as water, with or without tea, coffee, or cacao, which are chiefly stimulants.

The Energy Requirements of the Body.-The food must not only make up for the substances eliminated from the body but must also supply the potential energy of heat and motion set free in the living body. The amount of heat is measured in terms of calories, or more often in large Calories. The work energy may be expressed in gram-centimeters, or in kilogrammeters. Since one calorie of heat is the equivalent of 42,670 gramcentimeters of work, the two units may be computed interchangeably.

The source of the heat and work energy which is produced in the body is from the metabolic changes of the tissues, the chief part of which is in the nature of oxidation, since it may be supposed that the oxygen of the atmosphere taken into the system is ultimately combined with carbon and hydrogen. Any change, indeed, which occurs in the protoplasm of the tissues, resulting in an exhibition of protoplasmic function, is attended by the evolution of heat and the formation of carbon dioxide and water. The more active the changes the greater is the amount of heat produced. In order that the protoplasm may perform its functions, the waste of its own destructive metabolism must be repaired by the due supply of food material to be built up in some way into the protoplasmic molecule. In the tissues, as we have several times remarked, two processes are continually going on: the building up of the protoplasm from the food, anabolism, which is not accompanied by the evolution of heat; and the oxidation of the protoplasmic materials, katabolism, resulting in the production of energy, by which heat is set free. Food is therefore necessary for the production of heat. It is not neccessary to assume that the combustion processes, indeed, are as simple as the bare statement of the fact might seem to indicate. But complicated as the vari- 
ous stages may be, the ultimate result is as simple as in ordinary combustion outside the body, and the products are the same.

This view, that the maintenance of the temperature of the living body depends on continual chemical change, chiefly by oxidation of combustible materials in the tissues or by the tissues, has long been established. The quantity of carbon and hydrogen supplied as food, chiefly in the form of carbohydrates and fats which, in a given time, unites in the body with oxygen, is sufficient to account for the amount of heat generated in the animal within the same period, page 454 . This amount is capable of maintaining the temperature of the body at from $36.8^{\circ}$ to $38.7^{\circ} \mathrm{C}$., notwithstanding a large loss by radiation and evaporation. This estimation depends upon the chemical axiom that when a body undergoes a chemical change the amount of energy set free is the same, supposing the resulting products are the same, whether the change takes place suddenly or gradually. If a certain number of grams of different substances are introduced as food, and if they undergo complete oxidation, the amount of kinetic energy, as shown in the amount of heat and mechanical work, is the same as would be developed if the same bodies were completely oxidized outside the body. If one gram of fat be taken into the body and is completely oxidized, resulting in the production of a definite amount of carbon dioxide and water, it may be supposed to have produced the same amount of heat as it would have produced outside the body. In the case of protein food it is a little different, since it is never completely oxidized within the body, but may be supposed to give rise to a definite amount of urea and other lower nitrogenous compounds not completely oxidized in the body. In this case the gram of protein may be considered to liberate the same amount of heat as the protein would outside the body minus the amount which would be obtained from the complete oxidation of the resulting urea, etc.

The actual amount of heat produced per diem has been experimentally ascertained in the case of man and animals by the aid of an apparatus, the calorimeter. An animal is enclosed in a metal cage completely contained in a second cage containing water. Air is let into and out of the inner box by means of metal tubes so arranged that the inlet tubes maintain a constant temperature and the outlet tubes pass through water between the two chambers. The heat given out by the animal warms the water in the outside box, and may be estimated by the rise of its temperature, the amount of which is known. At the same time the carbon dioxide output is measured.

The rate of human metabolism has come to be of great importance in clinical diagnosis of nutritional states. The direct methods are too involved for rapid testing. Indirect determinations are quite accurate enough for such purposes. The indirect method rests on measurement 
of the rate of oxygen consumption, or carbon dioxide output, or both, as an index of heat production. The rate of heat production and of heat loss must, of course, be in balance in an animal of constant temperature, as in man. For this reason the rate of metabolism varies not with mass, as we would expect, but with the surface area. In man the unit used is expressed as calories per square meter of surface per hour. Measured at the minimal level this varies according to the following averages.

\section{Table of Basal Metabolic Rates.}

Rate of Minimal Metabolism in Calories per Square Meter per Hour, Aub and DuBois, Ages $\mathrm{I}_{2}-\mathrm{I} 3$ from DuBois, Logs. from Boothby and Sandiford.

\begin{tabular}{|c|c|c|c|c|}
\hline Age in years. & Males. & Log. cals. & Females & Log. cals. \\
\hline $14-16$ & 46.0 & 6,628 & 43.0 & 6,335 \\
\hline I6-I8 & 43.0 & 6,335 & 40.0 & $6,02 \mathrm{I}$ \\
\hline $18-20$ & $4 \mathrm{I} .0$ & 6,128 & 38.0 & 5,798 \\
\hline $20-30$ & $39 \cdot 5$ & 5,966 & 37.0 & 5,682 \\
\hline $30-40$ & $39 \cdot 5$ & 5,966 & 36.5 & 5,623 \\
\hline $40-50$ & 38.5 & 5,855 & 36.0 & 5,563 \\
\hline $50-60$ & $37 \cdot 5$ & 5,740 & 35.0 & $5,44 \mathrm{I}$ \\
\hline $60-70$ & 36.5 & 5,623 & 34.0 & 5,315 \\
\hline $70-80$ & $35 \cdot 5$ & $5,5 \circ 2$ & 33.0 & 5,185 \\
\hline
\end{tabular}

The surface area for man is computed from the formula of DuBois in

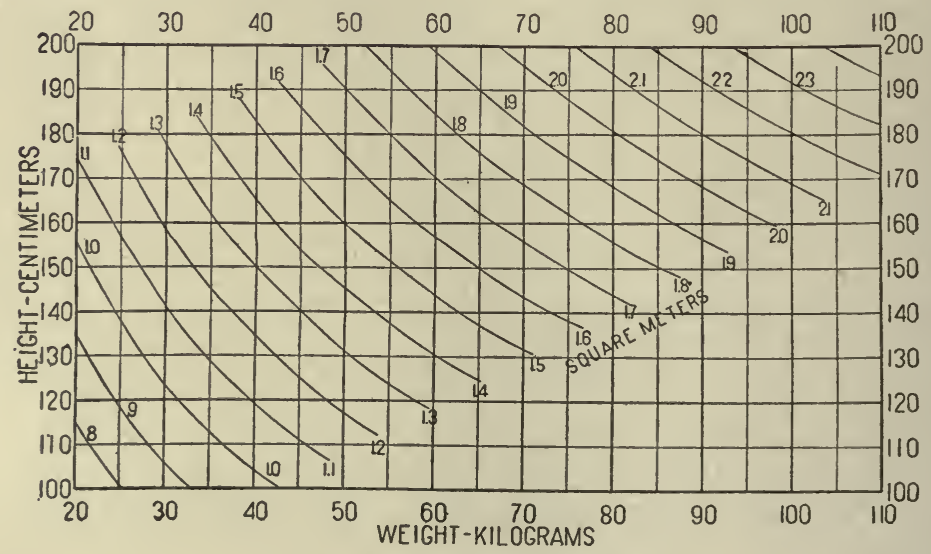

FIG. 3roa.-Height-weight Chart.

which the area is in square meters, the weight in kilograms, and the height in centimeters. 


$$
\text { Area }=\text { Weight }^{0.425} \times \text { Height }^{0.725} \times \text { Factor } 7 \mathrm{I} .84 .
$$

The area is more quickly determined from the height-weight-graph computed also by DuBois and DuBois.

The amount of heat evolved by the oxidation of various food stuffs has been carefully measured by numerous observers; the figures calculated by Rubner are perhaps most satisfactory.

Heat Value to the Body.

I gram carbohydrate................ Calories.

I gram fat.................. 9.3 Calories.

I gram protein............... 4. Calories.

One gram of dry protein has a total heat value of 5.754 Calories (Rubner), hence it is obvious that protein is not completely oxidized by the body. Each gram of protein yields at least one-third of a gram of urea, which has a heat value of 2.5 Calories per gram.

Atwater and Benedict have checked the energy value of the foods actually consumed against the actual liberation of heat and work energy of the human body. They find a wonderfully close agreement both for periods of rest and for periods of work. Atwater's estimate for the energy needs of man are as follows:

Man without muscular work........ 2,700 Calories.

Man with light muscular work........ 3,000 Calories.

Man with moderate muscular work..... 3,500 Calories.

Man with severe muscular work...... 4,500 Calories.

The daily output of energy for the adult man is as follows:

\begin{tabular}{|c|c|c|}
\hline & Kilogrammeters. & Calories \\
\hline Work of heart per day........ & 88,000 & \\
\hline Work of respiratory muscle...... & I 4,000 & \\
\hline 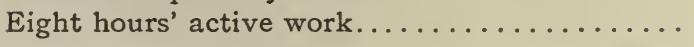 & $2 \times 3,344$ & \\
\hline lount of heat produced in 24 hours. & $\begin{array}{l}315,334 \text { or } \\
I, 582,700 \text { or }\end{array}$ & $\begin{array}{r}743 \\
3,724\end{array}$ \\
\hline & - & $\underline{30-1}$ \\
\hline & $\mathrm{r}, 898,034$ or & 4,467 \\
\hline
\end{tabular}

This estimate is relatively high for ordinary activity as determined by Atwater and others. It is indeed more energy than the standard diets in the table given on page 450 will yield to the body. For example, Voit's diet yields 3,055 Calories, and the average of the table is only 3,1 25 Calories. 


\section{FOOD SUPPLEMENTS, THE VITAMINES.}

Numerous workers have shown that it is not sufficient to supply the body with adequate proteins, fats, carbohydrates, inorganic salts and water meeting the standard requirements as regards the caloric value of foods. Formerly these facts were considered as physiologically settled, both by the medical profession and by those interested in animal nutrition. In the next chapter we will find that minute quantities of substances produced by the various ductless glands of the body may profoundly influence the process of metabolism. In like manner, observation indicates that the foods contain similar traces of material supposedly of chemical nature which have an influence on metabolism out of all proportion to their quantity or mass. Such substances have been given the name of vitamines by Funk. Most food sources, such as meat, milk, fish, vegetables and cereals contain these so-called vitamines. However, the amount of vitamines furnished by each varies widely.

The Nutritional Diseases.-A group of well known diseases of obscure etiology have been investigated on a large scale since the discoveries of Funk. At the present time the diseases of xerophthalmia, of the beriberi type of neuritis, and the scorbutic diseases are definitely attributed to lack of corresponding specific vitamines in the diet, namely, fat soluble $A$, water soluble $B$, and water soluble $C$ vitamines. Other factors in the food are necessary to its complete adequacy. Of these it is evident that a proper chemical balance among the inorganic salts of the diet, as well as a certain bulk and quality to which the digestive apparatus of the particular species of animal may be adapted, plays a vital part in the food sufficiency. Many of these questions are not yet adequately determined.

Beri-beri and the Antineuritic Vitamine B.-In the far East there is prevalent a characteristic nervous disease known as beri-beri. It is characterized by loss of nervous function which comes on relatively suddenly and proceeds to a state of motor paralysis. Beri-beri has been found to be associated with the use of an excessive rice diet and is prevalent in rice eating countries. Chickens, pigeons, and pigs which are relatively susceptible to a neuritis very similar to, if not identical with, beri-beri quickly recover when fed on rice polishings at an early stage of the disease. Such experiments have led to the conclusion that in the polishing of rice a food constituent is removed that is absolutely necessary to the normal growth of the nervous system. This material has been extracted and chemically examined by Funk who describes it as an organic crystalline substance melting at $233^{\circ} \mathrm{C}$., chemically resembling pyrimidin. Substances like the rice extract which cause recovery from nutritional diseases of the neuritic type have been given the name, antineuritic substances. Williams prepared an oxypyridine that cured neuritic pigeons in doses of one milligram. 
Scurvy and the Antiscorbutic Vitamine C.-Scurvy has long been known to be due to the inadequacies of a restricted diet, for example, in the siege of Paris, in long ship voyages, etc. The disease so prevalent in the southern part of the United States, and especially in the Mississippi Valley, known as pellagra seems to be in the same category. Through the work of Voegtlin, Koch and Sullivan it is found that solutions of flour, corn meal and vegetables contain a substance influencing metabolism. This has been tested on mice, and on man in the pellagra hospitals. The active principle is permanent in acid but destroyed in the alkaline solution. For example, breads that have been leavened by soda have lost this principle. Soda breads are detrimental to health not because of the manner of their cooking with excess of oil, but rather because of the destruction of the vitamines by the alkalinity of the soda. This active type of vitamine is called an antiscorbutic substance.

Koch and Voegtlin have studied the central nervous system of man dying of pellagra, also the nervous system of monkeys fed on inadequate diets. The results show a loss of lipoids, a tendency to a decrease in the proportion of proteins and an increase in the water content. There is a decrease in the cerebrosides, phosphatides and sulphatides with a relative decrease of cholesterol in the cerebrum and an increase in the spinal cord. There is an increase in extractives, especially the nitrogenous extractives. In general, "the spinal cord exhibits most striking chemical changes, a fact which is in perfect agreement with histological observations." The antiscorbutic substances are present in cabbage, tomatoes and the citrous fruits.

The Growth Producing and Antirachitic Vitamine A.-Osborne and Mendel have performed numerous feeding experiments on rats which tend to show that special growth stimulating substances are present in foods. They find, for example, that milk when its natural proteins are substituted by excess of casein will not support growth for a long time. When natural milk or milk albumins are resupplied, growth proceeds. In the growth producing food mixtures, if the lard which they used for fat is replaced by butter fat, growth proceeds more rapidly. It would seem that a fat soluble growth stimulating substance is present in the foods

If the fat soluble $A$, so richly present in butter fats, liver fat, etc., is deficient in the diet a characteristic malnutritional disease of the eye lids, cornea, etc., occurs known as xerophthalmia. This disease clears up when fats containing the vitamine $A$ are resupplied.

Source of the Normal Vitamines.-Voegtlin has emphasized the fact that the animal body can not manufacture its own vitamines from vitamine free food. The vitamines in eggs and milk, perhaps accounting for the 
relatively low content of milk in vitamines, apparently come from plant food sources. The plant can build up the vitamines from simpler compound.

Table Showing the Relative Richness in Antineuritic and Antiscorbutic Vitamines from Common Food Sources. (From Voegtim.)

\begin{tabular}{|c|c|c|c|}
\hline \multicolumn{2}{|c|}{ Antineuritic Properties } & \multicolumn{2}{|c|}{ Antiscorbutic Properties } \\
\hline Relatively rich & Relatively poor & Relatively rich & Relatively poor \\
\hline $\begin{array}{l}\text { Brewers' yeast } \\
\text { Egg yolk } \\
\text { Ox heart } \\
\text { Milk (fresh) } \\
\text { Beef and other fresh } \\
\text { meats } \\
\text { Fish } \\
\text { Beans } \\
\text { Peas } \\
\text { Oats } \\
\text { Barley } \\
\text { Wheat } \\
\text { Corn } \\
\text { Other cereals }\end{array}$ & $\begin{array}{l}\text { Sterilized milk } \\
\text { Sterilized meat } \\
\text { Cabbage } \\
\text { Turnips } \\
\text { Carrots and other } \\
\text { vegetables of this } \\
\text { type } \\
\text { Highly milled cereals } \\
\text { Starch } \\
\text { Molasses } \\
\text { Corn syrup }\end{array}$ & $\begin{array}{l}\text { Fresh vegetables } \\
\text { Fresh fruit } \\
\text { Raw milk } \\
\text { Raw meat } \\
\text { Cereals, sprouting. }\end{array}$ & $\begin{array}{l}\text { Dried vegetables. } \\
\text { Dried fruit } \\
\text { Sterilized milk } \\
\text { Canned meat } \\
\text { Dried cereals } \\
\text { Pork fat } \\
\text { Starch } \\
\text { Molasses } \\
\text { Corn syrup }\end{array}$ \\
\hline
\end{tabular}

Methods of milling rice, wheat flour and corn products whereby the outer hull of the grain which contains the major portion of the vitamines is lost should undergo vital modification. Both the kernel of the grain and the bran which formerly have been largely discarded as human food are rich in vitamines. They should be retained in the cereal products. The public agitation for the use of whole wheat and whole grain products has its biological justification chiefly in the conservation of the natural vitamine supplies. It is also evident that in cooking and in the preparation of food care should be taken not to destroy the normal vitamines by an excessive use of alkali. Heat in moderate degree somewhat reduces the normal vitamines. When such a reduction is unavoidable then the cooked foods should be supplemented by vegetables and citrous fruits in which the vitamines are preserved.

\section{THE INFLUENCE OF THE DUCTLESS GLANDS ON METABOLISM.}

A further question to be considered is the relationship between the metabolism of the tissues and the products of the metabolism of other tissues.

It is well known that all tissues elaborate substances, either end products or pure waste products, which may more or less sharply influence the reactions in the other tissues of the body. Many of the waste products are far from 
inert in this sense, though little physiological consideration is given to this class of material in this regard. On the other hand, many end products of the metabolism of certain tissue, the ductless glands in particular, are thrown back into the blood stream to influence the reactions of other tissues, just as do blood constituents from outside sources. The tissues and organs have become adapted to reaction in the presence of chemicals of this type and in many instances the relation is so firmly established as to become an interdependent one. Adaptations have taken place as between the tissue and its special environment of material derived from its neigh-

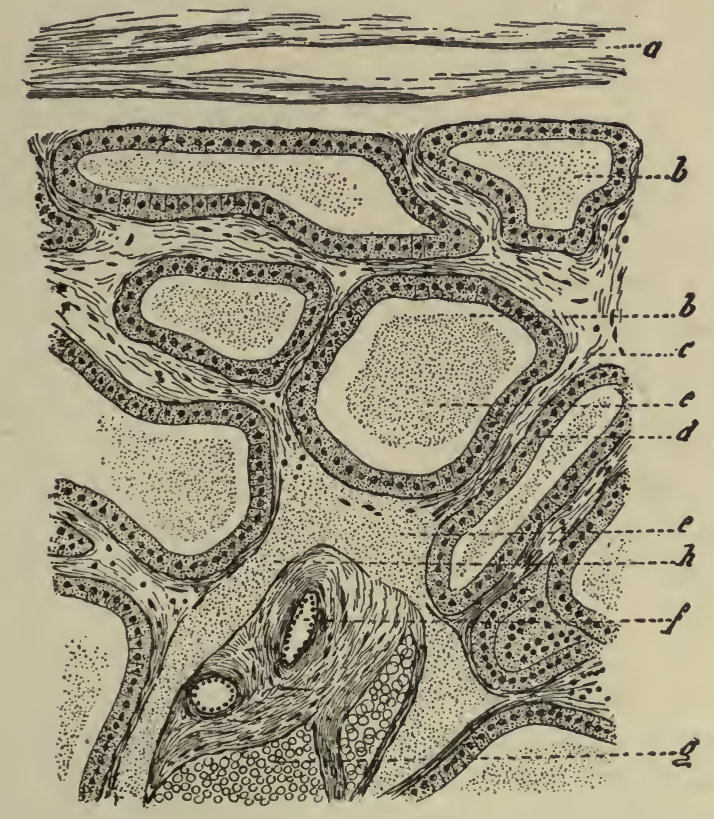

FIG. 3 II. - Part of a Section of the Human Thyroid. $a$, Fibrous capsule; $b$, thyroid vesicles filled with, $e$, colloid substances; $c$, supporting fibrous tissue; $d$, short columnar cells lining vesicles; $f$, arteries; $g$, veins filled with blood; $h$, lymphatic vessel filled with colloid substance. (S. K. Alcock.)

boring sources. Such substances are called internal secretions, and the active principle, either isolated and identified or assumed, goes by the name hormone (Starling). A hormone may be defined, therefore, as a chemical substance produced by one tissue or organ to which some other portion of the body has become biologically adapted to such an extent that its normal function can proceed only under the influence of the substance. Hormones are more or less specific in character. A hormone for one tissue acts particularly on that tissue to the practical exclusion of all others. 
The more important organs which, according to our present views, produce internal secretions are as follows: thyroid, parathyroid; pituitary posterior lobe, pituitary anterior lobe; thymus; suprarenal cortex, suprarenal medulla and chromaffine tissues; the islands of Langerhans of the pancreas; duodenal mucosa; liver; kidney; testicular interstitial tissue; ovary interstitial tissue and corpus luteum; placenta, and fetus.

\section{THE FUNCTION OF THE THYROIDS.}

\section{The Thyroid and Accessory Thyroids.}

The thyroid glands with certain structures called accessory thyroids are situated in the neck to either side of the trachea. The gland consists of two lobes, one on each side of the trachea extending from the thyroid cartilage to the level of the clavicle or lower. Often the lobes are connected across the mid line by a middle bar or isthmus. The thyroid is covered by the muscles of the neck It is highly vascular and varies greatly in size especially in different parts of the country. The gland vessels are each lines with a single layer of cubicle cells producing different sized follicles filled with a transparent colloid material containing nucleo albumen and the specific secretion of the gland.

The accessory thyroids are detached portions of thyroid tissue in the neighborhood of the lateral lobes. They are not different in function from the rest of the gland.

\section{The Function of the Thyroids.}

The thyroids now are known to secrete a specific chemical hormone which tremendously influences the rate of metabolism of the body as a whole. This secretion is produced by the cells of the follicle and diffuses out into the lymph and blood stream on the one hand or into the colloid material on the other. Ultimately, however, it enters the blood stream and is generally distributed throughout the body.

It has been a long and difficult task to determine the influence of the thyroid upon metabolic processes. This is due in part to the fact that the early work did not distinguish between the thyroids and parathyroids, and in the later work it has been found extremely difficult to eliminate the parathyroids without injury to the thyroids.

The present view is that the thyroid hormone increases the rate of metabolism throughout the body. This it does largely by virtue of the fact that the iodine containing compound acts as a deamidizer thus hastening nitrogen elimination and the process dependent upon nitrogenous metabolism. This last conception rests chiefly on the work of Kendall, 
who has further purified the hormone and determined its chemical structure. He applies the term thyroxin to the compound. Thyroxin is a compound containing 60 per cent. of iodine and its rate of production depends upon the presence of iodine in the intake of the body foods. The determination of the influence of the thyroid has been a long and interesting process. The steps have been progressive and cumulative. The methods used have been four. First, the study of metabolism in thyroidectomy. Second, the grafting of thyroid. Third, the feeding of thyroid tissue. Fourth, the isolation and physiological administration of the purified thyroid hormone. The specific dynamic action of the thyroid hormone is of such definite character that at the present time the chief dependence of medical men for the determination of variations in the gland function is on scientific measurement of the metabolic rate under the conditions known as basal metabolism.

a. Thyroidectomy.--It was noted in the middle of the last century that complete removal of the thyroid glands produced a marked derangement of the functions of the body and generally resulted in death. Later studies have shown that death was due to the fact that the parathyroids were also removed. The influence which is now attributed strictly to the removal of thyroids consists of a condition of malnutrition characterized by marked myxedema, a trouble that also appears in certain thyroid diseases. Failure of the hormone or hypothyroidism leads to a depression of the neuromuscular tone, and malnutrition of the vascular and lymphatic systems. In the early atrophy of this gland the body fails to develop and cretinism is the result.

b. Thyroid Grafting.-If a portion of the thyroid gland be engrafted in any other locality in an animal and the graft be allowed to grow before the thyroids are removed, then the characteristic symptoms no longer develop. The small amount of grafted tissue is able to produce enough of the thyroid hormone to supply the needs of the body. Successful and beneficial results have now been obtained by transplanting thyroid gland in man (McPherson). If in experimental cases, the thyroid graft be removed, then the usual symptoms immediately appear.

c. Thyroid Feeding. - If the thyroid is fed either as the purified hormone or merely as the gland itself, it seems to stimulate certain types of metabolism, as indicated by an increase in nitrogenous wastes, and by more healthful reaction of the nerve tissue. There is also an increased oxidation of fats, a property that has brought extracts of this gland into extensive but more or less dangerous use as an antifat remedy. Thyroid feeding has the same effect on the body as hvperthyroidism characteristic of certain types of enlarged or hypertrophied gland, exophthalmic goiter, or in hypothyroidism from any cause, extraneous use of the thyroid hormone in any form antagonizes the symptoms of malnutrition. Even following thyroidectomy if the gland or its extract be fed by way of the mouth the symptoms disappear or are reduced. 
The Nature of the Thyroid Hormone.-The active principle produced by the thyroid gland is an iodine compound as was first shown by Baumann in 1895 . He prepared an iodine-protein compound called thyroiodine, which contained as much as 9.3 per cent. of iodine. The compound was stable, readily soluble in dilute alkalies, and did not decompose with high heat or when the gland was digested in strong acid. Kendel's thyroxin we now know to be the pure principle.

Marine who has extensively studied the thyroid finds that certain types of hyperplasia are associated with a decreased supply of the active iodine compound. If iodine as such be given to such an animal, the symptoms are improved and the iodine compound content of the gland is increased.

Interesting biological experiments showing the influence of thyroid feeding on immature animals have been performed by Gudernatsch. Dried
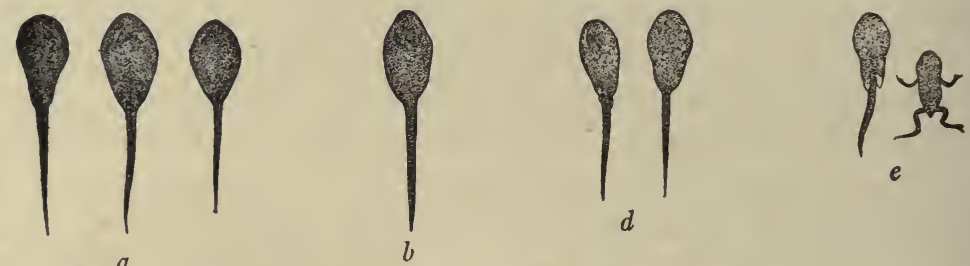

$a$
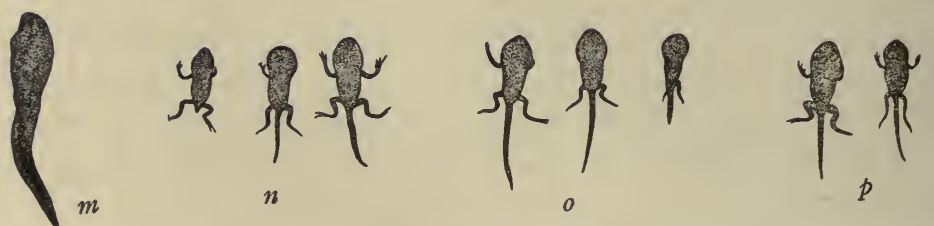

FIG. 3r2.-The influence of thyroid feeding on metamorphosis of tadpole larvæ of Bufo vulgaris. $a$, original size June 5 th. $b$, control. $d$, and $e$, thyroid and plants, $d$, thyroid given three days, $e$, thyroid given five days, taken June 26 th. $m$, control, $n$, fed thyroid and muscle, $o$ fed thyroid and plants, $p$, thyroid and hypophysis, taken July 6, I 912. (After Gudernatsch.)

thyroid gland fed to different species of tadpoles stopped growth and stimulated differentiation. Tadpole larvæ no matter how young were forced into metamorphosis, i.e., the development of fore and hind limbs and the assumption of the adult frog form. Gudernatsch by this means stimulated even tiny tadpoles only a few millimeters long, forcing metamorphosis "within eighteen days after hatching, while normally they would require ten to twelve weeks to reach such a stage. The results of this premature metamorphosis are perfect frogs of minute size, pygmy frogs." The thyroid as food stops tissue growth during this process. "The thyroid possesses a quality that stimulates differentiation, not contained in any other food used." The influence of the thyroid is somewhat modified by the food given with the gland, but one food would prevent the thyroid influence, namely thymus. 
derangement of calcium metabolism suggests the thought that the symptoms might be relieved by supplying this substance. The matter is presented in the following words of Berkeley and Beebe: "Probably the most striking argument in favor of Macallum's theory in respect to tetany parathyreoprivus is that it may be relieved almost instantly by the intravenous injection of a soluble calcium salt. We have repeated the experiment and find that intravenous injections of a soluble calcium salt will relieve tetany almost instantly; intramuscular injections may be effective after a period of thirty to forty-five minutes, and a similar period is required for the beneficial effect after subcutaneous injection. The effect of the calcium salt when given intravenously to an animal in advanced states of acute tetany is one of the most striking in the range of physiological experimentation."

Koch in I9I 3 studied the metabolism of dogs after parathyroidectomy, using physiological chemical methods, supplemented by histological examination. The urine of these dogs contained the toxic substances methylguanidine and other guanidine bases in large quantities. These are purin compounds. The examination of the tissues showed extensive chromatolysis, degenerating epithelial cells in the intestinal tract, and nuclear disintegration in the liver and especially the kidney. These led him to the view that the absence of the parathyroids resulted in disturbance of nuclear metabolism, a "nuclear atrophy." It has already been shown that nucleoproteins are richly present in the parathyroids and their extracts. Injections of nucleoproteins freshly prepared also relieve the symptoms of acute tetany in dogs. Berkeley and Beebe state: "We believe that the essential fact in the production of symptoms following complete thyroparathyroidectomy is the deranged metabolism giving rise to some active poison, and not the abnormal excretion of calcium which we regard as an accompanying phenomenon, perhaps due in part to the starvation cachexia which ensues in most cases if the animal is tided over the acute condition." This view, it will be noted, was borne out by the later work of Koch.

The Suprarenal Capsules or Adrenals.-These are two flattened, more or less triangular or cocked-hat shaped glandular bodies, resting by their lower border upon the upper border of the kidneys.

The gland tissue proper consists of an outside firmer cortical portion, and an inside soft dark medullary portion, figure 3 I 5 .

The adrenals are very abundantly supplied with nerves, chiefly composed of non-medullated fibers. These fibers are derived from the solar and renal plexuses and the vagi, but the method of their termination is unknown.

A vast amount of information has been given concerning the function of the suprarenal capsules within the last few years by the researches of Oliver and Schafer, Zyboulski, Abel, Aldrich, Dakin, Dale, Elliot, Cannon, and others. Brown-Séquard, it is true, showed by experiment as early 
as 1856 that removal of the suprarenals is followed by the death of the animal, but his experiments were repeated by others unfortunately on less susceptible animals. These men did not obtain the same results; and it was concluded that the suprarenal capsules had no function, or at least that their function was not known. Death was preceded in the case of Brown-Séquard's animals by symptoms somewhat analogous to those of the disease of man known as Addison's disease. The failures to reproduce these symptoms have probably resulted from incomplete removal or the presence of accessory bodies. Accessory suprarenallike tissues, chromofile tissues, are commonly present in animals. Further, if one gland is removed, the other hypertrophies. The experiments of all recent observers confirm the original experiments of Brown-Séquard and show that the removal of the suprarenal capsules is fatal.

Oliver and Schäfer found that injections of suprarenal extract produced marked effects upon the muscular layer of the arteries, the muscular tissue of the heart, and the skeletal muscles. The muscular layer of the arteries is markedly contracted, causing a rise of blood-pressure.

The contractions of the heart with its nerves intact are small although they are increased both in force and amplitude. If the vagi are sectioned, the slowing does not occur. Instead, the heart rate is accelerated through the
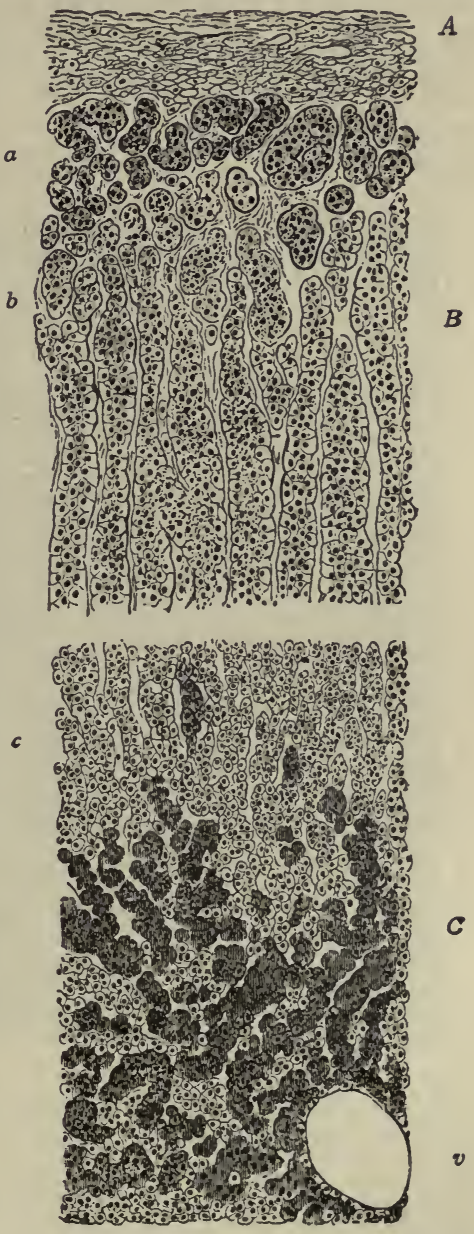

FIg. 3I5.-Vertical Section of Adrenal. $A$, Capsule; $B$, cortex; $C$, medulla; $a$, glomerular zone; $b$, fascicular zone; $c$, reticular zone; $v$, vein in medulla. (Merkel-Henle.) accelerator nerves, the movements of the alimentary canal are decreased, the splanchnic vascular region is sharply constricted, the urogenital musculature is stimulated to contraction, and the pupils are dilated. In fact, Elliot and others after him have shown that all those structures which are stimulated or inhibited by the action of sympathetic mechanisms are stimulated by the active principle of this gland and in 
the same manner. The conclusion follows that the active principle, epinephrin, specifically stimulates the sympathetic and autonomic mechanisms. The point of action is the nerve endings in the peripheral tissue and the reaction is sympathomimetic.

a. The Nature of the Active Principle of the Suprarenal Gland.-Early workers, notably Oliver and Schäfer, studied this gland by extracts of its dried tissue. Thus they stated that less than $\frac{1}{31 \frac{1}{00}}$ gram ( $\frac{1}{800}$ grain) of the

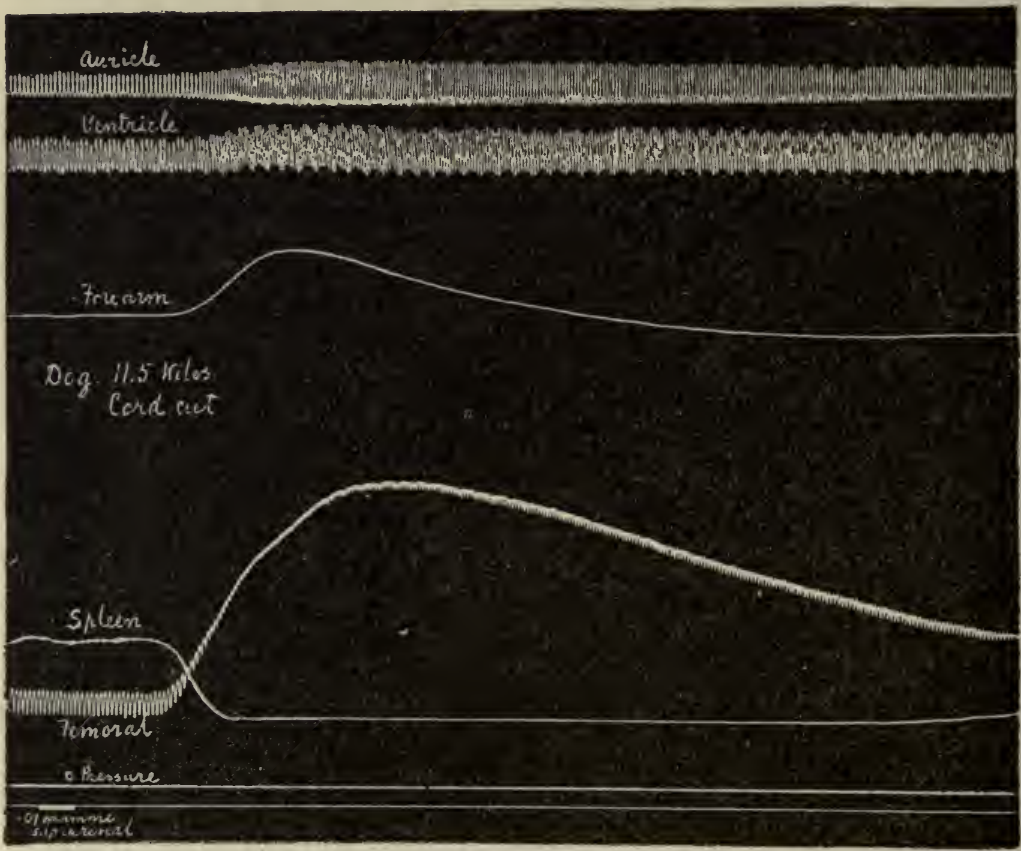

Fig. 316.-Injection of Suprarenal Extract. Effect upon the heart, limb, spleen and blood-pressure, after section of cord and vagi. (Oliver and Schäfer.)

desiccated gland is sufficient to produce an effect upon the heart and arteries of an adult man.

It is a curious fact that only extracts of the medullary portion of the gland are active. It has been further shown, by Christiani and others, that if only small portions of the medulla remain, the animal operated upon survives; while if all the medullary substance be removed, even though large portions of the cortex remain, the animal invariably dies.

Abel has succeeded in separating the blood-pressure-raising constituent of the gland extract, and calls it epinephrin, $\mathrm{C}_{10} \mathrm{H}_{13} \mathrm{NO}_{3} \frac{1}{2} \mathrm{H}_{2} \mathrm{O}$. Takamine isolated an active principle to which he assigned the formula $\mathrm{C}_{9} \mathrm{H}_{13} \mathrm{NO}_{3}$, and the name adrenalin. This product is now known to be an aminoalcohol with a pyrocatechin base as follows: 


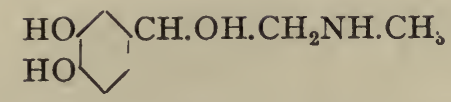

This compound is the active principle or hormone of the epinephros. Epinephrin is now prepared synthetically and commercially, and is an important and useful medicinal product.

b. Secretory Nerves for the Adrenals.-Dreyer was first to give evidence that the products of this gland are discharged into the blood of the adrenal vein in increased quantity on splanchnic stimulation. Cannon has extensively studied the relation of the secretion of epinephrin to the autonomic reactions of the body. His evidence indicates that during fright, anger, etc., which in animals is accompanied by vigorous activity of the mechanisms innervated through the automatic system, the suprarenal glands are strongly stimulated. The result is a production and discharge of an increased amount of epinephrin into the circulation. As the nervous mechanisms tend to fatigue the increase in the secretion of epinephrin brings about a hormone stimulation supplementing and strengthening the nerve control during this critical time.

This gland furnishes, on the whole, most conclusive and typical evidence of the presence of an internal secretion that is absolutely necessary to the normal metabolism of other organs.

c. Epinephrin in Relation to Carbohydrate Metabolism.-The action of the hormone, epinephrin, which seems so specific in relation to the function of special types of nerve endings is bound up with the metabolism of carbohydrates. Following the removal of the suprarenal glands the store of glycogen in the liver is quickly discharged and glycogenesis is no longer possible. This has been shown by removing the suprarenals in rats which stand the operation more readily than most animals, if the glands are removed in successive operations.

The Internal Secretion of the Pancreas.-Minkowski and von Mering have shown that total extirpation of the pancreas is followed in all cases by the appearance of sugar in the urine in the course of a few hours. The amount of sugar which appears is considerable, from 5 to ro per cent. This experimental disease is accompanied by an increase in the quantity of urine and by abnormal thirst and appetite. It proves fatal in fifteen days or less. These results are obtained only when the entire gland or more than nine-tenths of it have been removed. If one-tenth or more of the gland be left behind, sugar appears in the urine when carbohydrates are eaten, but not otherwise. Nor is it necessary that the remaining portion of the gland be in its normal situation. Successful grafts of pancreas under the skin of the abdomen or elsewhere will prevent the appearance of sugar in the urine and the other symptoms. If, however, the graft be subsequently removed, the sugar in the urine and the other symptoms reappear, and the experimental 
disease proceeds to a rapidly fatal issue. Opie and others have shown also that in most cases of diabetes mellitus, there are pathological changes in the islands of Langerhans.

The symptoms produced by total extirpation of the pancreas do not depend upon the loss of the pancreatic juice proper to the organism. This secretion may be diverted from the intestine through a pancreatic fistula without the production of diabetes. Moreover, Hédon and Thiroloix have rendered the acini of the gland functionally inactive, and ultimately destroyed them, by the injection of paraffin or other substances into the duct of Wirsung, without the supervention of diabetes.

These experiments have shown that the ordinary secreting cells degenerate and the islands of Langerhans increase in size, leading to the conclusion that the islands are the structures that produce a special internal secretion which influences or controls carbohydrate metabolism in the body. When the dextrose cannot be readily burned in the body, the content of this substance in the blood is increased and this excess is eliminated by the kidneys. Products of incomplete oxidation of the sugar are also thrown into the circulation and eliminated into the urine. In diabetic cascs oxybutyric acid, aceto-acetic acid, acetone, etc., are found in the blood and urine. Neither pancreatic nor muscle extracts have the power of burning sugar.

Banting and Best, I922, have announced in the Journal of Laboratory and Clinical Medicine a most brilliant demonstration of the isolation and function of an active internal secretion from the pancreas. They performed a two stage operation on dogs, the first operation consisting of ligation of pancreatic ducts to induce degeneration of the pancreatic acini. Ten months later they removed this degenerated pancreas and injected watery extracts of it into the circulation of the animal after the development of the usual pancreatic diabetic glycæmia. The blood sugar rapidly augmented after removal of the pancreas, from .o9 to over . 30 per cent. There was a corresponding glycosuria, as indicated in figure 3 r $6 a$. On injecting 5 cubic centimeters of a four day old extract of the degenerated pancreas, the blood sugar dropped from .27 to .I5 per cent. but slowly returned to the higher level. Repeated injections of degenerated pancreatic extract continued to depress the amount of blood sugar. These injections greatly reduced the associated glycosuria, see figure $3 \mathrm{r} 6 a$. Dr. Macleod, in whose laboratory these experiments were performed, has announced before a scientific medical assembly the extension of these observations. In his laboratory it has been shown that the active principle is easily destroyed by the digestion enzymes and by heat. The active principles must be injected into the blood stream to produce its physiological effect. Furthermore, if the active principle is injected into the blood stream along with an excess of glucose the liver 
will store up glycogen. This epoch making point has never before been demonstrated in experimental pancreatic diabetes. Preliminary tests on the human disease show marked improvements in the physical and mental symptoms with a reduction of glycaemia and glycosuria. If, as the authors express the hope, quantity production of the active internal secretion of the pancreas can be perfected, it will give for the first time a direct method of supplementing the physiological deficit in the necessary internal secretion for controlling the metabolism of carbohydrates in the human body.

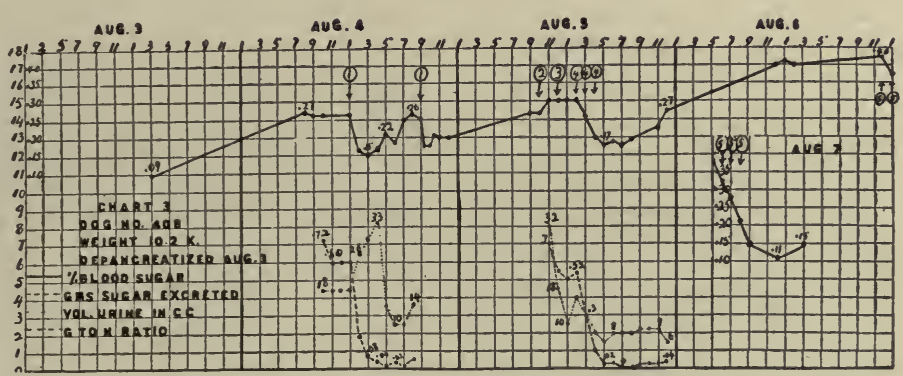

FIG. 3 r $6 a$.-(I) 5 cc. 4 day old extract of degenerated pancreas. (2) 5 cc. extract of liver. (3) 5 cc. extract of spleen. (4) 5 cc. extract of degenerated pancreas. (Banting and Best.)

The Liver as an Internal Secreting Organ.- That the liver is an organ of manifold function is well known. The secretion of bile, the glycogenic, lipogenic, urea, and uric acid forming functions have already been discussed. The glycogenic function as a mechanism for maintaining a comparatively constant supply of carbohydrate in the blood is a typical internal secretory process though the secretion is in this case not specific and not a typical hormone. Lipogenesis is of the same class. The urea, and in birds uric acid, are waste products. The liver carries on these functions by the aid of enzymes, i.e., oxidases, etc. There is also increasing evidence that the liver is concerned with the metabolism of the amino acids though the problem is not yet adequately understood.

The Kidney.-The proof of an internal secretion by the kidney is not so clear, chiefly because of the disturbance in the elimination of waste products when this organ is removed. However, the convulsions which follow nephrectomy are said to be diminished in intensity by the use of extracts of the kidney. It is claimed therefore that the kidney produces an internal secretion in addition to its primary function as an excretory organ. 
The Intestinal Mucosa.- The duodenal mucosa under the stimulating influence of hydrochloric acid produces a secretion, secretin, which acts as a chemical hormone to the pancreas. The enzyme, enterokinase, is activating for the trypsinogen of the pancreatic juice although the reaction takes place within the intestine.

The Thymus Gland.-The thymus is a ductless gland which is of relatively large size during embryological development. It, like the thyroids

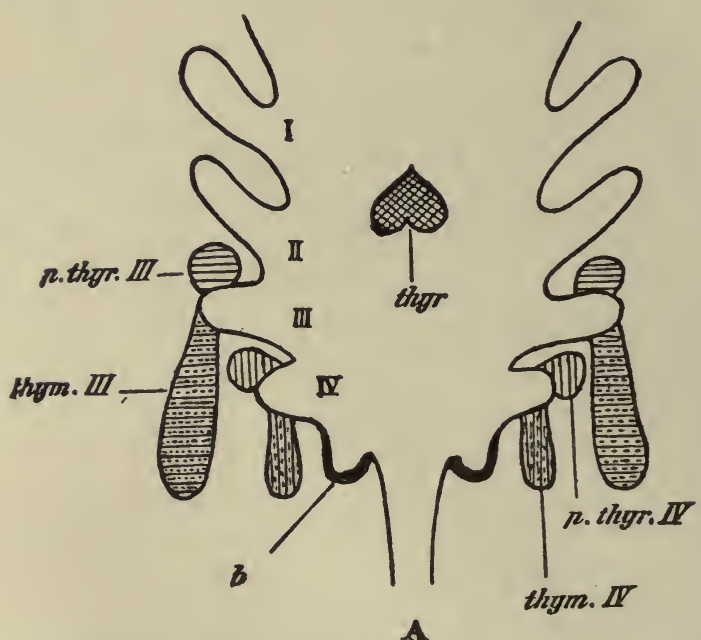

FIG. 317.-Diagram representing the embryological origin from the branchial organ of the thyroids, parathyroids, thymus, and posterior branchial body. (Vincent.)

and parathyroids, arises from the invagination of the epithelium of the third and fourth branchial arches. Little is known directly of the functions of the thymus in the human. Certain cases of excessive lymphoid development in adults are associated with persistence or enlargement of the thymus. Thyroidectomy interferes with the full development of the thymus and is associated with other evidences of disturbed nutrition, i.e., muscular, nervous, skeletal, etc.

Important information has been recently yielded by the biological studies of Gudernatsch referred to in the discussion of the thyroid gland. This observer fed dried thymus to frog tadpoles with the result that growth was strongly stimulated, whereas metamorphosis was indefinitely delayed. Tadpoles with ordinary food begin the differentiation that leads to metamorphosis upon reaching a certain maximum size and age. The thymus-fed tadpoles continue to grow beyond the normal size without showing signs of differentiation. Whereas the thyroid stops growth and stimulates differentiation, the thymus favors growth and seems to prevent differentiation.

The Pituitary Body.-This structure is a small reddish-gray mass occupying the sella turcica of the sphenoid bone. It consists of the pituitary gland 
proper or anterior lobe, the pars anterior, the posterior lobe, pars nervosa, and an intermediate epithelial zone known as the pars intermedia.

The pars anterior or glandular lobe of the pituitary and also the pars intermedia are derived from tissue that has its embryological origin from the epithelial pouch of the roof of the buco-pharyngeal cavity. The pars intermedia differentiates out of this common mass and comes to invest the infundibular portion, apparently sending cells into its trabeculæ. Three types of cells have been described for the pars anierior; I, neutrophiles, 2, acidophiles, and 3 , basophiles. The pars nervosa consists of a loose neuroglia like network arising out of the base of the third ventricle. The net exhibits open spaces more or less filled with ingrowths of pars intermedia cells. The pars intermedia (see Fig. 319) shows cavities filled with colloid being

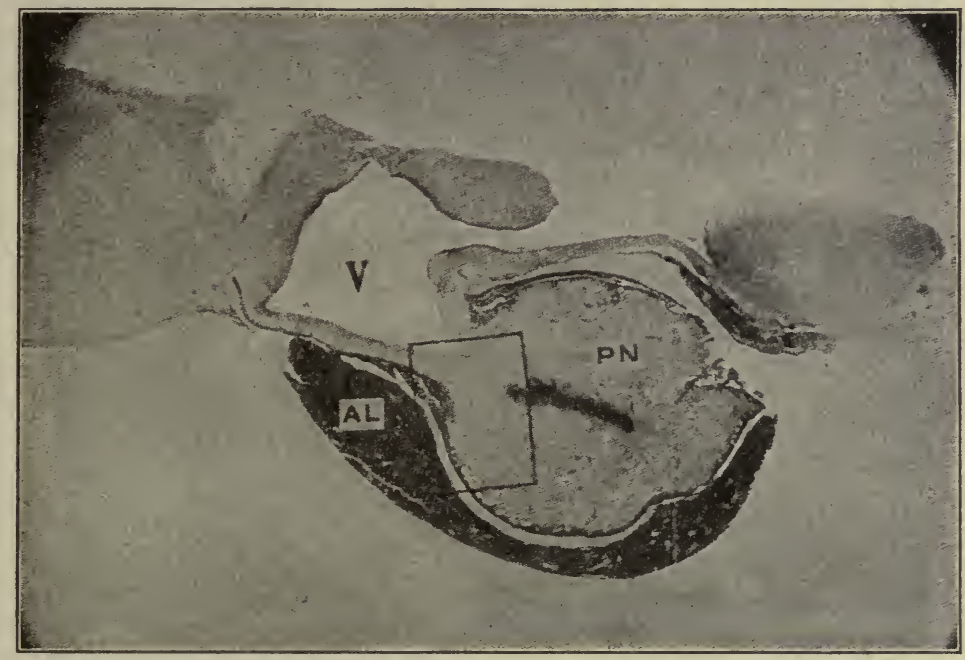

FIG. 318.-Mesial sagitta! section of the pituitary. $B$, third ventricle. $A L$ and $P L$, anterior and posterior lobes respectively. (Cushing and Goctsch.)

discharged into the spaces of the pars nervosa. It is thought that this colloid may be directly or indirectly the source of the active hormones of the posterior division of the pituitary.

a. The Function of the Pituitary.-The whole problem of the function of the pituitary body has been extensively studied physiologically and surgically by Cushing. This author has obtained concordant results by his operative study on dogs, and by the operative and clinical treatment of a long series of human patients. That the pituitary is absolutely necessary to life is now recognized. A number of functional disturbances have been traced to abnormal states of this organ, and evidence is accumulating to 
show that the anterior and posterior lobes are not concerned with identical functions. Attempts to arrive at the knowledge of the proper physiology of the organ have been made primarily along three experimental lines; I, Removal of the gland in whole or in part; 2, Transplantation; 3, The feeding or the injection of extracts.

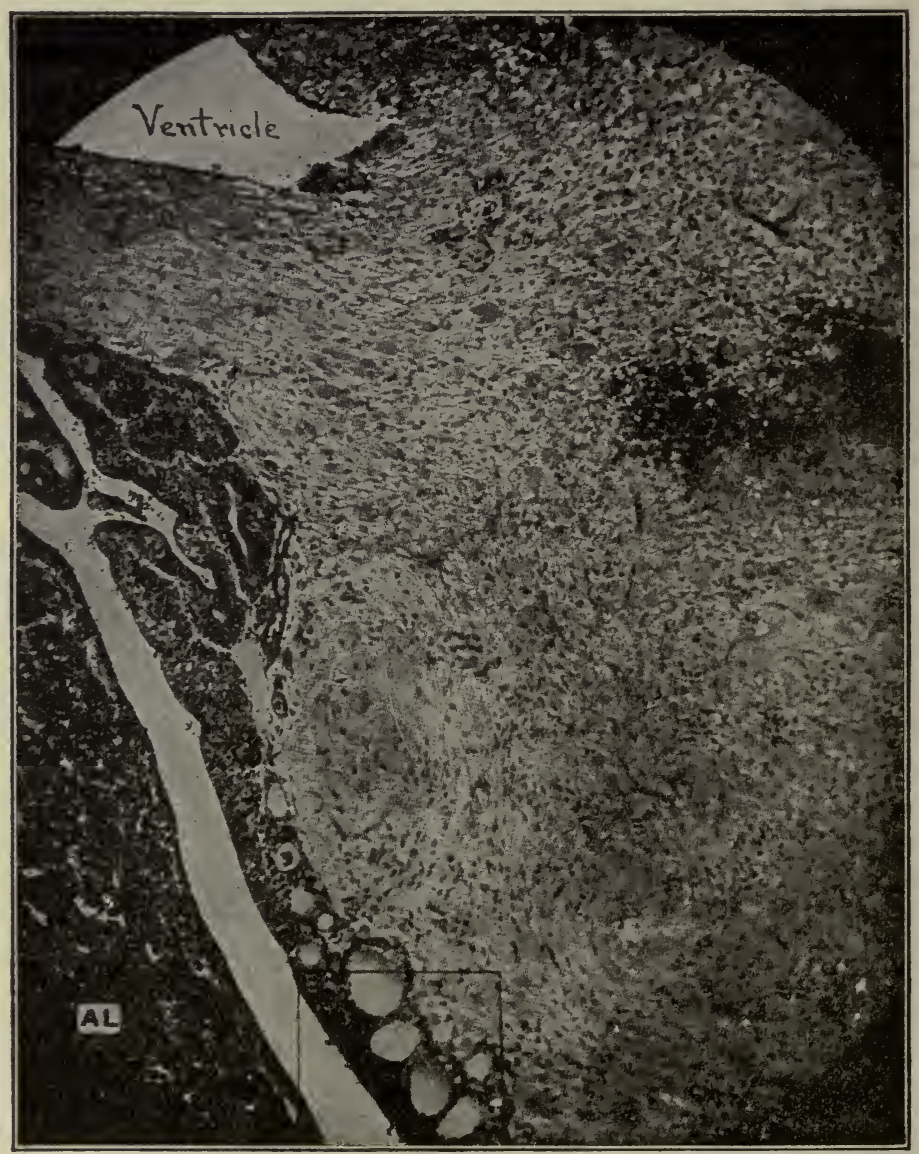

Fic. 319.-Enlarged view of the square marked on Fig. 318. (Cushing and Goetsch.)

b. Functional Disturbances After Complete Removal of the Pituitary.Removal of the gland is followed by death of the operated animals, in old dogs in from three to five days, but puppies survive ten to thirty days (Cushing). The acute symptoms are: insensitiveness, slow pulse and respiration, fall in body temperature, apathy, coma, and death.

If a small part of the gland is left so that only a condition of hypopituitarism is induced then the symptoms are more pronounced and chronic. 
These are summarized by Cushing: "Widespread adiposity, nutritional changes in the skin and its appendages, disturbance of carbohydrate metabolism, of body temperature, of growth and of renal secretion. Sexual inactivity or actual atrophy of the reproductive glands was observed; and, indeed, modification of most of the other ductless glands proved to be histologically demonstrable." In puppies there was persistent "sexual infantilism," "skeletal undergrowth" and "psychic disorders." These experimental results throw light on a great mass of clinical evidence which Cushing has gathered and interpreted.

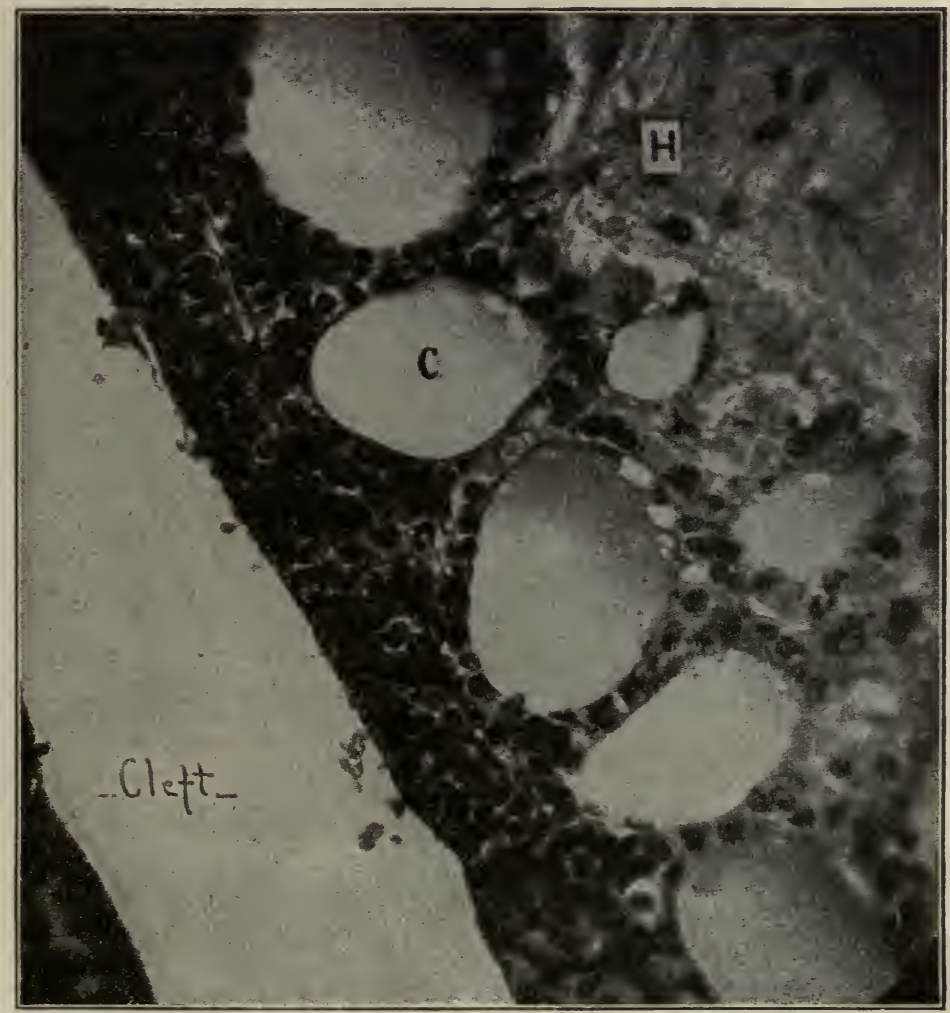

FIG. 320.-High magnification of the square marked on Fig. 3r9. C, Colloidol vesicles apparently discharging from $C$ into the tissue spaces of the pars nervosa. $H$, Hyaline bodies. (Cushing and Goetsch).

c. Action of Piluitary Grafts, Extracts, Etc.-Transplantation of the pituitary into the cerebral cortex prolonged the life of Cushing's animals. The gland gave histological evidence of viability for a month. Grafts have not, however, been uniformally and permanently beneficial, and do not produce evidences of hyperpituitarism. 
In Igr $x$ Cushing for the first time transplanted the pituitary of a child into an adult man suffering from lack of pituitary secretion. A pituitary cyst had been removed. The patient was temporarily but completely aroused from the "profound torpor" of hypopituitarism to a temporary state of normal mental and physical vigor by injections of gland extracts. After the gland was grafted the extracts were ceased and the man remained in his recovered state of health.

In addition to the general nutritional improvement from the action of extracts it is now known that smooth muscular tissues are stimulated, in this case by posterior lobe extracts. Uterine contractions in time of atonia in particular, are sharply stimulated, pituitrin producing this action strongly. The reaction in the blood vessels leads to rise of blood-pressure, increase of vagus tone, and augmentation of the heart beat. Pituitrin and posterior lobe extracts are vigorous galactogogues.

The feeding of extracts has been less positive, though prolonged feeding has seemed to aid in preventing the symptoms of hypopituitarism in alleviating obesity in particular.

e. Acromegaly or Hyperpituitarism.-Hypertrophy of the pituitary leads to a great acceleration in skeletal growth or gigantism. This we have not been able to prove by experimental means but must now assume to be established by clinical and pathological findings. The accelerated skeletal growth may occur either in childhood, youth or after full development, for example, Cushing's cases numbers XXXII and I, which began to grow rapidly at fifteen and twenty-five years of age respectively.

Hyperpituitarism with its giant skeletal growth shows a tendency to pass over to hypopituitarism with its attendant nutritional disorders of obesity, carbohydrate tolerance, and disturbance of correlated internal secreting glands and sex gonads.

Acromegaly as such seems due to a disturbance of the pars anterior, while the other nutritional derangements are attributed to disturbance of the pars intermedia and pars nervosa.

The Reproductive Glands.-The ovary and the testis are undoubtedly concerned with metabolism in the body. Numerous observations, not only of the sex gonads but of other parts of the reproductive system, show an intimate relation of this apparatus to the health and normal activities of other parts of the body.

a. The Testis.-It has been shown repeatedly that extracts of the tissue of the testis fed, or hypodermically injected when purified, increased the vigor both of the muscular and of the nervous systems. Ergograms show an increase in muscular power. Spermin isolated from the testis is claimed by its discoverer to produce these beneficial effects. Sterilization by the removal of the testis in domestic animals is followed by an entire change in the physical development of the animal, especially in the so-called secondary sexual 
characters. Such animals show less vigor and less muscular power. Sterilization of the male without removal of the gonads is not followed by the usual loss of vigor and of the typical male form and characteristics. This is ascribed to the preservation of the testis which is thought to produce internal secretion reacting through the nutritional processes of the body.

b. The Ovaries. -The ovaries not only possesses the germ cells, the ova, but their structure is characterized by other types of cells-the interstitial, stroma, and follicular cells. When a graafian follicle ruptures and discharges its ovum, cells rapidly grow and fill the cavity forming the corpus luteum.

The surgical removal of the ovaries results in disturbance of the periodic menstrual phenomenon, not because of the loss of the ova, but because the secretin fails which maintains the normal periodic reactions of the uterus. There are also in the human disturbances in nutrition from this cause, i.e., a decrease in oxidations, tendency toward obesity, marked nervous symptoms,

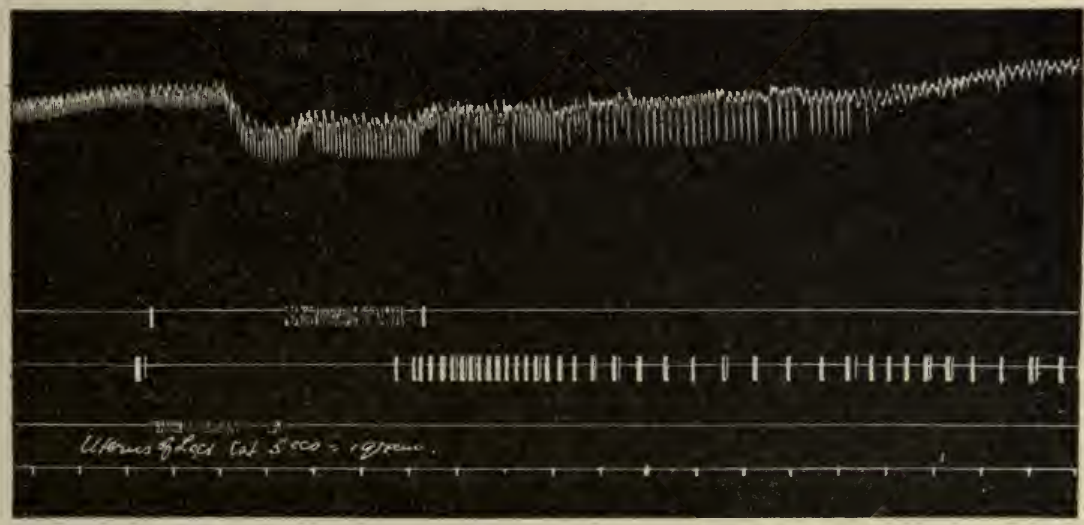

Fig. 321. - The galactagogic action of involuting uterus of the cat after intravenous injection of saline extracts. First tracing from above, blood-pressure; second, drops of milk from a hollow needle inserted into a mamma; third, drops from a wick inserted into two mamma that have been split open to remove the effect of the contractions of the muscles of the gland. (Mackenzie.)

etc., These symtoms are reduced or entirely disappear on grafting a portion of the gland, and the disturbed menstruation becomes regular again. Human ovaries have been transplanted to patients after previous ovariotomy with a recovery of normal body functions and improvements in health. Experiments by Lowey and Richter indicate that oxidations in the body are greatly increased on feeding ovarian extract to ovariotomized animals. Removal of the ovaries of hens leads to a loss of feminine characters, i.e., the development of spurs, cock-like comb and wattles, hackle, etc. Castrated female ducks are also said to develop male-like characters. Guthrie suggests that this may be "an unmasking of characters," since we do not know the asexual type in the higher animals by any positive evidence. 
c. Corpus Luteum.-The cells of the corpus luteum are now believed to produce an internal secretion or hormone. This body which develops rapidly after the rupture of the graafian follicles ordinarily persists only two or three weeks. When, however, the ovum becomes fertilized and attaches to the uterine wall and its development proceeds, then the corpus luteum enlarges and persists throughout the gestation period. Extracts of this body were found by Mackenzie to be galactagogues.

The Phenomena of Gestation and Lactation in Relation to Internal Secretions.-Physiologists are accumulating more and more evidence supporting the view that hormones exert a strong influence in the control of the process of gestation and lactation.

During gestation, aside from the mere fact of the development of the embryo, there are the following special conditions to be given consideration, namely, the persistence of the corpus luteum, the great enlargement of the uterine mass, the development of the mammary glands, to which may be added for this consideration, the development of the placenta and of thefetus itself. During this period there is a profound disturbance of many nutritional factors especially affecting the correlations of the digestive, the muscular, and the nervous systems.

After parturition the following changes in the picture occur, namely, the discharge of the placenta which implies retrogression in this organ, involuting changes in the uterus, and active lactation. The latter phenomenon is of special physiological interest. The growth of the gland without lactation during gestation and the abrupt development of the phenomenon of lactation apparently depends strictly upon the hormones. As will be seen later, the mammary gland tissue is poorly supplied with nerves, apparently having no secretory nerves. It would seem that we have here in the first period a group of hormones stimulating growth, possibly associated with inhibitive hormones for milk secretion. After gestation active galactagogue hormones control the phenomenon. Such hormones are known to come from the involuting uterus, corpus luteum, posterior lobe of the pituitary, and from the mammary gland itself. The present view is that an inhibitive influence is exerted by the hormones from the placenta and the developing fetus on milk production during the gland growth. Our information concerning this complicated question is, however, at the present time inadequate.

There are other organs whose function is still obscure but in which the indirect evidence points to an influence on metabolism at one stage or another of the existence of the animal body. Enough has been given here to show that the interrelation of the organs of the body is extremely complex in so far as metabolism is concerned. It is not enough simply to know the foods and their composition. The whole complex of intermediary metabolisms and metabolites and their influences must constantly be taken into consideration. 


\section{CHAPTER XII.}

\section{ANIMAL HEAT.}

HEAT is produced by the metabolism of the tissues of the body. In man and in such animals as are called warm-blooded, i.e., only mammals and birds, there is an average body temperature which is maintained with only slight variations in spite of changes in their environment. The possible variations above and below this average are comparatively slight. The average temperature in all mammals and birds is not the same, for, as we shall see, the average temperature of man is $37^{\circ} \mathrm{C}$. ( $98.6^{\circ} \mathrm{F}$.), in some birds it is as high as $44^{\circ} \mathrm{C}$., while in the wolf it is said to be under $36^{\circ} \mathrm{C}$.

The average temperature of the human body in those internal parts which are most easily accessible, as the mouth and rectum, is from $36.9^{\circ}$ to $37.4^{\circ} \mathrm{C}$. $\left(98.5^{\circ}\right.$ to $99.5^{\circ} \mathrm{F}$.). In different parts of the external surface of the human body the temperature varies only to the extent of one or two degrees centigrade, when all are alike protected from cooling influences; and the difference which under these circumstances exists depends chiefly upon the different degrees of blood supply. In the axilla and in the groin, the most convenient situations, under ordinary circumstances, for examination by the thermometer, the average temperature is $37^{\circ} \mathrm{C}$. $\left(98.6^{\circ} \mathrm{F}\right)$. In different internal parts, the variation is one or two degrees; those parts and organs being warmest which contain most blood, and in which there occurs the greatest amount of chemical change, e.g., the muscles and the glands. The temperature is highest when they are in a condition of activity. Those tissues which subserve only a mechanical function and are the seat of least active circulation and chemical change are the coolest. These differences of temperature, however, are actually but slight, on account of the provisions which exist for maintaining uniformity of temperature in different parts.

The average temperature of a healthy body varies somewhat according to age, sex, time of day, climate, etc. The mean temperature is said to be slightly higher, $0.5^{\circ} \mathrm{C}$., in young children and in old persons than in adults. It is perhaps very slightly higher in women than in men, in warm climates than in cold, in winter than in summer. It varies slightly at different times in the day, especially during sleep when metabolism is at a low ebb.

Diurnal Temperature Variations.-The discussion presented in this paragraph is abstracted from an excellent paper on the subject by Professor Gibson. "Certain features of daily rhythm are generally recognized, such as the rise of temperature during the forenoon and afternoon, and 
the fall during the evening and early morning hours. A study of the literature indicates considerable differences in the incidence of the maximum and minimum temperature, depending undoubtedly upon a variety of causes. Despite differences as regards dietetic habits, work, age, etc., the time of maximum temperature may be limited broadly between 4 and 8 P.M., and that of the minimum between 2 A.M. and 7 A. M., with an average range of variation of over $\mathrm{I}^{\circ} \mathrm{C}$. in rectal observations. While a hot or cold climate may determine minimal variations in the peripheral temperature of the body, the internal temperature, as measured in the rectum, appears to suffer only very slight modifications from such causes. The rise in temperature incidental to muscular exertion is transitory. The following quotations serve to indicate prevalent views regarding the diurnal rhythm. Pembrey writes: 'As regards the causes of the laily variation in temperature, muscular activity and food appear to be the most important factors. In ordinary life man is most active and takes food during the day, and is least active during the night. . . . We may conclude that the daily variation in temperature is one of the features of a corresponding variation in the activity of the tissues of the body, as shown by the rate of the contraction of the heart, the frequency of respiration, the intake of oxygen, the output of carbon dioxide, the discharge of urea, and the capacity for muscular work.' Jürgensen's observations on fasting subjects in which a more or less "normal" curve of temperature variations was maintained exclude the diet factor from a preponderating rôle. In estimating the relative importance of the bodily activities as distinguished from other environmental conditions, studies on the influence of the inversion of the daily routine have been made. Those of Benedict claim special interest, because the observations were practically continuous over long periods of time. Such an arrangement was made possible by the use of a specially devised electric resistance thermometer reading to $0.0 \mathrm{I}^{\circ} \mathrm{C}$, , which can be inserted $\mathrm{Io} \mathrm{cm}$. to $15 \mathrm{~cm}$. in the rectum and retained there without inconvenience during both waking and sleeping hours. Two subjects were carefully observed by Benedict: one a person usually working during the day, but made to work at night and sleep during the day for a series of consecutive days; the other, an individual long accustomed to night-work in the capacity of a night watchman. From the results of these observations it has been assumed that the general form of the night curve remains practically intact, even when the daily routine is inverted, indicating a fixity of rhythm that is difficult to explain. As Benedict says: 'Why the temperature of the human body reaches a minimum at 2 A.M. to 6 A.M., independent of whether the subject is sleeping soundly in the recumbent position or whether he is awake and sitting, or even standing and walking, is a problem that calls for extended research.' However, the transposition of the daily routine through a period of practically half a day, experienced as the result of the time changes 
during the trip from New Haven to Manila, produces a corresponding and coincident shifting of the rhythmic temperature changes, so that the normal character of the variations is always preserved."

Heat-producing Organs.-Heat is liberated in the body wherever oxidative metabolism takes place. Of all the tissues of the body muscular tissue is conspicuous for its mass and for its activity. It is evidently the great heat-producing tissue. The manifestation of muscular energy is always accompanied by the evolution of heat and the production of carbon dioxide. This production of carbon dioxide goes on while the muscles are in mechanical rest, only in a less degree than that which is noticed during muscular activity, and so it is certain that an active katabolism is going on in resting as well as in contracting muscles. This katabolism is a source of much heat, and so the total amount of heat produced in the muscular tissues per day must be very great. It has been calculated that, even neglecting the heat produced by the quiet katabolism of muscular tissue, the amount of heat generated by muscular activity would supply the principal part of the total heat produced within the body. The heart, as a special muscle, deserves particular mention since it is in constant vigorous activity. All its energy is ultimately converted into heat, accounting for about 5 per cent. of the total heat of the body. The secreting glands, and principally the liver as being the largest and most active, come next to the muscles and heart as heat-producing tissues. It has been found by experiment that the blood leaving the glands is considerably warmer than that entering them. The metabolism in the glands is very active; and the more active the katabolism, the greater the heat produced.

It must be remembered, however, that although the organs mentioned are the chief heat-producing parts of the body, all living tissues contribute their quota, and this in direct proportion to their activity. The blood itself is also the seat of katabolism and, therefore, of the production of heat; but the share which it takes in this respect, apart from the tissues in which it circulates, is very inconsiderable.

Regulation of the Temperature of the Human Body.-The average temperature of the body is maintained under different conditions of external circumstance by mechanisms which permit of (I) variation in the loss of heat, and (2) variations in the production of heat. In healthy warm-blooded animals the loss and gain of heat are so nearly balanced one by the other that, under all ordinary circumstances, a uniform temperature, within a degree or two, is preserved.

Variation in the Loss of Heat.-The loss of heat from the human body is principally regulated by the amount given off by radiation and conduction from its surface, by means of the constant evaporation of water from the same part, heat being thus rendered latent, and to a much less degree by loss from the air-passages. In each act of respiration, heat is lost to a greater or less extent according to the temperature of the atmosphere; unless indeed 
the temperature of the surrounding air exceeds that of the blood. We must remember, too, that all food and drink which enter the body at a lower temperature abstract a small measure of heat; while the urine, and feces which leave the body at about its own temperature are also means by which a certain small amount of heat is lost.

Heat Lost from the Surface of the Body.-By far the most important loss of heat from the body, probably 90 per cent. and upward of the whole amount, is that which takes place by radiation, conduction, and the evaporation of moisture from the skin. The actual figures for ordinary conditions are as follows: For every Ioo calories of heat produced, 2.6 are lost in heating the food and drink; 2.6 in heating the air inspired; 14.7 in evaporation; and 80. . I by radiation and conduction. During increased activity of the body the proportion of heat lost by evaporation is greatly increased. The means by which the skin is able to act as one of the most important organs for regulating the temperature of the blood are, I, that it offers a large surface for radiation, conduction, and evaporation; 2 , that it contains a large but adjustable amount of blood, and the quantity of blood is greater under those circumstances which demand a loss of heat from the body, and vice versa, which gives a means for varying the loss of heat by radiation and conduction; 3 , that it contains the sweat glands, which discharge a quantity of moisture to be evaporated from its surface.

The circumstance which directly determines the quantity of blood in the skin is that which governs the supply of blood to all the tissues and organs of the body, namely, the power of the vaso-motor nerves to cause a greater or less tension of the muscular element in the walls of the arteries, and, in correspondence with this, a lessening or increase of the caliber of the vessel, accompanied by a less or greater current of blood. A warm or hot atmosphere so acts on the sensory nerves of the skin as to lead to a reflex relaxation of the muscular fiber of the blood vessels; as a result, the skin becomes full-blooded, relatively hot, and moist from sweating; and much heat is lost. With a low temperature the blood vessels shrink, and with the consequently diminished blood supply, the skin becomes pale, cold, and dry, an effect produced through the vascular centers in the medulla and spinal cord.

The activity of the sweat glands of the skin is also regulated reflexly through the sweat centers. The increased blood supply just described is favorable to increased production of sweat by the sweat glands. Thus, by means of the self-regulation the skin becomes the most important of the means by which the temperature of the body is regulated.

The relative loss of heat by the means given, i.e., radiation, conduction, and evaporation, will depend on two factors: first, the relative temperature of the body to the surrounding air; and, second, the humidity of the air. If the atmospheric temperature is the same as that of the body, of course there will be no loss of heat by radiation and convection; if the air temperature is 
higher, there will be an actual gain. When the humidity of the air is great, there will be reduced evaporation of perspiration and consequent diminished heat loss. If we assume a moisture-saturated air at the body temperature, then heat loss becomes impossible and the temperature of the body will rise. This is why a hot moist climate is so oppressive, while a hot but dry atmosphere is readily borne by the human body. The amount of heat required to evaporate I c.c. of water is 536 small calories, hence an increase in the evaporation of perspiration readily compensates for a decrease in the loss of heat by radiation and convection.

Many examples may be given of the power which the body possesses of resisting the effects of a high temperature, in virtue of evaporation from the skin. Blagden and others supported a temperature varying between $92^{\circ}$ to $100^{\circ} \mathrm{C}$. $\left(198^{\circ}-212^{\circ} \mathrm{F}\right.$.) in dry air for several minutes; and in a subsequent experiment he remained eight minutes in a temperature of $126.5^{\circ} \mathrm{C}$. $\left(260^{\circ} \mathrm{F}\right.$.). "The workmen of Sir F. Chantrey were accustomed to enter a furnace, in which his molds were dried, while the floor was red-hot, and a thermometer in the air stood at $177.8^{\circ} \mathrm{C}$. $\left(350^{\circ} \mathrm{F}\right.$.), and Chabert, the fire king, was in the habit of entering an oven the temperature of which was from $205^{\circ}-315^{\circ} \mathrm{C}$. $\left(400^{\circ}-\right.$ $600^{\circ}$ F.)." (Carpenter.)

But such heats are not tolerable when the air is moist as well as hot, so as to prevent evaporation from the body. C. James states that in the vapor baths of Nero he was almost suffocated in a temperature of $44.5^{\circ} \mathrm{C}$. ( $\mathrm{II}^{\circ}{ }^{\circ} \mathrm{F}$.), while in the caves of Testaccio, in which the air is dry, he was but little incommoded by a temperature of $80^{\circ} \mathrm{C}$. ( $\left(76^{\circ} \mathrm{F}\right.$.). In the former, evaporation from the skin was impossible; in the latter it was abundant, and the layer of vapor which would rise from all the surface of the body would, by its very slowly conducting power, defend it for a time from the full action of the external heat.

Man is able by suitable clothing to increase or to diminish the amount of heat lost by the skin. There are baths and other means which man and animals instinctively adopt for lowering the temperature when necessary.

Although under any ordinary circumstances the external application of cold only temporarily depresses the normal temperature to a slight extent, it is otherwise in cases of high temperature in fever. In these cases a cool bath may reduce the temperature several degrees, and the effect so produced lasts in some cases for many hours.

Extreme heat and cold produce effects too powerful, either in raising or lowering the heat of the body, to be controlled by the proper regulating apparatus. Walther found that rabbits and dogs kept exposed to a hot sun, reached a temperature of $46^{\circ} \mathrm{C}$. (II4. $8^{\circ} \mathrm{F}$.), and then died. Cases of sunstroke furnish us with several examples in the case of man; for it would seem that here death ensues chiefly or solely from elevation of the temperature.

The effect of mere loss of bodily temperature in man is less well known than the effect of heat. From experiments by Walther it appears that rabbits can be cooled down to $8.9^{\circ} \mathrm{C}$. $\left(48^{\circ} \mathrm{F}\right.$.) before they die, if artificial respiration be kept up. Cooled down to $17.8^{\circ} \mathrm{C}$. $\left(64^{\circ} \mathrm{F}\right.$.), they eannot recover 
unless both external warmth and artificial respiration be employed. Rabbits not cooled below $25^{\circ} \mathrm{C}$. $\left(77^{\circ} \mathrm{F}\right.$.) recover by external warmth alone.

Loss of Heat from the Lungs. - The lungs and air-passages are very inferior to the skin as a means for lowering the temperature. In giving heat to the air breathed, the lungs stand next to the skin in importance. As a regulating power, the inferiority is very marked. The air which is expelled from the lungs leaves the body at about the temperature of the blood, and is always saturated with moisture. No inverse proportion, therefore, exists, as in the case of the skin, between the loss of heat by radiation and conduction, on the one hand, and by evaporation, on the other. The colder the air and the drier, for example, the greater will be the loss in all ways. Neither is the quantity of blood which is exposed to the cooling influence of the air diminished or increased in the lungs, so far as is known, in accordance with any need in relation to temperature. It is true that by varying the number and depth of the respirations, the quantity of heat given off by the lungs may be made to vary also for a few minutes. But the respiratory passages, while they must be considered important means by which heat is lost, are altogether subordinate, in the power of actively regulating the temperature.

The loss of heat used to warm foods is an obvious method of expenditure of heat which may be resorted to, especially in certain fevers. The loss of heat by the excreta discharged from the body at a high temperature must be of no use as a means of regulating the temperature, since the amount so lost must be capable of little variation.

Variation in the Production of Heat.-It may seem to have been assumed, in the foregoing pages, that the only regulating apparatus for temperature required by the human body is one that shall, more or less, produce a cooling effect; as if the amount of heat produced were always, therefore, in excess of that which is required. Such an assumption would be incorrect. The body has the power of regulating the production of heat, as well as its loss.

The production of heat in the body is apparently different for each animal; i.e., the absolute amount of heat set free by different animals in a given period varies. Each individual has his own coefficient of heat production. From all that has been said on the subject it will be seen that the amount of heat for all practical purposes depends upon the metabolism of the tissues of the body; everything, therefore, which increases that metabolism will increase the heat production; so, therefore, the absolute amount of heat produced by a large animal, having a larger amount of tissues in which metabolism may go on, will be, ceteris paribus, greater than that of a small animal. But the activity of the tissue change in a small animal may be greater than in a large one, as measured per kilo of body weight, and naturally no strict line can be drawn between the two. 
Heat Produced per Kilo per Hour. (Munk.)

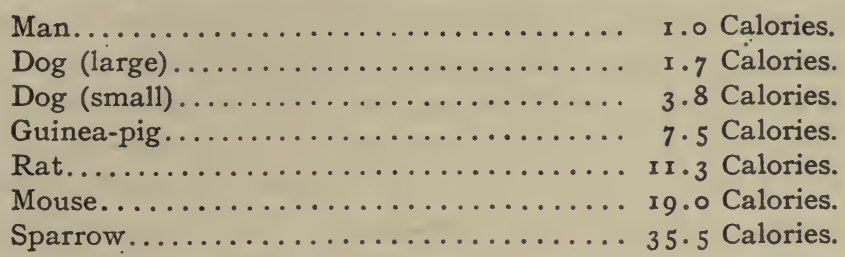

The ingestion of foods increases the metabolism of the tissues. As one would expect, the rate of heat production is found by experiment upon the dog to be increased after a meal, reaching its height about six hours after a meal.

It has also been experimentally ascertained that the rate of heat production varies with the kind of food taken: for example, if sugar be added to the meal of meat given to the dog, the height of maximum production is reached. It is often said that the various nations have found by experience what food is most suitable for the climate in which they live, and that such experience can be trusted to regulate the quantity consumed. Although there have been no very conclusive experiments to prove the view, yet it is a matter of general observation that in northern climates and in colder seasons the quantity of food taken is greater than in warmer climates or in warmer seasons. Moreover, the kind of food is different. For example, persons living in the colder climates require much fat in order to produce the requisite amount of heat.

Influence of the Nervous System on Heat Production.-The influence of the nervous system in modifying the production of heat must be very important, as upon the nervous influence depends the amount of the metabolism of the tissues. The experiments and observations which best illustrate it are those showing, first, that, when the supply of nerves to a part is cut off, the temperature of that part falls below its ordinary degree after a time; and, second, that when there is severe injury to or removal of the nervous centers the temperature of the body rapidly falls, even though artificial respiration be performed, the circulation maintained, and to all appearance the ordinary conditions for chemical changes in the body be completely maintained.

There is a heat-regulating nervous apparatus closely comparable to that which regulates the secretion of saliva or of sweat, by means of which the production of heat in the warm-blooded animals is increased or diminished, as occasion requires. This apparatus probably consists of a center or centers in the brain which may be reflexly stimulated, as, for example, by impulses from the skin, and which act through special nerves supplied to the various tissues. The evidence upon which the existence of this regulating apparatus is assumed is the marked effect in the increase of the oxygen consumed by 
a warm-blooded animal when exposed to cold, and the corresponding increase in the output of carbon dioxide, indicating that there is an increase of the metabolism and so an increased production of heat under such circumstances, and not a mere diminution of the amount of heat lost by the skin, etc. A cold-blooded animal reacts very differently to exposure to cold; instead of increasing the metabolism as in the case of the warm-blooded animal, cold diminishes the metabolism of its tissues. It is clear, therefore, that in warmblooded animals there is some apparatus not possessed by cold-blooded animals, which counteracts the effects of cold. In warm-blooded animals poisoned by curara, or in which section of the medulla has been done, it has been found that this regulating apparatus is no longer in action, and under such circumstances no difference appears to exist between such animals and those which are naturally cold-blooded. Warmth increases their temperature, and cold lowers it, and with this there is, of course, evidence of diminished metabolism.

The explanation of these experiments is that in such animals the connection between the skin and the muscles through the nervous chain, such as a thermotaxic nervous apparatus might be supposed to afford, is broken either at the termination of the nerves in the muscles (curara) or at the sectioned point of the bulb.

The position of these hypothetical centers is a matter of some difference of opinion. It has been demonstrated that stimulation of certain parts of the brain may, among other symptoms, produce increased metabolism of the tissues with increased output of carbon dioxide and a raised temperature: the parts of which this may be asserted are parts of the corpus striatum and of the optic thalamus. The general thermogenic centers are probably closely associated with the motor centers of the cord and brain stem. The thermoregulative centers are the nuclei in the corpus striatum and optic thalamus. Assuming a constant or tonic activity of the thermogenic regulative centers, it is easy to understand the fall of temperature on their destruction or on the destruction of the nerve paths to the active tissues.

Experimental observations, such as have been made upon animals, receive confirmation from the observations on patients who suffer from fever or pyrexia; in them the temperature of the body may be raised several degrees, as we have already pointed out. This increase of temperature might, of course, be due to diminished loss of heat from the skin, but this, although a factor, is not the only cause. The amount of oxygen taken in and the amount of carbon dioxide given out are both increased. With this there must be increased metabolism of the tissues, and particularly of the muscular tissues, since at the same time the amount of urea in the urine is increased. Every one is familiar with the rapid wasting which is such a characteristic of high fever; it indicates not only too rapid metabolism of the body, but also insufficient time for the tissues to again build themselves 
up. In certain fevers, therefore, there may be supposed to be some interference with the ordinary cutaneous reflex channels by which the usual temperature sensory stimuli in the skin are prevented from producing the reflexes that result in diminished production of heat in the muscles and other tissues. In consequence of this, and in spite of the condition of increased heat of the body, both at the surface and in the deeper tissues, the production of heat goes on at an abnormal rate. It is not certain whether the pathological condition is one which stimulates the thermogenic center by means of which the metabolism of the tissues is increased, or whether the normal reflexes which ordinarily inhibit the activity of the center when the temperature rises fail to bring about their usual reactions. The first is the probable explanation of the high fevers of certain toxemias.

Drugs may markedly interfere with the function of the thermogenic mechanism. For example, in anesthesia for operative purposes the temperature of the body falls below the normal unless heat loss is prevented. If too great loss occurs so that the body temperature as measured in the rectum (determined on cats) falls to $25^{\circ} \mathrm{C}$., and lower, the heat regulating mechanism ceases to be operative. It does not regain its function without the aid of artificial heat. But recovery of the thermogenic function may be accomplished by artificial aid even after the rectal temperature has fallen to as low as $16^{\circ} \mathrm{C}$. 


\section{CHAPTER XIII.}

\section{MUSCLE-NERVE PHYSIOLOGY.}

The structure of muscle, of nerve, and of nerve relations to muscle are all given in considerable detail in Chapter II, to which the reader is referred

\section{CHEMICAL COMPOSITION OF MUSCLE.}

Muscle Plasma.-The principal substance which can be extracted from muscle, when examined after death, is the protein body, myosin. This body appears to bear somewhat the same relation to the living muscle that fibrin does to the living blood, since the coagulation of muscle after death is due to the formation of myosin. Thus, if coagulation be delayed by removing the muscles immediately that an animal is killed, and rapidly cooling them to a temperature below $0^{\circ} \mathrm{C}$. before the muscles themselves lose their irritability, it is possible to express a viscid fluid of slightly alkaline reaction, called muscle plasma (Kühne). Muscle plasma, if exposed to the ordinary temperature of the air, undergoes coagulation much in the same way as does blood plasma under similar circumstances when separated from the blood corpuscles at a low temperature. The appearances presented by the fluid during the process are also very similar to the phenomena of blood-clotting, viz., first of all an increased viscidity appears on the surface of the fluid, and at the sides of the containing vessel, which gradually extends throughout the entire mass, until a fine transparent clot is obtained. In the course of some hours the clot begins to contract, and to squeeze out of its meshes a fluid corresponding to blood serum. In the course of coagulation, therefore, muscle plasma separates into muscle clot and muscle serum. The muscle clot contains the substance myosin. It differs from fibrin in being easily soluble in a ro per cent. solution of sodium chloride. It is insoluble in distilled water, and its solutions coagulate on application of heat; in short, it is a globulin. During the process of clotting the reaction of the fluid becomes distinctly acid.

The coagulation of muscle plasma can be prevented not only by cold, but also, as Halliburton has shown, by the presence of neutral salts in certain proportions; for example, of sodium chloride, magnesium sulphate, or sodium sulphate. It will be remembered that this is also the case with blood plasma. Dilution of the salted muscle plasma will produce slow coagulation.

It is highly probable that the formation of muscle clot is due to the presence of a ferment, myosin ferment. 
The relation between the proteins of living and dead muscle is represented by Halliburton as follows:

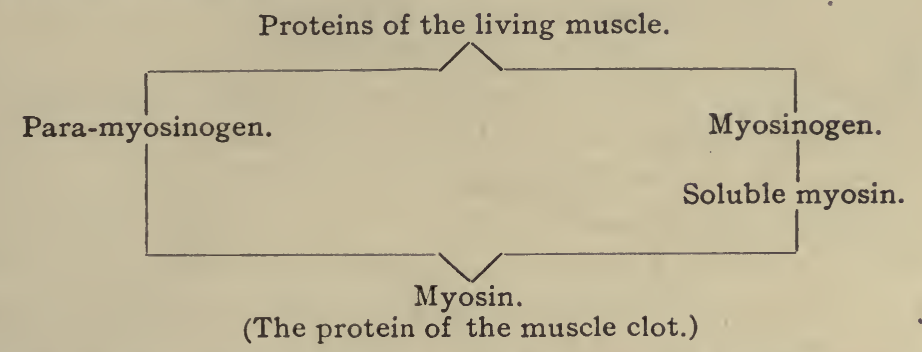

About 75 per cent. of the total protein content of living muscle is myosinogen and the remaining 25 per cent. is para-myosinogen. These proteins may be separated by subjecting the muscle plasma to fractional coagulation. The para-myosinogen coagulates at $47^{\circ} \mathrm{C}$. and the myosinogen at $56^{\circ} \mathrm{C}$. Para-myosinogen is a globulin since it responds to the precipitation tests of this group of proteins and is insoluble in water. Myosinogen, on the contrary, is not a typical globulin since it is soluble in water, but is a pseudo-globulin.

Other Constituents of Muscle.-In addition to muscle ferments already mentioned, muscle contains certain proteolytic enzymes, as do other tissues, an amylolytic ferment, a maltase and a lactic-acid forming enzyme.

Certain acids are also present, particularly sarco-lactic acid.

Of carbohydrates, glycogen and dextrose are present. Glycogen is present in considerable amount, especially in the muscles of well-nourished animals.

Nitrogenous crystalline bodies, such as creatin, creatinin, xanthin, hypoxanthin, carnin, guanin, urea in very small amount, uric acid, and inosinic and phospho-carnic acids, are all found on extracting dead muscle.

Chlorides and salts of potassium, calcium, magnesium, and iron are present in muscle, the chief of which is potassium phosphate.

In extracts of muscles, especially of red muscles, there is a certain amount of hemoglobin, and also of a pigment special to muscle, called by McMunn myo-hematin, which has a spectrum quite distinct from hemoglobin, viz., a narrow band just before $\mathrm{D}$, two very narrow bands between $\mathrm{D}$ and $\mathrm{E}$, and two other faint bands, near $\mathrm{E} b$, and between $\mathrm{E}$ and $\mathrm{F}$ close to $\mathrm{F}$.

Fats occur in the connective tissue around and between the muscle fibers, and lipoids and fat droplets also are present within the individual muscle fibers.

\section{THE PROPERTIES OF LIVING MUSCLE.}

Elasticity.-Muscle has a certain amount of elasticity during rest. It admits of being considerably stretched, but returns readily and completely 
to its normal condition. In the living body the muscles are always stretched somewhat beyond their natural length, they are always in a condition of slight tension: an arrangement which enables the whole force of the contraction to be utilized in approximating the points of attachment. If the extensibility of a given muscle be measured by adding to it equal increments of weight, it will be found that the extension or stretching is considerable at first, but that the amount decreases with each additional weight. If the figures obtained be plotted on co-ordinate paper, a curve approaching a parabola is obtained, whereas a steel spring is perfectly elastic and gives a straight line. When the weights are removed from a stretched muscle, one by one, the muscle regains its original length, though slowly. Extreme fatigue greatly decreases the elasticity, while an increase of temperature increases it.

Cardiac muscle and smooth muscle both manifest elasticity in the same manner as skeletal muscle. In fact, the elasticity of the arterioles is chiefly due to the smooth muscle in their walls, a fact that is of great importance in the adaptability of the circulatory apparatus. The flexibility of the stomach, the urinary bladder, etc., is traceable to the same property of their muscular walls.

Contractility and Irritability of Muscle.-The property of muscular tissue by which its peculiar functions are exercised is its contractility, which is excited by all kinds of stimuli applied either directly to the muscles or indirectly to them through the medium of their motor nerves. The property of the muscle which enables it to respond to a stimulus is called its irritability. This property, although commonly brought into action through the nervous system, is inherent in the muscular tissue. This is proven: $\mathrm{x}$. By the fact that contractility is manifested in a muscle which is isolated from the influence of the nervous system by division of the nerves supplying it so long as the natural tissue of the muscle is duly nourished. 2. It is manifested in a portion of muscular fiber in which, under the microscope, no nerve fiber can be traced. 3. Substances such as curara, which paralyze the nerve endings in muscles, do not at all diminish the irritability of the muscle itself. 4 . When a muscle is fatigued, a local stimulation is followed by a contraction of a small part of the fiber in the immediate vicinity, without any regard to the distribution of nerve fibers.

Forms of Stimuli for Muscle or Nerve.-The power of contraction in voluntary muscles is normally called forth in the body by nerve impulses which reach the muscles over the motor nerves. But a muscle will respond to stimuli of various kinds, and these stimuli may be applied directly to the muscle or indirectly to the nerve supplying it. There are distinct advantages, however, in applying the stimulus to the nerve, as it is more convenient as well as more potent. The stimuli which will produce contraction in a muscle are:

I. Mechanical Stimuli.-A blow, pinch, prick of the muscle or its nerve 
will produce a contraction, repeated on the repetition of the stimulus. If applied to the same point for a number of times such stimuli will soon destroy the irritability of the preparation.

2. Thermal Stimuli. - If a needle or glass rod be heated and applied to a muscle or its nerve, the muscle will contract. A temperature of over $45^{\circ} \mathrm{C}$. will cause the muscles of a frog to pass into a condition known as heat rigor. The sudden change of temperature acts as a stimulus.

3. Chemical Stimuli.-A great variety of chemical substances will excite the contraction of muscles, some substances being more potent in irritating the muscle itself and other substances having more effect upon the nerve. Of the former may be mentioned dilute acids, salts of certain metals, e.g., zinc, copper, and iron; to the latter belong strong glycerin, strong acids, ammonia, bile salts in strong solution, etc.

4. Electrical Stimuli.-Any form of electrical current may be employed to stimulate a muscle to contract, but either galvanism or the induced current is usually chosen. For experimental purposes electrical stimuli are most frequently used, as the strength of the stimulus may be conveniently regulated. In order that a stimulus shall be effective, it must have a certain amount of energy and the application to the muscle must have a certain abruptness. For example, a comparatively weak galvanic current suffices to stimulate a muscle to action when suddenly applied in full force. But if the electric current be applied very gradually, a current many times stronger will fail to arouse contraction of a muscle.

Conductivity in Muscle.-In an ameba or other simple undifferentiated contractile protoplasmic unit a stimulus applied at any point is quickly transmitted throughout the entire mass. Just so is it with differentiated muscle. A stimulus applied at any point of a muscle will quickly be propagated through the mass as far as there is protoplasmic continuity. In cardiac muscle and in smooth muscle there is uninterrupted conduction from cell to cell. But in voluntary muscle each fiber is physiologically isolated from its neighbors. When a voluntary muscle fiber is stimulated either at the extremities or at its middle, the effect of the stimulus quickly passes through the entire fiber, whether it arouses a distinct act of contraction or not.

The rate at which conduction takes place when a contraction accompanies it has been carefully measured by numerous observers. It varies greatly in the different kinds of muscle, from two-tenths of a meter per second in the rabbits' ureter (Engelmann) to ten meters per second in the voluntary muscles of man.

\section{SINGLE MUSCLE CONTRACTIONS.}

Characteristics of a Single Contraction.-The Myogram.-The contraction of a muscle in response to a single effective stimulus of short duration is called a simple muscle contraction. A record of such a contraction 
is called a myogram. The character of the myogram, and therefore the facts revealed by it, are dependent on whether or not the record is made on a rapidly moving recording surface. If the myogram is made on a recording surface that is standing still, then it shows merely the extent of shortening of the muscle. The amount of shortening for a given muscle will depend on a series of conditions, such as nutrition, load, temperature, etc., all of which will be discussed presently.

When the record is made on a rapidly moving drum or on the pendulum myograph, it is revealed that the simple contraction occupies a definite period of time with well-marked periods or phases. Although the stimulus may be practically instantaneous, the contraction lasts a considerable fraction of a second, in the frog's gastrocnemius about 0 . I of a second.

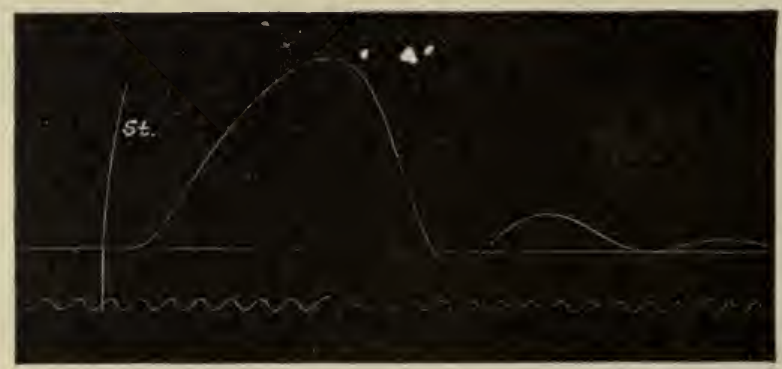

Fig. 322 - Record of a Simple Contraction of the Gastrocnemius of the Frog. Time in 0.01 second. St, Moment of stimulation. Record taken on a rapid drum that was provided with an automatic key.

It will be observed that after the stimulus has been applied, as indicated by the vertical line $S t$, there is an interval before contraction commences. This interval, termed the latent period, when measured by the number of vibrations of the tuning-fork directly beneath, is found to be about $0.0 \mathrm{I}$ of a second. The latent period is longer in some muscles than in others and differs also according to the condition of the muscle and the kind of stimulus employed. During the latent period there is no apparent change in the muscle. The second part of the record shows the contraction phase proper. The lever is raised by the sudden shortening of the muscle. The contraction is at first very rapid, but then progresses more slowly to its maximum. It occupies on an average 0.04 of a second in the frog's gastrocnemius. The third stage is the relaxation phase. After reachirg its highest point, the lever begins to descend, in consequence of the elongation of the muscle. At.first the fall is rapid, but it then becomes more gradual until the lever reaches the abscissa or base line, when the muscle has attained its precontraction length. The stage occupies 0.05 of a second. Usually after the contraction proper is over the lever oscillates below and above the base line in a series of diminishing waves, the elastic rebound following movement of the simple contrac- 
tion. These are, of course, wholly passive and would occur equally well if we should lift the weight to the height of the contraction, then simply let it fall while taking a record.

Change in Shape during Muscular Contraction.-There is a considerable difference of opinion as to the mode in which the transversely striated muscular fibers contract. The most probable account is that the contraction is effected by an approximation of the constituent parts of the fibrils, which, at the instant of contraction, without any alteration in their general direction, become closer, flatter, and wider, a condition which is rendered evident by the approximation of the transverse striæ seen on the surface of the fasciculus, and by its increased breadth and thickness. The appearance of the zigzag lines into which it was supposed the fibers are thrown in contraction is due to the relaxation of a fiber which has been recently contracted and is not
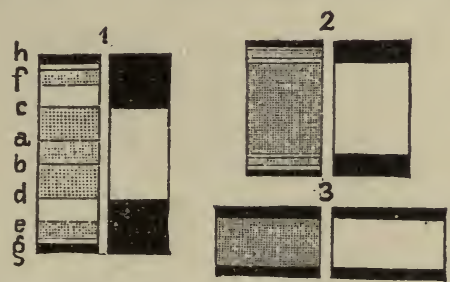

FIG. 323. - The Microscopic Appearances During a Muscular Contraction in the Individual Fibrillæ, after Engelmann. I. A passive muscle fiber; $c$ to $d=$ doubly refractive discs, with median disc $a b$ in it; $h$ and $g$ are lateral discs; $f$ and $e$ are secondary discs, only slightly doubly refractive; figure on right same fiber in polarized light. The bright part is doubly refracted, black ends not so. 2. Transition stage. 3. Stage of entire contraction. In each case the right-hand figure represents the effect of polarized light. (Landois, after Engelmann.)

at once stretched again by some antagonist fiber or whose extremities are kept close together by the contractions of other fibers. The contraction is therefore a simple and, according to Edward Weber, a uniform, simultaneous, and steady shortening of each fiber and its contents. What each fibril or fiber loses in length, it gains in thickness. The contraction is a change of form, not of size; it is, therefore, not attended with any diminution in bulk from condensation of the tissue. This has been proved for entire muscles by making a mass of muscles, or many fibers together, contract in a vessel full of water, with which a fine, perpendicular, graduated tube communicates. Any diminution of the bulk of the contracting muscle would be attended by a fall of fluid in the tube, but when the experiment is carefully performed, the level of the water in the tube remains the same, whether the muscle be contracted or not.

In thus shortening, muscles appear to swell up, becoming rounder, more prominent, harder, and apparently tougher. But this hardness of muscle in the state of contraction is not due to increased firmness or condensation of the muscular tissue, but to the increased tension to which the fibers, as well as 
their tendons and other tissues, are subjected from the resistance ordinarily opposed to their contraction. When no resistance is offered, as when a muscle is cut off from its tendon, not only is no hardness perceived during contraction, but the muscular tissue is even softer and more extensible than in its ordinary uncontracted state. During contraction in each fiber it is said that the anisotropous or doubly refractive elements become less refractive and the singly refractive more so, figure $32 \mathrm{I}$.

Chemical Changes in Contracting Muscle.-I. The reaction of the muscle, which is normally alkaline or neutral, becomes decidedly acid during contraction, from the development of sarcolactic acid. 2. The muscle gives out carbon dioxide gas and takes up oxygen. The amount of the carbon dioxide given out does not appear to be entirely dependent upon the oxygen taken in, and so doubtless in part arises from some other source. Muscle contracts in an atmosphere of hydrogen, showing that oxygen is present in fixed combination. A muscle, however, contracts for a longer time in an atmosphere of oxygen. 3. Certain imperfectly understood chemical changes occur, in all probability connected with I and 2 , in which glycogen is converted into dextrose and the latter oxidized.

Electrical Changes in Contracting Muscle.-Resting muscles uninjured in the body have a uniform potential, i.e., are isoelectric. But when removed from the body they are more or less injured and, therefore, show differences of electrical potential between different points on the muscle, called currents of injury or demarcation currents. It is necessary to use non-polarizable and not metallic electrodes in this experiment, as otherwise there is no certainty that the whole of the current observed is communicated from the muscle itself and not derived from the metallic electrodes and arising in consequence of the action of the saline juices of the tissues upon them. Non-polarizable electrodes are usually some modification of Du Bois Reymond's apparatus, which consists of a cylinder filled with china clay moistened with saline solution, part of which projects as a drawn-out point for contact with the muscle. The rest of the cylinder is filled with a saturated solution of zinc sulphate into which dips a well-amalgamated piece of zinc connected by wire with the galvanometer. In this way the zinc sulphate and the sodium chloride form a non-polarizable conductor between the zinc and the live muscle. Recently Porter has devised a bootshaped clay electrode that is burned, and hence has the immense advantage of permanency.

Currents of Injury, or Demarcation Currents.-If a segment is cut out of a living gastrocnemius, its cut ends present regions of maximal injury. Such a preparation is called. a muscle prism.

If the points on the surface of a muscle prism be connected with the galvanometer by non-polorizable electrodes, it will be found that the currents pass from point to point, as shown in the diagram, figure 324 . 
The strongest currents pass from the equator to a point representing the middle of the cut ends; currents also pass from points nearer the equator to those more remote, but not from points equally distant, which are isoelectric points. The cut ends are always negative to the equator. The currents are in all probability due to chemical changes going on in the muscles at the injured ends.

Action Currents. - When a muscle is made to contract the demarcation current undergoes a sharp decrease, as shown by the deflection of the galvanometer needle, which swings back in the direction of equilibrium. This deflection, originally called the current of negative variation, has been shown to be due to the processes going on in the muscle during contraction, and is therefore more properly called the action current. It occurs where no previous demarcation current exists.

For the study of the action current the capillary electrometer is very convenient. The hearts of cold-blooded animals, because of their slow contraction, serve well for demonstration. The muscle contraction passes over

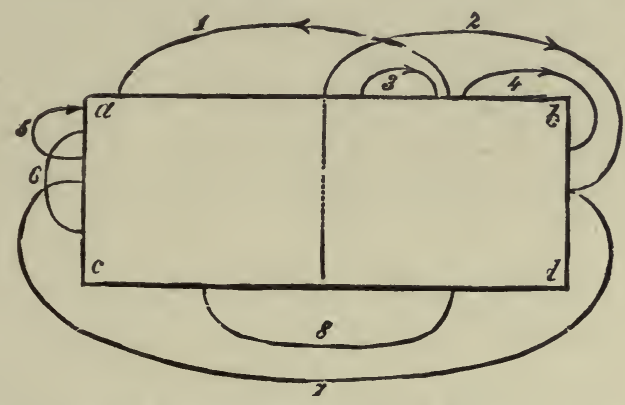

Fig. 324.-Diagram of the Currents in a Muscle Prism. (Du Bois Reymond.)

the ventricle in the form of a wave, the electric potential of the muscle changing as it becomes active or passive. For any two points on the heart muscle, therefore, there will be two changes of potential, the active part first becoming negative to the inactive, and then, as the wave passes down and the inactive part becomes active, the current is reversed. This is known as a diphasic current.

In certain fishes definite electrical organs exist, organs which are derived from muscle-like tissues and which may be regarded morphologically as muscles highly specialized for the production of energy in the form of electricity.

Heat Produced in a Simple Contraction.-Becquerel and Breschet found, with the thermo-multiplier, about $0.5^{\circ} \mathrm{C}$. of heat produced by each forcible contraction of a man's biceps; and when the actions were long continued, the temperature of the muscle increased $\mathrm{I}^{\circ} \mathrm{C}$. In the frog's muscle a considerable number of contractions have been found to produce an elevation of temperature equal on an average to less than $0.2^{\circ} \mathrm{C}$., while a single contraction produces, according to R. Heidenhain, from $0.00 \mathrm{I}^{\circ}$ to $0.005^{\circ} \mathrm{C}$. 
One gram of frog's muscle will produce in a single maximal contraction about 0.003 calorie or the equivalent of 128 gramcentimeters of work energy (since I calorie $=0.4267$ kilogrammeter of work). The cause of the rise of temperature is the increased chemical activity at the time of contraction. As we have already seen, in the chapter on Animal Heat, muscles have the power of producing heat even when not contracting, i.e., changing shape.

The amount of heat energy developed during a single contraction will vary sharply according to the tension under which the muscle contracts. The heat production follows closely the energy of work produced, and apparently obeys the same laws.

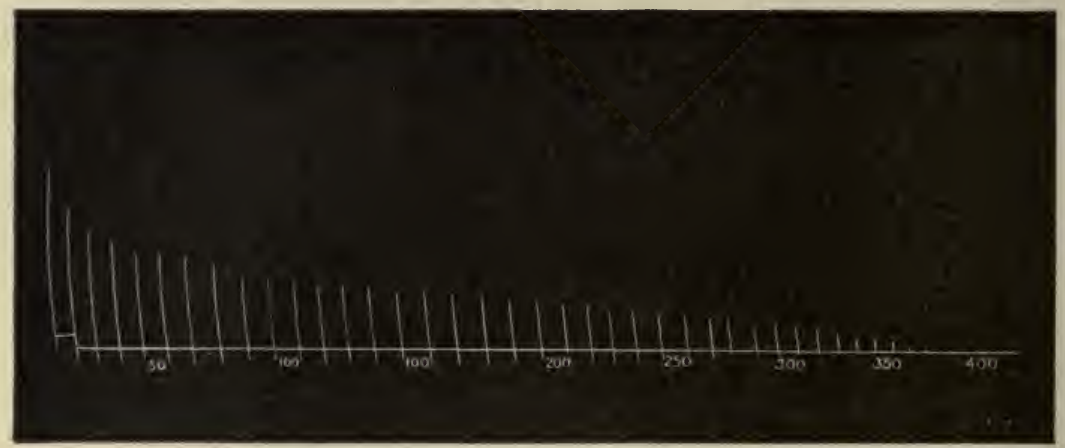

Fig. 325. - Figure for Work Energy, Showing Height of the Contraction of the Gastrocnemius of the Frog with Loads Increased by Ten Grams at a Time.

The Work Energy Liberated by a Simple Muscle Contraction.When a muscle contracts against a resistance and a load is moved, work energy is liberated. In fact, the liberation of work energy and heat energy are the specific functions of the muscles among the warm-blooded animals. A frog's gastrocnemius weighing I gram and loaded with 50 grams will contract from 0.5 to $0.6 \mathrm{~cm}$; i.e., will do 25 to 30 gramcentimeters of work

Table Showing the Relation Between Load and Work.

\begin{tabular}{c|c|c}
\hline $\begin{array}{c}\text { Load or tension. } \\
\text { Grams. }\end{array}$ & $\begin{array}{c}\text { Height lifted. } \\
\text { Centimeters. }\end{array}$ & $\begin{array}{c}\text { Work done. } \\
\text { Gramcentimeters. }\end{array}$ \\
\hline & & \\
40 & 1.2 & 32 \\
80 & 0.8 & 40 \\
120 & 0.5 & 48 \\
160 & 0.4 & 32 \\
200 & 0.2 & 20 \\
240 & 0.1 & 0 \\
\hline
\end{tabular}


for each simple contraction. The amount of work done is intimately associated with the tension under which the muscle contracts. As the tension increases from no load up to I00 or 150 grams (for a I-gram muscle), the work increases. But as the tension continues to increase, the work falls off until a point is reached at which the load is not lifted at all.

\section{CONDITIONS WHICH AFFECT THE IRRITABILITY OF THE MUSCLE AND THE CHARACTER OF THE CONTRACTION.}

There are a number of conditions which influence both the irritability of a muscle and the power and character of its contractions. Irritability and contractility may vary independently, but as a rule any condition which decreases one also decreases the other. The most important of these conditions are: relation of the muscle to the central nervous system, condition of nutrition, stimulus, temperature, fatigue, drugs, disease, etc.

Effect of the Strength of Stimulus.-A strength of current that is just sufficient to give the contraction of a muscle is called a minimal stimulus.

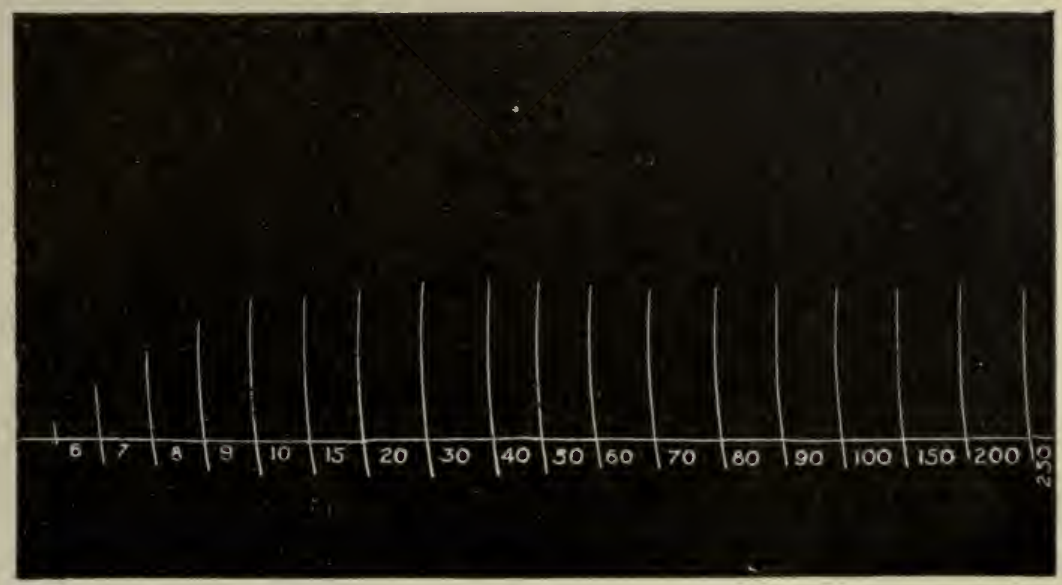

FIG. 326.-Contraction of the Gastrocnemius Under the Intluence of Variation of Strength of Stimulus. The muscle was stimulated by Petzold's inductorium, graduated to show units of current. The figures $6,7,8,9$, ro, etc., indicate relative strength of stimulus.

This is a comparatively weak induction current, one which can scarcely be detected by the tip of the tongue. As the strength of the current is very gradually increased, the height of the contraction curve increases until the maximal stimulus is reached, which produces a contraction of an amplitude beyond which no increase occurs even though the strength of the stimulus be multiplied many fold. The range between the strengths of the minimal and maximal stimuli is very restricted indeed. The absolute strength of a mini- 
mal stimulus varies exceedingly for a given muscle, depending on its degree of irritability. This narrow range between minimal and maximal stimuli serves as a convenient means for detecting the variations in irritability. One should count on a continued decrease in irritability in isolated muscles, hence should choose a supramaximal stimulus for all such preparations when other conditions surrounding the muscle are under investigation.

The Influence of Repeated Activity.-The irritability of muscle is decreased by undue functional activity. The cause of the diminished irritability is twofold: when a muscle contracts, part of its substance is expended. part of its store of nutriment is exhausted, and it cannot contract so

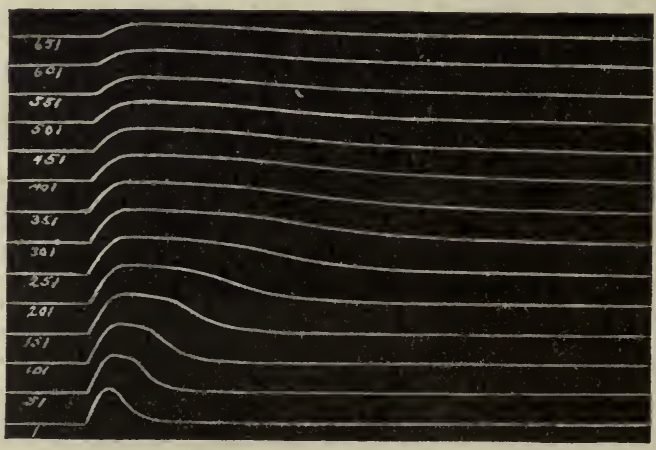

FIG. 327.-Contractions of the Gastrocnemius Muscle to Show Fatigue. The numbers printed on the figure indicate the contractions in the series which is recorded. (Lee.)

vigorously again until the loss is made up. To this extent fatigue has much the same effect as cutting off or diminishing the blood supply. The other cause for the diminution of irritability is the accumulation of poisonous products in the muscle, substances generated during contraction, probably sarcolactic acid chiefly. In a living animal these poisonous products exert their influence not only upon the muscle or muscles immediately concerned in contraction, but upon the musculature of the body generally, and the effect remains until they are eliminated from the body. Massage of the muscles increases the passage of waste products into the general blood stream and the rapidity of their elimination.

In the first few simple contractions, repeated in series, there is an increase in the amplitude of the contractions resulting in the phenomenon known as staircase contractions or "Treppe." This stage is followed by a period of sustained contractions, and this finally by a diminishing series of amplitudes until the muscle fails to respond. After a few minutes' rest a muscle will again give strong contractions, but only for a brief series.

If the time of the simple contractions is measured, it will be found, figure 327 , that not only is the amplitude decreased, but the duration is greatly 
increased as the contractions are repeated. The latent period changes very little. The contraction phase is considerably prolonged, but the relaxation phase is very greatly increased. As fatigue progresses, the total time of the simple contraction may be two or three times longer than the normal. The ability of the muscle to do work falls off rapidly, of course; and the greater the load during the time fatigue is coming on, the more quickly complete fatigue approaches.

The Influence of Temperature.-The irritability of muscle is increased by raising its temperature slightly above that of the animal from which it has been taken, while it is decreased by cooling. If, however, the temperature be raised too high $\left(40^{\circ} \mathrm{C}\right.$. for frog, $50^{\circ} \mathrm{C}$. for mammal), the muscle enters into a condition of heat rigor and its irritability is forever lost.

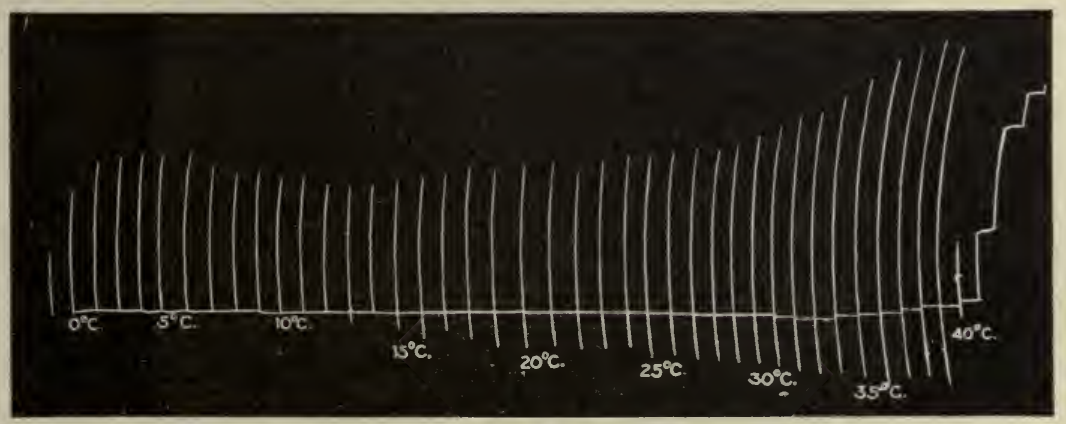

Fig. 328.-Contractions of the Gastrocnemius Muscle to Show the Influence of Temperature on the Amplitude of the Contractions. At $40^{\circ} \mathrm{C}$. the muscle has begun to pass into rigor mortis, yet is able to give short contractions. The steps on the curve of rigor at the right occur at temperatures of $4 \mathrm{I}^{\circ}, 42^{\circ}$, and $43^{\circ} \mathrm{C}$.

After cooling, unless the cold be too severe and prolonged, the irritability returns as the temperature is raised. A series of vertical records of simple contractions beginning at room temperature and decreasing with a contraction at each fall of one degree reveals the fact that the amplitude falls off slowly until a temperature of $12^{\circ}$ to $10^{\circ} \mathrm{C}$. is reached, then as gradually increases until $4^{\circ}$ to $2^{\circ} \mathrm{C}$., after which the amplitude drops off sharply to about $-\mathrm{I}^{\circ} \mathrm{C}$. However, this phenomenon is partly one of irritability, since a very strong stimulus will produce a vigorous contraction until the muscle begins to freeze. If at the freezing temperature the muscle be slowly and carefully increased in temperature it will recover from the effects of the cooling without apparent injury, and will give a reverse series to the one obtained by decreasing the temperature. As the increase of temperature is continued above room temperature the amplitude of the contractions very greatly increases (also the elasticity), reaching a maximum in the frog's gastrocnemius at about $35^{\circ}$ to $36^{\circ} \mathrm{C}$. The amplitude sharply decreases above 
$35^{\circ} \mathrm{C}$. up to $37^{\circ}$ to $38^{\circ} \mathrm{C}$, where heat rigor begins and the muscle permanently shortens. Heat rigor is usually complete at $40^{\circ}$ to $4 \mathrm{I}^{\circ} \mathrm{C}$. A muscle cannot recover its irritability after heat rigor has set in strongly.

If the time of the contraction is measured at different temperatures it will be found to be greatly delayed at $2^{\circ}$ to $4^{\circ} \mathrm{C}$., and very much quicker than normal at $33^{\circ}$ to $35^{\circ} \mathrm{C}$. As in fatigue, the effect falls chiefly on the contraction and relaxation phases and only slightly on the latent period. The latent period is more sharply influenced by temperature than by fatigue.

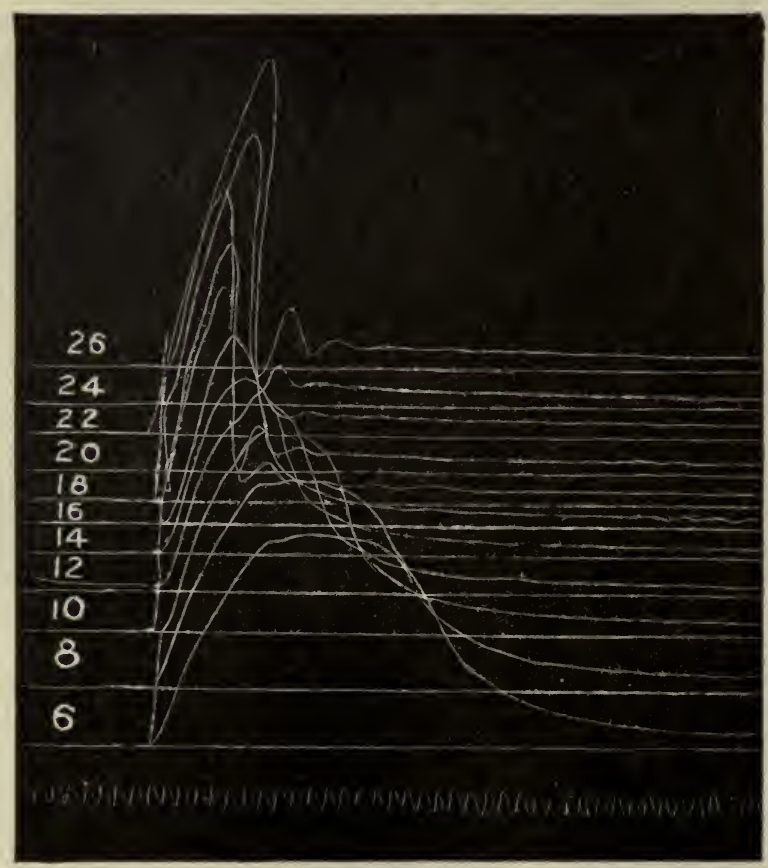

Fig. 329.-Influence of Temperature on the Duration of the Contraction of the Frog's Gastrocnemius.

Influence of Blood Supply. - In the normal human muscle there is a delicately balanced vaso-motor mechanism by which the amount of blood flowing through a muscle is immediately increased when the muscle is in contraction. This blood stream is of course carrying nutritive materials to the muscle and taking away wastes. If the blood supply to a muscle is cut off, then the muscle can only draw on its stored supply of potential energy, which in active contraction is sooner or later exhausted. Under such conditions the muscle increases in irritability for a few minutes and then gradually loses both its irritability and its power to contract. Even mammalian muscles have been kept alive and normal in their activity for several hours by irri- 
gating them with defibrinated and aerated blood (von Frey). Mammalian muscles will remain irritable for 30 minutes, or longer if cooled, after being shut off from their blood supply and isolated from the body, but both irritability and contractility soon disappear entirely.

Effect of Nerve Supply.-The voluntary or skeletal muscle normally contracts in the body only when stimulated through its motor nerve. If the motor nerve is severed, the muscle is cut off from its normal source of activity, hence will undergo the changes resulting from disuse, which will be presently discussed. Aside from this, it is held by most observers that there are distinct nutritive or trophic nerves which exercise a controlling influence over the growth, development, and general nutritive processes going on in muscle.

When a motor nerve is cut, the muscle at first exhibits heightened irritability to all forms of stimuli. In a couple of weeks it decreases in its power to respond to rapidly changing stimuli like induced currents. It responds more readily to mechanical shocks and to galvanic currents for six or seven weeks, then gradually loses the power of contracting through as many months. The changes are due to protoplasmic degeneration. It is not clear in what degree these changes are due to loss of trophic nerve influence, to inactivity, and to changes in nutritive conditions. Since degeneration occurs when the vascular supply is maintained, it would seem that the nutritive condition must be chargeable to one or the other of the first two factors, probably to both.

Use of muscle increases its power and also its irritability. A properly regulated exercise is well known to contribute to the health and development of muscles. In cases of paralysis, mechanical or electrical stimulation is applied directly to the muscle in an effort to supply artificial exercise until the nerves are regenerated and motor connections re-established. If such stimulation is not applied, the muscle degenerates from disuse and loses its irritability often before the nerves regenerate.

The Effect of Drugs.-Drugs affect the irritability of muscle, some augmenting, others depressing it. Voluntary muscle, which does not ordinarily contract except when stimulated, can be made so irritable by certain salts that it contracts automatically like heart muscle, and the converse. Ether, chloroform, etc., anesthetize muscle just as they do nerve, suppressing both irritability and contractility. Suprarenal extract increases the amplitude of contraction, as do also caffeine, digitalis, nicotine, and others. Veratrine is well known greatly to prolong the relaxation phase of the simple contraction without materially affecting the contraction phase or the latent period.

\section{TETANIC AND VOLUNTARY MUSCULAR CONTRACTIONS.}

Effect of Rate of Stimulation.-If we stimulate the muscle-nerve preparation with two induction shocks, one immediately after the other, when the 
point of stimulation of the second one corresponds to the crest of the contraction of the first, a second curve, figure 330 , will occur, which will commence near the highest point of the first and will rise nearly as much higher, so that the sum of the height of the two curves almost exactly equals twice the height of the first. This phenomenon is called summation. If a third and fourth shock be passed, a similar effect will ensue, and curves one above the other will be traced, the third being slightly lower than the second, and the fourth than the third. If a continuous series of shocks occur, however, the lever after a time ceases to rise any further, and the contraction, which has reached

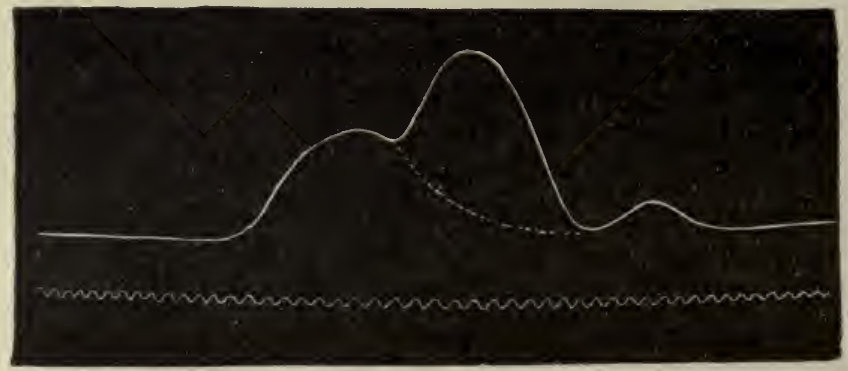

FIG. 330.-Tracing of a Double Muscle Curve. To be read from left to right. While the muscle was engaged in the first contraction (whose complete course, had nothing intervened, is indicated by the dotted line), a second induction shock was thrown in, at such a time that the second contraction began just as the first was beginning to decline. The second curve is seen to start from the first, as does the first from the base line. (M. Foster.)

its maximum, is maintained. The condition which ensues is called Tetanus. A tetanus is really a summation of contractions, but unless the stimuli become very rapid indeed, the muscle will still be in a condition of vibratory contraction and not of unvarying contraction.

If the shocks, however, be repeated at very short intervals, varying, in the frog, from eighteen to thirty per second, the muscle contracts to its utmost at once and continues at its maximum contraction for some time. The lever rises almost perpendicularly and then describes a straight line, figure $33 \mathrm{I}, c$. The rate of stimulation required increases with the rapidity of the simple contraction. If the stimuli are not so rapid the line of maximum contraction becomes wavy, indicating a tendency of the muscle to relax during the intervals between the stimuli, figure $33 \mathrm{I}, b$. As the muscle becomes fatigued, a less rapid rate of stimulation is required to produce a complete tetanus, owing to the prolongation of the relaxation period in such a muscle. The height of the contraction, however, is lessened. This condition of prolonged relaxation is known as contracture.

Co-ordinated Muscular Contractions. - In the human body the skeletal muscles contract only on stimulation through their motor nerves; i.e., under the influence of nerve impulses that have their origin in the central 
nervous system. Such motor impulses may arise through reflexes, through automatic activity of the nerve center, or by higher cerebral origin associated with conscious psychic effort. In either case the apparatus consists of one or more central neurones, an anterior-horn motor cell, and the muscle itself. Conscious or voluntary effort may be taken as a type.

Simple contractions are possible to human muscles, but undoubtedly tetanic contractions are the rule. If one holds the arm out at right angles to
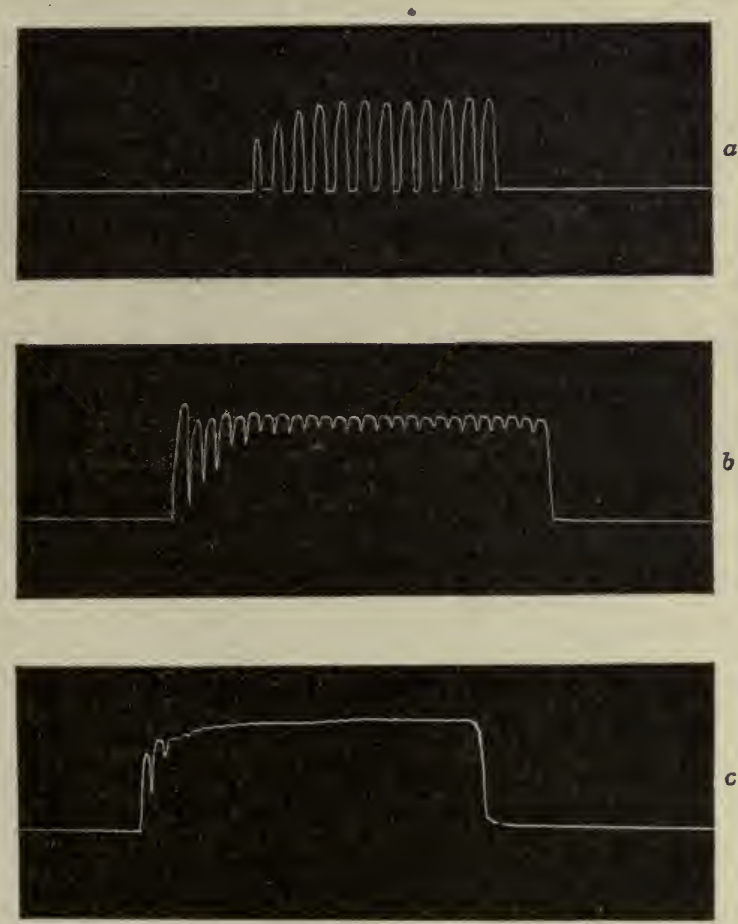

FIG. 33r.- $a$, Frog's gastrocnemius muscle stimulated with four induction shocks per second, showing complete relaxation between stimuli; $b$, same muscle stimulated eight times per second, showing partial relaxation between stimuli (incomplete tetanus); $c$, same muscle stimulated twelve times per second, showing development of an almost complete tetanus.

the trunk, the movement requires the continuous or tetanic contraction of the deltoid and the series of extensor muscles. If the arm is retained in the extended position long enough, extreme fatigue is felt and presently one can no longer maintain the position. Yet, if the muscles involved are immediately stimulated directly with an electric current, they contract, showing that such exhaustion as exists is not wholly due to the muscle.

Mosso's ergograph was devised for the specific purpose of studying the character of fatigue of voluntary effort. This apparatus is adapted to the 
study of the fatigue of the flexors of the middle finger or, in the newer instrument devised by Storey, to the abductor of the index finger. Numerous studies have shown, apparently, that the fatigue of voluntary effort involves, first, the nervous apparatus and, later, the muscle; that the muscle still retains a considerable reserve of energy when the apparatus as a whole is exhausted.

Muscle in Rigor Mortis.-After the muscles of the dying body have lost their irritability or capability of being excited to contraction by the application of a stimulus, they spontaneously pass into a state of shortening apparently identical in effect with that which ensues during life. It affects all the muscles of the body, and, when external circumstances do not prevent it, commonly fixes the limbs in that which is their natural posture of equilibrium or rest. From the simultaneous contraction of all the muscles of the trunk, a general stiffening of the body is produced, which constitutes the rigor mortis or postmortem rigidity.

When this condition has set in, the muscle becomes acid in reaction (due to development of sarcolactic acid), gives off carbonic acid in great excess, diminishes in volume slightly, becomes shortened and opaque, its substance sets in a firm coagulation. Rigor mortis comes on much more rapidly in death after muscular activity, and is hastened by warmth.

The immediate cause of rigor mortis seems to be a chemical one, namely, the coagulation of the muscle plasma. We may distinguish three main stages: I, gradual coagulation; 2, contraction of the coagulated muscle clot (myosin) and, 3, squeezing out of muscle serum. During the first stage, restoration is possible, by the circulation of arterial blood through the muscles, and even when the second stage has set in, vitality may be restored by dissolving the coagulum of the muscle by salt solution and by passing arterial blood through the vessels. After the second stage is advanced, recovery is impossible.

It has been noticed that the relaxation in muscles after rigor sometimes occurs too quickly to be caused by putrefaction. The suggestion that in such cases the relaxation is due to a ferment action is very plausible. Subjecting.fresh muscle to the action of heat $\left(50^{\circ}\right.$ to $60^{\circ} \mathrm{C}$.) or immersing it in distilled water causes a similar coagulation to that of rigor mortis. The former is known as heat rigor, and the latter as water rigor.

The muscles are not affected simultaneously by rigor mortis. It affects the neck and lower jaw first; next, the upper extremities, extending from above downward; and, lastly, reaches the lower limbs. In some rare instances only it affects the lower extremities before or simultaneously with the upper extremities. It usually ceases in the order in which it begins: first at the head, then in the upper extremities, and lastly in the lower extremities. It never ordinarily commences earlier than ten minutes, and never later than seven hours after death; and its duration is greater in pro- 
portion to the lateness of its accession. Heat is developed during the passage of a muscular fiber into the condition of rigor mortis.

Since rigidity does not ensue until muscles have lost the capacity of being excited by external stimuli, it follows that all circumstances which cause a speedy exhaustion of muscular irritability induce an early occurrence of the rigidity, while conditions by which the disappearance of the irritability is delayed are succeeded by a tardy onset of the rigidity of rigor. This is the explanation of its speedy occurrence, and equally speedy departure, in the bodies of persons exhausted by chronic diseases; and its tardy onset and long continuance after sudden death from acute diseases. In some cases of sudden death from lightning, violent injuries, or paroxysms of passion, rigor mortis has been said not to occur at all; but this is not always the case. It

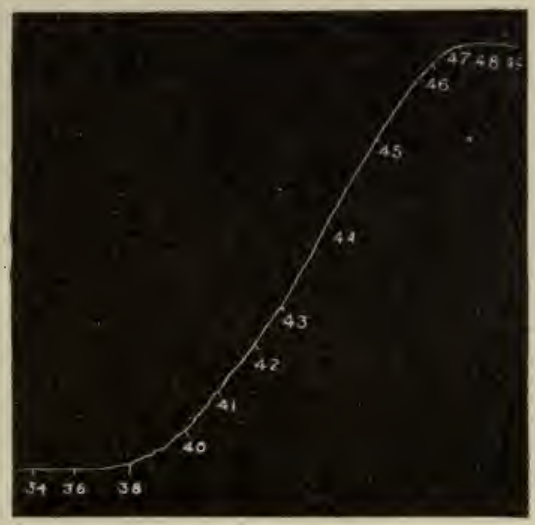

FIG. 332.-Curve of Shortening of the Gastronemius Muscle of the Frog, During Heat Rigor. The numbers indicate degrees centigrade.

may, indeed, be doubted whether there is really a complete absence of the postmortem rigidity in any such cases; for the experiments of BrownSéquard make it probable that the rigidity may supervene immediately after death, and then pass away with such rapidity as to be scarcely observable.

The occurrence of rigor mortis is not prevented by the previous existence of paralysis in a part, provided the paralysis has not been attended with very imperfect nutrition of the muscular tissue.

The rigidity affects the involuntary as well as the voluntary muscles, whether they be constructed of striped or unstriped fibers. The rigidity of involuntary muscles with striped fibers is shown in the contraction of the heart after death. The contraction of the muscles with unstriped fibers is shown by an experiment of Valentin, who found that if a graduated tube be connected with a portion of intestine taken from a recently killed animal, 
and the intestine be tied at the opposite end, and filled with water, the water will in a few hours rise to a considerable height in the tube, owing to the contraction of the intestinal walls. It is still better shown in the arteries, of which all that have muscular coats contract after death, and thus present the roundness and cord-like feel of the arteries of a limb lately removed or those of a body recently dead. Subsequently they relax, as do all the other muscles, and feel lax and flabby and lie as if flattened and with their walls nearly in contact.

Muscular Metabolism During Contraction.-The question of the metabolism of muscle both in a resting and in an active condition has for many years occupied the attention of physiologists. It cannot be said even now to be thoroughly understood. Most of the facts with reference to the subject have been already mentioned. We may shortly recapitulate them here: First, muscle during rest absorbs oxygen and gives out carbon dioxide. This has been shown by an analysis of the gases of the blood going to and leaving muscles. During activity, e.g., during tetanus, the same interchange of gases takes place, but the quantities of the oxygen absorbed and of the carbon dioxide given up are increased, and the proportion between them is altered thus:

\begin{tabular}{|c|c|c|}
\hline Venous blood. & $\begin{array}{c}\mathrm{O}_{2}, \text { less than in arterial } \\
\text { blood. }\end{array}$ & $\begin{array}{l}\mathrm{Co}_{2}, \text { more than in arterial } \\
\text { blood }\end{array}$ \\
\hline Of resting muscle ........ & 9 per cent. & 6.7 I per cent. \\
\hline Of active muscle ....... & 12.26 per cent. & Io.79 per cent. \\
\hline
\end{tabular}

There is, then, a greater proportion of carbon dioxide produced in muscle during activity than during rest.

During rigor mortis there is also an increased production of carbon dioxide.

Second, muscle during rest produces nitrogenous crystallizable substances, such as creatin, from the metabolism which is constantly going on in it during life; in addition there are formed, in all probability, sarcolactic acid and other non-nitrogenous matters.

During activity the nitrogenous substances, such as creatin, undergo very slight, if any, increase above the amount produced during rest-but the sarcolactic acid is distinctly increased. The glycogen stored in the muscle is gradually converted into dextrose and the latter oxidized to furnish the energy developed in the contraction. 
During rigor mortis the sarcolactic acid is increased, and in addition myosin is formed.

From these data it is assumed that the processes which take place in resting and active muscles are somewhat different, at any rate in degree, from actively contracting muscle. Also, there are obtained an increased amount of heat and mechanical work; potential energy is converted into kinetic energy.

\section{THE TYPE OF CONTRACTION IN INVOLUNTARY MUSCLE AND IN CILIA.}

Cardiac Muscle.-Some detail concerning the action of cardiac muscle has already been given in connection with the chapter on Circulation. As compared with the activity of skeletal muscle, cardiac muscle differs most strikingly in that it is automatic. A strip of heart muscle taken from any part of the heart, under proper conditions, gives off a series of contractions, whether it receives any special stimulus or not, whereas we have just found that skeletal muscle under similar conditions remains quiet unless stimulated in some special way. The fibers of skeletal muscle are more or less physiologically isolated from each other, and one fiber may contract without involving contractions of the others. Cardiac muscle, on the other hand, when stimulated at any point conducts the change produced throughout the continuity of the mass. Cardiac muscle contractions are influenced-by tension, temperature, fatigue, etc., apparently, in the same way as skeletal muscle.

When the contraction occurs it is always maximal. The actual amplitude of the contraction is dependent on the condition of nutrition of the cardiac muscle. If the contractions are at a rapid rate they will be relatively of less amplitude. If an extra contraction is induced in an automatic series, so that the interval between two contractions is similar, then the amplitude will be correspondingly reduced. Such an extra contraction is followed by a delayed automatic contraction, the phenomenon of compensatory pause. The contractions in cardiac muscle are simple contractions. In fact, it is said to be impossible to produce a tetanus except in certain invertebrate hearts. This possibility depends upon the fact that during the time of a single contraction there is a certain interval between the beginning and the crest of the contraction, figure $I 74$, in which the heart muscle is not irritable. This is known as the refractory phase.

The duration of the contraction of heart muscle is much greater than the contraction of skeletal muscle. The total time of a contraction in a frog's gastrocnemius is 0 . I of a second, while the time of a contraction of the ventricle in the same animal is at least 0.7 to 0.8 of a second. In the terrapin's cardiac muscle the time of a contraction is over a second, but in the warm- 
blooded cardiac muscle the time is shorter, perhaps from 0.4 to 0.5 of a second for the human ventricular muscle.

Smooth Muscle.-The physiology of smooth muscle has been given to some extent in previous chapters, particularly in connection with the movements of the stomach and intestines. As compared with skeletal and cardiac muscle it is a much more slowly acting contractile tissue. Isolated strips of smooth muscle, as a rule, contract only when stimulated, though preparations

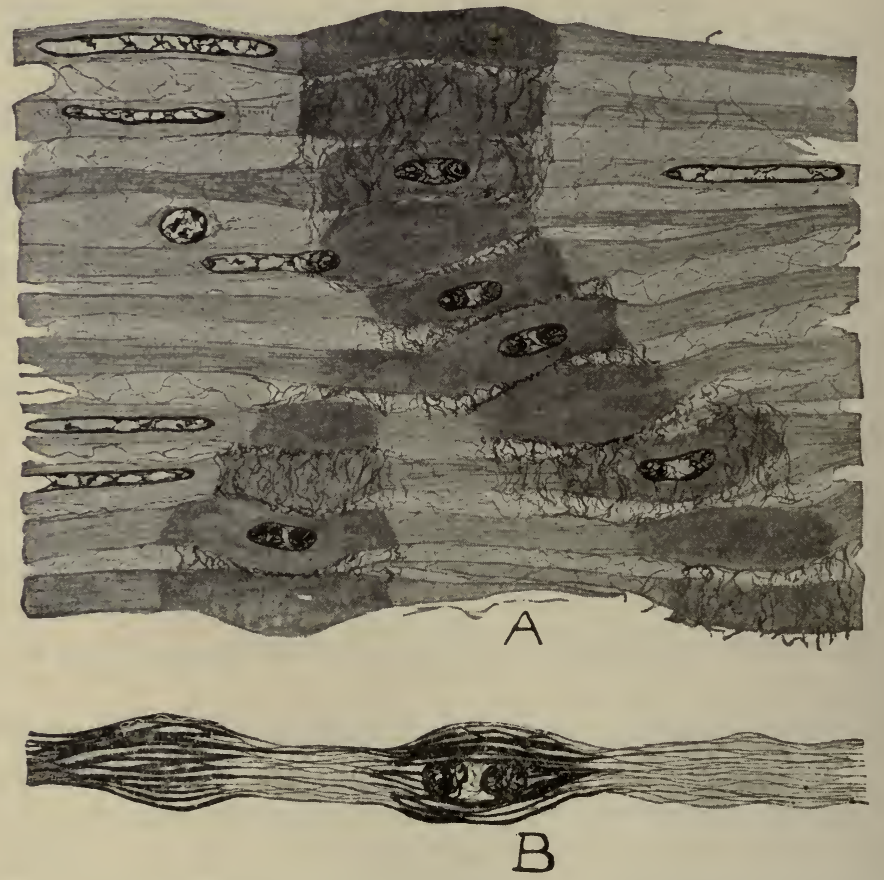

FIg 333.-Contraction Area in Smooth Muscle. A, Showing the contraction nodes of the fibers, the deep staining of the nodes, the condensation of surrounding connective tissue; $B$, diagrammatic, showing the thickening of the longitudinal fibrillæ. Intestine of dog. (Caroline McGill.)

of certain tissues, like the stomach muscle of the frog, give off rhythmic contractions occasionally. In this regard smooth muscle stands intermediate between skeletal and cardiac muscle; the former is normally never automatic, the latter always.

Smooth muscle requires a different type of stimulus to produce contraction; the stimulus must be more prolonged and more intense. For example, smooth muscle is not readily responsive to induction currents of short duration, but is readily stimulated by galvanic currents or induction currents of longer duration. The stimulus must be applied through a longer interval of time. Preparations of the stomach muscle can scarcely be made to 
contract by a single induction current, no matter how intense. Such muscle in the body is always associated with the local nervous apparatus which plays an indeterminate part in its activity.

The ureters and gall-bladder are the parts most difficult to excite by stimuli; they do not act at all till the stimulus has been long applied, and then contract feebly and to a small extent. The contractions of the cecum and stomach are quicker, and still quicker those of the iris and of the urinary bladder. The contractions of the small and large intestines, of the vas deferens, and of the pregnant uterus are yet more regular and more sustained.

Changes During the Contraction of Smooth Muscle.-The duration as well as type of contraction in smooth muscle is very markedly different from that of voluntary muscle. A contraction in smooth muscle is

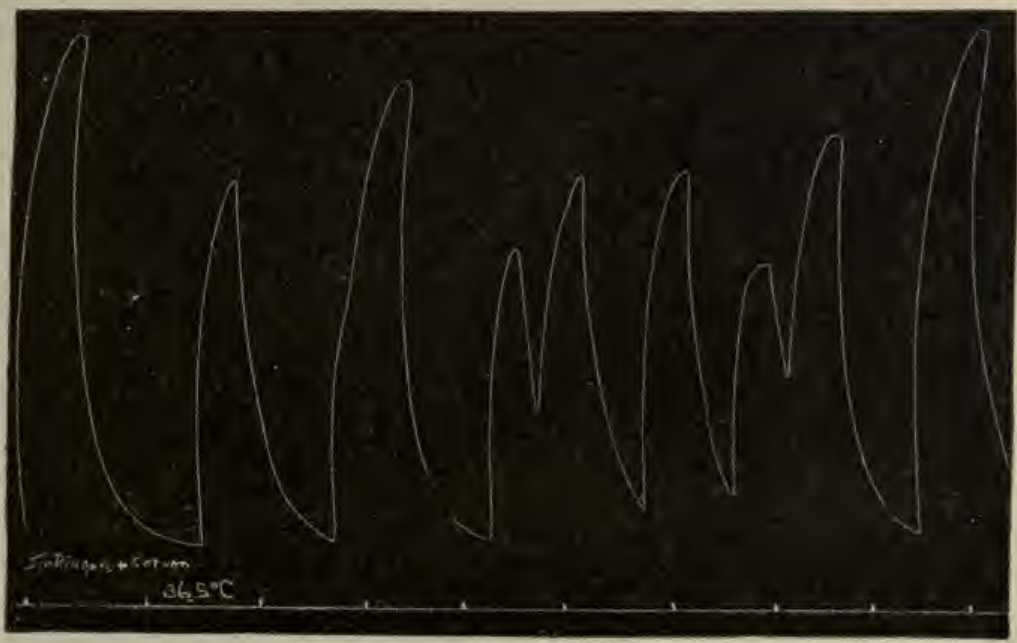

Frg. 334.- Showing periodic contractions of an isolated half of the uterus and Fallopian tube of the rat. The contractions occur spontaneously in serum and warm oxygenated Ringer's solution. Experiment by Bonham. Time in minutes.

characterized by a very long latent period, a slowly developed contraction phase, and an extremely delayed relaxation, figure $356, A$. The amount and duration of the contractions are dependent upon the strength and duration of the stimulus, though the curve of contraction itself does not in other respects differ sharply from the type of curve of the simple muscle contraction.

Owing to the apparently different structural type of smooth muscle, especial interest attaches to the changes which occur during its contraction. Caroline McGill has recently re-examined the histological structure and investigated the function of this type of muscle, and we are able to present a figure showing the changes. The longitudinal fibrillæ, which are readily stained with iron hematoxylin, show distinct shortening and thickening at the 
nodes of contraction of the muscle, figure $333, B$. The whole fiber is thickened at the contraction nodes and stains very readily and usually uniformly. However, by certain stains the fibrillæ can be traced through the node. The node is an apparent area of chemical differentiation. There is a marked condensation of the intermuscular fibrous tissue, which is doubtless purely a passive phenomenon. The most striking change during contractions is observed in the nucleus, figure $333, A$, and figure 334. The nucleus during rest is a long slender oval or spindle with a general chromatic network. "During contraction, the smooth muscle nuclei shorten and thicken by an active process. The chromatin collects, chiefly at the two ends of the nucleus, leaving a relatively clear area in the center."

Ciliary Motion.-Ciliary motion, which is closely allied to ameboid and muscular motion, is alike independent of the will, of the direct influence of the nervous system, and of muscular contraction. It may continue for several hours after death, or removal of the ciliated tissue, provided the portion of tissue under examination be kept moist. Its independence of the nervous system is shown also in its occurrence in the lowest invertebrate animals which are apparently unprovided with anything analogous to a nervous system, and in its persistence when the ciliated cells are completely separated from each other by teasing out in serum or other physiological solution. The vapor of chloroform arrests the motion; but it is renewed on the discontinuance of the application of the anesthetic. The movement ceases when the cilia are deprived of oxygen (although it may continue for a time in the absence of free oxygen), but is revived on the admission of this gas. Carbon dioxide also stops the movement. The contact of various substances, e.g., bile, strong acids, and alkalies, will stop the motion altogether; but this depends chiefly on destruction of the delicate substance of which the cilia are composed. Temperatures above $45^{\circ} \mathrm{C}$. and below $0^{\circ} \mathrm{C}$. stop the movement, whereas, moderate heat and faintly alkaline solutions are favorable to the action and revive the movement after temporary cessation. The exact explanation of ciliary movement is not known. Whatever may be the exact explanation, the movement must depend upon some changes going on in the cells of which the cilia are a part and not on changes limited to the cilia themselves, since, when the latter are cut off from the cell the movement ceases, and when severed so that portions of the cilia are left attached to the cell, the attached and not the severed portions continue the movement. Ciliary contraction is to be regarded as a type of motor activity carried out in a specialized form of motor apparatus. The changes going on in the cell must be classed with similar changes in heart or skeletal muscle. Ciliary tissue is like cardiac in at least two characteristics: the cells are capable of conducting a stimulus from cell to cell, and ciliary activity is automatic. As a special illustration of cilia-like action may be mentioned the motion of spermatozoa, which are cells with a single cilium. 


\section{THE FUNCTION OF NERVE FIBER.}

The Nerve Impulse.-The motor nerve fibers of the muscle-nerve preparation are of the medullated type described on page 66. But the essential structure, possessed by all fibers, is the axis cylinder. The peculiar function of the nerve fiber, i.e, of the axis cylinder, is its power to conduct a physiological change along its extent, a phenomenon known as a nerve impulse. A normal nerve impulse in a motor nerve has its origin in the motor cell of the central nervous system of which the fiber is an outgrowth. The manner in which such discharge from the cell takes place will be discussed later. But nerve impulses may be aroused by various artificial means, they are influenced by certain conditions in the environment, and possess certain other properties that may be discussed at this point.

Nerve Stimuli.-Nerve fibers like skeletal muscle require stimulation before they can manifest any of their properties, since they have no power of themselves of originating nerve impulses. The stimuli which are capable of exciting nerves to action are, as in the case of muscle, very diverse. The mechanical, chemical, thermal, and electrical stimuli which may be used in the case of muscles are also, with certain differences in the methods employed, efficacious in stimulating the nerve. The chemical stimuli are chiefly these: withdrawal of water as by drying; strong solutions of neutral salts of potassium, sodium, etc.; free inorganic acids, except phosphoric; and some organic acids. The electrical stimuli employed are the induction and continuous currents concerning which the observations in reference to muscular irritability should be consulted. Galvanic currents stimulate nerves only at the moment of turning on the current and of turning it off. Weaker electrical stimuli will excite nerves than will excite muscles; the nerve impulse appears to gain strength as it descends, and a weaker stimulus applied far from the muscle will have the same effect as a slightly stronger one applied to the nerve near the muscle.

Characteristics of the Nerve Impulse.-When a nerve impulse is aroused in a motor nerve, as by stimulating a nerve in its course by an induced current of medium strength, it is propagated along the axis cylinder to the muscle where it arouses a contraction of the muscle fiber. In the contraction of the muscle we have indirect but conclusive evidence of the passage of the nerve impulse, for it can be readily proven that the electrical current does not escape to the muscle. In this instance it can be shown that there is a nerve impulse passing from the point of stimulation in the direction away from the muscle; i.e., the artificially aroused nerve impulse passes over the entire extent of the fiber stimulated. In fact, a nerve impulse is known to travel from its point of origin over the entire neurone affected. This antidromal nerve impulse, of course, does not exist in the normal case, since the 
normal nerve impulse arises in the nerve-cell body and passes out over the fiber from its origin to its extremity.

The nerve impulse travels over the nerve fiber with a velocity that was first determined by Helmholtz. He found that in the sciatic of the frog the nerve impulse travels at the rate of twenty-seven meters per second. The rate has been measured in a number of animals and varies between wide limits. In human nerves the rate is variously given, but Helmholtz' figure of thirty-four meters per second may be taken as a fair average.

The presence of the nerve impulse can be detected by the action current, which exists in nerve as in muscle (see page 494 for methods of detecting the action current).

Rheoscopic Frog.-The action current may be demonstrated by means of the following experiment:

The muscle current produced by stimulating the nerve of one muscle-nerve preparation may be used to stimulate the nerve of a second muscle-nerve preparation. The hind leg of a frog with the nerve going to the gastrocnemius cut long is placed upon a glass plate and arranged in such way that its nerve touches in two places the gastrocnemius muscle, exposed but preserved in situ in the opposite thigh of the frog. The electrodes from an induction coil are placed behind the sciatic nerve of the second preparation, high up. On stimulating it with a single induction shock, the muscles not only of the same leg are found to undergo a twitch, but also those of the first preparation, although this is not near the electrodes. The stimulation cannot be due to an escape of the stimulating current into the first nerve, but is due to the action current of the second muscle. This experiment is known under the name of the rheoscopic frog.

When the nerve impulse is studied by means of the action current it is found that a nerve impulse can be aroused by a weaker stimulus than is required to produce a minimal contraction of a muscle. The response of the nerve to graduated strengths of the stimulus is increased very rapidly with slight increase of strength of the stimulus, the augmentation extending through a somewhat greater range than for muscle. If the stimulus is still further increased there is only slight increase of the resulting nerve impulse.

Fatigue of Nerve Fiber.-Many efforts have been made to discover evidences of fatigue of nerve fiber, with practically completely negative results. A difficulty has been to secure means of measuring change in intensity of the nerve impulse. The muscle quickly fatigues so that the character of the muscle response cannot be taken when measured in the ordinary way. An effective method used by Howell, Budgett, and Leonard consists in cooling a segment of nerve to suspend its conductivity, during stimulation of the free end, and periodically warming up the cooled segment of nerve to test the strength of nerve impulse passing through it to the unfatigued muscle beyond. By this and other methods it has been found that 
a motor nerve is not fatigued by at least ten hours' continuous stimulation with induction currents.

One must hesitate to draw the conclusion, however, that the nerve fiber conducts the nerve impulse without loss of energy. The fiber can be anesthetized, it responds to temperature changes, and gives other evidences of susceptibility to conditions which influence metabolism in other forms of protoplasm. Perhaps the nerve fiber is capable of repairing its wastes as rapidly as they occur.

The Effects of Battery Currents on Nerve Fiber.-Galvanic currents influence nerves in ways that call for special discussion. A constant current, say from a Daniell battery, can be introduced into the nerve of a musclenerve preparation by means of a pair of non-polarizable electrodes, figure 323 , and a convenient key arranged for turning the current on or off the nerve. It will be found that with a current of moderate strength there will be a contraction of the muscle, both at the closing and the opening of the key (called, respectively, making and breaking contractions), but that during the interval between these two events the muscle remains flaccid, provided the battery current continues of constant intensity. If the current be a very weak or a very strong one, the effect is not quite the same; one or the other of the contractions may be absent. Which of these contractions is absent depends upon another circumstance, viz., the direction of the current. The direction of the current may be ascending or descending: if ascending, the anode or positive pole is nearer the muscle than the cathode or negative pole, and the current to return to the battery has to pass up the nerve; if descending, the position of the electrodes is reversed. It will be necessary before considering this question further to return to the apparent want of effect of the constant current during the interval between the make and the break contraction. To all appearances no change is produced, but in reality a very important alteration of the irritability and conductivity is brought about in the nerve by the passage of this constant or polarizing current.

A second way of showing the effect of the polarizing current is by stimulating the nerve by a pair of electrodes from an induction coil, while the polarizing current from the battery is flowing through the nerve. If the strength of stimulus required in order that a minimum contraction be obtained by the induction shock before the polarizing current is applied and the secondary coil be removed slightly further from the primary, the induction current cannot now produce a contraction. If, now, the polarizing current be sent in a descending direction, that is to say, with the cathode nearest the muscle, and the induction current which was before insufficient be applied between the cathode and the muscle, it will now prove sufficient to cause a contraction. This indicates that with a descending current the irritability of the nerve is increased at the cathode. If, instead of applying the induction electrodes below the polarizing electrodes, they are applied above them, the irritability of 
the nerve is found to be decreased. If the polarizing current is reversed, i.e., made ascending, then the condition of irritability of the nerve is reversed. Both methods show that the polarization consists in an increase in irritability at the cathode, called catelectrotonus, and a decrease at the anode called anelectrotomus. The total change is called by the term electrotonus. As there is between the electrodes both an increase and a decrease of irritability on the passage of a polarizing current, it is evident that there must be a neutral point where there is neither increase nor decrease of irritability. The position of this neutral point is found to vary with the intensity of the polarizing current; when the current is weak the point is nearer the anode, when strong nearer the

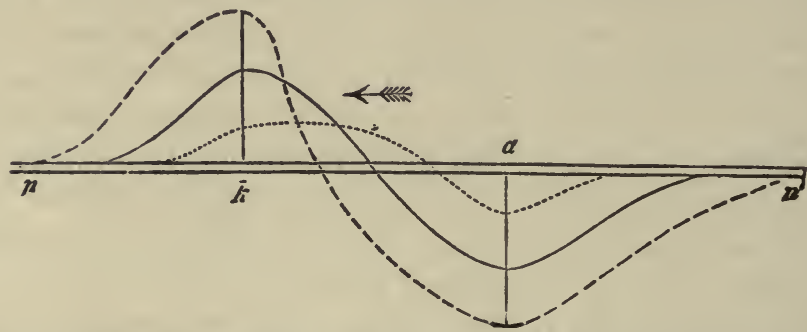

Fig. 335.-Diagram Illustrating the Effects of Various Intensities of the Polarizing Currents. $n, n^{\prime}$, Nerve; $a$, anode; $k$, cathode; the curves above indicate increase, and those below decrease of irritability, and when the current is small the increase and decrease are both small, with the neutral point near $a$, and so on as the current is increased in strength.

cathode, figure 335. When a constant current passes into a nerve, therefore, if a contraction result, it may be assumed that it is due to the increased irritability produced in the neighborhood of the cathode, but the breaking contraction must be produced by a rise in irritability from a lowered state to the normal in the neighborhood of the anode.

The contractions produced in the muscle of a muscle-nerve preparation by a constant current have been arranged in a table which is known as Pfluger's Law of Contractions. It is really only a statement as to when a contraction may be expected:

\begin{tabular}{|c|c|c|c|c|}
\hline \multirow{2}{*}{ Strength of current used } & \multicolumn{2}{|c|}{ Descending current } & \multicolumn{2}{|c|}{ Ascending current } \\
\hline & Make & Break & Make & Break \\
\hline 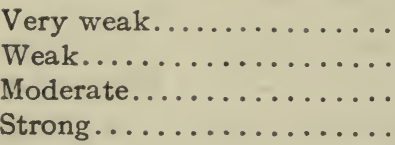 & $\begin{array}{l}\text { Yes } \\
\text { Yes } \\
\text { Yes } \\
\text { Yes }\end{array}$ & $\begin{array}{l}\text { No } \\
\text { No } \\
\text { Yes } \\
\text { No }\end{array}$ & $\begin{array}{l}\text { No } \\
\text { Yes } \\
\text { Yes } \\
\text { No }\end{array}$ & $\begin{array}{l}\text { No } \\
\text { No } \\
\text { Yes } \\
\text { Yes }\end{array}$ \\
\hline
\end{tabular}


During the passage of a constant current through a nerve and immediately after its cessation, there is a change in the conductivity as well as of the irritability of the nerve at the anode and cathode, respectively. During the passage of the current, the conductivity is increased at the cathode and decreased at the anode. After the passage of the current, the effect is reversed. With strong currents the area of decreased conductivity may be sufficient to act as a block, preventing the passage of impulses over it.

The foregoing statements concerning the changes produced in a nerve by the passage of a constant current may be briefly summarized as follows:

I. A nerve is more irritable to the closing of a constant current than it is to the opening of a constant current.

II. During the passage of the current through the nerve, both its irritability and conductivity are increased at the cathode and decreased at the anode.

III. After the passage of the current, the irritability and conductivity are both decreased at the cathode and increased at the anode.

The Effect of Battery Currents on Deep-seated Nerves.-The following account is condensed from Lombard in "An American Text-book of Physiology."

As an electric current cannot be applied to living human nerves directly, it is applied to the skin along the course of the nerve. The current passes
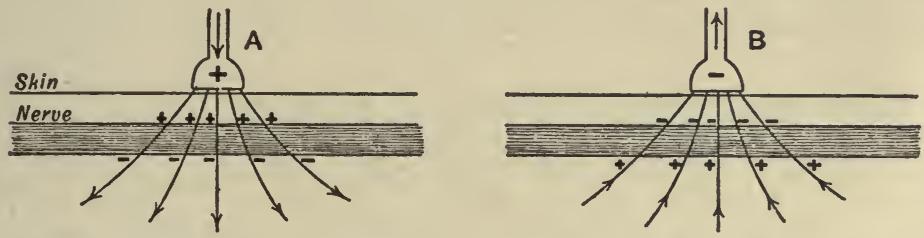

Fig. 335a.-Diagram of Skin and Subjacent Nerve. $A$, the positive electrode or phvsical anode; $B$, the negative electrode or physical cathode. Signs, + physiological anodes; signs - physiological cathodes. (After Waller.)

from the anode or positive pole through the skin, and spreads out in the tissues much as the bristles of a brush; it then gradually concentrates and leaves the skin at the cathode or negative pole.

In addition to the physical anode and cathode of the battery, there are what are called physiological anodes and cathodes. There is a physiological anode at every point where the current enters a nerve, and a physiological cathode at every point where it leaves it.

Generally when the current is applied to nerves through the skin, only part of it flows longitudinally along the nerves; most of it passes diagonally across them to the tissues below. Thus it happens that in that part of the nerve beneath either the physical anode or cathode, groups of physiological anodes and cathodes are found.

The contraction which occurs when the current is closed (closing contraction) represents irritation at the physiological cathode, while the opening 
contraction represents irritation at the physiological anode. Since there are physiological anodes and cathodes beneath each electrode, one or more of four conditions may arise:

I. Anodic closing contraction, i..e., the effect of the change developed at the physiological cathode, beneath the physical anode (positive pole).

2. Anodic opening contraction, ie., the effect of the change developed at the physiological anode, beneath the physical anode (positive pole).

3. Cathodic closing contraction, i.e., the effect of the change developed at the physiological cathode, beneath the physical cathode (negative pole).

4. Cathodic opening contraction, i.e., the effect of the change developed at the physiological anode, beneath the physical cathode (negative pole).

The following abbreviations of these contractions are used: ACC, AOC, $\mathrm{KCC}, \mathrm{KOC}$.

The closing contractions, $\mathrm{KCC}$ and $\mathrm{ACC}$, are stronger than the opening contractions, $\mathrm{KOC}$ and $\mathrm{AOC}$. Of the closing contractions, $\mathrm{KCC}$ is stronger than ACC. Of the opening contractions, AOC is stronger than KOC. These facts are also shown in a table of the effects of gradually increasing the strength of the current.

Weak currents.

$\mathrm{KCC}$

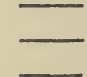

Medium currents.

$\mathrm{KCC}$

ACC

$\mathrm{AOC}$
Strong currents.

$\mathrm{KCC}$

$\mathrm{ACC}$

$\mathrm{AOC}$

$\mathrm{KOC}$

Sometimes AOC is stronger than ACC.

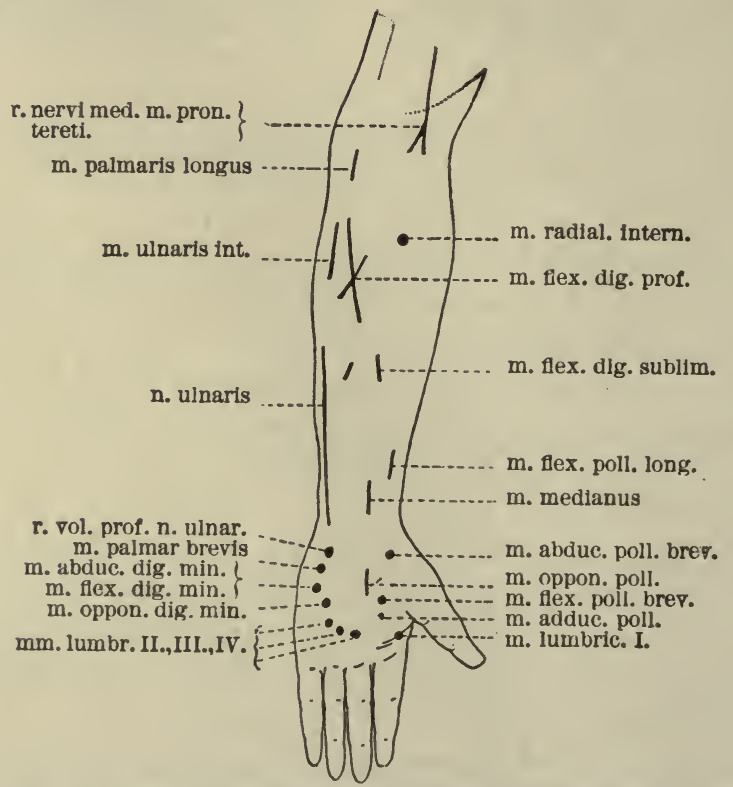

Fig. 336.-Figure Showing Motor Points in the Forearm. 
In diseases which cause degeneration of the nerves going to a muscle, stimulation causes results different from the above, and we get what is known as the reaction of degeneration.

The intensity of the anodic or cathodic effects is increased by using small electrodes, and decreased by electrodes of large surface. In fact in practice it is usual to apply the indifferent electrode to an extended surface, thus reducing its effect below the stimulating intensity. This gives only one active stimulating electrode and is known as the method of unipolar stimulation.

\section{SOME SPECIAL COORDINATED MOTOR ACTIVITIES.}

\section{LOCOMOTION.}

The greater number of the more important muscular actions of the human body, those, namely, which are arranged harmoniously so as to subserve some definite purpose in the animal economy, are described in various parts of this work in the sections which treat of the physiology of the processes by which these muscular actions are resisted or carried out. There are, however, some very important and somewhat complicated muscular acts which may be best described in this place.

Walking. - The coordinated movements of the body are carried out by the skeletal muscles acting on the skeletal elements as a system of levers. Even the bones of the skull are levers in so far as their relations to muscles are concerned.

Examples of the Three Orders of Levers in the Human Body.-All levers have been divided into three kinds, according to the relative position of the power, the weight to be moved, and the axis of motion or fulcrum. In a lever of
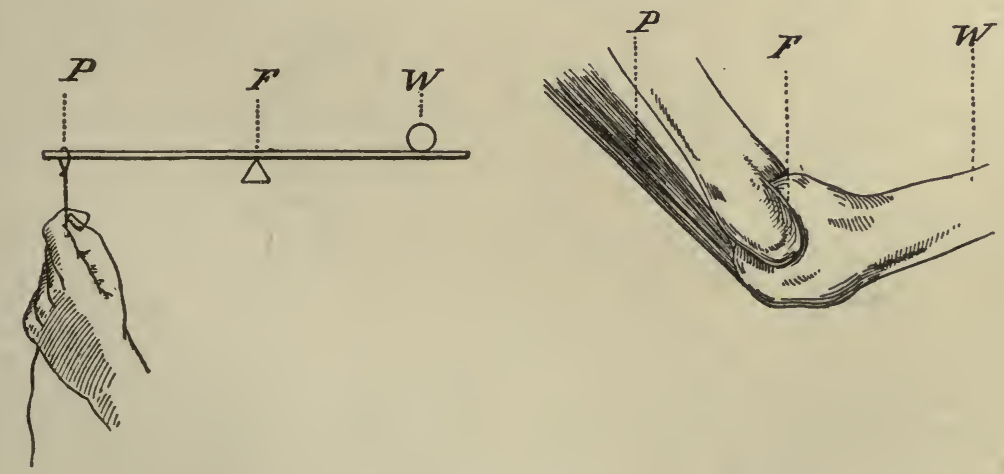

Fig. 337 .

the first kind the power is at one extremity of the lever, the weight at the other, and the fulcrum between the two. If the initial letters only of the power, weight, and fulcrum be used, the arrangement will stand thus: P. F. W. A poker as ordinarily used, or the bar in figure 337 , may be cited as an example of this variety of lever; while, as an instance in which the bones of the human 
skeleton are used as a lever of the same kind, may be mentioned the act of raising the body from the stooping posture by means of the hamstring muscles attached to the tuberosity of the ischium or of the triceps which extends the forearm by action at the elbow, figure 337 .

In a lever of the second kind, the arrangement is thus: P. W. F.; and this leverage is employed in the act of raising the handles of a wheelbarrow, or in stretching an elastic band, as in figure 338 . In the human body the act of opening the mouth by depressing the lower jaw is an example of the same kind
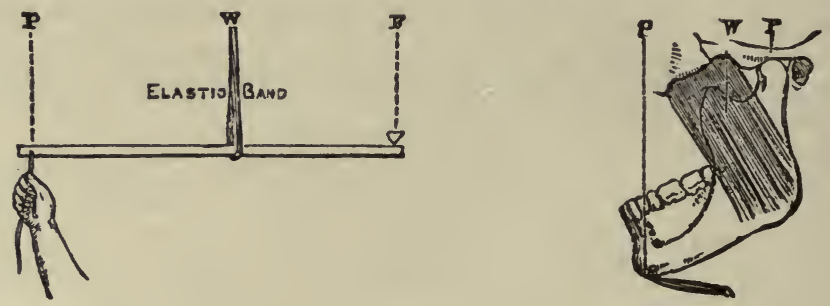

Fig. 338 .

-the tension of the muscles which close the jaw representing the weight, figure $33^{8}$.

In a lever of the third kind the arrangement is F. P. W., and the act of raising a pole, as in figure 339 , is an example. In the human body there are numerous examples of the employment of this kind of leverage. The act of bending the forearm may be mentioned as an instance, figure 339 . The act of biting is another example.

At the ankle we have examples of all three kinds of lever. rst kind-Extending the foot. $3 \mathrm{~d}$ kind-Flexing the foot. In both these cases the foot
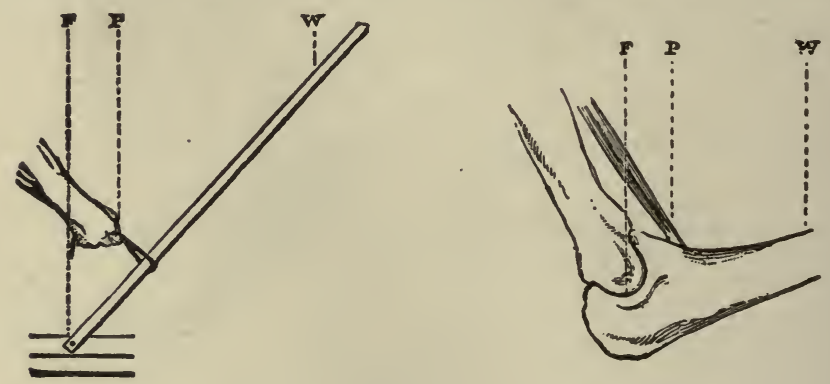

FIG. 339 .

represents the weight, the ankle joint the fulcrum, the power being the gastrocnemius muscles in the first case and the tibialis anticus in the second case. 2nd kind-When the body is raised on tiptoe. Here the tip of the toe is the fulcrum, the weight of the body acting at the ankle joint the weight, and the gastrocnemius muscles the power.

In the human body, levers are most frequently used at a disadvantage as regards power, the latter being sacrificed for the sake of a greater range of motion. Thus in the diagrams of the first and third kinds it is evident that the power is so close to the fulcrum that great force must be exercised in order to produce motion. It is also evident, however, from the same diagrams, that 
by the closeness of the power to the fulcrum a great range of movement can be obtained by means of a comparatively slight shortening of the muscular fibers.

In the act of walking, almost every voluntary muscle in the body is brought into play, either directly for purposes of progression, or indirectly for the proper balancing of the head and trunk. The muscles of the arms are least concerned; but even these are for the most part instinctively in action to some extent.

Among the chief muscles engaged directly in the act of walking are those of the calf, which, by pulling up the heel, pull up also the astragalus, and with it, of course, the whole body, the weight of which is transmitted through the tibia to this bone, figure 340 . When starting to walk, say with the left leg this raising of the body is not entirely dependent on the muscles of the left

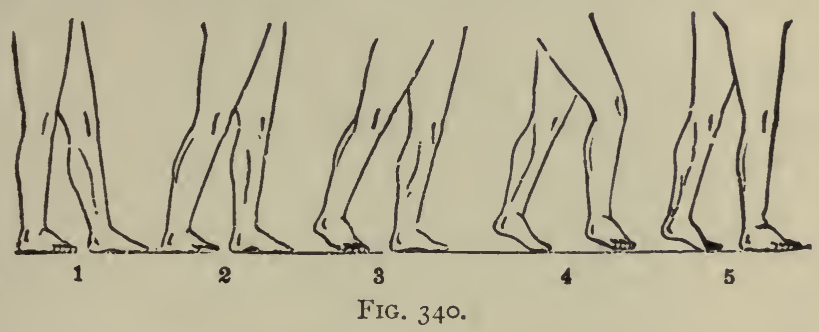

calf, but the trunk is thrown forward in such a way that it would fall prostrate were it not that the right foot is brought forward and planted on the ground to support it. Thus the muscles of the left calf are assisted in their action by those muscles on the front of the trunk and legs which, by their contraction, pull the body forward; and, of course, if the trunk form a slanting line, with the inclination forward, it is plain that when the heel is raised by the calf muscles, the whole body will be raised, and pushed obliquely forward and upward. The successive acts in taking the first step in walking are represented in figure $340,1,2,3$, etc.

Now it is evident that by the time the body has assumed the position No. 3 , it is time that the right leg should be brought forward to support it and prevent it from falling prostrate. This advance of the right leg is effected partly by its mechanically swinging forward, pendulum-wise, and partly by muscular action; the muscules used being- $\mathrm{I}$, those on the front of the thigh, which bend the thigh forward on the pelvis, especially the rectus femoris, with the psoas and the iliacus; 2 , the hamstring muscles, which slightly bend the leg on the thigh; and, 3 , the muscles on the front of the leg, which raise the front of the foot and toes, and so prevent the latter in swinging forward from striking the ground.

The second part of the act of walking, which has been just described, is shown in the diagram, 4 , figure 340 . 
When the right foot has reached the ground the action of the left leg has not ceased. The calf muscles of the latter continue to act, and, by pulling up the heel, throw the body still more forward over the right leg, now bearing nearly the whole weight, until the time when the left leg should again swing forward, and the left foot be planted on the ground to prevent the body from falling prostrate. As at first, while the calf muscles of one leg and foot are preparing, so to speak, to push the body forward and upward from behind by raising the heel, the muscles on the front of the trunk and the same leg (and of the other leg, except when it is swinging forward) are helping the act by pulling the legs and trunk, so as to make them incline forward, the rotation in the inclining occurring mainly at the ankle joint. Two main kinds of leverage are, therefore, employed in the act of walking, and if this idea be firmly grasped, the details will be understood with comparative ease. One kind of leverage employed in walking is essentially the same with that employed in pulling forward the pole, as in figure 339 . And the other, less exactly, is that employed in raising the handles of a wheelbarrow. Now, supposing the lower end of the pole to be placed in the barrow, we should have a very rough and inelegant, but not altogether bad representation of the two main levers employed in the act of walking. The body is pulled forward by the muscles in front, much in the same way that the pole might be by the force applied at $\mathrm{P}$, while the raising of the heel and pushing forward of the trunk by the calf muscles are roughly represented on raising, the handles of the barrow. The manner in which these actions are performed alternately by each leg, so that one after the other is swung forward to support the trunk, which is at the same time pushed and pulled forward by the muscles of the other, may be gathered from the previous description.

There is one more thing to be especially noticed in the act of walking. Inasmuch as the body is being constantly supported and balanced on each leg alternately, and therefore on only one at the same moment, it is evident that there must be some provision made for throwing the center of gravity over the line of support formed by the bones of each leg, as, in its turn, it supports the weight of the body. This may be done in various ways, and the manner in which it is effected is one element in the differences which exist in the walking of different people. Thus it may be done by an instinctive slight rotation of the pelvis on the head of each femur in turn, in such a manner that the center of gravity of the body shall fall over the foot of this side. Thus when the body is pushed onward and upward by the raising, say, of the right heel, as in figure 340,3 , the pelvis is instinctively by various muscles made to rotate on the head of the left femur at the acetabulum, to the left side, so that the weight may fall over the line of support formed by the left leg at the time that the right leg is swinging forward, and leaving all the work of support to fall on its fellow. Such a 
"rocking" movement of the trunk and pelvis, however, is accompanied by a movement of the whole trunk and leg over the foot which is being planted on the ground, figure $34 \mathrm{I}$, the action being accompanied with a compensatory outward movement at the hip, more easily appreciated by looking at the figure (in which this movement is shown exaggerated) than from the description.

Thus the body in walking is continually rising and swaying alternately from one side to the other, as its center of gravity has to be brought alternately over one or the other leg; and the curvatures of the spine are altered in cor-

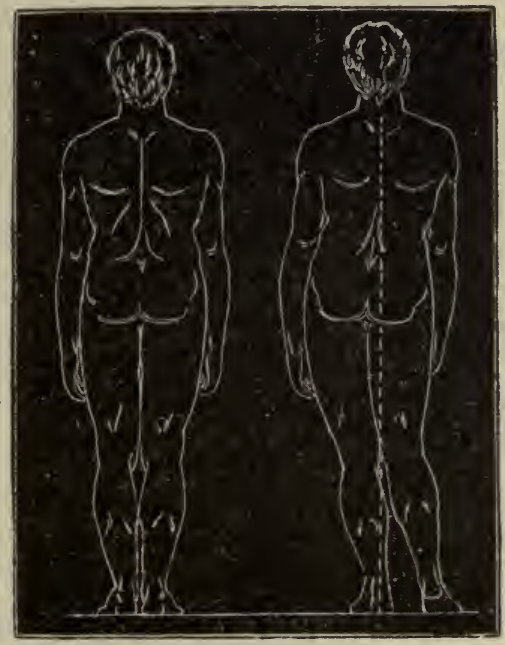

FIG. 34I.

respondence with the varying position of the weight which it has to support. The extent to which the body is raised or swayed differs much in different people.

In walking, one foot or the other is always on the ground. The act of leaping or jumping consists in so sudden a raising of the heels by the sharp and strong contraction of the gastrocnemius muscles that the body is jerked off the ground. At the same time the effect is much increased by first bending the thighs on the pelvis, and the legs on the thighs, and then suddenly straightening out the angles thus formed. The share which this action has in producing the effect may be easily known by attempting to leap in the upright posture, with the legs quite straight.

Running.-Running is performed by a series of rapid low jumps produced by each leg alternately; so that, during each complete muscular act concerned, there is a moment when both feet are off the ground.

In all these cases, however, the description of the manner in which any given effect is produced, can give but a very imperfect idea of the infinite 
number of combined and harmoniously arranged muscular contractions which are necessary for even the simplest acts of locomotion.

\section{THE PRODUCTION OF THE VOICE.}

Before commencing the consideration of the nervous system and the special senses it will be convenient to consider first speech, the production of the human voice, and the physiology of the larynx as a muscular apparatus.

The Larynx.-In nearly all air-breathing vertebrate animals there are arrangements for the production of sound, or voice, in some parts of the respiratory apparatus. In many animals, the sound admits of being variously modified and altered during and after its production; and, in man, one such modification occurring in obedience to dictates of the cerebrum, is speech.

It has been proven by observations on living subjects, by means of the laryngoscope, as well as by experiments on the larynx taken from the dead body, that the sound of the human voice is the result of the vibration of the inferior laryngeal ligaments, or the true vocal cords which bound the glottis, caused by currents of expired air impelled over their edges. If a free opening exist in the trachea, the sound of the voice ceases, but it returns if the opening is closed. An opening into the air-passages above the glottis, on the contrary, does not prevent the voice being produced. By forcing a current of air through the larynx in the dead subject, clear vocal sounds are elicited, though the epiglottis, the upper ligaments of the larynx or false vocal cords, the ventricles between the upper ligaments and the inferior ligaments, and the upper part of the arytenoid cartilages, be all removed. But the true vocal cords must remain entire with their points of attachment, and be kept tense and so approximated that the fissure of the glottis may be narrow.

The vocal ligaments or cords, therefore, are regarded as the proper organs for the production of vocal sounds. The modifications of these sounds are effected, as will be presently explained, by other parts, viz., by the tongue, teeth, lips, etc. The structure of the vocal cords is adapted to enable them to vibrate like tense membranes, for they are essentially composed of elastic tissue; and they are so attached to the cartilaginous parts of the larynx that their position and tension can be variously altered by the contraction of the muscles which act on these parts.

Thus it will be seen that the larynx is the organ of voice. It may be said to consist essentially of the two vocal cords and the various cartilaginous, muscular, and other apparatus by means of which not only can the aperture of the larynx (rima glottidis) be closed against the entrance and exit of air to or from the lungs, but also by means of which the cords themselves can be stretched or relaxed, brought together and separated in accordance with the conditions that may be necessary for the air in passing over them to set them vibrating to produce the various sounds. Their action in respiration has been already referred to. 
Anatomy of the Larynx.-The principal parts entering into the formation of the larynx, figures 342 and 343 , are-the thyroid cartilage; the cricoid cartilage; the two arytenoid cartilages; and the two true vocal cords. The epiglottis, figure 343 , has but little to do with the voice, and is. chiefly useful in protecting the upper part of the larynx from the entrance of food and drink in
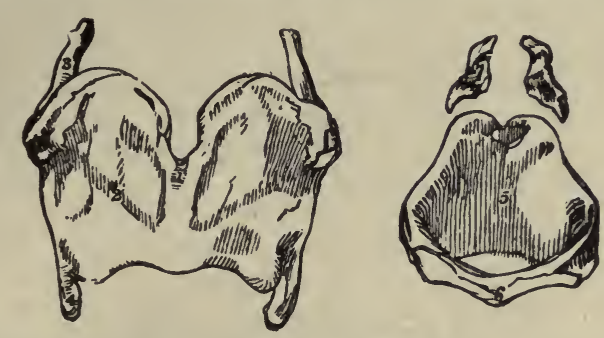

FIG. 342.-Cartilages of the Larynx Seen from the Front. I to 4, Thyroid cartilage; I, vertical ridge or pomum Adami; 2 , right ala; 3 , superior, and 4 , inferior cornu of the right side; 5,6 , cricoid cartilage; 5 , inside of the posterior part; 6 , anterior narrow part of the ring; 7 , arytenoid cartilages. $\times \frac{3}{4}$.

deglutition. The false vocal cords and the ventricle of the larynx, which is a space between the false and the true cord of either side, need be only referred to.

Cartilages. $-a$, The thyroid cartilage, figure 342 , I to 4 , does not form a complete ring around the larynx, but only covers the front portion. $b$, The cricoid cartilage, figure $342,5,6$, on the other hand, is a complete ring; the

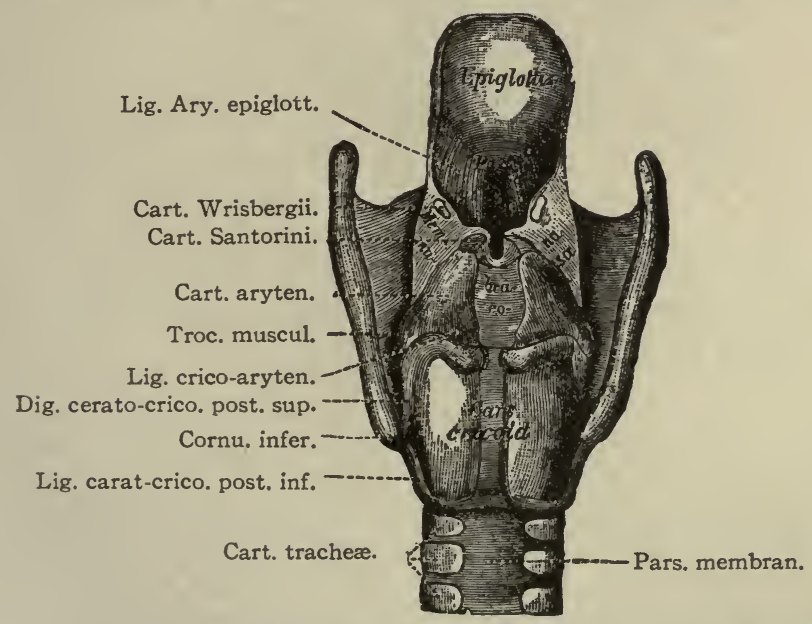

FIG. 343. - The Larynx as Seen From Behind after Removal of the Muscles. The cartilages and ligaments only remain. (Stoerk.)

back part of the ring being much broader than the front. On the top of this broad portion of the cricoid are, $c$, the arytenoid cartilages, figure 342,7 , the connection between the cricoid below and arytenoid cartilages above being a joint with synovial membrane and ligaments, the latter permitting tolerably free motion between them.

Joints and Ligaments.- The thyroid cartilage is also connected with the 
cricoid, not only by ligaments, but also by joints with synovial membranes; the lower cornua of the thyroid clasping the cricoid between them, yet not so tightly but that the thyroid can revolve, within a certain range, around an axis passing transversely through the two joints. The vocal cords are attached behind to the front portion of the base of the arytenoid cartilages, and ir front to the re-entering angle at the back part of the thyroid; it is evident, therefore, that all movements of either of these cartilages must produce an effect on them of some kind or other. Inasmuch, too, as the arytenoid cartilages rest on the top of the back portion of the cricoid cartilage, and are connected with it by capsular and other ligaments, all movements of the cricoid cartilage must move the arytenoid cartilages, and also produce an effect on the vocal cords.

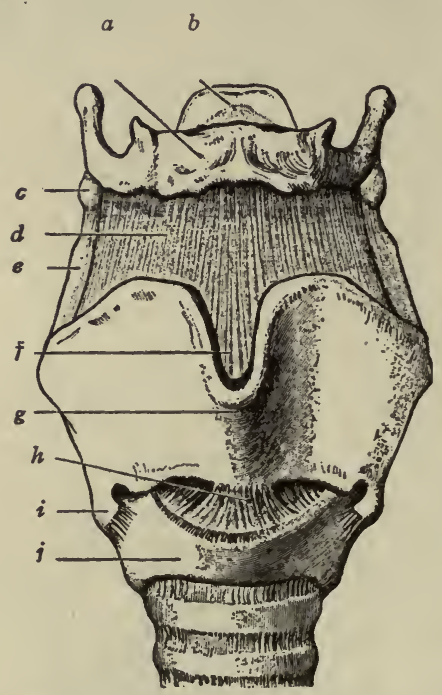

FIG. 344. - The Cartilages and Ligaments of the Larynx, Viewed from the Front. $a$, Epiglottis; $b$, hyoid bone; $c$, cartilago tritica; $d$, thyro-hyoid membrane; $e$, superior cornu of thyroid cartilage; $j$, thyroid notch; $g$, pomum Adami; $h$, crico-thyroid membrane; $i$, inferior cornu of thyroid cartilage; $f$, cricoid cartilage. (Cunningham.)

Intrinsic Muscles.-The intrinsic muscles of the larynx are so connected with the laryngeal cartilages that by their contraction alterations in the condition of the vocal cords and glottis are produced. They are usually divided into four classes according to their action, viz., into abductors, adductors, sphincters and tensors. The abductors, the crico-arytenoidei, widen the glottis by separating the cords; the adductors, consisting of the thyro-aryepiglottici, the arytenoideus posticus seu transversus, the thyro-arytenoidei externi, the crico-arytenoidei laterals, and the thyro-arytenoidei interni, approximate the vocal cords, diminish the rima glottidis, and act generally as sphincters and supporters of the glottis. Finally, the tensors of the cords put the cords on the stretch, with or without elongating them; the tensors are the crico-thyroidei and the thyro-arytenoidei interni.

The attachments and the action of the muscles will be readily understood from the following table. All the muscles are in pairs except the arytenoideus posticus. 
Table of the Several Groups of the Intrinsic Muscles of the LarynX and THEIR ATtACHMENTS.

\begin{tabular}{|c|c|c|c|}
\hline Group & Muscle & Attachments & Action \\
\hline $\begin{array}{c}\text { I. } \\
\text { Abductors. }\end{array}$ & $\begin{array}{l}\text { Crico-aryt- } \\
\text { enoidei } \\
\text { postici. }\end{array}$ & $\begin{array}{l}\text { This pair of muscles arises, on either side, } \\
\text { from the posterior surface of the cor- } \\
\text { responding half of the cricoid cartilage. } \\
\text { From this depression their fibers con- } \\
\text { verge on either side upward and out- } \\
\text { ward to be inserted into the outer angle } \\
\text { of the base of the arytenoid cartilages } \\
\text { behind the cricoarytenoidei laterales. }\end{array}$ & $\begin{array}{l}\text { Draw inward and } \\
\text { backward the outer } \\
\text { angle of arytenoid } \\
\text { cartilages, and so } \\
\text { rotate outward the } \\
\text { processus vocalis and } \\
\text { widen the glottis. }\end{array}$ \\
\hline $\begin{array}{l}\text { II. and III. } \\
\text { Adductors } \\
\text { and } \\
\text { Sphincters. }\end{array}$ & $\begin{array}{l}\text { In } \mathrm{three} \\
\text { layers: } \\
\text { a. Outer }\end{array}$ & $\begin{array}{l}\text { A pair of muscles. Flat and narrow, } \\
\text { which arise on either side from the pro- } \\
\text { cessus muscularis of the arytenoid car- }\end{array}$ & $\begin{array}{l}\text { Help to narrow or } \\
\text { close the rima } \\
\text { glottidis. }\end{array}$ \\
\hline
\end{tabular}

Thyroaryepiglottici. cross one another in the middle line to be inserted into the upper half of the lateral border of the opposite arytenoid cartilage and the posterior border of the cartilage of Santorini. The lower fibers run forward and downward to be inserted into the thyroid cartilage near the commissure. The fibers attached to the cartilage of Santorini are continued forward and upward into the aryepiglottic fold.

b. Middle A single muscle. Half-quadrilateral, layer.

i. Arytenoideus posticus.

ii. Thyroattached to the borders of the arytenoid cartilages, its fibers running horizontally between the two. arytenoidei ex terni.
A pair of muscles. Each of which consists of three chief portions. The lower and principal fibers arise from the lower half of the internal surface of the thyroid cartilage, close to the angle, and from the fibrous expansion of the cricothyroid ligament, and are inserted into the lateral border of the arytenoid cartilage. The inner fibers to the lower half of this border, and the outer fibers into the upper half, some pass to the cartilage of Wrisberg and the aryepiglottic fold.
Draws together the arytenoid cartilages and also depresses them. When the muscle is paralyzed, the inter-cartilaginous part of the cords cannot cume together. 
Table of the Several Groups of the Intrinsic Muscles of the Larynx and THEIR ATtaCHMENTS.-Continued.

\begin{tabular}{|c|c|c|c|}
\hline Group & Muscle & Attachments & Action \\
\hline & $\begin{array}{l}\text { c. Inner- } \\
\text { most layer, } \\
\text { Th y ro- } \\
\text { arytenoi- } \\
\text { dei in- } \\
\text { terni. }\end{array}$ & $\begin{array}{l}\text { A pair of muscles. They arise on either } \\
\text { side from the middle third of the upper } \\
\text { border of the cricoid cartilage and are } \\
\text { inserted into the whole anterior margin } \\
\text { of the base of the arytenoid cartilage. } \\
\text { Some of their fibers join the thyroid- } \\
\text { aryepiglottici. } \\
\text { A pair of muscles. They arise on either } \\
\text { side, internally from the angle of the } \\
\text { thyroid cartilage, internal to the last } \\
\text { described muscle, b. iii., and, running } \\
\text { parallel to and in the substance of the } \\
\text { vocal cords, are attached posteriorly to } \\
\text { the processus vocalis and to the outer } \\
\text { surface of the arytenoid cartilages. }\end{array}$ & $\begin{array}{l}\text { Approximate the vocal } \\
\text { cords by drawing the } \\
\text { processus muscularis } \\
\text { of the arytenoid car- } \\
\text { tilages forward and } \\
\text { downward and so } \\
\text { rotate the processus } \\
\text { vocalis inward. } \\
\text { Render the vocal cords } \\
\text { tense and rotate the } \\
\text { arytenoid cartilages } \\
\text { and approximate the } \\
\text { processus vocalis. }\end{array}$ \\
\hline Tensors. & $\begin{array}{c}\text { Thyro-ary- } \\
\text { te noidei } \\
\text { interni. }\end{array}$ & $\begin{array}{l}\text { A pair of fan-shaped muscles attached on } \\
\text { either side to the cricoid cartilage below; } \\
\text { from the mesial line in front for nearly } \\
\text { one-half of its lateral circumference } \\
\text { backward the fibers pass upward and } \\
\text { outward to be attached to the lower } \\
\text { border of the thyroid cartilage and to } \\
\text { the front border of its lower cornea. } \\
\text { The most posterior part is almost a dis- } \\
\text { tinct muscle and its fibers are all but } \\
\text { horizontal: sometimes this muscle is } \\
\text { described as consisting of two layers, } \\
\text { superficial with cortical fibers, deep } \\
\text { with oblique fibers, described under } \\
\text { Group III. }\end{array}$ & $\begin{array}{l}\text { The thyroid cartilage } \\
\text { being fixed by its } \\
\text { extrinsic muscles, the } \\
\text { front of the cricoid } \\
\text { cartilage is drawn } \\
\text { upward, and its back, } \\
\text { with the arytenoids } \\
\text { attached, is drawn } \\
\text { down. Hence the } \\
\text { vocal cords are elon- } \\
\text { gated antero-poste- } \\
\text { riorly and put upon } \\
\text { the stretch. Paral- } \\
\text { ysis of these muscles } \\
\text { causes an inability } \\
\text { to produce high } \\
\text { notes. }\end{array}$ \\
\hline
\end{tabular}

Nerve Supply.-The sensory filaments of the superior laryngeal branch of the vagus supply the epithelial lining of the larynx, giving it that acute sensibility by which the glottis is guarded against the ingress of foreign bodies, or of irrespirable gases. The contact of these stimulates the nerve endings; and the sensory nerve impulse conveyed to the medulla oblongata, whether accompanied by sensation or not, arouses motor impulses through the filaments of the recurrent or inferior laryngeal branch, which excite contraction of 
the muscles that close the glottis. Both these branches of the vagi cooperate also in the production and regulation of the voice. The inferior laryngeal determines the degree of contraction of the muscles that vary the tension of the vocal cords, and the superior laryngeal conveys to the brain the sensation which indicates the state of contraction of these muscles. Both the branches cooperate also in the actions of the larynx in the ordinary slight dilatation and contraction of the glottis in the acts of expiration and inspiration, more evidently in the acts of coughing and other forcible respiratory movements.

The Laryngoscope.-This is an instrument employed in investigating the condition of the pharynx, larynx, and trachea. It consists of a large concave mirror with perforated center and of a smaller mirror fixed in a long handle. In use the patient is placed in a chair, a good light (argand burner, or lamp) is arranged on one side of, and a little above his head. The operator fixes the

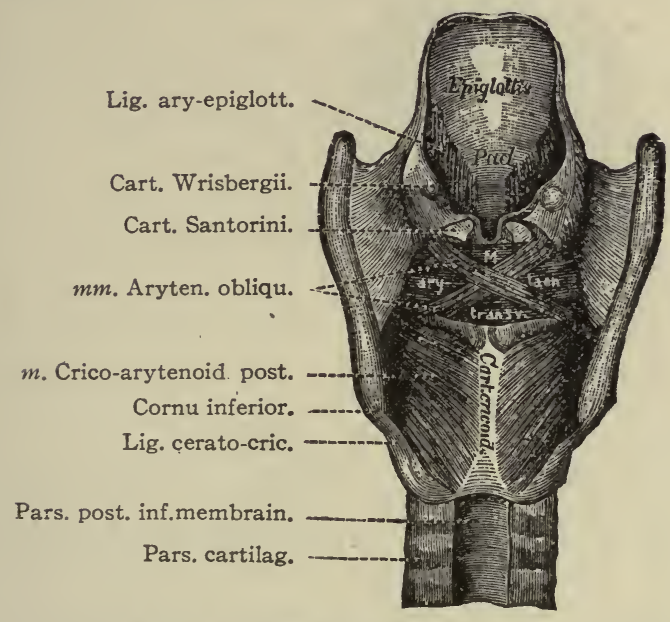

FIG. 345.-The Larynx as Seen from Behind. To show the intrinsic muscles posteriorly. (Stoerk.)

concave mirror round his head in such a manner that he looks through the central aperture with one eye. He then seats himself opposite the patient, and so adjusts the position of the mirror, which is for this purpose provided with a ball and socket joint, that a beam of light is reflected on the lips of the patient.

The patient is now directed to throw his head slightly backward, and to open his mouth; the reflection from the mirror lights up the cavity of the mouth, and by a little alteration of the distance between the operator and the patient the point at which the greatest amount of light is reflected by the mirror-in other words its focal length-is readily discovered. The small mirror fixed in the handle is then warmed, either by holding it over the lamp, or by putting it into a vessel of warm water; this is necessary to prevent the condensation of breath upon its surface. The degree of heat is regulated by applying the back of the mirror to the hand or cheek, rrhen it should feel warm without being painful.

After these preliminaries the patient is directed to put out his tongue, which is held by the left hand of the operator gently but firmly against the 
lower teeth by means of a handkerchief. The warm mirror is passed to the back of the mouth, until it rests upon and slightly raises the base of the uvula, and at the same time the light is directed upon it: an inverted image of the

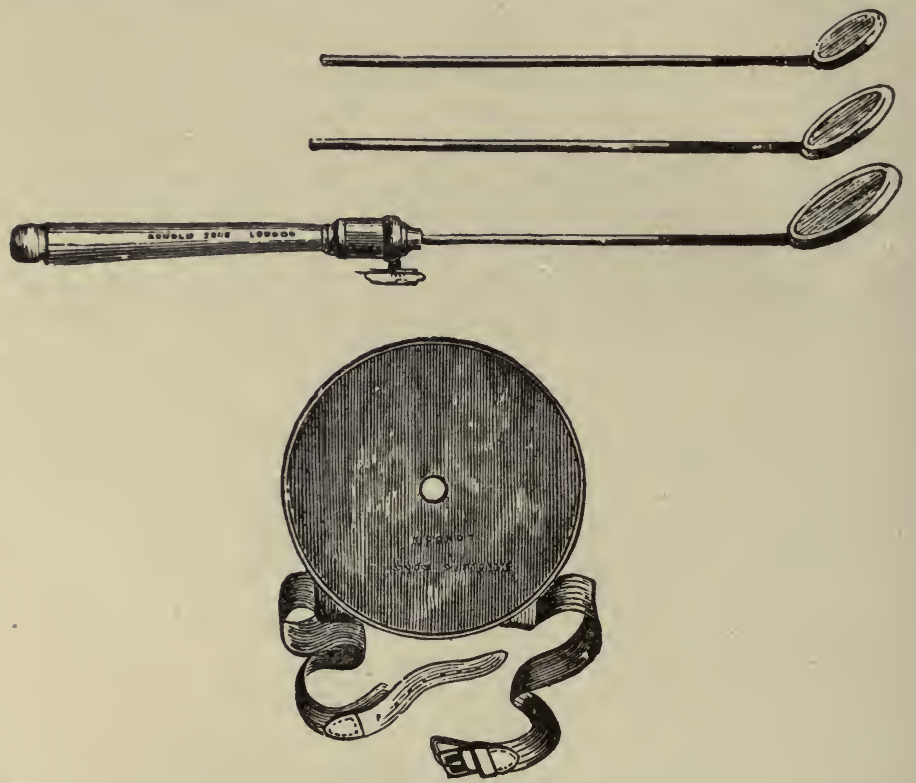

FIG. 346.-The Parts of the Laryngoscope. .

larynx and trachea will be seen in the mirror. If the dorsum of the tongue be alone seen, the handle of the mirror must be slightly lowered until the larynx comes into view; care should be taken, however, not to move the mirror

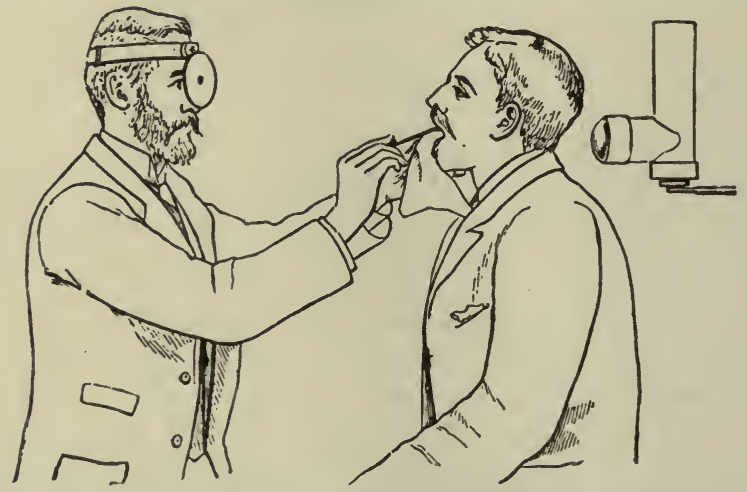

Fig. 347.-To Show the Position of the Operator and Patient when Using the Laryngoscope.

upon the uvula, as it excites retching. The observation should not be prolonged, but should rather be repeated at short intervals.

The structures seen will vary somewhat according to the condition of the parts as to inspiration, expiration, phonation, etc. They are the following: 
first, and apparently at the posterior part, the base of the tongue, immediately below which is the accurate outline of the epiglottis, with its cushion or tubercle, figure 348 . Then are seen in the central line the true vocal cords, white and shining in their normal condition. In the inverted image the cords are closer together posteriorly. Between them is left an open slit, narrow while a high note is being sounded, wide during a deep inspiration. On each side of the true vocal cords, and on a higher level, are the false vocal cords. Still more externally than the false vocal cords is the aryteno-epiglottidean fold, in which are situated upon each side three small elevations; of these the most external is the cartilage of Wrisberg, the intermediate is the cartilage of Santorini, while in front and somewhat below the preceding is the summit of the arytenoid cartilage seen only during deep inspiration. The rings of the trachea, and even the bifurcation of the trachea itself, if the patient be directed to draw a deep breath, may be occasionally seen.

Movements of the Vocal Cords. - The position of the vocal cords in ordinary tranquil breathing is so adapted by the muscles that the opening of the glottis is wide and triangular, figure $348, B$, becoming a little wider at each inspiration, and a little narrower at each expiration. On making a rapid and deep inspiration the opening of the glottis is widely dilated, figure $348, C$, and somewhat lozenge-shaped.

In Vocalization. - At the moment of the emission of a note the opening is narrowed, the margins of the arytenoid cartilages being brought into contact and the edges of the vocal cords approximated and made parallel at the same time that their tension is much increased. The higher the note produced, the tenser do the cords become, figure $348, A$; and the range of a voice depends, of course, in the main, on the extent to which the degree of tension of the vocal cords can be thus altered. In the production of a high note the vocal cords are brought well within sight, so as to be plainly visible with the help of the laryngoscope. In the utterance of low tones, on the other hand, the epiglottis is depressed and brought over the vocal cords, figure 349. The epiglottis, by being somewhat pressed down so as to cover the superior cavity of the larynx, serves to render the notes deeper in tone and at the same time somewhat duller, just as covering the end of a short tube placed in front of caoutchouc tongues lowers the tone. In no other respect does the epiglottis appear to have any effect in modifying the vocal sounds.

The degree of approximation of the vocal cords also usually corresponds with the height of the note produced; but probably not always, for the width of the aperture has no essential influence on the pitch of the note, as long as the vocal cords have the same tension; only with a wide aperture the tone is more difficult to produce and is less perfect, the rushing of the air through the aperture being heard at the same time.

No true vocal sound is produced at the posterior part of the aperture of the glottis, the part of the aperture which is formed by the space between the arytenoid cartilages. For if the arytenoid cartilages be approximated in such a manner that their anterior processes tourh each other, but yet leave an 
opening behind them as well as in front, no second vocal tone is produced by the passage of the air through the posterior opening, but merely a rustling sound. The pitch of the note produced is the same whether the posterior part of the glottis be open or not.

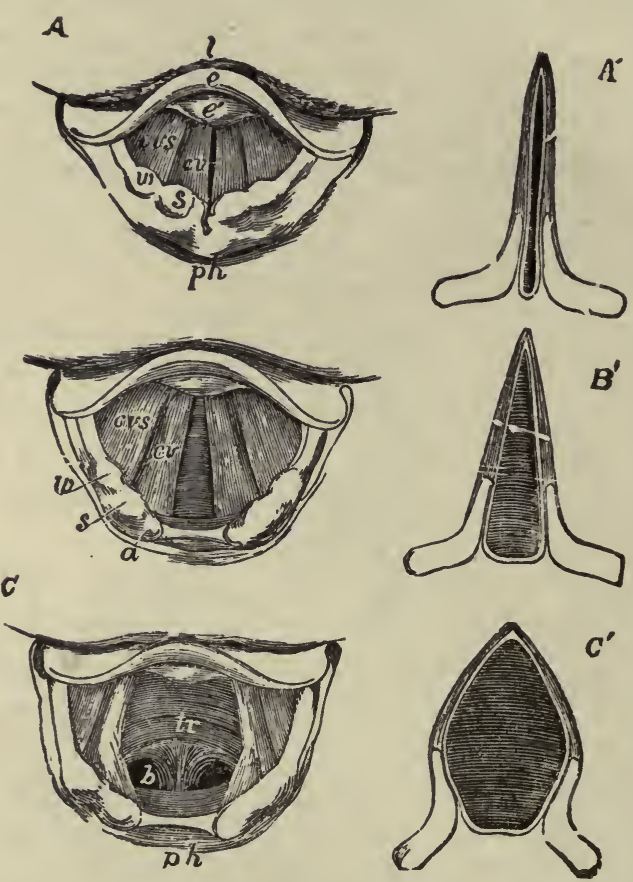

Fig. 348.-Three Laryngoscopic Views of the Superior Aperture of the Larynx and Surrounding Parts. $A$, The glottis during the emission of a high note in singing; $B$, in easy and quiet inhalation of air; $C$, in the state of the widest possible dilatation, as in inhaling a very deep breath. The diagrams $A^{\prime}, B^{\prime}$, and $C^{\prime}$, show in horizontal sections of the glottis the position of the vocal ligaments and arytenoid cartilages in the three several states represented in the other figures. In all the figures, so far as marked, the letters indicate the parts as follows, viz.: $l$, the base of the tongue; $e$, the upper free part of the epiglottis; $e^{\prime}$, the tubercle or cushion of the epiglottis; ph, part of the anterior wall of the pharynx behind the larynx; in the margin of the aryteno-epiglottidean fold $w$, the swelling of the membrane caused by the cartilages of Wrisberg; $s$, that of the cartilages of Santorini; $a$, the tip or summit of the arytenoid cartilages; $c v$, the true vocal cords or lips of the rima glottidis; cvs, the superior or false vocal cords; between them the ventricle of the larynx; in $C, t r$ is placed on the anterior wall of the receding trachea, and $b$ indicates the commencement of the two bronchi beyond the bifurcation which may be brought into view in this state of extreme dilatation. (Quain, after Czermak.)

The Voice in Singing.- The laryngeal tones may be produced in three different kinds of sequence. The first is the monotonous, in which the notes have nearly all the same pitch as in ordinary speaking; the variety of the sounds of speech being due to articulation in the mouth. In speaking, occasional syllables receive a higher intonation for the sake of accent. The second mode of sequence is the successive transition from high to low notes, 
and vice versa, without intervals; such as is heard in the crying in children and in the howling and whining of dogs. The third mode of sequence of the vocal sounds is the musical, in which each sound has a determinate number of vibrations, and the numbers of the vibrations in the successive sounds have the same relative proportions that characterize the notes of the musical scale.

The different sounds made by the musical voice are characterized by the three properties of tones in general, viz., the pitch, which is dependent on the rate of vibration of the vocal cords; the loudness, which depends on the force of

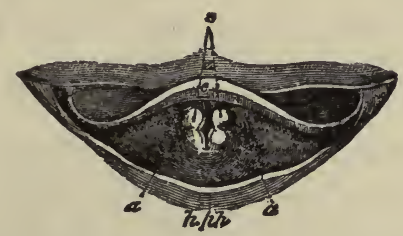

Fig. 349.-View of the Upper Part of the Larynx as Seen by Means of the Laryngoscope during the utterance of a grave note. $c$, Epiglottis; $s$, tubercles of the cartilages of Santorini; $a$, arytenoid cartilages; $z$, base of the tongue; $p h$, the posterior wall of the pharynx. (Czermak.)

the vibration, and the quality or timber, which is dependent on the resonance of the cavities of the respiratory apparatus, particularly of the mouth, pharynx, and nasal cavities.

The Vocal Range of the Voice.-In different individuals this comprehends one, two, or three octaves. In singers, that is, in persons trained in singing, it extends to three or more octaves. But the male and female voices commence and end at different points of the musical scale. The lowest note of the female voice is about an octave higher than the lowest of the male voice; the highest note of the female voice about an octave higher than the highest of the male. The entire scale of the average human voice includes, from the lowest male note to the highest female, about three to three and a half octaves. Some remarkable musical voices have had a range of three and a half octaves. A principal difference between the male and female voice is, therefore, in their pitch. But they are also distinguished by the quality of the tone. The voices of men and of women differ among themselves, both in the general pitch and in the quality. There are two kinds of male voices, technically called the bass and tenor, and two of female voices, the contralto and soprano, all differing from each other in general pitch. The bass voice reaches lower than the tenor, and its strength lies in the low notes. The contralto voice is of lower range than the soprano, and is strongest in the lower notes of the female voice. The barytone and mezzo-soprano voices are intermediate in range; the barytone being intermediate between bass and tenor, the mezzo-soprano between the contralto and soprano. The difference in the pitch of the male and the female voices depends primarily on the different size of the larynx and the 
length of the vocal cords in the two sexes; their relative lengths in men and women are as three to two.

The boy's larynx resembles the female larynx. His vocal cords before puberty are not two-thirds the length of the adult cords; and the angle of the thyroid cartilage is as little prominent as in the female larynx. Boy's voices are alto and soprano, resembling in pitch those of women, but louder, and differing somewhat from them in tone. But, after the larynx has undergone the change produced during the period of development at puberty, the boy's voice becomes bass or tenor. While the change of form is taking place the voice becomes imperfect, frequently hoarse and crowing, and is unfitted for singing until the readjustment of the larynx is complete and the muscles which control the vocal cords are again coordinated. In eunuchs who have been deprived of the testes before puberty, the voice does not undergo this change. The voice of most old people is deficient in tone, unsteady, and more restricted in extent. The first defect is owing to the ossification of the cartilages of the larynx and the altered condition of the vocal cords; the want of steadiness arises from the loss of nervous power and command over the muscles, the result of which is here, as in other parts, a tremulous movement. These two causes combined render the voices of old people void of tone, unsteady, and weak.

Most persons have the power, if at all capable of singing, of modulating their voices through a double series of notes of different character: namely, the notes of the natural voice, or chest-notes, and the falsetto notes. The natural voice, which alone has been hitherto considered, is fuller, and excites a distinct sensation of much stronger vibration and resonance than the falsetto voice, which has more of a flute-like character.

The Quality of the Voice.-The difference in quality of voices, seen when two or more persons sound the same note, is due to differences in resonance in the cavities of the mouth and larynx and also of the nose. The shape of the roof of the mouth, the regularity of the teeth, and the size of the tongue, and the size and clearness of the nasopharynx are all factors. The size and shape of the larynx and mouth cavity which influence the voice quality can be controlled to some, extent during singing, and this is a special point of training in voice culture.

Speech.-Besides the musical tones formed in the larynx a great number of other sounds can be produced in the vocal tubes, between the glottis and the external apertures of the air-passages, the combination of which sounds into different groups to designate objects, properties, actions, etc., constitutes language. The languages do not employ all the sounds which can be produced in this manner, the combination between certain sounds being often difficult. Those sounds which are easy of combination enter, for the most part, into the formation of the greater number of languages. Each language contains a certain number of such sounds, but in no one are all 
brought together. On the contrary, different languages are characterized by the prevalence in them of certain classes of these sounds, while other sounds are less frequent or altogether absent.

Articulate Sounds. - The sounds produced in speech, or the articulate sounds, are commonly divided into vowels and consonants: the distinction between which is that the sounds for the former are generated by the larynx, while those for the latter are produced by interruption of the current of air in some part of the air-passages above the larynx. The term consonant has been given to these because several of them are not properly sounded, except, consonantly with a vowel. Thus, if it be attempted to pronounce aloud the consonants b, d, and $g$, or their modifications, p, t, k, the intonation follows them only in their combination with a vowel. To recognize the essential properties of the articulate sounds, it is necessary first to examine them as they are produced in whispering, and then investigate which of them can also be uttered in a modified character conjoined with vocal tone. By this procedure we find two series of sounds: in one the sounds are mute, and cannot be uttered with a vocal tone; the sounds of the other series can be formed independently of voice, but are also capable of being uttered in conjunction with it.

All the vowels can be expressed in a whisper without vocal tone, that is, mutely. These mute vowel sounds differ, however, in some measure, as to their mode of production, from the consonants. All the mute consonants are formed in the vocal tube above the glottis, or in the cavity of the mouth or nose, by the mere rushing of the air between the surfaces differently modified in disposition. But the sound of the vowels, even when mute, has its source in the glottis, though its vocal cords are not thrown into the vibrations necessary for the production of voice; and the sound seems to be produced by the passage of the current of air between the relaxed vocal cords. The same vowel sound can be produced in the larynx when the mouth is closed, the nostrils being open, and the utterance of all vocal tone avoided. The sound when the mouth is open, is so modified by varied forms of the oral cavity as to assume the characters of the vowels $\mathbf{a}, \mathbf{e}, \mathbf{i}, \mathbf{o}, \mathbf{u}$, in all their modifications.

The cavity of the mouth assumes the same form for the articulation of each of the mute vowels as for the corresponding vowel when vocalized; the only difference in the two cases lies in the kind of sound emitted by the larynx. It has been pointed out that the conditions necessary for changing one and the same sound into the different vowels are differences in the size of two parts - the oral canal and the oral opening; and the same is the case with regard to the mute vowels. By oral canal is meant here the space between the tongue and palate: for the pronunciation of certain vowels both the opening of the mouth and the space just mentioned are widened; for the pronunciation of other vowels both are contracted; and for others one is wide, the other contracted. Admitting five degrees of size, both of 
the opening of the mouth and of the space between the tongue and palate, Kempelen thus states the dimensions of these parts for the following vowel sounds:

Vowel. Sound.

a as in "far"
a as in "name"
e as in "theme"
o as in "go"
oo as in "cool"

a as in "far"

a as in "name"

e as in "theme"

oo as in "cool"
Size of oral opening.

Size of oral canal.

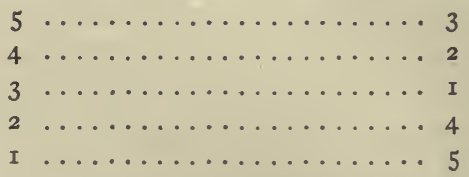

Another important distinction in articulate sounds is that the utterance of some is only of momentary duration, taking place during a sudden change in the conformation of the mouth, and being incapable of prolongation by a continued expiration. To this class belong $b, p, d$, and the hard $g$. In the utterance of other consonants the sounds may be continuous; they may be prolonged, ad libitum, as long as a particular disposition of the mouth and a constant expiration are maintained. Among these consonants are $\mathrm{h}, \mathrm{m}, \mathrm{n}$, $\mathrm{f}, \mathrm{s}, \mathrm{r}, \mathrm{l}$. Corresponding differences in respect to the time that may be occupied in their utterance exist in the vowel sounds, and principally. constitute the differences between long and short syllables. Thus the a as in far and fate, the $o$ as in go and fort, may be indefinitely prolonged; but the same vowels (or more properly different vowels expressed by the same letters), as in can and fact, in dog and gotten, cannot be prolonged.

All sounds of the first or explosive kind are insusceptible of combination with vocal tone (intonation), and are absolutely mute; nearly all the consonants of the second or continuous kind may be attended with intonation.

The tongue, which is usually credited with the power of speech, plays only a subordinate, although very important, part. This is well shown by cases in which nearly the whole organ has been removed on account of disease. Patients who recover from this operation talk imperfectly, and their voices are considerably modified; but the loss of speech is confined to those letters in the pronunciation of which the tongue is particularly concerned, namely, c, d, g, h, j, k, etc.

Stammering depends on a want of harmony between the action of the muscles (chiefly abdominal) which expel air through the larynx, and that of the muscles which guard the orifice (rima glottidis) by which it escapes, and of those (of tongue, palate, etc.) which modulate the sound to the form of speech. Over either of the groups of muscles, by itself, a stammerer may have as much power as other persons, but he cannot harmoniously arrange their conjoint actions. 


\section{LABORATORY EXPERIMENTS ON MUSCLE AND NERVES.}

Physiological experiments on living muscle serve to demonstrate many of the most fundamental particulars of physiology. The muscles of cold-blooded animals isolated from the body retain their living attributes for hours under laboratory conditions. They illustrate practically all the facts shown by the muscles of warm-blooded animals.

I. The Use of the Induction Coil.-The induced current obtained by means of an apparatus called an induction coil is the most convenient and reliable means of stimulating the muscle or the nerve. The arrangement of the batteries, keys, and coils for the ordinary muscle work is shown in figure 350. The strength of the induced current decreases with the distance of the secondary coil from the primary, but according to a logarithmic curve. For ordinary work the centimeter scale is adequate guide for the proportionate strength of the induction current.

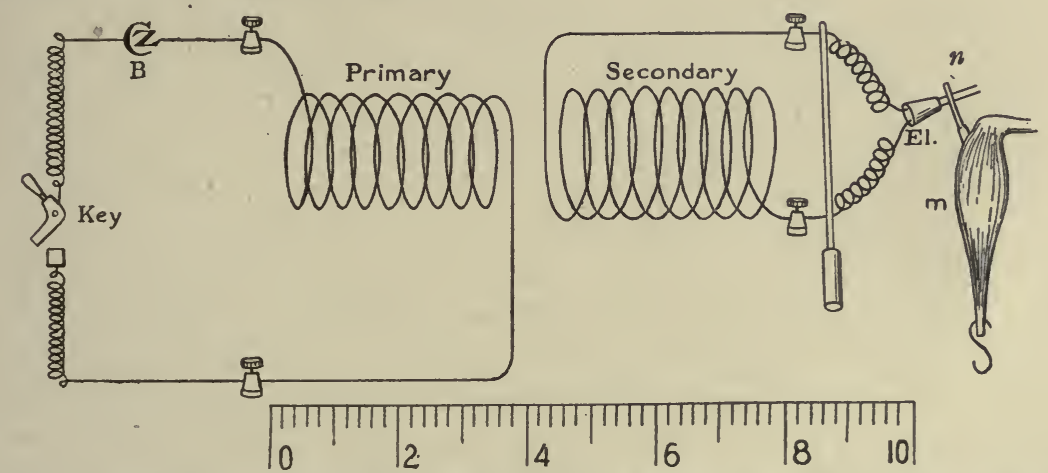

FIG. 350.-Diagram illustrating the relations of the battery, keys, coils, and electrodes as used for physiological stimulation.

At the moment of closure of the primary key, a current of electricity is induced in the secondary coil, the make current. The induced current is only momentary in duration, i.e., does not continue though the primary circuit is complete. Also when the primary current is broken by opening the key a second current is induced, the break current. The former is in the opposite direction to, the latter in the same direction as the primary current. In ordinary coils the break current is stronger. The induction coil may be used to produce a rapid series of shocks by means of a magnetic interrupter, as in the Harvard inductorium.

$a$. Test the strength of a series of single make induction shocks beginning with the weakest possible position of the secondary coil, and going toward the primary coil $\mathrm{I} \mathrm{cm}$. after each test. Test the stimulating effect by applying the electrodes to the tip of the tongue. Record the results as directed by the table below. 
b. Repeat using the break induction currents.

\begin{tabular}{|c|c|c|}
\hline \multirow{2}{*}{$\begin{array}{l}\text { Position of the secondary } \\
\text { coil in centimeters from } \\
\text { the primary }\end{array}$} & \multicolumn{2}{|c|}{$\begin{array}{l}\text { Relative stimulating strength on the tongue, i. e., "none, } \\
\text { trace, faint, mild, strong, very strong, etc." }\end{array}$} \\
\hline & Make Shock & Break Shock \\
\hline & & \\
\hline & & \\
\hline & & \\
\hline
\end{tabular}

2. The Muscle-nerve Preparation.-The classical muscle-nerve preparation is the gastrocnemius muscle and the sciatic nerve. Prepare it as follows: $a$. Kill the frog by pithing. This is done by grasping the frog firmly in one hand and with the other making a cut with a blunt scalpel through the cranium just over the medulla, turning the scalpel so as completely to destroy the medulla. Now take a slender knitting needle, quickly run it up into the cranial cavity to destroy the brain, and down the spinal canal to destroy the cord. After a brief spasmodic contraction of the muscles of practically the entire body, the frog remains limp and motionless. $b$. In making the muscle-nerve preparation it is better to isolate the tendon Achilles and insert its hook first, then expose the nerve, and finally the femur. The nerve should be prepared of as long a length as possible and should not be allowed to come in contact with the skin. If the preparation is to be used in a moist chamber, the skin should be entirely removed; if it is to be used in the open air, the skin should be left on. Use care not to stretch the nerve, and protect it from evaporation. Cut the femur long.

3. The Irritability of Nerve.-Prepare a muscle-nerve with its skin on and do not cut away the foot. Mount it by inserting the femur in a muscle clamp, letting the leg extend vertically upward, and the foot hang over. The nerve should lie along the exposed moist femur, one end being slightly free. Stimulate the nerve in the following ways:

a. Electrical Stimuli.-Apply the electrodes of the secondary coil of an induction apparatus to the tip of the nerve. When an induction current of sufficient strength is produced, the muscle to which the nerve is attached will give contractions, thus moving the foot. Notice that contractions occur with both make and break inductions. Apply the electrodes from the two poles of a dry battery. When the current of the battery is established a contraction will occur, but does not continue during the time of the flow of the current. When the current is stopped a second contraction occurs. The nerve is irritable to both galvanic and faradic currents. 
b. Mechanical Stimuli.-Pinch the nerve lightly with forceps, or give it a sudden stroke with the scalpel handle. With each mechanical impact there is a single contraction of the muscle.

c. Thermal Stimuli. - Touch the end of the nerve with a glass rod heated in boiling water. At each time the nerve is brought in contact with the rod there will be muscular contraction, as in the preceding cases. The experiment succeeds better if the nerve comes in contact with the rod for several millimeters of length. If the tip of the nerve has ceased to respond, then snip it off with the scissors, and repeat the experiment on the fresh end.

d. Chemical Stimulation.-Many chemical substances when brought in contact with living nerve fibers produce nerve impulses. Try crystals of sodium chloride, magnesium sulphate, dilute ammonia, acetic acid, ro per cent. nitric acid, I per cent. mercuric chloride.

Tabulate your observations on all the forms of stimulation used above, by the following outline:

\begin{tabular}{l|l}
\hline Kind of stimulation. & Effect produced. \\
\hline & \\
\hline
\end{tabular}

4. Irritability of Muscle.-Repeat the experiments in number 2 above, applying the stimuli, electricity, etc., directly to the muscle substance, choosing as far as possible portions of muscle which do not exhibit nerve fiber. The muscle will usually respond by a contraction to each of the above forms of stimulation.

These tests do not fully demonstrate the direct irritability of muscle substance, since in each case it is possible that nerves may have been stimulated. The nerve influences over the muscle can be eliminated by the use of drugs, as will be shown in the next experiment.

5. Independent Irritability of Muscle.-The influence or curara on the muscle-nerve preparation is demonstrated as follows: Ligate one leg of a frog near the thigh to stop the circulation on that side. Now inject under the skin of the back three drops of I per cent. curara, allowing twenty to thirty minutes for absorption. Make the following observations: Place the animal on a glass plate with back up and dissect out the sciatic nerve in each leg. Use care not to injure in any way the accompanying femoral artery.

$a$. Stimulate the muscles of the ligatured leg, also the muscles of the curarized leg, each will contract. 
$b$. Stimulate the sciatic nerve of the ligated leg above the ligature; also the sciatic of the opposite side, both of which have come in contact with the curara. Stimulation of the first nerve produces contraction of its muscle; of the second nerve does not produce contraction of its muscle.

From this experiment of Claude Bernard's it is evident that the curara does not destroy the irritability of nerve fiber nor the irritability of the muscle fiber, yet it does destroy the influence of the nerve over the muscle, probably acting as a specific poison for the motor end-plates. If the motor end-plates are destroyed, then forms of stimuli which produce contractions of the muscle must act directly on muscle substance, proving that muscle substance, as such, is irritable.

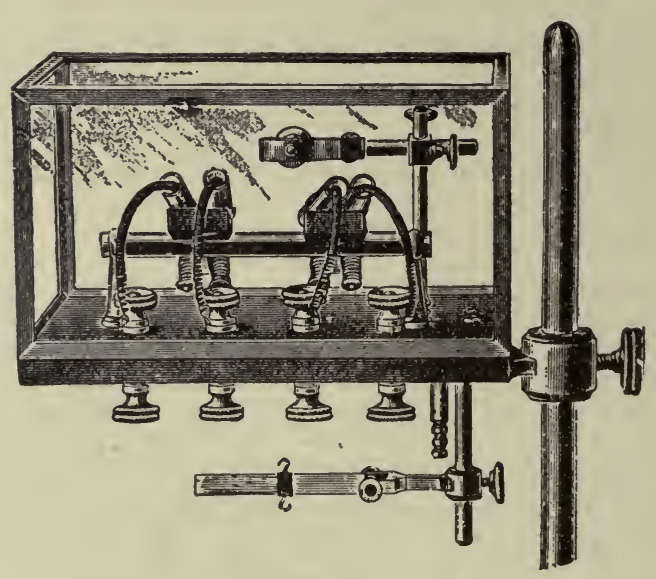

Fig. 351.-Moist Chamber.

6. The Relation of the Contraction to the Strength of the Stimulus.-Minimal and Maximal Stimuli.-a. Prepare a recording cylinder for making vertical records of the contractions. Adjust the writing point of the muscle lever to the drum and set the drum to be moved by hand $\mathrm{I} \mathrm{cm}$. after each succeeding contraction. Set the secondary coil of the induction apparatus so that it will be too weak to produce a stimulus.

$b$. Prepare a muscle nerve of the frog and mount in the moist chamber and arrange for stimulating the muscle directly by means of the secondary current of the induction coil, stimulating apparatus adjusted as in fig. $35^{\circ}$.

c. Now attempt to stimulate the muscle, then move the induction coil toward the primary $\mathrm{I} \mathrm{cm}$. at a time and repeat until the first slight contraction appears. Continue to slide the secondary coil toward the primary, stimulate at each new position, moving the drum forward for each stimulus as directed, until a series of contractions is obtained through the range of variation of induction of which the apparatus is capable, usually twenty or thirty contractions. 
A typical tracing, figure 326 , shows that as the strength of the stimulus is increased the amplitude of the contractions quickly mounts from the minimal to a maximal, after which all further increase in the strength of the stimulus produces contractions of practically the same height. The first perceptible contraction is called the minimal contraction, the strength of the current which produced it is a minimal stimulus for that preparation. The contractions of the greatest amount are called maximal contractions. The weakest stimulus which produces a maximal contraction is called the maximal stimulus, and all stronger stimuli supramaximal.

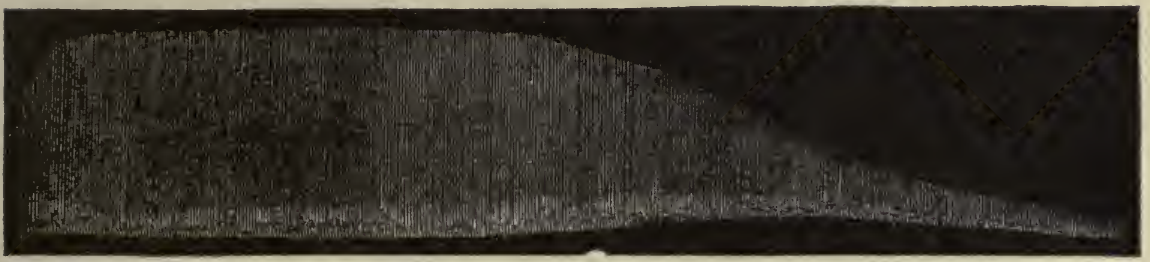

Fig. 352.-The Type of Contractions given by the Gastrocnemius of the Frog to a series of stimuli occurring at regular recurrent intervals. (Taskinen.)

7. The Effect of Fatigue on the Amplitude of a Series of Simple Muscle Contractions. - $a$, Arrange the recording apparatus and set the induction coil for single stimuli. Adjust the recording lever of the muscle to a smoked-paper kymograph and set the speed of the kymograph to revolve at the rate of $\mathrm{I} \mathrm{mm}$. per second.

$b$. Prepare a gastocnemius muscle for direct stimulation and mount it in a moist chamber.

c. Now stimulate the muscle with the make induction (short-circuiting the break) once every two seconds. The contractions will be recorded as vertical marks on the drum in regular order, at a distance of $2 \mathrm{~mm}$. apart, hence very slight changes in amplitude are readily detected. The contractions gradually increase in height for the first ten or twenty contractions, the phenomenon of treppe, then run for from fifty to one hundred contractions of practically uniform amplitude, after which there is a gradual but sharp decrease known as fatigue. Repeat on the second gastrocnemius.

$d$. Repeat the experiment after ten minutes' rest. The former variations occur now very rapidly, indicating that the fatigue effects are only, partially recovered from.

\section{Fatigue of Voluntary Muscular Contraction, Demonstration.-} The human voluntary muscles are used to demonstrate this experiment. Use a Mosso's ergograph, or any one of its numerous modifications. If the original form is used, then the muscle should be loaded with about 3 kilos, and contractions once a second recorded until the muscle can no longer lift the load. The load may have to be adjusted to the individual, but should be chosen so that exhaustion will be obtained with about thirty contractions. 
This experiment does not demonstrate complete exhaustion, but merely fatigue down to a certain level. If an apparatus is previously arranged for direct stimulation of the muscles by electric currents it will be found that the contractions of the muscles still occur after the voluntary power is lost, showing that at least a part of the phenomenon of fatigue, possibly the chief part, is located in the nervous tissue rather than in the muscle substance.

9. Effect of Load on the Height of the Contraction and on the Work of Voluntary Muscle.-a. Make a muscle-nerve preparation and arrange it for stimulation, as in Experiment 6 above. Set the induction coil of the stimulating apparatus for an effective supramaximal stimulus. Record the contractions as pairs of vertical lines on the kymograph, the pairs separated by a distance of $\mathrm{I} \mathrm{cm}$. Begin with the load of the lever only for the first contraction, then increase the load by steps of 20 grams each until the muscle is no longer able to lift the weight used. Record two contractions for each weight. Use care that no mechanical changes of the apparatus are recorded so as to confuse the record of the contraction on the smoked cylinder.

$b$. Repeat the experiment on a fresh muscle, supporting the lever with a tension of 20 grams on the muscle.

The amount of work done by the muscle at each contraction is the product of the load in grams times the height in centimeters. The height of the lift can be obtained in this experiment from the height of the record on the drum and the lengths of the recording and power arms of the lever, in which r.l., the length of the recording lever, is to p.l., the length of the power lever, as $h$., the height of the record obtained, is to $c$., the actual shortening of the muscle. Compute the exact amount of work done by each contraction ( $c$ the contraction times $w$ the weight gives the work), and tabulate on coordinate paper. Compare the variation in work done with the variation in amplitude of the contraction.

Io. The Effect of Temperature on the Amplitude of the Muscle Contractions.-Prepare a muscle-nerve and mount it in Porter's latest form of temperature apparatus. Insert the thermometer bulb beside the muscle. Adjust the levers for vertical records on the smoked paper of the kymograph. Begin with a temperature of tap water and gradually lower the temperature of the preparation by adding small amounts of crushed ice at first; later, add ice and salt crystals to the external chamber, siphoning off excess of fluid into a cup. Take care to lower the external temperature very slowly and gradually, say about one degree in two minutes. Stimulate the muscle with a supramaximal stimulus twice in rapid succession, for $\mathrm{I}^{\circ} \mathrm{C}$. of change. Record these contractions as pairs of vertical marks on the drum I $\mathrm{mm}$. apart, separating each pair by a space of $\mathrm{I} \mathrm{cm}$. When $0^{\circ} \mathrm{C}$. is reached, or before if the muscle fails to contract at a higher temperature, reverse the direction of the temperature change, gradually but slowly increasing it until the muscle goes into heat rigor, which begins at from $38^{\circ}$ to $40^{\circ} \mathrm{C}$. 
While the muscle is entering rigor, move the drum $\mathrm{I} \mathrm{cm}$. for each degree, as before, so as to record the development of that process.

Ir. The Form of the Simple Muscle Contraction.-Striated muscle responds to electrical stimuli even though of almost instantaneous duration. The response which the muscle gives to a single stimulus is called a simple muscle contraction, and is demonstrated as follows:

a. Arrange an induction coil with its keys, battery, and electrodes connected, so as to stimulate the muscle by the automatic key of the Guthrie

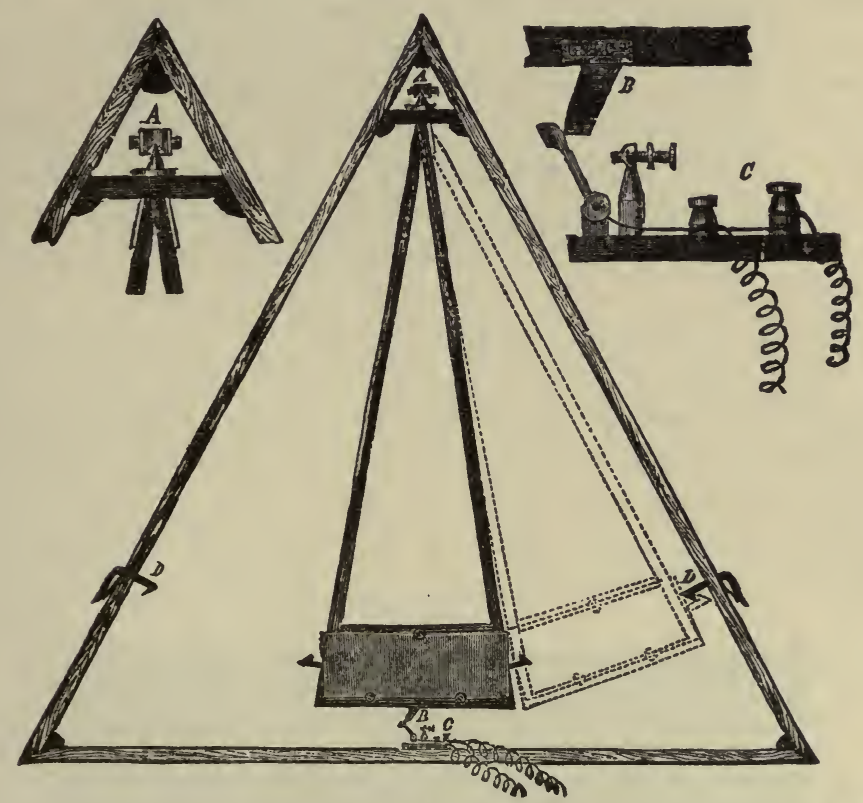

Fig. 353.-Simple Form of Pendulum Myograph and Accessory Parts. A, Pivot upon which pendulum swings; $B$, catch on lower end of myograph opening the key, $C$, in its swing; $D$, a spring-catch which retains myograph, as indicated by dotted lines, and on pressing down the handle of which the pendulum swings along the arc to $D$ on the left of figure, and is caught by its spring.

apparatus. Set the secondary coil at a position which will give a strong contraction of the muscle, and record this contraction on the smoked paper of an ordinary recording cylinder. Revolve the drum at a rapid rate using the weight-driven attachment, allowing the automatic key to be opened while the drum is turning at its highest speed. Or use the pendulum myograph, which is especially constructed for this experiment, figure 353 .

b. Make a muscle-nerve preparation with the tendon isolated and the skin removed, Experiment 2, and mount it in a moist chamber, figure 35I. Lay the nerve across a pair of platinum electrodes, shake a little water on the inside of the cover of the moist chamber, and place it over the preparation so as to prevent drying of the nerve and of the muscle. 
The muscle contraction now is recorded as a wave which shows some considerable duration in time, figure 354 . Introduce a roo double vibration tuning fork to record the speed of the drum, and take care to mark the exact point on the record where the automatic key is opened. (Instead of the automatic key one may use a hand key in connection with a signal magnet to mark the point of stimulation.) In this record the muscle contraction shows three different periods or phases. 'The first, a period of no activity, called the latent period, taking about $0.0 \mathrm{r}$ of a second; the second, the period of rapid shortening known as the contraction phase, taking about 0.04 of a second on the average; and the third, a period of rapid relaxation or return to the normal, which takes about 0.05 of a second, see figure 354 .

The time and character of the simple muscle contraction will be influenced by: $a$, load or tension; $b$, the exact temperature; $c$, by the amount of work it has previously done, or fatigue; $d$, by the time since it was isolated from the circulation. Perform a series of experiments varying these effects, and record the results by the following outline:

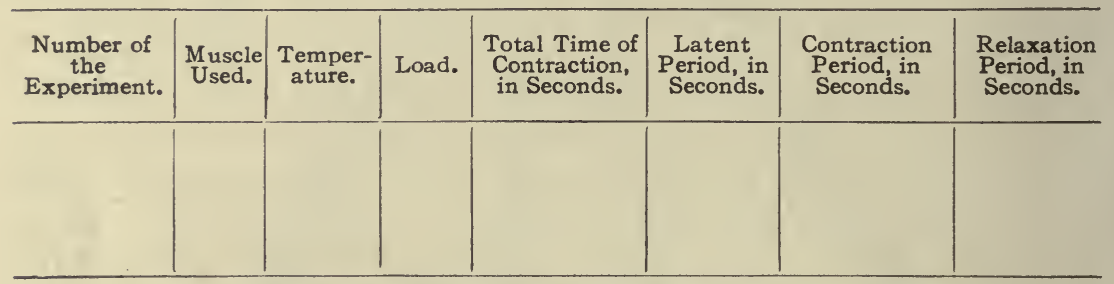

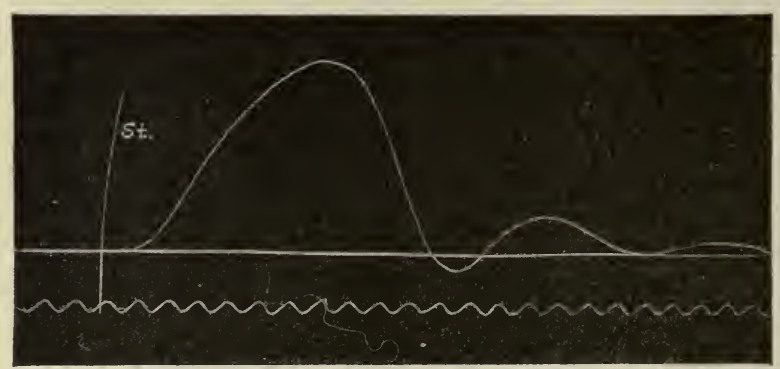

Fig. 354.--Record of a Simple Contraction of the Gastrocnemius of the Frog. Time in .or of a second. St, Moment of stimulation. Record taken on a rapid drum that was provided with an automatic key.

I2. The Effect of Fatigue on the Time of the Simple Contraction. Prepare a muscle nerve and mount it in the moist chamber, arrange for the record as directed under 5 above. Make a series of records of the simple contraction when automatically stimulated, recording only every tenth or twentieth contraction-the intermediate contractions should be shunted and are used merely to produce fatigue. After a time the contractions will not only diminish in amplitude, but there will be a gradual increase in the time 
consumed by the contraction. This increase in time falls very slightly on the latent period, is more pronounced in the contraction phase, but is very marked in the relaxation phase, figure 355 .

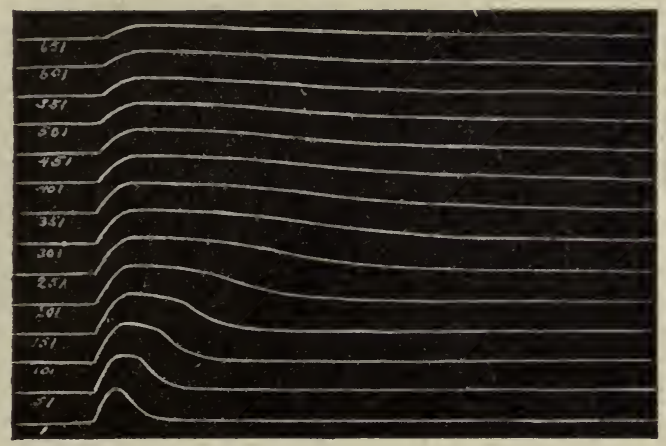

Fig. 355- - Contractions of the Gastrocnemius Muscle to Show Fatigue. The numbers printed on the figure indicate the contraction in the series which is recorded. (Lee.)

\section{The Effect of Temperature on the Time of the Simple Con-} traction.-Repeat Experiment ro but record the contractions by the method described in Experiment II above, recording a contraction for every change of $5^{\circ} \mathrm{C}$. Measure the time and amplitude of the different contractions, and the phases of the simple contractions, and tabulate them as shown in Experiment II.

I4. Tetanus.-A continued contraction of a voluntary muscle can be shown to be a fusion of simple muscle contractions. This is called a tetanus. Arrange the induction coil for stimulating with a series of rapidly repeated stimuli. Set the secondary coil so the break inductions only will stimulate thus securing one stimulus per vibration of the tetanometer key. The rate of the stimulation is obtained from the Harvard tetanometer, a form of key for rapidly interrupting the current, which should be connected with the primary coil instead of the key, K, figure 350. Prepare a muscle-nerve in the moist chamber and stimulate the muscle at a rate of ro per second, record the contractions on the drum moving at a speed of about $2 \mathrm{~cm}$. per second. Use care not to overfatigue the muscle, i.e., stimulate it only 2 seconds at a time controlling the time by the extra key in the primary circuit. Repeat this test, increasing the rate of stimulation each time by 5 , that is stimulate at $10,15,20$, etc., per second. In the first stimulation there will be a series of simple contractions with almost complete intervening relaxations. As the rate is increased these relaxations become less and less until presently a rate is found which produces continuous, apparently uninterrupted contractions. This is a tetanus. The others are incomplete tetani. The frog's gastrocnemius at a temperature of $20^{\circ} \mathrm{C}$. is tetanized with a stimulation of from 25 to 30 per second. 
I 5. DuBois-Reymond's Law of Galvanic Stimulation.-Connect a single dry cell, a key switch, and a meter-rheocord in circuit. To the zero end of the rheocord attach a lead-off through a pole-changer to one of the non-polarizable boot electrodes of a Harvard moist chamber. Attach the free wire of the lead-off to the other boot electrode. Make a musclenerve preparation with a long nerve. Mount in a moist chamber so that the nerve will lie across the two boot electrodes, one quite close to the muscle but not allowed to touch it, and the other as far from the muscle as the length of the nerve will permit. Record on a drum but move the drum by hand.

The galvanic current (when of moderate intensity) stimulates only at the time of changing intensity, i.e., on making and on breaking the current. Connect the free wire of the lead-off with the rheocord between the 70 and $100 \mathrm{~cm}$. points. The exact point will vary, the stronger the battery the nearer to point of attachment to the $70 \mathrm{~cm}$. mark. Note the responses of the muscle, $(a)$ on closing the circuit, $(b)$ during the flow of the current, and $(c)$ at the breaking of the circuit. Make records covering these facts known as DuBois-Reymond's Law of Stimulation.

r6. Pfluiger's Law of Irritability.-Both strength of current and direction of flow of current are factors in the determination of the physiological effects of the galvanic current on irritability. This law is tabulated as follows:

\begin{tabular}{l|l|c|c|c}
\hline \multirow{2}{*}{$\begin{array}{c}\text { Strength of galvanic } \\
\text { current }\end{array}$} & \multicolumn{2}{|c|}{ Ascending current } & \multicolumn{2}{c}{ Descending current } \\
\cline { 2 - 3 } & Making & Breaking & Making & Breaking \\
\hline $\begin{array}{l}\text { Very weak currents........ } \\
\text { Medium currents......... } \\
\text { Very strong currents...... }\end{array}$ & $\begin{array}{l}\text { Contraction } \\
\text { Contrartion }\end{array}$ & $\begin{array}{l}\text { No contraction } \\
\text { Contraction } \\
\text { Contraction }\end{array}$ & $\begin{array}{l}\text { Contraction } \\
\text { Contraction } \\
\text { Contraction }\end{array}$ & $\begin{array}{l}\text { No contraction } \\
\text { Contraction } \\
\text { No contraction }\end{array}$ \\
\hline
\end{tabular}

Determine the direction of flow of galvanic current in your apparatus and demonstrate the several parts of Pflüger's Law as given in the table. Begin with the very weak ascending currents, use one battery with the lead-off attached between 20 and $40 \mathrm{~cm}$. for very weak currents. Reverse the direction of the current by the pole-changer. Use two batteries with lead-off between 50 and $80 \mathrm{~cm}$. for medium currents; and two batteries with lead-off at $90 \mathrm{~cm}$. for very strong current, or four batteries with leadoff at $80 \mathrm{~cm}$. Make records of each contraction and label as to its type and direction.

17. Electrotonus. - The change in irritability at the physiological poles of a nerve through which a galvanic current is passing is called electrotonus. Mount a fresh muscle nerve preparation in a Harvard moist 
chamber with a long nerve and nonpolarizable electrodes. Use a small platinum stimulating electrode arranged to stimulate the nerve at different points, $a$, on the muscle side of the boot electrode, $b$, next to the muscle but as close to the boot electrode as possible. Use single induction currents for stimulation of the nerve during the flow of a galvanic current as in Experiment I 7 .

Record and check the change in nerve irritability during electrotonus against the diagram, figure 335 .

I8. The Action Current of Muscle. a. The Rheoscopic Frog.-The action current produced by a single contraction of a gastrocnemius muscle is enough to stimulate the sciatic nerve of a second preparation when the second nerve is laid across the first muscle in such a way as to be a conductor of its action current. Expose the two gastrocnemius muscles of a frog pinned horizontally to a frog board. These need not be separated from the skeleton. Bring the two near to each other and pin the knees to the frog board. Attach the tendon of the second to a lever and bring its long sciatic nerve in contact with the tendon and the belly of the first muscle. Now stimulate the nerve of the first muscle. When the first muscle contracts in response to the nerve impulse from its nerve, the action current developed will flow through the loop of the second nerve in physical contact only with the first muscle. Stimulation of the nerve is indicated by the contraction of the second muscle as recorded on the drum.

b. By careful manipulation the "current of injury" or "demarcation current" which develops in the first muscle when cut across the middle will also stimulate the second nerve. Use a glass rod to adjust the nerve. Bring the middle of the nerve in contact with the proximal tendon of the cut muscle, then suddenly drop the free end of the nerve onto the cut end of the muscle. The nerve will be stimulated and the second muscle will contract.

c. Action Current of the Heart.-Prepare a perfectly fresh gastrocnemius with a long sciatic. Expose a large turtle's heart. Support the sciatic by a glass rod so that it touches the heart at the base and the apex only. The cardiac action current will stimulate the sciatic at each contraction.

I9. Unipolar Stimulation.-The stimulating effect of an electric current is proportional to the intensity of the current per unit area of the physiological electrode. When one electrode is made indifferent by spreading the current over a large surface, then the stimulations will be limited to the smaller electrode. Use the Harvard apparatus, bind the indifferent electrode on the neck and with the other verify the motor points in the arm shown in figure 336 .

This method can be used to demonstrate DuBois-Reymond's law on human nerves. Apply the stimulating electrode over the median nerve at the elbow. Stimulation with a medium current will occur only on clos- 
ing the descending current or on opening the ascending current. On reversing the current the opening stimulus will not be effective.

20. Cardiac Muscle.-Cardiac muscle differs from voluntary in that the contractions occur rhythmically and automatically. This is shown by the isolated frog's heart, which continues to contract when bathed with blood or salt solution, often for hours. This isolated heart, however, has a complicated local nervous mechanism. The apex of the ventricle of the terrapin's heart is practically free from nerve ganglia and is used to demonstrate the characteristics of pure cardiac muscle.

a. Cut a strip off the apex of the terrapin's ventricle, as shown in figure 2I4, and mount it by means of light silk-thread ligatures tied around the two ends of a strip and attached to the apparatus shown in figure 356. The loop of the lower thread should be $\mathrm{I} \mathrm{cm}$. long, of the upper thread long enough to

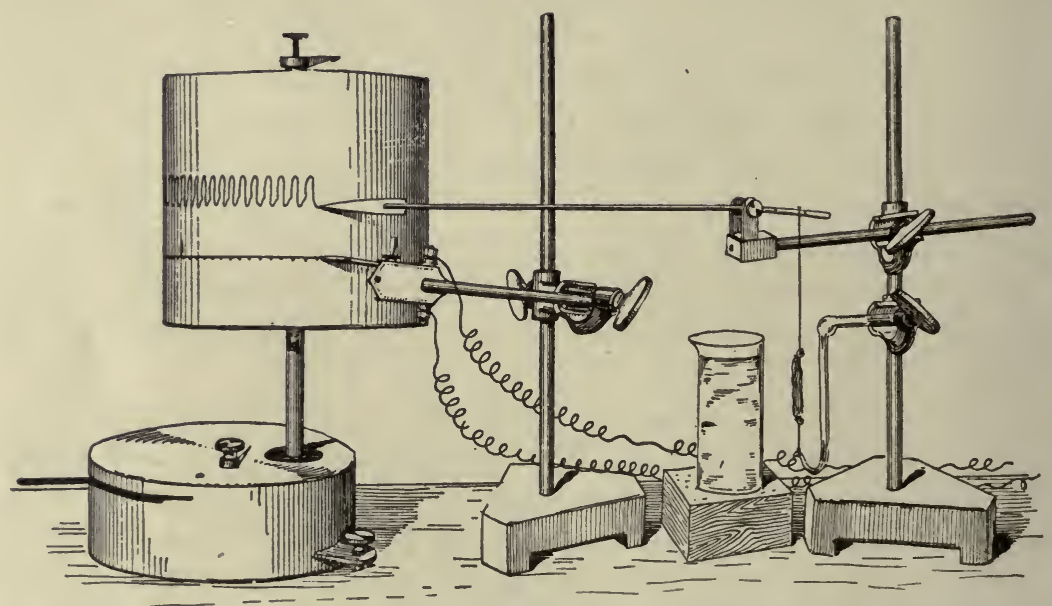

FIG. 356.-Arrangement of Apparatus for Studying the Contractions of the Strip of the Apex of the Ventricle.

reach to the lever above. When such ventricular strips are immersed in ordinary 0.7 per cent. sodium chloride they will begin contractions in a few minutes, twenty minutes or so. The contractions are regular in rate and will continue through two or three hours, gradually becoming smaller and smaller until a standstill is reached. If the strip is immersed in its own serum it will give only occasional contractions, but it remains irritable and capable of contracting at any moment. If changed to salt solution, the salt solution apparently brings out the automatic rhythm by an increase in its irritability.

$b$. Portions of the auricle and of the sinus, especially the latter, are more highly rythhmic than portions of the ventricle, due to a specific difference in the muscle cells themselves rather than to the nervous mechanism contained. Prepare a strip from the auricle and compare it with the ventricle. 
c. Repeat with sinus strips using care to compensate the weight of the lever.

Refer to the experiments on cardiac muscle at the end of the chapter or. Circulation.

21. Involuntary Muscle, Coldblooded Animal.-Strips of smooth or involuntary muscle cut from the stomach of the frog will show the physiological reactions of this type of muscle. Mount a spiral strip of frog stomach muscle in the Harvard warm chamber holder. Use a wire to connect the muscle with the lever as in figure 356 . Take care not to load too heavily, the ordinary muscle lever is often too great a tension. Mount a signal magnet to automatically mark the time of stimulation.

a. Frog stomach muscle will develop automatic contractions in a few moments. Record through two or three circuits of the drum. The speed should be I mm. per second.

$b$. If automatic contractions do not develop stimulate the muscle by interrupted currents of varying strength. After a variable latent period contractions will develop. If the automatic contractions are occurring, stimulation will increase the amount and sometimes the frequency of the contractions.

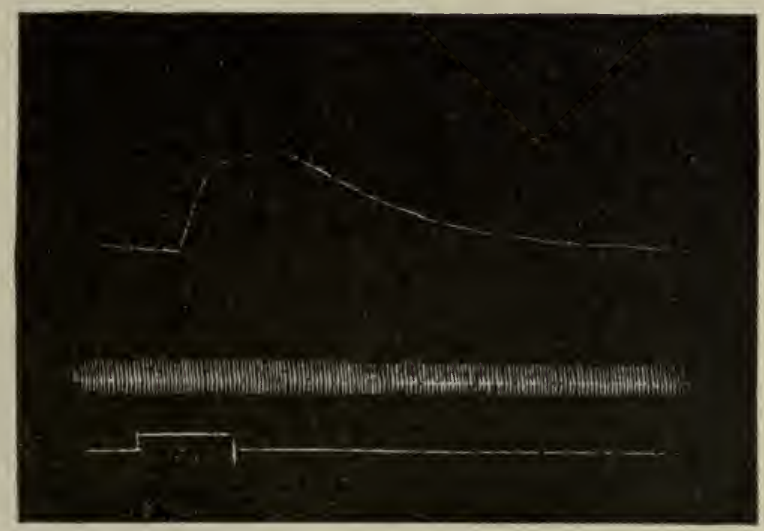

FIG. $356 a$.-Figure Showing the Type of Contraction of a Strip of Muscle from the Stomach of a Frog. The muscle was stimulated with an interrupted current during the time indicated by the signal tracing, immediately below the time tracing. Time in seconds.

c. A strip of terrapin stomach muscle will not develop automatic rhythm but will respond to comparatively strong stimulation. Increasing the temperature to $30^{\circ}$ will of ten initiate rhythm.

$d$. Smooth muscle, like voluntary muscle, responds to variation in temperature, fatigue, strength of stimulus, etc. It is more sensitive to injury. It shows a contraction amplitude proportional to the stimulus. 
It is kept in better condition in an atmosphere of oxygen. Test as many of these points as time permits.

$e$. Moisten the strip with I per cent. barium chloride.

$f$. Mount a section of the oviduct of the turtle. This tube displays a remarkably even and uniform but slow automatic rhythm.

22. Periodic Contractions of the Rat's Uterus.-A splendid preparation of smooth muscle in a warmblooded animal is obtained by splitting the rat's uterus longitudinally, giving one fallopian tube and one-half of the uterus as a preparation.

a. Mount a rat's uterus-fallopian tube in the Harvard warm chamber and immerse in a bath of oxygenated Ringer's solution at $37^{\circ} \mathrm{C}$. Rhythmic contractions of a comparatively uniform amplitude will begin promptly and continue through a long interval.

$b$. While the preparation is contracting rhythmically add to the 20 cc. of Ringer's solution I cc. of .oI per cent. epinephrin. This hormone stimulates the autonomic nerve endings producing inhibition. The effect passes away after a few minutes or on renewing the solution.

A similar preparation can be made from the uterus of a guineapig, cat or rabbit. Epinephrin stimulates the inhibitory mechanism of the virgin uterus, but produces contraction of the muscle of the gravid uterus.

23. Ciliary Contractions.-Ciliated Epithelium.-Make a preparation of ciliated epithelium by cutting out the esophagus of a terrapin or frog, slitting it open longitudinally, and smoothing it out on a cork block. The cilia of this membrane will drive in the direction down the esophagus. Test the rate at which different loads are moved and measure the distance on the preparation as follows: Cut pieces of clean white paper about 4 and $6 \mathrm{~mm}$. square. Select a favorable area on the ciliated surface as long as possible, place the $4 \mathrm{~mm}$. square paper at the beginning of the area, and measure the time which it takes to travel the distance. Measure the speed in terms of seconds per centimeter. Now replace the paper at the point of beginning and load it with small weighed cubes of paraffin. The rate at which the load is carried will slightly increase at first as the load is increased, but later will sharply decrease. Elevate one end of the ciliated membrane and repeat the experiment with different loads so that the cilia will now carry the load uphill. Calculate the work done in terms of gramcentimeters of work per square centimeter of ciliated surface acting on the load.

24. Rate of the Nerve Impulse.-Prepare a muscle-nerve of a large frog with the entire sciatic nerve. Sever the nerve where it leaves the cord, leaving it attached to the fascia. Mount in a moist chamber using two pairs of electrodes. Set one pair of electrodes on the nerve as near the muscle as possible, the other at the extreme end of the sciatic. Take several pairs of simple muscle contractions on the pendulum myograph stimulating in order, I, the long nerve, and 2, the short nerve. The latent periods must be marked 
with greatest accuracy and made as long as possible. The difference in the time of the two latent periods of each pair represents the time required for the nerve length between the two pairs of electrodes. Compute the rate of the nerve impulse in meters per second. Make a table of all the tests recorded and draw averages.

25. Relation of Total Work to Nutrition of Muscle.-Pith a frog and pin it prone on a frog board. Isolate one gastrocnemius and measure its total work as in experiment 7 . Inject 2 cc. of one per cent. glucose in Ringer into the circulation, or perfuse the heart directly with Ringer's solution containing 0.2 per cent. glucose. Now repeat the measurement of total work on the second gastrocnemius muscle. The experiment will be more striking if the records are made parallel on the same paper with the drum at constant speed.

Variations of this demonstration can be made by comparing the work of the two muscles of a frog, one while the normal circulation continues, the other with the circulation tied off by ligature around the thigh.

26. Production of Carbondioxide During Contraction.-Prepare a $\mathrm{CO}_{2}$-absorbing apparatus. Charge it with Ringer's solution containing phenolphthalein indicator. Reduce the hydrogen iron content to a constant, as indicated by the color of the solution matched against a pink standard, by aerating with $\mathrm{CO}_{2}$ free air. Prepare a small muscle and attach it to the holding device. Insert in the apparatus, and accurately measure the time until the color of the indicator is just discharged. This measures the rate of $\mathrm{CO}_{2}$ production in the resting muscle. Repeat until the readings are constant.

Now make the test while stimulating the muscle to a mild tetanus. The apparatus and solution are to be standardized. 


\section{CHAPTER XIV. \\ THE NERVOUS SYSTEM.}

The nervous system consists of an extremely complex anatomical mass of nerve cells and fibers. It is usually described as made up of two main divisions, the cerebro-spinal system and the sympathetic. These two divisions must be regarded as parts of one great whole, and in no sense coordinate. The gross subdivision of the nervous system may be given as the following: First, the cerebro-spinal axis, which consists of matter within the bony cranium and spinal column, constituting the brain and cord. Second, smaller masses for the most part in the abdominal and thoracic cavities, also in the neck and head, and constituting the sympathetic or autonomic ganglia. Third, the nerves or bundles of nerve fibers which connect the central nerve axis with the periphery and with the sympathetic ganglia. Fourth, there are special peripheral organs in connection with the beginnings and endings of the nerve fibers, the one for receiving nerve stimuli the receptors, the other for transmitting stimuli to other tissues, the effectors. These are properly parts of the nervous system. The peripheral organs for receiving stimuli constitute the sense organs and will be treated in a separate chapter.

\section{FUNCTION OF THE NERVE CELL.}

The Nerve Cell.-The nerve cell, the neurone, is considered the anatomical and physiological unit of the nervous system. Waldeyer introduced the term neurone to designate the nerve cell body and all its constituent processes. Much of the details of structure of types of neurones, both as to the structure of the cell body and all its processes, have already been given in Chapter II., see figures 83 to ro6. It is sufficient to recall that the types of nerve cells found in various parts of the nervous system vary extremely. The peculiar feature, however, consists in the fact that the cell body has one or more processes, branches, or arborizations, see figure 365 . Sometimes these processes are short but complexly branched, sometimes they are exceedingly long as compared with the extent of the cell body. The cell processes may or may not be medullated. In medullated nerves the adventitious structures are subdivided into nodes, but the axis-cylinder process is to be regarded as a continuation of the protoplasm of the cell body.

In recent years the structure of the cell body and its branches has been very carefully investigated, with the result that we have learned that the intimate structure is very complex. Networks of neurofibrillæ have been 
described not only in the cell body, but extending throughout the course of the processes and, in fact, from cell to cell. We are not in a position at the present time fully to determine what bearing these neurofibrillæ have on our accepted theories of nerve function, other than that they are assumed to be the conducting elements.
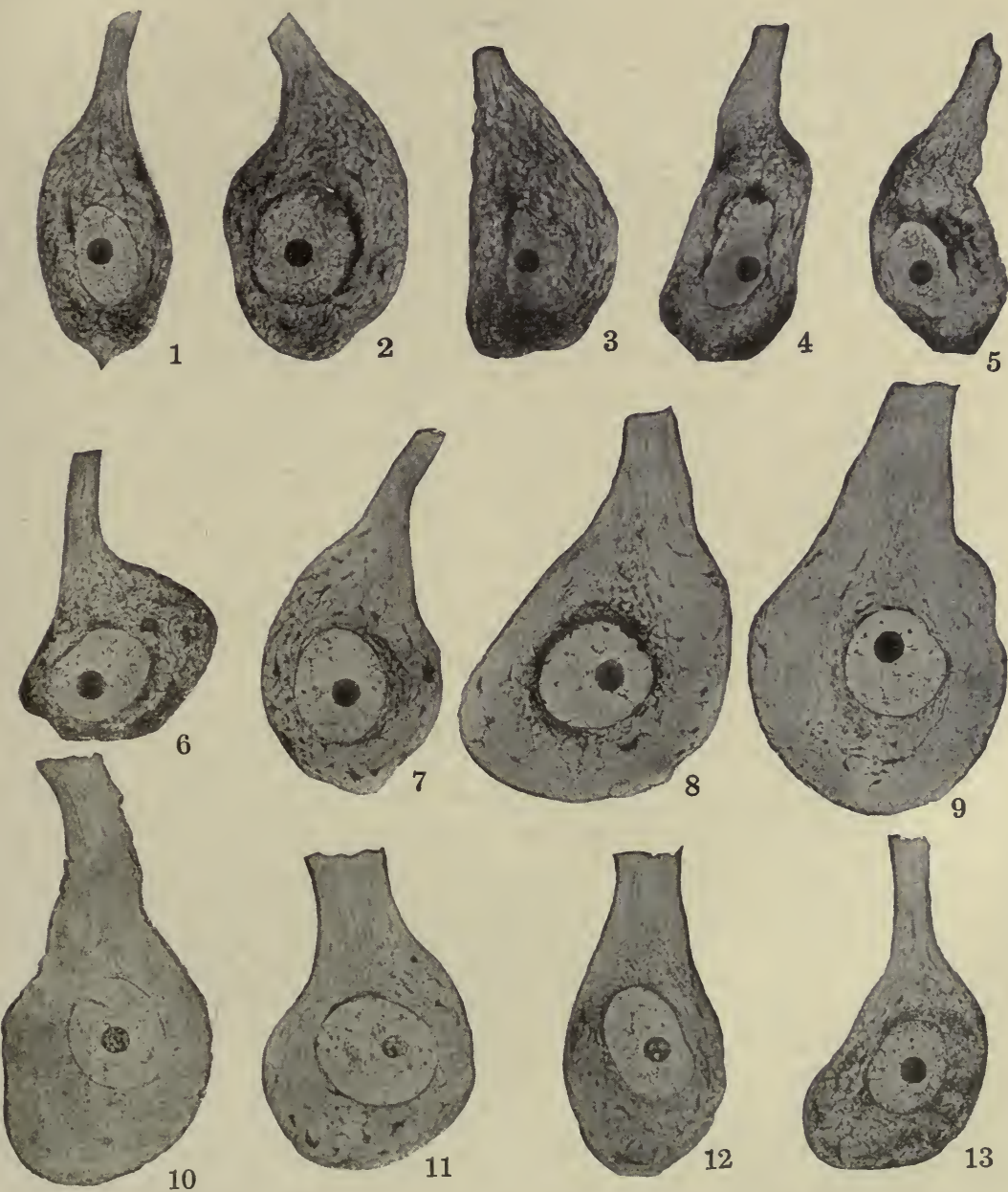

5

FIG. 357.-Morphological changes of nerve cells during function activity and during recuperation. I. Normal resting Purkinje cell. 2 to 9 . The main stages in progressive activity. Io to I3. The regular course of recovery. (Dolley.)

The Neurone Theory.-Our knowledge of the function of the nervous system is best explained on the basis of the neurone theory, which considers the neurone as a physiological unit. By this view each gross division of the nervous system is supposed to consist of a large number of in- 
dividual neurones,* each of which is a more or less complete morphological unit capable of carrying on certain functions of its own. Each of these neurones maintains physiological continuity with its associates, presumably
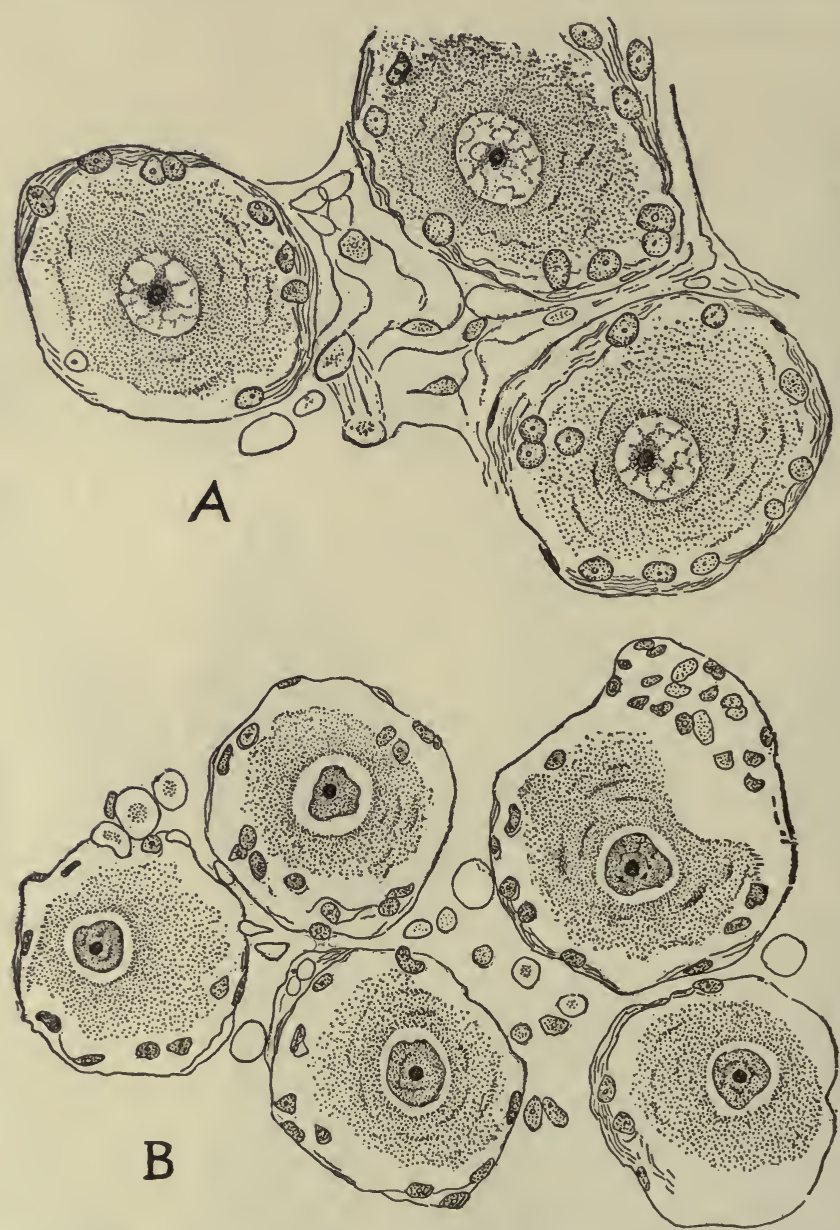

Fig. 358.- Spinal Ganglion Cells from the Cat. A, Normal taken before stimulation; B, taken after five hours' stimulation. From the right and left, eight thoracic ganglia. (Hodge.)

by protoplasmic contact rather than by continuity; so that well-marked paths of conduction are possible throughout the extent of the particular mass of

* "According to the estimations of Meynert, the cortex of the cerebral hemisphere alone contains twelve hundred millions of ganglion cells. Donaldson (The Growth of the Brain, a Study of the Nervous System in Relation to Education, I2mo, London, 1897, p. 159) states that for the total number of nerve cells in the central nervous system three thousand millions is a moderate estimate." (Barker, the Nervous System and its Constituent Neurones, p. 42, I899.) 
which the neurone is a part, and throughout the adjacent masses. By this view, paths of conduction are made up of series or chains of individual neurones which are in physiological continuity.

The Characteristics of the Individual Neurone.-The function of the nerve cell may be discussed under two headings: The function of the cell body, and the function of the cell processes.

The cell body of the neurone is the part that contains the nucleus and is the center of those activities which influence the metabolism of the cell itself. If the cell body be isolated from its processes, the processes will degenerate, while the body continues to live. In other words, the cell body may be considered as the center of those trophic influences which regulate the nutrition of the processes. Although the nerve cell as a whole is in many, perhaps in most, cases a conducting organ, still those physiological processes which go on in it produce marked changes in the protoplasm of the cell body. Hodge has demonstrated that nerve cells which have been active for several hours, in case of sparrows which have been flying about actively throughout the day, or in bees after a day's work, show marked evidences of fatigue. These evidences consist in the decrease in the size of the nucleus and the appearance of vacuoles in its structure, also in the shrinking of the protoplasm of the cell, which, in case of the cells of the spinal ganglia, draws away from its capsule. If the cells are examined early in the morning the fatigue changes will not be present, the cell having recuperated during the period of rest at night. It has also been found that the Nissl granules which are present in the cell body of resting cells decrease in number and show evidence of disintegration in cells that have been stimulated for several hours, or that have been in prolonged functional activity. Figure 357 presents the changes in nerve cell structure which can be determined from microscopical preparations after various stages of functional activity. In this series worked out by Dolley on the dog, I to 9 show these changes. Progressive recuperation is shown in figures Io to I3. As a matter of fact, the different individual brain cells are subjected to different degrees of activity during prolonged physiological strain. Hence all these cells are found in one and the same preparation, the relative number varying however.

Hodge studied the changes in spinal ganglion cells induced by artificial stimulation. The results are shown pictorially in figure $35^{8}$.

The nerve processes or fibers are primarily conducting structures. But fibers are susceptible to artificial stimulation, as shown in the previous chapter; that is, they are irritable. They are influenced by certain changes in the environment, but they do not show evidence of fatigue upon prolonged functional activity.

Nutritive Influence of the Cell Body over its Processes-Wallerian Degeneration. - The control of the cell body over the nutrition of the cell processes is demonstrated by the changes which occur when these proc- 
esses are severed from connection with the cell body. Under such conditions the axis-cylinder process completely degenerates. Howell and Huber have followed the degenerative changes in medullated nerve fibers. The medullated fiber in the course of three or four days, in mammals, breaks up into elliptical segments of myelin, containing small fragments of the axis-cylinder. These changes in the cut-off section of nerve occur simultaneously throughout its whole extent. In the course of a few weeks regenerative changes begin, apparently under trophic influence of the nuclei of the primitive sheath. These nuclei increase in number and form small masses of protoplasm which ultimately produce a strand of embryonic protoplasm, which is described as the "band fiber." If the ends of the sectioned nerve have originally been brought together and sutured in place, then the axis-cylinder processes of the portion of the nerve fiber still attached to the cell body will grow down into the peripheral fibers, thus forming new axis-cylinder processes along the course of the band fiber. If the stumps of the nerves are not so brought together, then apparently the band fiber again degenerates, especially in adult tissues, though it has been claimed by Bethe and others that complete regeneration of the peripheral fiber will take place in very young animals. Even if complete regeneration should take place in the peripheral fiber, unless connection were established between it and the central end of the fiber it would ultimately disintegrate and could only temporarily carry on any physiological function. Complete regeneration requires from three months to two years.

The central end of the divided nerve, that is, the part maintaining connection with the cell body, usually degenerates for a few nodes only, then regeneration and growth of the original stump proceed. Instances are observed in certain cases where the degeneration of the entire central fiber, including its cell body, takes place. This happens particularly in those relations where the original neurone forms a link in a conducting path. Such a conducting neurone would no longer perform its proper function so would atrophy just as would a muscle fiber when cut off from its nervous relations.

In conclusion, one may infer that the cell body exercises a nutritive or trophic control over the protoplasm of its branches, just as we have already seen the neurone as a whole exercises trophic control over the nutritive processes taking place in the tissue to which its branches are distributed, for example, the voluntary muscles.

Specific Energy of the Nerve Impulses.-We have already discussed the fact that a nerve fiber, also its cell body, is irritable to various forms of mechanical, electrical, and other stimuli. In the complex of activity of the nervous system it is found that whatever the form of the external stimulus applied to a nerve, the resulting nerve impulse produces the same effects in the central nervous system. The reaction in consciousness is constant and unvarying and independent of the character of the external stimulus. 
For example, if the temperature points on the skin be stimulated, as they may, by a number of widely different types of external stimuli, the resulting sensation is the same; stimulation of a "heat point" by ice produces a sensation of heat, not cold. This phenomenon has been called the specific energy of the nerve impulse, and the term was first advanced by Johannes Müller. Different views are presented in explanation. But it seems rational to believe that the gist of the matter rests in two factors: I. The highly differentiated sense organ is adapted especially to stimulation by a particular stimulus, as the eye by light. 2. The central apparatus is developed in response to and especially adapted to receive the specific stimulus. The interpretations that are made in consciousness in response to an inflow of nerve impulses from the sense organ are more or less constant. When the exceptional stimulus is applied to the special end organ it results in the usual change in the sense organ, the resulting nerve impulses reach the usual central area, and there is no physiological basis for other than the usual sensations which development and experience have associated with activity in the parts affected.

Transmission of Nerve Impulses through the Neurone.-The theory has been advanced that in the nerve cell the primary function of some processes is to carry nerve impulses toward the cell body, and of other processes to carry nerve impulses away from the cell body. At the present time this view is advocated by perhaps the ablest living anatomists and neurologists. The dendrites conduct toward the cell body, and the axones away from it. That is, the former are cellulipetal, the latter cellulifugal.

Impressions made upon the terminations or upon the trunk of an afferent nerve may cause, $a$, pain or some other kind of general sensation; $b$, special sensation; $c$, reflex action of some kind; or $d$, inhibition or restraint of action. Similarly impressions made upon an efferent nerve may cause, $a$, contraction of muscle (motor nerve); $b$, it may influence secretion (secretory nerve); $c$, it may influence nutrition (trophic nerve); or $d$, it may inhibit, augment, or stop any other efferent action.

By artificial stimulation nerve impulses can be made to pass in both directions in all classes of nerve processes. That is to say, if a motor axone is artificially stimulated in the middle of its course it will not only convey a nerve impulse to its distribution, but also a nerve impulse will pass back over the fiber to the cell body and out over the dendrites. Normally, in the complex of the body, it is probable that such a neurone will be stimulated only at its points of contact with other neurones chiefly through its dendrites, and especially by means of the sensory cells. The dendrites therefore will receive the nerve stimulus, carry it through the cell body to the axone and its distribution. In such cells there is isolated, or uninterrupted, conduction throughout the extent of the neurone. The nerve impulse is able to pass from a given neurone to adjacent ones only at the termination of the axone or its branches, 
and such terminations may be considered as special organs for the transference of the nerve impulses. This activity involves isolated conduction in nerve fibers bound in a common nerve trunk. It has been supposed that the myelin sheath of a medullated nerve acts as an insulator of the axis-cylinder, but this can be only relatively true, for the reason that non-medullated nerves do not possess the myelin sheath. In non-medullated nerves we must suppose that the primitive sheath is sufficient to give insulated conduction, or that it is an inherent property of the axis-cylinder itself to carry the nerve impulse without transmission to adjacent fibers.

We have already, page 500, discussed the rate of transmission of the nerve impulse in motor nerves which was given as from 27 to 30 meters per second. In sensory nerves the rate is said to be somewhat higher; in human nerve from 30 to 42 meters per second.

Physiological Types of Neurones.-Many classifications could be made of nerve cells, based on the differences in their functional relations but attention will be called to only one. Neurones may be classified as afferent or sensory, efferent or motor, and connecting or transmitting.

Under afferent neurones are classed all those neurones which transmit the effects of external stimuli received through the sense organs, both general and special sense organs. These neurones carry nerve impulses toward the central nervous system, which may ultimately produce those changes in the cerebral cortex which are associated with states of consciousness.

Under efferent neurones are included all those which transmit nerve impulses from any part of the central nervous system to the muscles, that is, motor nerves; or transmit nerve impulses to the glands, secretory nerves; or that transmit nerve impulse, which inhibit peripheral action, inhibitory nerves.

Under central or transmitting neurones are included those units which act as connecting links within the central organ, especially within coordinate parts of the central nervous system, between the afferent and efferent neurones.

Nerve Centers. - Whenever a number of neurones are gathered in one group to accomplish some specific function it is called a nerve center. The term usually applies to the aggregation of cell bodies and their dendritic processes in contradistinction to nerve trunks. There are aggregations of nerve cells into different specific groups, to which we cannot in every case ascribe a specific function. These groups are not called nerve centers, but are described by the general anatomical term, ganglia. Such ganglia are represented in the sympathetic chain, the spinal-root ganglia, the ganglia of certain cranial nerves, etc. The nerve centers are found in the spinal cord, the medulla, and the higher cranial groups. The medulla is particularly rich in nerve centers. The cerebro-spinal axis is in fact an aggregation of nerve centers of varying complexity in different parts. 
It is by means of the nerve centers that the activities of the differentiated parts of the human body are brought into intimate correlation. The nerve centers exercise their influence through the power of inhibiting or decreasing activity; or, on the other hand, of augmenting or increasing the activity in the

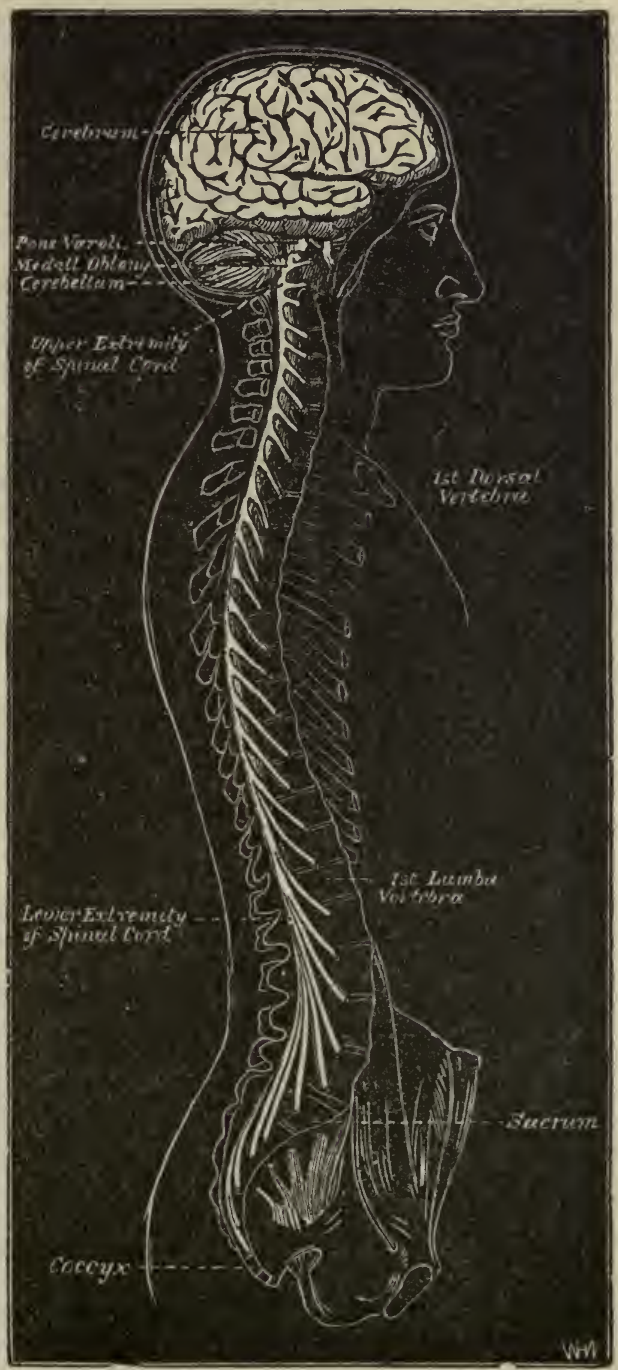

Fig. 359.- -View of the Cerebro-spinal Axis of the Nervous System. The right half of the cranium and trunk of the body has been removed by a vertical section; the membranes of the brain and spinal cord have also been removed, and the roots and first part of the fifth and ninth cranial, and of all spinal nerves of the right side, have been dissected out and laid separately on the wall of the skull and on the several vertebræ opposite to the place of their natural exit from the cranio-spinal cavity. (After Bourgery.) 
peripheral tissues or in other parts of the nervous system. For example, the vagus center regulates the activity of the heart muscle by its power to decrease or inhibit cardiac contractions. This center, we have already found, is in constant tonic activity; that is to say, in constant regulative control of the heart. The cardiac augmentory center, on the other hand, produces just the opposite effect, increasing the activity of the cardiac muscle. What is true for the heart is likewise true in general for other tissues of the body. The numerous nerve centers in the central nervous system are brought into correlation through an exceedingly complex system of communicating fibers. The cerebro-spinal axis may in fact be regarded as a segmental chain of nerve. centers, the complexity increasing from the cord toward the brain, and the coordinating control culminating in the cerebral cortex.

\section{THE STRUCTURE AND FUNCTION OF THE SPINAL CORD.}

\section{STRUCTURE.}

The spinal cord is a cylindrical column of nerve-substance connected above with the brain through the medium of the bulb, and terminating below in a slender filament of nerve substance, the filum terminale, which lies in the midst of the roots of the many nerves forming the cauda equina.

General Features.- The cord is composed of nerve fibers and nerve cells. The former are situated externally and constitute the chief portion of the cord, while the latter occupy its central or axial portion and are so disposed that on the surface of a transverse section of the cord two somewhat crescentic grayish masses connected by a narrower portion or isthmus appear, figure 358. Passing through the center of the cord in a longitudinal direction is a minute canal, the central canal, which is continued through the whole length of the cord, opening above into the space at the back of the medulla oblongata and pons Varolii called the fourth ventricle. The canal is lined by a layer of columnar ciliated epithelium.

The spinal cord consists of exactly symmetrical halves, separated anteriorly and posteriorly by vertical fissures (the posterior fissure being deeper but less wide and distinct than the anterior), and united in the middle by nervous matter which forms the commissures. The central part, which contains the central canal, is known as the gray commissure, and is bounded by the anterior white commissure in front. Each half of the spinal cord is marked on the sides (obscurely at the lower part, but distinctly above) by two longitudinal furrows, which divide it into three portions, funiculi, or tracts -an anterior, lateral, and posterior. From the groove between the anterior and lateral funiculi spring the anterior roots of the spinal nerves; and just 
in front of the groove between the lateral and posterior funiculi arise the posterior roots of the same; a pair of roots on each side corresponding to each segment of the cord.

The nerve tracts of the cord are made up of medullated nerve fibers of different sizes, arranged longitudinally, and of a supporting material of ordinary fibrous connective tissue and neuroglia, figure 105.

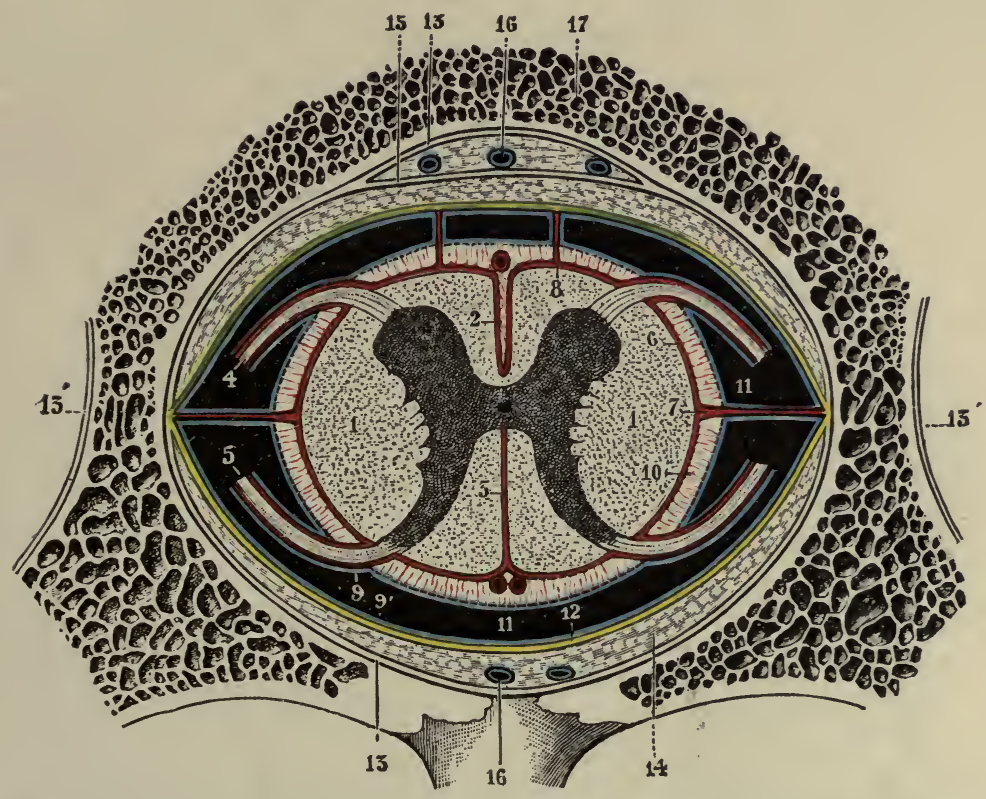

Fig. 360.-Horizontal Section of the Cord and its Envelopes, at the Middle of a Vertebral Body (Schematic). I, Spinal cord with 2, its anterior median fissure; 3, its posterior median fissure; 4, anterior roots; 5 , posterior roots; 6, pia mater (in red); 7 , ligamentum dentatum; 8 , connecting fibers passing from the pia to dura mater; 9 , visceral layer, and 9', parietal layer of the arachnoid (in blue); ro, subarachnoid space; II, arachnoid cavity; I2, dura mater (in yellow); I3, periosteum; I3', external periosteum; I4, cellular tissue situated between the dura mater and the wall of the vertebral canal; I5, $_{5}$ common posterior vertebral ligament; $I 6$, intraspinal veins; $I 7$, vertebra in section. (Testut.)

The general rule respecting the size of different segments of the cord appears to be that each is in direct proportion in this respect to the size and number of nerve roots given off from it, and has but little relation to the size or number of those given off below it. Thus the cord is very large in the middle and lower part of its cervical portion, whence arise the large nerve roots for the formation of the brachial plexuses and the nerve supply of the upper extremities; and again enlarges at the lowest part of its dorsal portion and the upper part of its lumbar, at the origins of the large nerves which, 
after forming the lumbar and sacral plexuses, are distributed to the lower extremities. The chief cause of the greater size at these parts of the spinal card is increase in the quantity of the gray matter; for there seems reason to believe that the white part of the cord becomes gradually and progressively larger from below upward, doubtless from the addition of a certain number of ascending fibers from each pair of nerves.

From careful estimates of the number of nerve fibers in a transverse section of the cord toward its upper end, and the number entering or issuing from it by the anterior and posterior roots of each pair of nerves, it has been shown that in the human spinal cord not more than half of the total number

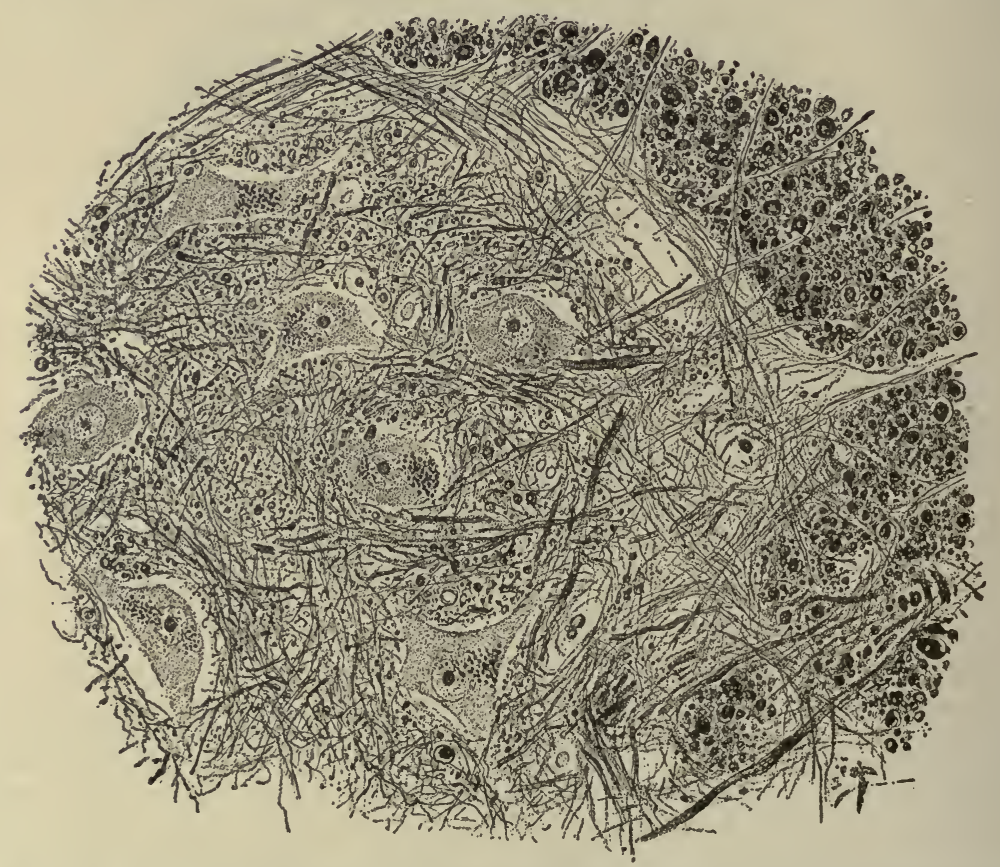

FIG. 36r.-From the Lower Lumbar Cord of Man, after a Preparation by Klönne and Müller stained by Weigert and Pal's method. A portion of the gray substance of the anterior column with the adjoining portions of the lateral funiculus is represented, showing anterior column cells and the fine medullated fibers which enter the gray substance from the lateral funiculi and surround the nerve cells, which here are provided with fine pigmented granules. High power. (Kölliker.)

of nerve fibers of all the spinal nerves are contained in a transverse section near its upper end. It is obvious, therefore, that at least half of the nerve fibers entering it must terminate somewhere within the cord itself.

The Arrangement of Nerve Cells in the Spinal Cord.- The gray matter of the spinal cord consists of numerous groups of nerve cells and of a close meshwork of nerve fibers, most of which are very fine and delicate. 
Medullated fibers mingled with the small gray fibers about the borders of the gray substance. Mingled with it and supporting it is the meshwork of the neuroglia.

The multipolar cells of the cord are either scattered singly or arranged in groups or columns in bilateral symmetry. The following are to be distinguished, certain of the groups being more or less marked in all of the regions of the cord, viz., $a$, those in the anterior columns; $b$, those in the lateral columns; $c$, those in the posterior columns; and $d$, intrinsic cells distributed throughout the gray matter.

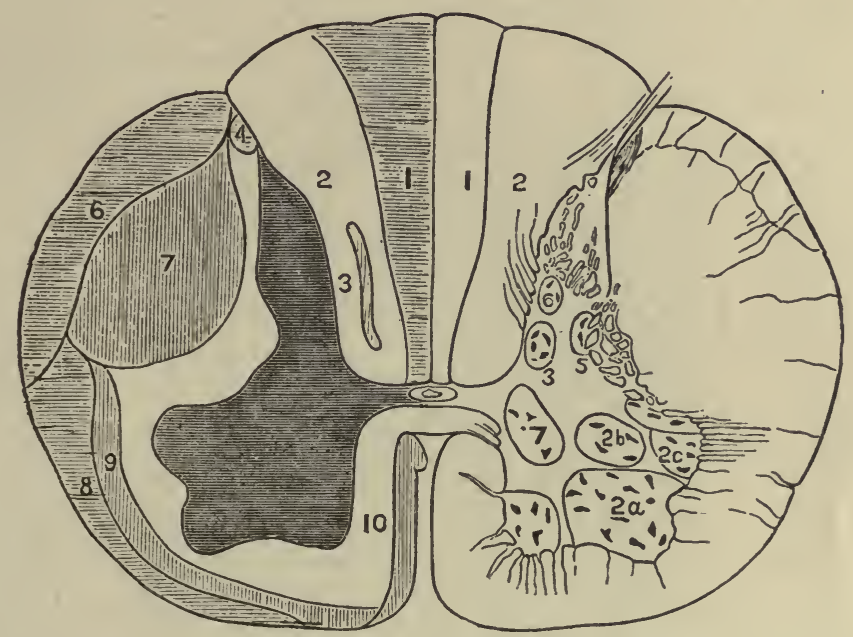

FIG. 362.- Section of Spinal Cord, One Half of Which (Left) Shows the Tracts of the White Matter, and the Other Half (Right) Shows the Position of the Nerve Cells in the Gray Matter. 7, I0, 9, and 3 are tracts of descending degeneration; $1,2,4,6$, and 8, of ascending degeneration. Semidiagrammatic. See the text for a description of the groups of nerve cells shown on the right. (After Sherrington.)

The cells in the anterior columns are large and branching, and each gives rise to an axis-cylinder process which passes out in the anterior nerve root. These cells are everywhere conspicuous, but are particularly numerous in the cervical and lumbar enlargements. In these districts they may be divided into several groups; 1 . A group of large cells close to the tip of the inner part of the anterior column, $\mathrm{x}$, figure 362 . This group is called the antero-mesial group of motor cells. It forms $a$ column the full length of the cord and is supposed to furnish motor innervation to the long muscles of the trunk. 2. The antero-lateral group of cells, 2a, forms a column which has its best development in the cervical and lumbo-sacral enlargements. It probably furnishes motor fibers to the muscles of the limbs including those of the shoulders and hips. 3. There are groups, $2 \mathrm{~b}, 2 \mathrm{c}$, the intermedio and lateral groups, which form slender columns in the cord, through the entire 
dorsal and the first two lumbar segments. The cells are small and closely aggregated and characteristic in appearance. These nuclei reappear in the upper cervical segments and in the sacral segments that give origin to the nervi erigens. They are supposed to be the motor nuclei for the vaso-motor, the pilo-motor nerves, and for the nerves of the sweat glands, i.e., the autonomic system. 4. The cells of the posterior vesicular column, or Clark's column, are in the posterior portion of the gray matter of the cord. They form a conspicuous column of cells, 3,5 , and 6 of figure 362 , extending from the last cervical to the second lumbar segments. These large cells contribute their axones to the ascending cerebello-spinal (direct cerebellar) fasciculus which ascends to the cerebellum and is believed to carry sensory impulses of prime importance in the coordinations of muscular movements. The nerve relations and the restricted location of the posterior vesicular column has also led to the suggestion that its function has to do with the visceral afferent or sensory nerve impulses. Sensory fibers from the posterior roots arborize around the cells of the posterior vesicular column, i.e., the dorsal nucleus, see figure 363 . The dorsal nucleus is considerably broken up by the passage of bundles of fibers through it, called the lateral reticular formation.

Besides these groups, which have their names largely on account of their location, there are distributed throughout the gray matter a very large number of other cells, which are known as intrinsic cells. These are of two types, those restricted to connections wholly within the gray matter Golgi cells of the second type and those that send out axones which pass into the adjacent ground bundles of the same or of the opposite side, and pass up and down the cord, to enter the gray matter again. They connect by their end-brushes with cells at different levels of the cord.

The functions of these connecting or intrinsic cells is to unite the posterior and anterior regions of the cord, to serve as conductors between the lateral halves, or to connect segments at different levels. They are also distributing fibers in that they bring a single or at least a small number of posterior sensory neurones into connection with a relatively large number of anterior or motor neurones.

The Fasciculi or Tracts in the White Matter of the Spinal Cord.We have already shown that the white matter of the cord is divided on each side into the anterior, lateral, and posterior funiculi. But evidence of various kinds discussed below has shown the following main conducting channels or tracts through the cord, see figures 363 and 364 for illustration.

\section{Tracts of the Cord.}

Funiculus posterior.

Fasciculus gracilis, tract of Goll (ascending).

Fasciculus cuneatus, tract of Burdach (ascending)

Comma, tract of Schultz (descending). 
Funiculus lateralis.

Fasciculus cerebello-spinalis, direct cerebellar (ascending).

Fasciculus antero-lateralis superficialis, tract of Gowers (ascending). Fasciculus cerebro-spinalis lateralis, crossed pyramidal tract (descending).

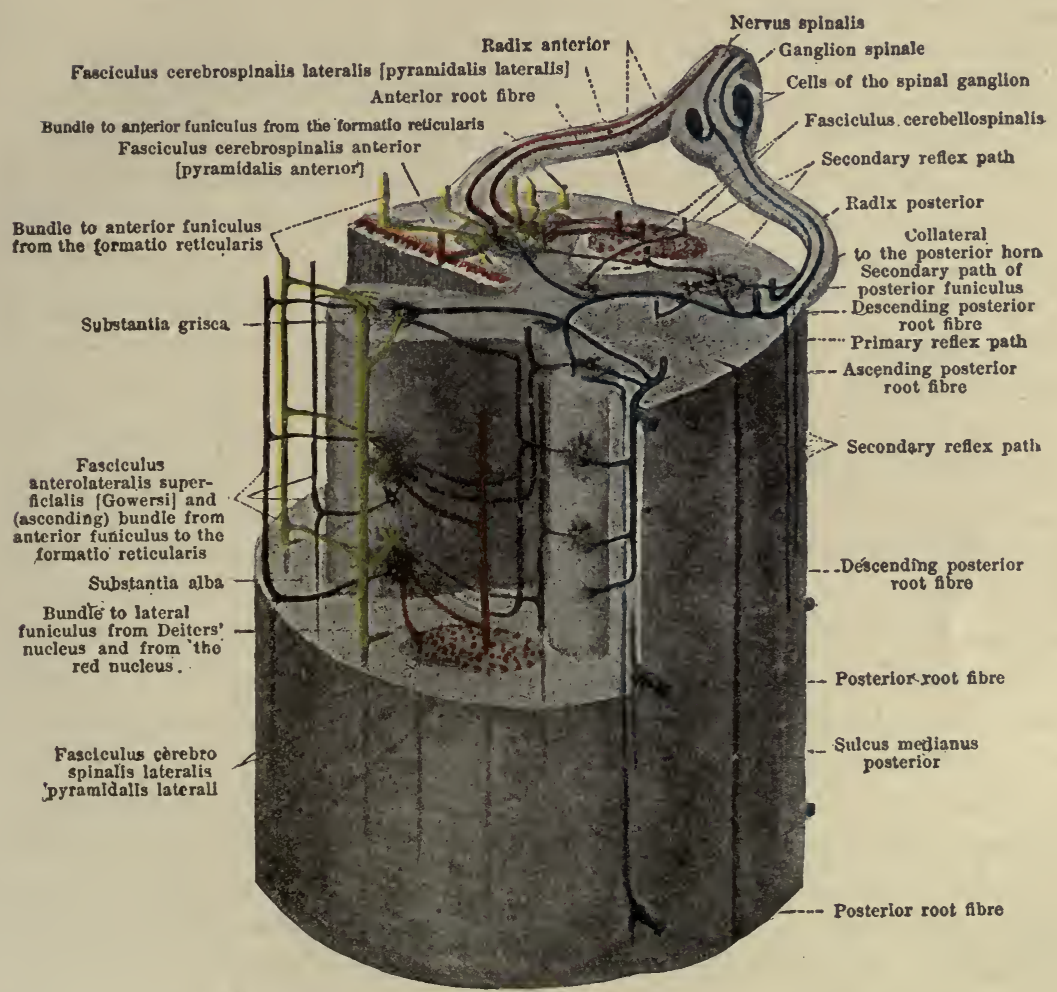

Fig. 363.-Reconstruction of a Segment of the Spinal Cord Representing Both a Transverse and Longitudinal Section. (Held, from Spalteholz's Anatomy.)

Fasciculus lateralis proprius, lateral ground bundles (ascending and descending).

Funiculus anterior.

Fasciculus cerebro-spinalis anterior, direct pyramidal tract (descending).

Fasciculus anterior proprius, anterior ground bundles (ascending and descending). 
The methods for determining the tracts in the cord and the evidence on basis of which function is ascribed are briefly the following:

The Embryological Method.-It has been found that, if the development of the spinal cord be carefully observed at different stages, certain groups of nerve fibers acquire their myelin sheath at earlier periods than others, and that the different groups of fibers can therefore be traced in various directions. This is known as the method of Flechsig.

Wallerian or Degeneration Method.-This method depends upon the fact already presented that if a nerve fiber is separated from its nerve cell it degenerates. It consists in tracing the course of tracts of degenerated fibers which result from an injury, or lesions in any part of the central nervous system. When fibers degenerate below a lesion the tract is said to be of descending degeneration, and when the fibers degenerate in the opposite direction the tract is one of ascending degeneration. By modern methods of staining of the nervous tissue, it has proved comparatively easy to distinguish degenerated parts in sections of the cord or of other portions of the central nervous system. Degenerated fibers have a differential staining reaction when the sections are treated by Marchi's method. Accidents resulting in loss of function and in degeneration in the central nervous system in man have given us much information as to its organization, but this has of late years been supplemented and largely extended by the experiments on animals, particularly upon monkeys. Considerable light has, by the method of section and degeneration, been shed upon the path of conduction of impulses to and from the various parts of the nervous system. Thus we not only have embryological evidence mapping out different tracts, but also confirmatory pathological and experimental observations.

The tracts which have been made out are the following:

Tracts of Descending Degeneration.-The Lateral Pyramidal Tract.This tract is situated to the outer side of the posterior cornu of gray matter, figure 360,7 . It originates in the cerebral cortex and extends throughout the whole length of the spinal cord; at the lower part it extends to the margin of the cord, but higher up it becomes displaced inward from this position by the interpolation of another tract of fibers, the direct cerebellar tract. The lateral pyramidal tract is large, and may touch the tip of the gray matter of the posterior gray column, but it is separated from it elsewhere. It is oval in shape on cross-section, and diminishes in size from the cervical region downward. The tract is particularly well marked out, both by the degeneration and by embryological methods. The fibers are supposed to pass off as they descend, and to join the various local nervous mechanisms of nerve cells and their branchings which are represented in the anterior columns of the cord. The fibers of which this tract is composed are moderately large, but are mixed with some that are smaller.

The Anterior Pyramidal Tract.-This tract is situated in the anterior 
funiculus by the sides of the anterior fissure, figure 360 , ro. It is smaller than the lateral tract and is not present in all animals, though conspicuous in the human cord and in that of the monkey. It can be traced upward to the cerebral cortex, and downward as far as the mid or lower thoracic region, where it ends.

Antero-lateral Descending Tract.-This is an extensive tract, elongated but narrow, and reaching from the lateral pyramidal to the anterior pyramidal tract. It is a mixed tract, since not all of its fibers degenerate below the lesions.

Comma Tract.-This is a small tract of fibers in the posterior funiculus which degenerates below the point of section of the cord. It consists of the descending collaterals of the posterior nerve roots as they pass into the cord.

Tracts of Ascending Degeneration.-The fasciculus gracilis, tract of Goll, and the fasciculus cuneatus, tract of Burdach. These tracts degenerate upward on section of the cord, also on section of the posterior nerve roots, figure 362 , I. They exist throughout the whole of the cord and can be traced into the bulb. They are sensory tracts, see page 586 .

The Fasciculus Cerebello-spinalis, or Direct Cerebellar Tract.-This tract is situated on the outer part of the cord between the lateral pyramidal tract and the margin. It is found in the cervical, thoracic, and uppur lumbar regions of the cord, and increases in size from below upward. It degenerates on injury or section of the cord itself, but not on section of the posterior nerve roots, since its fibers arise from the cells of the dorsal nucleus. As the name implies, it is believed to pass up into the cerebellum, see page 597 .

The Fasciculus Antero-lateralis, Tract of Gowers.-This tract lies at the margin of the lateral funiculus of the cord and extends the full length, see figure 364 .

It will thus be seen that the three general divisions of the white matter of the spinal cord-the anterior, the lateral, and posterior funiculi-are subdivided into tracts in which the fibers degenerate upward, those in which the fibers degenerate downward, and still others in which the fibers degenerate either way for only short distances when the cord is cut across. These latter parts of the cord are composed of association fibers which connect different levels of the cord. The association tracts form the antero-lateral columns and the lateral limiting layer. The arrangement of these tracts is shown well in figures 362 and 364 .

The Spinal Nerves. - The spinal nerves consist of thirty-one pairs, from the sides of the whole length of the cord, their number corresponding with the intervertebral foramina through which they pass. Each nerve arises by two roots, an anterior and a posterior, the latter being the larger. The roots emerge through separate apertures of the sheath of dura mater 
surrounding the cord; and directly after their emergence, where the roots lie in the intervertebral foramen, a ganglion is found on the posterior root. The anterior root lies in contact with the anterior surface of the ganglion, but none of its fibers intermingle with those in the ganglion, figure 36r. But immediately beyond the ganglion the two roots coalesce, and by the mingling of their fibers form a mixed spinal nerve; the spinal nerve, after issuing from the intervertebral canal, gives off anterior and posterior (or ventral and dorsal) branches, each containing fibers from both the roots. A third or visceral. branch of the spinal nerve, ramus communicans, joins the sympathetic chain.

The anterior root of each spinal nerve arises by numerous separate and

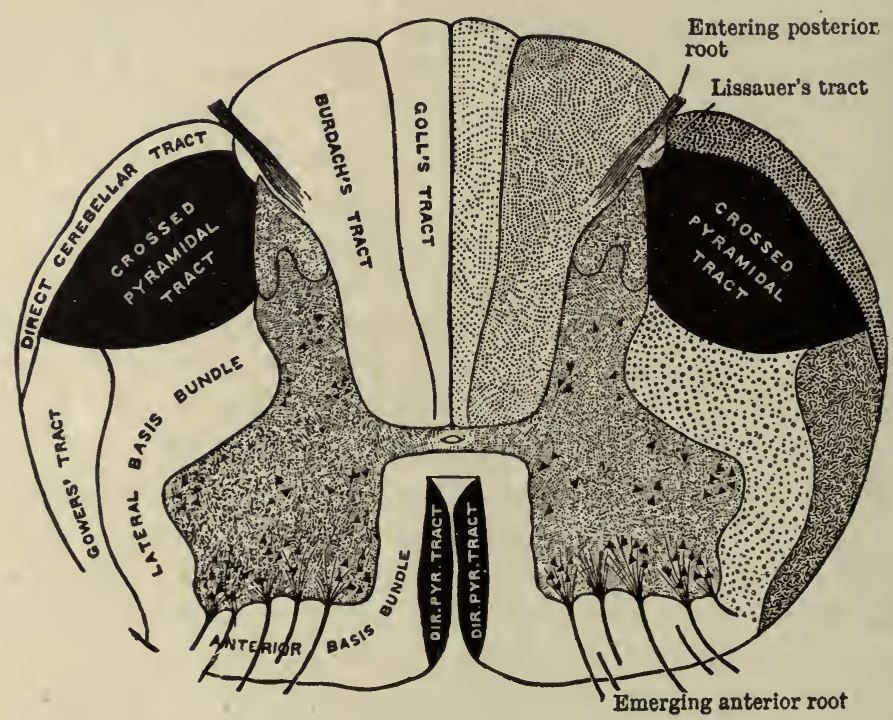

FIG. 364.-Diagrammatic Transverse Section of the Spinal Cord, Showing the Conduction Paths and Groups of Cells. (Cunningham.)

converging bundles from the anterior columns of the cord; the posterior root by more numerous parallel bundles, from the posterior column. The anterior roots of each spinal nerve consist chiefly of efferent fibers; the posterior exclusively of afferent fibers.

Course of the Fibers of the Spinal Nerve Roots.-The Anterior Roots. - The anterior roots leave the cord in several bundles, which may be called: I, Internal; 2, Middle; 3, External. All have their origin from the groups of multipolar cells in the anterior columns. The internal fibers are originated partly in the internal group of nerve cells of the anterior columns 
of the same side; but a few fibers can be traced through the anterior cornmissure to cells of the anterior column of the opposite side.

The Posterior Roots. - The fibers of the posterior roots enter the spinal cord to the inner or median side of the posterior column. The fibers, as soon as they reach the cord, divide in a fork-like fashion, one branch passing down a short distance, about three centimeters, the other branch passing up for a shorter or longer distance. This upper branch sometimes reaches . the whole extent of the cord, but generally it extends over only one or two segments of the cord. The divisions of the posterior root fibers give off

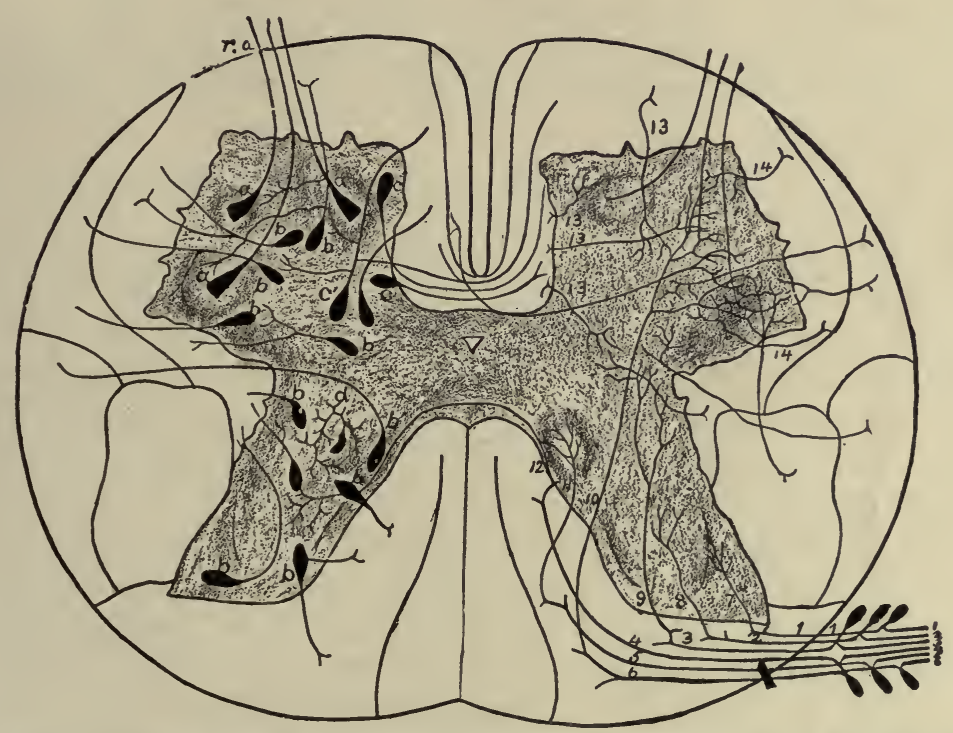

Fig. 365.-Section of the Spinal Cord, Showing the Grouping of Nerve Cells and the Course of Nerve Fibers Entering in Posterior and Anterior Roots. Numerals $I-6$ are different types of the sensory fibers. Their collaterals, $7-12$, connect with different regions of the gray substance; $\mathrm{I}_{3}$ and $\mathrm{I}_{4}$, collaterals from descending tracts of the cord; r.a., anterior root; $a$, anterior motor cells; $b$, cells contributing fibers to the various tracts; $c$, commissural cells; $d$, Golgi cells. (After Lenhossek.)

in their course numerous collaterals, figure 368 . The fibers of the posterior roots are divided into two sets, an internal or median, an external or lateral group. The lateral set consists mostly of small fibers which enter the cord opposite the tip of the posterior horn. The fibers pass in part to the marginal column of Lissauer, where they ascend and descend; in part they penetrate the posterior column, and come in relation with its cells. From the median set some fibers pass to Clarke's column, others pass by way of the posterior commissure to the median cells of the other side. Others pass through the gray matter to the anterior column cells of the same side, 
figure 365 . Besides this, they are connected through collaterals with the intrinsic cells of the gray matter at different levels of the cord. One can realize that each nerve root has, in this way, an effective grip upon a large extent of the cord. This is seen well by studying figures 363 and 365 .

The Peculiarities of Different Regions of the Spinal Cord.-The outline of the gray matter and the relative proportion of the white matter vary in different regions of the spinal cord, and it is, therefore, possible to tell approximately from what region any given transverse section of the spinal cord has been taken. The white matter increases in amount from below upward. The amount of gray matter varies; it is greatest in the cervical and lumbar enlargements, viz., at and about the 5 th lumbar and the 6th cervical nerves, and least in the thoracic region. The greatest development of gray matter corresponds with greatest number of nerve fibers passing from the cord.

In the cervical enlargement the gray matter occupies a large proportion of the section, the gray commissure is short and thick, the anterior column is blunt, while the posterior is somewhat tapering. The anterior and posterior roots run some distance through the white matter before they reach the periphery.

In the dorsal region the gray matter bears only a small proportion to the white, and the posterior roots in particular run a long course through the white matter before they leave the cord; the gray commissure is thinner and narrower than in the cervical region.

In the humbar enlargement the gray matter again bears a very large proportion to the whole size of the transverse section, but its posterior columns are shorter and blunter than they are in the cervical region. The gray commissure is short and extremely narrow.

At the upper part of the conus medullaris, which is the portion of the cord immediately below the lumbar enlargement, the gray substance occupies nearly the whole of the transverse section, as it is invested only by a thin layer of white substance. This thin layer is wanting in the neighborhood of the posterior nerve roots. The gray commissure is relatively thick.

At the level of the fifth sacral vertebra the gray matter is again in excess, and the central canal is enlarged, appearing T-shaped in section; while in the upper portion of the filum terminale the gray is uniform in shape without any central canal.

\section{THE FUNCTIONS OF THE CORD.}

The Reflex Arc and Reflex Action.-The spinal cord is morphologically a segmental or metameric structure. This is shown both by its development and by its comparative anatomy. The pairs of nerves are indicative of the component segments of the cord. The tracts of the cord are 
in a sense connectives from segment to segment, connecting the cells of both adjacent and of widely separated segments. The function of the cord

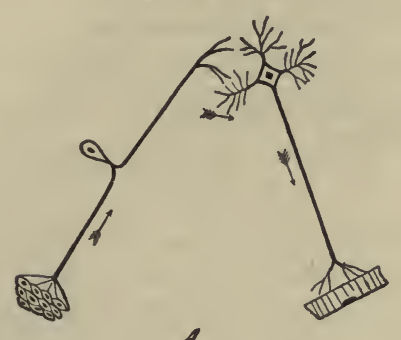

$A$

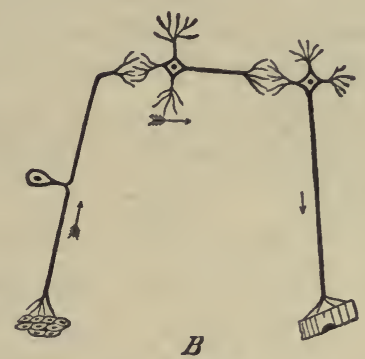

$\boldsymbol{B}$

Fig. 366.- Schematic Sketch of a Reflex Arc. $A$, With two neurones, an afferent and an efferent; $B$, with three neurones, an afferent, efferent, and a connecting or intracentral neurone.

is comprised in the function of the segments and in the function of the tracts.

From a physiological point of view, it may almost be considered as an axiom that before a nerve cell can send out a nerve impulse it must first receive a stimulus of some kind. This stimulus usually consists of an afferent impulse from the periphery. Its effect upon the receiving cell may be insufficient to cause any response, or the response may be delayed for a long period and may involve many complicated nervous activities and even psychological processes. Where the peripheral response is approximately immediate, the reaction is known as a reflex.

A reflex arc, reduced to its simplest terms, consists of the following anatomical elements: $a$, a sensory surface; $b$, an afferent neurone; $c$, an efferent neurone; $d$, a muscle or gland. The simplest form of reflex arc is schematically shown in figures 366 and 367 .

The interlocking of fibers between the termination of the afferent neurone and the dendrite of the efferent neurone shown in figure 366 is called a synapse. The reflex arc is probably seldom as simple as that shown in figure 367 , where only two neurones are involved.

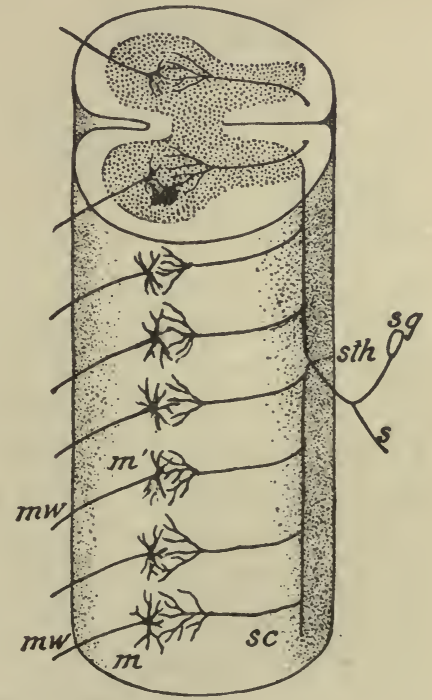

Fig. 367.-Showing the Arrangement of a Simple Reflex Mechanism Composed of a Motor and Sensory Neurone. $s g$, Posterior spinal gan. glion; $s$ and $s t h$, sensory root; $m$, motor-nerve cell; $m w$, motor root. (Kölliker.) 
More often, three or more neurones take part, as shown in figures $366 \mathrm{~B}$, and 368 .

The neurone connecting the afferent neurone with the efferent neurone belongs to the class of intracentral or connecting neurones. Since all parts of the cord, in fact of the entire cerebro-spinal axis, are directly or indirectly associated with one another by central neurones, figure 363 , the possibility of increasing the number of efferent limbs of the reflex arc can be readily understood.

An involuntary physiological reaction in a tissue produced by efferent nerve impulses which have been discharged from a nerve center under the

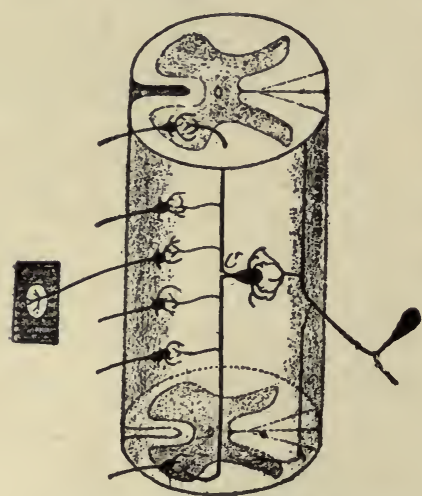

FIG. 368.- Showing the Arrangement of the reflex mechanism, with a neurone intercalated between the sensory and motor neurones. stimulus of a sensory or afferent nerve impulse, is called a reflex act. Where the nervous apparatus involved is of the type represented in figures 366 and 367 , the activity is called a simple reflex. Most reflexes are more complex in character. The afferent nerve impulse passes through more than one simple channel in the cord, so that in response to a sensory stimulus a series of coordinated acts occurs in what may be called a complex reflex.

The transmission of impulses within the cord occurs over the pathways of least resistance. Increasing a number of synapses, or the number of neurone links in the chain of conduction, increases the resistance so that reflexes will occur most readily, other conditions being equal, where the smallest number of neurones is involved, i.e., in the same segment of the cord in which the sensory impulse enters, or in immediately adjacent segments. In addition to the number of synapses in the reflex arc, certain strictly physiological factors are of importance in determining reflex reaction; e.g., the intensity of the exciting stimulus; the quality of the stimulus; the rapidity of the recurrence of the stimulus; and the duration of its application. Thus, a strong stimulus will bring about a reflex reaction sooner than a weak stimulus of the same kind. A single weak stimulus which will cause no reflex may do so if often enough and rapidly enough repeated, the phenomenon of summation of stimuli.

A reflex act once started may result in efferent impulses which continue for some time after the exciting cause has been removed. The same phenomenon is observed where groups of nerve cells are stimulated directly. It has been found, by observing electrical changes in nerve fibers by means of the capillary electrometer, that when their cells of origin are stimulated they discharge impulses in a rhythmical manner. 
Usually, impulses are transmitted to a nerve cell only over its dendrite, but it must be also assumed that such a conveyance of impulses may take place over the collaterals of its axone near the cell body, or the cell body may be stimulated directly by the afferent neurone. The peripheral fiber of the spinal ganglion cell, although it has the structure of an axone, may be looked upon physiologically as a dendrite, since homologues in lower vertebrates and in man himself (nerve cells of the ganglion of the cochlea of the auditory nerve) have this structure, the nerve cell body being situated near the sensory surface from which impressions are received.

Irradiation of Impulses within the Cord.-Taking as an example a frog whose brain has been destroyed, a simple reflex may be demonstrated by irritating the skin of one foot with a weak stimulus. In response to such a stimulus the foot is flexed upon the leg, due to a contraction of the muscles of the reflex arc corresponding to the sensory surface irritated. If the strength or duration of the stimulus be increased, other groups of muscles are involved in the following order: $\quad$ r. Those of the leg and thigh of the same side; 2, homologous muscles of the opposite side; 3 , the arms of the same side and of the opposite side.

The increasing complexity of the reflexes aroused by stimulation of one and the same sensory spot is not easy of explanation. We know that there is almost an infinite number of morphological paths in the cord, yet the responses are orderly and observe a certain sequence in their increasing complexity. The reflexes have a mechanical definiteness which, in a living structure, seems almost purposeful, yet there is no conscious action in a frog which has its brain destroyed.

The fact is that in the development of the

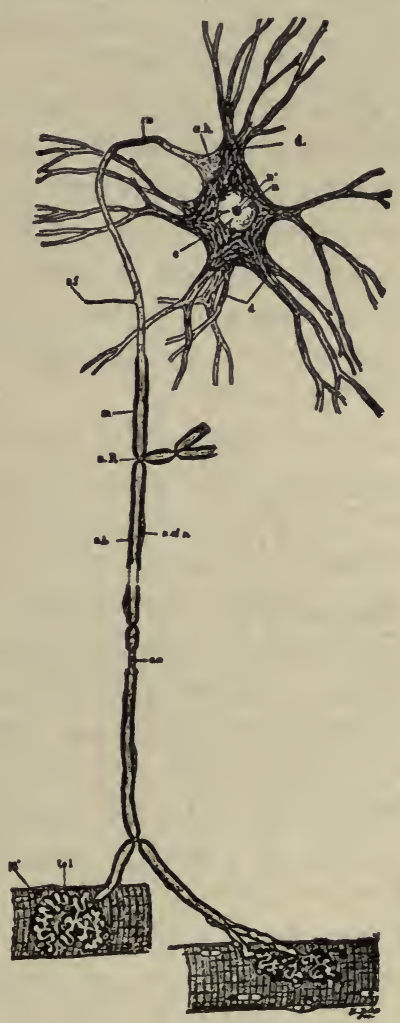

Fig. 369.-Scheme of Lower Motor Neurone. The cell body, protoplasmic processes, axone, collaterals, and terminal arborizations in muscle are all seen to be parts of a single cell and together constitute the neurone. (Barker.) c, Cytoplasm of cell body containing chromophilic bodies, neurofibrils, and perifibrillar substance; $n$, nucleus; $n^{\prime}$, nucleolus; $d$, dendrites; $a h$, axone hill free from chromophilic bodies; $a x$, axone; $s f$, side fibril (collateral); $m$, medullary sheath; $n R$, node of Ranvier where side branch is given off; $s l$, neurilemma and incisures of Schmidt; $m$, striated muscle fiber; tel, motor end plate. nervous system certain physiological paths of slight resistance have been established between the sensory areas and the muscles which move the parts 
for their protection. Apparently other physiological nerve pathways exist, but it requires a stronger sensory stimulus to arouse nerve impulses along these paths. In explanation we may suppose that the stronger afferent impulses are sufficient to overcome the resistance of increasingly complex paths, that they diffuse through greater and greater extents of the cord. But we may repeat that in the normal state of the cord this diffusion is in an orderly physiological sequence.

Orderly reflexes can be called forth only by stimulating sensory nerve endings, the first of the essential structures of the reflex arc. If artificial stimuli are applied to a nerve trunk, as the sciatic, unco-ordinated muscular responses occur because the sensory stimuli are diffuse and general and are not specific and local.

An involvement of multiple pathways may also be accomplished through decreasing the resistance within the cord, as through the use of some drug such as strychnine. In the strychninized frog a slight stimulus brings about multiple and violent reflex spasms. These contractions have lost their orderliness and are unco-ordinated. The entire musculature contracts. It is as though the strychnine removed all differences in the facility with which afferent stimuli spread through the cord, and that the resistance was reduced to the minimum. The strychnine effect is possibly due to a decrease in the resistance at the synapses, and possibly also to an increase in the irritability of the discharging nerve cells.

We must also suppose that the centers are particularly sensitive to certain kinds of stimuli, sometimes producing very extensive and violent muscular actions in response to a slight stimulus of a special kind. Such a condition is illustrated in the violent and general muscular spasms occurring when a small particle of food passes into the larynx, violent expiratory spasms accompanied by contractions of other muscles taking place.

The time taken in a reflex action for the eye in man has been found to be 0.066 to 0.058 of a second, but this estimate includes the entire time from the instant of stimulation to the beginning of the contraction of the muscle.

Functions of the Spinal Nerve Roots.-The anterior spinal nerve roots are efferent in function and the posterior are afferent. The fact is proved in various ways. Division of the anterior roots of one or more nerves is followed by complete loss of motion in the parts supplied by the fibers of such roots, but the sensation of the parts remains perfect. Division of the posterior roots destroys the sensibility of the parts supplied by their fibers, while the power of motion continues unimpaired. Moreover, stimulation of the ends of the distal portions of the divided anterior roots of a nerve excites muscular movements. There are sometimes slight evidences of sensory impulses due to recurrent fibers that are distributed through the anterior root to the spinal meninges. Stimulation of the proximal ends of the anterior roots, which are still in connection with the cord, is followed by no appreciable 
effect. It must be remembered, however, that in the anterior or efferent nerves other fibers besides motor are contained, e.g., vaso-motor, secretory, heat fibers, and when the distal end of a divided nerve is stimulated, the effects are exercised not only upon muscles, but upon glands, blood vessels, etc. Stimulation of the distal portions of the divided posterior roots, on the other hand, produces no muscular movements and no manifestations of pain; for, as already stated, sensory nerves convey impressions only toward the nerve centers. Stimulation of the proximal portions of these roots elicits signs of intense pain. Muscular movements also ensue; but these are the result of the reflex stimulation of the motor neurones of the anterior horn of the cord or are movements in response to the afferent impulses passing to higher centers from the roots stimulated.

Functions of the Ganglia on Posterior Roots.-The cells of the posterior ganglia act as centers for the nutrition of the nerve fibers given off from them. When these are cut, the parts of the nerves so severed degenerate, while the parts which remain in connection with the cells of the ganglia do not. Thus on section of the posterior nerve root beyond the ganglia the peripheral part degenerates and the central does not, and on section of the root between the ganglion and the cord the central part degenerates and the peripheral is unaffected. The number of nerve cells in the spinal ganglia far exceeds the number of nerve fibers in the corresponding root (Hardesty). The extra cells, see figure $4 \mathrm{I} 7$, serve at least in part as association neurones connecting the bipolar cells of the ganglion, cells of Dogiel, and to connect the ganglion with the sympathetic system of nerves.

Spinal Reflexes in Man and Mammals.-Much of our knowledge of the reflexes of the cord is derived from experiments on dogs, though paralysis of the lower extremities in man, by accident or otherwise, has given confirmatory information. In man the spinal cord is so much under the control of the higher nerve centers that its own individual functions in relation to reflex action are apt to be overlooked. But if the skin of the foot is stimulated, in a man whose lumbar cord is completely separated by injury or disease, the foot will be drawn away from the stimulus; or, if the stimulus be strong enough, tne entire leg will be moved. In both cases the movement may be orderly and well co-ordinated, and shows that the sensory stimulus has produced a co-ordinated reflex through the lumbar cord. The injured person feels no sensation of pain nor of action, and the phenomenon is independent of the higher nerve regions. The stimulus that is supplied to man must be carefully graded, since when too intense it calls forth muscular spasms or convulsive action.

When the cord of mammals is first cut, the shock is very great, and the lower or isolated portion of the cord remains for a time quite non-irritable. The vaso-motor and thermogenic centers are cut off from the periphery so that there is great vascular dilatation and marked fall of temperature, the 
effects of which are likely to lead to death unless the operated animal is carefully attended. But these effects are slowly recovered from, and man, as well as the lower mammals, soon regains the vascular tone. The general tonus of the muscular system, which is lost at first, is also regained.

In this partially recovered condition man, and such animals as the cat, the dog, and the monkey, perform certain of the lower functions with a remarkable degree of perfection. Of course these functions are under constant co-ordinative regulation and control in the normal animal, but experiments

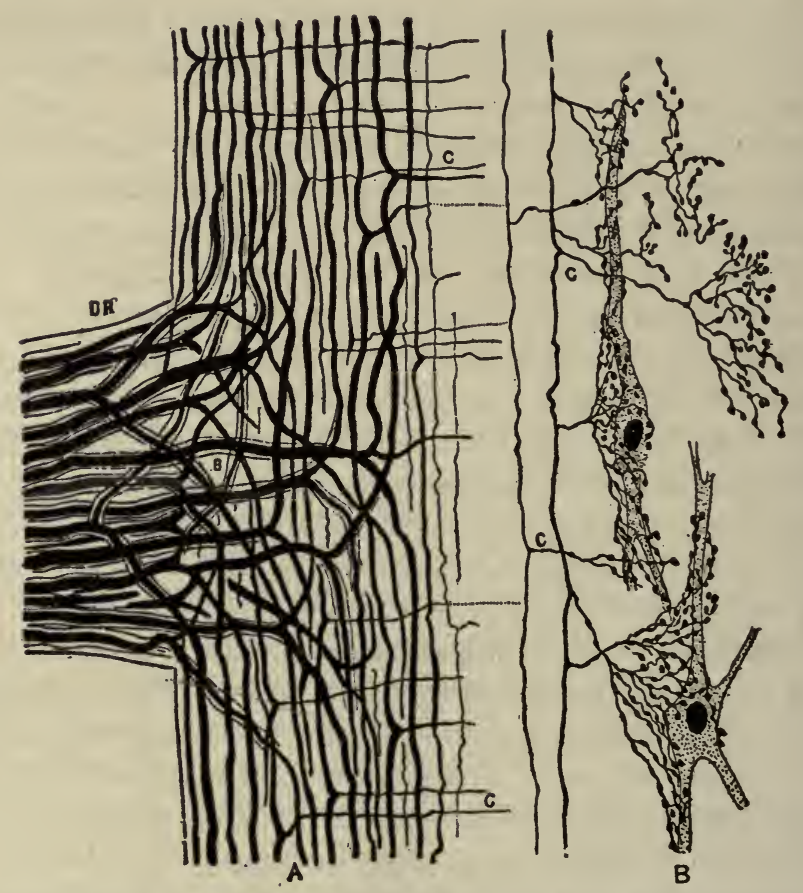

FIG. 370.-Scheme of the Relation of the Posterior Root Fibers upon Entering the Cord. $A$, The branch of the dorsal root fibers upon entering cord; $B$, terminal arborization about cell bodies of the cord; $D R$, axones of the dorsal root; $B$, their ascending and descending branches; $C$, collaterals. (After Cajal.)

and observation have shown that much of such activity really is a primary function of the cord. Of these activities the following may be especially mentioned: Muscular tonus, general reflexes, the special reflexes of micturition, defecation, erection and the sexual reflex, and parturition, some of which will be briefly discussed.

The Center of the Tone of Muscles.-The tonic influence of the spinal cord on the sphincter ani and sphincter urethræ will be presently mentioned. The cord maintains these muscles in permanent tonic contraction. The condition of the sphincters, however, is not altogether exceptional. Their contraction 
is the same in kind, though it exceeds in degree, that condition of the voluntary muscles which has been called tone, a condition of slight contraction which they always maintain during health. This tone of all the muscles of the trunk and limbs depends on the spinal cord, just as does the contraction of the sphincters. If an animal be killed by injury or removal of the brain, the muscles retain therr tenseness, but if the spinal cord be destroyed, all the muscles become loose, flabby, and atonic, remaining.so till rigor mortis commences.

If an animal, such as the dog, be held off the ground in the erect position assumed by the human body, when the trunk and hind limb muscles are not in voluntary contraction the limbs will assume a normal pendular position. In the pendular position the legs of a dog with cord severed hang more limp and are more completely extended. The muscles of the former exhibit that tone which keeps antagonistic muscles always slightly tense, the muscles of the latter have lost the tenseness.

Whether or not muscular tone is maintained through the constant subminimal action of sensory nerve impulses on the tonic centers of the cord, or whether these centers are automatic in their action, is a question that can be answered only by inference. The probability is that tone is a reflex activity, though it may be contributed to by the normal healthy nutritional condition of the muscles themselves-a condition which is itself dependent on the trophic influence of the nerve cells of the cord and brain stem.

The Ano-spinal or Defecation Center.-The mode of action of the anospinal center appears to be this: The mucous membrane of the rectum is stimulated by the presence of feces or of gas in the large bowel. The stimulus passes up by the afferent nerves of the hemorrhoidal and inferior mesenteric plexuses to the center situated in the lumbar enlargement of the cord, and is reflected through the pudendal plexus to the anal sphincter, and to the muscular tissue in the wall of the lower bowel. In this way there is produced a relaxation of the first and a contraction of the second, and expulsion of the contents of the bowel follows. The center in the spinal cord is partially under the control of the will, so that its action may be either inhibited or augmented. The action may be helped by the abdominal muscles, which are voluntary muscles, but are also stimulated to contract by reflex action.

The Vesico-spinal or Micturition Center.-The vesico-spinal center acts in a very similar way to that of the ano-spinal. The center is also in the lumbar enlargement of the cord. It is stimulated to action reflexly by the presence of urine in the bladder. The action may be voluntary and is excited by the sensation of distention of the bladder by the urine. The sensory fibers concerned are the posterior roots of the lower sacral nerves. The action of the spinal center is double, or it may be supposed that the center consists of two parts, one of which is usually in action and maintains the tone of the sphincter, and the other which causes contraction of the bladder and other 
muscles. When evacuation of the bladder occurs sensory impulses pass to that part of the center which discharges impulses to the bladder and to certain accessory muscles which cause their contraction; and impulses pass to that part of the center which inhibits the tonic action on the sphincter urethræ which procures its relaxation. The way having been opened by the relaxation of the sphincter, the urine is expelled by the combined action of the bladder and accessory muscles. The cerebrum may exert its influence on the reflex not only by stimulating the center to action, but also by inhibiting its action.

The Genito-spinal Center.-The presence of the genito-spinal center is proven by the fact that dogs, and even man, are known to discharge semen when the lumbar cord is severed and all voluntary motion and sensibility are lost. The center situated in the lumbar enlargement of the spinal cord is stimulated to action by sensory impressions from the glans penis. Efferent impulses from the center excite the successive and co-ordinate contractions of the muscular fibers of the vasa deferentia and vesiculæ seminales and of the accelerator urinæ and other muscles of the urethra; and a forcible expulsion of semen takes place, which, in cases of paraplegia, are not felt.

The Erectal Center.-This center is also situated in the lumbar region and is a vascular center, already described in the chapter on Circulation. It is reflexly excited to action by the sensory nerves of the penis, and also in the normal animal by impulses passing down from the cerebrum. Efferent impulses produce dilatation of the vessels of the penis.

The Parturition Center.-The center for the expulsion of the contents of the uterus in parturition is situated in the lumbar spinal cord rather higher up than the other centers already enumerated. The stimulation of the uterus may, under certain conditions, excite the center to send out impulses which produce a contraction of the uterine walls and expulsion of the contents of the cavity. The center is independent of the will since delivery takes place in paraplegic women, and also while a patient is under the influence of chloroform. Again, as in the cases of defecation and micturition, the abdominal and thoracic muscles assist; their action being for the most part reflex and involuntary.

Inhibition of Reflex Actions. - Movements such as are produced by stimulating the skin of the lower extremities in the human subject, after division or disorganization of a part of the spinal cord, do not always occur when the cerebrum is active and the connection between the cord and the brain is intact. The reflex which would occur in the animal with spinal cord only is suppressed or inhibited in the normal animal through the regulative action of the higher cerebral centers. When one is anxiously thinking, even slight stimuli may produce involuntary and reflex movements. So, also, during sleep, such reflex movements may be observed, when the skin is touched or tickled; for example, when one touches the palm of the hand of a sleeping 
child, the impression on the skin of the palm producing a reflex movement of the muscles which close the hand. But when the individual is awake no such reflex is produced.

Further, many reflex actions are capable of being more or less controlled or even altogether prevented by the will, of which the following may be quoted as familiar examples:

When the foot is tickled we can, by an effort of will, prevent the reflex action of jerking it away. So, too, the involuntary closing of the eyes and starting back, when a blow is aimed at the head, can be similarly restrained. Darwin has mentioned an interesting example of the way in which such an instinctive reflex act may override the strongest effort of the will. He placed his face close against the glass of the cobra's cage in the Reptile House at the Zoological Gardens, and, though of course thoroughly convinced of his perfect security, could not by any effort of the will prevent himself from starting back when the snake struck with fury at the glass.

It can be readily shown, by comparing a spinal frog and a normal uninjured frog, that stimuli which call forth definite reflexes in the first often produce no movement of the second.

Cutaneous and Muscle Reflexes as Diagnostic Signs.-In the human subject two classes of reflex actions dependent upon the spinal cord are usually distinguished, the alterations of which, either of increase or of diminution, are indications of some abnormality, and are used as a means of diagnosis in nervous and other disorders. They are termed, respectively, cutaneous reflexes and muscle reflexes. Cutaneous reflexes are set up by a gentle stimulus applied to the skin. The subjacent muscle or muscles contract in response. Although these cutaneous reflex actions may be demonstrated almost anywhere, yet certain of such actions as being most characteristic are distinguished, e.g., plantar reflex, gluteal reflex, i.e., a contraction of the gluteus maximus when the skin over it is stimulated; cremaster reflex, retraction of the testicle when the skin of the inside of the thigh is stimulated, and the like. The ocular reflexes, too, are important. They are contraction of the iris on exposure to light, and its dilatation on stimulating the skin of the cervical region. All of these cutaneous reflexes are true reflex actions. They differ in different individuals, and are more easily elicited in the young.

Muscle reflexes or tendon reflexes consist of a contraction of a muscle under conditions of more or less tension, when its tendon is sharply tapped. The so-called patellar-tendon reflex "knee-jerk" is the best known of this variety of reflexes. If one knee be slightly flexed, as by crossing it over the other, so that the quadriceps femoris is extended to a moderate degree, and the tendon of the patella be tapped with the fingers, the muscle contracts and the foot is jerked forward. Another variety of the same phenomenon is seen if the foot is flexed so as to stretch the calf muscles, and the tendo Achillis is tapped; the foot is extended by the contraction of the stretched muscles. It 
appears, however, that the tendon reflexes are not exactly what their name implies. The interval between the tap and the contraction is said to be too short for the production of a true reflex action. It is suggested that the contraction is caused by local stimulation of the muscle, but that this would not occur unless the muscle had previously been stimulated by the tension applied, and placed in a condition of excessive irritability. It is probable that the

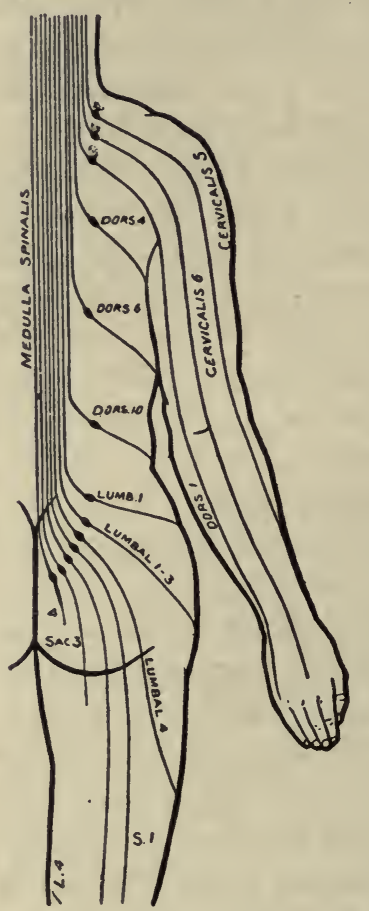

FIG. 371.-Diagram to Show the Manner in which the Fibers of the Posterior Nerve Roots Enter and Ascend the Posterior Columns of the Cord. (Edinger.) condition on which it depends is a reflex change in the spinal irritability acting on the muscle or exaggerated muscular tone, which is admitted to be a reflex phenomenon in the spinal cord.

Conduction in the Spinal Cord.-With the differentiation of the central nerve axis in vertebrates the conduction in the spinal cord becomes of increasing importance, reaching its maximum in man. It is evident that the cord is the path by which all nerve impulses arising in the trunk or in the arms and legs must reach the brain, or vice versa. Impulses of peripheral origin can and do produce reflexes, but they can arouse sensations and be perceived only after they have been conducted to the cerebral cortex. Motor impulses arising in the brain can reach the motor cells of the anterior columns of the cord only through the cord as a conducting path. The continuity of the cord, therefore, while not necessary for the execution of reflexes, is absolutely necessary for the higher co-ordinations of the reflexes and for the excitation and controlling influence of the brain.

Illustrations of this are furnished by various examples of paralysis, but by none better than by the common paraplegia, or loss of sensation and voluntary motion in the lower part of the body, in consequence of destructive disease or injury of a section including the whole thickness of the spinal cord. Such lesions destroy the communication between the brain and all parts of the spinal cord below the seat of injury, and consequently cut off from their connection with the brain the various organs supplied with nerves issuing from those parts of the cord.

It is not probable that the conduction of motor and of sensory impulses is effected under ordinary circumstances, to so great an extent as was formerly supposed, through the gray substance of the cord, i.e., from cell to cell through the short filaments lying wholly within the gray substance. But 
cells with fibers running for short distances in the ground bundles are numerous, and these short connectives are capable of conducting impulses along the cord. All parts of the cord are not alike able to conduct all impressions; and as there are separate nerve fibers for motor and for sensory impressions, so in the cord separate and determinate tracts serve to conduct always the same kind of impressions. The sensations of touch, and perhaps of temperature and pain, do not appear to have such sharply limited tracts as do the motor impulses.

Experimental and other observations point to the following conclusions regarding the conduction of sensory and motor impressions through the spinal cord. Many of these conclusions must, however, be received with considerable reserve.

Sensory Impulses.-The sensory impressions of touch, pain, heat and cold, and of the muscle sense are conducted to the spinal cord by the posterior nerve roots. Certain sensory impressions are then carried directly into the fasciculus gracilis on the same side, and thence up to the nucleus of this column in the medulla. It is mainly the impulses of the muscle sense and of the sense of touch that take this course through the cord, though the sense of touch is not wholly interrupted upon injury to the posterior columns. In lower animals it is scarcely interfered with at all. The posterior funiculi unquestionably are the primary muscle sensory paths. Visceral sensations are carried by the posterior root fibers to the cells of the column of Clarke in the posterior horn, figure 363 . From there the impulses pass to the direct cerebellar tract on the same side, and thence up through the medulla to the cerebellum, figure 388 . The impressions of pain, and of heat and cold, are conveyed to the nerve cells in the posterior cornua of the same side in part, and in part to the nerve cells in the posterior cornu and median gray matter of the apposite side. From this point, the impulses are taken up again by intermediary neurones and conveyed through the anterior and lateral columns of the cord to the brain in the ascending superficial antero-lateral tract, or tract of Gowers. By reason of the great number of collaterals and the interpolation in the course of the sensory path of many intermediary neurones, it has been difficult to make out very sharply defined tracts in the spinal cord for the conduction of the sensations of temperature, pain, and touch. If one set of fibers is destroyed by disease, others seem able, through the collaterals, to take up its functions. We can say that injury to the lateral columns has resulted in loss of the sense of pain, heat, and cold, but with only partial disturbance of touch sensations.

It is probable, also, that pain and temperature sensations cross over at once to a considerable extent and pass up in the opposite side of the cord to which they enter. Touch and the muscle sense impressions, especially the latter, pass up largely upon the same side until they reach the medulla or cerebellum. 
Motor Impulses.-Motor impulses are conveyed downward from the cerebral cortex of the brain along the pyramidal tracts, viz., the laterai and the anterior, chiefly the former. In the lateral pyramidal tract the impressions pass down chiefly on the side opposite to which they originate, having crossed over in the decussation in the medulla. But some motor impulses do not cross in the medulla, but descend in the anterior pyramidal tract to lower levels of the cord, where they cross in the anterior commissure. The motor fibers for the legs partially pass downward in the lateral columns of the same side without decussation. This is also probably the case with the bilateral muscles, i.e., muscles of the two sides that act together, such as the intercostal muscles and other muscles of the trunk.

It is quite certain, as was just now pointed out, that the fibers of the anterior nerve roots are more numerous than the fibers proceeding downward from the brain in the pyramidal tracts, the so-called pyramidal fibers. This is because each pyramidal fiber is really a very long nerve process or axone, and is supplied in its course with a large number of collaterals. These go off at different points, and thus put it in relation with different groups of nerve cells in the anterior columns at various levels. Each nerve fiber of the pyramidal tract, by means of its collaterals, can control a number of nerve cells, and can thus co-ordinate the action of impulses sent out through the anterior roots to a number of groups of muscles. In other words, the gray matter of the anterior columns contains an apparatus with various complicated co-ordinating powers, which apparatus is under the regulative control of the neurones whose cells of origin are in the cortex of the cerebrum. This is the same apparatus that is also reflexly influenced by sensory impressions passing to the cord from the periphery.

Division of a single anterior pyramid of the medulla at a point just above the decussation is followed by paralysis of voluntary motions in the muscles of the opposite side in all parts below. Disease or division of any part of the cerebro-spinal axis below the seat of decussation of the pyramids is followed by impairment or loss of voluntary motion on the same side of the body. The paralysis is never quite complete, and the opposite side usually shows some slight impairment of function of the muscle.

When one-half of the spinal cord is cut through in monkeys, the results are as follows (Mott): Motor paralysis of the muscles of the same side (never complete paralysis of the muscles used in bilateral associated action), followed by gradual recovery of muscular movement, except of the finer movements of the hand and foot; wasting and flabbiness of the muscles; partial sensory paralysis of the same side (temperature, touch, pain, and pressure); temporary vaso-motor paralysis on the same side. The temperature of the affected side is depressed I to $3^{\circ} \mathrm{F}$. 


\section{THE BRAIN.}

General Arrangement of Parts of the Brain.-The great relative and absolute size of the cerebral hemispheres in the adult man and in mammals to a great extent mask the real arrangement of the several parts of the brain. An examination of the accompanying diagram, figures 370,37 , reveals that the parts of the brain are disposed in a linear series, as follows (from before backward): Olfactory lobes, cerebral hemispheres, thalamencephalon (thalami and third ventricle), the mid-brain (corpora quadrigemina and crura cerebri, medulla oblongata, and cerebellum.

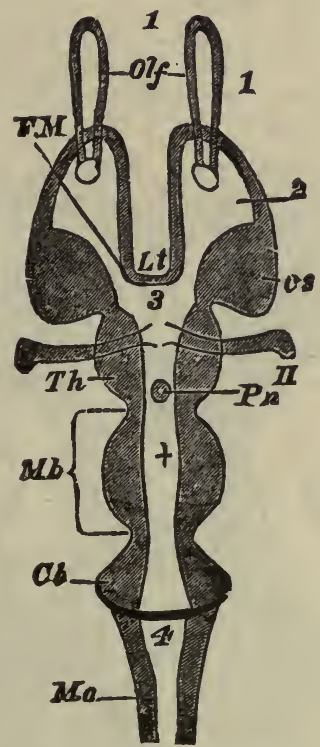

Fig. 372.-Diagrammatic Horizontal Section of the Vertebrate Brain. The figures serve both for this and the next diagram. $M b$, mid-brain; what lies in front of this is the fore-, and what lies behind the hind-brain; $L t$, lamina terminalis; Olf, olfactory lobes; Hemp, hemispheres; Th. E, thalamencephalon; $P n$, pineal gland; $P y$, pituitary body; $F$. M., foramen of Munro; $c s$, corpus striatum; $T h$, optic thalamus, $C C$; crura cerebri: the mass lying above the canal represents the corpora quadrigemina; $C b$, cerebellum; $I-I X$, the nine pairs of cranial nerves; $I$, olfactory ventricle; 2 , lateral ventricle; 3 , third ventricle; 4 , fourth ventricle; + , iter a tertio ad quartum ventriculum. (Huxley.)

The linear arrangement of parts actually occurs in an early stage of the development of the human fetus, and it is permanent in some of the lower vertebrata. In fishes the cerebral hemispheres are represented by a pair of ganglia intervening between the olfactory and the optic lobes, and considerably smaller than the latter, their adult development is fairly well represented by the figure 373. In Amphibia the cerebral lobes are further developed, and are larger than any of the other ganglia. 
In reptiles and birds the cerebral ganglia attain a still further development and in Mammalia the cerebral hemispheres exceed in weight all the rest of the brain. As we ascend the scale, the relative size of the cerebrum increases, till

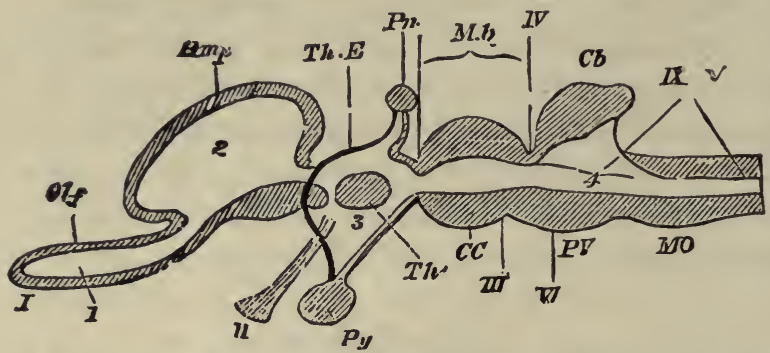

FiG. 373.- Longitudinal and Vertical Diagrammatic Section of a Vertebrate Brain. Letters as before. Lamina terminalis is represented by the strong black line joining $P_{n}$ and $P y . \quad$ (Huxley.)

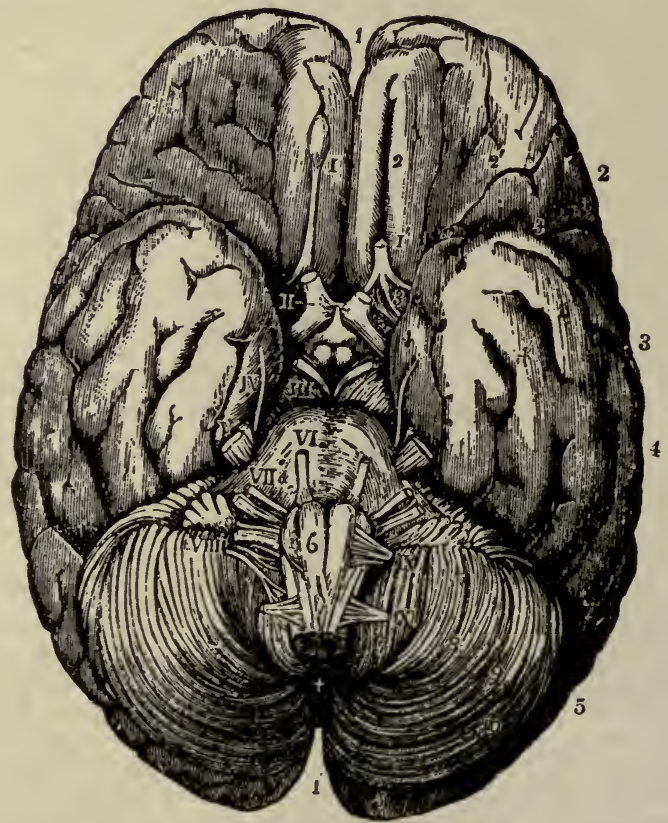

FIG. 374. -Base of the Brain. I, Superior longitudinal fissure; 2, $2^{\prime}, 2^{\prime \prime}$, anterior cerebral lobe; 3, fissure of Sylvius, between anterior and 4, 4', 4', middle cerebral lobe; $5,5^{\prime}$, posterior lobe; 6 , medulla oblongata. The figure is in the right anterior pyramid; 7 , 8,9 , Io, the cerebellum; + , the inferior vermiform process. The figures from $I$. to $I X$. are placed against the corresponding cerebral nerves; $I I I$. is placed on the right crus cerebri. $V I$. and $V I I$. on the pons Varolii; $X$., the first cervical or suboccipital nerve. (Allen Thomson.) $\times \frac{1}{2}$.

in the higher apes and man the hemispheres, which commenced as two little lateral buds from the anterior cerebral vesicle, having grown upward and backward, completely covering in and hiding from view practically all the 
rest of the brain. At the same time the smooth surface of the cerebral cortex of many lower mammalia, such as the rabbit, is replaced by the labyrinth of convolutions of the human brain.

When the cerebral hemispheres are removed, several large basal masses of nerve substance are revealed: the optic thalami, the corpora quadrigemina, and the crus cerebri. These structures, together with the pons and the medulla, form a direct continuation forward of the spinal cord and sometimes are designated under the general term of the brain stem.

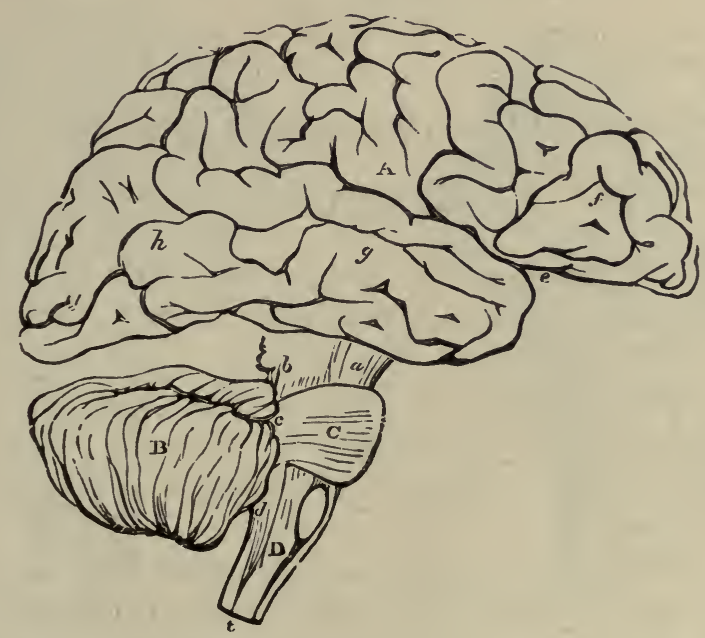

Fig. 375.- Plan in Outline of the Brain as seen from the Right Side. $\times \frac{1}{2}$. The parts are represented as separated from one another somewhat more than natural, so as to show their connections. A, Cerebrum; $f, g, h$, its anterior, middle, and posterior lobes $e$, fissure of Sylvius; $B$, cerebellum; $C$, pons Varolii; $D$, medulla oblongata; $a$, peduncles of the cerebrum; $b, c, d$, superior, middle, and inferior peduncles of the cerebellum. (From Quain.)

The human brain on superficial examination does not seem to follow the general plan outlined above, but when the cerebral hemispheres and the cerebellum are removed then it is found that what remains closely follows the plan presented. This central axis is shown in part in figure 379.

The morphological parts of the brain usually given are:

\section{The Brain or Encephalon.}

I. The hind-brain or rhombencephalon.

I. Myelencephalon.

a. Bulb or medulla oblongata.

2. Metencephalon.

b. Pons Varolii.

c. Cerebellum. 
II. Mid-brain or mesencephalon.
d. Corpora quadrigemina
e. Crura cerebri.

III. Fore-brain, prosencephalon.

3. Thalamencephalon.

f. Optic thalami.

4. Telencephalon.

g. Corpora geniculata.

h. Corpora striata.

i. Cerebral hemispheres.

\section{THE MEDULLA OBLONGATA AND PONS.}

\section{STRUCTURE.}

Anatomical Structure.-The medulla oblongata is continuous with the spinal cord at the upper end. It lies within the cranial cavity and forms the first part of the brain stem. The medulla consists of masses of nerve cells situated in the interior, but pretty generally distributed throughout the mass. The cell-masses are subdivided by laminæ of nerve fibers into groups, or nuclei, which give origin to or form the terminations of the various ranks of nerve fibers.

The nerve fibers are arranged partly in columns and partly in fasciculi traversing the central cellular matter. The medulla oblongata is larger than any part of the spinal cord. Its columns are pyriform, enlarging as they proceed toward the upper part of the brain, and are continuous with funiculi of the spinal cord. Each half of the medulla, therefore, may be divided into three columns or tracts of fibers, continuous with the three columns of funiculi or of the spinal cord. The columns are more prominent than those of the spinal cord, and are separated from each other by deeper grooves. The anterior, continuous with the anterior columns of the cord, are called the pyramids. The postero-median and external are represented at the posterior or dorsal aspect of the cord as the fasciculus gracilis and the fasciculus cuneatus. The posterior pyramids of the medulla, which include these two columns of white matter, soon become much increased in width by the addition of a new column of white matter outside the other two, which is known as the fasciculus of Rolando. In the upper portion of the medulla the fasciculi are replaced by the restiform bodies (the inferior peduncles of the cerebellum). The lateral columns of the cord are scarcely represented as such in the bulb.

It may be said then that the bulb at its commencement differs only slightly in size from the cord, with which it is continuous. It soon becomes larger both laterally and antero-posteriorly. It opens out on the dorsal surface into 
a space which is known as the fourth ventrical, and from being a cylinder with a central canal it is flattened out on the dorsal surface by the gradual approach of the central canal to that surface, where it is directly continuous with the fourth ventricle.

On the anterior or ventral surface of the bulb it is found that the anterior fissure is occupied at the most posterior part by fibers which are crossing from one side to the other. This is known as the decussation of the pyramids. It is formed of the lateral pyramidal fibers. The lateral pyramidal fibers of

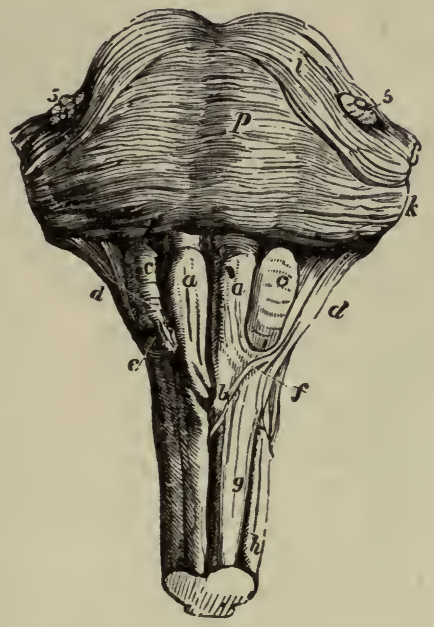

FIG. 376.

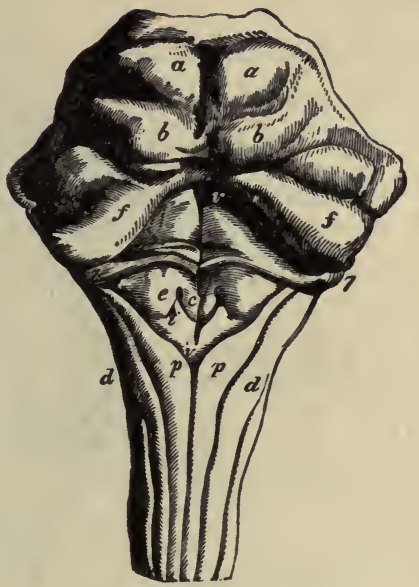

Fig. 377.

Fig. 376.-Ventral or Anterior Surface of the Pons Varolii and Medulla Oblongata, $a, a$, Anterior pyramids; $b$, their decussation; $c, c$, olivary bodies; $d, d$, restiform bodies; $e$, arciform fibers; $f$, fibers passing from the anterior column of the cord to the cerebellum; $g$. anterior column of the spinal cord; $h$, lateral column; $p$, pons Varolii; $i$, its upper fibers; 5,5 , roots of the fifth pair of nerves.

Fig. 377.- Dorsal or Posterior Surface of the Pons Varolii, Corpora Quadrigemina, and Medulla Oblongata. The peduncles of the cerebellum are cut short at the side. $a, a$, the upper pair of corpora quadrigemina; $b, b$, the lower; $f, f$, superior peduncles of the cerebellum; $c$, eminence connected with the nucleus of the hypoglossal nerve; $e$, that of the glosso-pharyngeal nerve; $i$, that of the vagus nerve; $d, d$, funiculus cuneatus; $p, p$, funiculus gracilis; $v, v$, groove in the middle of the fourth ventricle, ending below in the calamus scriptorius; 7,7 , roots of the auditory nerves.

either side after crossing the middle line become part of the pyramid of the opposite side; the rest of the pyramid is made up of the fibers which in the anterior column of the cord are known as the direct or anterior pyramidal tract. The pyramidal fibers are those which degenerate after lesions of the parts of the cerebrum known as the motor areas of the cortex. They are the descending fibers of communication between the cerebral motor cells of the cortex and the different segments of the spinal cord. The outer borders of the anterior pyramids of the bulb are marked by the exit from that part of the nervous axis of the twelfth or hypoglossal nerve. Still more later- 
ally there is on either side a rounded elevation or column, the olivary body. It begins at a level a little lower than the opening of the fourth ventricle. On the dorsal side of the olivary body is the line of origin of the eleventh, tenth, and ninth nerves, and from this to the posterior fissure is the region corresponding to the lateral and posterior columns of the cord.

The changes in structure which are noticed in a series of sections of the bulb from below upward may be summarized: In the dorsal or posterior region, the posterior columns are pushed more to each side by the large number of sensory fibers ascending in the posterior funiculus and terminating in the

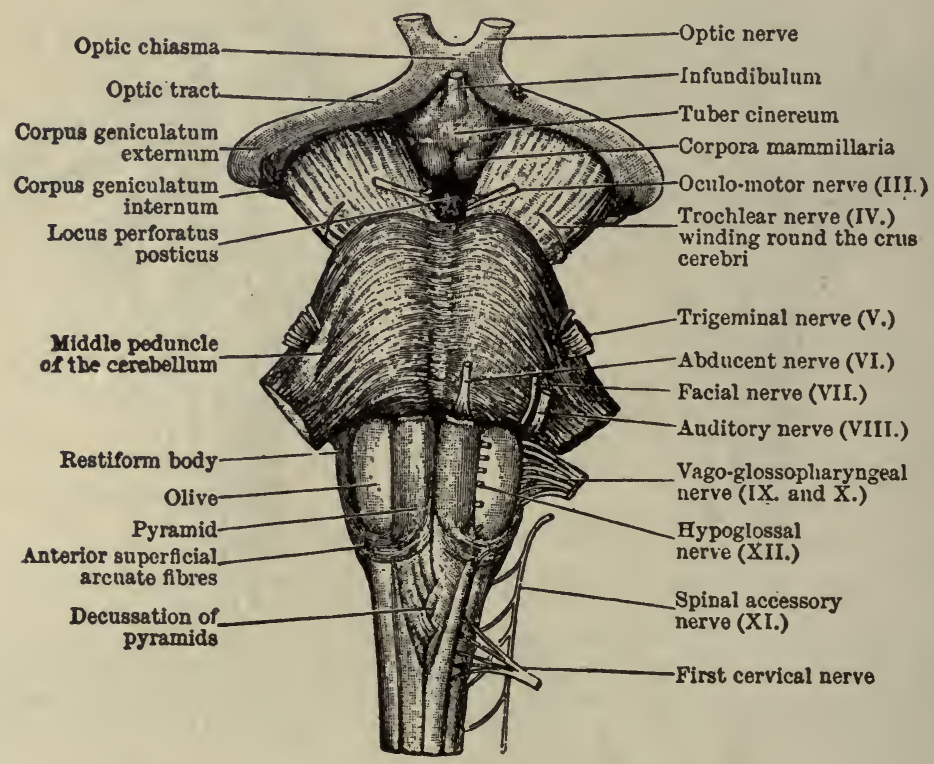

Fig. 378.-Front View of the Medulla, Pons, and Mesencephalon of a Full-term Human Fetus. (Cunningham.)

gracile and cuneate nuclei. The substance of Rolando is increased and becomes rounded, reaching almost to the surface of the bulb on each side, only a small tract of longitudinal fibers of the root of the fifth nerve intervening. There is a great increase of the reticular formation around the central canal. Then at the ventral or anterior aspect the decussation of the pyramids begins. By this crossing over of the fibers, the tip of the gray anterior cornu is cut off from the rest of the gray matter. The central canal is pushed farther toward the posterior surface, first of all by the decussation of the anterior pyramids just mentioned, and later on, i.e., above, by another decussation of more dorsal fibers. These fibers of the second decussation as they cross form a median raphe and also help to break up the remaining gray matter into what is called a reticular formation. These fibers arise from the nuclei of the funic- 


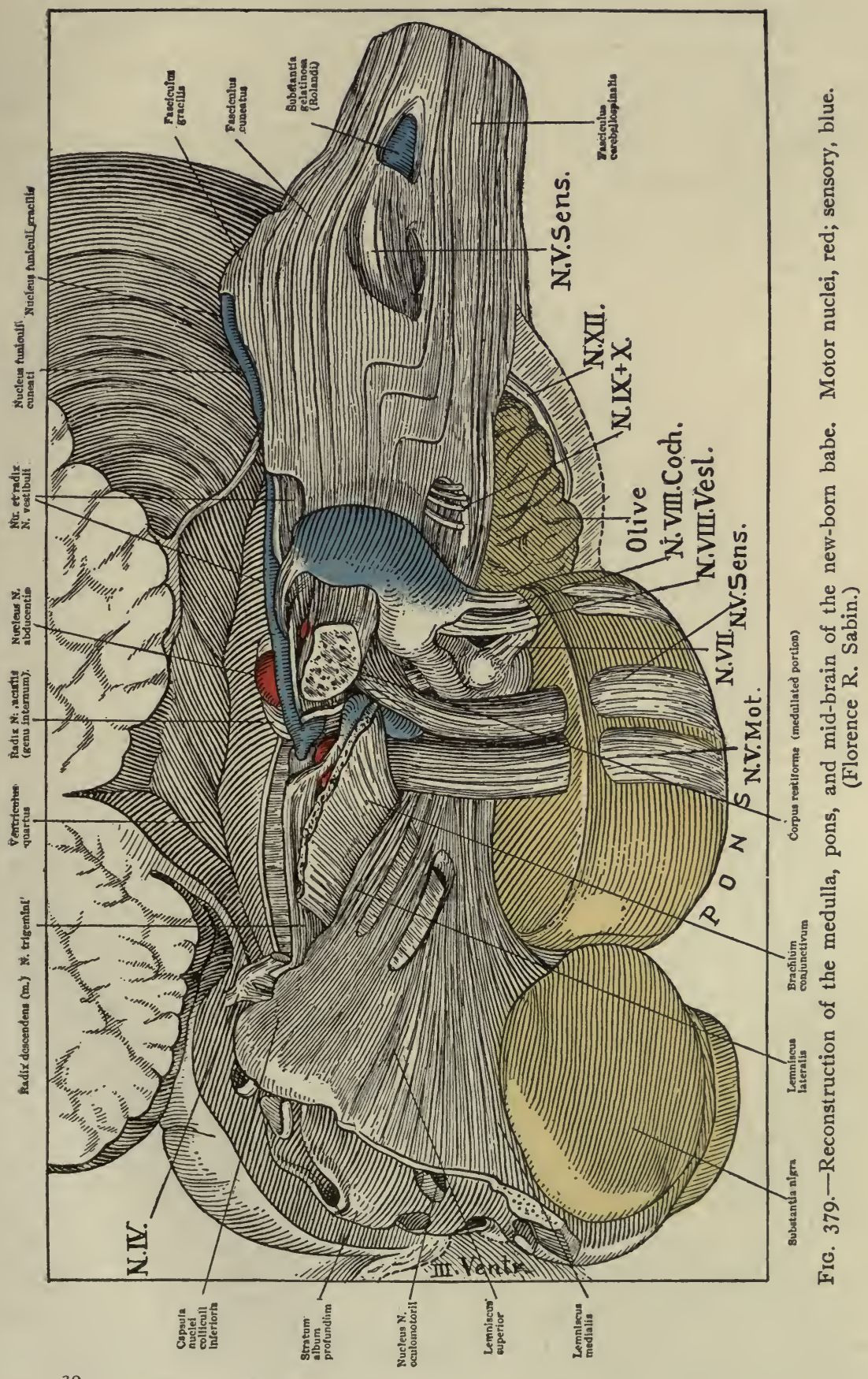


ulus gracilis and funiculus cuneatus of either side, and they are looked upon as a sensory decussation.

The olivary bodies extend forward almost to the level of the pons. They consist of both cells and fibers. The cellular matter consists of a plicated thinnish layer of small nerve cells, folded upon itself in the form of a loop, with the ends turned inward and slightly dorsal, figure $38 \mathrm{I}, \mathrm{O}$. The gray loop is filled with and covered by white fibers.

Internal to the olivary body on either side are two small masses of gray matter, one more ventral to the other, called accessory olives, external and

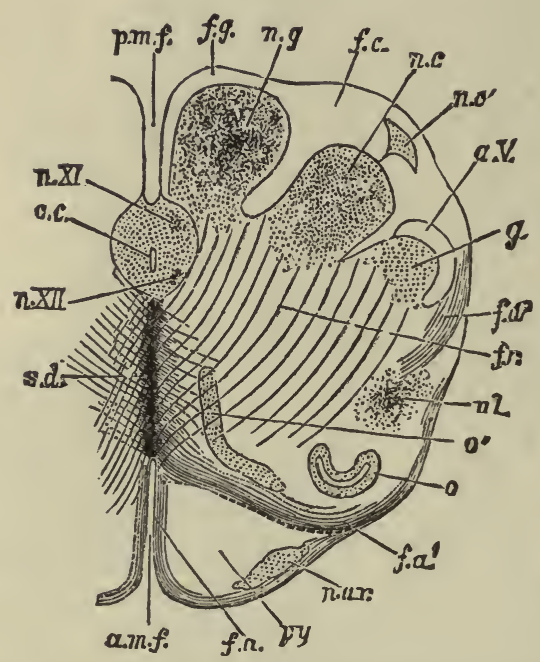

Fig. 380.-Anterior or Dorsal Section of the Medulla Oblongata in the Region of the Superior Pyramidal Decussation. a.m.f., Anterior median fissure; f,a., superficial arciform fibers emerging from the fissure; $p y$, pyramid; $n . a r$. , nuclei of arciform fibers; f.a., deep arciform becoming superficial; $o$, lower end of olivary nucleus; $n . l$., nucleus lateralis; f.r., formatio reticularis; $f . a^{2}{ }^{2}$, arciform fibers proceeding from the formatio reticularis; g., substantia gelatinosa of Rolando; $a . V$. , ascending root of fifth nerve; n.c., nucleus cuneatus; n.c.', external cuneate nucleus; $n . g$. , nucleus gracilis; $f . g .$, funiculus gracilis; p.m.f., posterior median fissure; c.c., central canal surrounded by gray matter, in which are $n . X I$., nucleus of the spinal accessory, and n.XII., nucleus of the hypoglossal; s.d., superior pyramidal decussation. (Modified from Schwalbe.)

internal, and on the surface of the anterior pyramid on either side a small mass of gray matter, external arcuate nucleus; laterally another mass of the same material, the representative of the lateral nucleus of the cord, is seen, viz., the antero-lateral nucleus, which gives origin to the spinal accessory nerve.

It will be necessary to follow as shortly as possible the fibers of the spinal cord upward into the bulb and beyond.

Tracts Through the Medulla.- The crossed and direct pyramidal tracts have already been described. Nothing definite is known of the antero-lateral descending tracts. The direct cerebellar tracts pass laterally into the resti- 
form bodies and go to the cerebellum. The antero-lateral-ascending tracts (Gowers) appear to have the same destination, but pass indirectly into the cerebellum by way of the superior medullary velum; some of the fibers probably pass upward to higher centers. The fibers of the tracts of Goll and Burdach, of the cord, end in the nuclei of the funiculus gracilis and funiculus cuneatus, respectively; at any rate, ascending degeneration of these columns cannot be traced above these nuclei. The rest of the fibers of the cord appear to end in the reticular formation of the bulb.

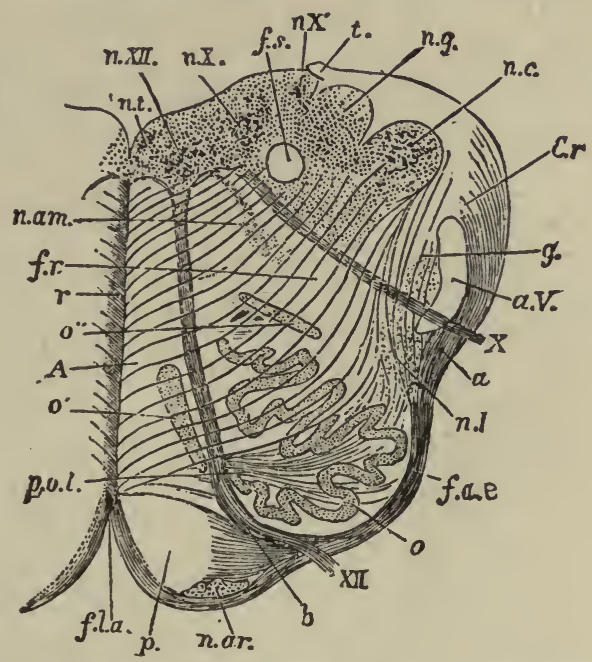

Fig. 381. - Section of the Medulla Oblongata at about the Middle of the Olivary Body. f.l.a., Anterior median fissure; n.ar., nucleus arciformis; p., pyramid; XII., bundle of hypoglassal nerve emerging from the surface; at $b$, it is seen coursing between the pyramid and the olivary nucleus, o.; f.a.e., external arciform fibers; n.l., nucleus lateralis; $a$. , arciform fibers passing toward restiform body, partly through the substantia gelatinosa, $g$., partly superficial to the ascending root of the fifth nerve, $a, V . ; X$., bundle of vagus root emerging; f.r., formatio reticularis; c.r., corpus restiforme, beginning to be formed, chiefly by arciform fibers, superficial and deep; n.c., nucleus cuneatus; n.g., nucleus gracilis; $t$, attachment of the ligula; f.s., funiculus solitarius; $n . X ., X n . .^{\prime}$, two parts of the vagus nucleus; n. $X I I$., hypoglossal nucleus; n.t., nucleus of the funiculus teres; n.am., nucleus ambiguus; $r .$, raphe; $A$., continuation of the anterior column of cord; $o^{\prime}, o^{\prime \prime}$, accessory olivary nucleus; p.o., pedunculus olivæ. (Modified from Schwalbe.)

Connections of the Bulb with the Cerebrum and Cerebellum.-The pyramidal tracts connect the bulb with the cerebrum; and the direct cerebellar and the antero-lateral ascending tract, tract of Gowers, connect it with the cerebellum. Other connections of the bulb with the cerebrum and with the cerebellum are:

i. Fibers from the nucleus gracilis and nucleus cuneatus, which, as we have said, are the endings of the fibers of the tracts of Goll and Burdach of the cord, pass in sets in the following manner:

a. Internal arcuate fibers pass down and inward to the opposite side in the reticular formation, composing in part the superior or sensory decussation, 
and in the inter-olivary region enter the mesial fillet, which passes upward through the pons to end about the cells in the mid-brain and in the optic thalami. These fibers are probably augmented by the addition of fibers from the anterior columns of the cord, and by fibers arising from cells in the sensory nuclei of the cranial nerves ending in the bulb.

$b$. External arcuate fibers, after decussating in the same way, pass outward superficially over the anterior pyramid and olivary body, reaching the restiform body and passing to the side of the cerebellum opposite to their nuclei of origin. These fibers appear to be interrupted, at least in part, in the external arcuate nuclei. They connect one side of the spinal cord with the opposite side of the cerebellum through the gracile and cuneate nuclei.

c. Direct lateral fibers pass to the restiform body and so to the same side of the cerebellum.

2. Fibers from the olivary body pass to the opposite side of the cerebellum through the reticular formation and restiform body.

3. Fibers from the vestibular nucleus of the eighth or auditory nerve in the floor of the fourth ventricle pass to the same side of the cerebellum.

\section{FUNCTIONS OF THE MEDULLA.}

The medulla is of great importance in the physiological economy of the body. Its functions can be classified in three groups. First should be given the function of conduction between the cord and the higher centers of the brain. Second, the medulla is a center of numerous reflex activities, especially those regulating the vital functions of the body, such as respiration, circulation, and the like. A third function of centers in the medulla is that of automatic activity.

The Medulla as a Conducting Path.-The medulla is the pathway of all ascending and descending nerve impulses between the spinal cord and most of the peripheral sensory and motor apparatus on the one hand, and the cerebellum and the cerebral centers on the other. These conducting paths are described in the tracts that have already been discussed at some length. They are represented graphically in the diagrams, figures 379,382 , and 388 .

Reflex Centers of the Medulla.-The larger number of the cranial nerves, as we shall presently see, take their origin from the medulla and pons. Some of these nerves have both sensory and motor roots, while others are either exclusively motor or sensory. A large percentage of the afferent or sensory impulses that enter the medulla produce reflex effects on the motor nuclei so richly represented in the medulla. The nuclei, or centers, regulating some of the most important functions of the body are among those in this group. When certain of these centers are interfered with, death follows.

Life may continue when the spinal cord is cut away in successive portions 
from below upward as high as the point of origin of the phrenic neryes from the cervical cord. In amphibia, the brain has been all removed from above, and the cord removed as far as the medulla oblongata from below; yet so long as the medulla oblongata was left intact, respiration and life were maintained. But if the medulla oblongata is wounded, particularly if it is wounded in its central part opposite the origin of the vagi, the respiratory movements cease,

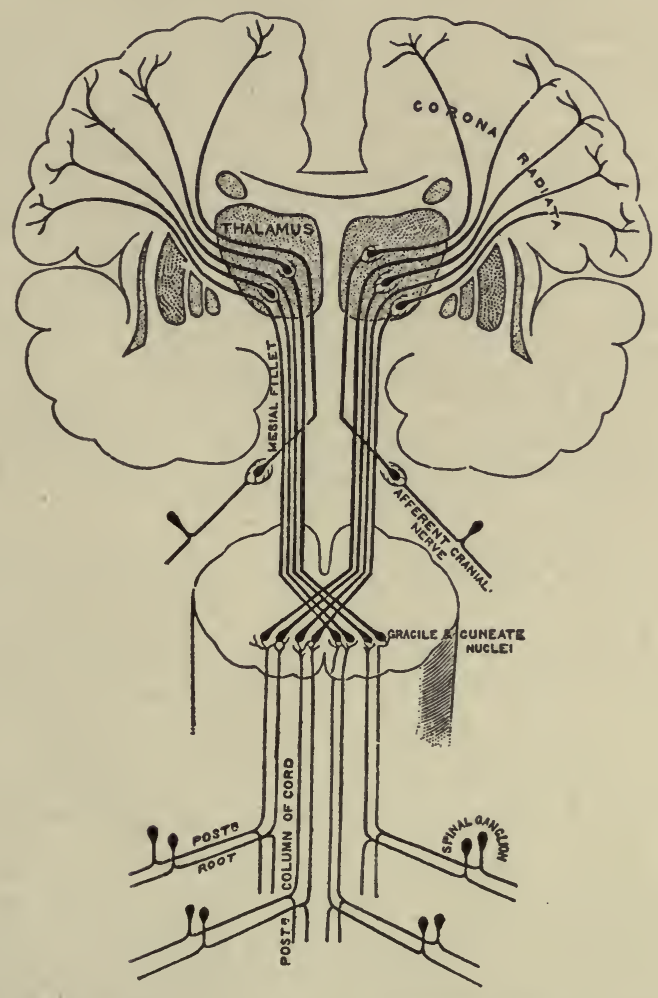

Fig. 382.-Diagram of Ascending Conduction Paths from the Cord through the Medulla and the Thalamus to the Cerebral Cortex. (Cunningham.)

and the animal dies from asphyxiation. This effect ensues even when all parts of the nervous system except the medulla oblongata are left intact.

Injury and disease in men are accompanied by the same nerve disturbances as are exhibited by these experiments on animals. Numerous instances are recorded in which injury to the medulla oblongata has produced instantaneous death; and, indeed, it is through injury to it, or of the part of the cord connecting it with the origin of the phrenic nerves, that death is commonly produced in fractures attended by sudden displacement of the upper cervical vertebræ. 
The majority of the medullary centers are reflex centers simply, and are stimulated by afferent or by voluntary impulses. Some of them are automatic centers and are capable of sending out efferent impulses without previous stimulation by afferent or by voluntary impulses. The automatic centers are, however, normally influenced by reflex or by voluntary impulses.

Some of these reflex centers which are bilateral are: I. Centers for the movements of deglutition. The medulla oblongata contains in the motor nuclei of the ninth and tenth nerves the centers whence are derived the motor impulses enabling the muscles of the palate, pharynx, and esophagus to produce the successive co-ordinated and adapted movements necessary to the act of deglutition, page 339. This is proved by the persistence of the act of swallowing in some of the lower animals after destruction of the cerebral hemispheres and cerebellum; its existence in anencephalous monsters, figure 400; and by the complete arrest of the power of swallowing when the medulla oblongata is injured in experiments.

2. Centers for the combined muscular movements of sucking, the nerves concerned being the facial for the lips and mouth, the hypoglossal for the tongue, and the inferior maxillary division of the fifth for the muscles of the jaw.

3. Centers for the secretion of saliva, which have been already mentioned, page 349 .

4. Centers for vomiting, page 376 .

5. Centers for coughing, which is a reflex act quite independent of the respiratory act.

6. Centers for the dilatation of the pupil, the fibers from which pass out through the spinal cord in the two upper dorsal nerves into the cervical sympathetic.

7. The respiratory center of the medulla has already been discussed as regards its automatic action. It is only necessary to repeat here that although it is automatic in its action, being capable of direct discharge of respiratory impulses with no other stimulus than the condition of the blood circulating within it, yet it is constantly reflexly influenced by afferent impulses. The respiratory center has been proven to be bilateral. It also consists of an inspiratory part and of an expiratory part. The center is influenced by voluntary impulses, but one cannot voluntarily control this center to the point of death. The vagus influence is probably the most constant of those stimulating the respiratory center. But the respiratory reflexes are taking place constantly in res, onse to afferent impulses flowing into the medulla from numerous other sensory nerves over the entire body.

8. The Cardio-inhibitory Centers. - The medulla contains the centers which maintain the proper rhythm of the heart, these centers acting through the vagus fibers. These terminate in a local intrinsic mechanism which has 
been already discussed. It is claimed that the center can be stimulated directly, as by the condition of the blood circulating within it. It is constantly exerting a tonic influence over the heart, which is the chief reason for considering it an automatic center. But the cardio-inhibitory center is primarily a reflex center. Sensory or afferent impulses arriving over the sensory paths in the vagus itself, by abdominal paths through the sympathetic, and through cutaneous nerves, are constantly causing reflex discharges of inhibitory impulses from this center.

9. Accelerator centers for the heart are present in the medulla. They are reflexly stimulated by sensory impulses arising from the same general source as in the preceding center.

Io. Vaso-motor centers which control the unstriped muscle of the arteries, are also situated in the medulla. The nerve cells constituting the center are under the constant influence of nerve impulses flowing in from the sensory and motor structures throughout the whole body. The reflexes produced by the afferent impulses bring about the variations in vaso-motor tone that not only regulate the general vascular responses of the body, but control and coordinate the local changes in the size of the blood vessels.

II. Centers for the secretion of sweat exist in the medulla. The medullary centers control the subsidiary spinal sweat centers. They may be excited unequally so as to produce unilateral sweating.

The reflex medullary centers described above are comparable to the spinal reflex centers previously described. If the medulla were completely isolated from the higher cerebral centers, and the spinal cord removed with the exception of those paths which are necessary to maintain respiration, these medullary reflex centers would be able to co-ordinate afferent impulses in the same general way that isolated segments of the cord do. In the living body, however, the medullary centers are under the influence of changes going on in regions of the nervous system both above and below, changes which constantly influence the details of the reactions. The activities are unconscious reflexes in the same sense that the motor reflexes of the spinal cord are unconscious and machine-like. The main difference is one of complexity and not of kind.

The Pons Varolii.-The pons Varolii is generally spoken of as a great commissure of fibers; of fibers which connect the two halves of the cerebellum and which connect the bulb and spinal cord with the cerebellum. It must not be forgotten that the pons contains several smaller collections of nerve cells. Sections reveal the following parts or structures, beginning with the anterior or ventral surface.

I. Transverse or commissural fibers connect one side of the cerebellum with the other through the middle peduncle. These fibers connect the cerebellar cortex with the cells of the pontine nuclei; some are afferent, some efferent; some end in the gray matter of the pons on the same side near the 
ventral surface; others cross to the opposite side of the pons and then become longitudinal, passing on to the tegmentum.

2. Fibers longitudinal in direction are arranged in larger or smaller bundles and are separated by gray matter. Most of these fibers are pyramidal fibers which pass down to the pyramids of the medulla.

3. The dorsal portion of the pons is made up to a considerable extent of the reticular formation of the tegmental region together with one or two distinct bundles of longitudinal fibers. The chief longitudinal bundle, situated at the junction of the ventral two-thirds with the dorsal third, is the

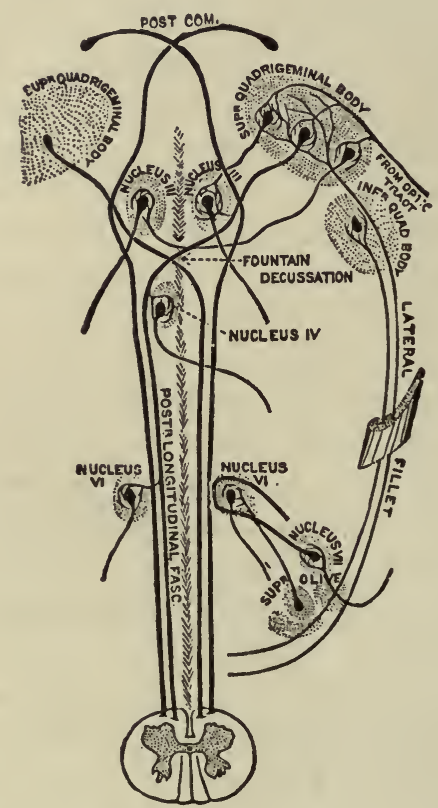

FrG. 383.-Scheme to show the connections of the Posterior Longitudinal Bundle. (Cunningham, modified from Held.)

fillet, including $a$, the larger mesial fillet, a sensory tract previously described arising in the gracile and cuneate nuclei, and $b$, the lateral fillet, an auditory tract, see figure $4 \mathrm{I} 4$. The second, the posterior longitudinal bundle, is situated on each side of the mid-line, just internal to the mesial fillet. Some of the connections of the posterior longitudinal bundle are shown in figure 383 .

4. In the upper part of the pons there is a mass of gray matter containing pigment, the locus ceruleus; and in the back part a second mass of gray matter, the superior olive, which is connected with the auditory conduction path, figure 4I4. 


\section{THE CEREBELLUM.}

The cerebellum is a large division of the brain, located just beneath the cerebrum and behind the medulla and pons. It is connected with the rest of the brain by three peduncles on each side: the superior, the middle, and the inferior peduncle, figure 384 .

The cerebellum is composed of white and gray matter, the latter being external as in the cerebrum, and, like it, infolded so that a larger area may be contained in a given space. The convolutions of the gray matter, however,

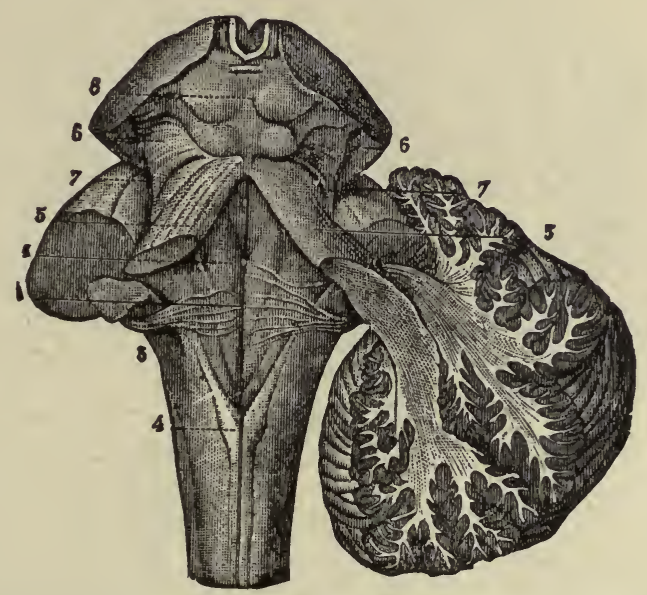

FIG. 384.-Cerebellum in Section and Fourth Ventricle, with the Neighboring Parts. I, Median groove of fourth ventricle, ending below in the calamus scriptorius, with the longitudinal eminences formed by the fasciculiteretes, one on each side; 2 , the same groove, at the place where the white streaks of the auditory nerve emerge from it to cross the floor of the ventricle; 3 , inferior crus or peduncle of the cerebellum, formed by the restiform body; 4, posterior pyramid; above this is the calamus scriptorius; 5 , superior crus of cerebellum, or processus e cerebello ad cerebrum (or ad testes); 6,6 , fillet to the side of the crura cerebri; 7,7 , lateral grooves of the crura cerebri; 8, corpora quadrigemina. (Fron) Sappey, after Hirschfeld and Leveillé.)

are arranged after a different pattern, as shown in figure 385 . Besides the gray substance on the surface, there is, near the center of the white substance of each hemisphere, a small capsule of gray matter called the corpus dentatum, figure 385 , resembling very closely the corpus dentatum of the olivary body of the medulla oblongata.

If a section be taken through the gray matter of the cerebellum, it will be found to be composed of two layers, an outer, or molecular, and an inner, or granular, layer. Each of these layers contains a large number of peculiarshaped nerve cells and very rich plexuses of nerve fibers. Recent studies of the cortex of the cerebellum by modern methods have revealed a most complex and beautiful arrangement of the parts. 
The General Structure of the Cerebellum.-The molecular layer of the cerebellum contains several peculiar types of nerve cells, of which may be specially mentioned Purkinje's cells and the basket cells. The cells of Purkinje lie along the internal margin of the molecular layer, being, in fact, practically at the boundary of the molecular and granular layers. They measure 40 to $60 \mu$ in diameter, and have large, round nuclei. Each cell gives off an enormous number of branching dendrites, which run up toward the surface of the cerebellum in the shape of a bush.

The cells of Purkinje give off at their deeper surface an axone which runs down into the white matter of the cerebellum. Recurrent collaterals occur.

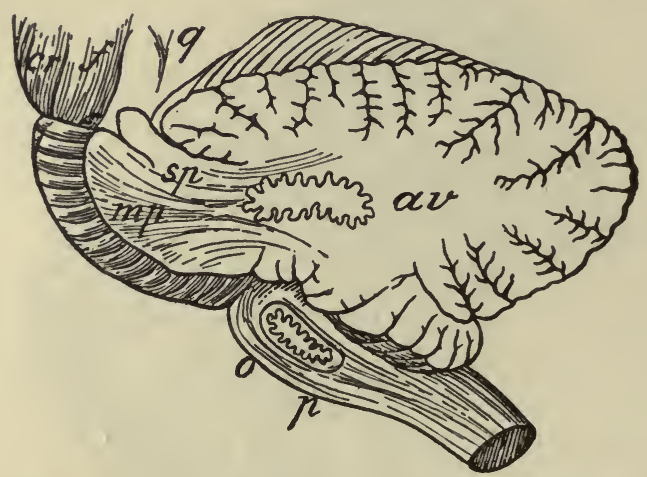

FIG. 385.- Outline Sketch of a Section of the Cerebellum, Showing the Corpus Dentatum. The section has been carried through the left lateral part of the pons, so as to divide the superior peduncle and pass nearly through the middle of the left cerebellar hemisphere. The olivary body has also been divided longitudinally so as to expose in section its corpus dentatum. $\quad c r$, Crus cerebri; $f$, fillet; $q$, corpora quadrigemina; $s p$, superior peduncle of the cerebellum, divided; $m p$, middle peduncle or lateral part of the pons Varolii, with fibers passing from it into the white stem; av, continuation of the white stem radiating toward the arbor vitæ of the folia; 0 , olivary body with its corpus dentatum; $p$, anterior pyramid. (Allen Thomson.)

Lying in the molecular layer, somewhat external to the Purkinje cells, are the cells of the type known as basket cells. These cells have a number of dendrites; they also send out an axone which runs parallel to the surface of the cortex and gives off numerous collaterals in its course that form baskets around the cell bodies of the Purkinje cells, figure $386, Z K$.

The granular layer contains a large number of very small granule-like cells that Golgi was the first to show are really nerve cells. They are only about $5 \mu$ in diameter, and they have a number of short dendrites which end in clubbed extremities. They give off a very slender axis-cylinder process or axone which runs up into the superficial part of the molecular layer and there divides in a $\mathrm{T}$-shaped fashion, the fibers run parallel to the surface of the convolution and pass in between the branches of the cells of Purkinje.

The white substance of the cerebellum consists of nerve fibers, which are 
of three kinds: I. Descending fibers, that are made up of the axis-cylinders of the cells of Purkinje, carrying impulses down from the cerebellar cortex. 2. Ascending fibers, which pass into the granular layer, and there end in a number of very short, finely divided brushes of fibers presenting a mossy appearance, so that these are known as the mossy fibers. These connect with the granular cells of this layer. 3. Ascending fibers, which pass up

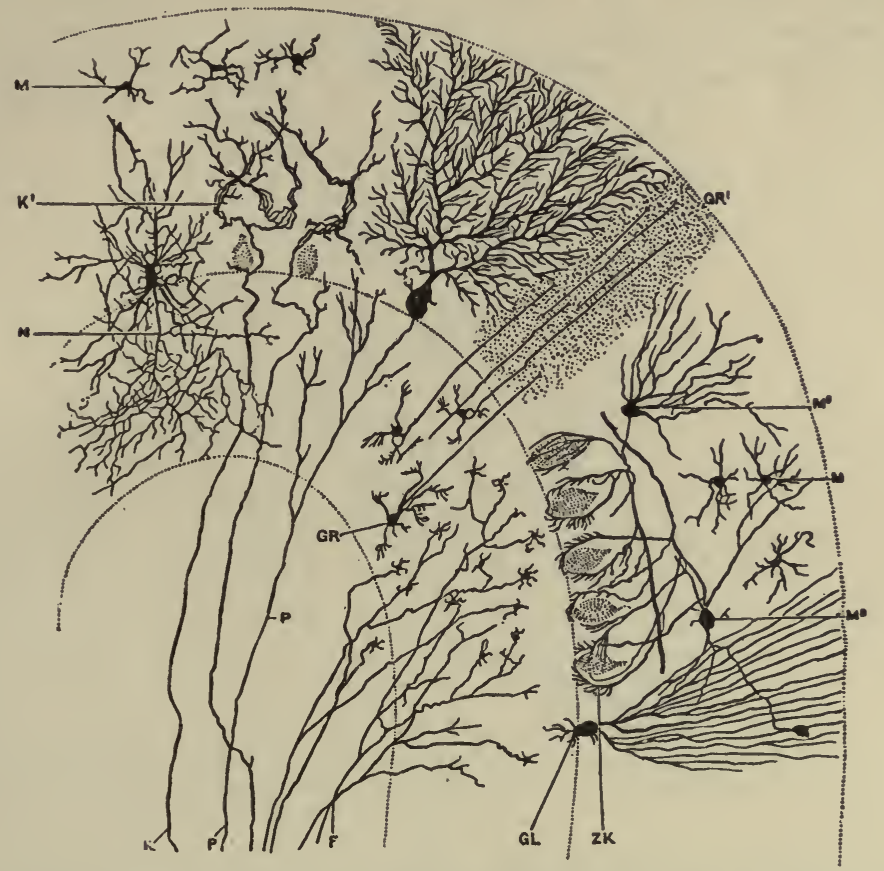

FIG. 386.-Transverse Section Through a Cerebellar Folium (after Kölliker). Treated by the Golgi method. $P$, Axone of cell of Purkinje; $F$, moss fibers; $K$ and $K^{\prime}$, fibers from white core of folium ending in molecular layer in connection with the dendrites of the cells of Purkinje; $M$, simple cell of the molecular layer; $G R$, granule cell; $G R^{1}$, axones of granule cells in molecular layer cut transversely; $M^{\prime}$, basket cells; $Z K$, basket work around the cells of Purkinje; GL, neuroglia cell; $N$, axone of an association cell.

through the granular into the molecular layer and there break up into a fine network which interlaces with the dendritic branches of the cells of Purkinje.

Paths through the Cerebellar Cortex.-It will be seen that the arrangements for the transmission and diffusion of nerve impulses and for the cooperation of different cells are extremely complicated and delicate. It is not possible to indicate absolutely by any schema the course of fibers and the course of impulses through the cerebellum, but approximately it is somewhat like that in the accompanying figure 387 .

Impulses pass up along the ascending fibers to the granular cells by way of the direct cerebellar, the fibers of the gracile and of the cuneatus, from the 
restiform body, etc. These cells, being stimulated, send the impulses by their axis-cylinders to the molecular layer, and through their $\mathrm{T}$-shaped divisions to the dendrites of the cells of Purkinje. Thence an impulse is sent out by the axis-cylinder process of this cell. Other ascending impulses are brought up by those fibers which pass directly to the molecular layer and send their terminals winding around the dendrites of the cells of Purkinje. Probably impulses pass up also through the ascending fibers which affect the basket cells, and, through them and their basket-like terminals, the cells of Purkinje. Purkinje cells send cerebeilar motor fibers to the nucleus dentatus

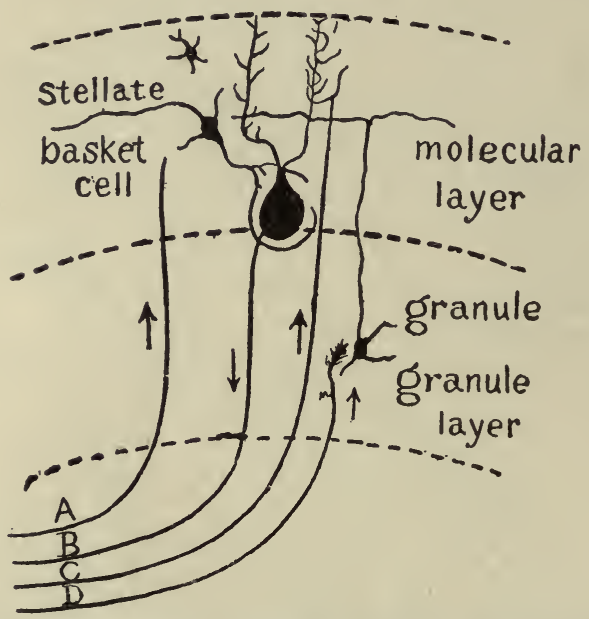

FIG. 387.-A, Afferent fiber to basket (stellate) cell; $B$, neuraxone of Purkinje cell; $\mathrm{C}$, afferent fiber to Purkinje cell; $D$, afferent (mossy) fiber to granule cell.

cerebelli and through the superior peduncles to the red nucleus and the thalamus, and to the ventro-lateral descending tract of the cord, to end about the anterior horn cells.

Functions of the Cerebellum.-With the exception of its middle lobe, the cerebellum is itself insensible to irritation and may be all cut away without eliciting signs of pain (Longet). Its removal or disorganization by disease is also generally unaccompanied by loss or disorder of sensibility; animals from which it is removed can smell, see, hear, and feel pain, to all appearances, as perfectly as before (Flourens; Magendie). It cannot, therefore, be regarded as a principal organ of sensation. Yet if any of its crura be touched, pain is indicated; and, if the restiform tracts of the medulla oblongata be stimulated, the most acute suffering appears to be produced.

These phenomena may properly be ascribed to the activity of the cerebral cortex, since the number of collaterals on the fibers that pass to cerebellar tracts is very great, and impulses arising from their stimulation may reach the sensorium by paths other than through the cerebellum. 
The experiments of Longet and many others agree in supporting the view that no stimulation of the cerebellar cortex leads to localized muscular contractions. In other words, there is no localization in the cerebellar cortex as in the cerebrum, the cerebellum apparently acting as a whole. If the cerebellum be removed, as was done by Flourens and numerous later physiologists, a very profound disturbance in motor functions occurs. With the removal

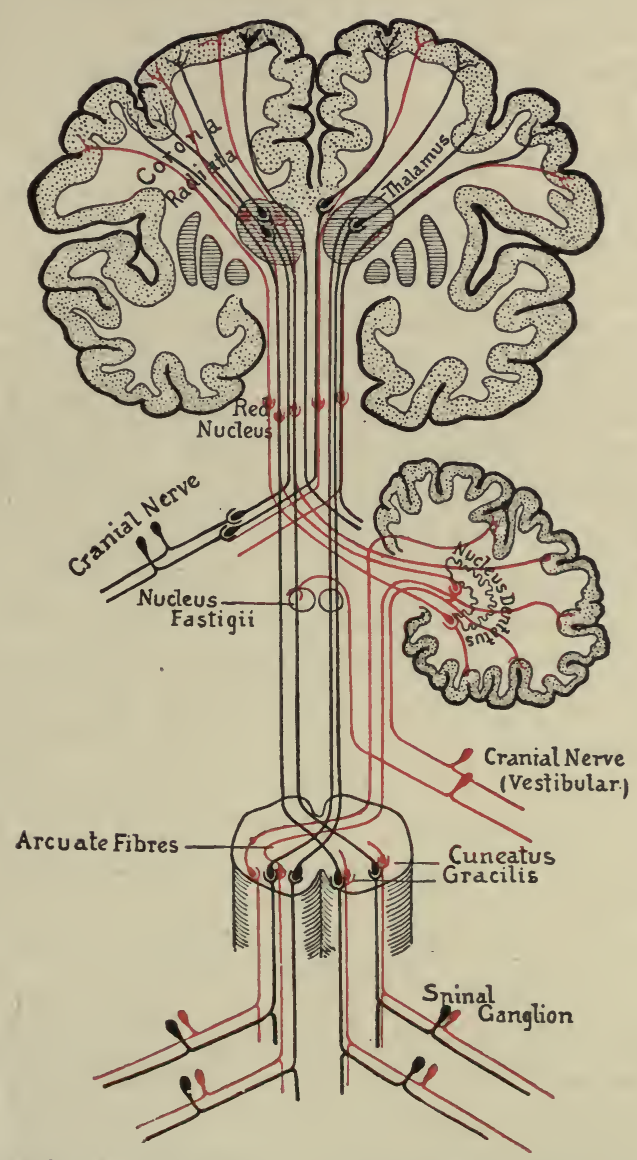

FiG. 388.-Scheme of Principal Ascending Cerebro-spinal (black) and Cerebellar (red) Conduction Paths. (Modified from Hardesty in Morris' Anatomy.)

of the superficial layers of the cerebellum, in pigeons particularly, there is increasing feebleness and lack of harmony of the muscles concerned in locomotion. When the entire organ is cut away in pigeons they lose the power of walking, flying, and of standing in the usual erect way. Their power of preserving equilibrium is lost, the most characteristic feature. Birds do not remain in a state of stupor, but attempt to carry out the usual muscular activi- 
ties. If a pigeon is laid on its back it cannot recover its erect position, though it make motions to do so. If set on its feet it will fall to one side or the other, and is not able to hold its head in the customary position. The endeavors of the animal to maintain its balance are insecure and uncertain, resembling the lack of muscular control of a drunken man.

Such an animal does not lose the power of perceiving sensations, nor of making voluntary efforts, as it will endeavor to avoid the blow that is threatened.

The experiments afford the same results when repeated on all classes of animals; and from them and the others before referred to, Flourens inferred that the cerebellum belongs neither to the sensory nor the intellectual apparatus; and that it is not the source of voluntary movements, although it belongs to the motor apparatus, but is the organ for the co-ordination of the voluntary movements, or for the excitement of the combined action of muscles.

Such evidence as can be obtained from cases of diseases of this organ confirms the view taken by Flourens; and, on the whole, it gains support from comparative anatomy-animals whose natural movements require most frequent and exact combinations of muscular contractions being those whose cerebella are most developed in proportion to the spinal cord.

We must remember, too, that the cerebellum is connected with the posterior columns of the cord through the cuneate and gracile nuclei as well as with the direct cerebellar tract, all of which probably convey to the middle lobe muscular sensations. It is also connected with the auditory nerves and bulb by the internal and external arcuate fibers; and with the tegmentum through the red nuclei. Its connection with the efferent tracts from the different cerebral lobes through the pons is also highly important. Movements of the eyes also occur on direct stimulation of the middle lobe. It seems, therefore, to be connected in some way with all of the chief sensory impulses which have to do with the maintenance of the equilibrium, and is generally included in the nervous apparatus which is supposed to govern this function of our bodies.

Foville supposed that the cerebellum is the organ of muscular sense, i.e., the organ by which the mind acquires that knowledge of the actual state and position of the muscles which is essential to the exercise of the will upon them; and it must be admitted that all the facts just referred to are as well explained on this hypothesis as on that of the cerebellum being the organ for combining movements. A harmonious combination of muscular actions must depend as much on the capability of appreciating the condition of the muscles with regard to their tension, and to the force with which they are contracting, as on the power which any special nerve center may possess of exciting them to contraction. And it is because the power of such harmonious movement would be equally lost, whether the injury to the cerebellum involved injury to the seat of muscular sense or to the center for combining muscular actions, that experiments on the subject afford no proof in one direction more than the other. 
Forced Movements.-The influence of each half of the cerebellum is directed to muscles on the opposite side of the body; and it would appear that, for the right ordering of movements, the action of its two halves must be always mutually balanced and adjusted. For if one of its crura, or if the pons on either side of the middle line, be divided, so as to cut off from the medulla oblongata and spinal cord the influence of one of the hemispheres of the cerebellum, strangely disordered movements ensue-forced movements. The animals fall down on the side opposite to that on which the crus cerebelli has been divided, and then roll over continuously and repeatedly; the rotation being always round the long axis of their bodies, and generally from the side on which the injury has been inflicted. The rotations sometimes take place with much rapidity; as often, according to Magendie, as sixty times in a minute, and may last for several days. Similar movements have been observed in men; as by Serres in a man in whom there was apoplectic effusion in the right crus cerebelli; and by Belhomme in a woman in whom an exostosis pressed on the left crus. They may, perhaps, be explained by assuming that the division or injury of the crus cerebelli produces paralysis or imperfect and disorderly movements of the muscles of the opposite side of the body. Such movements cease when the other crus cerebelli is divided; but probably only because the paralysis of the body is thus made almost complete. Other varieties of forced movements have been observed, especially those named "circus movement," when the animal operated upon moves round and round in a circle; and again those in which the animal turns over and over in a series of somersaults. Nearly all these movements may also result on section of one or other of the following parts: viz., medulla, pons, cerebellum, corpora quadrigemina, corpora striata, optic thalami, and even, it is said, of the cerebral hemispheres. But these structures are parts that involve tracts used in the co-ordination of complex muscular movements.

\section{THE MID-BRAIN.}

The mid-brain includes the crura cerebri and the corpora quadrigemina.

The Peduncles of the Cerebrum, or Crus Cerebri.-The crura diverge from the anterior edge of the pons Varolii and pass upward on either side toward the cerebral hemispheres. At their anterior termination each of them appears to have upon its dorsal surface, to the inner and outer sides, respectively, two large masses of gray matter which have been already spoken of, viz., the optic thalamus and the corpus striatum. The crus is made up of two principal parts. The crusta or pes is in the ventral position, and the tegmentum in the dorsal position. The two are separated by the substantia nigra.

The pes consists of longitudinal fibers which pass anteriorly between the optic thalamus and the posterior part (lenticular nucleus) of the corpus stria- 
tum. In this situation the fibers form a compact mass. This constitutes the internal capsule, and that portion of it which forms the angle at which the fibers are bent is called the genu of the capsule. The internal capsule spreads out dorsally in the corona radiata. The fibers thus have the form of a fan bent upon itself as they rise to pass into the cerebral hemisphere. The fibers of the internal capsule are connected with different districts of the cerebral cortex. Briefly the connections are, $a$, the fronto-pontine fibers are in the anterior limb of the capsule; $b$, the pyramidal fibers in the genu and the anterior part of the posterior limb; $c$, the temporo-pontine fibers in the

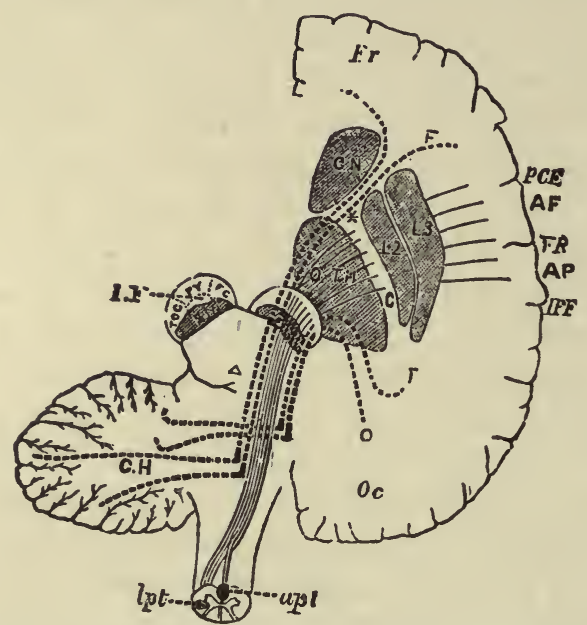

FIG. 389.-Diagram of the Motor Tract as Shown in a Diagrammatic Horizontal Section through the Cerebral Hemispheres, Crura, Pons, and Medulla. Fr., Frontal lobe; $O c$., occipital lobe; $A F$., ascending frontal, $A P$., ascending parietal convolutions; $P C F$., pre-central fissure, in front of the ascending frontal convolution; $F R$., fissure of Rolando; $I P F$., inter-parietal fissure, a section of crus is lettered on the left side; $S N$., substantia nigra; $P y$., pyramidal motor fiber which on the right is shown as continuous lines converging to pass through the posterior limb of $I C$., internal capsule (the knee or elbow of which is shown thus), upward into the hemisphere and downward through the pons to cross the medulla in the anterior pyramids. (Gowers.)

posterior part of the posterior limb. Fibers connecting the optic thalami and corpora striata with the cerebral cortex also run in the capsule. The pes, internal capsule, and the corona radiata form the great sensory and motor highway to and from the cerebral cortex.

The tegmentum is the continuation anteriorly of the reticular formation of the medulla. It ends for the most part in the neighborhood of the optic thalamus and in the parts beneath. The tegmentum of either side is supposed to be concerned chiefly with afferent impulses. It is made up to a very considerable extent of collections of gray matter, the most important of which are the substantia nigra, separating the pes and tegmentum, and the nucleus ruber, which is a rounded mass situated near the aqueduct of Sylvius. The 
latter serves as a way-station in the cerebello-cerebral conduction paths and also has important connections with the spinal cord. The locus niger extends back as far as the posterior corpus quadrigeminum. Posteriorly, the tegmentum is chiefly reticular in structure.

Corpora Quadrigemina.-There are two corpora quadrigemina on each side, the superior and inferior. They form prominences on the dorsal surface of the mid-brain, dorsal to the aqueduct of Sylvius. The inferior corpora quadrigemina receive through the lateral fillet fibers from the cochlear division of the eighth nerve, figure 4I4. They are closely associated with the median corpora geniculata, and, like these, give origin to fibers which continue the auditory conduction path upward to the auditory center. The superior corpus quadrigeminum receives fibers from the optic nerve, the mesial fillet, and also from the occipital cortex, as will be more fully described later. It is closely associated with the external corpus geniculatum. The corpora geniculata also form reflex centers for the eye muscles in the reflexes that result in the adjustments of the eye to vision at different distances. The nervous connections of these nuclei are discussed in presenting the optic tract and the auditory tracts.

\section{THE FORE-BRAIN.}

The fore-brain or prosencephalon consists of two divisons, the thalamencephalon or thalami, and the telencephalon, or corpora geniculata, corpora striata, and the cerebral hemispheres. These subordinate divisions will be discussed in order.

The Thalami.-The optic thalami are oval in shape, and rest upon the crura cerebri. They form part of the floor of the lateral ventricles and their inner sides bound the third ventricle. They are connected by a transverse tract, the middle commissure.

Each thalamus has several collections of gray matter, forming somewhat indistinctly defined masses separated by white fibers. These masses of gray matter are known as the nuclei of the thalamus, which are six in number. They are called the anterior nucleus, the median nucleus, the lateral nucleus, the ventral nucleus, the pulvinar, and the posterior nucleus. The corpora geniculata are also closely associated with the optic thalamus. The anterior nucleus is composed of large nerve cells which receive the terminations of axones of cells of the corpora mammillaria at the base of the brain (bundle of Vicq d'Azyr). There they meet the fibers of the fornix, which establish a relation between this tubercle of the thalamus and the hippocampal convolutions. The median nucleus is connected by its axones with the cortex of the island of Reil and the second and third frontal convolutions. The lateral nucleus is quite large and lies against the internal capsule, into which it sends fibers. It is connected with the central convolutions of the cortex. 
The ventral nucleus lies beneath the preceding; it is relatively small. It is connected with the cortex of the frontal lobe and with the operculum, the central convolutions, and the supramarginal gyrus. The fifth nucleus, known as the pulvinar, forms the posterior tip of the thalamus, and is connected with the optic tract, see figure 406 . The posterior nucleus, lying just below the pulvinar, is a small mass and is connected with the cortex of the interior parietal convolution. The cells of the thalamus are thus seen to be connected with a large area of the cerebral cortex. The axones spread out in a great fan in the corona radiata, the thalamus sending more fibers to the cortex than are received from it.

The collections of nerve cells in the thalamus are shown by anatomical investigations and by methods of physiological degeneration to be on the pathway of ascending or afferent nerve tracts. Large masses of sensory fibers pass through the thalami, the majority of which form synapses about the nerve cells in the thalamus. Even in those cases where there is no distinct ending of the nerve fiber, collaterals are given off which establish physiological connection with the nuclei.

The thalamus is thus closely connected with large areas of the cortex. It must at least form an important relay station in all those activities which involve the conscious perception of sensory stimuli wherever they may arise. Flechsig even claims that in the thalamus there are definite points of sensory localization corresponding to every sensory point in the periphery of the body (including the special senses). The thalamus also receives fibers from various parts of the cerebral cortex, thus establishing a double relation with this region.

Owing to the difficulty of those operations which establish isolation of the thalamus, it is not clear to what extent reflex actions may take place through its nuclei. It is probable, however, that extensive co-ordinations of afferent impulses may be mediated by the nuclei of the thalami. Such activities as walking, riding, writing, speaking, etc., are possibly reflexes co-ordinated through the thalami, perhaps with the assistance of the medulla in the case of walking.

Corpora Geniculata.-The corpora geniculata form prominences on each side of the peduncles of the cerebrum just ventral to the anterior corpora quadrigemina. There are two on each side, the external or outer, and median or inner. The external corpus geniculatum is at the side of the crus and appears to be a swelling on the lateral division of the optic tract and actually receives terminations of the optic fibers, thus constituting a way-station in the optic conduction paths. Similarly the median appears to be the termination of the median division of the optic tract, from which it receives some fibers, figure 406 , but it is more intimately connected with the auditory tracts, forming a way-station between the lateral fillet and the auditory cortical center, figure $4 \mathrm{r} 4$. 
Corpora Striata.-The corpora striata are situated in front and to the outside of the thalami, partly within and partly without the lateral ventricles.

Each corpus striatum consists of two parts: An intraventricular portion, the caudate nucleus, which is conical in shape, with the base of the cone forward (this consists chiefly of gray matter), and an extraventricular portion, the lenticular nucleus, separated from the other portion by the internal capsule. The lenticular nucleus is shown in a horizontal section of the hemisphere to consist of three parts, the two internal called globus pallidus major and minor, and the outer called the putamen.

The cells of the corpora striata are somewhat evenly distributed, and not grouped in nuclei. Their axones pass for the most part into the internal capsule. It is doubtful if these ganglia have any direct anatomical relations with the cortex of the brain, but they are intimately connected by fibers to and from the thalami, and are connected with the substantia nigra (Flechsig). These nuclei are developed from the walls of the embryonic brain tube and are probably therefore homologous with the areas of the cortex. Their lesion is said to be accompanied by disturbance in muscular co-ordination. Lesion of the left lenticular nucleus is said to cause some disturbance in the power of speech, though this has not been observed in the case of the right nucleus. Lesions of the corpora striata produce disturbances in heat regulation, causing a rise of body temperature, the rise amounting to as much as $2^{\circ}$ or $3^{\circ}$ C. in the rabbit. The rise of temperature in man after lesion of the corpus striatum on one side is said to be chiefly on the opposite side of the body (Kaiser).

\section{THE CEREBRUM.}

That portion of the brain which is concerned with all intellectual functions is the cerebrum or, more strictly speaking, the cerebral cortex. The cerebral cortex is the seat of those activities which we describe as intelligenceincluding states of consciousness, acts of idea formation and volition, and the phenomenon of memory.

The cerebrum includes the cerebral cortex, the mass of fibers connecting it with lower portions of the brain, the basal nuclei represented by the corpora striata, the thalami, etc. The structure and function of these basal nuclei have already been given briefly, so we may turn our attention now to the cerebral cortex.

Structure of the Cerebral Cortex.-The cerebral cortex forms a large part of the mass of the cerebrum, in fact of the whole brain. Its superficial appearance presents a series of ridges and folds, the gyri and sulci. For general convenience anatomists have divided the cerebral cortex into five lobes: the frontal, that portion in front of the fissure of Rolando extending down 
to the Sylvian fissure; the parietal, extending from the Sylvian fissure to the parieto-occipital fissure, and bounded below by the Sylvian fissure; the temporal lobe, just ventral to the parietal; the central lobe, or island of

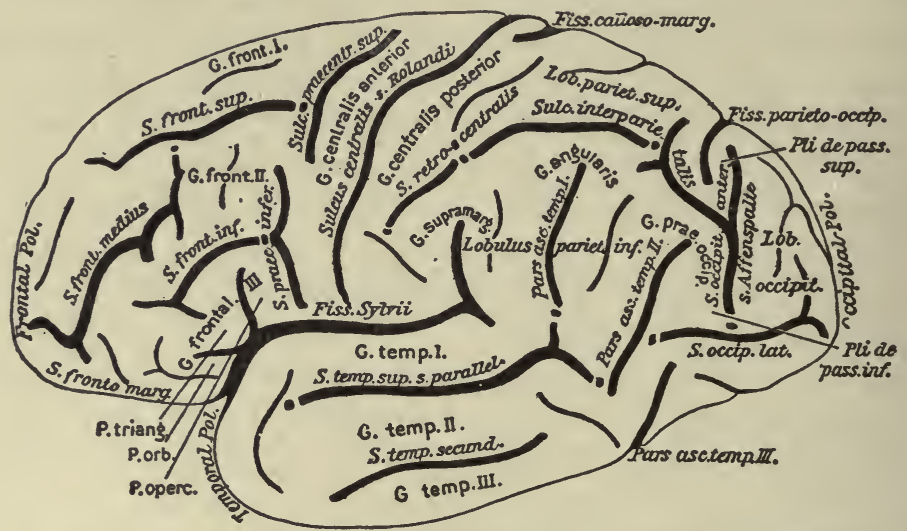

Frg. 390.-Left Hemisphere, from Without. (After Eberstaller.)

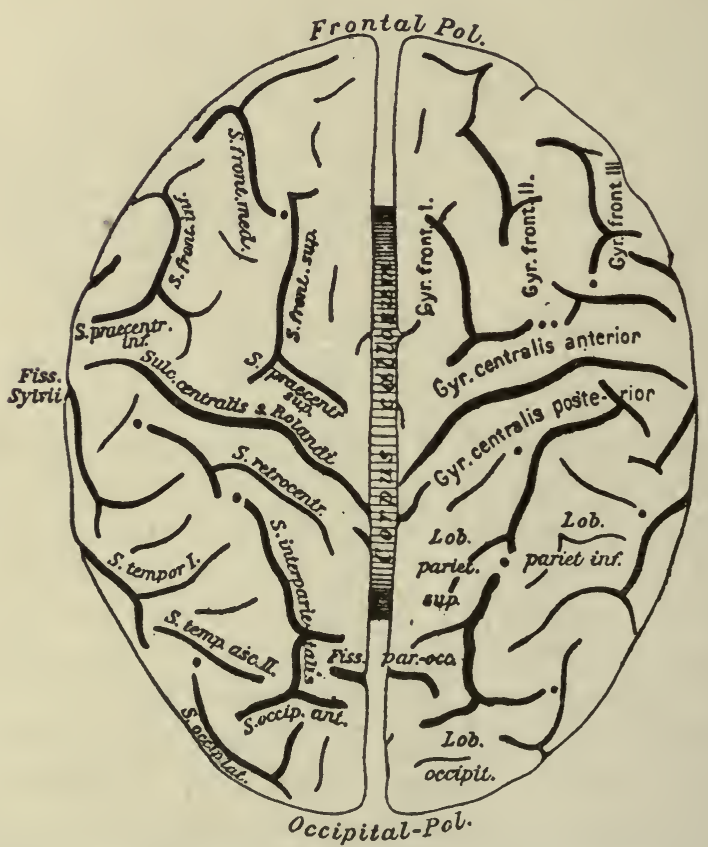

FIG. 391.-The Cerebrum, from Above. (After Eberstaller.)

Reil; and the occipital lobe, which includes the posterior portion of the cortex behind the parieto-occipital fissure. And, finally, the olfactory and limbic lobes together make up the olfactory division of the brain. For the 
detailed arrangements of the cortical gyri and sulci the reader is referred to figures 390,392 and to text-books of anatomy.

In a transverse section of the cerebral cortex there is shown an external gray layer chiefly composed of nerve cells and an internal white portion of nerve fibers. The folding of the cortex into convolutions increases the total mass of gray matter enormously.

The gray or cellular external part of the cerebral cortex has an average thickness of about $3 \mathrm{~mm}$; being thin in the occipital and frontal region, $2 \mathrm{~mm}$., and thick in the precentral, $4 \mathrm{~mm}$., and postcentral convolutions.

Several types of nerve cells have been described as present in the cortex, the exact type and relative proportion varying somewhat in different regions.

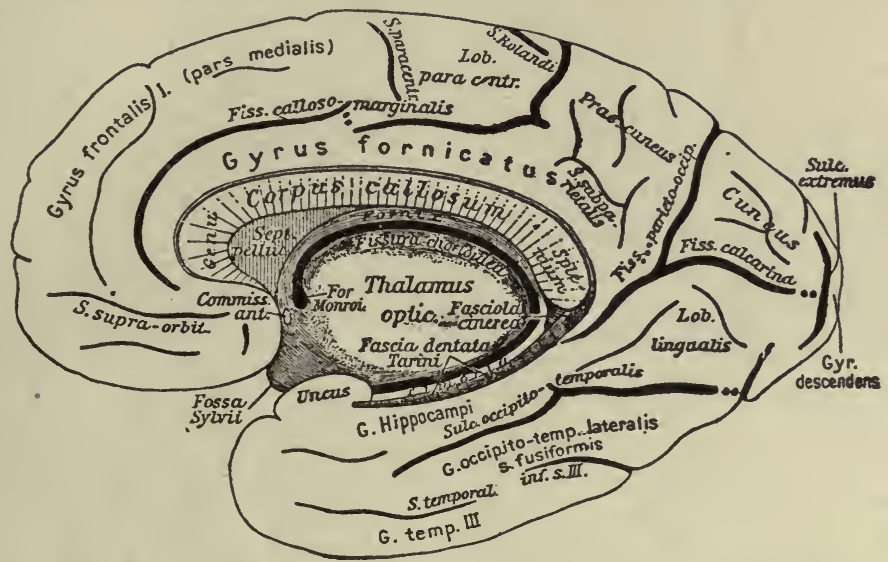

FIG. 392.-Right Hemisphere, from Within. (After Eberstaller.)

The typical characteristic cell, however, is the pyramidal cell. The pyramidal cell, as its name implies, has a pear-shaped cell body with numerous protoplasmic processes. The apex of the cell is directed toward the surface of the cortex, and supports numerous branches which extend out into the adjacent territory, bringing it into contact with a relatively large number of nerve cells. These processes are dendritic in character. The base of the pyramidal cell always has a single axis-cylinder process which is directed down into the white matter, and which in some cases ultimately finds its course through the corona radiata into the pyramids below. The axiscylinder processes give off collaterals both in the immediate neighborhood of the cell and somewhat deeper along its course.

In the superficial layer of the cortex there is a peculiar type of small cell, first described by Cajal. Most of these cells are fusiform in shape, with the long axis parallel to the surface of the convolution. They give off usually two axones which run along parallel to the surface and send down numerous fine collaterals at right angles. Another form of Cajal cell, triangular or 
quadrangular in shape, is also seen. Both forms have, as a rule, more than one axone. Their collaterals pass in a horizontal direction, forming a fine band of fibers, known as tangential fibers. A third type of cell is the fusiform or polymorphous. Some of these are strictly fusiform in shape and lie with their axes parallel to the surface of the convolution. They give off protoplasmic processes which pass down toward the white matter, some of

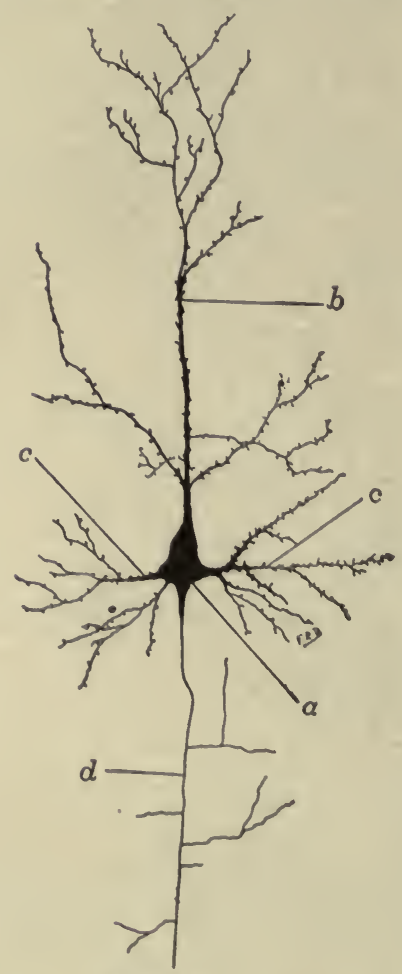

Fic. 393.

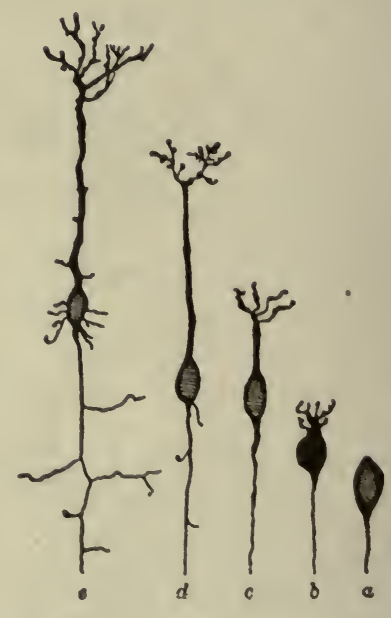

Fig. 394 .

FrG. 393.-Typical Pyramidal Cell from the Human Cortex. $a$, Cell body; $b$, main dendrites with gemmules; $c$, lateral dendrites; $d$, axone and collaterals. Only a small part of the axone is shown. (Bailey.)

Fig. 394. - Showing the Stages in the Development of a Pyramidal Cell. (Ramón y Cajal.)

them turning to run in a horizontal direction. The fusiform and polymorphous cells are grouped in the same layer.

Besides these cells we find scattered through the cortex a considerable number of the neuroglia cells. The character and position of these are shown in figure 395 .

The general arrangement of the layers of the cortex is described very differently by the various authors. It is not uniform in the different parts of the brain. The simplest and most representative type, however, of the arrange- 
ment is that in which the cortex is divided into four layers. The outermost, or superficial, known as the molecular layer, contains relatively few cells. It is composed of neuroglia tissue, embedded in which are a number of cells of the Cajal types, which have just been described. There are also in this layer many neuroglia cells. In the superficial part of the layer of some areas

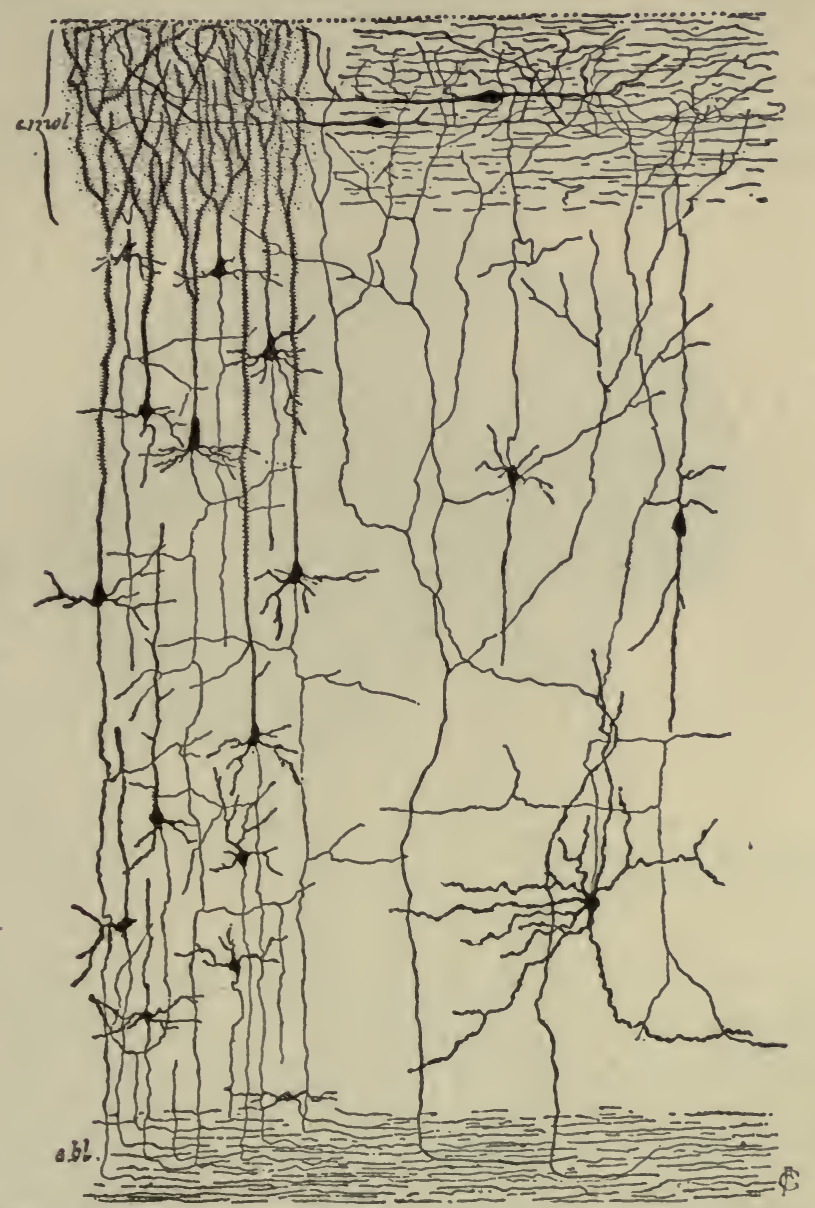

Fig. 395. - The Principal Constituent Elements of the Gray Cortical Layer of the Anterior Cerebrum. (After Ramón y Cajal.)

of the cortex are many tangential fibers. The second layer is composed of small pyramidal cells. In parts of the brain there are here interposed what are known as the vertical fusiform cells. The third layer is composed of large pyramidal cells, in which, however, one also sees many small pyramidal cells. The fourth layer is composed of the fusiform and polymorphous cells, beneath which is the white substance. This arrangement is shown 
in the accompanying figures, 395 and 397 . The gray matter of the brain contains, however, not only these layers and cells, but an infinitely rich mass of fibers, which can be shown to have a certain definite arrangement. Some of the fibers are vertical, passing directly up to the most superficial layers of cells; others have a horizontal direction, dividing the gray matter into different layers. These layers of fibers have received different names. A typical arrangement is shown in figure 398 . The most conspicuous fibers are those of certain large triangular or pyramidal cells.

The efferent or axone fibers from the cerebral cortex may be divided into three classes: I, the projection fibers, which descend through the corona

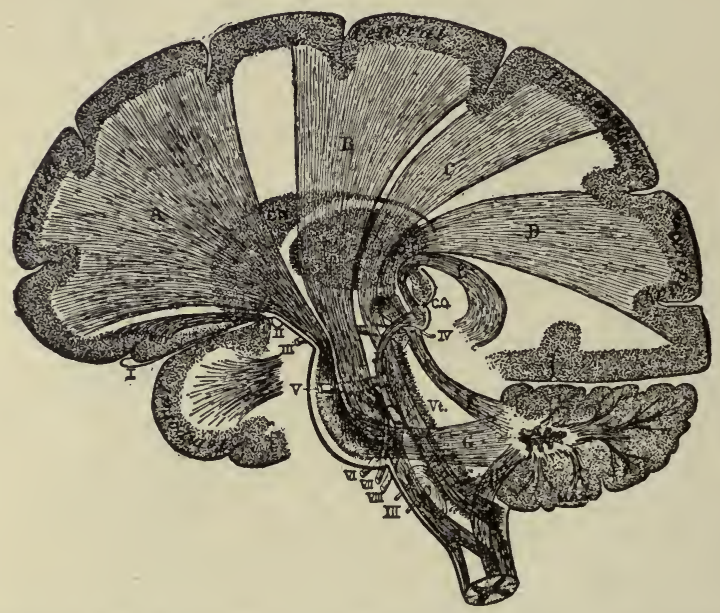

FIG. 396.-Scheme of Descending Conduction Pathways from the Cerebrum to Lower Nerve Centers.

radiata and internal capsule, to end in lower centers; 2, the commissural fibers, which cross to the opposite cerebral hemisphere, chiefly through the corpus callosum; 3, the association fibers, which pass in bundles beneath the cortex, to end in other regions of the same hemisphere.

It is by means of projection fibers and collaterals that associations are made with nerve cells in the thalamus, tegmentum, and pons, and through the latter region with tracts going to the cerebellum.

Weight of the Brain and Cord.-The brain of an adult man weighs from 48 to 50 oz., about 1,550 grams, or about 2 per cent. of the body weight. It exceeds in absolute weight that of all the lower animals except the elephant and whale. Its weight, relatively to that of the body, is exceeded only by that of a few small birds, and some of the smaller monkeys.

In the new-born child the brain (weighing ro to $4 \mathrm{oz}$.) is about ro per cent. of the total body weight. At the age of 7 years the weight of the brain already averages $40 \mathrm{oz}$., and about $\mathrm{I} 4$ years the brain not infrequently 


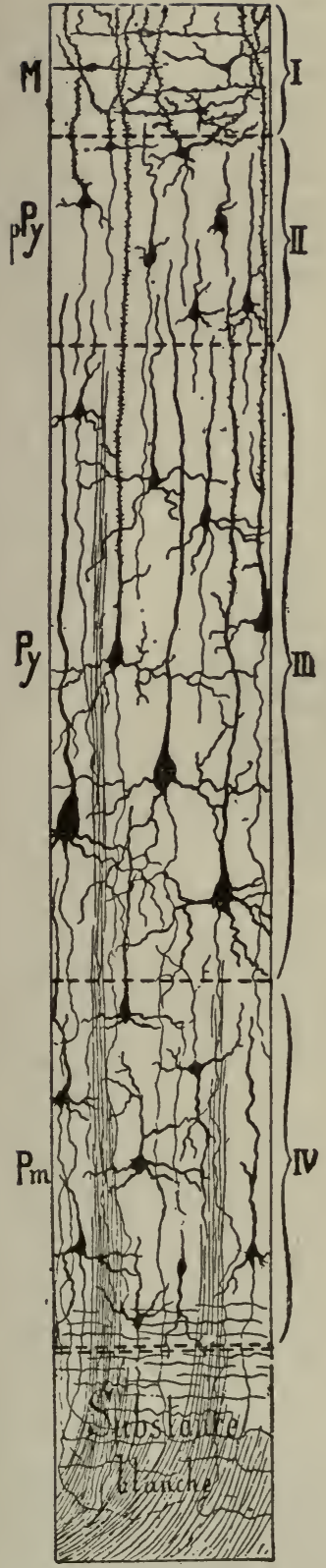

FIG. 397.

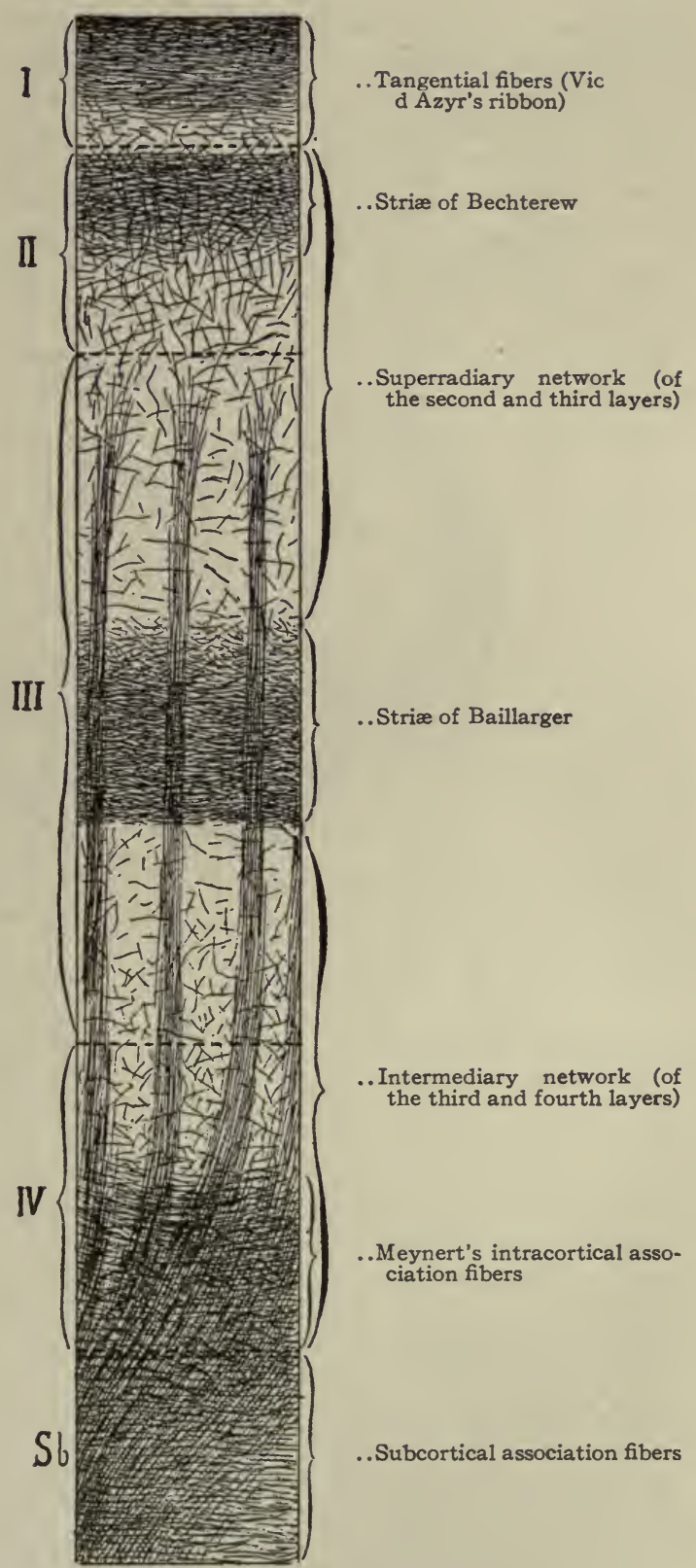

FIG. 398.

FIG. 397. - Schematic Diagram of the Different Layers of the Cerebral Cortex. (After Ramón y Cajal.) I, II, III, and IV, Layers of cortical cells. $M$, Molecular layer; $p P y$, layer of small pyramidal cells; $g P y$, layer of large pyramidal cells; $P m$, layer of polymorphous cells.

FIG. 398.- Schematic Diagram Showing the Arrangement of the Nerve Fibers in the Cerebral Cortex. The dotted lines separate the four cellular layers of Cajal. $S b$, White substance. 
reaches the weight of $48 \mathrm{oz}$. Beyond the age of forty years the weight slowly but steadily declines at the rate of about $\mathrm{I}$ oz. in ro years.

The average weight of the female brain is less than the male; and this difference persists from birth throughout life. The difference amounts to about $5 \mathrm{oz}$. Thus the average weight of an adult woman's brain is about $44 \mathrm{oz}$.

The brains of idiots are generally much below the average, some weighing less than $16 \mathrm{oz}$. Still the facts at present collected do not warrant more than a very general statement, to which there are numerous exceptions, that the brain weight corresponds to some extent with the degree of intelligence.

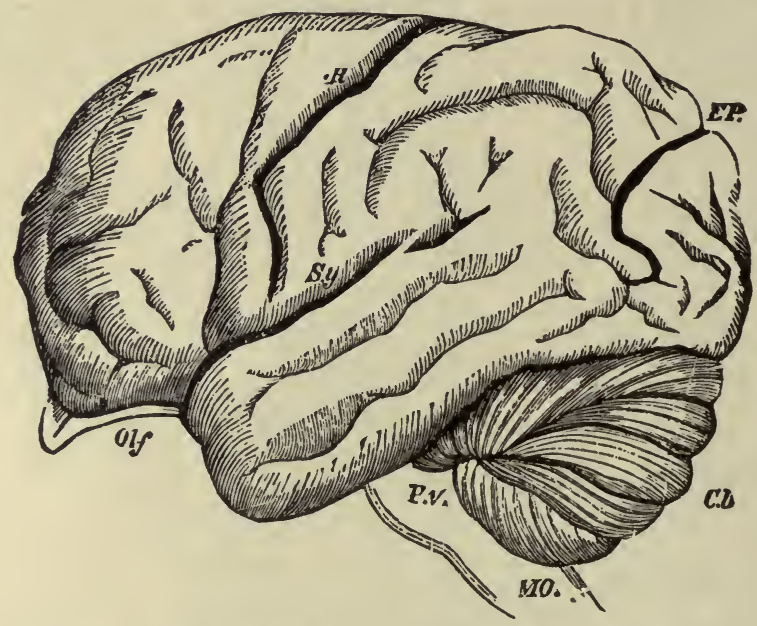

Fig. 399.-Brain of the Orang, $\frac{2}{3}$ Natural Size, Showing the Arrangement of the Convolutions. $S y$, Fissure of Sylvius; $R$, fissure of Rolando; $E P$, external perpendicular fissure; $O l f$, olfactory lobe; $C b$, cerebellum; $P V$, pons Varolii; $M O$, medulla oblongata. As contrasted with the human brain, the frontal lobe is short and small relatively, the fissure of Sylvius is oblique, the temporo-sphenoidal lobe very prominent, and the external perpendicular fissure very well marked. (Gratiolet.)

There can be little doubt that the complexity and depth of the convolutions, which indicate the area of the gray matter of the cortex, correspond with the degree of intelligence.

The spinal cord of man weighs from I to $I \frac{1}{2} \mathrm{Oz}$; its weight relatively to the brain is about $I: 40$ in the adult. As we descend the animal scale, this ratio constantly increases till in the mouse it is $I: 4$. In cold-blooded animals the relation is reversed, the spinal cord is the heavier. In the newt, I:I05; and in the lamprey, $x: 133$.

The most distinctive points in the human brain, as contrasted with that of apes, are: I. The much greater size and weight of the whole brain. The brain of a full-grown gorilla weighs only about I 5 oz. ( $450 \mathrm{grms}$.), which is less than one-third of the weight of the human adult male brain, and barely exceeds that of the human infant at birth. 2. The much greater complexity of the convolutions, especially the existence in the human brain of tertiary convolutions in the sides of the fissures. 3. The greater relative size and complexity and the blunted quadrangular contour of the frontal lobes in man, which are relatively broader, longer, and higher than in apes. In apes the frontal lobes project keel-like (rostrum) between the olfactory bulbs. 4. The much greater 
prominence of the temporo-sphenoidal lobes in apes. 5. The fissure of Sylvius is nearly horizontal in man, while in apes it slants considerably upward. 6. The distinctness of the fissure of Rolando.

Most of the above points are shown in the accompanying figure of the brain of the orang.

\section{GENERAL FUNCTIONS OF THE CEREBRUM.}

Evidence regarding the physiology of the cerebral hemispheres has been obtained, as in the case of other parts of the nervous system, from the study of anatomy, from pathology, and from experiments on the lower animals. The chief evidences regarding the functions of the cerebral hemispheres derived from these various sources are briefly these: I. Any severe injury of them, such as a general concussion, or sudden pressure as by apoplexy, may instantly deprive a man of all power of manifesting externally any mental faculty. 2. In the same general proportion as the higher mental faculties are developed in the vertebrates and especially in man at different ages, as well as in different individuals, the greater is the development of the cerebral hemispheres in comparison with the rest of the cerebro-spinal system. 3. No other part of the nervous system bears a corresponding proportion to the development of the mental faculties. 4. Congenital and other morbid defects of the cerebral hemisphere are, in general, accompanied by corresponding deficiency in the range or power of the intellectual faculties and the higher instincts. 5. Removal of the cerebral hemispheres in the lower animals produces effects corresponding with what might be anticipated from the foregoing facts.

Effects of the Removal of the Cerebrum.-The removal of the cerebrum in the lower animals appears to reduce them to the condition of a mechanism without spontaneity.

In the case of the frog, when the cerebral lobes have been removed, the animal appears similarly deprived of all power of spontaneous movement. But it sits up in a natural attitude and breathes quietly. When pricked it jumps away. When thrown into the water it swims. When placed upon a board it remains motionless, although, if the board be gradually tilted over till the frog is on the point of losing his balance, he will crawl up till he regains his equilibrium and comes to be perched quite on the edge of the board.

If the frog be turned on his back, he regains his normal position. If his back is stroked gently he will utter the usual croaking sound. These activities are carried on by the normal frog. There is one striking difference, however, between the brainless frog and the normal: the former, if placed in a position and left undisturbed, will. remain without moving for an indefinite time. It has apparently lost the power to initiate movements. Presumably any memory impressions or effects of former experiences have been lost. Even the more elemental stimuli, which come from tissue 
hunger and thirst, apparently do not affect the brainless frog. In other words, the operation has reduced the animal to the condition of an automaton capable of carrying on complex activities, but only upon receiving some definite stimulus. This condition contrasts sharply with that resulting from the removal of the entire brain, leaving only the spinal cord. In this spinal cord frog only the simpler reflex actions can take place. The frog does not breathe. It lies flat on the table instead of sitting up. When thrown into a vessel of water it sinks to the bottom. When its legs are pinched it kicks out, but does not leap away as in the normal.

If the cerebrum of the frog be removed, taking special care not to interfere with the optic nerves or the thalami, then it acts somewhat differently. Whereas with the entire cerebrum removed it makes no effort to take food, in this instance it is said to attempt to catch flies or other insects, and will show other signs of spontaneous activity. It will avoid an object and shows signs of responding to visual sensations, such as the attempt to feed just mentioned.

The cerebral lobes of the frog, however, are very low in the scale of development as compared with other vertebrates. The cortex is a simple layer of rather small cells, and the total volume of the cortex as compared with other portions of the brain is small.

The case of the pigeon, which represents a higher animal in the scale, has been extensively studied by Flourens and others. They have shown that when the cerebrum is carefully removed, leaving the basal nuclei undisturbed, and the animal has recovered from the immediate effects of the shock, it is able to carry on many co-ordinate activities. In the first place it can stand or perch without difficulty. If placed on its back it immediately regains its equilibrium. If tossed in the air it flies until it comes in contact with a firm support. If disturbed on its perch it will walk away, showing the power to co-ordinate not only wing muscles, but the leg muscles. If left undisturbed, such a pigeon will occasionally make motions, i.e., open its eyes, move its head, preen its feathers, or even take a step or two. It spends most of its time, however, sitting quietly as though asleep. If aroused, the animal shows little or no signs of excitement or fright.

After several months such pigeons are usually said to increase the motions of spontaneity or take short flights, avoiding obstacles in the way and alighting definitely on the perch. They will pick around among food for definite articles, apparently attempting to select the food. Early after the operation the pigeon will pick at objects indiscriminately, but does not take food unless it is placed in the mouth.

Apparently the main effect produced here is to diminish the complexity and efficiency of those activities which we call spontaneous. The surprising thing is that there is as little disturbance among the motor functions as is found.

In mammals it is difficult to remove the cerebral hemispheres, but in those 
animals in which the operation has been carried out, in the rabbit and rat, a result very similar to those observed in the case of the frog and pigeon has been obtained. The animal is able to maintain its equilibrium, to run or jump, and in fact successfully carry out the most complicated co-ordinated movements, but it is unable to originate them without stimulation. In the case of the dog, it has been found impossible to remove the whole brain at one operation. However, Goltz has succeeded in removing both the cerebral hemispheres of the dog by doing the operation in successive stages and taking extraordinary precautions to protect his animal against the great fall of temperature and the immediate shock of the operation. He kept his dog alive for some eighteen months and secured a complete recovery from the series of operations. Goltz's dog was able to walk about, it responded to a bright light by closing its eyes, and could be aroused by a sharp, loud sound. It spent, its time lying down in the cage, sleeping rolled up dog-fashion. When aroused by stimulation of the skin, it would move away from the stimulating object and would sometimes growl and snap at the object. If it snapped at the object it would do so without going toward it or making the usual effort to seize the object which we are accustomed to expect of a normal vicious dog. This dog did not spontaneously feed itself, but had to have food placed in its mouth before it would swallow. But the animal finally learned to take food, as in the case of the pigeon. This animal gave very definite responses to its condition of nourishment; it slept quietly and was peaceful when fully fed, but was restless and irritable when hungry.

Goltz's dog showed complete absence of those activities which we would call psychic. That is to say, it showed no memory signs, it was unable to learn the signal for feeding, it did not manifest any fondness or signs of pleasure at the presence of its caretaker. In short, there was a complete loss of memory and intelligence, and the animal, although performing some activities, was in fact reduced to a mere automaton. It would be difficult to imagine a more crucial experiment to elucidate the function of the cerebral cortex.

It is quite evident that the apparatus for carrying out co-ordinated movements in these animals is not localized either in the cerebrum or in the spinal cord. It must therefore be connected in some way with the parts of the brain below the cerebrum and above the cord. There is no reason why such an arrangement may not be supposed to exist in the human brain, although we must look upon the cerebrum as the originator of voluntary movements.

The Reactions of the Human without Cerebrum.-In I9I3 Edinger and Fischer reported a most interesting case of a child who without cerebrum lived to the age of three and three-fourths years, see figure 400 .

The cerebrum in this child consists only of a mass of cysts without nerve tissue. The optic and olfactory nerves were also lacking in nerve elements, though the optic chiasma had some nerve tissue. The cerebellum did not 
differ from the normal. The midbrain was diminished in size. The corpora quadrigemina had fewer nerve cells and fibers than the normal. The corpus striatum was normal on one side, but on the other lacked portions of the putamen and the nucleus caudatus. The thalamic ganglia which are well developed in a normal child were absolutely lacking except to a slight extent in the hypothalamic nuclei where a few fibers coming from the striatum were present. Of the cerebrum proper not the least trace remained, either macroscopic or microscopic except for the connective tissue vascular framework.

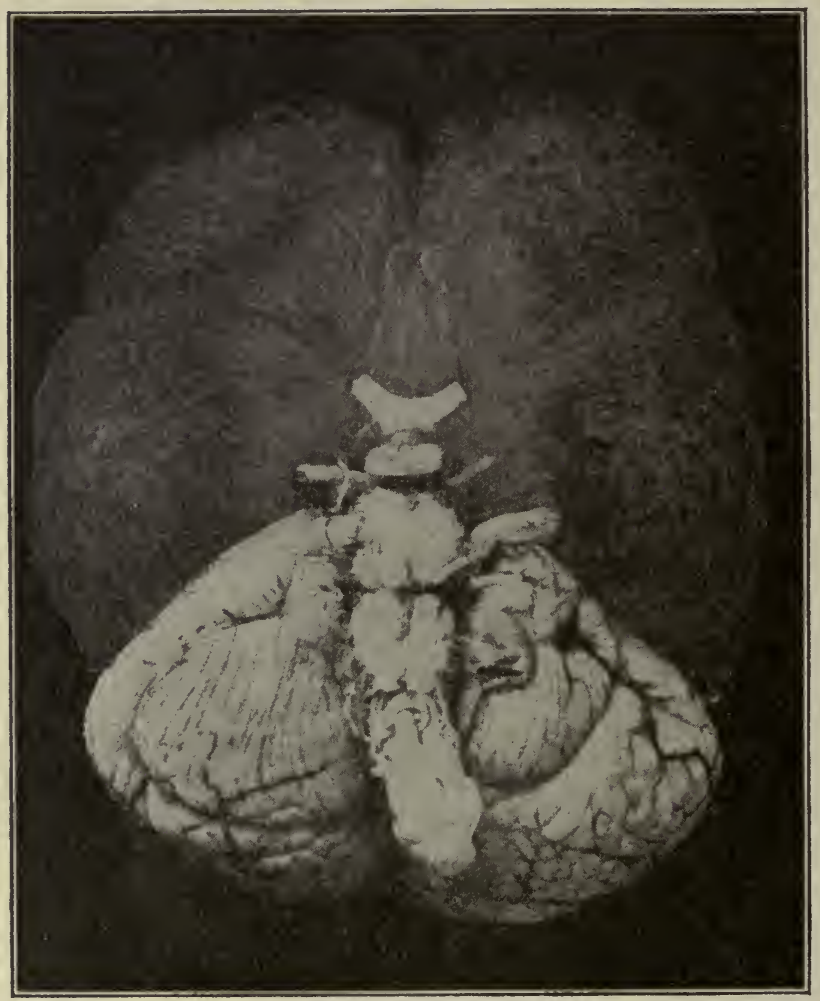

FIG. 400 .

This child showed no physiological development from birth until death. In the language of the authors, "It was astonishing how much less this child could accomplish than the dog without a cerebrum." While the operated dog showed the activities described above, the child remained quiet without conscious control over its limbs and body. It did not grasp with the hands, showed only a certain motility of the face, the lips were co-ordinated with the tongue in sucking; but otherwise the restlessness and motility shown by the 
dog were entirely absent in the child. The child kept up a perpetual crying from the second year on, but the crying could be stilled by pressure, especially about the head. The child had no control over its functions and showed no consciousness of physical discomfort. "This child without cerebrum was even less active than a decerebrate fish or frog."

In comparing the reactions of this child with those of the decerebrate dog, it must be remembered that the operated dog has not only a more mature cord and medulla, but also an intact and well developed thalamus. The nuclei of the thalamus may quite possibly carry on extensive nerve co-ordinations which were of course lacking to the child. However, the child possessed the necessary basic nervous mechanism for the control of respiration, circulation, digestion, alimentary motion, etc., adequate for the purely physical life. The case is only another link in the chain of evidence which more firmly establishes the view that the entire psychic life is inseparably associated with the normal physiological functioning of the cerebral cortex.

\section{LOCALIZATION OF THE MOTOR FUNCTION OF THE CEREBRAL CORTEX.}

The experiments upon the brains of various animals by means of electrical stimulation have demonstrated that there are definite regions of the cerebral cortex the stimulation of which produces definite movements of co-ordinated groups of muscles of the opposite side of the body. Fritsch and Hitzig were the first to show that the cerebral cortex responds to electrical irritation. They employed a weak constant current in their experiments, applying a pair of fine electrodes not more than one-twelfth inch apart to different parts of the cerebral cortex. The results thus obtained have been confirmed and extended by Ferrier, Sherrington, and many others, stimulating chiefly with induction currents.

The fundamental phenomena observed in all these cases may be thus epitomized:

I. Excitation of the same spot on the cortex is always followed by the same movement in the same animal. 2. The area of excitability for any given movement is extremely small, and admits of very accurate definition. 3. In different animals excitations of anatomically corresponding spots produce contractions in similar or corresponding muscles.

The various definite movements resulting from the electric stimulation of circumscribed areas of the cerebral cortex are enumerated in the description of the accompanying figures of the dog's and monkey's brains.

In the case of the dog the results obtained are summed up as follows by Hitzig: I. One portion, anterior, of the convexity of the cerebrum is motor; another portion, posterior, is non-motor. 2. Electric stimulation of the motor portion produces co-ordinated muscular contraction on the opposite side of the body. 3. With very weak currents, the contractions produced 
are distinctly limited to particular groups of muscles; with stronger currents the stimulus is communicated to other muscles of the same or neighboring parts. 4. The portions of the brain intervening between these motor centers are inexcitable.

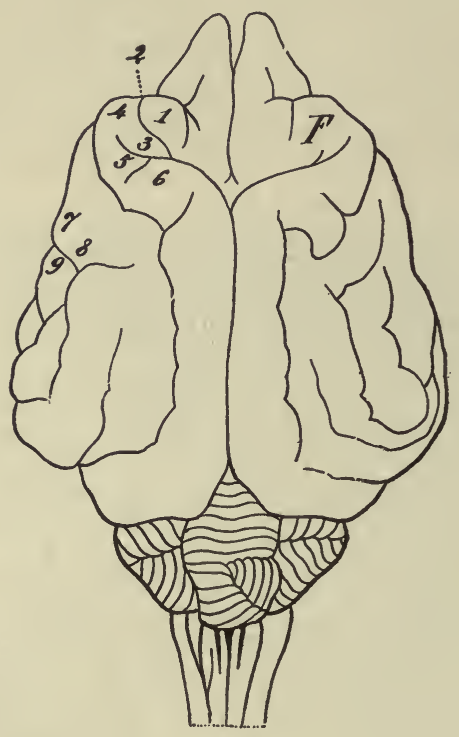

A

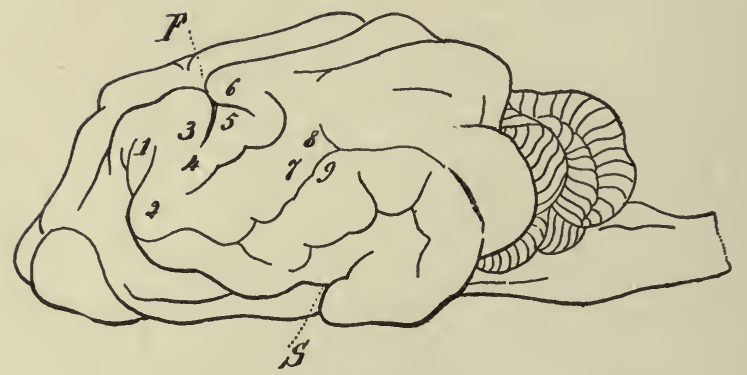

B

FIG. 40I.-Brain of Dog, $A$, Viewed from Above and $B$ in Profile. $F$, Frontal fissure sometimes termed crucial sulcus, corresponding to the fissure of Rolando in man; $S$, fissure of Sylvius, around which the four longitudinal convolutions are concentrically arranged; I, flexion of head on the neck, in the median line; 2, flexion of head on the neck, with rotation toward the side of the stimulus; 3,4 , flexion and extension of anterior limb; 5 , 6 , flexion and extension of posterior limb; $7,8,9$, contraction of orbicularis oculi and the facial muscles in general. (Dalton.)

Following strong stimulation of cortical motor centers other groups of muscles than those innervated by the centers stimulated may also take part in the contractions.

According to the further researches of Schäfer and Horsley, electrical 
stimulation of the marginal convolution internally at the parts corresponding with the ascending frontal and parietal convolutions, from the front backward, produces movements of the arm, of the trunk, and of the leg.

A good deal of doubt was thrown upon the experiments of Ferrier by Goltz and other observers, from the results of excising the so-called motor areas of the dog's brain. It was found that the part might be sliced away or washed away with a stream of water, but that no permanent paralysis ensued.

More extensive observations, however, have, in the main, confirmed Ferrier's original statement, at any rate with regard to the monkey's brain. Destruction of the motor areas for the arm produces some permanent paralysis of the arm of the opposite side, and similarly.of that for the leg, paralysis of the opposite leg. If both areas are destroyed, permanent hemiplegia

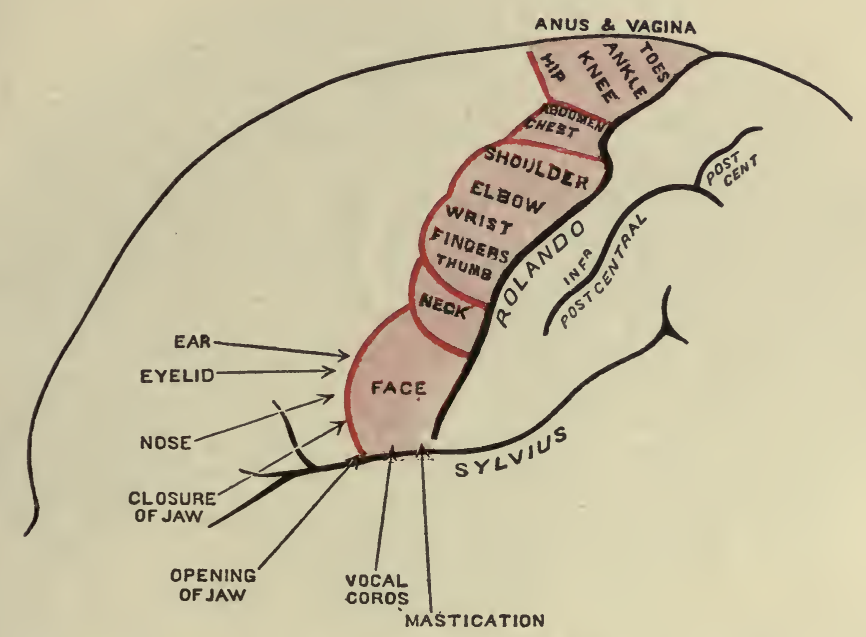

FIG. 402.- Scheme Showing the Motor Areas of the Brain. (Adapted from Grünbaum and Sherrington by Cunningham.)

ensues. Paralysis of so extensive and permanent a character does not, however, appear the rule when the brain of a dog is used instead of that of the monkey. It is suggested that in the animal lower in the scale the functions which in the monkey are discharged by the cortical centers may be subserved to a greater extent by the basal ganglia.

Motor Areas of the Human Brain.-It is naturally of great importance to discover how far the results of experiments upon the dog and monkey hold good with regard to the human brain. Evidence furnished by diseased conditions is not wanting to support the general idea of the existence of cortical motor centers in the human brain, figure 402 .

So far, however, it has been possible to localize motor functions only in the precentral convolutions and the walls of the adjacent sulci. 
The relative position of the centers is probably much the same as in the monkey's brain, those for the leg above, those of the arm, face, lips, and tongue from above downward. Destruction of these parts causes paralysis, corresponding to the district affected, and irritation causes contractions of the muscles of the same part. Again, a number of cases are on record in which aphasia, or the loss of power of expressing ideas in words, has been associated with disease of the posterior part of the lower or third frontal con-

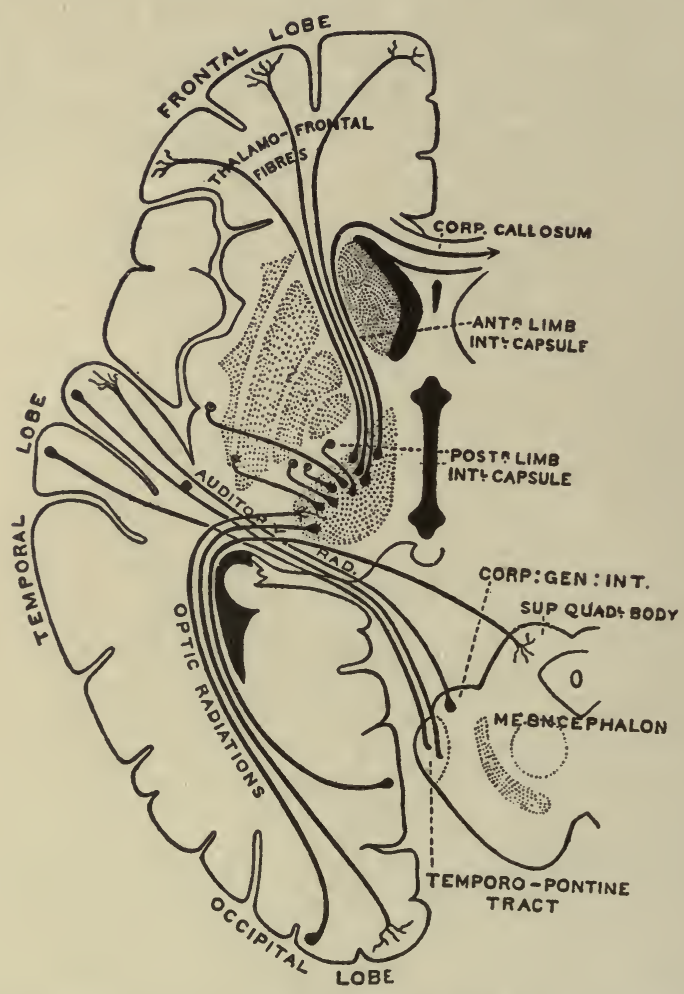

Fig. 403.-Diagram of Certain Connections of the Frontal, Temporal, and Occipital Lobes. Founded on the observations of Flechsig, Ferrier, and Turner. (Cunningham.)

volution on the left side. This condition is usually associated with motor paralysis on the right side of the body, right hemiplegia.

This district of the brain, particularly the convolutions bounding the fissure of Rolando anteriorly, is now generally known as the motor area. There is now no doubt whatever that this area gives origin to the nerve fibers which proceed to the spinal cord, and are there represented as the pyramidal tracts.

This is the reason that movements are produced on stimulation of the 
white matter after the superficial gray matter of the animal's brain has been sliced off.

These motor fibers are those which arise from the pyramidal cells of the cortex. From the motor area of the cortex they converge to the internal capsules, and pass down to the crus. In the internal capsule the fibers which pass to the pyramidal tracts of the spinal cord occupy that part known as the knee (genu) and the anterior two-thirds of the posterior limbs of the capsule, figure 404. In this district the fibers for the face, arm, and leg are in this relation: those for the face and tongue are just at the knee, and below or behind them come first the fibers for the arm and then those for the leg.

The more accurately known arrangements of these fibers in the monkey's brain, named in order, from above down, are those for the eye, head, tongue,

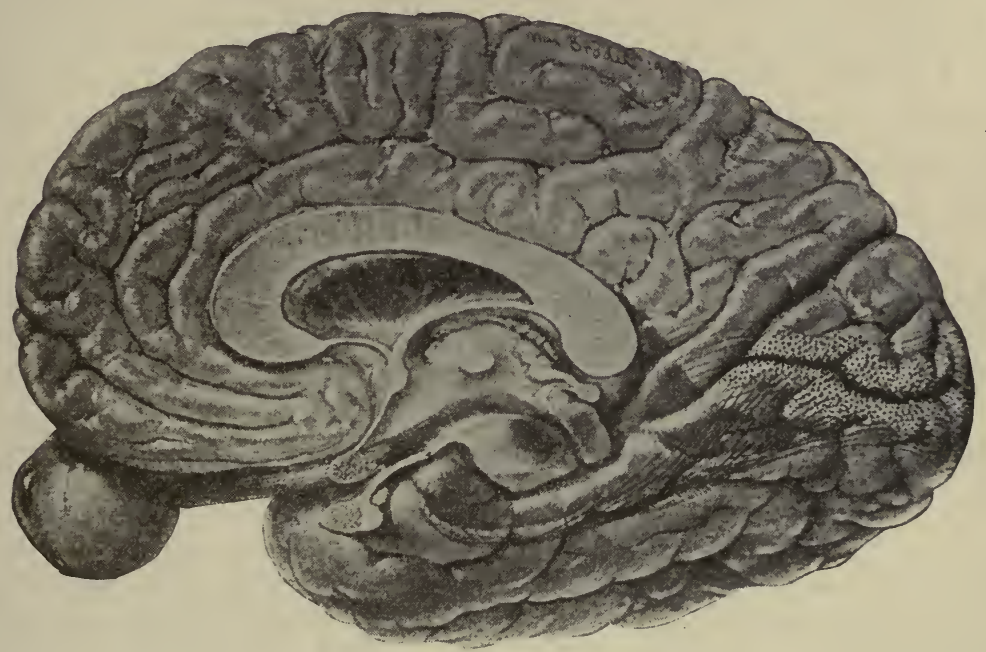

FIG. 404--Showing the geniculo-calcarine optic pathway. (Meyer, copied from Cushing.)

mouth, shoulder, elbow, digits, abdomen, hip, knee, digits. These fibers come for the most part from the portion of the cortex in front of the fissure of Rolando, chiefly from the precentral gyrus, hence called the Rolandic area. Those fibers, passing between the occipital lobe and the thalamus and superior corpora quadrigemina, are concerned with vision, and are called fibers of the optic radiation. In like manner, from the inferior corpora quadrigemina and the internal geniculate bodies, fibers which make up the auditory radiation pass to the auditory center in the superior temporal gyrus.

The term motor centers is applied to cortical areas which are concerned with the development of voluntary motor impulses. It must not be assumed that the motor cells of the area initiate such impulses. These centers react only in response to afferent nerve impulses which flow in upon them. This 
fact was conclusively proven by Mott and Sherrington who cut the sensory roots of the brachial plexus and found that the monkey so operated lost the power of voluntary use of the muscles on the side operated. The cortical motor apparatus here remains intact but lacking the necessary sensory stimulation the cortical cells are unable to initiate the motor impulses required for voluntary action.

It has already been shown that the motor fibers of the internal capsule of one side cross over to the opposite side in the decussation of the pyramids in the medulla. This decussation is not quite complete, as some fibers pass down on the same side in the direct pyramidal tract. A small portion of these direct fibers end around the motor neurones of the same side, but the great majority cross to the opposite side in the anterior commissure at some lower level of the cord. It follows that the motor areas of the cortex on one side control the muscular movements of the opposite side of the body, but also to a slight extent those of the same side. As a matter of fact, disease in the region of the fissure of Rolando is usually accompanied by a disturbance of the motor function on the opposite side of the body, although there is some slight motor disturbance on the same side.

\section{LOCALIZATION OF SENSORY FUNCTION IN THE CEREBRAL CORTEX.}

There is evidence that fibers from the nerves of special sense are specially connected with definite and distinct parts of the cerebral cortex, the sensory areas.

The fibers from the sensory nerves, we have found, are connected with the cerebral cortex by chains of neurones. These sensory paths, although complex, are definite and distinct. Their cortical connections have been mapped out with considerable definiteness.

The Body Sensory or Somesthetic Area.-The demonstrated motor function around the pre-Rolandic region for a long time obscured the fact that this region, especially the pre- and post-central convolutions, is intimately connected with the perception of general body sensations. Physiological and pathological observations supported this view, and recently Flechsig has much strengthened the view by his method of studying the progressive development of the brain. In figure 405 we produce Flechsig's diagram showing the body sensory area, aptly designated by Barker the somesthetic area. The borders of the area are more or less indefinite and less distinct than the main portion. This is indicated in the figure by the lighter shading. Lesions of this area in the cortex lead to loss of sensibility in definite regions of the opposite side of the body. Reaction in this cortical area evidently forms a necessary link in the chain of nerve impulses initiated by cutaneous sensory stimulation and leading to conscious sensations. 
Visual or Optic Center.-The termination of the optic nerve in each eye, the retina, to the structure of which we shall return when treating of the eye, is so arranged that when we look at an object with both eyes, symmetrical parts of the retinæ are used. For example, if we examine an object to the left of the center of vision, an image of that object is focused upon the right half of both retinæ, viz., upon the temporal side of the right retina, and upon the nasal side of the left retina. The optic nerve fibers of these symmetrical parts of the retinæ are gathered together behind where the optic nerves decussate, viz., in the optic chiasma. The fibers which come from the right side of both eyes are contained in the optic tract of the same side, viz., the right, those from the right eye being outside of the others. In the same way the left optic tract contains internally fibers from the left side of the right eye and externally those from the left side of the left eye. The optic tract thus formed then passes backward and terminates in three distinct nuclei, viz.,
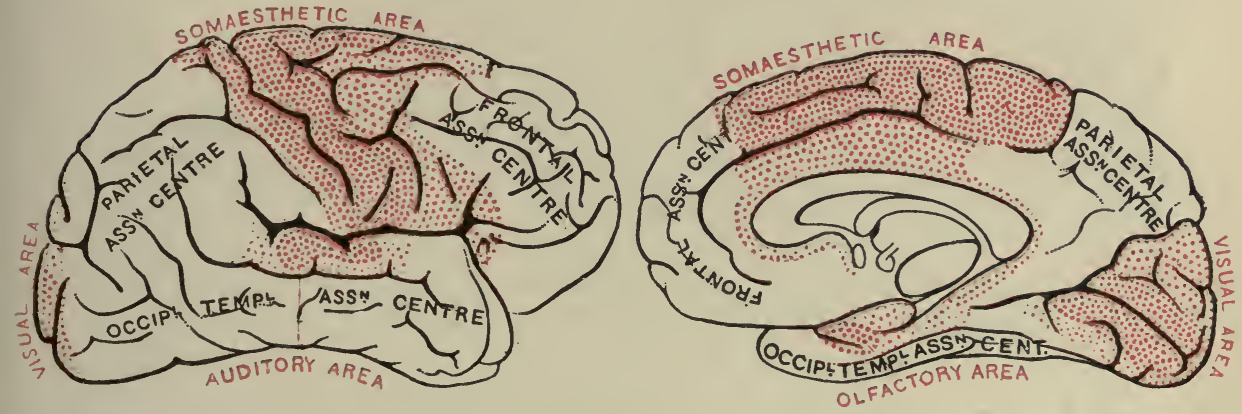

Fig. 405.-Diagrams to Show Flechsig's Sensory and Association Areas on the Surface of the Cerebral Hemisphere. (From Cunningham, after Flechsig.)

the pulvinar of the thalamus, the anterior corpus quadrigeminum, and the lateral corpus geniculatum. These nuclei atrophy if the eyes are removed from an adult animal; and if the eyes are removed from a newly-born animal, they do not fully develop. Through the superior corpora quadrigemina the optic tract establishes synapses that bring it into relation with the nucleus of the third nerve, and which form the basis of the eye reflexes to light stimulation.

It appears that some of the fibers of the optic tract pass directly into the cerebral cortex without joining with the thalamus, corpus quadrigeminum, or corpus geniculatum.

It was shown above that the fibers of the cerebral cortex, known as the optic radiation, pass from the occipital region to the three nuclei about which we are speaking, viz., into the pulvinar of the thalamus, the anterior corpus quadrigeminum, and lateral corpus geniculatum, and it is known that when the occipital cortex is removed, these three 
atrophy. It has been further shown that in a newly-born animal the removal of such a region is followed by imperfect development of the parts in question.

If one optic nerve be divided, blindness of the corresponding eye results; but if one optic tract be divided there is a half blindness in both eyes, which is called hemianopsia, or hemiopia, right or left, according as the right or left field of vision is cut off. It is also evident that the occipital lobe, figures 403 , 406 , and particularly the cuneus, is concerned as a visual center. Not only is the occipital lobe connected with the optic nerves, as we have seen, but the removal of the right occipital lobe in an animal (monkey) is followed by bilateral hemiopia of the right retinæ. Removal of the left occipital lobe is followed by left bilateral hemiopia. Removal of both occipital lobes is followed by total blindness. Clinical observations give convincing proof that in man also the occipital lobes are the cortical centers of vision. Disturbance of the optic lobes or tumors involving the optic tracts, figure 404 , lead to disturbance and blindness in the retinal field.

Olfactory Center in the Cortex.-The olfactory nerve differs from the other cranial nerves. In reality it is a representative of the olfactory lobes of other animals, which are part of the cerebrum. The olfactory lobe originates as an offshoot from the cerebral vesicle, the front part of which is developed into the bulb of the olfactory nerve, while the back part forms its peduncle. The nerve, the cavity of which in man is filled up in the fully developed condition with neurogliar substance, lies upon the cribriform plate of the ethmoid bone, and is contained in a groove on the under surface of the frontal lobe. On examination of the lobe it is found to be thus made up: Beneath the neurogliar layer is a layer of longitudinal fibers and a few nerve cells, next to this is a layer of small cells, nuclear layer, fibers from the layer of nerve fibers passing through it.

The nuclear layer is also separated into groups of cells by an interlacing of the fibers. The next layer is thick and is composed of neuroglia and nerve fibers, some of which are medullated, as well as of cells more or less pyramidal in shape. Below this layer is the layer of olfactory glomeruli. These glomeruli are small synapses of olfactory fibers. The larger also includes small cells and granular matter. A further description of the anatomy of these parts is given later, page 656 .

Fibers of the olfactory nerve proper are found below this layer, and pass through the cribriform plate to be distributed to the olfactory mucous membrane. They arise from cells in the olfactory mucous membrane, and end in the glomeruli. The peduncle of the nerve or the olfactory tract, as it is sometimes called, is made up of longitudinal fibers originating in the bulb, with neuroglia and some nerve cells.

The fibers of the olfactory tract have been traced into the nucleus amygdalæ and its juncture with the hippocampal gyrus in the temporal lobe, 
figure 392. The hippocampus must be in some way connected with smell, since a lesion of it, leaving the olfactory tract uninjured, seriously interferes with that sense.

Taste Center of the Cortex.-It is very uncertain where the taste center is situated, if such exist. It has been placed in the anterior portion of the inferior temporal convolution, not far from that of smell, figure 405 .

Auditory Center in the Cortex.-This center has been localized in

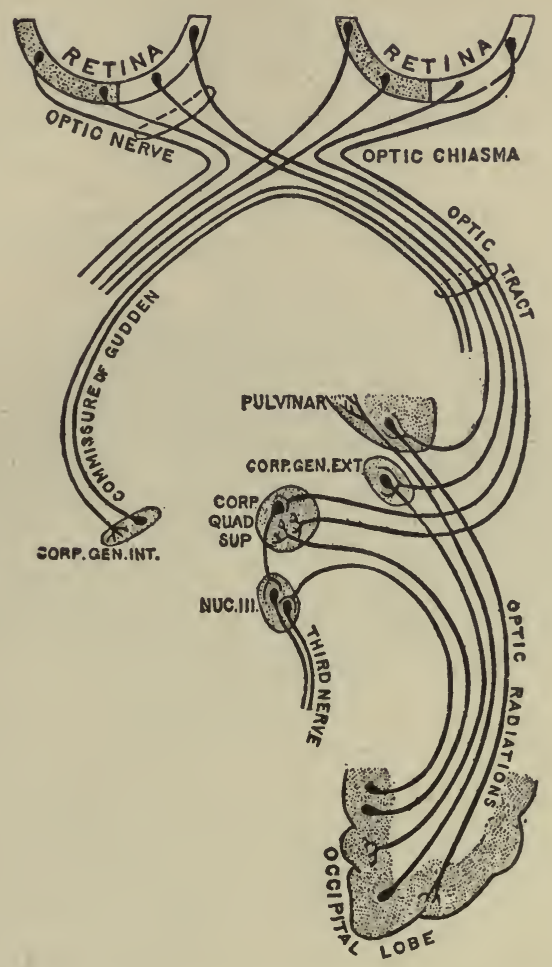

Fig. 406.-Scheme of the Central Connections of the Optic Fibers. (Cunningham.)

the superior temporal convolution. Experiments have been made which connect auditory impulses on either side with the inferior corpus quadrigeminum and the median corpus geniculatum, for when the internal ear is destroyed there results atrophy of these bodies as well as of the lateral fillet of the opposite side. On the other hand, destruction of the part of the temporal lobe above indicated is similarly followed by atrophy of the nuclei of the same side. These nuclei bear much the same relation to the sense of hearing as do the superior corpora quadrigemina and the lateral corpora geniculata to the sense of sight, figures 406 and $4 \mathrm{I} 4$. 


\section{ASSOCIATION CENTERS OF THE CEREBRAL CORTEX.}

The theory of localization of the function of different parts of the cerebral cortex has received substantial support from the study of the motor and the sensory areas in man and the mammals. But when the exploration of the cortex is complete and the motor and sensory areas are bounded as definitely as may be, there still remain great areas in which stimulation is apparently non-effective so far as our present means of interpretation reveal. Traumatic and pathological lesions produce no sensory or motor disturbances. The areas of this class which are most extensive, i.e., which cover the greatest amount of cortex, are three in number-the frontal lobe, the parietal lobe, and a large part of the temporal lobe below the superior temporal convolution.

Flechsig has made a study of the development of the human brain, paying especial attention to the progressive development of the great tracts of fibers. He has shown that the tracts appear in a certain order of sequence, also that the myelinization takes place progressively.

These observations give justification to the assumption that effective functionization is attained with the acquirement of the myelin sheath. $\mathrm{He}$ showed a close correspondence in time between the development of the tracts and the manifestation of functions known to involve the tracts in question. The great somesthetic area and its tracts are first to develop; then tracts to the occipital or visual center, to the auditory and other sensory centers, and, finally, to those great areas whose functions remain obscure.

Basing his deductions on the facts of development, on the isolated cases of lesion and disease, and on the careful comparative studies of the brains of certain men of unusual intellectual powers, whose personal characteristics and intellectual genius are known, Flechsig has advanced the hypothesis that the areas of the cortex not concerned directly with motor or sensory functions are association areas.

The Association Centers of Flechsig.-The great association centers are the frontal, parietal, and temporal, figure 405. These regions of the cortex are apparently not directly connected with tracts of the brain stem and cord, but they are richly connected with the areas that do have connection with the cord. Short association fibers connect neighboring convolutions within the centers, fibers which are chiefly the axones of the polymorphous cells of the fourth layer of the cortex. Long association fibers run from one center to another, such as the cingulum, superior and inferior longitudinal fasciculi, etc. The longer connectives run from association to association centers, and from association to sensory and motor centers. Flechsig believes that the sensory centers are not connected directly with each other, but only indirectly through the association areas.

Cases of injury and of disease of the human brain in the association areas are not numerous, but such as there are tend to confirm Flechsig's hypothesis that the function of these areas is that of the higher psychic activity. 
The Anterior or Frontal Association Center.-The frontal area is more closely connected with the motor areas and the centers for the somesthetic sense. With injury to this area the individual shows weakness in attention, in reflection, and in control over the expressions of anger, self-appreciation, and other activities that are expressive of personal volitions and emotions.

The American crowbar case is a classical instance of lesion of the frontal lobe. A young man of twenty-five had an iron bar, an inch and a quarter in diameter and over three feet long, driven through his skull and brain by the premature explosion of a blast of powder. He not only recovered, but lived for twelve years afterward. At the post-mortem examination the puncture was found to be through the prefrontal lobe, anterior to the coronal suture.

This man was considered a most efficient workman and foreman before

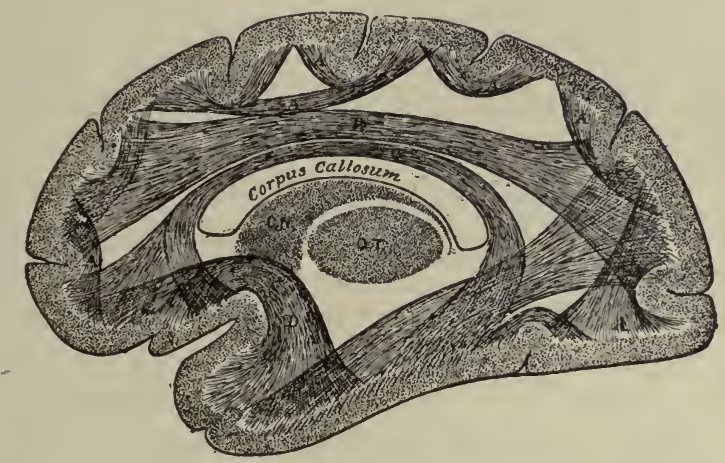

FIG. 407.-The Association Fibers in the Centrum Ovale. $A$, Between adjacent convolutions; $B$, between frontal and occipital lobes; $C$, between frontal and temporal lobes, the cingulum; $D$, between temporal and frontal lobes-lesion of this tract causes paraphasia; $E$, between occipital and temporal lobes-lesion of this tract causes word-blindness; C.N, caudate nucleus; O.T, optic thalamus.

the injury. After his recovery he was fitful, impatient of restraint, capricious, obstinate. He was most inconsiderate of his associates, profane and passionate. From a shrewd business man he was changed to the intellectual level of a child and was regarded by his associates as mentally unbalanced.

A summary of fifty cases of pathological lesions of the prefrontal areas of the human brain is given by Williamson. The mental traits of thirty-two are summarized in the following terms: "A condition of mental decadence; a dull mental state; loss of power of attention; loss of memory; loss of spontaneity; the patient takes no heed of his surroundings; sleeping during the greater part of the day, or remaining semi-comatose." Yet these patients are able to walk about and execute well co-ordinated muscular activities of all kinds that do not involve complex intellectual activity. 
The Parietal Association Center.-Special mention is made of this association area because there is increasing evidence that it is the parietal region of the brain, rather than the frontal, as popularly believed, that is most intimately concerned with the higher acts and powers of imagination, idealization, and reasoning. It is the region through which the individual maintains his interests and relations with the external world as against his own body. The parietal association center is more closely related to the visual, auditory, and speech centers of the cortex. The great musician Bach had an exceptionally well developed parietal region.

On the Cortical Centers in General.-For purposes of clearness in presentation, the cortical centers have been disclissed one by one, but the reader is guarded against the thought that their activities are in any sense isolated. A motor area does not usually act in the absence of sensory or afferent stimulation in the actual living body, whether it may do so on occasion or not. Neither do sensory impressions arising in the peripheral sense organ make their way over definite tracts to the brain and cortex and arouse sensations alone. Sensations do not occur independent of motor activities on the one hand, and of intellectual acts through the association centers on the other.

The association centers are the highest co-ordinating regions of the nervous system. They are to the sensory and motor centers what these latter are to the reflex centers of the cord. The difference is one of degree and not of kind. Further, the association centers are probably set into activity by the complex of inflowing or afferent impulses in just the same sense that the spinal reflex centers are set in activity by sensory or afferent stimuli; the condition is, of course, a thousand times more complex.

\section{THE CRANIAL NERVES.}

The cranial nerves consist of twelve pairs; they appear to arise (superficial origin) from the base of the brain in a bilateral series, which extends from the under surface of the anterior part of the cerebrum to the lower end of the medulla oblongata. Traced into the substance of the brain and medulla, the roots of the nerves are found to take origin from various masses of gray maiter.

The roots of the first or olfactory and of the second or optic nerves are discussed elsewhere. The third and fourth nerves arise from gray matter beneath the corpora quadrigemina; and the roots of origin of the remainder of the cranial nerves can be traced to gray matter in the floor of the fourth ventricle, and in the more central part of the medulla, around its central canal, as low down as the decussation of the pyramids.

According to their several functions the cranial nerves may be thus arranged: 
Nerves of special sense ........ $\left\{\begin{array}{l}\text { Olfactory, optic, auditory, parts of } \\ \text { the facial, glosso-pharyngeal, and of } \\ \text { the trigeminal. }\end{array}\right.$ Nerves of common sensation...... $\left\{\begin{array}{c}\text { The greater portion of the trigeminal, } \\ \text { and part of the facial. }\end{array}\right.$ (The motor oculi, trochlearis, lesser diNerves of motion........... $\left\{\begin{array}{l}\text { vision of the tri-geminal, abducens, } \\ \text { hypoglossal, and spinal accessory. }\end{array}\right.$

Mixed nerves............. Facial, glosso-pharyngeal, and vagus.

The physiology of the first, second, and eighth nerves will be considered with the Organs of Special Sense, see also figure $4 \mathrm{I} 6$.

The Third Nerve or Motor Oculi.-Origin.-The third nerve arises in

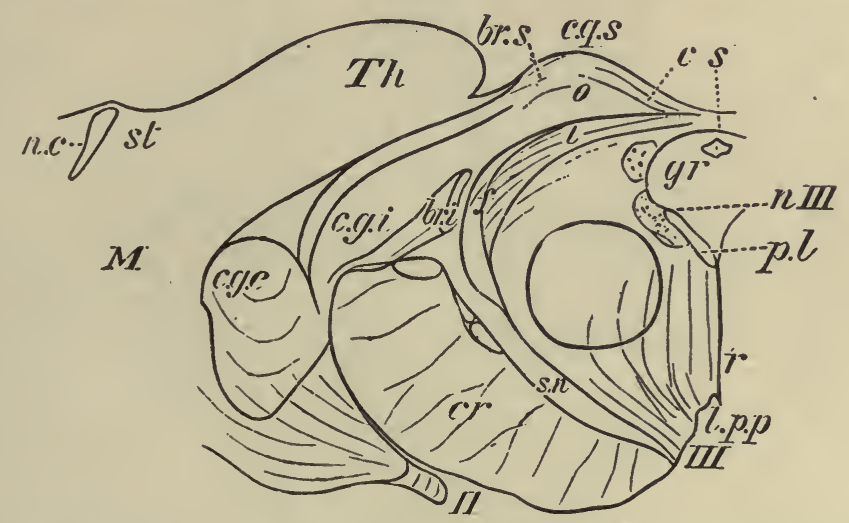

Fig. 408.-Section through Superior Corpus Quadrigeminum and Part of the Thalamus. $s$, Aqueduct of Sylvius; $g r$, gray matter of the aqueduct, c.q. $s$., superior corpus quadrigeminum; $l$, stratum lemnisci; $o$, stratum opticum, $c$, stratum cinereum; $T h$, pulvinar of optic thalamus; c.g.e, c.g.i, lateral and median corpora geniculata; $b r . s, b r . i$, superior and inferior brachia ' $f$, fillet; $p . l$, posterior longitudinal bundle; $r$, raphé; III, third nerve, and $n . I I I$, its nucleus; l.p.p, posterior perforated space; s.n, substantia nigra-above this is the tegmentum with the circular area of the red nucleus; $c r$, crusta; $I I$, optic tract; $M$, medullary center of hemisphere; n.c, nucleus caudatus; st, stria terminalis. (After Quain, from Meynert.)

three distinct bands of fibers from the gray nuclei surrounding the aqueduct of Sylvius near the middle line, but ventral to the canal, figure 408 . The nucleus of origin consists of large multipolar ganglion cells, and extends to the back part of the third ventricle as far as the level of the superior corpora quadrigemina. The fibers pass from their origin partly through the red nucleus to their superficial origin in front of the pons at the median side of each crus. The third nerve does not decussate.

Function. - The third nerve supplies the levator palpebræ superioris muscle, and all of the muscles of the eyeball, except the superior oblique, to which the fourth nerve is distributed, and the rectus externus which receives the sixth nerve. Through the medium of the ophthalmic or lenticular 
ganglion, of which it forms what is called the short root, it also supplies motor filaments to the iris and ciliary muscle. The fibers which subserve the three functions, accommodation, contraction of the pupil, and nervesupply to the external ocular muscles, arise from three distinct groups of cells. Optic reflexes involving movements of the eyeballs are mediated through fibers from cells of the superior corpora quadrigemina (which receive fibers from the optic nerve). These fibers from the corpora quadrigemina descend, chiefly through the posterior longitudinal bundle, figure

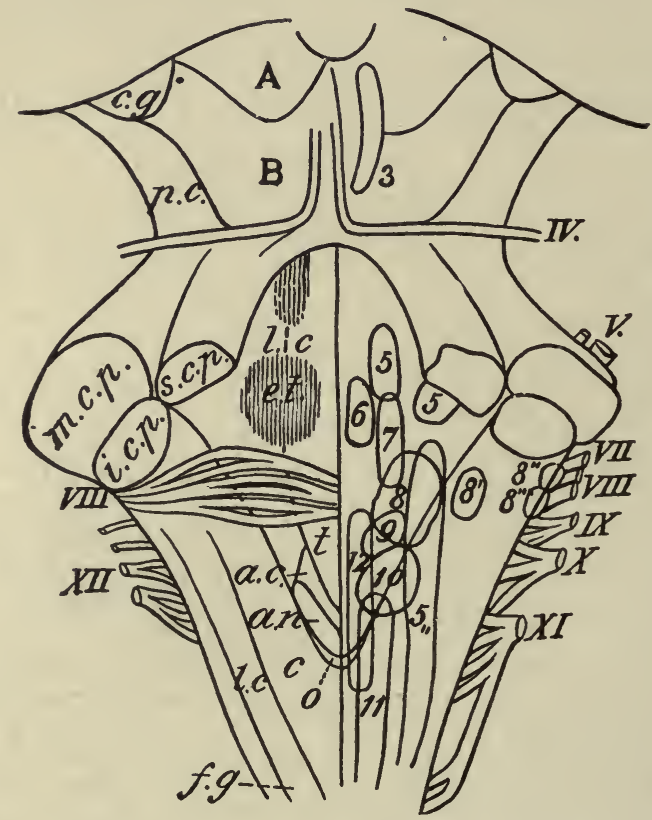

Fig. 409.-Fourth Ventricle with the Medulla Oblongata and the Corpora Quadrigemina. The Roman numbers indicate superficial origins of the cranial nerves, while the other numbers indicate their deep origins, or the position of their central nuclei. $8,8^{\prime}, 8^{\prime \prime}$, Auditory nuclei nerves; $t$, funiculus teres; $A, B$, corpora quadrigemina; $c . g$, corpus geniculatum; p.c. pedunculus cerebri; m.c.p, middle cerebellar peduncle; s.c.p, superior cerebellar peduncle; i.c.p, inferior cerebellar peduncle; l.c, locus ceruleus; e.t, eminentia teres; $a . c$, ala cinerea; $a . n$, accessory nucleus; $o$, obex; $c$, clava; f.c, funiculus cuneatus; $f . g$, funiculus gracilis.

383 , to the nuclei of the third, fourth, and sixth nerves, thus rendering possible co-ordinated reflex movements of all the eye muscles.

When the third nerve is stimulated within the skull, all those muscles to which it is distributed are contracted. When it is paralyzed or divided, the following effects ensue: $r$. The upper eyelid can be no longer raised by the levator palpebræ, but droops, ptosis, and remains gently closed over the eye, under the unbalanced influence of the orbicularis palpebrarum, which is supplied by the facial nerve. 2. The eye is turned outward and downward, 
external strabismus, by the unbalanced action of the rectus externus and superior oblique, to which the sixth nerve is appropriated; and hence, from the irregularity of the axes of the eyes, double sight, diplopia, is often experienced when a single object is within view of both the eyes. 3. The eye cannot be moved upward, downward, or inward. 4. The pupil becomes dilated, mydriasis. 5. The eye cannot accommodate for short distances.

The Fourth Nerve, or Trochlearis.-Origin.-The fourth nerve arises from a nucleus consisting of large multipolar ganglion cells situated ventral to the aqueduct of Sylvius, and the inferior corpus quadrigeminum.

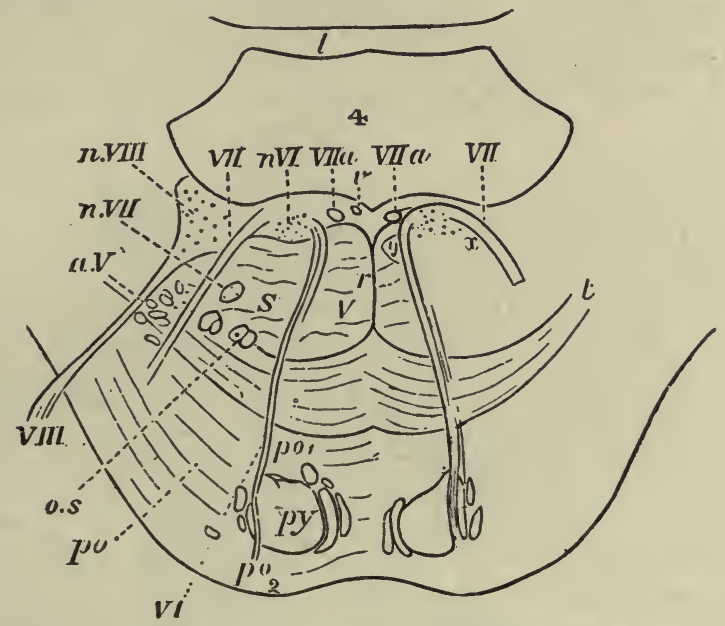

FIg. 4ro.-Section Across the Pons, About the Middle of the Fourth Ventricle. $p y$, Pyramidal bundles; $p o$, transverse fibers passing $p o_{1}$, behind, and $p o_{2}$, in front of $p y ; r$, raphé; o.s, superior olive; $a . V$, bundles of ascending root of $V$. nerve enclosed in a prolongation of the substance of Rolando; VI, the sixth nerve; $n V I$, its nucleus; VII, facial nerve; VII.a, intermediate portion; $n V I I$, its nucleus; VIII, auditory nerve; $n V I I I$, lateral nucleus of the auditory. (After Quain.)

The fibers from both sides sweep dorsally around the central gray matter, and reach the valve of Vieussens, where they decussate in the mid-line of the roof, then pass forward along the lateral aspect of the crus. The nucleus of the fourth nerve on either side is connected with those of the third and sixth nerves and with the optic reflex center previously described.

Functions. - The fourth nerve is exclusively motor, and supplies only the trochlearis or superior oblique muscle of the eyeball.

The Fifth Nerve, or Trigeminal.-Origin.-The fifth or trigeminal nerve resembles the spinal nerves in that it has two roots; namely, the larger or sensory, in connection with which is the Gasserian ganglion, and the small or motor root, which has no ganglion, and which passes under the ganglion of the sensory root. The fibers of origin of the fifth nerve come from the floor of the fourth ventricle. The motor root arises to the inside of the sensory about the middle of each lateral half of the fourth ventricle. The 
sensory fibers, however, can be traced down in the medulla oblongata as far as the upper part of the cord. The motor nucleus stretches forward as far as the superior corpus quadrigeminum, giving rise to a bundle of long fibers termed the descending root. The sensory nucleus receives a tract of sensory fibers from the trigeminus extending as low as the second cervical nerve, and this forms a tract at the tip of the posterior cornu, between it and the restiform body. The cells of origin of the sensory tract are in the Gas-

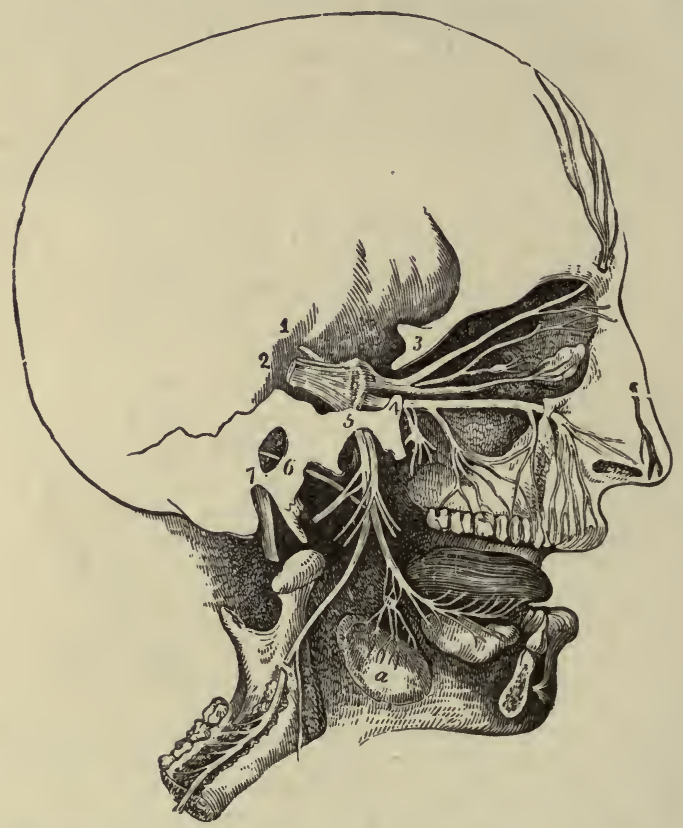

Fig. 4II.-General Plan of the Branches of the Fifth. $\times \frac{1}{3}$. I, Lesser root of the fifth; 2, greater root passing forward into the Gasserian ganglion; 3, placed on the bone above the ophthalmic nerve, which is seen dividing into the supra-orbital, lachrymal, and nasal branches, the latter connected with the ophthalmic ganglion; 4, placed on the bone close to the foramen rotundum, marks the superior maxillary division, which is connected below with the spheno-palatine ganglion, and passes forward to the infra-orbital foramen; 5 , placed on the bone over the foramen ovale, marks the inferior maxillary nerve, giving off the anterior auricular and muscular branches, and continued by the inferior dental to the lower jaw, and by the gustatory to the tongue; $a$, the submaxillary gland, the submaxillary ganglion placed above it in connection with the gustatory nerve; 6 , the chorda tympani; 7 , the facial nerve issuing from the stylomastoid foramen. (Charles Bell.)

serian ganglion. The nerve appears at the ventral surface of the pons near its front edge, at some distance from the mid-line.

Motor Functions. - The first and second divisions of the nerve, which arise wholly from the larger root, are purely sensory. The third division is joined by the motor root of the nerve and is of course both motor and sensory.

Motor branches of the lesser or non-ganglionic portion of the fifth supply the muscles of mastication, namely, the temporal, masseter, two pterygoid, anterior part of the digastric and mylohyoid. Filaments are also said to 
supply the tensor tympani and tensor palati (Kölliker). The motor function of these branches is proved by the violent contraction of all the muscles of mastication in experimental irritation of the third or inferior maxillary division of the fifth nerve; by paralysis of the same muscles when the nerve is divided or disorganized; and by the retention of the power of these muscles when the facial nerve is paralyzed. Whether the branch of the fifth nerve which is supplied to the buccinator muscle is entirely sensory, or in part motor also, must remain for the present doubtful. From the fact that this muscle, besides its other functions, acts in concert or harmony with the muscles of mastication in keeping the food between the teeth, it might be supposed from analogy that it would have a motor branch from the same nerve that supplies them. However, the so-called buccal branch of the fifth is, in the main, sensory.

Sensory Functions. - All the anterior and antero-lateral parts of the face and head, with the exception of the skin of the parotid region, acquire common sensibility through branches of the ganglionic division of the fifth nerve. The muscles of the face and lower jaw acquire muscular sensibility through the filaments of the ganglionic portion of the fifth nerve distributed to them with their proper motor nerves.

Through its ciliary branches and the branch which forms the long root of the ciliary or ophthalmic ganglion, it exercises some influence on the movements of the iris. When the trunk of the ophthalmic portion is divided, the pupil becomes, according to Valentine, contracted in men and rabbits, and dilated in cats and dogs, but in all cases becomes immovable even under all the varieties of the stimulus of light. How the fifth nerve affects the iris is unexplained; it has been suggested the influence of the fifth nerve on the movements of the iris may be ascribed to the affection of vision in consequence of the disturbed circulation or nutrition in the retina.

Trophic Influence.-The morbid effects which division of the fifth nerve produces in the organs of special sense make it probable that the fifth nerve exercises some special or trophic influence on the nutrition of all these organs, although the effects may in part be due to the loss of sensibility which is the natural protective safeguard. Thus, after such division and within a period varying from twenty-four hours to a week, the cornea begins to be opaque and later it grows completely white. A low destructive inflammatory process ensues in the conjunctiva, sclerotic coat, and in the interior parts of the eye. The sense of smell may be at the same time lost or gravely impaired. Commonly, whenever the fifth nerve is paralyzed, the tongue loses the sense of taste in its anterior and lateral parts, and according to Gowers in the posterior part as well.

In Relation to Taste.-The tactile sensibility of the tongue is due to the lingual branch of the fifth nerve, which supplies the anterior and lateral parts of the tongue. The sense of taste in the lateral and anterior portions of the tongue 
has recently been traced back to the pars intermedia and chorda tympani of the seventh, figures $4 \mathrm{I} 2$ and $4 \mathrm{I} 3$. It forms also one chief sensory link in the nervous circle for reflex action in the secretion of saliva. But, deferring this question until the glosso-pharyngeal nerve is to be considered, it may be observed that in some brief time after complete paralysis or division of the fifth nerve, the power of all the organs of the special senses may be impaired. They may lose not merely their sensibility to common impressions,

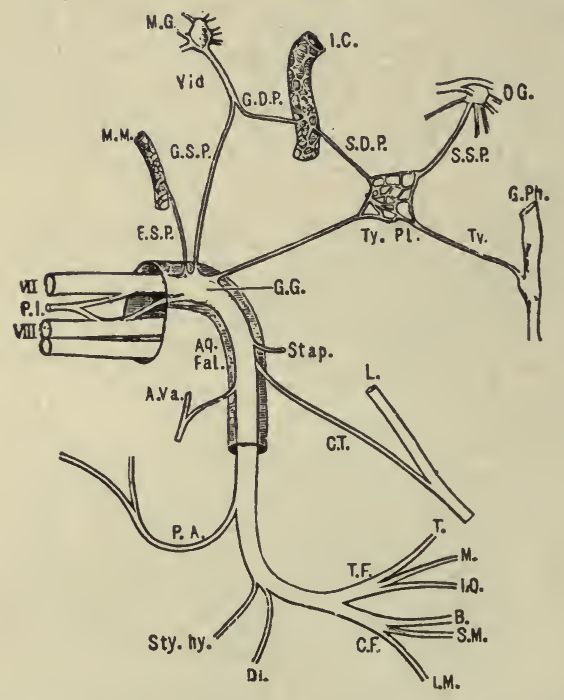

FIG. 4I2.-The Seventh Nerve and Its Branches. VII, Facial nerve; P.I, pars intermẹdia; VIII, auditory nerve; Aq.Fal, aqueduct of Fallopius; $G . G$, geniculate ganglion; E.S.P, external superficial petrosal nerve; M.M, middle meningeal artery; G.S.P, great superficial petrosal nerve; G.P.D, great deep petrosal nerve; I.C, internal carotid artery; $V i d$, Vidian nerve; M.G., Meckel's ganglion; Ty.Pl, tympanic plexus; S.D.P, small deep petrosal nerve; G.Ph, Glosso-pharyngeal nerve; Ty, tympanic branch; S.S.P., small superficial petrosal nerve; O.G., optic ganglion; Stap, nerve to stapedius; C.T, chorda tympani nerve; $L$, lingual nerve; $A . V a$, communication with auricular branch of vagus; P.A, posterior auricular nerve. Sty.hy, nerve to stylo-hyoid; $D i$, nerve to digastric (posterior belly); T.F, temporal-facial division; $C . F$, cervico-facial division; $T$, temporal; $M$, malar; $I . O$, infra-orbital; $B$, buccal, $S . M$, supra-mandibular; $I . M$, infra-mandibular branches. (Cunningham.)

for which they all depend directly on the fifth nerve, but also their sensibility to the special stimuli to which they are adapted.

The Sixth Nerve, the Abducens.-Origin.-The sixth nerve arises from a compact oval nucleus, situated somewhat deeply at the back part of the pons near the middle of the floor of the fourth ventricle. The eminentia teres marks its position. It contains moderately large cells with large nerve axis-cylinder processes. It is connected, figure 375 , with the nuclei of the third, fourth, and seventh nerves, and with reflex centers of the optic tracts, as previously mentioned. The root is thin, and passes ventrally and laterally 
through the reticular formation, to the surface, which it reaches at the lower edge of the pons, opposite the front end of the pyramid.

Functions. - The sixth nerve is exclusively motor, and supplies only the rectus externus muscle of the eye. The muscle is paralyzed when the nerve is divided. In all such cases of paralysis the eye squints inward and cannot be moved outward.

The Seventh Nerve, or Facial.-Origin.-The facial or seventh pair of nerves arises from the floor of the central part of the fourth ventricle, behind and in line with the motor nucleus of the fifth, to the outside of and deeper down than the nucleus of the sixth. The nucleus is narrower in front than behind, and consists of large motor cells with well-marked axis-cylinder processes, which are gathered up at the dorsal surface of the nucleus to form a root. The root describes a loop around the nucleus of the sixth nerve, running forward for some little distance dorsal to the nucleus, then descending vertically, passing to the outside of its own nucleus, between it and the

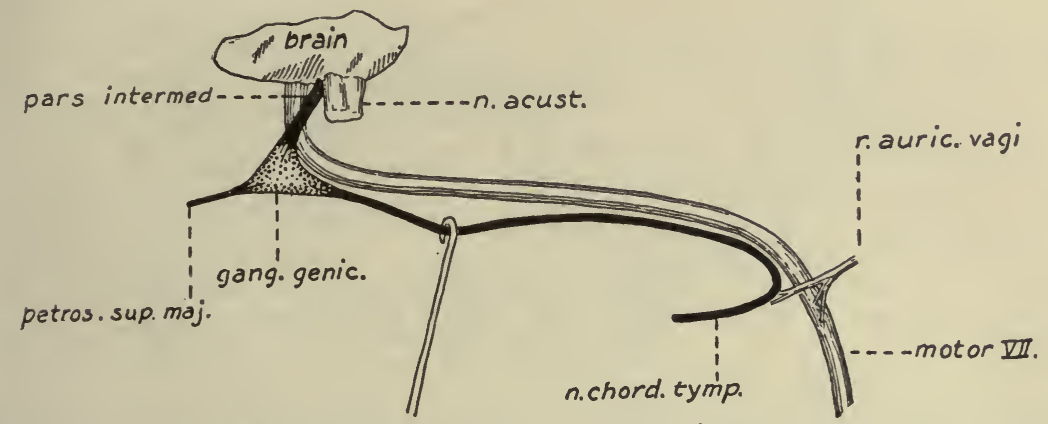

Fig. 4I3.-Dissection of the Sensory and Motor Divisions of the Facial in a 20-cm. Embryo (Pig). (Streeter.)

descending root of the fifth nerve. It emerges at the lower margin of the pons, lateral to the sixth nerve, opposite the front edge of the groove between the olivary and restiform bodies. The second or sensory root is smaller and is called the pars intermedia, figure $4 \mathrm{I} 2$. It is the portion which is connected with the chorda tympani and geniculate ganglion, figure 4I3. The pars intermedia terminates within the brain in the fasciculus solitarius in common with the glosso-pharyngeal.

Functions. - The seventh nerve is the motor nerve of all the muscles of the face, including the platysma, but not including the muscles of mastication. It supplies the stapedius, the stylo-hyoid and the posterior part of the digastric. Its branches also supply the rudimentary muscles of the external ear.

Fibers from the chorda tympani are distributed to the submaxillary gland and produce secretion when stimulated.

When the facial nerve is divided or in any other way paralyzed, the loss of 42 
function in the muscles which it supplies interferes with the perfect exercise of the organs of the special senses. Thus, in paralysis of the facial nerve the orbicularis palpebrarum being powerless, the eye remains open through the unbalanced action of the levator palpebrx. The conjunctiva is thus continually exposed to the air and dust and is liable to repeated inflammation, which may end in thickening and opacity of the cornea.

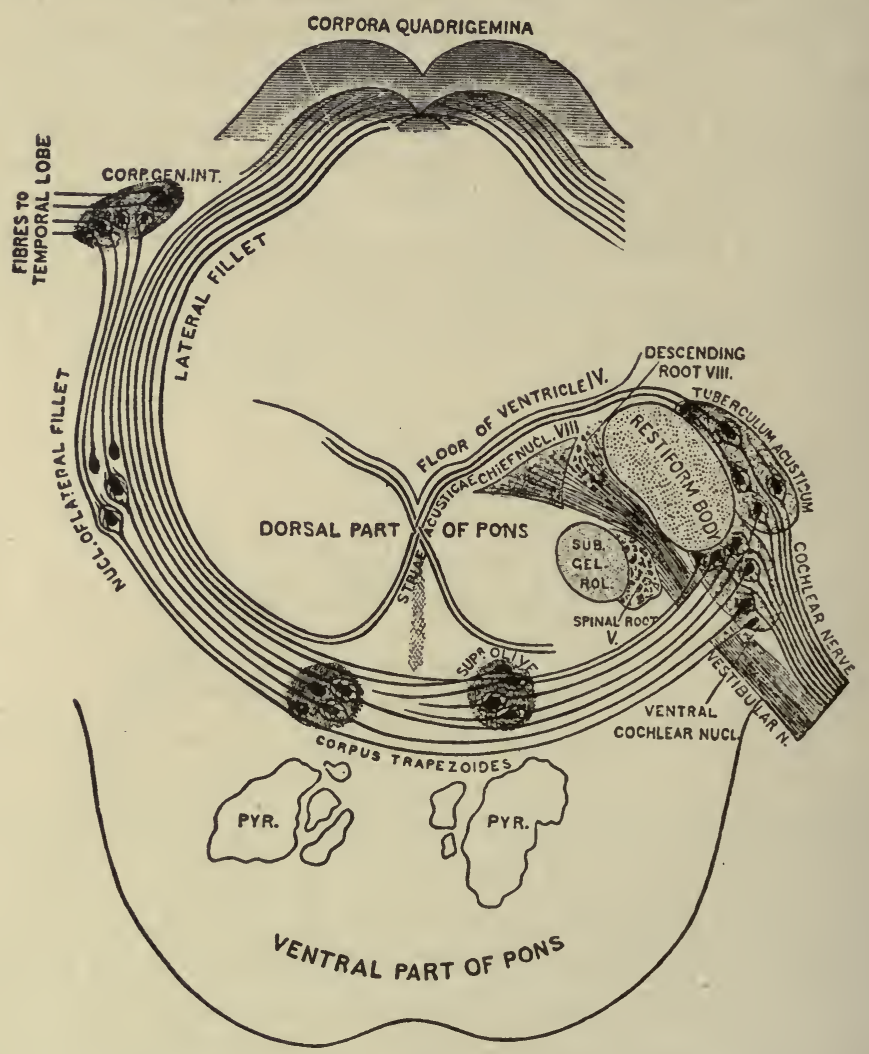

FIG. 4I4. - The Nuclei of Origin and Central Connections of the Auditory and Vestibular Nerve. (Cunningham.)

The sense of taste may be weakened or wholly lost in paralysis of the facial nerve, which involves the chorda tympani. This result, which has been observed in many instances of disease of the facial nerve in man, appears explicable on the supposition that the chorda tympani is the nerve of taste to the anterior two-thirds of the tongue, its fibers being distributed with the so-called gustatory or lingual branch of the fifth. Streeter has just published a study of the development of the seventh and eighth nerves in which he traces the chorda tympani through the pars intermedia, as shown in figure $4 \mathrm{I} 3$, thus settling this oft-disputed question. 
The Eighth Nerve, or Auditory.-The eighth nerve consists of two divisions, anatomically distinct and functionally independent. These are the vestibular and the cochlear divisions of the auditory nerve.

The cochlear division arises in the spiral ganglion and passes to the medulla to establish immediate connections with the ventral cochlear nucleus and the tuberculum acusticum. The central relations of these nuclei are established by the striæ acusticæ, the trapezoideus, and the lateral fillet with the internal corpus geniculatum and the inferior corpus quadrigeminum of the opposite side, as told by figure $4 \mathrm{I} 4$. These latter nuclei send tracts to the auditory center in the superior temporal gyrus.

The vestibular division arises in the vestibular ganglion, which is entirely distinct from the cochlear ganglion, and enters the medulla, passing to the lateral or chief auditory nucleus. From this point the relations are not fully established, but apparently fibers pass to the nucleus fastigii of the opposite side and to the vermis, where they are brought into relations with motor descending paths.

Functions. - The cochlear branch is the auditory nerve proper, and the vestibular is the nerve of equilibrium. The reader is referred to the chapter on Hearing for the details of function.

The Ninth Nerve, or Glosso-pharyngeal.-Origin.-The glosso-pharyngeal nerves, figure $378, I X$, arise by nuclei intimately associated with those of the vagus and spinal accessory nerves. The union of the nuclei is indeed so intimate that it will be as well to consider the origins of the ninth, tenth, and eleventh nerves together.

These three nerves emerge from the bulb and spinal cord in their numerical order from above downward, the bulbar portion from the lateral aspect of the bulb in a line between the olivary and restiform bodies; and the spinal portion from a line intermediate between the anterior and posterior nerve roots as far down as the sixth or seventh cervical spinal nerves.

The combined glosso-pharyngeal-accessory-vagus nucleus appears to consist of two parts, viz., one median or common origin, having conspicuous nerve cells of moderate size, and three lateral origins, having but few cells of small size. These are: I, the nucleus ambiguns, which lies on the lateral side of the reticular formation and is the motor origin of the glosso-pharyngeal, the vagus, and the spinal accessory; 2, the fasciculus solitarius, situated in the bulb, ventral and a little lateral to the combined nucleus, is also called the ascending root of the glosso-pharyngeal nerve or the respiratory bundle; and 3 , the spinal portion, which takes origin from a group of cells lying in the extreme lateral margin of the anterior cornu. This is the origin of the spinal accessory; it corresponds to the antero-lateral nucleus of the bulb, and the lateral part of the gray matter of the spinal cord.

The fibers of the spinal origin of the nerve pass from these cells through the lateral column to the surface of the cord. The fibers from the median 
part pass in a ventral and lateral direction through the reticular formation, then ventral to or through the gelatinous substance and strand of fibers connected with the fifth nerve, to the surface of the bulb.

The fibers from the nucleus ambiguus join the combined nerve, chiefly the vagus and the glosso-pharyngeal.

The bundles of fibers of the fasciculus solitarius start in the lateral gray matter of the cervical cord and higher in the reticular formation of the bulb, run longitudinally forward, to pass into the roots of the ninth nerve. It is composed of sensory fibers, chiefly of the glosso-pharyngeal, and of the pars intermedia of the facial.

The glosso-pharyngeal nerve gives filaments through its tympanic branch (Jacobson's nerve), to the fenestra ovalis and fenestra rotunda, and the Eustachian tube; also to the carotid plexus, and through the petrosal nerve, to the spheno-palatine ganglion. After communicating with the vagus and, soon after it leaves the cranium, with the sympathetic, with the digastric branch of the facial, and the accessory nerve, the glosso-pharyngeal divides into the two principal divisions indicated by its name. These divisions supply the mucous membrane of the posterior and lateral walls of the upper part of the pharynx, the Eustachian tube, the arches of the palate, the tonsils and their mucous membrane, and the tongue as far forward as the foramen cecum in the middle line, and to near the tip at the sides and inferior part.

Functions. - The glosso-pharyngeal nerve contains some motor fibers, together with fibers of common sensation and the sense of taste.

Motor fibers are distributed to the palato-pharyngeus, the stylo-pharyngeus, palato-glossus, and constrictors of the pharynx.

Sensory fibers of touch and of common sensation are distributed to the pharynx, the tonsils, and posterior palate. Nerves of taste are supplied to the taste buds on the posterior third of the tongue and to the fauces.

The Tenth Nerve, Vagus or Pneumogastric Nerve. - The origin of the vagus nerve is, as we have just seen, situated in the lower half of the floor of the fourth ventricle, figure 378 . Its nucleus is said to represent the homologue of the cells of Clarke's column of the spinal cord. In origin it is closely connected with the ninth, eleventh, and the twelfth. The combined glosso-pharyngeal-vago-accessory nuclei lie outside of, close to, and parallel with the nucleus of the twelfth. There are two main vagal nuclei, one motor, the other sensory. The motor nucleus gives rise to a large group of efferent fibers of wide distribution in the body all belonging to the bulbar autonomics of Langley.

Distribution.-It has, of all the nerves, the most varied distribution and functions, either through its filaments, or through sensory fibers which are mingled in its branches, and give rise to most varied reflex reactions. The vagus supplies sensory branches, which accompany the sympathetic 


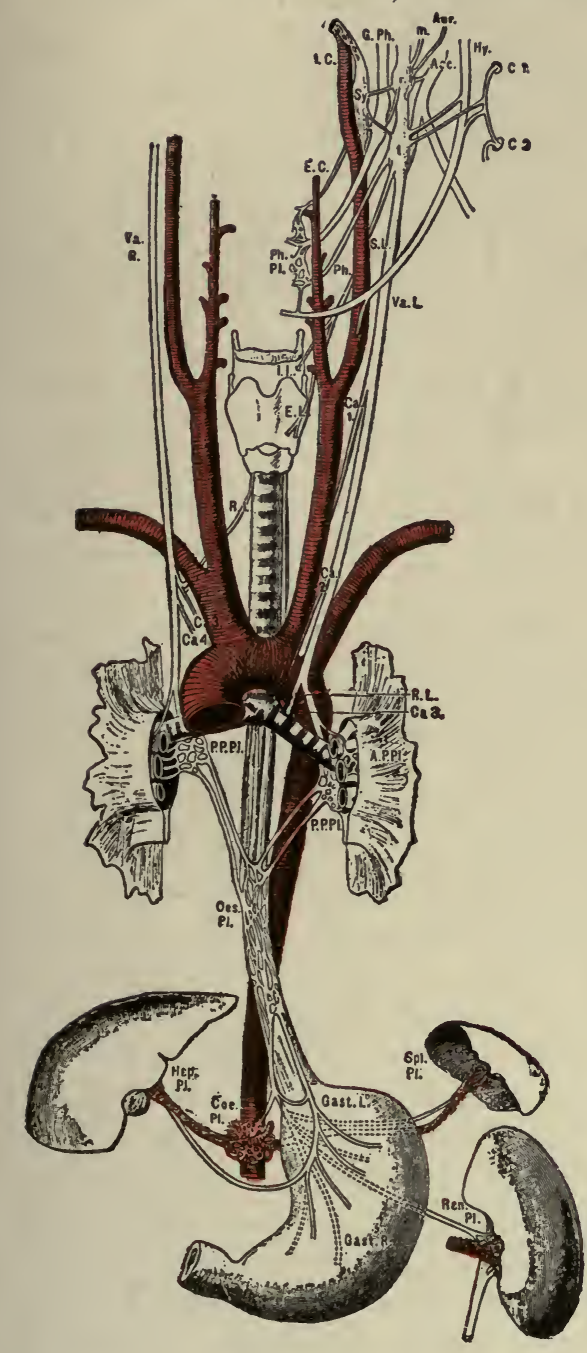

FIG. 4I5.

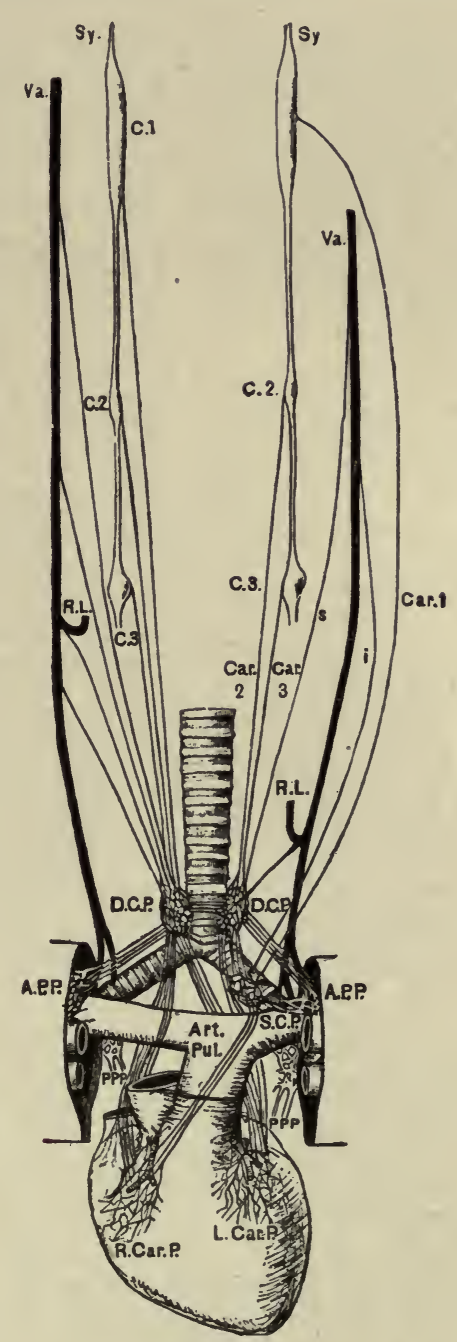

FIG. 4 I6.

Fig. 4I5.-The Distribution of the Tenth or Vagus Nerve. Va.R, Va.L., Right and left vagi; $r$, ganglion of the root and connections with $S y$., sympathetic, superior cervical ganglion; g.Ph., glosso-pharyngeal; $A c c$., spinal accessory nerve; $m$, meningeal branch; $A u r$. auricular branch; $t$, ganglion of the trunk and connections with $H y$., hypoglossal nerve; $C_{\mathrm{I}}, C_{2}$, loop between the first two cervical nerves-Sy., sympathetic, $A c c$., spinal accessory nerve; $P h$., pharyngeal branch; $P h . P l$., pharyngeal plexus; S.L., superior laryngeal nerve; I.L., internal laryngeal branch; E.L., external laryngeal branch; I.C., internal, and E.C., external carotid arteries; $C a$ I, superior cervical cardiac branch; $\mathrm{Ca}$, inferior cervical cardiac branch; R.L., recurrent laryngeal nerve; $\mathrm{Ca}_{3}$, cardiac branches of recurrent laryngeal nerve; $\mathrm{Ca}_{4}$, thoracic cardiac branch (right vagus); A.P.Pl., anterior, and P.P.Pl., posterior pulmonary plexuses; Oes.Pl., esophageal plexus; Gast.R. and Gast.L., gastric branches of vagus (right and left); Coe.Pl., celiac plexus; $H e p . P l$., hepatic plexus; $P l$., splenic plexus; Ren.Pl, renal plexus. (Cunningham.)

FIG. 4I6. - The Constitution of the Cardiac Plexus. Sy., Cervical sympathetic cord; C.I, superior, $C .2$, middle, and $C .3$, inferior cervical ganglia; $C a r . I$, superior, $C a r .2$, middle, and $C a r .3$, inferior cervical cardiac sympathetic branches; $V a$., vagus nerve; R.L., recurrent laryngeal nerve; $s$, superior, and $i$, inferior cervical cardiac branches of vagus; D.C.P., deep cardiac plexus; S.C.P., superficial cardiac plexus; A.P.P., anterior pulmonary plexus; P.P.P., posterior pulmonary plexus; R.Car.P., right, and L.Car.P., left coronary plexuses; $A r t . P u l_{\text {., }}$ pulmonary artery. (Cunningham.) 
on the middle meningeal artery supplying the back part of the meatus and the adjoining part of the external ear. It is connected with the petrous ganglion of the glosso-pharyngeal and with the spinal accessory which contributes motor fibers for the larger and upper portion of the esophagus, and inhibitory fibers for the heart. It is connected also with the twelfth; with the superior cervical ganglion of the sympathetic; and with the cervical plexus. The organs supplied by the branches of the vagus are as follows:

I. A large portion of the mucous membrane and some of the muscles of the pharynx are supplied by its pharyngeal branches, through the pharyngeal plexus.

2. The mucous membrane of the under surface of the epiglottis, and of the greater part of the larynx, and the crico-thyroid muscle, by the superior laryngeal nerve.

3. The mucous membrane and muscular fibers of the trachea, the lower part of the pharynx and larynx, and all the muscles of the larynx except the crico-thyroid are supplied by the inferior laryngeal nerve. It also supplies the first segment of the esophagus.

4. The mucous membrane and muscular coats of the esophagus receive fibers from the esophageal branches.

5. The branches of the vagus form the supply of inhibitory nerves to the heart and the sensory distribution of depressor fibers to the great arteries.

6. The sensory fibers of the lungs and the bronchial muscle motor nerves are supplied through the anterior and posterior pulmonary plexuses.

7. The stomach, the intestines, the spleen, and the liver are supplied by the gastric, splenic, and hepatic vagus branches.

Functions.- Throughout its whole course the vagus contains both sensory and motor fibers. To summarize the many functions of this nerve, which have been for the most part considered in the preceding chapters, it may be said that it supplies, I, motor fibers to the pharynx and esophagus, to the stomach and intestines, to the larynx, trachea, bronchi, and lungs; 2 , sensory fibers to the same regions; 3 , inhibitory fibers to the heart; 4, inhibitory afferent fibers, i.e., depressors, to the vaso-motor center.

Surgical division of both vagi or of both their recurrent branches is often ultimately fatal in young animals; but in old animals the division of the recurrent nerve is not generally fatal, and that of both the vagi, even, is not always fatal.

The Eleventh Nerve, or Spinal Accessory.-This nerve arises by two nuclei, one the nucleus ambiguus from a center in the floor of the fourth ventricle, partly but chiefly in the medulla and continuous with the glossopharyngeal-vagus nucleus; the other, from the outer side of the anterior cornu of the spinal cord as low down as the fifth or sixth cervical nerve. 
The fibers from the two origins come together at the jugular foramen, but separate again into two branches. The inner arises from the medulla and joins the vagus, to which it supplies fibers, consisting of small medullated nerve fibers. The outer consists of large medullated fibers and supplies the trapezius and sterno-mastoid muscles. The muscles of the larynx, all of which are supplied, apparently, by branches of the vagus, are said to derive their motor nerves from the accessory; and Vrolik makes the very significant statement that in the chimpanzee the internal branch of the accessory does not join the vagus at all, but goes direct to the larynx.

The Twelfth Nerve, or Hypoglossal.-Origin and Connections.-The nerve arises from a large-celled and very long nucleus in the bulb, extending from the floor of the fourth ventricle to the level of the olivary bodies close to the mid-line and inside the nucleus ambiguus. Fibers from this nucleus run from the ventral surface through the reticular formation in a series of bundles passing between the olivary nucleus laterally and the pyramid and accessory olive medially, to gain the ventral surface. The nerve emerges from a groove between the pyramid and olivary body. The fibers of origin are continuous with the anterior roots of the spinal nerves.

This nerve is the motor nerve to the muscles connected with the hyoid bone, including those of the tongue. It supplies the sterno-hyoid, sternothyroid, and omo-hyoid through its desceriding branch, descendens hypoglossi; the thyro-hyoid through a special branch; and the genio-hyoid, stylo-glossus, hyo-glossus, and genio-hyoglossus and linguales through its lingual branches.

Functions.- The function of the hypoglossal is exclusively motor. In cases of hemiplegia involving the functions of the hypoglossal nerve the tongue when protruded deviates toward the paralyzed side, when withdrawn it turns away from the paralyzed side. Occasionally it is not possible to observe any deviation in the direction of the protruded tongue; probably because the tongue is so compact and firm that the muscles on either side can push it straight forward or turn it for some distance toward either side. In hypoglossal paralysis from cerebral lesions or lesions of the peduncles the paralysis is contralateral.

\section{THE AUTONOMIC SYSTEM.}

In the introductory statement of this chapter we emphasized the fact that the outlying anatomical nerve divisions long known as the sympathetic system is an organic and constituent part of the general nervous system. Histologically there is close relation of the constituent parts, embryonically there is a common origin of the neurones, and physiologically the mechanisms are dependently related. The main portion of this group of neurones we discuss under the title The Autonomic System. 
In the discussion the intimate anatomical relationships must be constantly kept in view.

Organization and Distribution.-Strictly speaking, the name autonomic nervous system emphasizes physiological groupings of nerve cells and pathways rather than anatomical structures. However, the fact is that outside the brain and spinal cord and exclusive of the somatic nerves there are numerous nerve ganglia. These ganglia are connected by tracts with each other and with the cerebro-spinal axis.

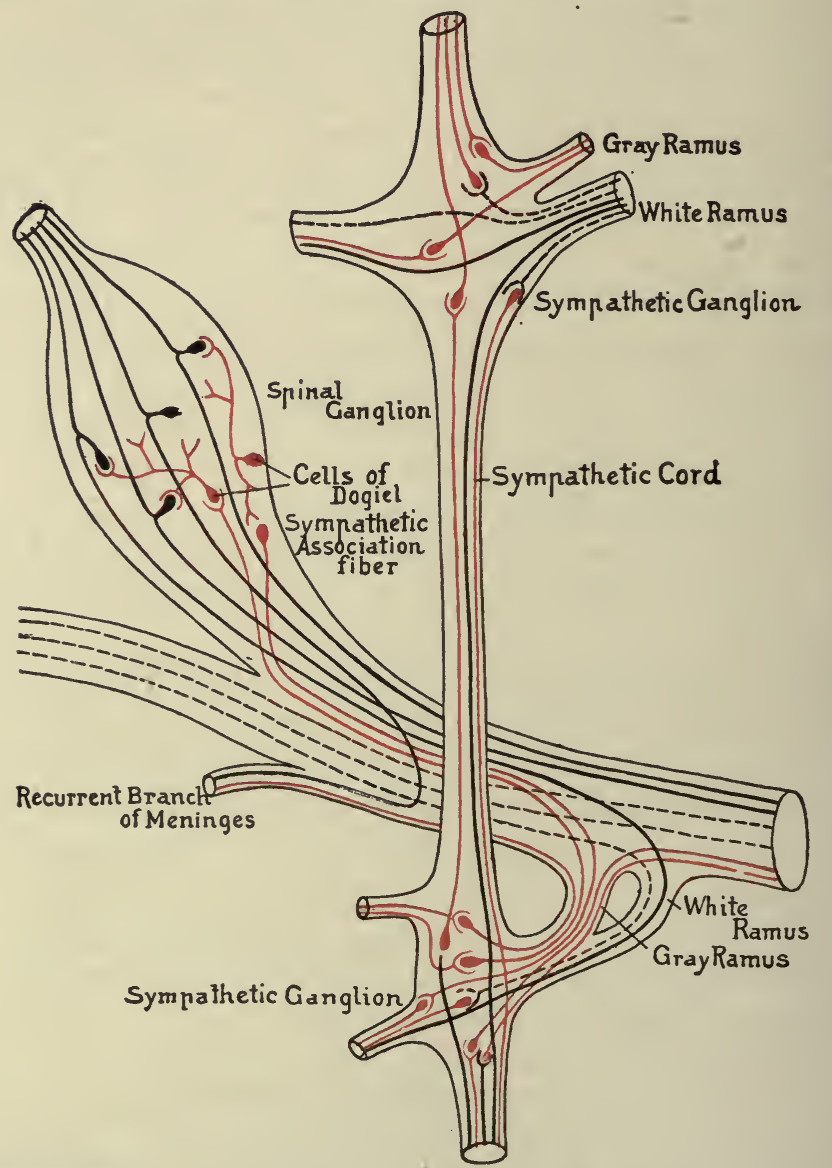

FIG. 4I7.-Schematic Representation of the Relation of the Constituents of the Sympathetic Chain and the Spinal Nerve. (Modified from Hardesty, in Morris' Anatomy.)

The chief gross constituent unit to be mentioned is a bilaterally symmetrical double chain of ganglia and connecting nerves extending from the cranium to the pelvis and along each side of and in front of the vertebral column. A ganglion is connected with each of the spinal nerves 
below the region of the first dorsal, but there are only three cervical ganglia in the neck and certain ganglia of questionable homology in the head region. This chain of ganglia is known in the historical anatomical literature and in the older physiological literature as the sympathetic system.

There are, however, numerous other outlying ganglia such as the ganglia of the celiac axis, the superior mesenteric and hypogastric plexuses, as well as such secondary or collateral plexuses as the aortic, renal, etc. From these ganglia nerves are distributed to other parts of the system and to the motor end mechanisms. The small ganglia in connection with those branches of the fifth and other cerebral nerves which are distributed in the neighborhood of the organs of special sense, namely, the ophthalmic, otic, spheno-palatine, and submaxillary ganglia, etc., all belong to the cranial autonomics as we shall presently see. Similar small ganglia are found on the sacral autonomic pathways.

Physiologically there are two functional types of nerve fibers, afferent and efferent, which run in autonomic pathways. The former or afferent fibers arise in the sensory epithelium of the alimentary canal, lungs or blood vessels, and from special sense organs such as the pacinian corpuscles of the mesentery. The second or efferent group constitutes the autonomic system proper. It is to this group that the cells of the sympathetic ganglia of all kinds belong.

There is still a third order of nerve-cell collections or ganglia found in the walls of hollow organs. The chief and best known of these ganglionic networks are found in the walls of the stomach and intestinal canal and are known as the plexuses of Auerbach, and of Meissner. Smaller collections of this type are found in the walls of the urinary bladder, the ureter, the uterus and vagina, and in different divisions of the male genital system. The term enteric system is applied to this type of nerve structure, specifically that part typical of the alimentary canal. These nets, which are for the most part microscopic, are also freely connected with other parts of the system, as well as with the cerebro-spinal axis.

Gross Anatomical Relations. - No special interest attaches to the gross anatomical relations outside of the fact that these factors are guides to the complicated neurone relations in which our present interest in the autonomic system lies. The ganglia of the sympathetic chain typically correspond segment by segment with the spinal nerves with which they are connected. The type arrangement is shown in figure 4I7. Each ganglion is connected with its corresponding spinal nerve by two nerve branches, the white and gray rami.

The secret of the presence of the two kinds of rami is revealed by the work of Gaskell on the type and character of the constituent neurones of the white and gray rami and of the spinal nerves, and of Langley and Anderson on the neurone connections and neurone pathways. It has been 
determined that the cord neurones contributing fibers to the autonomic system have their cell bodies of origin in the lateral and antero-lateral gray columns of the cord. Their axones are small medullated fibers which compose the major portion of the white rami. They are a third or a fourth of the diameter of ordinary medullated fibers, measuring $1.8 \mu$ to $2.7 \mu$ instead of $14 \mu$ to $19 \mu$. Such fibers are peculiar to the spinal nerve roots of the thoracic group and of the upper lumbar nerves. But they are also found in the second and third sacral nerves and constitute there the nervi erigentes which pass directly to the hypogastric plexus. These fibers end in arborizations about the cell bodies of the sympathetic ganglia.

White rami are lacking in the entire cranial and cervical regions. They are absent also in the lumbo-sacral cord below the fourth lumbar segment. Since the superior, middle, and inferior cervical ganglia are the only cervical representatives of the chain ganglia, it is evident that there is an atypical distribution of gray rami in the neck and head. The gray rami, distributed to cervical spinal nerves, arise in the first thoracic and inferior and the superior cervical ganglia.

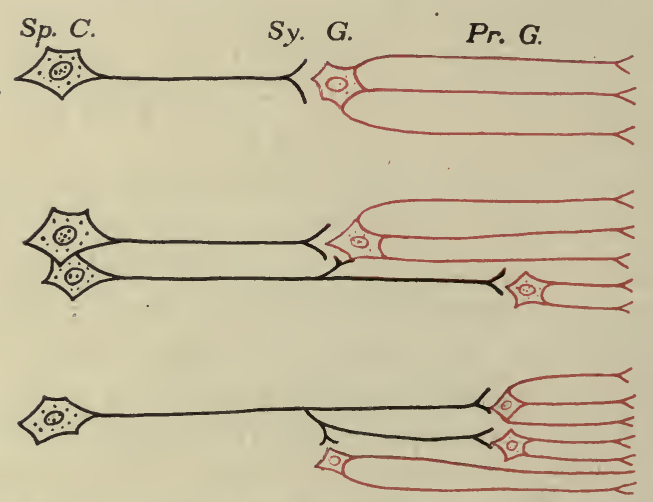

FIG. 4I8.-Langley's autonomic types of preganglionic, black, and postganglionic, red, neurones. Sp. C. = Spinal cord. Sy. G.= Sympathetic ganglia. Pr. G. = Peripheral ganglia, solius, hypogastric; etc. $\mathrm{Tr} .=$ Terminations in muscle, gland, etc.

The axones of the cells of the ganglia, fibers of the second order, constitute the continuation pathways either by the gray rami back to the somatic nerves and on to a distribution in the blood vessels, and the glands and skin of the trunk Or they often run by direct visceral branches to the lungs, heart, alimentary canal, urogenital system, etc.

The autonomic pathway, from the histological and functional standpoint, always consists of a two neurone chain. The last link in this chain is called by Langley the postganglionic neurone. The connecting link between the cord and brain stem and the sympathetic chain ganglia he designates the preganglionic neurone. Some of the ganglionic neurones 
do not form synapses immediately they enter a chain ganglion but pass on through to more distant ganglia, Pr. G., figure 4 I 8 .

The determination of the location of the synapses between pre- and postganglionic neurones was accomplished by the brilliant work of Langley and Anderson with the nicotine method. They used the discovery that nicotine readily poisons the synapses of preganglionic arborization on postganglionic neurones, thus effectively blocking physiological conduction through the synapse.

When nicotine is injected into the circulation or painted directly onto a ganglion it at first stimulates, then completely paralyzes the endings of any preganglionic arborizations about the cell bodies of the postganglionic neurones of the group. Nerve fibers as such do not lose their functions under these conditions. This observation enables one by the use of the nicotine method to determine in what particular ganglion nerve impulses to a peripheral organ are blocked. Langley and his students reëxplored the physiological distribution of fibers controlling visceral organs.

They found for example that the sympathetic nerves for the head and upper portion of the neck, which have their origin in the second to the fifth thoracic spinal nerve roots, have their pre-postganglionic synapses in the superior cervical ganglion. The outflow of autonomic nerves to the abdominal viscera which occurs in the cord from the seventh thoracic to the fourth lumbar have their preganglionic terminations in the ganglia of the celiac and the mesenteric plexuses. The pelvic viscera receive their fibers from the first four lumbar spinal nerves and the synapses are in the inferior mesenteric ganglia and certain smaller more distal ganglia. The type arrangement is diagrammatically presented by Langley as shown in figure $4 \mathrm{I} 8$.

The Double System.-The description of the outflow of efferent nerve fibers from the central nervous axis to autonomic mechanisms has thus far been based on the type region, i.e., from the first dorsal to the fourth lumbar inclusive. However, certain other regions of the central axis give rise to nerve fiber groups that bear both anatomical and physiological similarities to the dorsal region. These regions are that portion of the brain stem which gives rise to the oculomotor or third cranial nerve, especially those fibers which innervate the ciliary muscle and the iris; the motor roots of the seventh, ninth and tenth cranial nerves containing secretory, vasodilator, motor and inhibitory nerve fibers; and the region of the third and fourth segments of the sacral cord which gives rise to the fibers distributed to the urogenital system and muscles of the lower bowel. In each of these groups of anatomical nerves there is a pre- and postganglionic neurone in the physiological path to the terminal tissue. In the third cranial nerve, for example, the ciliary ganglion is the location of the pre-postganglionic synapse. In the inhibitory pathway of the vagus this 
synapse takes place in the cardiac ganglion. In short, the second group of ganglia described in the introductory paragraph on the autonomic system belongs to nerve mechanisms of this special group.

The efferent nerve groups of the cranial, thoracic and sacral regions are all similar in one functional regard, viz., there is no voluntary control of the functions which they influence. These nerves as a whole innervate the eyes, salivary glands, blood vessels, heart, bronchial tubes, stomach, intestine, colon, liver, pancreas, kidney, urinary bladder and genital organs, all so-called involuntary organs and mechanisms. All of these organs have a double innervation as shown in detail in figure 418. This similarity of neurone pathway and of involuntary functional control led Langley to reclassify the whole nerve outflow under the name, the autonomic system.

It will be seen that each visceral organ receives groups of fibers from two quite different sections of the brain or cord. However, physiologically these two sources of nerves provide the organ with diametrically opposed nerve influences controlling or regulating its function. For example, in the iris of the eye one set of fibers stimulates constriction of the pupil and the other dilation. One set of fibers, the vagus, inhibits the heart, the other set, the augmentors, accelerates it, etc. Autonomic fibers from the thoracico-lumbar cord in general have exactly the opposite influence on function which the fibers from the brain stem and the sacral cord exert. The similarity of functional differentiation is revealed also by the similarity of action of drugs on the cranial and sacral nerves, as contrasted with the thoracic and lumbar nerves. This group of autonomic nerves may therefore be subclassed into cranio-sacral and thoracico-lumbar autonomics. Langley originally classified the oculomotor and medullary outflow, and the sacral outflow as parasympathetic, in contrast with the thoracic, for which he retains the name sympathetic. It seems better to use the more logical classification in the functional grouping as outlined.

To the more diffuse innervation of the walls of hollow organs, such as the stomach and intestine, Langley gave the name enteric system, see page 669. The best known portion of the enteric system consists of the myenteric plexus (Auerbach) and the submucous plexus (Meissner). These diffuse nets of ganglia are the only groups of nerve cells outside of the central axis in the mammalia known to coordinate reflexes. The system is phylogenetically an older nerve differentiation. The enteric ganglia are also pathways for extrinsic autonomic nerves from the cerebrospinal axis in which it is assumed with little or no proof that the enteric ganglion cells are postganglionic in their homologies. Portions of the urogenital system, tubules and vesicles, possess a similar diffuse nerve mechanism. The whole schema is tabulated below. This classification is now rather 
broadly accepted in physiology and forms a more rational system around which we may group the divers physiological functions, see figure 4 I 9 .

\section{THE AUTONOMIC NERVOUS SYSTEM.}

I. The Thoracicolumbar Autonomics.

The First dorsal to the Fourth lumbar inclusive.

II. The Craniosacral Autonomics.

The midbrain autonomic nerves.

The $3 \mathrm{~d}$ cranial or oculomotor.

The bulbar autonomics.

The 7 th or facial.

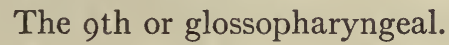

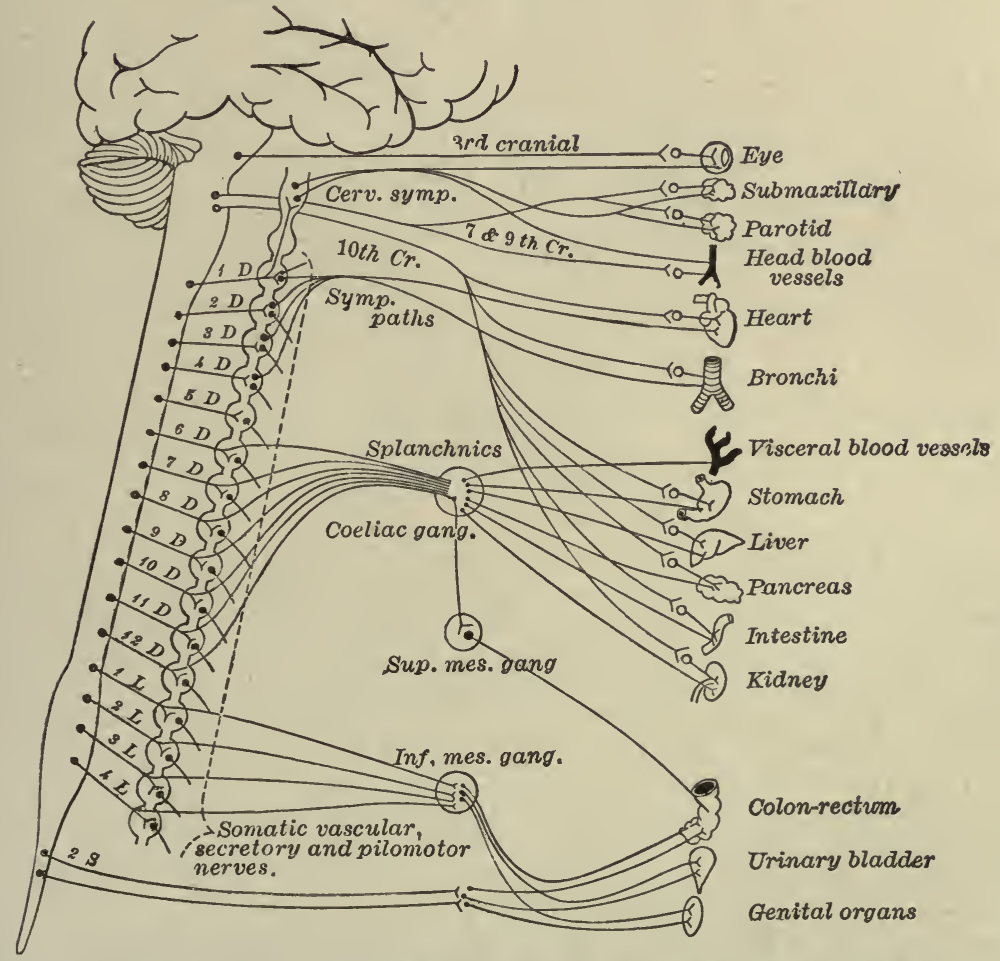

FIG. 4I9.-Diagrammatic representation of the paths of the autonomic nervous distribution. (Modified from Meyers and Gottlieb.)

The Ioth or vagus.

The inth or spinal accessory.

The sacral autonomics.

The $2 \mathrm{~d}$ and $3 \mathrm{~d}$ sacral or the nervus erigens.

III. The enteric or visceral autonomics.

Plexus of Auerbach.

Plexus of Meissner. 
Functions of the Autonomic Nerves.-The Thoracicolumbar Autonom$i c s$.-As we have already seen, the thoracicolumbar fibers have their spinal origin from the first thoracic to the fourth lumbar spinal segments. Yet these fibers are distributed to all portions of the body. They accomplish the following functions: vasomotors to the blood vessels of the entire body; vasodilators to the blood vessels of the body exclusive of the portions of the skin of the head; the salivary gland, gastric and pancreatic glands and urogenital system; they furnish motor nerves to the heart and inhibitory nerves to the bronchi, stomach and intestine, and both motor and inhibitory nerves to portions of the urogenital system; secretory nerves for the sweat glands throughout the body; and the pilomotor nerves of the skin. The details of functional control of these divisions of the thoracic autonomics have been discussed in presenting the physiology of the organs concerned.

The Third Cranial Nerve. - Langley has divided the cranial autonomics into the mid-brain autonomics-the third cranial nerves, and the bulbar autonomics - the seventh, ninth, tenth and eleventh cranial nerves.

The oculomotor or third cranial nerve is an efferent nerve distributed to the extraocular muscles and to the muscles of the iris and ciliary apparatus. The fibers to the extraocular muscles are of the usual skeletal motor type. The fibers of the second group belong to the autonomic system. These nerves run by way of the ciliary ganglia where they have their prepostganglionic synapses. They are distributed to the circular muscles of the eyes which on stimulation cause constriction of the pupils, and to the two sets of muscles in the ciliary apparatus which on stimulation lead to the act of accommodation.

The Seventh Cranial Nerve.-The intermediate nerve of Wrisberg contains the autonomic fibers of the facial, figure 4I3. Fibers of this group constitute the chorda tympani whose function was discovered by Bernard when he demonstrated the presence of secretory fibers to the submaxillary gland. He also proved the presence of vasodilator nerves through this same channel. The chorda tympani stands today as the typical example of a vasodilator and secretory nerve, both functions of the involuntary or autonomic motor type. The cell bodies of the postganglionic neurones lie in the ganglia at the hilus of the gland. The seventh apparently also distributes preganglionic fibers to the spheno-palatine ganglion from whence postganglionic fibers are distributed to the mucous membrane of the nose and upper respiratory passages.

The Ninth Cranial Nerve. - The tympanic branch of the ninth cranial nerve supplies vasodilator and secretory fibers to the parotid gland. 
The Tenth and Eleventh Cranial Nerves.-The vagus or tenth cranial nerve is perhaps the most composite of all the autonomic nerve pathways. It together with the roots distributed to it from the accessory supplies inhibitory fibers to the heart; motor fibers to the bronchi, esophagus, stomach and intestine; and secretory fibers to the gastric glands, pancreas. and liver. The pre-postganglionic unions are found in small terminal ganglia such as the local cardiac ganglia.

The Sacral Autonomics. - These fibers arise from the sacral cord at the level of the second and third sacral nerves. The fibers do not join the

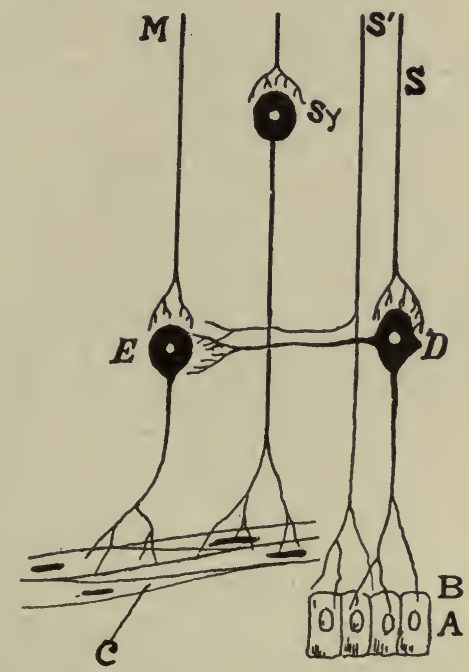

FIG. 4rgA.-Diagrammatic representation of the scheme of innervation of the alimentary canal. $A$, mucosa; $B$, sensory nerve endings; $C$, gastric or intestinal wall; $D$ and $E$, sensory and motor cell bodies in the enteric plexus; $M$, motor neurons, vagus fibers for the stomach and upper portion of the intestine, sympathetic fiber lower down; $S$ and $S^{\prime}$, afferent sensory nerves; $S y$, sympathetic ganglion, pre-postganglionic synapse, inhibitory path for the mammalian canal. The fiber $S^{\prime}$ is introduced to Dixon's original figure on the basis of his foot-note. (Greene, modified from Dixon.)

main sympathetic chain but are distributed in the nervus erigens by way of the ganglia of the pelvic plexus. The nerves furnish motor fibers to the bladder, the descending colon and rectum; inhibitory fibers to the sphincter muscles of the bladder. They also contain vasodilators for the rectum, anus and penis; also inhibitory fibers to the retractor penis muscle. The urogenital system obviously receives its double innervation in part from the thoracic autonomics and in part from the sacral autonomics.

The Enteric Nervous System.-The enteric autonomics consist of those ganglia and fibers lying between the muscle walls of such hollow organs as the stomach, intestine, etc. Among all the numerous peripheral ganglia of the body the ganglia in the stomach and intestinal wall are the only ones 
for which we have proof of local reflex control. The regulation of the pyloric sphincter through the acid control is an example of such local reflex. This reaction takes place in the isolated organ. In certain ways the enteric system seems to be most primitive in structure, comparable to the nervous system we find in lower organism, such as anemones and medusæ. However, the ganglion cells of this system have been proven to arise from the neural groove and to migrate to their adult location during embryonic development. Out of the investigations as to the function of these mechanisms we have the idea of control represented in figure $4^{\mathrm{I}} 9 \mathrm{~A}$.

According to Gaskell the functions of the main sympathetic ganglia are the following: r. The sympathetic ganglia are aggregates of large numbers of multipolar cells around which the medullated fibers of the white rami form synapses. The branches of the cells are of the non-medullated fiber type. Thus a medullated conduction is converted in the ganglia into a non-medullated path beyond the ganglia. 2. The ganglion cells exercise a nutritive influence over the tissues to which their fibers are distributed. 3. The number of preganglionic fibers entering the ganglia is not so great as that leaving, since the cells are multipolar (not shown in the schematic figure $4 \mathrm{I} 7$ ). This serves to multiply the influence of a relatively simple efferent preganglionic conduction path and to extend it over a larger area the parts of which are usually acting co-ordinatively.

The sympathetic ganglia are not nerve centers in the usual sense. It is better to regard them merely as distributing organs in which impulses of central origin and comparatively simple type are distributed over relatively large areas. These ganglia do not possess the power of reflex function except in the enteric system as noted above. A type of pseudo-reflex has been described depending on the law of neurone reaction. But it is not supposed that such reflexes occur in the normal animal.

Afferent Fibers in Sympathetic Paths.-Afferent or sensory fibers of the ordinary spinal-root ganglion cells are present in the sympathetic nerves of the splanchnic region. These fibers are the distal branches of posterior root ganglion cells and are distributed to the visceral region by way of the white rami and the sympathetic system. These fibers have their terminations in the mucosa of the alimentary and urogenital system or in the muscular walls of these organs. Their stimulation arouses the sensation of fullness (Hertz) that characterizes hollow organs, also sensations of pain and temperature as shown by Carlson for the stomach. It is the stimulating influence of operative conditions on this class of sensory fibers that plays so important a part in surgical shock. Modern practices in anesthesia are especially devised to prevent this dangerous state of depression. Such afferent paths are not a constituent part of the autonomic classification of Langley. However, Dogiel has described a type of true afferent sympathetic neurone. Their fibers arise from cells located in the 
sympathetic ganglia, and pass through the rami communicantes, to end by terminal arborizations in the spinal root ganglia, chiefly around cells of the Dogiel type. The number and significance of this class of afferent neurones remain as yet uncertain. Though their spinal relations suggest some association with the phenomenon of referred pain.

\section{THE PHYSIOLOGY OF SLEEP.}

All parts of the body which are the seat of active change require periods of rest. The alternation of work and rest is a necessary condition of their maintenance and of the healthy performance of their functions. These alternating periods, however, differ much in duration in different organs or regions of the body. But, for any individual instance they preserve a general and rather close uniformity. Thus, the periods of rest and work mentioned in the case of the heart occupy, each of them, about half a second. In the case of the ordinary respiratory muscles the periods are about four or five times as long. In many cases (as of the voluntary muscles during violent exercise), while the periods during active exertion alternate very frequently, yet the expenditure goes far ahead of the repair, and to compensate for this an after-repose of some hours becomes necessary, the rhythm being less perfect as to time than in the case of the muscles concerned in circulation and respiration.

Obviously, short periods of activity and repose, or in other words, of consciousness and unconsciousness would be impossible in the case of the brain. The repose must occur at long intervals and must be proportionately long. Hence the necessity for that condition which we call Sleep; a condition which, at first sight seeming exceptional, is only an unusually perfect example of what occurs at varying intervals in every actively working portion of our bodies.

By exposing the surface of the brain of a living animal at a circumscribed spot, and protecting the exposed part by a watch-glass, Durham was able to prove that the brain becomes visibly paler, anemic, during sleep. And the anemia of the optic disc during sleep, observed by Hughlings Jackson, may by analogy be taken as a strong confirmation of the same fact.

The Circulation During Sleep.-Blood is supplied to the brain in four distinct but anastomosing arteries. This efficient anatomical arrangement is obviously all the more important when it is remembered that the circulation in the brain has only an inefficient local device for regulating the blood-flow, and that the circulation of the brain is constantly influenced by the variations in general blood pressure, see page 242 .

Howell and others have studied the circulation by the plethysmographic method during sleep. The results show that with the loss of consciousness, 
and immediately following, there is a sharp dilatation of the blood vessels of the arm, probably chiefly of the skin, as shown by the increase in volume. The vessels remain dilated until the individual begins to awaken, then there is a rapid constriction with decrease of volume of the organ.

The dilatation of the general blood vessels draws off the supply of blood from the brain, and the resulting partial anemia contributes to loss of consciousness. The blood supply is ample for the growth, repair and rest of the nervous system. How efficient this rest period is for the rejuvenation of the nervous tissue is indicated even by the relatively gross means, figure 357 , shown in the histological preparations of nerve cells.

Somnambulism and Dreams. - What we term sleep occurs often in very different degrees in different parts of the nervous system; and in reference to some parts the expression cannot be used in the ordinary sense. For example, during the most profound cerebral sleep the medulla is discharging rhythmic nerve impulses to maintain respiratory movements.

The phenomena of dreams and somnambulism are examples of differing degrees of sleep in different parts of the cerebro-spinal nervous system. In the former case the cerebrum is still partially active; but the activity is no longer corrected by the reception, on the part of the sleeping sensorium, of impressions of objects belonging to the outer world. Neither can the cerebrum in this half-awake condition control the centers of reflex action of the voluntary muscles so as to cause the latter to contract in close coordination with the changing cerebral reactions as during waking hours -a fact within the painful experience of all who have suffered from nightmare.

In somnambulism the higher centers are capable of co-ordinating that train of reflex nervous action which is necessary for progression, while the nerve center of the muscular equilibrium sense (in the cerebellum) is, presumably, fully awake; but the sensorium is still asleep, and impressions made on it are not sufficiently felt to rouse the cerebrum to a comparison of the difference between mere ideas or memories and sensations derived from external objects. 


\section{LABORATORY EXPERIMENTS ON THE NERVOUS SYSTEM.}

I. Irritability of the Neurone.-Repeat the tests outlined in Experiment 3 under Laboratory Experiments in Muscle. Apply these tests both to nerve trunk and to any ganglion, for example, the ganglion on the posterior root of the tenth spinal nerve of the frog, or the superior cervical or the celiac ganglion of the cat.

2. Conductivity of the Neurone. $-a$. Conductivity can be demonstrated quantitatively by the method of Experiment 19, under Muscle Nerve Physiology, using the muscle as an index of nerve activity.

$b$. A better method is to measure the latency of the action current of an isolated sciatic nerve. Connect one end of the nerve by means of nonpolarizable electrodes with a delicate galvanometer. Stimulate the nerve at the far end, then at the near end.

c. That conduction is in either direction can also be demonstrated on branched motor nerves. Dissect out the sartorius muscle of the frog with its motor nerve, which has two branches. Split the muscle between the branches. Pick up one end and stimulate its nerve branch. Both muscles will contract. The nerve impulse developed at the point of stimulation also travels away from its muscle to the point of branching of the axone, then down the second nerve branch to stimulate the second division of the muscle. Branched axones are involved, not separate neurones. This is the type of reaction in a pseudo reflex.

3. Differentiation Polarity in Neurones.-Neurones have a polarity because of two facts, first, their anatomical relations, and second, their physiological contacts. Afferent or sensory nerves cannot be stimulated normally except at the sense organs. The nerve impulses developed must pass in the centripetal direction, affecting such other neurones as are in physiological contact, until a motor organ is reached.

Differentiations can be demonstrated by certain drugs, for example, paint a nerve trunk with nicotine. No effect follows. Paint a ganglion, the nicotine stimulates, then poisons, the cell bodies of the ganglion. Or, paint the accelerator nerve trunks to the heart with o.I per cent. adrenalin, no obvious change occurs. Paint the heart or perfuse the heart with adrenalin to bring the hormone in contact with the cardiac motor nerve endings. The endings are specifically stimulated and the heart beats faster and stronger.

4. The Afferent and Efferent Functions of the Spinal Nerve Roots.Skilfully dissect and expose the two roots of the eighth, ninth, and tenth spinal nerves of the right side of a large frog. Reserve the left side for a second try. Insert silk threads under each root using care not to stretch or injure the delicate nerves. The posterior root is the smaller and its orange-colored ganglion aids in identifying it. 
a. Stimulate the posterior root with weak induction currents. Vary the strength till muscular contractions occur in the leg on the same side.

$b$. Section the root in the middle. Stimulate the peripheral end. Stimulate the central end. Do not allow escape of the stimulating current to other tissues nor to the anterior root.

c. Stimulate the anterior root and note the contractions that result.

$d$. Section the anterior root in its middle and stimulate the peripheral end, using as weak a stimulus as you can for effective contractions. Now stimulate the central end.

$e$. Demonstrate the crossed reflex if possible by stimulating the central end of a posterior root strongly. Stimulate the central end of the brachial nerve and look for crossed reflexes.

$f$. Expose the third spinal nerve, cut it and stimulate the central end in an attempt to show both crossed and descending reflexes that shall include movements of the hind legs.

5. The Reflex Act, Spinal Frog.-Prepare a spinal frog by transecting the cord where it joins the medulla, the posterior border of the tympanum, and pith the brain. This spinal animal will remain quiet in whatever position.

a. Place in the prone position with the hind legs stretched full length, pinch the tip of the toe, after a short latent period the leg of that side will be drawn up into the usual position by its side. If the stimulation is continued and strong enough contractions of the opposite muscles will occur.

$b$. Suspend the frog by its jaws and stimulate different points of the skin with 4 per cent. sulphuric acid applied by wet bits of blotting paper. Complicated though orderly contractions occur in the muscles of now one, now the other, or both legs according to which spot is stimulated by the acid.

c. Stimuli applied in the median line of the body lead to symmetrical contractions. These contractions fail if the spinal cord is destroyed. Hence they are reflex acts, simple, or coordinated; unilateral or crossed, etc. Convulsive reactions occur when the stimulation is too violerit.

6. Decerebrate Frog.-Prepare a series of frogs by destroying portions of the brain as follows:

a. The upper portion of the cerebrum, not injuring the olfactory or optic tracts.

$b$. All the cerebrum without injury to the optic lobes and tracts.

c. Destroy the cerebrum and optic lobes, without injury to the base of the brain or optic tracts.

$d$. Destroy all parts of the brain in front of the medulla.

$e$. Section at the point of union of the spinal cord and medulla and destroy the brain completely. 
Compare this series of frogs with the behavior of the normal frog as regards the following points: $a$. position; $b$. spontaneous movements; $c$. movements in response to stimuli of different types and intensities, i.e., electrical, chemical, disturbed position, etc. These notes should be carefully tabulated and reported.

7. The Decerebrate Pigeon.-Observe the behavior of a young pigeon, then remove the entire cerebral cortex. The operation must be done aseptically under ether and 1/100 grain of atropine. Scoop the cortex out with care not to injure the brain ganglia, optic lobes and tracts. Control bleeding by hot tampons. Close the wound aseptically.

Keep the animal for several weeks, feeding and watering by placing food in the back of the mouth. Healing will quickly occur and the animal will show certain normal behaviors from the moment it recovers from ether. Examine:

a. Its spontaneous activity.

b. Its ability to take feed, swallow, etc.

c. Its responses to light, mechanical and other stimuli.

$d$. Its ability to balance on a perch and in the air.

$e$. Any daily improvement in its reactivity.

8. Stimulation of the Cerebral Cortex in the Dog or Cat.-Sensory and motor localizations of the cortex of the mammal have been described for certain areas. Under chloral and ether anesthesia expose the anterior surface of the cerebral hemisphere of the dog. Identify the crucial fissure. Stimulate points anterior, lateral and posterior to this fissure, recording and labeling the exact points on a map prepared for the purpose. Keep records of all muscular reactions. Areas can be identified which on stimulation produce contractions of muscles of the fore leg, of the hind leg, or of various parts of the trunk and tail, etc., verify such areas. Stimulate for short periods only. Avoid fatigue and protect the area from cooling. Especial care must be given to the degree of anesthesia.

9. Spinal Transection and Motor Control.-Under chloral and ether and with aseptic technic transect the spinal cord of a female dog at the level of the thirteenth dorsal. Aseptically close the wound and take hospital care of the animal while it is under observation.

$a$. All voluntary movements of the hind legs and pelvic musculature cease. It may be necessary to support the hind quarters.

$b$. Certain reflexes still take place through the isolated lumbar segments of the cord, for example, micturition and defecation.

c. Vasomotor control is at first lost, but is slowly regained in a few days.

Io. Spinal Hemisection.-Expose the spinal cord under chloral and ether. Carefully hemisect the right half of the cord in the twelfth segment and close as in the preceding test. Use surgical and aseptic technic.

$a$. Carefully map the area and degree of analgesia. 
$b$. Analyze and record the extent of motor paralysis.

c. Analyze all other disturbances.

II. Spinal Analgesia.-Temporary effects similar to complete section of the cord are produced on injecting cocaine into the spinal canal at the same level. Inject I cc. of 2 per cent. cocaine hydrochlorate into the spinal canal of the twelfth dorsal segment of a dog. Complete analgesia and loss of motor control occur in the hind legs and pelvic parts. This condition passes off in 20 to 40 minutes but is adequate for major surgical operations.

I2. Reflex Reaction Time of Man.-Arrange an electric buzzèr signal in circuit with a lead off from a sixty cycle alternating current lighting system. The circuit is controlled by a spring key with pin pricking attachment. Use a rapid moving Harvard drum for recording. No time signal is needed, since the buzzer not only records the opening and closing of the key but also the duration of the latent period in $1 / 120$ seconds. Have the reagent close the key quickly with his index finger. The resulting pinprick will be sufficient stimulus to produce reflex removal of the finger, particularly if he is left in ignorance of the pin. An electric shock can readily be arranged to stimulate the sensory surface only.

I3. Voluntary Reaction Time Conditioned by Simple Choice.- - Use the signal and recording apparatus arranged as outlined above. For the pin - substitute a simple spring key with flag signal device arranged to be used or not at the discretion of the experimentor. The reagent is instructed to close the key and open it instantly provided he sees the flag signal.

- Read off the reaction time from the buzzer vibrations. Any other sensory channel may be chosen instead of sight:

In extended experimental work more and more complicated nerve reactions should be measured. 


\section{CHAPTER XV.}

\section{THE SENSES.}

THROUGH the medium of the nervous system man obtains a knowledge of the existence both of the various parts of his body and of the external world. This knowledge is based upon sensations resulting from the stimulation of certain centers in the brain by nerve impulses conveyed to them by afferent nerves. Under normal circumstances the following structures are necessary for the physical development and mental perception of a sensation: $a$, A peripheral organ for the reception of the impression; $b$, a nerve for conducting it; $c$, a nerve center for feeling or perceiving it.

The senses may be conveniently classified according to the sensation which is experienced. Each sense organ when stimulated is supposed to lead to a sensation of distinctive character. Yet many of the sensations are vaguely defined, such as thirst, fatigue, etc. Other sensations have a very definite and readily identified quality such as sight, taste, etc. Sensations, whether definite or vague, are referred by us to some source or origin either in the body, $\mathrm{I}$, the internal or so-called common sensations, or outside the body, 2, the external or special senses. No sharp line can be drawn in this classification.

Internal Senses.-Under this head fall all those senses which produce sensations that are referred to an origin within the body, such as fatigue, discomfort, faintness, satiety, nausea, together with hunger, thirst, the muscle sense, and pain. In hunger and thirst there is a general bodily discomfort, but in many persons also a distinct sensation referred to the stomach or to the fauces. In this class must also be placed the various stimulations of the mucous membrane of the bronchi, which give rise to coughing, and also the sensations derived from various viscera. It is by means of the muscle sense that we become aware of the condition of the muscles, and thus obtain the information necessary for their adjustment to various purposes-standing, walking, grasping, etc. This muscular sensibility is shown in our power to estimate the differences between weights by the different muscular efforts necessary to raise them. It must be carefully distinguished from the sense of contact or pressure, of which the skin is the organ. When standing erect, we can feel the 'ground contact, and there is a sense of pressure, due to our feet being pressed against the ground by the weight of the body. Both these are derived from the skin of the sole of the foot. If now we raise the body on the toes, we are conscious, through the muscular sense, of a muscular 
effort made by the muscles of the calf. But the muscle sense will be discussed further, page 687 .

It is manifestly impossible to draw a very clear line of demarcation between some of these senses.

Special Senses.-The special senses include Touch, Temperature (Heat and Cold), Taste, Smell, Hearing, Sight.

The most important distinction between common and special sensations is that by the former we are made aware of certain conditions of various parts of our bodies, while from the latter is gained a knowledge of the external world. This difference will be clear if we compare the sensations of pain and touch, the former. of which is a common, the latter a special, sensation. "If we place the edge of a sharp knife on the skin, we feel the edge by means of our sense of touch; we perceive a sensation, and refer it to the object which has caused it. But as soon as we cut the skin with the knife, we feel pain, a feeling which we no longer refer to the cutting knife, but which we feel within ourselves, and which communicates to us the fact of a change of condition in our own body. By the sensation of pain we are neither able to recognize the object which caused it nor its nature."

It is important in studying the phenomena of sensation clearly to understand that the sensorium, or seat of sensation, is in the brain, and not in the particular organ through which the sensory impression is received. In common parlance we are said to see with the eye, hear with the ear, etc., but in reality these organs are only adapted to receive stimuli which produce changes that are, through their respective nerves, conducted to the sensorium, to give rise to sensation of sight, hearing, etc.

Hence, if the optic nerve is severed, vision is no longer possible. Although the image falls on the retina as before, the sensory impulse can no longer be conveyed to the sensorium. When any given sensation is felt, all that we can with certainty affirm is that some part of the brain is excited. The exciting cause may be some object of the external world, producing an objective sensation; or the condition of the sensorium may be due to some excitement within the brain itself, in which case the sensation is termed subjective. The mind habitually refers sensations to external causes; and hence, whenever they are subjective we can hardly divest ourselves of the idea of an external cause, and an illusion is the result.

Sensory Illusions.-Numberless examples of such illusions might be quoted. As familiar cases may be mentioned humming and buzzing in the ears caused by some irritation of the auditory nerve center. These stimuli may even be interpreted as musical sounds, or voices, sometimes termed auditory spectra. So-called optical illusions in which objects are described as seen, although not present, may be caused by changes going on in some part of the visual apparatus beyond the eye. Such illusions are most strikingly exemplified in cases of delirium tremens or other forms of 
delirium, and may take the form of animals such as cats, rats, or creeping loathsome forms, etc.

One uniform internal cause, which may act on all the nerves of the senses in the same manner, is capillary congestion. This one cause excites in the retina, while the eyes are closed, the sensations of light and luminous flashes; in the auditory nerve, the sensation of humming and ringing sounds; in the olfactory nerve, the sense of odors; and the nerves of feeling, the sensation of pain. In the same way a chemical substance introduced into the blood may excite in the nerves of each sense peculiar symptoms: In the optic nerves, the appearance of luminous sparks before the eyes; in the auditory nerves, tinnitus aurium; and in the common sensory nerves, the sensations of creeping over the surface. So, also, among external causes, the stimulus of electricity, or the mechanical influence of a blow, concussion, or pressure, excites in the eye the sensation of light and colors; in the ear, a sensation of a sound or of ringing; and in the tongue, a saline or acid taste.

Sense Perceptions.-The habit of constantly referring our sensations to external causes leads us to interpret the various modifications which external objects produce in our sensations, as properties of the external bodies themselves. Thus we speak of certain substances as possessing a disagreeable taste and smell; whereas, the fact is their taste and smell are only disagreeable to us. It is evident, however, that on this habit of referring our sensations to causes outside ourselves, perception, depends the reality of the external world to us; and more especially is this the case with the senses of touch and sight. By the co-operation of these two senses, aided by the others, we are enabled gradually to attain a knowledge of external objects which daily experience confirms, until we come to place unbounded confidence in what is termed the evidence of the senses.

We must draw a distinction between mere sensations, and the judgments based, often unconsciously, upon them. Thus, in looking at a near object, we unconsciously estimate its distance and say it seems to be ten or twelve feet off. But the estimate of its distance is in reality a judgment based on many things besides the appearance of the object itself; among which may be mentioned the number of intervening objects and their relative size, the number of steps which from past experience we know we must take before we can touch it, etc.

\section{THE SENSES OF TOUCH, TEMPERATURE, PAIN, AND THE MUSCLE SENSE.}

The Sense of Touch.-The sense of touch, like all the special senses, possesses a special end-organ for the initiation of a nerve impulse in this instance through the stimulus of contact with external objects. The sense organ of touch is not confined to particular parts of the body of small extent, 
like the organ of sight, for example, but is found in all parts of the skin and its inversions, the stomodeum and proctodeum. The nerves of touch sensation are contained in the same trunks with other sensory nerves. They are found in the posterior or sensory roots of the spinal nerves and in the sensory divisions of the cranial nerves, especially the fifth, seventh, ninth, and tenth.

All parts of the epidermis supplied with sensory nerves are thus, in some degree, organs of touch, yet the sense is exercised in greatest perfection in certain parts, the sensibility of which is extremely delicate, e.g., the skin of the hands, the tongue, and the lips, which are provided with abundant touch papillæ. A peculiar and very acute sense of touch is exercised through the medium of the nails and teeth, and, to a less extent, the hair may be considered an organ of touch, as in the case of the eyelashes. It has been computed that the human body possesses over 500,000 touch spots.

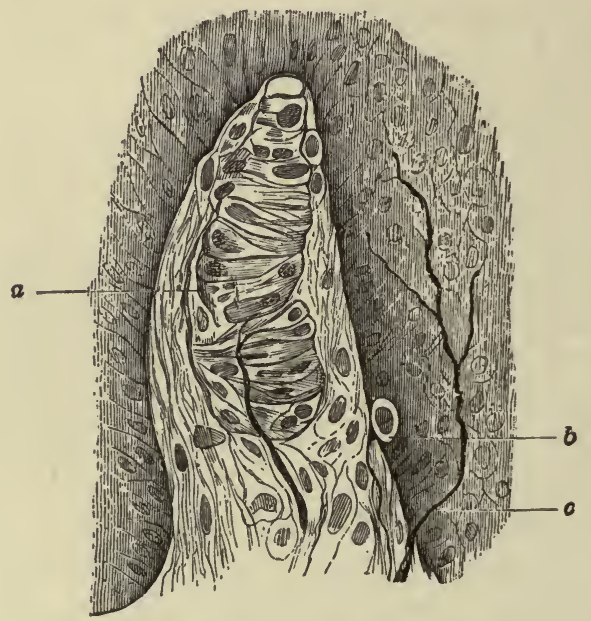

FIG. 420.-Touch Corpuscle.

The sense of touch renders us conscious of the presence of a contact stimulus, from the slightest to the most intense degree of its action. The modifications of this sense often depend on the extent of the parts affected. The sensation of pricking, for example, is produced when the sensitive fibers are intensely affected in a small extent; the sensation of pressure indicates a slighter affection of the parts over a greater extent and depth. It is by the depth to which the parts are affected that the feeling of pressure is distinguished from that of mere contact.

In almost all parts of the body which have delicate tactile sensibility the epidermis, immediately over the dermal papillæ, is moderately thin. When its thickness is much increased, as over the heel, the sense of touch is very much dulled. On the other hand, when it is altogether removed, and the 
cutis laid bare, the sensation of contact is replaced by one of pain. Further, in all highly sensitive parts, the papillæ are numerous and highly vascular, and the sensory nerves are connected with special end-organs which have been described on page 74 et seq.

The special endings of the nerves which have to do with touch may, however, be again mentioned here. They are of two kinds, viz.: r. Touch corpuscles, which are found chiefly in the hands and feet, particularly on the palmar surface of the hands and fingers, but also on the under surface of the forearm, on the nipple, eyelids, lips, and the genital organs. Touch corpuscles are situated in the cutis vera. 2. End bulbs are found in the conjunctiva and other mucous membranes, the lips, genital organs, tongue, rectum, and elsewhere, but not in the skin proper. As regards the Pacinian corpuscles and similar end-organs, which are so widely distributed, and which may be in some way connected with the development of the sensation of touch, when they are found in the skin they are situated very deeply in the cutis vera or in the subcutaneous tissue. They are extremely numerous on the nerves of the palmar surface of the fingers. In addition to these special nerve endings in sense organs, nerve fibers terminate everywhere in the skin between the cells of the Malpighian stratum of the epidermis.

The acuteness of the sense of touch depends in no small degree on the cutaneous circulation and is of course greatly influenced by external temperature. This explains the numbness familiar to everyone that is produced by the application of cold to the skin.

Acuteness of the Sense.-The perfection of the sense of touch on different parts of the surface is proportional to the minimal pressure required to stimulate the point, i.e., the threshold stimulus. Or it can be measured by the power which such parts possess of distinguishing and isolating the sensations produced by two stimulating points placed close together. This latter is in a degree a measure of the power of localization. This power depends, at least in part, on the number of primitive nerve fibers; for the fewer the primitive fibers which an organ receives, the more likely is it that several impressions on different contiguous points will act on only one nerve fiber, and hence be confounded, and perhaps produce but one sensation. Experiments have been made to determine the tactile properties of different parts of the skin, as measured by this power of distinguishing distances between points of simultaneous contact. These consist in touching the skin with the points of a pair of compasses sheathed with cork, and in ascertaining how close the points of the compasses may be brought to each other and still be felt as two bodies. 
Table of Variations in the Tactile Sensibility of the Different Parts OF THE SKIN.

The measurement indicates the least distance at which the two blunted points of a pair of compasses can be separately distinguished as two. (E. H. Weber.)

Tip of tongue.................... I $\mathrm{mm}$.

Palmar surface of third phalanx of forefinger...... $2 \mathrm{~mm}$.

Palmar surface of second phalanges of fingers.... $4 \mathrm{~mm}$.

Red surface of under-lip.................. $4 \mathrm{~mm}$.

Tip of nose.....................

Middle of dorsum of tongue.............. $8 \mathrm{~mm}$.

Palm of hand.......................

Center of hard palate.................

Dorsal surface of first phalanges of fingers........ $44 \mathrm{~mm}$.

Back of hand........................

Dorsum of foot near toes................. $37 \mathrm{~mm}$.

Gluteal region........................

Sacral region........................ $37 \mathrm{~mm}$.

Upper and lower parts of forearm............ $37 \mathrm{~mm}$.

Back of neck near occiput................ $50 \mathrm{~mm}$.

Upper dorsal and mid-lumbar regions.......... $50 \mathrm{~mm}$.

Middle part of forearm...................62 $\mathrm{mm}$.

Middle of thigh.......................

Mid-cervical region ...................

Mid-dorsal region.................... $62 \mathrm{~mm}$.

In the case of the limbs, before the points are recognized as two, they have to be separated further when the line joining them is in the long axis of the limb than when in the transverse direction.

According to Weber the mind estimates the distance between two points by the number of unexcited nerve endings which intervene between the two points touched. It would appear that a certain number of intervening unexcited nerve endings is necessary before two points touched can be recognized as separate, and the greater this number the more clearly are the points of contact distinguished as separate. The delicacy of the sense of touch may be very much increased by practice. A familiar illustration occurs in the case of the blind, who, by constant practice, can acquire the power of reading raised letters the forms of which are almost, if not quite, undistinguishable by the sense of touch to an ordinary person.

The different degrees of sensitiveness possessed by different parts may give rise to errors of judgment in estimating the distance between two points where the skin is touched. Thus, if blunted points of a pair of compasses (maintained at a constant distance apart) be slowly drawn over the skin of the cheek toward the lips, it is almost impossible to resist the conclusion that the distance between the points is gradually increasing. When they reach the lips they seem to be considerably farther apart than on the cheek. Thus, 
too, our estimate of the size of a cavity in a tooth is usually exaggerated when based upon sensations derived from the tongue alone. Another curious illusion may here be mentioned. If we close the eyes, and place a small marble or pea between the crossed fore and middle fingers, we seem to be touching two marbles, figure 480 . This illusion is due to an error of judgment. The marble is touched by two surfaces which, under ordinary circumstances, could be touched only by two separate marbles, hence the mind, taking no cognizance of the fact that the fingers are crossed, forms the conclusion that two sensations are due to two marbles.

Sense of Temperature.-The whole surface of the body is more or less sensitive to differences of temperature. The sensation of heat is distinct from that of touch, hence it would seem reasonable to suppose that there are special nerves and nerve endings for temperature. At any rate the power of discriminating temperature may remain unimpaired when the sense of touch is temporarily in abeyance. Thus if the ulnar nerve be compressed at the elbow till the sense of touch is very much dulled in the fingers which it supplies, the sense of temperature remains quite unaffected. And in certain diseases of the cord the sense of touch may be impaired in a part, and temperature remain undisturbed, or the converse.

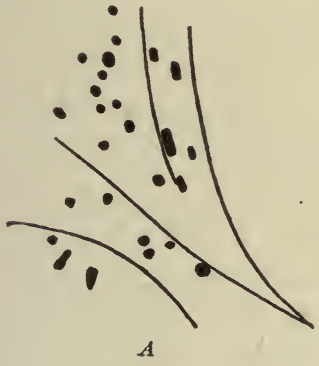

$A$

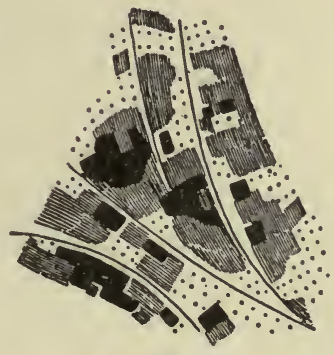

$B$

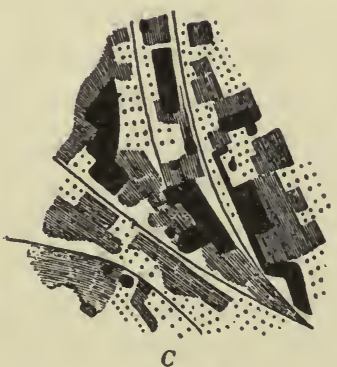

C

FIG. 42I.-Diagram of a Part of the Hand, Showing Distribution of Sense Spots; for touch; $A$; for heat, $B$; and for cold, $C$. In $A$ the skin is sensitive except at the parts marked with black; in $B$ and $C$, the intensity of the shading represents the relative sensitiveness. (Goldscheider.)

The mapping of the surface of a part of the skin with reference to its sensibility to temperature reveals the fact that there are definite heat and cold spots. Furthermore, the areas do not coincide, leading us to conclude that there are two distinct sense organs concerned, figure $42 \mathrm{I}, B$ and $C$.

The sensations of heat and cold are often exceedingly fallacious, and in many cases are no guide at all to the absolute temperature as indicated by a thermometer. All that we can with safety infer from our sensations of temperature is that a given object is warmer or cooler than the skin. Thus 
the temperature of our skin is the standard; and as this varies from hour to hour according to the activity of the cutaneous circulation, our estimate of the absolute temperature of any body must necessarily vary too. If we put the left hand into water at $5^{\circ} \mathrm{C}$. $\left(40^{\circ} \mathrm{F}\right.$.) and the right into water at $45^{\circ} \mathrm{C}$. $\left(\mathrm{I} \mathrm{O}^{\circ} \mathrm{F}\right.$.), and then immerse both in water at $27^{\circ} \mathrm{C}$. $\left(80^{\circ} \mathrm{F}\right.$.), it will feel warm to the left hand, but cool to the right. Again, a piece of metal which has really the same temperature as a given piece of wood will feel much colder, since it conducts away the heat much more rapidly. For the same reason air in motion feels very much cooler than air of the same temperature at rest.

In some cases we are able to form a fairly accurate estimate of absolute temperature. Thus, by plunging the elbow into a bath, a practiced bathattendant can tell the temperature sometimes within half a degree centigrade.

The temperatures which can be readily discriminated are between $10^{\circ}$ and $45^{\circ} \mathrm{C}$. $\left(50^{\circ}\right.$ and $I 15^{\circ} \mathrm{F}$.); very low and very high temperatures alike produce a burning sensation. A temperature appears higher according to the extent of cutaneous surface exposed to it. Thus, water of a temperature which can be readily borne by the hand is quite intolerable if the whole body be immersed.

The delicacy of the sense of temperature coincides in the main with that of touch, though at the elbow where the skin is thin, and the sense of temperature is delicate, that of touch is not remarkably so. Weber has further ascertained two points so near together on the skin that they produce but a single impression, at once give rise to two sensations when one is hotter than the other. Moreover, of two bodies of equal weight, that which is the colder feels heavier than the other.

As every sensation is attended with a perception and leaves behind it an idea in the mind which can be reproduced at will, we are enabled to compare the idea of a past sensation with another sensation really present. Thus we can compare the weight of one body with another which we had previously felt, of which the idea is retained in our mind. Weber was indeed able to distinguish in this manner between temperatures experienced one after the other, better than between temperatures to which the two hands were simultaneously subjected. This power of comparing present with past sensations diminishes, however, in proportion to the time which has elapsed between them. After-sensations left by impressions on nerves of common sensibility or touch are very vivid and durable. As long as the condition into which the stimulus has thrown the organ endures, the sensation also remains, though the exciting cause should have long ceased to act. Both painful and pleasurable sensations afford many examples of this fact.

Sense of Pain.-As regards painful sensations, three views can be taken, I, That it is a special sensation provided with a special conducting apparatus in each part of the body; 2, that it is produced by an over-stimulation of the 
special nerves concerned with touch or temperature, or of the other nerves of special sense; or 3 , that it is an over-stimulation of the nerves of common sensation, which tell us of the condition of our bodies, both of the surface and also of the internal organs. There seems to be much in favor of all of these views. The weight of evidence is, however, rather against there being any special pain sense with a special end-organ and fibers, though Barker in his own arm experienced the presence of pain sensations while there was absence of sensations of touch and temperature. It is, indeed, certain that, even if any variety of pain be a special sensation, some kind of pain may be produced by stimulation of the bare sensory nerves apart from any special form of nerve termination. It is said that the main difference between the common sensory apparatus which tells us of the condition of all parts of the body of which thirst and hunger are but examples, and the special sense of touch and temperature, is that the latter are provided with a special local apparatus. By means of this apparatus we are able to localize the sensation. Such a special apparatus is evidently not absolutely essential for the sensation of pain, but this does not exclude the idea that pain may result from over-stimulation of a nerve of special sense or of its termination.

The Muscular Sense.-The estimate of a weight is usually based on two sensations: I, of pressure on the skin, and 2, the sense of muscular resistance.

The estimate of weight derived from a combination of these two sensations (as in lifting a weight) is more accurate than that derived from the former alone (as when a weight is laid on the hand); thus Weber found that by the former method he could generally distinguish $19 \frac{1}{2} \mathrm{oz}$. from $20 \mathrm{oz}$, but not I $9 \frac{3}{4} \mathrm{oz}$. from 20 , while by the latter he could at most distinguish only $14 \frac{1}{2} \mathrm{oz}$. from $15 \mathrm{oz}$. It is not the absolute, but the relative, amount of the difference of weight which we have thus the faculty of perceiving.

It is not, however, certain, that our idea of the amount of muscular force used is derived solely from the muscular sense. We have the power of estimating very accurately beforehand, and of regulating, the amount of nervous influence necessary for the production of a certain degree of movement. When we lift a vessel, with the contents of which we are not acquainted, the force we employ is determined by the idea we have conceived of its weight. If it should happen to contain some very heavy substance, as quicksilver, we would probably fail in the attempt; the amount of muscular action, or of nervous energy, which we exerted being insufficient. It is possible that in the same way the idea of weight and pressure in raising bodies, or in resisting forces, may in part arise from a consciousness of the amount of nervous energy transmitted from the brain rather than from a sensation in the muscles themselves. The mental conviction of the inability longer to support a weight must also be distinguished from the actual sensation of fatigue in the muscles. 
So, with regard to the ideas derived from sensations of touch combined with movements, it is doubtful how far the consciousness of the extent of muscular movement is obtained from sensations in the muscles themselves. The sensation of movement attending the motions of the hand is very slight; and persons who do not know that the action of particular muscles is necessary for the production of given movements, do not suspect that the movement of the fingers, for example, depends on an action in the forearm. The mind has, nevertheless, a very definite knowledge of the changes of position produced by movements; and it is on this that the ideas which it conceives of the extension and form of a body are in great measure founded.

There is no marked development of common sensibility to be made out in muscles: they may be cut without the production of pain. On the other hand, there is no doubt that afferent impulses must pass upward from muscles and tendons to the brain on the basis of which we become conscious of their condition. This, then, must be a special sense. It has been suggested that the minute end-bulbs of Golgi found in tendons, and that the Pacinian corpuscles in the neighborhood of joints, are the terminal organs of this special sense.

Cutaneous Sensibility and Differential Innervation of the Skin.Studies on the regeneration of cutaneous or sensory nerve trunks after section or after degeneration from disease have revealed the interesting fact that the skin possesses two types of sensory nerves. In the recovery of sensibility to touch and temperature and to pain Head and Rivers have discovered that after a relatively short time, from seven to twenty-six weeks, a certain degree of sensitiveness appears. However, the sensations are different from ordinary sensibility-less distinct, more diffuse, different in quality and cannot be localized with the usual definiteness. The stimulus to evoke them must be coarser and more general. Only relatively wide extremes of heat are perceived, heat above $38^{\circ} \mathrm{C}$. or below $24^{\circ} \mathrm{C}$. Pain sensations are more disturbing, of a peculiar type. This type of sensibility has been designated as protopathic to distinguish it from the usual type or epicritic sensibility. In regeneration epicritic sensibility returns much later, in two years more or less, after nerve section or degeneration. With the return of the epicritic sensory function the usual accurate discrimination of temperature variations within the narrower limits between $24^{\circ}$ and $38^{\circ} \mathrm{C}$., and of delicate touch sensibility, accurate spatial localization, and discrimination replace the protopathic type.

These experiments lend strength to the view that cutaneous sensibility depends on a double or at least a differential innervation. However, Head's later experiments seem to show that the two classes of nerves probably run in common tracts in the cord and brain stem. In cord lesions that affect the epicritic sensibility the protopathic is also lost. 
Touch Sensations and Judgment of the Form and Size of Bodies.-By the sense of touch the mind is made acquainted with the size, form, and other external characters of bodies. And in order that these characters may be easily ascertained, the sense of touch is especially developed in those parts which can be readily moved over the surface of bodies. Touch, in its more limited sense, or the act of examining a body by the touch, consists merely in a voluntary employment of this sense combined with movement, and stands in the same relation to the sense of touch, or common sensibility, generally, as the act of seeking, following, or examining odors does to the sense of smell. The hand is the best adapted for it, by teason of its peculiarities of structure-namely, its capability of pronation and supination, which enables it, by the movement of rotation, to examine rhe whole circumference of a body; the power it possesses of opposing the thumb to the rest of the hand, and the relative mobility of the fingers; and lastly from the abundance of the sensory terminal organs which it possesses. In forming a conception of the figure and extent of a surface, the mind multiplies the size of the hand or fingers used in the inquiry by the number of times which it is contained in the surface traversed; and, by repeating this process with regard to the different dimensions of a solid body, acquires a notion of its cubical extent, but, of course, only an imperfect notion, as other senses, e.g., the sight, are required to make it complete.

It is impossible in this consideration to say how much of our knowledge of the thing touched depends upon pressure and how much upon the muscular sense.

\section{TASTE AND SMELL.}

The special sense organs for taste and smell are stimulated by chemical substances, the former by chemicals in solution, the latter by volatile materials. They are also closely associated in action and we do not always differentiate between the two.

\section{THE SENSE OF TASTE.}

The conditions for the perceptions of taste are: I, the presence of a sense organ, a nerve, and a nerve center with special endowments; 2 , the excitation of the sense organ by the sapid matters, which for this purpose must. be in a state of solution; 3 , a temperature of about $37^{\circ}$ to $40^{\circ} \mathrm{C}$. ( $98^{\circ}$ to $100^{\circ} \mathrm{F}$.).

The Nerves and Organs of Taste.-The principal organ of the sense of taste is the tongue. But the soft palate and its arches, the uvula, tonsils, and probably the upper part of the pharynx, are also endowed with taste. These parts, together with the base and posterior parts of the tongue, are supplied with branches of the glosso-pharyngeal nerve, and evidence has 
been already adduced that this is the principal nerve of the sense of taste. The anterior parts of the tongue, especially the edges and tip, are innervated by fibers from the lingual branch of the fifth, but which arise in the ganglion of the pars intermedia and are distributed in the chorda tympani, figures $25^{\circ}$ and $4 \mathrm{I} 2$.

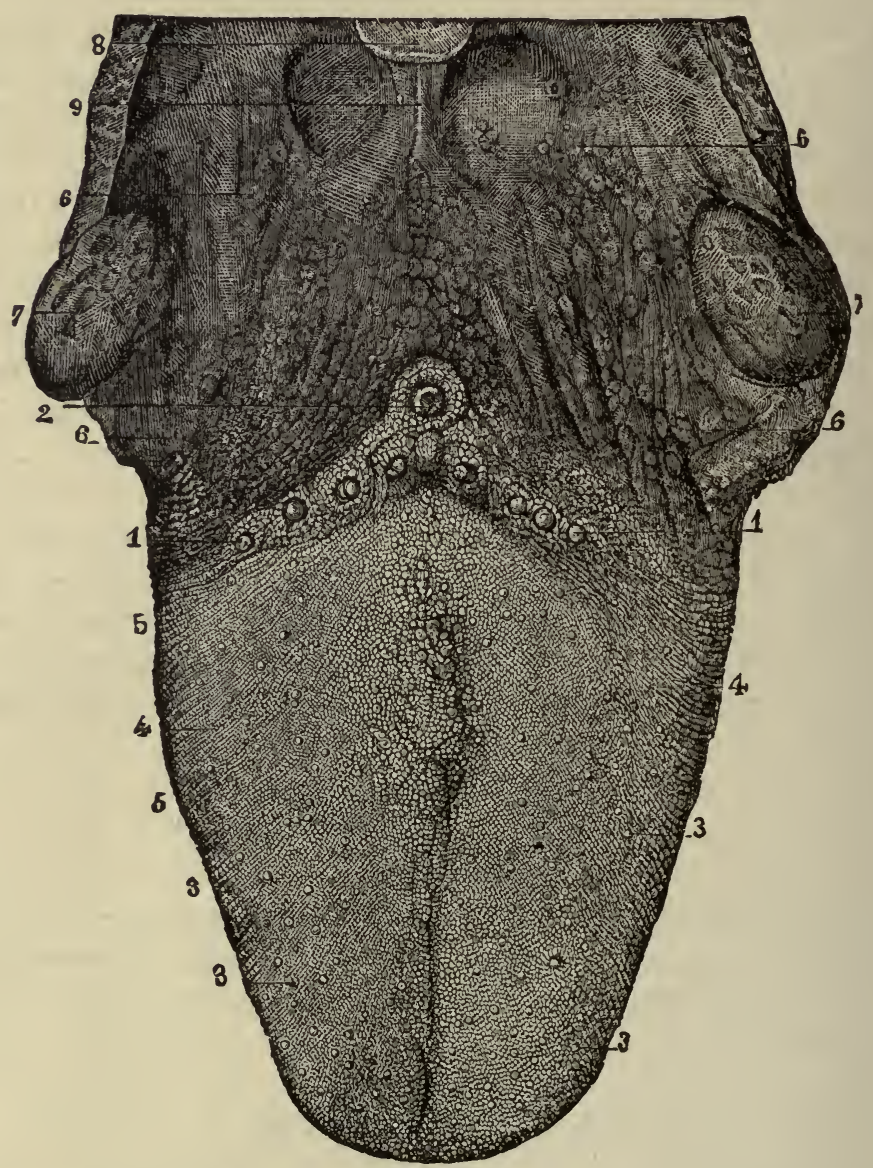

FIG. 422. - Papillar Surface of the Tongue, with the Fauces and Tonsils. I, Circumvallate papillæ, in front of 2 , the foramen cecum; 3 , fungiform papillæ; 4 , filiform and conical papillæ; 5 , transverse and oblique rugæ; 6 , mucous glands at the base of the tongue and in the fauces; 7 , tonsils; 8 , part of the epiglottis; 9 , median glosso-epiglottidean fold (frenum epiglottidis). (From Sappey.)

The mucous membrane in the regions just mentioned possesses special epithelial structures called taste buds. The taste buds are very abundant in the side walls of the circumvallate papillæ. They are also present in the fungiform papillæ, in the foliate papillæ, and in the mucous membrane. The taste bud is located at the deeper part of the stratified epithelium, is 
ovoid in shape, and its free end abuts on the surface or opens to the surface by a short canal. It is composed of two kinds of modified epithelial cellsthe supporting cells, which are long, spindle-shaped cells that form a sheath around the special gustatory cells; and the taste cells, which are neuro-epithelial cells that are found in the center of the taste bud. They are very slender cells that project on the surface by a delicate process. A bundle of nerve fibrils enters the base of each taste bud and forms a net about the taste cells.

$a$

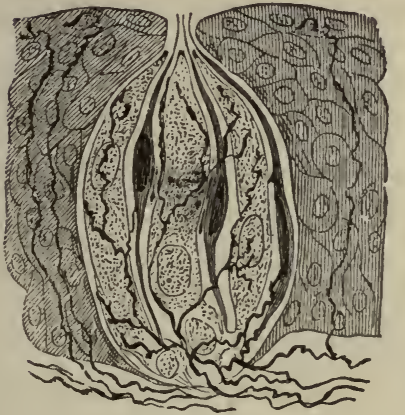

FIG. 423 .

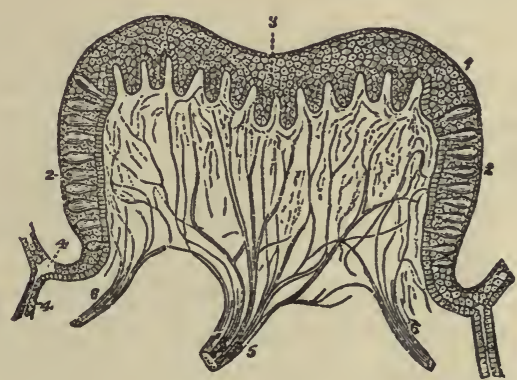

FIG. 424 .

Fig. 423.-Taste Bud from Side Wall of Circumvallate Papillæ. (Merkel-Henle.) $a$, Taste pore; $b$, nerve fibers, some of which enter the taste bud, intrageminal fibers, while others end freely in the surrounding epithelium, intergeminal fibers.

FIG. 424.- - Vertical Section of a Circumvallate Papilla of the Calf. I and 3, Epithelial layers covering it; 2 , taste goblets; 4 , and $4^{\prime}$, duct of serous gland opening out into the pit in which the papilla is situated; 5 and 6 , nerves ramifying within the papilla. (Engelmann.)

The circumvallate, the fungiform, and the filiform papillæ, shown in figure 422 , are special structures that facilitate the stimulation of the taste buds by sapid substances. They are all formed by a projection of the mucous membrane, and contain special branches of blood vessels and nerves. In details of structure, however, they differ considerably one from another.

Circumvallate Papilla.-These papillæ, figure 424, eight or ten in number, are situated in two V-shaped lines on the base of the tongue. They are circular elevations from I to $2 \mathrm{~mm}$. in diameter each, with a central depression, and surrounded by a circular fissure, at the outside of which is a slightly elevated ring. Both the central elevation and the ring are formed of close set simple papillæ.

Fungiform Papilla.-The fungiform papillæ are scattered chiefly over the sides and tip, and sparingly over the middle of the dorsum, of the tongue; the name is derived from their being usually narrower at the base than at the summit. They also are supplied with loops of capillary blood vessels, and nerve fibers. 
Conical or Filiform Papilla.-These, which are the most abundant papillæ, are scattered over the whole surface of the tongue, but especially over the middle of the dorsum. They vary in shape somewhat, but for the most part are conical.

Taste Sensations. - The occurrence of two kinds of special sensibility, i.e., touch and taste in the same part, makes it sometimes difficult to determine whether the impression produced by a substance is perceived through the ordinary tactile sensitive fibers, or through those of the sense of taste. In many cases, indeed, it is probable that both sets of nerve fibers are concerned, as when irritating acrid substances are introduced into the mouth.

Many of the so-called tastes are due to the sapid substances being also odorous, and exciting the simultaneous action of the sense of smell. This is shown by the insipid taste of certain substances when their action on the olfactory nerves is prevented by closing the nostrils. Many of the popular drinks lose much of their apparent excellence if the nostrils are held close while they are drunk.

When these accessory sensations are taken into account it is found that the clearly defined tastes are reduced to four: sweet, bitter, acid, and salt. These taste sensations are produced by the respective substances when in solution. If dry salt or quinine is placed on the surface of the tongue, no taste appears until solution takes place in the secretions of the tongue. A piece of metal, as a silver coin, gives rise to a seemingly distinct taste sensation, called metallic, but it is probably not to be accepted as co-ordinate with the others. The acid taste may be excited by electricity. If a piece of zinc be placed beneath and a piece of copper above the tongue, and their ends brought into contact, an acid taste (due to the feeble galvanic current) is produced. The delicacy of the sense of taste is sufficient to discern one part of sulphuric acid in 10,000 of water, or one part of quinine in 200,000 of water. But it is far surpassed in acuteness by the sense of smell.

Acuteness of the Sense of Taste. (Hall.) The average of ro individuals.

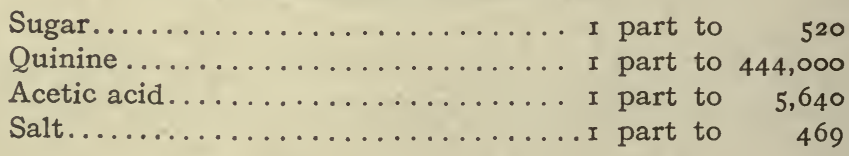

Exploration of the taste areas reveals the fact that regions of the tongue and mouth are not equally sensitive to the sapid substances. Sweet tastes are especially developed at the tip and sides of the tongue, while bitter tastes are almost absent in the front, but especially developed on the basal region, and in the fauces and pharynx. Salts are more stimulating to the tip of the tongue, and acids along the sides. Individual tests of the fungiform papillæ 
by Oehrwall showed that about half the papillæ reacted to sweet, bitter, and acid, but that certain ones reacted only to sweet, or to sweet and bitter, or to acid and bitter. This suggests the specific nature of the taste sensations and tends to prove that there may be a special organ for each kind of stimulus. Experiments have also shown that it is possible to do away with the power of tasting bitters and sweets while the taste for acids and salts remains. This is done by chewing the leaves of an Indian plant, Gymnema sylvestre. It has also been shown that the power of tasting sweet substances disappears before that of tasting bitter. Other experiments have shown that the mechanisms for salt and acid tastes are distinct.

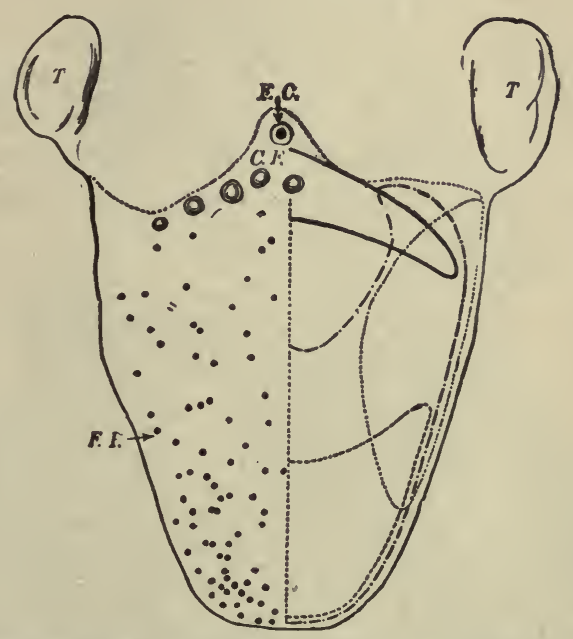

FIG. 425. - Localization of Taste. Bitter-_ acid ....; salt,-._.-; sweet -----; $T$, tonsils; $F C$, foramen cecum; $C F$, circumvallate papillæ; $F P$, fungiform papillæ. (Hall.)

After-tastes and Contrasts.-Very distinct sensations of taste are frequently left after the substances which excited them have ceased to act on the nerve, as the after-taste of metallic bitter, which remains after breaking the stimulating current. Such sensations often endure for a long time, and modify the taste of other substances applied to the tongue. Thus, the taste of sweet substances is intensified after the tasting of common salt. After rinsing the mouth with water containing salt, it is said that sweet solutions are perceived that are too dilute to be detected ordinarily. Many other chemicals produce similar results. The application of a sapid substance, acid for example, to one side of the tongue intensifies the sensation produced by a sapid substance applied to the other side. There is a simultaneous contrast which suggests that the same relation exists between tastes as between colors, of which those that are opposed, i.e., complementary, render each other more vivid, though no general principles governing this relation have 
been discovered in the case of tastes. In the art of cooking, however, attention has at all times been paid to the consonance or harmony of flavors in their combination or order of succession, just as in painting and music the fundamental principles of harmony have been employed empirically while the theoretical laws were unknown.

Frequent and continued repetitions of the same taste render the perception of it less and less distinct, in the same way that a color becomes more and more dull and indistinct the longer the eye is fixed upon it. There is fatigue of the taste organ at some point.

\section{THE SENSE OF SMELL.}

The sensation of smell is produced by the action of odorous particles on a special end-apparatus, which in turn causes nerve impulses that arouse changes in a special area in the sensorium. The stimulating cause is the direct action of chemical substances as in the sense of taste. In this case

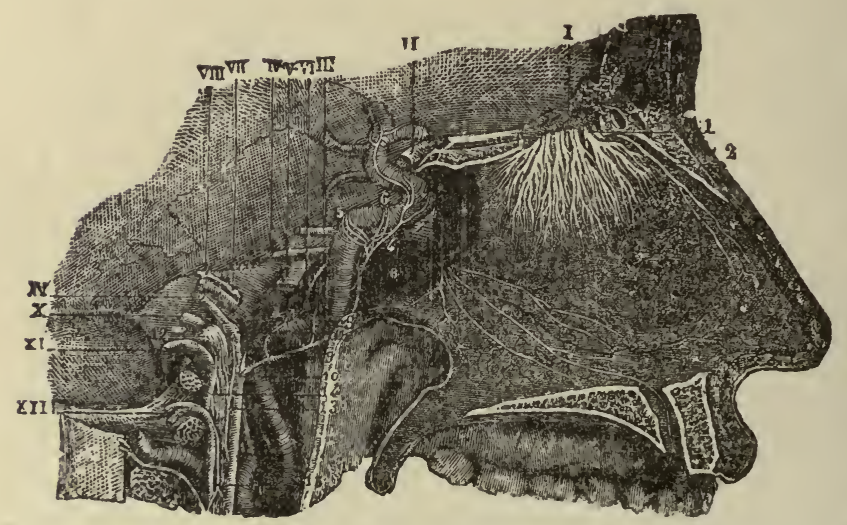

Fig. 426.-Nerves of the Septum Nasi, Seen from the Right Side. $\times \frac{3}{4}-1$, The olfactory bulb; $\mathrm{I}$, the olfactory nerves passing through the foramina of the cribriform plate, and descending to be distributed on the septum; 2, the internal or septal twig of the nasal branch of the ophthalmic nerve; 3, naso-palatine nerves. (From Sappey, after Hirschfeld and Leveillé.)

however, the substances must reach the sensory membrane in a gaseous state or in extremely fine division, so that it can quickly enter into solution in the moisture on the sensitive mucous surface. The odorous particles are carried to the membrane by inspiratory currents of air.

The Olfactory Apparatus. - The essential parts of the olfactory apparatus are the nasal sensory or olfactory membrane to receive the special stimuli, and the nervous apparatus to conduct the olfactory nerve-impulse to the sensory area in the cortex cerebri for its perception. 
The nose is not entirely an organ for the seat of smell. In fact the nasal cavities are divided into three districts called, respectively: I, Regio vestibularis which is the entrance to the cavity. It is lined with a mucous membrane very closely resembling the skin, and guarded by hairs and by sebaceous glands. 2, Regio respiratoria, which includes the lower and middle meatus of the nose. It is covered with mucous membrane of stratified columnar ciliated epithelium. The mucosa is thick and consists of fibrous connective tissue; it contains a certain number of tubular mucous and serous glands. 3 , Regio

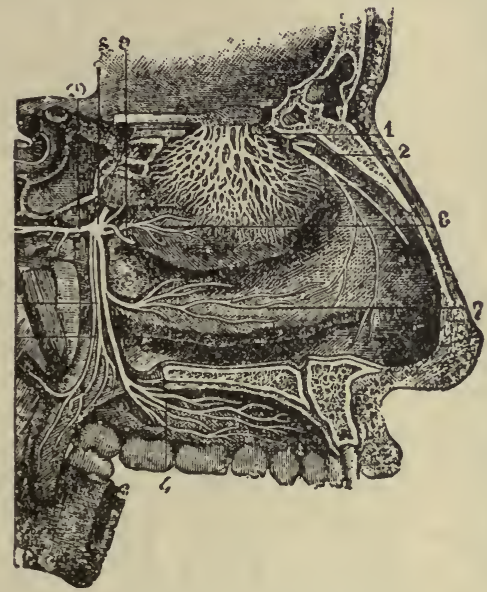

FIG. 427.-Nerves of the Outer Walls of the Nasal Fossæ. I, Network of the branches of the olfactory nerve, descending upon the region of the superior and middle turbinated bones; 2, external twig of the ethmoidal branch of the nasal nerves; 3 , spheno-palatine ganglion; 4, ramification of the anterior palatine nerves; 5 , posterior, and 6, middle divisions of the palatine nerves; 7 , branch to the region of the inferior turbinated bone; 8 , branch to the region of the superior and middle turbinated bones; 9 , naso-palatine branch to the septum cut short. (From Sappey, after Hirschfeld and Leveillé.)

olfactoria. This includes the anterior two-thirds of the superior meatus, the middle meatus, and the upper half of the septum nasi, figures 427 and 428 . It is of a yellowish color. It consists of a thicker mucous membrane than in 2, made up of loose, areolar connective tissue covered by epithelium of a special variety, resting upon a basement membrane. The cells of the epithelium are of two principal kinds: $a$, columnar epithelial cells whose function is to support $b$, the bipolar olfactory cells. The epithelial cells are prismatic in shape and have upon their surfaces facets into which the olfactory cells fit themselves, figure $428, e$. They are thus analogous to the cells of Müller of the retina. The olfactory cells have an oblong or fusiform shape, which is mainly determined by the large nucleus. The thin protoplasmic body has two processes, an external and an internal. The external is large and passes up to the free surface, to end in a small bunch of fibrils that are not vibratile. The internal process is very fine, often varicose, and passes through the 
cribriform plate to form a glomerular basket with the branches of the mitral cells of the olfactory bulb.

The olfactory bulb must be studied in relation with the nerve fibers and olfactory cells with which it is connected. These parts together form a sensory end-organ which resembles in many respects the retina. The discovery

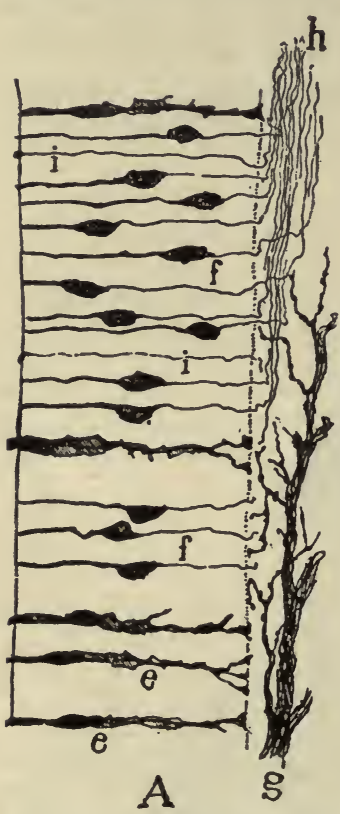

FIG. 428.-Bipolar Olfactory Cells from the Nasal Fossæ of the Rat (Full-term Fetus). $A$, Epithelium of the olfactory mucosa; $e$, epithelial cells; $f, f$, nerve cells; $i$, nerve fibers terminating freely on the epithelial surface; $h$, olfactory nerve fibers; $g$, sensory nerve derived from the trigeminus. (Cajal.) of its true structure has thrown a flood of light on the architecture of the nerve centers as a whole.

The olfactory bulb is not a nerve, but a modification of the brain cortex. A transection shows it to be made up of four layers: I. Peripheral fibers. 2. Olfactory glomerules. 3. Layer of mitral cells. 4. Layer of granular cells and deep nerve fibers.

The first and external layer is composed of the fine nerve fibrils of the olfactory nerves. They pass through the cribriform plate of the ethmoid, arising from the olfactory cells of which they are processes.

The glomerular layer contains numbers of small round bodies whose structure shows that they are made up of the interlocking expansions of the olfactory fibers, on the one hand, and of the branches of the "mitral" cells, on the other. These are mingled in a close network, but do not anastomose. It was by the study of these bodies in part that the fact of the non-continuity of the neurones was demonstrated, figure 429 . This layer also contains small fusiform cells with branching dendrites that extend outward to the glomeruli. Each has an axis-cylinder process which passes inward to join the fibers of the internal olfactory nerves.

The layer of mitral cells contains large cells, some of them triangular and some in the shape of a miter. They have numerous dendrites, one of which passes into a glomerulus and then breaks up in a fine arborization. An axis-cylinder process passes off from the inner surface and is continued as an internal olfactory nerve fiber in the olfactory tract.

The layer of granules and central fibers contains a large number of very small nerve cells, which are peculiar in that they have no axis-cylinder. Their dendrites extend chiefly into the layer of mitral cells. They resemble the spongioblasts of the retina and probably have commissural functions. This layer has also some small star-shaped cells whose dendrites end in the 
mitral-cell layer. Among these cells run numerous fibers, chiefly from the mitral cells and the fusiform cells of the glomerular layer. The general arrangement is shown in figure 429.

The Stimulation of the Olfactory Membrane.-The extent of the nasal mucous surfaces, and of the frontal and antral sinuses connected with them, might suggest that the sensory olfactory surface is widely distributed, but such is not the case. Air impregnated with vapor of camphor has been injected into the frontal sinus through a fistulous opening, and odorous substances have been injected into the antrum of Highmore; but in neither case

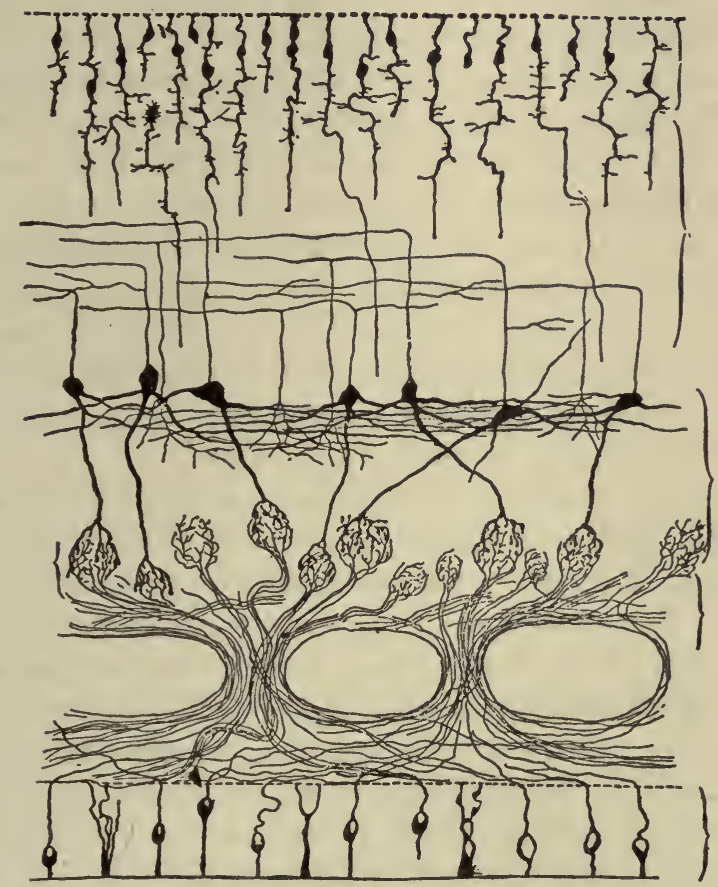

Fig. 429.-Principal Constituent Elements of the Olfactory Bulb of a Mammal. (Van Gehuchten.)

was any odor perceived by the patient. All parts of the nasal cavities are endowed with cutaneous sensibility by the nasal branches of the first and second divisions of the fifth nerve, hence the sensations of cold, heat, itching, tickling, and pain, and the sensation of tension or pressure in the nostrils. That these nerves cannot perform the functions of the olfactory nerves is proved by cases in which the sense of smell is lost, while the mucous membrane of the nose remains susceptible to the various modifications of the sense of touch. But it is often difficult to distinguish the sensation of smell from that of mere feeling, and to ascertain what belongs to each separately. This is true particularly of the sensations excited by acrid vapors in the nose, 
as of ammonia, horse-radish, mustard, etc., and the difficulty is the greater when it is remembered that these acrid vapors have nearly the same action upon the mucous membrane of the eyelids.

The true olfactory membrane is limited to the small area on either side of the superior meatus and supplied by the olfactory nerve. It is stimulated by odorous substances when they penetrate the upper chamber of the nose. Currents of air can be drawn over this membrane more certainly and effectively by sniffing the air, as noticed in the acts of a dog following the trail. The odorous particles must come into contact with the olfactory cells when in solution in the moisture over the surface and produce the stimulus by chemical change. Mere presence in solution is not always adequate to a stimulation. It seems that movement over the surface is necessary, at least to effective stimulation. Haycraft has repeated some of the older experiments and finds that eau de Cologne can be introduced into the nasal cavity in warm saline solutions without producing a sensation of smell even when Io per cent. solutions are used. He also showed that Cologne, bergamot, etc., can be slowly diffused into the nasal cavity without producing a stimulus. If, while the vapor is thus in the nasal cavity, the nostril be closed and the person goes into pure air and breathes, then an odorous sensation is at once experienced. This shows that even odorous gases "must be moved over the olfactory surface" in order to produce a stimulus.

The presence of bodies in quantities so minute as to be undiscernible even by spectrum analysis, $0.000,000,03$ of a grain of musk, can be distinctly smelt (Valentin). Opposed to the sensation of an agreeable odor is that of a disagreeable or disgusting odor, which corresponds to the sensations of pain, dazzling and disharmony of colors, and dissonance in the other senses. Thercause o1 this difference in the effect of different odors is unknown; but this much is certain, that odors are pleasant or offensive in a relative sense only, for many animals pass their existence in the midst of odors which to us are highly disagreeable. A great difference in this respect is, indeed, observed among men. Many odors, generally thought agreeable, are to some persons intolerable; and different persons describe differently the sensations that they severally derive from the same odorous substances. There seems also to be in some persons an insensibility to certain odors, comparable with that of the eye to certain colors; and among different persons, as great a difference in the acuteness of the sense of smell as among others in the acuteness of sight. We have no exact proof that a relation of harmony exists between odors as between colors and sounds, though it is probable that such is the case, since it certainly is so with regard to the sense of taste. Such a relation would account in some measure for the different degrees of perceptive power in different persons; for as some have no ear for music, so others have no clear appreciation of the relation of odors, and therefore little pleasure in them. 
Most of the substances taken as foods into the mouth give off odorous particles that stimulate the olfactory membrane. In fact, the chief elements in food flavors are not tastes, but smells, or combinations of the two. This is particularly true of meats. Meats are especially prized for their delicate flavors, and cooking is performed to bring out these flavors. Yet meat has little taste other than salt; the so-called tastes are due to odorous particles entering the nostril and stimulating the olfactory membrane at the same moment the taste buds of the mouth are stimulated.

Subjective sensations occur frequently in connection with the sense of smell. Often a person smells something which is not present, and which other persons cannot smell; this is very frequent with nervous persons, but it occasionally happens to every one. In a man who was conscious of a bad odor, the arachnoid was found after death to be beset with deposits of bone, and a lesion in the middle of the cerebral hemispheres was also discovered. Dubois was acquainted with a man who, ever after a fall from his horse, which occurred several years before his death, believed that he smelt a bad odor.

\section{HEARING AND EQUILIBRATION.}

\section{THE ANATOMY OF THE EAR.}

For descriptive purposes, the ear, or organ of hearing, is divided into three parts, I, the external, 2, the middle, and 3, the internal ear. The first two are only accessory structures to the third, which contains the essential parts of the organ of hearing. The accompanying figure, 430, shows very well the relation of these divisions to each other.

The External Ear.-The external ear consists of the pinna or auricle and the external auditory canal or meatus.

The principal parts of the pinna, figure 430, are two prominent rims enclosed one within the other, the helix and antihelix, and inclosing a central hollow named the concha; in front of the concha, a prominence directed backward, the tragus, and opposite to this one directed forward, the antitragus. From the concha, the auditory canal passes inward and a little forward to the membrana tympani, to which it thus serves to convey the vibrations of the air. It consists of a fibro-cartilage tube lined by skin continuous with that of the pinna, and extending over the outer part of the membrana tympani. Fine hairs and sebaceous glands are present toward the outer part of the canal, while deeper in the canal are small glands, resembling the sweat glands in structure, which secrete the cerumen.

Regarding the external ear, therefore, as a collector and conductor of sonorous vibrations, all its inequalities, elevations, and depressions become of evident importance; for those elevations and depressions upon which the undulations fall will tend to intensify certain sound waves while not affecting 


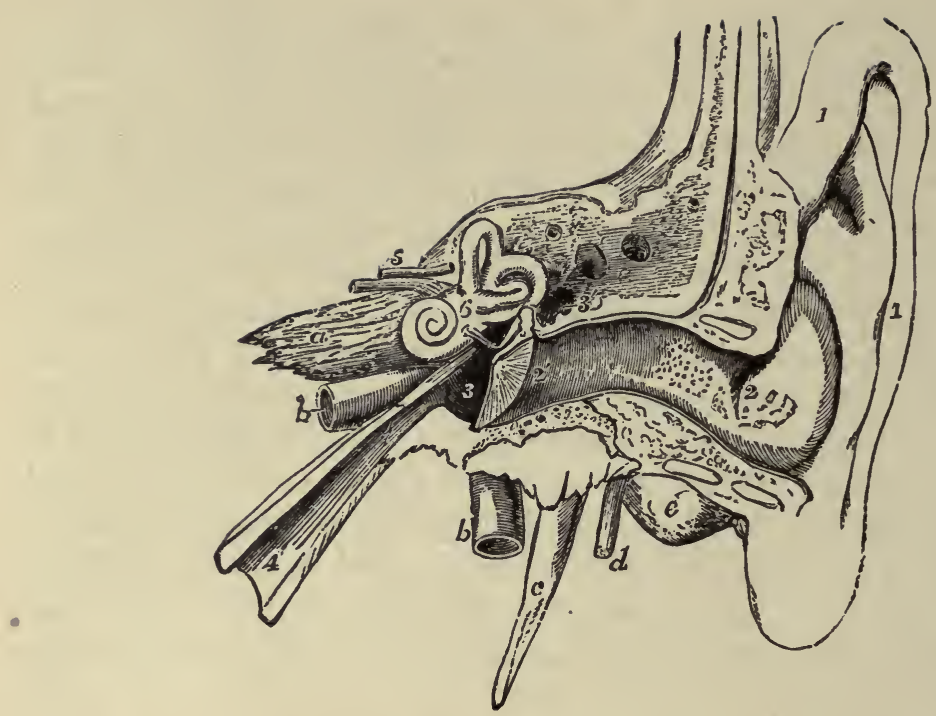

FIG, 430.-Diagrammatic View from Before of the Parts Composing the Organ of Hearing of the Left Side. The temporal bone of the left side, with the accompanying soft parts, has been detached from the head, and a section has been carried through it transversely, so as to remove the front of the meatus externus, half the tympanic membrane, the upper and anterior wall of the tympanum and Eustachian tube. The meatus internus has also been opened, and the bony labyrinth exposed by the removal of the surrounding parts of the petrous bone. I, The pinna and lobe; $2,2^{\prime}$, meatus externus; $2^{\prime}$, membrana tympani; 3 , cavity of the tympanum; $3^{\prime}$, its opening backward into the mastoid cells; between 3 and $3^{\prime}$, the chain of small bones; 4 , Eustachian tube; 5 , meatus internus, containing the facial (uppermost) and the auditory nerves; 6 , placed on the vestibule of the labyrinth above, the fenestra ovalis; $a$, apex of the petrous bone; $b$, internal carotid artery; $c$, styloid process; $d$, facial nerve issuing from the stylo-mastoid foramen; $e$, mastoid process; at squamous part of the bone covered by integument, etc. (Arnold.)

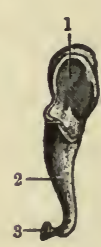

A.

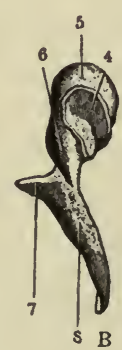

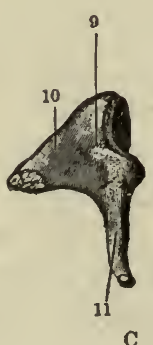

C
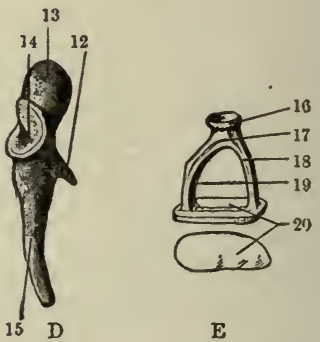

E

Fig. 43r.-Tympanic Ossicles of Left Ear. $\quad A$, Incus seen from the front; $B$, malleus, viewed from behind; $C$, incus, and $\mathrm{D}$, malleus, seen from inner aspect; $E$, stapes. I, Body of incus, with articular surface for head of malleus; 2, processus longus; 3 , processus lenticularis; 4 , articular surface for incus; 5 , head, 6 , neck; 7 , processus brevis; 8 , manubrium; 9, body; Io, short process; II, long process; I2, processus longus; I3, head, I4, facet for incus; I5, manubrium; I6, head; I7, neck; I8, crus anterius; I9, crus posterius; 20, foot plate. 
others. It is thought that this forms at least an aid in determining the direction whence a sound comes.

The Middle Ear or Tympanum.-The middle ear, or tympanum, 3, figure 430 , is separated by the membrana tympani from the external auditory canal. It is a cavity in the temporal bone, opening through its anterior and inner wall into the Eustachian tube.

The Eustachian canal establishes communication between the tympanic cavity and pharynx, thus equalizing the air pressure on the sides of the tympanic membrane, serving the same mechanical purpose as the vent-hole in a snare or bass drum. The cavity of the tympanum communicates posteriorly with air cavities, the mastoid cells, in the mastoid process of the tem-

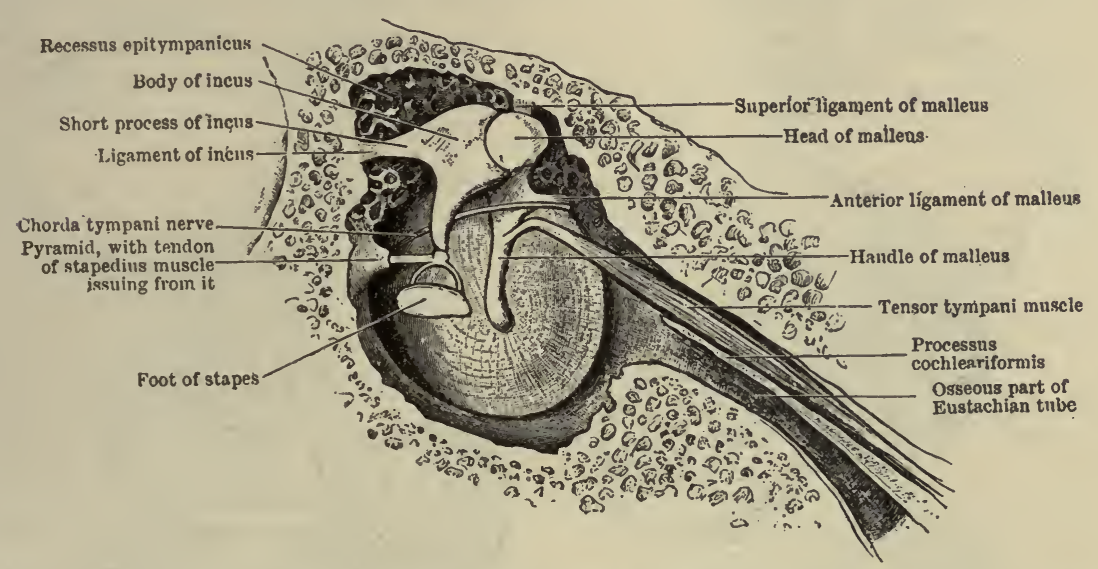

Fig. 432.-Left Membrana Tympani and Chain of Tympanic Ossicles (Seen from Inner Aspect). (Cunningham.)

poral bone; but its only opening to the external air is through the Eustachian tube. The cavity of the tympanum is lined with mucous membrane, the epithelium of which is ciliated and continuous with that of the pharynx. It contains a chain of small bones, ossicula auditus, which extends from the membrana tympani to the fenestra ovalis.

The Membrana Tympani.-The tympanic membrane is placed in a slanting direction at the bottom of the external canal, its plane being at an angle of about forty-five degrees with the lower wall of the canal. It is formed chiefly of a tough and tense fibrous membrane, the edges of which are set in a bony groove. Its outer surface is covered by a continuation of the epithelial lining of the auditory canal, its inner surface with part of the mucous membrane of the middle ear.

The Tympanic Ossicles.-The ear bones, or ossicles, are named the malleus, incus, and stapes. The malleus is attached by a long slightly curved process, called its handle, to the membrana tympani, the line of attachment 
being vertical, including the whole length of the handle, and extending from the upper border to the center of the membrane. The head of the malleus is irregularly rounded; its neck, or the line of boundary between it and the handle, supports a short conical process which receives the insertion of the tensor tympani muscle. The incus, shaped like a bicuspid molar tooth, is articulated by its broader part to the malleus. Of its two fang-like processes, one directed backward has a free end lodged in a depression in the mastoid bone; the other, curved downward and more pointed, articulates by means of a roundish tubercle with the stapes. The stapes is a little bone shaped exactly like a stirrup, of which the base or bar fits into the fenestra ovalis. The stapedius muscle is attached to the neck of the stapes.

The bones of the ear are covered with mucous membrane reflected over them from the wall of the tympanum. They are movable both altogether and one upon the other. The malleus moves and vibrates with every move-

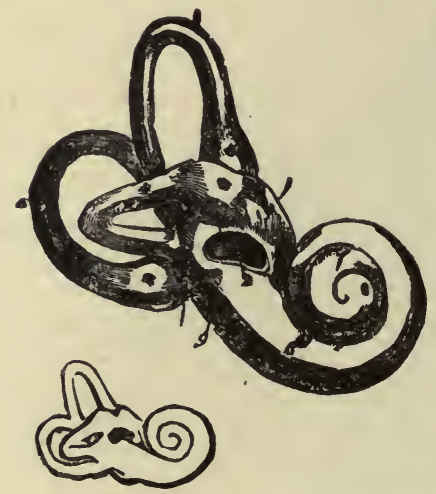

FIG. 433 .

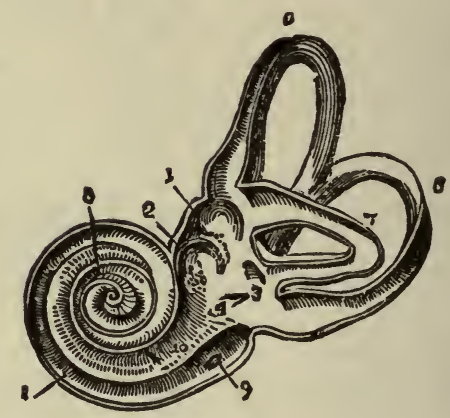

Fig. 434 .

FiG. 433.-Right Bony Labyrinth, Viewed from the Outer Side. The specimen here represented is prepared by separating piecemeal the looser substance of the petrous bone from the dense walls which immediately enclose the labyrinth. I, The vestibule; 2, fenestra ovalis; 3, superior semicircular canal; 4, horizontal or external canal; 5, posterior canal; *, ampullæ of the semicircular canals; 6 , first turn of the cochlea; 7 , second turn; 8 , apex; 9, fenestra rotunda. The smaller figure in outline below shows the natural size. $\times 2.5$. (Sömmering.)

Fig. 434.-View of the Interior of the Left Labyrinth. The bony wall of the labyrinth is removed superiorly and externally. I, Fovea hemielliptica; 2, fovea hemispherica; 3 , common opening of the superior and posterior semicircular canals; 4 , opening of the aqueduct of the vestibule; 5 , the.superior, 6 , the posterior, and 17 , the external semicircular canals; 8 , spiral tube of the cochlea (scala tympani); 9 , opening of the aqueduct of the cochlea; ro, placed on the lamina spiralis in the scala vestibuli. $\times 2.5$. (Sömmering.)

ment and vibration of the membrana tympani, and its movements are communicated through the incus to the stapes, and through the stapes to the membrane closing the fenestra ovalis. The malleus, also, is movable in its articulation with the incus. The membrana tympani which moves the long process of the malleus is altered in its degree of tension by the degree of con- 
traction of the tensor tympani muscles. The stapes is movable on the process of the incus, the contractions of the stapedius muscle draws it outward. The axis round which the malleus and incus rotate is the line joining the processus gracilis of the malleus and the posterior process of the incus.

The Internal Ear.- The internal ear, or labyrinth, constitutes the proper organ of hearing. It contains special epithelial structures to which are distributed the auditory nerves. The organ is located in a cavity in the
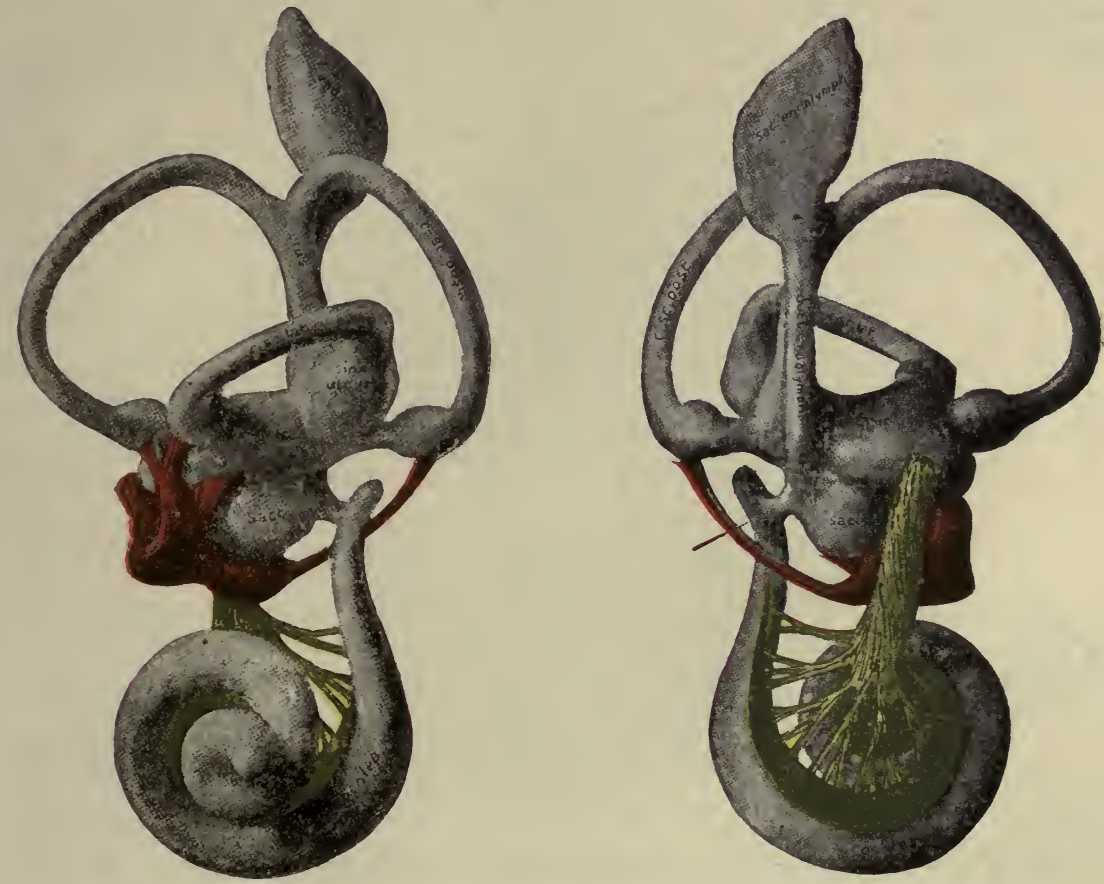

Fig 435.-Membranous Labyrinth of a $30 \mathrm{~mm}$. Human Fetus. $A$, Viewed from its Lateral Aspect; $B$, viewed from the mesial aspect. (Streeter.)

petrous bone, called the osseous labyrinth. The auditory organ within is called the membranous labyrinth. The membranous labyrinth contains a fluid called endolymph; while outside it, between it and the osseous labyrinth, is a fluid called perilymph. This is not a pure lymph, as it contains mucin.

The osseous labyrinth consists of three principal parts, namely the vestibule, the cochlea, and the semicircular canals, containing the respective divisions of the membranous labyrinth. The osseous labyrinth possesses openings on its inner wall for the entrance of the divisions of the auditory nerve from the cranial cavity, in its outer wall the fenestra ovalis, 2, figure 433 , an opening filled by the base of the stapes, and the fenestra rotunda. The vestibule also presents an opening, the orifice of the aqueductus vestibuli. 
The Membranous Labyrinth.-The membranous labyrinth corresponds generally with the form of the osseous labyrinth, so far as regards the vestibule and semicircular canals, but is separated from the walls of these parts by perilymph, except where the nerves enter into connection within it. The labyrinth is a closed membrane containing endolymph.

The Utriculus and the Sacculus.- The vestibular portion of the inner ear consists of membranous sacs, the upper, the utriculus, the lower called the sacculus. The former is connected with the semicircular canals, the latter with the cochlea by the cochlear canal. The utriculus and the sacculus have on their floors each a special patch of sensory epithelium called the macula. The fibers of the vestibular divisions of the auditory nerve end in the maculæ, figure 435 . In the cavities of the sacculus and utriculus are small masses of calcareous particles called otoliths.

The Semicircular Canals. - There are three semicircular canals for each ear, one horizontal and two vertical ones placed almost at right angles to each other. The three canals, therefore, occupy the three planes of space. Each has a considerable enlargement or swelling, called an ampulla. The epithelium of the ampulla is modified at the point of entrance of the nerve into a thickened hillock called the crista acustica. This epithelium is composed of rod cells or supporting cells which extend the full thickness of the

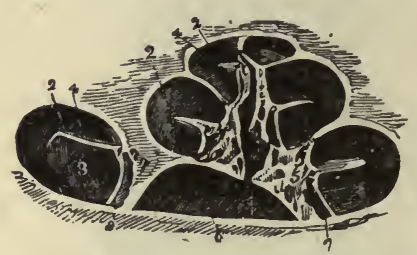

FIG. 436.-View of the Osseous Cochlea Divided through the Middle. I, Central canal of the modiolus; 2, lamina spiralis ossea; 3 , scala tympani; 4 , scala vestibuli; 5 , porous substance of the modiolus near one of the sections of the canalis spiralis modioli. $\times 5$. (Arnold.) crista, and of hair cells, which occupy the inner or free half of the crista. The hair cells are the sensory cells. They have hairlike processes which project from the free ends of the cells out into the endolymph of the cavity. Nerve fibrils run up into the crista and apparently form terminal arborizations about the hair cells, or, according to some observers, end in the cells.

The Cochlea and the Organ of Corti.The membranous cochlea is located in the spiral canal in the petrous bone, called the cochlear canal. It is attached to the wall of the cavity between the fenestra ovalis and the fenestra rotunda, and to the outer wall of the canal and the free border of the lamina spiralis almost, but not quite, to its summit. A small cavity is thus left around the upper end of the cochlea connecting the scala vestibuli above with the scala tympani below. A cross-section through the cochlear canal shows the relations of the cochlear canal which was named scala media by the earlier anatomists. The free portion of the membranous wall above is called the membrane of Reisner, while that below is called the basilar membrane. The basilar membrane supports the special sensory apparatus for the reception of stimuli of sound waves. 
Organ of Corti.-The basilar membrane supports cells of several types. About midway between the outer edge of the lamina spiralis and the outer wall of the cochlea are situated the rods of Corti. Viewed sideways, they are seen to consist of an external and internal pillar, each rising from an expanded foot or base on the basilar membrane, figure 438. They slant inward toward each other, and each ends in a swelling termed the head, the head of the inner pillar overlying that of the outer, figure 438 . Each pair of pillars forms, as it were, a pointed roof arching over a space, and by a succession of them a little tunnel is formed. It has been estimated that there

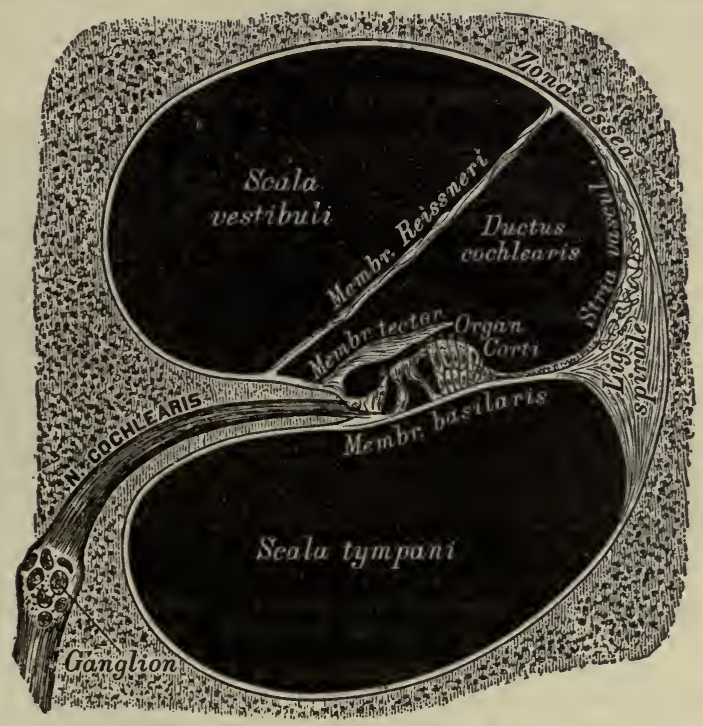

FIG. 437.-Semidiagrammatic Section of a Cochlear Whorl. (After Heitzmann.)

are about four thousand of these pairs of rods of Corti between the base of the cochlea and its apex, in man 3,848 outer and 5,590 inner rods (Retzius). They are found progressively to increase in length, and become more oblique; in other words, the tunnel becomes wider, but diminishes in height as we approach the apex of the cochlea.

Leaning against the rods of Corti and apparently supported by them are sensory cells or hair cells. The hair cells are in two series, the inner and the outer hair cells. The former consist of a single layer, the latter of three or four layers, figure 438 . There are two additional types of supporting cells, the cells of Deiters and of Hensen. The whole structure when viewed from above bears a remarkable resemblance to the keyboard of a piano.

The cochlear division of the auditory nerve enters the base of the modiolus and sends a spiral whorl of fibers out under the spiral lamina. The ganglionic cells of the cochlear division of the auditory nerve are located in the 
base of the lamina where they form the spiral ganglion. The nerve fibers from the ganglion cells pass out through small holes in the periphery of the spiral plate of bone, to enter the organ of Corti. Here they form small longitudinal bundles that quickly end about the hair cells.

\section{THE SENSE OF HEARING.}

All the acoustic contrivances of the organ of hearing are means for conducting sound. Since all matter is capable of propagating sonorous vibrations, the simplest conditions must be sufficient for mere hearing; since all substances surrounding the auditory apparatus would stimulate it. The complex development of the organ of hearing, therefore, must have for its

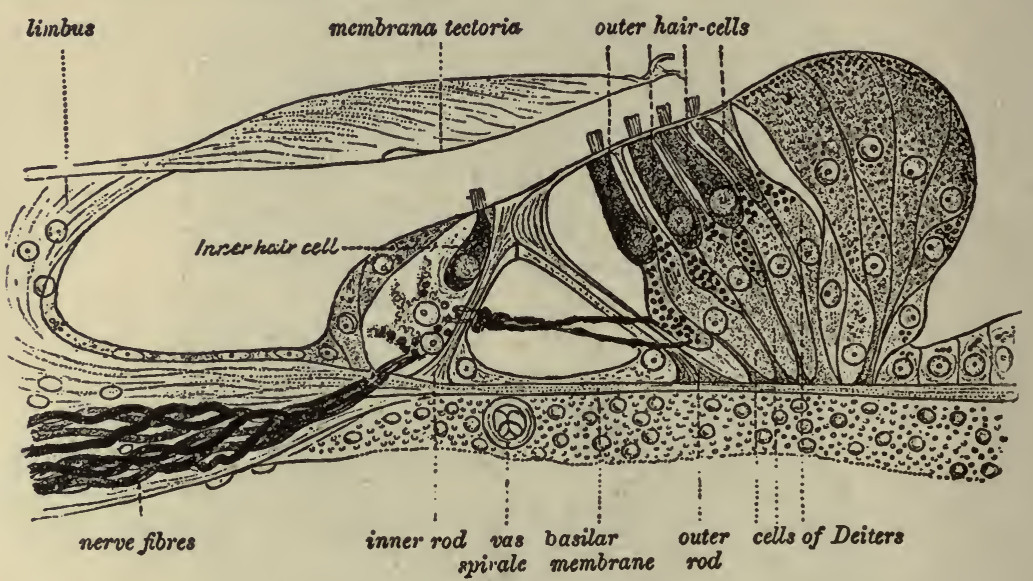

FIG. 438.-Semidiagrammatic Representation of the Organ of Corti and Adjacent Structures. (Merkel-Henle.) $a$, Cells of Hensen; $b$, cells of Claudius; $c$, internal spiral sulcus; $x$, Nuel's space. The nerve fibers (dendrites of cells of the spinal ganglion) are seen passing to Corti's organ through openings (foramina nervosa) in the bony spiral lamina. The black dots represent longitudinally running branches, one bundle lying to the inner side of the inner pillar, a second just to the outer side of the inner pillar within Corti's tunnel, the third beneath the outer hair cells.

object the more effective propagation of the sonorous vibrations and their intensification by resonance; and, in fact, the whole of the acoustic apparatus may be shown to have reference to these principles.

The external ear and the auditory passages influence the propagation of sound to the tympanum by collecting from the atmosphere the sonorous undulations that strike against the external ear and by transmitting them by the air in the passage to the membrana tympani.

In animals living in the atmosphere, the sonorous vibrations are conveyed to the auditory epithelium through three different media in series; namely, the air of the external ear and meatus, which sets in vibration the tympanic membrane, the solid chain of auditory ossicles, and the fluid of the labyrinth. Sonorous vibrations are imparted too imperfectly from air to 
the solid structures of the body as a whole for the propagation of sound to the internal ear to be adequately effected by that means alone. In passing from air directly into water, sonorous vibrations suffer also a considerable diminution of their strength; but if a tense membrane exists between the air and water, the sonorous vibrations are communicated from the former to the latter medium with very great intensity. This fact, of which Müller gives experimental proof, furnished at once an explanation of the use of the fenestra ovalis and of the membrane closing it. It is the means of communicating, in full intensity, the vibrations of the ear bones, or, in their absence, of the air in the tympanum, to the fluid of the labyrinth. The vibration of the fluids, the perilymph and endolymph, of the internal ear, sets the basilar membrane in vibration and in consequence stimulates the sensory apparatus resting upon it. This last is the essential stimulating act, while all that precedes is more or less accessory or contributory to this act. Just what the accessory apparatus contributes can be best understood by an examination of the stimulus and the sensation which results from its action.

Sound.-Any elastic body, e.g., air, a membrane, or a string, performing a certain number of regular vibrations per second, gives rise to what is termed a musical sound or tone. We must, however, distinguish between a musical sound and a mere noise; the latter being due to irregular vibrations.

Musical sounds are distinguished from each other by three qualities: I. Strength or intensity, which is due to the amplitude or length of the wave of vibrations. 2. Rate, the number of vibrations in a second. 3. Quality, or timbre, the peculiar property by which we distinguish the same note sounded on two instruments, e.g., a piano and a flute. It has been proved by Helmholtz to depend on the number of secondary tones, termed harmonics, which are present with the predominating or fundamental tone; that is, rhythmic vibrations are either simple in form, like the vibrations of a reed or tuning-fork, or compound, like the vibrations of a violin or piano string. If the string of a violin is plucked it not only vibrates as a whole, but in segments in the ratio of one, two, three, etc. The form of air wave that is produced by several such vibrating bodies is very complex indeed, as, for example, when an orchestra is playing.

The compound wave can be analyzed into its constituent elements by a system of resonators, on the principle of sympathetic vibration. If one sounds a series of musical notes before such a system of resonators it will be found that the tones and overtones are selected by the resonators and made more prominent so that they can be identified.

The sensation of sound has in it certain elements that correspond closely with the physical properties of sound, i.e., loudness, pitch, and quality. Loudness is dependent merely on the intensity of the stimulation. A sound wave of great energy, for example, produces a larger movement of the tympanic membrane, and it, through the chain of bones and the fluid of the 
internal ear, a larger swing of the basilar membrane, hence a more intense stimulus of the organ of Corti.

Function of the External and Middle Ears.-It has already been stated that the external ear collects the sound waves and conducts them against the membrana tympani. This membrane vibrates as a whole to the compound waves that impinge upon it, and thus serves for the transmission of sound from the air to the chain of ossicles of the middle ear. It is often compared to the membrane of a drum, but there are fundamental differences.

When a drum is struck, a certain definite fundamental tone is elicited; similarly a drum is thrown into vibration when certain tones are sounded in its neighborhood, while it is quite unaffected by others. In other words, it can take up and vibrate in response to only those tones whose vibrations

$\Delta$

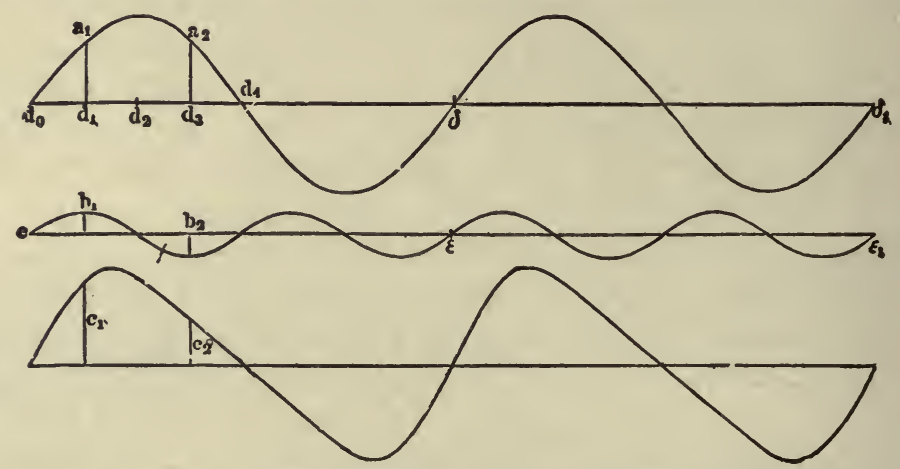

Frg. 439. - Showing $A$ and $B$, Simple Pendular Vibrations, Separated by One Octave. $C$ The form of the curve produced by the combination of $A$ and $B$.

correspond in number with those of its own fundamental tone. The tympanic membrane can vibrate in response to a wide range of tones, thus transmitting vibration frequencies ranging between 30 to 4,000 or 5,000 per second, or even higher. The limits of hearing as regards pitch are in the ordinary average ear represented by tones of these vibration frequencies. In some individuals the scale extends to 30,000 or more. This range is made possible by the fact that the membrana tympani is by no means under constant tension, but is connected with the chain of auditory ossicles, the contractions of the muscle of which produce variations in tension, especially on the handle of the malleus. If the membrane were quite free in its center, it would be like a drum which goes on vibrating for some time after it is struck, and each sound would be prolonged, leading to considerable confusion. This evil is obviated by the ear bones, which check the continuance of the vibrations like the "dampers" in a piano.

The vibrations of the membrana tympani are transmitted by the chain of ossicles to the fenestra ovalis and the fluid of the labyrinth, their 
dispersion in the tympanum being prevented by the difficulty of the transition of vibrations from solid to gaseous bodies. The necessity of the presence of air on the inner side of the membrana tympani is obvious. Without this provision, neither would the vibrations of the membrane be free nor the chain of bones isolated so as to propagate the sonorous undulations with concentration of their intensity. While the oscillations of the membrana tympani are readily communicated to the air in the cavity of the tympanum, those of the solid ossicles will not be conducted away by the air, but will be propagated to the labyrinth without being dispersed in the tympanum. The existence of the membrane over the fenestra rotunda permits vibration of the stapes to and from the labyrinth. When the membrane of the fenestra ovalis is pressed toward the labyrinth by the stapes, the pressure is communicated through the fluid of the labyrinth toward the cavity of the tympanum at the fenestra rotunda which yields.

The propagation of sound through the auditory ossicles to the labyrinth must be effected by oscillations of the bones as a whole. The long process of the malleus receives the undulations of the membrana tympani, figure $440, a, a$, in a direction indicated by the arrows and nearly perpendicular to itself. The vibrations of the long process of the malleus sets the chain of bones vibrating about the axis fixed by the attachment of the anterior ligament of the malleus and the ligaments of the incus, see

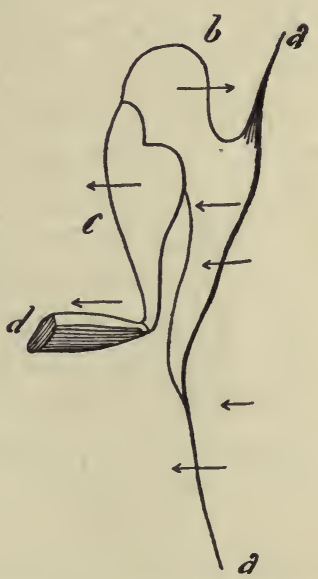

FIG. 440.-Diagram to Illustrate the Action of the Ossicles of the Middle Ear in the Conduction of Sound to the Internal Ear. figure 432. From the long process of the incus, which is parallel with the long process of the malleus, the undulations are communicated to the stapes and by the stapes to the fenestra ovalis in a perpendicular direction. Increasing tension of the membrana tympani diminishes the facility of transmission of sonorous undulations from the air to it. It has been inferred, therefore, that hearing is rendered less acute by increasing the tension of the membrana tympani. This is accomplished by the contractions of the tensor tympani muscle. The exact influence of the stapedius muscle in the act of hearing is unknown. It acts upon the stapes in such a manner as to make it rest obliquely in the fenestra ovalis, depressing that side of the stapes on which it is attached and elevating the other side to the same extent. It seems to prevent too greal a movement of the bone.

The pharyngeal orifice of the Eustachian tube is usually shut. During swallowing, however, it is opened; which may be shown as follows: If the 
nose and mouth be closed and the cheeks blown out, a sense of pressure is produced in both ears the moment we swallow. This is due, doubtless, to the bulging out of the tympanic membrane by the compressed air, which at that moment enters the Eustachian tube. The principal office of the Eustachian tube has relation to the prevention of the effects of increased tension of the membrana tympani. Its existence and openness will provide for the maintenance of the equilibrium between the air within the tympanum and the external air, so as to prevent the inordinate tension of the membrana tympani which would be produced by too great or too little pressure on either side. While discharging this office it serves as an outlet for mucus. If the tube were permanently open, the sound of one's own voice would probably be greatly intensified, a condition which would of course interfere with the perception of other sounds. At any rate, it is certain that sonorous vibrations can be propagated up the tube to the tympanum by means of a catheter inserted into the pharyngeal orifice of the Eustachian tube.

The Function of the Internal Ear.-The fluids of the labyrinth receive the sonorous vibrations at the fenestra ovalis and, we must assume, conduct the same throughout the cavity. In all forms of organs of hearing even to the simplest, liquid is the medium through which the auditory sensory epithelium is stimulated. We have already seen that in the mammalian ear there is a special mechanical arrangement to intensify the vibrations of the fluid in the cochlear canal.

The utriculus, sacculus, and semicircular canals are probably not concerned with auditory function, but with the sense of equilibrium; hence they will be discussed separately a little later.

The cochlea is the special organ of hearing. When it is set in vibration the movement stimulates the sensory hair cells on the basement membrane, producing a sensory impulse which is transmitted along the paths to the brain and there produces an auditory sensation. If the stimulus results from a disturbance of an explosive or non-harmonic nature, the sensation is interpreted as a noise. If the disturbance is rhythmic or harmonic and repeated in sequence within certain limits of rate, then a tone is perceived.

The intensity of sound, the energy of the disturbance, affects the basilar membrane by producing motion of varying amplitude. This stimulates the hair cells with greater or less intensity, which can be detected by the sensorium as loudness. Loudness of the sound sensation is interpreted as intensity of sound wave.

The interpretation of pitch is accomplished by the ear through a wide range of rates of vibration that produce sensations of tone. The average person can perceive musical tones over a range of vibration of from sixty-four double vibrations per second for the lower notes, to four thousand and ninetysix for the higher notes. These limits may be extended to thirty per second 
and forty thousand per second, respectively, but only a small number of tones can be perceived outside of the narrower limits given above. This extraordinary range of tone is conceivable only on the supposition of localization of the stimulus in some part of the organ. Most physiologists look to the basilar membrane and the organ of Corti for the localization.

Suppose a simple tuning-fork to be vibrating with a frequency of sixtyfour per second, then these waves will be conducted through the auditory apparatus until they fall on the basilar membrane, and will set it in vibration at the same rate. The exact type of the vibration is at present a matter of inference. The piano theory of Helmholtz gives probably the most satisfactory explanation. It assumes that the basilar membrane vibrates as would a number of strings set in its transverse dimension. In support of this assumption it is asserted that the membrane is taut in the transverse and loose in the longitudinal plane. Retzius has estimated that it contains about 24,000 fibers, and that it measures in width at the base $0.135 \mathrm{~mm}$. and at the apex $0.234 \mathrm{~mm}$. In the above illustration the vibration frequency of sixty-four would supposedly set in sympathetic vibration that part of the apex of the basilar membrane which vibrated in the same frequency, and the sensory cells of the organ of Corti, located over the vibrating fiber, would be stimulated accordingly. In the same way notes of medium and of high frequency stimulate localized areas of sensory cells in the middle and basal parts of the organ of Corti and produce sensations of corresponding pitch.

This idea of localization of auditory sensory stimulation makes it easier to understand the analysis by the ear of compound sonorous waves. Such waves impinge on the membrana tympani and are transmitted through the conducting media unanalyzed, and may be supposed to fall on the basilar membrane as compound waves. The basilar fibers acting like so many resonators, take up the constituent sonorous elements in sympathetic vibration. In short, the basilar membrane is an analyzer in which the compound wave is reduced to its simple components, each of which stimulates its corresponding portion of the organ of Corti. The auditory nerve impulses are conducted through the cochlear nerves to the sensorium where they produce auditory sensations with the same definiteness of pattern as cutaneous or optical stimuli produce sensations that correspond to the patterns of stimulation. The audition is so definite that one can consciously pick out one or the other of the constituent stimulating elements and follow and examine the same to the exclusion of the others, as when one follows a single instrument in an orchestra or a single voice in a group of chattering children.

Bernstein says of this wonderful organ:

"In the cochlea we have to do with a series of apparatus adapted for performing sympathetic vibrations with wonderful exactness. We have here before us a musical instrument which is designed not to create musical 
sounds, but to render them perceptible, and which is similar in construction to artificial musical instruments, but which far surpasses them in the delicacy as well as the simplicity of its execution. For, while in a piano every string must have a separate hammer by means of which it is sounded, the ear possesses a single hammer of an ingenious form in its ear bones, which can make every string of the organ of Corti sound separately."

Auditory Judgments. - Direction.-The power of perceiving the direction of sounds is not a faculty of the sense of hearing itself, but is an act of the mind judging by experience previously acquired. From the modifications which the sensation of sound undergoes according to the direction in which the sound reaches us, the mind infers the position of the sounding body. The only true guide for this inference is the more intense action of the sound upon one than upon the other ear. But even here there is room for much deception, by the influence of reflexion or resonance, and by the propagation of sound from a distance, without loss of intensity, through curved conducting tubes filled with air. By means of such tubes, or of solid conductors, which convey the sonorous vibrations from their source to a distant resonant body, sounds may be made to appear to orginate in a new situation. The direction of sound may also be judged of by means of one ear only; the position of the ear and head being varied, so that the sonorous undulations at one moment fall upon the ear in a perpendicular direction, at another moment obliquely. But when neither of these circumstances can guide us in distinguishing the direction of sound, as when it falls equally upon both ears, its source being, for example, either directly in front or behind us, it becomes impossible to determine whence the sound comes.

Distance.-The judgment of the distance of the source of sounds is inferred from their intensity. The sound is interpreted as coming from an exterior sonorous body. When the intensity of the voice is modified in imitation of the effect of distance, it excites the idea of its originating at a distance. Ventriloquists take advantage of the difficulty with which the direction of sound is recognized, and also the influence of the imagination over our judgment, when they modulate the voices, and at the same time pretend, themselves, to hear sounds as coming from a certain direction.

Duration of the Auditory Stimulus.-By removing one or several teeth from the toothed wheel of a vibrator, the fact has been demonstrated that in the case of the auditory organ, as in that of the eye, the sensation continues longer than the impression which causes it; for a removal of the tooth produced no interruption of the sound. The gradual cessation of the sensation of sound renders it difficult to determine its exact duration beyond that of the impression of the sonorous impulses.

Binaural Sensations.-Sound stimulates each of the two ears yet ordinarily only one sound sensation is perceived, just as in binocular vision , only a single object is seen. Analogous to the double vision dependent on 
unequal refraction is the double hearing of a single sound owing to the sound coming to the ear through media of unequal conducting power. The phenomenon depends on the unequal conducting power of two media through which one and the same sound is transmitted to the ear. If a small bell be sounded in water while the ears are closed by plugs, and a solid conductor be interposed between the water and one ear, two sounds will be heard differing in intensity and tone, one being conveyed to the ear through the medium of the atmosphere, the other through the conducting-rod.

Subjective Sensations. - Subjective sounds are the result of a state of irritation or excitement of the auditory nerve produced by other causes than sonorous impulses. A state of excitement of this nerve or its tracts gives rise to the sensation of sound, hence the ringing and buzzing in the ears heard by persons of irritable and exhausted nervous system, and by patients with cerebral disease, or disease of the auditory nerve itself; hence also the noise in the ears heard for some time after a long journey in a rattling, noisy vehicle. Ritter found that electric currents also excite sounds in the ears. From the above truly subjective sound we must distinguish those dependent, not on a state of the auditory nerve itself merely, but on sonorous vibrations excited in the auditory apparatus. Such are the buzzing sounds attendant on vascular congestion of the head and ear or on aneurysmal dilatation of the vessels. Frequently even the simple pulsatory circulation of the blood in the ear is heard. To the sounds of this class belong also the buzz or hum heard during the contraction of the palatine muscles in the act of yawning, during the forcing of air into the tympanum so as to make tense the membrana tympani.

Irritation or excitement of the auditory nerve may give rise to movements in the body and to sensations seemingly from other organs of sense. In both cases it is probable that associated centers of the brain stem and connected areas of the cortex come into play. An intense and sudden noise excites in every person closure of the eyelids, and in nervous individuals a start of the whole body or an unpleasant sensation through the body like that produced by an electric shock.

\section{THE SENSE OF EQUILIBRIUM.}

Although the utriculus, sacculus, and semicircular canals form the major part of the labyrinth and are closely associated with the cochlea in development, there is increasing evidence that these structures are not concerned with hearing, but rather with a sense of equilibrium. This view has been strengthened by recent investigations into the anatomical relations of the different elements in the auditory nerve, figure 435 . 
These structures have each a special modification of the sensory epithelium which receives the vestibular branch of the eighth nerve. These epithelial areas are differentiations of the embryonic ear pit, which is derived from the epiblast. In fishes which have well-developed semicircular canals and vestibule this sensory epithelium has a common origin from the embryonic anlage which gives rise to the ear, the branchial sense organ, and the lateral line organs, all of which probably have static functions.

The Semicircular Canals. - The semicircular canals are connected with the utriculus, are three in number on each side, and have been already shown to lie in space practically at right angles to one another. Each is filled with endolymph, and each has a special organ, the crista acustica, which receives a division of the vestibular branch of the eighth nerve.

The function of the semicircular canals has long been believed to be to give rise to sensations by which we determine the motions of the body in space. But Maxwell has shown that motion and position are both mediated by the combined reactions of the labyrinthine sense organs. It was shown long ago that if one closes his eyes and turns rapidly around the vertical axis, then suddenly stops and opens the eyes, surrounding objects seem to be rotating around the same vertical axis. If the face is in the horizontal plane and the rotation around the vertical axis, then, upon suddenly raising the head into the ordinary position and opening the eves, objects seem to be moving about the head around the horizontalaxis. In both these cases the direction of the apparent motion of objects depends upon the actual motion of the body that preceded it and is in the opposite direction. In the first case the rotation is in the plane of the horizontal semicircular canal. It is assumed here that, at the beginning of such a movement, the endolymph, being fluid and inert, tends to remain still for a moment and the effect is to produce an increase in pressure in the funnel of the ampulla. This relative increase of tension on the hair cells of the crista acustica, stimulates the hair cells and gives rise to sensory nerve impulses. When the head suddenly stops rotating the situation is just reversed and stimulation is in the opposite direction. Considering the position of the three semicircular canals, it will be seen that movement of the head in any direction will stimulate one or more of the cristæ, giving rise to either simple or complex sensory impulses. Stimuli on the recessus utriculi adds to the effect.

This theory is borne out by the effects of operation and stimulation of the exposed semicircular canals. Injury to the semicircular canals causes disturbances in muscular co-ordination, especially in movements that take place in the plane of the injured canal. If a horizontal canal in a pigeon be sectioned, the animal supports its head in the vertical position very well, but is unable to co-ordinate its horizontal movements. It tends to produce rotary motions around the vertical axis. If a vertical canal is sec- 
tioned, the head falls to one or the other side according to the canal, and the animal shows instability of position in that plane. It has been shown that stimulation of a sectioned canal produced reflex movements in that plane.

Muscular co-ordination is a complex phenomenon and involves the operation of numerous sensory impulses from other organs of the body, especially from the eyes and general skin. Some confusion has arisen from the fact that there are associated with the disturbance in the semicircular canals movements of the eyes and head in higher animals, and of the eyes, head, and fins in such animals as fishes-the so-called compensatory movements. Without going into details it is sufficient to state that the sense organs of the semicircular canals probably form only one of the series of sensory structures concerned in the co-ordination of body movements.

The Utriculus and Sacculus.-The utriculus and sacculus each have a sensory area, the maculæ, over which there rests in the human ear and in most animals small particles of calcareous matter, otoliths. These otoliths, therefore, lie among the projecting hairs of the sensory cells. This is characteristic of these sensory areas and differentiates them from the arrangement present in the cristæ. There would seem to be close agreement in function between the maculæ and cristæ, and we naturally look to the influence of the otoliths on the processes which result in the stimulation of the maculæ. Attempts have been made to remove the otoliths, with the result that in such animals there is apparent inability to maintain a constant position in space. The experiments have been performed which have suggested the theory that the otoliths take an active part in stimulating the sensory cells, probably by their mere pressure. If the head is inclined in one or the other direction, the pressure of the otoliths will shift on the hair cells, and that is sufficient to stimulate them. If this view is correct, then we may regard these structures as static in function as compared with the semicircular canals, which are dynamic. The anatomical separation of the nerves for the cochlea from the division for the utriculus, sacculus, and semicircular canals itself suggests isolation in function, figures 389 and 435 . It is conceivable that loud noises of an explosive nature may cause sufficient vibration of the endolymph to affect the otoliths and thus stimulate the cristæ. Yet, if such stimulation takes place it is probably only of secondary importance.

\section{THE SENSE OF SIGHT.}

\section{THE EYE.}

The eye, the organ of vision, is the most complex and most highly developed of the organs of special sense. It consists not only of a special sensory epithelium, the retina, sensitive to light stimulation, but of a series of special structures which intensify and localize the stimulus. There are also accessory organs for the protection of the eye. 
The Eyelids and Lachrymal Apparatus.-The eyeball is kept moist over its free surface and protected from external injury by the eyelids, by the glands that secrete the lachrymal fluid to moisten the surface of the cornea, and by the oil glands that secrete oil on the margins of the lids.

The conjunctiva, or lining membrane of the lids, which is reflected on to the free surface of the eyeball, protects the eye from injury by its extraordinary sensitiveness to irritation by dust or other external substance. Its stimulation produces reflex secretion of the lachrymal fluid that flows over the surface of the eye, and tends to wash away the stimulating substance.

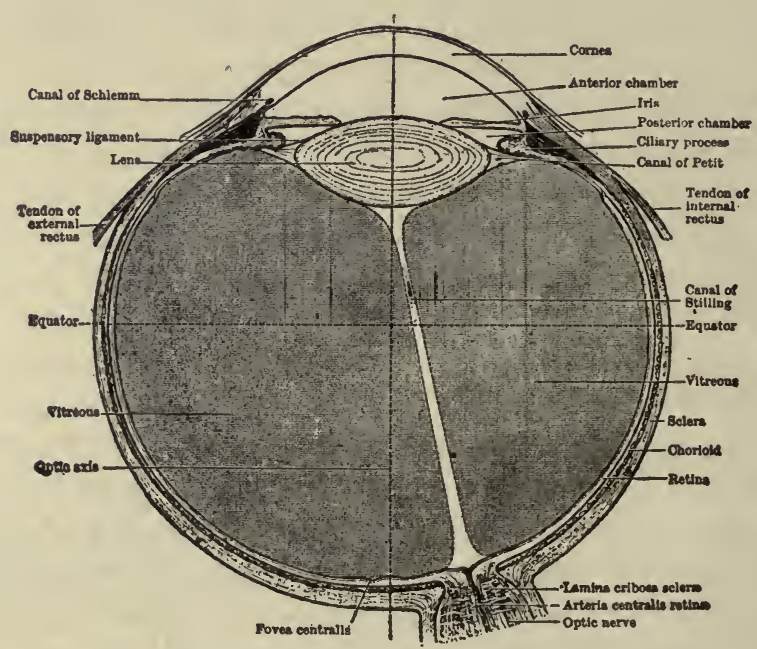

Fig. 44r.-Section of the Eyeball.

The Eyeball and its Parts.-The detail of the structure of the eyeball is too complex to be given here except in so far as seems necessary for a clearer presentation of the physiological facts. A gross dissection reveals the tough, white, outer coat, the sclera; the intermediate thin, vascular pigmented coat, the choroid; and the inner nervous coat, the retina.

The section also shows that the eyeball is specialized in structure in its anterior region and that its contained cavity is divided into two parts, viz., the anterior and posterior chambers. The anterior chamber is filled with the transparent aqueous fluid. This fluid is like lymph in its composition. The posterior chamber between the lens and the retina is filled with the clear jelly-like vitreous substance.

The Cornea.-The sclera is continuous with the cornea in front of the eyeball, but the cornea is transparent and its radius of curvature is less than that of the main portion of the eye. It is composed of stratified epithelial cells, and is richly supplied with sensory nerves that form an intraepithelial 
plexus of delicate varicose f'Jrils. The cornea has no blood vessels, but has a rich network of lym $\because+1$ tic spaces.

The Lens. - The lers is a special modification of epithelium composed of highly refractive material, situated just behind the iris. It is enclosed in a capsule and supported in its place by the suspensory ligament, which

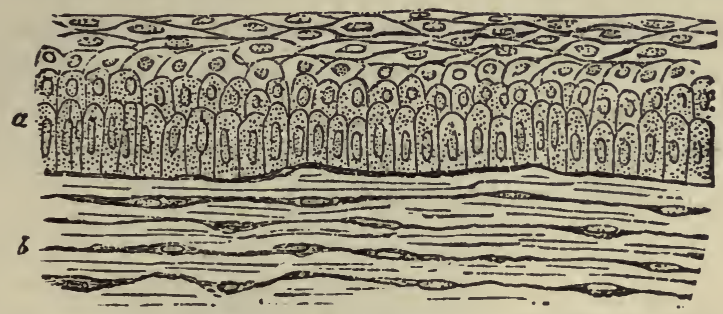

FIG. 442.-Vertical Section of Rabbit's Cornea. $a$, Epithelium of cornea, showing the different shapes of the cells at various depths from the free surface; $b$, portion of the proper substance of the cornea. (Klein.)

is fused into the capsule around its equator. The lens is a biconvex structure composed of transparent fibers which are arranged in concentric layers. Its posterior curvature is greater than the anterior, the radii being 6 and Io $\mathrm{mm}$. respectively.

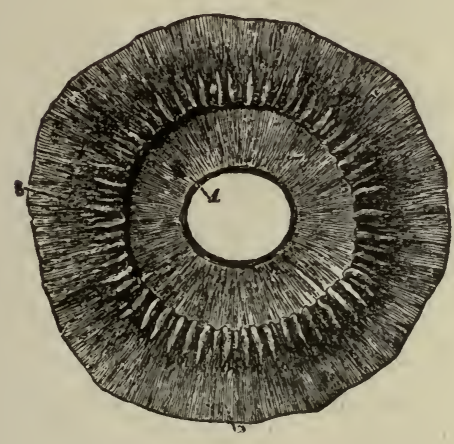

FIG. 443.

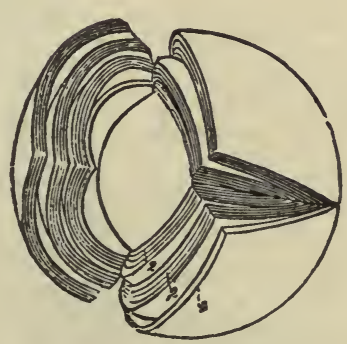

FIG. 444 .

FIG. 443.-Ciliary Processes, as Seen from Behind. I, Posterior surface of the iris, with the sphincter muscle of the pupil; 2, anterior part of the choroid coat; 3 , one of the ciliary processes, of which about seventy are represented. $\times \frac{1}{2}$.

FIG. 444.-Laminated Structure of the Crystalline Lens. The laminæ are split up after hardening in alcohol. I The denser central part or nucleus; 2 , the successive external layers. $\times 4$. (Arnold.)

The Ciliary Apparatus and the Iris.-These structures are a continuation and modification of the choroid coat in the anterior portion of the eye. Around the circumference of the cornea the choroid coat is considerably thickened and folded in the modification known as the ciliary apparatus. 
A radial layer of muscle, figure 445 , is knitted into the base of the cornea, on the one hand, and extends back into the choroid, on the other. Thick bundles of the circular fibers are also present in this mass of muscle. From the ciliary processes extending over the lens is the iris. It is a sheet of connective tissue and muscle lined with epithelium and highly pigmented.

In the middle anterior portion of the iris is a round aperture, the pupil. The muscle fibers are of the unstriated muscle type and are arranged in two layers one circularly and the other radially. Contractions of the circular muscles of the iris produce constriction of the pupil, while contractions of the radial fibers produce dilatation. Both the ciliary apparatus and the iris are supplied with motor nerves.

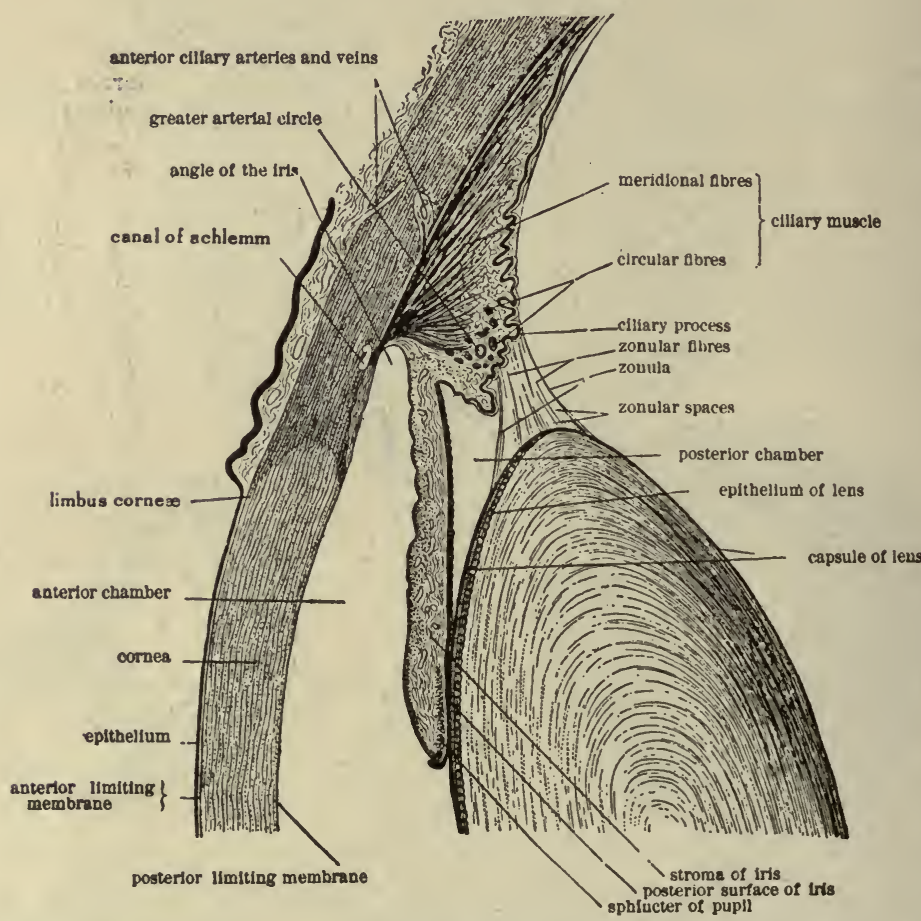

FIG. 445.-Meridional Section of a Portion of the Anterior Part of the Eyeball. (Toldt.)

Fibers of the third cranial nerve are distributed to the ciliary muscles, apparently to both radial and circular muscles, and when these nerves are stimulating the resulting contractions of the muscles tend to remove the tension from the capsule of the lens. These nerve fibers pass through the ciliary ganglion where they form synapses with the ganglionic cells. Motor fibers from the third cranial nerve also supply the circular muscle of the iris, which produces constriction of the pupil. The radial muscles of the iris are 
supplied by nerves by way of the cervical sympathetic, superior cervical ganglion, and the ophthalmic branch of the fifth cranial nerve.

Structure of the Retina.-The retina occupies the deeper half of the cup of the eyeball. It extends forward as far as the ora serrata, where its complex structure changes the form to a simple epithelial layer, which lines the anterior portion of the eyeball and the ciliary processes. In the center of the retina is a round yellowish spot having a minute depression in its center, called the yellow spot of Sommering. The depression in its center is

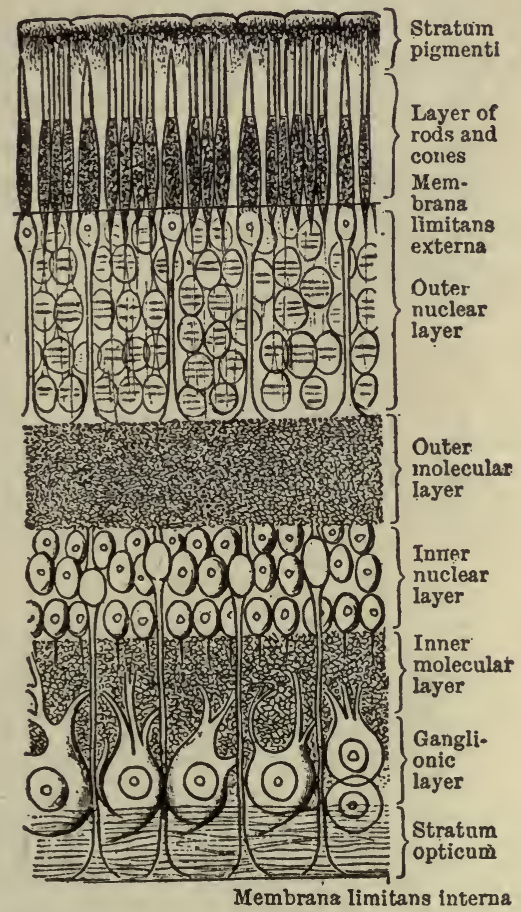

Fig. 446.-Section of Human Retina. (Cunningham, modified from Schulze.)

the fovea centralis. About $2.5 \mathrm{~mm}$. to the inner side of the yellow spot is the point at which the optic nerve enters and spreads out its fibers into the retina.

The optic nerve arises from the base of the brain and passes forward toward the orbit, being covered by the membranes which cover the brain. The fibers of the optic nerve are exceedingly fine, and are surrounded by the myelin sheath, but do not possess the ordinary external nerve sheath. As they pass into the retina they lose their myelin sheaths and proceed as axis-cylinders (the cells of origin of these fibers are in the retina). Neuroglia supports the nerve fibers in the optic nerve trunk. In the center of the nerve is a small artery, the arteria centralis retince. The number of fibers in the 
optic nerve is said to be upward of 500,000 in each nerve (Salzer). The fibers of the optic nerve spread out over the inner surface of the retina as far as the ora serrata.

The retina itself consists of layers of nerve elements supported by delicate connective tissue. The older descriptions recognize some eight or ten layers in the retina. These appear in the ordinary microscopic preparations and are shown in figure 446. But the newer investigations of Cajal, Golgi, Retzius, and others have shown that the retina is a much simpler structure than heretofore described. The retina is formed of essentially three layers of nerve and sense cells. Naming from the center of the eye outward, they are: The ganglionic layer; the layer of bipolar cells; and the layer of rods and cones, figure 447. The cells of these layers have numerous fibrous processes which interlock in such a way that they seem to form different areas when studied in cross-section, figure 446 . If we recognize the strata of interlacing fibers, then the following may be made out:

The layer of ganglion cells ....

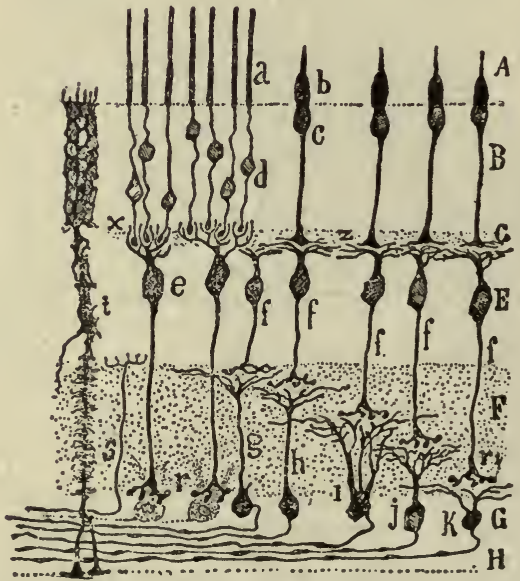

FIG. 447.-Transverse Section of a Mammalian Retina. A, Layer of rods and cones; $B$, bodies of visual cells (external granular); $C$, external molecular layer; $E$, layer of bipolar cells (internal granular); $F$, internal molecular layer; $G$, layer of ganglionic cells; $H$, layer of optic-nerve fibers; $a$, rod; $b$, cone; $c$, body of the cone cell; $d$, body of the rod cell; $e$, bipolar rod cells; $f$, bipolar cone cells; $g, h, i, j, k$, ganglionic cells ramifying in the various strata of the internal molecular zone; $r$, inferior arborization of the bipolar rod cells, connecting with the ganglionic cells; $r$, inferior arborization of the bipolar cone cells; $t$, epithelial or Müller cells; $x$, point of contact between the rods and their bipolar cells; $z$, point of contact between the cones and their bipolar cells; $s$, centrifugal nerve fiber. (Cajal.) 
The Nerve Fiber and Nerve Cell Layers. - The inner surface of the retina is formed of a layer of nerve fibers which have their origin in the adjacent large nerve cells and converge toward the exit of the optic nerve. Externally the ganglionic cells send up numerous processes, or dendrites, which interlace with the fibers of the bipolar cells of the third or inner nuclear layer and form the inner molecular layer of the older writers.

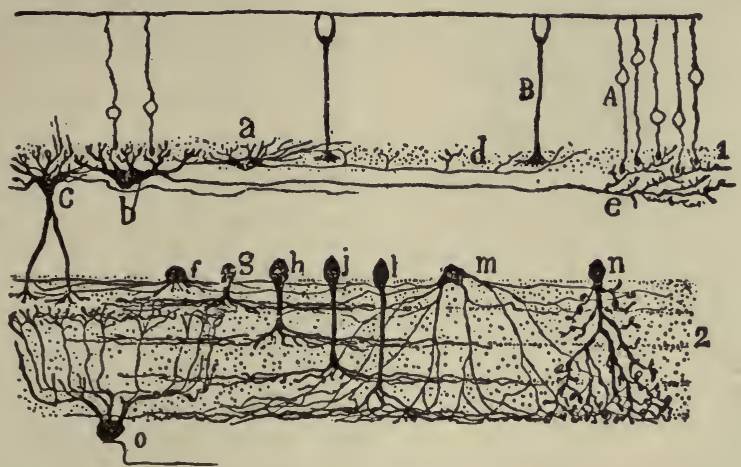

Fig. 448.-Perpendicular Section o, the Retina of a Mammal. A, External grains or bodies of rods; $B$, bodies of cones; $a$, horizontal external or small cell; $b$, horizontal internal or large cell; $c$, horizontal internal cell with descending protoplasmic appendages; $e$, flattened arborization of one of the large cells; $f, g, h, j, l$, spongioblasts ramifying in the various strata of the internal molecular zone; $m, n$, diffuse spongioblasts; $o$, ganglionic cell; I, external molecular zone; 2, internal molecular zone. (Cajal.)

The Middle Layer.-The middle layer consists of bipolar cells which send one process toward the ganglionic layer to interlace with the dendrites of the ganglionic cells, and one process externally. This process is often divided into many branches, which separate out into a horizontal brush, interlacing

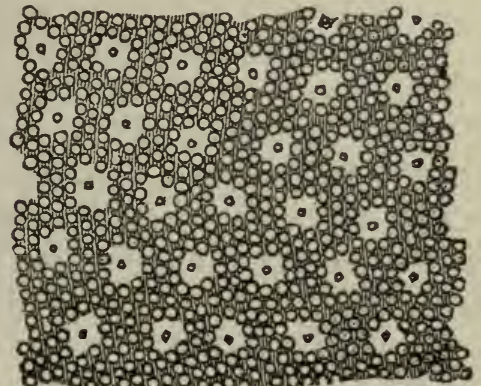

A

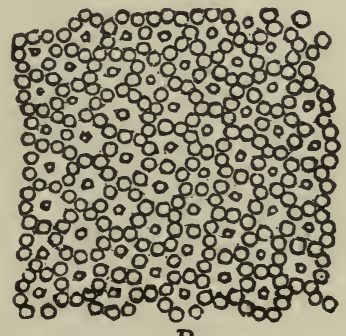

B

FIG. 449.-Distribution of the Rods and Cones. $A$, In the peripheral part of the retina; $B$, from the region of the macula lutea.

with the processes of the rods and cones. Special cells have been described for this layer of the retina, as, for example, the spongioblasts of Cajal.

The External Layer of Rods and Cones. - The rod cells are composed of two parts quite different in structure, known as the outer and inner limbs. 


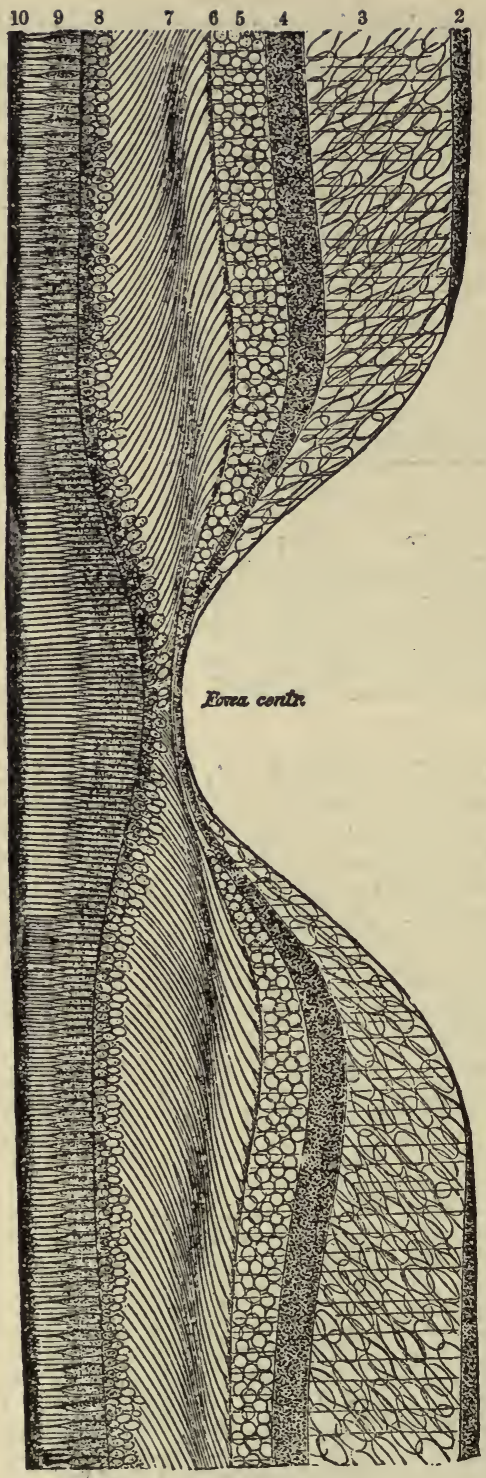

Fig. 450.-Diagrammatic Section of the Macula Lutea and Fovea Centralis, 2, Layer of nerve fibers; 3 , layer of multipolar cells; 4 , internal molecular layer, composed of intertwining arborescent processes; 5, layer of bipolar cells; or internal granular layer; 6 , external molecular layer, composed of intertwining arborescent processes; 7 , nuclei of epithelial cells, or external granular layer; 8, frillwork formed by processes from fibers of Müller, often called the "external limiting membrane"; 9 , layer of rods and cones; to, layer of pigment epithelium.
The outer limb is a cylindrical rod about $30 \mu$ long by $2 \mu$ in diameter. It is transparent and composed of doubly refractive material. The inner limb of the cell is about the same length as the outer, is similar, and is longitudinally striated, and contains a nucleus on its course, figure $447, d$.

The cone cells are also made up of two limbs, the outer of which is conical instead of cylindrical as in the case of the rods. In other respects they are similar to the rods in structure, with the exception that the inner limb ends in a brush of fibrils which interlace with the bipolar cells of the middle layer. In man and mammals the number of rod cells is much greater than the cones, but it is said that in birds cones predominate. Even in man the center of the fovea centralis is devoid of rods and consists of cones only, figure $45^{\circ}$.

All the elements of the retina are sustained and isolated by large cells lying vertically which are known as the fibers of Miiller. The nucleus of the fiber of Müller is found at the level of the layer of bipolar cells. The two extremities of the protoplasm or cell body are condensed in two homogeneous layers, known as the external and the internal limiting layer. The external limiting layer is placed just between the two segments of the rod and cone cells, forming a fenestrated membrane. The internal limiting layer is situated upon the internal surface of the retina.

At the ora serrata the highly specialized structure of the retina disappears. The nerve fibers and ganglion cells disappear, the connecting cells are fewer, the cones more sparse, and the rods shorten 
and disappear. The structure is quickly reduced to that of a simple epithelial membrane known as the pars ciliaris retinæ.

At the pars ciliaris retinæ, the retina is represented by a layer of columnar

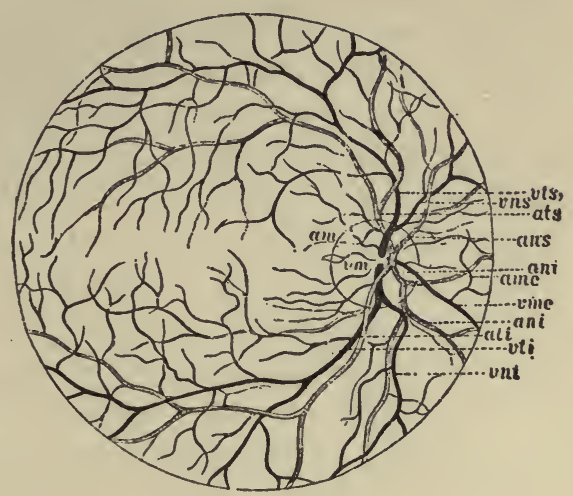

FIG. 45I.-Diagram of the Blood Vessels of the Human Retina. (Leber, after Jaeger.) $a n s$, vns, Superior nasal artery and vein; ats, vts, superior temporal artery and vein; ani, $u n i$, inferior nasal artery and vein; ati, vti, inferior temporal artery and vein; am, vm, macular artery and vein; ane, vme, median artery and vein.

cells, derived from the fusion of the nuclear layers which are in contact with the pigment layers of the retina and continued over the ciliary processes.

Pigment Layer.-This layer, which was formerly considered part of the

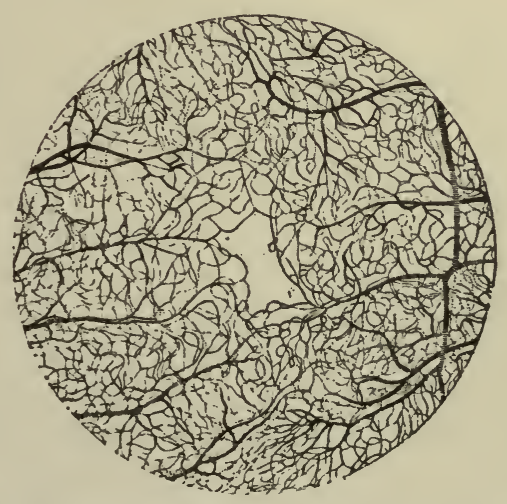

Fig. 452.-Blood Vessels of the Macula Lutea. The part that is totally free from vessels is the fovea centralis.

choroid, consists of cells which cover and entirely surround the outer limbs of the rods and cones.

Blood Vessels of the Eyeball.-The eye is very richly supplied with blood vessels. In addition to the conjunctival vessels; which are derived from the 
palpebral and lachrymal arteries, there are at least two other distinct sets of vessels supplying the tunics of the eyeball: I, the vessels of the sclera, choroid, and iris, and 2, the vessels of the retina. The first are the short and long posterior ciliary arteries. which pierce the sclerotic in the posterior half of the eyeball, and the anterior ciliary which enter near the insertions of the recti. These vessels anastomose and form a very rich choroidal plexus; they also supply the iris and ciliary processes, forming a very highly vascular circle round the outer margin of the iris and adjoining portion of the sclerotic. The distinctness of these vessels from those of the conjunctiva is well seen in the difference between the bright red of blood-shot eyes (conjunctival congestion), and the pink zone surrounding the cornea which indicates deep-seated ciliary congestion.

The central artery of the optic nerve enters the retina from the center of the optic disc and sends out branches over the retinal cup lying in the nerve fiber layer, figure 45I. These blood vessels, however, are absent from the fovea centralis and reduced in size in the macula lutea, figures $45 \mathrm{I}$ and $45^{2}$.

\section{THE OPTICAL APPARATUS.}

The optical apparatus may be supposed, for the sake of description, to consist of several parts: I, a system of transparent refracting surfaces and media by means of which images of external objects are brought to a focus upon the back of the eye; 2, a sensitive screen, the retina, which is a specialized sensory apparatus in connection with the terminations of the optic nerve, and capable of being stimulated by luminous objects, and of sending such impressions as to produce in the brain visual sensations. To these main parts may be added, 3 , an apparatus for focusing light from objects at different distances from the eye, and 4 , since both eyes are usually employed in vision, an arrangement of muscles by means of which the eyes may be turned in the same direction so that binocular vision is possible. The arrangement of the optic nerve fibers, and of the continuation of these backward in the optic chiasma, and thence to special districts of the brain have already been discussed, page 620 .

The eye may be compared to a photographic camera, in which the transparent refracting media correspond to the photographic lens. In a camera images of external objects are thrown upon a screen, the sensitive plate, at the back of the camera box. In the eye, the camera proper is represented by the eyeball with its choroidal pigment, the sensitive screen by the retina, and the lens by the refracting media. In the case of the camera, the screen is adjusted to receive clear images of objects at different distances by a mechanical apparatus for focusing. The corresponding adjustment in the eye is accomplished by the accommodating mechanism.

Refractive Media and Surfaces. - At first sight it would seem as if the refracting apparatus of the eye were very complicated, since it consists 
of so many parts. These parts are: the anterior surface of the cornea itself, the posterior surface of the cornea, the aqueous humor, the anterior surface of the lens, the substance of the lens itself (which is unequally refractive), the posterior surface of the lens, and the vitreous humor. Thus there are four surfaces, and at least, including the air, five media. For all practical purposes, however, we may leave out of consideration all but three refracting surfaces and their adjacent media. These are: the anterior surface of the cornea, separating the air and the corneal substance; the anterior surface of the lens, separating the aqueous humor and the lens substance; and the posterior surface of the lens, separating the lens surface from the vitreous humor.

Image Formation.-In the refraction through a simple transparent spherical surface there are certain cardinal points to be considered. The rays of light which fall perpendicularly on such a surface pass through without refraction. All such rays cut the center of the radius of curvature of the

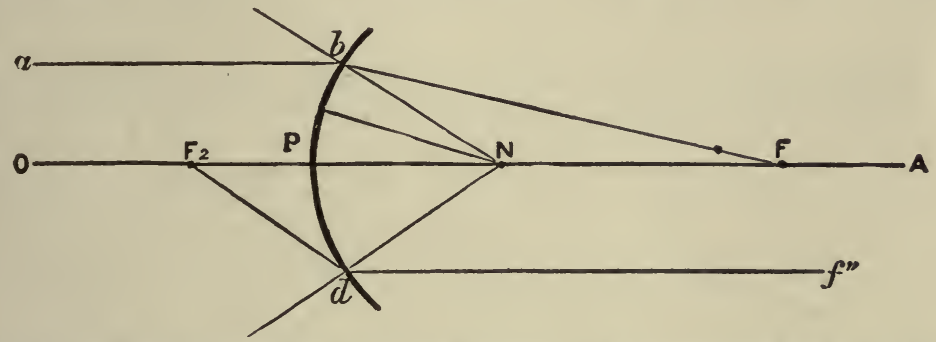

FIG. 453.-Diagram of a Simple Optical System. The curved surface, $b d$, is supposed to separate a less refractive medium toward the left from a more refractive medium toward the right. $\mathrm{N}$, the center of curvature or nodal point; $\mathrm{OA}$, principal axis; $\mathrm{P}$, principal point; $a b$ and OP, rays from an infinite distant point; $b \mathrm{~N}$ and $d \mathrm{~N}$, secondary axes; $\mathrm{F}$ and $\mathrm{F}_{2}$, posterior and anterior principal foci; $\mathrm{PA}$ and $d f^{\prime \prime}$, parallel rays that meet in an infinite distance only.

lens, called the nodal point. A line $O A$ that passes through the center of curvature of a lens and thus pierces the nodal point $N$ is called the optical axis, and the point on the surface pierced by the optical axis $P$ is the principal point. In every optical system there are certain other cardinal facts to be considered. All rays which do not strike vertical to the curved surface are refracted toward the optical axis. Rays which impinge upon the spherical surface of a lens parallel to the optical axis will meet at a point upon the axis called the posterior principal focus, figure $453, F$. The posterior principal focus is outside of the nodal point. Again, there is a point in the optical axis in front of the surface, rays of light from which strike the surface so that they are refracted in a line parallel with the axis, $F_{2} d f^{\prime \prime}$; such a point, $F_{2}$, is called the anterior principal focus.

In any given system the principal foci can be found by erecting verticals at the nodal and principal points of the optical axis and laying off lengths on each, $a$ and $b$, proportional to the refractive indices of the media. A line 
drawn through $a$ on the principal vertical and $b$ on the nodal vertical will cut the optical axis at the posterior principal focus, and vice versa.

If a luminous point outside the anterior principal focus is considered, it is obvious that rays from it will be so refracted when they enter the convex surface that they will become converging and will ultimately meet again in a point or focus. Two such points form conjugate foci, figure 454 . If the anterior focus of a conjugate is moved away from the anterior principal focus, then the posterior conjugate will move toward the posterior principal focus, and the converse. If one conjugate is known, the other can be found as

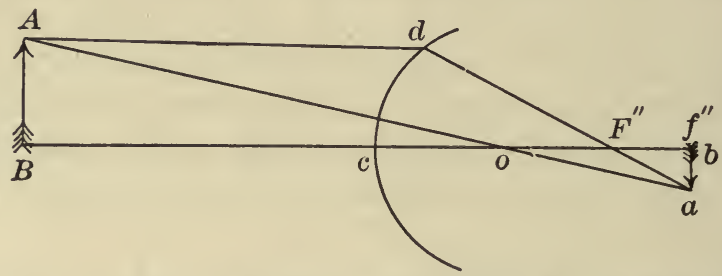

Frg. 454.-Diagram to Show the Relations of Conjugate Foci. $c d$, Refracting surface; $A B$ and $b a$, conjugate foci; $o$, nodal point; $F^{\prime \prime}$, posterior principal focus.

follows: From a point in the plane of the known conjugate, but outside the principal axis, draw two rays, one perpendicular to the refracting surface which will pass through the nodal point, the other parallel to the principal axis. The latter will be refracted through the posterior principal focus and when prolonged will meet the first ray in the plane of the second conjugate, figure 454, $a$. This relationship between conjugate foci is played upon in the focusing of a camera.

It is quite obvious that the eye, even considering only the three surfaces above indicated, is a much more complicated optical apparatus than the one described in the figure. It is, however, possible to reduce the refractive surfaces and media to a simpler form when the refractive indices of the different media and the curvature of each surface are known. All of these data have been very carefully collected. They are as follows:

Index of refraction of aqueous and vitreous.............

Index of refraction of the lens....................

Radius of curvature of cornea.........................

Radius of curvature of anterior surface of lens........ $=10.0 \mathrm{~mm}$.

Radius of curvature of posterior surface of lens........

Distance between anterior surface of cornea and anterior sur-

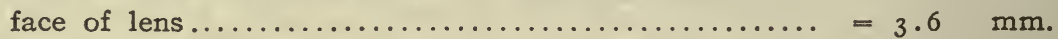

Distance between anterior surface of cornea and posterior

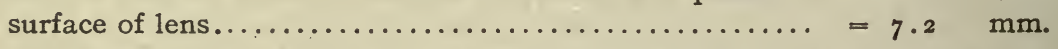

With these data it has been found comparatively easy by mathematical calculation to reduce the different refractive surfaces of the different curva- 
tures into one mean curved surface of known curvature, and the different refracting media into one mean medium the refractive power of which is known.

The simplified or so-called schematic eye, formed upon this principle, suggested by Listing as the reduced eye, has the following dimensions: .

From the anterior surface of the cornea to the principal point $=2.3448 \mathrm{~mm}$. From the nodal point to the posterior surface of lens...... $=0.4764 \mathrm{~mm}$. Posterior principal focus lies behind cornea.......... $=22.8237 \mathrm{~mm}$. Anterior principal focus in front of cornea............ $=12.8326 \mathrm{~mm}$. Radius of curvature of ideal surface..............

In this reduced or simplified eye the principal posterior focus, about $22.8 \mathrm{~mm}$. behind the spherical surface, would correspond to the position of the retina behind the anterior surface of the cornea. The ideal refracting surface would be situated about midway between the posterior surface of the cornea and the anterior surface of the lens.

The optical axis of the eye is a line drawn through the centers of curvature of the cornea and lens, and when prolongéd backward it cuts the retina between the optic disc and fovea centralis. This differs somewhat from the visual axis which passes through the nodal point of the reduced eye to

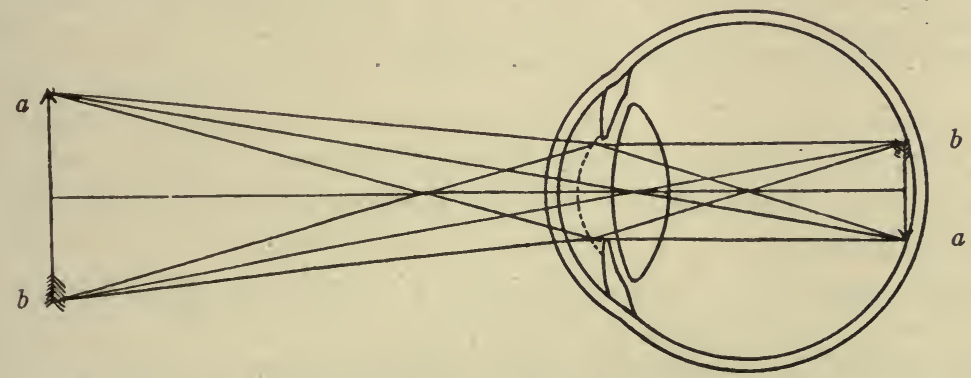

Fig. 455.-Diagram of the Method of the Formation of an Inverted Image Exactly Focused upon the Retina. The dotted line is the ideal surface of curvature.

the fovea centralis, and forms an angle of five degrees with the optical axis. The visual or optical angle is the angle included between the lines drawn from the opposite borders of any object through the nodal point. It has been shown by Helmholtz that the smallest angular distance between two points which can be appreciated is fifty seconds, the size of the retinal image being $3.65 \mu$. This practically corresponds to the diameter of the cones at the fovea centralis which is $3 \mu$, the distance between the centers of two adjacent cones being $4 \mu$.

The image of an object formed upon the retina may be considered as a series of points, from each of which a pencil of light diverges to the eye, and this pencil has for its center or axis a ray which impinging upon the refractive surface perpendicularly to the surface is not refracted, but passes 
through the nodal point and is prolonged backward to the retina. The diverging rays are refracted to converge to a posterior conjugate focus behind the lens on the chief axis of the pencil of light proceeding from the point in question. This focus, if the image is to be clear, should fall on the retina.

Thus from each point of an object a corresponding image is formed on the retina, so that an image of the distant object is produced. It is an inverted image. Whether the image is blurred or not depends upon the refractive power of the media and upon the distance of the anterior surface of the cor-

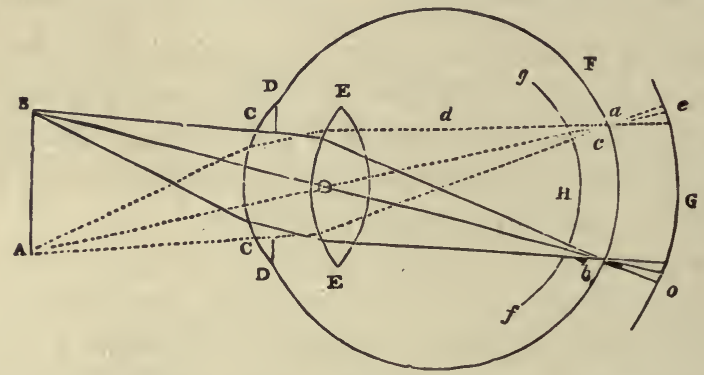

Fig. 456.-Diagram of the Course of a Ray of Light, to Show how a Blurred or Indistinct Image is Formed if the Object be not Exactly Focused upon Retina. The surface $C C$ should be supposed to represent the ideal curvature. The nodal point should be nearer the posterior surface of lens as in figure 455 .

nea from the retina. If the refractive media are too powerful or the eye too long, the image is formed in front of the retina, figure 456 ; if the reverse, the image is formed behind the retina, and in both cases an indistinct and b.urred image is the result.

Accommodation.-The distinctness of the image formed upon the retina is mainly dependent on the perfection with which the rays emitted by each luminous point of the object are brought to a focus upon the retina. If this focus occurs at a point either in front of or behind the retina, indistinctness of vision ensues, in the way we have just described, with the production of a halo. The focal distance, i.e., the distance of the point at which the luminous rays are collected from a lens, besides being regulated by the degree of convexity and density of the lens, varies with the distance of the object from the lens, being greater as the distance is shorter, and vice versa. In other words, the luminous points on the object and the focal points on the retina are conjugate foci. Hence, since objects placed at various distances from the eye can within certain limits be seen with almost equal distinctness, there must be some provision by which the eye is enabled to adapt itseli, so that, at whatever distance the luminous object may be, the focal point may always fall exactly upon the retina.

Accommodation is the act of adapting the eye to vision at different distances. It is obvious that the effect might be produced in either of two ways, viz., 
I, by altering the convexity, and thus the refracting power, either of the cornea or of the lens; or 2, by changing the position of the lens relative to the retina, as in the focusing of a camera, so that whether the object be near or distant, the focal points to which the rays are converged by the lens may always fall exactly on the retina. The amount of either of these changes which is required in even the widest range of vision is extremely small, for from the refractive powers of the media of the eye the difference between the focal distances of the images of an object at a distance and of one at four inches is only about $3.5 \mathrm{~mm}$. On this calculation the change in the distance of the retina from the lens required for vision at all distances, supposing the cornea and lens to remain the same, would not be more than about $2.5 \mathrm{~mm}$. Beer has shown that the second method is indeed the type of accommodative apparatus in fishes. But in man and the higher animals accommodation occurs by the first method, i.e., by changing the convexity of the refracting surface.

The accommodation of the human eye for objects at different distances is primarily due to a varying shape of the lens, its front surface

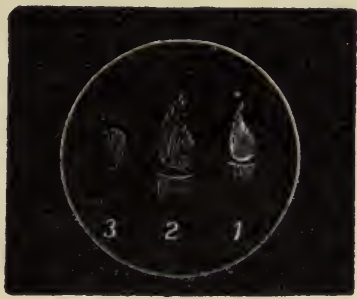

FIG. 457.-Diagram Showing Three Reflections of a Candle. $r$, From the anterior surface of cornea; 2, from the anterior surface of lens; 3 , from the posterior surface of lens. For further explanation, see text. The experiment is best performed by employing an instrument invented by Helmholtz, termed a Phakoscope.

becoming more or less convex, according as the distance of the object looked at is near or far. The nearer the object, the more convex the front surface of the lens, up to a certain limit, and vice versa; the back surface takes little or no share in accommodation. The following simple experiment illustrates this point: If a lighted candle be held a little to one side of a person's eye, an observer looking at the eye from the other side sees three distinct images of the flame, figure 457. The first and brightest, $\mathrm{x}$, is a small erect image formed by the anterior convex surface of the cornea; the second, 2, is also erect, but larger and less distinct than the preceding, and is formed at the anterior convex surface of the lens; the third, 3, is smaller, inverted, and indistinct; it is formed at the posterior surface of the lens, which is concave forward, and therefore, like all concave mirrors, gives an inverted image. If the eye under observation be made to look at a near object, the second image becomes smaller, clearer, and approaches the first. If the eye be now adjusted for a far point, the second image enlarges again, becomes less distinct, and recedes from the first. In both cases alike the first and third images remain unaltered in size, distinctness, and relative position. This proves that during accommodation for near objects the curvature of the cornea, and that of the posterior surface of the lens, remain unaltered, while the anterior surface of the lens becomes more convex and approaches the cornea. 
The experiment, figure $45^{8}$, is more striking when the two prisms of the phakoscope which form two images of the candle are used. The pair of images of the candle from the front surface of the lens not only approach those from the cornea during accommodation, but also approach one another, and become somewhat smaller, Sanson's images.
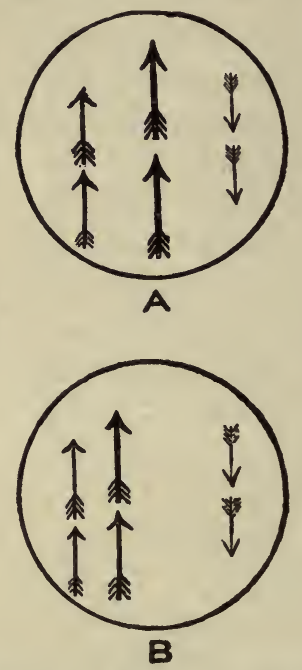

Fig. 458.-Diagram of Sanson's Images. $A$, When the eyes are focused for far objects, and $B$, when they are focused for near objects. The figure to the right in $A$ and $B$ is the inverted image from the posterior surface of the lens.

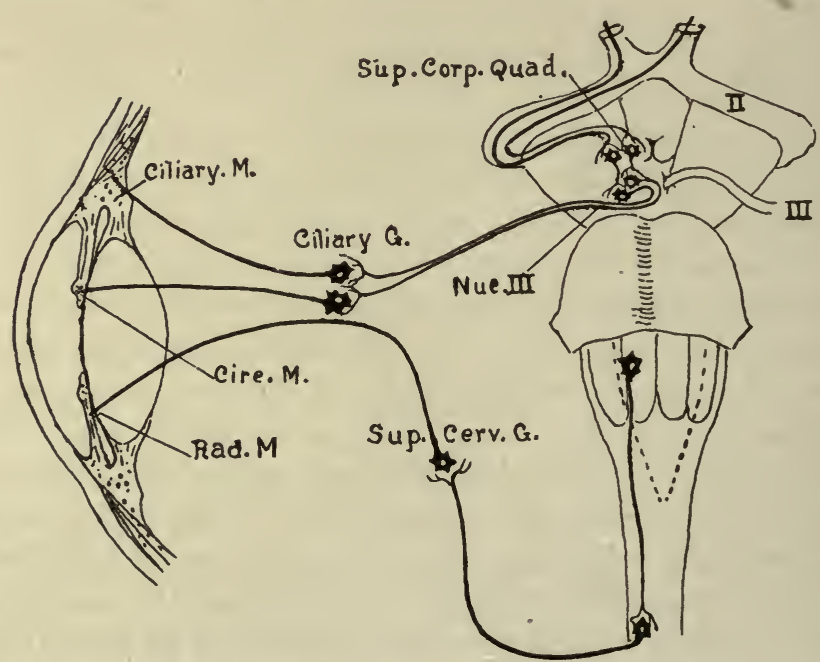

FIG. 459.-Diagrammatic representation of the nerves of the intrinsic muscles of the eye. Sup. Corp.Quad., superior corpora quadrigemina. Nuc. III, nucleus of the third cranial nerve. Sup. Cerv. G., superior cervical ganglion. Circ. M., circular muscles of the iris. Rad. M., radial muscles of the iris. Ciliary $G_{\text {., }}$ ciliary ganglion (Greene). 
The Mechanism of Accommodation.-The mechanism of accommodation depends primarily upon the inherent tendency of the lens to approximate the shape of a sphere. When the eye is at rest the intra-ocular tension is such as to put stress on the suspensory ligament around its equator, which compresses the elastic lens in its antero-posterior dimension. The elasticity of the lens can make itself apparent when the tension of the suspensory ligament is relaxed. This takes place completely after a division of the fibers of the zonula. When we remove the lens from the eye of a young person, we see it assume the spherical shape immediately upon the division of its capsule. In life the slackening of the tension of the suspensory ligament of the lens is brought about by the active contractions of the muscle fibers of the ciliary body, the combined contractions of the radial and the circular fibers. This allows the surfaces of the lens by its own elastic powers to become more convex, thus focusing entering rays of light from a nearer object upon the retina, figure 460 . It therefore appears that when the eye

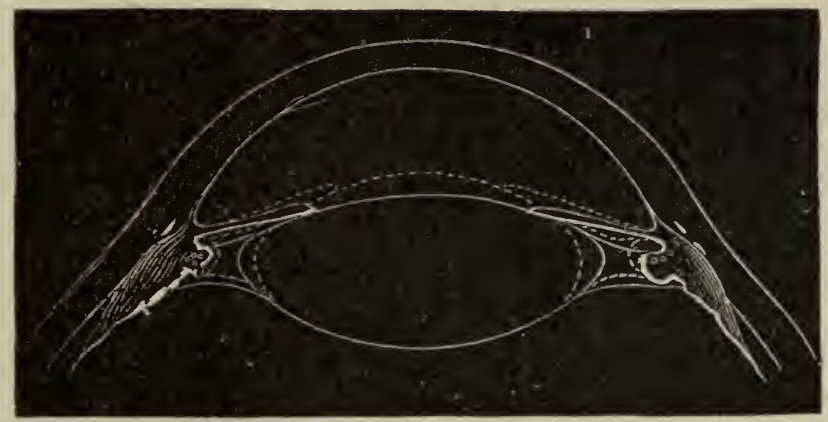

Fig. 460.-Diagram Representing by Dotted Lines the Alteration in the Shape of the Lens on Accommodation for Near Objects. (E. Landolt.)

is at rest it is focused for distant objects, in as much as the suspensory ligament is taut and the anterior surface of the lens more flattened. The normal eye is therefore passive when in focus for distant objects. It is the active contraction of the muscles of accommodation that focuses for near objects.

The iris acts in co-ordination with the accommodative contractions of the ciliary muscles. In viewing near objects the pupil contracts, and upon viewing distant ones it dilates.

Range of Distinct Vision.-Near-point.-In every eye there is a limit to the power of accommodation. If a book be brought nearer and nearer to the eye, the type at last becomes indistinct, and cannot be brought into focus by any effort of accommodation, however strong. The printed letters appear gray and with shadowy outlines. At a certain distance the 
letters are black and distinct but just within this point the outlines present a just perceptible indistinctness, a loss of sharpness of border. This limit is termed the near-point of vision. The near-point can be determined by the experiment of Scheiner. Two small holes not more than $2 \mathrm{~mm}$. apart are pricked in a card with a pin; at any rate their distance from each other must not exceed the diameter of the pupil. The card is hela with the holes close in front of the eye, and a small needle viewed through the pin-holes. At a moderate distance it can be clearly focused, but when brought nearer, within a certain point, the image appears double and more or less blurred. This point where the needle ceases to appear single is the near-point of vision. Its distance from the eye can of course be readily measured. It is usually about five or six inches, I 2 to $I 5 \mathrm{~cm}$. In the accompanying figure, $46 \mathrm{I}$, the lens $b$ represents the eye; $e, f$, the two pin-holes in the card; $n n$ the retina; $a$ represents

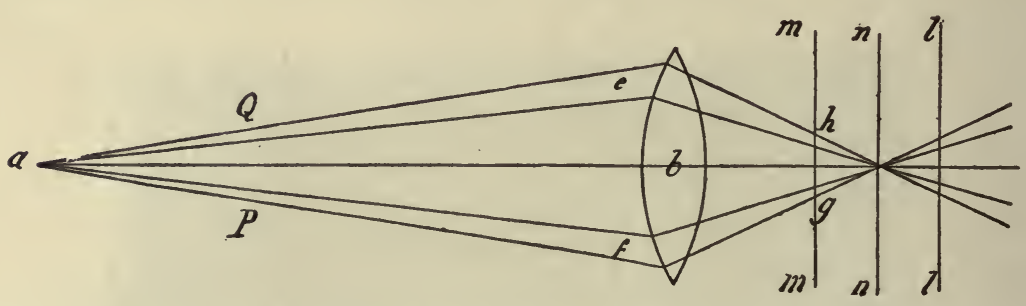

Fig. 461.-Diagram of Experiment to Ascertain the Minimum Distance of Distinct Vision.

the position of the needle. When the needle is at a moderate distance, the two pencils of light coming through the holes $e$ and $f$ are focused at a single point on the retina $n n$. If the needle be brought nearer than the near-point, the strongest effort of accommodation is not sufficient to focus the two pencils, they meet at a point behind the retina. The effect is the same as if the retina were shifted forward to $\mathrm{mm}$. Two indistinct images, $h, g$, are formed by the converging pencils of light, one from each hole. It is interesting to note that when in this way two shadowy images are produced, the lower one, $g$, really appears out in space in the position $Q$, while the upper one appears in the position $P$. This may be readily verified by covering the holes in succession. This is due to the fact that when points on the retina outside the visual axis are stimulated the sensation is referred to an object in space along the line of the secondary optic axis that cuts the retina at the point stimulated.

During accommodation two other changes take place in the eyes. The two eyes converge by the action of the extra-ocular muscles, chiefly by the internal and inferior recti or internal and superior recti. The pupils also contract. 
Movements of the Eye. The eyeball possesses movement around three axes indicated in figure 462 , viz., an antero-posterior, a vertical, and a transverse, passing through a center of rotation a little behind the centre of the optic axis. The movements are accomplished by pairs of muscles.

Direction of Movement.

Inward..................

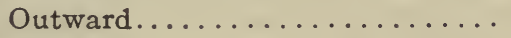

Upward.................

Downward...................

Inward and upward...........

Inward and downward..........

Outward and upward..........

Outward and downward.........

\section{By what muscles accomplished.}

Internal rectus.

External rectus.

Superior rectus.

Inferior oblique.

Inferior rectus.

Superior oblique.

Internal and superior rectus.

Inferior oblique.

Internal and inferior rectus.

Superior oblique.

External and superior rectus.

Inferior oblique.

External and inferior rectus.

Superior oblique.

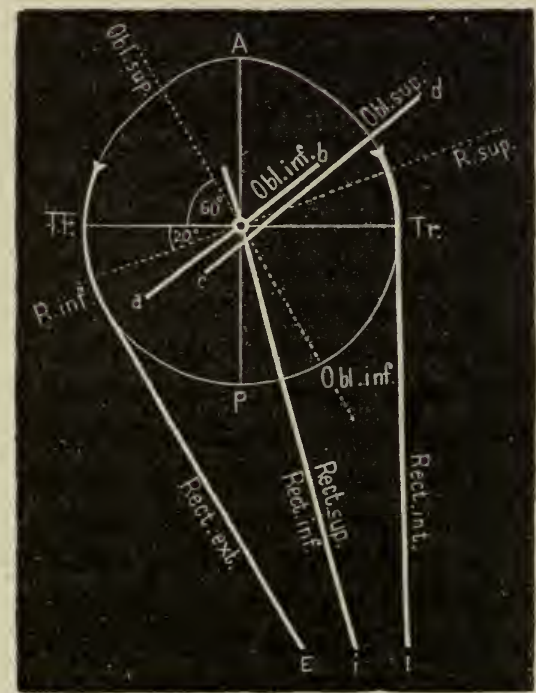

Fig. 462.-Diagram of the Axes of Rotation of the Eye. The thin lines indicate axes of rotation, the thick the position of muscular attachment.

The contraction of all of the muscles during the act of accommodation, viz., of the ciliary muscles, of the recti muscles, and of the sphincter pupillæ, is under the control of the fibers of the third nerve. But the superior oblique may also be employed, in which case the fourth nerve is concerned.

Reflexes of the Pupil.-Contraction of the iris may occur under the following circumstances: I. On exposure of the eye to a bright light. On 
the local application of eserine (active principle of Calabar bean). 3. On the administration internally of opium, aconite, and in the early stages of chloroform and alcohol poisoning. 4. On division of the cervical sympathetic or of stimulation of the third nerve. Dilatation of the pupil occurs,. , in a dim light; 2, when the eye is focused for distant objects; 3 , on the local application of atropine and its allied alkaloids; 4 , on the internal administration of atropine and its allies; 5 , in the later stages of poisoning by chloroform, ether, and other drugs; 6 , on paralysis of the third nerve; 7 , on stimulation of the cervical sympathetic, or of its center in the floor of the front of the aqueduct of Sylvius. The contraction of the pupil is under the control of a center in the floor of the aqueduct beneath the anterior corpora quadrigemina. This center is reflexly stimulated by a bright light, and the dilatation when the center is not in action is due to the stimulation of the radial fibers of the iris by sympathetic nerves. In addition, it appears that both contraction and dilatation may be produced by a local action of certain drugs which is independent of, and probably often antagonistic to, the action of the central apparatus of the third and sympathetic nerves.

The close co-ordination between the two eyes is nowhere better shown than by the condition of the pupil. If one eye be shaded by the hand its pupil will of course dilate; the pupil of the other eye will also dilate, though unshaded, due to crossed reflex action.

Defects in the Optical Apparatus.-Under this head we may consider the defects known as: I, Spherical Aberration; 2, Chromatic Aberration; 3, Astigmatism; 4, Myopia; 5, Hypermetropia.

The normal or emmetropic eye is so perfect that parallel rays are brought exactly to a focus on the retina without any effort of accommodation, figure 466. Hence all objects except near ones (in practice all objects at a distance of twenty feet or more) are seen without any effort of accommodation; in other words, the far-point of the normal eye at rest is at an infinite distance. In viewing near objects we are conscious of the effort (the contraction of the ciliary muscle) by which the anterior surface of the lens is rendered more convex, and rays which would otherwise be focused behind the retina are converged upon the retina.

Spherical Aberration.-The rays in a cone of light from a point on an object situated in the field of vision do not all meet in the same point in the retina, owing to the greater refraction of the rays which pass through the margin of a lens than those traversing its central portion. This defect is spherical aberration. In the camera, telescope, microscope, and other optical instruments it is remedied by the interposition of a screen with a circular aperture in the path of the rays of light, cutting off all the marginal rays and allowing the passage only of those near the center. Such correction is effected in the eye by the iris, which forms a diaphragm to cover the circumference of the lens, and prevents the rays from passing through any 
part of the lens but its center, which corresponds to the pupil. The iris is pigmented to prevent the passage of rays of light through its substance. The image of an object will be most clearly defined and distinct when the pupil is small, if the light is abundant; so that, while a sufficient number of rays are admitted, the narrowness of the pupil may prevent the production of indistinctness of the image by spherical aberration. But even the image formed by the rays passing through the circumference of the lens, when the pupil is much dilated, as in the dark or in a feeble light, may be well defined. Some types of optical apparatus are corrected for this defect by a central instead of marginal diaphragm.

Distinctness of vision is further secured by the pigment of the outer surface of the retina and of the posterior surface of the iris and the ciliary processes. This absorbs any rays of light that may be reflected within the eye and prevents their being thrown again upon the retina so as to interfere with the images formed there. The pigment of the retina is especially important in this respect; for with the exception of its outer layer the retina is very transparent; and if the surface behind it were not of a dark color, but capable of reflecting the light, the luminous rays which had already acted on the retina would be reflected again and would fall upon other parts of the same membrane, producing indistinctness of the images.

Chromatic Aberration.-In the passage of light through the periphery of an ordinary convex lens decomposition of each ray into its elementary colors commonly ensues, and a colored margin appears around the image, owing to the unequal refraction which the elementary colors undergo. This is termed chromatic aberration. It is corrected by the use of lenses constructed of alternate layers of glass of different refractive indices so ground that they produce chromatic dispersion in opposite directions and thus mutually correct any chromatic aberration which may have resulted. The human eye has considerable chromatic aberration, as may readily be demonstrated, Experiment I3, page $72 \mathrm{I}$.

An ordinary ray of white light in passing through a prism has its constituent rays refracted in unequal degrees, and therefore appears as colored bands fading off into each other, known as the spectrum. The colors of the spectrum are arranged as follows; red, orange, yellow, green, blue, indigo, violet; of these the red ray is the least, and the violet the most refracted. Hence, as Helmholtz has shown, the rays from a point of white light cannot be accurately focused on the retina, for if we focus for the red rays, the violet are out of focus, and vice versa; such objects, if not exactly focused, are often seen surrounded by a pale yellowish or bluish fringe.

For similar reasons a red surface looks nearer than a blue one at an equal distance, because, the red rays being less refrangible, a stronger effort of accommodation is necessary to focus them, and the eye is ad- 
justed as if for a nearer object, and therefore the red surface appears nearer, Experiment 13 .

Astigmatism.-The formation of perfect images becomes impossible when either of the refractive surfaces of the eye has unequal curvatures in the different meridians. This defect is called astigmatism. It was first discovered by Airy. An astigmatic eye cannot form a perfect image of a luminous point, and since images of objects are built up of the images of the infinite number of points on their surfaces it follows that all images are distorted. Luminous points appear with imperfect or star-shaped radii or haloes. If these radii overlap in an image they tend to neutralize the diffuse stimulating effects and such regions appear more distinct, while in the converse condition the images are more blurred, figure 465 .

This defect, which is generally present in a slight degree in all eyes, is usually seated in the cornea, but occasionally in the lens as well.

The plane of greatest curvature in the cornea is usually in the vertical meridian, a fact which doubtless comes from the pressure of the eyelids during development. If one looks at figure $463, A$ or $B$, with one eye, the three ines in the radii of the figure will be seen with unequal distinctness. Certain sets will stand out sharp and black and others dim and with indistinct outlines, and if the astigmatism is great enough the three lines may not be distinguished. Figures $C$ and $D$ of this series enable one to detect minute traces of astigmatism with great accuracy.
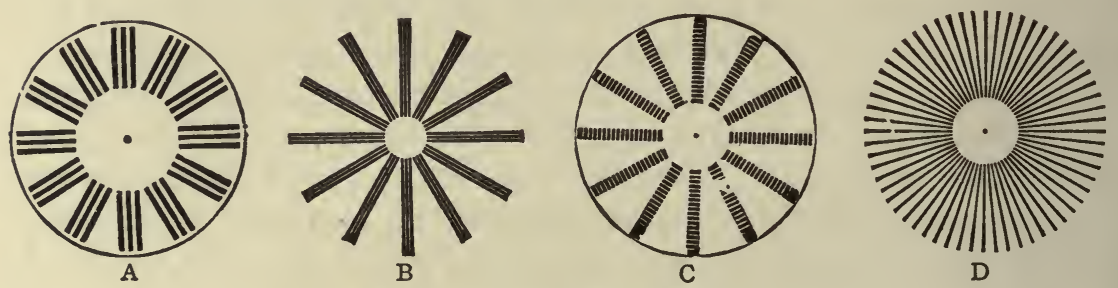

FIG. 463.-Astigmatic Charts.

It is somewhat difficult to picture the rays from a luminous point in their course through eyes which have this defect, but an examination of figure 464 will show their refraction in astigmatism. In this figure four of the total sphere of rays diverging from the point $L$ in the arrows are represented as striking on the refractive surface of the eye at $A, B, C, D$, and being converged toward a focus. The rays $A, C$, separated by a vertical line on the refractive surface, are focused at $f_{1}$, while the rays $A, B$, separated by the horizontal distance on the refractive surface, are brought to a focus at $f_{2}$. Rays from the point $L$, therefore, have two apparent focal points, one point composed of the rays that strike the refractive surfaces in a horizontal plane, $f_{2}$, the other of rays that strike in a vertical plane, $f$. If the retina of the eye be placed at $f_{1}$, it will receive an image of a luminous point with indistinct horizontal halos 
composed of the unfocused rays of all other meridians than the vertical which are in focus. If placed at the position $f_{2}$ it will receive a luminous point with indistinct halos in the vertical plane. If the series of points in the arrow $M N$ be considered, it is evident that at the position $f_{1}$ the rays which fall in the vertical plane will form distinct foci, while those that fall in the horizontal plane will form overlapping diffuse images in that plane. Since they are overlapping, they will not appear separate except at the ends of the image of the arrow, and the arrow will therefore be seen distinctly. If the position $f_{2}$ is

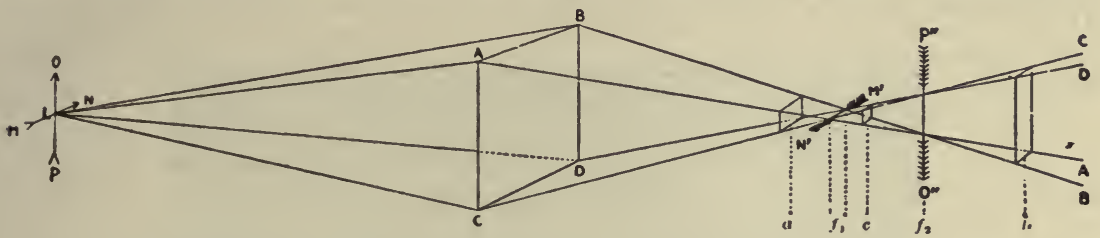

Fig. 464. - The Unequal Refraction of Rays in an Astigmatic Eye. (John Green.)

considered where the rays of the horizontal plane are focused, then it is evident that the points in the arrow $M N$ will present a series of rays or halos in the vertical plane, thus rendering its outline very dim or indistinct. The condition with the arrow $O P$ is exactly the reverse. Hence, in the astigmatic eye the images of the horizontal arrow $M N$ will be distinct at the focus $f_{1}$, while the image of the vertical arrow $O P$ will be distinct in the focus $f_{2}$, and the eye cannot see the two lines distinctly at the same time. This condition is further illustrated in figure 465 which represents the type of image formed at the position $f$, shown in figure 464 .

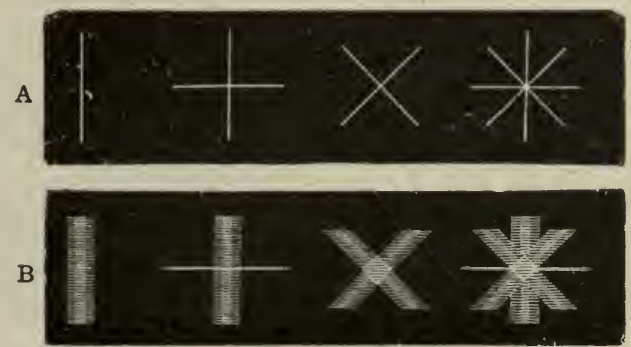

FIG. 465.-Diagram of Character of Retinal Images in Astigmatism. (John Green.)

Myopia.-This is that refractive condition of the eye in which parallel rays are brought to focus in front of the retina when the eye is at rest, 4, figure 466. It is due either to an abnormal elongation of the eyeball antero-posteriorly or to an increase in the convexity of the refracting surfaces, or to both of these conditions. Parallel rays from a

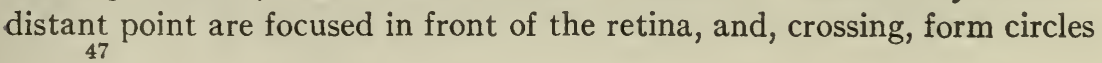


on the retina. Thus, the images of distant objects are blurred and indistinct. The eye is, as it were, permanently adjusted for a near point. Rays from a point near the eye are exactly focused on the retina. But those which issue from any object beyond a slight distance, the myopic far-point, which is less than twenty feet, cannot be distinctly focused. This defect is corrected by concave glasses, which cause parallel rays to diverge before entering the eye. Such glasses of course are needed only to give a clear vision of distant objects. For near objects they are not required.

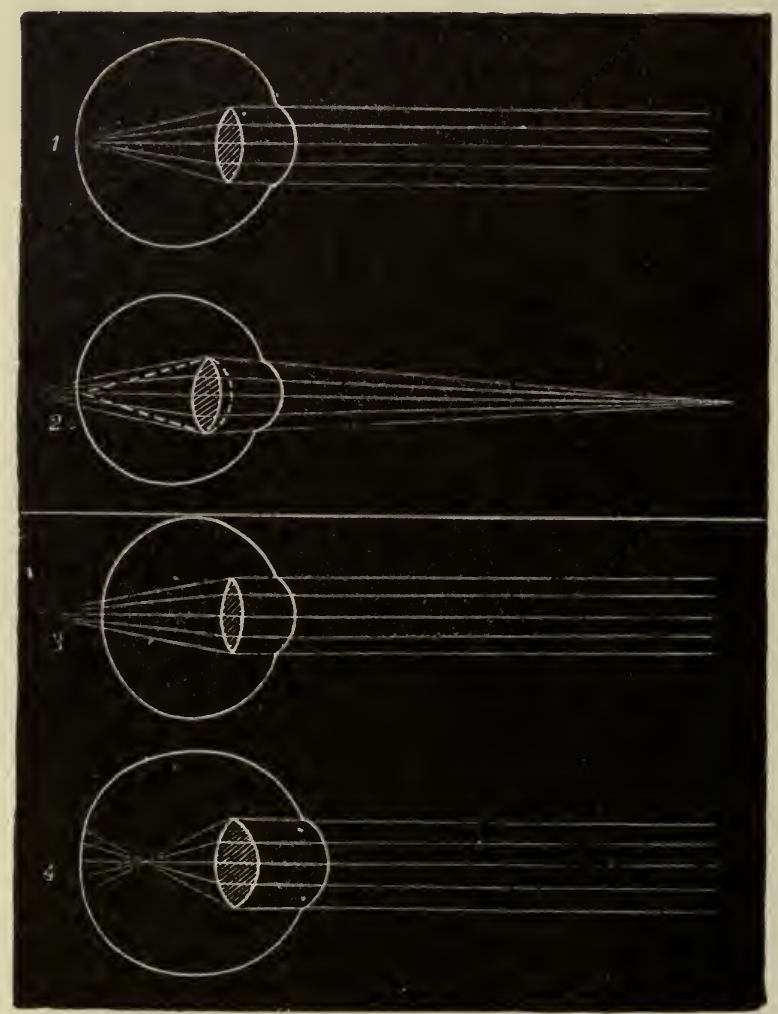

Fig. 466.-Diagram Showing: I, Normal or emmetropic eye bringing parallel rays exactly to a focus on the retina; 2 , normal eye at rest, showing that light from a near point is focused behind the retina, but by increasing the curvature of the anterior surface of the lens (shown by dotted lines) the rays are focused on the retina; 3 , hypermetropic eye. In this case the axis of the eye is shorter and the lens normal (or the lens may be flatter than normal and the eyeball normal); parallel rays are focused behind the retina; 4, myopic eye. In this case the lens is too convex (or the axis of the eye is abnormally long); parallel rays are focused in front of the retina.

Hypermetropia.-This is that refractive condition of the resting eye in which parallel rays are still converging but not yet focused at the retina, are brought to a focus behind the retina, 3 , figure 466 . It is the opposite 
of myopia, and is due either to an abnormal shortening of the eyeball antero-posteriorly or to a decrease in the convexity of the refracting surfaces, or both. Parallel rays entering the eye at rest are focused behind the retina. An effort of accommodation is therefore required to focus parallel rays on the retina. When the rays are sharply divergent, as in viewing a very near object, the accommodation is insufficient to focus them. Thus, not only do distant objects, normally seen without effort, require an act of accommodation, but near objects are focused only by the maximal muscular contraction of the accommodative mechanism. The eye is under a constant strain which produces in the end various nervous, as well as ocular, disorders. This defect is obviated by the use of convex glasses, which render the pencils of light more convergent. Such glasses are especially needed for near objects, as in reading, etc. They are also required for distant vision to rest the eye by relieving the ciliary muscle from constant work.

Presbyopia.-Presbyopia is a condition of diminished range of accommodation. It takes place with considerable uniformity from youth to old age. It is not a disease, but a physiological process which every eye undergoes as its owner grows older. It is due to a gradual diminution of elasticity of the lens by a sort of sclerosis from the center toward the periphery. It begins even in childhood, but advances so slowly that it is not until the age of twenty-five or so that a distinct, though small, nucleus is present. With advancing years the process goes on until finally the lens becomes inelastic and is unable to assume a shape convex enough to focus rays from a near object upon the retina, as in reading. The defect is remedied by the use of convex lenses equivalent to the loss in accommodation.

Visual Sensations, from Excitation of the Retina.-Light is the normal agent in the excitation of the retina. The only portion of the retina capable of reacting to the stimulus is the rod and cone layer. The proofs of this statement may be summed up thus: $I$. The point of entrance of the optic nerve into the retina, where the rods and cones are absent, is insensitive to light and is called the blind spot. The phenomenon itself is very readily demonstrated. If we close one eye, and direct the other upon a point at such a distance to the side of any object that the image of the latter must fall upon the retina at the point of entrance of the optic nerve, its image is lost. If, for example, we close the left eye, and direct the axis of the right eye steadily toward the circular spot in figure 467 , while the page is held at a distance of about six inches from the eye, both dot and cross are visible. On gradually increasing the distance between the eye and the object, by removing the book farther and farther from the face, keeping the right eye steadily on the dot, it will be found that suddenly the cross disappears from view, while on removing the book still farther it suddenly comes into view 
again. The cause of this phenomenon is simply that the portion of retina which is occupied by the entrance of the optic nerve is quite blind; and therefore that when it alone occupies the field of vision objects cease to be visible.

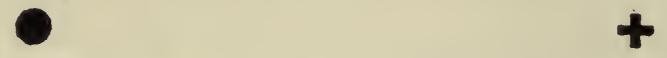

FIG. 467.-Diagram for Demonstrating the Blind Spot.

2. In the fovea centralis and macula lutea, which contain rods and cones but no optic-nerve fibers, light produces the greatest effect. In the latter, cones occur in large numbers, and in the former cones without rods are found, whereas in the rest of the retina, which is not so sensitive to light, there are fewer cones than rods. We may conclude, therefore, that cones are even more important to vision than rods. 3. If a small lighted candle be moved to and fro at the side of and close to one eye in a dark room while the eyes look steadily forward into the darkness, a remarkable branching figure Purkinje's figures, is seen floating before the eye, consisting of dark lines on a reddish ground. As the candle moves, the figure moves in the opposite direction, and from its whole appearance there can be no doubt that it is a reversed picture of the retinal vessels projected before the eye. The two large branching arteries passing up and down from the optic disc are clearly visible, together with their minutest branches. A little to one side of the disc there is an area free from vessels. This corresponds to the yellow spot, or macula lutea, figure $45^{2}$. This remarkable appearance is due to shadows of the retinal vessels cast by the candle. The branches of these vessels are chiefly distributed in the nerve fiber and ganglionic layers; and since the light of the candle falls on the retinal vessels from in front, the shadow is cast behind them. It follows that those elements of the retina which perceive the shadows must also lie behind the vessels. Here, then, we have a clear proof that the light-perceiving elements of the retina are not the fibers of the optic nerve forming the innermost layer of the retina, but the external layers of the retina, the rods and cones.

When light falls on the rods and cones it produces changes which develop nerve impulses that are transmitted by the chain of neurones extending through the retina, the optic nerve and chiasma, the geniculate bodies, etc., to the cerebral cortex of the occipital lobe, which is the sensorium for visual sensations, figures 405 and 406 . We have already seen that the eye possesses a wonderful mechanical perfection for receiving and focusing light on definite parts of the retina. A comparison of visual sensations shows that there are corresponding qualities in the sensation, as, for example, its intensity, duration, localization, complexity, etc.

Duration of Visual Sensations. - The duration of the sensation produced by a luminous impression on the retina is always greater than that of the stimulus which produces it. However brief the luminous impression, 
the effect on the retina always lasts for about one-twentieth of a second. Thus, suppose an object in motion, say a horse, to be revealed on a dark night by a flash of lightning, the image remaining on the retina during the time of the flash. The object is really revealed for such an extremely short period (a flash of lightning being almost instantaneous) that no appreciable movement could have taken place in the period during which the stimulus was produced on the retina of the observer. The horse would appear standing in the position of motion for about a twentieth of a second, though he would not be seen to make any motions. And the same fact is proved in a reverse way. The spokes of a rapidly revolving wheel are not seen as distinct objects, because at every point of the field of vision over which the revolving spokes pass, a given impression has not faded before another comes to replace it. Thus every part of the interior of the wheel appears filled.

The duration of the after-sensation produced by an object is greater in a ratio proportionate to the duration of the impression which caused it. Hence, the image of a bright object, as of the light of a window, may be perceived in the retina for a brief period, the positive after-image. If, however, the primary stimulation is sharp and intense there will follow presently an appearance of the window in which all the contrasted lights are reversed, the negative after-image.

Intensity of Visual Sensations.-It is quite evident that the more luminous a body the more intense is the stimulus it produces. But the intensity of the sensation is not directly proportional to the intensity of the luminosity of the object. It is necessary for light to have a certain intensity before it can excite the retina, but it is impossible to fix an arbitrary limit of the power of excitability. As in other sensations so also in visual sensations, a stimulus may be too feeble to produce a sensation. If it be increased in amount sufficiently, it reaches a point that is intense enough to produce an effect; this is a minimal or threshold stimulus. The amount of increase in the stimulus that produces a perceptible change in the sensation is at first very slight, but later quite great. It does not depend on the absolvite change of intensity of the stimulus, but is proportional to the intensity of the stimulus already acting, Weber's law.

This law, which is true only within certain limits, may be best understood by an example. When the retina has been stimulated by the light of one candle, the light of two candles will produce a difference in sensation which can be easily and distinctly felt. If, however, the first stimulus is that of an electric arc-light, the addition of the light of a candle will make no difference in the sensation. So, generally, for an additional stimulus to be felt, it may be proportionately small if the original stimulus is small, and must be greater if the original stimulus is great. The stimulus increases as the numbers expressing its strength, while the sensation increases as the logarithms. 
Retinoscopy. Everyone is familiar with the fact that it is quite impossible to see the fundus or back of another person's eye by simply looking into it. The interior of the eye forms a perfectly black background to the pupil. The same remark applies to an ordinary photographic camera,

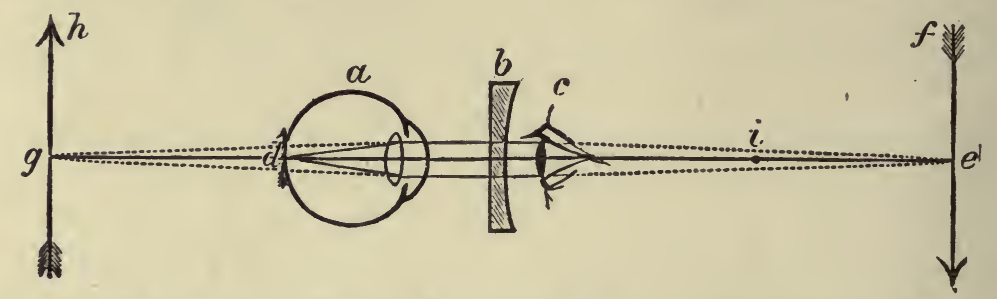

FIG. 468.-Diagram to Illustrate the Action of the Ophthalmoscope when a Plane Concave Glass is Used. c, Observer's eye. The light reflected from any point, $d$, on retina of $a$, would naturally be focused at $e$; if the lens $b$ is used is would be focused at $i$, in other words, at back of $c$. The image would be enlarged, as though of $g$, and would be inverted. (After McGregor Robertson.)

and may be illustrated by the difficulty we experience in seeing into a room from the street through the window unless the room be lighted from within. In the case of the eye this fact is partly due to the feebleness of the light reflected from the retina, most of it being absorbed by the retinal pigment. But the difficulty is due more to the fact that every such ray is reflected back to the source of light and cannot be seen by the unaided eye without intercepting the incident light as well as the reflected rays from the retina. The difficulty is surmounted by the use of the ophthalmoscope.

The ophthalmoscope, brought into use by Helmholtz, consists in its simplest form of a concave mirror with a hole in it. The one described is one of the less intricate of the modern instruments. It consists of, $a$, a slightly concave mirror of metal or silvered glass perforated in the center, and fixed

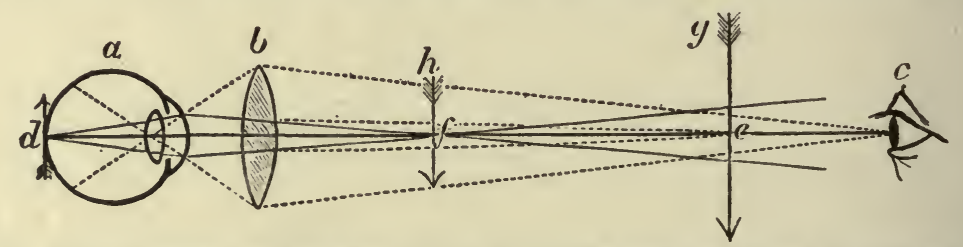

Fig. 469.-Diagram to Illustrate Action of Ophthalmoscope when a Biconvex Glass is Used. The figure $d$ on retina of $a$ is under ordinary conditions focused at $f$ and inverted. If the lens $b$ be placed between eyes, the image $h$ is seen by the eye $c$ as an enlarged image. (After McGregor Robertson.)

into a handle; and $b$, a biconvex lens of 6 to $8 \mathrm{~cm}$. focal length. Two methods of examining the eye with this instrument are in common use-the direct and the indirect: both methods of investigation should be employed. A normal eye should be examined. A drop of a solution of atropine (two grains to the ounce) or of homatropine hydrobromate should be dropped into the right eye only about twenty minutes before the examination is commenced; the ciliary muscle is thereby paralyzed, the power of accommodation is abolished, 
and the pupil is dilated. This will materially facilitate the examination; but it is quite possible to observe all the details to be presently described without the use of this drug. The room being now darkened, the observer seats himself in front of the person whose eye he is about to examine, placing himself upon a somewhat higher level. A subdued but steady light is placed close to the left ear of the patient in the examination of the right eye. Guiding the mirror in his right hand, and looking through the central hole, the operator directs a beam of light into the eye of the patient. A red glare, called in practice the reflex, due to the illumination of the retina, is seen. The patient is then told to look at the little finger of the observer's right hand as he holds the mirror; to effect this the eye is rotated somewhat inward, and at the same time the reflex changes from red to a lighter color, owing to the reflection from the optic disc. The observer now approximates the mirror, and with it his eye to the eye of the patient, taking care to keep the light fixed upon the pupil, so as not to lose the reflex. At a certain distance, which varies with the refractive power in different eyes, but is usually an interval of about two or three inches between the observed and the observing eye, the vessels of the retina will become visible as lines running in different directions. The smaller and brighter red arteries can be distinguished from the larger and darker colored veins. An examination of the fundus of the eye reveals the optic disc and the entrance of the blood vessels, the macula lutea, and the fovea centralis. No blood vessels are seen in the fovea. This constitutes the direct method of examination, figure 468 ; by it the various details of the fundus are seen as they really exist, and it is this method which should be adopted for ordinary use.

If the observer is ametropic, i.e., is myopic or

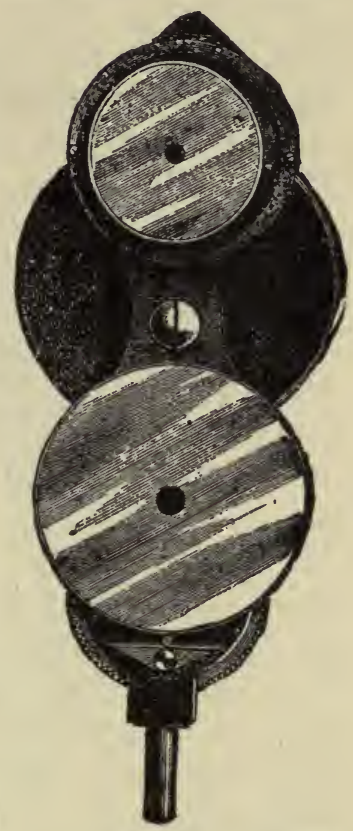

FIG. 470.-The Ophthalmoscope. The small upper mirror is for direct, the larger for indirect, illumination. hypermetropic, he will be unable to employ the direct method of examination until he has remedied his defective vision by the use of proper glasses.

In the indirect method, figure 469 , the patient is placed as before, and the operator holds the mirror in his right hand at a distance of 30 to $40 \mathrm{~cm}$. from the patient's right eye. At the same time he rests his left little finger lightly upon the patient's right temple, and holding the lens between his thumb and forefinger, two or three inches in front of the patient's eye, directs the light through the lens into the eye. The red reflex, and subsequently the white one, having been gained, the operator slowly moves his mirror, and with it his eye, toward or away from the face of the patient, until the outline of one of the retinal vessels becomes visible, when very slight movements on the part of the operator will suffice to bring into view the details of the fundus above described, but the image will be much smaller and inverted. The lens should be kept at a fixed distance of two or three inches, the mirror being alone moved until the disc becomes visible: should the image of the mirror obscure the disc, the lens may be slightly tilted. 
The Field of Vision.-The field of vision of an eye is that part of the external world which can be seen by it when the eye is fixed. Under such circumstances objects near the axis of vision stimulate points in the retina near the fovea or on it, while objects at an angle of $60^{\circ}$ to $90^{\circ}$ from the axis of vision stimulate regions of the opposite side of the retinal cup, i.e., the retinal field is inverted.

The perimeter is an instrument for measuring the field of vision in terms of angular measure. When a field is charted by means of the perimeter it is revealed that objects can be seen further out in the field in some directions

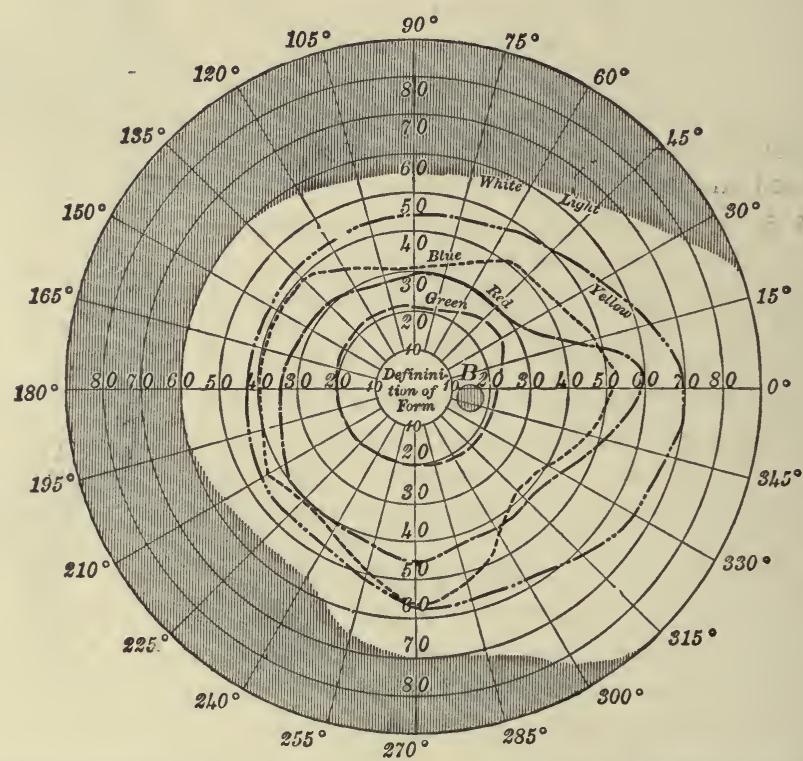

FIG. 47r.-Perimeter Chart, Showing Extent of Field of Vision for White Light and to the Colors Red, Green, Yellow, and Blue. (Krapart.)

than in others. For example, objects in the temporal field can be seen at an angle of $90^{\circ}$ to $100^{\circ}$, while on the nasal side they are seen only $60^{\circ}$ to $70^{\circ}$ If the head is turned to the right or the left while keeping the eye fixed, it is found that objects are seen at a greater angle. This shows that the limitations are due to the facial boundaries of the eye preventing the light from entering the eye and not from lack of sensitiveness of the retina. In fact, the retina is sensitive to light out to the ora serrata.

Localization in the Retina.-Careful exploration of the retina with the perimeter gives a measure not only of the extent of the visual field, but of its acuteness and localization in different areas toward the periphery. Considering the minimal distance apart which two luminous points must be to be distinguished as two, it is found that when the image falls on the fovea 
the two points may be very near together, as little as one minute or even less. Two stars can be seen only at a somewhat greater angular distance, two to three minutes. One minute angular measure covers an area on the retina of a trifle over $4 \mu$. The diameter of the cones is about $2 \mu$, so that the stimuli in the fovea fall on at least two separate cones. The inference seems reasonable that the retina in its most sensitive part can localize stimuli that fall on adjacent cones.

The area of the fovea centralis is small, from 0.5 to $1.5 \mathrm{~mm}$. Outside of its area the acuteness of vision quickly falls off. The fact is roughly estimated by fixing the vision on a letter in the printed line in the book before the reader and then determining the number of letters to either side that can be identified. The height of these letters is $1.5 \mathrm{~mm}$.; by measuring the distance of the page from the eye one can quickly calculate the area of distinct vision on the retina. Test types are printed on the basis of an angle of five minutes.

In the outer limits of the retina the power of localizing stimuli is very slight; in fact, in the extreme borders of the field it is difficult to determine other than general form.

Visual Purple.-The method by which a ray of light is able to stimulate the endings of the optic nerve in the retina is not yet understood. It is supposed that the change effected by the agency of the light which falls upon the retina is in fact a chemical alteration in the protoplasm, and that this change initiates a nerve impulse that is transferred to the optic nerve endings. The discovery of a certain temporary reddish-purple pigmentation of the outer limbs of the retinal rods in certain animals, e.g., frogs, which had been killed in the dark, forming the so-called rhodopsin or visual purple, appeared likely to offer some explanation of the matter, especially as it was also found that the pigmentation disappeared when the retina was exposed to light and reappeared when the light was removed, and that it underwent distinct changes of color when other than white light was used. It was also found that if the operation were performed quickly enough and in the dark, the image of an object, optogram, might be fixed in the pigment on the retina by soaking the retina of an animal in alum solution.

The visual purple cannot, however, be absolutely essential to the production of visual sensations, as it is absent from the retinal cones, and from the macula lutea and fovea centralis of the human retina, and does not appear to exist at all in the retinæ of some animals, e.g., bat, dove, and hen, which are, nevertheless, possessed of good vision.

However, the fact remains that light falling upon the retina bleaches the visual purple, and this must be considered as one of its effects. It has been found that certain pigments, also sensitive to light, are contained in the inner segments of the cones. These colored bodies are said to be oil globules of various colors-red, green, and yellow-called chromophanes, and are found 
only in the retinæ of animals other than mammals. The rhodopsin at any rate appears to be derived in some way from the retinal pigment, since the color is not renewed after bleaching if the retina be detached from its pigment layer. The second change produced by the action of light upon the retina is the movement of the pigment cells. On the stimulation by light the granules of pigment in the cells which overlie the outer part of the rod and cone layer of the retina become diffused into the parts of the cells between the rods and cones, the melanin granules, as they are called, passing down into the processes of the pigment cells A movement of the cones and
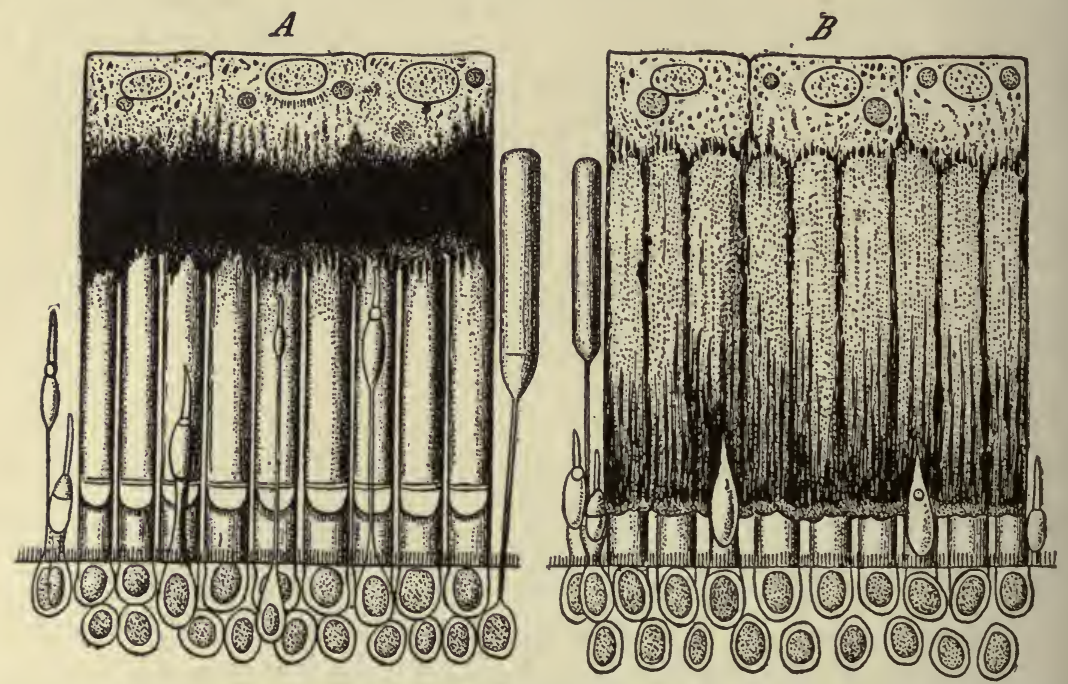

Fig. 472. - Sections of Frog's Retina Showing the Action of Light upon the Pigment Cells and upon the Rods and Cones. (von Gendesen-Stort.) A, From a frog which had been kept in the dark for some hours before death; $B$, from a frog which had been exposed to light just before being killed. Three pigment cells are shown in each section. In $A$ the pigment is collected toward the nucleated part of the cell, in $B$ it extends nearly to the basis of the rods. In $A$ the rods, outer segments, were colored red (the detached one green); in $B$ they had become bleached. In $A$ the cones, which in the frog are much smaller than the rods, are mostly elongated; in $B$ they are all contracted.

possibly of the rods is also said to occur, as has been already incidentally mentioned. Under the influence of the stimulus of light the outer parts of the cones, which in an eye protected from light extend to the pigment layer, are retracted. In is even thought by some that the contraction is under the control of the nervous system. Finally, according to the careful researches of Dewar and McKendrick, and of Holmgren, it appears that the stimulus of light is able to produce an action current in the retina. McKendrick believes that this is the electrical expression of those chemical changes in the retina of which we have already spoken.

Color Sensations. - When a ray of sunlight enters the eye it produces a sensation of white light. But if the ray first passes through a prism, then it 
produces sensations corresponding to the colors of the spectrum. As is well known, white light is produced by vibrations of the luminiferous ether through a wide range of vibration rates. When a beam of white light is passed through a dispersing.prism those vibration rates of low frequency are refracted less than those of higher frequency, giving rise to the spectrum. Vibrations of the luminiferous ether of rates just outside of the spectral rates exist, those which have a lower rate giving rise to heat rays, and those of higher rate to the so-called actinic or chemical rays, because they exert a powerful chemical action. Those spectral colors which stimulate the retina to produce sensations of color presumably affect the retinal elements through chemical changes which they produce there. But this matter will be discussed under theories of color vision.

The examination of color sensations reveals certain correspondences between the physical color of the stimulus and the resulting color perception. If a pure spectral color be allowed to fall on the retina, a corresponding simple sensation is produced. If two colors fall on the same portion of the retina at the same time, a sensation is produced that is different from that which occurs when either color alone stimulates. The same fact holds true for three colors or more. In fact, three spectral colors can be selected which by proper combination can be used to produce sensations of all the colors of the spectrum. Such colors are called the fundamental colors, and while the choice is more or less arbitrary, red, green, and violet are the colors usually considered.

Extent of the Visual Field for Color.-The retina is most sensitive to color in the region of the macula lutea. If by means of the perimeter one explores the retina to spectral red, for example, it is found that the color can be identified only at a distance of from $30^{\circ}$ to $50^{\circ}$ from the macula; the limits extending out somewhat farther on the nasal side of the retina; that is, the part corresponding to the temporal visual field. In the same way yellow can be identified for from $40^{\circ}$ to $70^{\circ}$, blue from $40^{\circ}$ to $50^{\circ}$. The visual field for green is quite restricted, usually extending only from $20^{\circ}$ to $30^{\circ}$. The extent of the color visual field varies greatly in different individuals.

Complemental Colors, and After-images of Color.-Certain colors, when allowed to stimulate the retina at the same time, tend to neutralize each other. That is, they produce sensations approaching white, usually some shade of gray, which will have a tinge of one or the other primary colors according to the proportion of stimulation. These pairs of colors are called complemental colors. Each spectral color has its complemental color, a fact that is represented in figure 473. The complemental colors of greatest physical significance are red and green (greenish-blue), yellow and deep blue (indigo blue), green (greenish-yellow), and violet.

Positive after-images of color exist for a brief moment, but the greatest significance attaches to the negative after-images. The negative after-images 
of color following the stimulus of colored light upon the retina are not the sensation of color produced by the color of an object, but are the opposite or complemental color. The after-image of red is, therefore, green, and that of green, red; that of violet, yellow, and of yellow, violet, etc. The same relation holds with the other colors. A condition for the development of a strong after-image is that the primary image shall have continued to a certain degree of fatigue. The colors which reciprocally excite each other in the retina are those placed at opposite points in the color table, figure 473. The after-images of color are most intense in the axis of the visual field and are not always present in the periphery of the retina, as can readily be seen by examining the chart, figure $47 \mathrm{r}$.

Color sensations may also be produced by contrast. Thus, a very small dull gray strip of paper, lying upon an extensive surface of any bright color, does not appear gray, but has a faint tint of the color which is the comple-

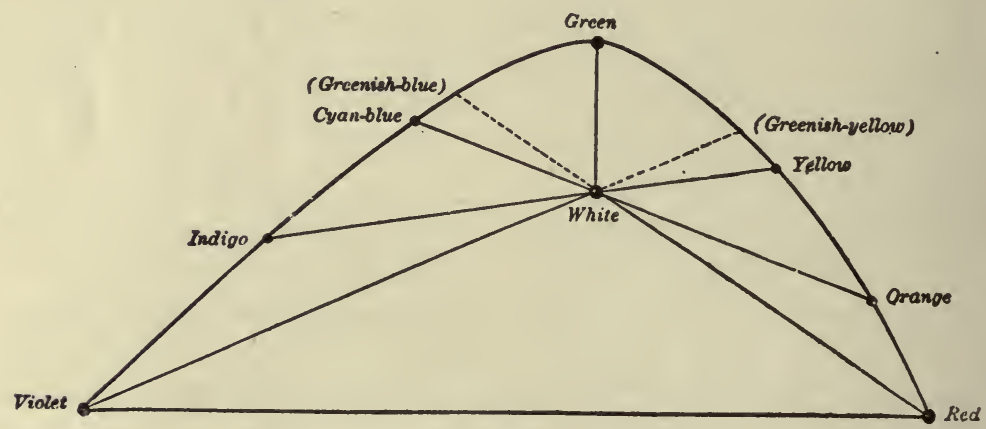

FIG. 473.-Geometrical Color Table for Determining the Complemental Colors.

ment of that of the surrounding surface. A strip of gray paper upon a green field, for example, appears to have a tint of red, and when lying upon a red surface, a greenish tint; it has an orange-colored tint upon a bright blue surface, and a bluish tint upon an orange-colored surface; yellowish color upon a bright violet, and a violet tint upon a bright yellow surface. The color excited thus must arise as an opposite or antagonistic condition of the retina, and the opposite conditions of which it thus becomes the subject, would seem to balance each other by their reciprocal reaction. A necessary condition for the production of the contrast colors is that the part of the retina in which the new color is to be excited shall be in a state of comparative repose; hence the small object itself must be gray. A second condition is that the color of the surrounding surface shall be very bright.

Color-blindness.-Many persons are unable to distinguish one or mo"e of the fundamental colors, and therefore have different perceptions of the color combinations from that of the normal individual. It is said that 
from 4 to 5 per cent. of men and about I per cent. of women are defective in color vision. The defect is called color-blindness.

In very rare cases complete color-blindness exists. Such individuals distinguish lights and shades only, that is, form. A more common defect, however, is the absence of one or more of the fundamental color sensations, the most common of all being the green blind, or the red-green blind. The red-green blind individual cannot distinguish red and green colored yarns from each other or from shades of gray which reflect light with the same intensity. When they are given the color test by the Holmgren yarns, they indiscriminately mix the reds, greens, and grays. Cases have been described in which the individual was red-blind alone, or green-blind alone. A less common color defect is the inability to distinguish yellows and blues, yellowblue blindness.

Color-blindness may occasionally arise from disease or accident, but it is usually congenital. The individual often does not discover his defect until examined especially for his color vision. He may have learned to apply the terms green and red to surrounding objects, such as the grass, bricks, etc., but he distinguishes these objects by slight differences in intensity of lumination, form, etc., and not by the sensations of color which the normal individual experiences.

Theories of Color Vision.-We have no way of determining the method by which the colors stimulate the retina other than our inferences from indirect evidence. It is probable that the energy of light vibration is transformed in the retinal structures into either physical or chemical change, perhaps the latter. Those interested in the phenomena of color vision generally accept one of two theories, or their modifications, in explanation of the facts.

The Young-Helmholtz Theory of Color Vision.-This theory assumes that there are three fundamental sensory elements in the retina which correspond to and are stimulated primarily by the three primary colors-red, green, and violet. The theory in its present form further assumes that each color-perceiving element is slightly stimulated by others of the spectral rays, as shown in figure 474. When red rays fall upon the retina, they stimulate the red-perceiving elements strongly and the green and violet very feebly. The resulting sensation is that of red. So also is it with green and violet rays. When the retina is stimulated by both red and green rays, the two corresponding color-perceiving elements are strongly stimulated. The resulting color perception, however, is a combination of the two sensations and corresponds to some region of the spectrum between the red and green, according to the relative intensity of the two stimuli. When all three color-perceiving elements are stimulated at the same time, the theory assumes that white light will be perceived. In a similar manner all the various color sensations are arrived at. 
Hering's Theory of Color Vision.-This theory is based on the assumption that there are chemical substances in the retina, photogenic substances, which are stimulated by the colors of the spectrum. It assumes three photogenic substances which are called the red-green, the yellow-blue, and the

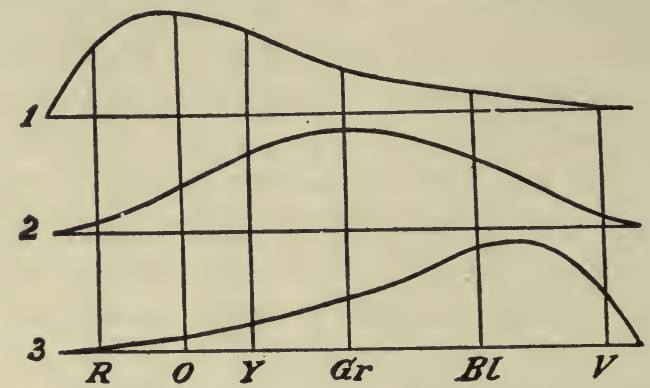

Fig. 474.-Diagram to Illustrate the Stimulating Effects of the Three Primary Colors, (Young-Helmholtz theory.) I is the red; 2, green, and 3, violet, primary color sensations. The lettering indicates the colors of the spectrum. The diagram indicates by the height of the curve to what extent the several primary sensations of color are excited by vibrations of different wave lengths. (Helmholtz.)

white-black substances. By the theory, when the red-green substance is stimulated by red or green light, respectively, the former produces destructive or katabolic changes, the latter constructive or anabolic changes in the substance. When red light falls upon the retina, it produces katabolism in the red-green substance, which in turn develops a nerve impulse that arouses

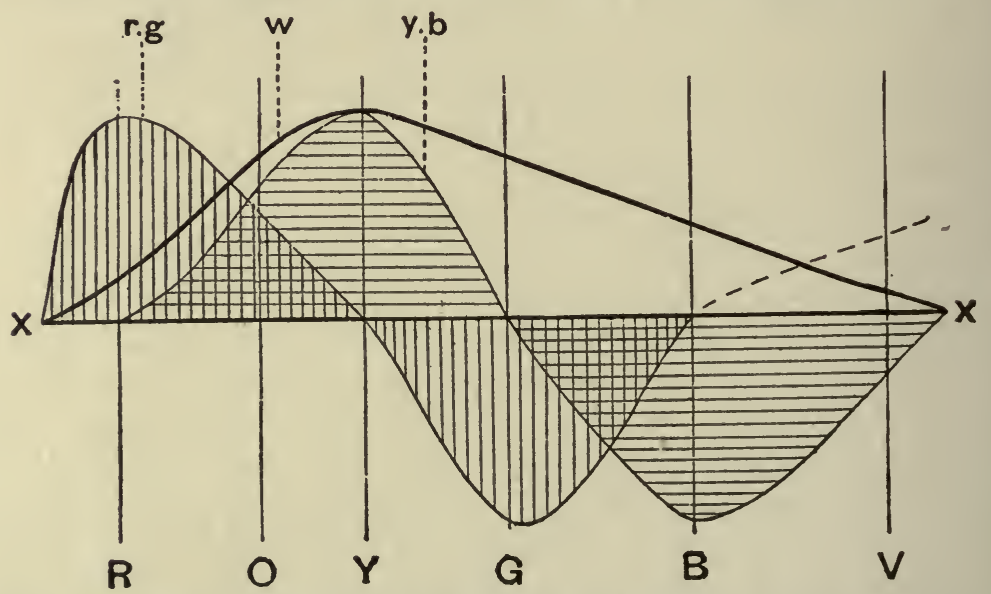

FIG. 475.-Diagram to Illustrate the Reactions of the Three Photogenic Substances, according to Hering's Theory. (Foster.)

the sensation of red. When green light, on the other hand, stimulates the retina, it produces anabolism of the red-green substance and the sensation of green. The same rule holds with the other two substances. It will be noticed that this theory is based on the complemental colors. 
When we apply the theories mentioned above to the phenomena of colorcontrast and color-blindness, we find that each is defective in some point. By the Young-Helmholtz theory it is difficult to understand the perception of the sensation of black, for by the theory black could be perceived only as the absence of all colors, and it is generally granted that there is a distinct black sensation other than and different from mere darkness. This theory explains more satisfactorily those cases of blindness to one color, as redblindness, for example. The Hering theory, on the other hand, gives us a rational explanation for positive black sensation, and is particularly applicable to the observed facts of color-contrast and negative color after-images.

Color after-images, as for instance the after-images of green following stimulation by red light, are readily explained by Hering's theory, since the strong katabolism in the red-green substance will be followed immediately by anabolism to bring this substance up to its normal in the eye, thus producing the after-image. This phenomenon can be explained by the YoungHelmholtz theory only by assuming that following the stimulation by red light and the consequent fatigue of red-perceiving elements there is sufficient light entering the eye to stimulate the relatively sensitive green and violet perceiving elements, thus producing an after-image. Strong after-images are perceived in the dark room, so that the Hering theory is most applicable in the explanation of these cases.

Binocular Vision.-When one looks at an object with a single eye, the eye is so adjusted that the axis of vision is directed toward the object investigated. This is called ocular fixation. The ocular fixation is accomplished by the co-ordinated contractions of the six ocular muscles. Its purpose is to bring the image of the object examined in the external visual field as nearly as possible upon the macula lutea. In binocular vision both eyes are fixed on the same point in the visual field. A projection of the visual axis of each eye will pierce the point of fixation in the external object. It is evident that objects to either side of the point of fixation will give off rays which will enter the eyes, stimulating fields in the retina on the opposite side of the visual axis. An examination of figure 476 will show that each point in the visual field, $A, B, C, D$, stimulates corresponding points, $a, b, c, d, a^{\prime}, b^{\prime}, c^{\prime}, d^{\prime}$, in the retinas of the two eyes. $a, b, c, d$, and $a^{\prime} b^{\prime}, c^{\prime}, d^{\prime}$, are corresponding points in the two retinas. When $a$ and $a^{\prime}$ are stimulated at one and the same time, the resulting sensation is attributed to one object in the visual field, $A$, and these are corresponding points. This can be shown by pressing one eye out of its normal fixation so that the primary axes of the two eyes are not directed toward the same point. If one eye is pressed lightly by the thumb while examining a given object, as soon as the pressure is applied two objects will appear. This phenomenon is known as diplopia. Diplopia is due to the fact that the images of visual objects do not fall on corresponding points in the two retinæ. 
The parts of the retinæ in the two eyes which thus correspond to each other in the property of referring the images which affect them simultaneously to the same spot in the field of vision, are, in man, just those parts which would correspond to each other if one retina were placed exactly in front of and over the other, as in figure 477. Thus, as we have noticed in speaking of the distribution of the optic nerve fibers, the temporal portion of one eye corresponds to or is identical with the nasal portion of the other

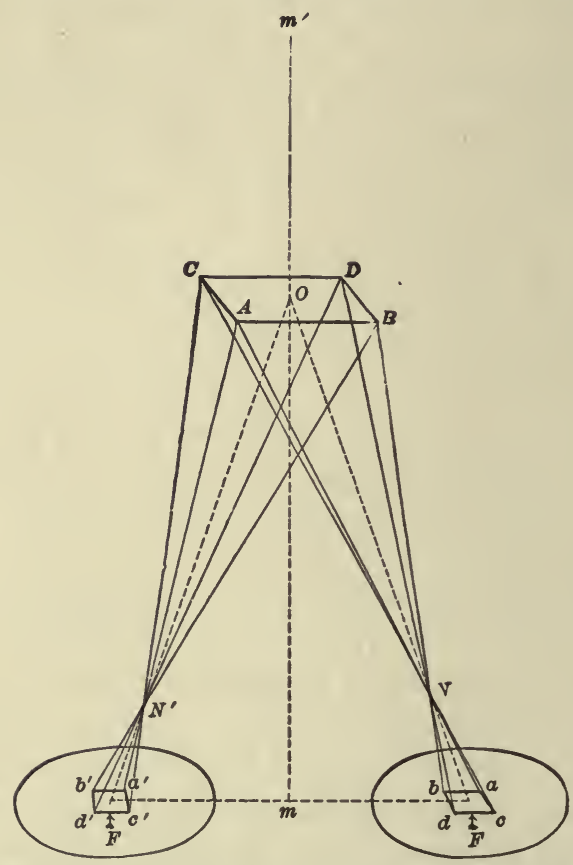

FIg. 476.-Diagram Showing the Symmetrical Correspondence of the Retinal Fields. $N$, Nodal point; $F$, fovea centralis. The observer is supposed to be looking down upon the optical apparatus from above. Note that the line $C D$, which is on the lower side of the object, is the upper side of the image; and that the line $B D$, which is the right side of the object, is the left side of the image, which brings it at the inner segment of the right retina and the outer segment of the left retina.

eye. The upper part of one retina is also identical with the upper part of the other; and the lower parts of the two eyes are identical with each other. The distribution of the optic nerve fibers corresponds with the distribution of the identical points. The identical points on the upper and lower parts of the retinæ may also be shown by the following simple experiment.

Pressure upon any part of the ball of the eye, so as to affect the retina, produces a luminous circle, seen at the opposite side of the field of vision to that on which the pressure is made. If, now, in a dark room, we press with the finger at the upper part of one eye, and at the lower part of the other, two luminous circles are seen, one above the other; so, also, two figures are 
seen when pressure is made simultaneously on the outer or the inner sides of both eyes. But if pressure be made with the fingers upon both eyes simultaneously at their lower part, one luminous ring is seen at the middle of the upper part of the field of vision. If the pressure be applied to the upper part of both eyes, a single luminous circle is seen in the middle of the field of vision below. So, also, if we press upon the outer side of one eye and upon the inner side of the other eye, a single luminous spot is produced, and is apparent at the extreme right of the field of vision. The hemispheres of the two retinæ may, therefore, be regarded as lying one over the other, as in $C$, figure 477 . If the axes of the eyes, $A$ and $B$, figure 478 ,

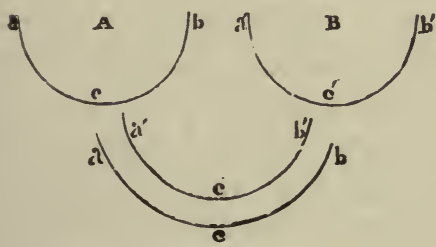

FIG. 477 .

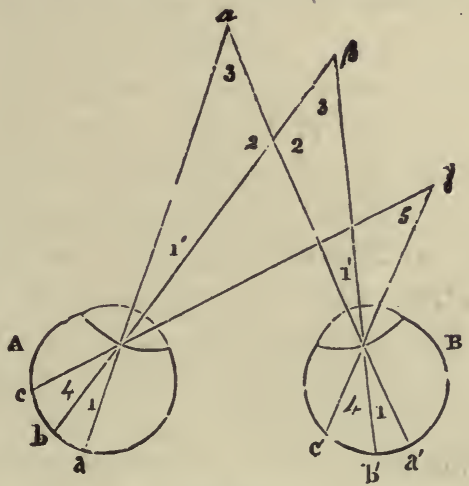

Fig. 478 .

FIG. 477.-Diagram to Show the Corresponding Parts of the Retinæ.

Fig. 478.-Diagram to Show the Simultaneous Action of the Eyes in Viewing Objects in Different Directions.

be so directed that they meet at $\alpha$, an object at $\alpha$ will be seen singly, for the point $a$ of the one retina and $a^{\prime}$ of the other are identical. So, also, if the object $\beta$ be so situated that its image falls in both eyes at the same distance from the central point of the retina-namely, at $b$ in the one eye and at $b^{\prime}$ in the other $-\beta$ will be seen single, for it affects identical parts of the two retinæ. The same will apply to the object $\gamma$

The reason why the impressions on the identical points of the two retinæ give rise to but one sensation, and the perception of but a single image, must either lie in the structural organization and relations of the deeper or cerebral portions of the visual apparatus, or it must be the result of a mental operation; for in no other case is it the property of corresponding nerves of the two sides of the body to refer their sensations to one spot.

Many attempts have been made to explain this remarkable relation between the eyes, by referring it to anatomical relation between the optic nerves. The circumstance of the inner portion of the fibers of the two optic nerves decussating at the commissure, and passing to the eye of the opposite side, while the outer portion of the fibers continue their course to the eyes of the 
same side, so that the left side of both retinæ is formed from one root of the nerves, and the right side from the other root, naturally led to an attempt to explain the phenomenon by this distribution of the fibers of the nerves. And this explanation is favored by cases in which the entire half of one side of the retina sometimes becomes insensible.

Visual Judgments.-Form and Solidity.-The estimation of the form of bodies by sight is the result partly of the visual sensations and partly of the association of ideas. The form of the image perceived by the retina depends wholly on the outline of the part of the retina affected; the sensation alone is adequate only to the distinction of superficial forms from each other which lie in one plane, as of a square from a circle. But the idea of a solid body, as a sphere, or a body of three or more surfaces, e.g., a cube, can be attained only by the action of the mind in constructing it from the different superficial images seen in different positions of the eye with regard to the object, and (as shown by Wheatstone and illustrated in the stereoscope), from two different perspective projections of the body being presented simultaneously to the mind by the two eyes. Hence, when, in adult age, sight is suddenly restored to persons blind from infancy, all objects in the field of vision appear at first as if painted flat on one surface; and no idea of solidity is formed until atter long exercise of the sense of vision combined with that of touch. The clearness with which an object is perceived, irrespective of accommodation, would appear to depend largely on the definiteness of stimulation of the rods and cones which its retinal image covers. Hence, the nearer an object is to the eye, within the limits of vision, the more clearly are all its details seen. Moreover, if we want carefully to examine any object, we always direct the eyes straight toward it, so that its image shall fall on the yellow spot, which has already been shown to be the area of the most acute vision.

In binocular vision the images of an object, while they fall in approximately corresponding points on the two retinæ, are never absolutely the same.

When an object is placed so near the eyes that to view it the optic axes must converge, a different perspective projection of it is seen by each eye, these perspectives being more dissimilar as the convergence of the optic axes becomes greater. Thus, if any figure of three dimensions, an outline cube, for example, be held at a moderate distance before the eyes, and viewed with each eye successively while the head is kept perfectly steady, $A$, figure 479 , will be the picture presented to the right eye, and $B$ that seen by the left eye. Wheatstone has shown that on this circumstance depends in a great measure our conviction of the solidity of an object, or of its projection in relief. If different perspective drawings of a solid body, one representing the image seen by the right eye, the other that seen by the left, for example, the drawing of a cube, $A, B$, figure 479 , be presented to corresponding parts of the two retinæ, as may readily be done by means of the stereoscope, the mind will 
perceive not merely a single representation of the object, but a body projecting in relief, the exact counterpart of that from which the drawings were made.

Judgments of Size and Distance.-The estimation of the size of an object and its distance away from the observer is based in part upon the visual image and in part upon judgments due to past experience. The elements are inseparable and mutually dependent. Thus, a lofty mountain many miles away may subtend the same visual angle as a small hill near at hand.

A

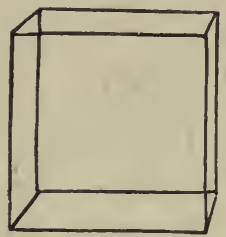

B

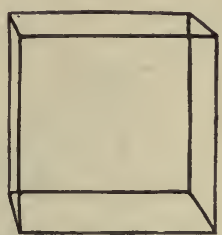

Fig. 479.-Diagrams to Illustrate how a Judgment of a Figure of Three Dimensions is Obtained.

While the size and shape of the two images may be identical, yet the image of the hill near at hand is more distinct, its details are perceived, and its outlines are sharper than in the image of the mountain. If the atmosphere be charged with moisture or with dust, the image of the mountain will be still more indistinct and dim. From previous experiences we have learned that the dimness and indistinctness of the one and the definiteness of the other are associated with distance.

If two objects are very near at hand then there will be a difference in the convergence of the two eyes in binocular vision. It is now well known that the ocular muscles are possessed of a very delicate muscle sense. This muscle sense leaves the impression which enables us to judge that the one object is nearer and the other farther. In the common and familiar objects about us we have from long experience and intimate contact learned their actual size and the character of the retinal image formed at definite but known distances. When such an object forms an image of the common size and usual distinctness on the retina the judgment as to its distance is quickly made.

In the case of unknown objects which are associated with known objects, the judgment of the size and distance of the latter is used in forming a judgment of the size and distance of the former by comparison. Many visual deceptions are based on these comparisons, a fact that is often taken advantage of by photographers. It is also well known that people living in a moist, hazy climate are utterly unable accurately to estimate distances when suddenly transferred to a clear mountain climate. 


\section{LABORATORY DIRECTIONS FOR EXPERIMENTS ON THE SENSE ORGANS.}

I. Touch.-Use the small compasses with rounded tips provided for the purpose, and determine the power of localization of the sense of touch as follows: Have the person observed close his eyes, then touch different parts of the skin, of the hand, arm, face, neck, etc., and let the observed one announce the exact point touched.

The localization can also be determined by touching two points on the skin with the points of the compasses separated by varying distances. Examine especially the skin on the forearm, on the back of the hand, on the

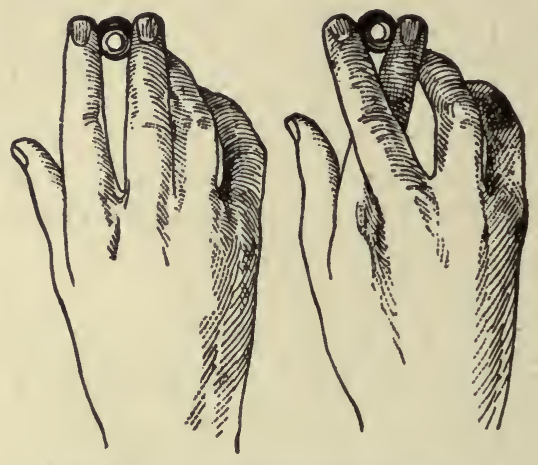

Frg. 480.-Aristotle's Experiment.

palm of the hand, the tips of the fingers, and at different points on the face, including the lips and tip of the tongue. Touch these regions of the skin with either one or with two points of the compasses, and allow the person observed to announce results, drawing your conclusions according to the principle of trial and error. Make a table showing the power of local discrimination in the different regions.

2. Aristotle's Touch Experiment.-Roll the tips of the middle and index fingers over a marble and note that the sensation from the two fingers is interpreted as that of a single object. Now cross the fingers and repeat the experiment. This time there is the sensation of touching two spheres.

3. Temperature Sensations. - It is a common experience that the hand brought in the neighborhood of a warm or a cold object develops the sensation of warmth or cold. Examine a given small area of the back of the hand, or a square centimeter on the palmar surface of the wrist. Use the thermoesthesiometer, or a pencil with large diameter of the carbon warmed in a sand bath. Certain points will give stronger sensation of heat than others. Map these out carefully. Examine the same area for the cold. A large number of cold spots will be found and they will not coincide with the warm spots, figure $42 \mathrm{I}$. 
The stimulation for the hot and cold spots does not depend upon the absolute temperature, but on the relative temperature. Insert the hand in water that feels lukewarm. Place the same hand in a cup of quite warm water for a moment, then reinsert it in the lukewarm water. This will now feel cold.

4. Sensations of Taste.-The distribution of taste organs in the tongue is shown in figure 48I. Examine your own tongue for organs of sweet, acid, saline, and bitter, using solutions of $\mathrm{I}$ to 2 per cent. salt, Io per cent. sugar, I to 2 per cent. sulfuric acid, or 5 per cent. acetic acid, and 0. I per cent. quinine.

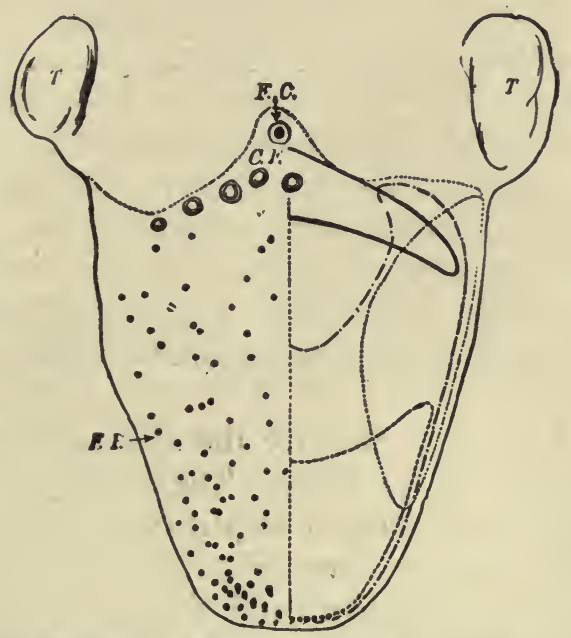

FIG. 481. - Localization of Taste. Bitter...; acid ...; salt, -._-_; sweet ...; $T$, tonsils; $F C$, foramen cecum; $C F$, circumvallate papillæ; $F P$, fungiform papillæ. (Hall.)

Wipe the tongue dry and apply the solution named from the tip of a glass rod. The best form of rod is about $15 \mathrm{~cm}$. long by $0.5 \mathrm{~cm}$. in diameter, and has one end drawn out to a slender pencil-shaped tip and of a size which will suspend a very small drop. Too large a drop diffuses over too great an area of the tongue. Occasionally small crystals of sugar, salt, etc., give more satisfactory results.

Perform the experiments on yourself before a mirror and map the results as shown in figure $48 \mathrm{r}$.

If the experiments are done with care certain papillæ will be found which give one or two of the taste sensations, but not all.

5. Sensations of Smell.-Quantitative experiments on the sense of smell are difficult to determine. Inhale vapor of ammonia so dilute that it can just be detected. Note that the sensation is strongest at the moment of drawing the vapor into the nostril. Fill the nostrils with the diluted vapor and close the external opening; the sensation quickly disappears. Keeping 
the nostrils closed, walk into the open air, then inhale fresh air. At the moment of the inhalation of fresh air the ammonia is again perceptible. Repeat with bergamot, rose water, etc.

6. The Limits of the Sense of Hearing.-Use a set of tuning forks for the purpose, and determine the lowest vibration per second which can be perceived as sound. Determine the highest limits in the same way.

7. Acuteness of the Sense of Hearing.-Listen to the vibrations of a tuning fork, or, better, to the ticking of a watch which is moved back and forth from the ear. Measure the distance at which it can just be distinguished. This experiment should be performed with the person blindfolded, and extraneous noise should, of course, be suppressed.

7a. Dynamic and Static Functions of the Labyrinth.-Maxwell working with sharks and Woodworth with pigeons have demonstrated new points in the physiology of the semicircular canals and of the utriculus and sacculus. Compensating motions are produced on stimulating the sense organs of the semicircular canal, easily demonstrated in the shark and pigeon. Destruction of these sense organs results in loss of co-ordination of types depending upon the organs affected. The semicircular canals must be operated in pairs to induce complete loss of co-ordination in the particular plane.

a. Operate on the Pigeon.-Remove the sensory ampullæ of the horizontal semicircular canals on each side. The operation is performed under careful ether anesthesia and must be aseptic throughout. Quick recovery occurs. The animal loses the power of controlling movements, the compensatory movements, in the horizontal plane. It will walk round and round in a circle, or move the head without the usual quick co-ordinative control of the skeletal musculature.

$b$. Remove the right upper and the left lower ampullæ in a second bird. Study its disturbances of co-ordination as in the first.

In the case of these pigeons, particular attention must be given to supplying water and feed for the first few days following the operation. The birds will soon learn to take care of themselves. They do not recover power of co-ordination as generally supposed. This was shown by the demonstrations of Dr. Woodworth at the St. Louis meeting of the American Medical Association, I922. Dr. Woodworth's pigeons had been operated many months before.

8. Refraction.--Light passes out from a luminous point in straight lines so long as the line of propagation is in a medium of uniform density. If the rays pass from a transparent medium, of one density into a second medium of different density, they will usually be turned out of their course, or refracted. If the rays enter the second medium at right angles to its surface, they will continue in straight lines, but if they enter at any other angle they will be refracted. If the second medium is denser than the first, the rays will 
be refracted toward the perpendicular; if it is less dense, away from the perpendicular.

Use a Hall's refraction-measuring apparatus (constructed of a carpenter's try square). Adjust it in a water-pan, and fill to the exact level with clear water. Clamp a rule to the vertical limb of the apparatus at an angle of 45 degrees and in line with the axial point of the instrument. Read the horizontal scale of the instrument along the edge of the clamped rule. Remove the instrument from the pan, using care not to disturb the adjustment of the ruler, and construct the angle of refraction on coordinate paper. Determine the relation of the angle of incidence and of refraction, and compute the refractive index of the water, the air having a refractive index of one.

Repeat the determination using a block of glass. Draw two sharp lines at right angles across a white sheet of paper. Lay a block of glass with polished surfaces on the paper with one margin coinciding with the horizontal line. Insert one pin at the intersection of the lines but in contact with the glass. Insert a second pin at the opposite margin of the glass and at an angle of about 40 degrees from the intersection of the lines on the paper. Now looking through the glass determine a third point out on the paper away from the glass and where the two pins seem to be in line. Remove the glass and construct a diagram showing the angles of incidence and of refraction.

The index of refraction is found by the formula:

$$
\frac{\sin \mathrm{i}}{\sin \mathrm{r}}=\text { Refractive index }
$$

If equal distances are laid off on the hypotenuses then one may substitute the actual measurements of the vertical legs for the sins in the formula and calculate. Repeat several times.

9. To Determine the Refractive Power of a Convex Lens. - Use a meter stick which is provided with a movable diaphragm or screen, and a holder for a lens. Measure the focal distance of lens number I as furnished from the optical set. Put the lens in its holder and focus the image of the sun or of an electric bulb on the screen, moving the screen back and forth until the sharp focus is determined. If the lens is accurately ground, the focus will be at a distance of one meter, which is the refractive power of a one-diopter lens by definition. In the same way determine the refractive power of lenses numbers 2,3 , and 4. . If a source of light of a finite distance is used, then correction must be made for the divergence of the rays by the formula:

$$
\frac{\mathrm{r}}{\mathrm{f}}+\frac{\mathrm{r}}{\mathrm{f}^{\prime}}=\frac{\mathrm{r}}{\mathrm{F}}
$$

Where $f$ and $\mathrm{f}^{\prime}$ are the conjugate foci and $\mathrm{F}$ the principal focal distances in meters. 
Construct a diagram showing the path of the light in the formation of the image in these cases.

If the measurement in the above case is made through two parallelopenings or diaphragms about $5 \mathrm{~mm}$. in diameter each, and separated by $\mathrm{I}$ or $2 \mathrm{~cm}$., the point of focus can be more accurately determined (see Scheiner's Experiment, No. I4). Construct the mathematical figure showing the course of both cones of rays in this test.

I0. Determination of Near and Far Limits of Vision.-Support a meter stick in a horizontal position at a comfortable level for the eye. Mount

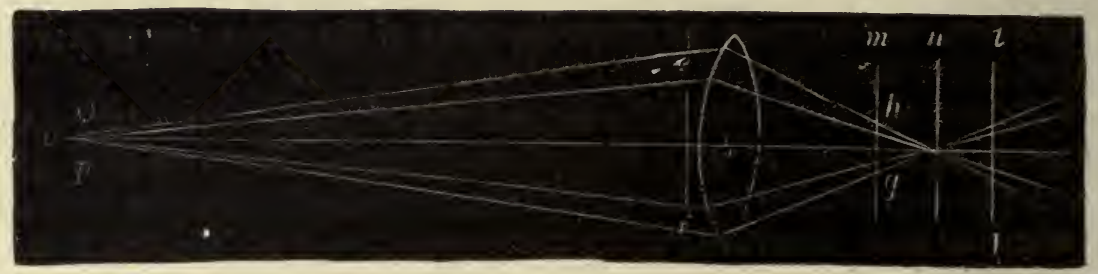

FIG. 482.-Diagram of Experiment to Ascertain the Minimum Distance of Distinct Vision.

a needle in a cork and set it on the meter stick about $25 \mathrm{~cm}$. in front of the eye. Make two pin-holes in a card at a distance of about $2 \mathrm{~mm}$. from each other. Hold this card with the pin-holes close in front of the right eye, and bring the eye up to the end of the meter stick; cover the other eye. Observe that when the needle is brought nearer and nearer to the eye, at a certain distance it becomes double. Determine this distance very accurately. It is the near-point of accommodation for the right eye. Make the same determination for the left eye.

Hold the punctured card in front of the right eye, and move the needle (it is better to use something larger) farther and farther a way until it becomes again double, if it does so. This is the far-point of accommodation. In normal eyes infinity is the far limit. In practice an eye that has no far limit under twenty feet is considered normal. This test should be made on each eye.

Test near vision with printed letters the size of those on this page. Mount a test paper on a small cube and run it along the meter stick as above. Test one eye at a time. When the printed letters begin to lose their sharp black outlines the near-point is reached. To test the far limit use letters large enough to be clearly seen at a distance of 20 feet by the normal eye, see the test chart.

II. Inverted Image on the Retina.-Dissect off a segment of the sclerotic of a fresh ox eye, or use a fresh eye from an albino rabbit. Make a tube of black paper of the size of the eye, and insert the eye in one end, with the cornea directed into the tube. In the dark room examine the image 
of the candle flame as formed on the retina of the eye in the tube. In a favorable experiment, a clear inverted image of the candle can be seen on the retina through the semi-transparent membranes of the eye. The same experiment can be demonstrated with the camera, or with a small lens, using a ground-glass plate to make the image more apparent.

12. Spherical Aberration.-In physical optics it is found that it is difficult to grind lenses so that they will refract equally in the center or optical axis and in the periphery. Unequal refraction of these two regions is called spherical aberration. It is corrected in optics by diaphragms which shut out the light, either from the borders of the lens or from its center. The former method is used in the eye. To demonstrate spherical aberration, look at an object two meters from the eye, such as a part of the window moulding. Pass a card close in front of the eye until the light enters only at the margin of the pupil, i.e., the borders of the lens. It will be found that the object is no longer in focus and the outlines are dim and diffused. Step nearer and nearer the window, when quite close the outlines of the moulding become clear again. Normal eyes are nearsighted for the rays that are refracted by the borders of the lens.

I3. Chromatic Aberration.-Look toward the borders between the sash and the bright light of an open window, at a distance of twenty feet or more. Use the right eye only. Bring a card across the pupil approaching from the side of the light until the eye is almost covered with the card. The window sash will seem to have a blue-violet fringe. If the card is brought across from the opposite side, the sash will have a reddish-yellow fringe.

Make a cross of two strips of Bradley's pure color paper, one red and the other blue, on a black surface. When held at the proper distance the red appears nearer than the blue. This phenomenon is brought out more strongly by covering the colored papers with very thin white tissue paper. The judgment of distance is based on the effort of accommodation which is greater for the red than for the blue and violet rays.

I4. Scheiner's Experiment.-Use two needles on corks, the method described in Experiment Io, placing one at a distance of $20 \mathrm{~cm}$., and the other about $60 \mathrm{~cm}$. from the eye. Use only the right eye, look through two pinholes in a card at the far needle. The near needle will appear double but the images will be somewhat blurred. While looking at the far needle, bring a cardboard across the right hole, note that the left image of the near needle disappears, and vice versa. If one accommodates for the near needle, the far needle appears double, and upon covering the right hole with the card the right image of the far needle disappears. This is known as Scheiner's Experiment. It depends on the diffuse stimulation of points on the retina outside of the principal axis. The apparent images are referred out in space along the corresponding secondary axes. Construct a diagram to explain these phenomena. 
I5. Purkinje-Sanson's Images.-Examine the eye of another person in a dark room as follows: With the observing eye focus for a far object, let the observer hold a candle slightly to one side of the axis of vision and about one foot from the eye. If the observer looks into the other eye from the side opposite the candle, he will be able to see three reflected images, figures 457 and 458 . One, from the anterior surface of the cornea, is bright and distinct, and of medium size and erect. In the middle of the pupil there will be a second image, larger and quite dim. This is a reflection from the front of the lens. The third image, reflected from the posterior surface of the lens, will seem to be farther back in the eye, quite small and inverted. These images can all three be seen at once with careful adjustment of the relative positions of the candle and the observer, with reference to the axis of vision of the eye observed.

If the observer protects his own eye from the direct light of the candle by a blackened cardboard between his eye and the candle, and asks the observed person to accommodate now for near objects, now for far, keeping the axis of vision constant, he will be able to note that the middle image, i.e., the one from the anterior surface of the lens, changes in size and in relative position with reference to the other two, which are essentially constant. With near accommodation this image becomes smaller and seems to move toward the image from the cornea; with far accommodation it becomes larger and appears to move to the image reflected from the posterior surface of the lens. This shows that the act of accommodation consists in a change in the convexity of the front of the lens.

I6. The Phakoscope of Helmholtz.-This classical instrument was invented by Helmholtz to demonstrate the act of accommodation, as outlined in the second paragraph of the preceding experiment. Repeat the preceding experiment, using this instrument in a dark room.

I7. Astigmatism.-Astigmatism is a term used to describe the condition of unequal curvature of the refracting surfaces of the eye in the different meridia. The cornea is the surface which usually shows the greatest astigmatism. The defect is demonstrated by numerous forms of astigmatic charts, the most serviceable of which are the barred-letter test type, the clock dial, or the dials shown in figure $46_{3}$ or 483 . Hang an astigmatic dial at a distance of six meters and test the right and left eyes separately, as follows: When the vision is focused on the center of the dial, if the eye is normal, the three bars in each radius of the clock dial will be seen with equal distinctness and have sharp black lines. In an astigmatic eye one or more of these radii will appear sharp and distinct, while the other will appear dim and indistinct, the relative difference depending upon the degree of astigmatism. Note the meridian of astigmatism in the right and left eyes separately. Use 
the test set, and find the cylinder necessary to correct the astigmatism in each eye and determine its meridian.

Astigmatism is commonly shown by the presence of radii when one looks at the stars at night, or by the ragged outline of a pin-hole in a card, when

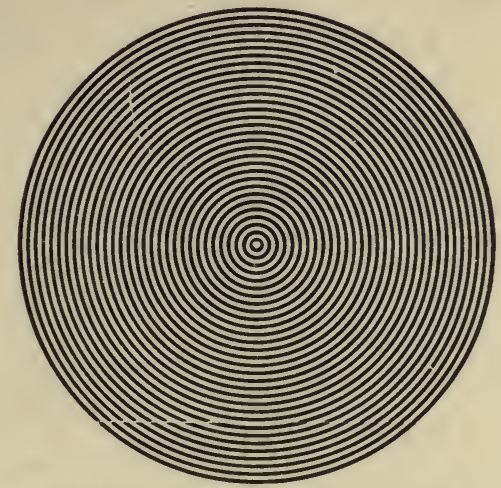

FIG. 483.-Disc of Concentric Lines for the Astigmatic Test.

held at arm's length against a white sky. In extreme cases outlines like the bars in the window sash or checks in clothing may be distorted, or some of the lines may not even be seen.

18. The Blind Spot.-Look with the right eye at the spot in the accompanying figure at a distance of about 20 to $25 \mathrm{~cm}$., covering the left eye. Hold the spot in the line of direct vision and move the book to and from the eye; in some cases it is necessary to rotate the book slightly. It will be found that the cross to the right will, at a certain position, completely disappear. This happens when its image falls on the retina directly over the entrance of the optic nerve, which has no visual cells, and is, therefore, the blind spot. This area is large enough to cause a man completely to disappear from the field of vision at a distance of about one hundred meters.

Place a sheet of white paper at a distance of $30 \mathrm{~cm}$. in front of the eye, holding the head in a fixed position against the special support furnished; look with the right eye at the top of the cross made on the left of the sheet

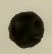

FIG. 484.-Diagram for Demonstrating the Blind Spot.

of paper. Covering the sharpened portion of a lead pencil with white paper, leaving the black tip exposed, move this pencil across the paper from the visual center to the right. At a certain distance the black lead will suddenly disappear. Mark this point. Continue to move the pencil until the lead reappears. Mark this point. These two points represent 
the limits of the blind spot in the horizontal plane, as magnified by the conditions of the experiment. Mark the limits in the other meridians in the same manner. Compute from the figures obtained the exact size of

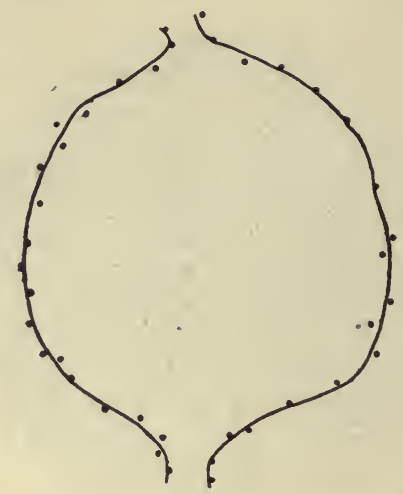

Fig. 485.-The Blind Spot with the Eye $30 \mathrm{~cm}$. from the Paper. The irregularity of outline is due to the larger blood vessels.

the blind spot in your right eye, figure 485. Repeat on the left eye. Usually these areas are not symmetrical. The computation may be based on the following proportion: $a$, the distance of the map from the nodal point of the eye, is to $b$, the diameter of the map of the blind spot, as $c$, the distance from the nodal point to the retina, which is $1.5 \mathrm{~cm}$., is to $x$, the diameter of the actual blind spot in the retina, $a: b:: c: x . x$ varies from $\mathrm{I} .5$ to 3 or more $\mathrm{mm}$.

I9. Relations of the Size of the Retinal Image to Distance.-Compute the size of the retinal images of familiar objects by the equation given in the last experiment. Compute the size of the image formed on the retina by a man six feet tall at a distance of roo feet. Compute the size of the image formed by a tower 125 feet tall at a distance of 575 feet.

20. Purkinje's Shadows. - Stand in front of a blackened wall in the dark room. While looking toward the wall with the right eye accommodated for distant objects, move a lighted candle back and forth about Io to $20 \mathrm{~cm}$. to the right of the eye and a little below its level. Presently many branching shadows will be seen as though they stood in space in front of the individual. These are the shadows of the blood vessels cast upon the retina. A careful examination will show that these shadows seem to converge to a point to the right of the center of vision of the right eye. By moving the candle up and down or from side to side, the shadows seem also to move slightly. Many persons can readily see Purkinje's figures by looking through the narrow spaces between the fingers of the hand moved close in front of the eye, when the vision is directed toward a bright sky. One can demonstrate by this means that the macula is free from blood vessels, since 
the pattern of the blood vessels around the borders of the macula is very readily determined. This is especially true if there is slight retinal congestion.

21. Duration of the Retinal Image.-When a beam of light falls upon the retina for an instant it produces a stimulus which endures for a time after the stimulus is removed. This interval can be measured by the proper mechanical device. Place on the color wheel a disc, which has a small segment cut out at one point on the periphery. Put a printed page behind the segment with the observer standing in front. Rotate the segment faster and faster until the printed page is seen continuously. At this point the visual image made at one revolution of the disc lasts until the next impression on the same spot. The speed of the revolution of the color wheel can be measured by attaching an electric contact key and signal magnet to the disc wheel and measuring the rate of interruptions against the known vibrations of a tuning fork. The same phenomenon may be determined by placing on the disc two complemental colors and judging the speed of revolution required for complete fusion.

22. Limits of the Field of Vision.-The limits of the visual field are determined by direct measurement with the perimeter. Set the person whose retina is to be measured in a comfortable erect position, with one eye at the center of the arc of the perimeter a $d$ the other covered by an eyeshade. The observed eye must be fixed on the center of the field of vision, and care must be used to prevent obstruction of the field. The examination is made with greatest accuracy by bringing an object into the field of vision from behind the person observed. When the individual examined first detects the presence of the object, he announces it and the angle is read off from the arc of the perimeter and recorded on the chart for the purpose. These readings should be made in about twelve radii. They should be made for each eye.

23. Limits for the Field of Vision for Color.-To measure the limits of the field of vision for color one should proceed as in the preceding experiment, except that small squares of colored papers are brought into the field from the rear. The retina should be mapped for red, green, yellow, and blue. Use Bradley's pure color papers. Take four penholders and mount on the end of one a centimeter square of red paper, on the others green, yellow, and blue. To make a determination bring the color up from behind and, as soon as it is certainly detected and announced, remove it from the field of vision. Examine the eye for all four colors at one sitting, mixing them indeterminately in the individual tests. Occasionally an eye will be found which exhibits a well-marked restriction of the color field, though the individual himself may not be completely color-blind.

24. Color-blindness. - Make an examination for color-blindness, using Holmgren's colored yarns. Spread the yarns out on a table in the best 
of light. Place the three confusion skeins in front of the individual to be examined and ask him to match them quickly from the skeins on the table, paying no attention to lights and shades of the same color. A color-blind individual will confuse colored skeins, most usually the reds, greens, and grays.

25. Color Mixing.-Use Bradley's color wheel and test the effect of simultaneous stimulation of the retina with two or more colors, by placing on the wheel two or more colored discs, rotating the wheel at a speed sufficient to cause complete fusion. The sensation produced by two colors applied simultaneously will be entirely different from that produced by either alone. Red and green (or greenish-blue), when mixed in the proper proportion, produce a sensation of gray. The same effect may be had from yellow and blue, orange and violet, or any of the complementary colors chosen according to the geometrical color table, figure 473. By mixing three colors, red, green, and violet, in the proper proportion one can produce a sensation almost the same as that produced by white light.

26. Color After-images.-Color after-images can be demonstrated by looking continuously at the center of one of the primary colors of Bradley's color charts against a white or gray wall until there- is apparent fatigue, then suddenly removing the chart. An after-image of approximately the complementary color will appear in the course of a few seconds. Occasionally these images are very vivid. The experiments are brilliant if performed in the dark room, using colored gelatin screens through which an intense light shines. When the light is turned off, a brilliant after-image of the complementary color appears.

27. Retinoscopy. -Use the ordinary small ophthalmoscope and examine the retina of the eye of a cat or rabbit. Dilate the pupil by the use of atropine. Place the animal whose eye is to be examined on a support in front of a bright but uniform light (an Argand burner). Reflect the light from the mirror of the ophthalmoscope through the pupil into the retinal cup of the animal. Usually the ophthalmoscope has to be focused for a cat's retina. When a good light is secured, the retinal cup will appear as a brilliantly colored disc, with the branching blood vessels, and usually with some brilliant bluish-green pigment in the lower portions of the retinal disc.

After some practice on the cat or rabbit, the student should examine the retina of one of his mates, preferably an eye that has an unusually wide pupil. In some cases a light dosage of homatropine may be used on one eye. This will dilate the pupil and the examination will be much easier.

Students are not recommended to use atropine unless under conditions which permit the eye to rest for two or three days following.

28. Corneoscopy.-Astigmatism is usually a defect of the cornea. It is measured most accurately by the Javal-Schiotz pattern of ophthal- 
moscope. Seat the patient erect with his head supported in the head rest. and eyes level with the instrument and one covered. Set the pointer of the telescope at 90 and focus for a clear image of the mires from the corneal. surface of the exposed eye raising or lowering the telescope if necessary.

Turn the telescope until the meridian lines of the adjacent images form an unbroken line and the spurs an exact cross. This is the primary position and the radius of curvature is read off the side scale in millimeters. and tenths.

Set the primary position indicator on the left side wheel at " $\circ$," then turn the telescope through 90 degrees or until the secondary position is found. If there is no astigmatism the cross will still be perfect. If there is astigmatism then adjust the right hand wheel to reform the cross and read the right scale as before and compute the astigmatism.

29. Visual Acuity. - The localizing power of the retina is measured by the angle formed at the nodal point by the rays from the opposite bordersor limits of an object that can just be identified. The standard test is based on the size of an object at a distance of twenty feet which will subtend an angleof one minute. The letters of the standard test chart are constructed on a total angle of five minutes, but the identifying marks subtend an angle of one minute. The visual acuity of the eye should be tested first for the right eye, then for the left. Hang a test chart at a distance of twenty feet, so that its disc is well illuminated, and allow the individual tested to read off the letters on the chart, beginning with the larger ones at the top. The letters on this chart are constructed on the basis of a visual angle of five degrees. When the letters marked "twenty feet" or "six meters" represent the limit of accurate identification, the visual acuity is said to. be $\frac{20}{20}$, i.e. I, or normal. If the line marked "thirty feet" is the limit that can be read at the normal distance of twenty feet the eye is subnormal and the visual acuity is measured by the fraction $\frac{20}{30}$, i.e. $2 / 3$ that of the standard. If the "fifteen feet" test can be read, then the visual acuity is $\frac{20}{15}$, or $4 / 3$ the standard.

If the eyes tested are astigmatic, or have other optical defects, these must first be corrected before testing for visual acuity.

30. The Test Set.-The student is recommended to close the experiments on the eye by fitting glasses for himself and at least two others. He should correct for the defects that have been revealed in the preceding experiments, especially for astigmatism; myopia, or hypermetropia; and presbyopia. Of course each eye must be tested and fitted separately. 


\section{CHAPTER XVI.}

\section{THE REPRODUCTIVE ORGANS.}

\section{THE REPRODUCTIVE ORGANS OF THE MALE.}

THE male reproductive organs comprise the Testis, the Ductus Deferens the Vesicula Seminalis, the Prostate, and the Penis.

The Testis.-The testis consists of two parts, I, the testicle proper, which is covered by the tunica albuginea and secretes the germinal cells, and 2 , the conducting tubules, which compose the epididymis and ductus deferens.

The testicle is divided by connective-tissue septa into lobules, each of which is an aggregation of tubuli seminiferi. Each tubule is limited by a membrana propria on which rests the germinal epithelium.

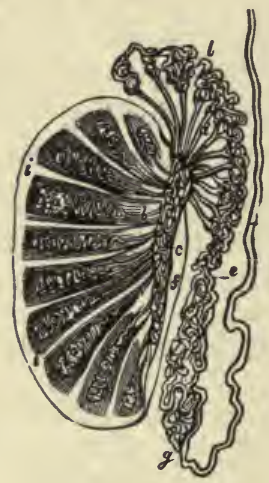

FIG. 486 .

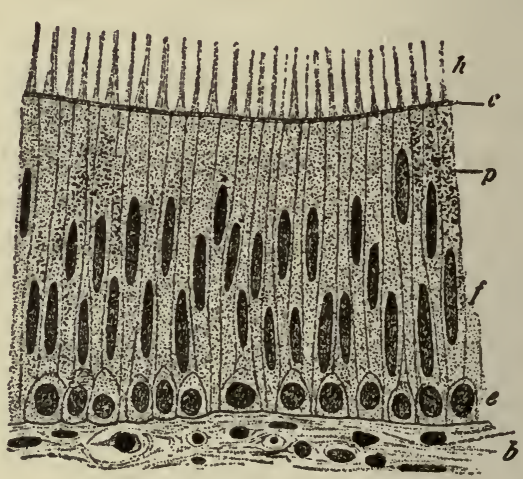

FIG. 487 .

FIg. 486.-Plan of a Vertical Section of the Testicle, Showing the Arrangement of the Ducts. The true length and diameter of the ducts have been disregarded. $a, a$, Tubuli seminiferi coiled up in the separate lobes; $b$, tubuli recti; $c$, rete testis; $d$, ductuli efferentes ending in the coni vasculosi; $l, e, g$, convoluted canal of the epididymis; $h$, vas deferens; $f$, section of the back part of the tunica albuginea; $i, i$, fibrous processes running between the lobes; $s$, mediastinum.

FIG. 487.- - Vertical Section through the Wall of the Tubules of Epididymis. $\times 700$. (Kölliker.) b, Connective tissue and smooth muscle cells; $e$, basal layer of epithelial cells; $f$, high columnar cells; $p$, pigment granules in columnar cells; $c$, cuticula; $h$, cilia.

The male reproductive cells are all descended from primitive germ cells, the archispermiocytes, that become differentiated during the fetal life.

On the approach of sexual maturity the process of spermatogenesis begins. The germinal cells multiply rapidly, and, by a complex series of mitotic 
divisions or stages, form ultimately the male reproductive cells, or sperm cells.

The important stages in order are: archispermiocyte, spermatogonia, primary and secondary spermatocytes, spermatids, and spermatozoa. The spermatogonia stage is the stage of rapid multiplication; the spermatocyte, that of maturation, comparable to the maturation stage of the ovum.

The spermatozoa, or sperm cells, are the essential male reproductive cells. Each mature spermatozoon consists of a minute oval head, a middle piece, and

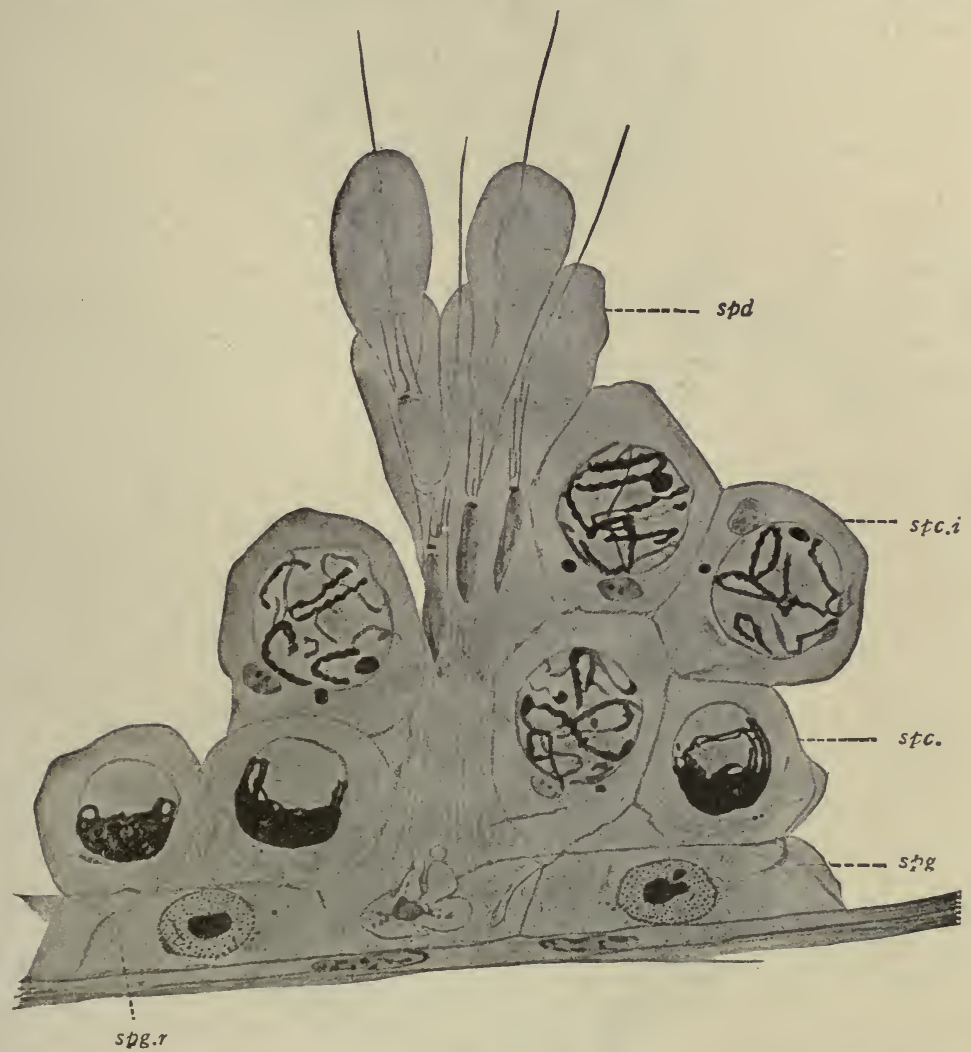

FIG. 488.- - Later Stages in Spermatogenesis of the Bull. spg.r, Reserve spermatogonium; $s p g$, spermatogonium; $s p c . g$, spermatocyte in late synapsis stage; $s p c . i$, spermacyte in stage just preceding the maturation divisions; $s p d$, spermatids in advanced stage of histogenesis, with heads deeply embedded in Sertoli cell. Highly magnified. (After Schoenfeld.)

a tail. The head consists almost entirely of the cell nucleus, while the middle piece and tail are cytoplasmic structures. The head is $4 \mu$ by $2.5 \mu$. The middle piece and tail are about 50 to $60 \mu$ long. The tail is essentially a cilium, and exhibits the power of flagellate movement.

The Ductus Deferens. - This is the single duct proceeding from each testicle to join its fellow at the base of the bladder. Each has an ampulla or 


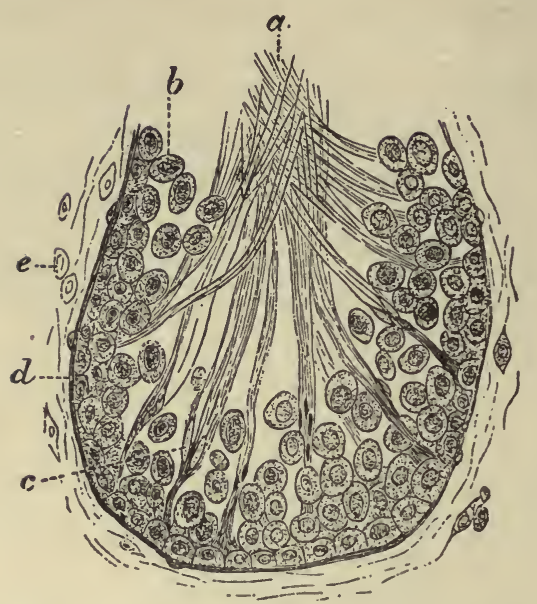

FIG. 489.-Section of a Tubule of the Testicle of a Rat, to Show the Formation of the Spermatozoa. $a$, Spermatozoa; $b$, seminal cells; $c$, spermatoblasts, to which the spermatozoa are still adherent; $d$. membrana propria; $e$, fibro-plastic elements of the connective tissue. (Cadiat.)

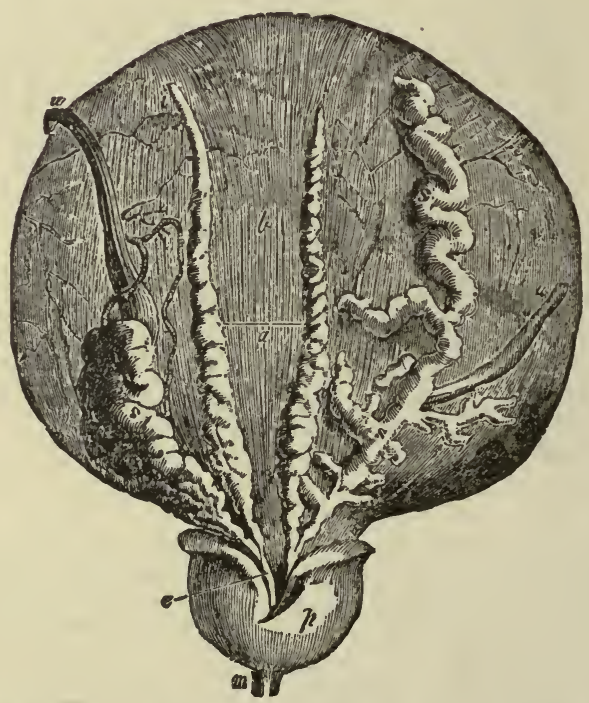

Fig. 490.-Dissection of the Base of the Bladder and Prostate Gland, Showing the Vesiculæ Seminales and Ductus Deferens. $a$, Lower surface of the bladder at the place of reflection of the peritoneum; $b$, the part above covered by the peritoneum; $i$, left.ductus deferens, ending in $e$, the ejaculatory duct; the ductus deferens has been divided near $i$, and all except the vesical portion has been taken away; $s$, left vesicula seminalis joining the same duct; $s, s$, the right ductus deferens and right vesicula seminalis, which has been unraveled; $p$, under side of the prostate gland; $m$, part of the urethra; $u, u$, the ureters (cut short), the right one turned aside. (Haller.) 
enlargement just before it unites with its fellow. The ductus deferens has muscular walls and is lined with ciliated epithelial cells.

The Vesiculæ Seminales. - The seminal vesicles have the appearance of outgrowths from the base of the deferent ducts. Each deferent duct just before it enters the prostate gland, through part of which it passes to terminate in the urethra, gives off a side branch which bends back from it at an acute angle. This branch, dilating, variously branching, and pursuing in both itself and its branches a tortuous course, forms the vesicula seminalis. Each vesicle is a single-branching, convoluted, and sacculated tube. The microscopic structure resembles closely that of the ductus deferens.

The Penis.-The penis is attached to the symphysis pubis by its root. It is composed of three long, more or less cylindrical masses enclosed in remarkably firm fibrous sheaths. Two, the corpora cavernosa, are alike and are firmly joined together. They receive below and between them the third part, or corpus spongiosum. The urethra passes through the corpus spongiosum. The enlarged extremity, or glans penis, is continuous with the corpus spongiosum. Cowper's glands are at its base, and their ducts open into the base of the urethra.

The Prostate Gland.-The prostate is situated at the neck of the urinary bladder, and encloses the base of the urethra. The prostate is made up of small compound tubular glands embedded in an abundance of muscular fibers and connective tissue. The glandular substance consists of numerous small saccules, opening into elongated ducts, which unite into a smaller number of excretory ducts. The acini of the upper part of the prostate are small and hemispherical, in the middle and

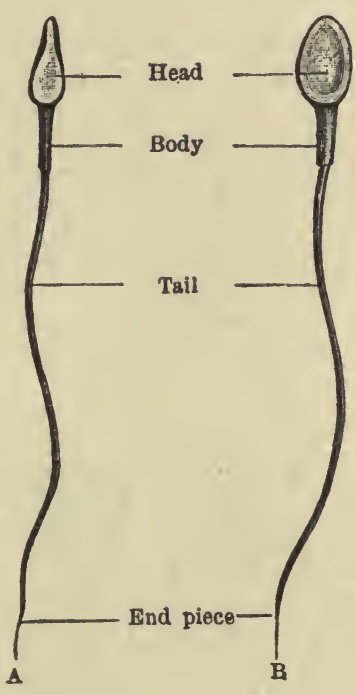

Fig. 491.-Human Spermatozoa (after Retzius). $A$, Side view, $B$, front view. lower parts the tubes are longer and more convoluted. The ducts, twelve to twenty in number, open into the urethra. They are lined by a layer of columnar cells, beneath which is a layer of small polyhedral cells.

The muscular tissue of the prostate not only forms the chief part of the stroma of the gland, but also forms a continuous layer inside the fibrous sheath, as well as a layer surrounding the urethra continuous with the sphincter of the bladder.

The Seminal Fluid.-The sperm cells of the testes are joined on their way to the exterior by the fluids secreted by the mucous lining of the various tubules and glands. Of the fluids the chief ones are the secretions of the 
seminal vesicles, of the prostate gland, and of Cowper's glands. The sperm cells and the secretions together constitute the seminal fluid.

After the period of puberty the seminal fluid is secreted constantly but slowly, except under sexual excitement. It is ordinarily received into the seminal vesicles, whence it is expelled at the time of coitus. In celibates the seminal fluid may at times escape in small quantity into the urethra to be washed away by the urine, or periodic reflex emissions may occur. The seminal vesicles contribute a secretion. as well as a vesicle to receive the sperm.

The secretion of the seminal vesicles and that of the prostate gland are in some way concerned in maintaining the activity and prolonging the life of the spermatozoa probably owing to the alkalinity of the secretions. These cells remain alive in the fluid for as much as forty-eight hours after removal from the body, and remain alive quite indefinitely in the vesicles in the body. The secretions have been proven necessary to the life and function of the spermatozoa by the results of operations in which the seminal vesicles and the prostate were removed, whereby the animals became sterile.

\section{THE REPRODUCTIVE ORGANS OF THE FEMALE.}

The female genital organs consist of the Ovarium, the Tuba Uterina, the Uterus, and the Vagina.

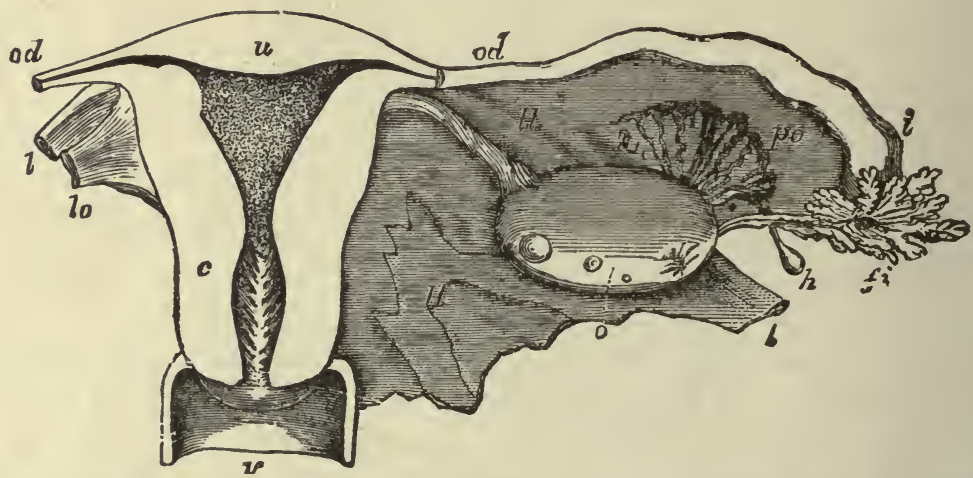

Fig. 492.-Diagrammatic View of the Uterus and Its Appendages, as Seen from Behind. The uterus and upper part of the vagina have been laid open by removing the posterior wall; the Fallopian tube, round ligament, and ovarian ligament have been cut short, and the broad ligament removed on the left side. $u$, The upper part of the uterus; $c$, the cervix opposite the os internum; the triangular shape of the uterine cavity is shown, and the dilatation of the cervical cavity with the rugæ termed arbor vitæ; $v$, upper part of the vagina; od, Fallopian tube or oviduct; the narrow communication of its cavity with that of the cornu of the uterus on each side is seen; $l$, round ligament; $l o$, ligament of the ovary; $o$, ovary; $i$, wide outer part of the right Fallopian tube; $f$, its fimbriated extremity; po, parovarium; $h$, one of the hydatids frequently found connected with the broad ligament. $\frac{1}{2}$. (Allen Thomson.)

The Ovaries.- The ovaries are paired bodies, situated in the cavity of the pelvis, and adherent to the posterior surface of the broad ligament. The 
attached border of the ovary is called the hilum, and it is at this point that the blood vessels and nerves enter it. Each ovary is about $4 \mathrm{~cm}$. long, $2 \mathrm{~cm}$. wide, and $I .25 \mathrm{~cm}$. thick. It is supported by the suspensory ligament.

The internal structure of the ovary in all mammals consists of a peculiar soft fibrous connective tissue, stroma, abundantly supplied with blood vessels. The surface of the ovary is covered with cubical epithelium. Embedded in the stroma in various stages of development are numerous minute follicles or vesicles, the vesicular ovarian follicles, containing the ova, figure 494 . They are small and numerous near the surface of the ovary, either arranged as a continuous layer, as in the cat or rabbit, or in groups, as in the human ovary. Nearer the center are large and fully developed follicles.

Each follicle has an external membranous envelope, or tunica externa, which is lined with a layer of nucleated cells, forming a kind of epithelium

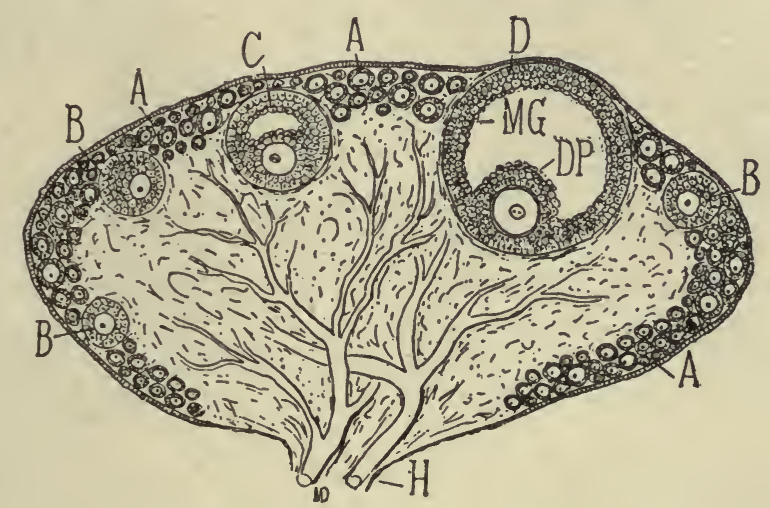

FIG. 493.-Diagrammatic Section of the Ovary, Showing its Cortical or Ovigenous Layer, Formed of Ovisacs in Various Stages of Evolution. (Duval.) $A, A, A$, Primordial ovisacs; $B, B, B$, ovisacs further developed; $C$, ovisac approaching maturity; $D$, ripe ovisac with its proligerous disc $(D P)$ containing the ovum; $M G$, membrana granulosa; $H$, hilum of ovary.

or internal coat and named the tunica interna. The cavity of the follicle contains the ovule, or immature egg cell, enclosed in a very delicate membrane. The large spherical nucleus contains one or more nucleoli. The nucleus is known as the germinal vesicle, and the nucleolus as the germinal spot.

The human ovum measures about $0.2 \mathrm{~mm}$. in diameter. Its external investment, or the zona pellucida, or vitelline membrane, is a transparent membrane, about Io $\mu$ in thickness, which under the microscope appears as a bright ring, figure 495 . The ovum itself has the characteristic structure of the typical cell, with the exception that its cytoplasm is filled with numerous yolk granules. The larger granules or globules, which have the aspect of fat-globules, are in greatest number at the periphery of the yolk.

The nucleus, or germinal vesicle, is about $0.05 \mathrm{~mm}$. in diameter. The vesicle is of greatest relative size in the smallest ova. 
These ova are descended from primitive germ cells which become differentiated very early in the embryo. In some vertebrates, such as the chick, there is evidence that the primitive germ cells become distinct from all other cells in the body even before the formation of the embryonic mesoderm has been completed. 'Later they become located in a membrane of short columnar cells, the so-called germinal epithelium, covering the surface of the embryonic ovary.

The Graafian follicles of the human ovary are formed in the following manner: The cells of the germinal epithelium undergo proliferation so as to form several strata, and grow into the ovarian stroma as longer or shorter
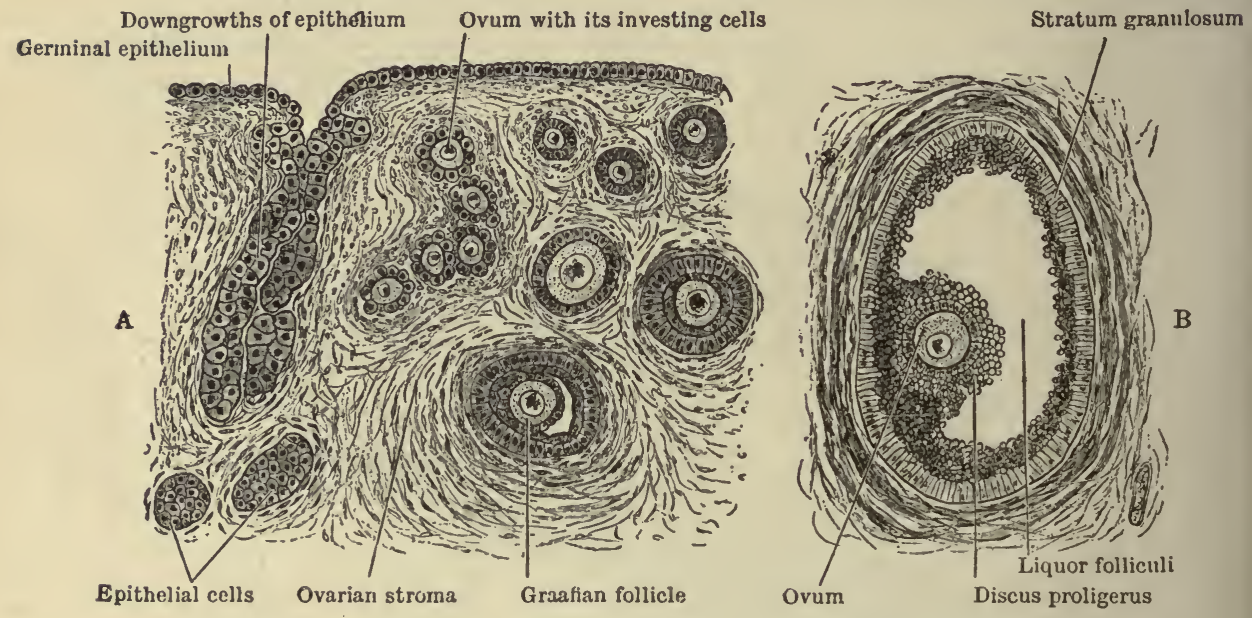

FIG. 494.- $A$, Diagrammatic Representation of the Manner in which the Vesicular Ovarian Follicles Arise During the Development of the Ovary. $B$, Diagram Illustrating the Structure of a Ripe Vesicular Ovarian Follicle. (Cunningham.)

columns or tubes. By degrees these tubes become cut off from the surface epithelium, and form cell nests, small if near the surface, larger if in the depth of the stroma. The nests increase in size from multiplication of their cells. Inside these nests, certain cells which are descended from the primitive germ cells enlarge and form ova. The small cells of a nest surround the ova, and form their membrana granulosa, and the stroma growing up separates the surrounded ova into so many Graafian follicles.

The smallest follicles are formed at the surface, and make up the cortical layer. It is said by some that the superficial follicles as they begin to ripen become more deeply placed in the ovarian stroma; and, again, that as they increase in size they make their way back toward the surface. The development of all eggs that are destined to mature is carried as far as the early follicular stage in the ovary of the child, previous to the birth of the child, or within a relatively short period thereafter. Conditions indicative of the for- 
mation of new follicles have never been observed in a person more than two years of age.

When the vesicular ovarian follicles mature, they form little prominences on the exterior of the ovary covered only by a thin layer of condensed fibrous tissue and epithelium. From the earliest infancy, and through the whole fruitful period of life, there appears to be a constant development and maturing of ovarian vesicles, with their contained ova. Until the period of puberty, however, the process is comparatively inactive. But, coincident with the

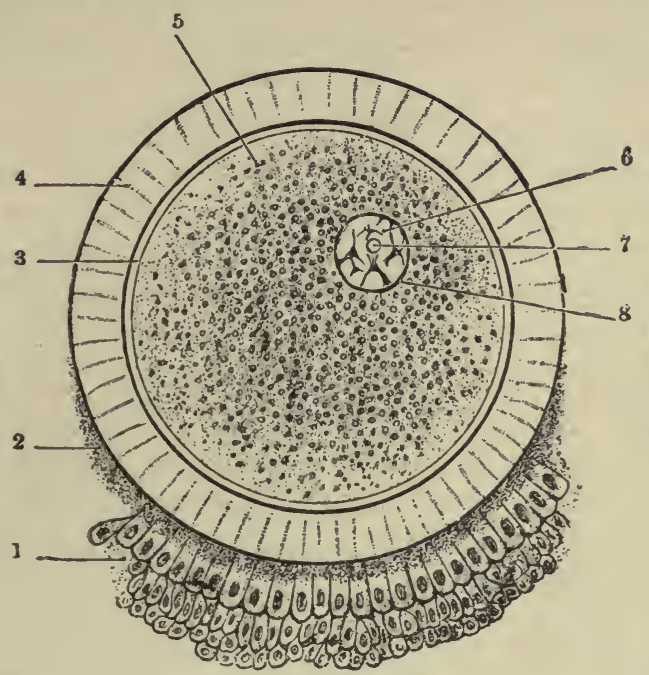

FIG. 495.-Diagrammatic. Representation of a Human Ovum and Its Coverings. (Cunningham.)

The corona radiata, which completely surrounds the ovum, is represented only in the lower part of the figure.
I, Corona radiata;
2, granular layer;
5 , vitellus or yolk;
3 , vitelline membrane;
4, zona pellucida (oolemma);
6, germinal vesicle (nucleus);
7 , germinal spot (nucleolus);
8 , nuclear membrane.

other changes which occur in the body at the time of puberty, the ovaries enlarge and become very vascular, the development of ovarian vesicles is more abundant, the size and degree of development attained by them are greater, and the ova are capable of being fertilized. The follicles receive their nourishment from the surrounding capillaries in the stroma. They are never penetrated by blood vessels, and are never entered by nerve fibers, so far as is known.

The Uterine Tubes or Oviducts.-The uterine tubes are about ro $\mathrm{cm}$. in length and extend between the ovaries and the upper angles of the uterus. At the point of attachment to the uterus each tube is very narrow; but in its course to the ovary it increases to about $3 \mathrm{~mm}$. in thickness. At 
its distal extremity, which is free and floating, it bears a number of elongated lobes, or fimbrice, one of which is longer than the rest and is attached to the ovary. The canal of the tube is narrow, especially at its point of entrance into the uterus. Its other extremity is wider and opens into the cavity of the abdomen at the fimbriæ. The uterine tube is invested with peritoneum, and its canal is lined with ciliated epithelium.

The Uterus.- The uterus, $u, c$, figure 492 , is a somewhat pear shaped organ, and is about $7.5 \mathrm{~cm}$. in length, $5 \mathrm{~cm}$. in breadth at its upper part or fundus, but at the neck or cervix only about $\mathrm{I} .25 \mathrm{~cm}$. The part between the fundus and neck is termed the body of the uterus; it is about $2.5 \mathrm{~cm}$. in thickness.

The uterus is constructed of three principal layers, or coats: serous, fibrous and muscular, and mucous. The serous coat, which has the same general structure as the peritoneum, covers the organ except the front surface of the neck. The middle coat is a thick mass of unstriped muscle. The muscle fibers become enormously developed during pregnancy. The arteries and veins are found in large numbers in the outer part so as to form almost a special vascular coat. The mucous membrane of the uterus is composed of columnar ciliated epithelium, which extends also to the interior of the tubular glands, of which the mucous membrane is largely made up. In the cervix of the uterus the mucous membrane is arranged in permanent longitudinal folds, plica palmate. The glands of this part branch repeatedly, and extend deeply into the substance of the cervix. The body has numerous simpler tubular glands. The glands are also lined with ciliated epithelium. They secrete a thick glairy mucus, resembling white of egg.

The Vagina.-The vagina is a membranous canal 8 to $10 \mathrm{~cm}$. long, extending obliquely downward and forward from the neck of the uterus, which it embraces, to the external organ of generation. It is lined with mucous membrane, covered with stratified squamous epithelium, which in the ordinary contracted state of the canal is thrown into transverse folds. External to the mucous membrane, the walls of the vagina are constructed of unstriped muscle and fibrous tissue, within which in the submucosa, especially around the lower part of the tube, is a layer of erectile tissue. The lower extremity of the vagina is embraced by an orbicular muscle, the sphincter vagina. The external organs of generation are the clitoris, the labia interna or nympha; and, the labia externa or pudenda, formed of the external integument, and lined internally by mucous membrane. Numerous mucous follicles are scattered beneath the mucous membrane of the external organs of generation; and two larger lobulated glands, the glands of Bartholin, analogous to Cowper's glands in the male, are located at the sides of the lower part of the vagina. The ducts of these glands are about $12 \mathrm{~mm}$. long and open immediately external to the hymen at the mid-point of the lateral wall of the vaginal orifice. 
Ovulation and Menstruation.-In the process of development in the ovary, the individual vesicular ovarian follicle increases in size and gradually approaches the surface of the ovary. When fully ripe or mature, it forms a little projection on the exterior. Coincident with the increase in size, which is caused by the augmentation of its liquid contents, the external envelope of the distended vesicle becomes very thin and eventually bursts. The ovum and fluid contents of the vesicle escape on the exterior of the ovary, whence they pass into the uterine tube.

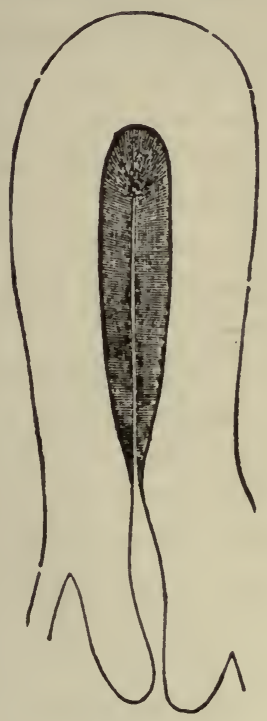

FIG. 496.

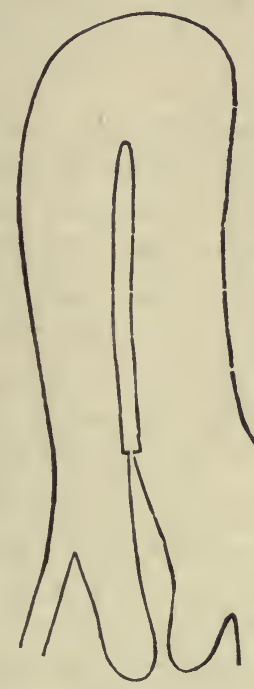

FIG. 497 .

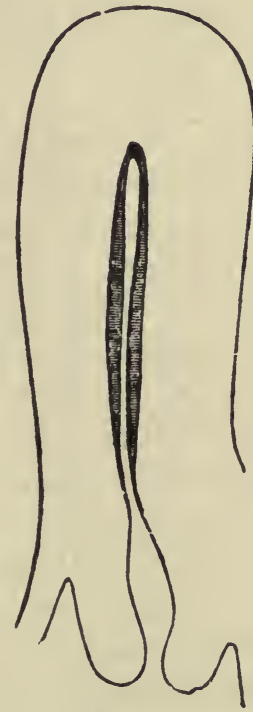

FIG. 498.

Fig. 496.-Diagram of Uterus just Before Menstruation. The shaded portion represents the thickened mucous membrane.

Fig. 497.-Diagram of Uterus when Menstruation has just Ceased, Showing the Cavity of the Uterus Deprived of Mucous Membrane.

Fig. 498.-Diagram of Uterus a Week After the Menstrual Flux has Ceased. The shaded portion represents renewed mucous membrane. (J. Williams.)

In man and mammals ovulation apparently occurs only at certain periods. These periods are closely connected with the changes in the woman that constitute the phenomenon of menstruation, or, in the lower mammals, of astrus, or heat.

That ovulation and discharge occur periodically, and only during the phenomenon of heat, in the lower mammalia, is indicated by the facts that, in all instances in which ovarian vesicles have been found presenting the appearance of recent rupture, the animals were at the time or had recently been in heat. There are few authentic and detailed accounts of ovarian vesicles being found ruptured or of ova being fertilized in the intervals 
between periods of heat. Although conception is not confined to the periods of menstruation in the human species, yet it is more likely to occur about a menstrual epoch than at other times.

The exact relation between the discharge of ova and menstruation is not very clear. In animals, the physiological analogy of menstruation appears to be found in the proæstral processes, which are the preliminary stages of heat, and occur immediately before ovulation. It was formerly believed that menstruation was the result of a congestion of the uterus arising in association with the enlargement and rupture of a vesicular ovarian follicle; but though a vesicular ovarian follicle is, as a rule, ruptured at each menstrual epoch, yet instances are recorded in which menstruation has occurred where no ovarian follicle can have been ruptured, and cases where ova have been discharged in amenorrheic women. It must therefore be admitted that menstruation is not strictly dependent on the maturation and discharge of ova.

Observations made after death, and facts obtained by clinical investigation, support the view that rupture of a vesicular ovarian follicle does not happen on the same day of the monthly period in all women. In the minority of cases it may occur toward the close or soon after the cessation of a flow. On the other hand, in almost all subjects examined after death, of which there is record, rupture of the follicle appears to have taken place before the commencement of the menstrual flow.

However, the presence of the ovaries seems necessary for the performance of the menstrual function; for women do not menstruate when both ovaries have been removed by operation. See page 498 for a discussion of the functional effects of removal of the ovary.

Source and Character of Menstrual Changes.-The menstrual periods usually occur at intervals of a lunar month, the duration of each being from three to six days. In some women the intervals are so short as three weeks or even less; while in others they are longer than a month. The periodical return is usually attended by pains in the loins, a sense of fatigue in the lower limbs, and other symptoms, which vary extremely in different individuals.

The menstrual discharge is a thin sanguineous fluid, and consists of blood, epithelium, and mucus from the uterus and vagina. The menstrual flow is preceded by a general engorgement of all the pelvic organs with blood. The cervix and vagina become darker in color and softer in texture, and the quantity of mucus secreted by the glands of the cervix and body is increased. The uterine mucous membrane is swollen and the glands are enlarged. The discharge of blood, the source of which is the mucous membrane of the body of the uterus, is probably associated with uterine contractions. There is great difference of opinion as to whether or not any of the uterine mucous membrane is normally shed during the process of menstruation. John Williams believes that the whole of the mucous membranes of the body of the uterus is thrown off at each monthly period, 
forming a true decidua menstrualis, figure 496, while Möricke and others believe that the mucous membrane remains intact. Leopold believes that red blood corpuscles escape from the congested capillaries and undermine the superficial epithelium, and that in this way the superficial layer of the mucous membrane is eroded and subsequently regenerated. There is a period of regeneration followed by a period of rest before the next repetition. Minot distributes the variations in time as follows:

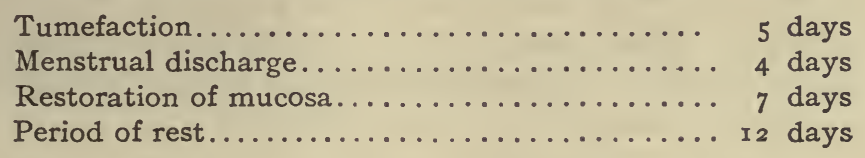

The menstrual period is often accompanied by profound disturbances in other parts of the body, especially of the vascular and of the nervous systems, and of the nutritive processes.

Corpus Luteum. - Immediately before, as well as subsequent to, the rupture of an ovarian follicle and the escape of its ovum, changes ensue in the interior of the follicle, which result in the production of a yellowish mass, termed a corpus luteum.

When fully formed, the corpus luteum of mammals is a roundish solid body, of a yellowish or orange color, and composed of a number of lobules, which surround, sometimes a small cavity, but more frequently a small stelliform mass of substance, from which delicate processes pass as septa between the several lobules. The processes gradually change till they nearly fill the cavity of the follicle, and even protrude from the orifice in the external covering of the ovary. Subsequently this orifice closes, but the fleshy growth within still increases during the earlier period of pregnancy, the color of the substance gradually changing to yellow, and its consistency becoming firmer. After the orifice of the follicle has closed, the growth of the yellow substance continues during the first half of pregnancy, till the cavity is reduced to a comparatively small size or is obliterated; in the latter case, merely a white stelliform cicatrix remains in the center of the corpus luteum.

The first changes of the internal coat of the ovarian follicle in the process of formation of a corpus luteum seem to occur in every case in which an ovum escapes. If the ovum is impregnated, the growth of the yellow substance continues during nearly the whole period of gestation and forms the large corpus luteum commonly described as a characteristic mark of impregnation.

The significance of the corpus luteum is found in the belief that it is the portion of the ovary especially concerned in the production of an internal secretion that affects the uterus, especially stimulating it at and before the menstrual period. 
Menstrual Life.-The occurrence of a menstrual discharge is one of the most prominent indications of the commencement of puberty in the female sex; though its absence even for several years is not necessarily attended with arrest of the other characters of this period of life or incapability of impregnation. The average time of its first appearance in females of this country and others of about the same latitude is from fourteen to fifteen: but it is much influenced by the kind of life to which girls are subjected, being accelerated by habits of luxury and indolence, and retarded by contrary conditions. Its appearance may be slightly earlier in persons dwelling in warm climates than in those inhabiting colder latitudes. The menstrual functions continue through the whole fruitful period of a woman's life, and usually cease between the forty-fifth and fiftieth years, which time is known as the climacteric. Menstruation does not usually occur in pregnant women. 


\section{CHAPTER XVII.}

\section{DEVELOPMENT.}

Maturation of Male Germ Cells, the Spermatozoon.-The general effect of maturation of germinal cells is to reduce the number of chromosomes, in order that there may not be more than the normal supply in the new cell that will be formed by the union of the ovum and the sperm in the fertilization of the ovum. We shall describe the process of maturation in terms that will apply to the production of spermatozoa in nearly all animals.

In each species of animals the number of chromosomes in every cell nucleus is constant. Although they are of various sizes and shapes, each nucleus in the body appears to have the same assortment as every other. This is explained by the fact that whenever a cell divides, each chromosome divides individually, and the two resulting daughter chromosomes from each original chromosome are distributed to the two new cells. Thus the chromosomes can be traced back individually through innumerable cell divisions to the assortment that existed in the egg cell at the very beginning of its development. The source of this supply of chromosomes found in the developing egg is double, one set having been derived from the original nucleus of the egg, and the other provided by the sperm. Now the developed organism, which has obtained in this way a double set of chromosomes in every cell, must like its ancestors be able to produce germ cells that each contain a single set.

This maturation reduction of chromosomes, from a double to a singleset, is always carried out by the joint effect of a process of synapsis and of two peculiar cell divisions known as the maturation divisions. In the nucleus of the primary spermatocyte the chromatin materials become crowded together, and pass through the stage known as synapsis, during which the chromosomes become closely united with each other in pairs. The double set of simple, i.e., uncompounded chromosomes occurring in the spermatogonia is thus transformed in the primary spermatocyte into an apparently single set of double chromosomes. Each of these double structures is believed to represent the union of corresponding chromosomes out of the two sets previously existing. They grow immediately into a four-parted condition, which has given them the name tetrads. In the cell division of the primary spermatocytes, the first maturation division, the tetrads are divided equally, and the result is two secondary spermatocytes, each containing a single set, called diads. The second maturation division follows promptly, producing two 
spermatids from each secondary spermatocyte. Each spermatid receives half the chromosomes of each diad, and as a result a spermatid possesses but
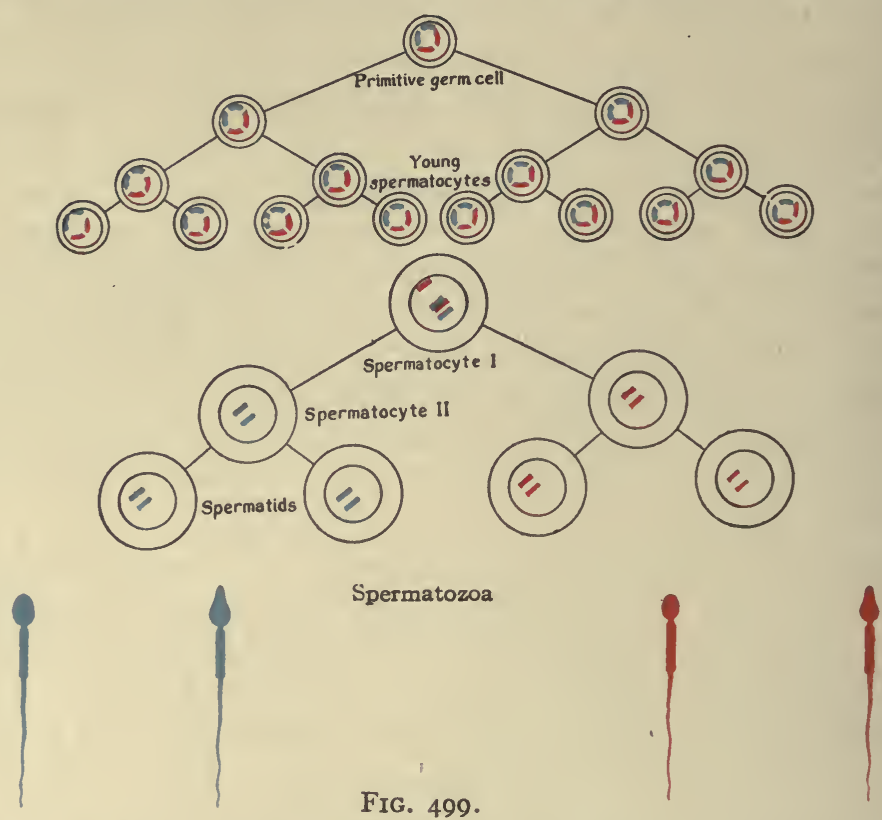

a single set of simple chromosomes. It is then said to have the reduced or gametic number, characteristic of sexual germ ceils, as contrasted with the

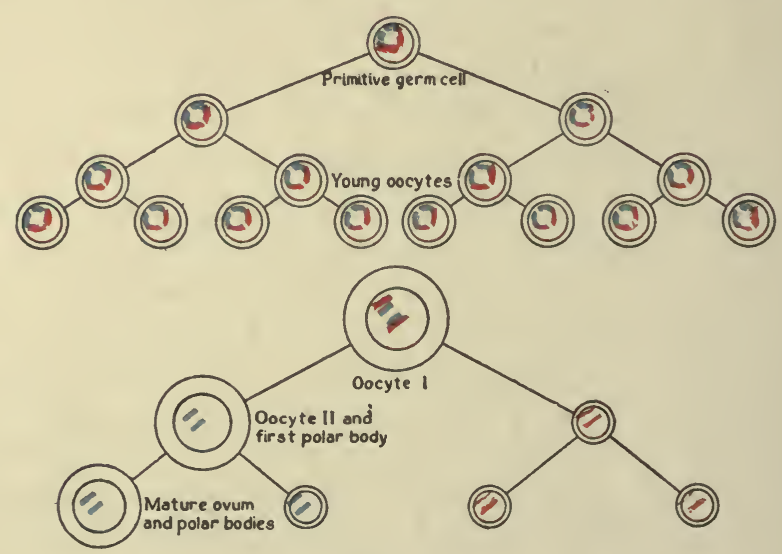

FIG. 500 .

Figs. 499 and 500.-Diagrams Showing the Scheme of Development and Chromatin Reduction in the Growth of the Spermatozoa and Ova. (From Cunningham.)

unreduced or somatic number, characteristic of the cells of the body.

The spermatid is metamorphosed directly into a spermatozoon. The 
process consists in the development of a tail or cilium, the transformation of the nucleus into the form of a spermatozoon head, and the sloughing off of nearly all the remaining cell protoplasm. The centrosome, a minute body closely concerned with the power of cell division, is apparently retained, but the power of cell division is impossible in the presence of so little cytoplasm, figure 499.
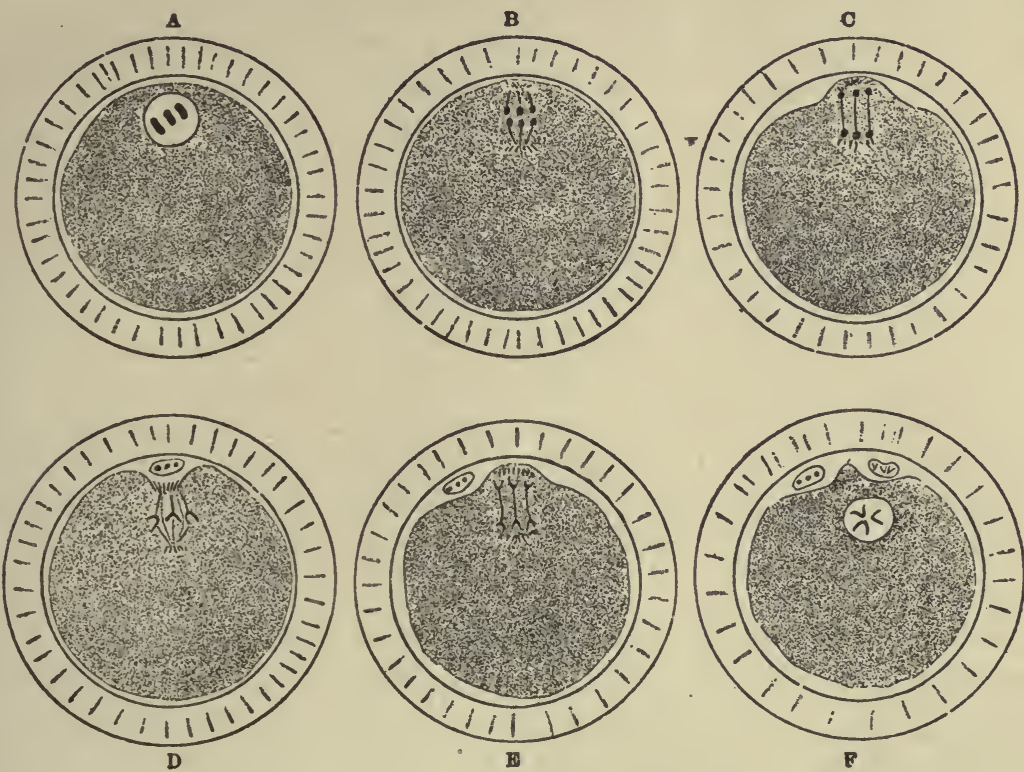

FIG. 50r.-The Maturation of the Ovum; Extrusion of the "Polar Bodies." (Diagrammatic.) $A$, An ovum at the commencement of the process; $B$, after the formation of the spindle. The chromosomes are gathered at the equator of the spindle. $C$, One apex of the spindle has projected into a bud on the surface, and half of the divided dyads have passed to each pole; $D$, the separation of the first polar body; $E$, the commencement of the second polar body; $F$, the completion of the second polar body. (Cunningham.)

Maturation of the Ovum-the Female Germ Cell.-The ovum when liberated from the ovary is a single cell enclosed within the zona pellucida, and containing the germinal vesicle or nucleus and germinal spot or nucleolus. It still possesses the somatic or double number of chromosomes, and must undergo maturation divisions in order to reduce their numbers. Previous to the maturation divisions it should more correctly be called a primary oocyte.

Synapsis now occurs in the nucleus. It is not fundamentally different from the synapsis in the spermatocyte. Meanwhile the nucleus migrates to the surface of the oocyte. After synapsis, the chromosomes are in the form of a single set of tetrads as in the male. They are liberated by the disappear- 
ance of the nuclear membrane, and become arranged upon a nuclear spindle, as if for an ordinary cell division. The two maturation divisions occur in rapid succession. In the first of these the oocyte is divided extremely unequally, into a secondaryoccyte of nearly the same size as the primary, and the first polar body, morphologically equivalent to a minute, degenerant secondary oocyte. Each secondary oocyte receives a single set of diads, which are the division products of the set of tetrads. The second maturation division is similarly unequal, and its products are the ripe ovum and the second polar body. Each of these receives a single set of simple chromosomes. Those that are in the ovum become the female pronucleus, possessing the reduced chromosomal number, figure 500. The centrosome disappears, and the egg is left incapable of division. It remains unchanged unless fertilized by the sperm.

Material Basis of Heredity.-In considering the relations of parent and child to each other through heredity, it is necessary to distinguish sharply between that which comes to the child as an inherent part of its nature, and all else which may be added through conditions of nourishment, physical surroundings, infection with disease-producing parasites, and the like. The former is the only true heredity, in a scientific sense, although it is obvious that until birth, or even for some time after birth, the well being and development of the germ cells, fetus, and child are directly dependent upon the parental organism.

It has been shown that every cell in a child is descended from one cell, the fertilized ovum. This fertilized egg is the product of the union of male and female germ cells, cells which are not derived from the active body tissues of the parents, but can be traced back to cells that were set aside for the purpose of reproduction at a very early stage of the parent's embryonic life. Thereafter the influences which the vicissitudes of the parent's life may have exerted upon the germ cells he produces are limited to what can be carried by diffusion into the germ cells of substances from the lymph.

Relative Influence of Mother and Father in Heredity.-We may use a more concrete form of this question as follows: If two races are crossed, does it make any difference which form was the mother's stock, and which the father's? This comparison has been made very extensively in domesticated animals and garden plants, and to a less extent in man. Good examples are the inheritance of color peculiarities in mammals, and peculiarities of the skeleton. Excepting in certain special cases, like color blindness in man, which will be treated later, it is found that these "reciprocal crosses" produce identical results in heredity; hence, that the average influence of father and mother upon inheritance is in general equal. We have already seen that the two sexes make very unequal contributions to the substance of the fertilized egg. The following table summarizes these differences and similarities. 
Ripe ovum Spermatozoon

Fertilized ovum
Much cytoplasm Trace of cytoplasm

Single set of chromosomes Much cytoplasm
No centrosome

One centrosome

One centrosome

We see that virtually all the cytoplasm of the beginning embryo comes from the mother, and represents her variety. The minute centrosome comes from the spermatozoon. But the nucleus represents the two stocks half and half, and is the only part of the germ cell that fulfills the conditions for equal inheritance from the two parental stocks. The conclusion has been drawn, that the transmission of such differences as we find between different breeds of the same species is due to peculiarities in the nuclei.

Mendelian Inheritance and Chromosomes.-Two types of inheritance have long been recognized, the blending, and the alternative or Mendelian forms. In the former, the offsprings are intermediate between the stocks of their parents. In the latter, a certain characteristic from one stock is manifested by the child to the exclusion of the alternative trait shown in the other stock. Many supposed cases of blending are now believed to be complex instances of alternative inheritance. Albinism, or the lack of pigment in hair, skin, and eyes, is a good example of an alternatively heritable trait.

When two pure bred animals that carry two opposite and alternative traits are crossed with each other, one type "dominates," or shows in full force in all the young. Thus in most mammals color dominates over albinism, and all the young from the cross look like thoroughbred colored animals. We conclude that if either egg or sperm carries color, the offspring is always colored in appearance. But if these animals are now crossed with the white stock, half of the offspring will be white and the others colored. This indicates that the dark colored hybrids are producing equal numbers of two kinds of germ cells, one of which possesses, and the other lacks the color-producing factor. These last colored young are really hybrids producing two kinds of germ cells, like their hybrid parent. But their white brothers have received white from both their parents, and are essentially a pure stock once more.

Thus in simple alternative (Mendelian) inheritance, the hybrid looks like a pure-bred representative of the "dominant" form, the other, or "recessive" character not coming to view. And its germ cells are not hybrid, but half of them are pure for one form, and the other half pure for the other form. The character of the offspring depends upon the particular germ cells that produced it. If they are both alike, the young is "pure," no matter how hybrid its parents.

These facts lead to certain statistical relations known as the Mendelian ratios, in the proportionate numbers of pure dominant, mixed, and pure recessive young that will be found in each kind of cross. When two pairs of Mendelian characters are crossed at once, as for example, two colors of hair and two contrasting lengths of hair, each pair of characters usually follows these Mendelian rules quite independent of the other pair. From one such 
hybrid animal, germ cells will result that carry white short hair, white long, dark short, and dark long.

The theory of inheritance through the agency of chromosomes is based upon this type of fact, and the close similarity to the manner of distribution of chromosomes in the maturation of the germ. By the theory the color hybrid is supposed to have a color-producing chromosome from one parent, but from the other parent a corresponding chromosome which lacks this power. In the maturation of the germ cell one of these two corresponding chromatin elements is left alone in each mature cell, otherwise the chromosomes would not have been reduced from a double to a single set. And for any one germ cell the chances are equal whether it receives the colorbearing or the color-lacking chromosome. In either case the cell is "pure" for whichever trait it carries.

Germ Cells in Relation to the Determination of Sex.-A peculiar chromosome or pair of chromosomes has been found in man, and some other vertebrates, as well as in insects, nematode worms and other types of lower animals, which is closely connected with the determination of sex. It is called the heterochromosome, or X chromosome. In its simplest form this chromosome is single in the male, but paired in the female. In synapsis in the male cells it remains separate, because of the lack of a mate. As a result, before the first maturation division it is a triad instead of a tetrad. It can divide only once in the two maturation divisions, and at the other division it passes bodily over to one of the daughter cells, leaving the other cell unsupplied. Thus there are produced equal numbers of two types of spermatozoa, those with and those without an $\mathrm{X}$ element.

In the corresponding female maturation stages a tetrad is formed, because there are two of these chromosomes present. They behave normally in maturation, and every ripe egg receives one of them. Therefore, after fertilization there will be two types of embryos; (I) those receiving two $\mathrm{X}$ chromosomes, one of which came from each parent. These develop into females. (2) Those receiving one $\mathrm{X}$ chromosome from the mother, and none from the father. These are the males.

"Sex-linked" Inheritance.-This is a peculiar and instructive type of inheritance, which behaves in a way suggesting that the trait may be carried in association with the $\mathrm{X}$ chromosome. Its chief distinction is that it is never carried from father to son, the son never receiving an $\mathrm{X}$ chromosome from the father. The best human example is hereditary color-bindness, which is a "sex-linked" recessive character, due to some lack in the X chromosome. The presence of one normal color bearing $\mathrm{X}$ chromosome is sufficient to establish color vision.

Changes Following Impregnation.-The process of impregnation of the ovum has been observed most accurately in the lower types. The process is as follows: The head of a single spermatozoon joins with an elevation of the yolk substance, the tail remaining motionless and 
then disappearing. The head enveloped in the protoplasm then sinks into the yolk and becomes a nucleus, from which the yolk substance is arranged in radiating lines. This is the male promucleus. The middle piece of the sperm is believed to furnish a new centrosome to the ovum, thus restoring its capacity for cell division. The centrosome now divides and moves to either side the two pronuclei, and a segmentation spindle is formed, which bears all the chromosomes from both pronuclei. The first segmentation occurs, and divides the egg into two cells, in each of which there is the unreduced chromosomal number.

The process of segmentation begins almost immediately in each half of the divided egg, and cuts it also in two. The process is repeated until at last by continued cleavages the whole yolk is changed into a mulberry-like mass, still enclosed by the zona pellucida, figure 502 . Fertilization probably takes place in the Fallopian tubes, and segmentation of the fertilized ovum occurs on its passage to the uterus.

The passage of the ovum from the ovary to the uterus occupies probably eight or ten days in the human.

The peripheral cells, which are formed first, arrange themselves at the surface of the yolk into a membrane, the ectoderm. The deeper cells of the interior pass gradually toward the surface, thus increasing the thickness of the membrane already formed by a second, or entoderm, layer of cells, while the central part of the yolk, the blastoderm cavity, remains filled only with a clear fluid. By this means the yolk is shortly converted into a kind of secondary vesicle, the walls of which are composed externally of the original vitelline membrane, and within by the two newly formed cellular layers, the blastoderm or germinal membrane, as they are

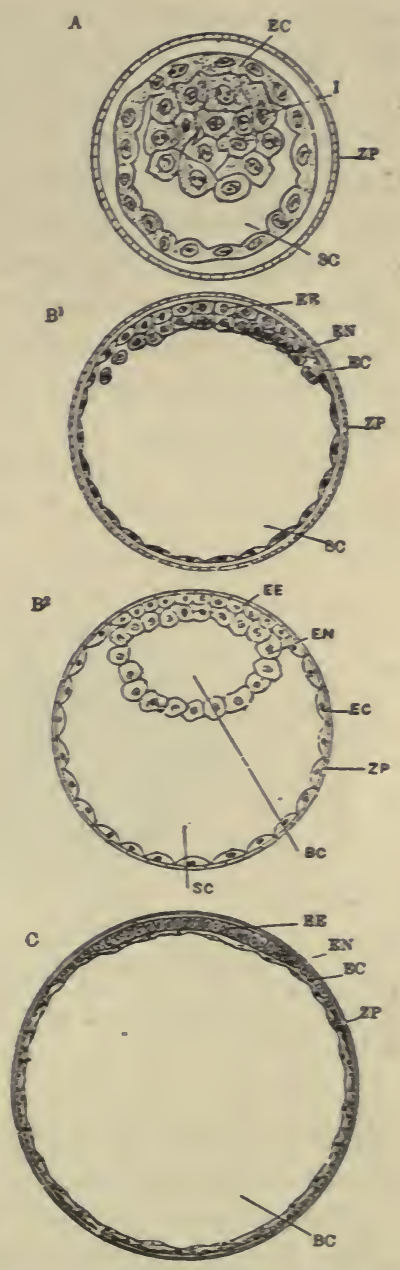

FIG. 502.-Conversion of the Morula to the Blastula. Formation of Blastodermic Vesicle and Membrane. A, Appearance of segmentation cavity and attachment of inner cell mass to ectoderm at upper pole of ovum; $B^{1}$, extension and flattening of inner cell mass as it occurs in rabbits and some other mammals; $B^{2}$, extension of entoderm as it occurs in insectivora, monkeys, apes, and man; $C$, completion of bilaminar blastodermic vesicle; $B C$, blastodermic cavity; $E C$, ectoderm; $E E$, embryonic ectoderm; $E N$, entoderm; $I$, inner cell mass; $S C$, segmentation cavity; $Z P$, zona pellucida. (Cunningham.) 
called. A third cellular layer, the mesoderm, is soon developed between the other two. The fetus results from the harmonious growth of these three layers, each of which is the source of certain tissues and organs.

Important changes occur in the structure of the mucous membrane of the uterus. The epithelium and subepithelial connective tissue, together with the tubular glands, increase rapidly, and there is a greatly increased vascularity of the whole mucous membrane, while a substance composed chiefly of nucleated cells fills up the interfollicular spaces in which the blood vessels are contained. The effect of these changes is an increased thickness, softness, and vascularity of the mucous membrane, the superficial part of which itself forms the membrana decidua.

The object of this increased development is the production of nutritive materials for the ovum; for the cavity of the uterus shortly becomes filled

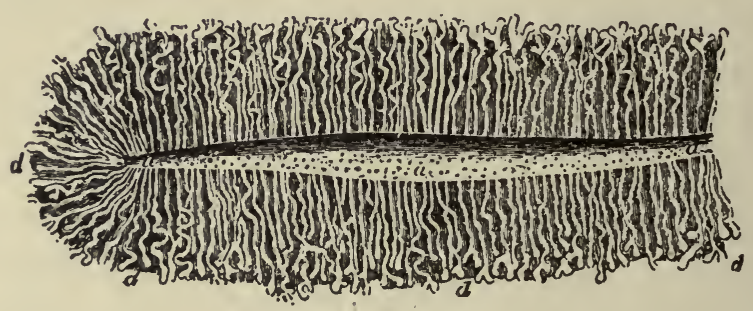

FIG. 503.- Section of the Lining Membrane of a Human Uterus at the Period of Commencing Pregnancy, Showing the Arrangement and Other Peculiarities of the Glands, $d, d, d$, with Their Orifices, $a, a, a$, on the Internal Surface of the Organ. Twice the natural size.

with secreted fluid, consisting almost entirely of nucleated cells in which the chorion villi are embedded.

When the ovum first enters the uterus it becomes embedded in the structure of the decidua, which is yet quite soft, and in which soon afterward three portions are distinguishable. These have been named the decidua vera, the decidua basalis, and the decidua capsularis.

In connection with these villous processes of the chorion there are developed depressions or crypts in the decidua vera, which correspond in shape to the villi they are to lodge; and thus the chorionic villi become more or less embedded in the maternal structures. These uterine crypts, it is important to note, are not, as was once supposed, merely the open mouths of the uterine follicles.

The Placenta.-During these changes the deeper part of the mucous membrane of the uterus, at and near the region where the placenta is placed, becomes hollowed out by sinuses, or cavernous spaces, which communicate on the one hand with arteries and on the other with veins of the uterus. Into 
these sinuses the villi of the chorion protrude, pushing the thin walls of the sinuses before them, and so come into intimate relation with the blood contained in them. There is no direct communication between the blood vessels of the mother and those of the fetus; but the layer or layers of membrane intervening between the blood of the one and of the other offer no obstacle to a free interchange of matters between them by diffusion and osmosis. Thus the villi of the chorion, containing fetal blood, are bathed or soaked in maternal blood contained in the uterine sinuses.

The placenta, therefore, of the human subject is composed of a fetal part and a maternal part-the term placenta properly including all that entanglement of fetal villi and maternal sinuses, by means of which the

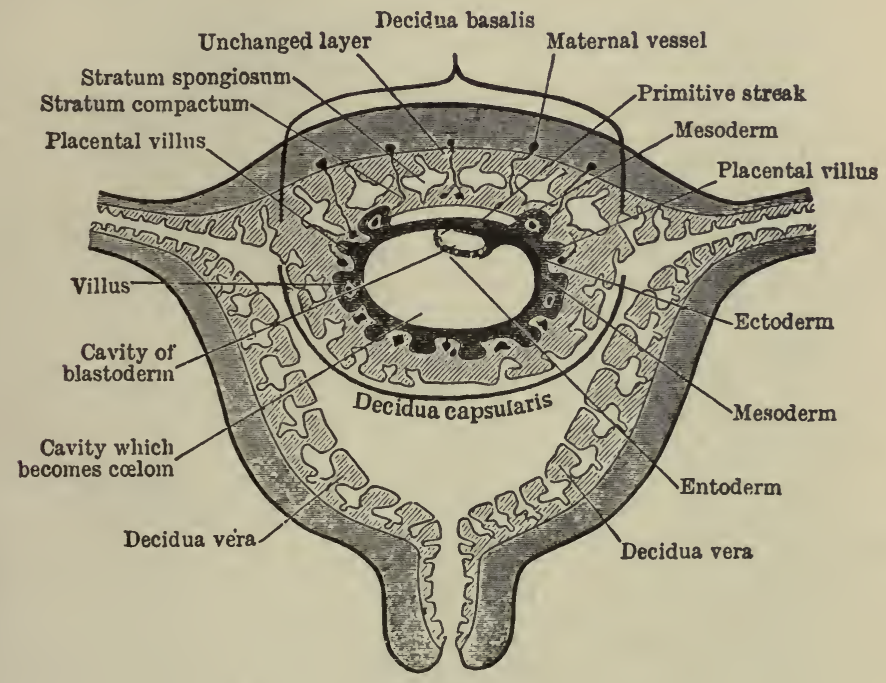

Fig 504.-Diagram of the Early Stage of Human Embryo in Relation to the Uterus (Cunningham.)

blood of the fetus is enriched and purified after the fashion necessary for the proper growth and development of those parts which it is designed to nourish.

The whole of this structure is not, as might be imagined, thrown off immediately after birth. The greater part, indeed, comes away at that time; as the after-birth; and the separation of this portion takes place by a rending or crushing through of that part at which its cohesion is least strong, namely, where it is most burrowed and undermined by the cavernous spaces before referred to. In this way it is cast off with the fetal membrane. The remaining portion is either gradually absorbed, or thrown off in the uterine dis- 
charges which occur at this period. A new mucous membrane is of course gradually developed.

Circulation of Blood in the Fetus.-The circulation of blood in the fetus differs considerably from that of the adult.

Returning from the placenta by the umbilical vein the blood is first conveyed to the under surface of the liver, where the stream is divided - a part of the blood passing straight on to the inferior vena cava through a venous canal called the ductus venosus, while the remainder passes into the portal vein and reaches the inferior vena cava only after circulating through the liver. It is carried by the vena cava to the right auricle of the heart, into which cavity

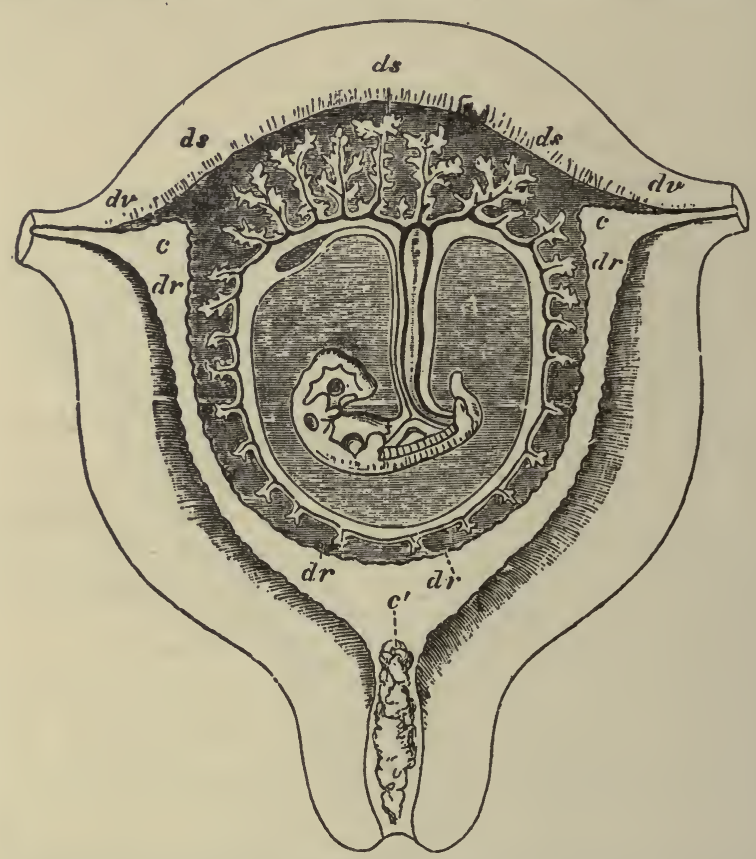

FIG. 505.-Diagrammatic View of a Vertical Transverse Section of the Uterus at the Seventh or Eighth Week of Pregnancy. $c, c, c^{\prime}$, Cavity of uterus, which becomes the cavity of the decidua, opening at $c, c$, the cornua, into the Fallopian tubes, and at $c^{\prime}$ into the cavity of the cervix, which is closed by a plug of mucus; $d v$, decidua vera; $d r$, decidua reflexa, with the sparser villi embedded in its substance; $d s$, decidua serotina, involving the more developed chorionic villi of the commencing placenta. The fetus is seen lying in the amniotic sac. The umbilical cord and its vessels pass up from the umbilicus to the distribution of the blood vessels in the villi of the chorion- and the pedicle of the yolk-sac the cavity between the amnion and chorion. (Allen Thomson.)

the blood is also pouring that has circulated in the head and neck and arms, and has been brought to the auricle by the superior vena cava. It might be naturally expected that the two streams of blood would be mingled in the right auricle, but such is the case only to a slight extent. The blood from the 
superior vena cava - the less pure fluid of the two-passes almost exclusively into the right ventricle, through the auriculo-ventricular opening, just as it does in the adult. The blood of the inferior vena cava is directed by a fold of the lining membrane of the heart, called the Eustachian valve, through the foramen ovale into the left auricle and into the left ventricle, and out of this into the aorta, and thence to all the body, but chiefly to the head and neck. The blood of the right ventricle is sent out in small amount through the pulmonary artery to the lungs, and thence to the left auricle, as in the adult,

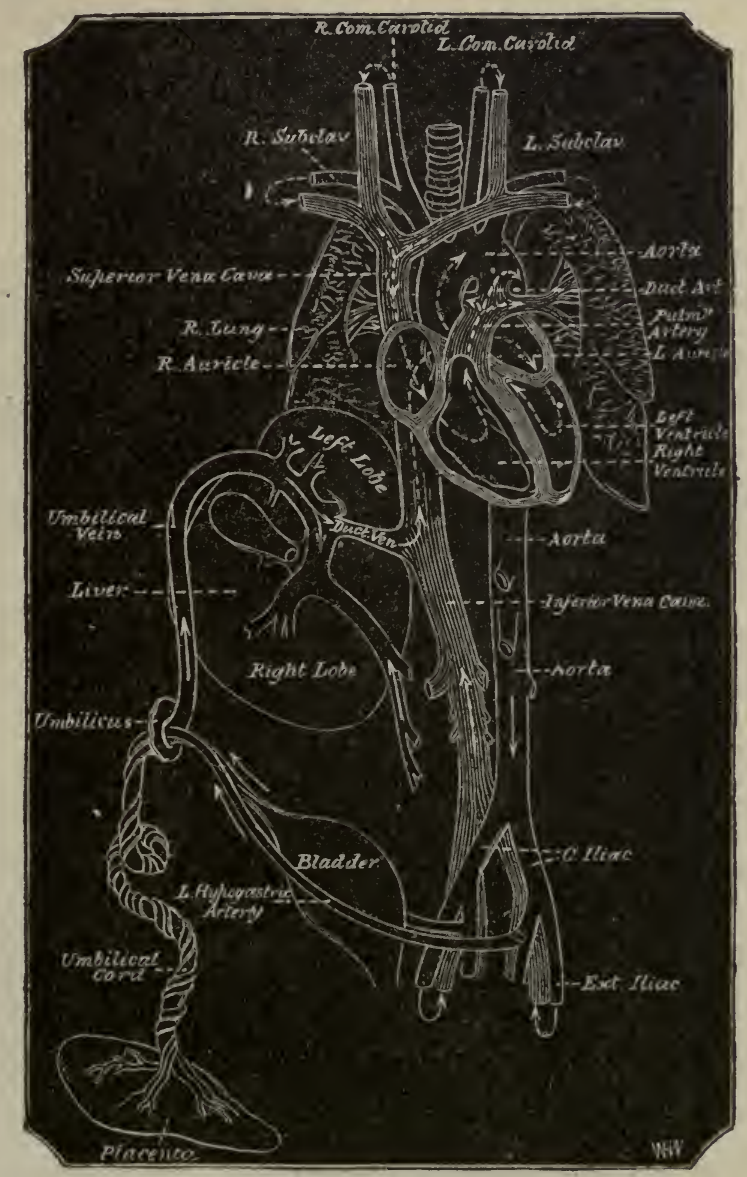

FIG. 506.-Diagram of the Fetal Circulation.

but the greater part by far passes through a canal, the ductus arteriosus, leading from the pulmonary artery into the aorta just below the origin of the three great vessels which supply the upper parts of the body, and is distributed 
to the trunk and lower parts of the body. A large portion passes out by way of the two umbilical arteries to the placenta. From the placenta it is returned by the umbilical vein to the under surface of the liver, from which the description started.

After birth the foramen ovale, the ductus arteriosus, and ductus venosus all close, and the umbilical vessels are tied off, so that the two streams of blood which arrive at the right auricle by the superior and inferior vena cava, respectively, thenceforth mingle in this cavity of the heart, and pass into the right ventricle, by way of the pulmonary artery to the lungs, and through

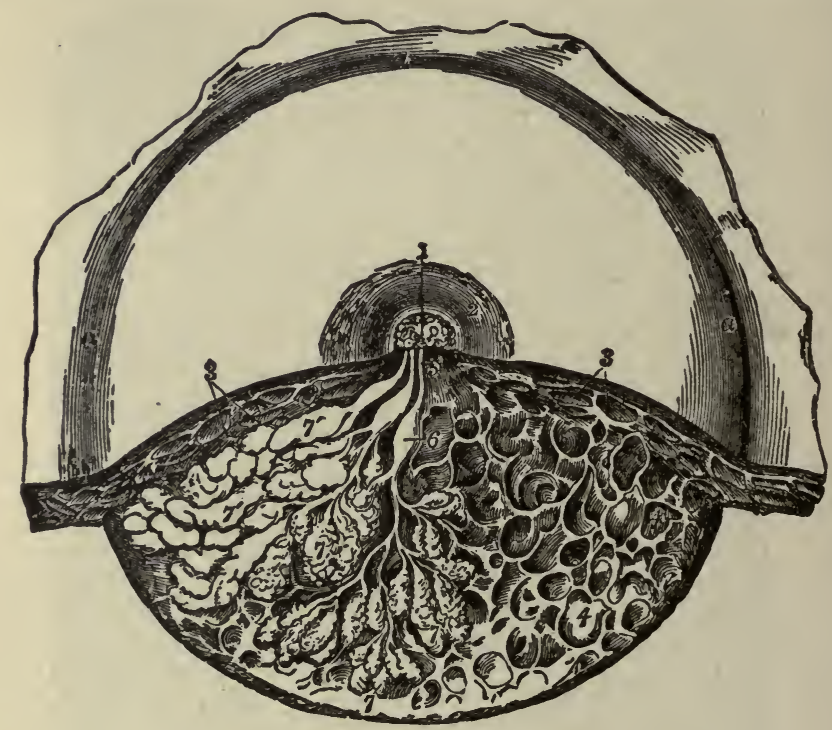

Fig. 507.-Dissection of the Lower Half of the Female Mamma During the Period of Lactation. 2. - In the left-hand side of the dissected part the glandular lobes are exposed and partially unravelled, and on the right-hand side the glandular substance has been removed to show the reticular loculi of the connective tissue in which the glandular lobules are placed. I, Upper part of the mammilla or nipple; 2, areola; 3, subcutaneous masses of fat; 4, reticular loculi of the connective tissue which support the glandular substance and contain the fatty masses; 5 , one of three lactiferous ducts shown passing toward the mammilla, where they open; 6 , one of the sinus lactei or reservoirs; 7 , some of the glandular lobules which have been unravelled; $7^{\prime}$, others massed together. (Luschka.)

these, after aeration, to the left auricle and ventricle, to be distributed over the body.

Parturition. - With the implantation of the embryo and the development of the placenta, the uterus grows rapidly until the end of pregnancy. The muscles of its walls increase enormously in volume, apparently by an increase in the size of the fibers, and the whole structure may become thirty or forty times its size in the resting period. Many changes take place also in other parts of the body, changes which are dependent on the 
presence of the fetus and a change in the number or quantity of hormones developed during gestation. Full-term pregnancy occurs when the uterus is isolated from the nervous system, hence it has been inferred that there is some sort of special secretion or hormone possibly of the embryo itself, that makes its way into the blood and influences the organs of the mother.

At the end of the period of pregnancy the strong uterine walls begin periodic contractions which ultimately result in the delivery of the fetus. These contractions are at first weak and at long intervals, but gradually. become very strong and even violent and follow each other in rapid succession. The uterine contractions are supported by reflex contractions of the abdominal and thoracic muscles. After the fetus is delivered the uterine contractions become milder, but still continue until the placenta is finally expelled.

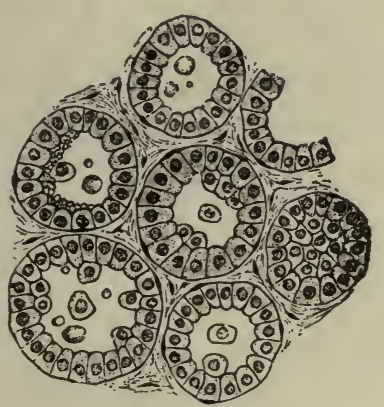

FIG. 508 .

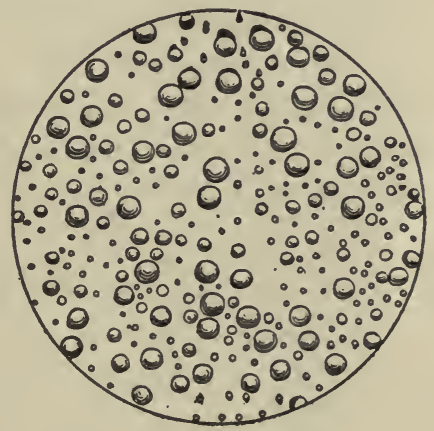

FIG. 509 .

FIG. 508.-Section of Mammary Gland of Bitch, Showing Acini, Lined with Epithelias Cells of a Polyhedral or Short Columnar Form. $\times 200 . \quad$ (V. D. Harris.)

Fig. 509.-Globules and Molecules of Cow's Milk. $\quad \times 400$.

The initiation of the contractions of the uterus at delivery probably depends on the chemical stimulation of some substance or substances produced in the uterus itself or in the fetus; substances that react on the nervous mechanism and on the uterine muscles themselves. This view cannot be said to be proven, but it is supported by certain observed facts and experiments.

Lactation.-There is a marked development of the mammary glands, during the period of gestation, especially in the later weeks. No milk is secreted during this time and the change is sharply limited to the processes of growth in the glands. Upon delivery of the fetus the gland immediately enlarges very sharply and an abundant secretion is formed. During gestation it is thought that an inhibitive hormone prevents actual secretion. After parturition these hormones are removed and secretion begins under the stimulative influence of definite hormones from the corpus luteum, the involuting uterus, and the mammary gland tissue, see Chapter on ductless glands. 
The secretion of the first few days is called the colostrum. It contains a larger per cent. of solids, has the large granular colostral corpuscles, is more alkaline than ordinary milk, and has a specific gravity of 1040 to 1046.

The mammary glands have been isolated from the nervous system to determine whether or not the association in time between their changes and the changes in the uterus were of a nervous nature. The isolated mammæ develop and begin lactation at parturition as in the normal. It would seem that there is some special form of stimulation through the medium of the blood, i.e., by the hormones as suggested in the preceding paragraph. Yet one must not draw the conclusion that the nervous system exerts no influence on the mammary gland. Stimulation of the nerves to the gland produces vascular changes that increase or decrease the quantity of milk secreted. Many observations have been noted in women, which show that the secretion of milk is sharply influenced, or even completely suppressed by nervous states.

The Composition of Milk.-Milk has a specific gravity of 1028 to 1034. Its fat is held in emulsion. Under the microscope, it is found that the milk globules vary in size, the majority being from 2 to $3 \mu$ in diameter. The old view that they have an investing membrane of albuminous material is now generally discarded.

Composition of Colostrum (Pfeiffer).

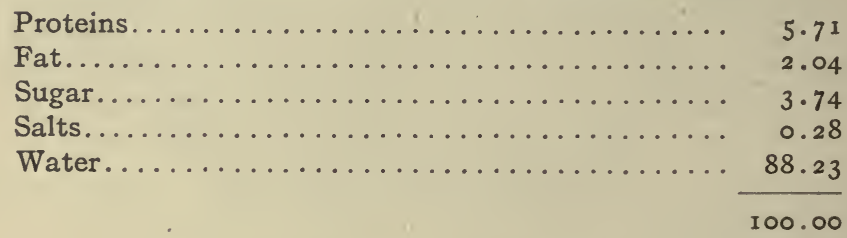

Salts in Woman's Milk (Rotch).

Calcium phosphate.................. 23.87

Calcium silicate.................... I.27

Calcium sulphate................... 2.25

Calcium carbonate..................... 2.85

Magnesium carbonate................ 3.77

Potassium carbonate................. 23.47

Potassium sulphate................... 8.33

Potassium chloride................... 12.05

Sodium chloride.................... 21.77

Iron oxide and alumina................. 0.37

100.00

In addition to the oil or butter fat, milk contains certain proteins, milksugar, and several salts. Its percentage composition is given in the tables appended. 
Chemical Composition of Milk. (After Foster, Harrington, et al.) Human. Cow. Mare. Bitch.

\begin{tabular}{|c|c|c|c|c|}
\hline Water & $87 \cdot 3 \circ$ & 87 & 90 & 76 \\
\hline Solids. . & 12.70 & I 3 & Io & 24 \\
\hline Fats. & 4.00 & 4.0 & 2.0 & 10.0 \\
\hline Proteins. & I. 50 & 4.0 & 2.5 & 10.0 \\
\hline Sugar.. & 7.00 & $4 \cdot 3$ & 5.0 & $3 \cdot 5$ \\
\hline Salts.... & 0.20 & 0.7 & 0.5 & 0.5 \\
\hline
\end{tabular}




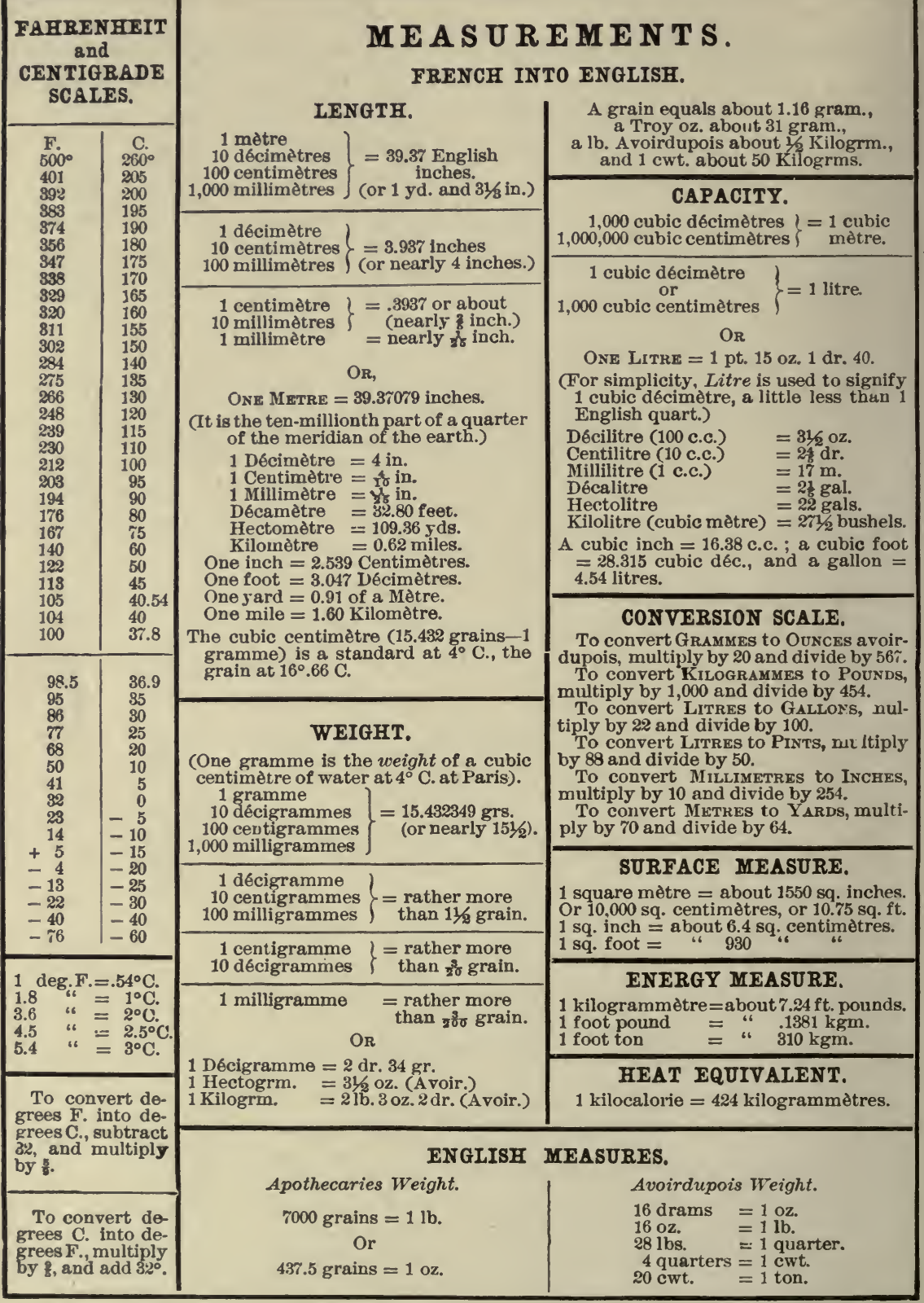

Measure of 1 décimetre, or 10 centimetres, or 100 millimetres.

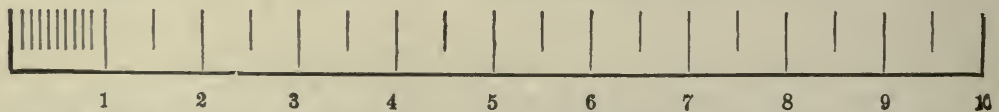

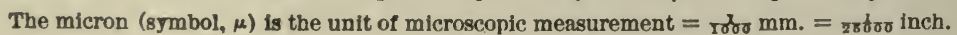




\section{INDEX}

Abdominal viscera, vascular nerves for, 257

Abducens nerve, 66I

Aberration, chromatic, 760 spherical, 760

Absorption, 409 coefficient of, 304 conditions for, 409 methods of, 409 of carbohydrates, 408

of fats, $4 \mathrm{I} 6$ of minerals, $4 \mathrm{I} 7$ of protein, $4 \mathrm{I} 4$ of water, 4I 7 places for, 409 rapidity of, 408 through the intestines, 4I 2, 4I4 the lungs, $4 \mathrm{r} 8$ the mouth, 4ro the skin, $4 \mathrm{I} 8$ the stomach, 4 ro

Accelerator centers for heart, 210,620 Accessory olives, 6 I 5 thyroids, 484

Accommodation of vision, 728,730

Achromatic layer, 18 spindle, 20

Achromatin, 18

Achroodextrin, 356

Acromegaly, 498

Activating ferments, 347

Adamkiewicz reaction, 107

Adenase, 94

Adenine, 93

Adenoid tissue, 36

Adipose tissue, 37,38

Adrenalin, 490

Adrenals, 488

Afferent fibers in the sympathetics, 672 After-birth, 788 -images, 741, 748, 765 -sensations, 686

Agglutinative substances, ${ }_{5}$ I

Air, 284 analysis, 297
Air, changes in, during respiration, 297, 298

complemental, 294, 328

composition of, 297

diffusion of, 301

pressure of, 3 or , 325,328

quantity breathed, 294, 328

reserve, 295, 328

residual, 295

supplemental, 295

tidal, 294, 328

volume breathed, 328

Alanine, 8I

Albumin, egg, I05

properties of, I I

serum, 88

in urine, 435,453

Albuminate, acid, I 12

Albuminoids, 87,89

tests for, I IO

Albumins, 86, 88

reactions of, I ro

Albumoses, 96

Alcohol as a food, 345

Alexin, I 50

Alkali metaprotein, I I 2

Amboceptor, I 50

Ameba, 3

Ameboid leucocytes, I 33 movement, $3,4, \mathrm{I} 34$

Amino-acids, $8 \mathrm{r}$

Amitosis, I9

Ammonia, effect of breathing, 3 I 8 in expired air, $30 \mathrm{I}$ in the urine, 433

Ammonium carbamate, 43I, 462

Amylolytic ferments, 347

Amylopsin, 347, 382

Amylose, IOI

Anabolism, 7, 454

Anabolites, 454

Anacrotic limb, 239

wave, 239

Anaphase, 21

Anaphylaxis, 152

Anelectrotonus, 536 
Animal heat, 501 starch, IO2

Animals differentiated from plants, 12 Anode, 537

Ano-spinal center, 597

Anterior association center, $65^{\circ}$

Antiperistalsis, 395

Antiscorbutic substance, 48I, 482

Antisubstances in blood, I52

Antithrombin, I 24

Antitoxins in blood, 151

Apnea, 316, 333

Arborization, interepithelial, 74

Archoplasm, I 9

Areolar tissue, 35

Arginine, 82

Aristotle's experiment, $75^{6}$

Arrhythmia, sinus, 213

Arterial blood, analysis of, I34

flow, 229

rhythmic, 230

velocity of, $23 \mathrm{I}$

gases of, 301

pulse, 272

blood-pressure and nervous regulation in, 274

tone, 244

Arteries, I 73

blood-pressure in, 21 8, 269, 274

coats, $\mathrm{I} 73$

nerves of, $\mathrm{I} 74$

tone of, 244

Arterioles, I 73

Articulate sounds, 555

Aspartic acid, 82

Asphyxia, 3I6, 333

Assimilation, 6, 7

Association centers of cerebral cortex, 648

Aster, 20

Astigmatism, 736, 762

Atmosphere, composition of, 297

Atmospheric pressure, 301

Attraction sphere, 19

Auditory center, 647

judgments, $7 \mathrm{I} 2$

nerve, 659

Auricles, action of, 179

Autolytic substances, 150

Autonomic system, 663, 669

Axis cylinder, 67

Axone, 583

Bacteria in digestive tract, $39 \mathrm{I}$
Basal metabolism, 476

Basement membrane, 23, 336

Basket cells, 6 I9

Basophile, I 33

Beri-beri, 480

von Bezold's ganglia, 201

Bidder's ganglia, 201

Bile, 386, 408

acids, 386,408

action of, on peptic digestion, 404

chemical composition of, 386

coloring matter of, 386

functions of, 387

mode of discharge of, 388

of secretion of, 388

pigments of, 387

salts, 386

secretion of, 388

Bilirubin, 387

Biliverdin, 387

Binaural sensations, 7 I 2

Binocular vision, 75I

Bioplasm, 2

Biuret reaction, 108

Bladder, urinary, 427

Blastema, 2

Blastoderm, Io

Blind spot, 73I, 763

Blood, II 7

agglutinative substances in, 150

analysis of, man, I 35

anaphylaxis, 152

antisubstances in, 152

antitoxins in, I5I

arterial flow, 229

buffy coat, 120

capillary flow, 23I

carbon dioxide of, 307

chemical composition of, 134

circulation of, 166,208

experiments on, 260

in fetus, 790

coagulation of, II 8, I6I

calcium in, 122

conditions affecting, I24, I6 2

fibrin in, I 19

rapidity of, II9, I $24, I 62$

theories of, I 2 I

composition of, table, I 34, I 35

tissue extracts on, ${ }^{6} 63$

corpuscles of, II 7, I26, I3 I, I64

chemical composition of, 138 
Blood, corpuscles of, colorless, I 3 I

ameboid movement of, 133,222

chemical composition of, 138

number of, 126

phagocytosis, $\mathrm{r}_{5} 8$

varieties of, I3I

enumeration of, $15^{8}$

percentage of, 160

red, $\mathrm{x} 26$

characters of, $\mathrm{I} 26$

chemical composition of, I39

corpuscles of, red, action of reagents on, $\mathrm{I} 57$

development of, I 29

number of, I 26

origin of, 129

rouleaux formation, $\mathrm{I} 28$

defibrinated, $\mathrm{I} 20$

differences between arterial and venous, 148

elimination of carbon dioxide by, 307

examination of, ${ }_{57}$

ferments in, 137

flow, arterial, 229

capillary, 23 I

regulation of, 242

velocity of, 235

in arteries, $23 \mathrm{I}$

in capillaries, 234

in veins, 235

venous, 234

gas apparatus, 303

gases of, 301

hemoglobin, 139

hurnan types, 150

isotonicity of, I6I

laboratory experiments on, $\mathrm{I}_{57}$

microscopical examination of, $\mathrm{I}_{57}$

morphology of, I 26

opsonins in, I $5 \mathrm{I}$

oxygen of, 307

phagocytes, I35

plasma, II 7

chemistry of, I36, r6 3

composition of, 136

percentage of, 160

physical factors of, $\mathrm{I}_{52}$

reaction of, I6I

platelets, I 35

portal, 148

precipitins in, $\mathrm{I}_{5} \mathrm{I}$
Blood, pressure, 218

apparatus, 226

arterial, 218, 269, 27I, 272

capillary, 227, 273

in $\operatorname{man}, 226,227$

model, $27 \mathrm{I}$

respiratory undulations of, 222

variations in, 228

venous, 227

production of heat by the, $5 \circ 3$

quantity of, II 7

influence on secretion, 339

respiratory changes in, 289

serum, II $8, x_{3} 8, I_{52}$

chemistry of, 163

composition of, $\mathrm{I} 35$

globulicidal properties of, 149

specific gravity of, I6I

thrombocytes, I 35

transfusion, $\mathrm{I} 49$

uses of, II 7

variations in composition of, $\mathrm{I} 48$

velocity of flow, 235

venous flow, 234

whipped, 162

Blushing, 247

Body, chemical composition of, 79

energy requirements of, 476

experiments on the chemistry of, 105

Bone, 42

blood-vessels of, 43

canaliculi of, 44

cells, 45

compact, 50

development of, 46

growth of, 5 I

Haversian canals of, 44

lacunæ of, 44,45

lamellæ of, 44,45

marrow, 42

microscopic structure of, 44

ossification in carti'age, 47

in membrane, 46

periosteum of, 43

structure of, 44

Bowman's sarcous elements, 6 r theory of urinary secretion, 436

Brain, 603, 605, 6r 3

arrangement of different parts, $6 \circ 3$

association centers of, 648

distinctive characters of human, $605,64 \mathrm{I}$ 
Brain, fore-, 605,625

hind-, 605

mid-, 606, 623

motor areas of, 641

centers, $64 \mathrm{I}$

tracts in, $62 \mathrm{I}$

Rolandic area of, 644

sensory areas of, 644

vascular nerve-supply of, 253

weight of, 632

Bread, composition of, 344

Bronchi, 280

Buffy coat, I 20

Bulb, the, 6II; and see Medulla.

Bundle of His, 203

Burdach, tract of, 587

Caffeine, 93

Cajal, cells of, 629

Calcification, 47, 5I

Calcium salts in the body, 103

in coagulation of the blood, I 24 tests for, II 5

Calorimeter, 477

Canaliculi, 44

Cane sugar, roo

Capillaries, 174

blood-pressure in, 227, 273

structure of, I 75

Capillary circulation, 272

flow, 23I

velocity of, 234

Carbohydrates, 98, I 33

absorption of, by intestines, 417

as foods, 344

chemical reactions of, I I 3

energy value of, 467

metabolism of, 467

relation of epinephrin to, 49 I

Carbon, amount excreted, 456

dioxide, determination of, 329

elimination of, 309,320

monoxide, effect of breathing, 318 hemoglobin, 142, I6 5

in proteins, 106

Carbonates in the body, 104

Cardiac force of action, 195

conducting system, 203

conduction stimulus, 206 rate of, 207

contractions, automatic, 265

experiments on, 260
Cardiac force of action, contractions, maximal, 199

cycle, I79, I93

fibrillation, 209

glands, of stomach, 363

impulse, 185

muscle, 62, 195, 529, 568

action of, 529

automatic contractions of, 265

development of, 65

properties of, I 95

refractory phase, 52

nerves, 210,266

orifice of stomach, 374

Cardiac nodes, 203

Cardio-accelerator centers, 213, 6r 5

Cardiogram, 187, 261

Cardiograph, 185

Cardio-inhibitory centers, 213,614

Cartilage, 39

development of, $4 \mathrm{I}$

elastic, 4 I

embryonic spongy bone, 48

hyaline, 39

temporary, 4I

vascularization of, 48

white fibro-, 4I

Catacrotic limb, 239

wave, 240

Catelectrotonus, 536

Cathode, 535,538

Cauda equina, 580

Caudate nucleus, 627

Cell, I, 8, I7, I8

body, I 7

connection, modes of, 23

difference between plant and animal, I 2

division of, Io, Ig

functions of, II, 14

growth, 7, io

mutiplication, 19

nucleus of, 18

structure of, I 7, I 8

Cells, decay and death of, 23

modes of connection, 23

origin of, 23

shapes of, 22

types of, 22

Cellulose, I $3, \mathrm{I}_{0} 3$

Center for muscle tone, 596

Centers, motor, 639,641 
Centers, sensory, 644 spinal, 596

Centrosome, I9

Cerebellar cortex, paths through, 6 I9

Cerebellum, $6 \mathrm{r} 7$ connection with bulb, 6I I, 6I 5

functions of, 620 general structure of, 6 I 8

Cerebral cortex, association centers of, 648

localization of motor function of, 639 of sensory function of, 644

nerve cells in, 629

structure of, 627

Cerebrum, 627

connection with bulb, 6 I I

effects of removal of, 635,637

functions of, 635 motor areas of cortex, 639,641

peduncles of, 623

sensory areas of, 644

weight of, 632

Cerumen, 444

Ceruminous glands, 444

Chemical composition of the body, 79 elements in the body, 79

structure of proteins, 80

Chemistry of the body, experiments on, 105

Chest, changes in diameter of, during respiration, 325,327

Cheyne-Stokes breathing, 3I 7

Chlorides in the body, 103

in the urine, $435,45^{\circ}$

tests for, II 5

Chlorine, effect of breathing, 318

Chlorophyll, $\mathrm{I}_{3}$

Cholesterol, 98, II 4

Choline, 98

Chorda tympani, $35 \circ, 399$

Chordæ tendineæ, I 73

Chromatic aberration, $735,76 \mathrm{r}$

Chromatin, 18

Chromophanes, 745

Chromophiles, 72, 73

Chromoplasm, i 8

Chromoproteins, 92

Chromosomes, 20, 785

and Mendelian inheritance, 785

Chyme, 374

Cilia, 3 I

Ciliary apparatus, $7 \mathrm{I} 7$

contraction, 532, 570
Circulation, coronary, 215

atmospheric pressure on, 318

during sleep, 673

effect of respiration on, 323

fetal, 790

in brain, 253

in erectile structures, 258

laboratory experiments on, 260

of blood, $\mathrm{x} 66,2 \mathrm{I} 8$

regulation of blood flow, 242

through blood-vessels, 2 I8

time of, 270

vegetable, 5

velocity of, 234

Coagulated proteins, 96

Coagulating ferments, 347

Coagulation of blood, I 18, r6 1

calcium salts in, 122

conditions affecting, I 24, I 62

rapidity of, II9, I $24, I 62$

theories of, I2I

Cochlea, 704

Cohnheim's areas, 62

Cold, influence of extreme, 505

Collagen, 90, iro

Collaterals, 69

Colloidal solution, 85

Colloids, $85, \mathrm{I}_{52}$

Color, after-images, 747,765

-blindness, $748,76_{3}$

complemental, 748

extent of visual field for, 747

Hering's theory of, $75^{\circ}$

limits of field of vision for, 747,765

-mixing, 766

sensations of, 748

vision, theories of, 749

Young's and Helmholtz's theory, 749

Colorless corpuscles, I3 I

Colostrum, 794

Comma tract, $5^{87}$

Common sensations, 679

Compact bone formation, 50

Complement, I 50

Complemental air, 294, 328 colors, 747

Conducting system, cardiac, 203

Conductivity of muscle, $5 \mathrm{r} 3$

Conjugated proteins, 87,92

Conjunctiva, 7 I 6

Connective tissues, 33

adenoid, 36 
Connective tissues, adipose, 37

areolar, 35

cells of, 33

fibrous, 37

gelatinous, 36

general structure of, 33

intercellular substance of, 34

lymphoid, 36

retiform, 36

varieties of, 34

white fibrous, 34,37

yellow elastic, 35,37

Consonants, 555

Contractility of muscle, 5 I 2

Contraction phase of muscle, 5 I4

Contracture, 524

Convoluted tubule, 423

Cooking, effects of, 345

Cornea, 7 I 6

Corneoscopy, 766

Corona radiata, 624

Coronary circulation, 2I 5

Corpora cavernosa, $25^{8}$

geniculata, 626

quadrigemina, 625

striata, 627

Corpus Arantii, 173

dentatum, 6I 7

luteum, 500, 779

internal secretion of, 500

spongiosum, $25^{8}$

Corpuscles, blood, I I 7, I 26, I3 I , I64

of Golgi, 77

of Krause, 76

of Meissner, 75

of Pacini, 75, 379

Corti, organ of, 704

Coughing, center for, 614

Cranial nerves, 650

Crassamentum, i 8

Creatinin, 433, 459, 463

Cretinism, 485

Crura cerebri, 623

Crusta, 623

petrosa, 53

phlogistica, I 20

Crystalloids, 152

Crystals, protein, I05

Cutis vera, 44I

anserina, 58

Cystin, 8r

in urine, 435
Cytolysis, 149

Cytoplasm, 9, I 7

Cytosine, 93

Death, 7

Decay, 7

Decidua basalis and capsularis, 788 menstrualis, 779 vera, 788

Decussation of the pyramids, 607

Defecation, 396 center for, 597

Degeneration in spinal cord, 586 reaction of, 539

Wallerian, 575,586

Deglutition, 358

center for, 6r 4

nervous mechanism of, 360

time occupied in, 359

Demarcation currents, 516

Dendrites, 65, 577

Dental papilla, 56

Dentine, 53

Depressor nerve, 248

Development, 78I

Dextrin, IoI

tests for, II 3

Dextrose, 99

tests for, Ir 3

in urine, 435

Diabetes mellitus, 470

Dialysis, 152

Diapedesis, 234

Diaphragm in respiration, 288

Diastole of heart, I79, I8I

Dicrotic notch, 239 pulse, 242

Diet, normal, requisites of, 454,474 tables, 475

Diffusion, 152

Digestion, 34I, 346

enzymes in, 346

experiments in, 399

in intestines, 376, 390, 406

in mouth, 347,399

in stomach, 360,370

Digestive enzymes, 347

Diphasic current, $5 \mathrm{I} 7$

Diplopia, 653, 75I

Disaccharides, 99

Disassimilation, 7

Distance, estimation of, 755 
Diuretics, action of, 439

Dogiel's cells, 202

Dreams, 673

Ductless glands, influence on metabolism, 482

Ductus arteriosus, 790

deferens, 769

venosus, 790

Dyspnea, 315, 333

Ear, cochlea of, 704

external, 708

function of, 708

internal, 703

function of, 710

membranous labyrinth, 704

middle, 708

function of, 708

organ of Corti, 705

ossicles of, 701

semicircular canals, 704

tympanum, 701

Edestin, 105, I10

Egg albumin, ro5

Eggs, composition of, 343

Eighth cranial nerve, 659

Elasticity of muscle, 5 II

Elastin, 90, II I

Electrocardiogram, normal, 205 of extraventricular systole, 209 of fibrilation, 208

Electrotonus, 536, 566

Elements, chemical, in body, 79

Eleventh cranial nerve, $66_{2}$

Embryonic spongy bone cartilage, 48

Emulsification, I I 4,382

Enamel, 54

cap, 57

germ, 56

organ, 56

papilla, 56

Encephalon, 605

End-brushes, 70

-bulbs, 76

-plates, 63

Endocardiac pressure, 187, I90

Endocardium, I68

Endomysium, 60

Endoneurium, 69

Endothelium, 26

Energy, income and output of, 474

requirements for body, 476
Enteric system, 67 I

Enterokinase, 347, 381, 390, 409

Enzymes, 347

activating, 347

amylolytic, 347

coagulating, 347

digestive, 347

lipolytic, 347

of pancreatic juice, 383,407

proteolytic, 347

Eosinophile, Ir6, I33

Epiblast, II

Epidermis, 44I

Epiglottis, 280

Epinephrin, 490

Epineurium, 69

Epithelial tissues, 24

Epithelium, 24

ciliated, 30,32

classification of, 24

columnar, 25, 27, 29

cubical, 24

functions of, 25, 32

glandular, 30

simple, 24,25

situations of, 24,3 I

specialized, 30

squamous, 24,28

stratified, 24, 27

transitional, 29

Equilibrium, sense of, 68

Erection center, 598

Erepsin, 347, 390

Ergograph, 525

Erythroblasts, I 30

Erythrocytes, I 26

Erythrodextrin, 356

Eustachian tube, 701, 709 valve, 169,791

Excreta, analysis of, 456 channels of elimination of, 456

Excretion, 42I during starvation, 473

from skin, 444

laboratory experiments in, 448

Expiration, 290, 297

forced, 297

muscles of, force of, 288

relative time of, 293

Expired air, carbon dioxide of, 298, 330 oxygen of, 298

External genitals, vascular nerves for, ${ }_{25} 8$ 
Eye, 7 I 5

anatomy of, $7 \mathrm{r} 6$

astigmatism, 73I, 736, 762

chromatic aberration of, $76 \mathrm{I}$

image formation, 725

movements of, 733

muscles concerned in, 73.3

optical apparatus, 724

axis, 727

refractive media and surfaces, 724

schematic, 727

spherical aberration of, $76 \mathrm{I}$

Eyeball, 7I 6

blood-vessels of, 723

ciliary apparatus, $7 \mathrm{I} 7$

cornea, 716

iris and lens, 7I 7

retina, 7 I 9

Eyelids, 716

Facial nerve, 657

function of, 657

paralysis of, 657

relation to taste, $65^{8}$

secretory fibers, 657

Falsetto voice, 554

Far-point, 760

Fasciculus antero-lateralis, 587

cerebello-spinalis, 587

cuneatus and gracilis, 587,606

of Rolando, 606

solitarius, 659

Fasting, 472

Fatigue, effect on muscular contraction, $56 \mathrm{I}, 564$

of nerve fiber, 534

Fats, 96, II4

absorption of, by intestines, 518

as food, 34I, 344

destination of, 467

digestion of, 382,387

emulsification of, $114,382,387$

energy value of, 464

metabolism of, $46_{3}$

mobilization of, in body, 466

saponification of, I I 4,382

source of, in body, $46_{5}$

tests for, II 4

Fatty acids, tests for, II 4

Feces, 393

composition of, 393

excretion by, 455
Fermentation, 39I

Ferments in the blood, I 37 unorganized, 346, and see Enzymes.

Fetus, circulation of blood in, 790

Fibers of Remak, 67

Fibrillation, auricular, 209

Fibrin, I 20 ferment, I 2 I

Fibrinogen, I 21 , I 37

Fictitious feeding, $36_{5}$

Fifth cranial nerve, 653

Fillet $6 r 6$

Filtration, 4I I

Filum terminale, 55

Finger, vasomotor changes in, 276

Fish, composition of, 342

Flutter, auricular, 209

Food, and digestion, 34I

effects of deprivation of, 472

mastication of, 348

salts of, 344

time of passage through alimentary canal, 397

Foods, 34I

carbohydrates, 344

classification of, 34I

effect of cooking, 345

fats, 344

fuel value of, $34^{2}$

heat production from, 342,479

income and output of energy, 455

inorganic, 344

liquid, 345

mineral, 344

nitrogenous, $34 \mathrm{I}$

percentage composition of, 342

salts, 344

water, 345

Forced movements, 623

Fore-brain, 625

Form, estimation of, $653,783,754$

Fossa ovalis, 169

Fourth cranial nerve, 653

Fovea centralis, 7 99

Frontal association center, 649

Fructose, roo

Galactose, 100

Gall-bladder, 386 emptying of, 388

Gallstone, $3^{89} 9$

Ganglia, 578 
Ganglia, Bezold's, 20I

Bidder's, 20I

Remak's, 20r

spinal, functions of, 595

Ganglion cells, 73

Gases in alimentary canal, 394

in blood, determination of, 334

Gastric digestion, 360, 402

changes in food in, 373

circumstances influencing, 372

cleavage products of, 404

products of, $37 \mathrm{I}$

time of, 373

hormone, 366

juice, 368,402

acid of, 368,370

action on milk, 372

on proteins, $370,37 \mathrm{I}, 404$

artificial, 404

chemical composition of, 368,403

digestive action of, 402

enzyme action of, 372, 404

fictitious meals, action on, 365

hydrochloric acid in, 370

pepsin in, 372

psychic secretion of, 403

quantity of, 368

reaction of, 368

secretion of, 365,402

quantity, 368

lipase, action of, 372

secretion, changes in glands during, 365

Gelatin, II I

nervous mechanism of, 366

effect of diet of, 460

Gelatinous tissue, 36

Genito-spinal center, 598

Germinal membrane, 787

matter, 2

spot, 775

vesicle, 773

Gestation, relation of to internal secretions, 500

Giant cells, 42

Glands, cardiac, 363

gastric, 364

mammary, 793

pyloric, 364

reproductive, relation to metabolism, 498

salivary, 348
Glands, sebaceous, 444

secreting, 336

sudoriferous, 443

types of, 336

Gliadin, 83

Globin, I 45

Globulins, 86,89 , r г

Glomerulus, 422

Glosso-pharyngeal nerve, $6_{59}$

in respiration, $3 \mathrm{I}_{2}$

Glotțis, 544

respiratory movements of, 291

Glucose, 99

Glutaminic acid, 82

Glutelins, 86

Glutenin, 83

Glycemia, 470

Glycocoll, 8I, 386

Glycogen, 468

destination of, 470

formation of, 468

from carbohydrate, 462

from protein, 459

relation to metabolism, 469

sources of, 468,469

tests for, II 3

Glycogenesis, 468 hormone control, 468

Glycoproteins, 87, 95, I I I

Glycosuria, 469, 470

Goblet cells, 27

Golgi, corpuscles of, 77

Goll, tract of, $5^{8} 7$

Gowers' tract, 587

Graafian follicles, 774

Grape sugar, 99

Guanase, 94

Guanilic acid, 93

Guanine, 94

Haversian canals, 44

Head, vascular nerve supply of, 253

Hearing, 699

acuteness of, $75^{8}$

limits of, $708,75^{8}$

Heart, 167

accelerator nerve of, $2 I_{3}, 6 I_{5}$

action of, I79, 195

anatomy of, 167

automaticity of, 20.4, 263, 265

rate of, 217,26 I

sequence, $26 \mathrm{I}$ 
Heart, automaticity of, theories of, 200 -block, 202 capacity of, 170 chambers of, 168 character of contraction, 195,199 compensatory pause, 200 conducting bundle, 203 coronary circulation of, 2I 5 cycle of, I79, 193 depressor nerve of, 248 development of, I 7 I endocardiac pressure, 187 excised, experiments on, 263,264 force of action, r95

frequency of action, 179,217 ganglia of, 201

impulse of, 185

influence of accelerator nerve on, 210, 615

of coronary circulation on, 215

of drugs on, $2 \mathrm{I} 7$

of inhibitory nerves on, 210, 6I4

of mechanical tension on, 2I 6

of nervous system on, 210,266

of nutrient fluids on, $26_{3}$

of sympathetic system on, 210

of temperature on, 216

of vagus on, 2 IO

inhibitory nerve of, 210,6 I 4

irritability of, 199

isolated, 200, 263, 264

metabolism of, 204

muscle, 62

properties of, 195

production of heat by, 503

relation of rhythm to nutrition, 204

rhythmic contraction of, 196, 204

size of, $\mathrm{I} 7 \mathrm{O}$

sounds of, 183

causes of, 184

structure of, 17 I

tonicity of, 198

valves of, $\mathrm{I} 72$

action of, I8I

volume of, 264

weight of, 170

work, per diem, 479

Heat, animal, $50 \mathrm{r}$

dissipation of, 503

from lungs, 506

from skin, 504

influence of extreme, 505
Heat, of nervous system on production of, $5 \circ 7$

produced in muscular contraction, $50 \mathrm{I}$ -producing organs, 503

production of body-, 503, 506

regulation of body-, 503

centers for, 507

-rigor, $5^{1} 3,5^{26}$

variations in, 506,507

in loss of, $5 \circ 3$

in production of, 506

Heidenhain's experiments on urine secretion, 436

Height weight chart, 479

formula for area, 479

Heller's ring test, I08

Hemacy tometer, 159

Hematin, 146

Hematoblasts, $\mathbf{2} 28$

Hematoidin, I 46

Hematoporphyrin, I46

Hemianopsia, 646

Hemin, 146

Hemiopia, 646

Hemochromogen, 145

Hemoglobin, 87, 95, I39, 160, 307

action on gases, 142

combining power with oxygen, 305 , 306

crystals, I40, I6.4

preparation of, 105

derivatives of, $\mathrm{I} 45, \mathrm{I} 64$

estimation of, $142, \mathrm{I} 60$

reduced, $\mathrm{I}_{42}$

Hemoglobinometer, 142 , 160

Dare's, 144

Fleischl's, I42

Talquist's, I44

Hemolysis, I49

Henle's membrane, I 74

loop, 423

Hepatolytic sera, 150

Heredity, 784

Hind-brain, 605

Hippuric acid, 433

formation of, $433-463$

His, bundle of, 202

Histidine, 82

Histones, 87, 9 r

Hopkins-Cole reaction, IO7

Hormones, 483

gastric, $366,36 i$ 
Hormones, pancreatic, 49I thyroid, 486

Hyaline cartilage, 39 cells, I33 leucocytes, 133

Hyaloplasm, 9, $\mathrm{x} 7$

Hydrochloric acid, 370, 37 I combined, $37 \mathrm{I}$ digestive action of, $37 \mathrm{I}$ test for free, $37 \mathrm{I}$

Hydrogen, amount excreted, 455 in proteins, 106

Hydrolytic cleavage, 83

Hypermetropia, 738

Hyperpituitarism, 496, 497, 498

Hyperpnea, 3 I 6, 3I 7

Hyperthyroidism, 485

Hypertonic solutions, I 54

Hypoblast, I I

Hypoglossal nerve, $66_{3}$

Hypopituitarism, 496

Hypothyroidism, 485

Hypotonic solutions, I 54

Hypoxanthine, 94

Immune body, I49

Impregnation, changes in ovum following, 786

Indican in urine, test for, $45^{2}$

Indol, 408

Induction coil, 557

Infundibulum, 284

Inheritance, sex-linked, 786

Inhibition of reflex actions, 598

Inorganic foods, 344 substances, I03

Insalivation, 348

Inspiration, 287,288

forced, 288

muscles of, 288

quiet, 288

relative time of, 293

Intercellular substance, 23, 34

Interepithelial arborizations, 74

Internal, capsule, 624

ear, 703

secretions, 483

Intestinal digestion, 376,390 rôle of bile in, 387

gases, 394

juices, 406, 408

secretion, 390
Intestines, absorption in, $4 \mathrm{I} 6$

action of microorganisms in, 39I

digestion in, 376

feces in, 393

fermentation in, 39 I

gases in, 394

large, summary of digestive changes in, 391

movements of, 394

influence of nervous system on, 396

putrefaction in, 392

small, summary of digestive changes in, 390

vascular nerves for, 257

Intonation, $55^{2}$

Inulin, 102

Invertase, 347

Inverted image on retina, 760

Involuntary muscle, 529, 569

Iodine, I04

Iris, $7 \mathrm{I} 7$

contraction of, 733

Iron, I04

tests for, II 5

Irritability, 5

of heart-muscle, 199

of muscle, $5^{12}$, $5^{1} 9,559$

Islands of Langerhans, 378, 379, 492

Isotonic solutions, 154

Isotonicity of blood, I6I

Ivory, 53

Judgment of form and size of bodies, 689

of form and solidity, 754

of size and distance, 755

Jumping, 543

Karyokinesis, I9

Karyolymph, 18

Karyoplasm, i 8

Karyosomes, 18

Katabolism, 7, 454

Katabolites, 454

Keratin, 89, г 10

Kidneys, $42 \mathrm{I}$

action of diuretics on, 439

blood supply of, 424

effect of blood pressure on, 448

factors affecting excretion from, 436

function of, 421,422 
Kidneys, internal secretion of, 493

Malpighian bodies of, 425,426

nerves of, 426

plethysmogram of, 277

structure of, $42 \mathrm{I}$

tubuli uriniferi of, 422

vasa efferentia of, 426

recta of, 426

vascular nerves of, 257

volume of urine, 428

Krause, corpuscles of, 76

membrane of, $6 \mathrm{I}$

Kronecker-Meltzer theory of deglutition, $35^{8}$

Kymograph, 220

Labyrinth, 703

Lachrymal apparatus, 716

Lactase, 347

Lactation, 793

relation of internal secretion to, 500

Lacteals, 413

Lactose, ror

Lacunæ, 44, 45

Langerhans, islands of, $378,379,492$

Large intestine, summary of digestive changes in, 391

Laryngoscope, 549

Larynx, 279, 544

Latent period of muscle, $5^{1} 4,5^{64}$

Law, DuBois, 566

Pflüger's, 536, 566

Leaping, 543

Lecithins, 95, 97, II4

Lecithoproteins, 87,95

Legumes, composition of, 344

Lens of eye, 7I 7

Lenticular nucleus, 627

Leucin, 82

Leucocytes, II6, I3I

number, $\mathrm{I} 3 \mathrm{I}$

phagocytic action, 133

varieties, $\mathrm{I} 32$

Leucolytic sera, 150

Leucosin, 83

Levers, action of, in the body, 540

Levulose, 100

Lichinin, 102

Liebermann's reaction, 107

Life, phenomena of, I

Limbs, vascular nerves for, $25^{8}$

Linin, 18
Lipase, $347,382,383$

Lipogenesis, 466, 493

Lipoids, 98, II 4

Lipolytic ferments, 347

Liquid foods, 345

Liquor sanguinis, 117

Liver, 385

glycogenic function of, 460

internal secretion of, 493

secretions of, 384,388

structure of, 385

urea formation in, 46 I

vascular nerves for, 257

Localization, cerebral, 639, 644

Locomotion, 539

Locus ceruleus, 6 I 6

Ludwig's theory of urine secretion, 436

Lungs, 282

absorption from, 420

blood supply of, 286

excretion by, 456

interchange of gases in, 309

loss of heat from, 506

lymphatics of, 286

nerves of, 287

structure of, 288

Luxus consumption, $45^{8}$

Lymph, I 55

chemical composition of, I 55

flow, ${ }_{5} 6$

formation of, 155

Lymphagogues, ${ }_{5} 6$

Lymphatic sheaths, perivascular, I 79

spaces, in blood-vessels, I 79

Lymphocyte, II6, I33

Lymphocytes, variety of, I 32

Lymphoid tissue, 36

Lysine, 82

Lysis, 149

Lytic substances, I 49

Magnesium salts in the body, I03, II 5

Malpighian bodies, 426

Maltase, $347,355,379,382$

Maltose, 100, 356

Malt sugar, 100,356

Mammary glands, 792, 793, 794

Manometer, 192, 219

Marrow, bone, 42

Mast cells, I32

Mastication, 348

nervous mechanism of, 349 
Mastoid cells, 70I

Maximal stimulus, 5 I9

Measures and weights, table of, 796

Meat, composition of, 342

Medulla oblongata, $605,606,6$ 1 0

as a conducting path, 6 ro

connection of, with cerebrum and cerebellum, 6i I

functions of, $6 \mathrm{I}_{2}$

reflex centers of, $6 \mathrm{r}_{2}$

tracts through, 6 I $_{2}$

Medullary sheath, 66

Medullated fibers, 66

Meissner's corpuscles, 75

Melanins, 96

Membrana decidua, 788

propria, 23

tympani, 701

Membranous labyrinth, 704

Mendelian inheritance and chromosomes, 785

Menstrual discharge, 778

Menstruation, 777

Mesoblast, I I

Mesothelium, 26

Metabolism, 7, 454

basal rate, 478

calories per square meter per hour, 478

constructive, 7

destructive, 7

during fasting, 472

endogenous, 459

exogenous, 459

height, weight chart, 478

influence of ductless glands on, 482 of reproductive glands on, $49^{8}$

intermediate, 459

nutrition and diet, 454

of carbohydrates, 467

of fats, 463

of proteins, 458

Metaphase, 20

Metaplasm, I 7

Metaproteins, 87, 95, I I 2

Methemoglobin, ${ }^{4} 42$

Microcytes, I 28

Micro-organisms in intestines, 39I

Microsomes, 9

Micturition, 440

center for, 597

Mid-brain, 606, 623
Milk, composition of, 343,794 sugar, IOI

Millon's reaction, I07

Mineral foods, 34I, 344, 47 I

absorption of, in intestines, 419

Minimal stimulus, $5^{19}$

Mitosis, 19

Molisch reaction, 108

Monaster, 20

Monosaccharides, 99

Mossy fibers, 6 I 9

Motor areas of cortex, $639,64 \mathrm{I}$

of human brain, $64 \mathrm{I}$

end-plates, 63

impulses, 602

-oculi nerve, $65 \mathrm{I}$

tracts in human brain, 643

Mouth, absorption in, 4I 2 digestion in, 347

in speech, 554

Movement, ameboid, 3

gliding, 5

streaming, 4

Movements, circus, 623

forced, 623

of stomach, 374

Mucigen, 353

Mucinogen, 349

Mucins, 95, 353

Mucoids, 95, III

Mucous membranes, 336

Mucus in urine, 434

Murexide test, 432

Muscle, blood supply of, 63

cardiac, 62, 195, 529

chemical changes of, $5 \mathrm{I} 6$

composition of, 5 I I

coagulation of, 5 ro

conditions affecting irritability of, 519

conductivity of, $5^{\mathrm{I}} 3$

contractility of, $5 \mathrm{I} 2$

contraction of, 5 I $2,53 \mathrm{I}, 560$

phase of, $5^{1} 3$

contracture, 524

currents, demonstration of, 516

development of, 59, 64, 65

effect of blood supply on, 522

of drugs on, 523

of nerve supply, 523

of temperature on, 521

of use on, 520 
Muscle, elasticity of, 5 I I electrical phenomena of, 5 I 6

end-plates, 63

experiments on, 557

ferments, 5 io

in rigor mortis, 526

involuntary, $58,530,569$

compared with skeletal and cardiac, $53 \mathrm{r}$

irritability of, 5 I $2,519,559^{2}$

-nerve physiology, 5 10

preparation, $55^{8}$

nerve supply of, 63

non-striated, 58

plain, 58

plasma, 5 10

properties of, 5I I

record of contraction of, $52 \mathrm{I}$

serum, 5 ro

skeletal, 60

smooth, 53r

spindles, 78

stimuli, 5 I9

striated, 60

tetanus, 525,565

-tone, center of, 596

voluntary, 60

Muscular action as heat producer, 503

center for tone of, 596

contraction, 524

action currents, $5 \mathrm{I} 6$

apparatus for producing and recording, 514

changes in shape during, $5^{15}$

characteristics of single, 513

chemical changes during, 516

conditions affecting character of, 579

co-ordinated, 524

differences between voluntary and involuntary, 530

effect of blood supply on, 522

of drugs on, 523

of fatigue on, $564,56 \mathrm{r}$

of load on, $5^{62}$

of nerve supply on, 523

of rate of stimulation on, 523

of repeated activity on, 520

of strength of stimulus on, 519 , 560

of temperature on, 521,562 , $5^{6} 5$
Muscular action, effect of use on, 520

electrical changes during, ${ }_{51}^{16}$

energy liberated during, $5^{1} 8$

heat produced during, $5^{\mathrm{I}} 7$

latent period of, $5 \mathrm{I} 4,564$

metabolism during, 528

refractory phase of, 529

response to stimuli in voluntary and involuntary, 530

simple, 564

summation of contractions, 524

tetanic, $523,5^{6} 5$

voluntary, 523

contracture, 524

co-ordination, $5^{24}$

energy, $5_{1} 8$

sense, $68 \mathrm{r}$

tissue, $5^{8}$

Musculi papillares, I 73

pectinati, 169

Mydriasis, 663

Myelin sheath, 66

Myelocyte, I06, I I6, I34

Myeloplaxes, 42

Myogenic theory of heart beat, 202

Myogram, 5I3

Myograph, pendulum, $5^{6} 3$

Myohematin, 5 II

Myopia, 737

Myosin, 5II

ferment, $5^{\text {I I }}$

Myosinogen, 5 I I

Myxedema, 485

Near-point, 73I, 760

Nephrolytic sera, 150

Nerve cells, 65, 572

arrangement in spinal cord, $5^{82}$

body, 72

characteristic of individual, 575

functions of, 575

in cerebral cortex, 527

neurone theory, 573

nutritive influence of, 575

transmission of impulses through, 577

centers, 578

collaterals, 69

end-brushes, 70

fibers, 65,66

effect of battery current on, 535 
Nerve fibers, fatigue of, 575

functions of, 534

medullated, 66

non-medullated, 67

impulses, 533

cellulifugal, 577

cellulipetal, 577

character of, 533

rate of, 570

specific energy of, 576

transmission through neurone, 577

velocity of, 534

stimuli, 5I 2, 533

terminations, 74

Nerves, cardiac, 214, 266

cranial functions of, $65^{\circ}$

depressor, 248

effect of currents on human, 535, 537

experiments on, 557

irritability of, $55^{8}$

vasoconstrictor, 246,254

vasodilator, $249,25 \mathrm{I}, 252$

vasomotor, $243,246,249$

Nervous system, 572

axones of, 65,577

dendrites of, 65,577

functions of, 572

ganglia of, 578

influence on secretion, 339,446

laboratory experiments on, 675

neuroglia of, 65,78

spinal hemisection, 677

sympathetic, $66_{3}$

Neurilemma, 66

Neurofibrils, 72

Neurogenic theory of heart beat, $20 \mathrm{I}$

Neuroglia, 65,78

Neurone, 65,572

characteristics of, 575

irritability, 675

polarity in, 675

theory, 573

transmission of nerve impulses through, 577

types of, 578

Neutrophiles, I 16 , I32

Ninth cranial nerve, 659

Nitrogen in proteins, 106

Nitrogenous equilibrium, 457

food, 34I

output, 459

substances in body, 79
Nitrous oxide, effect of breathing, 318

Nodes, cardiac, 204 of Ranvier, 66

Nostrils, respiratory movements of, 29 I

Nuclei of optic thalamus, 625

Nucleic acid, 92

Nucleoalbumins, 95

Nucleoli, 18

Nucleoproteins, 87,92

Nucleus, 9, 18 ambiguus, 657

ruber, 624

Nutritional diseases, 480

Obesity, 467

Ocular fixation, 75I

Odontoblasts, 57

Oils, as food, 344

Olfactory apparatus, 694

bulb, 696

center, 646

glomeruli, 646

nerve and tract, 646

Olivary bodies, $608,6 \mathrm{IO}$

Olive, accessory, 610 superior, 616

Onkograph, 438

Onkometer, 437

Ophthalmoscope, 742, 766

Opsonic index, $\mathrm{I}_{5} \mathrm{I}$

Opsonins, 15 I

Optic center, 645

nerve, 7 I 9

thalami, 625

Optical apparatus, 724

defects in, 734

Organ of Corti, 705

Osmosis, ${ }^{1} 5^{2}$

Osmotic pressure, $85, \mathrm{I} 53$

Osseous labyrinth, 703

Ossicles of ear, 70I

Ossification, 46 center of, 47 in membrane, 46

Osteoblasts, 47

Osteoclasts, 49

Osteogenetic fibers, 47

Output of energy, 479

Ovaries, 772

relation to metabolism, 499

Oviducts, 775

Ovulation, 777 
Ovum, 783

changes following impregnation, 786

prior to impregnation, 783

Oxalic acid in urine, 435

Oxygen, amount excreted, 456

determination of, in air, 329

in blood, 302, 334

in expired air, 298

in tissues, 308

of the blood, 302

Van Slyke apparatus, 301

Oxyhemoglobin, I39, I64, 306

Pacinian corpuscles, 75, 379

Pain, sense of, 68I

Pancreas, 377

enzymes of, 38I

extirpation of, 492

internal secretion of, $49 \mathrm{I}$

islands of Langerhans in, 379,492

secretion of, 379

structure of, 377

Pancreatic digestion, 372, 406

cleavage products of, 407

fistula, 379

glycemia, 470

juice, 379,406

artificial, 407

chemical characters of, 406 composition of, 379

enzymes of, 381,407

action of, 38I, 407

conditions influencing action of, 383

secretion of, 406

action of nerves on, 380

influence of secretin on, 406

Papillæ of skin, $44^{2}$

of tongue, $69 \mathrm{I}$

Paralytic secretion of saliva, 35 I

Paramyosinogen, 5 I I

Parathyroidectomy, 487

Parathyroid glands, 487

Parietal association center, 650

Parotid gland, 348

nerves of, $35^{2}$

Parturition, 792

center, 598

Peduncles of cerebrum, 623

Pellagra, 48I

Pelvic viscera, vascular nerves for, 257

Penis, 77 I
Pepsin, 347,370

action of, 370

Pepsinogen, 370

Peptides, 88, 96

Peptone plasma, I 26

Peptones, 88, 96, I I 2, 370

Perforating fibers of Sharpey, 46

Pericardium, 167

Perichondrium, 39, 47

Perimysium, 60

Perineurium, 69

Periosteum, 43

Peristalsis, intestinal, 395

of stomach, 375

reversed, 395

Perivascular lymphatic sheaths, 179

Perspiration, 445

Pes, 623

Pflüger's law of contractions, 536

Phagocytes, 158

Phagocytosis, 158

Phakoscope of Helmholtz, 762

Phenomena of life, I

Phenomenon of treppe, $56 \mathrm{I}$

Phenylalanine, 8I

Phosphates, I03, II 5

Phosphoproteins, 87,95

Phosphoric acid in urine, 434,45 I

Phosphorus in proteins, I06

Phrenic nerve, influence on respiration, 332

Pigments, bile, 408

in urine, 433,452

Pituitary body, 494

action of grafts, extracts, etc., 497

effect of removal of, 496

function of, 495

Placenta, 788

Plants differentiated from animals, I 2

Plasma, I I 7, I36

chemistry of, I 63

composition of, ${ }_{13} 6$

Plasmosomes, I8

Plethysmogram, 277

Plethysmograph, 245, 276

Pleuræ, 282

Pneumogastric nerve, 660; and see Vagus.

Pneumograph, 292

Polysaccharides, 99

Pons Varolii, 605, 6 5

Postdicrotic wave, 239

Posterior, longitudinal bundle, 6 I 6 
Posterior, pyramids, 606 roots of spinal nerves, 489,594

Postganglionic fibers, 666

Potassium salts in the body, ro3, 104

Poultry, composition of, 343

Precipitins, I5I

Predicrotic wave, 239

Preganglionic nerve fibers, 666

Presbyopia, 739

Pressor nerves, 249

Pressure, endocardiac, 187

Pronucleus, female, 784 male, 787

Prostate gland, $77 \mathrm{I}$

Protamines, 87,9 I

Proteins, 80 absorption of, from intestines, 416 action of trypsin on, $38 \mathrm{I}$

as fat formers, 459

as foods, $34 \mathrm{r}$

as glycogen formers, 459

circulating, 458

classification of, 86,88

coagulation, rog

color reactions of, 107

crystals, I05

decomposition products, $38 \mathrm{I}$

derived, 87,95 , I 2

digestion of, $369,38 \mathrm{I}$

experiments on, 105, 109

hydrolytic cleavage of, $8_{3}, 84$

metabolism of, $45^{8}$

nitrogen and phosphorus in, Io6

precipitation, reactions of, I08

preparation of, ro5

properties of, 85

reactions of, 107

salting out experiments on, I09

simple, 86

sulphur in, 106

tissue, $45^{8}$

wheat, 83

Proteolytic ferments, 347

Proteoses, 88, 96, I I 2, 370

Prothrombin, I 24

Protoplasm, I, 2

chemistry of, 3

differentiation of, 8 , I0

growth of, 7

irritability of, 5

metabolism in, 7

movement of, 3
Protoplasm, physiological characteristics of, 3

reproduction of, 8

Ptosis, $65^{2}$

Ptyalin, action of, $347,355,402$

Pulse, 236

arterial, 270,272

dicrotic, 240

variations in rate of, $2 \mathrm{I} 2$

-wave, rate of propagation of, 272

Pulvinar, 626

Pupil, 718

contraction of, 733

dilatation of, 733

center for, 6 I 4

Purines, 94

Purkinje's cells, 61 8, 620

fibers, I 7 I, 203

figures, 740

shadows, 764

Purkinje-Sanson's images, 762

Putamen, 627

Putrefaction in intestines, 399

Pyloric glands, 364

orifice, 374

Pyramids, 606

decussation of, 607

Pyramidines, 93

Racemose glands, 337

Ranvier, nodes of, 66

Reaction of degeneration, 539

Red corpuscles, I 26

action of reagents on, 157

chemical composition of, $\mathbf{I} 39$

development of, I 29

origin of, 129

Red nucleus, 624

Reflex action, 590

time of, 594

arc, 590

centers in medulla, $6 \mathrm{r} 2$

Reflexes, complex, 592

cutaneous, 599

inhibition of, 598

muscle, 599

simple, 592

special centers for, 596

spinal, 595

Refraction, 759

Refractory period, 199

phase, 529 
Relaxation phase of muscle, $5^{1} 4$

Remak's fibers, 67

$$
\text { ganglia, } 196
$$

Rennin, 347, 373

action of, 373,406

Reproductive glands, relation of to metabolism, 498

organs, 768

of female, 772

of male, 768

Reserve air, 295, 328

Residual air, 295

Respiration, 278

changes in diameter of chest, during, 327

effect of altitude on, 318

effect on circulation, 323

of atmospheric pressure on, $3 \mathrm{I} 8$

of various gases on, 318

of vitiated air on, 3I 7

expiration, 290

influence of cutaneous nerves on, 331

of general sensory nerves on, 313 , 331

of glosso-pharyngeal nerves on, 3r 3

of phrenic nerves on, 332

of superior laryngeal nerves on, 312

of vagus nerves on, $3 \mathrm{II}$, 33 I

inspiration, 287

internal, 279

laboratory experiments in, 327

mechanism of, 287

nervous apparatus of, $310,33 \mathrm{I}$

rate, 327

rhythm of, 29I

special types of, 315

tissue, 279

volume of air breathed, 299, 328

Respiratory apparatus, 279

elimination of carbon dioxide by, 309

nervous regulation of, 3 10

capacity, 295, 328

circumstances affecting, 296

center, 310, 6I 4

automatic action of, 313

stimulation of, $3 \mathbf{r} 3$

changes in air breathed, 297

in the blood, $30 \mathrm{r}$
Respiratory changes in the tissues, 308

interchange, 330

movements, 287

character of, 326

establishment of, at birth, 315

nervous mechanism of, $33 \mathrm{I}$

of nostrils and glottis, 29I

rate and character of, $33 \mathrm{I}$

recording of, $29 \mathrm{I}$

relative time of, 293

murmur, 294

muscles, force of, 297

pressure, 301,328

quotient, 300

rate, 296,327

rhythm, 293

action of stimuli on, 3 I I

terms for quantity of air breathed, 294

Resuscitation from drowning, $32 \mathrm{I}$

from electric shock, 32 I

Rete mucosum, 442

Reticular formation in medulla, 608

Reticulum, 9, I 7

Retina, 719

cones of, $72 \mathrm{I}$

inverted image on, 760

layers of, 720

localization in, 744

movements of pigment cells, 746

rods of, $.72 \mathrm{I}$

Retinal image, duration of, 765

relation of size to distance, 764

Retinoscopy, 766

Rheoscopic frog, 534

Rhodopsin, 745

Rhythmical contractility of heart, 196

Rhythmicity of arterial flow, 230

Ribs, movement of, in respiration, 287

Rigor mortis, 526

heart, 180

heat, $5^{27}$

order of occurrence, 527

Rima glottidis, 279

Rolandic area, 642

Running, 543

Saccharose, 100

Sacculus, 704, 7I 5

Sacral autonomics, $67 \mathrm{I}$

Saliva, 354

action of, on starch, $355,356,40 \mathrm{I}$ 
Saliva, chemical composition of, 354

function of, 355

properties of, 355

ptyalin in, 355

quantity of, 354

secretion of, center for, 349

nerve mechanism of, 349

rate of, 354

Salivary digestion in stomach, $35^{8}$

influence of acids and alkalies on, 401

of temperature on, $40 \mathrm{r}$

glands, 348

changes in during secretion, $35^{2}$, 400

nerves of, 399

structure of, 348

secretion, $35^{2}$

reflex, 399

Salting out proteins rog

Salts, absorption of, by intestines, 4 I 9

as foods, 344

bile, 386, 408

in the body, 103

tests for, II 5

Sanson's images, 730

Saponification, Ir 4,382

Sarcode, 2

Sarcolemma, 6I

Sarcoplasm, 62

Sarcostyles, 6I

Sarcous elements of Bowman, 6r

Schaefer's method of resuscitation, 322

Scheiner's experiment, 760

Schwann, sheath of, 66

Scurvy and vitamine, C, 48I

Sebaceous glands, 444

Secretin, 390

influence on pancreatic secretion, 390,406

Secreting glands, 335

production of heat by, $5 \circ 3$

types of, 336

Secretion, 390

circumstances influencing, 339

discharge of, 339

external, 335

internal, 335,482

organs and tissues of, 336

process of, 338

psychic, 403

Segmentation, 787
Semicircular canals, 703, 714

Semilunar valves, 183

action of, 183

Seminal fluid, $77 \mathrm{I}$ vesicles, 772

Sensations, binaural, 7 72

common, 679

objective, 68 o

of color, 746

special, 680

subjective, 680

Sense, hearing, 699, 706

muscular, 687

of equilibrium, $7 \mathrm{I} 3$

of pain, 686

of sight, 715

of smell, 694, 757

of taste, $659,689,757$

of temperature, 685,756

of touch, 68I

organs, directions for experiment

$$
\text { on, } 756
$$

perceptions, 68I

Senses, the, 679

internal, 679

special, 680

Sensorium, 680

Sensory areas of brain, 644

illusions, 680

impulses, 601

Serine, $8 \mathrm{I}$

Serous membranes, 336

Serum, II 8, I35

agglutinative substances, I 50

blood, I 8 , I 35

chemistry of, I 63

composition of, I 36

globulicidal action of, 149

hemolytic action of, I49

muscle, 5 Io

precipitins of, I 5 I

Seventh cranial nerve, 657

Sex, germ cells and determination of, 786

Sex-linked inheritance, 786

Sharpey's fibers, 46

Sight, 715

Silicon, I04

Sino-auricular node, 204

Sinus arrhythmia, 213

Sixth cranial nerve, 656

Size, estimation of, 689,755

Skin, absorption from, 420 
Skin, carbon dioxide exhaled by, 446 excretion by, 444,456 excretory function of, 44I, 444 functions of, 44I, 444

glands of, 424

loss of heat from, 504

structure of, $44 \mathrm{I}$

water excreted by, 445

Sleep, 673

Small intestine, absorption in, 4I4 digestion in, 390

Smell, center for, 646 sensation of, 757 sense of, 694

Sodium salts in the body, 103, 104

Solidity, judgment of, 754

Solutions, isotonic, 154

Somesthetic area of brain, 644

Somnambulism, 674

Sound, 707

Sounds, articulate, 555

localization of, 7 I I

of the heart, 183

pitch of, 707

Speech, 544, 554

action of tongue in, 555 of mouth in, 555

Spermatids, 782

Spermatocytes, $78 \mathrm{I}$

Spermatogonia, $78 \mathrm{I}$

Spermatozoa, $783,785,786$

Spherical aberration, 734, 761

Sphygmogram, 239

Sphygmograph, 238

Sphygmomanometer, 226, 239

Sphygmometer, 238

Spinal accessory nerve, 662

centers, $596,597,598$

cord, 580

anterior pyramidal tract, $5^{86}$

antero-lateral descending tract, 587

arrangement of nerve cells in, 582 ascending degeneration, tracts of, 587

comma tract of, 587

conduction in, 600

course of motor impulses in, 602

of sensory impulses in, $60 \mathrm{r}$

descending degeneration, tracts of, 586

direct cerebellar tract, 587
Spinal cord, fasciculi of, $5^{84}$

functions of, 590

general features of, 580

Gowers' tract, $5^{6} 2$

irradiation of impulses in, 593

lateral pyramidal tract, 586

peculiarities of different regions,

590

reflex action in, 590

tracts of, 584

weight of, 632

nerve-roots, functions of, 588

nerves, 588

anterior roots, 588

course of fibers, 588

posterior roots, 589

reflexes, 595

Spirem, 20

Spirometer, 295

Spleen, vascular nerves for, 257

Spongioplasm, 9, I 7

Staircase contractions, 520

Stammering, 556

Starch, IOI

action of amylopsin on, 382

of ptyalin on, 356

animal, I02

chemical reactions on, II 3

hydrolysis of, I I 3

Starvation, 472

death from, 474

effect on body temperature, 473

influence of, on excretions, 474

symptoms of, 473

Steapsin, 347,382

Stercobilin, 387

Stereoscope, 754

Stethograph, 292

Stimuli, forms of, $5^{\text {I } 2}$

maximal and minimal, 510,560

Stokes' fluid, I4 I

Stomach, 360

absorption from, 4I 2

action of pylorus, 374

blood-vessels of, 364

changes in glands during secretion, 365

digestion in, $358,360,372$

gases in, 394

glands of, $36_{3}, 36_{5}$

hunger contraction of, 375

lymphatics of, 364 
Stomach, movements of, 373

nerves of, 360

pancreatic digestion in, 372

peristalsis of, 374

secretion in, 365

structure of, $36 \mathrm{I}$

vascular nerves for, 257

Stomata, 26

Stratum granulosum, 442

l ucidum, 442

Malpighii, 442

Striated muscle, 60 development of, 64

Sublingual gland, 348

Submaxillary gland, 348

action of atropine on, $35 \mathrm{I}$

influence of nerves on, $35^{\circ}$

paralytic secretion of, $35 \mathrm{I}$

secretion of, 354

Substantia nigra, 623

Succus entericus, 390

Sucking, center for, 614

Sudoriferous glands, 443

Sugar, test for, in urine, 453

Sulphates in body, II 5

Sulphur in proteins, I06

Sulphureted hydrogen, effect of breathing, 318

Sulphuric acid in urine, $431,434,45^{\circ}$

Sulphurous acid, effect of breathing, 318

Summation, 524

of stimuli, 592

Superior laryngeal nerve in respiration, 309

Supplemental, air, 295

Suprarenal capsules, 488

functions of, 489

internal secretion, 490

nerves of, $49 \mathrm{I}$

Swallowing, $35^{2}$

Sweat, 445

centers, 615

chemical composition of, 445

glands, 443

influence of nervous system on secretion of, 446, 449

Sympathetic ganglia, functions and structure, $66_{3}, 666,668$

afferent fibers in, 672

functions, 670

Synapse, 59I
Synovial membranes, 663

Systole of heart, I 79

duration of, $\mathbf{1} 8 \mathbf{r}$

extra ventricular, 209

Tactile corpuscles, 76,683

of Meissner, 75

menisques, 77

Taste, 689

acuteness of, 69I

after-, 693

buds, 280,690

center, 693

contrasts, 693

influence of fifth nerve on, 655

nerves of, 689

seat of, 693

sensation of, 692,757

sense of, 689

varieties of, 692

Teeth, 5I

dentine of, 53

development of, 56

enamel of, 54

ivory of, 53

permanent, 52

structure of, 52

temporary, 5I

Tegmentum, $6 \mathrm{r} 6$

Telophase, $2 \mathrm{I}$

Temperature, body; $5 \circ 3$

dissipation of, 5 이

influence of extreme heat and cold on, 505

of starvation on, 472

regulation of, $5 \circ 3$

sense of, 685,756

variations in, 5 or

influence of, on muscular contraction, $5^{6}{ }_{5}$

Tenth cranial nerve, 660

Testes, 768

relation to metabolism, 499

Tetanometer, $5^{6} 5$

Tetanus, 524, 565

Thalami, 625

Thermogenic centers, 509

Third cranial nerve, $65 \mathrm{I}$

Thoracic viscera, vascular nerves for, 257

Thoracic autonomics, 669

Thoracograph, 327 
Thorax, respiratory changes in diameter, 289, 327

Thrombin, 121

Thrombocytes, 135

'Thrombogen, 122

Thrombokinase, I 22, 347

Thymine, 94

Thymus gland, 494

Thyroidectomy, 485

Thyroid feeding, 485

gland, 484 accessory, 484

functions of, 484

grafting, 485

hormone, 486

Thyroiodine, 486

Tidal air, 282, 328

Tissues, connective, 33

elementary, 23

epithelial, 23

interchange of gases in, 308

lymphoid, 36

muscular, 58

nervous, 65,67

Tone, of artery, 244 of muscle, 596

Tongue, 685

action of, in speech, $55^{6}$ papillæ of, 683

Tonicity of heart muscle, 198

Tonometer, 304

Tooth-pulp, 52

Touch corpuscles, $75,76,683$

sense of, 68I

acuteness of, 683

Trabeculæ carnex, I 78

Trachea, 280

Tract of Burdach, 587

of Goll, 587

of Gowers, 587

Traube-Hering curves, 249

Treppe, phenomenon of, $56 \mathrm{I}$

Tricuspid valve, action of, $\mathrm{I} 8 \mathrm{I}$

Trigeminal nerve, 653

Tri-olein, 97

Tri-palmitin, 97

Tri-stearin, 97

Trochlearis nerve, 653

Trunk, vascular nerves for, $25^{8}$

Trypsin, 379, 38I

action of, $38 \mathrm{I}$

Tryptophane, 82
Tubular glands, 337

Tubuli seminiferi, 768

uriniferi, 42 I

Twelfth cranial nerve, $66_{3}$

Tyco sphygmomanometer, 226

Tympanum, 70I

Tyrosine, 8I

Unorganized ferments, 346; and see Enzymes.

Unstriped muscle, $5^{8}$

Uracil, 94

Urea, amount in tissues, $43 \mathrm{I}$

antecedents of, 462

determination of, $45^{I}$

formation of, 431,442

preparation of, $45 \mathrm{I}$

properties of, 430

quantity excreted, 432

Ureters, 427

Uric acid, 94, 432, 45 I

condition of, in urine, 432

formation of, 463

properties of, 432

tests for, $45^{2}$

Urinary bladder, 427

Urine, 427

abnormal constituents of, $45^{2}$

albumin in, 435,453

ammonia in, 433

analysis of, 449

average daily quantity of constituents, 429

chlorides in, $435,45^{\circ}$

composition of, 428

creatinin in, 433,452

cystin in, 435

dextrose in, 435

discharge of, 440

diuretics, action of, 439

excretion by, 455,456

experiments on, 437

factors affecting secretion of, 436

general properties of, 427

hippuric acid in, 433,463

indican in, 452

mucus in, 434

nitrogenous substances in, 430,452

occasional constituents of, 435

oxalic acid in, 435

phosphates in, 434, 45 I

pigments in, $433,45^{2}$ 
Urine, quantity of, 429,449

rcaction of, 428,449

relation of blood pressure to secretion of, 448

saline matter in, 434

secretion of, theories of, 436

solids of, $45^{\circ}$

specific gravity of, 428,449

sugar in, 453

sulphates in, 434,450

urea in, 430, 43I, $45 \mathrm{I}$

uric acid in, 410,432

variations in quantity of constituents, 430

in specific gravity, 429

Uriniferous tubules, 422

Urobilin, 433, $45^{2}$

Urochrome, 433

Uroerythrin, 434

Uterine tubes, 775

Uterus, 776

Utriculus, 704, 7I 5

Vagina, 776

Vagus nerve, 660

distribution of, 660

effects of section, 662

functions of, 662

relation to deglutition, 660

to gastric secretion, $66_{2}$

to heart's action, 662

to respiration, 31 2, 332

Valine, 82

Valves of heart, 169 action of, $\mathrm{r} 8 \mathrm{r}$ of veins, $\mathrm{r} 78$

Van Slyke blood gas apparatus, 303

Vasoconstrictor activity, $25^{\circ}$ center, 245

nerves, 244, 246, 252, 259

reflexes, 247

Vasodilator activity, $25^{\circ}$

center, 250

nerves, 249, 252

reflexes, 250

Vasomotor centers, 247

changes, 275

nerves, 243

tone, 246

Veins, 177

blood pressure in, 227

structure of, $\mathrm{x} 78$
Veins, valves of, $\mathrm{x} 78$ vasoconstrictor nerves in, 259

Venous blood, analysis of, 302

$$
\begin{aligned}
& \text { flow, } 234 \\
& \text { velocity of, } 235
\end{aligned}
$$

Ventilation, $3 \mathrm{I} 7$

Ventricles of heart, action of, 180

Vesico-spinal center, 597

Vesiculæ seminales, $77 \mathrm{I}$

Vesicular breathing, 294

Vicq d' Azyr, bundle of, 625

Villi, 4r 4

Visceral sensations, 6or

Vision, accommodation of, 728, $73 \mathrm{I}$

binocular, $75 \mathrm{r}$

field of, 744

limits of, 760,765

localization of, 744

mechanism of accommodation, 73I

range of distinct, $73 \mathrm{I}$

Visual acuity, 767

center, 645

image, projection of, 732

judgments, 754

purple, 745

sensations, 739

after-images, $74 \mathrm{I}$

duration of, $74 \mathrm{I}$

- sense, 7I5

Vital capacity, 3 r 3,328

phenomena, I

Vitamine A, 48r

Vitamine $\mathrm{B}, 480$

Vitamine C, $48 \mathrm{I}$

Vitamines, 480

Vitiated air, effects of, 3 r 7

Vocal cords, 279, 545

movements of, $55^{\mathrm{I}}$

Vocalization, $55 \mathrm{I}$

Voice, 544

difference between male and female, 553

production of, 544

quality of, 554

in singing, and speaking, 552

vocal range of, $55 \mathrm{r}$

Vomiting, 376

center for, 376

nervous mechanism of, 376

Vowels, 555

Walking, 539, 54 I 
Wallerian degeneration, 575, 586

Water, I04

absorption of, in intestines, 4I9 amount excreted, $445,455,456$ in expired air, 300 of the body, I04

as food, 345

rigor, 526

Weights and measures, tables of, 796

White fibrous tissue, 34 chemical composition of, 90
White fibrous tissue, development of, 37

Xanthine, 94

Xantho-proteic reaction, I07

Yellow elastic tissue, 35 chemical composition of, $9 \mathrm{r}$ development of, 37

Zymogens, 378 




THE LIBRARY

UNIVERSITY OF CALIFORNIA

San Francisco Medical Center

THIS BOOK IS DUE ON THE LAST DATE STAMPED BELOW

Books not returned on time are subject to fines according to the Library Lending Code.

Books not in demand may be renewed if application is made before expiration of loan period.

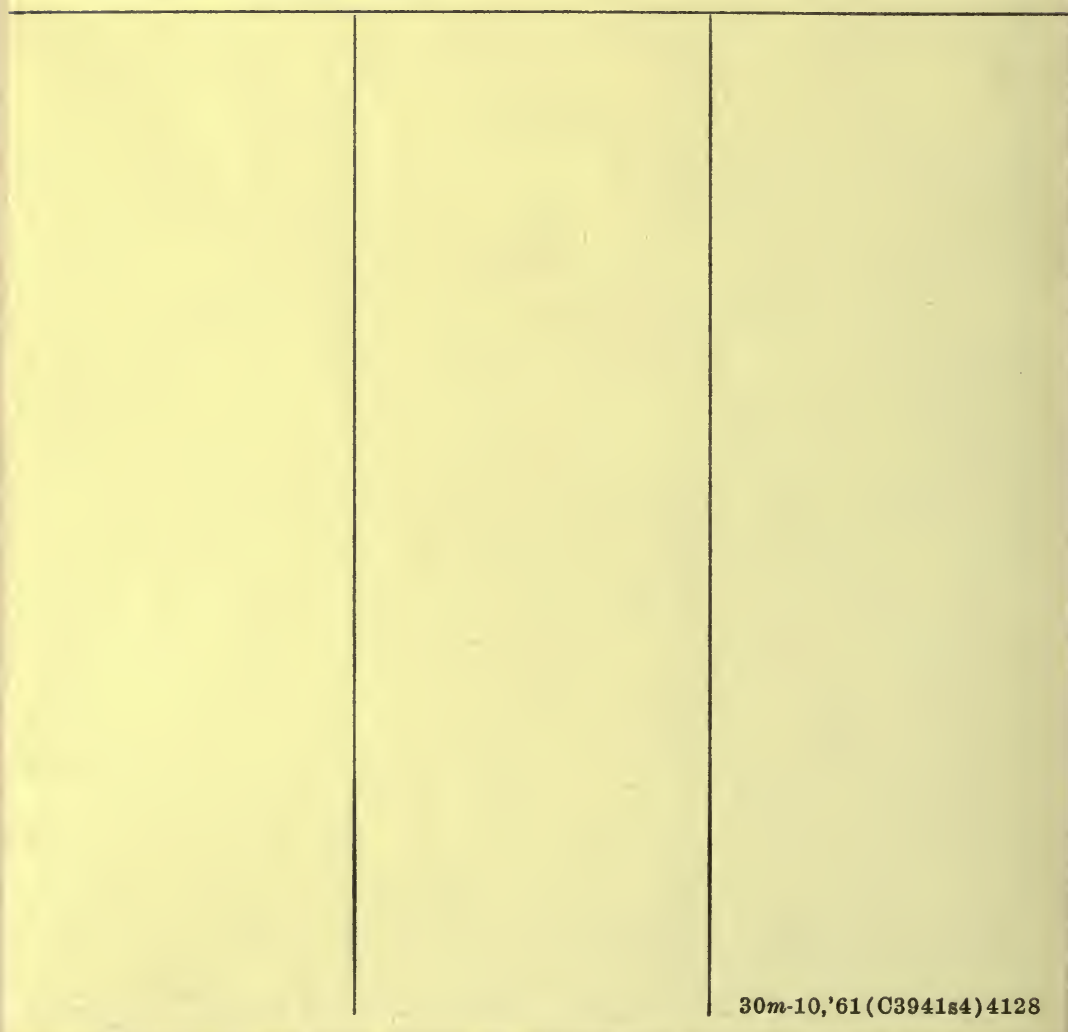


$4^{15}$

194801 
